\title{
Local Modification to Phononic Properties at Solid-Solid Interfaces: Effects on Thermal Transport
}

\author{
A Dissertation Presented to the
}

Faculty of the School of Engineering and Applied Science University of Virginia

In Partial Fulfillment

of the Requirements for the Degree of

Doctor of Philosophy

Mechanical and Aerospace Engineering

Justin L. Smoyer

May 2015 


\section{APPROVAL SHEET}

The dissertation is submitted in partial fulfillment of the requirements for the degree of

Doctor of Philosophy in Mechanical and Aerospace Engineering

Justin L. Smoyer

Justin L. Smoyer

This dissertation has been read and approved by the Examining Committee:

Pamela M. Norris

Pamela M. Norris, Advisor

Patrick E. Hopkins

Patrick E. Hopkins, Chairman

Jerry Floro

Jerry Floro

Gavin Garner

Gavin Garner

Leonid V. Zhigilei

Leonid V. Zhigilei

Accepted for the School of Engineering and Applied Science:

James H. Aylor

James H. Aylor, Dean

School of Engineering and Applied Science 


\begin{abstract}
The development of nano-devices relies on a combination of internal solid-solid interfaces between materials with different elemental and crystallographic structures to provide the functional operation of the device. Traditionally, these devices have been designed with the operational performance and efficiency in mind, with consideration of thermal energy management and heat dissipation only as a design afterthought. Therefore, the current methodology in dealing with thermal management issues begins with external device structures and scales upwards. However, as the feature size of most nano-devices continues to diminish, the impact of thermal transport across solid-solid interfaces on device performance, reliability, and lifetime becomes increasingly important.

Developed starting in the 1980 's, the transient thermoreflectance/time-domain thermoreflectance technique has been a primary tool in the measurement of nanoscale thermophysical properties and in particular thermal boundary conductance. Over the past several decades there have been a number of improvements made to refine and extend the applicability of the technique to nanoscale systems. In this work, the theory for modeling the measured results is extended from a sinusoidal to a pulsed waveform analysis which includes the effects of higher harmonics in the measured signal and allows for variations in the duty cycle of the modulation waveform. The inputs to the thermal model were critiqued and convergence criteria for the numerical analysis were established for high and low repetition rate laser systems to ensure accurate modeling. In addition, methods to quantify the sensitivity of input parameters in the thermal model were defined and the characterization of noise in the raw data and statistical outliers in the deduced parameter presented. To assist in the statistical interpretation of experimental results, a series of simulated data sets were analyzed to develop a set of empirical relations for the anticipated standard deviation in the collected results, based on the sensitivity of the model to the parameter being deduced and the amount of noise in the data.
\end{abstract}

Finally, an experimental study to test the theory of vibrational bridging through the use of an intermediate layer between two solids as a means to enhance thermal boundary 
conductance is presented and discussed. The thermal boundary conductance between $\mathrm{Pt} / \mathrm{Si}$ and $\mathrm{Pt} / \mathrm{Ge}$ is measured using the transient thermoreflectance technique and the results compared to measurements made with a Ni intermediate layer of varying thickness between the Pt and $\mathrm{Si}$ (or Ge). The measured results are compared to theoretical calculations of the contributions of interface bonding and electron transport across the metal-semiconductor interface. It is found that the contributions of electron transport are minimal, and that while it is assumed that bonding plays some role in the increased boundary conductance observed with the addition of the intermediate layer, it alone cannot explain the trend in the data. Overall, the results in the measured data support the hypothesis of vibrational bridging via a metallic intermediate layer and support the concept as a possible means to phononically engineer thermal conductance across solid-solid boundaries in nanodevices. 
This work is dedicated to my family.

Those that came before and those who are the future, for their everlasting support and inspiration. 


\section{Forward}

There is a saying that you should never go back and reread your dissertation after it is completed because there will always be mistakes to be found or things you would have presented differently. While this may be the case, this body of work is part of an evolving process that will continue with the aim of refining the methods in measuring and understanding nanoscale heat transfer. Therefore, any comments and/or suggestions from interested readers are always welcome. 


\section{Acknowledgements}

As the clock on my graduate career begins its final countdown after hundreds of pages, figures and plots, after countless hours spent with the laser, rebuilding it, maintaining it, eating with it, sleeping next to it, after countless trips the to the Corner and the Castle, and about 3,500 miles spent walking back and forth to the lab I find that this is the hardest part of the dissertation to write. Not because I lack for people to acknowledge, quite the opposite, I fear that I could never do justice to all the people who have supported and inspired me on this journey.

First and foremost, my sincerest thanks and everlasting gratitude to my advisor Dr. Pamela Norris for accepting me on this journey. Over the course of my graduate career I have learned that I am one of only two people that Dr. Norris has hired without interviewing in person first, and I can only hope that I have fulfilled her expectations. As an advisor Dr. Norris has gone above and beyond in every sense of the word and has continued to be a constant source of inspiration to me both personally and professionally. The opportunities that Dr. Norris have provided me have allowed me to grow professionally and as a person, have literally taken me around the world, have given me countless new experiences, and have most importantly given me an invaluable friendship.

I would like to thank Dr. Patrick Hopkins, Dr. Jerrold Floro, Dr. Gavin Garner, and Dr. Leonid V. Zhigilei for taking the time to serve on my doctoral committee. My interactions with each and every member of the committee have been invaluable and has only bolstered my desire to remain in academia and to continue to evolve as a teacher. I would like to acknowledge Louis Steva, Kevin Knight, and Claude Mitchell for helping to keep the lab up and running, facilitating the construction of the new lab space, giving me an opportunity to get my hands dirty from time to time, and for always supporting me and being good friends. In addition, I would like to thank Lisa Stanton for all her assistance and for being just plain awesome. Lisa is the kinda person you can depend on 110\%. If you need to know how to get something done, you ask Lisa, if you need it to be done no matter what, you ask Lisa, and while I am sure it causes her to be pestered by graduate students like myself who need things done, it is greatly appreciated.

Nobody has a complete understanding of what it takes to be a graduate student other 
than those who have undertaken the journey themselves. The kind of research that one does is only half as important as the comradery shared amount labmates whom you will work with, travel with, and spend inordinate amounts of time with, and in this aspect I have also been extremely fortunate. Any accomplishments that I have achieved as a graduate student could not have been completed without the support and guidance of past and present members of the Nanoscale Energy Transport Lab: Chris Baker, Matt Bauer, Casey Bauer, John Duda, Timothy English, Michael Fish, Christina Haden, Patrick Hopkins, LeighAnn Larkin, Nam Le, Christina Pappas, Quang Pham, MacKenzie Redding, Rich Salaway, Jennifer Simmons. In particular, I'd like to thank Christina Pappas for being my support system and my sounding board which has carried me through the ups and downs of research and lab life. Christina is the unsung hero of the lab, always going above and beyond, and always self-sacrificing. Likewise I'd like to thank MacKenzie Redding, not a better colleague to work with and to rebuild and run a laser system can be found. MacKenzie's knowledge, skill, patience, and attention to detail have been nothing short of exemplary, and along with his friendship have carried me through some tough times in the lab, and in life, and have made me ever grateful that our paths crossed at U.Va.

Last and in no way least I would like to thank my family. In no way could any of these accomplishments have been possible without their unconditional love and support. My dad Scott Smoyer, who has always been my inspiration, fostered my love of knowledge, and provided me with every ounce of work ethic that I have. I have had many professors to teach me science in my lifetime but the most important lessons I have learned have been riding the jump seat in Woolly. My mom Brenda Smoyer, who will deny it, but has always been my source of inner strength and who has given me my patience and attention to detail, two of the largest requirements for working with lasers. My brother Jeremy, who never let me forget that I was still in school, but who has also continued to support me, foster my love of science, and has provided me with nephews and nieces (Aidyn, Walker, Maiya) to make me smile even when things got tough. To Brittany and Madison whom I met along the way, I am forever grateful for the love and support you have provided, the encouragement you have shown me, and for making the future seem brighter than I ever thought possible. 


\section{CONTENTS}

Forward

Acknowledgements

List of Figures

xiv

List of Tables

$\mathrm{xl}$

Nomenclature

xliii

1 Introduction 1

1.1 Microprocessor Heating Trends . . . . . . . . . . . . . . . . 1

1.2 So What if It's a Little Hot? . . . . . . . . . . . . . . . . 6

1.3 How Big Can Such a Little Problem Be? . . . . . . . . . . . . . . . . 8

1.4 Levels of Thermal Management . . . . . . . . . . . . . . . . . . . 10

1.5 Chip/Module Level Thermal Management . . . . . . . . . . . . . . . . 11

1.6 Transistor-Level Thermal Management . . . . . . . . . . . . . . . . . . 17

1.7 Outline and Objectives . . . . . . . . . . . . . . 21

2 Thermal Transport at Nanoscale Solid-Solid Interfaces 24

2.1 Introduction . . . . . . . . . . . . . . . . . . 25 
2.2 Mechanisms of Thermal Boundary Conductance - Electrons and Phonons . 25

2.3 "Predicting" Thermal Boundary Conductance at Solid-Solid Interfaces . 28

2.3.1 Phonon Transport - Semi-classical Approach . . . . . . . . . . . 29

2.4 General Phonon Flux Across an Interface . . . . . . . . . . . . . . . . . 30

2.4 .1 Acoustic Mismatch Model . . . . . . . . . . . . . . . . . . 32

2.4.2 Breakdown of the AMM . . . . . . . . . . . . . . 33

2.4 .3 Diffuse Mismatch Model . . . . . . . . . . . . . . . . . . . . . 34

2.4 .4 Electron Transport - eDMM . . . . . . . . . . . . . . . 36

2.5 Failures of the DMM in Predicting Thermal Boundary Conductance . . . . 38

2.6 Interface Effects on Thermal Boundary Conductance at Solid-Solid Interfaces 42

2.6.1 Mismatch of Vibrational Properties . . . . . . . . . . . . 42

2.6.2 Inelastic Scattering Contributions _ . . . . . . . . . . . . . . 45

2.6 .3 Bonding at the Interface . . . . . . . . . . . . 46

2.6.4 Strain, Defects and Dislocations at the Interface . . . . . . . . 48

2.6.5 Roughness at the Interface . . . . . . . . . . . . . 51

2.6.6 Compositional Inter-diffusion at the Interface . . . . . . . . . . 54

2.7 Chapter Summary . . . . . . . . . . . . . . . . 56

$\begin{array}{lll}3 & \text { The Experimental System } & 57\end{array}$

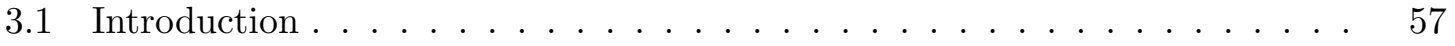

3.2 Optical Methods for Thermal Measurement . . . . . . . . . . . . 59

3.2 .1 Photothermal Techniques . . . . . . . . . . . . . 60

3.2.2 Femtosecond Thermoreflectance Techniques . . . . . . . . . . . 61

3.2.3 Energy Transport/Conversion Following Pulsed Laser Heating . . 63

3.3 The General System and Components . . . . . . . . . . . . . . . 69

3.4 Summary . . . . . . . . . . . . . . . . . . . . . 79

4 Measurement Theory and Data Analysis $\quad 80$

4.1 Introduction . . . . . . . . . . . . . . . . 80

4.2 Pulsed Laser as a Linear Time-Invariant (LTI) System . . . . . . . . . . . . 81

4.2.1 Assumptions of Linearity and Time Invariance . . . . . . . . . . . 82 
4.2.2 Frequency Domain Response - TTR/TDTR . . . . . . . . . . . . . 82

4.2 .3 Lock-in Amplifier Signal . . . . . . . . . . . . . . . . . . . . . . 92

4.2.4 Comparison of Sine and Square Waveforms . . . . . . . . . . . . 94

4.3 Heat Transfer Analysis . . . . . . . . . . . . . . . . . . . . . . . 96

4.3.1 Previous Modeling - Crank-Nicolson Analysis . . . . . . . . . . . . . 96

4.3.2 Multilayer Analysis via Feldman - Temperature Profile . . . . . . . . 101

4.3.3 Surface Temperature Response in the Frequency Domain - H $(\omega)$. . 109

4.3.4 Criteria for 1D vs 3D Modeling . . . . . . . . . . . . . . . 113

4.4 Chapter Summary . . . . . . . . . . . . . . . . . . 115

5 Practical Considerations in Data Deduction 118

5.1 Introduction . . . . . . . . . . . . . . . . . . . . . . 119

5.2 Proper Modulation to Eliminate Phase Drift _ . . . . . . . . . . . . 119

5.3 Convergence Criteria in Modeling the Lock-in Amplifier Response During TTR/TDTR Experiments . . . . . . . . . . . . . . . 126

5.3.1 Impacts of Non-convergence in $M \ldots \ldots \ldots$. . . . . . . 126

5.3.2 Impact of Series Convergence - Sinusoidal Modulation Waveform . . 129

5.3.3 Impact of Series Convergence - Square Modulation Waveform . . . . 137

5.3.4 Impact of Series Convergence - Pulsed Modulation Waveform . . . . 142

5.3.5 Summary of Convergence Criteria . . . . . . . . . . . . . . . 147

5.4 Fitting to Deduce Thermophysical Parameters . . . . . . . . . . . . 147

5.4.1 Gibbs Oscillations at $t_{o}-$ M-Fourier Components . . . . . . . . 148

5.4 .2 Normalization of Data . . . . . . . . . . . . . . . . 155

5.5 Sensitivity Analysis . . . . . . . . . . . . . . . . . 157

5.5.1 Sensitivity Coefficient _. . . . . . . . . . . . . . . 160

5.5.2 Statistical Sensitivity Analysis . . . . . . . . . . . . . . . 164

5.6 Defining Noise in Data . . . . . . . . . . . . . . . 166

5.6.1 Measuring Signal Noise via the Lock-in Amplifier . . . . . . . . . . . 167

5.6.2 Defining a Signal-to-Noise Parameter . . . . . . . . . . . . . . . 168

5.7 Post-processing of Data to Remove Outliers . . . . . . . . . . . . . . 171 
5.7 .1 Removing Statistical Outliers . . . . . . . . . . . . . . 172

5.8 Chapter Summary . . . . . . . . . . . . . . . . . . . 174

6 Robust Data Collection and Management $\quad 176$

6.1 Introduction . . . . . . . . . . . . . . . . . . 177

6.2 Confidence in Results - Accuracy vs. Precision . . . . . . . . . . . . . . 180

6.3 Statistically Reporting Results . . . . . . . . . . . . . . . 183

6.4 Assumptions of the Sampling Distribution . . . . . . . . . . . . . 187

6.5 Quantification of Confidence in Prediction of the Population Mean . . . . . 190

6.5.1 Choice of Sample Size for Normally Distributed Data with Known Variance . . . . . . . . . . . . . . . . . . 192

6.6 Predicting Population Statistics of Thermoreflectance Data Through Large Scale Simulations . . . . . . . . . . . . . . . . . . . . . 194

6.6.1 Modeling a Range of Fitting Sensitivities . . . . . . . . . . . 196

6.6 .2 Simulating Noise in the Data . . . . . . . . . . . . . . . 197

6.6.3 Fitting Simulated Data and Results . . . . . . . . . . . . . 201

6.6.4 Standard Deviation Simulation Results . . . . . . . . . . . 205

6.7 Statistical Analysis via Bootstrapping . . . . . . . . . . . . . . . . . 212

6.7.1 Notation and Principles of Bootstrapping . . . . . . . . . . 213

6.7.2 Bootstrapping Diagnostics of the Sampling Distribution . . . . . . . 215

6.7.3 Bootstrapping Standard Error and Confidence Intervals . . . . . . . 217

6.7.4 Two-sample Bootstrap Comparison . . . . . . . . . . . . . . . . . . . 219

6.7.5 Effect of Sample Size on Bootstrapping . . . . . . . . . . . . 220

6.7.6 How Many Bootstrap Resamplings? . . . . . . . . . . . . . . . . 223

6.7.7 Summary: Why bootstrap? . . . . . . . . . . . . . . 224

6.8 Accuracy of Results . . . . . . . . . . . . . . . . 225

6.9 Statistical Example $\mathrm{Pt} / \mathrm{Si} \ldots \ldots \ldots \ldots \ldots \ldots . \ldots \ldots$

6.9.1 Preliminary Sensitivity Analysis _ . . . . . . . . . . . . . . . 229

$6.9 .2 \quad$ Estimated Precision Analysis . . . . . . . . . . . . . . 231

6.9 .3 Estimated Accuracy Analysis . . . . . . . . . . . . . . . . 232 
6.9.4 Experimental and Simulated Results . . . . . . . . . . . . . 233

6.10 Chapter Summary . . . . . . . . . . . . . . . . . . 236

7 Vibrationally Mediating Materials $\quad 237$

7.1 Introduction . . . . . . . . . . . . . . . . . . . . 238

7.2 The Concept of Vibrational Bridging . . . . . . . . . . . . . . . . 239

7.3 Simulating Phonon Transport Across Intermediate Layers by Molecular Dynamics Simulations . . . . . . . . . . . . . . . . . . 240

7.4 Thermal Bridging via Metallic Intermediate Layers . . . . . . . . . . . . 246

7.4.1 Metallic vs. Semiconductor Intermediate Layers . . . . . . . . . . . 246

7.4.2 Sample Preparation . . . . . . . . . . . . . . . . . 248

7.5 Experimental Procedure and Results . . . . . . . . . . . . . . . . 250

7.5.1 Resistor Network Analysis . . . . . . . . . . . . . . . . . 251

7.5.2 Sensitivity Analysis . . . . . . . . . . . . . . . . . 253

7.5 .3 Raw Data . . . . . . . . . . . . . . . . . . . 254

7.5 .4 Accuracy Analysis . . . . . . . . . . . . . . . . . 257

7.5.5 Final Results . . . . . . . . . . . . . . . . . 258

7.6 Results and Interpretation . . . . . . . . . . . . . . . . . 259

7.6 .1 Bonding Analysis . . . . . . . . . . . . . . . . . 259

7.6.2 Electron Boundary Conductance . . . . . . . . . . . . . . 261

7.7 Chapter Summary . . . . . . . . . . . . . . . . 271

8 Summary, Conclusions, Outlook, and Suggestions 272

8.1 Summary and Conclusions . . . . . . . . . . . . . . . . . 272

8.2 Outlook and Suggested Future Work . . . . . . . . . . . . . . 275

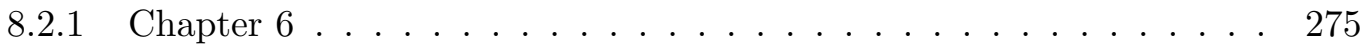

8.2 .2 Chapter $7 \ldots \ldots \ldots \ldots \ldots \ldots \ldots$

$\begin{array}{lr}\text { A Fundamentals of Solid State Physics } & 279\end{array}$

A.1 Introduction . . . . . . . . . . . . . . . . . . . 279

A.2 Crystallography . . . . . . . . . . . . . . . . . . 280 
A.2.1 Crystals in Real Space . . . . . . . . . . . . . . . . . 281

A.2.2 Crystals in Reciprocal Space . . . . . . . . . . . . . . . . . 284

A.3 Phonons . . . . . . . . . . . . . . . . . . . . . . . . 289

A.3.1 Phonon Polarizations _. . . . . . . . . . . . . . . . 290

A.4 Phonon Dispersion . . . . . . . . . . . . . . . . . . . . . . . . . . . 291

A.4.1 Vibrations of a 1D Lattice with a Monatomic Basis . . . . . . . . . . 291

A.4.2 Phonon Behavior in the Brillouin Zone . . . . . . . . . . . . 294

A.4.3 Vibrations of a 1D Lattice with a Diatomic Basis . . . . . . . . . 298

A.4.4 Phonon Behavior in the Brillouin Zone . . . . . . . . . . . . 300

A.4.5 Generalized Discussion 3D Crystal with Z Atoms in the Basis . . . . 302

A.5 Phonon Density of States _ . . . . . . . . . . . . . . . . 304

A.5.1 Debye Assumptions . . . . . . . . . . . . . . . . 307

A.6 Phonon Scattering . . . . . . . . . . . . . . . . . . . . . 309

A.6.1 Transport Processes - Boltzmann Transport Equation . . . . . . . . 310

A.6.2 Heat Flux in 1-Dimension - Thermal Conductivity . . . . . . . . . . 312

A.6.3 Phonon Scattering Events . . . . . . . . . . . . . . . . . . 314

A.7 Appendix Summary . . . . . . . . . . . . . . . . 317

B Laser System Basics $\quad 318$

B.1 Reflectivity vs Reflectance . . . . . . . . . . . . . . . . . 318

C Signal Analysis and Interpretation $\quad 322$

C.1 Square Wave of Arbitrary Duty Cycle . . . . . . . . . . . . . . . . . 322

C.2 Frequency Domain Signal Analysis - Reduction of Pulsed Modulation to Sinusoidal Modulation . . . . . . . . . . . . . . . . 326

C.3 Frequency Domain Signal Analysis - Sinusoidal Modulation . . . . . . . . . 328

C.3.1 Lock-in Amplifier Signal . . . . . . . . . . . . . . . . . . . . 330

C.4 Feldman Analysis - Layer Temperature . . . . . . . . . . . . . . . . . . . 332

C.5 Feldman Analysis - Probe Through Glass . . . . . . . . . . . . . . . 333 
D Fundamentals of Statistical Inference $\quad 336$

D.1 Testing for Normality . . . . . . . . . . . . . . . 336

D.1.1 Graphical Methods for Testing Normality . . . . . . . . . . . . 337

D.1.2 Numerical Methods for Testing Normality . . . . . . . . . . . . . . . 337

D.1.3 Hypothesis Testing for Normality . . . . . . . . . . . . . . . . 339

E Modeling Analysis of Pt/Ni/Si(Ge) System 342

E.1 Averaged Sensitivity Parameter . . . . . . . . . . . . . . . . . . . 342

E.2 Sensitivity Parameter as a Function of Time . . . . . . . . . . . . . . 343

$\begin{array}{ll}\text { Bibliography } & 349\end{array}$

S Modeling and Fitting of Data $\quad 372$

S.1 Simulation Parameters . . . . . . . . . . . . . . . . . . 375

S.2 Simulation Parameter Sensitivity Coefficient $-h_{\mathrm{BD}} \ldots \ldots \ldots 76$

S.3 Simulation Parameter Sensitivity Coefficient - Time . . . . . . . . . 378

S.4 Simulation Summary - Al/Si 1.00 MW Series . . . . . . . . . . . . . . 399

S.5 Simulation Summary - Al/Si 1.29 MW Series . . . . . . . . . . . . . 400

S.6 Simulation Summary - Al/Si 1.67 MW Series . . . . . . . . . . . . . . 401

S.7 Simulation Summary - Al/Si 2.15 MW Series . . . . . . . . . . . . . . 402

S.8 Simulation Summary - Al/Si 2.78 MW Series . . . . . . . . . . . . . . 404

S.9 Simulation Summary - Al/Si 3.59 MW Series . . . . . . . . . . . . . 405

S.10 Simulation Summary - Al/Si 4.64 MW Series . . . . . . . . . . . . 406

S.11 Simulation Summary - Al/Si 5.99 MW Series . . . . . . . . . . . . 408

S.12 Simulation Summary - Al/Si 7.74 MW Series . . . . . . . . . . . . . 409

S.13 Simulation Summary - Al/Si 10.00 MW Series . . . . . . . . . . . . . 410

S.14 Simulation Summary - Al/Si 12.90 MW Series . . . . . . . . . . . . . . 412

S.15 Simulation Summary - Al/Si 16.70 MW Series _. . . . . . . . . . . . 413

S.16 Simulation Summary - Al/Si 21.50 MW Series . . . . . . . . . . . . . . 414

S.17 Simulation Summary - Al/Si 27.80 MW Series . . . . . . . . . . . . . 417

S.18 Simulation Summary - Al/Si 35.90 MW Series . . . . . . . . . . . . 419 
S.19 Simulation Summary - Al/Si 46.40 MW Series _ . . . . . . . . . . . . 421

S.20 Simulation Summary - Al/Si 59.90 MW Series . . . . . . . . . . . . . 424

S.21 Simulation Summary - Al/Si 77.40 MW Series _ . . . . . . . . . . . 426

S.22 Simulation Summary - Al/Si 100.00 MW Series . . . . . . . . . . . . . . 428

S.23 Simulation Summary - Al/Si 129.00 MW Series . . . . . . . . . . . . . . 431

S.24 Simulation Summary - Al/Si 167.00 MW Series . . . . . . . . . . . . . 433

S.25 Simulation Summary - Al/Si 215.00 MW Series . . . . . . . . . . . . 435

S.26 Simulation Summary - Al/Si 278.00 MW Series . . . . . . . . . . . . . 438

S.27 Simulation Summary - Al/Si 359.00 MW Series . . . . . . . . . . . . . . 440

S.28 Simulation Summary - Al/Si 464.00 MW Series . . . . . . . . . . . . . . . . 442

S.29 Simulation Summary - Al/Si 599.00 MW Series . . . . . . . . . . . . . 445

S.30 Simulation Summary - Al/Si 774.00 MW Series . . . . . . . . . . . . . . 447

S.31 Simulation Summary - Al/Si 1000.00 MW Series . . . . . . . . . . . . . 449

S.32 Simulation Summary - Al/AlN 1.00 MW Series . . . . . . . . . . . . . . 452

S.33 Simulation Summary - Al/AlN 1.29 MW Series . . . . . . . . . . . . . 453

S.34 Simulation Summary - Al/AlN 2.78 MW Series . . . . . . . . . . . . . . 454

S.35 Simulation Summary - Al/AlN 5.99 MW Series . . . . . . . . . . . . 455

S.36 Simulation Summary - Al/AlN 12.90 MW Series . . . . . . . . . . . . 456

S.37 Simulation Summary - Al/AlN 27.80 MW Series . . . . . . . . . . . . . 457

S.38 Simulation Summary - Al/AlN 59.90 MW Series . . . . . . . . . . . . 458

S.39 Simulation Summary - Al/AlN 100.00 MW Series . . . . . . . . . . . . . 460

S.40 Simulation Summary - Al/AlN 129.00 MW Series . . . . . . . . . . . . . 461

S.41 Simulation Summary - Al/AlN 167.00 MW Series . . . . . . . . . . . . . 463

S.42 Simulation Summary - Al/AlN 215.00 MW Series . . . . . . . . . . . . . 464

S.43 Simulation Summary - Al/AlN 278.00 MW Series . . . . . . . . . . . . 465

S.44 Simulation Summary - Al/AlN 359.00 MW Series . . . . . . . . . . . . . 467

S.45 Simulation Summary - Al/AlN 464.00 MW Series . . . . . . . . . . . . 468

S.46 Simulation Summary - Al/AlN 599.00 MW Series . . . . . . . . . . . . . 469

S.47 Simulation Summary - Al/AlN 774.00 MW Series . . . . . . . . . . . . . 471

S.48 Simulation Summary - Al/AlN 1000.00 MW Series . . . . . . . . . . . . . 472 
S.49 Simulation Summary - Al/Ge 1.00 MW Series . . . . . . . . . . . . . . 474

S.50 Simulation Summary - Al/Ge 2.15 MW Series . . . . . . . . . . . . . 475

S.51 Simulation Summary - Al/Ge 3.59 MW Series . . . . . . . . . . . . . 476

S.52 Simulation Summary - Al/Ge 7.74 MW Series . . . . . . . . . . . . . 477

S.53 Simulation Summary - Al/Ge 16.70 MW Series . . . . . . . . . . . . 478

S.54 Simulation Summary - Al/Ge 46.40 MW Series . . . . . . . . . . . . . 480

S.55 Simulation Summary - Al/Ge 100.00 MW Series _ . . . . . . . . . . . . 482

S.56 Simulation Summary - Al/Ge 129.00 MW Series . . . . . . . . . . . . . 484

S.57 Simulation Summary - Al/Ge 167.00 MW Series . . . . . . . . . . . . 485

S.58 Simulation Summary - Al/Ge 215.00 MW Series . . . . . . . . . . . . 486

S.59 Simulation Summary - Al/Ge 359.00 MW Series . . . . . . . . . . . . 488

S.60 Simulation Summary - Al/Ge 464.00 MW Series . . . . . . . . . . . . . 489

S.61 Simulation Summary - Al/Ge 599.00 MW Series . . . . . . . . . . . . . 491

S.62 Simulation Summary - Al/Ge 774.00 MW Series . . . . . . . . . . . . . 492

S.63 Simulation Summary - Al/Ge 1000.00 MW Series . . . . . . . . . . . . . 493 


\section{LIST OF FIGURES}

1.1 Heat flux trends of high performance computers since the 1950s for bipolar transistor and complimentary metal-oxide-semiconductor (CMOS) technologies. Plot taken from $[1] \ldots \ldots \ldots \ldots \ldots$

1.2 Number of transistors per die for various commercial processors developed over the past 4 decades. Plot taken from $[2] \ldots \ldots \ldots \ldots \ldots$

1.3 Power densities for various commercial processors developed over the past 4 decades. Plot taken from $[2] . \ldots \ldots \ldots \ldots$

1.4 Thermal mapping of a dual-core AMD Athlon II processor under various CPU workloads. The color scale represents the temperature in degrees Celsius. Image taken from $[3] \ldots \ldots \ldots \ldots \ldots \ldots$

1.5 The 4 primary packaging levels, that are of interest in thermal management (a) the transistor-level, (b) the chip/module-level, (c) the board-level (image from $[4]$ ), and $(\mathrm{d})$ the-system level (image from $[5]) . \ldots \ldots$

1.6 Methods of thermal management solutions, for various power dissipation levels and system complexities. Image reproduced with permission from [6]. . .

1.7 Cross-section view of a typical module found in most consumer grade desktop machines, denoting the primary components and the internal and external

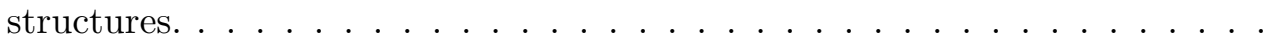


1.8 Temperature regions and primary thermal resistances in typical microproces-

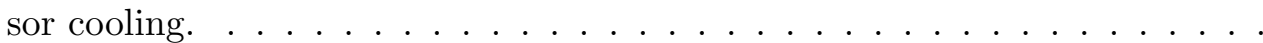

1.9 (a) Schematic of mechanical contact between two materials, showing the surface asperities and reduced contact between the surfaces, as well as an example of an ideal TIM. (b) Schematic of the temperature distribution of two materials in contact with the addition of a TIM. . . . . . . . . .

1.10 Schematic of a microscope contact area of a TIM between two materials, showing areas of wetting and non-wetting contact. . . . . . . .

1.11 Schematic illustrating the physical differences between (a) thermal contact resistance, and (b) thermal boundary resistance. . . . . . . . . . . .

1.12 Typical physical structure of a SOI-MOSFET with an overlaid thermal resistor network showing the primary interfacial thermal resistances. Where $R_{C-S}$ is the contact-to-source resistance, $R_{S-O}$ is the source-to-oxide resistance, $R_{C-D}$ is the contact-to-drain resistance, etc. [7]. . . . . . . .

2.1 Schematic of the various thermal boundary conductance channels for electron and phonon transport at (a)-(c) metal-semiconductor (e) metal-metal and (f) semiconductor-semiconductor interfaces. Electrons and phonons are the dominate energy carries at (e) metal-metal and (f) semiconductor-semiconductor interfaces respectively. At metal-semiconductor interfaces the total conductance is the sum of (a) phonon-phonon (b) electron-electron and (c) mixed electron-phonon and phonon-electron transport. . . . . . . . . . . . . . . 26

2.2 Specularity parameter, calculated from Equation 2.8, as a function of temperature for a variety of phonon group velocities, $v_{g}$, assuming a asperity parameter of $\delta=1 \mathrm{~nm} . \ldots \ldots \ldots \ldots$

2.3 Specularity parameter, calculated from Equation 2.8, as a function of asperity parameter, $\delta$, for a phonon velocity of $v_{g}=10,000 \mathrm{~m} / \mathrm{s}$, at several temperatures. 34 
2.4 Ratio of experimentally measured $h_{\mathrm{BD}}$ to that predicted by the DMM versus the ratio of Debye temperatures of the film and substrate. The data plotted is taken from Stoner \& Marris, [8] Lyeo \& Cahill, [9] Stevens et al., [10] and Hopkins et al. $[11,12] \ldots \ldots \ldots \ldots \ldots$

2.5 The phonon dispersion relation for solid Argon in the directions of high symmetry at $0 \mathrm{~K}$, modeled with the lattice dynamics program, General Utility Lattice Program (GULP) $[13] . \ldots \ldots \ldots \ldots$

2.6 A solid-solid system with (a) a finite temperature drop at the interface as described by the DMM and (b) a step function dependence on material properties, e.g. atomic mass, etc. due to the infinitesimal physical extent attributed to the interface. . . . . . . . . . . . . . .

2.7 (a) Bulk occupied phonon density of states for material A (40 amu) and material B (120 amu), with the shaded region representing the overlap in the bulk density of states. (b) Occupied phonon density of states in the z-direction calculated from the monolayers on either side of the interface between material A and B. Data and figures reproduced with permission from English et al. [14]. . . . . . . . . . . . . . . . .

2.8 (a) Anharmonic inelastic model predictions of $h_{\mathrm{BD}}$ for $\mathrm{Pb}$ on diamond as a function of temperature and $n$-phonon processes for $n=2-17$. (b) Comparison of $h_{\mathrm{BD}}$ predicted from the DMM and the AIM along with experimental data for $\mathrm{Pb} / \mathrm{H} /$ diamond, $\mathrm{Pb} /$ diamond, and $\mathrm{Au} /$ diamond. Figures and data from Hopkins et al. [15]. . . . . . . . . . . . . . . . .

2.9 (a) Crystal structure of the $\mathrm{SiO}_{2} / \mathrm{Si}$ system, with the $\mathrm{Si}$ denoted in grey and the $\mathrm{O}$ atoms in red. (b) $h_{\mathrm{BD}}$ at the $\mathrm{SiO}_{2} / \mathrm{Si}$ interface as a function of the length of the interface junction for both weak and strong interfacial coupling. Figures and data reproduced with permission from Chen et al. [16]. . . . .

2.10 (a) Relaxed Si/Ge interface formed from an $8 \%$ lattice mismatch. (b) Phonon transmission as a function of frequency for different lattice mismatches. Figures and data reproduced with permission from Li and Yang [17]. . . . . . 
2.11 Phonon transmission from Si-to-Ge (a) and thermal conductance from Sito-Ge (b) based on Green's function calculations using DFT force constants from Tian et al. as a function of the thickness of the roughened interface layer. Figures reproduced with permission from [18] . . . . . . . . . . . 53

2.12 (a) Alloyed Si/Ge layer created via melting and quenching of the interface region with an $8 \%$ lattice mismatch. (b) Phonon transmission as a function of frequency for different thicknesses of the alloyed region. Figures and data reproduced with permission from Li and Yang $[17] \ldots \ldots \ldots$

3.1 Basic schematic of the primary components of the pump-probe thermoreflectance experimental system. . . . . . . . . . . . . . .

3.2 Schematic defining and comparing the relaxation and collision length of energy carriers. For a collection of carriers, e.g. electrons, phonons, photons, the mean free path is the average distance between scattering events. The relaxation length is the length required to reach thermal equilibrium. Note: Perspective is for illustration only. In reality mean free paths can be orders of magnitude longer than the average carrier separation. . . . . . . . . .

3.3 Schematic depicting the 4 primary time frames of ultrafast laser interaction with metals, 1) the deposition of laser radiation energy into the electron subsystem of the metal, 2) the thermalization of the electron sub-system from a non-equilibrium to an equilibrium state, 3) the exchange of energy between the electron and phonon sub-systems, and 4) the diffusive propagation of thermal energy through the system. . . . . . . . . . . .

3.4 Schematic describing the distribution of electrons and the interaction of the laser and material system (a)-(b) after pulse deposition but before electronelectron thermalization and (c)-(d) after the thermalization of the electron

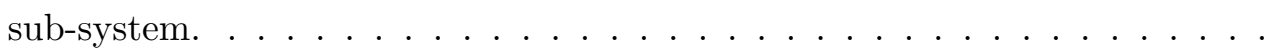

3.5 A schematic of the primary components in the TTR/TDTR system in the University of Virginia Nanoscale Energy Transport lab. . . . . . . . . . . . 70 
3.6 Schematic of the optical isolator used to prevent optical feedback into the MIRA or RegA cavity. . . . . . . . . . . . . . . . .

3.7 Schematic of the polarizing beam splitter system. The incoming p-polarized light from the laser is rotated off access by the $\lambda / 2$-waveplate, and the vector components are then separated by the PBC to scatter the s-polarized light and pass the p-polarized light. . . . . . . . . . . . . .

3.8 Schematic of the electro-optical modulator (EOM) driven by a square wave for (a) low logic and (b) high logic. . . . . . . . . . . . . . . . 75

3.9 Gaussian beam diameter as a function of the direction of propagation, z. . . 76

3.10 Percentage change in the pump beam radius as a function of stage delay, based on Equation 3.7, for various expansion powers. . . . . . . . . . . .

3.11 Schematic of the optical delay system used to create temporal resolution between the pump and probe beams. . . . . . . . . . . . . . .

3.12 Schematic of the filtering methods to block scattered pump light from reaching the photodetector (a) using the color filtering scheme and (b) using the spatial filtering scheme.

4.1 This figure illustrates the basic process flow in pump-probe spectroscopy. Input from the modulated pump source is converted to the output response of the lock-in amplifier via a complex transfer function $Z(\omega)$, containing information on the spectroscopy system and the sample response. . . . . .

4.2 Impulse-train modeled from Equation 4.1 for (a) the MIRA and (b) RegA, for $Q=1 \mathrm{~J}, T=13.2 \mathrm{~ns}$ and $4 \mu \mathrm{s}$ for the MIRA and RegA respectively. . .

4.3 Generalization of the pulsed waveform used in the LTI modeling of the pumpprobe spectroscopy experiment, with the functional form in the time domain given by Equations 4.11a-4.11c. . . . . . . . . . . . . . .

4.4 Modulated pump impulse-train, from the MIRA, given by Equation 4.14b for $Q_{\mathrm{pm}}=1 \mathrm{~J}, D=0.05, T_{s}=13.2 \mathrm{~ns}, \omega_{o}=4.22 \mathrm{MHz}$, and $\tau_{d}=0 \ldots \ldots$

4.5 Maximum film thickness as a function of film thermal conductivity and $h_{\mathrm{BD}}$ for the assumptions of lumped capacitance to be valid. . . . . . . . . . . . 100 
4.6 Geometry and indexing of the multilayered structure utilized in Feldman analysis. . . . . . . . . . . . . . . . . . . 102

4.7 Example of a film on finite substrate system with indexing for Feldman analysis. .......................... 106

4.8 Example of a film on a semi-infinite substrate system with indexing for Feldman analysis. . . . . . . . . . . . . . . . . . 108

4.9 Radial spreading of laser energy in a confined film given by Equations 4.88 and 4.89 for $D=1 \times 10^{-3}$ and an initial pump waist of $10 \mu \mathrm{m}$. Pane (a) shows the Gaussian distribution of heat at $t=0$ and $t=8 \mathrm{~ns}$, with the dashed lines representing the $1 / e$ radius and (b) the distribution of temperature as a function of time and radial distance with the color scale representing the normalized temperature. The black marks providing the $1 / e$ location as a

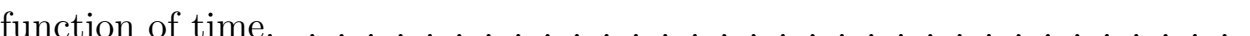

4.10 Percentage of $1 / e$ heat spreading as a function of the initial pump waist for several thermal diffusivities, covering a range from on the order of quartz to

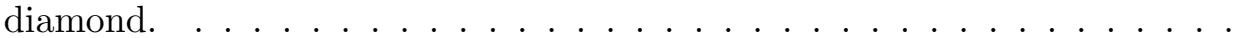

5.1 Simulated laser impulse-train, $f_{s}=250 \mathrm{kHz}$, modulated by a square waveform at $f_{o}=125 \mathrm{kHz} . \ldots \ldots \ldots \ldots \ldots$

5.2 Simulated TDTR data of $100 \mathrm{~nm}$ of $\mathrm{Al}$ on $\mathrm{Si}\left(h_{\mathrm{BD}}=215 \mathrm{MW} / \mathrm{m}^{2} \mathrm{~K}\right)$, normalized at $50 \mathrm{ps}$, for modulation rates of $f_{o 1}=38 \mathrm{MHz}(\mathrm{red})$ and $f_{o 2}=3 . \overline{45}$ $\mathrm{MHz}$ (blue), with $f_{s}=76 \mathrm{MHz}, D=0.5, M=3,500$, and $k=10,000$. . .

5.3 Generalization of the pulsed waveform, where $T_{o}$ is the modulation period, $D$ is the duty cycle, and $d$ is the width of the "on" portion of the modulation

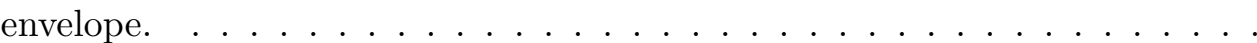

5.4 Simulated laser impulse-train, $f_{s}=76 \mathrm{MHz}$, modulated by a square waveform at (a) $f_{o}=38 \mathrm{MHz}, 1$ pulse per envelope, and (b) $f_{o}=3 . \overline{45} \mathrm{MHz}, 11$ pulses

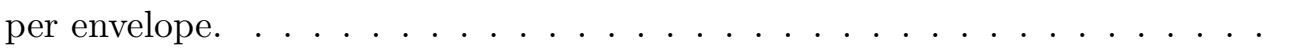


5.5 Simulated TDTR data of $100 \mathrm{~nm}$ of $\mathrm{Al}$ on $\mathrm{Si}\left(h_{\mathrm{BD}}=215 \mathrm{MW} / \mathrm{m}^{2} \mathrm{~K}\right)$, normalized at $50 \mathrm{ps}$, for modulation rates of $f_{o 1}=38 \mathrm{MHz}$ (red) and $f_{o 2}=37$ $\mathrm{MHz}$ (blue), with $f_{s}=76 \mathrm{MHz}, D=0.5, M=3,500$, and $k=10,000$. . .

5.6 Simulated laser impulse-train, $f_{s}=76 \mathrm{MHz}$, modulated by a square waveform at $f_{o}=37 \mathrm{MHz} \ldots \ldots \ldots \ldots \ldots \ldots$

5.7 Comparison of the lock-in amplifier output for a TTR signal, with the pump laser modulated via a traditional function generator (blue) and a phase-locked circuit (red), showing (a) the signal magnitude and (b) the signal phase. The error bars shown are \pm one standard deviation from 300 measurements. The signal to noise ratio of the magnitude with the phase-locked circuit is nearly four times greater than when using the traditional function generator. . . .

5.8 The number of laser pulses per "on" portion of the modulation envelope as a function of modulation frequency, $f_{o}$, assuming a $50 \%$ duty cycle and a laser repetition frequency of $f_{s}=76 \mathrm{MHz} \ldots \ldots \ldots \ldots$

5.9 Simulated data for a $100 \mathrm{~nm} \mathrm{Al} / \mathrm{Si}$ system $\left(h_{\mathrm{BD}}=200 \mathrm{MW} / \mathrm{m}^{2} \mathrm{~K}\right)$ for $k=$ $\{-1,1\}, f_{s}=250 \mathrm{kHz}, f_{o}=125 \mathrm{kHz}$, a $50 \%$ duty cycle of the modulation waveform, and $M=50,000,200,000$, and $2,000,000 \ldots \ldots \ldots$

5.10 Schematic of modeled data highlighting the oscillations in the modeled data for a low summation in $M$, and defining the time period between peaks, $T_{\text {peak }} .128$ $5.11 T_{\text {peak }}$ of the Gibbs oscillations in modeled TTR/TDTR data as a function of thermophysical property perturbation for a modeled system of $100 \mathrm{~nm}$ of $\mathrm{Al} / \mathrm{Si}\left(h_{\mathrm{BD}}=215 \mathrm{MW} / \mathrm{m}^{2} \mathrm{~K}\right)$ with $M=50,000$. Each assumed thermophysical value was perturbed one-at-a-time, with the error bars representing plus/minus one standard deviation of the data collected over the first 30 peaks. 128

5.12 Real signal $X$ at 100 ps as a function of $M$ summation for a modeled system of $100 \mathrm{~nm} \mathrm{Al} /$ Glass $\left(h_{\mathrm{BD}}=30 \mathrm{MW} / \mathrm{m}^{2} \mathrm{~K}\right)$, with $k=\{-1,1\}, f_{s}=250 \mathrm{kHz}$, $f_{o}=125 \mathrm{kHz}$, and a $50 \%$ duty cycle of the modulation waveform. . . . . . 130

5.13 Form of the Gaussian factor, from Equation 5.2, as a function of frequency, $f$, for $f_{\max }=200 \mathrm{GHz} \ldots \ldots \ldots \ldots \ldots$ 
5.14 Real signal $X$ at 100 ps as a function of $M$ summation for a modeled system of $100 \mathrm{~nm} \mathrm{Al} /$ Glass $\left(h_{\mathrm{BD}}=30 \mathrm{MW} / \mathrm{m}^{2} \mathrm{~K}\right)$, with $k=\{-1,1\}, f_{s}=250 \mathrm{kHz}$, $f_{o}=125 \mathrm{kHz}$, and a $50 \%$ duty cycle of the modulation waveform. With the inclusion of the Gaussian factor, see Equation 5.2, to the lock-in analysis, see Equations $4.37 \mathrm{a}$ and $4.37 \mathrm{~b} . \ldots \ldots \ldots \ldots \ldots$

5.15 Real signal $X$ of modeled data for a system of $100 \mathrm{~nm} \mathrm{Al} /$ Glass $\left(h_{\mathrm{BD}}=30\right.$ $\mathrm{MW} / \mathrm{m}^{2} \mathrm{~K}$ ), with $k=\{-1,1\}, f_{s}=250 \mathrm{kHz}, f_{o}=125 \mathrm{kHz}$, and a $50 \%$ duty cycle of the modulation waveform for various levels of $M$ summation, with and without the Gaussian factor. . . . . . . . . . . . . . . .

5.16 Fit of $h_{\mathrm{BD}}$ for a modeled system of $100 \mathrm{~nm}$ of $\mathrm{Al} / \mathrm{Si}\left(h_{\mathrm{BD}}=215 \mathrm{MW} / \mathrm{m}^{2} \mathrm{~K}\right)$, with $k=\{-1,1\}, f_{s}=250 \mathrm{kHz}, f_{o}=125 \mathrm{kHz}$, a $50 \%$ duty cycle of the modulation waveform, and generated with $M=1,000,000$, with increasing values of $M$ during fitting. Note: the red line indicated the true $h_{\mathrm{BD}}$ value.

5.17 Number of summations in $M$ required for fit convergence to the known value of $h_{\mathrm{BD}}$, for a modeled system of $100 \mathrm{~nm}$ of $\mathrm{Al} / \mathrm{Si}\left(h_{\mathrm{BD}}=215 \mathrm{MW} / \mathrm{m}^{2} \mathrm{~K}\right)$, with $k=\{-1,1\}, f_{s}=250 \mathrm{kHz}, f_{o}=125 \mathrm{kHz}$, a $50 \%$ duty cycle of the modulation waveform, and generated with $M=1,000,000$. The red line represents a logarithmic fit of the data. . . . . . . . . . . .

5.18 Real signal $X$ at $100 \mathrm{ps}$ as a function of $M$ summation for a modeled system of $100 \mathrm{~nm} \mathrm{Al} /$ Glass $\left(h_{\mathrm{BD}}=30 \mathrm{MW} / \mathrm{m}^{2} \mathrm{~K}\right)$, with $k=\{-1,1\}, f_{s}=76 \mathrm{MHz}$, $f_{o}=1 \mathrm{MHz}$, and a $50 \%$ duty cycle of the modulation waveform. . . . . .

5.19 Real signal $X$ at 100 ps as a function of $M$ summation for a modeled system of $100 \mathrm{~nm} \mathrm{Al} /$ Glass $\left(h_{\mathrm{BD}}=30 \mathrm{MW} / \mathrm{m}^{2} \mathrm{~K}\right)$, with $k=\{-1,1\}, f_{s}=76 \mathrm{MHz}$, $f_{o}=1 \mathrm{MHz}$, and a $50 \%$ duty cycle of the modulation waveform. With the inclusion of the Gaussian factor, see Equation 5.13, to the lock-in analysis, see Equations $4.37 \mathrm{a}$ and $4.37 \mathrm{~b} . \ldots \ldots \ldots \ldots$

5.20 Fit of $h_{\mathrm{BD}}$ for a modeled system of $100 \mathrm{~nm} \mathrm{of} \mathrm{Al} / \mathrm{Si}\left(h_{\mathrm{BD}}=215 \mathrm{MW} / \mathrm{m}^{2} \mathrm{~K}\right)$, with $k=\{-1,1\}, f_{s}=76 \mathrm{MHz}, f_{o}=0.1 \mathrm{MHz}$, a $50 \%$ duty cycle of the modulation waveform, and generated with $M=10,000$, with increasing values of $M$ during fitting. Note: the red line indicated the true $h_{\mathrm{BD}}$ value. . . . . . 
5.21 Fit of $h_{\mathrm{BD}}$ for a modeled system of $100 \mathrm{~nm}$ of $\mathrm{Al} / \mathrm{Si}\left(h_{\mathrm{BD}}=215 \mathrm{MW} / \mathrm{m}^{2} \mathrm{~K}\right)$, with $k=\{-1,1\}, f_{s}=76 \mathrm{MHz}, f_{o}=38 \mathrm{MHz}$, a $50 \%$ duty cycle of the modulation waveform, and generated with $M=10,000$, with increasing values of $M$ during fitting. Note: the red line indicated the true $h_{\mathrm{BD}}$ value. . . . . .

5.22 Number of summations in $M$ required for fit convergence to the known value of $h_{\mathrm{BD}}$, within a tolerance of $1,0.1$, and $0.01 \%$, for a modeled system of 100 $\mathrm{nm}$ of $\mathrm{Al} / \mathrm{Si}\left(h_{\mathrm{BD}}=215 \mathrm{MW} / \mathrm{m}^{2} \mathrm{~K}\right)$, with $k=\{-1,1\}, f_{s}=76 \mathrm{MHz}$, a $50 \%$ duty cycle of the modulation waveform, and generated with $M=10,000$, as a function of laser modulation frequency, $f_{o}$. Note: the lines are a guide to the eye only. . . . . . . . . . . . . . . . .

5.23 Real signal $X$ at $100 \mathrm{ps}$ as a function of $k$ summation for a modeled system of $100 \mathrm{~nm} \mathrm{Al} / \mathrm{Si}\left(h_{\mathrm{BD}}=215 \mathrm{MW} / \mathrm{m}^{2} \mathrm{~K}\right)$, with $M=850,500, f_{s}=250 \mathrm{kHz}$, $f_{o}=125 \mathrm{kHz}$, and a $50 \%$ duty cycle of the modulation waveform. . . . . .

5.24 Fit of $h_{\mathrm{BD}}$ for a modeled system of $100 \mathrm{~nm}$ of $\mathrm{Al} / \mathrm{Si}\left(h_{\mathrm{BD}}=215 \mathrm{MW} / \mathrm{m}^{2} \mathrm{~K}\right)$, with $M=850,500, f_{s}=250 \mathrm{kHz}, f_{o}=125 \mathrm{kHz}$, and a $50 \%$ duty cycle of the modulation waveform, with increasing values of $k$ during fitting. Note: the red line indicated the true $h_{\mathrm{BD}}$ value. . . . . . . . . . .

5.25 Impulse-train of laser pules for $f_{s}=250 \mathrm{kHz}$, and a square modulation waveform at $f_{o}=125 \mathrm{kHz}$, for a summation in $k$ of 1,3 , and $5 . \ldots \ldots$

5.26 Real signal $X$ at 100 ps as a function of $k$ summation for a modeled system of $100 \mathrm{~nm} \mathrm{Al} / \mathrm{Si}\left(h_{\mathrm{BD}}=215 \mathrm{MW} / \mathrm{m}^{2} \mathrm{~K}\right)$, with $M=3,500, f_{s}=76 \mathrm{MHz}$, $f_{o}=0.1 \mathrm{MHz}$, and a $50 \%$ duty cycle of the modulation waveform. . . . . . 140

5.27 Real signal $X$ at 100 ps as a function of $k$ summation for a modeled system of $100 \mathrm{~nm} \mathrm{Al} / \mathrm{Si}\left(h_{\mathrm{BD}}=215 \mathrm{MW} / \mathrm{m}^{2} \mathrm{~K}\right)$, with $M=3,500, f_{s}=76 \mathrm{MHz}$, $f_{o}=38 \mathrm{MHz}$, and a $50 \%$ duty cycle of the modulation waveform. . . . . . 140 
5.28 Number of summations in $k$ required for fit convergence to the known value of $h_{\mathrm{BD}}$, within a tolerance of 5,1 , and $0.5 \%$, for a modeled system of 100 $\mathrm{nm}$ of $\mathrm{Al} / \mathrm{Si}\left(h_{\mathrm{BD}}=215 \mathrm{MW} / \mathrm{m}^{2} \mathrm{~K}\right)$, with $M=3,500, f_{s}=76 \mathrm{MHz}$, a $50 \%$ duty cycle of the modulation waveform, and generated with $k=10,000$, as a function of laser modulation frequency, $f_{o}$. Note: the lines are a guide to the eye only. . . . . . . . . . . . . . . . . . .

5.29 Modulated impulse-train from the MIRA, $f_{s}=76 \mathrm{MHz}$, modulated by a square waveform, $D=50 \%$, at a modulation frequency of $f_{o}=3.8 \mathrm{MHz}$. .

5.30 Modeled system of $100 \mathrm{~nm}$ of $\mathrm{Al} / \mathrm{Si}\left(h_{\mathrm{BD}}=215 \mathrm{MW} / \mathrm{m}^{2} \mathrm{~K}\right)$, with $k=100$, $f_{s}=250 \mathrm{kHz}, f_{o}=125 \mathrm{kHz}, M=1,000,000$, and for duty cycles of $D=$ $0.5000,0.2500,0.1250$ and $0.0625 \ldots \ldots \ldots \ldots$

5.31 Laser impulse-train at $f_{s}=250 \mathrm{kHz}$ modulated by a pulsed waveform at $f_{o}=125 \mathrm{kHz}$ for duty cycles of $D=0.5000,0.2500,0.1250$ and $0.0625 \ldots$.

5.32 Real signal $X$ at 100 ps as a function of $k$ summation for a modeled system of $100 \mathrm{~nm} \mathrm{Al} / \mathrm{Si}\left(h_{\mathrm{BD}}=215 \mathrm{MW} / \mathrm{m}^{2} \mathrm{~K}\right)$, with $M=3,500, f_{s}=76 \mathrm{MHz}$, $f_{o}=7.6 \mathrm{MHz}$, and duty cycles of (a) $D=0.5000$, (b) $D=0.2500$, (c) $D=0.1250$, (d) $D=0.166 \overline{7}$, and (e) $D=0.1000 \ldots \ldots \ldots$

5.33 Required summation in $k$ for $0.5 \%$ convergence as a function of duty cycle from fit $h_{\mathrm{BD}}$ results from a modeled system of $100 \mathrm{~nm} \mathrm{Al} / \mathrm{Si}\left(h_{\mathrm{BD}}=215\right.$ $\left.\mathrm{MW} / \mathrm{m}^{2} \mathrm{~K}\right)$, with $M=3,500, f_{s}=76 \mathrm{MHz}$ and $f_{o}$ as shown. . . . . . 146

5.34 Time required to generate a TTR/TDTR model (320 data points), as a function of M summation. Program run in LabVIEW ${ }^{\mathrm{TM}} 2012$ on an Intel ${ }^{\circledR}$ Core $^{\mathrm{TM}_{2}}$ quad core CPU running at $2.40 \mathrm{GHz}$ with $4 \mathrm{~GB}$ of RAM. The red line indicates a $2^{\text {nd }}$ order polynomial fit of the data, with the fitted results

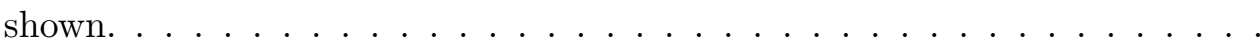

5.35 Schematic of modeled data highlighting the oscillations in the modeled data for a low summation in $M$, and defining the time period between peaks, $T_{\text {peak }}$, and the node spacing. . . . . . . . . . . .

5.36 The time between the peaks in the Gibbs oscillations, $T_{\text {peak }}$, as a function of the summation bounds $M$ for several laser repetition frequencies, $f_{s} \ldots \ldots$ 
5.37 Generated TTR model of $100 \mathrm{~nm}$ of $\mathrm{Al}$ on $\mathrm{Si}\left(h_{\mathrm{BD}}=215 \mathrm{MW} / \mathrm{m}^{2} \mathrm{~K}\right)$, with $M=1,000,000, f_{s}=250 \mathrm{kHz}, f_{s}=125 \mathrm{kHz}$, and a $50 \%$ duty cycle of the modulation waveform (blue), and the interpolated data using Equations 5.4 and 5.6 for $M=50,000$.

5.38 TTR fit of a modeled system of $100 \mathrm{~nm}$ of $\mathrm{Al}$ on $\mathrm{Si}\left(h_{\mathrm{BD}}=215 \mathrm{MW} / \mathrm{m}^{2} \mathrm{~K}\right)$, with $M=1,000,000, f_{s}=250 \mathrm{kHz}, f_{s}=125 \mathrm{kHz}$, and a $50 \%$ duty cycle of the modulation waveform, as a function of increasing summation in $M$, with the data spacing for each $M$ adjusted based on Equations 5.4 and 5.6. The horizontal dashed lines represent plus/minus 1\% deviation from the known

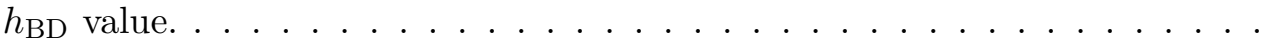

5.39 TTR fit of a modeled system of $100 \mathrm{~nm}$ of $\mathrm{Al}$ on $\mathrm{Si}\left(h_{\mathrm{BD}}=215 \mathrm{MW} / \mathrm{m}^{2} \mathrm{~K}\right)$, with $M=3,500, f_{s}=76 \mathrm{MHz}, f_{s}=1 \mathrm{MHz}$, and a $50 \%$ duty cycle of the modulation waveform, as a function of increasing summation in $M$, with the data spacing for each $M$ adjusted based on Equations 5.5 and 5.6. The horizontal dashed lines represent plus/minus $1 \%$ deviation from the known

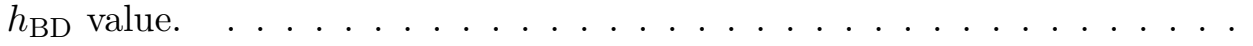

5.40 Fit $h_{\mathrm{BD}}$ as a function of repetition frequency, $f_{s}$, for modulation of $f_{o}=f_{s} / 2$, with the time spacing interpolated using Equations 5.5 and 5.6, for $f_{s}=250$ $\mathrm{kHz}$ in each case. . . . . . . . . . . . . . . . . . .

5.41 Fit of $h_{\mathrm{BD}}$ as a function of the selected scaling time between the data and the model, for simulated data of $100 \mathrm{~nm}$ of $\mathrm{Al}$ on $\mathrm{Si}\left(h_{\mathrm{BD}}=215 \mathrm{MW} / \mathrm{m}^{2} \mathrm{~K}\right)$, with $M=1,000,000, f_{s}=250 \mathrm{kHz}, f_{s}=125 \mathrm{kHz}$, and a $50 \%$ duty cycle of the modulation waveform, with added Gaussian noise for a signal to noise parameter of 8.5. The red line indicates the fit value using the free normalization routine. . . . . . . . . . . . . .

5.42 Scaling time as a function of fitting iteration from the TTR fitting program with the free normalization routine, for the fitting of $h_{\mathrm{BD}}$ for the data shown in Figure $5.41 \mathrm{a} . \ldots \ldots \ldots$

5.43 Schematic of the inputs required for the TTR/TDTR thermal model. . . . 157 
5.44 Modeled data of $100 \mathrm{~nm}$ of $\mathrm{Al}$ on $\mathrm{Si}\left(h_{\mathrm{BD}}=1 \mathrm{MW} / \mathrm{m}^{2} \mathrm{~K}\right)$, with $k=\{-1,1\}$, $f_{s}=250 \mathrm{kHz}, f_{o}=125 \mathrm{kHz}$, and a $50 \%$ duty cycle of the modulation waveform, for (a) the baseline case of $h_{\mathrm{BD}}=100 \mathrm{MW} / \mathrm{m}^{2} \mathrm{~K}$ and for the perturbed case of $90 \%$ of the baseline case, and (b) the difference between the perturbed case and the baseline case as a function of time. . . . . . .

5.45 Modeled data of $100 \mathrm{~nm}$ of $\mathrm{Al}$ on $\mathrm{Si}\left(h_{\mathrm{BD}}=1 \mathrm{MW} / \mathrm{m}^{2} \mathrm{~K}\right)$, with $k=\{-1,1\}$, $f_{s}=250 \mathrm{kHz}, f_{o}=125 \mathrm{kHz}$, and a $50 \%$ duty cycle of the modulation waveform, for (a) the baseline case of $L 1_{C}=2.44 \times 10^{6} \mathrm{~J} / \mathrm{m}^{3} \mathrm{~K}$ and for the perturbed case of $90 \%$ of the baseline case, and (b) the difference between the perturbed case and the baseline case as a function of time. . . . . . . 159

5.46 Average sensitivity parameter, $S_{h_{B D}}^{X}$, over $100-8000$ ps as a function of the percentage that $h_{\mathrm{BD}}$ is perturbed $\ldots \ldots \ldots \ldots$

5.47 Sensitivity coefficient, see Equation 5.8, as a function of time from 130-8050 ps, for (a) non-normalized and (b) normalized data. The modeled system is $100 \mathrm{~nm}$ of $\mathrm{Al}$ on $\mathrm{Si}\left(h_{\mathrm{BD}}=1 \mathrm{MW} / \mathrm{m}^{2} \mathrm{~K}\right)$, the details of the modeled system can be found in Appendix S.1. . . . . . . . . . . . .

5.48 Sensitivity to $h_{\mathrm{BD}}$ of a system of $100 \mathrm{~nm}$ of $\mathrm{Al}$ on $\mathrm{Si}$ as a function of time and the assumed $h_{\mathrm{BD}}$ at the $\mathrm{Al} / \mathrm{Si}$ interface. . . . . . . . .

5.49 Averaged sensitivity as a function of parameter, $p$, for a modeled system of $100 \mathrm{~nm}$ of Al on Si for (a) $S_{\mathrm{hBD}}^{X}$ as a function of $h_{\mathrm{BD}}$ and (b) $S_{\mathrm{kS}}^{X}$ as a function of the substrate thermal conductivity, $k_{S z} \ldots \ldots \ldots$

5.50 Examples of the distribution of input values for a modeled system of $100 \mathrm{~nm}$ of $\mathrm{Al}$ on Si with $k=\{-1,1\}, f_{s}=76 \mathrm{MHz}, f_{o}=1 \mathrm{MHz}$, and a $50 \%$ duty cycle of the modulation waveform for (a) substrate thermal conductivity with a nominal value of $k_{z}=148 \mathrm{~W} / \mathrm{mK}$ and (b) $h_{\mathrm{BD}}$ with a nominal value of $h_{\mathrm{BD}}=215 \mathrm{MW} / \mathrm{m}^{2} \mathrm{~K} \ldots \ldots \ldots \ldots \ldots \ldots \ldots \ldots \ldots \ldots \ldots \ldots \ldots \ldots \ldots \ldots$ 
5.51 Resulting distributions of the average sensitivity coefficient for substrate thermal conductivity and thermal boundary conductance based on the stochastic sensitivity analysis example in Section 5.5.2. The distributions represent the results for $N=5,000$ iterations of the sensitivity model, and the red vertical lines represent the sensitivity results using the nominal input parameters. . 165

5.52 Modeled data sets of $100 \mathrm{~nm}$ of $\mathrm{Al}$ on $\mathrm{Si}$ with $h_{\mathrm{BD}}=2.15 \mathrm{MW} / \mathrm{m}^{2} \mathrm{~K}$ for 3 different signal-to-noise parameters a) 4.18 , b) 8.65 , and c) $12.12 \ldots$. . . . .

5.53 Distribution of noise in 10 trials of the modeled system of $100 \mathrm{~nm}$ of $\mathrm{Al}$ on Si $\left(h_{\mathrm{BD}}=1 \mathrm{MW} / \mathrm{m}^{2} \mathrm{~K}\right)$, with $k=\{-1,1\}, f_{s}=250 \mathrm{kHz}, f_{o}=125 \mathrm{kHz}$, and a $50 \%$ duty cycle of the modulation waveform, with Gaussian noise added to achieve signal-to-noise parameters of 4.18, 8.65, and 12.12 for panes (a)-(c) respectively. . . . . . . . . . . . . . . . . . . 170

5.54 Theoretical $h_{\mathrm{BD}}$ values for a data set containing 58 data points. . . . . . . . 171

5.55 Normality testing of the data shown in Figure 5.54 by (a) a histogram plot with the distribution skew and kurtosis denoted and (b) a quantile-quantile plot with the results of the Shapiro-Wilk test for normality displayed. . . . 173

6.1 Results of molecular dynamics simulations of $h_{\mathrm{BD}}$ as a function of temperature taken from a predominate heat transfer journal (impact factor $\approx 2.5$ ). Error bars represent plus/minus one standard deviation. . . . . . . . . . 178

6.2 Representation of the population of all potential $h_{\mathrm{BD}}$ values and several example sub-populations of potential material systems, from which a random sample set will be selected. . . . . . . . . . . . . . . . . . .

6.3 Examples of normal distributions, see Equation 6.1, for $\mu=100 \mathrm{MW} / \mathrm{m}^{2} \mathrm{~K}$, and $\sigma=1,5$, and $10 \mathrm{MW} / \mathrm{m}^{2} \mathrm{~K} \ldots \ldots \ldots \ldots$

6.4 Schematic of the standard normal curve with percentages for each standard deviation denoted. . . . . . . . . . . . . . . . . . 183 
6.5 Sampling distribution of the mean $h_{\mathrm{BD}}$ values for a sampling size of $n=$ 10 selected from a normal population with mean $\mu=100 \mathrm{MW} / \mathrm{m}^{2} \mathrm{~K}$ and standard deviation $\sigma=5 \mathrm{MW} / \mathrm{m}^{2} \mathrm{~K}$, for a total number of repeated samplings

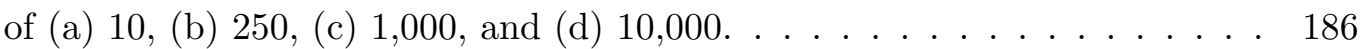

6.6 Distributions of 1,000 samples from a normal distribution with $\mu=100 \mathrm{MW} / \mathrm{m}^{2} \mathrm{~K}$ and $\sigma=5 \mathrm{MW} / \mathrm{m}^{2} \mathrm{~K}$ and (a) $\beta_{\text {skew }}=-0.6$ and (b) $\beta_{\text {skew }}=-1.1 \ldots \ldots$.

6.7 Error, precision, and accuracy of estimating the true mean of a distribution $\mu$ using the sample mean $\bar{X}[19] \ldots \ldots \ldots$. . . . . . . . . . . . . 191

6.8 Mean (a) and standard deviation (b) of a random sampling of $h_{\mathrm{BD}}$ values from a normally distributed population of $\mu=100.00 \mathrm{MW} / \mathrm{m}^{2} \mathrm{~K}$ and $\sigma=$ $20 \mathrm{MW} / \mathrm{m}^{2} \mathrm{~K}$ as a function of the sample size $n . \ldots \ldots \ldots$

6.9 Sensitivity parameter as a function of boundary conductance for the modeled $100 \mathrm{~nm} \mathrm{Al}$ on Si system, where $\mathrm{L}_{1}$ denotes the $\mathrm{Al}$ film and $\mathrm{L}_{\mathrm{S}}$ the substrate. 196

6.10 Examples of ideal data created for the $\mathrm{Al} / \mathrm{Si}$ system with various $h_{\mathrm{BD}}$ values. 197

6.11 (a) Five examples of experimental data taken with the low-rep TTR system on $100 \mathrm{~nm}$ of $\mathrm{Pt}$ on $\mathrm{Si}$, (b) distribution of normalized noise in 152 trials of Pt/Si data and (c) Q-Q plot and Shapiro-Wilk analysis of noise in the experimental data. . . . . . . . . . . . . . . . . . . . . 198

6.12 Process flow for adding normally distributed noise to modeled data. . . . . 199

6.13 (a) Five examples of simulated data of $100 \mathrm{~nm}$ of $\mathrm{Al}$ on $\mathrm{Si}\left(h_{\mathrm{BD}}=215\right.$ $\left.\mathrm{MW} / \mathrm{m}^{2} \mathrm{~K}\right), f_{s}=250 \mathrm{kHz}$, and $f_{o}=125 \mathrm{kHz}$ with added noise (b) distribution of normalized noise in 152 trials of $\mathrm{Al} / \mathrm{Si}$ data and (c) Q-Q plot and Shapiro-Wilk analysis of noise in the simulated data. . . . . . . . . . . . . 199

6.14 Simulated data of (a) $100 \mathrm{~nm}$ of $\mathrm{Al}$ on $\mathrm{AlAlN}\left(h_{\mathrm{BD}}=1,000 \mathrm{MW} / \mathrm{m}^{2} \mathrm{~K}\right)$, $f_{s}=250 \mathrm{kHz}$, and $f_{o}=125 \mathrm{kHz}$ with added noise SNP $=1.21$ and (b) 100 $\mathrm{nm}$ of $\mathrm{Al}$ on $\mathrm{Si}\left(h_{\mathrm{BD}}=1 \mathrm{MW} / \mathrm{m}^{2} \mathrm{~K}\right), f_{s}=250 \mathrm{kHz}$, and $f_{o}=125 \mathrm{kHz}$ with added noise $\mathrm{SNP}=113.53 \ldots \ldots \ldots \ldots$. . . . . . . . . . 200

6.15 Example of the fit summary file generated by the TTR/TDTR fitting program recording the file name, best fit value, sum of squared errors, and any program warnings. . . . . . . . . . . . . . . . . . . . . 201 
6.16 Results of fitting simulated TTR data to deduce $h_{\mathrm{BD}}$ for a system of 100 $\mathrm{nm}$ of $\mathrm{Al}$ on $\mathrm{Si}\left(h_{\mathrm{BD}}=215 \mathrm{MW} / \mathrm{m}^{2} \mathrm{~K}\right), f_{s}=250 \mathrm{kHz}, f_{o}=125 \mathrm{kHz}$, and $\mathrm{SNP}=6.37$ (a) showing the raw data including errors and outliers and (b) with the errors and statistical outliers removed. . . . . . . . . . . . . . . 202

6.17 Analysis of the results of fitting simulated TTR data to deduce $h_{\mathrm{BD}}$ for a system of $100 \mathrm{~nm}$ of $\mathrm{Al}$ on $\mathrm{Si}\left(h_{\mathrm{BD}}=215 \mathrm{MW} / \mathrm{m}^{2} \mathrm{~K}\right), f_{s}=250 \mathrm{kHz}, f_{o}=125$ $\mathrm{kHz}$, and $\mathrm{SNP}=6.37$ for normality $(\mathrm{a})-(\mathrm{b})$ and the mean and standard deviation of the results as a function of the number or trials considered (c)(d). The red horizontal line in (c)-(d) represents the average mean and average standard deviation across all trials. . . . . . . . . . . . .

6.18 Relative population standard deviation as a function of signal-to-noise parameter for $100 \mathrm{~nm}$ of $\mathrm{Al} / \mathrm{Si}$ with $h_{\mathrm{BD}}=215.00 \mathrm{MW} / \mathrm{m}^{2} \mathrm{~K}$. Error bars represent a $95 \%$ confidence interval of the measured $\mathrm{SD}_{\mathrm{R}}$ and the solid line represents a best fit of a power law to the data, of the form $\mathrm{SD}_{\mathrm{R}}=a \times \mathrm{SNP}^{b}$, with parameter $a$ provided for a fixed $b=-1$, along with the $95 \%$ confidence level in $a . \ldots \ldots \ldots \ldots$. . . . . . . . . . . . . . . . . . . . 204

6.19 Fitting coefficient of the relative standard deviation analysis as a function of $h_{\mathrm{BD}}$ for the modeled systems of $\mathrm{Al} / \mathrm{Si}, \mathrm{Al} / \mathrm{AlN}$, and $\mathrm{Al} / \mathrm{Ge} \ldots \ldots . . . . .205$

6.20 Interface time constant, $\tau_{\mathrm{int}}$, as a function of $h_{\mathrm{BD}}$, see Equation 6.18. . . . 206

6.21 Sensitivity of the real signal, $X$, as a function of extended time for the thermal parameters in the $100 \mathrm{~nm} \mathrm{Al}$ on Si system for $h_{\mathrm{BD}}=1.00 \mathrm{MW} / \mathrm{m}^{2} \mathrm{~K}$. . . . 207

6.22 Standard deviation coefficient as a function of sensitivity coefficient for the $100 \mathrm{~nm}$ of $\mathrm{Al}$ on $\mathrm{Si}$ simulated data series. . . . . . . . . . . . . . . 207

6.23 Fitting coefficient as a function of the absolute value of the sensitivity coefficient for various parameter fits in the diffusive regime. The data was fit using a double exponential of the form $f(x)=a * \exp (b x)+c * \exp (d x)$ with the fitting coefficients listed. The bounds on the coefficients and the grey shaded area represent a $95 \%$ confidence interval in the fitted values and the model respectively. . . . . . . . . . . . . . . . . . . . . . . . 208 
6.24 Estimated standard deviation of the population of $h_{\mathrm{BD}}$ values for a modeled system of $100 \mathrm{~nm}$ of $\mathrm{Al}$ on $\mathrm{Si}\left(h_{\mathrm{BD}}=30 \mathrm{MW} / \mathrm{m}^{2} \mathrm{~K}\right)$ as a function of the SNP in the data as calculated from Equation 6.19. The blue data points represent the analysis based on the best fit coefficients and the gray shaded region the $95 \%$ confidence region in the model. . . . . . . . . . . . . . . . . . . . 210

6.25 Fitting coefficient as a function of the absolute value of the sensitivity coefficient for $h_{\mathrm{BD}}$ in the AlSi, AlAlN, and AlGe sample series in the effusive regime. The data was fit using a exponential of the form $f(x)=a * \exp (b x)$ with the coefficients AlSi $(a=97.76 \pm 5.14, b=-1.93 \pm 0.25)$, AlAlN $(a=51.65 \pm 4.40, b=-1.13 \pm 0.26)$, and AlGe $(a=186.40 \pm 13.20$, $b=-1.55 \pm 0.30)$. The bounds on the coefficients and the grey shaded area represent a 95\% confidence interval in the values and model respectively. 211

6.26 Schematic in the non-parametric bootstrapping of experimental data to de-

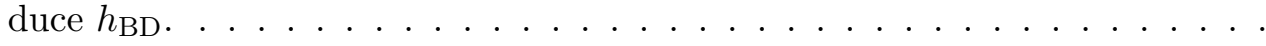

6.27 Distribution of 50,000 and 5,000 random samples respectively selected from (a) a normal distribution with $\beta_{\text {kurt }}=3$ and a skewness coefficient of $\beta_{\text {skew }}=$ 0 and (b) a skewed distribution with $\beta_{\text {kurt }}=2.9$ and $\beta_{\text {skew }}=-0.8$. The skew and kurtosis listed in the plots are the calculated values from the data sets.

6.28 Distribution of bootstrap means from 10 random samples selected from a normal and skewed population with $\beta_{\text {kurt }}=3, \beta_{\text {skew }}=0$ and $\beta_{\text {kurt }}=2.9$, $\beta_{\text {skew }}=-0.8$ respectively, see Figure 6.27 , for $B=10,000$ resamplings. The skew and kurtosis listed in the plots are the calculated values from the data

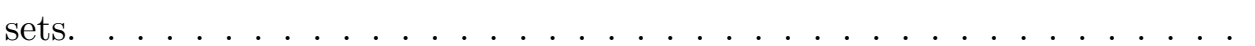

6.29 Example of the standard error of the bootstrap distribution of the means, where (a) is the distribution of the random sample of $n=50$ selected from a normal distribution, $F_{\mu, \sigma}$ with mean $\mu=215 \mathrm{MW} / \mathrm{m}^{2} \mathrm{~K}$ and standard deviation $\sigma=2 \mathrm{MW} / \mathrm{m}^{2} \mathrm{~K}$, and (b) is the bootstrap distribution of the

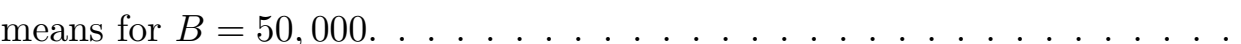


6.30 Distribution of the difference in the means between sample 1 and sample 2, $\bar{x}_{1}-\bar{x}_{2}$, with the means calculated from random samplings with replacement of $n=10$ from all 20 values shown in Table 6.4. The arrow denotes the location of the difference in the means calculated for the original data samples. 220

6.31 Possible number of combinations of $n$ values with repetition and independent of order, see Equation 6.24 . . . . . . . . . . . . . . . . 221

6.32 Four random samples of size $n=8$ selected from a normal distribution $F_{\mu, \sigma}$ with mean $\mu=215 \mathrm{MW} / \mathrm{m}^{2} \mathrm{~K}$ and standard deviation $\sigma=2 \mathrm{MW} / \mathrm{m}^{2} \mathrm{~K}$ are shown in the left column. In the right column, the bootstrap distribution of the sample means for each corresponding sample, $B=50,000$. . . . . . .

6.33 Four random samples of size $n=30$ selected from a normal distribution, $F_{\mu, \sigma}$, with mean $\mu=215 \mathrm{MW} / \mathrm{m}^{2} \mathrm{~K}$ and standard deviation $\sigma=2 \mathrm{MW} / \mathrm{m}^{2} \mathrm{~K}$ bootstrapped to establish the bootstrap distribution of sample means for each corresponding sample, $B=50,000 \ldots \ldots \ldots \ldots$

6.34 Distribution of $h_{\mathrm{BD}}$ values fit using the quasi-stochastic accuracy analysis for input parameters (a) normally distributed with a $1 \sigma$ uncertainty given by the percentages in Table 6.5 and (b) uniformly distributed between a maximum and minimum given by $\mu \pm 1 \sigma \ldots \ldots \ldots \ldots \ldots \ldots \ldots$

6.35 Sensitivity coefficient per parameter as a function of time for the $100 \mathrm{~nm}$ of Pt on Si system where $L_{1}$ denotes the Pt film and $L_{S}$ the Si substrate. . . . 229

6.36 For the quasi-stochastic sensitivity analysis of the Pt/Si system (a) the distribution of the input $h_{\mathrm{BD}}$ values and (b) the resulting distribution of the average sensitivity coefficient for thermal boundary conductance based on $N=1,000$ iterations of the sensitivity model, with the red vertical line representing the sensitivity results using the nominal input parameters. . . 231

6.37 Sensitivity parameter as a function of $h_{\mathrm{BD}}$ for the modeled $100 \mathrm{~nm} \mathrm{Pt}$ on $\mathrm{Si}$ system, where $L_{1}$ denotes the Pt film and $L_{S}$ the Si substrate. . . . . . . . 231 
6.38 Calculated anticipated standard deviation of the population of $h_{\mathrm{BD}}$ values for a system of $100 \mathrm{~nm}$ of $\mathrm{Pt}$ on $\mathrm{Si}\left(h_{\mathrm{BD}}=140 \mathrm{MW} / \mathrm{m}^{2} \mathrm{~K}\right),\left|S_{h_{\mathrm{BD}}}^{\mathrm{avg}, X}\right|=0.4770$, as a function of SNP for calculations from both the diffusive and effusive regimes, given by Equations 6.19 and 6.20 respectively. The shaded regions represent a $95 \%$ confidence region in the predicted values. Note: Data points

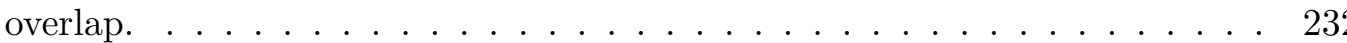

6.39 Distributed of deduced $h_{\mathrm{BD}}$ values for a modeled system of $100 \mathrm{~nm}$ of $\mathrm{Pt}$ on $\mathrm{Si}$ $\left(h_{\mathrm{BD}}=140 \mathrm{MW} / \mathrm{m}^{2} \mathrm{~K}\right)$ for the assumed uncertainty in the input parameters provided by Table 6.8. The red vertical line represents the nominal $h_{\mathrm{BD}}$ value, and the green lines plus/minus one standard deviation. . . . . . . .

6.40 Relative population standard deviation as a function of signal-to-noise parameter for $100 \mathrm{~nm}$ of $\mathrm{Pt} / \mathrm{Si}$ with $h_{\mathrm{BD}}=140.00 \mathrm{MW} / \mathrm{m}^{2} \mathrm{~K}$. Error bars represent a $95 \%$ confidence interval of the measured $\mathrm{SD}_{\mathrm{R}}$ and the solid line represents a best fit of a power law to the data, of the form $\mathrm{SD}_{\mathrm{R}}=a \times \mathrm{SNP}^{b}$, with parameter $a$ provided for a fixed $b=-1$, along with the $95 \%$ confidence level in $a . \ldots \ldots \ldots \ldots \ldots \ldots \ldots$

6.41 Fitting coefficient as a function of the absolute value of the sensitivity coefficient for $h_{\mathrm{BD}}$ in the $\mathrm{Al} / \mathrm{Si}$ sample series in the effusive regime. The data is fit using an exponential of the form $f(x)=a * \exp (b x)$ with the coefficients $(a=97.76 \pm 5.14, b=-1.93 \pm 0.25)$. The bounds on the coefficients and the grey shaded area represent a $95 \%$ confidence interval in the values and model respectively. The data point for the $\mathrm{Pt} / \mathrm{Si}$ system $\left(h_{\mathrm{BD}}=140 \mathrm{MW} / \mathrm{m}^{2} \mathrm{~K}\right)$ corresponds to the developed exponential trend. . . . . . . . . . . . . . . . 234

6.42 Distributed of deduced $h_{\mathrm{BD}}$ values for an experimental system of $100 \mathrm{~nm}$ of Pt on Si using the input parameters provided by Table 6.8. The red vertical line represents the average $h_{\mathrm{BD}}$ value, and the green lines plus/minus one standard deviation. . . . . . . . . . . . . . . . . . 235 
7.1 Representation of the phonon density of states as a function of frequency $\omega$ for (a) a solid-solid system in which $\Theta_{\mathrm{D} \text {,film }}($ Red $)<\Theta_{\mathrm{D} \text {, substrate }}($ Blue) and (b) a solid-solid system with an intermediate layer between the film and substrate such that $\Theta_{\mathrm{D} \text {,film }}($ Red $)<\Theta_{\mathrm{D} \text {,int }}($ Yellow $)<\Theta_{\mathrm{D} \text {,substrate }}($ Blue $) . \ldots . .$.

7.2 Schematic of the computational domains used by English et al. [14] for (a) the baseline case and (b) the case of an intermediate film of varying mass and thickness. Image reproduced with permission from [14] . . . . . . . .

7.3 Measured ratio of $h_{\mathrm{BD}}$ with the addition of the intermediate layer to the baseline case without the intermediate layer as a function of the mass and thickness of material $\mathrm{C}$ for (a) $0.1 T_{m}$ and (b) $0.5 T_{m}$. Image reproduced with permission from $[14] \ldots \ldots \ldots \ldots \ldots$

7.4 Isotropic occupies phonon density of states for (a) the baseline interface with the gray shaded region denoting the vibrational overlap and (b) with the addition of the intermediate layer at the interface between material $\mathrm{A}$ and $\mathrm{C}$ and $\mathrm{C}$ and $\mathrm{B}$ with the hatched region indication the added area of vibrational overlap. Image reproduced with permission from [14]. . . . . . . . . . . . . 242

7.5 Example computation domains from the work of English et al. showing a varying degree of compositional disorder added to the interface region. Image reproduced with permission from $[14] \ldots \ldots \ldots \ldots$

7.6 Measured ratio of $h_{\mathrm{BD}}$ with the addition of the intermediate layer to the baseline case without the intermediate layer as a function of the mass and thickness of material $\mathrm{C}$ for (a) $0.1 T_{m}$ and (b) $0.5 T_{m}$ for a system with simulated interface disorder. Image reproduced with permission from [14]. .

7.7 Isotropic occupied phonon density of states for (a) the added intermediate layer with an abrupt interface with the vibrational overlap denoted and (b) with the added intermediate layer with compositional disorder added with the hatched region indicating the added area of vibrational overlap. Image reproduced with permission from $[14] \ldots \ldots \ldots \ldots$ 
7.8 (a) The ratio of thermal boundary resistance with the intermediate layer to the baseline case as a function of the ratio of the Debye temperatures between the intermediate layer material and material B for several intermediate layer thicknesses. (b) The ratio of thermal boundary resistance with the intermediate layer to the baseline case as a function of the intermediate layer thickness for several Debye temperature ratios between the intermediate layer and material B. Image reproduced with permission from [20]. . . . . . . . .

7.9 Table of potential ideal Debye matches, see Equation 7.1, for the vibrational bridging effect. The left hand column provides the available metals and their respective Debye temperatures in $\mathrm{K}$, and the row across the top the available substrates and Debye temperatures. In the field are the Debye temperatures of the ideal intermediate layers. . . . . . . . . . . .

7.10 Two examples of randomized raster patterns of 45 locations used to scan the sample surfaces in the TTR/TDTR experiment. . . . . . . . . . . . . . 251

7.11 Theoretical thermal resistor network for a Pt film on a Si substrate with a $\mathrm{Ni}$ intermediate layer. . . . . . . . . . . . . . .

7.12 Thermal conductivity as a function of film thickness, for data from Hopkins et al. [21], Li-Dan et al. [22], and Caffrey et al. [23]. Image reproduced from Li-Dan et al. $[22] . \ldots \ldots \ldots \ldots \ldots \ldots \ldots$

7.13 (a) The sensitivity coefficient as a function of time and parameter and (b) the sensitivity coefficient as a function of $h_{\mathrm{BD}}$ and parameter for $100 \mathrm{~nm}$ of Pt on $\mathrm{Si}$ with a $5 \mathrm{~nm} \mathrm{Ni} \mathrm{intermediate} \mathrm{layer.} \mathrm{.} \mathrm{.} \mathrm{.} \mathrm{.} \mathrm{.} \mathrm{.} \mathrm{.} \mathrm{.} \mathrm{.} \mathrm{.} \mathrm{.} 253$

7.14 Total conductance from Pt to Si based on the thermal resistor network model, see Equation 7.2, as a function of the $\mathrm{Ni}$ intermediate layer thickness. The data points represent the average $h_{\mathrm{BD}}$ value and the error bars represent a $95 \%$ confidence in the precision of the deduced value based on repeated samplings. . . . . . . . . . . . . . . . . . . . . 254 
7.15 Thermal boundary conductance from the $\mathrm{Ni}$ intermediate layer to the $\mathrm{Si}$ substrate as a function of the Ni intermediate layer thickness. The horizontal line represents an independently measured system of $100 \mathrm{~nm}$ of $\mathrm{Ni}$ on $\mathrm{Si}$. The data points represent the average $h_{\mathrm{BD}}$ value and the error bars represent a $95 \%$ confidence in the precision of the deduced value based on repeated samplings. . . . . . . . . . . . . . . . . . . . 255

7.16 Total conductance from $\mathrm{Pt}$ to Ge based on the thermal resistor network model, see Equation 7.2, as a function of the Ni intermediate layer thickness. The data points represent the average $h_{\mathrm{BD}}$ value and the error bars represent a $95 \%$ confidence in the precision of the deduced value based on repeated samplings. . . . . . . . . . . . . . . .

7.17 Thermal boundary conductance from the Ni intermediate layer to the Ge substrate as a function of the Ni intermediate layer thickness. The horizontal line represents an independently measured system of $100 \mathrm{~nm}$ of Ni on Ge. The data points represent the average $h_{\mathrm{BD}}$ value and the error bars represent a $95 \%$ confidence in the precision of the deduced value based on repeated samplings. . . . . . . . . . . . . . . . .

7.18 Distribution of deduced $h_{\mathrm{BD}}$ values for the system $100 \mathrm{~nm}$ of Pt on Si with a intermediate Ni of (a) $5 \mathrm{~nm}$ and (b) $10 \mathrm{~nm}$ with the assumed uncertainty

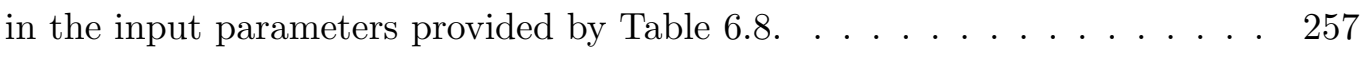

7.19 Total conductance from Pt to Si based on the thermal resistor network model, see Equation 7.2, as a function of the Ni intermediate layer thickness. The data points represent the average $h_{\mathrm{BD}}$ value and the black error bars represent a $95 \%$ confidence in the precision of the deduced value based on repeated samplings. The green error bars represent the uncertainty in the accuracy of the deduced results based on uncertainties in the input parameters, see Table $7.4 \ldots \ldots \ldots \ldots \ldots \ldots \ldots$ 
7.20 Total conductance from Pt to Ge based on the thermal resistor network model, see Equation 7.2, as a function of the Ni intermediate layer thickness. The data point represents the average $h_{\mathrm{BD}}$ value and the black error bar represents a $95 \%$ confidence in the precision of the deduced value based on repeated samplings. The green error bar represents the uncertainty in the accuracy of the deduced results based on uncertainties in the input parameters, see Table $7.4 . \ldots \ldots \ldots \ldots \ldots \ldots \ldots \ldots$. . . . . . . . . . . . . . . . . . . . .

7.21 Effective conductance of a $\mathrm{Ni}$ film, $h_{\mathrm{BD}, \mathrm{eff}}=k_{\mathrm{Ni}} / d_{\mathrm{Ni}}$, as a function of film thickness, with the thermal conductivity of Ni taken from Li-Dan et al. [22], see Figure $7.12 \ldots \ldots \ldots \ldots \ldots \ldots \ldots$

7.22 Total conductance as a function of $\mathrm{Ni}$ intermediate layer thickness assuming a Ni-Si boundary conductance of $h_{\mathrm{BD}}=38.8 \mathrm{MW} / \mathrm{m}^{2} \mathrm{~K}$ (the measured value for the $\mathrm{Pt} / 5 \mathrm{nmNi} / \mathrm{Si}$ sample) and the total conductance of the thicker $\mathrm{Ni}$ layers driven by the $\mathrm{Ni}$ conductance only. . . . . . . . . . . . . . . . . . . . 261

7.23 Energy band diagrams of (a) an isolated metal and isolated n-type semiconductor adjacent to each other and (b) a metal-semiconductor (n-type) contact at thermal equilibrium. . . . . . . . . . . . . 263

7.24 Work function for various clean metals in vacuum $[24,25] \ldots$. . . . . . . 264

7.25 Tunneling condition as a function of barrier height. Tunneling occurs if $d$ is much less than the tunneling condition, based on Equation 7.9. . . . . . .

7.26 Barrier width as a function of barrier height for various substrate doping

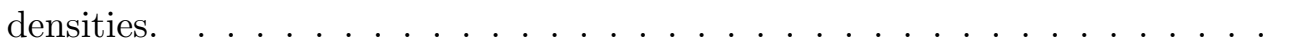

7.27 Tunneling probability as a function of metal-semiconductor interface barrier height for various semiconductor doping concentration. . . . . . . . . . .

7.28 Electron thermal boundary conductance assuming electron tunneling as a function of metal-semiconductor interface barrier height, for various semiconductor doping concentrations. . . . . . . . . . . . . . . .

7.29 Electron thermal boundary conductance assuming electron thermionic emission as a function of metal-semiconductor interface barrier height independent of semiconductor doping concentrations. . . . . . . . . . . . . . . 269 
A.1 A two-dimensional lattice with vectors, $a_{1}$ and $a_{2}$, highlighting three examples of primitive lattice vectors, which also define three examples of primitive unit cells, shaded in grey. Additionally vectors $a_{1}^{*}$ and $a_{2}^{*}$ provide two examples of sets of non-primitive lattice vectors, which define two examples of conventional unit cells, shaded in blue. . . . . . . . . . . . . . . . 282

A.2 Schematic of the construction of the Wigner-Seitz unit cell for a two-dimensional lattice with a monatomic basis. . . . . . . . . . . . . . 283

A.3 The 14 types of Bravais lattices, representing 7 crystal systems: triclinic, monoclinic, orthorhombic, tetragonal, cubic, hexagonal, and trigonal. . . . .

A.4 A schematic representation of x-ray diffraction for (a) a single row of periodic atoms, (b) a 2D array of atoms in a square lattice, (c) the real space lattice stretched in the vertical direction and $(d)$ the real space lattice compressed in the vertical direction. . . . . . . . . . . . . . . . .

A.5 Schematic of a periodic one-dimensional chain of atoms of uniform mass $m$, and interatomic spacing $a \ldots \ldots \ldots \ldots \ldots$

A.6 Representation of a real space square lattice in reciprocal space showing the construction of the first (red), second (blue) and third (yellow) Brillouin zones.289

A.7 Schematic of the types of phonon polarizations available in a two-dimensional lattice, (a) longitudinal and (b) transverse. . . . . . . . . . . . . . .

A.8 Schematic of a one-dimensional monatomic chain of atoms. Here $m$ is the mass of the atoms, $K$ is the force constant between atoms, $a$ is the equilibrium interatomic spacing, and $j$ is the index of the atom. The top row of atoms is the equilibrium configuration, with the lower row depicting atoms displaced from equilibrium by a distance $u_{j+i} \ldots \ldots \ldots \ldots \ldots$

A.9 Phonon dispersion relation assuming a sine-type relation, as in Equation A.23, extended from $-4 \pi / a \leq k \leq 4 \pi / a \ldots \ldots \ldots$

A.10 Phonon dispersion relation assuming a sine-type relation, as in Equation A.23, within the extent of the first Brillouin zone. . . . . . . . . . . 295 
A.11 Elastic stiffness constants, $C_{i j}$, for the longitudinal and transverse phonon polarizations in the 3 primary crystallographic directions. Note that in the [100] and [111] directions, the transverse polarization modes are degenerate. 297

A.12 The phonon dispersion relation for solid Argon in the directions of high symmetry at $0 \mathrm{~K}$, modeled with the lattice dynamics program, General Utility Lattice Program (GULP) $[13] \ldots \ldots \ldots \ldots$. . . . . . . . . . 298

A.13 Schematic of a one-dimensional diatomic chain of atoms. Here $m$ and $M$ are the masses of the two types of atoms, $K$ is the force constant between atoms, $a$ is the equilibrium interatomic spacing, and $j$ is the index of the atom. The top row of atoms is the equilibrium configuration, with the lower row depicting atoms displaced from equilibrium by a distance $u_{j+i}$ and $v_{j+i}$.

A.14 Schematic of the motion in (a) a transverse optical mode, i.e the two masses moving $180^{\circ}$ out of phase with each other, and (b) a transverse acoustic mode, i.e. the two masses moving in phase with each other, assuming an equal wavelength for both modes. . . . . . . . . . . . . . .

A.15 Phonon dispersion in half of the Brillouin zone, for a diatomic chain of atoms of masses $m$ and $M$, connected by massless springs of force constant $K$. The acoustic branch is shown in blue and the optical branch in red, with the cutoff frequencies at the Brillouin zone boundaries denoted. . . . . . . . . .

A.16 Phonon dispersion of $\mathrm{Si}$ in the directions of high symmetry, image from [26]. The lines are calculated from [26], and the circles are experimental data. Note: The frequency is given in terms of $\nu$ which is related to $\omega$ by a factor of $2 \pi$, and the wavevector is denoted $q$, which is equivalent to $k$ in the notation of this dissertation. . . . . . . . . . . . . . . . . . . . 304

A.17 Schematic representation a constant energy surface in k-space. . . . . . . . 305

A.18 Schematic of the first quadrant of a constant energy surface in k-space depicting (a) the determination of the number of quantum states that fit into that volume and (b) the differential expansion of the spherical volume in k-space. 305

A.19 (a) Debye density of states for FCC Cu from Equation A.53 and (b) DOS of solid Ar at $0 \mathrm{~K}$ calculated from lattice dynamics, GULP [13] . . . . . . . . 308 
A.20 Schematic of phonon-phonon scattering processes within the first Brillouin zone for (a) normal phonon processes and (b) Umklapp phonon processes. .

B.1 Optical penetration depth in $\mathrm{Pt}$ as a function of incident photon wavelength, calculated using Equation B.1. Optical constant data provided from [27]. .

B.2 Reflectance as a function of film thickness for $\mathrm{Al}, \mathrm{Au}, \mathrm{Pt}$, and $\mathrm{Ni}$ on $\mathrm{Si}$ substrates in air, based on Equations B.4a-B.4j. The dashed lines are the reflectance of the given bulk metals using Equation B.2. Optical properties used in the calculations are shown in Table B.1. . . . . . . . . . . . .

C.1 Generalization of the pulsed wavefunction that we seek, given by Equation

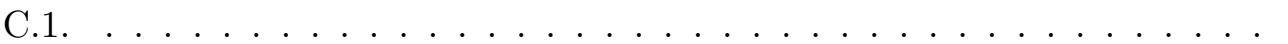

C.2 Plot of the waveform given by Equations C.12 and C.11 for a frequency of 1 Hz, i.e. $f=1 \mathrm{~Hz}, \tau_{d}=0$, for a duty cycles (a) $D=0.10$, (b) $D=0.25$, (c) $D=0.50$, and $(\mathrm{d}) D=0.75 \ldots \ldots \ldots \ldots \ldots$

C.3 Amplitude as a function of time, for the pulsed wave formulation, Equations C.11 and C.12, showing the sum of $1,3,5,7$, and 5000 harmonic terms, $k$. Assuming $f=1 \mathrm{~Hz}, \mathrm{D}=0.5$, and $\tau_{d}=0 \ldots \ldots \ldots 326$

C.4 Fourier coefficients, $a_{k}$, for the first 61 harmonic terms, assuming $D=0.50$ and $\tau_{d}=0 \ldots \ldots \ldots \ldots \ldots \ldots \ldots \ldots \ldots \ldots \ldots \ldots \ldots \ldots \ldots$

C.5 Schematic of the indexing scheme used in the thermal analysis for a sample configuration where heating and probing are through a glass layer. . . . . . 334

D.1 Normal data, $\mu=100 \mathrm{MW} / \mathrm{m}^{2} \mathrm{~K} \sigma=1 \mathrm{MW} / \mathrm{m}^{2} \mathrm{~K} \ldots \ldots . \ldots . \ldots 338$

D.2 Normal data, $\mu=100 \mathrm{MW} / \mathrm{m}^{2} \mathrm{~K} \sigma=1 \mathrm{MW} / \mathrm{m}^{2} \mathrm{~K}, n=20 \quad \ldots \ldots . \ldots 338$

D.3 Normal data, skew and kurtosis as a function of the number of data points. 339

E.1 The sensitivity coefficient as a function of time for $100 \mathrm{~nm}$ of $\mathrm{Pt}$ on Si. . . . 343

E.2 The sensitivity coefficient as a function of time for $100 \mathrm{~nm}$ of $\mathrm{Ni}$ on Si. . . 343

E.3 The sensitivity coefficient as a function of time for $100 \mathrm{~nm}$ of $\mathrm{Pt}$ on $\mathrm{Si}$ with a $5 \mathrm{~nm} \mathrm{Ni} \mathrm{intermediate} \mathrm{layer.} \mathrm{.} \mathrm{.} \mathrm{.} \mathrm{.} \mathrm{.} \mathrm{.} \mathrm{.} \mathrm{.} \mathrm{.} \mathrm{.} \mathrm{.} \mathrm{.} \mathrm{.} \mathrm{.} \mathrm{.} \mathrm{.} \mathrm{.} \mathrm{.} \mathrm{.} 344$ 
E.4 The sensitivity coefficient as a function of time for $100 \mathrm{~nm}$ of Pt on Si with a $10 \mathrm{~nm} \mathrm{Ni} \mathrm{intermediate} \mathrm{layer.} \mathrm{.} \mathrm{.} \mathrm{.} \mathrm{.} \mathrm{.} \mathrm{.} \mathrm{.} \mathrm{.} \mathrm{.} \mathrm{.} \mathrm{.} \mathrm{.} \mathrm{.} \mathrm{.} \mathrm{.} \mathrm{.}$

E.5 The sensitivity coefficient as a function of time for $100 \mathrm{~nm}$ of $\mathrm{Pt}$ on $\mathrm{Si}$ with a $20 \mathrm{~nm} \mathrm{Ni} \mathrm{intermediate} \mathrm{layer.} \mathrm{.} \mathrm{.} \mathrm{.} \mathrm{.} \mathrm{.} \mathrm{.} \mathrm{.} \mathrm{.} \mathrm{.} \mathrm{.} \mathrm{.} \mathrm{.} \mathrm{.} \mathrm{.} \mathrm{.}$

E.6 The sensitivity coefficient as a function of time for $100 \mathrm{~nm}$ of $\mathrm{Pt}$ on $\mathrm{Si}$ with a $30 \mathrm{~nm} \mathrm{Ni} \mathrm{intermediate} \mathrm{layer.} \mathrm{.} \mathrm{.} \mathrm{.} \mathrm{.} \mathrm{.} \mathrm{.} \mathrm{.} \mathrm{.} \mathrm{.} \mathrm{.} \mathrm{.} \mathrm{.} \mathrm{.} \mathrm{.} \mathrm{.} \mathrm{.}$

E.7 The sensitivity coefficient as a function of time for $100 \mathrm{~nm}$ of $\mathrm{Pt}$ on Si with a $50 \mathrm{~nm} \mathrm{Ni} \mathrm{intermediate} \mathrm{layer.} \mathrm{.} \mathrm{.} \mathrm{.} \mathrm{.} \mathrm{.} \mathrm{.} \mathrm{.} \mathrm{.} \mathrm{.} \mathrm{.} \mathrm{.} \mathrm{.} \mathrm{.} \mathrm{.} \mathrm{.} \mathrm{.}$

E.8 The sensitivity coefficient as a function of time for $100 \mathrm{~nm}$ of Pt on Si with a $100 \mathrm{~nm} \mathrm{Ni} \mathrm{intermediate} \mathrm{layer.} \mathrm{.} \mathrm{.} \mathrm{.} \mathrm{.} \mathrm{.} \mathrm{.} \mathrm{.} \mathrm{.} \mathrm{.} \mathrm{.} \mathrm{.} \mathrm{.} \mathrm{.} \mathrm{.} \mathrm{.} \mathrm{.} \mathrm{.} \mathrm{.} 345$

E.9 The sensitivity coefficient as a function of time for $100 \mathrm{~nm}$ of $\mathrm{Pt}$ on Ge. . . 346

E.10 The sensitivity coefficient as a function of time for $100 \mathrm{~nm}$ of $\mathrm{Ni}$ on Ge. . . 346

E.11 The sensitivity coefficient as a function of time for $100 \mathrm{~nm}$ of Pt on Ge with a $5 \mathrm{~nm} \mathrm{Ni} \mathrm{intermediate} \mathrm{layer.} \mathrm{.} \mathrm{.} \mathrm{.} \mathrm{.} \mathrm{.} \mathrm{.} \mathrm{.} \mathrm{.} \mathrm{.} \mathrm{.} \mathrm{.} \mathrm{.} \mathrm{.} \mathrm{.} \mathrm{.} 346$

E.12 The sensitivity coefficient as a function of time for $100 \mathrm{~nm}$ of $\mathrm{Pt}$ on Ge with a $10 \mathrm{~nm} \mathrm{Ni} \mathrm{intermediate} \mathrm{layer.} \mathrm{.} \mathrm{.} \mathrm{.} \mathrm{.} \mathrm{.} \mathrm{.} \mathrm{.} \mathrm{.} \mathrm{.} \mathrm{.} \mathrm{.} \mathrm{.} \mathrm{.} \mathrm{.} \mathrm{.} \mathrm{.} \mathrm{.} 347$

E.13 The sensitivity coefficient as a function of time for $100 \mathrm{~nm}$ of Pt on Ge with a $20 \mathrm{~nm} \mathrm{Ni} \mathrm{intermediate} \mathrm{layer.} \mathrm{.} \mathrm{.} \mathrm{.} \mathrm{.} \mathrm{.} \mathrm{.} \mathrm{.} \mathrm{.} \mathrm{.} \mathrm{.} \mathrm{.} \mathrm{.} \mathrm{.} \mathrm{.} \mathrm{.} \mathrm{.} 347$

E.14 The sensitivity coefficient as a function of time for $100 \mathrm{~nm}$ of $\mathrm{Pt}$ on Ge with a $30 \mathrm{~nm} \mathrm{Ni} \mathrm{intermediate} \mathrm{layer.} \mathrm{.} \mathrm{.} \mathrm{.} \mathrm{.} \mathrm{.} \mathrm{.} \mathrm{.} \mathrm{.} \mathrm{.} \mathrm{.} \mathrm{.} \mathrm{.} \mathrm{.} \mathrm{.} \mathrm{.} \mathrm{.} 347$

E.15 The sensitivity coefficient as a function of time for $100 \mathrm{~nm}$ of Pt on Ge with a $50 \mathrm{~nm} \mathrm{Ni} \mathrm{intermediate} \mathrm{layer.} \mathrm{.} \mathrm{.} \mathrm{.} \mathrm{.} \mathrm{.} \mathrm{.} \mathrm{.} \mathrm{.} \mathrm{.} \mathrm{.} \mathrm{.} \mathrm{.} \mathrm{.} \mathrm{.} \mathrm{.} \mathrm{.} \mathrm{.} 348$

E.16 The sensitivity coefficient as a function of time for $100 \mathrm{~nm}$ of $\mathrm{Pt}$ on Ge with a $100 \mathrm{~nm} \mathrm{Ni} \mathrm{intermediate} \mathrm{layer.} \mathrm{.} \mathrm{.} \mathrm{.} \mathrm{.} \mathrm{.} \mathrm{.} \mathrm{.} \mathrm{.} \mathrm{.} \mathrm{.} \mathrm{.} \mathrm{.} \mathrm{.} \mathrm{.} \mathrm{.} \mathrm{.} \mathrm{.} \mathrm{.} \mathrm{.} 348$ 


\section{LIST OF TABLES}

1.1 Properties of various types of TIMs $[28-31] \ldots \ldots \ldots \ldots$

5.1 Estimation of the summation in $M$ required to reach a specified convergence level for the low-rep (RegA) system, based on the log fit of the data in Figure 5.17. Additionally the time required to generate a 320 point data set, at the summation level is provided for the TTR/TDTR program run in LabVIEW ${ }^{\mathrm{TM}} 2012$ on an Intel ${ }^{\circledR}$ Core $^{\mathrm{TM}_{2}}$ quad core CPU running at 2.40 $\mathrm{GHz}$ with $4 \mathrm{~GB}$ of RAM. . . . . . . . . . . . . . . .

5.2 Estimation of the summation in $M$ required to reach a specified convergence level for the high-rep (MIRA) system, based on the data in Figure 5.22. Additionally the time required to generate a 320 point data set, at the summation level, is provided for the TTR/TDTR program run in LabVIEW ${ }^{\mathrm{TM}} 2012$ on an Intel ${ }^{\circledR}$ Core $^{\mathrm{TM}_{2}}$ quad core CPU running at $2.40 \mathrm{GHz}$ with 4 GB of RAM. 137

5.3 Practical duty cycles available for a high-rep system, $f_{s}=76 \mathrm{MHz}$, modulated at $f_{o}=3.8 \mathrm{MHz}$ and the width of the "on" portion of the modulation

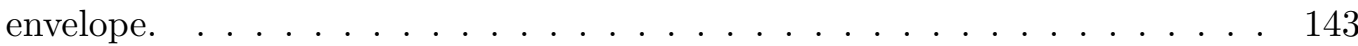

5.4 Results of fitting of the data in Figure 5.36 using a power law of the form,

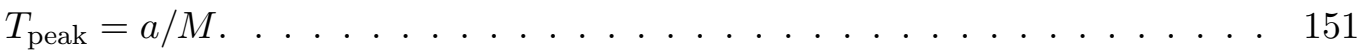


5.5 Uncertainty in TTR/TDTR input parameters for the example of a modeled system of $100 \mathrm{~nm}$ of $\mathrm{Al}$ on $\mathrm{Si}\left(h_{\mathrm{BD}}=215 \mathrm{MW} / \mathrm{m}^{2} \mathrm{~K}\right)$ in Section 5.5.2 demonstrating the implementation of stochastic sensitivity analysis, where $\mathbf{L} \mathbf{1}$ and LS denote the film and substrate layer respectively. . . . . . . . . . . . .

5.6 Tabulated parameters of the generalized Studentized extreme deviate test for outlier based on the data in Figure 5.54 assuming $r=5$ potential outliers and a significance level of $\alpha=0.05 \ldots \ldots \ldots \ldots \ldots$

6.1 Calculated $p$-values based on Welch's t-test between the data points shown in Figure 6.1 where the null hypothesis is that the two means are equal. . .

6.2 Summary of simulation data $-100 \mathrm{~nm} \mathrm{Al} \mathrm{on} \mathrm{Si} h_{\mathrm{BD}}=215 \mathrm{MW}$ Series. Error bounds represent a $95 \%$ confidence level. . . . . . . . . . . . . . . . . . . 204

6.3 Fitting coefficients for estimating the relative standard deviation in the deduced parameters calculated for the effusive regime, see Equation 6.20, for the three system substrates used in this study along with the $R^{2}$ values of the model fits. . . . . . . . . . . . . . . . .

6.4 Example of two sample sets of $n=10$ randomly selected from normal distributions of $\mu=215 \mathrm{MW} / \mathrm{m}^{2} \mathrm{~K}$ and $\mu=220 \mathrm{MW} / \mathrm{m}^{2} \mathrm{~K}$ for sample 1 and sample 2 respectively with both distributions having a dispersion of $\sigma=4 \mathrm{MW} / \mathrm{m}^{2} \mathrm{~K} \ldots \ldots \ldots \ldots \ldots \ldots \ldots \ldots \ldots$

6.5 Uncertainty in the input parameters for a modeled system of $100 \mathrm{~nm}$ of $\mathrm{Al}$ on $\mathrm{Si}\left(h_{\mathrm{BD}}=215 \mathrm{MW} / \mathrm{m}^{2} \mathrm{~K}\right)$ and the corresponding change in the deduced $h_{\mathrm{BD}}$ using the uncertainty analysis described in Equation 6.25. . . . . . 226

6.6 Thermophysical properties used in modeling a Pt film on Si substrate. . . . 229

6.7 Averaged sensitivity coefficient, $S_{p}^{\mathrm{avg}, X}$, for the parameters in the simulated Pt/Si system. . . . . . . . . . . . . . . . . . . 230

6.8 Uncertainty in the TTR/TDTR input parameters for the example of a system of $100 \mathrm{~nm}$ of Pt on $\mathrm{Si}\left(h_{\mathrm{BD}}=140 \mathrm{MW} / \mathrm{m}^{2} \mathrm{~K}\right)$ where $\mathbf{L} \mathbf{1}$ and $\mathbf{L S}$ denote the Pt film and Si substrate layer respectively. . . . . . . . . . . . . . . 230 
6.9 Standard deviation in $h_{\mathrm{BD}}$ calculated from Equation 6.20, simulated from 1,000 data sets of a modeled system of $100 \mathrm{~nm}$ of $\mathrm{Pt}$ on $\mathrm{Si}\left(h_{\mathrm{BD}}=140 \mathrm{MW} / \mathrm{m}^{2} \mathrm{~K}\right)$ with $\mathrm{SNP}=25$, and from 152 experimentally collected scans analyzed to deduce $h_{\mathrm{BD}}$. The upper and lower bounds represent a $95 \%$ confidence level in the respective values. . . . . . . . . . . . . . . . 235

7.1 Overview of samples fabricated at the US Army Research Laboratory to test the hypothesis of the phonon bridging effect. The deposition rate was $1 \mathrm{~nm} / \mathrm{s}$ for all samples. . . . . . . . . . . . . . . . . . . . . 249

7.2 Thermophysical properties used in modeling of a Pt film on Si substrate. . . 251

7.3 Averaged sensitivity coefficient, $S_{p}^{\mathrm{avg}, X}$, for the parameters in the system of $100 \mathrm{~nm}$ of $\mathrm{Pt}$ on $\mathrm{Si}$ with a $5 \mathrm{~nm} \mathrm{Ni} \mathrm{intermediate} \mathrm{layer.} \mathrm{.} \mathrm{.} \mathrm{.} \mathrm{.} \mathrm{.} \mathrm{.} \mathrm{.} \mathrm{.} \mathrm{.} \mathrm{.} 254$

7.4 Uncertainty in the TTR/TDTR input parameters for the example of a system of $100 \mathrm{~nm}$ of $\mathrm{Pt}$ on $\mathrm{Si}\left(h_{\mathrm{BD}}=140 \mathrm{MW} / \mathrm{m}^{2} \mathrm{~K}\right)$ where $\mathbf{L} \mathbf{1}$ and $\mathbf{L S}$ denote the Pt film and Si substrate layer respectively. . . . . . . . . . . . . . 257

7.5 Electron affinity, $q \chi$, of various semiconductors. . . . . . . . . . . 264

7.6 Properties of semiconductor elements used to model electron boundary con-

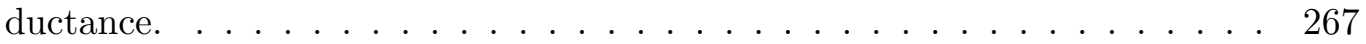

A.1 Room temperature Debye temperatures, $\Theta_{D}$, for various elements. Data taken from $[32] \ldots \ldots \ldots \ldots \ldots \ldots \ldots$

B.1 Optical and laser properties used in the calculation of reflectance and reflectivity of Al,Au,Pt, and Ni shown in Figure B.2. . . . . . . . . . . . . 320

E.1 Averaged sensitivity parameter for the $100 \mathrm{~nm} \mathrm{Pt} \mathrm{on} \mathrm{Si} \mathrm{system.} \mathrm{.} \mathrm{.} \mathrm{.} \mathrm{.} \mathrm{.} \mathrm{.} \mathrm{.} 342$

E.2 Averaged sensitivity parameter for the $100 \mathrm{~nm} \mathrm{Pt}$ on Ge system. . . . . . . 343 


\section{NOMENCLATURE}

\section{Glossary}

$3 \mathrm{TM}$

AES

AFM

AGF

AIM

$\mathrm{AMM}$

$\mathrm{BBO}$

BLT

BOE

BTE

CMOS

DFT

$\mathrm{DMM}$
Three-temperature Model

Auger Photoelectron Spectroscopy

Atomic Force Microscope

Atomistic Green's Function

Anharmonic Inelastic Model

Acoustic Mismatch Model

Beta Barium Borate

Bond Line Thickness

Buffered Oxide Etch

Boltzmann Transport Equation

Complimentary Metal-Oxide-Semiconductor

Density Functional Theory

Diffuse Mismatch Model 


\begin{tabular}{|c|c|}
\hline DPSS & Diode-pumped Solid-state \\
\hline EBPVD & Electron Beam Physical Vapor Deposition \\
\hline eDMM & Electron Diffuse Mismatch Model \\
\hline eDOS & Electron Density of States \\
\hline ENIAC & Electronic Numerical Integrator and Computer \\
\hline $\mathrm{EOM}$ & Electo-optic Modulator \\
\hline FCC & Face-centered Cubic \\
\hline HHIM & Higher Harmonic Inelastic Model \\
\hline HMETs & High-Electron-Mobility Transistors \\
\hline JFDMM & Join Frequency Diffuse Mismatch Model \\
\hline $\mathrm{LBO}$ & Lithium Triborate \\
\hline LJ & Lennard-Jones \\
\hline LTI & Linear Time-Invariant \\
\hline MAD & Mean Average Deviation (Chapter 5) \\
\hline MD & Molecular Dynamics \\
\hline MFP & Mean Free Path \\
\hline MOSFET & Metal-oxide-semiconductor Field Effect Transistor \\
\hline MTM & Maximum Transmission Model \\
\hline NEMD & Nonequilibrium Molecular Dynamics \\
\hline $\mathrm{PBC}$ & Polarizing Beam Cube \\
\hline PDOS & Phonon Density of States \\
\hline POS & Parabolic One-step \\
\hline
\end{tabular}




\begin{tabular}{|c|c|}
\hline PUE & Power Utilization Effectiveness \\
\hline $\mathrm{QCM}$ & Quartz Crystal Monitor \\
\hline $\mathrm{RCA}$ & Radio Corporation of America \\
\hline rms & Root Mean Square \\
\hline SAM & Self-assembled Monolayer \\
\hline SOI-MOSFET & Silicon-on-insulator MOSFET \\
\hline TCC & Thermal Contact Conductance \\
\hline TCR & Thermal Contact Resistance \\
\hline TDTR & Time-domain Thermoreflectance \\
\hline TIM & Thermal Interface Material \\
\hline TTM & Two-temperature Model \\
\hline TTR & Transient Thermoreflectance \\
\hline $\mathrm{UC}$ & Unit Cell \\
\hline XDS & X-ray Diffuse Scattering \\
\hline XRR & X-ray Reflectivity \\
\hline
\end{tabular}

$\alpha$

$\alpha_{1 \rightarrow 2}$

$\beta$

$\beta_{\text {kurt }}$
Significance level (Chapter 6)

Phonon transmission probability, acoustic mismatch model (Chapter 2)

Thermoreflectance constant that contains information about the thermoreflectance coefficient of the sample and the gain in the measurement electronics (Chapter 4)

Population kurtosis (Chapter 6) 


\begin{tabular}{|c|c|}
\hline$\beta_{\text {skew }}$ & Population skewness (Chapter 6) \\
\hline$\chi$ & Electron affinity - [V] \\
\hline$\delta$ & Radiation penetration depth - $[\mathrm{m}]$ \\
\hline$\delta$ & $\begin{array}{l}\text { Root mean square roughness of the interface, also known as the } \\
\text { asperity parameter (Chapter } 2)-[\mathrm{m}]\end{array}$ \\
\hline$\delta()$ & Dirac delta function (Chapter 4) \\
\hline$\delta_{i}$ & Cube root of the atomic volume for the $i^{\text {th }}$ impurity atom in its own \\
\hline & lattice (Appendix A) - [m] \\
\hline$\epsilon$ & Electron energy (Chapter 2) - $[\mathrm{eV}]$ \\
\hline$\epsilon_{o}$ & Free space permittivity $8.854 \times 10^{-12}($ Chapter 7$)-[\mathrm{F} / \mathrm{m}]$ \\
\hline$\epsilon_{r}$ & Relative semiconductor permittivity (Chapter 7) \\
\hline$\epsilon_{\mathrm{F}, \mathrm{i}}$ & Fermi energy of material $i$ (Chapter 2$)-[\mathrm{eV}]$ \\
\hline$\gamma$ & Average Grüneisen anharmonicity parameter (Appendix A) \\
\hline$\gamma$ & Sommerfeld constant (electron heat capacity constant) - $\left[\mathrm{J} / \mathrm{m}^{3} \mathrm{~K}^{2}\right]$ \\
\hline$\gamma_{i}$ & $\begin{array}{l}\text { Electron heat capacity constant, also known as the Sommerfeld con- } \\
\text { stant }\end{array}$ \\
\hline$\Gamma_{M}$ & Atomic form factor (Appendix A) \\
\hline$\lambda_{i}$ & Outlier ESD critical value (Chapter 5) \\
\hline$\mu$ & Population mean (Chapter 6) \\
\hline$\Omega$ & Small frequency shift (Chapter 4) \\
\hline$\omega$ & Phonon angular frequency - [rads/s] \\
\hline$\omega(\vec{k})$ & Phonon dispersion relation -[rads/s] \\
\hline$\omega_{\max }$ & Maximum angular frequency in thermal analysis (Chapter 5$)-[\mathrm{rad} / \mathrm{s}]$ \\
\hline
\end{tabular}




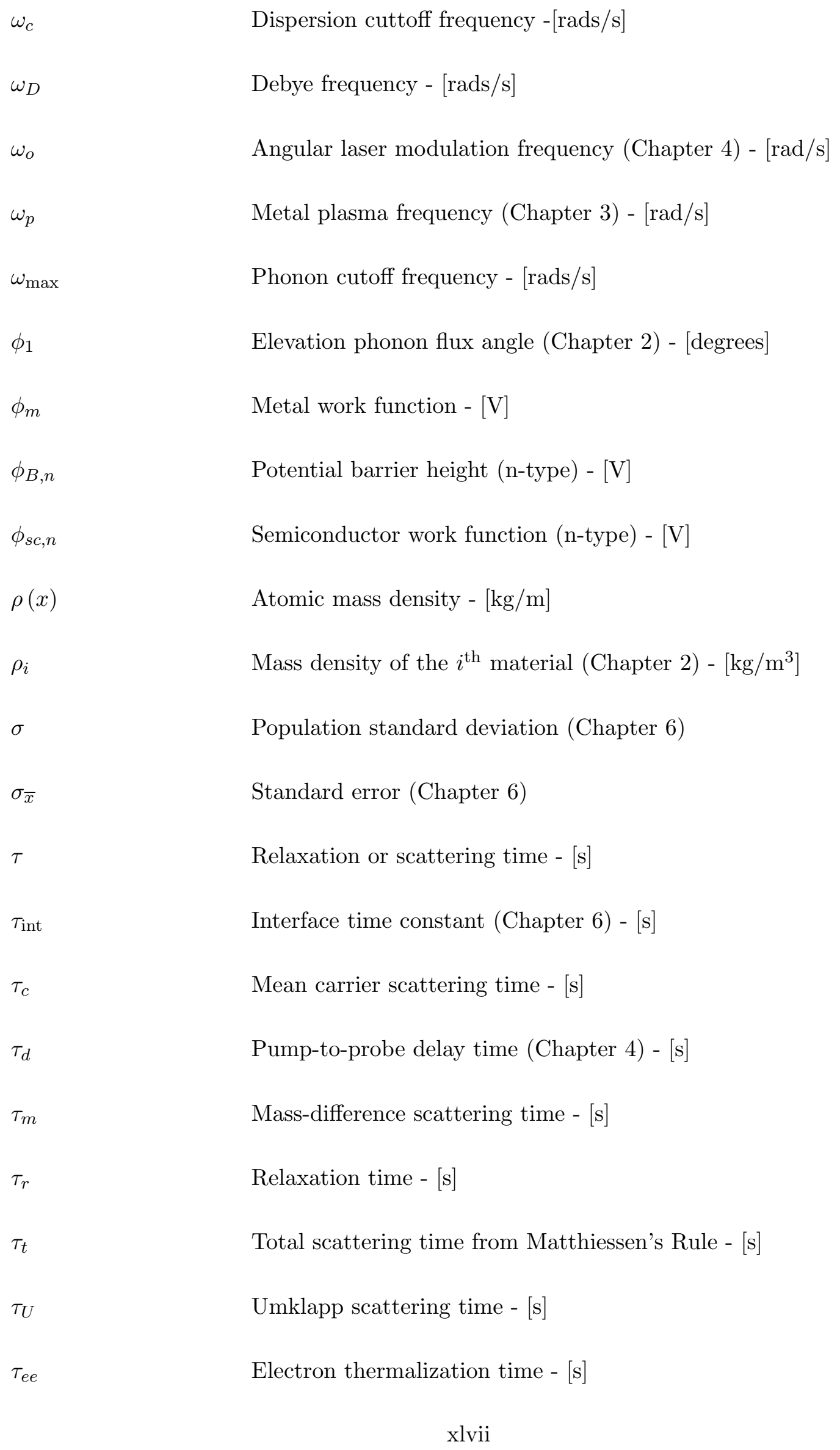




$\begin{array}{ll}\tau_{e p} & \text { Electron-phonon thermalization time - }[\mathrm{s}] \\ \theta_{1} & \text { Azimuthal phonon flux angle (Chapter } 2)-[\text { degrees }] \\ \Theta_{D} & \text { Debye temperature }-[\mathrm{K}] \\ \zeta^{1 \rightarrow 2} & \text { Phonon transmission coefficient, material } 1 \text { to material } 2 \\ \zeta_{e}^{1 \rightarrow 2} & \text { Electron transmission coefficient for the eDMM }\end{array}$

\section{Superscripts}

$A$

$B$

$I L$

$j$

$j$

$z$

\section{Variables}

$\bar{M}$

$\Delta T$

$\dot{q}$

$\frac{\partial f}{\partial t}$

$\hbar$

$h_{\mathrm{BD}}$

$\left(\frac{\partial f}{\partial t}\right)_{c}$
Material A in molecular dynamics simulation (Chapter 7)

Material B in molecular dynamics simulation (Chapter 7)

Intermediate material in molecular dynamics simulation (Chapter

7)

Atomic index (Appendix A)

Phonon polarization (Chapter 2)

z-direction of transport

Average atomic mass - $[\mathrm{kg}]$

Temperature drop - $[\mathrm{K}]$

Heat flux - $\left[\mathrm{W} / \mathrm{m}^{2}\right]$

Change in the distribution function with time (Appendix A)

Reduced Plank's constant - $1.055 \times 10^{-34}$ Js

Thermal boundary conductance - $\left[\mathrm{W} / \mathrm{m}^{2} \mathrm{~K}\right]$

Scattering term of the Boltzmann transport equation (Appendix A) 
$\mathrm{SD}_{\mathrm{R}, \mathrm{D}}$

$\mathrm{SD}_{\mathrm{R}, \mathrm{E}}$

$\mathrm{SD}_{\mathrm{R}}$

$\bar{x}$

$\overrightarrow{\mathrm{G}}$

$\vec{p}$

$\overrightarrow{\mathbf{R}}$

$\overrightarrow{\mathbf{r}}$

$\overrightarrow{a_{i}^{*}}$

$\overrightarrow{a_{i}}$

$\overrightarrow{b_{i}}$

$\vec{k}$

A

$a$

$a_{k}$

$a_{O}$

$c$

$C_{\omega}$

$C_{e}$

$C_{p}$
Relative standard deviation in the diffusive regime (Chapter 6) [\%]

Relative standard deviation in the effusive regime (Chapter 6) - [\%]

Relative standard deviation (Chapter 6) - [\%]

Sample mean (Chapter 6)

Reciprocal-space translation vector - $\left[\mathrm{m}^{-1}\right]$

Momentum vector - $[\mathrm{kg}-\mathrm{m} / \mathrm{s}]$

Real-space translation vector - $[\mathrm{m}]$

Position vector - $[\mathrm{m}]$

Non-primitive lattice vector in the $i^{\text {th }}$ direction - [m]

Primitive lattice vector in the $i^{\text {th }}$ direction - $[\mathrm{m}]$

Primitive reciprocal lattice vector in the $i^{\text {th }}$ direction $-\left[\mathrm{m}^{-1}\right]$

Phonon wavevector - $\left[\mathrm{m}^{-1}\right]$

Process- and geometry-dependent constant in Black's equation (Chapter 1$)$

Interatomic spacing - $[\mathrm{m}]$

AC Fourier components of the pulsed waveform (Chapter 4)

DC Fourier component of the pulsed waveform (Chapter 4)

Speed of sound in a crystal (Appendix A) - [m/s]

Phonon heat capacity - $\left[\mathrm{J} / \mathrm{m}^{2} \mathrm{~K}\right]$

Electron heat capacity - $\left[\mathrm{J} / \mathrm{m}^{3} \mathrm{~K}\right]$

Phonon heat capacity - $\left[\mathrm{J} / \mathrm{m}^{3} \mathrm{~K}\right]$ 


\begin{tabular}{|c|c|}
\hline$C_{i j}$ & Elastic stiffness constant $-\left[\mathrm{N} / \mathrm{m}^{2}\right]$ \\
\hline$D$ & Duty cycle (Chapter 5)- [\%] \\
\hline$d$ & Temporal width of the modulation envelope (Chapter 5)- [s] \\
\hline$D(\omega)$ & $\begin{array}{l}\text { Phonon density of states - \# of phonon states between } \omega \text { and } \omega+d \omega \\
\text { per unit volume, per unit wavevector, per polarization - }\left[\mathrm{s} / \mathrm{m}^{3}\right]\end{array}$ \\
\hline$D(\vec{k})$ & $\begin{array}{l}\text { Phonon density of states - \# of phonon states between } k \text { and } k+d k \\
\text { per unit volume, per unit wavevector, per polarization - }\left[\mathrm{m}^{2}\right]\end{array}$ \\
\hline$D(\epsilon)$ & Electron density of states (Chapter 2$)-\left[\frac{1}{\mathrm{eVm}^{3}}\right]$ \\
\hline$d_{B}$ & Potential barrier width (Chapter 7$)$ - $[\mathrm{m}]$ \\
\hline$e_{c}$ & Electronic charge - $[\mathrm{C}]$ \\
\hline$E_{f}$ & Fermi energy (Chapter 3) - $[\mathrm{eV}]$ \\
\hline$E_{o}$ & Potential barrier height - $[\mathrm{eV}]$ \\
\hline$E_{o, J}$ & Potential barrier height - $[\mathrm{J}]$ \\
\hline$f$ & Distribution function (Appendix A) \\
\hline$f(x, \mu, \sigma)$ & Normal distribution of mean $\mu$ and standard deviation $\sigma$ (Chapter \\
\hline & $6)$ \\
\hline$f_{\max }$ & Maximum frequency in thermal analysis (Chapter 5)- $[\mathrm{Hz}]$ \\
\hline$f_{i}$ & Fractional proportion of atoms with mass $M_{i}$ \\
\hline$f_{o}$ & Equilibrium distribution function (Appendix A) \\
\hline$f_{o}$ & Equilibrium phonon distribution, i.e. Bose-Einstein distribution \\
\hline$f_{o}$ & Laser modulation frequency (Chapter 5)- $[\mathrm{Hz}]$ \\
\hline$f_{s}$ & Laser repetition frequency (Chapter 5$)-[\mathrm{Hz}]$ \\
\hline$G$ & Atomic stiffness constant of the host lattice $-\left[\mathrm{N} / \mathrm{m}^{2}\right]$ \\
\hline
\end{tabular}




\begin{tabular}{|c|c|}
\hline$G$ & Electron-phonon coupling factor $-\left[\mathrm{W} / \mathrm{m}^{3} \mathrm{~K}\right]$ \\
\hline$G_{i}$ & $\begin{array}{l}\text { Average atomic stiffness constant between an impurity atom and } \\
\text { the nearest-neighbor atoms - }\left[\mathrm{N} / \mathrm{m}^{2}\right]\end{array}$ \\
\hline$h$ & Plank's constant $-6.626 \times 10^{-34} \mathrm{Js}$ \\
\hline$H(\omega)$ & Frequency domain temperature response (Chapter 4) \\
\hline$h(t)$ & Time domain temperature response (Chapter 4) \\
\hline$h_{\mathrm{BD}, \mathrm{eff}}$ & Total effective conductance from resistor network analysis (Chapter \\
\hline & 7) $-\left[\mathrm{W} / \mathrm{m}^{2} \mathrm{~K}\right]$ \\
\hline$h_{\mathrm{BD}, \mathrm{e}}$ & Electron dominated thermal boundary conductance (Chapter 2) - \\
\hline & {$\left[\mathrm{W} / \mathrm{m}^{2} \mathrm{~K}\right]$} \\
\hline$h_{\text {layer }}$ & Effective layer conductance (Chapter 7 ) - $\left[\mathrm{W} / \mathrm{m}^{2} \mathrm{~K}\right]$ \\
\hline$h_{C}$ & Thermal contact conductance $-\left[\mathrm{W} / \mathrm{m}^{2} \mathrm{~K}\right]$ \\
\hline$h_{P}$ & Plank's constant (Chapter 7) - [Js] \\
\hline$I_{\mathrm{DS}}$ & Transistor source-to-drain current - $[\mathrm{A}]$ \\
\hline$J$ & Average DC current in Black's equation (Chapter 1) - $[\mathrm{A}]$ \\
\hline$J$ & Laser Fluence - $\left[\mathrm{J} / \mathrm{m}^{2}\right]$ \\
\hline$J_{q x}(x)$ & Heat flux in the $\mathrm{x}$ direction $-[\mathrm{J} / \mathrm{s}]$ \\
\hline$K$ & Atomic force constant - $[\mathrm{N} / \mathrm{m}]$ \\
\hline$k(x, y, z)$ & Thermal parameters (Chapter 1$)-\left[\mathrm{W} / \mathrm{m}^{3}\right]$ \\
\hline$k_{\mathrm{TIM}}$ & Thermal conductivity of TIM material - $[\mathrm{W} / \mathrm{mK}]$ \\
\hline$k_{B}$ & Boltzmann constant - $[\mathrm{J} / \mathrm{K}]$ \\
\hline$L$ & Phonon coherence length (Chapter 2) - [m] \\
\hline$l$ & Phonon mean free path $-[\mathrm{m}]$ \\
\hline
\end{tabular}




\begin{tabular}{|c|c|}
\hline$L_{\mathrm{CI}}$ & Confidence interval length (Chapter 6) \\
\hline$l_{c}$ & Mean free path, also called collision length - $[\mathrm{m}]$ \\
\hline$l_{r}$ & Relaxation length - [m] \\
\hline$M$ & Atomic mass of large atom (Appendix A) - $[\mathrm{kg}]$ \\
\hline$m$ & Atomic mass of small atom (Appendix A) - $[\mathrm{kg}]$ \\
\hline$m_{e, s c}$ & Effective mass of the electrons/holes in a semiconductor (Chapter \\
\hline & $7)-[\mathrm{kg}]$ \\
\hline$N$ & Donor/acceptor concentration (Chapter 7$)-\left[\mathrm{m}^{-3}\right]$ \\
\hline$n(\omega)$ & $\begin{array}{l}\text { \# of phonon states between } \omega \text { and } \omega+d \omega \text { per unit volume per } \\
\text { polarization }-\left[\mathrm{s}^{2} / \mathrm{m}^{3}\right]\end{array}$ \\
\hline$N(\vec{k})$ & $\#$ of phonon states between $k$ and $k+d k$ per polarization \\
\hline$n(\vec{k})$ & $\begin{array}{l}\text { \# of phonon states between } k \text { and } k+d k \text { per unit volume per } \\
\text { polarization }-\left[1 / \mathrm{m}^{4}\right]\end{array}$ \\
\hline$N_{p}$ & $\begin{array}{l}\text { Number of pulses per "on" portion of the modulation envelope } \\
\text { (Chapter } 5)\end{array}$ \\
\hline$p$ & Degrees of freedom (Appendix A) \\
\hline$P(\omega)$ & Sampling function in the frequency-domain (Chapter 4) \\
\hline$p(t)$ & Sampling function in the time-domain (Chapter 4) \\
\hline$P(x, y, z)$ & Volumetric power distribution - $\left[\mathrm{W} / \mathrm{m}^{3}\right]$ \\
\hline$p_{\mathrm{sn}}(t)$ & Sinusoidal modulation waveform (Chapter 4) \\
\hline$p_{\mathrm{sq}}(t)$ & Pulsed modulation waveform (Chapter 4) \\
\hline$Q$ & $\begin{array}{l}\text { Activation energy for grain-boundary diffusion in Black's equation } \\
\text { (Chapter } 1)-[\mathrm{eV}]\end{array}$ \\
\hline
\end{tabular}




\begin{tabular}{|c|c|}
\hline$q \chi$ & Electron affinity - $[\mathrm{eV}]$ \\
\hline$q \phi_{m}$ & Metal work function - $[\mathrm{eV}]$ \\
\hline$q \phi_{B, n}$ & Potential barrier height (n-type) - $[\mathrm{eV}]$ \\
\hline$q \phi_{s c, n}$ & Semiconductor work function (n-type) - $[\mathrm{eV}]$ \\
\hline$Q_{\mathrm{pm}}$ & Pump energy (Chapter 4$)-[\mathrm{J}]$ \\
\hline$Q_{\mathrm{pr}}$ & Probe energy (Chapter 4$)-[\mathrm{J}]$ \\
\hline$R$ & Reflectance \\
\hline$R^{i \rightarrow j}$ & Probability of phonon reflection at an interface \\
\hline$R_{\mathrm{th}}$ & Absolute Thermal Resistance - $[\mathrm{K} / \mathrm{W}]$ \\
\hline$R_{\mathrm{TIM}}$ & Thermal resistance of TIM material - $\left[\mathrm{m}^{2} \mathrm{~K} / \mathrm{W}\right]$ \\
\hline$R_{\mathrm{BD}}$ & Thermal boundary resistance $-\left[\mathrm{m}^{2} \mathrm{~K} / \mathrm{W}\right]$ \\
\hline$R_{C}$ & Thermal contact resistance $-\left[\mathrm{m}^{2} \mathrm{~K} / \mathrm{W}\right]$ \\
\hline$R_{i}$ & Outlier ESD test statistic (Chapter 5) \\
\hline$R_{\mathrm{BD}, e e}$ & Electron-electron boundary resistance (Chapter 7$)-\left[\mathrm{m}^{s} \mathrm{~K} / \mathrm{W}\right]$ \\
\hline \multirow[t]{2}{*}{$R_{\mathrm{BD}, e l, t e}$} & Electron boundary resistance due to thermionic emission (Chapter \\
\hline & 7) $-\left[\mathrm{m}^{s} \mathrm{~K} / \mathrm{W}\right]$ \\
\hline$R_{\mathrm{BD}, e l, t n}$ & Electron boundary resistance due to tunneling (Chapter 7$)-\left[\mathrm{m}^{s} \mathrm{~K} / \mathrm{W}\right]$ \\
\hline$R_{\mathrm{BD}, e l}$ & Electrical boundary resistance (Chapter 7$)-\left[\Omega / \mathrm{m}^{2}\right]$ \\
\hline$R_{\mathrm{BD}, e p}$ & Electron-phonon boundary resistance (Chapter 7$)-\left[\mathrm{m}^{s} \mathrm{~K} / \mathrm{W}\right]$ \\
\hline$R_{\mathrm{BD}, p e}$ & Phonon-electron boundary resistance (Chapter 7$)-\left[\mathrm{m}^{s} \mathrm{~K} / \mathrm{W}\right]$ \\
\hline$R_{\mathrm{BD}, p p}$ & Phonon-phonon boundary resistance (Chapter 7$)-\left[\mathrm{m}^{s} \mathrm{~K} / \mathrm{W}\right]$ \\
\hline 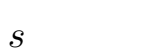 & Sample standard deviation (Chapter 6) \\
\hline
\end{tabular}




\begin{tabular}{|c|c|}
\hline$S(x, t)$ & Laser source term - $\left[\mathrm{W} / \mathrm{m}^{3}\right]$ \\
\hline$S_{P}$ & $\begin{array}{l}\text { Specularity parameter, i.e. the probability of specular phonon scat- } \\
\text { tering at an interface }\end{array}$ \\
\hline$S_{p}^{\Psi}$ & Sensitivity coefficient (Chapter 5)- $[\mathrm{s}]$ \\
\hline$T$ & Temperature - $[\mathrm{K}]$ \\
\hline$T(x, y, z)$ & Temperature distribution - $[\mathrm{K}]$ \\
\hline$t_{\min }$ & Minimum time delay considered (Chapter 5)- $[\mathrm{s}]$ \\
\hline$T_{\text {Node }}$ & Time between Gibbs nodes (Chapter 5 )- $[\mathrm{s}]$ \\
\hline$T_{\text {peak }}$ & Time period between Gibbs oscillations (Chapter 5)- [s] \\
\hline$T_{\text {Start }}$ & Time of first Gibbs node (Chapter 5)- $[\mathrm{s}]$ \\
\hline$T_{a}$ & Ambient temperature - $[\mathrm{K}]$ \\
\hline$T_{e}$ & Effective electron sub-system temperature - $[\mathrm{K}]$ \\
\hline$T_{j}$ & Junction temperature at microprocessor surface - $[\mathrm{K}]$ \\
\hline$T_{m}$ & Crystal melting temperature (Chapter 7$)-[\mathrm{K}]$ \\
\hline$T_{o}$ & Initial system temperature - $[\mathrm{K}]$ \\
\hline$T_{o}$ & Laser modulation period (Chapter 5 )- $[\mathrm{s}]$ \\
\hline$T_{p}$ & Phonon sub-system temperature - $[\mathrm{K}]$ \\
\hline$T_{s}$ & Laser repetition period (Chapter 5$)-[\mathrm{s}]$ \\
\hline$t_{\alpha / 2, n-1}$ & $\begin{array}{l}\text { t-Student distribution at the } \alpha / 2 \text { point for } n-1 \text { degrees of freedom } \\
\text { (Chapter } 6)\end{array}$ \\
\hline$T_{D, x}$ & $\begin{array}{l}\text { Molecular dynamics Debye temperature of material } x \text { (Chapter } 7 \text { ) } \\
-[\mathrm{K}]\end{array}$ \\
\hline$T_{e, \max }$ & Peak electron temperature (Chapter 3 ) - $[\mathrm{K}]$ \\
\hline
\end{tabular}




\begin{tabular}{|c|c|}
\hline$u_{j}$ & Displacement of the $j^{\text {th }}$ atom of mass $m-[\mathrm{m}]$ \\
\hline$V_{\text {Noise }}^{\Psi}$ & Lock-in amplifier noise voltage (Chapter 5) - [V] \\
\hline$V_{\mathrm{Sig}}^{\Psi}$ & Lock-in amplifier signal voltage (Chapter 5) - [V] \\
\hline$v_{\mathrm{F}}$ & Fermi velocity - $[\mathrm{m} / \mathrm{s}]$ \\
\hline$V_{\mathrm{TH}}$ & Transistor threshold voltage - [V] \\
\hline$v_{D}$ & Debye velocity - $[\mathrm{m} / \mathrm{s}]$ \\
\hline$v_{g}$ & Phonon group velocity - $[\mathrm{m} / \mathrm{s}]$ \\
\hline$v_{j}$ & Displacement of the $j^{t h}$ atom of mass $M-[\mathrm{m}]$ \\
\hline$V_{o}$ & Atomic volume (Appendix A) - $\left[\mathrm{m}^{3}\right]$ \\
\hline$W$ & Depletion layer width (Chapter 7) - [m] \\
\hline$w(z)$ & Laser beam waist as a function of distance - $[\mathrm{m}]$ \\
\hline$w_{o}$ & Laser beam waist (Chapter 3$)-[\mathrm{m}]$ \\
\hline$X(\omega)$ & Function to be sampled in the frequency-domain (Chapter 4 ) \\
\hline$x(t)$ & Function to be sampled in the time-domain (Chapter 4) \\
\hline$x_{j}$ & Atomic position of the $j^{\text {th }}$ atom - $[\mathrm{m}]$ \\
\hline$x_{j}^{o}$ & Equilibrium atomic position of the $j^{\text {th }}$ atom - $[\mathrm{m}]$ \\
\hline$X_{p}(\omega)$ & Sampled function in the frequency-domain (Chapter 4) \\
\hline$x_{p}(t)$ & Sampled function in the time-domain (Chapter 4) \\
\hline$Z$ & Atomic basis number (Appendix A) \\
\hline$Z(\omega)$ & Lock-in amplifier transfer function (Chapter 4) \\
\hline$Z_{i}$ & Acoustic impedance of material $i$ (Chapter 2$)-\left[\frac{\mathrm{kg}}{\mathrm{m}^{2} \mathrm{~s}}\right]$ \\
\hline . & Rayleigh distance (Chapter 3$)$ - $[\mathrm{m}]$ \\
\hline
\end{tabular}



6)

$d R / d T$

ENBW

MTTF

SNP

SNR

TDP
Thermoreflectance coefficient - $[1 / \mathrm{K}]$

Equivalent noise bandwidth (Chapter 5)

Mean time to failure - $[\mathrm{s}]$

Signal-to-noise parameter (Chapter 5)

Signal-to-noise ratio (Chapter 5)

Thermal Design Power - [W] 
[This page is intentionally left blank] 


\section{CHAPTER 1}

\section{INTRODUCTION}

1.1 Microprocessor Heating Trends . . . . . . . . . . . . . . . . . . . . . . . 1

1.2 So What if It's a Little Hot? . . . . . . . . . . . . . . . 6

1.3 How Big Can Such a Little Problem Be? . . . . . . . . . . . . . . . . 8

1.4 Levels of Thermal Management . . . . . . . . . . . . . . . . . 10

1.5 Chip/Module Level Thermal Management . . . . . . . . . . . . . . . . . . . 11

1.6 Transistor-Level Thermal Management . . . . . . . . . . . . . . . 17

1.7 Outline and Objectives . . . . . . . . . . . . . . 21

\subsection{Microprocessor Heating Trends}

The story of thermal management in electronic, micro-electronic, and nano-electronic devices $^{1}$ is as old as the story of the electronic computer itself. One of the first electronic computer systems was the Electronic Numerical Integrator and Computer, ENIAC, developed in the early 1940's at the University of Pennsylvania. The ENIAC weighed in at 30 tons, with a footprint of $1,500 \mathrm{ft}^{2}$, contained approximately 17,500 vacuum tubes, 70,000 resistors, 10,000 capacitors, about 5,000,000 solder joints, and consumed around $150 \mathrm{~kW}$

\footnotetext{
${ }^{1}$ Micro will generally be used to describe systems with characteristic lengths on the order of $0.1-100 \mu \mathrm{m}$, and nano will generally describe systems with characteristic lengths on the order of 1-100 nm [33].
} 
of electricity [34-36]. The nearly 18,000 vacuum tubes were cooled by a forced air/air conditioning system. While one of only a handful of computer systems at the time, the ENIAC was a major milestone in computer development and increased the speed at which calculations could be completed by over a factor of 1,000 compared to calculations using traditional hand held-devices [37].

However, while the vacuum tube based electronic computer was a major step forward in computational power, vacuum tubes were plagued with a number of issues. Vacuum tubes in the ENIAC were constantly failing, at a rate from several a day to around one tube every other day, leading to a maximum period of continuous operation of only 116 hours [38]. Most of the tube failures occurred during the time of warm-up or shutdown when the thermal stresses on the tubes were greatest.

In the 1960's, computer technology was shifted from vacuum tube based components to the recently developed bipolar transistor technology $[36,39]$. The bipolar transistor had the advantages of being smaller, consumed less power, and therefore produced less heat as compared to the vacuum tube. At the time, this lead to the thought that thermal management would no longer be an issue in device development, since "no heat [was] developed as in a vacuum tube" [35]. In order to increase computational power however, device engineers worked to improve packing density by adding more and more transistors to the circuits. This continual increase in the transistor density, while increasing system performance, had the added cost of increasing the thermal density as well. In Figure 1.1 the red data points show the heat flux produced by high performance computers during the mid 1960's through the late 1980's using bipolar transistor technologies. After 1980, of the data points shown in red in Figure 1.1, only the Fujitsu M380 and the IBM 4381 were air-cooled, while the rest, in order to manage the high thermal loads, were water-cooled systems [40]. Computer engineers were quickly reaching a point where their ability to increase the speed of high performance computers was limited by the ability to keep the systems $\mathrm{cool}^{2}$.

Thermal management received a brief respite in the early 1990's when circuit technology was shifted once again, from the bipolar transistor to the complimentary metal-oxide-

\footnotetext{
2 "Cool" here refers to operating temperatures generally in the range of 60 to $100{ }^{\circ} \mathrm{C}$. As temperature is increased, switching speeds within the device are reduced due to increased resistance of the interconnect materials. More on this later.
} 


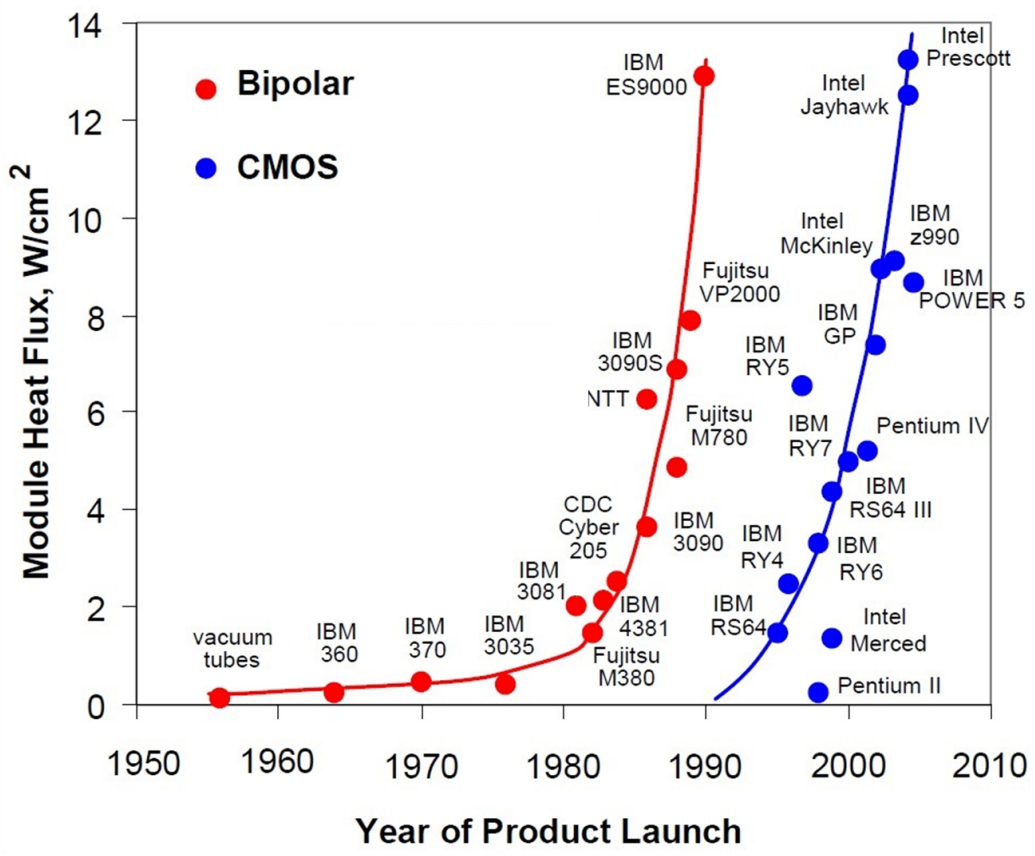

Figure 1.1: Heat flux trends of high performance computers since the 1950s for bipolar transistor and complimentary metal-oxide-semiconductor (CMOS) technologies. Plot taken from [1].

semiconductor (CMOS) transistor. CMOS transistors were cheaper to produce and required less power to operate, and therefore produced far less heat than their bipolar counterparts. However, just as in the past, although even more rapidly since the introduction of the CMOS transistor, the demand for increased performance drove increased packing densities, and pushed higher the heat flux once again, see the blue data points in Figure 1.1.

Similar trends have been observed in the consumer sector. For more than 4 decades the development of microprocessors and microprocessor technology has surpassed the trends set forth by Moore's Law [41]. "Moore's Law" states that the density of transistors in a circuit will double every 18 months $^{3}$. Figure 1.2 provides the trend in the number of transistors per die for processors released over the past 4 decades, showing that developments in the past approximately 5 years have pushed the number of transistors per die past the 1 billion mark.

\footnotetext{
${ }^{3}$ Moore's Law is in quotes, because while this statement is commonly held as the definition of Moore's Law, the statement was not made by Gordon Moore. In 1965 Moore stated that "we had about doubled every year the amount of components we could put on a chip"[41]. In 1975 he refined he prediction stating "we'd only be doubling every couple of years" [42]. The quoted "Law" is a combination of Moore's predictions and that of David House who predicted that computer performance was going to double every 18 months.
} 


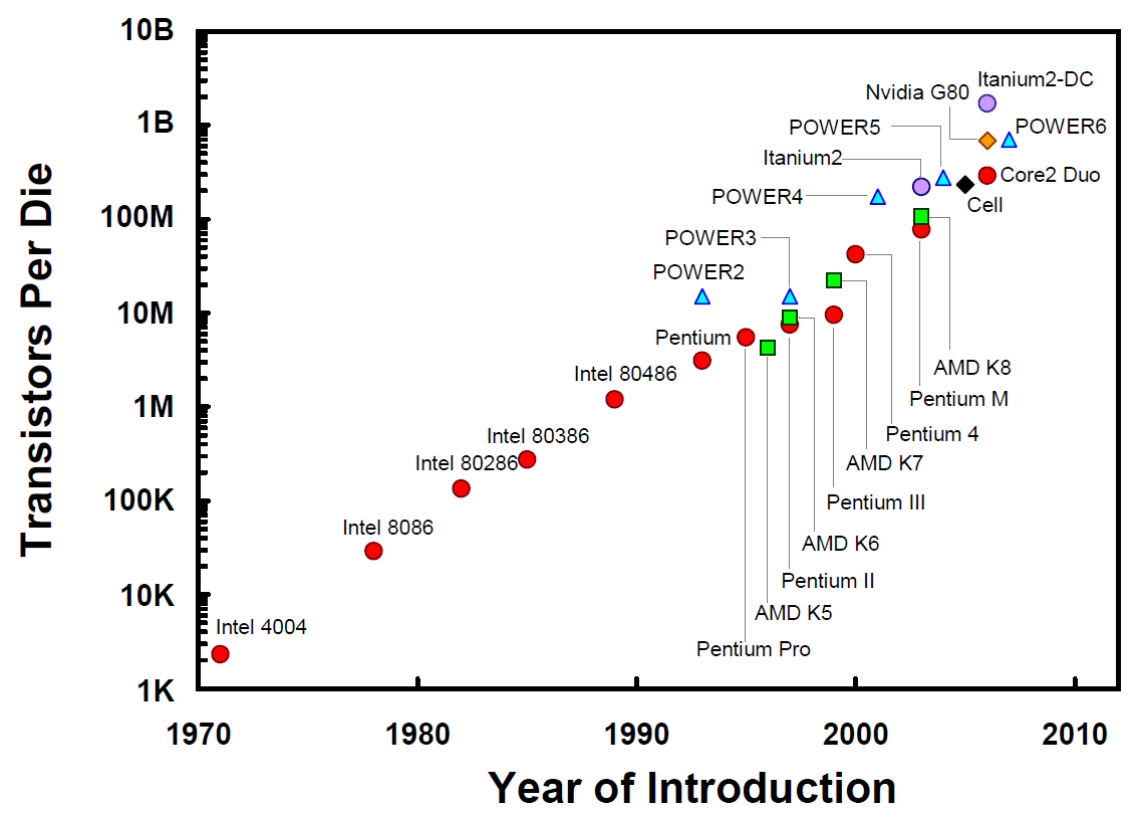

Figure 1.2: Number of transistors per die for various commercial processors developed over the past 4 decades. Plot taken from [2].

While the number of transistors on a chip has continued to increase at the rate given by "Moore's law", the size of the die itself has remained relatively unchanged. This has led to equally significant increases in the transistor density, and therefore the power and thermal densities as well. As shown in Figure 1.3, the power densities of microprocessors have continued to increase steadily since the mid-1980's. To put these power densities into perspective, the power density of the thermal energy impinging on earth, from the sun, is on the order of $0.1 \mathrm{~W} / \mathrm{cm}^{2}$, a hot plate is on the order of $10 \mathrm{~W} / \mathrm{cm}^{2}$, and a nuclear reactor has a power density on the order of $100 \mathrm{~W} / \mathrm{cm}^{2}$ [43]. It appears that device designers are reaching a limit, in commercial components, around the $100 \mathrm{~W} / \mathrm{cm}^{2}$ power density level, and are rapidly again reaching a point where one of the major roadblocks to increased device performance will be the ability to keep the devices sufficiently cool $[44,45]$.

High power densities repeatedly arising over the decades as a roadblock to device design, begs the questions as to where all this heat originates? While the events that cause the generation of heat can be complex, the underlying principle is relatively straight forward. The heat is generated as current carrying electrons (set in motion by an established potential) encounter resistance, and a portion of their energy is coupled to their surroundings 


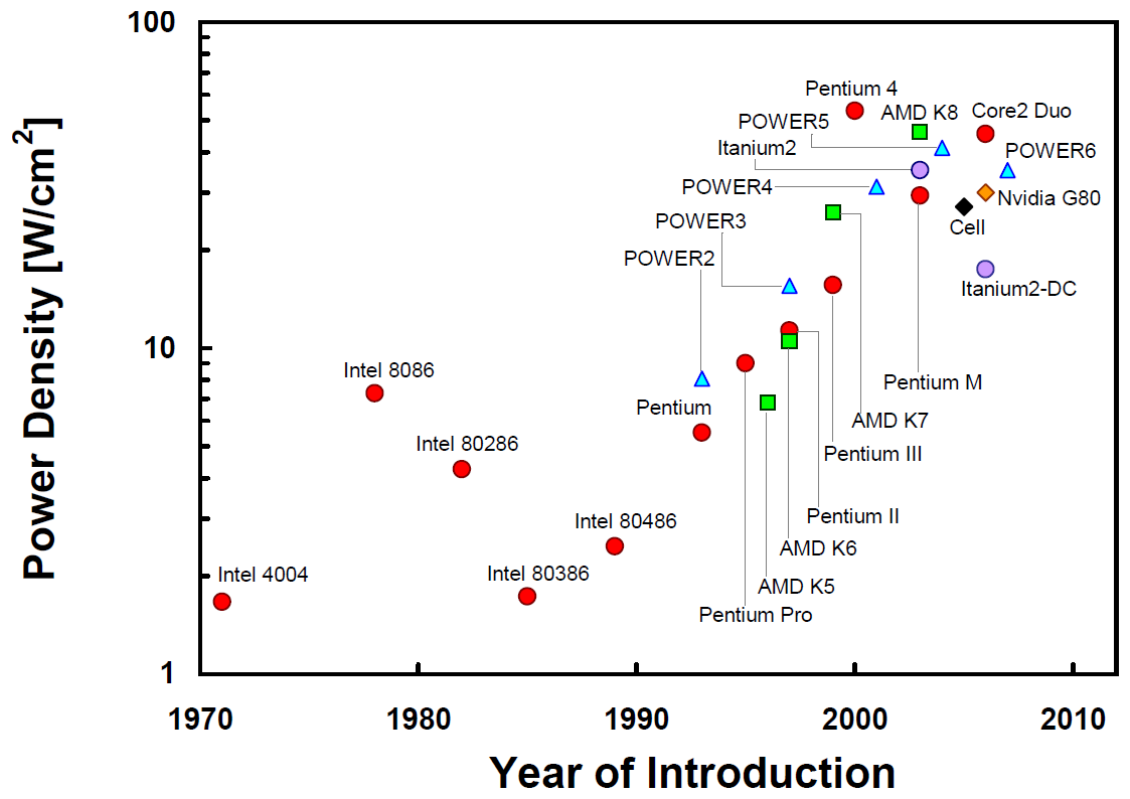

Figure 1.3: Power densities for various commercial processors developed over the past 4 decades. Plot taken from [2].

in a process known as Joule heating [44]. In CMOS structures, current is consumed from three primary sources: 1) during the dynamic switching between "on" and "off" states, 2) subthreshold leakage current, i.e. current flow from the source to drain, even in the "off" state, and 3) short-circuit current, i.e. current generated due to finite rise/fall times leaving two sections "on" simultaneously for a brief period with a direct path from the source to ground $[45,46]$. Typically, device engineers tackle the thermal management problem at the scale of the chip-level or larger [36, 47-49]. However, as history once again repeats itself, and we are rapidly approaching the power density limit in the current generation of technology, engineers are beginning to consider the thermal management problem at the scale of the individual transistors $[50,51]$, shifting the focus into the nanoscale length regime. Regardless of the size of the application, even thermal energy generated on the nanoscale is eventually passed "upward" until it is expelled to the surrounding macroscale environment.

The remainder of this Chapter, will focus on describing and illustrating the size and scope of the "thermal management problem" and how the impact is felt across various sectors of the electronic and computer industries as motivation for the fundamental science presented in this dissertation. Special care will be taken to help establish the dichotomy 
between more macro-scopically driven and nano-scopically driven affects, and establish the various levels of thermal management consideration. Finally, the remainder of the chapters presented in this dissertation will be outlined.

\subsection{So What if It's a Little Hot?}

With the increased performance and packing density in microprocessors comes another important consequence beyond just the high power density. Along with increased packing density comes an increase in the non-uniformity of the power distribution, and thereby the thermal distribution as well. Figure 1.4 shows the thermal mapping of a dual-core AMD Athlon II processor ran under various CPU load scenarios from a SPEC CPU2006 benchmark program [3]. From the figure, there are obvious regions of increased temperature under operating conditions. These regions of high heat flux, typically refereed to as
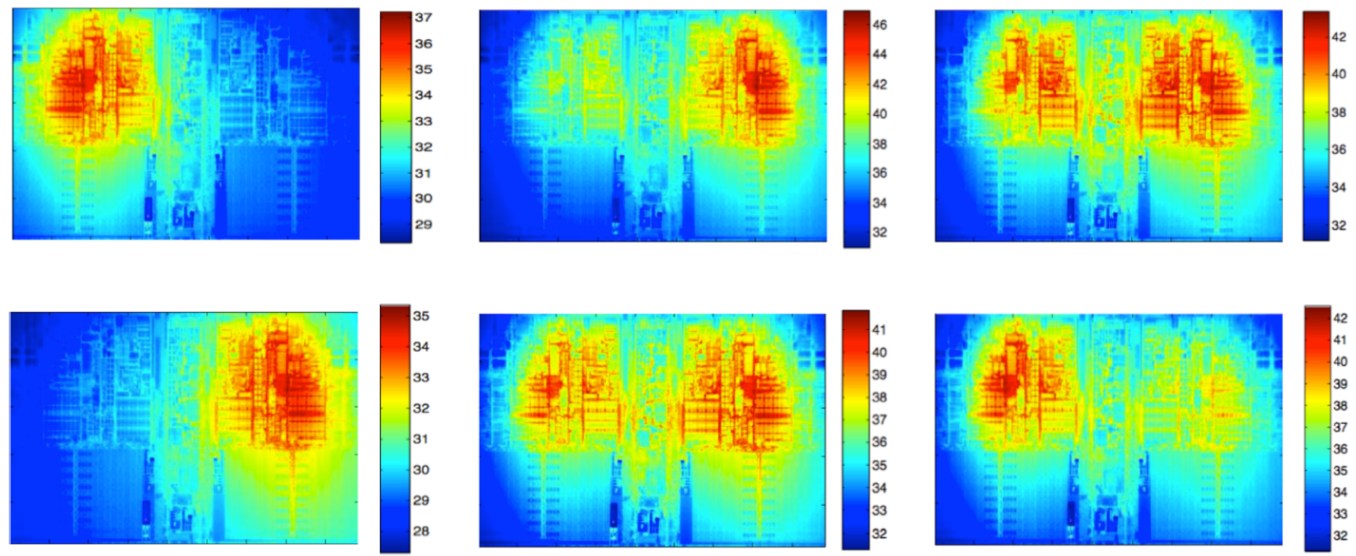

Figure 1.4: Thermal mapping of a dual-core AMD Athlon II processor under various CPU workloads. The color scale represents the temperature in degrees Celsius. Image taken from [3].

"hotspots", can have heat fluxes several times higher than those presented in Figure 1.1, on the order of $0.3-1 \mathrm{~kW} / \mathrm{cm}^{2}[48,52,53]$. These hotspots can create temperature variations across the microprocessor surface on the order of $5-30{ }^{\circ} \mathrm{C}[52,53]$. These high temperature hot spots, and large thermal gradients, are a major factor in determining the reliability of semiconductor devices $[3,46,54,55]$. If the heat generated in a microprocessor cannot be effectively removed at a rate greater than the rate of generation, the temperature of the device will rise, reducing the mean time to failure (MTTF) [3, 46]. 
The temperature of a device has a direct impact on nearly every metric used to rate the devices performance including: speed, power dissipation, device lifetime, and cost [3, 46]. The increased cost required for more robust thermal management techniques is an intuitive relationship. As an example, the limit of air cooling techniques is around $150 \mathrm{~W} / \mathrm{cm}^{2}$ on average, with an anticipated cost of approximately $\$ 3.5$ per Watt above $60 \mathrm{~W}$ [56]. The impact of temperature on the other metrics of device performance are not as intuitive, and are rooted in the design and operation of the device itself.

The reliability of a microprocessor is directly related to its temperature, particularly through the reliability of the interconnects between transistors. With increasing temperature, the integrity of the metal interconnects is degraded due to a process known as electron migration $[46,54]$. As the temperature is increased, due to increased current density, the dense flow of electrons between the power and ground tracks causes metal ions within the interconnects to migrate. This migration causes metal ions to build up in one location to form hillocks, and disappear in another to form voids, ultimately leading to open- and short-circuit failures $[46,54]$. The MTTF due to electron migration trends exponentially with temperature and can be estimated by Black's equation $[46,57]$ :

$$
\mathrm{MTTF}=A J^{-n} e^{Q / k_{\mathrm{B}} T}
$$

where $A$ is a process- and geometry-dependent constant, $J$ is the average DC current, $n=2$ under normal operating conditions, $Q$ is the activation energy for diffusion, $k_{\mathrm{B}}$ is the Boltzmann constant, and $T$ is the metal temperature. A typical goal is to achieve a 10 year lifetime, i.e without electron migration failure, at a device temperature of $100{ }^{\circ} \mathrm{C}$ [58].

The performance of a processor is determined in part, by the ability of electrical signals to travel between transistors and other parts of the chip, via the metal interconnects. This is yet another motivation for increasing the packing density of transistors, to increase speeds. Increased temperature can affect the performance of a processor in two ways, 1) reducing the carrier mobility, and 2) increasing the resistance of the interconnects. For a $130 \mathrm{~nm}$ generation processor ${ }^{4}$, the logic gate delay changes by $4 \%$ for a $40{ }^{\circ} \mathrm{C}$ change in temperature,

\footnotetext{
${ }^{4}$ The feature size is generally used to define the size of the different elements on a microprocessor chip. Because of the many different components making up the chip, it is not necessarily the smallest, therefore
} 
and the resistance of a metal interconnect changes by about $5 \%$ for the same temperature change [46]. Additionally, the non-uniformity of the temperature distribution can cause the clock frequency to become skewed across different portions of the processor. For example, the clock timing can be skewed as much as $10 \%$ for a temperature gradient of $40{ }^{\circ} \mathrm{C}$ [46].

Finally, the temperature has a direct impact on the power consumed by the device. As discussed previously, one of the major sources of current consumption in a CMOS device is subthreshold leakage. As the geometries of microprocessor design continue to decrease, the path between the source and drain in the transistor continues to shrink leading to increased leakage current. For the $90 \mathrm{~nm}$ generation of processor technology, leakage current accounted for $25-45 \%$ of the overall power consumed [46]. With the introduction of the 65 $\mathrm{nm}$ generation of processors, that number jumped to $50-70 \%$ of the total power consumed [46]. The subthreshold leakage current, i.e. the source-to-drain current, $I_{\mathrm{DS}}$, is a function of a number of parameters of the transistor, including the threshold voltage, $V_{\mathrm{TH}}$. As the temperature of the processor increases $V_{\mathrm{TH}}$ decreases, leading to a "weaker" off state, and causing an increase in leakage current $[46,59]$. It has been shown that the leakage current increases $2 \%$ for each ${ }^{\circ} \mathrm{C}$ increase in temperature, across multiple processor generations [55].

These are just a few ways in which increased temperature reduce the functionality and reliability of microprocessor devices. What is evident from these examples is that increased temperature does not have an isolated affect on processors. The cost of thermal management cannot simply be weighted in terms of sacrificing processor speed, or processor lifetime, but will impact all aspects of the final product. Making matters worse, the microprocessor is usually only one component, in a much larger structure.

\subsection{How Big Can Such a Little Problem Be?}

It is estimated that in 2008 there were 108 million PCs in offices across the US, costing $\$ 4.2$ billion in energy consumption [45], and that is only a fraction of the story. One of the major challenges of thermal management in the micro- and nano-electronics industry currently being faced is that the problem is not isolated to just individual processors. The

$45 \mathrm{~nm}$ "generation" chips will have features smaller than $45 \mathrm{~nm}$. The generation classification is generally the half-pitch, or half the distance between metallic connections. 
invention and proliferation of the internet and its subsidiary services such as online banking, online shopping, cloud computing, etc. have created a huge demand for data centers. The video sharing website YouTube alone reports 72 hours of video content is uploaded every minute [60].

In 2005 the worldwide electricity demand for power in data centers reached 152 billion $\mathrm{kWh}$ per year, representing $0.97 \%$ of the total worldwide electricity consumption [61]. This consumption is expected to increase dramatically in the near future as developing nations increase technology consumption. Currently $58 \%$ of the world's computers are found in the US, Japan, and Eastern Europe, while these countries only contain $15 \%$ of the world's population [45]. The United States alone consumed 45 billion $\mathrm{kWh}$ in 2005 to power its data centers, costing around $\$ 2.7$ billion and representing around $1.2 \%$ of the total electricity consumption. This value is expected to increase in the coming years, with one-third of the total US power consumption expected to be due to electronic systems by 2025 [45].

Of the electricity consumed in data centers, only 50\% is used by the IT systems [61]. That is the systems that actually are responsible for the storage, retrieval, manipulation and transmission of data. The remaining electricity is used by the thermal management structure, $33 \%$, and for the distribution of the electrical power, $17 \%$ [61]. The energy efficiency of a data center is typically represented by the power utilization effectiveness, the PUE, which represents the ratio of the total energy consumed by the center to that used by only the IT equipment. For data centers, depending on the type of cooling systems employed, the PUE ranges from 1.1-2.7 [61]. Based on the current electricity budget allocated to thermal management and the high cost per $\mathrm{kWh}$, thermal management represents a significant cost to keeping the worlds "technological infrastructure" running efficiently.

In thermal management one of the major challenges is simply the size and scope of the problem. Considering that the generation of the parasitic heat originates in the interconnects of the transistor device itself, with lengths on the order of nanometers, and that this heat scales be a major issue for data centers with length scales on the order of 10s of meters, it is obvious that a one size fits all approach will not be sufficient to tackle the problem. The design of microprocessors up-to data centers, combines disciplines and integrates electronic, thermal, electrical, materials, electromagnetic, chemical, mechanical, fluid, etc. fields. 


\subsection{Levels of Thermal Management}

In the field of thermal management we can typically define 4 packaging levels: 1) the chip/module-level, 2) the board-level, 3) the system-level, and 4) the more recently established transistor-level, as discussed previously due to the continued size reduction and the increased packing density of transistors in a processor, see Figure 1.5. The transistor-

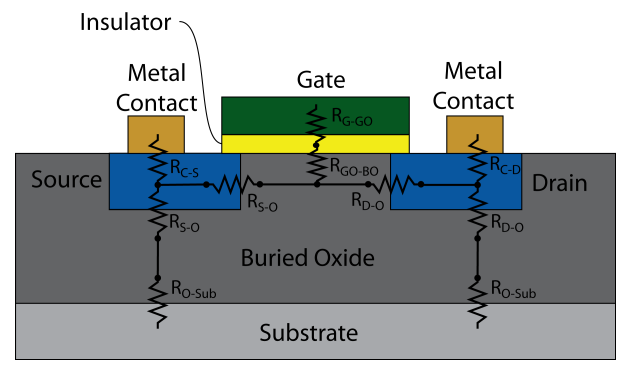

(a)

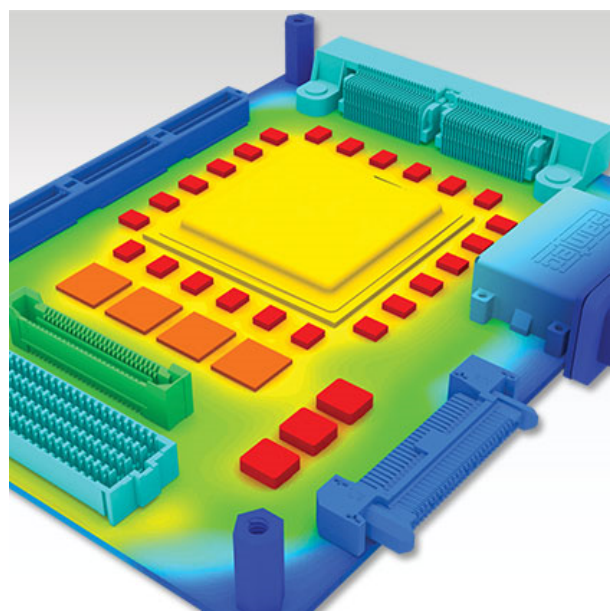

(c)

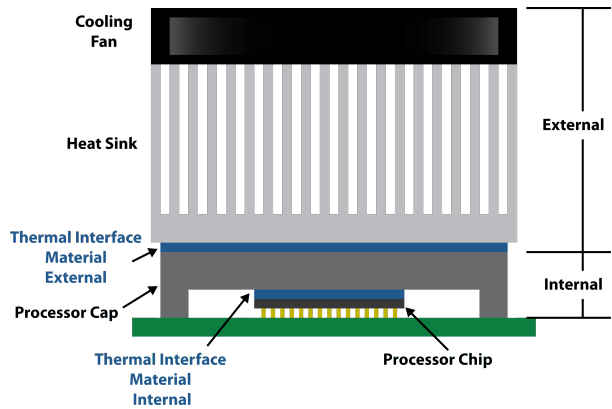

(b)

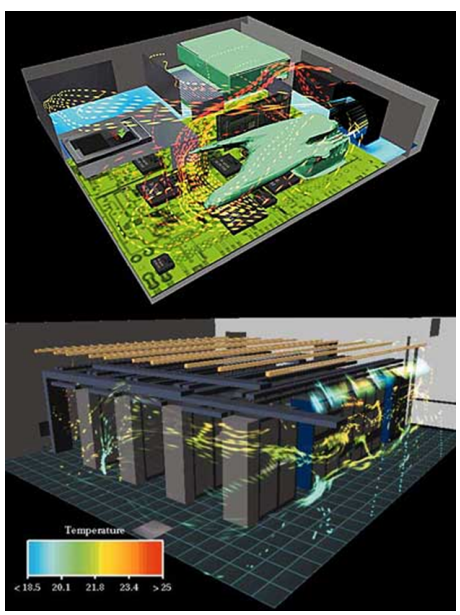

(d)

Figure 1.5: The 4 primary packaging levels, that are of interest in thermal management (a) the transistorlevel, (b) the chip/module-level, (c) the board-level (image from [4]), and (d) the-system level (image from $[5])$.

level focuses on the solid-solid interfaces of the semiconductor components, and the metal connections and interconnects between transistors, see Figure 1.5a. The chip/module-level focuses on the processor as a whole and its packaging up to the first level of the heat sink, see Figure 1.5b. The board-level considers the placement of active thermal components on a larger board structure, e.g. a motherboard, to reduce the concentration of the thermal load, see Figure 1.5c. The definition of the system-level can be more ambiguous and can 
depend on the particular device. The system-level can represent for example; the case of a smartphone, the case of a desktop computer, or a room full of racks in a data center, see Figure 1.5d. Regardless of the size, the system-level typically represents the largest amount of gathered heat being exchanged with the surrounding environment.

While all of these levels share a common issue of excess heat, the particular challenges facing device designers at these levels vary. At the system-level the challenge is to remove the most heat, using the least volume and/or mass. Management at the board-level requires the removal of a large amount of heat with minimal thermal gradients, and the chip-level challenges arise from localized hot-spots and size effects [6]. The transistor-level is dominated by size effects, where vibrational mismatch between materials, and energy carrier bottlenecks increase thermal resistance [44, 45].

For the purposes of this dissertation, the primary focus and motivation will be based on the transistor- and chip/module-level of a system. For typical computer systems, this will encompass a scale from the heat sink on a processor on down to the individual transistors. While the fundamental science developed in this dissertation will be focused on solid-solid interfaces such as those found in the transistors, the principles will in general scale to the interface between the processor and the heat sink.

\subsection{Chip/Module Level Thermal Management}

While engineers have done well with keeping up with Moore's Law, an interesting picture arises when observing the power density trends shown in Figure 1.3. Over the past decade, even though the number of transistors per die has nearly exponentially increased, microprocessor power densities have begun to cluster around the $100 \mathrm{~W} / \mathrm{cm}^{2}$ level. Device designers appear to be running into a "power density wall" at this level $[45,56]$. In part, the reason for this limit can be seen in Figure 1.6. The figure shows that at the $100 \mathrm{~W} / \mathrm{cm}^{2}$ power density level and below, the thermal energy can be managed by relatively simple heat spreaders and heat sinks. Above that level, more complex and therefore more expensive, methods would be required, which are not practical at the consumer level.

To better understand the mechanisms behind this roadblock, and in order to develop new 


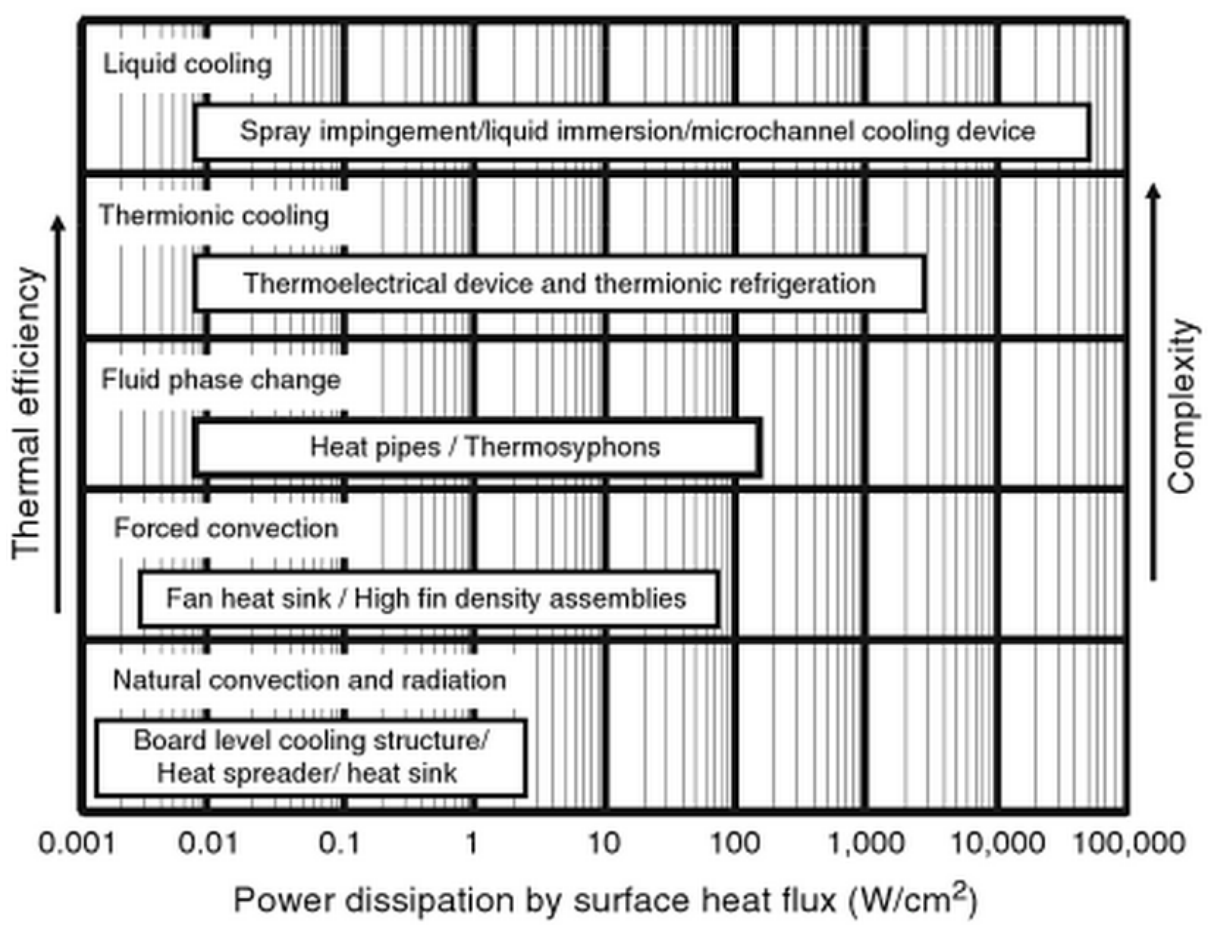

Figure 1.6: Methods of thermal management solutions, for various power dissipation levels and system complexities. Image reproduced with permission from [6].

strategies to move beyond these limitations, the source of the issue must be investigated. Consider the most classical view of thermal conduction, assuming diffusive processes, in a microprocessor through a steady state Fourier's law analysis [56]

$$
\nabla(-k(x, y, z) \nabla T(x, y, z))-P(x, y, z)=0
$$

where $P(x, y, z)$ is the volumetric power distribution, $T(x, y, z)$ is the temperature distribution, and $k(x, y, z)$ is the thermal parameters of the package. Equation 1.2 shows that the average temperature of the microprocessor chip is a function of the total dissipated power within the device. In order to better quantify this relationship in terms of needs for thermal management, thermal designers use a metric designated as the thermal design power, or TDP. The TDP is the maximum amount of power dissipated by the microprocessor during "realistic operations" 5 .

The primary task for thermal engineers, and the major thermal management problem

\footnotetext{
${ }^{5}$ This is not the maximum power that the chip will ever draw. Brief periods of increased activity are acceptable, provided that they fall within the thermal time constant of the system [62].
} 
at this scale, is to remove the TDP from the chip surface and expel it to the surroundings. The temperature at the chip is typically referred to as the junction temperature, $T_{j}$, and the temperature of the ambient surroundings, typically the air inside the case, is given by $T_{a}$. For the device to perform efficiently $T_{j}$ has to be maintained below certain specifications, such that the TDP can be effectively removed. Removal of the TDP from the microprocessor surface is traditionally where thermal management solutions have begun. Figure 1.7 shows a cross-section of a typical consumer level module. The module can typically be broken into two regions of interest, the internal (passive) and external (active) sections, where conduction and convection are the primary thermal transport mechanism in each section respectively [53].

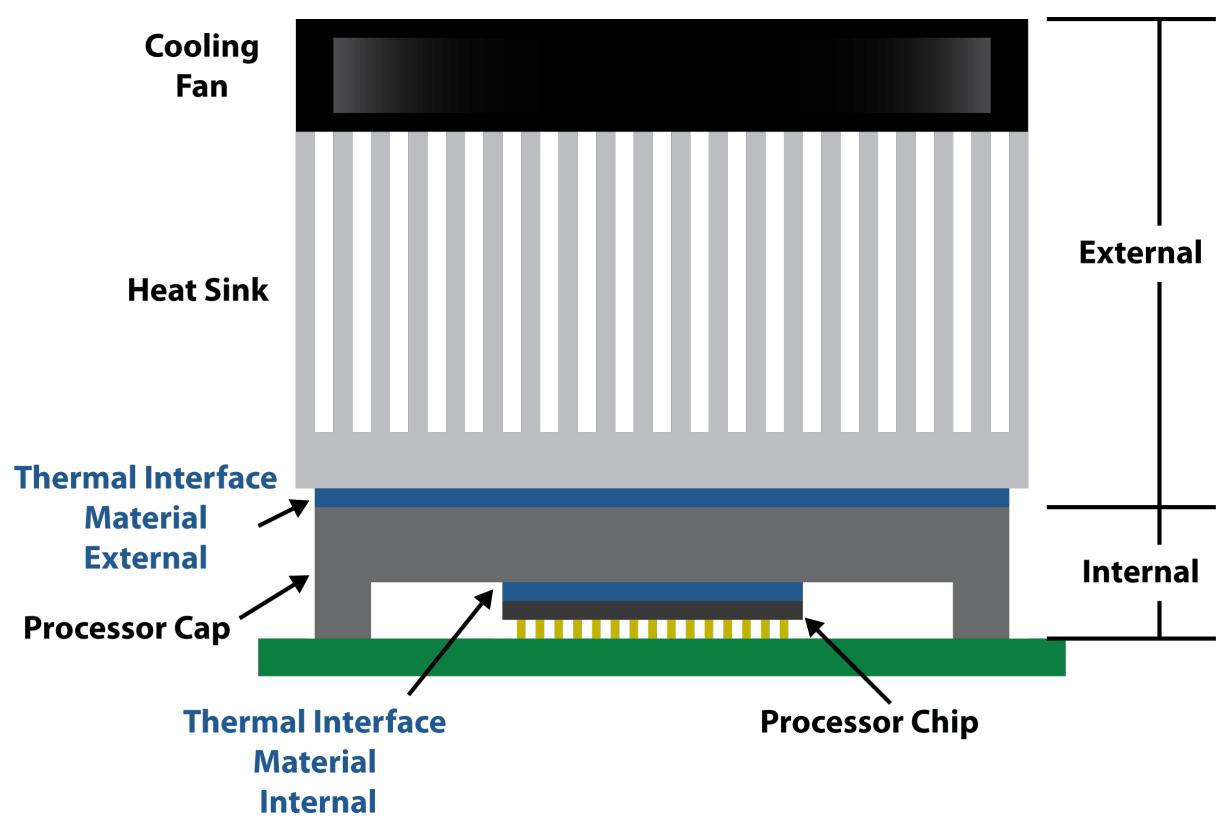

Figure 1.7: Cross-section view of a typical module found in most consumer grade desktop machines, denoting the primary components and the internal and external structures.

To be removed effectively, the TDP must conduct through various solid layers and interfaces from the microprocessor unit, into the heat sink, and then finally into the ambient surroundings. Heat flow is impeded through these various materials, structures, and interfaces. Therefore, there is an absolute thermal resistance, $R_{\mathrm{th}}$, dictated by the module's physical structure and the thermophysical properties of its components. In the most general 
sense, the required absolute thermal resistance for a device is dictated by [48]:

$$
R_{\mathrm{th}}=\frac{\left(T_{j}-T_{a}\right)}{\mathrm{TDP}}
$$

More specifically $R_{\mathrm{th}}$ can be broken into two primary regions, 1) resistance in the solid structure of the microprocessor and through the interface to the system packaging, and 2) across the interface from the packaging to the heat sink and into the surrounding atmosphere, see Figure 1.8. A large body of research in the field of thermal management

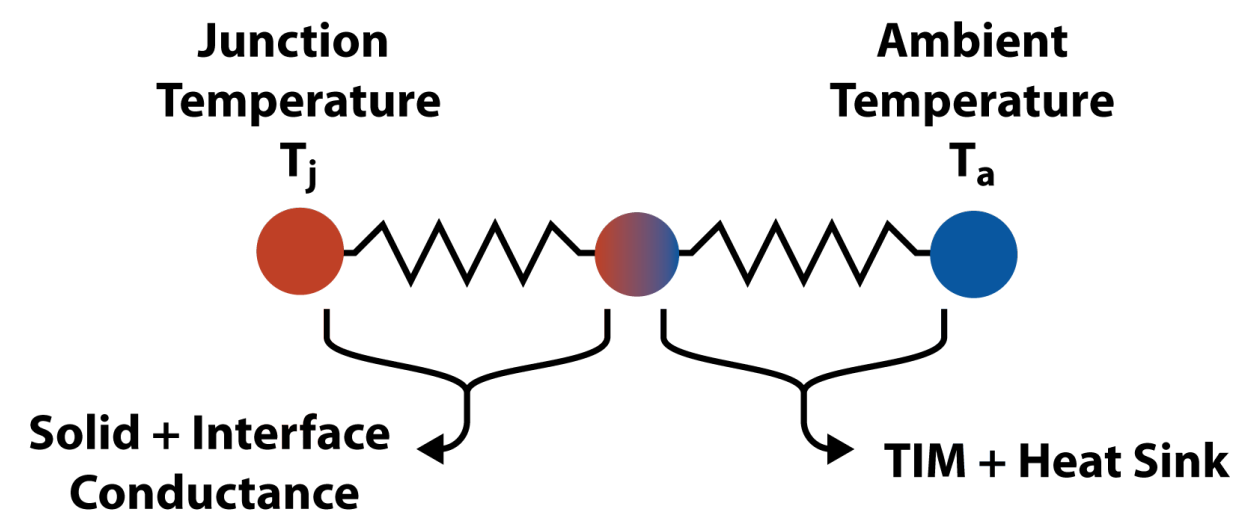

Figure 1.8: Temperature regions and primary thermal resistances in typical microprocessor cooling.

has been dedicated to increasing the efficiency of cooling solutions primarily by increasing thermal conduction across the module junctions via thermal interface materials, TIMs, and by increasing the efficiency of heat sinks via air- and liquid-cooled systems.

The resistance to the flow of thermal energy from a hot spot on a processor chip to the heat sink, see Figure 1.7, is dominated by what is defined as thermal contact resistance (TCR), its inverse quantity being the thermal contact conductance (TCC). As heat flows across the mechanical joints in the system, a temperature drop across the interface is realized. Therefore the TCR across an interface can be defined as:

$$
R_{C}=\frac{\Delta T}{\dot{q}}=\frac{1}{h_{\mathrm{C}}}
$$

where $\Delta T$ is the temperature drop, $\dot{q}$ is the heat flux, and $h_{\mathrm{C}}$ is the thermal contact conductance. The TCR is a function of several geometric and thermophysical properties, 


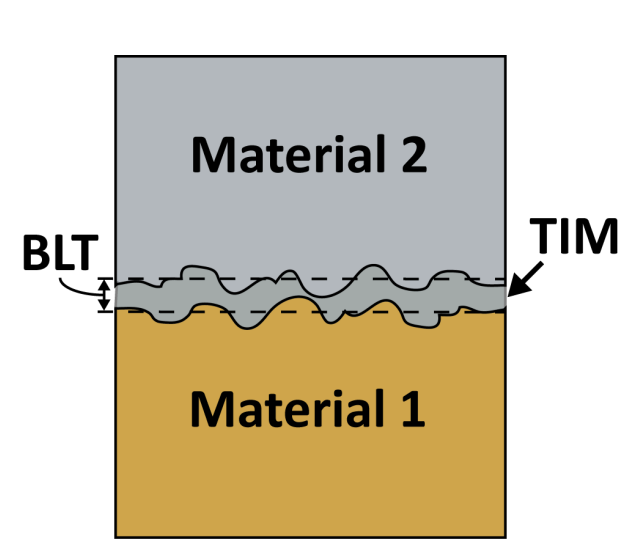

(a)

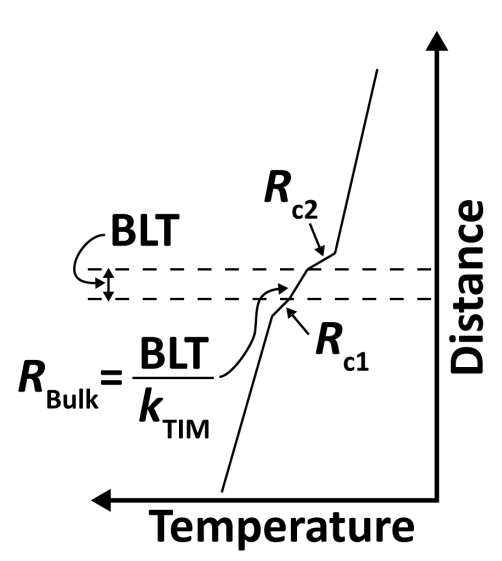

(b)

Figure 1.9: (a) Schematic of mechanical contact between two materials, showing the surface asperities and reduced contact between the surfaces, as well as an example of an ideal TIM. (b) Schematic of the temperature distribution of two materials in contact with the addition of a TIM.

including the surface roughness, surface flatness, the thermal conductivity of the materials and the surface microhardness [63]. One of the largest contributors to TCR between two materials is microscopic asperities on each surface. When two surfaces are brought into contact, these asperities limit the actual area of contact, which can be as little as 1-2\% depending on loading [28], see Figure 1.9a. Therefore the total resistance is a combination of the resistance across the actual contact area, and the resistance across the fluid occupying the area between contact points. In order to reduce the overall resistance across the joint, the gaps between the asperities that are normally filled with air, are replaced by a higher thermal conductivity material, typically refereed to as a thermal interface material.

The overall effectiveness of the TIM, is based on 3 different factors: 1) the thermal conductivity of the TIM material, 2) the bond line thickness (BLT), i.e. the RMS thickness of the TIM material, and 3) the contact resistance between the individual components [28]. Therefore the total resistance of the mechanical joint is given by [28]:

$$
R_{\mathrm{TIM}}=\frac{\mathrm{BLT}}{k_{\mathrm{TIM}}}+R_{\mathrm{c} 1}+R_{\mathrm{c} 2}
$$

where BLT is the bond line thickness, $k_{\text {TIM }}$ is the thermal conductivity of the TIM material, and $R_{\mathrm{c} 1, \mathrm{c} 2}$ is the contract resistance between the materials on either side of the interface and the TIM material. From Equation 1.5, we can see that there are 3 primary methods 
Table 1.1: Properties of various types of TIMs [28-31].

\begin{tabular}{llll}
\hline $\begin{array}{l}\text { Material } \\
\text { Type }\end{array}$ & Typical Composition & $\begin{array}{l}\text { BLT } \\
{[\mathbf{m m}]}\end{array}$ & $\begin{array}{l}k_{\mathrm{TIM}} \\
{[\mathbf{W} / \mathbf{m K}]}\end{array}$ \\
\hline $\begin{array}{l}\text { Grease } \\
\text { Gel }\end{array}$ & AlN1, Ag, ZnO, Silicon Oil & 2.2 & $0.5-7.5$ \\
$\begin{array}{l}\text { Phase Change } \\
\text { Material }\end{array}$ & $\begin{array}{l}\text { Polyolefins, epoxies, polyesters, acrylics, BN, Alu- } \\
\text { mina, Al, Carbon Nanotubes }\end{array}$ & $1-1.5$ & $3-4$ \\
$\begin{array}{l}\text { Phase Change } \\
\text { Metallic Alloy }\end{array}$ & Pure In, In/Ag, Sn/Ag/Cu, In/Sn/Bi & $0.5-5$ \\
Solder & Pure In, In/Ag, Sn/Ag/Cu, In/Sn/Bi & $2-5$ & $30-50$ \\
Adhesives & Ag particles in epoxy matrix & $2-5$ & $30-50$ \\
& & $* * * *$ & $* * * *$ \\
\hline
\end{tabular}

which can be used to reduce the thermal resistance across the joint: 1) decrease the BLT, 2) increase the thermal conductivity of the TIM, and 3) reduce the contact resistance between the TIM and the two mating surfaces.

The thermal conductivity of the TIM can be increased by using a high thermal conductivity material, or using a lower thermal conductivity base material that is loaded with a percentage of high thermal conductivity materials such as metals or carbon nanotubes [64-67]. Properties of some of the common types of TIM materials are provided in Table 1.1. The thermal resistance across the joint can also be reduced by reducing the bond line thickness. This can be achieved by using materials with a low elastic modulus. Typically these two properties run in opposition of each other. Materials such as solders and highly laden polymers have high thermal conductivities, but are generally less mechanically compliant leading to increased bond line thicknesses. On the other hand, polymers and low density polymer composites generally have good mechanical compliance, but low thermal conductivity. The ideal properties of a TIM would have both high thermal conductivity and high mechanical compliance.

Finally, the last property to change the resistance in Equation 1.5 is the contact resistance between the base materials and the TIM, $R_{\mathrm{c} 1}$ and $R_{\mathrm{c} 2}$. These contact resistances arise from two sources; 1) analogous to the original problem, but at a smaller scale, air gaps between the TIM and the base material due to imperfect wetting of the surface, see Figure 
1.10, and 2) differences in the thermophysical properties between the base material and the TIM. This implies that even for an ideal TIM, i.e. one that perfectly wets the material's surface, there will still be a resistance to the flow of thermal energy due to the presence of the boundary between the dissimilar materials. It is this boundary resistance, between two ideally wet interfaces, that will be the focus of the work in this dissertation, as it is the macroscopic analog of the type of interface resistance observed in transistor-level thermal management.

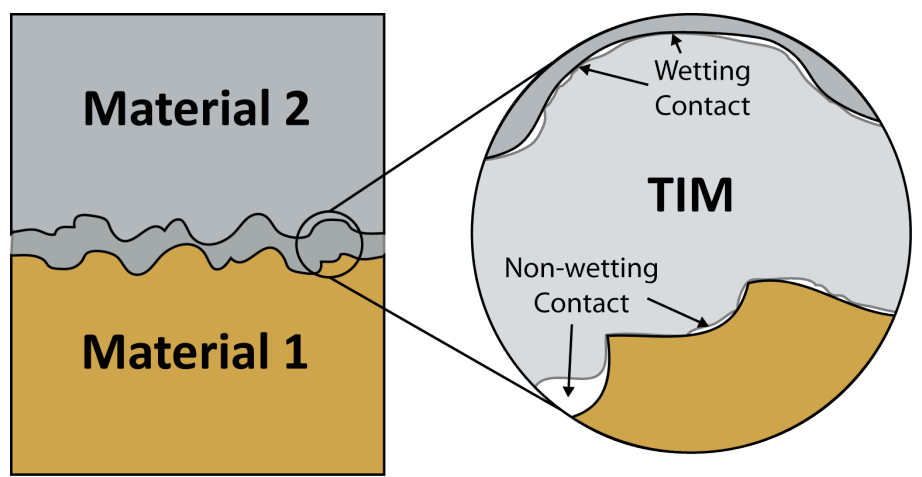

Figure 1.10: Schematic of a microscope contact area of a TIM between two materials, showing areas of wetting and non-wetting contact.

\subsection{Transistor-Level Thermal Management}

While much work has gone into dealing with chip level hotspots $[28,29,36,40,48,52$, $65,66,68,69]$, as the size of device features continues to shrink into the nanometer length scale thermal management becomes more complex. Consider that the Intel ${ }^{\circledR} 386$ processor released in 1985 contained 275,000 transistor on a die about the size of a postage stamp, with feature sizes on the order of $1 \mu \mathrm{m}$ [70]. Since 2007 the most widely implemented chipsets operate with $45 \mathrm{~nm}$ feature sizes (although $32 \mathrm{~nm}$ and $22 \mathrm{~nm}$ architectures are already in place) with the Intel ${ }^{\circledR}$ Penryn quad-core processor containing 820 million transistors in roughly the same size die as the 386 processor [70]. Due to this continued reduction in the size of device components, feature sizes are shrinking below the mean free path $^{6}$ of the

\footnotetext{
${ }^{6}$ The mean free path of an energy carrier is the average distance the carrier will travel between collisions with another "object", i.e. another energy carrier, impurity, vacancy, etc. Typically at room temperature the mean free path of electrons and phonons is on the order of $10 \mathrm{~s}$ and $100 \mathrm{~s}$ of nm respectively.
} 
primary carriers of thermal energy, electrons and phonons ${ }^{7}$. The question arises as to if the "classical" view of heat and thermal transport can be applied to systems on such a small length scale?

The development of nano-devices relies on a combination of internal solid-solid interfaces between materials with different elemental and crystallographic structures to provide the functional operation of the device. Traditionally these devices have been designed with the operational performance and efficiency in mind, with consideration of thermal energy management and heat dissipation as a design afterthought. Therefore the current methodology in dealing with thermal management issues is relocated to the external device structures and beyond [28, 36, 48, 71]. However, as the feature size of most nano-devices continues to diminish, the impact of thermal transport across solid-solid interfaces becomes ever increasingly important.

The solid-solid interfaces created within these nano-devices provide additional scattering sites and impede the propagation of thermal energy carriers [72]. The hindrance to thermal transport is quantified in terms of the thermal boundary conductance $\left(h_{\mathrm{BD}}\right)$, the inverse of thermal boundary resistance $\left(R_{\mathrm{BD}}\right)$. Thermal boundary conductance is defined as [33]:

$$
h_{\mathrm{BD}}=\frac{\dot{q}}{\Delta T}=\frac{1}{R_{\mathrm{BD}}}
$$

where $\dot{q}$ is the applied thermal flux, and $\Delta T$ is the temperature drop across the interface ${ }^{8}$. Thermal boundary conductance has been demonstrated to be the limiting thermal conductance (dominant thermal resistance) in a wide range of nano-devices and nano-structures $[73]$.

We notice that the formulation of Equation 1.6 is the same as Equation 1.4 where we discussed thermal contact resistance. While mathematically these formulations are similar, the subtleties of their meaning are very important. We defined the contact resistance, $R_{C}$, previously, as the resistance to thermal flow across a mechanical joint, primarily due to geometric properties such as surface roughness. On the other hand, the thermal boundary

\footnotetext{
${ }^{7}$ Phonons are quantized vibrations of elastic waves within a solid lattice. Phonons are the analog of photons which are quantized energies or perturbations of the electromagnetic field.

${ }^{8}$ The temperature drop at the interface is generally assumed to be discrete, however as will be discussed in Chapter 2, there is a spatial extent to the temperature gradient.
} 
resistance, $R_{\mathrm{BD}}$, is an inherent property of the interface between two materials with dissimilar thermophysical properties. This implies that $R_{\mathrm{BD}}$ is a fundamental property that is present even for ideal interfaces, see Figure 1.11. In terms of chip-level thermal management,

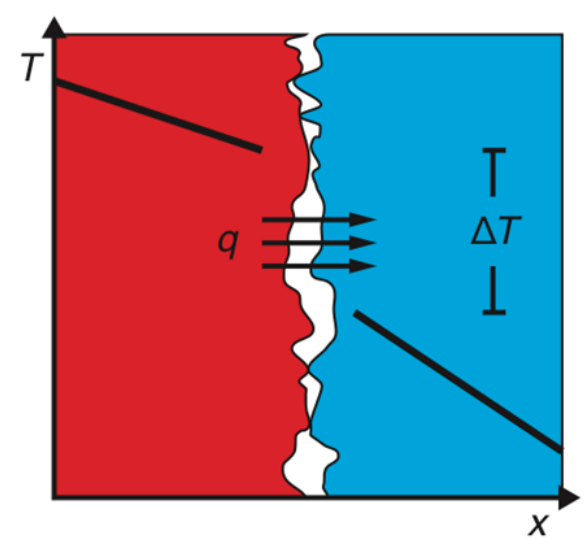

(a)

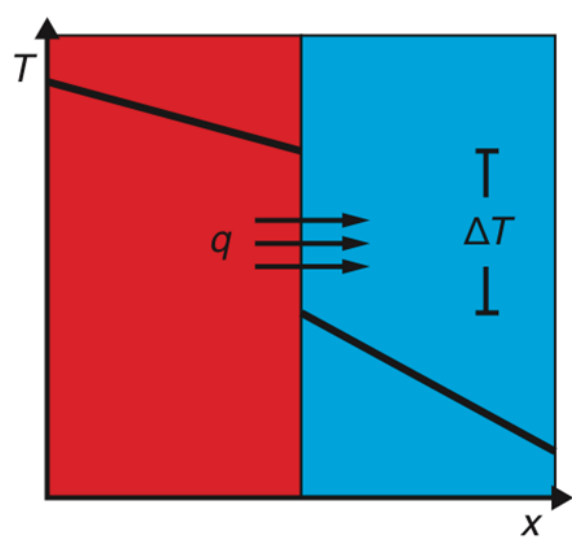

(b)

Figure 1.11: Schematic illustrating the physical differences between (a) thermal contact resistance, and (b) thermal boundary resistance.

this would be analogous to a perfect TIM, i.e. completely wetting. On the transistor-level however, this represents a thermal resistance at every junction between dissimilar solid materials.

At the transistor-level, devices are complex systems of interconnected atomic substructures with various layers of metals and non-metals, i.e. semiconductors and dielectrics, forming numerous solid-solid interfacial structures. Figure 1.12 shows the typical structure of a silicon on insulator metal-oxide-semiconductor field-effect transistor (SOI-MOSFET), overlaid with a resistor network illustrating the primary thermal resistances in the structure. While a MOSFET is an example of a small subset of nano-devices, it illustrates the wide variety of interfaces, i.e. metal-metal, metal-semiconductor, and semiconductor-semiconductor that thermal energy carriers can encounter. It is commonly held that electrons are the dominant thermal energy carriers in metals, while phonons are the dominate carriers in semiconductors and dielectrics [74-77]. This dictates that the dynamics of the dominate thermal energy carriers will depend, in part, on the types of interfaces encountered, and the overall thermal performance of the device will be governed by the interplay of these dynamics. 


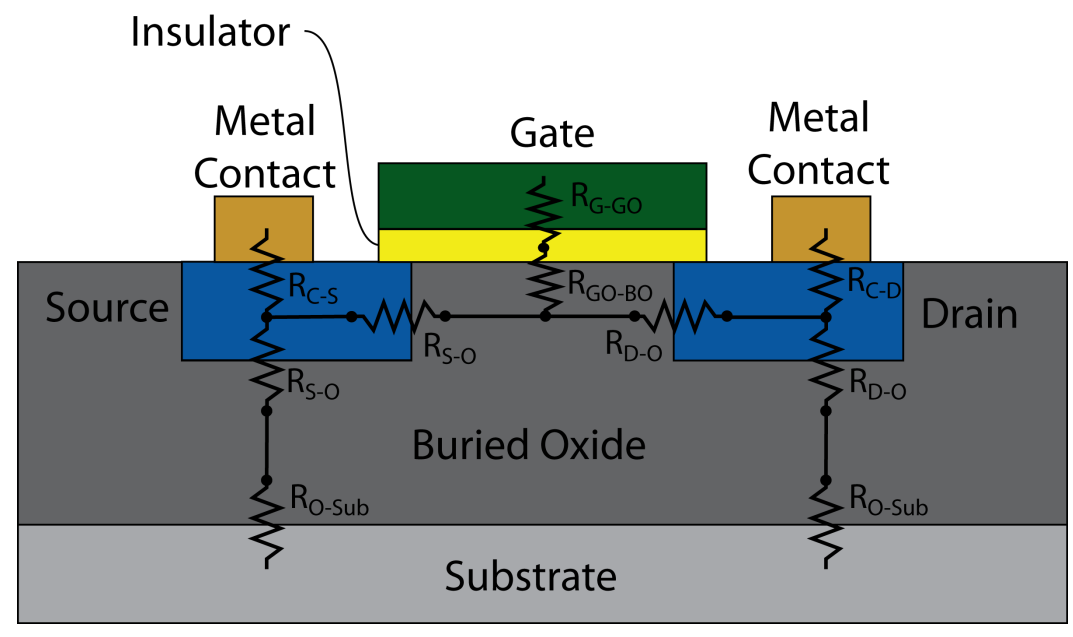

Figure 1.12: Typical physical structure of a SOI-MOSFET with an overlaid thermal resistor network showing the primary interfacial thermal resistances. Where $R_{C-S}$ is the contact-to-source resistance, $R_{S-O}$ is the source-to-oxide resistance, $R_{C-D}$ is the contact-to-drain resistance, etc. [7].

Despite the numerous applications affected by $h_{\mathrm{BD}}$, and the advances made in understanding phonon transport at the nanoscale, the ability to manipulate structures and properties to control $h_{\mathrm{BD}}$ has remained limited [78]. While the ability to control the electrical conductivity of a material spans over 20 orders of magnitude, the ability to control the thermal conductivity and thermal boundary conductance of a structure spans only around 5 orders of magnitude [78]. Since the birth of the micro- and nanoscale thermal transport field, in conjunction with advances in the design and fabrication of nanoscale structures, the breath of knowledge in thermal transport has grown rapidly, while the inherent understanding of how material properties are affecting the foundations of phonon thermal transport has developed more slowly. In order to move forward, to continue to develop faster and more efficient nanoscale devices, and to move towards phononically engineered devices, our understanding of the very fundamentals of phononic thermal transport across solid-solid interfaces must continue to expand.

While the microprocessor is a cogent example of a device with nanometer feature sizes for which development and performance is greatly affected by thermal management issues, it is certainly not the only relevant nano-device of interest. Thermal boundary conductance has been shown to affect the performance and properties of optoelectronic devices, [79, 80] supperlattice structures and devices, [81-85] and is a major design criteria for the 
development of thermal interface materials (TIMs), [28, 86, 87] nanoelectronic coolers, [45] phase change memory, [88, 89] and high-electron-mobility transistors (HMETs) [90].

As device components become more complex, and their size continues to decrease, there is a paradigm shift required in the science and methodology of managing high thermal loads. Solutions can no longer be post-design considerations, as it is no longer sufficient to simply add a bigger fan after the fact to deal with thermal issues. The miniaturization of device components has shifted the focus of thermal management, reducing the role of convective heat transfer and focusing more on the role of conduction within the system components. Motivated by the shift towards conductive heat transfer, this dissertation will focus on the methods of measurements and analysis of thermal boundary conductance at solid-solid interfaces.

\subsection{Outline and Objectives}

The work in this dissertation is essentially broken up into four primary parts. The first part focuses on the basics of phonon transport and a historical perspective of the theoretical and experimental bodies of work in phonon transport at solid-solid interfaces. The second part introduces the experimental technique, pump-probe thermoreflectance spectroscopy. The underlying principles and historical development are presented along with the details of the current system in the UVa Nanoscale Energy Transport Lab. The theoretical model used to analyze the spectroscopy results is presented, and while built off previous contributions the formulation is extended to be applicable for square modulation waveforms of an arbitrary duty cycle. The third part focuses on the often omitted details of the application of the pump-probe thermal analysis and establishes robust methods for handling data and predicting the standard deviation of the fitted results prior to collecting the data. In the final part, the robust methods developed are applied to an investigation of tuning thermal transport between two solids via the addition of an intermediate layer of mediating vibrational properties. 


\section{Specifically the remaining chapters present:}

In Chapter 2 we present some of the current theories of phonon transport at solidsolid interfaces, with a specific focus on how the properties of the interface affect thermal boundary conductance.

In Chapter 3 we describe, in detail, the experimental thermoreflectance system in the Nanoscale Energy Transport Lab at UVa. The primary beam paths, starting from the lasers, are followed with descriptions of the purpose and operation of the major system components provided. When appropriate, any special techniques to increase functionality are highlighted.

In Chapter 4 the theoretical framework of modeling the results of the temperature decay in the pump-probe experiment are presented. The theoretical lock-in response in the frequency domain is provided. The formulation of the lock-in model is re-derived for clarity from previous work, but is extended from the simple sinusoidal modulation waveform to that of a square wave of arbitrary duty cycle. A diffusive heat transfer model for multilayered systems in the frequency domain is presented as an extension of the previous single-layer lumped capacitance model. Finally the conditions for 1D and 3D transport are discussed.

In Chapter 5 some practical issues in the application of the model discussed in the previous chapter are presented. The effects of the modulation waveform and duty cycle are presented and convergence criteria for the infinite summations within the lock-in model established. Issues are discussed in the current implementation of the sensitivity coefficient utilized by the field, and corrections are suggested. A method for characterizing noise in the raw thermoreflectance data is established, and finally a procedure for removing statistical outliers from the fitted results is presented.

In Chapter 6 we look at establishing robust methods for the collection and management of the thermoreflectance data. After a brief introduction to statistical methods and terminology, large scale simulations of thermoreflectance data are presented, and an empirical formulation relating the signal-to-noise in the data and the sensitivity to fitting a parameter of interest to the expected standard deviation in the results is given. The concept of statistical bootstrapping is presented as a method to verify the statistical assumptions made in the analysis of the thermoreflectance data. Finally the accuracy of the model, based on the 
uncertainty in the input parameters, is compared to the precision results presented based on the simulated data.

In Chapter 7 the concept of tuning the total effective thermal boundary conductance of a system of thin films on a bulk substrate is presented. The theoretical framework and fundamental phononic principles of the tuning effect are presented based on previously completed molecular dynamics simulations. Based on the theoretical work, the selection of a metallic $(\mathrm{Ni})$ intermediate layer is motivated. The structure of the prepared samples is presented based on characterization via Auger photoelectron spectroscopy. Thermoreflectance measurements on Pt-Ni-Si and Pt-Ni-Ge systems are presented, and the effective film-to-substrate thermal boundary conductance presented as a function of Ni thickness. The results provide preliminary evidence of enhanced conductance due to the presence of the Ni layer and raises several interesting questions based on the observed trends in the data. Finally, we review some of the potential challenges in interpreting the Pt-Ni system results, looking at the effects of solid-solid bonding and electron transport on the the overall conductance.

Finally, Chapter 8 summarizes the information provided in this dissertation and draws general conclusions based on the original work presented. Suggestions for expansion on existing work and/or potential future projects are also presented. 


\title{
CHAPTER 2
}

\author{
THERMAL TRANSPORT AT \\ NANOSCALE SOLID-SOLID \\ INTERFACES: MODELING AND \\ IMPACTS OF INTERFACE \\ NONIDEALITIES
}

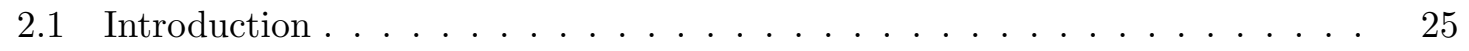

2.2 Mechanisms of Thermal Boundary Conductance - Electrons and Phonons . 25

2.3 "Predicting" Thermal Boundary Conductance at Solid-Solid Interfaces . 28

2.4 General Phonon Flux Across an Interface . . . . . . . . . . . . . . . . . 30

2.5 Failures of the DMM in Predicting Thermal Boundary Conductance . . . . 38

2.6 Interface Effects on Thermal Boundary Conductance at Solid-Solid Interfaces 42

2.7 Chapter Summary . . . . . . . . . . . . . . . . 56 


\section{$2.1 \quad$ Introduction}

The transport of thermal energy in solid materials by phonons is a fundamental branch of solid-state physics. At this point it is assumed that the reader has a background in the fundamentals of crystallography and phonon physics. If this is not the case, Appendix A has been provided as a brief introduction to these topics. The interaction and scattering of phonons within a constituent material, i.e. phonon-phonon scattering, are the primary mechanisms leading to finite thermal conductivity. Variations from a perfect crystal system such as impurities and defects are additional mechanisms which reduce the inherent thermal conductivity of a material. Along similar lines, in Chapter 1 the concept of thermal boundary conductance, $h_{\mathrm{BD}}$, the inverse of thermal boundary resistance, $R_{\mathrm{BD}}$, was introduced as a quantification of the interaction and transmission of phonon energy across a boundary between two materials with dissimilar thermophysical properties. In this chapter, we will begin by first discussing what energy carriers are dominate in several material systems, i.e. metals, semiconductors, and insulators, and then discuss several semi-classical models that have been developed in an attempt to predict $h_{\mathrm{BD}}$. In a similar manner as the thermal conductivity of a material is impacted by scattering caused by impurities and defects in the surrounding material, so too is $h_{\mathrm{BD}}$ impacted by the localized properties of the interface. Therefore, we will take time to discuss some of the pertinent literature and theories dealing with the impacts of vibrational mismatch, inelastic scattering, bonding, dislocations, defects, roughness, and compositional inter-diffusion at the solid-solid interface on the propagation of phonon energy across a boundary.

\subsection{Mechanisms of Thermal Boundary Conductance - Elec- trons and Phonons}

Before presenting some of the methods used to model $h_{\mathrm{BD}}$ and discussing some specific examples of how different interface structures can impact $h_{\mathrm{BD}}$, it is important to understand when discussing heat flux across an interface, $\dot{q}$, the contribution of the primary energy carriers. In general, the primary thermal energy carriers in solid materials are electrons and phonons [33]. However, as discussed in Chapter 1, the constituent materials that 
make up solid-solid interfaces in most modern micro- and nano-devices are various pairings of metals, semiconductors, and dielectrics (electrical insulators). The selection of like or dissimilar pairs of materials to comprise the interface will determine whether transport across the interface is dominated by either phonons or electrons.

In metals, the atoms are bonded together in a lattice and the electrons in the outer orbitals of each atom are free from the bonding of the nucleus and can move throughout the metal. Phonons and free electrons are therefore both available in metals to carry thermal energy. The velocity of the free electrons however, is nearly three orders of magnitude greater than the average phonon velocity and therefore the electrons in a metal carry significantly more thermal energy than the phonons [33]. Therefore, in metals it is assumed that electrons are the primary energy carriers, and that electrons are the dominant energy carriers across the interface between two metals, see Figure 2.1e. At the metal-metal interface it is estimated that phonons contribute less than $10 \%$ to the overall thermal conductance [75]. From experimental literature, $h_{\mathrm{BD}}$ in metal-metal systems has been shown to be on the order of 100's-1,000's of MW/ $\mathrm{m}^{2} \mathrm{~K}$ [75, 91-93], which is several orders of magnitude higher than the average phonon dominated conductance. Metals also typically have high thermal

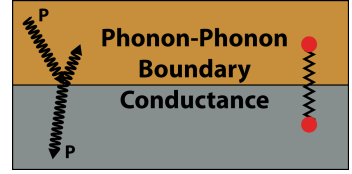

(a)

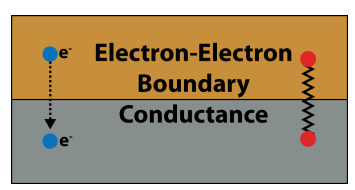

(b)

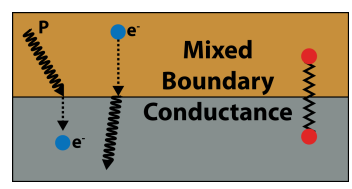

(c)

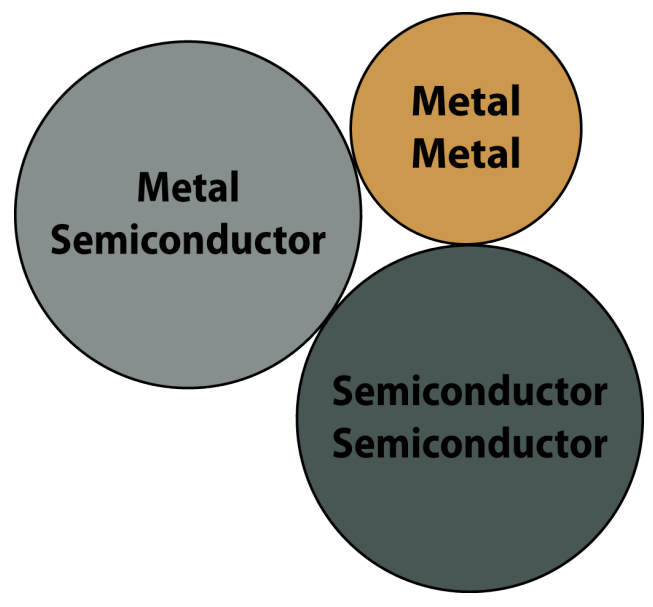

(d)

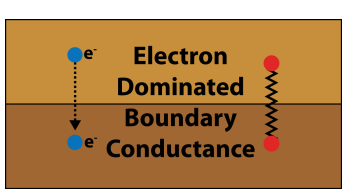

(e)

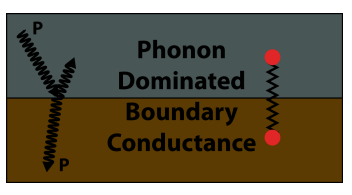

(f)

Figure 2.1: Schematic of the various thermal boundary conductance channels for electron and phonon transport at (a)-(c) metal-semiconductor (e) metal-metal and (f) semiconductor-semiconductor interfaces. Electrons and phonons are the dominate energy carries at (e) metal-metal and (f) semiconductor-semiconductor interfaces respectively. At metal-semiconductor interfaces the total conductance is the sum of (a) phononphonon (b) electron-electron and (c) mixed electron-phonon and phonon-electron transport. 
conductivities, due to the high velocity of free electrons present. Despite the high thermal conductivity in metals, the effective conductance of a thin metal layer can be greater than the conductance across the metal-metal interface, making the interface the dominate resistive pathway.

In dielectric (insulating) materials, there are very few available free electrons and therefore thermal transport is dominated by phonons. Semiconductors, while having a large free electron population $\left(\sim 10^{18} \mathrm{~cm}^{-3}\right)$ in comparison to insulators $\left(\sim 10^{6} \mathrm{~cm}^{-3}\right)$, still have significantly less free electrons compared to metals $\left(\sim 10^{23} \mathrm{~cm}^{-3}\right)$ [33]. Therefore, for normally doped semiconductors thermal transport is still dominated by phonons ${ }^{1}$. Only for heavily doped semiconductors does electron transport become appreciable. At the interface between two semiconducting materials, phonon transport across the interface is assumed to be the dominant transport mechanism, see Figure 2.1f. The majority of theoretical modeling and simulation over the past two decades has focused on phonon dominated thermal transport across metal-semiconductor and semiconductor-semiconductor interfaces [10, 72, 73, 94].

At the interface between a metal and a semiconductor or dielectric, the picture of thermal transport is more complicated. In this type of system there are populations of electrons and phonons available for thermal transport in both the film and the substrate. In addition to the potential of electron-electron and phonon-phonon transport across the interface, there is also the potential for electrons in the film to couple with phonons in the substrate, and phonons in the film to couple with electrons in the substrate, see Figure 2.1a-2.1c. Typically, for the sake of simplicity it is assumed that there is no direct energy exchange between electrons and phonons across the interface. That is not to say that the phenomena has not been studied however.

Huberman and Overhauser studied the contribution of direct energy transfer between electrons and phonons in $\mathrm{Pb}$ and diamond respectively, as a means to describe the failure of the acoustic mismatch model to predict $h_{\mathrm{BD}}$ across the Pb-diamond interface [95]. They concluded in the case of $\mathrm{Pb}$ on diamond, that there was direct energy transfer between $\mathrm{Pb}$ and diamond but caution that their results were specific to the material system being

\footnotetext{
${ }^{1}$ Since phonons are the dominate energy carriers in both semiconductors and insulators, when metalsemiconductor and semiconductor-semiconductor interfaces are discussed, it is implied that from the perspective of phonons equivalent relations will hold for metal-dielectric and dielectric-dielectric interfaces.
} 
studied. Hopkins et al. investigated the impacts of electron-substrate energy transfer in $\mathrm{Au} / \mathrm{Si}$ and $\mathrm{Au} / \mathrm{glass}$ systems as a function of gold thickness via a modified version of the two-temperature model (TTM) which was designated the three-temperature model (3TM) $[96,97]$. Their results showed that the influence of energy transfer with the substrate became more significant for film thicknesses on the order of the thermal penetration depth.

When assuming no direct energy transfer between electrons and phonons on either side of a metal-semiconductor interface, the electrons in the metal must first couple their energy to phonons in the metal prior to energy being transferred across the interface. There has been some debate as to whether this added electron-phonon interaction impacts the overall thermal resistance in the metal-semiconductor system. Majumdar and Reddy proposed that the total resistance between a metal and substrate is the sum of two series resistors: 1) the interfacial phonon-phonon resistance and 2) the volumetric electron-phonon coupling resistance in the metal [74]. Singh et al. considered the same resistor network, but calculated the contribution of electron-phonon resistance independent of the assumed phononic thermal properties of the metal, and concluded that the electron-phonon coupling thermal resistance is not significant in metal-semiconductor systems for temperature above $200 \mathrm{~K}$ [76].

In this chapter, the primary focus will be on phonon-mediated transport in semiconductorsemiconductor systems, and electron-mediated transport in metal-metal systems. In Chapter 7 , we will return to the issue of mixed transport in metal-semiconductor systems and take a closer look at the possibility of electron-electron transport across the metal-semiconductor interface and thermal resistance due to electron-phonon coupling in a metal film, as they apply more specifically to the material systems under study.

\section{3 "Predicting" Thermal Boundary Conductance at Solid- Solid Interfaces}

As can occur in scientific research, experimental observation of $h_{\mathrm{BD}}$ predated theory of its existence or prediction of its values. Since it was first observed, much work has gone into predicting $h_{\mathrm{BD}}$ for solid-liquid and solid-solid interfaces, based on the thermophysical properties of the materials. 


\subsubsection{Phonon Transport - Semi-classical Approach}

In terms of modeling $h_{\mathrm{BD}}$, there are a variety of methods to choose from all with a varying level of computational expense. Rigorous methods such as molecular dynamics (MD) simulations, have been used to model trends in thermal transport for a number of systems [14, 98-116]. MD is a classical mechanical technique in which the time-evolved position and velocity of a group of atoms within a computational domain are tracked and recorded. One of the biggest advantages to $\mathrm{MD}$ simulations, is they do not require $a$ priori knowledge of the properties of the system. Instead only the equilibrium positions of the atoms and the interatomic potentials between them ${ }^{2}$ need be specified. The classical nature of the calculations results in the restriction that simulations are only strictly valid at temperatures at or above the Debye temperature, $\Theta_{D}$, of the constituent materials. The computational expense for MD simulations can be high, with simulations taking on the order of hours to days to complete depending on the level of complexity.

In contrast to MD simulations, Atomistic Green's Function (AGF) methods are based on a quantum mechanical description of the phonon energy distribution [117]. AGF simulations, similar to MD simulations, have the advantage that no a priori information on the phonon density of states is required, only the atomic positions and interatomic potential(s) [117]. Unlike MD simulations, the results from the AGF simulations are only strictly valid at low temperatures [118]. The computational expense of AGF methods can vary from on the order of several minutes to several hours, depending on the resolution of the mesh required for the solutions to converge [117].

In addition to these more complex models, there have been semi-classical methods developed in order to predict $h_{\mathrm{BD}}$ across an interface, namely the Acoustic Mismatch Model (AMM) and the Diffuse Mismatch Model (DMM). While not overly rigorous, these models are often turned to as relatively simple formulations that provide insight into the key fundamental processes that are driving or restricting thermal transport across an interface. Because the focus of this dissertation is centered more on experimental methodology and results, we will not belabor the details of the more rigorous modeling techniques. However,

\footnotetext{
${ }^{2}$ Note however, that the results of MD simulations depend heavily on the choice of interatomic potential, which in itself is generally as much a function of the property to be "measured" as it is the material system itself.
} 
because of their usefulness in gaining insight into the processes driving or restricting thermal transport, and in as much provided the motivation for the experimental studies in this work, we will take the time to discuss some of the merits and shortcomings of the DMM.

\subsection{General Phonon Flux Across an Interface}

Regardless of the semi-classical model used to predict $h_{\mathrm{BD}}$, the general ambition is to predict the amount of heat transported across an interface. Therefore, assuming phonons as the primary energy carries, a discussion of thermal transport should most logically begin with a general expression for the transmitted phonon flux. Defining the two materials on either size of the interface as material 1 and material 2 , the phonon flux from $1 \rightarrow 2$ in the z-direction, $q_{z}^{1 \rightarrow 2}$, is given by: [33]:

$$
\begin{aligned}
q_{z}^{1 \rightarrow 2}=\frac{1}{(2 \pi)^{3}} \sum_{j} \int_{0}^{\frac{\pi}{2}} \int_{0}^{2 \pi} \int_{k_{x, 1}} \int_{k_{y, 1}} \int_{k_{z, 1}>0} \hbar \omega_{j, 1}\left(k_{j, 1}\right) \zeta^{1 \rightarrow 2} & \\
& \left|v_{j, 1}\left(k_{j, 1}\right)\right| f_{0} \sin \left(\theta_{1}\right) \cos \left(\theta_{1}\right) d k_{z, 1} d k_{y, 1} d k_{x, 1} d \theta_{1} d \phi_{1}
\end{aligned}
$$

where $z$ is the direction of transport, $j$ is the phonon polarization, $\theta_{1}$ and $\phi_{1}$ are the azimuthal and elevation angles of the phonon flux in material 1 approaching material 2 relative to the direction of transport, $\zeta^{1 \rightarrow 2}$ is the transmission coefficient, $v_{1}$ is the phonon group velocity in material $1, f_{0}$ is the equilibrium distribution of phonons in material 1 , and $k$ is the wavevector. Since we are dealing with phonons as the primary energy carries, the equilibrium distribution function, $f_{0}$, is given by the Bose-Einstein distribution, $f_{0}=1 /\left(\exp \left(\hbar \omega(k) / k_{B} T\right)-1\right)$. Typically, only phonon flux approaching the interface from material 1 is considered, and therefore the direction dependence can be simplified by integrating over half the Brillouin zone and taking the absolute value of the group velocity, $v_{1}$, which gives:

$$
q_{z}^{1 \rightarrow 2}=\frac{1}{8 \pi^{2}} \sum_{j} \int_{k_{x, j, 1}} \int_{k_{y, j, 1}} \int_{k_{z, j, 1}>0} \hbar \omega_{j, 1}\left(k_{j, 1}\right) \zeta^{1 \rightarrow 2}\left|v_{j, 1}\left(k_{j, 1}\right)\right| f_{0} d k_{z, j, 1} d k_{y, j, 1} d k_{x, j, 1}
$$


Furthermore, under the assumption that the materials have isotropic properties, and therefore an isotropic phonon dispersion, this relation can be further simplified to:

$$
q_{z}^{1 \rightarrow 2}=\frac{1}{8 \pi^{2}} \sum_{j} \int_{k_{j, 1}>0} \hbar \omega_{j, 1}\left(k_{j, 1}\right) k_{j, 1}^{2} \zeta^{1 \rightarrow 2}\left|v_{j, 1}\left(k_{j, 1}\right)\right| f_{0} d k_{j, 1}
$$

Recall from Chapter 1 that we defined $h_{\mathrm{BD}}$ as a proportionality constant relating the heat flux across an interface to the temperature drop within the interfacial region. Therefore, we can define $h_{\mathrm{BD}}$ in a modified version of the Fourier law, given by:

$$
q_{z}^{1 \rightarrow 2}=h_{\mathrm{BD}}^{1 \rightarrow 2} T^{1 \rightarrow 2} \Rightarrow h_{\mathrm{BD}}^{1 \rightarrow 2}=\frac{\partial q_{z}^{1 \rightarrow 2}}{\partial T^{1 \rightarrow 2}}
$$

Combining Equations 2.3 and 2.4 provides a formulation for $h_{\mathrm{BD}}$ given by:

$$
h_{\mathrm{BD}}^{1 \rightarrow 2}=\frac{1}{8 \pi^{2}} \sum_{j} \int_{k_{j, 1}} \hbar \omega_{j, 1}\left(k_{j, 1}\right) k_{j, 1}^{2} \zeta^{1 \rightarrow 2}\left|v_{j, 1}\left(k_{j, 1}\right)\right| \frac{\partial f_{0}}{\partial T} d k_{j, 1}
$$

It is important to note, so it will be reiterated, the formulation of $h_{\mathrm{BD}}$ was derived assuming small temperature perturbations at the interface, an isotropic phonon dispersion, and a spherical Brillouin zone.

Outside of these assumptions, the primary element that delineates the different methods of modeling $h_{\mathrm{BD}}$ is the formulation of the transmission coefficient $\zeta^{1 \rightarrow 2}$. One of the primary metrics used to determine the appropriate choice of methodology used to model $\zeta^{1 \rightarrow 2}$ is the assumption of the dominate phonon scattering mechanism at the interface. The two most common assumptions are that either the scattering at the interface is completely diffuse ${ }^{3}$ or completely specular ${ }^{4}$, which in turn is used in the formulation of the DMM and AMM respectively.

\footnotetext{
${ }^{3}$ Diffuse scattering typically occurs at rough surfaces/interfaces, and the angle at which an incident wave reflects or transmits is independent of the incident angle. It is therefore said that in diffuse scattering, an incoming wave "loses all memory" of it's incident properties.

${ }^{4}$ Specular scattering, typically occurs at a smooth surface/interface, and the angle at which the incident wave reflects or transmits, is dependent on incident angle the properties of the material(s).
} 


\subsubsection{Acoustic Mismatch Model}

The phenomenon of thermal resistance at an interface was first discovered by Kapitza in 1941 at the interface of $\mathrm{Cu}$ and $\mathrm{He}$ II during an experimental investigation into the superfluidity of He II [119]. It was originally hypothesized that the thermal resistance at the $\mathrm{Cu} / \mathrm{He}$ II interface was a unique property of the superfluid helium, and it was not until later experiments were conducted that it was found that the resistance exists between any pairs of materials with dissimilar properties [73]. Kapitza's experiments were conducted at temperatures in the range of approximately 1-3 K. At these temperatures, the wavelengths of phonons are much larger than the atomic spacing between the atoms and therefore the assumption can be proposed that phonons can be treated as plane waves and that the interface can be treated as a smooth plane between the two materials. This assumption implies that the materials on either side of the interface can be treated as continua, rather than lattices of individual atoms. These assumptions are the basis of the acoustic mismatch model which was proposed in general form by Little in 1959 [120].

Under the assumptions of the AMM, i.e. a smooth interface, plane wave phonons, and continuous media, phonons incident on the interface can only react in a finite number of ways: 1) reflect back to material 1,2 ) reflect back to material 1 and change polarization modes, 3) refract in material 2, and 4) refract in material 2 and change polarization modes [72]. The angle at which a transmitted phonon leaves the interface, assuming it transmits, can be calculated from an analog of Snell's law used for electromagnetic waves, given by $[72]:$

$$
\sin \theta_{2}=\frac{v_{2}(\omega, j)}{v_{1}(\omega, j)} \sin \theta_{1}
$$

where $\theta_{2}$ is the angle of transmission in material $2, v_{i}(\omega, j)$ is the phonon velocity as a function of phonon frequency $\omega$, of the polarization $j$ on the $i^{\text {th }}$ side of the interface, and $\theta_{1}$ is the incident angle. Similarly to Snell's law, there is a critical angle above which the probability of phonon transmission across the interface reaches zero. The collection of all these critical angles defines a cone of transmittance, from within which there is a finite probability of phonons transmitting across the interface.

In order to calculate the probability of phonon transmission across the interface, Little 
turned to an acoustic analog of the Fresnel equations for the transmission and reflection of light [120]. Under this formulation, each material is prescribed an acoustic impedance, $Z_{i}$, which is the product of the materials mass density, $\rho$, and phonon velocity, $v$, i.e. $Z_{i}=\rho_{i} v_{i}$. The transmission coefficient as a function of incident angle is then given by [120]:

$$
\alpha_{1 \rightarrow 2}\left(\theta_{1}\right)=\alpha_{2 \rightarrow 1}\left(\theta_{2}\right)=\frac{4 Z_{1} Z_{2} \cos \theta_{2} \cos \theta_{1}}{\left(Z_{1} \cos \theta_{1}+Z_{2} \cos \theta_{2}\right)^{2}}
$$

\subsubsection{Breakdown of the AMM}

Outside of the continuum assumption, the major assumption of the AMM that affects its applicability is the assumption of completely specular scattering at the interface. This assumption will hold, in general, for very low temperatures and very low interface roughnesses, but will break down as either of these conditions increases. In order to provide a rough estimate of the effectiveness of these assumptions, i.e. the percentage of specularly scattered phonons, Ziman derived an approximated relation known as the specularity parameter, $S_{P}$, given by [121]:

$$
S_{P}=\exp \left[\frac{-16 \pi^{3} \delta^{2}}{L^{2}}\right]
$$

where $\delta$ is the RMS roughness of the interface, also known as the asperity parameter, and $L$ is the phonon coherence length given by [33]:

$$
L=\frac{h v_{g}}{k_{\mathrm{B}} T}
$$

where $h$ is Planck's constant, $v_{g}$ is the phonon group velocity, $k_{\mathrm{B}}$ is the Boltzmann constant, and $T$ is the temperature. To illustrate the functional dependence of $S_{P}$, Figure 2.2 provides $S_{p}$ as a function of temperature for a selected variety of phonon velocities assuming an asperity parameter of $\delta=1 \mathrm{~nm}$. As a particular example, for the highest velocity phonons in $\mathrm{Si}, v_{g}=8,192 \mathrm{~m} / \mathrm{s}$, impingent on an interface with an RMS roughness of $1 \mathrm{~nm}$ there is a less than $1 \%$ chance of specular scattering at temperatures above $40 \mathrm{~K}$. Additionally, we can look at $S_{P}$ as a function of interface roughness, i.e. the asperity parameter, for 


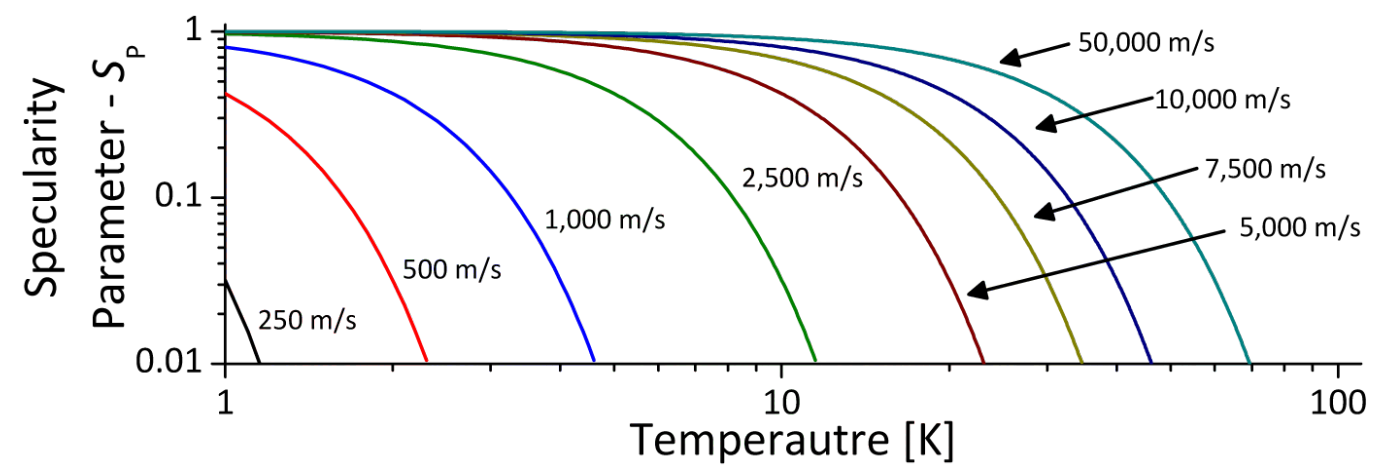

Figure 2.2: Specularity parameter, calculated from Equation 2.8, as a function of temperature for a variety of phonon group velocities, $v_{g}$, assuming a asperity parameter of $\delta=1 \mathrm{~nm}$.

various temperatures assuming a phonon group velocity of $v_{g}=10,000 \mathrm{~m} / \mathrm{s}$, see Figure 2.3. These results indicate that the AMM will only be applicable in situations of very low temperature and very low interface roughness. For temperatures above approximately 40 $\mathrm{K}$ and for phonon frequencies above $100 \mathrm{GHz}$ the assumptions of the AMM break down, and the interaction at the interface is dominated by diffusive scattering [74, 122].

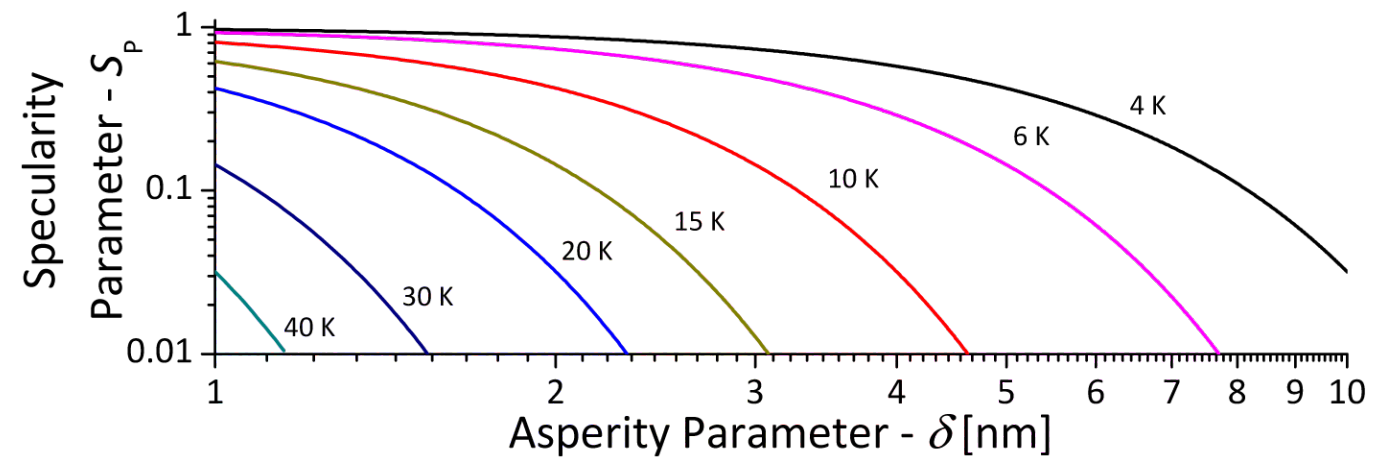

Figure 2.3: Specularity parameter, calculated from Equation 2.8, as a function of asperity parameter, $\delta$, for a phonon velocity of $v_{g}=10,000 \mathrm{~m} / \mathrm{s}$, at several temperatures.

\subsubsection{Diffuse Mismatch Model}

Due to the low temperature restrictions of the AMM, the most commonly referenced model for the prediction of thermal boundary conductance across a solid-solid interface, under the assumption of diffusive scattering, is the diffuse mismatch model developed by Swartz and Pohl in the late 1980's [72, 123]. By definition of its diffusive nature, the 
DMM implies that phonons ${ }^{5}$ lose memory of their incident polarization and direction after scattering at the interface. This has the implication that the probability of the energy carried by the phonons being transmitted from material 1 to material 2 , is the same as the probability of the phonon energy reflecting from material 2 to material 1. Mathematically this can be represented by [33]:

$$
\zeta^{1 \rightarrow 2}=R^{2 \rightarrow 1}=1-\zeta^{2 \rightarrow 1}
$$

where $\zeta^{i \rightarrow j}$ and $R^{i \rightarrow j}$ are the probability of phonon transmission and reflection from material $i$ to material $j$ respectively. As with the AMM, the major assumption that goes into the development of the DMM is the formulation of the transmission coefficient, $\zeta^{1 \rightarrow 2}$.

Somewhat counter-intuitively, the transmission coefficient of phonon flux across an interface is calculated using the concept of local thermal equilibrium. Again, invoking the assumption of small temperature perturbations, the DMM assumes a local thermal equilibrium at the interface, i.e. $T_{1}=T_{2}$, and therefore there is no net heat flux across the interface. Through the application of the principle of detailed balance, it is possible to equate heat fluxes from both sides of the interface, i.e. $q_{z}^{1 \rightarrow 2}=q_{z}^{2 \rightarrow 1}$, and therefore solve for the transmission coefficient $\zeta^{1 \rightarrow 2}$ explicitly which is given by:

$$
\zeta^{1 \rightarrow 2}=\frac{\sum_{j}\left|\nu_{1, j}^{-2}\left(k_{2, j}\right)\right|}{\sum_{j}\left|\nu_{1, j}^{-2}\left(k_{2, j}\right)\right|+\sum_{j}\left|\nu_{1, j}^{-2}\left(k_{1, j}\right)\right|}
$$

The formulation of the transmission coefficient depends heavily on the assumptions invoked in the application of detailed balance. A more thorough review of the different assumptions and their impact on calculating $h_{\mathrm{BD}}$ can be found elsewhere [124]. In the case of Equation 2.11 , the primary assumptions are that phonons scatter elastically ${ }^{6}$ at the interface, and that phonons can scatter into any available phonon polarization, i.e. longitudinal or transverse. It is important to note, that the assumptions made in the application of detailed balance,

\footnotetext{
${ }^{5}$ In reality, the formulation for $h_{\mathrm{BD}}$ across an interface proposed by the DMM is applicable for phonons, electrons, and even photons [91]. However, the majority of the time it is only applied to phonons, as it will be the focus of our discussion.

${ }^{6}$ In an elastic phonon scattering process, a phonon from the film of frequency $\omega$ can only transfer energy across the solid-solid interface by scattering with a phonon in the substrate, also of frequency $\omega$.
} 
i.e. small temperature perturbations, isotropic phonon dispersion, etc., were the same assumptions made in the formulation of the expression for $h_{\mathrm{BD}}$, see Equation 2.5. When applying the DMM it is important to keep assumptions consistent for both the formulation of $h_{\mathrm{BD}}$ and $\zeta^{1 \rightarrow 2}$.

With the established formulation of the DMM, there is a key issue that should be pointed out. In the purely diffusive regime, the phonon transmission probability predicted by the DMM in the limit of the same material on either side of the interface is $50 \%$. This may be viewed as an incorrect result of the model, but is in fact a correct description assuming the most rigorous definition of diffusive scattering. Given an interface between two identical materials and assuming that phonons scatter diffusely, thereby loosing all memory of incident direction, phonons will have an equal probably of scattering in all directions, i.e. forward and backward, and therefore the transmission probability of the incident phonon flux will be $50 \%$. In Chapter 2, a closer look is taken into some of the issues facing the DMM and how the predicted values compared to experimental data.

\subsubsection{Electron Transport - eDMM}

When the two materials comprising an interface are both metals, it is expected that electrons will be the dominate energy carries facilitating thermal transport across the interface $[75,93]$. Recall that it has been estimated that phonons contribute less than $10 \%$ to the overall thermal transport between typical metals [75]. Therefore, formulations such as the DMM would not be appropriate for modeling thermal transport at metal-metal interfaces. However, there have been several series of experimental and theoretical studies that have shown that electrons do scatter diffusely at an interface ${ }^{7}$ between two metals [75, 125-129]. The assumption of diffuse scattering at the interface is one of the major assumptions of the phonon-mediated DMM discussed previously. This correlation lead Gundrum et al. to develop an electron-mediated diffuse mismatch model (eDMM) to model thermal transport across an Al-Cu interface [75].

Recalling the formulation of $h_{\mathrm{BD}}$ for phonon-mediated thermal transport, see Equation

\footnotetext{
${ }^{7}$ Note: The diffuse scattering in these studies is upheld in part to do to the presence of a disorder region at the interface, with a spatial extent on the order of a few atomic-layers.
} 
2.5, the development of a model for electron-mediated thermal transport, under the diffusive assumption, is a matter of the appropriate choice of electronic over phononic properties. Therefore, the electron dominated thermal boundary conductance, $h_{\mathrm{BD}, \mathrm{e}}$, is given by ${ }^{8}[92]$ :

$$
h_{\mathrm{BD}, \mathrm{e}}=\frac{1}{4} \int_{0}^{\infty}\left(\epsilon-\epsilon_{\mathrm{F}, 1}\right) D(\epsilon) \frac{\partial F_{1}}{\partial T} v_{\mathrm{F}, 1} \zeta_{e}^{1 \rightarrow 2} d \epsilon
$$

where $\epsilon$ is the electron energy, $\epsilon_{\mathrm{F}, 1}$ is the Fermi energy of metal 1, $D(\epsilon)$ is the electron density of states (eDOS), $f$ is the electron distribution function, $T$ is the electron temperature, $v_{\mathrm{F}}$ is the Fermi velocity, and $\zeta_{e}^{1 \rightarrow 2}$ is the transmission coefficient from metal 1 to metal 2. As with the development of the AMM and DMM, the challenge in implementing the eDMM is the formulation of the transmission coefficient, $\zeta_{e}^{1 \rightarrow 2}$. Recall that in the development of the DMM, care was taken care to ensure the same assumptions were made in the derivation of $h_{\mathrm{BD}}$ and the transmission coefficient. The same restrictions are valid for the development of the eDMM, and therefore in the formulation of the electron transmission coefficient it is assumed that scattering is diffuse and elastic, and there is a local thermal equilibrium at the interface. Under these assumptions, the principle of detailed balance can then be used to formulate the transmission coefficient [92]:

$$
\int_{0}^{\infty}\left(\epsilon-\epsilon_{\mathrm{F}, 1}\right) D_{1}(\epsilon) f_{1} v_{\mathrm{F}, 1} \zeta_{e}^{1 \rightarrow 2} d \epsilon=\int_{0}^{\infty}\left(\epsilon-\epsilon_{\mathrm{F}, 2}\right) D_{2}(\epsilon)\left(1-f_{2}\right) v_{\mathrm{F}, 2} \zeta_{e}^{2 \rightarrow 1} d \epsilon
$$

Under the assumption of detailed balance, i.e. no temperature gradient at the interface, the Fermi levels between the two metals will be equivalent on an absolute scale [92], i.e. $\epsilon_{\mathrm{F}, 1}=\epsilon_{\mathrm{F}, 2}$. Therefore the transmission coefficient can be written as [92]::

$$
\zeta_{e}^{1 \rightarrow 2}=\frac{D_{2}(\epsilon)\left(1-f_{2}\right) v_{\mathrm{F}, 2}}{D_{1}(\epsilon) f_{1} v_{\mathrm{F}, 1}+D_{2}(\epsilon)\left(1-f_{2}\right) v_{\mathrm{F}, 2}}
$$

Under the low temperature assumption, i.e. typically below a few thousand $\mathrm{K}(<3,000 \mathrm{~K}$

\footnotetext{
${ }^{8}$ Note: This is a modified version of the original eDMM proposed by Gundrum et al. to take into account sub-conduction electrons at elevated temperatures. However, it will be shown that this formulation reduces to the original derivation by Gundrum in the low temperature limit.
} 
for $\mathrm{Cu}$ ), Equation 2.14 can be reduced and the expression for $h_{\mathrm{BD}, \mathrm{e}}$ simplified to [92]:

$$
h_{\mathrm{BD}, \mathrm{e}}=\frac{1}{4} \frac{\gamma_{1} v_{\mathrm{F}, 1} \gamma_{2} v_{\mathrm{F}, 2}}{\gamma_{1} v_{\mathrm{F}, 1}+\gamma_{2} v_{\mathrm{F}, 2}} T
$$

where $\gamma_{i}$ is the electron heat capacity constant, also known as the Sommerfeld constant.

\subsection{Failures of the DMM in Predicting Thermal Boundary Conductance}

While the DMM is a very computationally efficient tool for understanding the fundamental processes of phonon transport across an interface and predicting $h_{\mathrm{BD}}$, the question arises as to how accurate of a predictor the DMM really is? Taking a subset of experimental data from available literature and comparing the measured values of $h_{\mathrm{BD}}$ along with those predicted by the DMM, we find that the results are generally not well correlated, see Figure 2.4. Despite a few cases where the experimental data and DMM predictions match, in general, half of the data is over-predicted by the DMM and half is under-predicted. Typically, the values of $h_{\mathrm{BD}}$ for systems with phonon dominated thermal transport, e.g. metal-semiconductor systems, only range from 10's to 100 's of $\mathrm{MW} / \mathrm{m}^{2} \mathrm{~K}$. Recalling again Figure 2.4, we see that for the data presented the predictions are only within plus-or-minus one order of magnitude in accuracy. With the data and the model varying by roughly the same orders of magnitude, there is little chance of any accurate predictive calculations or correlations between the DMM and experimental data.

Since the development of the DMM by Swartz and Pohl, there have been many attempts at modifying the DMM in order to more accurately match experimental data. Investigations and modifications to the development of the models $[105,122,124,130,131]$, the role of optical phonons [132], the accuracy of the phonon dispersion used [132-134], methods to deal with crystal anisotropy $[15,135,136]$, and the role of inelastic phonon scattering processes $[11,12,137-141]$, all have been considered as potential ways to improve the accuracy of the DMM. While these modifications highlight important considerations that are not found in the original development of the DMM, they are focused on modeling a specific case 


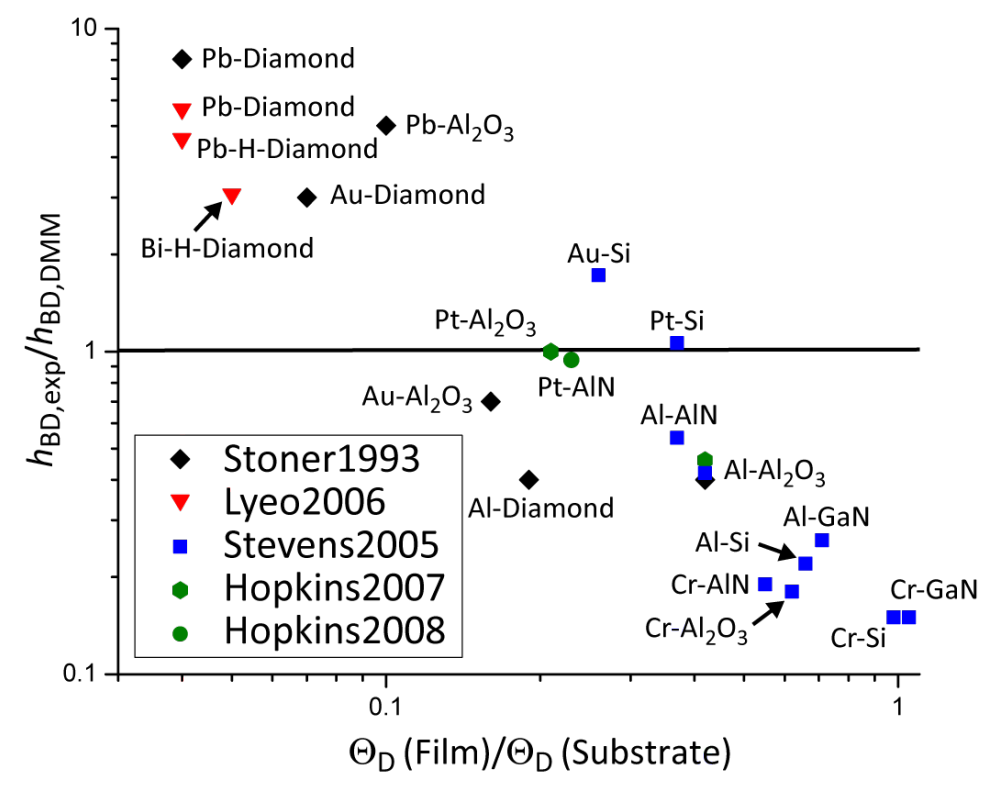

Figure 2.4: Ratio of experimentally measured $h_{\mathrm{BD}}$ to that predicted by the DMM versus the ratio of Debye temperatures of the film and substrate. The data plotted is taken from Stoner \& Marris, [8] Lyeo \& Cahill, [9] Stevens et al., [10] and Hopkins et al. [11, 12].

targeted at a specific subset of data. Therefore, while the modifications appear to enable the model to match well with experimental data for which it was developed, they are not applicable globally, or in conjunction with each other, and the DMM has remained largely a post-dictive tool with little accuracy as a predictive model.

To investigate potential mechanisms of failure that led to the under or over prediction of $h_{\mathrm{BD}}$ via the DMM, we turn back to its formulation. Recalling Equations 2.5 and 2.11 we notice that the majority of the variables, the phonon frequency, $\omega_{1, j}\left(k_{1, j}\right)$, the phonon wavevector, $k_{1, j}$, the phonon group velocity, $\nu_{1, j}\left(k_{1, j}\right)$, and the transmission coefficient, $\zeta^{1 \rightarrow 2}$, are a function of the phonon wavevector $k$. The relationship between all these properties can be ascertained from the phonon dispersion relation, see Figure 2.5. The phonon dispersion in itself is a mapping relationship between the phonon wavevector $k$ and the phonon frequency $\omega$, where the phonon group velocity is taken as the slope of the dispersion at a given wavevector, i.e. $v_{g}=d \omega / d k$. Therefore with knowledge of the phonon dispersion relation for the two materials adjacent to an interface the DMM should enable the prediction of $h_{\mathrm{BD}}$.

What we find is lacking in Equations 2.5 and 2.11 are properties defined to quantify the 


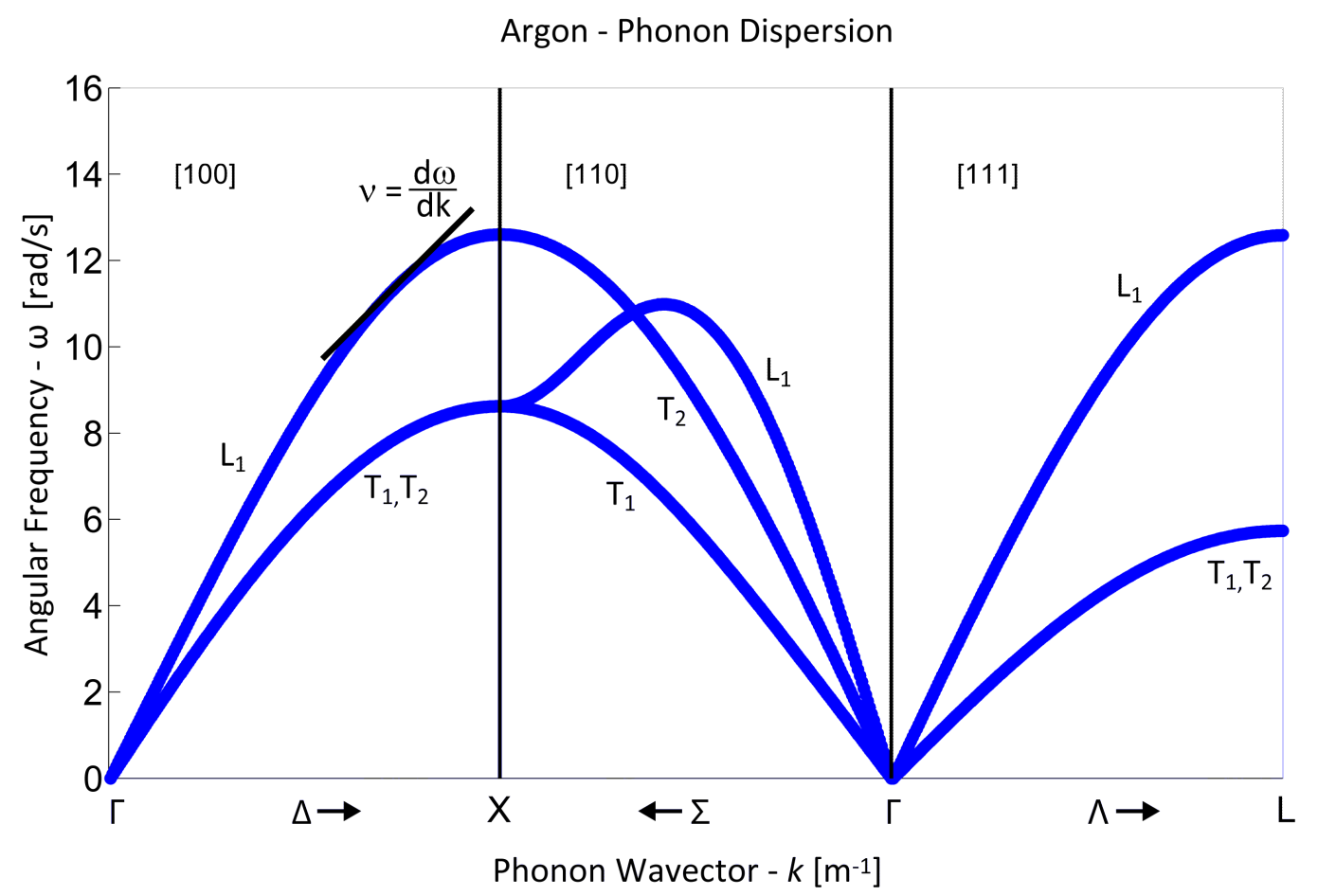

Figure 2.5: The phonon dispersion relation for solid Argon in the directions of high symmetry at $0 \mathrm{~K}$, modeled with the lattice dynamics program, General Utility Lattice Program (GULP) [13].

"interface" ${ }^{9}$. While the transmission coefficient, $\zeta^{1 \rightarrow 2}$, gives the probability of a phonon from material 1 transmitting to material 2, it is formulated based on the inherent, isolated, properties of the materials on either side of the interface. As the name implies, the DMM assumes that all phonons approaching the interface scatter diffusely, and while $\zeta^{1 \rightarrow 2}$ gives the probability of forward transmission to material 2, it assumes that all phonons scatter at a single spatial location. Recalling the definition of $h_{\mathrm{BD}}$ from Equation 2.4, this scattering of phonons at the interface creates a finite temperature drop between the two sides of the interface, see Figure 2.6a. Throughout these formulations, there is no definition of what the "interface" actually consists of.

In the mathematical formulation of $h_{\mathrm{BD}}$ in the DMM, the interface is assumed to be an infinitesimally thin boundary between two solid materials. No spatial extent is given to the interface, and therefore there are no inherent properties attributed to this region. The two solids are assumed to have inherent thermophysical properties based on their

\footnotetext{
${ }^{9}$ Interface is written in quotes, because there is no coherent definition as to the extent of the interface or its properties, as will be discussed shortly.
} 
bulk dispersions, to be in close proximity to each other, to be non-interacting, and to be unperturbed by the presence of the adjoining solid. This means that the thermophysical properties of the solid-solid system i.e., atomic mass, force constants, the phonon group velocity, etc., follow a step function with the point of inflection at the interface, see Figure $2.6 \mathrm{~b}$.

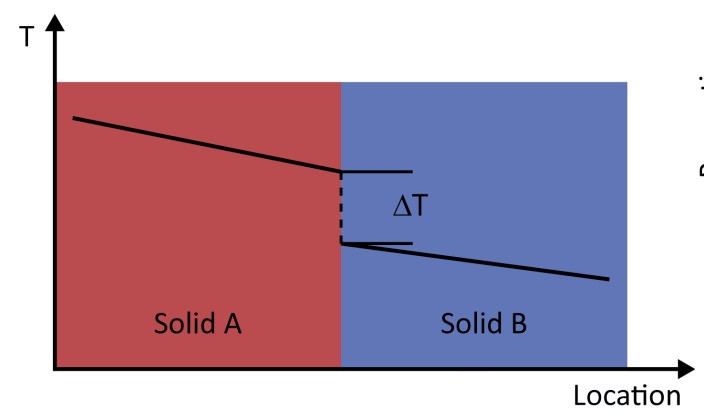

(a)

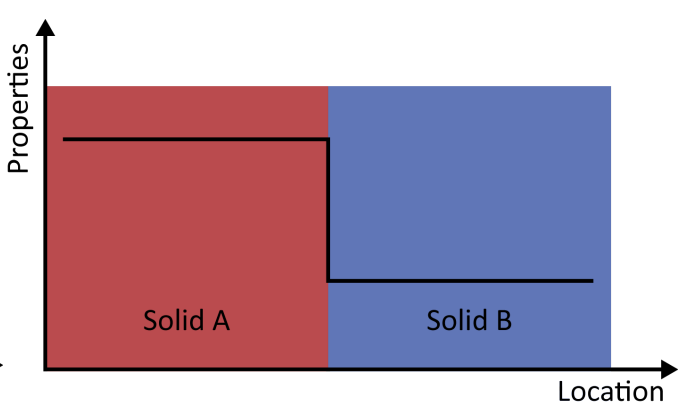

(b)

Figure 2.6: A solid-solid system with (a) a finite temperature drop at the interface as described by the DMM and (b) a step function dependence on material properties, e.g. atomic mass, etc. due to the infinitesimal physical extent attributed to the interface.

As may be expected, this is an idealized case and realistic solid-solid systems do not behave in this manner. The closest representation of this type of system would be the epitaxial growth of a well matched system such as $\mathrm{TiN} / \mathrm{MgO}$ [142]. In reality, solid-solid systems fabricated with more common sputtering and evaporation processes used in the microfabrication of nanoscale devices will not form perfect interfaces. Due to the energetics of the fabrication process, the lattice mismatch between the two solids, thermodynamically driven interactions, etc., solid-solid interfaces can deviate greatly from the "ideal" picture. In realistic nanofabricated devices, species interdiffusion and reaction can occur on the order of the devices' characteristic length scale, even at room temperature [143]. In most cases, the realistic interface between two solids will have varying extents of interdiffusion, amorphization, and reaction based on the nature of the materials and fabrication processes. To better understand how the properties of the interface affect phonon transmission, we will look at several subsets of studies and experimental data focused on particular properties of the interface. A basic understanding of how these various properties affect $h_{\mathrm{BD}}$ will be important when analyzing the results in Chapter 7. 


\subsection{Interface Effects on Thermal Boundary Conductance at Solid-Solid Interfaces}

Formulations such as the AMM and DMM assume that the interface between two materials is an infinitely thin plane with no unique properties. In contrast to this, the development of the transient thermoreflectance technique by Paddock and Eesley [144], which will be discussed in detail in Chapters 3-4, was done in part to allow the measurement of the thermal properties of a thin film, supported by a substrate, without the influence of the film-substrate interface. Paddock and Eesley cite that while the thermal modeling considers an ideal interface, in reality there are many factors that alter the properties of the interface relative to the bulk. Over the past two decades much scientific effort has gone into better understanding the impacts of properties in the vicinity of the solid-solid interface on $h_{\mathrm{BD}}$. Recently, there has been several excellent reviews that have summarized these efforts, and have highlighted some of the major issues currently facing the field of nanoscale energy transport $[91,94,145,146]$. Here we will briefly introduce several topics related to phonon transport across a solid-solid interface, which will be pertinent when considering the experimental results in Chapter 7. While not an exhaustive review, a selection of theoretical work, simulations, and experimental results are presented to provide insights on how several key interface properties affect $h_{\mathrm{BD}}$.

\subsubsection{Mismatch of Vibrational Properties}

The formulation and dependence of both the AMM and the DMM relies in large part on the transmission coefficient, given by Equations 2.7 and 2.11 for the AMM and the DMM respectively. In either case, the transmission coefficient, in part, describes a numerical comparison between the thermophysical (vibrational) properties of material 1 and material 2. When the vibrational properties between the materials are similar, the transmission coefficient approaches a maximum. Conversely, when there is a large mismatch between the vibrational properties the transmission coefficient approaches a minimum. Beyond the considerations in the AMM and DMM, Pettersson and Mahan [147] used lattice dynamics theory to investigate the impact of dissimilar lattice properties, i.e. atomic masses, force 
constants, and lattice constants, on $h_{\mathrm{BD}}$. Their results showed decreased conductance with deviation from the ideal case of matching properties for both materials comprising the interface.

Much more work has been undertaken studying the impacts of mismatched vibrational properties on $h_{\mathrm{BD}}$ via molecular dynamics. $\mathrm{MD}$ is ideally suited for this task due to the ability to individually control the properties of the crystal systems. Twu and Ho [111] studied $h_{\mathrm{BD}}$ between two FCC crystals defined by a Lennard-Jones (LJ) and Morse potential respectively. They tuned the mismatch between the crystals by varying the width of the interatomic potential well of each crystal, and found a nearly exponential decrease in interface conductance with an increase in the ratio of the widths of the potential wells. Stevens et al. [110] used the mass ratio between two Lennard-Jones FCC solids to tune the vibrational mismatch between the two crystals, finding that interface conductance decreased with increasing mass mismatch as expected from the DMM theory. Similar results were found by Wang and Liang [148] who showed a nearly linear dependence on $h_{\mathrm{BD}}$ with mass ratio. Landry and McGaughey [105] used a more complex Stillinger-Weber (SW) potential to study $h_{\mathrm{BD}}$ between Si and "heavy-Si" and also found a monotonic increase in $h_{\mathrm{BD}}$ with decreasing mass ratio. Hu et al. [149] varied the vibrational mismatch between Si and an amorphous polyethylene polymer through the atomic spring constant in Si. This in turn quantified the elastic modulus of the Si which varied from the baseline Si to that similar to diamond. They found $h_{\mathrm{BD}}$ decreased with increasing elastic modulus, as it increased the vibrational mismatch between the $\mathrm{Si}$ and the polymer. Lyver and Blaisten-Barojas [112] directly compared the vibrational mismatch between two LJ crystals by comparing the calculated phonon density of states (PDOS) in each material as a function of the ratio of LJ parameters in each material. They found that as the ratio of $\epsilon$ increased, phonon modes were shifted from lower to higher frequencies. Increasing the mass difference ratio on the other hand shifted mid range frequency phonons down to the lower frequency range. However, ultimately in both cases $h_{\mathrm{BD}}$ decreased as the ratio of LJ parameters increased.

As molecular dynamics techniques have been expanded, it has become possible to find the phonon density of states near the region of the interface. Shin et al. [150] compared the bulk PDOS in Si and In to the PDOS in a region confined to the Si/In interface. They found 
that the PDOS in In shifted to higher frequencies locally at the interface, while the PDOS of Si shifted towards lower frequencies. Similar redistribution of the PDOS at an interface was found by English et al. [14], see Figure 2.7, and Liang et al. [20, 151]. The locally modified PDOS at the interface region was later used to attempt to tune $h_{\mathrm{BD}}$ through the use of an intermediate layer, which will be the focus of Chapter 7 .

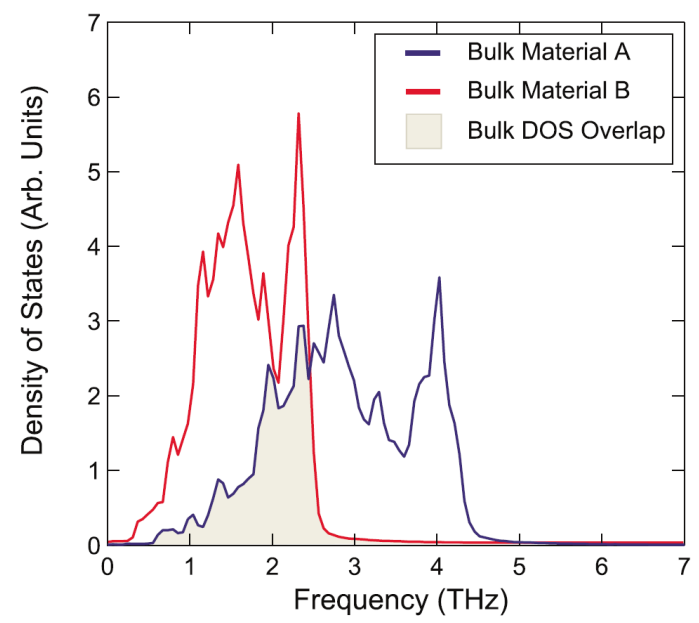

(a)

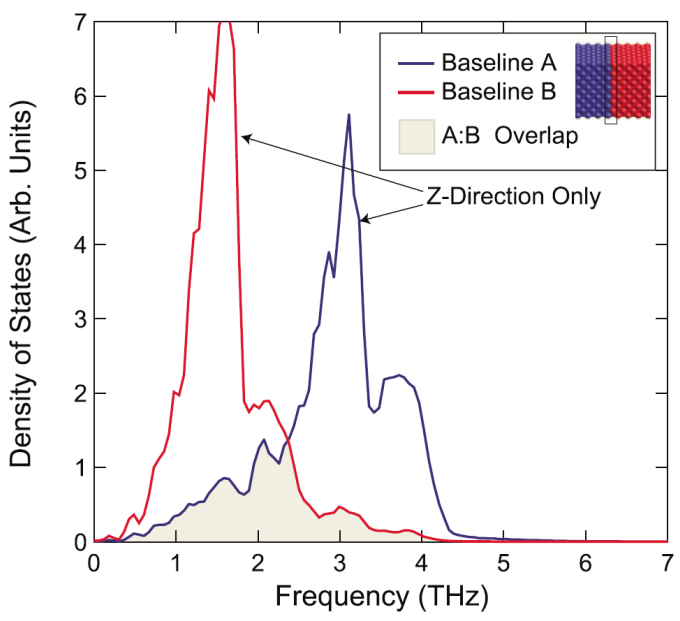

(b)

Figure 2.7: (a) Bulk occupied phonon density of states for material A (40 amu) and material B (120 amu), with the shaded region representing the overlap in the bulk density of states. (b) Occupied phonon density of states in the z-direction calculated from the monolayers on either side of the interface between material A and B. Data and figures reproduced with permission from English et al. [14].

Experimentally, the variation in vibrational properties between two materials is difficult to quantify, as there is no simple method to vary system parameters or determine the PDOS. As a proxy for the overlap of the PDOS in two materials, Stoner and Maris [8] used the ratio of the Debye temperatures. Using picosecond thermoreflectance to measure $h_{\mathrm{BD}}$, they found that $h_{\mathrm{BD}}$ decreased as the Debye temperature mismatch between the film and substrate increased. Similar results were found by Stevens et al. [10] for metalsemiconductor systems of interest to microelectronic device designers. Lyeo and Cahill [9] found very low $h_{\mathrm{BD}}$ for the highly vibrationally mismatched system of $\mathrm{Bi}$ on diamond, but found that their measurements exceeded the conductance predicted by the phonon radiation limit, i.e. the theoretical upper limit for thermal conductance. They attributed this discrepancy to the possibility of inelastic phonon scattering processes, which we will focus on next. 


\subsubsection{Inelastic Scattering Contributions}

Most semi-classical treatments of $h_{\mathrm{BD}}$, such as those formulated in the AMM and DMM only consider elastic phonon interactions. Under this assumption the phonon transmission coefficient, see Equation 2.11, is independent of temperature. Therefore, $h_{\mathrm{BD}}$ is expected to remain constant above the Debye temperature of one or both materials, depending on the underlying assumptions of phonon interaction. To test this assumption Stevens et al. [110], using nonequilibrium molecular dynamics (NEMD), found $h_{\mathrm{BD}}$ between two mismatched Lennard-Jones crystals as a function of temperature. Their results showed a strong linear dependence of $h_{\mathrm{BD}}$ with temperature which was attributed to inelastic ${ }^{10}$ phonon scattering. This work was further corroborated by molecular dynamics work from Landry and McGaughey [105], Luo and Lloyd [152], Ju et al. [114], and Duda et al. [131]. The linear dependence of $h_{\mathrm{BD}}$ was supported experimentally for a wide range of material systems by measurements of $h_{\mathrm{BD}}$ at elevated temperature by Lyeo and Cahill [9] and by Hopkins et al. $[11,12]$.

There have been numerous theoretical models developed and existing models modified in an attempt to describe the contributions of inelastic scattering to the total thermal conductance across an interface. In 2004 Dames and Chen introduced the maximum transmission model (MTM) as an extreme upper bound to $h_{\mathrm{BD}}$, which included interaction of all possible phonon frequencies on either side of the interface and therefore all inelastic processes. Hopkins and Norris [138] proposed a joint frequency diffuse mismatch model (JFDMM) which considered a weighted portion of the phonon spectra in the substrate to interact with the incident phonons from the film, as a means to estimate the contributions of inelastic scattering without specifying inelastic transmission probabilities. In an attempt to specifically quantify the contributions of inelastic scattering on the transmission coefficient of phonons at an interface, Hopkins [140] developed the higher harmonic inelastic model (HHIM). The HHIM considers higher order phonon interactions, i.e. three-phonon, four-phonon processes, etc., with reduced probability of each higher order event. Because the HHIM considers only integer combination phonon process, it represents a lower bound

\footnotetext{
${ }^{10}$ Inelastic, sometimes called anharmonic, phonon scattering involves two or more phonon which combine (or separate) to form a phonon with a frequency that is the sum (or difference) or the incident phonons.
} 
to the contribution of inelastic processes. To more rigorously consider inelastic phonon processes, of any combination, Hopkins et al. [15] further modified the HHIM to integrate the available phonon flux over all possible combinations of n-phonon processes when calculating the transmission coefficient. The new model, denoted as the anharmonic inelastic model (AIM), showed that for samples with a high degree of vibrational mismatch at elevated temperatures, higher order processes (up to 10-phonon processes) contributed significantly to $h_{\mathrm{BD}}$, see Figure 2.8 .

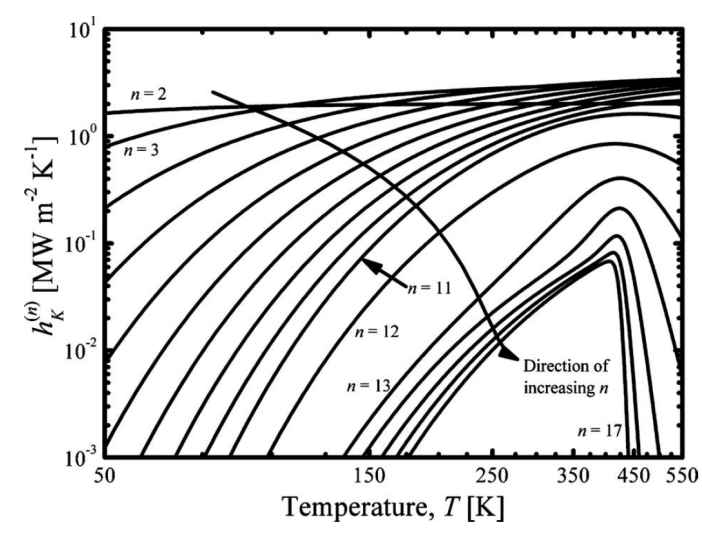

(a)

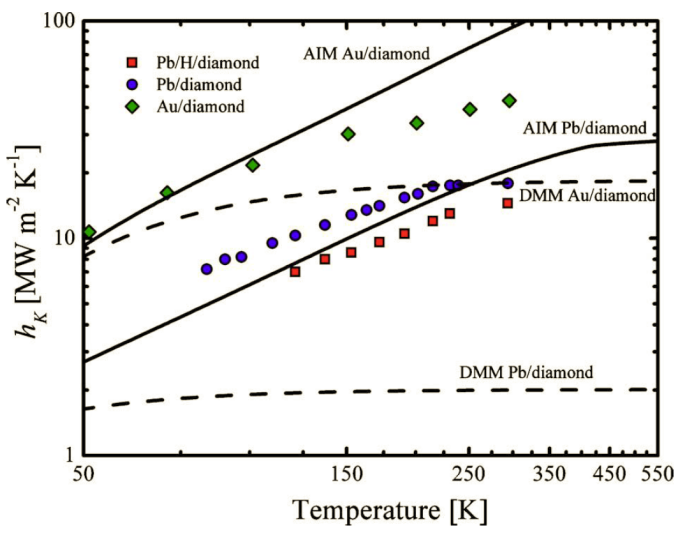

(b)

Figure 2.8: (a) Anharmonic inelastic model predictions of $h_{\mathrm{BD}}$ for $\mathrm{Pb}$ on diamond as a function of temperature and $n$-phonon processes for $n=2-17$. (b) Comparison of $h_{\mathrm{BD}}$ predicted from the DMM and the AIM along with experimental data for $\mathrm{Pb} / \mathrm{H} /$ diamond, $\mathrm{Pb} /$ diamond, and $\mathrm{Au} /$ diamond. Figures and data from Hopkins et al. [15].

\subsubsection{Bonding at the Interface}

As discussed more thoroughly in Appendix A, the bonding between atoms contributes in part to the behavior of phonons in a crystal. It is therefore reasonable to surmise that the bonding between two crystal types will create a modified local dispersion with properties inherently different than the surrounding bulk, and in turn impact $h_{\mathrm{BD}}$.

To study the impact of interface bonding on $h_{\mathrm{BD}}$, Prasher [153] developed a modified version of the AMM, which assumes perfect contact, to take into account weaker van der Waals forces at the interface. His results showed large deviations from the AMM for interface adhesion energies below $200 \mathrm{~mJ} / \mathrm{m}^{2}$, with weaker adhesion reducing $h_{\mathrm{BD}}$. Persson et al. [154] used linear elasticity theory to study $h_{\mathrm{BD}}$ across the solid-solid interface as a function 


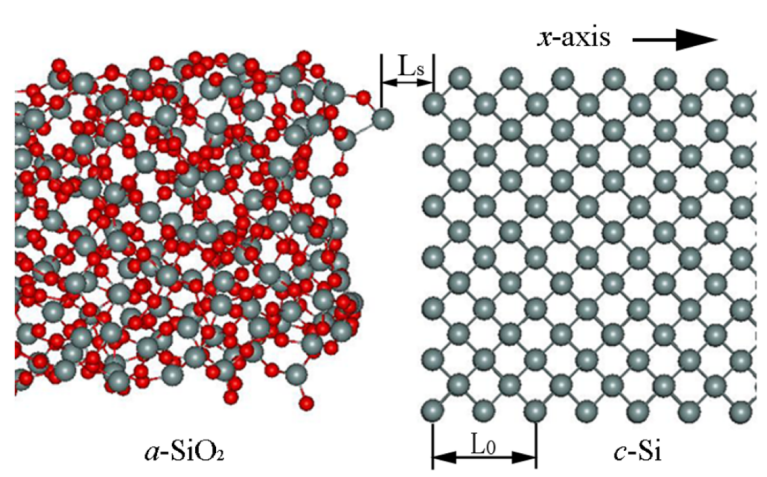

(a)

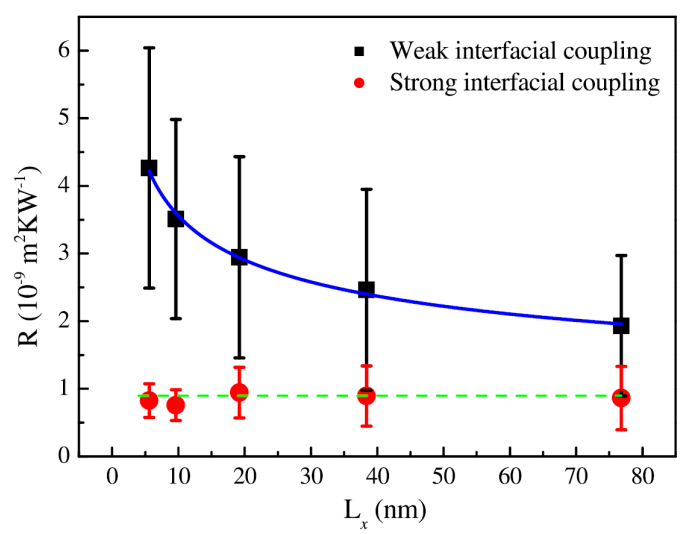

(b)

Figure 2.9: (a) Crystal structure of the $\mathrm{SiO}_{2} / \mathrm{Si}$ system, with the $\mathrm{Si}$ denoted in grey and the $\mathrm{O}$ atoms in red. (b) $h_{\mathrm{BD}}$ at the $\mathrm{SiO}_{2} / \mathrm{Si}$ interface as a function of the length of the interface junction for both weak and strong interfacial coupling. Figures and data reproduced with permission from Chen et al. [16].

of temperature. They found for very low temperatures, $T<3 \mathrm{~K}$, that a weakly and strongly bonded interface transmitted phonons equally well. However, they found that at room temperature and above, phonon transport across the interface may be reduced by a factor of 100 or more for the weakly bonded interface as compared to the strong interface interaction.

Using molecular dynamics simulations, Chen et al. [16] studied the impact of the strength and physical organization of bonds on $h_{\mathrm{BD}}$ at the interface between amorphous and crystalline Si. It was found that $h_{\mathrm{BD}}$ increased monotonically with increasing bonding strength at the interface. In the limit of weak interfacial coupling, it was found that the physical organization of the bond, i.e. bond angle and the distance between bonds, significantly impacted $h_{\mathrm{BD}}$. In contrast, in the limit of strong coupling, $h_{\mathrm{BD}}$ was shown to be nearly independent of the details of the bond structure, see Figure 2.9. A monotonic relationship between $h_{\mathrm{BD}}$ and interface bond strength was also observed by $\mathrm{Hu}$ et al. [149] at the interface between single-crystal Si and amorphous polyethylene using molecular dynamics simulations. Shen et al. [155] investigated using MD simulations the roles of interface boding and pressure on $h_{\mathrm{BD}}$ between two Lennard-Jones FCC crystals. They found that increasing the pressure at the interface was essentially the same as increasing the interface bond strength, with the increased pressure stiffening the interface bonds. Therefore, in the case of weak bonds $h_{\mathrm{BD}}$ increased with increasing pressure while for the case of strong bonds 
$h_{\mathrm{BD}}$ was pressure independent. Similar trends of increasing $h_{\mathrm{BD}}$ with increasing pressure were also showed by Liu et al. [156] for simulations of $h_{\mathrm{BD}}$ between a single open-ended carbon nanotube and crystalline Si.

Collins et al. [157] and Monachon and Weber [158] both experimentally studied $h_{\mathrm{BD}}$ between $\mathrm{Al}$ and diamond with various surface chemistry configurations on the diamond surface to introduce different bonding conditions. Both investigated various surface pretreatments to the diamond substrate, including oxygen and hydrogen treatments. The results showed that the samples that were oxygen treated had significantly higher (by a factor of 4-5) $h_{\mathrm{BD}}$ than those with the hydrogen treatment. Both groups attributed the increased $h_{\mathrm{BD}}$ to more open bonds at the interface and therefore better wetting of the deposited Al. In a similar manner, Hopkins et al. [159] measured $h_{\mathrm{BD}}$ between $\mathrm{Al}(\mathrm{Au})$ and a functionalized single layer of graphene using time domain thermoreflectance. The single graphene layers were functionalized with either an oxygen or hydrogen plasma prior to deposition of the metal, to control the number of covalent bonds at the metal-graphene interface. Their results showed increased conductance across the oxygen functionalized interface, attributed in an increased density of covalent bonds. To more directly control the number of covalent bonds, Losego et al. [160] investigated $h_{\mathrm{BD}}$ between Au and quartz with an intermediate layer of a self-assembled monolayer (SAM) with varying termination chemistries. For a methyl:thiol SAM layer with varying thiol termination, $h_{\mathrm{BD}}$ was shown to increase $80 \%$ as the fraction of thiol termination (which corresponds to increasing covalent bond density) increased from 0 to $100 \%$.

\subsubsection{Strain, Defects and Dislocations at the Interface}

In the next few subsections we will discuss physical nonidealities at the solid-solid interface. The major factor in determining the separation of the various phenomena into categories will be the scale of the phenomenon. In reality, the challenge is all these categories are interdependent. Compositional inter-diffusion can be viewed as a form of roughness, and any roughness is likely to introduce crystallographic dislocations, vary lattice strain, and can be viewed as a form of defects. However, in an attempt to categorize the phenomena, we will consider dislocations/defects, roughness, and inter-diffusion to be increasing scales 
of interface nonidealities, not to be confused with their importance in determining $h_{\mathrm{BD}}$.

For the smallest deviations from the ideal interface, we will consider the impacts of lattice strain, defects, and dislocations on $h_{\mathrm{BD}}$. These are considered physically small phenomena that are a natural product of the interaction between the two species at the interface. While the selection of material pairs and fabrication conditions can be use to mitigate these effects, in general these factors will almost always be present to some degree.

Kozorezov et al. [161] theoretically derived the probability of phonon transmission between two solids with an intermediate region of material with properties differing from either solid to act as an analog for interface defects, mixing, etc. They found that scatteringmediated transmission is possible at high phonon frequencies, but is less frequency dependent for low frequencies. Prasher and Phelan [162] created a scattering-mediated acoustic mismatch model to account for scattering at the interface between two materials. Their formulation was generalized to account for any non-bulk like properties at the interface through an effective index of refraction at the interface. Meng et al. [163] studied the effect of a strain field at the interface on phonon conductance. For low frequency phonons below a certain cutoff frequency they found no scattering at the interface, with the magnitude of the cutoff frequency increasing with increasing interface strain.

Using molecular dynamics simulations Stevens et al. [110] studied the impact of interface defects on $h_{\mathrm{BD}}$ using a Lennard-Jones solid-solid lattice structure with large lattice mismatch, which was relaxed to form a disordered interface structure a few atomic layers thick. Their results showed that the presence of defects at the interface reduced $h_{\mathrm{BD}}$, with the impact of the reduction being greatest for systems with well matched Debye temperatures. For highly mismatched systems, the results of added defects were not significant. Li and Yang [17] used a combination of molecular dynamics simulations and atomistic Green's function calculations to determine the impact of lattice strain and the presence of vacancy defects at the interface between $\mathrm{Si}$ and Ge-like crystals on $h_{\mathrm{BD}}$. For the impact of lattice strain, Li and Yang investigated strain induced by a lattice mismatch of up to $16 \%$ and found that $h_{\mathrm{BD}}$ decreased with increasing lattice mismatch, see Figure 2.10. While the presence of lattice strain has been shown to increase the allowed mode conversion at the interface, and therefore $h_{\mathrm{BD}}$ [147], the disorder introduced at the relaxed interface negated these effects 


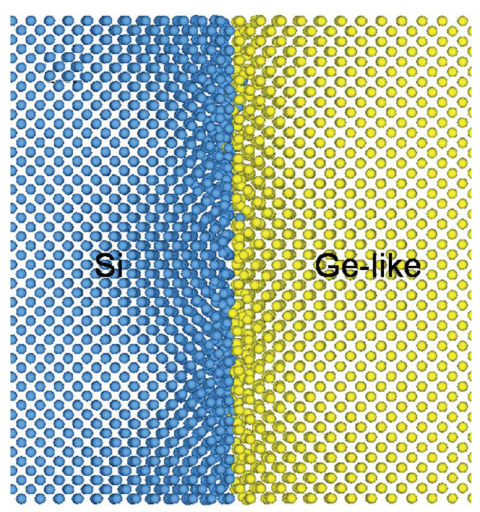

(a)

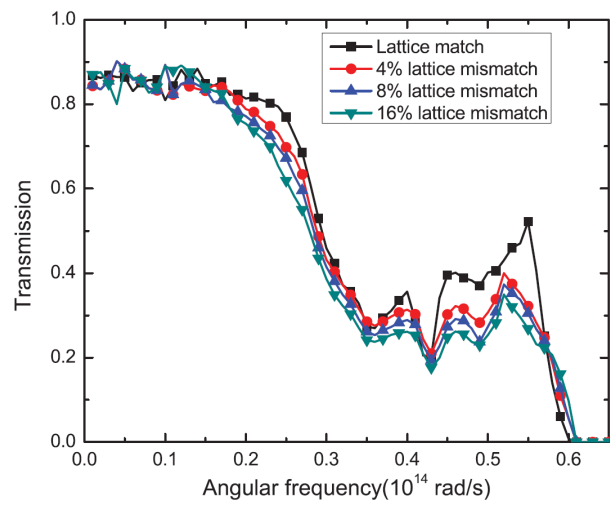

(b)

Figure 2.10: (a) Relaxed Si/Ge interface formed from an $8 \%$ lattice mismatch. (b) Phonon transmission as a function of frequency for different lattice mismatches. Figures and data reproduced with permission from Li and Yang [17].

and reduced the overall phonon transmission, agreeing with the results of Stevens et al. [110]. Additionally Li and Yang studied the affects of two atomic layers at the Si/Ge-like interface with randomly distributed spherical vacancy defects of varying size and distribution. The simulations showed that the defect size had a larger impact than the defect density, with the transmission of low frequency phonons being the most impacted. When the defect size was large enough, the changes to phonon transmission due to the defects were greater than the effects of the lattice mismatch. Duda et al. [164] investigated how the size and distribution of impurity atoms near the solid-solid interface of Lennard-Jones materials impacted $h_{\mathrm{BD}}$. In general, their findings in both cases showed a trend to reduce $h_{\mathrm{BD}}$ with increasing impurity distribution and mass. Their results did show increased $h_{\mathrm{BD}}$ for impurities with a mass that was the mean between the constituent masses, however uncertainty in their results make that claim inconclusive. Ju and Liang [165] used molecular dynamics and wave packet simulations to study the effects of grain boundary twists between Stillinger-Weber $\mathrm{Si}$ on $h_{\mathrm{BD}}$, for twist angles between $\sim 12-37^{\circ}$. The $h_{\mathrm{BD}}$ across the grain boundary interface was found to decrease with increasing twist angle and was attributed to increased grain boundary energy with increasing twist.

Hopkins et al. [166] experimentally studied the effects of ion implantation on $h_{\mathrm{BD}}$ between $\mathrm{Al} / \mathrm{Si}$ and $\mathrm{Al} / \mathrm{Al}_{2} \mathrm{O}_{3}$ systems. They found an order of magnitude reduction in $h_{\mathrm{BD}}$ for the highest ion dose $\left(5.79 \times 10^{17}\right.$ protons $\left./ \mathrm{cm}^{2}\right)$ in both systems, which was attributed 
to increased surface roughness, near surface damage, and bond breaking. Compared to the baseline samples, they found a large drop in $h_{\mathrm{BD}}$ for even the smallest ion dose $\left(5.79 \times 10^{14}\right.$ protons $/ \mathrm{cm}^{2}$ ). Additionally Hopkins et al. [167] measured $h_{\mathrm{BD}}$ across the $\mathrm{Al} / \mathrm{GaSb}$ and GaSb/GaAs interfaces for GaSb grown to promote varying densities of threading dislocations $\left(5 \times 10^{6}-5 \times 10^{8}\right.$ dislocations $\left./ \mathrm{cm}^{2}\right)$. It was found that $h_{\mathrm{BD}}$ decreased across both interfaces with increasing dislocation density. In contrast to Hopkins et al., HanischBlicharski et al. [168] found no difference in the $h_{\mathrm{BD}}$ for a $\mathrm{Bi} / \mathrm{Si}$ system with and without misfit dislocations at the interface. One possible explanation for the independence of $h_{\mathrm{BD}}$ is the $\mathrm{Bi} / \mathrm{Si}$ system had nearly 5 times fewer dislocations as compared to the work of Hopkins et al. Additionally the Bi/Si system has a high degree of vibrational mismatch (Debye ratio $=0.19$ ) compared to the $\mathrm{Al} / \mathrm{GaSb} / \mathrm{GaAs}$ system from Hopkins et al. (Debye ratio of 1.48 and 0.74 respectively), which may mask the effects of the misfit dislocations.

\subsubsection{Roughness at the Interface}

We will generally define a rough interface as one in which the interface is not planar, but where there is still a sharp ${ }^{11}$ compositional gradient from material 1 to material 2 . Quantifying the degree of roughness is generally accomplished via two quantities: 1) the root mean square (rms) roughness of the interface and 2) the correlation length. Either parameter on its own provides an incomplete picture of the surface roughness. Because the rms roughness is an average deviation from the mean plane, the same rms roughness can be accomplished by either very large deviations sparsely populated in the horizontal direction, or many smaller deviations in the same surface area. To add clarification, it is also usually helpful to consider the correlation length, which in the direction parallel to the interface is a measure of the average distance between similar features. Prior to depositing the film the surface roughness can be measured via microscopy techniques such as atomic force microscopy (AFM) [169]. After the film is deposited, the interface roughness can be characterized by X-ray techniques such as X-ray reflectivity (XRR) for rms roughness and X-ray diffuse scattering (XDS) for surface roughness and correlation length [170].

\footnotetext{
${ }^{11}$ We can see how categories are difficult to define as the difference between a rough interface and one that is compositionally inter-diffuse is a matter of semantics in the definition of sharp. Much more research will have to be conducted to determine if there are distinct trends delineating rough from inter-diffuse interfaces.
} 
There have been a number of studies that have focused on the theoretically determined contributions of atomic level interface roughness to variations in the transmission of phonons across an interface, and therefore $h_{\mathrm{BD}}$. In the early 1990's Kechrakos $[171,172]$ studied the transmission of elastic phonons, specularly and diffusely scattered, across the interface between two crystals with an atomic level roughness on the order of one monolayer using a simplified lattice dynamics approach. They showed an increase in interface conductance across all temperatures, as high as a factor of 3 above the perfect interface for vibrationally mismatched materials above the Debye temperature. Kazan [122] created a modified version of the phonon transmission coefficient based on the morphology of the solid-solid interface to weight the probability of specular and diffuse scattering and bridge the gap between the AMM and DMM. He found in general that the conductance between two solids with similar vibrational properties increased with increasing rms roughness. Conversely when the two solids have highly dissimilar vibrational properties, thermal conductance is increased only for low rms roughness.

Using Green's function methods, Fagas et al. [173] studied the transmission of elastic phonons across a disordered atomic monolayer between two perfect crystals, which was tuned by varying the mass of the atoms in the disordered layer. They found that the phonon transmission was a function of phonon frequency and the correlation length of the disordered region. Zhao and Freud [174] used a Green's function method to determine the scattering and transmission of phonons at the atomically rough interface between two FCC crystals, with phonon dispersions approximately equal to that of Si and Ge. They found that the proportion of specular scattering, as compared to diffuse scattering, was strongly dependent on the interface roughness and only weakly dependent on the correlation length. In contrast, they found that the overall phonon transmission across the interface was generally independent of both the interface roughness and the correlation length. Additionally, Tian et al. [18] combined Green's function methods with density functional theory (DFT) to calculate phonon transmission and phonon conductance across an ideal and roughened Si/Ge interface, see Figure 2.11. They found that the conductance across the interface can be increased when the thickness and profile of the interface roughness is properly controlled.

Molecular dynamics simulations have also been utilized to study interface roughness 


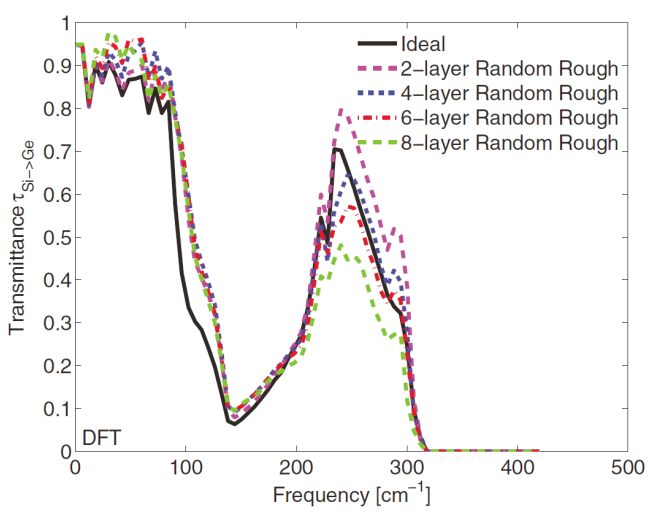

(a)

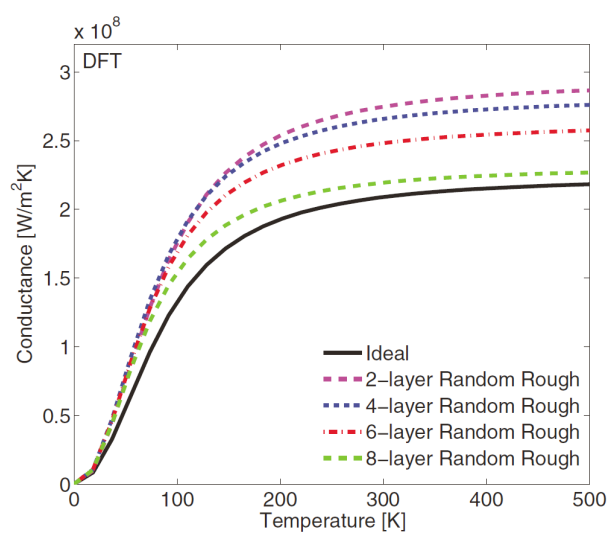

(b)

Figure 2.11: Phonon transmission from Si-to-Ge (a) and thermal conductance from Si-to-Ge (b) based on Green's function calculations using DFT force constants from Tian et al. as a function of the thickness of the roughened interface layer. Figures reproduced with permission from [18].

effects on $h_{\mathrm{BD}}$. Sun and Murthy [104] used the molecular dynamics wave-packet method to study the transmission of phonons across the $\mathrm{Si} / \mathrm{Ge}$ interface for an ideal interface, an interface with random roughness, and an interface with roughness organized in a regular pattern. The phonon transmission was shown to be a strong function of frequency compared to the interface roughness. For the ideal interface, the transmission predicted by the simulations agreed well with the expected values from the AMM. For the roughened interfaces, transmission decreased with increasing phonon frequency with the transmission for the regularly roughened interface showing complex mode conversion. Zhou et al. [175] used MD to study $h_{\mathrm{BD}}$ between $\mathrm{Al}$ and $\mathrm{GaN}$ for regularly arranged interface patterns including rectangular, sinusoidal, and triangular morphologies. Their results showed that when bonding at the interface between the two species was weak, and the correlation length between features long, that $h_{\mathrm{BD}}$ was proportional to the surface area of the interface which increases with increasing surface roughness.

Experimental studies of $h_{\mathrm{BD}}$ dependence on surface roughness have also been undertaken. Hopkins et al. found $h_{\mathrm{BD}}$ decreased as a function of interface roughness in an $\mathrm{Al} / \mathrm{Si}$ system for roughness caused by both chemical etching of the substrate prior to $\mathrm{Al}$ deposition [176] and the growth of quantum dots on the Si surface [177]. For quantum dot roughening on the order of $4 \mathrm{~nm}$, they found a decrease in $h_{\mathrm{BD}}$ on the order of 1.6 times compared to the case of no quantum dots. Chen and Hui [178] found decreased thermal 
conductivity in thin $\mathrm{Au}$ layers on $\mathrm{Si}(100)$ substrates with high $\mathrm{Si}$ surface roughness. The reduced thermal conductivity was attributed to increased electron scattering near the interface and increased grain-boundary scattering due to small grain sizes, caused by limited adatom mobility during $\mathrm{Au}$ deposition.

\subsubsection{Compositional Inter-diffusion at the Interface}

Similar to interface roughness, the degree of compositional inter-diffusion at the solidsolid interface can also impact $h_{\mathrm{BD}}$. While it is difficult to provide a clear definition of the delineation between roughness and inter-diffusion, generally the spatial extent of interdiffusion is larger than the surface roughness. In addition, inter-diffused species will intermix as opposed to conformal mapping of a film on a rough surface and can form compounds. Therefore, while the interface roughness was quantified by the rms roughness and the coherence length, the inter-diffused region will be quantified by the width of the mixed region and the compositional profile of the species throughout the region.

Theoretically, the treatment of disorder between two solids was treated as an extension of the DMM by Beechem et al. for crystalline [179] and non-crystalline [180] interphase layers. The former treated the inter-diffused region as a virtual crystal layer with properties based on the weighted average of the constituent material properties. The latter used a similar formulation, but considered the inter-diffused region as a non-crystalline (disordered) layer. The results of the modified version of the DMM agreed reasonably well with the experimental data with which it was compared.

Li and Yang [17] used a combination of MD simulations to create the relaxed crystal structure, and atomistic Green's function calculations to determine the phonon transmission across the interface between $\mathrm{Si}$ and Ge-like materials with a $\mathrm{Si} / \mathrm{Ge}$ alloy layer of varying thickness at the interface. Their results showed a reduction in the phonon transmission across the alloyed layer with increasing layer thickness, and the disappearance of peaks in the transmission for certain phonon frequencies which they attributed to phonon mode conversion, see Figure 2.12. The $h_{\mathrm{BD}}$ was also reduced with increasing layer thickness with a $50 \%$ drop for an alloy layer thickness of $1 \mathrm{~nm}$.

Using nonequilibrium molecular dynamics simulations Twu and Ho [111] investigated 


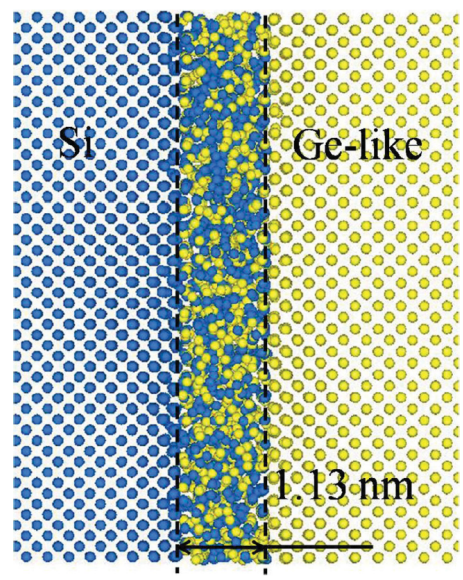

(a)

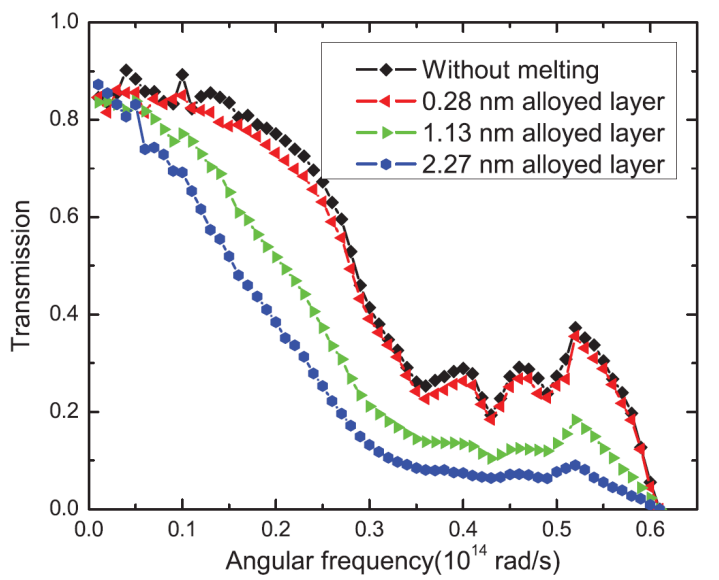

(b)

Figure 2.12: (a) Alloyed Si/Ge layer created via melting and quenching of the interface region with an $8 \%$ lattice mismatch. (b) Phonon transmission as a function of frequency for different thicknesses of the alloyed region. Figures and data reproduced with permission from Li and Yang [17].

$h_{\mathrm{BD}}$ across the interface of two FCC crystals with the crystal systems being described by a Lennard-Jones and Morse potential on either side of the interface respectively. An interdiffused region of varying thickness and compositional disorder was added between the two crystals, and it was found that in all cases $h_{\mathrm{BD}}$ was reduced as compared to the perfect interface. In contrast, Stevens et al. [110] found an increase in $h_{\mathrm{BD}}$ between dissimilar LJ solids by a factor of 1.4-1.8 for alloy layers between 2 and 20 atomic planes in thickness. It should be noted in the work of Twu and Ho the alloy layer had random mixing, while in the work from Stevens et al. the atoms in the alloy layer were systematically arranged. Choi et al. [181] used more realistic potentials and parameters to calculate the thermal conductance across atomically clean and disordered $\mathrm{Al} / \mathrm{Si}$ interfaces via NEMD. Their results showed decreasing $h_{\mathrm{BD}}$ with increasing disorder, and attributed the reduction to increased phononphonon scattering. In contrast once again, Zhou et al. [175] found that a disordered layer of $2.9 \mathrm{~nm}$ between $\mathrm{Al}$ and $\mathrm{GaN}$ increased $h_{\mathrm{BD}}$ by a factor of 1.6. They attributed the increase in $h_{\mathrm{BD}}$ to a phonon bridging effect, which was proposed by English et al. [14] and later by Liang and Tsai [20]. More details of this phonon bridging effect will be discussed in Chapter 7.

Hopkins et al. $[182,183]$ experimentally measured $h_{\mathrm{BD}}$ for a $\mathrm{Cr} / \mathrm{Si}$ system as a function of inter-diffusion layer thickness, which was created via backsputtering etching and heat 
treatment of the Si substrate prior to the deposition of Cr. Their results showed a reduction in $h_{\mathrm{BD}}$ as large as $40 \%$ for a diffusion layer thickness of $\sim 15 \mathrm{~nm}$. The results showed a dependence on both the width of the inter-diffused region and the slope of the compositional change between $\mathrm{Cr}$ and Si.

\subsection{Chapter Summary}

In this chapter the primary energy carriers of thermal energy in different material systems were discussed as well as some of the modeling techniques used in an attempt to model $h_{\mathrm{BD}}$. To highlight some of the current work in the field a brief review of literature was presented focused on various properties of the interface that can potentially alter $h_{\mathrm{BD}}$. It would appear from the review of the subsets of literature focused on $h_{\mathrm{BD}}$, that $h_{\mathrm{BD}}$ is in fact impacted by many different variables. In part this is true, with in essence $h_{\mathrm{BD}}$ being a complex function of the physical structure of the solid-solid interface. The important take away from this review is that while $h_{\mathrm{BD}}$ is impacted by many interface features, all these properties are interconnected and it is important not to necessarily consider each property, but rather decide which has the most dominate impact on $h_{\mathrm{BD}}$. For example, for highly dissimilar materials the vibrational mismatch has the greatest impact on $h_{\mathrm{BD}}$ overshadowing the contributions of crystal defects. Therefore, when analyzing and interpreting data it is important to consider which features will most directly impact the results. 


\section{CHAPTER 3}

\section{THE EXPERIMENTAL SYSTEM}

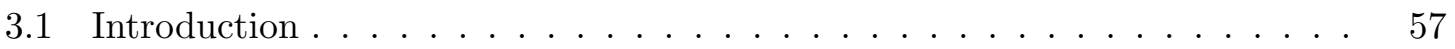

3.2 Optical Methods for Thermal Measurement . . . . . . . . . . . . . 59

3.3 The General System and Components . . . . . . . . . . . . . . . . 69

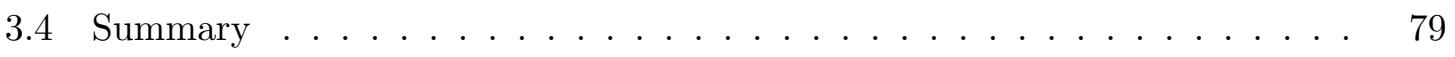

\subsection{Introduction}

In this chapter we will detail the components and configurations of the pump-probe thermoreflectance system used in the University of Virginia Nanoscale Energy Transport lab and in support of this dissertation. While the major components of the thermoreflectance system itself were in place and operational when I began my study at UVa it has been one of my primary goals during my time as a graduate student to improve the performance of the system, provide standards for consistent operation, and introduce methodologies to reduce the time needed to realign the system after laser maintenance or other changes to the system optics.

In an effort to achieve these goals, a series of improvements were implemented to both the physical design of the experimental system and the formulation and methods of deducing 
thermophysical parameters of interest from the experimental data. Chapters 4-6 will cover the details of the improvements to the analytic side of the experiment including improved computational models, methods for increasing the accuracy and efficiency of the modeling process, and methods to ensure robust statistical treatment of the data. In this chapter, the focus will be on the physical improvements and configuration of the experimental system.

After learning to operate and maintain the thermoreflectance system for a few years I was fortunate to have the opportunity to make a major upgrade to the experimental system. Physically this included breaking the system down and moving to a new location, adding optical table space, improving the infrastructure for the ancillary equipment, building a partitioning wall for increased laser safety, improving the electrical service, and improving the HVAC management of environmental conditions. For the laser system itself, the layout and design of the system was reconfigured to include improved thermal management of the primary laser systems, doubling of the temporal capacity of the pump-to-probe delay, implementation of a colinear pump-probe geometry, adding CCD imaging capability of the sample surface, installing xyz motion control of the sample stage for alignment and raster scanning, and implementation of a continuous flow cryostat system for temperature dependent measurements. One of the primary goals of the redesign and re-implementation of the thermoreflectance system was to make the system versatile, allowing the experiment to be switched from a low-rep to a high-rep configuration with minimal optical realignment, the ability to switch between a down-probe and side-probe configuration for room temperature and temperature dependent measurements via the cryostat respectively, and to minimize the system down time after laser realignment or other system maintenance and repair.

In this chapter a brief introduction into the history and fundamental properties of the pump-probe thermoreflectance technique are provided to assist in the understanding of the modeling methods used to deduce nanoscale thermophysical parameters, see Chapters 46, and the experimental studies conducted, see Chapter 7. The more specific design and implementation of the pump-probe thermoreflectance system used in this work and housed in the Nanoscale Energy Transport Lab at UVa is also provided. Traditionally, there is very little information on the details of the setup and operation of thermoreflectance experiments that makes its way into literature making for a steep learning curve in the operation and/or 
setup of such a system. It is the goal of this chapter to provide a useful guide on the background, setup, and operation of the thermoreflectance experiment so that this work can be duplicated and/or extended without each new generation having to start from the beginning and learn from experience and mistakes alone ${ }^{1}$.

\subsection{Optical Methods for Thermal Measurement}

As highlighted in Chapter 1, as the nanotechnology revolution continues structures in technologies ranging from the most basic home computer to advanced computational systems are continually decreasing in size and operating at ever-increasing frequencies. Thermoelectrics [184], thermal interface materials [28, 185], optical storage media [186], and solid-state transistors [187] all have device dimensions equal to, or smaller than, carrier mean free paths (MFPs) at room temperature. Some such devices, like high electron mobility transistors [188] and quantum dot cascade lasers [189] not only employ structures at these length scales, but also operate within or above the terahertz frequency regime. Measurement of thermophysical properties in such systems requires a measurement system with both fine temporal and spatial resolution.

For solid materials, the opto- and electro-mechanical properties that are observed, e.g. refractive index, resistance, dielectric constant, etc., arise from the interaction of the lattice and electronic structure that comprise the solid. The measurement and characterization of these phenomena have been completed using a variety of techniques [190]. Properties such as electrical resistance and dielectric constant [191] can be determined using electrical characterization techniques. Electrical techniques can be used because the signals generated during the characterization fall within the bandwidth of electronic measurement devices. The resolution or band-width of electronic measurements, determined by the $\mathrm{RC}$ time constant of the system, is such that when the measured (material) response is much slower than the time constant, the measurement can be accurately determined by the experiment. If signals received by the electronic components are much faster than the $\mathrm{RC}$ time constant,

\footnotetext{
${ }^{1} \mathrm{I}$ have made plenty mistakes along the way in my understanding of thermoreflectance and would be only happy, if others can avoid them and/or learn from them to be able to take the system further than I ever could.
} 
the signals will be quickly damped out by the electronics and the signal will be lost. This limitation sets a lower bound to the timescale that electronic devices can measure of about $0.1 \mathrm{~ns}(10 \mathrm{GHz})$ [192]. In order to measure signals generated by processes on sub nanosecond time scales, non-electrical methods must be utilized.

Advances in laser technology have made optical pump-probe spectroscopy an attractive choice for the measurement of thermophysical properties in systems with micro- and nanoscale feature sizes. Modern pump-probe spectroscopy systems typically operate utilizing an ultrafast ${ }^{2}$ laser system which provides sub-picosecond temporal resolution. The lateral spatial resolution is given by the diffraction limited spot size of the focused laser beam (on the order of microns) and the depth resolution is typically on the order of nanometers determined by the thermal penetration depth. Optical techniques are ideally suited for investigation of nanoscale thermal properties as they require no physical heaters and/or temperature sensors and can work through optically transparent system environments.

\subsubsection{Photothermal Techniques}

The primary purpose of pump-probe spectroscopy is the detection of thermal waves within a medium. Any time there is periodic excitation of a solid medium, thermal waves are created $[193,194]$. While for pump-probe spectroscopy the source of excitation is laser energy, thermal waves in non-contact systems can be created by many periodic sources ranging from electron beams to chopped sunlight. The thermal waves generated by the periodic source leave the heated region as diffusive critically damped waves traveling only a few wavelengths before losing most of their intensity [195]. As these waves propagate, they interact with thermal features ${ }^{3}$ in the material causing the thermal waves to scatter and reflect. Thermal features arise from variations in the local lattice structure caused by impurities, vacancies and other defects. These lattice disruptions can greatly affect thermal transport properties, such as thermal conductivity, but have a negligible effect on local optical and elastic parameters due to statistical averaging over a large number of lattice

\footnotetext{
${ }^{2}$ Ultrafast laser systems typically refer to systems with pulse widths on the order of attoseconds to nanoseconds $\left(10^{-18}-10^{-9} \mathrm{~s}\right)$.

${ }^{3}$ Thermal features are defined are regions in an otherwise homogeneous surrounding which exhibit variations in thermal parameters such as density, specific heat, and thermal conductivity [195].
} 
bonds.

Several methods have been developed for the detection of thermal waves [194], these methods include: 1) gas-microphone photoacoustic detection, 2) photothermal measurement of emitted infrared radiation, 3) optical laser beam deflection, 4) interferometric and optical detection of thermoelastic surface deformations, and 5) piezoelectric detection of thermoacoustic signals. One of the earliest methods developed for the detection of thermal waves was photoacoustic spectroscopy [196]. The premise of this technique is based in part on the work by Alexander Graham Bell in 1880, who detected an audible signal from a solid in a closed gas cell when a periodically interrupted beam of sunlight was impingement on the surface [197]. Modern techniques use a gas microphone to inspect local surface temperatures by monitoring variations of gas pressure in a closed photoacoustic cell as a result of periodic conduction from the sample surface to the gas. While the photoacoustic technique works well for samples with thicknesses in the micron range, the limit of modulation frequencies in the range of $10 \mathrm{~s}$ of $\mathrm{kHz}$ limits the applicability for thinner samples.

\subsubsection{Femtosecond Thermoreflectance Techniques}

With new advances in the development of picosecond and femtosecond laser systems in the early 1980's, a combination of high repetition rates and small pulse durations allowed for the investigation of thinner samples and faster thermal phenomena. Paddock and Eesley [144] are credited with implementing the first modern transient thermoreflectance system for measuring diffusive thermal properties. Their implementation utilized two pumped ring die lasers with a pulse width on the order of 8 ps and a $246 \mathrm{MHz}$ repetition rate to measure the thermal diffusivity of metal films on the order of $100 \mathrm{~nm}$ in thickness, independent of the properties of the supporting substrate. Over the past several decades many improvements have been made to the original system configuration to improve the accuracy, resolution, and ease of implementation of the thermoreflectance experiment. The basic principles behind the thermoreflectance technique however, have remained relatively unchanged. 


\section{Basics of Pump-probe Thermoreflectance Spectroscopy}

In general, the pump-probe thermoreflectance technique uses an ultra-fast pulsed laser source split into a high intensity pump beam which imparts a time varying heat flux on a sample surface, and a lower intensity probe beam that interrogates the surface temperature as a function of time through the temperature dependent changes to the optical reflectance ${ }^{4}$ of the sample surface. Because of the dependence in the thermoreflectance experiment on the relationship between the temperature and optical properties of the material, metals are always used as the top layer in a thermoreflectance sample. The metal film can be a primary component of the sample system or a thin layer to act as a thermal transducer only. The change in optical reflectance of the metal surface with temperature is given by the thermoreflectance coefficient, $d R / d T$. For a given metal, the thermoreflectance coefficient will be dependent on the electron band structure of the metal and the incident photon wavelength. Typical thermoreflectance coefficients are on the order of $10^{-4}-10^{-5}$ for most metals. More details on specific thermoreflectance coefficients for metals and photon wavelengths of interest in pump-probe thermoreflectance experiments can be found in [198].

A schematic of the major components in a pump-probe thermoreflectance system is shown in Figure 3.1. After the output from the initial laser source is split, the impulse-train of pump pulses is further modulated by an optical modulator to impart a "heat frequency" to the system and allow for a lock-in detection scheme for increased signal-to-noise. The frequency at which the pump beam is modulated is a function of the original repetition frequency of the laser system and will determine several properties of the thermoreflectance analysis as will be discussed in further detail in Chapter 5. Typically, thermoreflectance experiments are separated into two categories based on the repetition frequency of the laser system. Laser repetition frequencies in the $\mathrm{kHz}$ regime are typically denoted as lowrep systems, while systems with repetition frequencies in the $\mathrm{MHz}$ regime are denoted as high-rep systems. The major difference between the two types of systems will be whether it can be assumed that after thermal excitation due to a single laser pulse, the system returns to thermal equilibrium before the arrival of the next laser pulse. While there is no agreed upon nomenclature established in the nanoscale thermal community, for the

\footnotetext{
${ }^{4}$ See Appendix B for brief discussion on the difference between reflectance and reflectivity.
} 


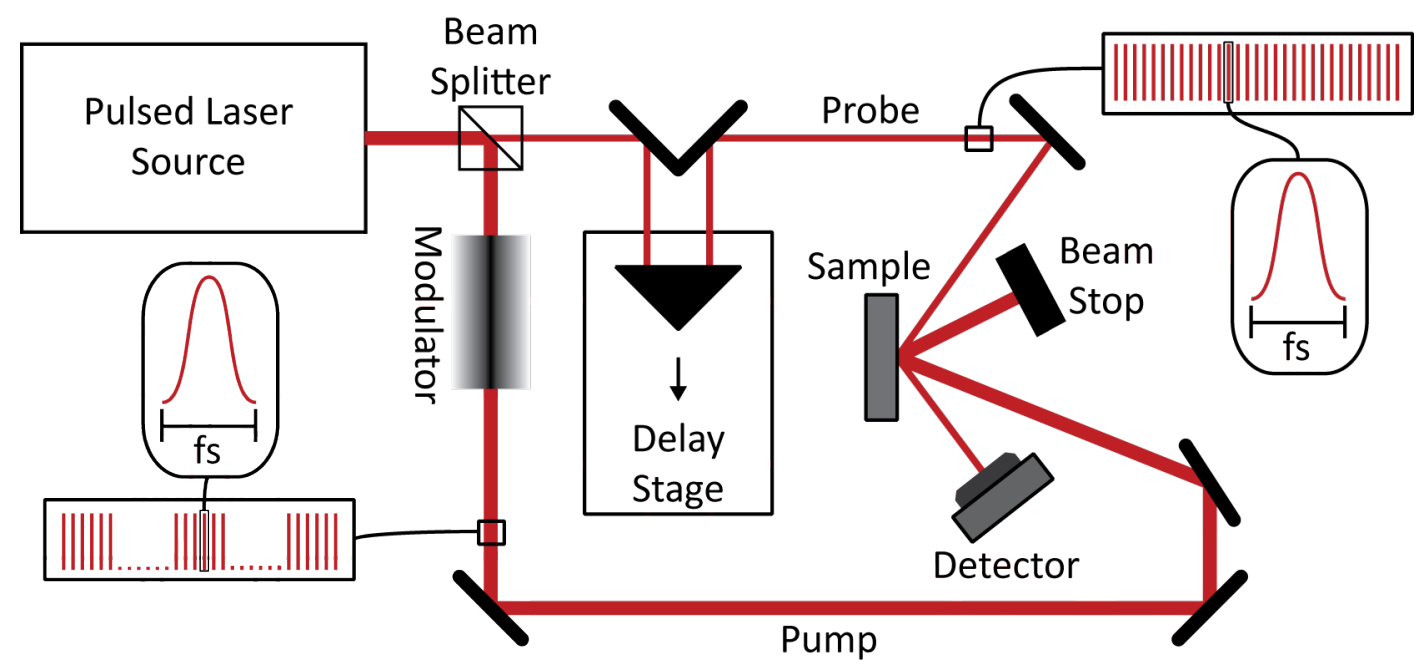

Figure 3.1: Basic schematic of the primary components of the pump-probe thermoreflectance experimental system.

purposes of this dissertation the low-rep configuration of the experiment will be denoted as the transient thermoreflectance (TTR) experiment, while the high-rep configuration will be denoted as the time-domain thermoreflectance experiment (TDTR). When the discussion of a particular topic is independent of the laser repetition rate, the system will be described as the TTR/TDTR experiment.

The length of the probe path is varied using a mechanical delay stage to provide temporal resolution of the surface temperature decay from the time of the arrival of the pump pulse, to typically on the order of a few ns after the initial heating event. The resolution of typical commercially available delay stages is on the order of microns. This translates into a temporal resolution of on the order of a few femtoseconds, which is typically much less than the duration of the lases pulses. Therefore, the laser pulse width is the limiting factor determining the temporal resolution of the thermoreflectance technique. The laser pulse width will then determine the different types of thermal phenomena that can be deduced from the thermoreflectance experiment.

\subsubsection{Energy Transport/Conversion Following Pulsed Laser Heating}

As discussed in more detail in Chapter 2, the primary energy carriers in solids are electrons and phonons. From the theory of wave/particle duality we know that these carriers 
can be treated as either particles or waves. The choice of how we analyze these energy carriers depends on both the length and time scale of interest. In the regime of thermal transport, the shortest time scale that is generally of interest is the time between energy carrier collisions, $\tau_{c}$, also known as the mean scattering time. The shortest length scale of interest is typically the collision length, $l_{c}$, also known as the mean free $\operatorname{path}^{5}$. Traditionally, the dominant phonons that contribute to thermal transport have been considered to have MFPs on the order of 10-100 $\mathrm{nm}$ at room temperature [78]. However, recent advances in simulation and experimental methods have shown that phonons cover a much longer MFP spectrum, with the majority of thermal energy being carried by phonons with MFPs greater than $1 \mu \mathrm{m}[199,200]$. Classically, the mean free path and the time between carrier collisions is related through the carrier velocity [33]:

$$
l_{c}=v \tau_{c}
$$

Carrier velocities are typically on the order of $\sim 10^{6}, \sim 10^{3}$, and $\sim 10^{8} \mathrm{~m} / \mathrm{s}$ for free electrons, phonons, and photons respectively [33]. Excited carriers typically require on the order of 5-20 collisions to reach a local thermal equilibrium [201]. This is characterized by the relaxation time, $\tau_{r}$, and the volume in which the local thermal equilibrium can be defined is related to the relaxation length, $l_{r}[201]$, see Figure 3.2. The relation between relaxation time and length, and the physical and temporal constraints of the system of interest will determine the type of carrier modeling required to describe the system.

When the length and time scales of the system are much shorter than the relaxation length and time, i.e $L \ll l_{c}$ and $t \ll \tau_{c}$, the wavelength of the carriers must be taken into account and the transport described by wave mechanics. When the length of the system is on the order of the relaxation length, i.e $L \approx l_{r}$, and the time scale is much greater than the relaxation time, i.e. $t \gg \tau_{r}$ the carrier transport is ballistic and no thermal equilibrium can be defined. When the system length is much greater than the relaxation length, and the system time is on the order of the relaxation time, i.e. $L \gg l_{r}, t \approx t_{c}$, a local thermal equilibrium can be defined, but time-dependent properties must be used. Under these

\footnotetext{
${ }^{5}$ Note: The scattering time and scattering length are both statistical quantities, and represent the average time or distance an energy carrier will travel between collisions respectively.
} 


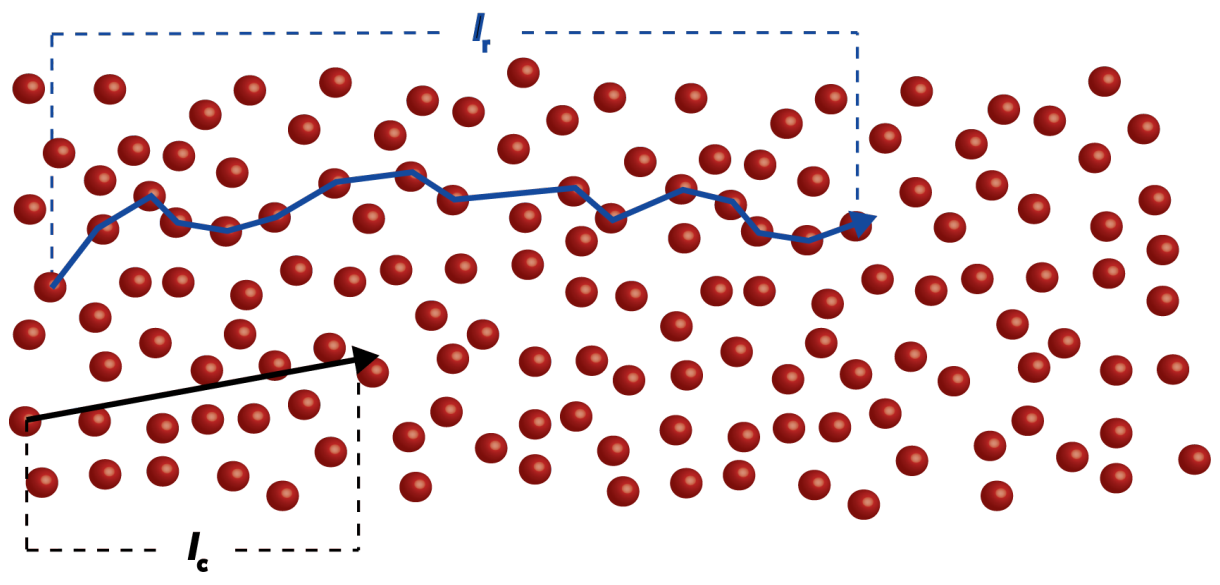

Figure 3.2: Schematic defining and comparing the relaxation and collision length of energy carriers. For a collection of carriers, e.g. electrons, phonons, photons, the mean free path is the average distance between scattering events. The relaxation length is the length required to reach thermal equilibrium. Note: Perspective is for illustration only. In reality mean free paths can be orders of magnitude longer than the average carrier separation.

conditions, transport is modeled utilizing the Boltzmann transport equation. When both the length and time are much greater than the relaxation length and time respectively, a thermal equilibrium can be defined in both space and time, and therefore carrier transport can be model with classical conduction laws, e.g. Fourier law for phonons and Ohm's law for electrons [201].

As mentioned previously, the focus of experiments in this work is on non-contact methods of measuring thermal properties, specifically using an ultrafast laser system interacting with a metal film. In the analysis of laser heating of the metal, we can consider 4 primary timeframes: 1) the deposition of laser radiation energy into the electron sub-system of the metal, 2) the thermalization of the electron sub-system from a non-equilibrium to an equilibrium state, 3) the exchange of energy between the electron and phonon sub-systems, and finally 4) the diffusive propagation of thermal energy through the system, see Figure 3.3.

For laser wavelengths in the UV to near IR range, the radiation energy interacts with the metal through the excitation of free/bound electrons and electron-phonon interaction. For lasers with pulse durations in the sub-picosecond range, the electrons are initially in a highly non-equilibrium state immediately after laser absorption $[202,203]$. The electron sub-system thermalizes through two potential processes which occur simultaneously, see Figure 3.4. The most general case is the collision of the excited electrons with other excited electrons and 


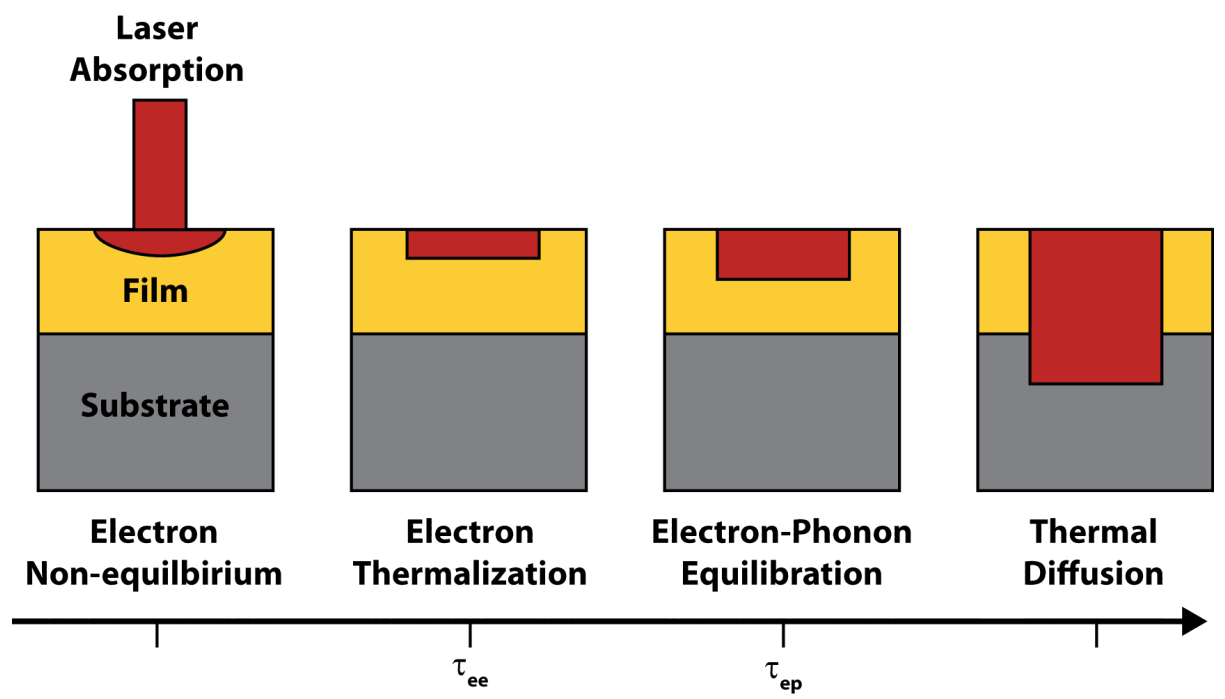

Figure 3.3: Schematic depicting the 4 primary time frames of ultrafast laser interaction with metals, 1) the deposition of laser radiation energy into the electron sub-system of the metal, 2) the thermalization of the electron sub-system from a non-equilibrium to an equilibrium state, 3) the exchange of energy between the electron and phonon sub-systems, and 4) the diffusive propagation of thermal energy through the system.

electrons near the Fermi level [204-208]. The second path of electron thermalization is through ballistic transport of electrons through the metal film. Assuming that the ballistic electrons travel at the Fermi velocity ${ }^{6}$, which for typical metals is on the order of $v_{F}=1 \times 10^{6}$ $\mathrm{m} / \mathrm{s}[206,209,210]$, during the pulse duration ( $100 \mathrm{fs})$ the ballistic electrons will travel $100 \mathrm{~nm}$ into the film.

The electron thermalization time, $\tau_{e e}$, can be experimentally determined using timeresolved two-photon photoemission spectroscopy [211]. The experimental results show general qualitative agreement with the electron thermalization time derived from Fermi liquid theory given by $[32,192]$ :

$$
\tau_{e e}=\frac{128}{\pi^{2} \sqrt{3} \omega_{p}}\left(\frac{E_{F}}{E-E_{F}}\right)^{2}
$$

where $\omega_{p}$ is the plasma frequency of the metal and $E_{F}$ is the Fermi energy. Equation 3.2 provides an upper bound for the e-e scattering rate in metals, with real scattering rates being much more complex due to electronic band structure and electronic screening [212]. The main focus being that the e-e scattering time is proportional to density of electrons in the conduction band [212]. This has been experimentally demonstrate in $\mathrm{Cu}(100), \mathrm{Cu}(110)$,

\footnotetext{
${ }^{6}$ The Fermi velocity, $v_{F}$, is related to the Fermi energy, $E_{F}$, through the classic kinetic energy relationship, $E_{F}=1 / 2 m v_{F}^{2}[32]$
} 


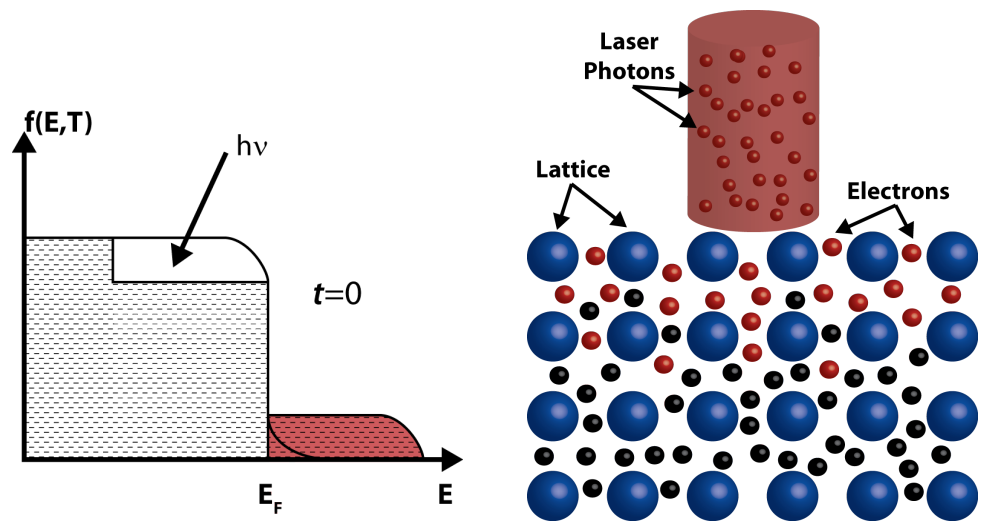

(a)

(b)

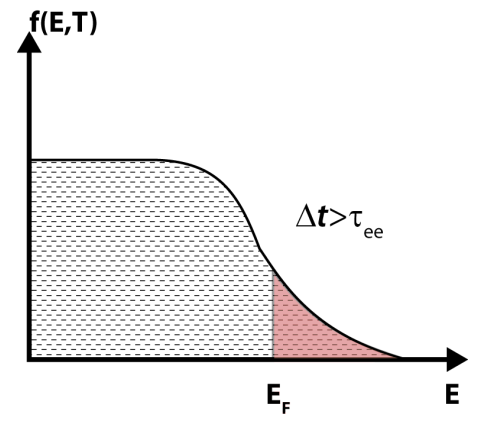

(c)

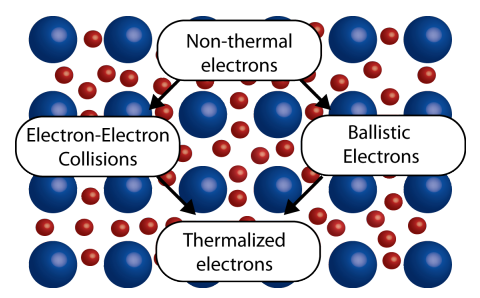

(d)

Figure 3.4: Schematic describing the distribution of electrons and the interaction of the laser and material system (a)-(b) after pulse deposition but before electron-electron thermalization and (c)-(d) after the thermalization of the electron sub-system.

and $\mathrm{Cu}(111)$, with scattering times ranging between $\sim 5-75$ fs depending on electron excitation levels [213]. Once the electron sub-system has relaxed to an equilibrium FermiDirac distribution a well-defined electron temperature, $T_{e}$, can be used to describe the electron system [204-206].

In comparison to the phonon sub-system, the electron sub-system has a relatively small heat capacity and therefore while the thermal input of the laser may only raise the lattice temperature of a sample by a few degrees Kelvin over the course of a measurement, during the initial non-equilibrium period shortly after laser heating the effective electron temperature can be on the order of several thousand Kelvin. Assuming that the pulse duration is very short and can be assumed to be instantaneous, and in the absence of any thermal 
coupling or diffusion, the peak electron temperature, $T_{e, \max }$, is given by [214]:

$$
T_{e, \max }=\sqrt{\frac{2(1-R) J}{\delta \gamma}-T_{o}^{2}}
$$

where $R$ is the reflectance of the metal, $J$ is the incident laser fluence, $\delta$ is the radiation penetration depth, $\gamma$ is the electron heat capacity or Sommerfeld constant, and $T_{o}$ is the initial temperature of the system. The high temperature electron sub-system, $T_{e}$, couples its energy to the low temperature phonon sub-system, $T_{p}$, through a series of scattering events at a rate that is proportional to the electron-phonon coupling factor, $G$. The electron-phonon coupling factor is dependent on several system properties including film thickness and grain size [215], contributions of inter- and intraband excitations [21, 216, 217], contributions of ballistic electron transport and substrate effects [96, 218], and the excitation of d-band electrons at high temperatures [219]. Examples of experimentally measured electron-phonon coupling factors are on the order of $G \approx 4 \times 10^{16} \mathrm{~W} / \mathrm{m}^{3} \mathrm{~K}, G \approx 12 \times 10^{16} \mathrm{~W} / \mathrm{m}^{3} \mathrm{~K}$, and $G \approx 22 \times 10^{16} \mathrm{~W} / \mathrm{m}^{3} \mathrm{~K}$ for $\mathrm{Au}, \mathrm{Cr}$, and $\mathrm{Al}$ respectively [215].

The interaction of the electron and phonon sub-systems due to the nonequilibrium temperature introduced during the ultrashort laser heating of the metal film, can be described by the two-temperature model (TTM) first proposed by Anisimov in 1974 [220], and given by:

$$
\begin{gathered}
C_{e}\left(T_{e}\right) \frac{\partial T_{e}}{\partial t}=-G\left[T_{e}-T_{p}\right]+S(x, t) \\
C_{p}\left(T_{p}\right) \frac{\partial T_{p}}{\partial t}=G\left[T_{e}-T_{p}\right]
\end{gathered}
$$

where $C_{e}$ is the temperature dependent electron heat capacity, given by $\gamma T_{e}, G$ is the electron-phonon coupling factor, $C_{p}$ is the temperature dependent heat capacity of the phonon sub-system, i.e. the lattice, and $S$ is the source term which describes the energy absorbed by the laser. The time constant for the electron and phonon sub-system to reach thermal equilibrium, $\tau_{e p}$, is given by [221]:

$$
\tau_{e p}=\frac{C_{e} C_{p}}{\left(C_{e}+C_{p}\right) G} \approx \frac{C_{e}}{G}
$$


Typically electron-phonon relaxation times have been found to be on the order of 1's to 10 's of picoseconds [215, 221-224].

Once the electron and phonon sub-systems have reached thermal equilibrium the thermal transport can be described by the parabolic one step (POS), given by [221]:

$$
C_{p} \frac{\partial T_{p}}{\partial t}=\frac{\partial}{\partial z}\left(k_{e q} \frac{\partial T_{p}}{\partial z}\right)+S(t)
$$

The POS is a special form of the standard heat diffusion equation and is generally applicable for lasers with pulse durations in the pico- or nanosecond regime and/or when considering thermal transport after the electron and phonon sub-systems have reached thermal equilibrium. Even though the measurements completed in this dissertation were performed with a femtosecond laser system, the primary interest was in diffusive phononic transport. Therefore the analysis was completed using thermal models of the form of the POS, with considerations restricted to times greater than $t>100$ ps after laser excitation. Much more detail on the thermal analysis is provided in Chapter 4.

\subsection{The General System and Components}

To begin the discussion of the physical experiment, a full schematic of the TTR/TDTR system in the University of Virginia Nanoscale Energy Transport lab is shown in Figure 3.5. The system is centered around a Coherent Ti:sapphire ultrafast laser oscillator, MIRA, and an ultrafast regenerative laser amplifier, $\operatorname{Reg} A$, both of which are optically pumped by a solid-state Coherent VERDI. To provide a robust description of the thermoreflectance system we will walk through the primary beam paths and highlight the major components, detailing their purpose and functionality as part of the system as a whole.

\section{Pump Laser - VERDI}

The primary laser power source to pump the Ti:sapphire lasers is a Coherent VERDI V18 (18 W) diode-pumped solid-state (DPSS) laser ${ }^{7}$. The VERDI itself is a pumped laser,

\footnotetext{
${ }^{7}$ The VERDI V18 is an upgrade from the original system configuration, replacing two VERDI DPSS lasers, a V5 $(5 \mathrm{~W})$ and V10 $(10 \mathrm{~W})$, that pumped the MIRA and RegA respectively.
} 


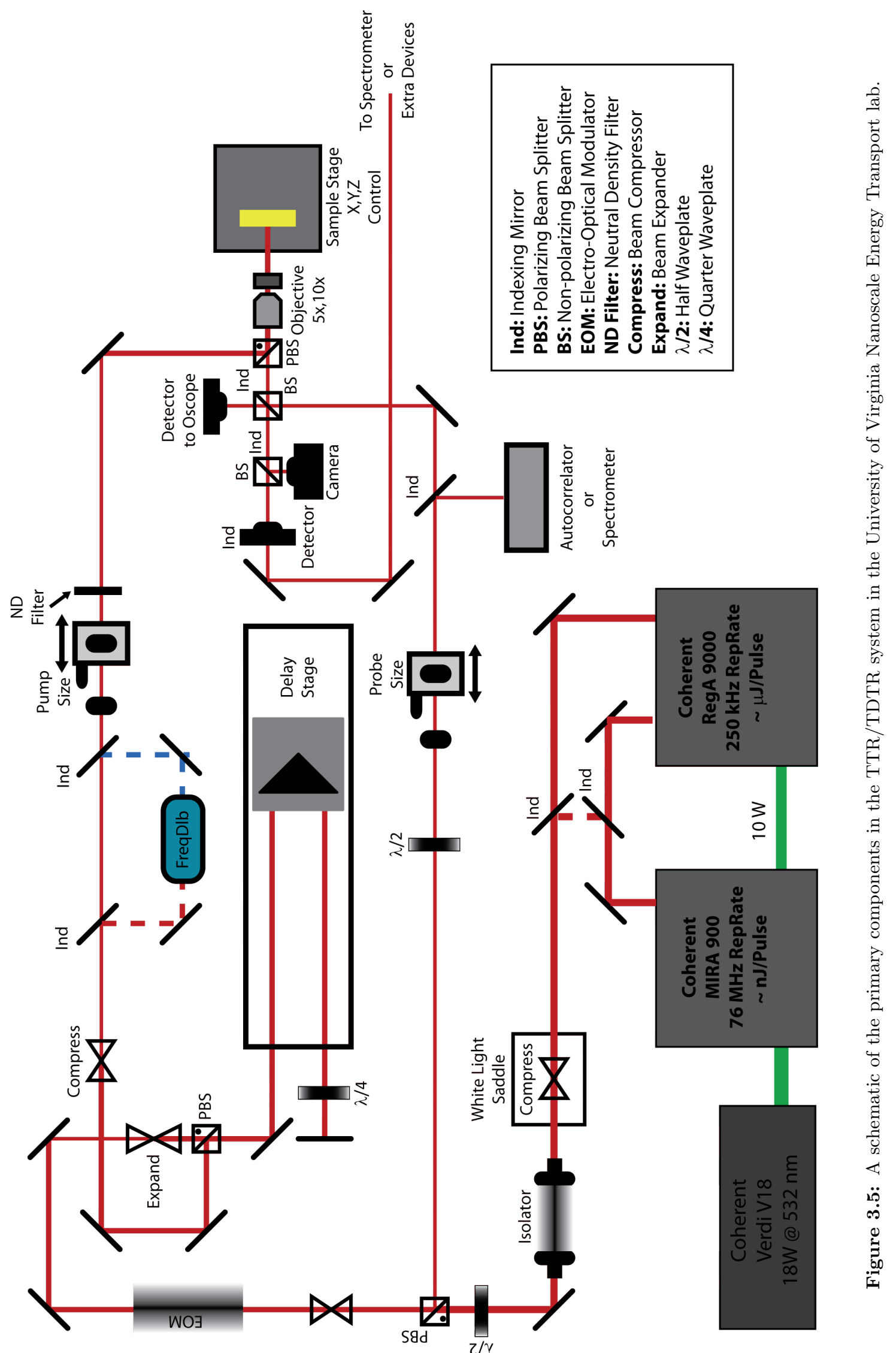


utilizing dual diode assemblies (Fiber Array Package - Integrated, FAP-I ${ }^{\mathrm{TM}}$ ), producing on the order of $40 \mathrm{~W}$ each at a wavelength of $808 \mathrm{~nm}$ to pump a ring laser configuration in the VERDI laser head. The primary gain medium in the laser head is a $\mathrm{Nd}: \mathrm{YVO}_{4}$ (neodymiumdoped yttrium orthovanadate, a.k.a vanadate) crystal producing $1064 \mathrm{~nm}$ output which is then frequency doubled by a birefringent lithium triborate (LBO) crystal to produce the final output of $532 \mathrm{~nm}$ at a power of $18 \mathrm{~W}$.

\section{Ultrafast Oscillator - MIRA}

The $18 \mathrm{~W}$ power output of the VERDI DPSS laser is used to optically pump both the MIRA and RegA systems. A beam splitter located in the MIRA is used to split the incoming laser power into $8 \mathrm{~W}$ and $10 \mathrm{~W}$ to pump the MIRA and RegA respectively. The MIRA is a modelocked ultrafast Ti:sapphire laser oscillator with a tunable output wavelength between 710-910 nm. The MIRA emits a train of laser pulses with a temporal width on the order of $200 \mathrm{fs}$, at a nominal repetition rate, or rep-rate, of $76 \mathrm{MHz}$ (approximately one pulse every $13.2 \mathrm{~ns}$ ). For our configuration the wavelength was centered at $785 \mathrm{~nm}$ providing an individual pulse energy on the order of 6-13 nJ per pulse ${ }^{8}$. The laser output from the MIRA has two potential paths: 1) using a pair of indexing mirrors the MIRA beam can be used directly for the high-rep rate configuration of the thermoreflectance experiment or 2) the output is seeded into the regenerative amplifier for amplification to be used in the low-rep rate configuration. The latter path is the one we will continue to follow as it is the configuration the system is in the majority of the time and the configuration used primarily in this work.

\section{Regenerative Amplifier - RegA}

The remaining $10 \mathrm{~W}$ from the V18 is used to optically pump the Ti:sapphire regenerative amplifier, RegA. The RegA increases the laser energy from on the order of nJ's per pulse, to on the order of $\mu \mathrm{J}$ 's per pulse. The RegA emits a train of laser pulses with a temporal pulse width on the order of $200 \mathrm{fs}^{9}$, at a repetition rate between $10 \mathrm{kHz}-300 \mathrm{kHz}$. For our

\footnotetext{
${ }^{8}$ The power output of the MIRA is in large part a function of the quality of the cavity alignment, which can vary significantly.

${ }^{9}$ Prior to the compression stage in the RegA, the pulse width is on the order of $40 \mathrm{ps,}$ and can be compressed to on the order of $200 \mathrm{fs}$.
} 
experiments the repetition rate was set at the maximum of the gain curve, or $250 \mathrm{kHz}$ (one pulse every $4 \mu \mathrm{s})$.

The RegA amplifies the energy of the incoming MIRA pulses seeded into the RegA cavity by first passing the MIRA pulses through a Faraday isolator. The Faraday isolator allows the MIRA pulses to be seeded into the cavity without optical feedback back into the MIRA and allows the amplified RegA pulses to leave on an alternate trajectory without attenuation. A single MIRA pulse is allowed into, or out of, the cavity using a $\mathrm{TeO}_{2}$ (tellurium dioxide) acousto-optic cavity dumper which uses a high frequency RF driver to send acoustic waves into the $\mathrm{TeO}_{2}$ crystal via a piezoelectric transducer (lithium niobate, $\mathrm{LiNbO}_{3}$ ), changing the index of refraction. Prior to injection of the MIRA pulse, or shortly after the ejection of the previous pulse, lasing in the RegA cavity is suppressed by a $\mathrm{TeO}_{2}$ acousto-optic Q-switch. Suppressing lasing in the cavity allows energy to build up in the Ti:Sapphire crystal. After injection, the MIRA pulse is amplified making 20-30 round trips within the RegA cavity before being ejected by the cavity dumper. After leaving the RegA cavity via the cavity dumper and through the Faraday isolator, the pulse width of the amplified pulse is on the order of $40 \mathrm{ps}$ due to temporal stretching during the amplification process. The laser pulse is recompressed to on the order of 200 fs via a quadruple passed compression stage utilizing a gold-coated holographic grating.

\section{Beam Conditioning}

The main output of either the MIRA or the RegA is passed through a beam compressor to shrink the beam diameter and reduce optical losses through the optical isolator and electro-optical modulator. The beam compressor is created using a pair of achromatic doublet lenses with focal lengths of $200 \mathrm{~mm}$ and $100 \mathrm{~mm}$ (Thorlabs AC254-200-B-ML and AC254-100-B-ML) to create a $2 \mathrm{X}$ beam compressor. One of the two lenses is mounted on a linear translation stage (Thorlabs PT1) to ensure accurate lens positioning to maintain beam collimation.

The compressed laser output is then passed through an optical isolator (Conoptics Model 713A) to prevent any laser energy from returning into the laser cavity via back reflections which can cause laser instability and/or damage. A schematic of the optical isolator is 


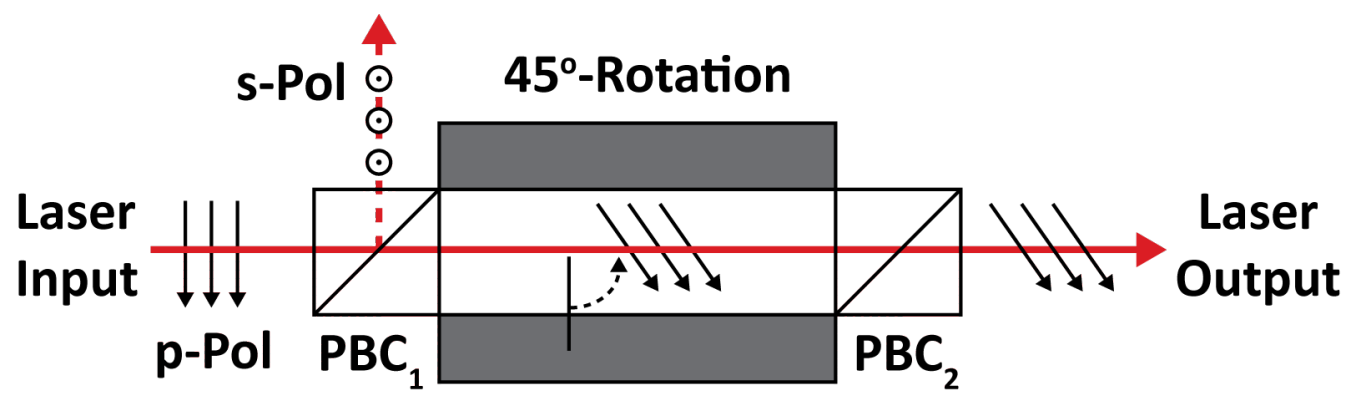

Figure 3.6: Schematic of the optical isolator used to prevent optical feedback into the MIRA or RegA cavity.

shown in Figure 3.6. Inside the isolator, the laser input first passes through a polarizing cube, $\mathrm{PBC}_{1}$, oriented to match the incoming laser polarization (p-pol). The Faraday rotator at the heart of the isolator utilizes an intense magnetic field to rotate the polarization of the incoming light $45^{\circ}$ off-axis compared to the incoming polarization. The output is passed through a second polarizer, $\mathrm{PBC}_{2}$, which is oriented $45^{\circ}$ compared to the incoming axis. Any feedback light that makes it through $\mathrm{PBC}_{2}$ on the return trip will be rotated an additional $45^{\circ}$ to s-polarization, and be rejected by $\mathrm{PBC}_{1}$ preventing feedback along the original beam path.

\section{Beam Splitting - Pump/Probe}

The laser light is then split into the pump and probe beam via a polarizing beam splitter system, see Figure 3.7. The polarizing beam splitter works by utilizing a variable $\lambda / 2$-waveplate and a polarizing beam cube (PBC). To split the beam, the horizontally polarized, i.e. p-polarized, light from the laser is rotated off-axis by the $\lambda / 2$-waveplate, which is mounted on a rotational stage to vary the degree of off-axis rotation. The beam is then passed through the $\mathrm{PBC}$ which separates the laser light by polarization, sending the s-polarized light in a direction perpendicular to the original beam path ${ }^{10}$, while the direction of the p-polarized light is unchanged. The percentage split between the pump and probe beam can be continuously varied by rotating the waveplate and changing the percentage of the beam in each of the orthogonal axes. In our system, typically $\sim 12 \%$ of

\footnotetext{
${ }^{10}$ Assuming the face of the cube is properly aligned perpendicular to the direction of the incoming laser light.
} 


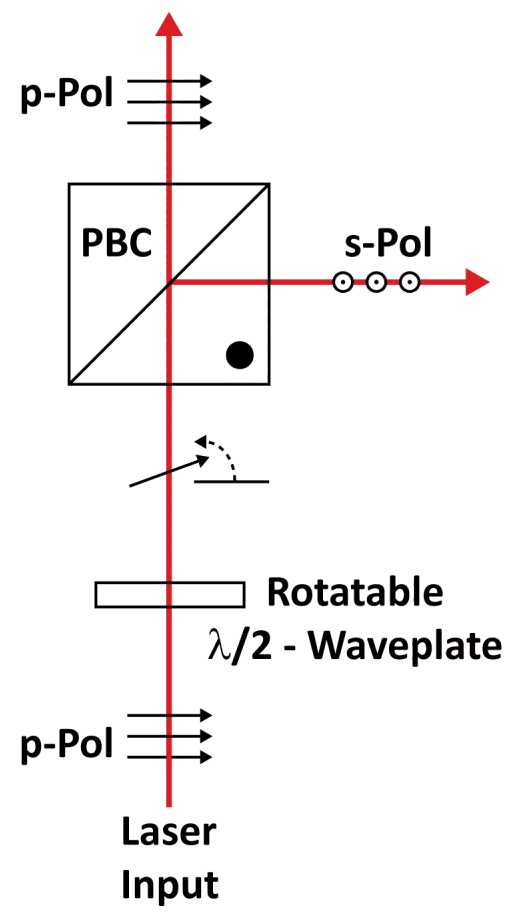

Figure 3.7: Schematic of the polarizing beam splitter system. The incoming p-polarized light from the laser is rotated off access by the $\lambda / 2$-waveplate, and the vector components are then separated by the PBC to scatter the s-polarized light and pass the p-polarized light.

the incoming laser power is sent down the probe arm, and the remainder is used for the pump $\operatorname{arm}^{11}$.

\section{Pump Modulation}

Continuing to follow the pump beam first, the beam is next sent through an electrooptical modulator (EOM) (Conoptics 350-160 EOM and 25D amplifier). The purpose of the EOM is to impart a modulation waveform onto the pump impulse-train to provide a reference frequency, besides the laser frequency, to allow for signal detection via a lock-in amplifier. Much more detail on the affects of the modulation waveform on system response and data analysis will be provided in Chapter 4 .

A schematic of the EOM system is shown in Figure 3.8. The EOM contains a non-linear crystal that essentially acts as a voltage controlled waveplate that can be used to rotate the polarization of the incoming laser light as a function of the driving voltage applied (called the Pockels effect), and in combination with an output polarizer can be used to select the

\footnotetext{
${ }^{11}$ For the low-rep system, utilizing both the MIRA and RegA.
} 


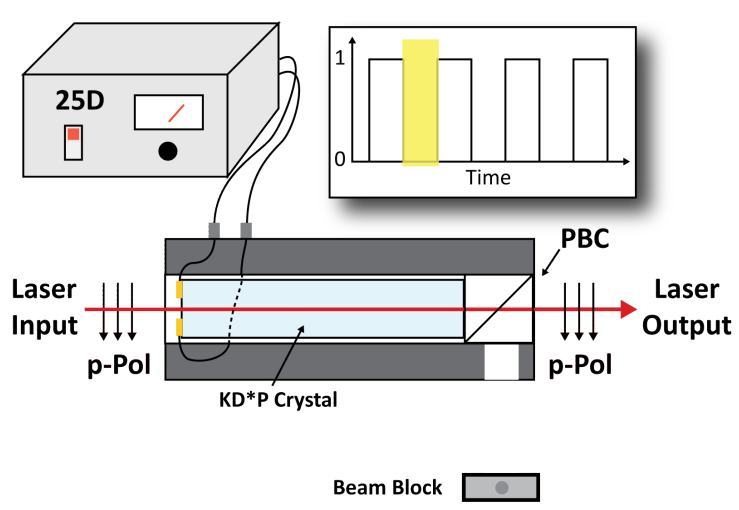

(a)

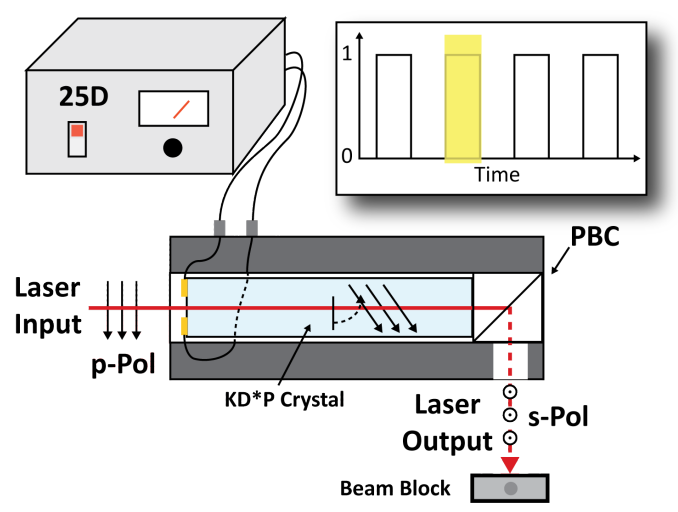

(b)

Figure 3.8: Schematic of the electro-optical modulator (EOM) driven by a square wave for (a) low logic and (b) high logic.

exit location of the beam. When the EOM is driven at low logic, the beam is unmodified and passes through the EOM, see Figure 3.8a. In the high logic state, the polarization of the laser light is rotated to s-polarization and therefore the output of the EOM is via a side aperture towards a beam block, see Figure $3.8 \mathrm{~b}^{12}$. In our system the non-linear crystal in the EOM is a potassium di-deuterium phosphate $\left(\mathrm{KD}^{*} \mathrm{P}\right)$ crystal, with the $25 \mathrm{D}$ amplifier supplying the crystal with a max $\pm 125 \mathrm{~V}$ at the driving waveform. The EOM can modulate from DC to $30 \mathrm{MHz}$ with a rise and fall time of $8 \mathrm{~ns}$, and in our system imparts a square waveform onto the laser pulse train.

\section{Temporal Delay - Pump Advance}

After leaving the EOM, the pump beam is sent through a $5 \mathrm{X}$ beam expander, increasing the $1 / e^{2}$ diameter from $1.3 \mathrm{~mm}$ to $6.5 \mathrm{~mm}$. The purpose of expanding the beam diameter is to reduce the beam divergence as the path length of the pump beam is varied. Even for a perfectly collimated Gaussian beam, the beam radius will diverge as it travels in the direction of propagation from the smallest point, known as the beam waist $w_{o}$, see Figure 3.9. The spot radius as a function of position in the direction of propagation is given by [225]:

$$
w(z)=w_{o}\left[1+\left(\frac{z}{z_{o}}\right)\right]^{1 / 2}
$$

\footnotetext{
${ }^{12}$ Note: The choice of EOM output based on low/high logic from the driving signal is arbitrary. Depending on the polarization of the light and the orientation of the EOM, it is possible to reject the light in the low logic condition and pass during the high logic condition.
} 


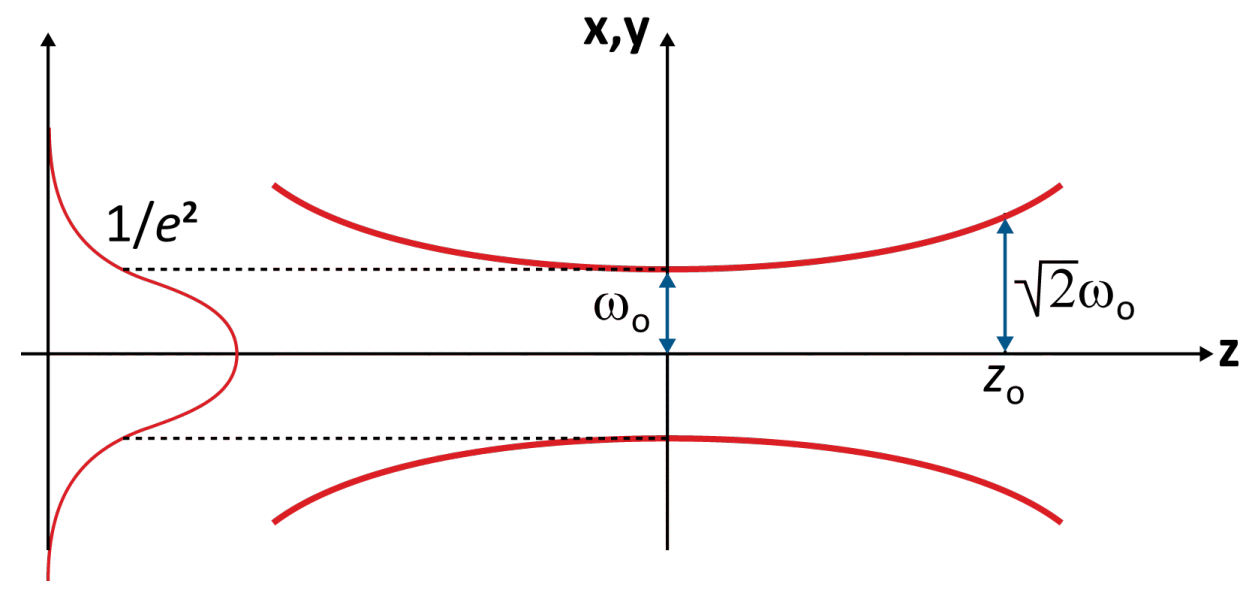

Figure 3.9: Gaussian beam diameter as a function of the direction of propagation, $z$.

where $z_{o}$ is known as the Rayleigh distance, i,e, the distance at which the cross sectional area of the beam has doubled (and the spot radius has increased by a factor of $\sqrt{2}$ ), and is given by:

$$
z_{o}=\frac{\pi w^{2}}{\lambda}
$$

where $\lambda$ is the wavelength of the laser. Due to this phenomena, the un-expanded beam (1.3 $\mathrm{mm}$ ) over the course of the delay travel would increase in size by $\sim 5 \%$. This in turn would change the focused pump spot size and therefore the incident fluence and pump-to-probe ratio causing inputs to the thermal model (discussed in Chapter 4) to vary as a function of delay time. Expanding the beam size prior to sending it onto the delay stage reduces the amount of beam divergence over the length of the stage travel, see Figure 3.10. By expanding the pump size prior to the delay stage the expansion over the length of the delay stage is reduced to under $0.02 \%$

Delay between the pump and probe beam is achieved using a linear mechanical delay stage (Newport IMS600PP linear stage) which provides $600 \mathrm{~mm}$ of linear movement. Mounted to the delay stage is an optical retroreflector (Newport UBBR2.5-1S) which provides a return beam that is parallel to the incoming beam trajectory. In this configuration a total of $1.2 \mathrm{~m}$ of optical delay provides a maximum of $4 \mathrm{~ns}$ of pump-to-probe delay. In order to increase the possible temporal pump-probe delay polarization "tricks" are used to double pass the delay stage. 


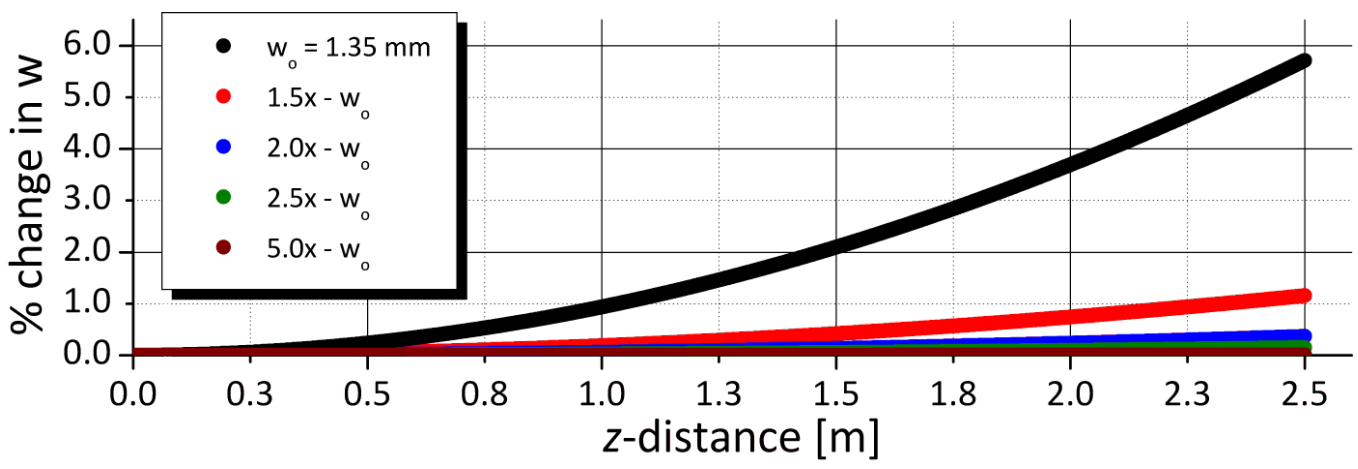

Figure 3.10: Percentage change in the pump beam radius as a function of stage delay, based on Equation 3.7, for various expansion powers.

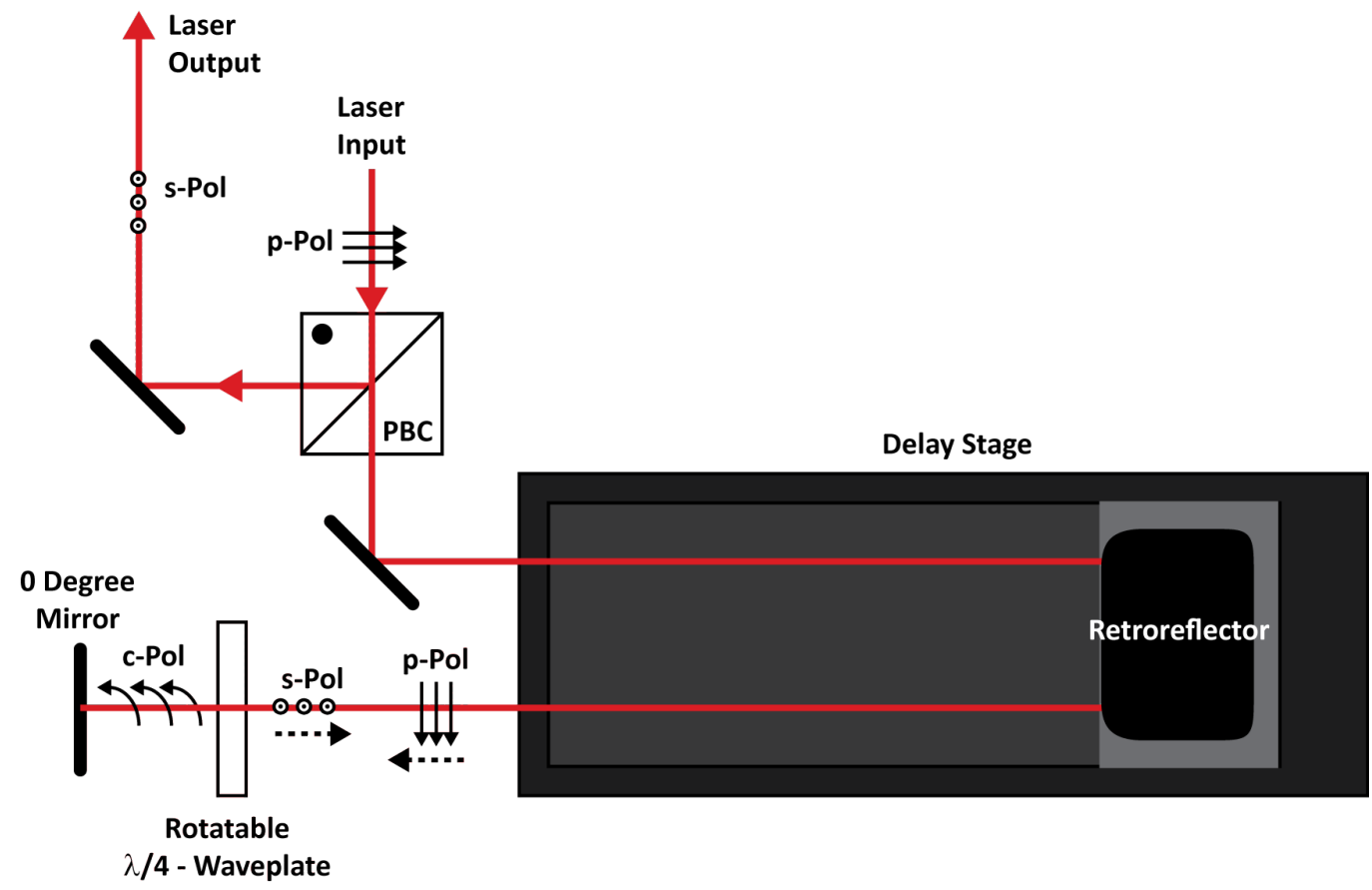

Figure 3.11: Schematic of the optical delay system used to create temporal resolution between the pump and probe beams.

The p-polarized pump light passes through a PBC before being sent onto the delay stage towards the retroreflector and is linearly shifted and sent back on a parallel return path. The beam is then passed through a $\lambda / 4$ waveplate which rotates the p-polarized light into a circular polarization and is impinged on a zero degree mirror. The pump beam is then sent back through the $\lambda / 4$ waveplate rotating the beam polarization from circular to s-polarization and sent back along the original beam path. When the pump beam reaches the $\mathrm{PBC}$ for the second time the beam path is shifted perpendicular to the original beam 
path. Using this polarization scheme the effective length of the delay stage is doubled to $2.4 \mathrm{~m}$ providing a maximum optical delay of $8 \mathrm{~ns}$.

\section{Sample Heating - Pump}

After leaving the delay stage, the pump beam is recompressed using another pair of lenses to set the final beam size before reaching the objective. The size and collimation of the beam as it enters the objective and the magnification of the objective will determine the focused beam size. In order for the lock-in detection scheme to be effective it is important that no pump light be allowed to reach the photodetector. For the thermoreflectance system at UVa there are two options for filtering scattered pump light, color and spatial filtering. For the color filtering scheme, before the pump beam reaches the sample stage it is routed through a frequency doubler via a set of indexing mirrors. The frequency doubler uses a non-linear beta barium borate (BBO) crystal to convert the $785 \mathrm{~nm}$ ("red") pump light to $392.5 \mathrm{~nm}$ ("blue") light. The pump and probe beams are then combined through a dichroic mirror which reflects the pump wavelength and passes the probe through the objective and onto the sample surface, see Figure 3.12a. Both the pump and probe beams return along the original beam path and again theoretically filtered by the dichroic mirror allowing the probe to pass through onto the photodetector and reject the pump light. To filter any pump light that makes it through the dichroic mirror a second bandpass filter is placed in front of the photodetector.

While the color filtering scheme is highly effective and particularly useful for samples

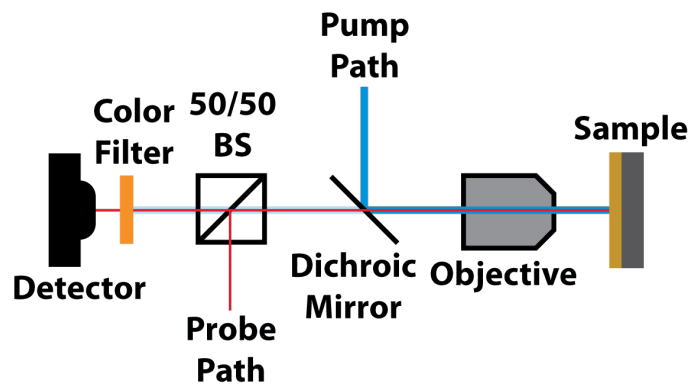

(a)

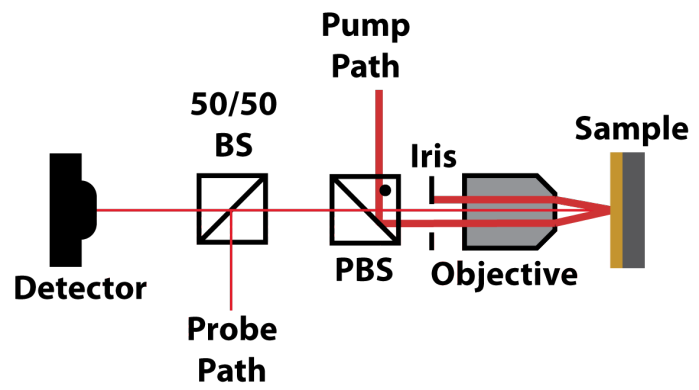

(b)

Figure 3.12: Schematic of the filtering methods to block scattered pump light from reaching the photodetector (a) using the color filtering scheme and (b) using the spatial filtering scheme. 
with a high degree of surface roughness which cause diffuse scattering of the pump beam, the trade-off is the laser power consumed during the frequency doubling process. For samples with a relatively smooth surface the pump beam can also be filtered spatially. By bypassing the frequency doubler the pump and probe beam are sent towards the objective through a polarizing beam cube. The pump beam is sent into the PBS off-axis compared to the probe beam and therefore enters the objective along the edge of the aperture, see Figure 3.12b. After reflecting off the sample surface the pump beam leaves the rear aperture of the objective equally off-axis but on the opposite side of the objective. An adjustable iris is then placed in the beam path so as to allow the probe and incoming trajectory of the pump to pass but blocking the return trajectory of the pump beam from the sample surface.

\section{Signal Detection - Probe}

In either filtering scheme the probe beam follows the same trajectory going through the center axis of the objective and returning on the same beam path after being reflected from the sample surface. The reflected probe signal which now contains a modulated response at the pump modulated frequency due to variations in the surface temperature of the sample, is sent through a long wavelength lens to focus the signal onto the photodetector. The photodetector is a high speed Si photodiode (ThorLabs DET10A) with a 1 ns response time and a $0.8 \mathrm{~mm}^{2}$ sensor area. The photodetector is connected to the lock-in amplifier (Stanford Research Systems SR844).

\subsection{Summary}

In this chapter the design and setup of the thermoreflectance experiment in the Nanoscale Energy Transport lab at the University of Virginia was described in detail. Many of the details that are all too often left out of literature but which are incredibly important to success in the application of the thermoreflectance technique have been provided with the hope that they may be of use to anyone who is working to reproduce these results or individuals who are new to the technique and need an introduction to the fundamental optical systems. 


\section{CHAPTER 4}

\section{MEASUREMENT THEORY AND DATA}

\section{ANALYSIS}

4.1 Introduction . . . . . . . . . . . . . . . . . . . 80

4.2 Pulsed Laser as a Linear Time-Invariant (LTI) System . . . . . . . . . . . . 81

4.3 Heat Transfer Analysis . . . . . . . . . . . . . . . . . . . . . . 96

4.4 Chapter Summary . . . . . . . . . . . . . . . . . . . 115

\subsection{Introduction}

In the most general sense, ignoring the details from Chapter 3, the primary process flow in pump-probe spectroscopy experiments is input from the modulated pump beam and output from the lock-in amplifier. Between the input of the pump laser and the output of the lock-in amplifier lies a complex transfer function, $Z(\omega)$, see Figure 4.1. The transfer function contains all the physical characteristics of the system, including the thermophysical properties of the sample and the properties of the laser system, as well as the thermal response of the system to the modulated heating. The primary task in the mathematical modeling of thermoreflectance data is to determine the transfer function. 


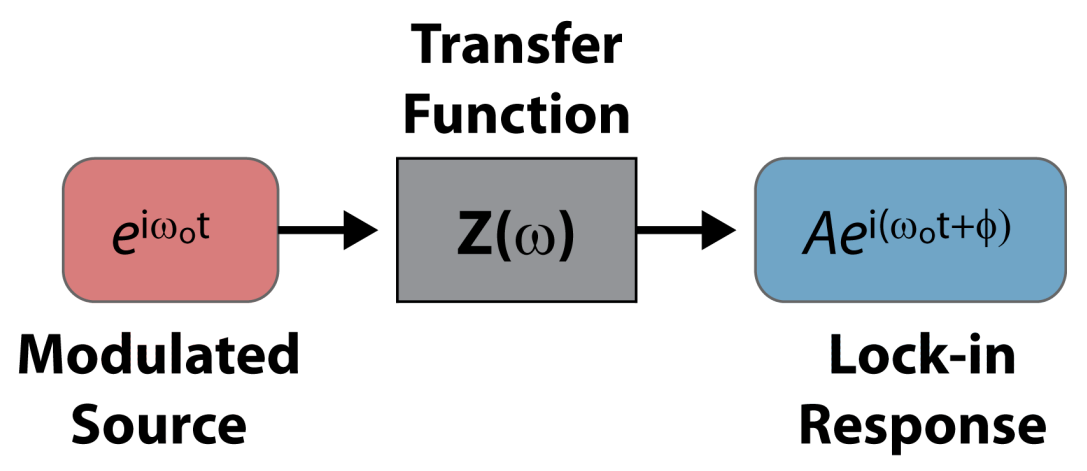

Figure 4.1: This figure illustrates the basic process flow in pump-probe spectroscopy. Input from the modulated pump source is converted to the output response of the lock-in amplifier via a complex transfer function $Z(\omega)$, containing information on the spectroscopy system and the sample response.

In this chapter we will detail the derivation of the transfer function, $Z(\omega)$. We will begin by treating the interaction of the pulsed pump and probe beams using linear time-invariant theory for a generic frequency domain temperature response, $H(\omega)$, and detail how $Z(\omega)$ is translated into the various output forms of a lock-in amplifier. We will deviate from the typical assumption that the waveform used to modulate the pump is sinusoidal, and provide an analysis for a pulsed modulation waveform, which more accurately represents the physical system configuration. In Chapter 5, we will go into more detail on how the properties of the pulsed waveform affect the system response. Before that however, in Section 4.3, we will provide the details of the thermal response, $H(\omega)$, and discuss the switch from a time-domain single film-on-substrate analysis, to a general multi-layer frequency domain analysis.

\subsection{Pulsed Laser as a Linear Time-Invariant (LTI) System}

In order to develop the transfer function, $Z(\omega)$, for the TTR/TDTR system, the theory of linear time-invariant (LTI) systems was utilized. LTI theory is well established and used in a wide variety of fields including circuit analysis and signal processing $[226,227]$ and has been used previously to describe TTR/TDTR systems ${ }^{1}[79,228-230]$. As the name suggests, systems studied utilizing the LTI theory have the properties of linearity and time invariance.

\footnotetext{
${ }^{1}$ The derivation presented here will expand primarily on the work of Schmidt, extending the analysis to pulsed waveforms.
} 


\subsubsection{Assumptions of Linearity and Time Invariance}

Linearity suggests that the system obeys the property of superposition. That is, if the input is a series of several weighted signals, then the output is simply the sum of the weighted responses to each of those individual signals. Mathematically this can be represented, if $y_{1}(t)$ is the system response to an input $x_{1}(t)$, and $y_{2}(t)$ is the system response to an input $x_{2}(t)$, then $a_{1} y_{1}(t)+a_{2} y_{2}(t)$ is the total system response to the summed input $a_{1} x_{1}(t)+a_{2} x_{2}(t)$. This idea can be extended to any number of inputs such that $y(t)=\sum_{k} a_{k} y_{k}(t)$ is the response of the input $x(t)=\sum_{k} a_{k} x_{k}(t)$.

Linearity for pump-probe spectroscopy experiments relies on small temperature perturbations. When focusing on the investigation of diffusive thermal processes, i.e. when the electron and phonon sub-systems have reached a thermal equilibrium, for small temperature perturbations $(\sim 10 \mathrm{~K})$ the systems response, i.e. the change in the reflectance coefficient, can be assumed to be linear [144].

Time invariance suggests that a time shift in the input signal will cause a subsequent time shift in the output signal by the same amount. Mathematically that is, if $y(t)$ is the output for an input signal $x(t)$, then $y\left(t-t_{o}\right)$ is the output of the input signal $x(t-$ $\left.t_{o}\right)$. The physical pump-probe system, as well as the mathematical analysis relies on the property of time invariance. One of the basic premises of using the pump-probe spectroscopy technique is that electronic devices do not have the bandwidth necessary to capture the entire thermoreflectance scan (on the order of nanoseconds) after the arrival of the pump pulse. We therefore rely on time invariance to allow us to sample the system response from many different, i.e. widely spaced temporally, pump pulses to build the time dependent change in surface temperature.

\subsubsection{Frequency Domain Response - TTR/TDTR}

As we apply the LTI theory to the pump-probe thermoreflectance system, we will focus our application on the frequency domain response of the system. Schmidt showed that the derivation can be carried out in either the time or frequency domain, and in fact the results are transforms of each other [228]. However, the thermal model that we will use, presented in Section 4.3, is a frequency domain model and we were therefore not consider the time 
domain derivation.

To begin, we will consider the output of the laser to be a periodic train of pulses. In our system, the laser pulses either originate from a laser oscillator (the MIRA) or the regenerative amplifier seeded by the oscillator (the RegA). In the former case, the pulses arrive at a frequency of $76 \mathrm{MHz}$, i.e. roughly one pulse every $13 \mathrm{~ns}$. In the latter case, the pulses arrive at a frequency of $250 \mathrm{kHz}$, or one pulse every $4 \mu \mathrm{s}$. In both cases, the individual pulse widths are on the order of 200 fs. Since typical delay times for pumpprobe spectroscopy experiments are on the order of pico- to nanoseconds, each pulse will be treated as an impulse with absorbed energy $Q$. The generic impulse-train, scaled by the impulse energy $Q$, is given by:

$$
q_{\text {gen }}(t)=Q \sum_{n=-\infty}^{\infty} \delta(t-n T)
$$

where $T$ is the time period between pulses, $t$ is time in the usual sense, and $\delta()$ is a delta function. A plot of an impulse-train representation of the MIRA and RegA is shown in Figure 4.2 .

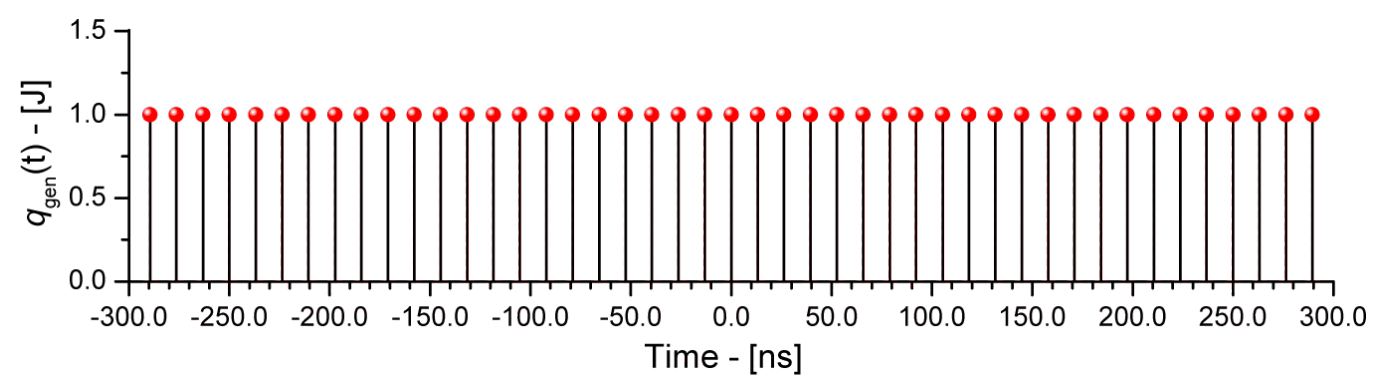

(a)

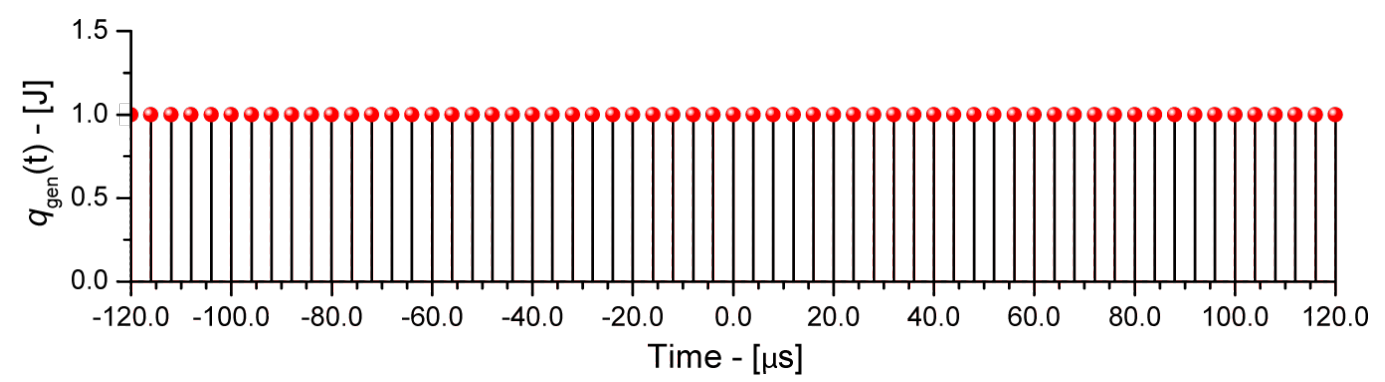

(b)

Figure 4.2: Impulse-train modeled from Equation 4.1 for (a) the MIRA and (b) RegA, for $Q=1 \mathrm{~J}$, $T=13.2 \mathrm{~ns}$ and $4 \mu \mathrm{s}$ for the MIRA and RegA respectively. 


\section{Sampling Theory}

To analyze the interaction of laser pulses in the TTR/TDTR experiment sampling theory is used. Sampling theory is commonly used in image and signal processing, turning the analog world into digital information that can be stored and manipulated. Mathematically, sampling theory in the time domain is represented by [226]:

$$
x_{p}(t)=p(t) x(t)
$$

where $x(t)$ is the function to be sampled, $p(t)$ is the sampling function, and $x_{p}(t)$ is the sampled function. In pump-probe spectroscopy, first the pump impulse-train will be sampled by the modulation waveform, and then the temperature response of the system, due to the modulated pump, will be sampled by the probe impulse-train ${ }^{2}$. It can be shown that while sampling theory in the time domain is represented by a product, in the frequency domain it is given by the convolution of the impulse and sampling functions such that:

$$
x_{p}(t)=p(t) x(t) \stackrel{\mathcal{F}}{\longleftrightarrow} X_{p}(\omega)=\frac{1}{2 \pi}[P(\omega) * X(\omega)]
$$

where $X, P$, and $X_{p}$ are the function to be sampled, the sampling function, and the sampled function in the frequency domain respectively and the convolution of $P(\omega)$ and $X(\omega)$ is given by:

$$
\begin{aligned}
{[P(\omega) * X(\omega)] } & =\int_{-\infty}^{\infty} X(\Omega) P(\omega-\Omega) d \Omega \\
& =\int_{-\infty}^{\infty} X(\omega-\Omega) P(\Omega) d \Omega
\end{aligned}
$$

where $\Omega$ is a small shift in frequency, and by definition the convolution of $P(\omega)$ and $X(\omega)$ is the sum of shifted impulses in the frequency domain $P(\omega-\Omega)$ weighted by the function at that frequency, i.e. $X(\Omega) d \Omega$ (also shown is the commutative equivalent). Combining Equations 4.3 and $4.4 \mathrm{a}-4.4 \mathrm{~b}$ we arrive at a generic expression for a sampled function in the

\footnotetext{
${ }^{2}$ Note: Since the sampling function $p(t)$ will first be the continuous modulation waveform and then the discrete probe impulses, the notation of the impulse-train will alternate. However, there will be no loss of generality since the functions used obey the commutative property.
} 
frequency domain (and its commutative equivalent):

$$
\begin{aligned}
X_{p}(\omega) & =\frac{1}{2 \pi} \int_{-\infty}^{\infty} X(\Omega) P(\omega-\Omega) d \Omega \\
& =\frac{1}{2 \pi} \int_{-\infty}^{\infty} X(\omega-\Omega) P(\Omega) d \Omega
\end{aligned}
$$

As stated previously, in the analysis of pump-probe spectroscopy experiments we will utilize the sampling theory twice. Beginning with the pump pulses sampled by the modulation waveform, we need expressions for the waveform and the impulse-train of the pump in the frequency domain. For generality, at this time we shall consider $P(\omega)$ to be a modulation waveform to be defined later. The modulation waveform and the pump impulse-train in the frequency domain, $P(\omega)$ and $X(\omega)$, are given by the Fourier transforms of the time domain representations of the modulation waveform and the pump impulse-train (given by Equation 4.1 in the specific form of the pump):

$$
\begin{gathered}
P(\omega)=\mathcal{F}\{p(t)\} \\
X(\omega)=\frac{2 \pi Q_{\mathrm{pm}}}{T_{s}} \sum_{M=-\infty}^{\infty} \delta\left(\omega-M \frac{2 \pi}{T_{s}}\right)
\end{gathered}
$$

where $Q_{\mathrm{pm}}$ is the pump energy and $T_{s}$ is the time between pump impulses. Inserting Equations $4.6 \mathrm{a}$ and $4.6 \mathrm{~b}$ into Equation $4.5 \mathrm{~b}$ we have:

$$
X_{p}(\omega)=\frac{Q_{\mathrm{pm}}}{T_{s}} \int_{-\infty}^{\infty} \sum_{M=-\infty}^{\infty} P(\Omega) \delta\left(\omega-\Omega-M \frac{2 \pi}{T_{s}}\right) d \Omega
$$

Using the property below, the integration can be eliminated:

$$
\int_{-\infty}^{\infty} f(x) \delta\left(x-x_{o}\right) d x=f\left(x_{o}\right)
$$


and we are left with a generic expression for the sampled function, i.e. the train of pump impulses modulated by a generic waveform $P(\omega)$ :

$$
X_{p}(\omega)=\frac{Q_{\mathrm{pm}}}{T_{s}} \sum_{M=-\infty}^{\infty} P\left(\omega-M \frac{2 \pi}{T_{s}}\right)
$$

\section{Pump Modulation Function}

At this point we will define the functional form of the modulation waveform that we left generic in the previous steps. In most of the prominent TTR/TDTR literature, the functional form of the waveform used to modulate the pump is sinusoidal [230, 231], i.e:

$$
p_{\mathrm{sn}}(t)=e^{i \omega_{o} t}=\cos \left(\omega_{o} t\right)+i \sin \left(\omega_{o} t\right)
$$

where $\omega_{o}$ is the angular modulation frequency. While a sinusoidal waveform is used in the analysis of these works, the true waveform analyzed by the lock-in amplifier is typically, in reality, a square wave. In the sinusoidal analysis it is assumed that the contributions of the higher harmonics comprising the square wave are either negligible or sufficiently filtered using inductive resonance filters [230-232]. A detailed derivation of the transfer function $Z(\omega)$ using a sinusoidal waveform as in Equation 4.10 is provided in Appendix C.3 for clarity and comparison.

In this work the modulation function of interest is a square wave with an arbitrary duty cycle $^{3}$, i.e. a pulsed waveform, see Figure 4.3. There are several requirements of such a modulation waveform:

- As with the sinusoidal waveform, we seek a modulation period of $T_{o}$.

- The function should act like a logic gate, with a 0 and 1 condition in the off and on state respectively.

- The time period that the waveform is in the "on" state is variable and given by $d$, which will adjust the duty cycle of the waveform given by $D=d / T_{o}$.

\footnotetext{
${ }^{3}$ The choice of duty cycle will not be completely arbitrary. While not a fixed $50 \%$ as considered in most analyses, there will be discrete choices of duty cycle based on the laser and modulation frequencies. More on this to come.
} 


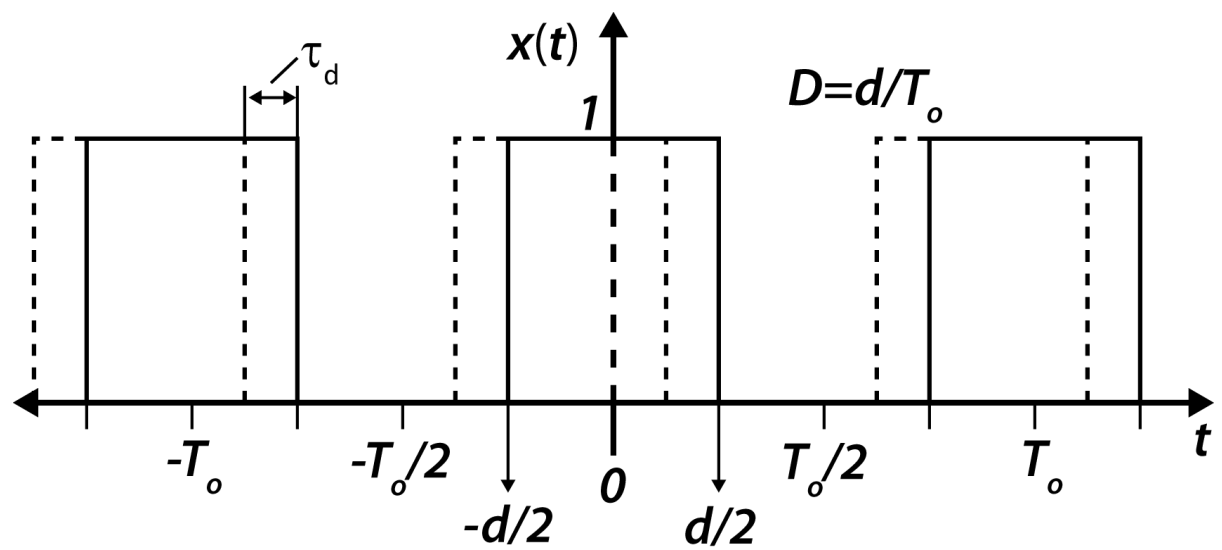

Figure 4.3: Generalization of the pulsed waveform used in the LTI modeling of the pump-probe spectroscopy experiment, with the functional form in the time domain given by Equations 4.11a-4.11c.

- The waveform must include a time delay $\tau_{d}$ to account for the temporal shift of the waveform relative to the pump pulses as the pump distance is varied via the delay stage in the TTR/TDTR experiment.

While adding some complexity to the analysis, using the pulsed waveform will have two benefits: 1) more accurate representation of the modulation waveform will eliminate the need for resonant filtering as the modulation frequency is changed, and 2) define the discrepancies between high and low rep rate systems where the duty cycle is not necessarily $50 \%$. The functional form of the pulsed waveform used in this analysis is given below, and the detailed formulation from Fourier Series analysis is provided in Appendix C.1:

$$
p_{\mathrm{sq}}(t)=\sum_{\substack{k=-\infty \\ k \neq 0}}^{\infty} a_{k} e^{i k \omega_{o} t}
$$

where $k$ is the index of the harmonic components, and the $a_{k}$ 's are the $\mathrm{DC}^{4}$ and $\mathrm{AC}$ Fourier coefficients given by:

$$
\begin{gathered}
a_{0}=\frac{d}{T_{o}} \\
a_{k}=\frac{1}{-2 \pi i k}\left[e^{-i k \pi d / T_{o}}-e^{i k \pi d / T_{o}}\right] e^{i k \omega_{o} \tau_{d}}
\end{gathered}
$$

where $d / T_{o}$ is the duty cycle, $D$, and $\tau_{d}$ is the pump-probe delay. In the frequency domain

\footnotetext{
${ }^{4}$ Note: In the practical application of the signal detection, the lock-in amplifier will filter out the DC portion of the signal, therefore the majority of our analysis will focus on the AC components of the signal.
} 
Equation 4.11a is given by:

$$
P(\omega)=\sum_{\substack{k=-\infty \\ k \neq 0}}^{\infty} a_{k} \delta\left(\omega-k \omega_{o}\right)
$$

where the Fourier coefficients, the $a_{k}$ 's, are $\omega$-independent and still given by Equations 4.11b-4.11c. Inserting Equation 4.12, the frequency domain representation of the pulsed waveform, into the generic expression for the sampled pump, Equation 4.9, we obtain the frequency domain solution for a periodic pump impulse-train modulated by a pulsed waveform:

$$
Q(\omega)=X_{p}(\omega)=\frac{2 \pi Q_{\mathrm{pm}}}{T_{s}} \sum_{M=-\infty}^{\infty} \sum_{\substack{k=-\infty \\ k \neq 0}}^{\infty} a_{k} \delta\left(\omega-M \omega_{s}-k \omega_{o}\right) e^{i k \omega_{o} \tau_{d}}
$$

As a check at this point we can take the inverse Fourier transform of Equation 4.13 and observe the pump impulse-train modulated by the pulsed waveform in the time domain. The inverse Fourier transform is given by:

$$
\begin{gathered}
q(t)=\mathcal{F}^{-1}\{Q(\omega)\} \\
q(t)=Q_{\mathrm{pm}} \sum_{n=-\infty}^{\infty} \sum_{\substack{k=-\infty \\
k \neq 0}}^{\infty} a_{k} \delta\left(t-n T_{s}\right) e^{i k \omega_{o} t} e^{i k \omega_{o} \tau_{d}}
\end{gathered}
$$

Figure 4.4 shows the MIRA impulse-train, see Figure $4.2 \mathrm{a}$, modulated by a square wave, i.e. a pulsed waveform with $D=0.5$, at a frequency of $\omega_{o}=4.22 \mathrm{MHz}$. Analysis of the plot shows that the functional form of the pump impulses, modulated by the pulsed waveform, meets the requirements of the desired pulsed waveform previously listed.

\section{Temperature Response}

While $Q(\omega)$ represents the heat input to the system by the modulated pump impulsetrain, what we are interested in for pump-probe spectroscopy is the system's temperature response to the periodic heat input. In the time domain the temperature response of the system to the heat input from the periodic pump train is given by the convolution of the heat input $q(t)$ and the system's thermal response $h(t)$. In the frequency domain the convolution 


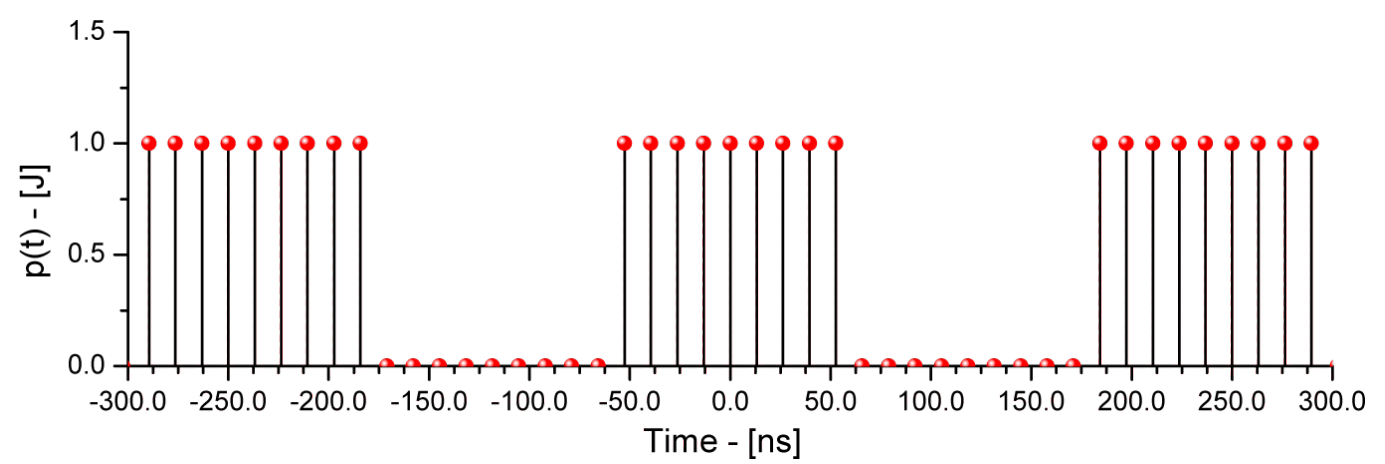

Figure 4.4: Modulated pump impulse-train, from the MIRA, given by Equation $4.14 \mathrm{~b}$ for $Q_{\mathrm{pm}}=1 \mathrm{~J}$, $D=0.05, T_{s}=13.2 \mathrm{~ns}, \omega_{o}=4.22 \mathrm{MHz}$, and $\tau_{d}=0$.

is simply a product of the two, i.e.:

$$
\theta(t)=q(t) * h(t) \stackrel{\mathcal{F}}{\longleftrightarrow} \Theta(\omega)=Q(\omega) H(\omega)
$$

where $Q(\omega)$ and $H(\omega)$ are the heat input and temperature response in the frequency domain respectively. Operating on Equation 4.13 we get the frequency domain temperature response of the system, $\Theta(\omega)$, due to periodic pump heating:

$$
\Theta(\omega)=\frac{2 \pi Q_{\mathrm{pm}}}{T_{s}} \sum_{M=-\infty}^{\infty} \sum_{\substack{k=-\infty \\ k \neq 0}}^{\infty} H(\omega) a_{k} \delta\left(\omega-M \omega_{s}-k \omega_{o}\right) e^{i k \omega_{o} \tau_{d}}
$$

At this point we will only describe $H(\omega)$ as a generic frequency domain temperature response, and in Section 4.3 will provide a formal definition of the model used in this work, based on a frequency domain solution for the heat diffusion equation in stratified mediums.

\section{Probe Sampling}

While we have formulated an expression for the temperature response of the system due to the periodic heating of the pump beam, the physical temperature cannot be measured without interaction interaction from the probe impulse-train. This is where the probe beam comes into play, and as discussed earlier, where the sampling theory will be applied for the second time. To begin, we give an expression for the periodic impulse-train of probe pulses 
in the time domain:

$$
p_{\mathrm{pr}}(t)=\beta Q_{\mathrm{pr}} \sum_{l=-\infty}^{\infty} \delta\left(t-l T_{s}-\tau_{d}\right)
$$

where $Q_{\mathrm{pr}}$ is the heat input of the probe pulses, $\beta$ is a constant that contains information about the thermoreflectance coefficient of the sample and the gain in the measurement electronics ${ }^{5}$, and $\tau_{d}$ is the time delay between the pump and probe pulses. In the frequency domain, the probe impulse-train is represented by:

$$
P(\omega)=\left[\frac{2 \pi \beta Q_{\mathrm{pr}}}{T_{s}} \sum_{l=-\infty}^{\infty} \delta\left(\omega-l \frac{2 \pi}{T_{s}}\right)\right] e^{-i \omega \tau_{d}}
$$

Recalling the formulation of the sampling theory in the frequency domain, we have the expression for the sampled function as being the convolution of the function to be sampled $X(\Omega)$ and the sampling function $P(\omega-\Omega)$, the probe in this instance:

$$
X_{p}(\omega)=\frac{1}{2 \pi} \int_{-\infty}^{\infty} X(\Omega) P(\omega-\Omega) d \Omega
$$

Inserting Equation 4.18 into Equation 4.19 we have:

$$
X_{p}(\omega)=\frac{\beta Q_{\mathrm{pr}}}{T_{s}} \int_{-\infty}^{\infty} \sum_{l=-\infty}^{\infty} X(\Omega) \delta\left(w-\Omega-l \frac{2 \pi}{T_{s}}\right) e^{-i(\omega-\Omega) \tau_{d}}
$$

and again using the property:

$$
\int_{-\infty}^{\infty} f(x) \delta\left(x-x_{o}\right) d x=f\left(x_{o}\right)
$$

we arrive at a generic expression for an arbitrary function $X$ sampled by the probe impulsetrain:

$$
X_{p}(\omega)=\frac{\beta Q_{\mathrm{pr}}}{T_{s}} \sum_{l=-\infty}^{\infty} X\left(\omega-l \omega_{s}\right) e^{-i l \omega_{s} \tau_{d}}
$$

In this case the function that we want to sample, via the probe, is the temperature response of the system due to the periodic heating from the pump, given by $\Theta(\omega)$, see

\footnotetext{
${ }^{5}$ When fitting the thermal model to the data to deduce a parameter of interest, the model will be normalized to the data, see Chapter 5.4.2, therefore we will not need specific knowledge of $\beta$, although thermoreflectance coefficients can be found in literature [198, 233, 234].
} 
Equation 4.16. Inserting $\Theta(\omega)$ into Equation 4.22 we have:

$$
\begin{aligned}
Z(\omega)=X_{p}(\omega)=\frac{2 \pi \beta Q_{\mathrm{pm}} Q_{\mathrm{pr}}}{T_{s}^{2}} \sum_{\substack{k=-\infty \\
k \neq 0}}^{\infty} \sum_{l=-\infty}^{\infty} \sum_{M=-\infty}^{\infty} \\
H\left(\omega-M \omega_{s}\right) \delta\left(\omega-l \omega_{s}-k \omega_{o}-M \omega_{s}\right) a_{k} e^{-i l \omega_{s} \tau_{d}} e^{i k \omega_{o} \tau_{d}}
\end{aligned}
$$

Simplifying:

$$
\begin{aligned}
Z(\omega)=\frac{2 \pi \beta Q_{\mathrm{pm}} Q_{\mathrm{pr}}}{T_{s}^{2}} \sum_{\substack{k=-\infty \\
k \neq 0}}^{\infty} \sum_{l=-\infty}^{\infty} \sum_{M=-\infty}^{\infty} \\
H\left(\omega-l \omega_{s}\right) \delta\left(\omega-k \omega_{o}-(l+M) \omega_{s}\right) a_{k} e^{-i l \omega_{s} \tau_{d}} e^{i k \omega_{o} \tau_{d}}
\end{aligned}
$$

The quantity $Z(\omega)$ will evaluate to zero, except where the terms in the delta function are equal to zero, i.e.:

$$
\omega-k \omega_{o}-(l+M) \omega_{s}=0
$$

Assuming $\omega$ is within the lock-in bandwidth (including higher harmonics), i.e. $\omega=k \omega_{o}$, we find that this condition is only satisfied when $l=-M$, and therefore we can eliminate $l$, and one of the summation terms from Equation 4.24:

$$
Z(\omega)=\frac{2 \pi \beta Q_{\mathrm{pm}} Q_{\mathrm{pr}}}{T_{s}^{2}} \sum_{\substack{k=-\infty \\ k \neq 0}}^{\infty} \sum_{M=-\infty}^{\infty} H\left(\omega+M \omega_{s}\right) \delta\left(\omega-k \omega_{o}\right) a_{k} e^{i M \omega_{s} \tau_{d}} e^{i k \omega_{o} \tau_{d}}
$$

Taking the inverse Fourier Transform we arrive at the time domain solution $z(t)$ :

$$
z(t)=\sum_{\substack{k=-\infty \\ k \neq 0}}^{\infty} Z\left(k \omega_{o}\right) a_{k} e^{i k \omega_{o} t}
$$

where $Z\left(k \omega_{o}\right)$ is the transfer function relating the frequency domain temperature response of the system to heat input from the pump, to the time domain response observed by the 
detector:

$$
Z\left(k \omega_{o}\right)=\frac{\beta Q_{\mathrm{pm}} Q_{\mathrm{pr}}}{T_{s}^{2}} \sum_{M=-\infty}^{\infty} H\left(k \omega_{o}+M \omega_{s}\right) e^{i M \omega_{s} \tau_{d}} e^{i k \omega_{o} \tau_{d}}
$$

\subsubsection{Lock-in Amplifier Signal}

Equation 4.27 provides the time domain representation of the complex temperature response of the system of interest to periodic pump heating as monitored by the train of probe pulses. However, in terms of TTR/TDTR analysis, what we are interested in is the lock-in amplifier's interpretation of this signal and the outputs provided. In most cases the lock-in will provide the $X$ and $Y$, real and imaginary, components of the mixture of the input and reference signal ${ }^{6}$.

In Equation 4.27, all the terms, $Z\left(k \omega_{o}\right), a_{k}$, and $e^{i k \omega_{o} t}$ are complex terms and therefore $z(t)$ is of the form:

$$
z(t)=\sum_{k}(a+i b)(c+i d)(e+i f)
$$

where

$$
\begin{gathered}
a=\mathfrak{R e}\left\{Z\left(k \omega_{o}\right)\right\} \\
b=\mathfrak{I m}\left\{Z\left(k \omega_{o}\right)\right\} \\
c=\mathfrak{R e}\left\{a_{k}\right\} \\
d=\mathfrak{I m}\left\{a_{k}\right\} \\
e=\mathfrak{R e}\left\{e^{i k \omega_{o} t}\right\}=\cos \left(k \omega_{o} t\right) \\
f=\mathfrak{I m}\left\{e^{i k \omega_{o} t}\right\}=\sin \left(k \omega_{o} t\right)
\end{gathered}
$$

\footnotetext{
${ }^{6}$ The lock-in will also typically provide the magnitude and phase of the signal, which can also be found from $X$ and $Y$ using the standard coordinate transforms.
} 
Expanding $z(t)$ we seek a solution of the form:

$$
z(t)=\sum_{k}(a+i b)(c+i d)(e+i f)=\sum_{k} a c e+i a c f+i a d e-a d f+i b c e-b c f-b d e-i b d f
$$

Therefore:

$$
\begin{aligned}
z(t)= & \sum_{k} \mathfrak{R e}\left\{Z\left(k \omega_{o}\right)\right\} \mathfrak{R e}\left\{a_{k}\right\} \cos \left(k \omega_{o} t\right)+i \mathfrak{R e}\left\{Z\left(k \omega_{o}\right)\right\} \mathfrak{R e}\left\{a_{k}\right\} \sin \left(k \omega_{o} t\right) \\
& +i \mathfrak{R e}\left\{Z\left(k \omega_{o}\right)\right\} \mathfrak{I m}\left\{a_{k}\right\} \cos \left(k \omega_{o} t\right)-\mathfrak{R e}\left\{Z\left(k \omega_{o}\right)\right\} \mathfrak{I m}\left\{a_{k}\right\} \sin \left(k \omega_{o} t\right) \\
& +i \mathfrak{I m}\left\{Z\left(k \omega_{o}\right)\right\} \mathfrak{R e}\left\{a_{k}\right\} \cos \left(k \omega_{o} t\right)-\mathfrak{I m}\left\{Z\left(k \omega_{o}\right)\right\} \mathfrak{R e}\left\{a_{k}\right\} \sin \left(k \omega_{o} t\right) \\
& -\mathfrak{I m}\left\{Z\left(k \omega_{o}\right)\right\} \mathfrak{I m}\left\{a_{k}\right\} \cos \left(k \omega_{o} t\right)-i \mathfrak{I m}\left\{Z\left(k \omega_{o}\right)\right\} \mathfrak{I m}\left\{a_{k}\right\} \sin \left(k \omega_{o} t\right)
\end{aligned}
$$

In terms of the lock-in analysis for the pump-probe spectroscopy experiment, we are interested in the physical signal to be measured, i.e. the real component of $z(t)$, therefore Equation 4.32 reduces to:

$$
\begin{aligned}
\mathfrak{R e}\{z(t)\}= & \sum_{k} \mathfrak{R e}\left\{Z\left(k \omega_{o}\right)\right\} \mathfrak{R e}\left\{a_{k}\right\} \cos \left(k \omega_{o} t\right)-\mathfrak{R e}\left\{Z\left(k \omega_{o}\right)\right\} \mathfrak{I m}\left\{a_{k}\right\} \sin \left(k \omega_{o} t\right) \\
& -\mathfrak{I m}\left\{Z\left(k \omega_{o}\right)\right\} \mathfrak{R e}\left\{a_{k}\right\} \sin \left(k \omega_{o} t\right)-\mathfrak{I m}\left\{Z\left(k \omega_{o}\right)\right\} \mathfrak{I m}\left\{a_{k}\right\} \cos \left(k \omega_{o} t\right)
\end{aligned}
$$

Grouping terms:

$$
\begin{aligned}
\mathfrak{R e}\{z(t)\}=\sum_{k} & {\left[\mathfrak{R e}\left\{Z\left(k \omega_{o}\right)\right\} \mathfrak{R e}\left\{a_{k}\right\}-\mathfrak{I m}\left\{Z\left(k \omega_{o}\right)\right\} \mathfrak{I m}\left\{a_{k}\right\}\right] \cos \left(k \omega_{o} t\right) } \\
& -\left[\mathfrak{R e}\left\{Z\left(k \omega_{o}\right)\right\} \mathfrak{I m}\left\{a_{k}\right\}+\mathfrak{I m}\left\{Z\left(k \omega_{o}\right)\right\} \mathfrak{R e}\left\{a_{k}\right\}\right] \sin \left(k \omega_{o} t\right)
\end{aligned}
$$

In our analysis of a symmetric pulsed waveform, it was found that the Fourier coefficients, $a_{k}$ are real for all harmonics, i.e. $\mathfrak{I m}\left\{a_{k}\right\}=0$, therefore Equation 4.34 can be simplified to:

$$
\mathfrak{R e}\{z(t)\}=\sum_{k} \mathfrak{R e}\left\{Z\left(k \omega_{o}\right) a_{k}\right\} \cos \left(k \omega_{o} t\right)-\mathfrak{I m}\left\{Z\left(k \omega_{o}\right)\right\} \mathfrak{R e}\left\{a_{k}\right\} \sin \left(k \omega_{o} t\right)
$$

Where in terms of the lock-in analysis, the real and imaginary components of the signal 
(also called the in-phase and out-of-phase components), $X$ and $Y$ are given by:

$$
\begin{gathered}
X=\sum_{k} a_{k} Z\left(k \omega_{o}\right) \\
Y=\sum_{k}-i a_{k} Z\left(k \omega_{o}\right)
\end{gathered}
$$

Inserting Equation 4.28 into Equations 4.36a and 4.36b we arrive at the final form of the lock-in amplifier's response, in channels $X$ and $Y$, to the sample's temperature response, monitored by the probe impulse-train, to the modulated heat input of the pump:

$$
\begin{gathered}
X=\frac{\beta Q_{\mathrm{pm}} Q_{\mathrm{pr}}}{T_{s}^{2}} \sum_{\substack{k=-\infty \\
k \neq 0}}^{\infty} \sum_{M=-\infty}^{\infty} a_{k} H\left(k \omega_{o}+M \omega_{s}\right) e^{i M \omega_{s} \tau_{d}} e^{i k \omega_{o} \tau_{d}} \\
Y=-i \frac{\beta Q_{\mathrm{pm}} Q_{\mathrm{pr}}}{T_{s}^{2}} \sum_{\substack{k=-\infty \\
k \neq 0}}^{\infty} \sum_{M=-\infty}^{\infty} a_{k} H\left(k \omega_{o}+M \omega_{s}\right) e^{i M \omega_{s} \tau_{d}} e^{i k \omega_{o} \tau_{d}}
\end{gathered}
$$

\subsubsection{Comparison of Sine and Square Waveforms}

As a check for the solutions provided in Equations $4.37 \mathrm{a}$ and $4.37 \mathrm{~b}$, we expect that in the limit that only the first harmonic is considered, i.e. $k=\{-1,1\}$, and for a duty cycle of $50 \%$, i.e. $D=0.5$, that the solution should reduce to the sinusoidal solution reported in literature [230, 235]. Under the assumption of the first harmonic, the Fourier coefficients are given by:

$$
\begin{gathered}
a_{k}=\frac{1}{-2 \pi i k}\left[e^{-i k \pi / 2}-e^{i k \pi / 2}\right] \\
a_{1}=a_{-1}=\frac{1}{\pi}
\end{gathered}
$$


Therefore for the sinusoidal modulation, the $X$ and $Y$ signal components from Equations 4.37a and $4.37 \mathrm{~b}$ are given by:

$$
\begin{aligned}
& X=\frac{1}{\pi} \frac{\beta Q_{\mathrm{pm}} Q_{\mathrm{pr}}}{T_{s}^{2}}\left[\sum_{M=-\infty}^{\infty} H\left(\omega_{o}+M \omega_{s}\right) e^{i M \omega_{s} \tau}\right. \\
& \left.+\sum_{M=-\infty}^{\infty} H\left(-\omega_{o}+M \omega_{s}\right) e^{i M \omega_{s} \tau_{d}}\right] e^{i \omega_{o} \tau_{d}} \\
& Y=\frac{-i}{\pi} \frac{\beta Q_{\mathrm{pm}} Q_{\mathrm{pr}}}{T_{s}^{2}}\left[\sum_{M=-\infty}^{\infty} H\left(\omega_{o}+M \omega_{s}\right) e^{i M \omega_{s} \tau}\right. \\
& \left.-\sum_{M=-\infty}^{\infty} H\left(-\omega_{o}+M \omega_{s}\right) e^{i M \omega_{s} \tau_{d}}\right] e^{i \omega_{o} \tau_{d}}
\end{aligned}
$$

If we compare these solutions to those given in Appendix C.3 for the results of the system response derived directly from the sinusoidal modulation, Equations C.42a and C.42b, we see that they only differ in the first term, the Fourier coefficient $a_{k}$ :

$$
\begin{aligned}
& \left.a_{1}=a_{-1}=\frac{1}{2} \quad \text { [sinudiodal }\right] \\
& a_{1}=a_{-1}=\frac{1}{\pi} \quad \text { [squarewave }-1^{\text {st }} \text { harmonic] }
\end{aligned}
$$

As detailed in Appendix C.3, we find that this discrepancy is expected given the formulation of the pulsed waveform, and that the Fourier coefficient of the pulsed waveform is larger than the sinusoidal due to the positive and negative contributions of the coefficients at higher harmonics and the restriction of the peak pulsed waveform to unity.

Therefore Equations $4.37 \mathrm{a}$ and $4.37 \mathrm{~b}$ provide an extension to the usual sinusoidal formulation of the lock-in response from LTI theory, to include the effects of higher harmonics and changes to the duty cycle, in a manner that is consistent with, and reduces to, the known sinusoidal results under the proper assumptions. In Chapter 5 we will provide more details on how the square vs sinusoidal modulation waveforms and changes to the duty cycle affect the TTR/TDTR signal. Before getting there though, we will first go back and define in detail, $H(\omega)$, the thermal response of the sample to the periodic heating. 


\subsection{Heat Transfer Analysis}

Equations $4.37 \mathrm{a}$ and $4.37 \mathrm{~b}$ provide a model of the lock-in amplifier response in the pump-probe spectroscopy experiment as laid out in the LTI theory, and includes a number of laser and system properties. Probably one of the most important terms, that to this point we have left undefined, is the frequency domain thermal response of the system to the periodic heat input, $H(\omega)$. The thermal model is where we will include the thermophysical properties of the sample of interest. We will begin by first reviewing the previous timedomain model used when I first arrived in the Nanoscale Energy Transport Lab at UVa, discuss its strengths and weaknesses, and motivate the adaptation of a frequency domain model that will be used for the majority of the work in this dissertation.

\subsubsection{Previous Modeling - Crank-Nicolson Analysis}

In nanoscale thermal analysis there are several time scales of interest in the metalsubstrate systems after the introduction of the ultrafast laser pulse. When I began in the lab, the primary interests were non-equilibrium electron-phonon coupling and diffusive thermal transport process. In this dissertation the focus is on diffusive transport, which occurs typically in the regime of $t>100$ ps after laser excitation, during which heat conduction can be described as diffusion in the film and substrate respectively, given by [10]:

$$
\begin{aligned}
& \frac{\partial T_{\mathrm{f}}(z, t)}{\partial t}=\alpha_{\mathrm{f}} \frac{\partial^{2} T_{\mathrm{f}}(z, t)}{\partial z^{2}} \\
& \frac{\partial T_{\mathrm{s}}(z, t)}{\partial t}=\alpha_{\mathrm{s}} \frac{\partial^{2} T_{\mathrm{s}}(z, t)}{\partial z^{2}}
\end{aligned}
$$

where $T_{\mathrm{f}}, \alpha_{\mathrm{f}}$ and $T_{\mathrm{s}}, \alpha_{\mathrm{s}}$ are the temperature and thermal diffusivity of the film and substrate respectively. The initial conditions for the film and the substrate are given by [10]:

$$
\begin{gathered}
T_{\mathrm{f}}(z, 0)=\frac{F(1-R)}{C_{\mathrm{p}, \mathrm{f}} \delta} \exp [-z / \delta] \\
T_{\mathrm{S}}(z, 0)=0
\end{gathered}
$$


where $F$ is the incident fluence, $R$ is the reflectance of the film, $C_{\mathrm{p}, \mathrm{f}}$ is the volumetric specific heat capacity of the film, and $\delta$ is the energy deposition depth. These conditions assume that all the laser energy is deposited into the film. This is a reasonable assumption when the film thickness is greater than the thermal penetration depth. Flux boundary conditions are applied at the interface between the film and substrate, that is to say the heat flux in the film and substrate are equal to the heat transport across the interface, i.e. [10]:

$$
\begin{gathered}
-k_{\mathrm{f}} \frac{\partial T_{\mathrm{f}}}{\partial z}(z=d, t)=h_{\mathrm{BD}}\left[T_{\mathrm{f}}(z=d, t)-T_{\mathrm{S}}(z=0, t)\right] \\
k_{\mathrm{s}} \frac{\partial T_{\mathrm{s}}}{\partial z}(z=d, t)=h_{\mathrm{BD}}\left[T_{\mathrm{f}}(z=d, t)-T_{\mathrm{S}}(z=0, t)\right]
\end{gathered}
$$

Equations $4.41 \mathrm{a}$ and $4.41 \mathrm{~b}$ assume thermal transport in only one direction, which is a reasonable assumption for a single shot system. A more in-depth discussion on the directionality assumptions will follow in Section 4.3.4. The other major assumption that was utilized when I began in the lab was that the metal film could be modeled using the lumped capacitance method. This assumes that at any point in time (past the beginning of modeling) there is no thermal gradient in the film [236]. Under this assumption, the conduction equations are given by [8]:

$$
\begin{gathered}
C_{\mathrm{p}, \mathrm{f}} d_{\mathrm{f}} \frac{\partial T_{\mathrm{f}}(t)}{\partial t}=-h_{\mathrm{BD}}\left[T_{\mathrm{f}}(t)-T_{\mathrm{S}}(z=0, t)\right] \\
\frac{\partial T_{\mathrm{s}}(z, t)}{\partial t}=\alpha_{\mathrm{s}} \frac{\partial^{2} T_{\mathrm{s}}(z, t)}{\partial z^{2}}
\end{gathered}
$$

where $C_{\mathrm{p}, \mathrm{f}}, d_{\mathrm{f}}$, and $T_{\mathrm{f}}(t)$ are the volumetric specific heat, thickness, and temperature of the film ${ }^{7}$, respectively, $h_{\mathrm{BD}}$ is the thermal boundary conductance between the film and the substrate, and $\alpha_{\mathrm{s}}$ is the thermal diffusivity of the substrate. To simplify the coding and analysis, the temperature in Equations $4.44 \mathrm{a}$ and $4.44 \mathrm{~b}$ can be nondimensionalized by the factor [237]:

$$
\phi_{\mathrm{f}}=\frac{T_{\mathrm{f}}-T_{o}}{T_{\mathrm{f}}(t=0)-T_{o}}
$$

\footnotetext{
${ }^{7}$ Note that we have dropped the spatial dependence of the properties of the film, given the assumption of lumped capacitance.
} 


$$
\phi_{\mathrm{s}}=\frac{T_{\mathrm{s}}-T_{o}}{T_{\mathrm{f}}(t=0)-T_{o}}
$$

where $T_{\mathrm{f}}(t=0)$ is the temperature of the film at laser excitation and $T_{o}$ is the ambient temperature. Therefore, the thermal model to be analyzed is given by [237]:

$$
\begin{gathered}
\frac{\partial \phi_{\mathrm{f}}(t)}{\partial t}=\frac{h_{\mathrm{BD}}}{C_{\mathrm{p}, \mathrm{f}} d_{\mathrm{f}}}\left[\phi_{\mathrm{s}}(z=0, t)-\phi_{\mathrm{f}}(t)\right] \\
\frac{\partial \phi_{\mathrm{s}}(z, t)}{\partial t}=\alpha_{\mathrm{s}} \frac{\partial^{2} \phi_{\mathrm{s}}(z, t)}{\partial z^{2}}
\end{gathered}
$$

Equations $4.46 \mathrm{a}$ and $4.46 \mathrm{~b}$ are subject to the initial conditions that the film is at an elevated temperature and the substrate is at ambient temperature at $t=0$ respectively. In the nondimensionalized formulation this is given by [237]:

$$
\begin{aligned}
& \phi_{\mathrm{f}}=1 \\
& \phi_{\mathrm{s}}=0
\end{aligned}
$$

Two boundary conditions are set for this system, the first being a flux boundary condition assuming that the rate of heat gained by the substrate is equal to the thermal flux across the interface, and the second being that the substrate is considered semi-infinite, i.e. the temperature gradient is zero at the far boundary. These boundary conditions in their nondimensional forms are given by [237]:

$$
\begin{gathered}
-k_{\mathrm{s}} \frac{\partial \phi_{\mathrm{s}}}{\partial z}=h_{\mathrm{BD}}\left[\phi_{\mathrm{f}}-\phi_{\mathrm{s}}\right] \quad \text { at } z=d \\
\frac{\partial \phi_{\mathrm{s}}}{\partial z}=0 \quad \text { at } x \rightarrow \infty
\end{gathered}
$$

The model in Equations $4.46 \mathrm{a}$ and $4.46 \mathrm{~b}$, subject to the initial and boundary conditions given in Equations 4.47a-4.47b and 4.48a-4.48b, respectively, are solved using a CrankNicolson methodology. The model is scaled to the data at a point where it is assumed that the electron and phonon sub-systems are in thermal equilibrium and there is no thermal gradient in the film, typically $t>100 \mathrm{ps}$. The $h_{\mathrm{BD}}$ is treated as a free parameter, and iteratively adjusted to minimize the error between the data and the model. 
The Crank-Nicolson model provided has the advantages of being relatively computationally efficient, requires no assumptions about the film thermal conductivity, and makes no assumptions about the properties of the pump laser. However, a disadvantage is that the formulation does not account for pulse accumulation effects and is therefore only suited for single shot measurements, e.g. low-rep lasers. The other disadvantage comes in the consideration of the lumped capacitance assumption.

\section{Assumptions of Lumped Capacitance}

In order for the assumptions of lumped capacitance to be appropriate, the resistance to conduction within the film, i.e. $1 / k_{\mathrm{f}}$, must be much less than the resistance to conduction across the solid-solid interface [236]. To give a quantitative value for this condition, the Biot number is provided [236]:

$$
B i=\frac{h_{\mathrm{BD}} d_{\mathrm{f}}}{k_{\mathrm{f}}}<0.1
$$

where it is assumed that the conditions of lumped capacitance are met when $B i<0.1$, which can also be rearranged to show the maximum film thickness under which these conditions will be met:

$$
d_{\mathrm{f}}<\frac{0.1 k_{\mathrm{f}}}{h_{\mathrm{BD}}}
$$

Figure 4.5 shows the maximum film thickness to maintain the lumped capacitance assumption as a function of $k_{\mathrm{f}}$ and $h_{\mathrm{BD}}$. The results in the figure show that assuming the bulk thermal conductivity of aluminum, and $h_{\mathrm{BD}}$ values on the order of $1 \mathrm{MW} / \mathrm{m}^{2} \mathrm{~K}$, the lumped capacitance assumption is appropriate for film thicknesses up to 100-200 nm. However, if we consider lower thermal conductivity metals (or take into account thermal conductivity reduction due to nanoscale size effects [22]) we can very rapidly reach situations where film thicknesses must be below $50 \mathrm{~nm}$ for the assumption of lump capacitance to hold. As the acceptable film thickness continues to decrease, care must be taken so that we can still assume that all the laser energy is deposited into the film, and therefore the initial conditions in Equations 4.42a-4.42b are still valid. The restrictions set forth by the Biot number in Equation 4.49 limit the choice of metals films and film thicknesses in which the standard diffusion model is applicable. While the diffusion model solved under the Crank-Nicolson 


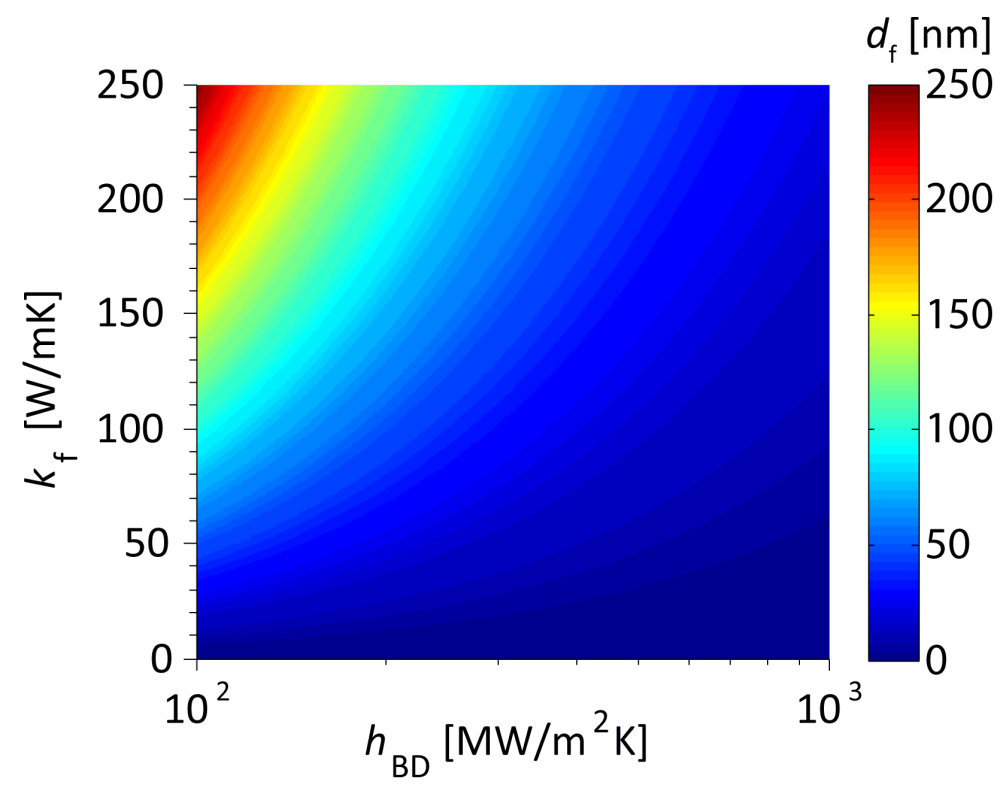

Figure 4.5: Maximum film thickness as a function of film thermal conductivity and $h_{\mathrm{BD}}$ for the assumptions of lumped capacitance to be valid.

formulation can be extended to account for diffusion in the film, it comes at the expense of increased computational cost (with a fine spatial and temporal spacing necessary in the nm scale film $)^{8}$.

In order to expand the ability to model different systems, a new model was implemented. The analysis by Feldman is an adaptation of Carslaw and Jaeger's solution to the heat diffusion equation for a stratified medium [238, 239]. The Feldman model has the advantages of: taking into account diffusion in both the film and substrate, being expandable to any number of layers, and being appropriate for any location of the heating source. In conjunction with the LTI analysis, the new formulation is applicable for both single shot and accumulated systems, and can account for cross-plane and in-plane thermal transport.

\footnotetext{
${ }^{8}$ More details regarding this will be presented in a future dissertation by Mr. MacKenzie Redding from our lab.
} 


\subsubsection{Multilayer Analysis via Feldman - Temperature Profile}

For the Feldman analysis we begin with the simplest case of one-dimensional heat diffusion in a semi-infinite region with the governing equation given by:

$$
\frac{\partial T(z, t)}{\partial t}+u_{z} \frac{\partial T(z, t)}{\partial z}=\alpha \frac{\partial^{2} T(z, t)}{\partial z^{2}}+\frac{Q}{\rho C_{p}}
$$

where $T(z, t)$ is the temperature in the z-direction as a function of time, $u_{z}$ is the velocity in the z-direction, $\alpha$ is the thermal diffusivity, i.e. $\alpha=k_{z} / \rho C_{p}$ (where $k_{z}$ is the thermal conductivity in the $z$-direction), $Q$ is the source term, $\rho$ is the density, and $C_{p}$ is the specific heat capacity. In the analysis used, it is assumed there is no convection, i.e. $u_{z}(\partial T(z, t) / \partial z)=0$, and no traditional source term ${ }^{9}$, i.e. $Q=0$. Therefore the heat diffusion equation simplifies to:

$$
\frac{\partial T(z, t)}{\partial t}=\alpha \frac{\partial^{2} T(z, t)}{\partial z^{2}}
$$

While work can be done in the time domain, for many heat conduction problems analytical solutions are readily available in the frequency domain, and therefore Equation 4.52 can be rewritten in the frequency domain using the temporal Fourier transform. In the frequency domain the heat equation is given by:

$$
\frac{d^{2} T(z)}{d z^{2}}=q^{2} T(z)
$$

where $q^{2}$ is given by:

$$
q^{2}=\frac{i \omega}{\alpha}=\frac{i \omega \rho C_{p}}{k_{z}}
$$

where $\omega$ is the angular frequency of the modulated heating source, and $T$ in this case is the complex temperature at frequency $\omega$. In the frequency domain, a generalized solution for multilayered slabs heated by a modulated source has been given by Feldman in 1999 [238]. The Feldman analysis is an adaptation of Carslaw and Jaeger's solution to the heat diffusion equation [239] whose matrix formulation described temperature and heat

\footnotetext{
${ }^{9}$ The Feldman analysis treats $\mathrm{Q}$ as a boundary condition similar to the methods used in the solution via the Crank-Nicolson method.
} 
flux vectors, whereas Feldman considered the vectors to be counter-propagating thermal waves. The Feldman analysis technique has been used in a variety of TDTR configurations [230, 240], frequency domain methods [241], 3 $\omega$ methods [242, 243], and for the measurement of the coefficient of thermal expansion [244]. A schematic representation of the layered structure and the indexing scheme is illustrated in Figure 4.6.

Equation 4.53a is a linear second order differential equation with a general solution given by:

$$
T(z)=T_{n}^{+} e^{\left(q_{n} z\right)}+T_{n}^{-} e^{\left(-q_{n} z\right)}
$$

where $T_{n}^{+}$and $T_{n}^{-}$are complex temperature constants, $q_{n}^{2}$ is the thermal wavevector, i.e. $q_{n}^{2}=i \omega / \alpha$, and $n$ denotes the $n^{\text {th }}$ layer in the solid. Utilizing this formulation, the complex temperature, $\tilde{\mathbf{T}}$, can be written as a vector given by:

$$
\tilde{\mathbf{T}}=\left[\begin{array}{c}
T_{n^{+}}(z) \\
T_{n^{-}}(z)
\end{array}\right]
$$

Considering a boundary between two layers $n$ and $n+1$, the boundary conditions required are constant temperature and constant heat flux at the interface, given by:

$$
T_{n}\left(\beta_{j}^{-}\right)=T_{n+1}\left(\beta_{j}^{+}\right)
$$

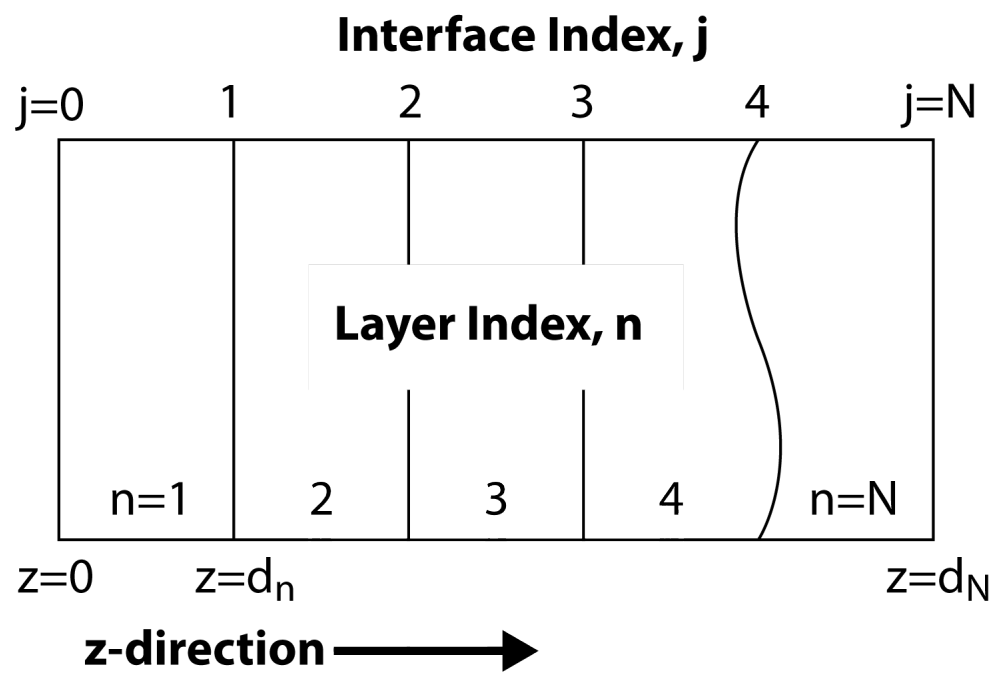

Figure 4.6: Geometry and indexing of the multilayered structure utilized in Feldman analysis. 


$$
\left.k_{n} \frac{d T_{n}}{d z}\right|_{z=\beta_{j}^{-}}=\left.k_{n+1} \frac{d T_{n+1}}{d z}\right|_{z=\beta_{j}^{+}}+Q
$$

where $\beta_{j}^{-}$is the limit as $z$ approaches the $j^{\text {th }}$ boundary from the left, $\beta_{j}^{+}$is the limit as $z$ approaches the $j^{\text {th }}$ boundary from the right, $k_{n}$ is the thermal conductivity of the $n^{\text {th }}$ layer, and $Q$ is the heat source term.

Based on the general form of the solution to Equation 4.52 and the prescribed boundary conditions, Equations 4.56a and 4.56b, several important relationships for diffusion through each layer and across the boundaries are formulated by Feldman [238].

\section{Layer Diffusion}

The temperature at $z_{n}=0$ in the $n^{\text {th }}$ layer in terms of the temperature at $z_{n}=d_{n}$ in the $n^{\text {th }}$ layer is given by:

$$
\tilde{\mathbf{T}}_{\mathbf{n}}\left(z_{n}=0\right)=\tilde{\mathbf{U}}_{\mathbf{n}}\left(d_{n}\right) \tilde{\mathbf{T}}_{\mathbf{n}}\left(z_{n}=d_{n}\right)
$$

where

$$
\tilde{\mathbf{U}}_{\mathbf{n}}\left(d_{n}\right)=\left[\begin{array}{cc}
\exp \left[-q_{n} d_{n}\right] & 0 \\
0 & \exp \left[q_{n} d_{n}\right]
\end{array}\right]
$$

therefore

$$
\left[\begin{array}{c}
T_{n^{+}}(0) \\
T_{n^{-}}(0)
\end{array}\right]=\left[\begin{array}{cc}
\exp \left[-q_{n} d_{n}\right] & 0 \\
0 & \exp \left[q_{n} d_{n}\right]
\end{array}\right]\left[\begin{array}{c}
T_{n^{+}}\left(d_{n}\right) \\
T_{n^{-}}\left(d_{n}\right)
\end{array}\right]
$$

\section{Boundary Diffusion}

The temperature of the $n^{\text {th }}$ layer adjacent to the boundary of the $n^{\text {th }}$ and $n+1$ layer is related to the temperature of the $n+1$ layer adjacent to the same boundary by:

$$
\tilde{\mathbf{T}}_{\mathbf{n}}\left(z_{n}=d_{n}\right)=\tilde{\boldsymbol{\Gamma}}_{\mathbf{n} \rightarrow \mathbf{n}+\mathbf{1}} \tilde{\mathbf{T}}_{\mathbf{n}+\mathbf{1}}\left(z_{n+1}=0\right)
$$

where

$$
\tilde{\boldsymbol{\Gamma}}_{\mathbf{n} \rightarrow \mathbf{n}+\mathbf{1}}=\frac{1}{2}\left[\begin{array}{ll}
1+\frac{\gamma_{n+1}}{\gamma_{n}}-\frac{\gamma_{n+1}}{h_{\mathrm{BD}, \mathrm{n} \rightarrow \mathrm{n}+1}} & 1-\frac{\gamma_{n+1}}{\gamma_{n}}+\frac{\gamma_{n+1}}{h_{\mathrm{BD}, \mathrm{n} \rightarrow \mathrm{n}+1}} \\
1-\frac{\gamma_{n+1}}{\gamma_{n}}-\frac{\gamma_{n+1}}{h_{\mathrm{BD}, \mathrm{n} \rightarrow \mathrm{n}+1}} & 1+\frac{\gamma_{n+1}}{\gamma_{n}}+\frac{\gamma_{n+1}}{h_{\mathrm{BD}, \mathrm{n} \rightarrow \mathrm{n}+1}}
\end{array}\right]
$$


and $\gamma_{n}=q_{n} k_{n}$. Therefore

$$
\begin{aligned}
{\left[\begin{array}{c}
T_{n^{+}}\left(d_{n}\right) \\
T_{n^{-}}\left(d_{n}\right)
\end{array}\right] } & \\
& =\frac{1}{2}\left[\begin{array}{ll}
1+\frac{\gamma_{n+1}}{\gamma_{n}}-\frac{\gamma_{n+1}}{h_{\mathrm{BD}, \mathrm{n} \rightarrow \mathrm{n}+1}} & 1-\frac{\gamma_{n+1}}{\gamma_{n}}+\frac{\gamma_{n+1}}{h_{\mathrm{BD}, \mathrm{n} \rightarrow \mathrm{n}+1}} \\
1-\frac{\gamma_{n+1}}{\gamma_{n}}-\frac{\gamma_{n+1}}{h_{\mathrm{BD}, \mathrm{n} \rightarrow \mathrm{n}+1}} & 1+\frac{\gamma_{n+1}}{\gamma_{n}}+\frac{\gamma_{n+1}}{h_{\mathrm{BD}, \mathrm{n} \rightarrow \mathrm{n}+1}}
\end{array}\right]\left[\begin{array}{c}
T_{n+1^{+}}(0) \\
T_{n+1^{-}}(0)
\end{array}\right]
\end{aligned}
$$

\section{Boundary Conditions}

The boundary conditions in the physical limits are given by:

$$
\begin{array}{r}
\tilde{\mathbf{T}}_{\mathbf{0}}=T_{0}\left[\begin{array}{l}
1 \\
0
\end{array}\right] \\
\tilde{\mathbf{T}}_{\mathbf{N}+\mathbf{1}}=T_{N+1}\left[\begin{array}{l}
0 \\
1
\end{array}\right]
\end{array}
$$

These boundary conditions insure that the temperature decays in the infinite limits, i.e. $T(z= \pm \infty)=0$.

\section{Heat Source Temperature}

For a heat source located in layer $n$ at $z=\xi$, the temperature on either side of the source is given by:

$$
\tilde{\mathbf{T}}_{\mathbf{n}}\left(\xi^{+}\right)-\tilde{\mathbf{T}}_{\mathbf{n}}\left(\xi^{-}\right)=-\frac{Q}{2 \gamma_{n, Q}}\left[\begin{array}{c}
1 \\
-1
\end{array}\right]
$$

where $\gamma_{n, Q}$ denotes the $\gamma$ term of the layer where the source is located. Using these relations, solutions for the temperature at any location within the structure can be determined. The temperature just to the left of the heat source is given by applying Equations 4.57 and 4.60 to Equation 4.63a:

$$
\tilde{\mathbf{T}}_{\mathbf{n}}\left(z_{j-1}+\xi^{-}\right)=T_{0}\left[\begin{array}{c}
A^{+} \\
A^{-}
\end{array}\right]
$$


and the temperature just to the right of the heat source is given by applying Equations 4.57 and 4.60 to Equation 4.63b:

$$
\tilde{\mathbf{T}}_{\mathbf{n}}\left(z_{j-1}+\xi^{+}\right)=T_{n+1}\left[\begin{array}{c}
B^{+} \\
B^{-}
\end{array}\right]
$$

where $A^{+}, A^{-}, B^{+}, B^{-}$are complex components of vectors $\tilde{\mathbf{A}}$ and $\tilde{\mathbf{B}}$ that contain the thermophysical parameters of each layer, i.e. $\tilde{\mathbf{U}}^{\prime} \mathbf{s}$ and $\tilde{\boldsymbol{\Gamma}}^{\prime} \mathbf{s}$. The formulation of $\tilde{\mathbf{A}}$ and $\tilde{\mathbf{B}}$ will be discussed in further detail shortly.

To obtain the temperature at either free surface in the z-direction Equations 4.65a and 4.65b are combined with Equation 4.64 to give:

$$
\begin{gathered}
T_{0}=\frac{Q}{2 \gamma_{n, Q}} \frac{B^{+}+B^{-}}{A^{+} B^{-}-A^{-} B^{+}} \\
T_{N+1}=\frac{Q}{2 \gamma_{n, Q}} \frac{A^{+}+A^{-}}{A^{+} B^{-}-A^{-} B^{+}}
\end{gathered}
$$

see Appendix C.4 for the derivation. Because we treated the heat input, $Q$, as part of the LTI analysis we will define $\Theta$ as the temperature profile that contains all the necessary information about the system's temperature response, independent of the heat input, i.e.:

$$
\begin{aligned}
\Theta_{0} & =\frac{1}{2 \gamma_{n, Q}} \frac{B^{+}+B^{-}}{A^{+} B^{-}-A^{-} B^{+}} \\
\Theta_{N+1} & =\frac{1}{2 \gamma_{n, Q}} \frac{A^{+}+A^{-}}{A^{+} B^{-}-A^{-} B^{+}}
\end{aligned}
$$

\section{$\tilde{\mathbf{A}}$ and $\tilde{\mathbf{B}}$ Matrices}

The $A$ and $B$ matrices contain the thermophysical properties of each layer and are formulated by successively applying Equations 4.57 and 4.60 along with the end boundary conditions, Equations 4.63a and 4.63b. As defined by Equations 4.65a and 4.65b, the matrix $\tilde{\mathbf{A}}$ accounts for layers to the left of the heat source (as denoted in Figure 4.6) going in the $-z$-direction, and the $\tilde{\mathbf{B}}$ matrix accounts for layers to the right of the heat source moving 
in the $+z$-direction. In general form, the matrices $\tilde{\mathbf{A}}$ and $\tilde{\mathbf{B}}$ are given by [238]:

$$
\begin{gathered}
\tilde{\mathbf{A}}=\tilde{\mathbf{U}}_{\mathbf{n}, \mathbf{q}}(\xi) \times \tilde{\boldsymbol{\Gamma}}_{\mathbf{n}, \mathbf{q} \rightarrow \mathbf{n}-\mathbf{1}} \times \cdots \times \tilde{\boldsymbol{\Gamma}}_{\mathbf{2} \rightarrow \mathbf{1}} \times \tilde{\mathbf{U}}_{\mathbf{1}}\left(-d_{1}\right) \times \tilde{\boldsymbol{\Gamma}}_{\mathbf{1} \rightarrow \mathbf{0}} \times\left(\begin{array}{l}
1 \\
0
\end{array}\right) \\
\tilde{\mathbf{B}}=\tilde{\mathbf{U}}_{\mathbf{n}, \mathbf{q}}\left(d_{n, q}-\xi\right) \times \tilde{\boldsymbol{\Gamma}}_{\mathbf{n}, \mathbf{q} \rightarrow \mathbf{n}+\mathbf{1}} \times \cdots \times \tilde{\boldsymbol{\Gamma}}_{\mathbf{N}-\mathbf{1} \rightarrow \mathbf{N}} \times \tilde{\mathbf{U}}_{\mathbf{N}}\left(d_{N}\right) \times \tilde{\boldsymbol{\Gamma}}_{\mathbf{N} \rightarrow \mathbf{N}+\mathbf{1}} \times\left(\begin{array}{l}
0 \\
1
\end{array}\right)
\end{gathered}
$$

\section{Example - Film on a Finite Substrate}

As an example, we will consider a basic case of a thin film on a finite substrate (see Figure 4.7), and construct the $\tilde{\mathbf{A}}$ and $\tilde{\mathbf{B}}$ matrices. The thicknesses of the film and substrate are assumed to be finite, and given by $L_{1}$ and $L_{2}$ respectively. The heat source is assumed to be deposited at $z_{1}=0$, as would be the case for a typical TTR/TDTR experiment. Considering first the $\tilde{\mathbf{A}}$ matrix, for the configuration shown in Figure 4.7 we have:

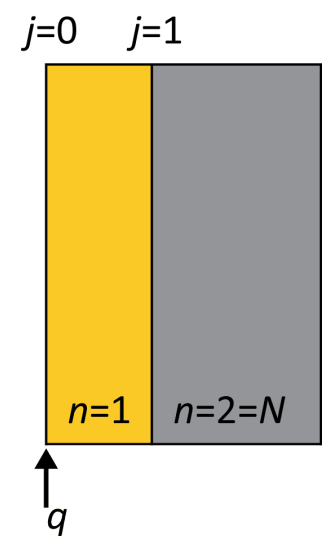

Figure 4.7: Example of a film on finite substrate system with indexing for Feldman analysis.

$$
\tilde{\mathbf{A}}=\tilde{\mathbf{U}}_{\mathbf{1}}(\mathbf{0}) \times \tilde{\boldsymbol{\Gamma}}_{\mathbf{1} \rightarrow \mathbf{0}} \times\left(\begin{array}{l}
1 \\
0
\end{array}\right)
$$

Expanding the terms we have:

$$
\tilde{\mathbf{A}}=\frac{1}{2} \times\left[\begin{array}{ll}
1 & 0 \\
0 & 1
\end{array}\right] \times\left[\begin{array}{cc}
1+\frac{\gamma_{0}}{\gamma_{1}}-\frac{\gamma_{0}}{h_{\mathrm{BD}, 1 \rightarrow 0}} & 1-\frac{\gamma_{0}}{\gamma_{1}}+\frac{\gamma_{0}}{h_{\mathrm{BD}, 1 \rightarrow 0}} \\
1-\frac{\gamma_{0}}{\gamma_{1}}-\frac{\gamma_{0}}{h_{\mathrm{BD}, 1 \rightarrow 0}} & 1+\frac{\gamma_{0}}{\gamma_{1}}+\frac{\gamma_{0}}{h_{\mathrm{BD}, 1 \rightarrow 0}}
\end{array}\right] \times\left[\begin{array}{l}
1 \\
0
\end{array}\right]
$$


Simplifying we have:

$$
\tilde{\mathbf{A}}=\frac{1}{2} \times\left[\begin{array}{c}
1+\frac{\gamma_{0}}{\gamma_{1}}-\frac{\gamma_{0}}{h_{\mathrm{BD}, 1 \rightarrow 0}} \\
1-\frac{\gamma_{0}}{\gamma_{1}}-\frac{\gamma_{0}}{h_{\mathrm{BD}, 1 \rightarrow 0}}
\end{array}\right]
$$

If we assume that the sample is held in vacuum (or in air assuming no heat loss from the surface, which was part of our original assumptions) $k_{0}=0$ and therefore $\gamma_{0}=0$. Under these assumptions the $\tilde{\mathbf{A}}$ matrix reduces to:

$$
\tilde{\mathbf{A}}=\left[\begin{array}{c}
1 / 2 \\
1 / 2
\end{array}\right]
$$

Following a similar methodology for the $\tilde{\mathbf{B}}$ matrix we have:

$$
\begin{gathered}
\tilde{\mathbf{B}}=\tilde{\mathbf{U}}_{\mathbf{1}}\left(d_{1}\right) \times \tilde{\boldsymbol{\Gamma}}_{\mathbf{1} \rightarrow \mathbf{2}} \tilde{\mathbf{U}}_{\mathbf{2}}\left(d_{2}\right) \times \tilde{\boldsymbol{\Gamma}}_{\mathbf{2} \rightarrow \mathbf{3}} \times\left(\begin{array}{l}
0 \\
1
\end{array}\right) \\
\tilde{\mathbf{B}}=\frac{1}{2} \times\left[\begin{array}{cc}
\exp \left[-q_{1} d_{1}\right] & 0 \\
0 & \exp \left[q_{1} d_{1}\right]
\end{array}\right] \\
\times\left[\begin{array}{ccc}
1+\frac{\gamma_{2}}{\gamma_{1}}-\frac{\gamma_{2}}{h_{\mathrm{BD}, 1 \rightarrow 2}} & 1-\frac{\gamma_{2}}{\gamma_{1}}+\frac{\gamma_{2}}{h_{\mathrm{BD}, 1 \rightarrow 2}} \\
1-\frac{\gamma_{2}}{\gamma_{1}}-\frac{\gamma_{2}}{h_{\mathrm{BD}, 1 \rightarrow 2}} & 1+\frac{\gamma_{2}}{\gamma_{1}}+\frac{\gamma_{2}}{h_{\mathrm{BD}, 1 \rightarrow 2}}
\end{array}\right] \times\left[\begin{array}{cc}
\exp \left[-q_{2} d_{2}\right] & 0 \\
0 & \exp \left[q_{2} d_{2}\right]
\end{array}\right] \\
\times\left[\begin{array}{cc}
1+\frac{\gamma_{3}}{\gamma_{2}}-\frac{\gamma_{3}}{h_{\mathrm{BD}, 2 \rightarrow 3}} & 1-\frac{\gamma_{3}}{\gamma_{2}}+\frac{\gamma_{3}}{h_{\mathrm{BD}, 2 \rightarrow 3}} \\
1-\frac{\gamma_{3}}{\gamma_{2}}-\frac{\gamma_{3}}{h_{\mathrm{BD}, 2 \rightarrow 3}} & 1+\frac{\gamma_{3}}{\gamma_{2}}+\frac{\gamma_{3}}{h_{\mathrm{BD}, 2 \rightarrow 3}}
\end{array}\right] \times\left[\begin{array}{l}
0 \\
1
\end{array}\right]
\end{gathered}
$$

Again assuming $k_{3}=0$ and therefore $\gamma_{3}=0$ it can be shown that:

$$
\begin{aligned}
B^{+}=\frac{1}{2} \times\left[\left(1+\frac{\gamma_{2}}{\gamma_{1}}-\frac{\gamma_{2}}{h_{\mathrm{BD}, 1 \rightarrow 2}}\right) \exp \left[-q_{1} d_{1}\right] \exp \left[-q_{2} d_{2}\right]+\right. & \left.\left(1-\frac{\gamma_{2}}{\gamma_{1}}+\frac{\gamma_{2}}{h_{\mathrm{BD}, 1 \rightarrow 2}}\right) \exp \left[-q_{1} d_{1}\right] \exp \left[q_{2} d_{2}\right]\right] \\
B^{-=} \frac{1}{2} \times\left[\left(1-\frac{\gamma_{2}}{\gamma_{1}}-\frac{\gamma_{2}}{h_{\mathrm{BD}, 1 \rightarrow 2}}\right) \exp \left[q_{1} d_{1}\right] \exp \left[-q_{2} d_{2}\right]+\right. & \left.\left(1+\frac{\gamma_{2}}{\gamma_{1}}+\frac{\gamma_{2}}{h_{\mathrm{BD}, 1 \rightarrow 2}}\right) \exp \left[q_{1} d_{1}\right] \exp \left[q_{2} d_{2}\right]\right]
\end{aligned}
$$




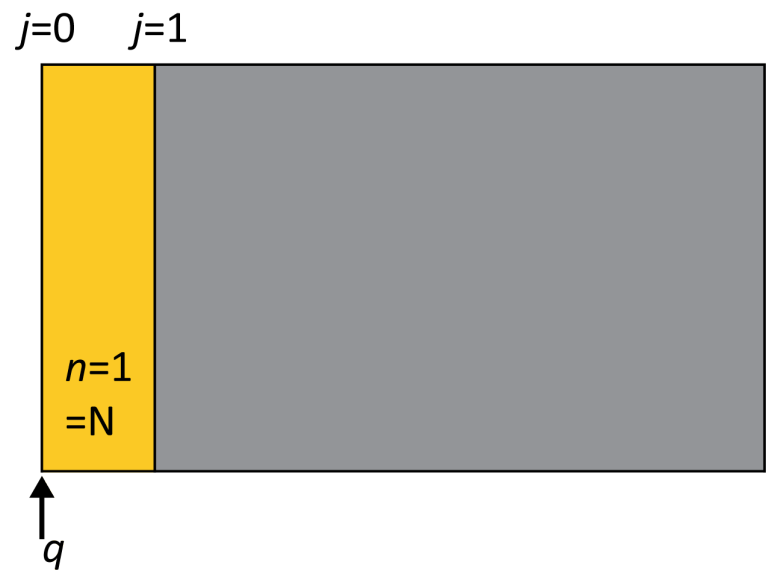

Figure 4.8: Example of a film on a semi-infinite substrate system with indexing for Feldman analysis.

We can take note already that the $\tilde{\mathbf{B}}$ matrix is quite complex for even a two-layer system, and can expect it to grow computationally complex rapidly with added layers. It is expected that the Feldman matrices will be solved as a series of numerical matrices rather than a closed form solution.

\section{Example - Film on Semi-infinite Substrate}

Next we will consider a film on a semi-infinite substrate, typically the simplest and most common TTR/TDTR sample configuration, see Figure 4.8, to illustrate the changes in formulation when making the assumption of a semi-infinite substrate. Referring back to Equations $4.68 \mathrm{a}$ and $4.68 \mathrm{~b}$, we see that the last term in each equation, prior to the boundary condition, represents heat flow across a boundary from the outermost layers (either top of bottom) to the surrounding "atmosphere" which is assumed to be infinite. When making the semi-infinite substrate assumption, we replace the "atmosphere" term with the properties of the substrate in the $\tilde{\mathbf{B}}$ matrix, i.e. the substrate properties are indexed as $N+1$. We can further illustrate this concept by constructing the $\tilde{\mathbf{A}}$ and $\tilde{\mathbf{B}}$ matrices for a film on a semi-infinite substrate.

In this case, the formulation of the $\tilde{\mathbf{A}}$ matrix does not change, and is given by:

$$
\tilde{\mathbf{A}}=\left[\begin{array}{c}
1 / 2 \\
1 / 2
\end{array}\right]
$$


In fact, the $\tilde{\mathbf{A}}$ matrix will have the form given in Equation $4.69 \mathrm{~d}$ anytime it is assumed that the heat is deposited in the topmost film and the adjacent boundary in the $-z$-direction is with the environment. The formulation of the $\tilde{\mathbf{B}}$ matrix is modified though under the new assumptions:

$$
\tilde{\mathbf{B}}=\tilde{\mathbf{U}}_{\mathbf{1}}\left(d_{1}\right) \times \tilde{\boldsymbol{\Gamma}}_{\mathbf{1} \rightarrow \mathbf{2}} \times\left(\begin{array}{l}
0 \\
1
\end{array}\right)
$$

Expanding we have:

$$
\begin{aligned}
\tilde{\mathbf{B}}=\frac{1}{2} \times\left[\begin{array}{cc}
\exp \left[-q_{1} d_{1}\right] & 0 \\
0 & \exp \left[q_{1} d_{1}\right]
\end{array}\right] \\
\times\left[\begin{array}{cc}
1+\frac{\gamma_{2}}{\gamma_{1}}-\frac{\gamma_{2}}{h_{\mathrm{BD}, 1 \rightarrow 2}} & 1-\frac{\gamma_{2}}{\gamma_{1}}+\frac{\gamma_{2}}{h_{\mathrm{BD}, 1 \rightarrow 2}} \\
1-\frac{\gamma_{2}}{\gamma_{1}}-\frac{\gamma_{2}}{h_{\mathrm{BD}, 1 \rightarrow 2}} & 1+\frac{\gamma_{2}}{\gamma_{1}}+\frac{\gamma_{2}}{h_{\mathrm{BD}, 1 \rightarrow 2}}
\end{array}\right] \times\left[\begin{array}{l}
0 \\
1
\end{array}\right]
\end{aligned}
$$

Simplifying

$$
\begin{gathered}
B^{+}=\frac{1}{2} \times\left[\left(1-\frac{\gamma_{2}}{\gamma_{1}}+\frac{\gamma_{2}}{h_{\mathrm{BD}, 1 \rightarrow 2}}\right) \exp \left[-q_{1} d_{1}\right]\right] \\
B^{-}=\frac{1}{2} \times\left[\left(1+\frac{\gamma_{2}}{\gamma_{1}}+\frac{\gamma_{2}}{h_{\mathrm{BD}, 1 \rightarrow 2}}\right) \exp \left[q_{1} d_{1}\right]\right]
\end{gathered}
$$

A final example, showing a formulation where the heat is not deposited in the topmost film, e.g. probing through glass, is provided for clarity and convenience in Appendix C.5.

By combining Equations 4.68a and 4.68b with Equation 4.67a the Feldman analysis provides a straight forward and adaptable algorithm for solving the heat diffusion equation for stratified mediums. Its adaptation in our lab, has allowed us to expand the complexity and variety of samples that can be measured, modeled, and analyzed. As a final step in determining the frequency domain thermal response of the system, we will apply a top surface boundary condition, based on the laser properties, to convert the surface temperature profile, $\Theta_{0}$, given by the Feldman analysis to the frequency domain temperature response, $H(\omega)$.

\subsubsection{Surface Temperature Response in the Frequency Domain - $H(\omega)$}

To develop the final temperature profile, we will consider the shape and intensity of the pump and probe beams. We will begin by considering the case of one-dimensional transport, 
and then specify how the Feldman analysis can be extended to include 3D and anisotropic effects.

Assuming no radial heating effects, i.e. a uniform intensity per unit area, the intensity distribution of the pump can be given by [245]:

$$
I_{\mathrm{pm}}=\frac{A_{\mathrm{pm}}}{\pi w_{\mathrm{pm}}^{2}}
$$

where $A_{\mathrm{pm}}$ is the power absorbed by the pump laser and $w_{\mathrm{pm}}$ is the $1 / e^{2}$ radius. Note that, we already include the energy of the pump and probe pulses in the LTI analysis. Therefore, to prevent double counting of the laser energy, we will replace $A_{\mathrm{pm}}$ with the product of the time between pulses, $T_{o}$, and the duty cycle, $D$, with the understanding that when $H(\omega)$ is included in Equations 4.37a-4.37b the input power of the laser energy will be properly accounted for. Combining Equations 4.75 and 4.66a we find the surface temperature due to the pump heating, given by:

$$
T_{0, p m}=\frac{T_{o} D}{\pi w_{\mathrm{pm}}^{2}} \Theta_{0}(z=0)
$$

The temperature response in the frequency domain is given by the fraction of the temperature change measured by the probe beam, assumed to be of uniform intensity and $1 / e^{2}$ radius $w_{\mathrm{pr}}$, and therefore $H(\omega)$ is given by [245]:

$$
H(\omega)=\frac{T_{s} T_{o} D w_{\mathrm{pr}}^{2}}{\pi w_{\mathrm{pm}}^{4}} \Theta_{0}(z=0)
$$

\section{Extension to 3D}

If the geometry of the laser spots are such that the 1D assumption is no longer valid, or we are interested in more advanced quantities such as in-plane thermal conductivity, and/or are considering samples with anisotropic properties, the analysis developed to this point must be extended to 3D. The lock-in transfer function $Z(\omega)$ (see Equation 4.28) is dimensionaly independent, however to extend the thermal analysis to $3 \mathrm{D}$ the analysis of the 
surface temperature profile, $\Theta_{o}$ (see Equation 4.67a), and the frequency domain temperature response, $H(\omega)$, must be modified.

Starting again from the heat diffusion equation, this time in cylindrical coordinates, we have:

$$
\frac{k_{r}}{r} \frac{\partial}{\partial r}\left(r \frac{\partial T}{\partial r}\right)-k_{z} \frac{\partial^{2} T}{\partial z^{2}}=\rho C_{p} \frac{\partial T}{\partial t}
$$

where $k_{r}$ and $k_{z}$ are the thermal conductivities in the radial and cross-plane directions respectively, $\rho$ is the material density, and $C_{p}$ is the specific heat capacity. Under the assumption that the pump and probe laser beams are co-linearly aligned, we can take advantage of the axial symmetry to simply the analysis through the use of Hankel transforms, as outline by Cahill [230]. Taking the Hankel transform of Equation 4.78 we have:

$$
-k_{r} \kappa^{2} T(\kappa, t, z)-k_{z} \frac{\partial^{2} T(\kappa, t, z)}{\partial z^{2}}=\rho C_{p} \frac{\partial T(\kappa, t, z)}{\partial t}
$$

where $\kappa$ is the transform variable. We can additionally transform into the frequency domain, through the application of the Fourier transform:

$$
-k_{r} \kappa^{2} T(\kappa, \omega, z)-k_{z} \frac{\partial^{2} T(\kappa, \omega, z)}{\partial z^{2}}=\rho C_{p} i \omega T(\kappa, \omega, z)
$$

Rearranging we have:

$$
\frac{\partial^{2} T(z)}{\partial z^{2}}=q^{2} T(z)
$$

where

$$
q^{2}=\frac{k_{r} \kappa^{2}+i \omega \rho C_{p}}{k_{z}}
$$

Note that Equation 4.81a is identical to Equation 4.53a that was derived from the onedimensional heat equation in Section 4.3.2. The only difference between the 1D and 3D solutions, is the formulation of the thermal wavevector:

$$
\begin{array}{r}
q^{2}=\frac{i \omega \rho C_{p}}{k_{z}} \\
q^{2}=\frac{k_{r} \kappa^{2}+i \omega \rho C_{p}}{k_{z}}
\end{array}
$$


Therefore, the Feldman analysis described in Section 4.3.2, can be used in the threedimensional analysis with the same formulation other than the replacement of the thermal wavevector in Equation 4.82a with Equation $4.82 \mathrm{~b}$ and the re-treatment of the heat input $Q$ which we will reconsider now following the work of Cahill and Schmidt [230, 233].

We begin by considering the pump laser beam to have a Gaussian intensity distribution given by:

$$
I(r)=\frac{2 A_{\mathrm{pm}}}{\pi w_{\mathrm{pm}}^{2}} \exp \left(\frac{-2 r^{2}}{w_{\mathrm{pm}}^{2}}\right)
$$

where $A_{\mathrm{pm}}$ is the power absorbed by the pump laser and $w_{\mathrm{pm}}$ is the $1 / e^{2}$ radius of the beam. Again, we will substitute $T_{0} D$ for $A_{\mathrm{pm}}$ to keep the units of power consistent. Taking advantage of the cylindrical symmetry, the Hankel transform of the pump distribution is given by:

$$
I(\kappa)=\frac{T_{0} D}{2 \pi} \exp \left(\frac{-\kappa^{2} w_{\mathrm{pm}}^{2}}{8}\right)
$$

Combining with Equation 4.66a we find the surface temperature in the Hankel domain:

$$
T_{0, H}(\kappa)=\Theta_{0}(z=0) \frac{T_{0} D}{2 \pi} \exp \left(\frac{-\kappa^{2} w_{\mathrm{pm}}^{2}}{8}\right)
$$

The temperature response of the system, as measured by the probe beam, is found by taking surface temperature $T_{0, H}(\kappa)$ and weighting it by the distribution of the Gaussian probe beam with a $1 / e^{2}$ waist of $w_{\text {pr }}$ :

$$
\begin{gathered}
H(\kappa)=\frac{T_{s}}{2 \pi} \exp \left(\frac{-\kappa^{2} w_{\mathrm{pr}}^{2}}{8}\right) T_{0, H}(\kappa) \\
H(\kappa)=\frac{T_{s}}{2 \pi} \exp \left(\frac{-\kappa^{2} w_{\mathrm{pr}}^{2}}{8}\right) \Theta_{0}(z=0) \frac{T_{o} D}{2 \pi} \exp \left(\frac{-\kappa^{2} w_{\mathrm{pm}}^{2}}{8}\right) \\
H(\kappa)=\frac{T_{s} T_{o} D}{4 \pi^{2}} \Theta_{0}(z=0) \exp \left(\frac{-\kappa^{2}\left(w_{\mathrm{pm}}^{2}+w_{\mathrm{pr}}^{2}\right)}{8}\right)
\end{gathered}
$$

Taking the inverse Hankel transform brings us back to the real space domain and gives the 
frequency domain response of the surface temperature:

$$
H(\omega)=\frac{T_{s} T_{o} D}{2 \pi} \int_{0}^{\infty} \Theta_{0}(z=0) \exp \left(\frac{-\kappa^{2}\left(w_{\mathrm{pm}}^{2}+w_{\mathrm{pr}}^{2}\right)}{8}\right) \kappa d \kappa
$$

As was discussed previously, the temperature profile developed using the Feldman analysis, $\Theta$, is solved numerically. Therefore, the integral in Equation 4.87 will also be solved numerically with a finite upper bound. Cahill provides that an upper bound of the integral of $2 / \sqrt{w_{\mathrm{pm}}^{2}+w_{\mathrm{pr}}^{2}}$ should provide a sufficient solution to Equation 4.87 [230]. However, it is probably prudent to check for system convergence before accepting the final results.

\subsubsection{Criteria for 1D vs 3D Modeling}

While the 3D model is inherently more robust than assuming a $1 \mathrm{D}$ model, there is of course an additional computational cost for the stronger assumption, mainly the numeric integration of Equation 4.87, for every term in the summation, see Equation 4.28. To save computational time, ideally we would prefer to test whether or not we need to consider radial effects in our thermal analysis. For high-rep systems, there have been several studies to indicate how radial spreading affects the TDTR model, and the criteria for 1D modeling $[231,245]$. These results don't clearly hold for the low-rep, single shot systems, so we will present a simplified single pulse analysis of radial heat spreading. To do so, we will consider the limit of a confined homogeneous and isotropic film, with temperature independent properties. The boundaries of the film will either be insulating or held at the initial temperature. At $t=0$, assuming a Gaussian distribution of the pump beam, the temperature distribution in the film is given by $[79,246]$ :

$$
\Delta T(r, t=0)=T_{o} \exp \left(\frac{-r^{2}}{w_{o}^{2}}\right)
$$

where $T_{o}$ is the initial temperature, and $w_{o}$ is the pump waist. As a function of time after the initial heating event, the temperature distribution is a function of the thermal diffusivity 
of the film, $D$, and is given by $[79,246]$ :

$$
\Delta T(r, t)=T_{o} \frac{w_{o}^{2}}{w_{o}^{2}+4 D t} \exp \left(-\frac{r^{2}}{w_{o}^{2}+4 D t}\right)
$$

Figure 4.9a shows the heat distribution due to a Gaussian pump pulse with an initial waist of $10 \mu \mathrm{m}$, in an insulated film with a thermal diffusivity of $D=1 \times 10^{-3} \mathrm{~m}^{2} / \mathrm{s}$, immediately after energy deposition and after 8 ns (the maximum about of time considered in our experiment). After the initial energy deposition, the heat spreads, reducing the

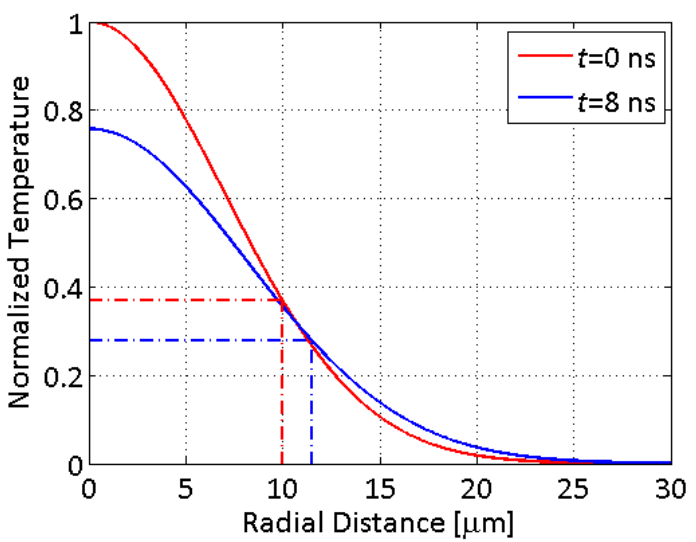

(a)

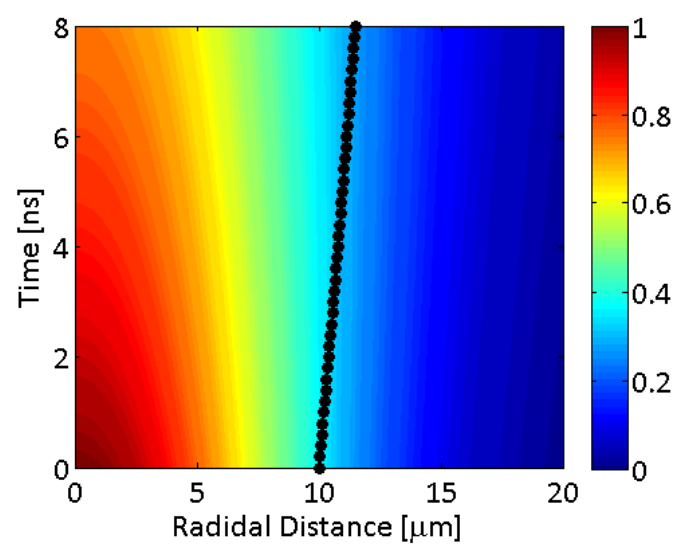

(b)

Figure 4.9: Radial spreading of laser energy in a confined film given by Equations 4.88 and 4.89 for $D=1 \times 10^{-3}$ and an initial pump waist of $10 \mu \mathrm{m}$. Pane (a) shows the Gaussian distribution of heat at $t=0$ and $t=8 \mathrm{~ns}$, with the dashed lines representing the $1 / e$ radius and $(\mathrm{b})$ the distribution of temperature as a function of time and radial distance with the color scale representing the normalized temperature. The black marks providing the $1 / e$ location as a function of time.

peak temperature at the center of the heating event, and moving the radial $1 / e$ location outward. In this example, the $1 / e$ distance shifts to $11.5 \mu \mathrm{m}$ over the $8 \mathrm{~ns}$, representing a $\sim 15 \%$ change. Figure $4.9 \mathrm{~b}$ provides the radial distance as a function of time, with the $1 / e$ location of the thermal energy noted in black. To give a general idea of how the pump waist and the thermal diffusivity of the film affect the amount of thermal spreading, Figure 4.10 shows the percent change in the $1 / e$ location of the thermal energy compared to the initial distribution, due to thermal spreading, as a function of the initial pump waist for several thermal diffusivity values.

The data in Figure 4.10 shows that for low diffusivity films, there is negligible radial 


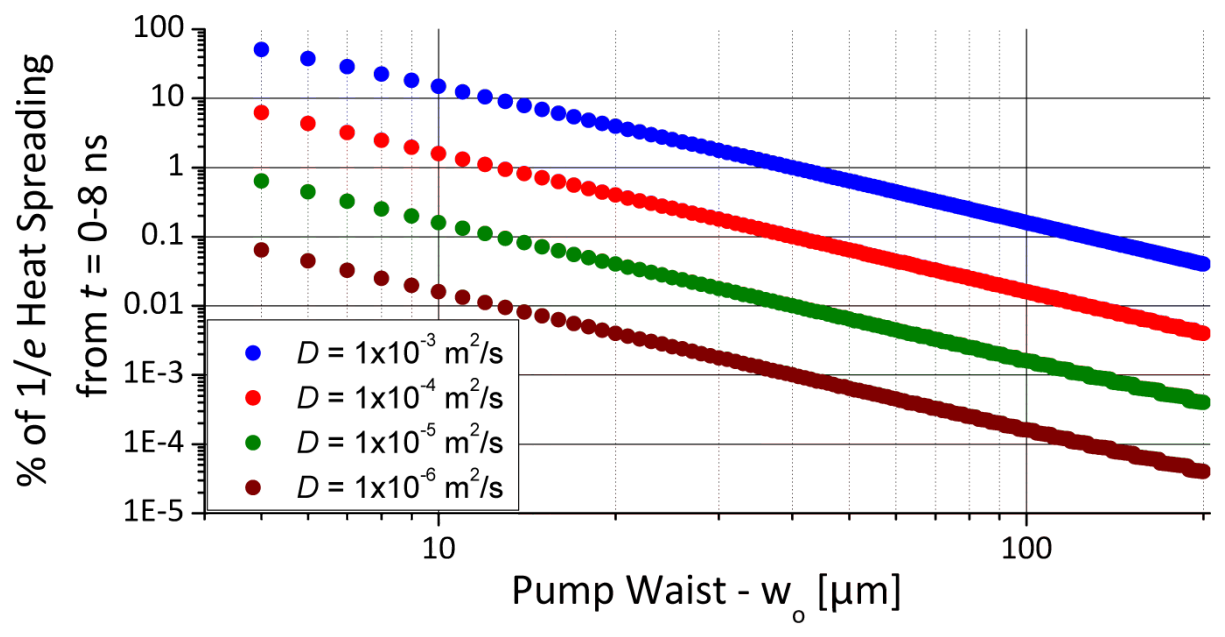

Figure 4.10: Percentage of $1 / e$ heat spreading as a function of the initial pump waist for several thermal diffusivities, covering a range from on the order of quartz to diamond.

heat spreading regardless of the initial pump size. As the thermal diffusivity of the film increases, a larger initial pump waist is required to ensure the effects of radial spreading are negligible. One of the primary advantages of the low-rep system is that the high pulse energy allows for larger pump waists, while still maintaining enough fluence to create a sufficient temperature perturbation. For layered structures, these assumptions hold provided that the substrate diffusivity is less than that of the film [79]. Care must be taken for systems with high substrate thermal diffusivity such as metals on HOPG. While for the film the 1D assumption may be made, as the thermal energy reaches the HOPG, the heat will spread rapidly in the radial direction due to the high thermal diffusivity.

\subsection{Chapter Summary}

In this chapter we took an in-depth look at the computational formulation of the analysis of TTR/TDTR data. A mathematical formulation of the complex signal returned by the lock-in amplifier was developed:

$$
z(t)=\sum_{\substack{k=-\infty \\ k \neq 0}}^{\infty} Z\left(k \omega_{o}\right) a_{k} e^{i k \omega_{o} t}
$$


extending on the work of Schmidt $[228,231]$ to model the modulation function as a pulsed waveform, i.e. a square wave of arbitrary duty cycle, with $Z\left(k \omega_{o}\right)$ being a complex transfer function describing the properties of the laser and sample and the response of their interaction. The real and imaginary components of the lock-in transfer function, $Z\left(k \omega_{o}\right)$, which will be the quantities compared to the experimental data, are given by:

$$
\begin{gathered}
X=\frac{\beta Q_{\mathrm{pm}} Q_{\mathrm{pr}}}{T_{s}^{2}} \sum_{\substack{k=-\infty \\
k \neq 0}}^{\infty} \sum_{M=-\infty}^{\infty} a_{k} H\left(k \omega_{o}+M \omega_{s}\right) e^{i M \omega_{s} \tau_{d}} e^{i k \omega_{o} \tau_{d}} \\
Y=-i \frac{\beta Q_{\mathrm{pm}} Q_{\mathrm{pr}}}{T_{s}^{2}} \sum_{\substack{k=-\infty \\
k \neq 0}}^{\infty} \sum_{M=-\infty}^{\infty} a_{k} H\left(k \omega_{o}+M \omega_{s}\right) e^{i M \omega_{s} \tau_{d}} e^{i k \omega_{o} \tau_{d}}
\end{gathered}
$$

where $H(\omega)$ is the frequency domain solution to the systems thermal response to the periodic heating, given by the work of Cahill and Schmidt in 1D and 3D by [228, 230, 231]

$$
\begin{gathered}
H_{1 \mathrm{D}}(\omega)=\frac{T_{s} T_{o} D w_{\mathrm{pr}}^{2}}{\pi w_{\mathrm{pm}}^{4}} \Theta_{0}(z=0) \\
H_{3 \mathrm{D}}(\omega)=\frac{T_{s} T_{o} D}{2 \pi} \int_{0}^{\infty} \Theta_{0}(z=0) \exp \left(\frac{-\kappa^{2}\left(w_{\mathrm{pm}}^{2}+w_{\mathrm{pr}}^{2}\right)}{8}\right) \kappa d \kappa
\end{gathered}
$$

For either solution of $H(\omega), \Theta_{0}$ is the temperature distribution at the surface given by the frequency domain analysis of the heat diffusion equation for stratified media as presented by Feldman [238]. This was introduce over the previous Crank-Nicolson solution, to add the ability to function with both high- and low-rep laser systems, increase the number of layers that can be considered in a sample, and allow for the modeling of both $1 \mathrm{D}$ and $3 \mathrm{D}$ transport. The temperature profile, $\Theta_{0}$, from the Feldman analysis is given by:

$$
\Theta_{0}=\frac{1}{2 \gamma_{n, Q}} \frac{B^{+}+B^{-}}{A^{+} B^{-}-A^{-} B^{+}}
$$

where $A^{+}, A^{-}, B^{+}, B^{-}$are complex values containing the thermophysical properties of the materials and interfaces in the system.

The information provided in this chapter, along with the set of equations summarized here, should provide the necessary mathematical tools to model the data collected during 
a TTR/TDTR scan. In the next chapter we will look into some practical issues in the implementation of this model. We will discuss the convergence criteria of the infinite summations, how the higher harmonics and duty cycle of the modulation waveform affect the thermal response, and introduce methods to increase fitting efficiency. 


\title{
CHAPTER 5
}

\author{
PRACTICAL CONSIDERATIONS IN \\ THE METHODS OF FITTING THE
}

THERMOREFLECTANCE MODEL TO

EXPERIMENTAL DATA FOR

\section{THERMOPHYSICAL PARAMETER}

DEDUCTION

5.1 Introduction . . . . . . . . . . . . . . . . . . . 119

5.2 Proper Modulation to Eliminate Phase Drift _ . . . . . . . . . . . . . 119

5.3 Convergence Criteria in Modeling the Lock-in Amplifier Response During TTR/TDTR Experiments . . . . . . . . . . . . . . 126

5.4 Fitting to Deduce Thermophysical Parameters . . . . . . . . . . . . . 147

5.5 Sensitivity Analysis . . . . . . . . . . . . . . 157 
5.6 Defining Noise in Data . . . . . . . . . . . . . . . . 166

5.7 Post-processing of Data to Remove Outliers . . . . . . . . . . . . . . . . 171

5.8 Chapter Summary . . . . . . . . . . . . . . . . . . . . . 174

\subsection{Introduction}

Chapters 3 and 4 provided details on the physical setup of the thermoreflectance technique and the details of the mathematical modeling used to analyze the data and deduce a parameter of interest. All too often many of the details that are required for accurate application of the thermoreflectance technique, either in the completion of the physical measurement or the application of the thermal analysis, are omitted from literature. The lack of these details prevents a consensus on the methods used among different scientific groups, and/or requires each group to take the time to create their own guidelines. To help eliminate these issues, this chapter focuses on describing the details and considerations in applying the thermal analysis described in Chapter 4 . We will begin by discussing the appropriate selection of modulation frequencies to optimize the signal-to-noise ratio in the data. That will be followed up with the development of convergence criteria to ensure accurate thermal modeling. Methods to speed up the model convergence and quantify the sensitivity in the thermal model are discussed. Finally, the quantification of noise in the measured data will be discussed and a method to identify statistical outliers in the fitted results presented.

\subsection{Proper Modulation to Eliminate Phase Drift}

In Chapter 3 we discussed the primary TTR/TDTR system as being modular between a high-rep (MIRA) or low-rep (RegA) based system. The choice between either configuration did not impact the development of the thermal model, see Chapter 4. However, the choice of the laser repetition rate will impact the application, both physically and analytically, of the TTR/TDTR analysis. One of the most immediate impacts of the choice of the laser repetition frequency, $f_{s}$, is the choice of the modulation frequency, $f_{o}$.

Typically for the low-rep (RegA) configuration, the modulation frequency is fixed at $f_{o}=125 \mathrm{kHz}$ assuming a nominal laser repetition frequency of $f_{s}=250 \mathrm{kHz}$. This one- 
shot-on/one-shot-off configuration leaves $8 \mu$ s between incident laser pulses, sufficient time for the system to return to thermal equilibrium before the arrival of the next pulse, see Figure 5.1. Because the system returns to thermal equilibrium between each pulse, there is little advantage to having multiple pulses per envelope, i.e. reducing $f_{o}$.

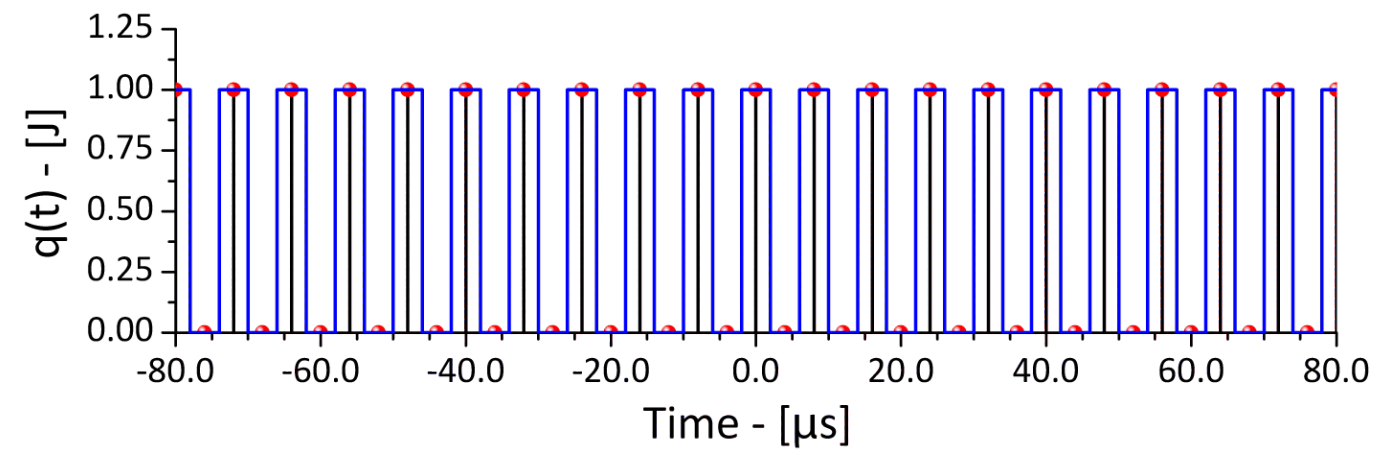

Figure 5.1: Simulated laser impulse-train, $f_{s}=250 \mathrm{kHz}$, modulated by a square waveform at $f_{o}=125$ $\mathrm{kHz}$.

For the high-rep (MIRA) configuration however, the choice of modulation frequency will impact the modeled results and the physical measurements made. In Figure 5.2, simulated $\operatorname{TDTR}\left(f_{s}=76 \mathrm{MHz}\right)$ data of $100 \mathrm{~nm}$ of $\mathrm{Al}$ on $\mathrm{Si}\left(h_{\mathrm{BD}}=215 \mathrm{MW} / \mathrm{m}^{2} \mathrm{~K}\right)$ for modulation rates of $f_{o}=38 \mathrm{MHz}$ and $f_{o}=3 . \overline{45} \mathrm{MHz}$ highlight the impact of the laser modulation frequency on the measured signal. Even though the simulated thermophysical properties are exactly the same between both data sets, the change in the laser modulation frequency impacts the decay of the surface temperature. This divergence between the two plots in Figure 5.2, occurs due to residual heating of the sample. Typical high-rep systems operate

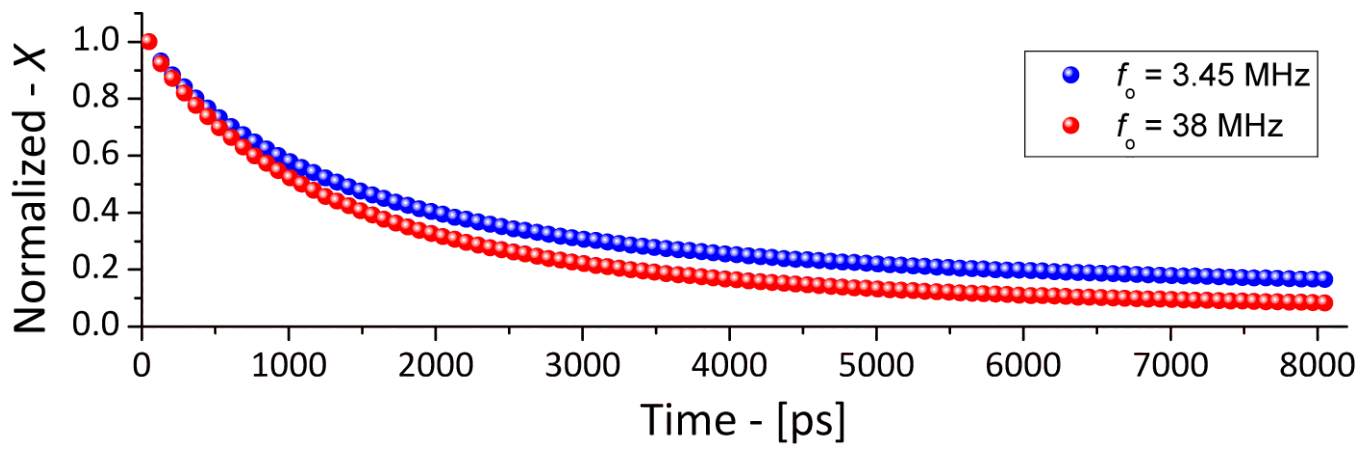

Figure 5.2: Simulated TDTR data of $100 \mathrm{~nm}$ of $\mathrm{Al}$ on $\mathrm{Si}\left(h_{\mathrm{BD}}=215 \mathrm{MW} / \mathrm{m}^{2} \mathrm{~K}\right)$, normalized at $50 \mathrm{ps}$, for modulation rates of $f_{o 1}=38 \mathrm{MHz}$ (red) and $f_{o 2}=3 . \overline{45} \mathrm{MHz}$ (blue), with $f_{s}=76 \mathrm{MHz}, D=0.5$, $M=3,500$, and $k=10,000$. 
with laser repetition frequencies on the order of $f_{s}=80 \mathrm{MHz}\left(f_{s}=76 \mathrm{MHz}\right.$ for the MIRA), leaving only on the order of $T_{s}=12.5 \mathrm{~ns}$ between each laser pulse. At this timescale, there is insufficient time between laser pulses for the system to return to thermal equilibrium before the arrival on the next pulse, leading to a residual heating effect [229, 231, 247]. Additionally, the modulation frequency also impacts the thermal penetration depth of the established thermal wave [248-251]. Current theories suggest that manipulation of the thermal penetration depth is directly related to the spectrum of phonon mean free paths sampled during the thermoreflectance measurement $[199,241,252,253]$. Therefore, for highrep (MIRA) based systems it is desirable to be able to select from a range of modulation frequencies, $f_{o}$, depending on the application and/or the measurement of interest. The choice of $f_{o}$ however is not arbitrary.

Physically, variations in the modulation frequency change the width of the "on" portion of the modulation envelope, given by $d$ in Figure 5.3. Note, the temporal width of the modulation envelope, $d$, can also be affected by changes to the duty cycle which is something that will be covered in more depth shortly. However, for now we will constrain our analysis to the square modulation waveform, i.e. $D=50 \%$. As an example, assuming a laser repetition frequency of $f_{s}=76 \mathrm{MHz}$, for a modulation frequency of $f_{o}=38 \mathrm{MHz}$ the temporal width of the modulation envelope is $d=13.6 \mathrm{~ns}$, allowing one laser pulse within the modulation envelope, see Figure 5.4a. If the modulation frequency is reduced to $f_{o}=3 . \overline{45} \mathrm{MHz}$, the modulation envelop width is $d=144.74$ ns, allowing 11 pulses per envelope, see Figure 5.4b.

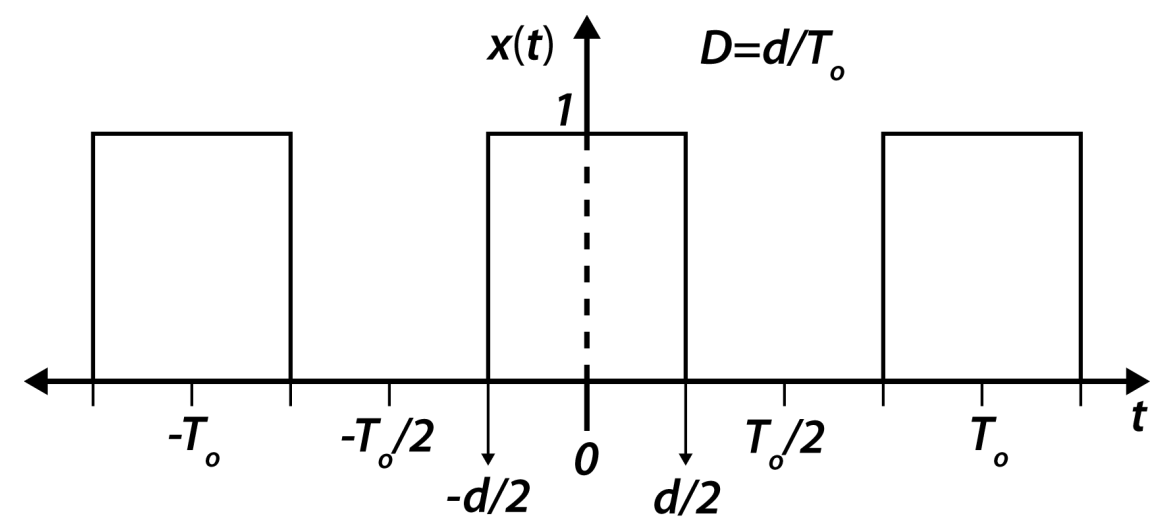

Figure 5.3: Generalization of the pulsed waveform, where $T_{o}$ is the modulation period, $D$ is the duty cycle, and $d$ is the width of the "on" portion of the modulation envelope. 


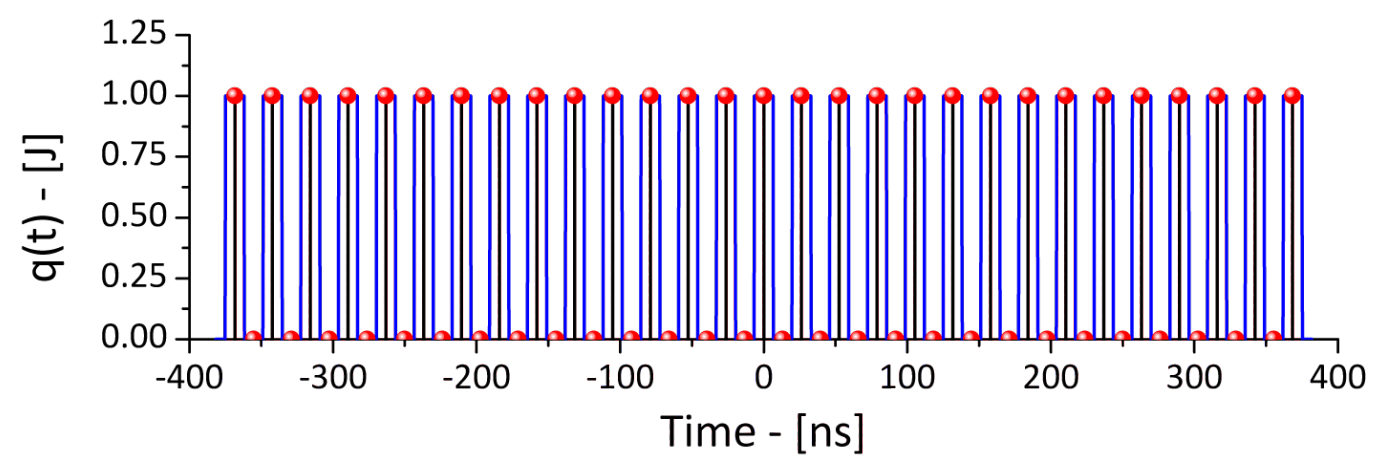

(a)

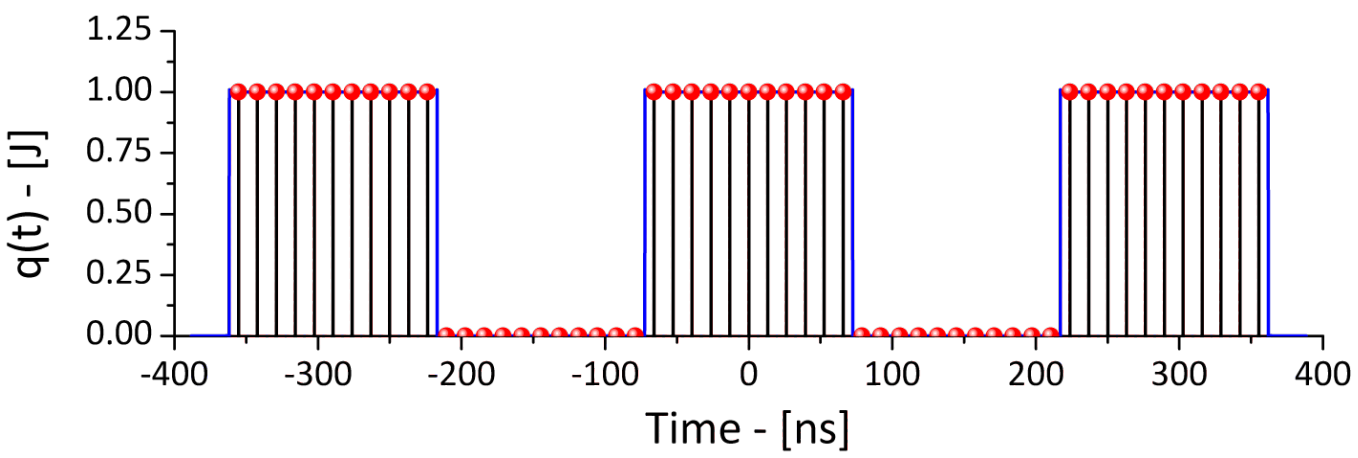

(b)

Figure 5.4: Simulated laser impulse-train, $f_{s}=76 \mathrm{MHz}$, modulated by a square waveform at (a) $f_{o}=38$ $\mathrm{MHz}, 1$ pulse per envelope, and (b) $f_{o}=3 . \overline{45} \mathrm{MHz}, 11$ pulses per envelope.

Because the modulation waveform is a continuous function, it is tempting to arbitrarily select modulation frequencies considering there to be an infinite spectrum to choose from. However, it is important to remember that we are modulating a discrete train of laser pulses. When setting the modulation frequency in the experiment via the frequency generator, it is easy enough for example to set either 37 or $38 \mathrm{MHz}^{1}$. However, there is no advantage to the choice of $f_{o}=37 \mathrm{MHz}$ over $f_{o}=38 \mathrm{MHz}$ because it will not change the number of laser pulses within the modulation envelope, and thereby does not influence the thermal signal, see Figure 5.5. In fact, there is a significant disadvantage to the choice of $f_{o}=37 \mathrm{MHz}$ as the modulation frequency.

At $f_{o}=38 \mathrm{MHz}$, the modulation frequency is an exact multiple of the laser repetition frequency, $f_{s}=76 \mathrm{MHz}$. Therefore at a $50 \%$ duty cycle, there is one laser pulse within the modulation envelope and one pulse removed from each modulation period. This con-

\footnotetext{
${ }^{1}$ Note: Typically, in a TDTR setup one would not choose such a high modulation frequency at all due to signal-to-noise issues, however the simulated data works well for the purposes of discussion
} 


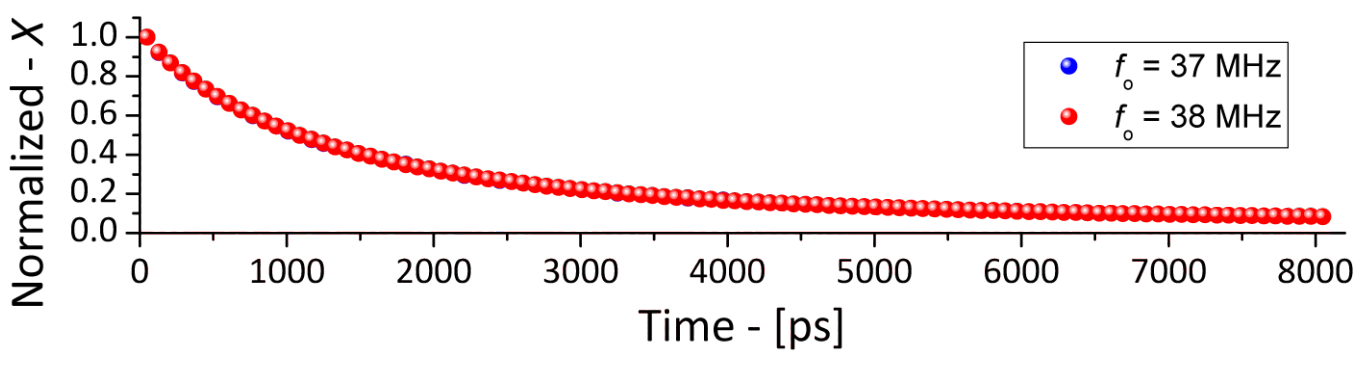

Figure 5.5: Simulated TDTR data of $100 \mathrm{~nm}$ of $\mathrm{Al}$ on $\mathrm{Si}\left(h_{\mathrm{BD}}=215 \mathrm{MW} / \mathrm{m}^{2} \mathrm{~K}\right)$, normalized at $50 \mathrm{ps}$, for modulation rates of $f_{o 1}=38 \mathrm{MHz}$ (red) and $f_{o 2}=37 \mathrm{MHz}$ (blue), with $f_{s}=76 \mathrm{MHz}, D=0.5, M=3,500$, and $k=10,000$.

figuration places the laser pulses directly in the center of the modulation window for each period, and this pattern is repeated infinitely in time, see Figure 5.4a. When the modulation frequency is not an integer multiple of the laser repetition frequency, the modulation envelope will outpace (or lag behind) the laser pulses in time, see Figure 5.6. While this does not appear to impact the modeled signals in Figure 5.5, it does have a direct impact on the physically measured signals.

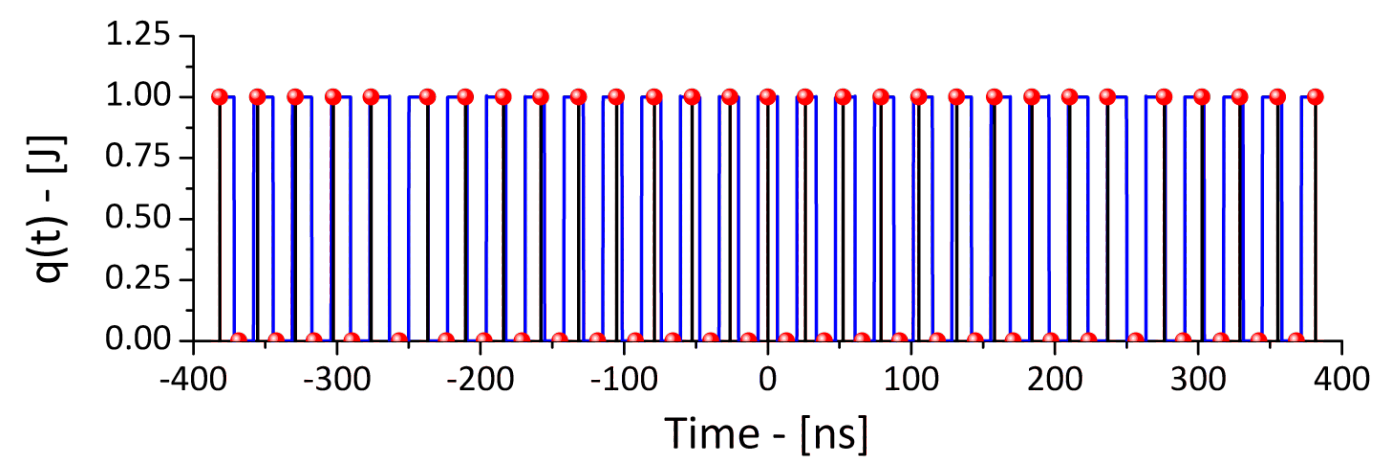

Figure 5.6: Simulated laser impulse-train, $f_{s}=76 \mathrm{MHz}$, modulated by a square waveform at $f_{o}=37$ $\mathrm{MHz}$.

Because the lock-in amplifier in the TTR/TDTR experiment, in addition to the signal magnitude, measures the phase difference between the reference signal (modulation frequency) and the input signal (thermoreflectance voltage at the modulated laser frequency) a dynamic time variation between the two signals is manifested as a time varying phase shift. This varying phase shift appears as noise in the $X$ and $Y$ channels, and impacts the signal magnitude as well due to the non-constant signal frequency. The magnitude of the added noise will be dependent on the relation between the laser and modulation frequencies. 
Figure 5.7 shows the results of the measurement of the signal magnitude and phase as a function of the modulation frequency, which is supplied by either a function generator (SRS DS345) or a phase-locked circuit ${ }^{2}$, see Norris et al. [254], for a sample of $100 \mathrm{~nm}$ of $\mathrm{Al}$ on a Si substrate. For the low-rep (RegA) TTR system, the laser repetition frequency is nominally set at $f_{s}=250 \mathrm{kHz}$. However, the measured laser frequency was found to be approximately $f_{s}=247.400 \mathrm{kHz}$, which implies an ideal modulation frequency of $f_{o}=123.700 \mathrm{kHz}$. As seen in Figure 5.7, the thermoreflectance signal is strongly detected by the lock-in amplifier at frequencies very close to the anticipated modulation frequency when driven by the function generator. However, there is a large increase in the noise in both channels of the lock-in amplifier signal (magnitude and phase) as $f$ approaches $f_{o}$. The design of the phase-locked circuit provides an exact $f_{o}=f_{s} / 2$ modulation that is clocked off the laser repetition frequency and is therefore directly dependent on $f_{s}$, and independent of laser frequency drift or jitter. The lock-in amplifier signal when modulated with the phase-locked circuit, see the red data points in Figure 5.7, shows a large noise (standard deviation) reduction in the data. This corresponds to a nearly four times increase in the signal-to-noise ratio in the TTR response compared to the best case scenario using the function generator.

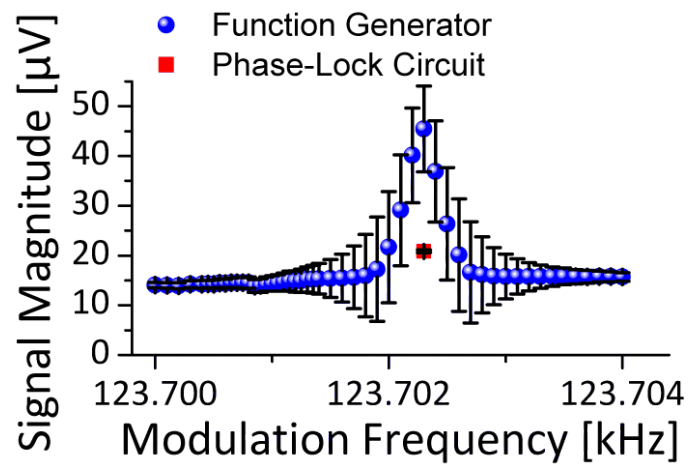

(a)

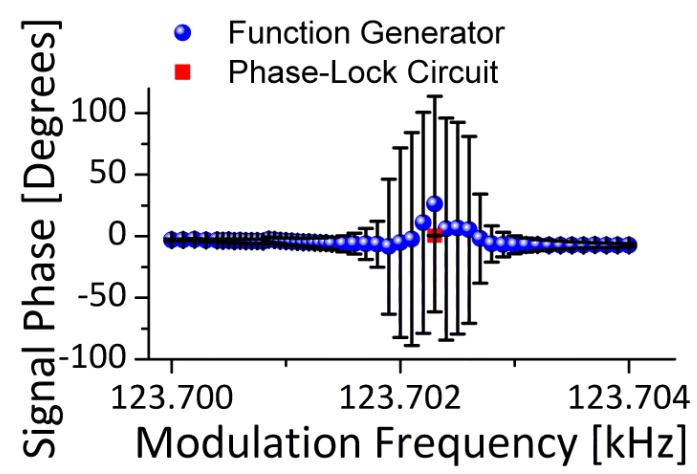

(b)

Figure 5.7: Comparison of the lock-in amplifier output for a TTR signal, with the pump laser modulated via a traditional function generator (blue) and a phase-locked circuit (red), showing (a) the signal magnitude and (b) the signal phase. The error bars shown are \pm one standard deviation from 300 measurements. The signal to noise ratio of the magnitude with the phase-locked circuit is nearly four times greater than when using the traditional function generator.

\footnotetext{
${ }^{2}$ More details regarding this will be presented in a future dissertation by Mr. MacKenzie Redding from our lab.
} 
The impact of the phase-locked circuit is most dramatic when considering one laser pulse per modulation envelope. For the high-rep (MIRA) system, as the number of laser pulses within the modulation envelope increases, the effects of non-ideal modulation frequencies are diminished due to the averaging across multiple pulses ${ }^{3}$. The challenge in implementing a phase-locked circuit as in Norris et al. [254] for the high-rep (MIRA) system is having appropriate circuit component bandwidth to handle the $\mathrm{MHz}$ switching speed necessary. Therefore, it may still be necessary to determine appropriate modulation frequencies based on the laser repetition frequency for function generator operation.

To prevent the noise and errors associated with arbitrary modulation of the laser impulse-train, we seek to only modulate at frequencies which are integer multiples of the fundamental laser frequency, thereby controlling the number of laser pulses within the modulation envelope. The number of pulses per "on" portion of the modulation envelope, $N_{P}$ is given by:

$$
N_{p}=D \times \frac{f_{s}}{f_{o}}
$$

where $D$ is the duty cycle of the modulation waveform, $f_{s}$ is the laser repetition frequency, and $f_{o}$ is the modulation frequency. The allowed pulses per envelope as a function of modulation frequency at a $50 \%$ duty cycle from $f_{s} / 2$ to $0.1 \mathrm{MHz}$ for the high-rep (MIRA) system is provided in Figure 5.8. As we move forward in discussing the criteria and methods for accurate application of the TTR/TDTR thermal analysis, we will use modulation frequencies based on Equation 5.1, particularly for the high-rep (MIRA) system.

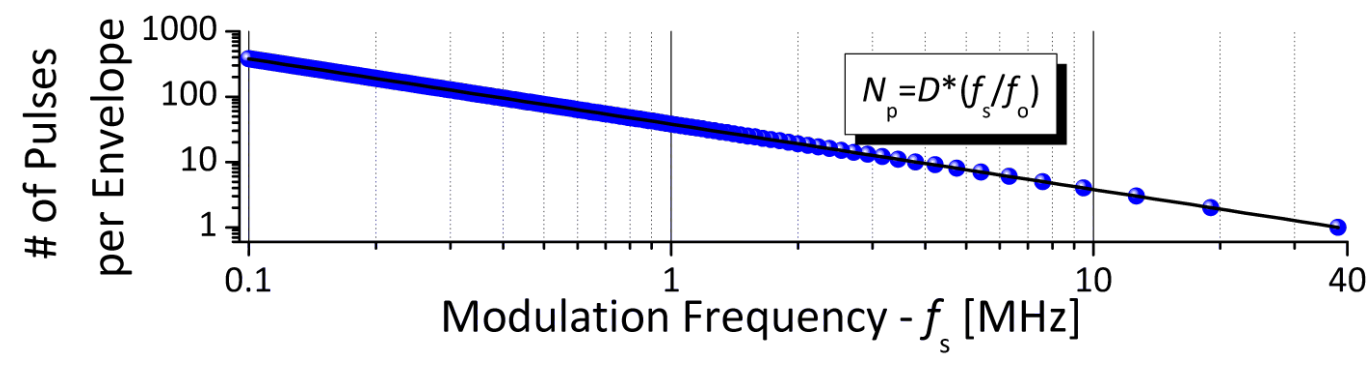

Figure 5.8: The number of laser pulses per "on" portion of the modulation envelope as a function of modulation frequency, $f_{o}$, assuming a $50 \%$ duty cycle and a laser repetition frequency of $f_{s}=76 \mathrm{MHz}$.

\footnotetext{
${ }^{3}$ Typically modulations frequencies in high-rep (MIRA) systems, range on the order of $19 \mathrm{MHz}$ to 0.1 $\mathrm{MHz}$, which for a $50 \%$ duty cycles, places in the range of 4-760 pules per envelope.
} 


\subsection{Convergence Criteria in Modeling the Lock-in Amplifier Response During TTR/TDTR Experiments}

In Chapter 4 we derived the lock-in amplifier's output in the $X$ and $Y$ channels to the sample's temperature response, monitored by the probe impulse-train, to the modulated heat input of the pump, see Equations $4.37 \mathrm{a}$ and $4.37 \mathrm{~b}$. When implementing these equations during a thermal analysis, we are left to handle two double bounded sets of infinite summations in $M$ and $k$ as a result of using the LTI theory to describe the laser system, and assuming a pulsed waveform for the modulation of the pump. In this particular case, the summation over $k$ is due to the higher harmonic components in the infinite Fourier series necessary to describe a pulsed waveform, see Equation 4.11a and/or Appendix C.1, and $M$ arises in the frequency domain representation of the infinite impulse-train of pump pulses, see Equation 4.6b. For the thermal analysis, Equations $4.37 \mathrm{a}$ and $4.37 \mathrm{~b}$ will be solved numerically and therefore the calculations will use non-infinite bounds on $M$ and $k$. To produce accurate modeling of the TTR/TDTR signal we must establish convergence criteria to ensure that either summation is sufficiently large as to not influence the final result. The cost of increasing $M$ and/or $k$ will be increased computational time necessary to model the system. Therefore, the establishment of convergence criteria will not only ensure accurate, but also efficient modeling. To begin, we will consider the simplified, although the most commonly implemented case of a sinusoidal waveform for the modulation of the pump beam to focus on the convergence criteria in $M$, independent of $k$. We will then consider the square modulation waveform, i.e. the pulsed waveform with at $50 \%$ duty cycle and the convergence criteria in $k$, and finally consider how the duty cycle impacts the convergence criteria in $k$.

\subsubsection{Impacts of Non-convergence in $M$}

The inherent manifestation of an insufficient summation over $M$ is shown in Figure 5.9. For low values of $M$ there are significant oscillations in the signal response, with the frequency of these oscillations increasing with increasing $M$. This oscillation in the data as a function of the summation $M$, is a Gibbs phenomena. 


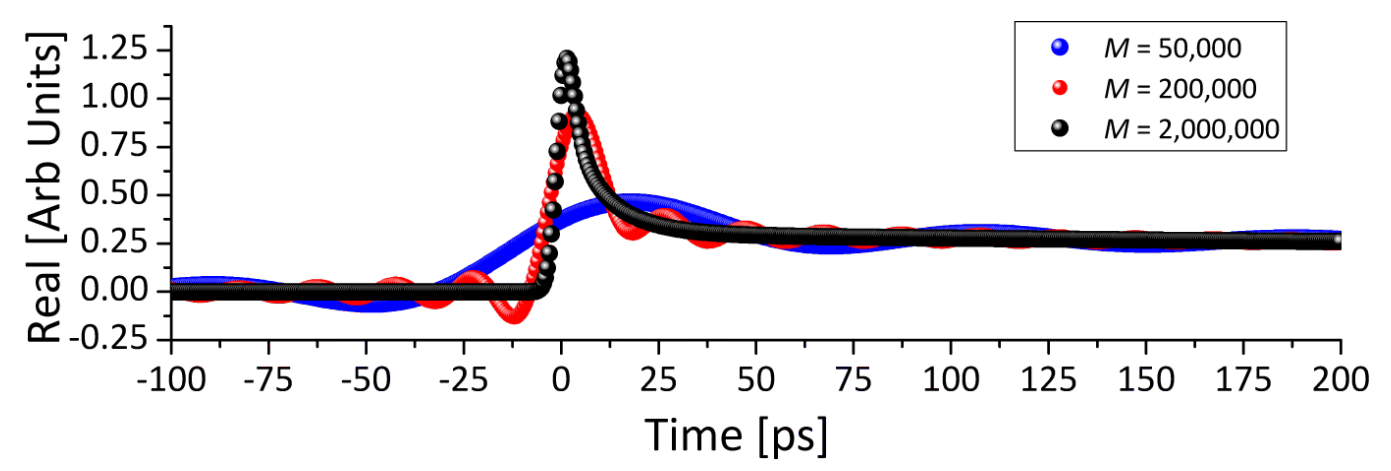

Figure 5.9: Simulated data for a $100 \mathrm{~nm} \mathrm{Al} / \mathrm{Si}$ system $\left(h_{\mathrm{BD}}=200 \mathrm{MW} / \mathrm{m}^{2} \mathrm{~K}\right)$ for $k=\{-1,1\}, f_{s}=250$ $\mathrm{kHz}, f_{o}=125 \mathrm{kHz}$, a $50 \%$ duty cycle of the modulation waveform, and $M=50,000,200,000$, and $2,000,000$.

By the nature of how the laser system was modeled, there is a jump discontinuity located at $t_{o}$, the time the pump and probe impulses arrive a the same instant in time ${ }^{4}$. This is due to the assumption that the laser pulses are perfect impulses, i.e. there is no temporal width to the pump pulses. Assuming this, and neglecting any electron thermalization time during pulse deposition, there is an instantaneous rise in temperature when the pump pulse arrives leading to an instantaneous jump in the modeled signal.

There are several important implications of the presence of the Gibbs phenomena at $t_{o}$. If we begin by considering arbitrary peaks in the oscillating solution, we find that as $M$ increases each peak moves closer to the discontinuity at $t_{o}$. As an example, if we consider the second peak (after $t_{o}$ ) of the data shown in Figure 5.9, as $M$ increases from $M=50,000$ to $M=200,000$, the temporal location of the peak is shifted from approximately 108 ps to 26.4 ps. As $M$ is increased, the frequency of the ringing in the data is increased, and we can define the time between peaks in the oscillations as $T_{\text {peak }}$, see Figure 5.10. It is important to note however, that the amplitude of the peaks is independent of the size of $M$. The overshoot of the primary peak is $9 \%$ of the actual height, with each subsequent oscillation reducing in amplitude [226]. Increasing $M$ does not decrease the amplitude of these oscillations, but rather compresses the high amplitude oscillations towards the discontinuity.

Because the primary thermal analysis will be focused on diffusive processes, we concentrate on the region of data where it is assumed that both the electron and phonon

\footnotetext{
${ }^{4}$ The arrival of the pump and probe laser pulses at the same instant in time is typically referred to as $t_{o}$ and corresponds to $t=0$. Physically during the experiment this is taken as the peak in the signal which is assumed to be greatest when the two pulses overlap temporally.
} 


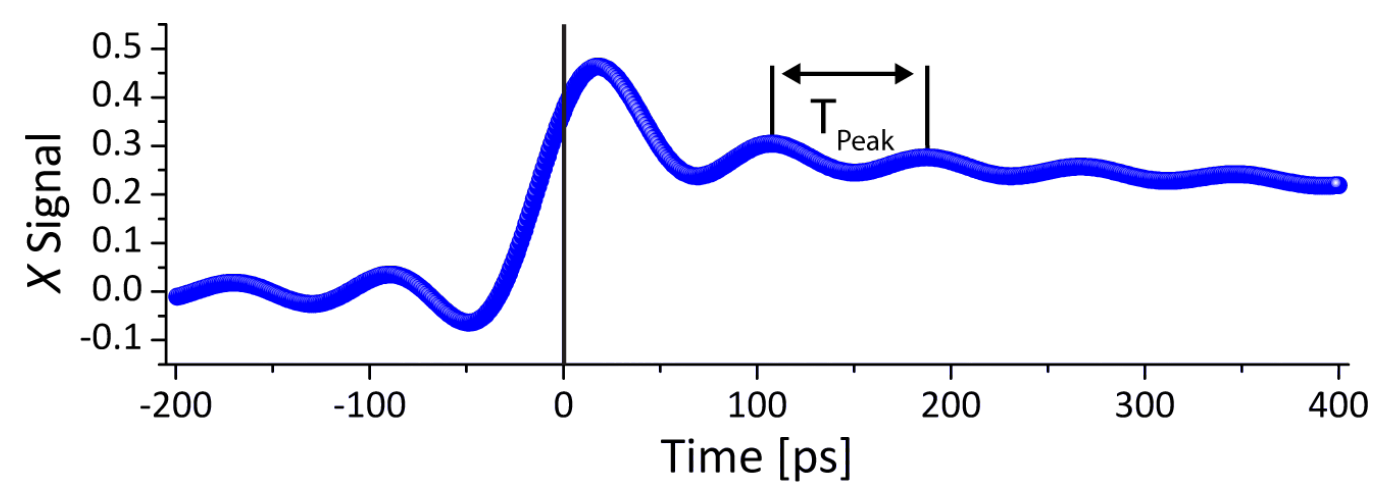

Figure 5.10: Schematic of modeled data highlighting the oscillations in the modeled data for a low summation in $M$, and defining the time period between peaks, $T_{\text {peak }}$.

sub-systems have thermalized with each other after ultrashort laser heating, which is typically taken to be after approximately $t=100$ ps. Therefore, we can envision a realistic convergence criteria to be a sufficient $M$ such that the primary oscillations in the data are "pushed" prior to 100 ps, with the remaining solution after 100 ps having sufficiently low oscillations as to not impact the deduction of the parameter of interest.

Ideally, we will establish a convergence criteria that is dependent on $M$ alone, independent of the thermophysical properties of the system. To test this theory, first the time between peaks in the oscillations of a modeled system of $100 \mathrm{~nm}$ of $\mathrm{Al}$ on $\mathrm{Si}\left(h_{\mathrm{BD}}=215\right.$ $\mathrm{MW} / \mathrm{m}^{2} \mathrm{~K}$ ) was determined to be approximately $T_{\text {peak }}=79.85 \pm 0.53 \mathrm{ps}$ for $M=50,000$. Then to check for independence, each thermophysical property of the system was varied using a one-at-a-time method and $T_{\text {peak }}$ was calculated for each permutation, see Figure

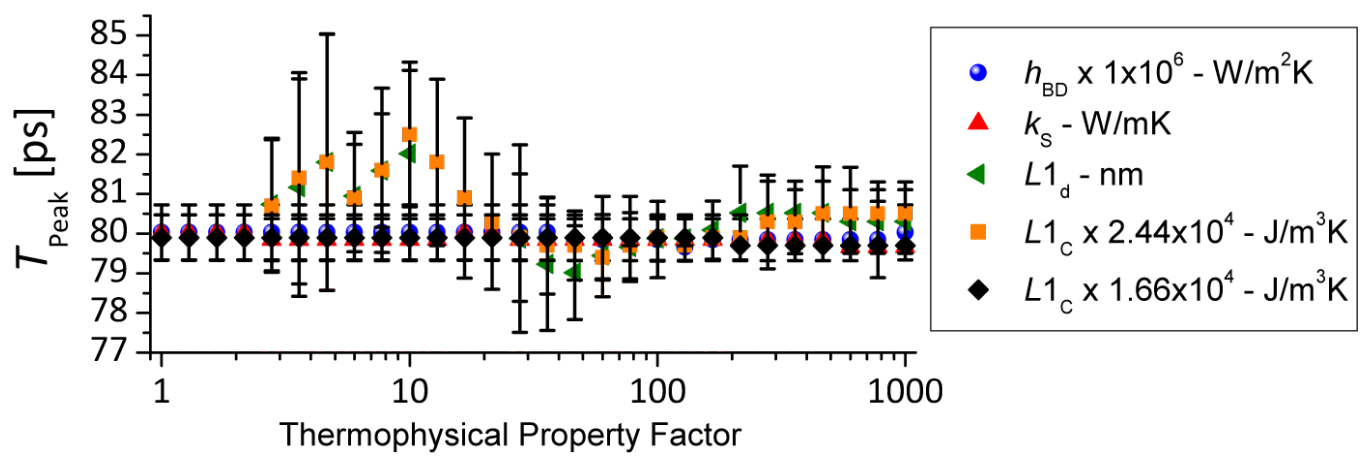

Figure 5.11: $T_{\text {peak }}$ of the Gibbs oscillations in modeled TTR/TDTR data as a function of thermophysical property perturbation for a modeled system of $100 \mathrm{~nm}$ of $\mathrm{Al} / \mathrm{Si}\left(h_{\mathrm{BD}}=215 \mathrm{MW} / \mathrm{m}^{2} \mathrm{~K}\right)$ with $M=50,000$. Each assumed thermophysical value was perturbed one-at-a-time, with the error bars representing plus/minus one standard deviation of the data collected over the first 30 peaks. 
5.11. The average $T_{\text {peak }}$ was collected over the first 30 peaks in the data after $t_{o}$, with the error bars representing plus/minus one standard deviation in the data. While there are some fluctuations in the data, overall the error bars in the results are overlapping, and therefore the results are statistically equivalent, supporting the independence of $T_{\text {peak }}$ on thermophysical properties. Therefore, moving forward we can consider the convergence criteria of the TTR/TDTR model to be a function of $M$ only, independent of the system being modeled. While the data in this dissertation was collected using a low-rep (RegA) system, we will consider the convergence criteria for both the low-rep (RegA) and high-rep (MIRA) systems in this analysis.

\subsubsection{Impact of Series Convergence - Sinusoidal Modulation Waveform}

As mentioned, we will begin by assuming a sinusoidal waveform for the pump modulation, implying that $k=\{-1,1\}$, i.e. the first harmonic components of the square (pulsed) waveform. Applying this assumption, Equations $4.37 \mathrm{a}$ and $4.37 \mathrm{~b}$, reduce to the form commonly reported in literature $[230,231,245,251]$, see Appendix C.3. This will allow the focus for the time being to be on the convergence criteria in $M$, independent on the summation in $k$. We will begin with convergence in the low-rep (RegA) system, to be followed up with the high-rep (MIRA) system. In both cases we will begin with a qualitative description of convergence by tracking a point in the model as a function of $M$, and then establish more quantitative criteria by fitting a converged model of known parameters with increasing bounds on $M$.

\section{Convergence in $M$ - Low-Rep $(\operatorname{Reg} A)$}

In order to estimate the convergence criteria in $M$ for the low-rep (RegA) system, the $X$ component of a modeled system, see Equation 4.39a, is tracked as a function of the size of the summation bounds, $M$, at a given time index ${ }^{5}$. In this analysis, 100 ps was chosen as the time point of interest as it typically represents the lower bound in the fitting time where it is assumed that the electron and phonon sub-systems are in thermal equilibrium

\footnotetext{
${ }^{5}$ Note: $M$ in Equations $4.37 \mathrm{a}$ and $4.37 \mathrm{~b}$ is given from $-\infty$ to $\infty$, therefore when $M$ is specified it is understand that in the analysis the summation will range from $-M: M$.
} 


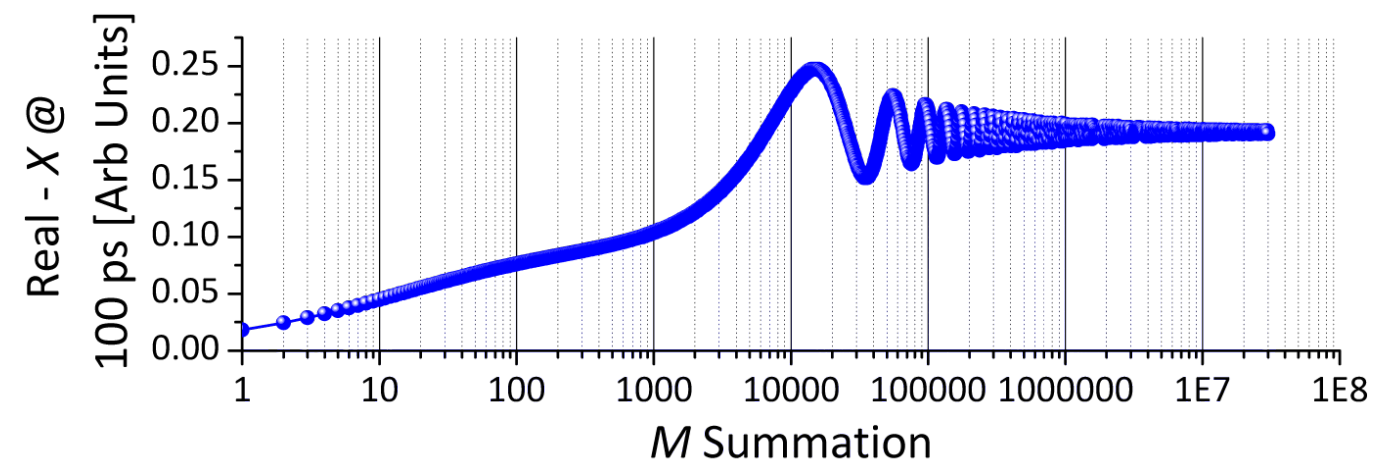

Figure 5.12: Real signal $X$ at $100 \mathrm{ps}$ as a function of $M$ summation for a modeled system of $100 \mathrm{~nm}$ $\mathrm{Al} /$ Glass $\left(h_{\mathrm{BD}}=30 \mathrm{MW} / \mathrm{m}^{2} \mathrm{~K}\right)$, with $k=\{-1,1\}, f_{s}=250 \mathrm{kHz}, f_{o}=125 \mathrm{kHz}$, and a $50 \%$ duty cycle of the modulation waveform.

and diffusion processes dominate thermal transport. The $X$ component of the model system at $100 \mathrm{ps}$ as a function of $M$ is shown in Figure 5.12. From a visual inspection of the graph, it appears that the solution at 100 ps does not converge until on the order of $M \approx 30 \times 10^{6}$. With the time to run a single simulation at this summation level taking over an hour, and a typical complete fit requiring 6-20 iterations ${ }^{6}$, fitting data for the low-rep (RegA) system becomes very computationally expensive.

In order to speed up convergence, Cahill proposed multiplying the terms in Equation 4.37 a by a Gaussian factor, given by [230]:

$$
\exp \left(\frac{-\pi f}{f_{\max }}\right)^{2}=\exp \left(\frac{-\pi \omega}{\omega_{\max }}\right)^{2}
$$

where $f$ is the frequency and $f_{\max }$ is a maximum frequency that will be considered in the thermal analysis. Cahill reports that they find accurate modeling with $f_{\max }=10 / t_{\min }$, where $t_{\min }$ is the minimum delay time that will be considered for a fit. For the analyses in this dissertation $t_{\min }$ is taken as $50 \mathrm{ps}$, which corresponds to a maximum frequency of $f_{\max }=200 \mathrm{GHz}$. The purpose of the Gaussian factor is to focus the analysis on the lower frequency terms and eliminate or greatly reduce the dependence on the higher frequency terms. The form of the Gaussian term is shown in Figure 5.13, for the maximum cutoff

\footnotetext{
${ }^{6}$ It is important to note that the number of iterations required for fitting is based on a number of factors, including the fitting routine used, quality of the initial guesses, sensitivity to the parameter of interest, and the tolerance of the stopping criteria. The specified range of iterations is based on observational evidence of the methods used in this dissertation, and does not represent a general rule.
} 
frequency of $f_{\max }=200 \mathrm{GHz}$. The impact of the Gaussian factor is evident by comparing Figure 5.12 with the same analysis using the Gaussian factor, shown in Figure 5.14.

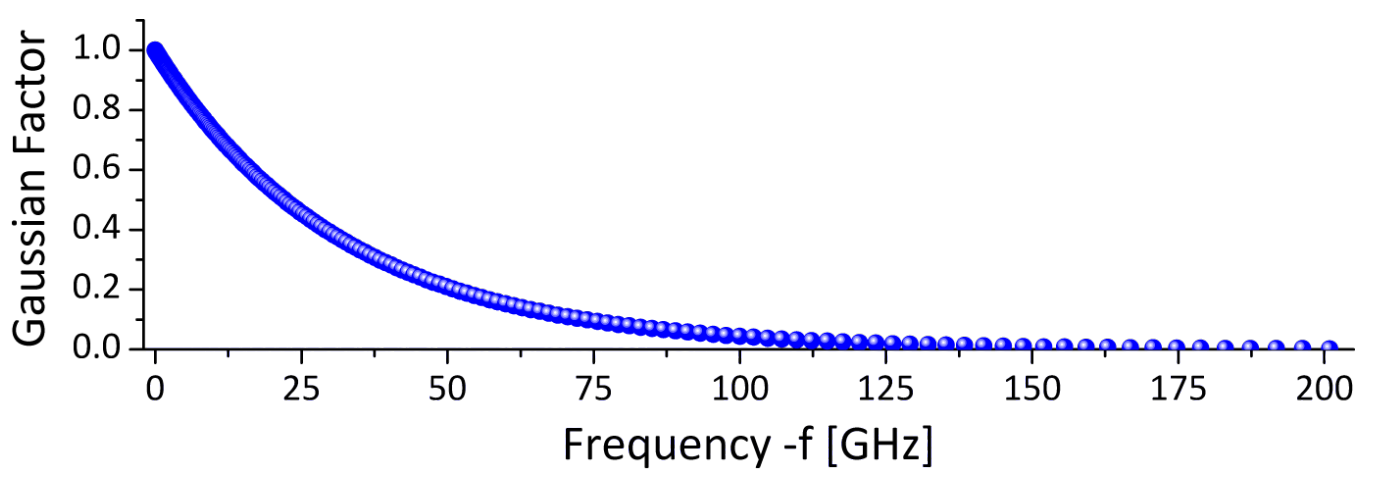

Figure 5.13: Form of the Gaussian factor, from Equation 5.2, as a function of frequency, $f$, for $f_{\max }=200$ $\mathrm{GHz}$.

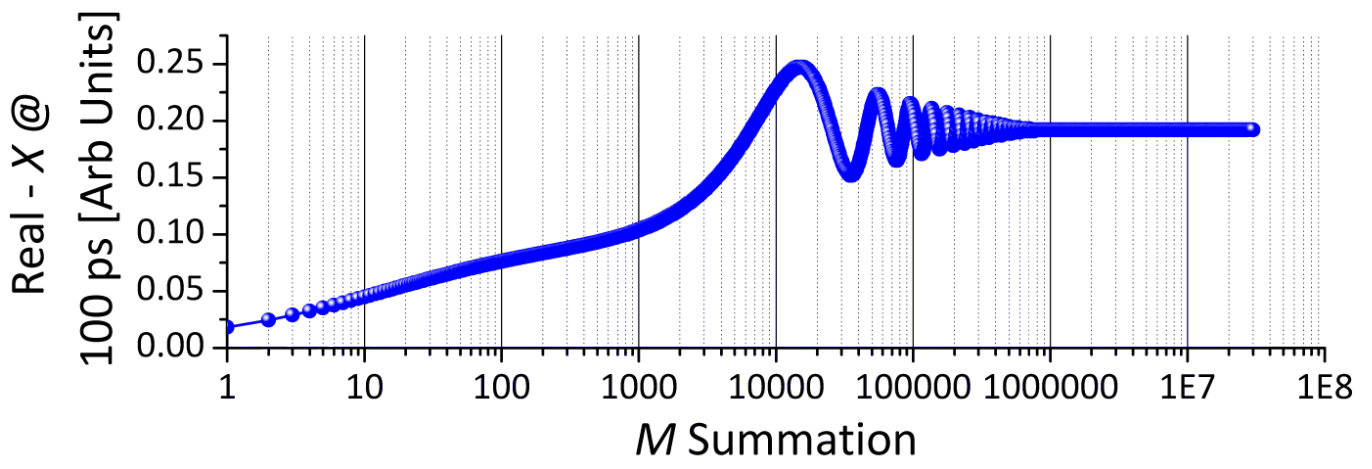

Figure 5.14: Real signal $X$ at $100 \mathrm{ps}$ as a function of $M$ summation for a modeled system of $100 \mathrm{~nm}$ $\mathrm{Al} /$ Glass $\left(h_{\mathrm{BD}}=30 \mathrm{MW} / \mathrm{m}^{2} \mathrm{~K}\right)$, with $k=\{-1,1\}, f_{s}=250 \mathrm{kHz}, f_{o}=125 \mathrm{kHz}$, and a $50 \%$ duty cycle of the modulation waveform. With the inclusion of the Gaussian factor, see Equation 5.2, to the lock-in analysis, see Equations $4.37 \mathrm{a}$ and $4.37 \mathrm{~b}$.

To ensure the use of the Gaussian factor does not alter the modeled results, a comparison of data generated with and without the factor for various $M$ summation levels is shown in Figure 5.15. The results in Figure 5.15 show no appreciable difference between the models at various $M$ summation levels, with and without the Gaussian factor. Comparing Figure 5.12 to 5.14 we see that the addition of the Gaussian factor decreased the $M$ summation required for visual convergence from over $M=30 \times 10^{6}$ to on the order of $M \approx 8.5 \times 10^{5}$. This in turn decreases the computational time required to generate the modeled data from 


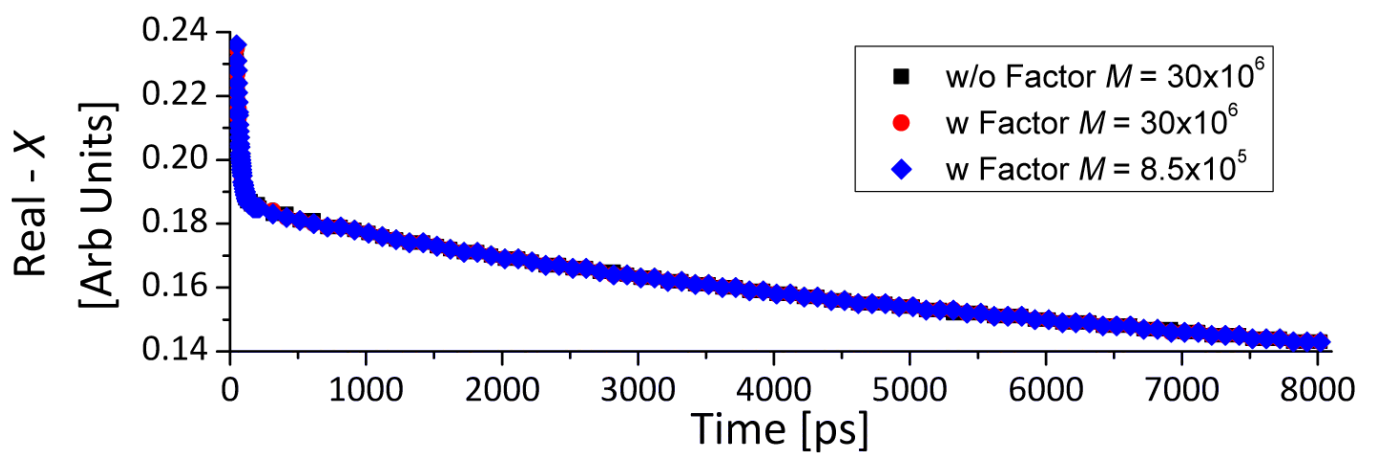

Figure 5.15: Real signal $X$ of modeled data for a system of $100 \mathrm{~nm} \mathrm{Al} / \mathrm{Glass}\left(h_{\mathrm{BD}}=30 \mathrm{MW} / \mathrm{m}^{2} \mathrm{~K}\right)$, with $k=\{-1,1\}, f_{s}=250 \mathrm{kHz}, f_{o}=125 \mathrm{kHz}$, and a $50 \%$ duty cycle of the modulation waveform for various levels of $M$ summation, with and without the Gaussian factor.

over an hour to under 3 minutes $^{7}$. The value of $X$ at $100 \mathrm{ps}$ for the $M=30 \times 10^{6}$ and $M=8.5 \times 10^{5}$ cases differs by less than $0.07 \%$. While this methodology provided a good method for visually determining the criteria for convergence as a function of the summation $M$, a more quantitative convergence criteria is desirable.

Using the general sense of the visual convergence criteria in $M$ from the analysis of the time index as a basis, see Figure 5.14, a more quantitative criteria can be established. This was accomplished by first modeling a TTR data set, $100 \mathrm{~nm}$ of $\mathrm{Al} / \mathrm{Si}\left(h_{\mathrm{BD}}=215\right.$ $\mathrm{MW} / \mathrm{m}^{2} \mathrm{~K}$ ), with a level of summation $M$ in which we could assume we would have appropriate convergence, from Figure 5.14, which in this case was taken as $M=1,000,000$. That data was then fit to deduce $h_{\mathrm{BD}}$ using the standard TTR/TDTR fitting routine, as the value of $M$ was iterated. This provided a known value of $h_{\mathrm{BD}}, h_{\mathrm{BD}}=215 \mathrm{MW} / \mathrm{m}^{2} \mathrm{~K}$, with which a numerical convergence criteria in $M$ can be established. The results of this process are shown in Figure 5.16 with the blue circles representing the fitted results and the horizontal red line indicating the known value. Again from visual inspection, we see that convergence occurs when $M$ is on the order of 300,000 .

To extend this to a quantitative analysis, the percent difference between the known $h_{\mathrm{BD}}$ value, $h_{\mathrm{BD}}=215 \mathrm{MW} / \mathrm{m}^{2} \mathrm{~K}$, and the fitted value at a given $M$ was calculated, the results of which are shown in Figure 5.17. In Figure 5.17, the red line represents a log fit of the data points of the form $y=a-b \ln (x+c)$, and the black lines represent the $95 \%$ confidence

\footnotetext{
${ }^{7}$ Any discussion of computational time will be dependent on a number of factors, including the computer used and the number of data points in the model. For the majority of calculations made the programs will be run in LabVIEW ${ }^{\mathrm{TM}} 2012$ on an Intel ${ }^{\circledR}$ Core $^{\mathrm{TM}} 2$ quad core CPU running at $2.40 \mathrm{GHz}$ with 4 GB of RAM.
} 


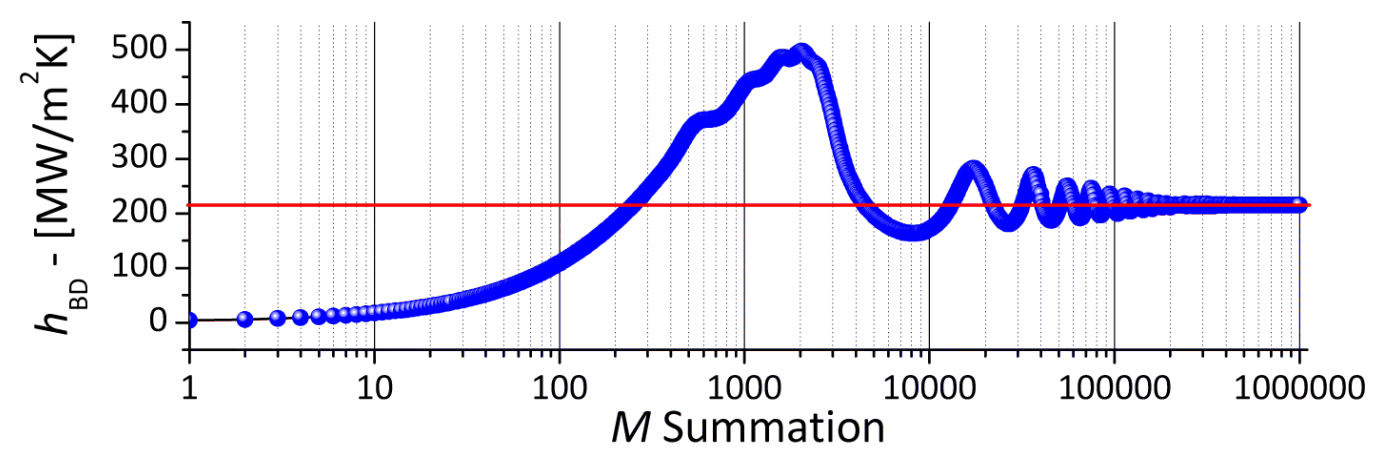

Figure 5.16: Fit of $h_{\mathrm{BD}}$ for a modeled system of $100 \mathrm{~nm}$ of $\mathrm{Al} / \mathrm{Si}\left(h_{\mathrm{BD}}=215 \mathrm{MW} / \mathrm{m}^{2} \mathrm{~K}\right)$, with $k=$ $\{-1,1\}, f_{s}=250 \mathrm{kHz}, f_{o}=125 \mathrm{kHz}$, a $50 \%$ duty cycle of the modulation waveform, and generated with $M=1,000,000$, with increasing values of $M$ during fitting. Note: the red line indicated the true $h_{\mathrm{BD}}$ value.

interval of the fit. From this formulation, the basic convergence levels are summarized in Table 5.1, along with the time required to model the data at the given summation level. It is important to note, that the estimated time to generate the thermoreflectance model in Table 5.1, is for a single iteration of the thermal model, while fitting to deduce a thermophysical parameter from measured data takes on the order of 6-20 iterations.

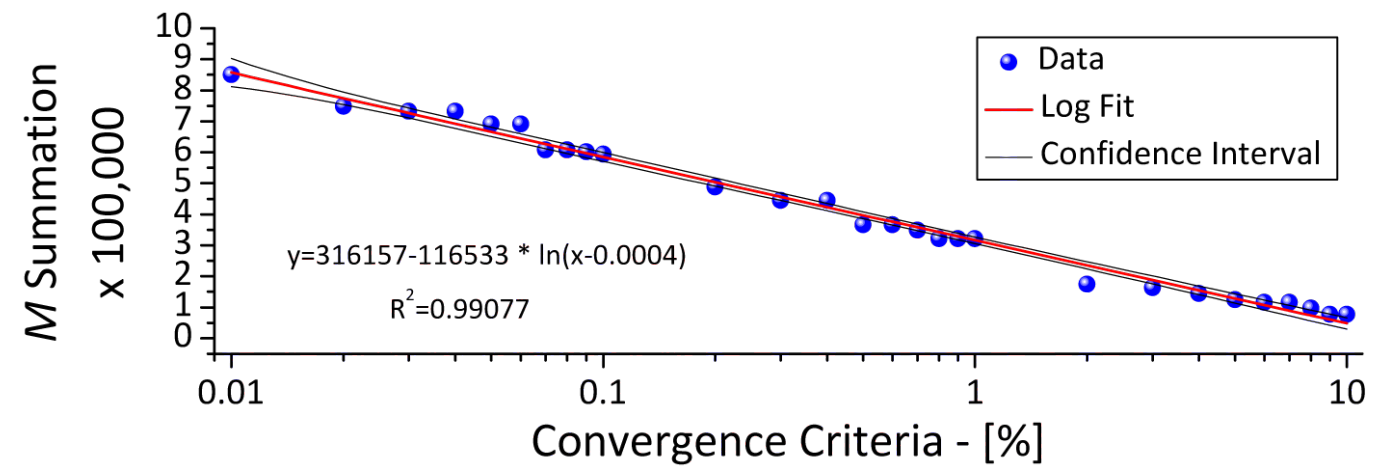

Figure 5.17: Number of summations in $M$ required for fit convergence to the known value of $h_{\mathrm{BD}}$, for a modeled system of $100 \mathrm{~nm}$ of $\mathrm{Al} / \mathrm{Si}\left(h_{\mathrm{BD}}=215 \mathrm{MW} / \mathrm{m}^{2} \mathrm{~K}\right)$, with $k=\{-1,1\}, f_{s}=250 \mathrm{kHz}, f_{o}=125 \mathrm{kHz}$, a $50 \%$ duty cycle of the modulation waveform, and generated with $M=1,000,000$. The red line represents a logarithmic fit of the data.

\section{Convergence in $M$ - High-Rep (MIRA)}

We can use a similar formulation as was done for the low-rep (RegA) system to find the convergence criteria in $M$ for the high-rep (MIRA) system. The major difference in the analysis will be that we will need to consider if, and to what degree, the modulation frequency will impact the conditions of the convergence criteria in $M$. To begin, we will 
Table 5.1: Estimation of the summation in $M$ required to reach a specified convergence level for the low-rep (RegA) system, based on the log fit of the data in Figure 5.17. Additionally the time required to generate a 320 point data set, at the summation level is provided for the TTR/TDTR program run in LabVIEW ${ }^{\mathrm{TM}} 2012$ on an Intel ${ }^{\circledR}$ Core $^{\mathrm{TM}} 2$ quad core CPU running at $2.40 \mathrm{GHz}$ with $4 \mathrm{~GB}$ of RAM.

\begin{tabular}{ccc}
\hline $\begin{array}{c}\text { Convergence } \\
\text { Level }\end{array}$ & $\begin{array}{c}\boldsymbol{M} \text { Summation } \\
\text { Required }\end{array}$ & $\begin{array}{c}\text { Time to } \\
\text { Generate [min] }\end{array}$ \\
\hline $10 \%$ & 47,800 & 0.139 \\
$1 \%$ & 316,000 & 0.919 \\
$0.10 \%$ & 585,000 & 1.701 \\
$0.01 \%$ & 856,000 & 2.489 \\
\hline
\end{tabular}

consider the $X$ component of a TDTR signal sinusoidally modulated at $1 \mathrm{MHz}$ for a pump to probe delay time of $100 \mathrm{ps}$, as a function of the summation bound $M$ with and without the Gaussian factor, shown in Figures 5.18 and 5.19. We can see that without the Gaussian factor the $X$ signal at 100 ps oscillates in $M$ and appears to converge visually for $M \approx$ 100,000. The introduction of the Gaussian factor, see Equation 5.13, reduces the number of $M$ necessary for visual convergence to around $M \approx 3,000$. The value of $X$ at $100 \mathrm{ps}$ for the $M=1 \times 10^{6}$ and $M=3 \times 10^{3}$ cases differs by less than $0.006 \%$, using the Gaussian factor.

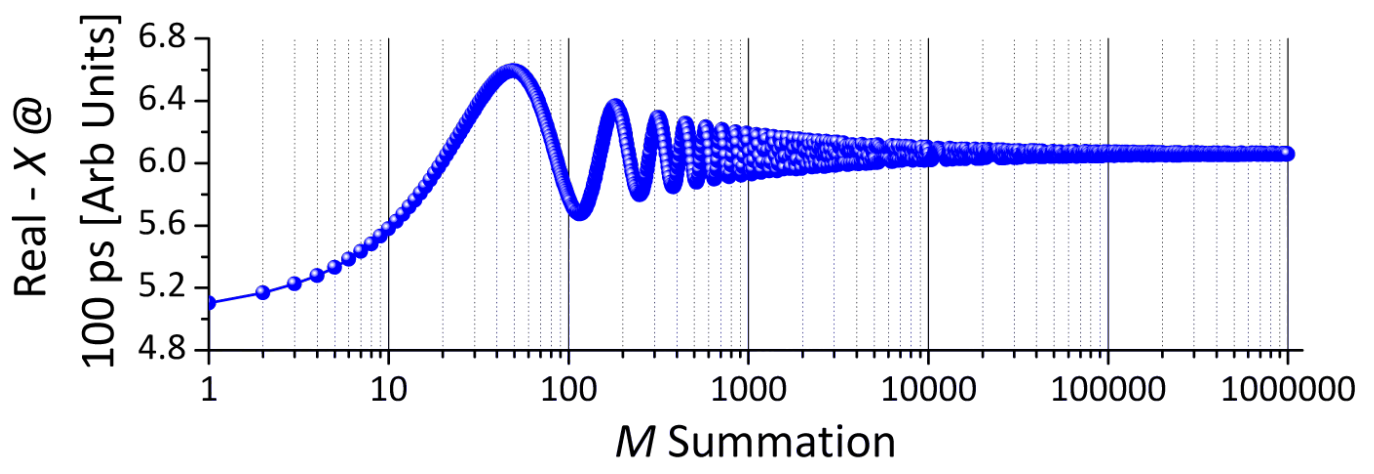

Figure 5.18: Real signal $X$ at $100 \mathrm{ps}$ as a function of $M$ summation for a modeled system of $100 \mathrm{~nm}$ $\mathrm{Al} /$ Glass $\left(h_{\mathrm{BD}}=30 \mathrm{MW} / \mathrm{m}^{2} \mathrm{~K}\right)$, with $k=\{-1,1\}, f_{s}=76 \mathrm{MHz}, f_{o}=1 \mathrm{MHz}$, and a $50 \%$ duty cycle of the modulation waveform.

In the same manner as was done with the low-rep (RegA) system, we can also compare the convergence criteria based on fitting a modeled system under varying summations in $M$ to establish quantitative guidelines. However, as mentioned for the high-rep (MIRA) system we have a greater choice of pump modulation frequencies, and therefore must include $f_{o}$ in our sample space, chosen appropriately based on the discussion in Section 5.2. The 


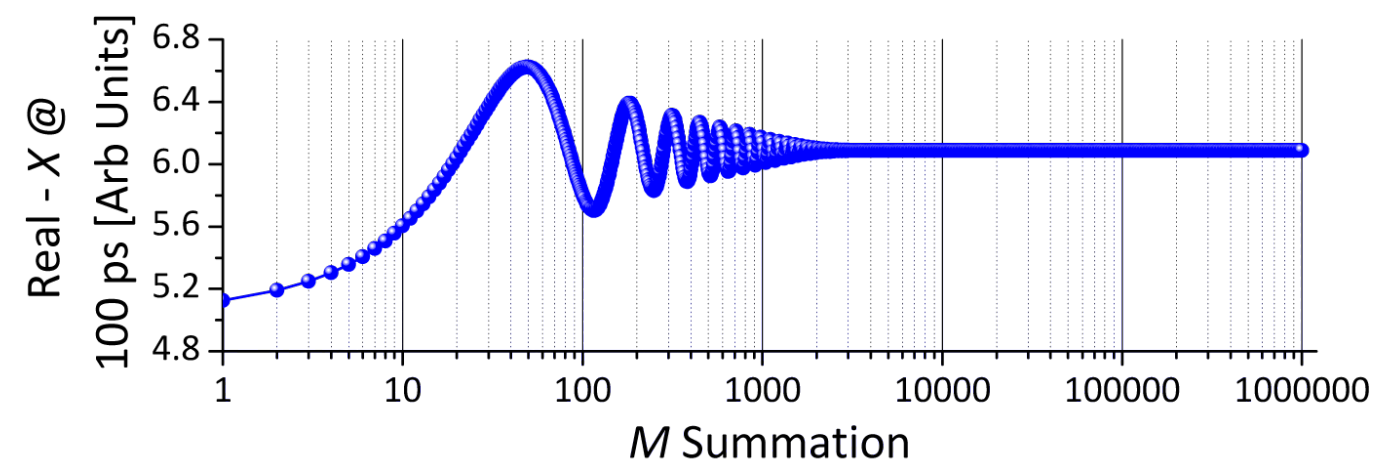

Figure 5.19: Real signal $X$ at $100 \mathrm{ps}$ as a function of $M$ summation for a modeled system of $100 \mathrm{~nm}$ $\mathrm{Al} /$ Glass $\left(h_{\mathrm{BD}}=30 \mathrm{MW} / \mathrm{m}^{2} \mathrm{~K}\right)$, with $k=\{-1,1\}, f_{s}=76 \mathrm{MHz}, f_{o}=1 \mathrm{MHz}$, and a $50 \%$ duty cycle of the modulation waveform. With the inclusion of the Gaussian factor, see Equation 5.13, to the lock-in analysis, see Equations $4.37 \mathrm{a}$ and $4.37 \mathrm{~b}$.

fitted $h_{\mathrm{BD}}$ results of a modeled system of $100 \mathrm{~nm}$ of $\mathrm{Al} / \mathrm{Si}\left(h_{\mathrm{BD}}=215 \mathrm{MW} / \mathrm{m}^{2} \mathrm{~K}\right)$, with $k=\{-1,1\}, f_{s}=76 \mathrm{MHz}$, a $50 \%$ duty cycle of the modulation waveform, and generated with $M=10,000$ as a function of $M$ summation is shown in Figures 5.20 and 5.21 for modulation frequencies of $f_{o}=0.1 \mathrm{MHz}$ and $f_{o}=38 \mathrm{MHz}$ respectively.

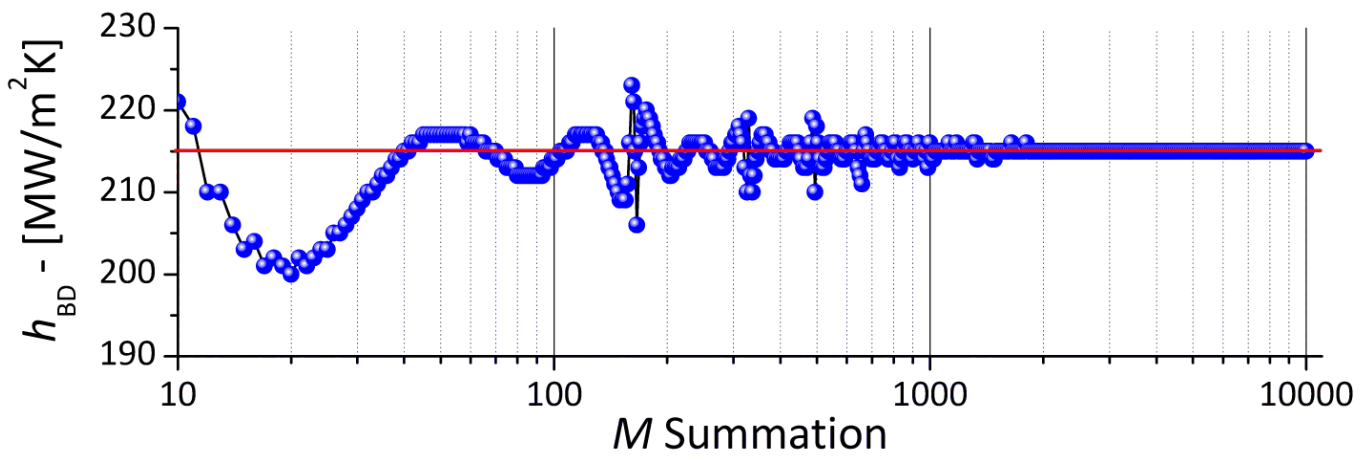

Figure 5.20: Fit of $h_{\mathrm{BD}}$ for a modeled system of $100 \mathrm{~nm}$ of $\mathrm{Al} / \mathrm{Si}\left(h_{\mathrm{BD}}=215 \mathrm{MW} / \mathrm{m}^{2} \mathrm{~K}\right)$, with $k=\{-1,1\}$, $f_{s}=76 \mathrm{MHz}, f_{o}=0.1 \mathrm{MHz}$, a $50 \%$ duty cycle of the modulation waveform, and generated with $M=10,000$, with increasing values of $M$ during fitting. Note: the red line indicated the true $h_{\mathrm{BD}}$ value.

From visual inspection of Figures 5.20 and 5.21 there does not appear to be a strong dependence in the convergence criteria in $M$ on the modulation frequency, $f_{o}$. To create quantitative convergence criteria in $M$, data from the fitted results is compared to the known $h_{\mathrm{BD}}$ value to find the percent difference as a function of modulation frequency. The results from this analysis are shown in Figure 5.22, and show only a weak dependence on $f_{o}$ as was anticipated. Because the $M$ summation required for convergence in the high-rep 


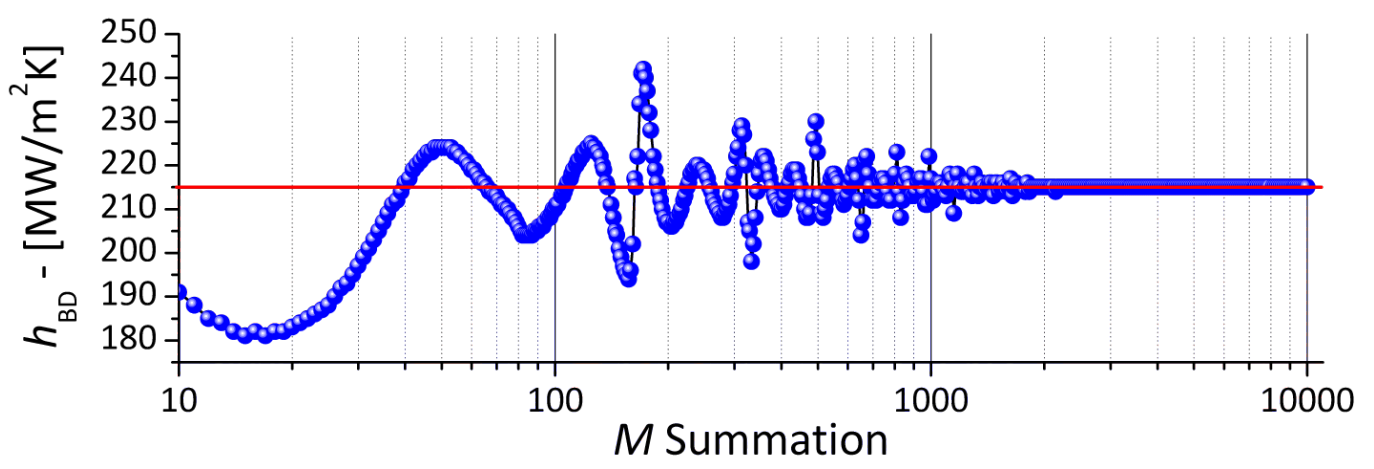

Figure 5.21: Fit of $h_{\mathrm{BD}}$ for a modeled system of $100 \mathrm{~nm}$ of $\mathrm{Al} / \mathrm{Si}\left(h_{\mathrm{BD}}=215 \mathrm{MW} / \mathrm{m}^{2} \mathrm{~K}\right)$, with $k=\{-1,1\}$, $f_{s}=76 \mathrm{MHz}, f_{o}=38 \mathrm{MHz}$, a $50 \%$ duty cycle of the modulation waveform, and generated with $M=10,000$, with increasing values of $M$ during fitting. Note: the red line indicated the true $h_{\mathrm{BD}}$ value.

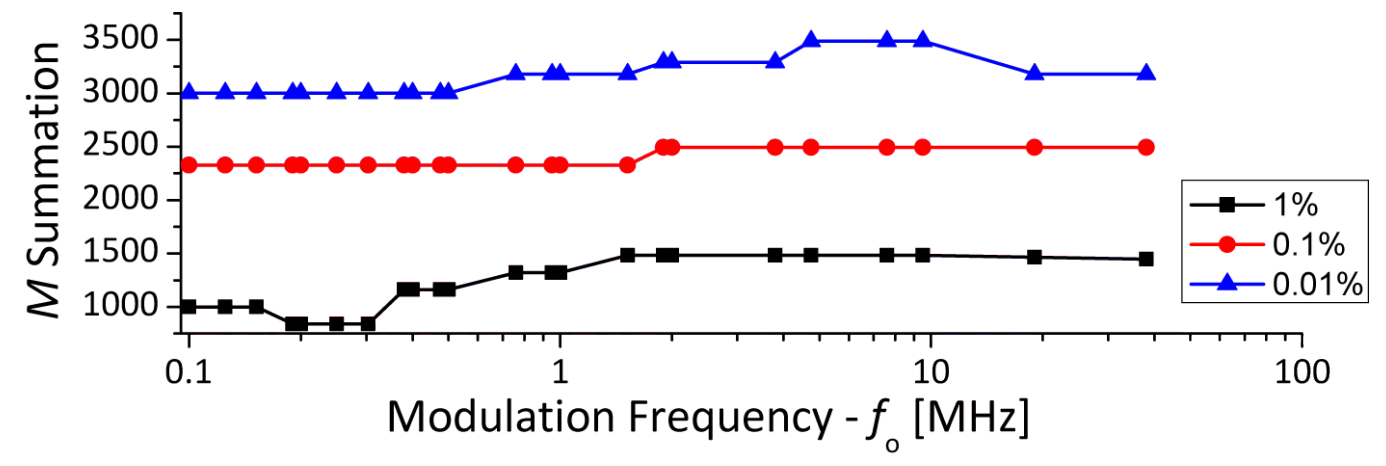

Figure 5.22: Number of summations in $M$ required for fit convergence to the known value of $h_{\mathrm{BD}}$, within a tolerance of $1,0.1$, and $0.01 \%$, for a modeled system of $100 \mathrm{~nm}$ of $\mathrm{Al} / \mathrm{Si}\left(h_{\mathrm{BD}}=215 \mathrm{MW} / \mathrm{m}^{2} \mathrm{~K}\right)$, with $k=\{-1,1\}, f_{s}=76 \mathrm{MHz}$, a $50 \%$ duty cycle of the modulation waveform, and generated with $M=10,000$, as a function of laser modulation frequency, $f_{o}$. Note: the lines are a guide to the eye only.

(MIRA) system is relatively small compared to the levels that are required for the low-rep (RegA) system, rather than defining the convergence criteria as a function of $f_{o}$, we will simply define a minimum value to cover all modulation frequencies at a given convergence level, independent of $f_{o}$. The results of this interpretation are summarized in Table 5.2. We will notice, as compared to the low-rep (RegA) convergence results in Table 5.1, the highrep (MIRA) system required significantly lower values of $M$ for convergence, and therefore takes significantly less time to generate the model.

\section{Summary of Convergence Criteria in $M$}

So far, we have established convergence criteria in $M$ assuming a sinusoidal modulation waveform for both the low-rep (RegA) and high-rep (MIRA) based systems. The primary 
Table 5.2: Estimation of the summation in $M$ required to reach a specified convergence level for the highrep (MIRA) system, based on the data in Figure 5.22. Additionally the time required to generate a 320 point data set, at the summation level, is provided for the TTR/TDTR program run in LabVIEW ${ }^{\mathrm{TM}} 2012$ on an Intel ${ }^{\circledR}$ Core $^{\mathrm{TM}_{2}}$ quad core CPU running at $2.40 \mathrm{GHz}$ with $4 \mathrm{~GB}$ of RAM.

\begin{tabular}{ccc}
\hline $\begin{array}{c}\text { Convergence } \\
\text { Level }\end{array}$ & $\begin{array}{c}\boldsymbol{M} \text { Summation } \\
\text { Required }\end{array}$ & $\begin{array}{c}\text { Time to } \\
\text { Generate }[\mathbf{s}]\end{array}$ \\
\hline $1 \%$ & 1,500 & 0.259 \\
$0.10 \%$ & 2,500 & 0.436 \\
$0.01 \%$ & 3,500 & 0.609 \\
\hline
\end{tabular}

purpose of this analysis was to establish quantitative guidelines to the required modeling parameters in $M$, and eliminating the guess work of deciding when $M$ is "sufficiently large". This will help in establishing both accurate and efficient guidelines for TTR/TDTR modeling. With these guidelines established, we can now turn our attention to a more complex modulation waveform, i.e. the square waveform, which includes the summation in $k$ as well.

\subsubsection{Impact of Series Convergence - Square Modulation Waveform}

To establish convergence guidelines in $k$, we will use a similar methodology as we did in determining the convergence criteria in $M$. We will begin by monitoring a data point in time as a function of the size of $k$, and use the results as a visual guideline to establish quantitative convergence criteria through fitting $h_{\mathrm{BD}}$ in a modeled system. During the course of these analyses we will use the established convergence criteria in $M$ as was developed in Section 5.3.2 to model the data.

\section{Convergence in $k$ - Low-Rep (RegA)}

Beginning again with the low-rep (RegA) system, the $X$ signal of a modeled system of $100 \mathrm{~nm} \mathrm{Al} / \mathrm{Si}\left(h_{\mathrm{BD}}=215 \mathrm{MW} / \mathrm{m}^{2} \mathrm{~K}\right)$, with $M=850,500, f_{s}=250 \mathrm{kHz}, f_{o}=125 \mathrm{kHz}$, and a $50 \%$ duty cycle of the modulation waveform at a time index of $t=100 \mathrm{ps}$ was tracked as a function of the summation ${ }^{8}$ in $k$, see Figure 5.23. Interpretation of Figure 5.23 places visual convergence in $k$ on the order of $k \approx 500$.

\footnotetext{
${ }^{8}$ The summation in $k$ in Equations $4.37 \mathrm{a}$ and $4.37 \mathrm{~b}$ arises from the use of the square modulation waveform, which is constructed via a Fourier series of an infinite summation of sine and cosine waves, where $k$ is the index of the harmonics. For the square modulation waveform only odd harmonics contribute in the Fourier series, and therefore the summation of $k$ is across odd numbers. (Note: this is only the case for the square modulation waveform, and not for a pulsed waveform.)
} 


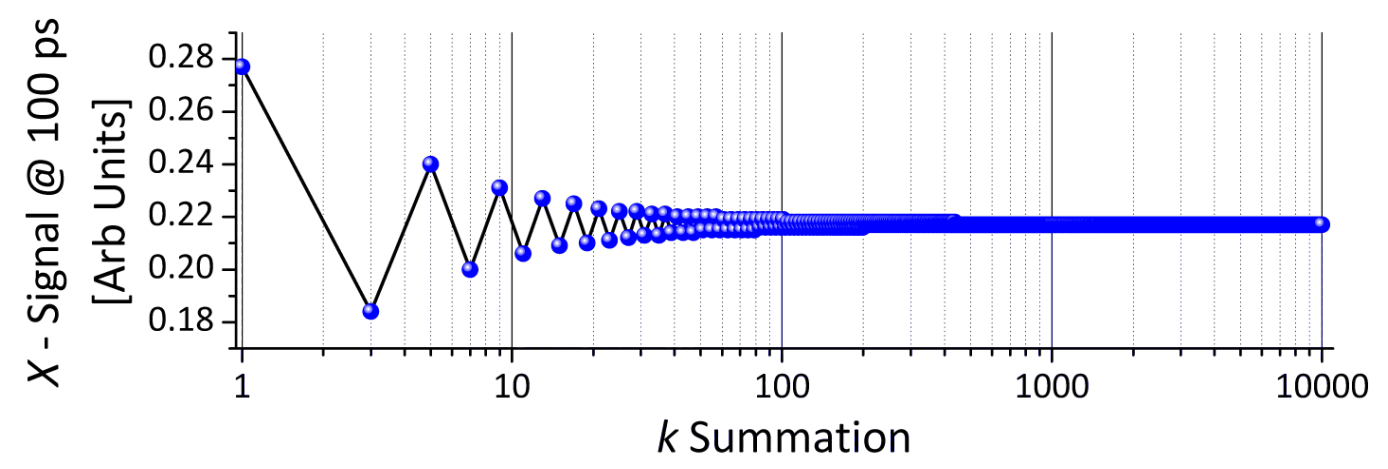

Figure 5.23: Real signal $X$ at $100 \mathrm{ps}$ as a function of $k$ summation for a modeled system of $100 \mathrm{~nm} \mathrm{Al} / \mathrm{Si}$ $\left(h_{\mathrm{BD}}=215 \mathrm{MW} / \mathrm{m}^{2} \mathrm{~K}\right)$, with $M=850,500, f_{s}=250 \mathrm{kHz}, f_{o}=125 \mathrm{kHz}$, and a $50 \%$ duty cycle of the modulation waveform.

Using the visual convergence as a guideline, the $\mathrm{Al} / \mathrm{Si}$ system was modeled with $k=$ 5,000 and the data set fitted using the standard TTR fitting routine to deduce $h_{\mathrm{BD}}$ with increasing bounds on $k$. The results of this analysis are shown in Figure 5.24. It is immediately apparent from these results that there is no dependence on the level of summation in $k$ necessary to deduce the $h_{\mathrm{BD}}$ value from the model. While this would seem counterintuitive compared to the results in Figure 5.23, if we consider how the modulation waveform interacts with the pump impulse-train, and how the TTR analysis is conducted, these results make sense.

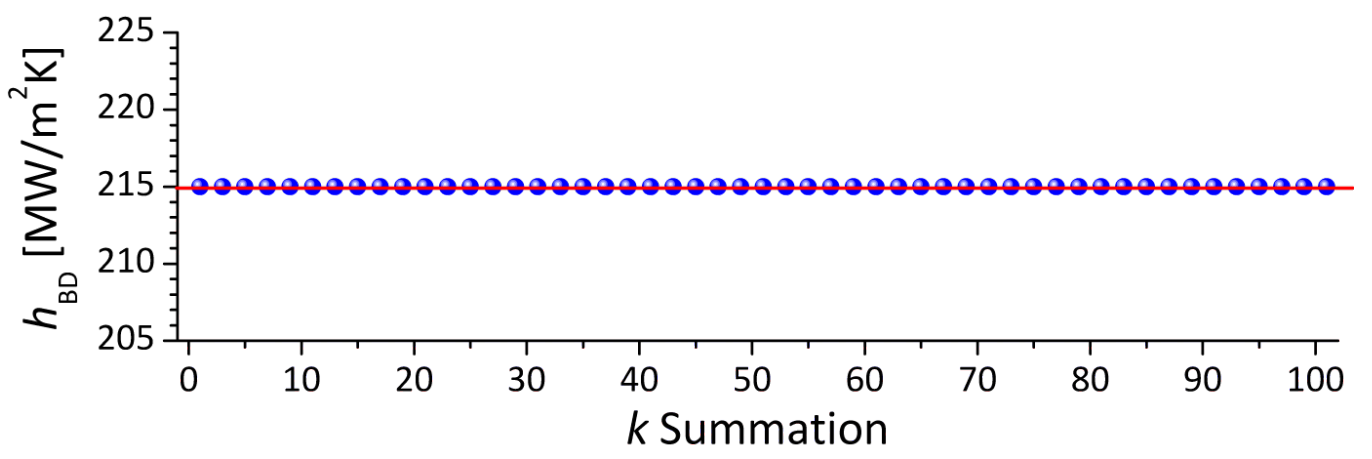

Figure 5.24: Fit of $h_{\mathrm{BD}}$ for a modeled system of $100 \mathrm{~nm}$ of $\mathrm{Al} / \mathrm{Si}\left(h_{\mathrm{BD}}=215 \mathrm{MW} / \mathrm{m}^{2} \mathrm{~K}\right)$, with $M=$ $850,500, f_{s}=250 \mathrm{kHz}, f_{o}=125 \mathrm{kHz}$, and a $50 \%$ duty cycle of the modulation waveform, with increasing values of $k$ during fitting. Note: the red line indicated the true $h_{\mathrm{BD}}$ value.

Figure 5.25 shows a pump laser impulse-train, $f_{s}=250 \mathrm{kHz}$, modulated by a square 
waveform of insufficient ${ }^{9} k$, at $f_{o}=125 \mathrm{kHz}$. The first 3 harmonics of the square waveform illustrate the interaction of the waveform and the laser pulses. As $k$ is increased, the shape of the waveform oscillates as it begins to converge towards the square shape that would be expected when $k$ is sufficiently large. It is important to note however, that for each value of $k$ the laser impulse is center in the envelope of the waveform. Due to this property, the only impact of insufficient summation in $k$ on the laser impulse is the modulation of the peak intensity. In essence, what Figure 5.23 is demonstrating is the oscillating magnitude of the signal as the intensity of the center portion of the square wave envelope oscillates while converging to the square wave solution.

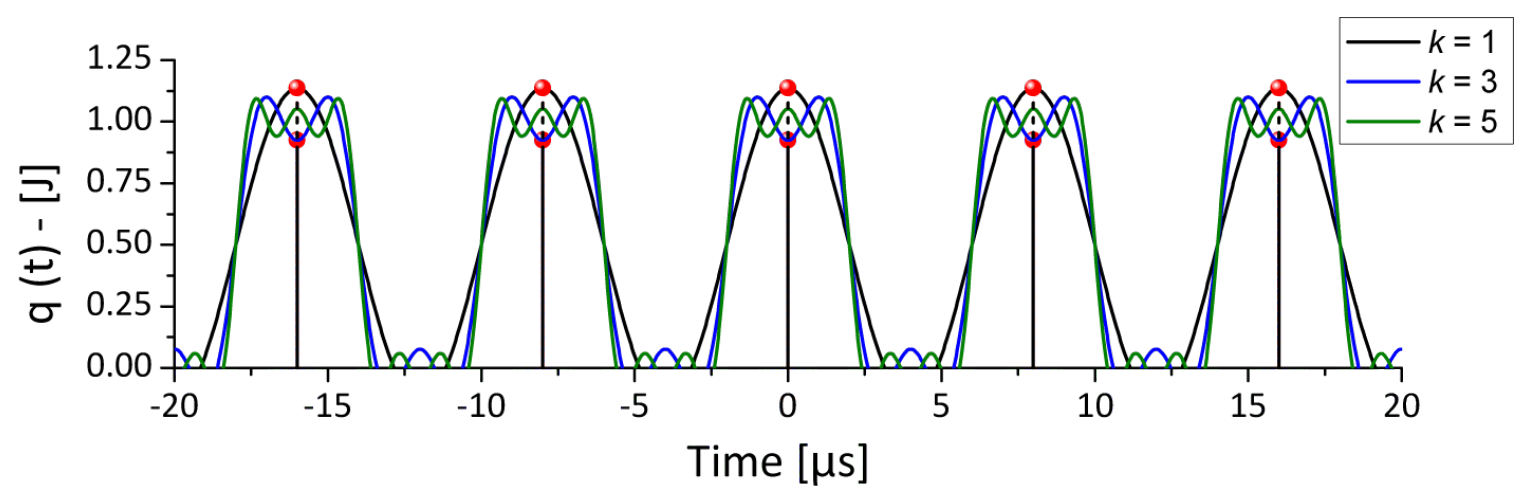

Figure 5.25: Impulse-train of laser pules for $f_{s}=250 \mathrm{kHz}$, and a square modulation waveform at $f_{o}=125$ $\mathrm{kHz}$, for a summation in $k$ of 1,3 , and 5 .

When modeling the TTR/TDTR analysis that was developed in Chapter 4, the intensity of the thermoreflectance signal is a function of the thermoreflectance coefficient of the metal being modeled, and the absorbed power of the pump laser. In practice, both of these factors are difficult to accurately measure during the experiment to use as inputs to the model. To eliminate this complexity when analyzing the TTR/TDTR data, after the model is generated using arbitrary values for the thermoreflectance coefficient and absorbed power, the model is scaled relative to the data for each fitting iteration. More details of this process will be presented in Section 5.4.2. Because of this normalization routine, the fitted results are insensitive to changes in the pump intensity. Therefore for the low-rep (RegA) system modulated via a square wave at $f_{o}=125 \mathrm{kHz}$, see Figure 5.23 , the results are independent

\footnotetext{
${ }^{9} \mathrm{~A}$ true square wave is constructed from an infinite summation of harmonics in $k$. Therefore, an insufficient summation in $k$ will fail to reproduce a true square wave with the severity of the failure increasing as $k$ decreases.
} 
of the summation in $k$. Note that this is a special case of the low-rep system due in part to the normalization routine and the absence of residual heating effects, and cannot be assumed for high-rep systems.

\section{Convergence in $k$ - High-Rep (MIRA)}

To establish convergence criteria in $k$ for the high-rep (MIRA) system, we again need to be concerned with the impact of the modulation frequency on the model convergence. Beginning with a modeled system of $100 \mathrm{~nm} \mathrm{Al} / \mathrm{Si}\left(h_{\mathrm{BD}}=215 \mathrm{MW} / \mathrm{m}^{2} \mathrm{~K}\right)$, with $M=3,500$, $f_{s}=76 \mathrm{MHz}$, and a $50 \%$ duty cycle of the modulation waveform, the $X$ signal at $100 \mathrm{ps}$ as a function of the summation in $k$ is shown in Figures 5.26 and 5.27, for modulation frequencies of $f_{o}=0.1 \mathrm{MHz}$ and $f_{o}=38 \mathrm{MHz}$ respectively. These results again based on

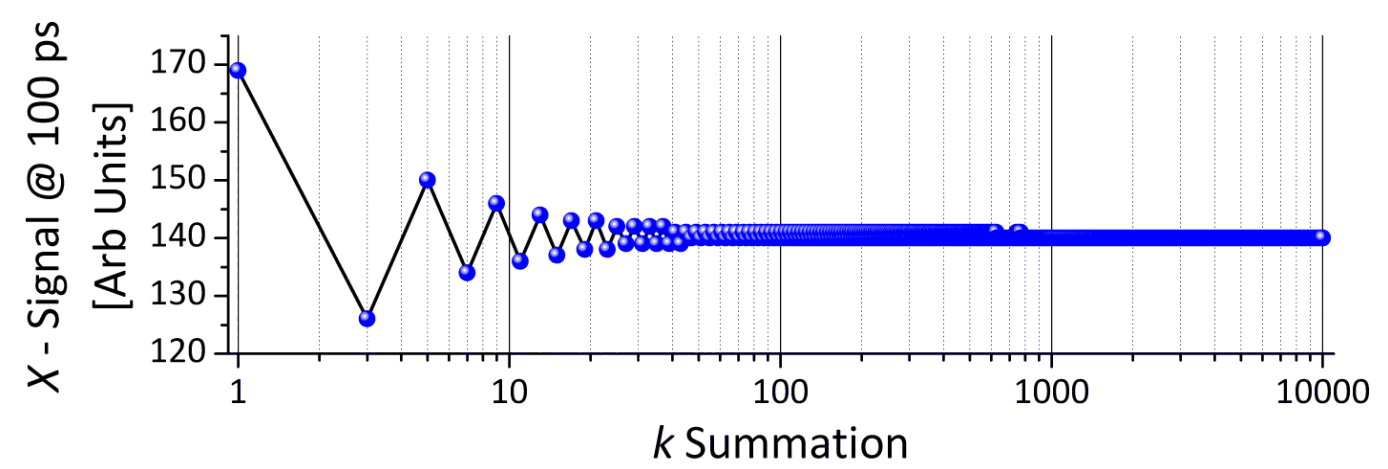

Figure 5.26: Real signal $X$ at $100 \mathrm{ps}$ as a function of $k$ summation for a modeled system of $100 \mathrm{~nm} \mathrm{Al} / \mathrm{Si}$ $\left(h_{\mathrm{BD}}=215 \mathrm{MW} / \mathrm{m}^{2} \mathrm{~K}\right)$, with $M=3,500, f_{s}=76 \mathrm{MHz}, f_{o}=0.1 \mathrm{MHz}$, and a $50 \%$ duty cycle of the modulation waveform.

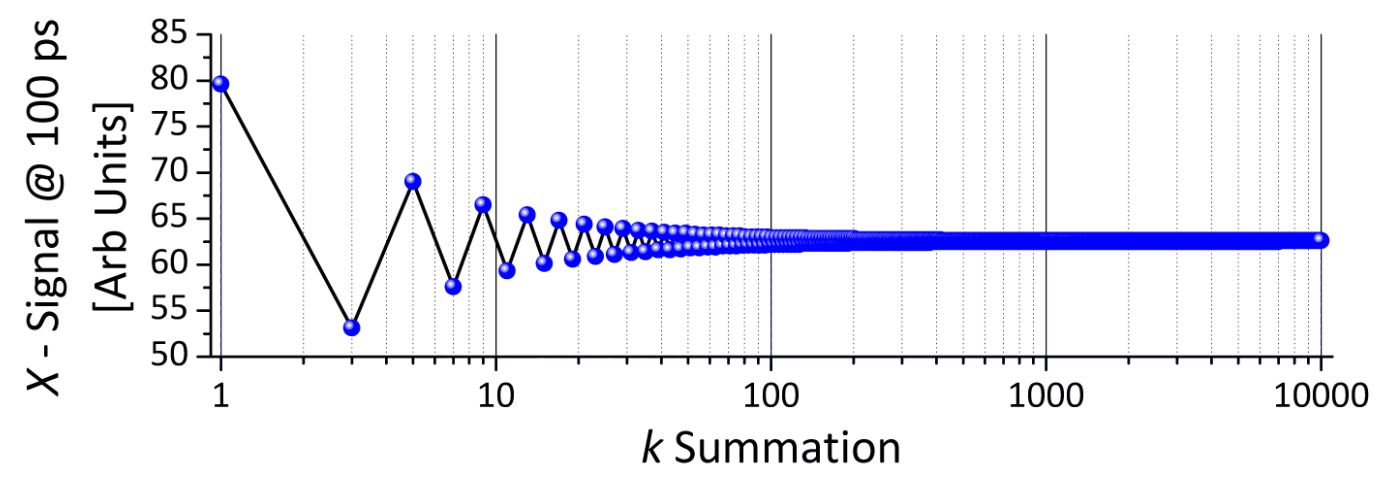

Figure 5.27: Real signal $X$ at $100 \mathrm{ps}$ as a function of $k$ summation for a modeled system of $100 \mathrm{~nm}$ $\mathrm{Al} / \mathrm{Si}\left(h_{\mathrm{BD}}=215 \mathrm{MW} / \mathrm{m}^{2} \mathrm{~K}\right)$, with $M=3,500, f_{s}=76 \mathrm{MHz}, f_{o}=38 \mathrm{MHz}$, and a $50 \%$ duty cycle of the modulation waveform. 
visual convergence criteria, show no strong dependence on the modulation frequency when determining the $k$ required for convergence.

To establish the quantitative convergence criteria based on the results from Figures 5.26 and 5.27, a model of $100 \mathrm{~nm} \mathrm{Al} / \mathrm{Si}\left(h_{\mathrm{BD}}=215 \mathrm{MW} / \mathrm{m}^{2} \mathrm{~K}\right)$, with $M=3,500, f_{s}=76 \mathrm{MHz}$, $\mathrm{MHz}$, and a $50 \%$ duty cycle of the modulation waveform was generated with $k=10,000$, and fit using the standard TDTR routine to deduce $h_{\mathrm{BD}}$. The results of the fitting analysis were then compared to the known $h_{\mathrm{BD}}$ value of $h_{\mathrm{BD}}=215 \mathrm{MW} / \mathrm{m}^{2} \mathrm{~K}$ to calculate the percent difference between the fitted and known results and establish quantitative convergence criteria in $k$, see Figure 5.28.

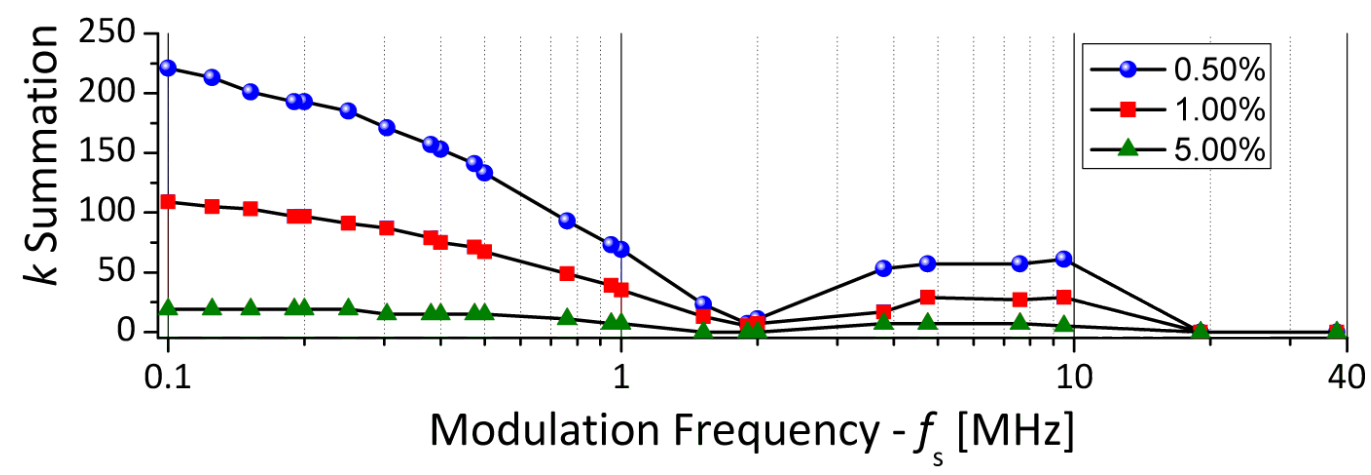

Figure 5.28: Number of summations in $k$ required for fit convergence to the known value of $h_{\mathrm{BD}}$, within a tolerance of 5,1 , and $0.5 \%$, for a modeled system of $100 \mathrm{~nm}$ of $\mathrm{Al} / \mathrm{Si}\left(h_{\mathrm{BD}}=215 \mathrm{MW} / \mathrm{m}^{2} \mathrm{~K}\right)$, with $M=3,500, f_{s}=76 \mathrm{MHz}$, a $50 \%$ duty cycle of the modulation waveform, and generated with $k=10,000$, as a function of laser modulation frequency, $f_{o}$. Note: the lines are a guide to the eye only.

We notice in Figure 5.28 that for high modulation frequencies, i.e. $f_{o}=38$ and 19 $\mathrm{MHz}$, the results are again independent of the summation in $k$. This is due to a similar phenomena as was discussed for the low-rep system, see Figure 5.25. As the modulation frequency is decreased, the dependence on $k$ becomes more complex as the number of pulses interacting with the waveform increases and residual heating effects become more dominate. Assuming convergence criteria of $M=3,500$ and $k=250$, the time to generate the model is on the order of 2.5 minutes. When accepting the $1 \%$ convergence tolerance in $k$, i.e. $k=125$, the modeling time drops to on the order of 1.25 minutes. In either case, assuming approximately 10 iterations required for a complete fit leads to a total time of 15-30 minutes as a worst case scenario for a single one-parameter fit. This time scale is very manageable considering current computational speeds. 


\subsubsection{Impact of Series Convergence - Pulsed Modulation Waveform}

In Section 5.3.3 we dealt with the simplest and most common case of a square modulation waveform, which by definition implies a $50 \%$ duty cycle. In Chapter 4 however, we developed the equations of the lock-in amplifier response, see Equations $4.37 \mathrm{a}$ and $4.37 \mathrm{~b}$, to be general and applicable to a modulation waveform with an arbitrary duty cycle. Therefore, it is important that we consider how the duty cycle will affect the convergence criteria in $k$. The square waveform contains jump discontinuities at the leading and trailing edges of the modulation envelop. Therefore, finite summation in $k$ produces ringing at either edge of the modulation envelope via the Gibbs phenomenon, in a similar manner as was discussed in Section 5.3.1. As $k$ is increased, the high amplitude oscillations are "pushed" towards the edges of the modulation envelope. It is conceivable then that as the width of the modulation envelope, $d$, is modified through variations in the duty cycle, $D$, that the convergence criteria in $k$ for the pulsed waveform will be modified due to the high amplitude Gibbs regions interacting with a larger percentage of the modulation envelope.

\section{What Duty Cycles are Allowed}

Before getting too deep into a discussion of how the duty cycle impacts the convergence criteria in $k$, we will briefly take the time to discuss what duty cycles are "allowed." Similar to the discussion that was presented in Section 5.2 for the modulation frequency, $f_{o}$, for a given system there are only certain duty cycles that are appropriate. Even though the modulation waveform is continuous, and therefore has an infinite number of potential duty cycles, we are modulating a discrete laser impulse-train and therefore will only consider duty cycles which add or remove an impulse from the "on" (or "off") portion of the modulation envelope. Under this constraint the allowable duty cycles, $D$, are given by:

$$
D=\frac{N_{P} f_{o}}{f_{s}} \quad \text { and } \quad 1 \leq N_{P} \leq \frac{f_{s}}{2 f_{o}}
$$

where $N_{P}$ is the number of pulses within the "on" portion of the modulation envelope, $f_{s}$ is the laser repetition frequency, and $f_{o}$ is the modulation frequency. 


\section{Examples}

As an example, consider the high-rep MIRA laser system with $f_{s}=76 \mathrm{MHz}$, modulated by a pulsed waveform at a frequency of $f_{o}=3.8 \mathrm{MHz}$ and a $50 \%$ duty cycle, see Figure 5.29. From inspection of the data in the figure it is apparent that there are 10 pulses per envelope for the given laser repetition and modulation frequency, supported by Equation 5.1, and therefore by Equation 5.310 possible duty cycles, see Table 5.3.

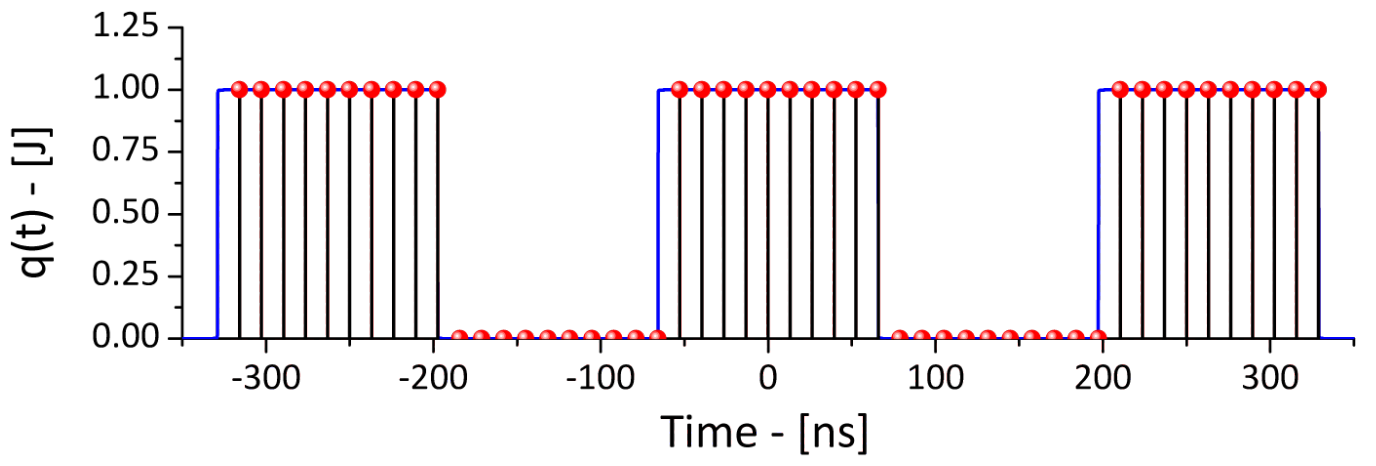

Figure 5.29: Modulated impulse-train from the MIRA, $f_{s}=76 \mathrm{MHz}$, modulated by a square waveform, $D=50 \%$, at a modulation frequency of $f_{o}=3.8 \mathrm{MHz}$.

Table 5.3: Practical duty cycles available for a high-rep system, $f_{s}=76 \mathrm{MHz}$, modulated at $f_{o}=3.8 \mathrm{MHz}$ and the width of the "on" portion of the modulation envelope.

\begin{tabular}{cccc|cccc}
\hline$D$ & Pulses On & Pulses Off & $d[\mu \mathbf{s}]$ & $D$ & Pulses On & Pulses Off & $d[\mu \mathbf{s}]$ \\
\hline 0.05 & 1 & 19 & 0.013 & 0.30 & 6 & 14 & 0.079 \\
0.10 & 2 & 18 & 0.026 & 0.35 & 7 & 13 & 0.092 \\
0.15 & 3 & 17 & 0.039 & 0.40 & 8 & 12 & 0.105 \\
0.20 & 4 & 16 & 0.053 & 0.45 & 9 & 11 & 0.118 \\
0.25 & 5 & 15 & 0.066 & 0.50 & 10 & 10 & 0.132 \\
\hline
\end{tabular}

\section{Convergence in $k$ - Low-Rep $(\operatorname{Reg} A)$}

For the low-rep (RegA) system, from Equation 5.3 the only duty cycle that is practical is $D=0.5$. There is no advantage to changing the duty cycle beyond this level as it does not alter the number of laser pulses within the modulation envelope. This is evident considering the modeled data shown in Figure 5.30 for a selection of duty cycles. For the range of duty cycles presented, the modeled TTR data overlaps perfectly with no change in the signal as a function of duty cycle. This if further confirmed by viewing the form of the laser 


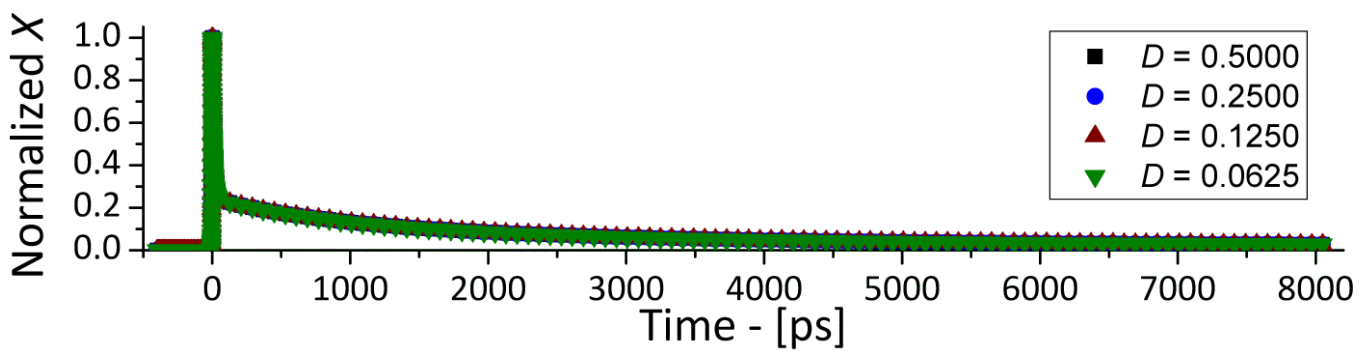

Figure 5.30: Modeled system of $100 \mathrm{~nm}$ of $\mathrm{Al} / \mathrm{Si}\left(h_{\mathrm{BD}}=215 \mathrm{MW} / \mathrm{m}^{2} \mathrm{~K}\right)$, with $k=100, f_{s}=250 \mathrm{kHz}$, $f_{o}=125 \mathrm{kHz}, M=1,000,000$, and for duty cycles of $D=0.5000,0.2500,0.1250$ and 0.0625 .

impulse-train and pulsed modulation waveform in Figure 5.31. By definition of how the pulsed waveform was derived, see Appendix C.1, based on a synchronous phase between the modulation waveform and thermoreflectance signal, regardless of the duty cycle the single laser pulse in the low-rep system remains in the center of the modulation waveform. Therefore, in terms of the thermal analysis for the low-rep system, there is no advantageous impact to changing the duty cycle of the modulation waveform and doing so only adds unneeded complexity to the modeling.

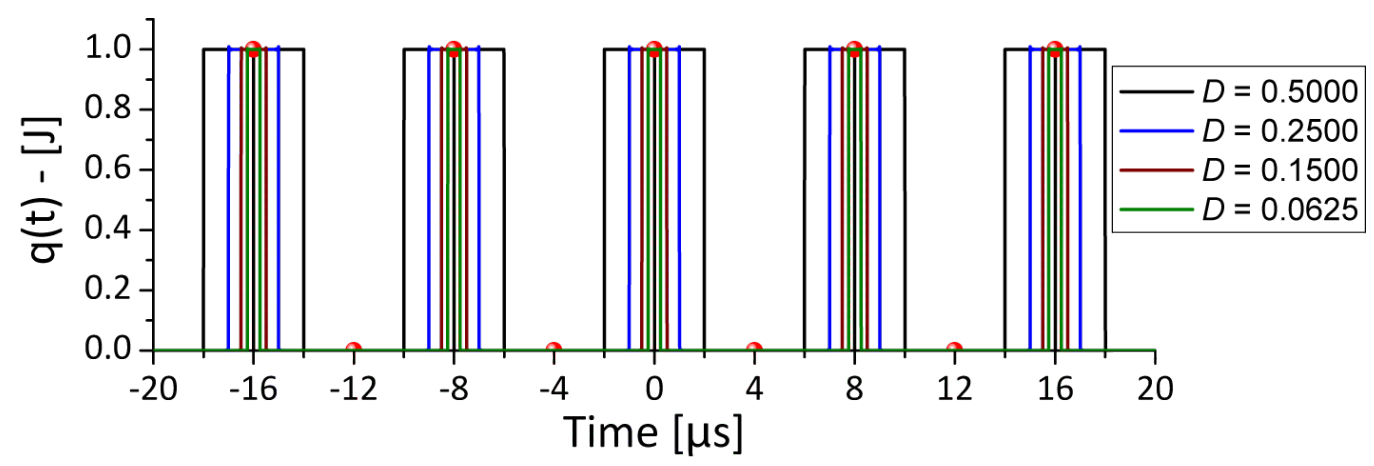

Figure 5.31: Laser impulse-train at $f_{s}=250 \mathrm{kHz}$ modulated by a pulsed waveform at $f_{o}=125 \mathrm{kHz}$ for duty cycles of $D=0.5000,0.2500,0.1250$ and 0.0625 .

\section{Convergence in $k$ - High-Rep (MIRA)}

The convergence criteria in $k$ for the pulsed waveform in the high-rep (MIRA) system has been more difficult to ascertain in a global form. Tracking the $X$ signal at $100 \mathrm{ps}$ for a modeled system of $100 \mathrm{~nm} \mathrm{Al} / \mathrm{Si}\left(h_{\mathrm{BD}}=215 \mathrm{MW} / \mathrm{m}^{2} \mathrm{~K}\right)$, with $M=3,500, f_{s}=76 \mathrm{MHz}$, and $f_{o}=7.6 \mathrm{MHz}$ as a function of the summation in $k$ for several duty cycles, we find as expected that as the duty cycle is lowered, i.e. the width of the modulation window is 


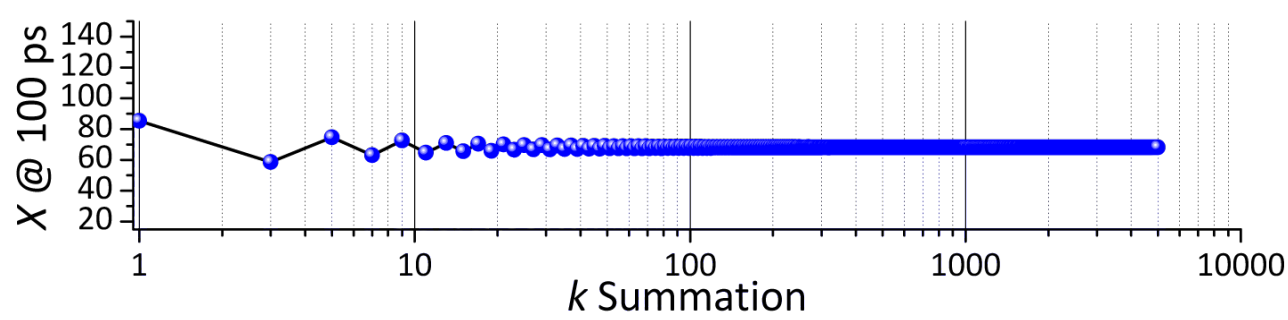

(a)

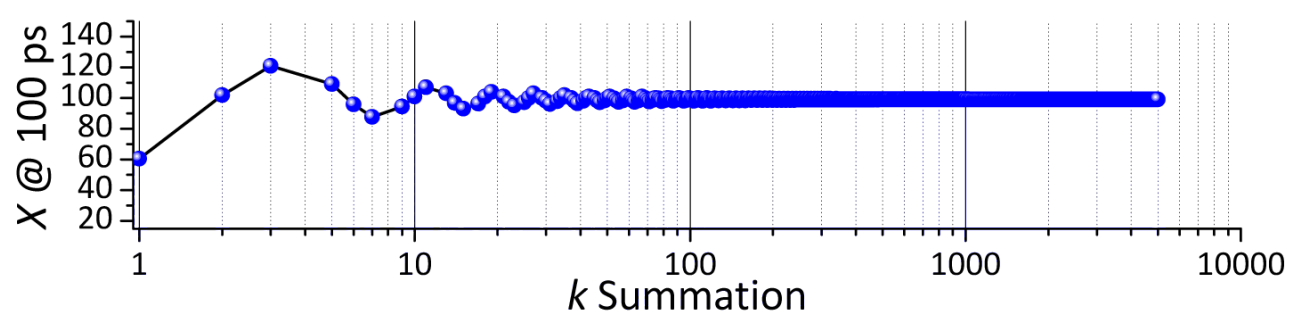

(b)

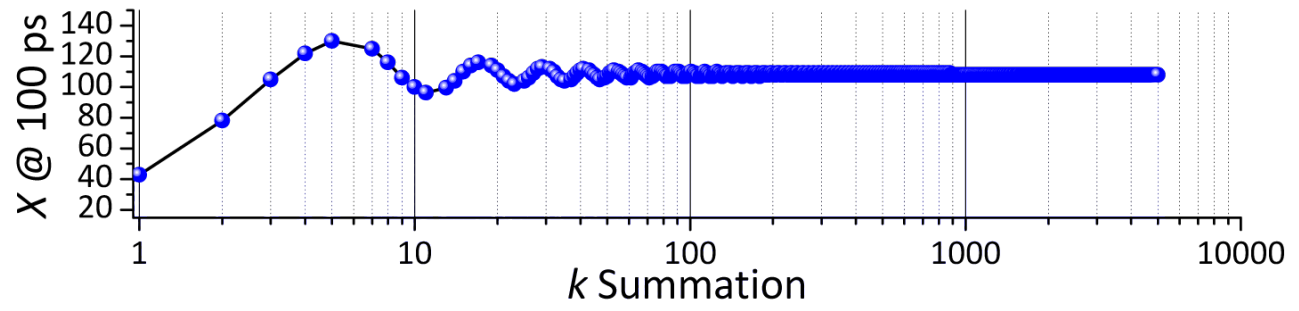

(c)

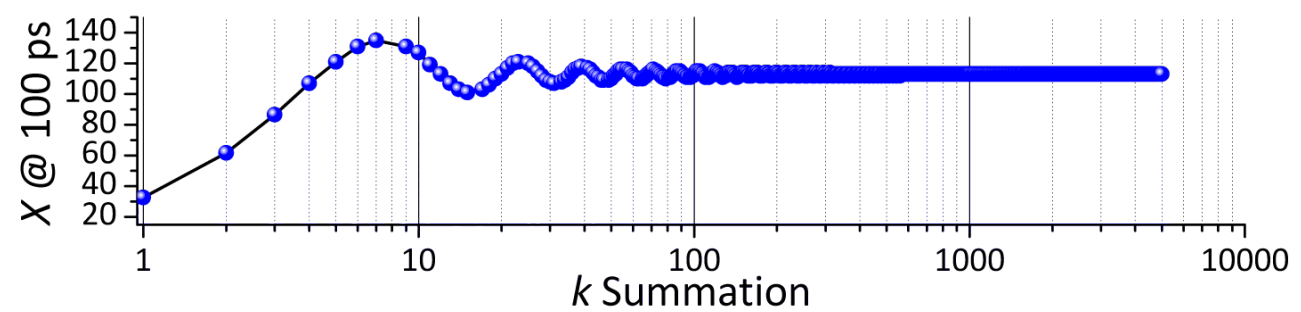

(d)

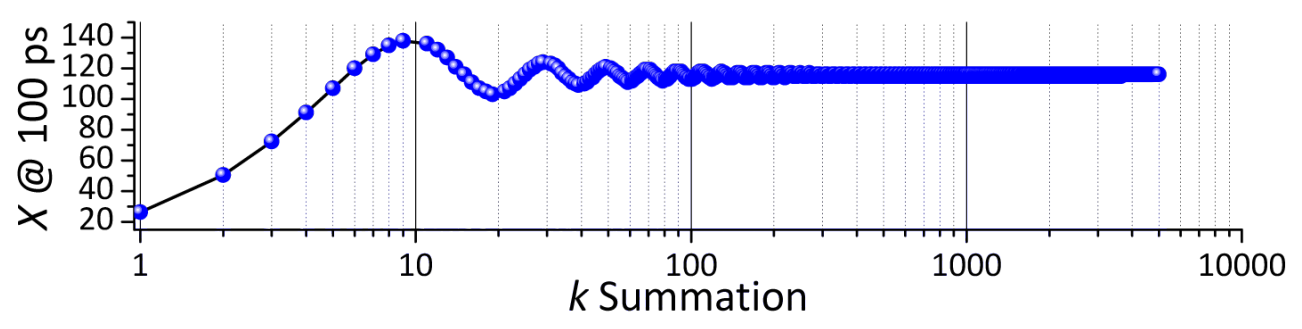

(e)

Figure 5.32: Real signal $X$ at 100 ps as a function of $k$ summation for a modeled system of $100 \mathrm{~nm} \mathrm{Al} / \mathrm{Si}$ $\left(h_{\mathrm{BD}}=215 \mathrm{MW} / \mathrm{m}^{2} \mathrm{~K}\right)$, with $M=3,500, f_{s}=76 \mathrm{MHz}, f_{o}=7.6 \mathrm{MHz}$, and duty cycles of (a) $D=0.5000$, (b) $D=0.2500$, (c) $D=0.1250$, (d) $D=0.166 \overline{7}$, and (e) $D=0.1000$ 
decreased, it appears that a greater summation in $k$ is required for convergence, see Figure 5.32. As the duty cycle is lowered not only does the magnitude of the fluctuations in the data increase, but the oscillations are stretched out over a larger range of $k$ values. These results become more complicated however when turning toward the fitted results.

In Figure 5.33 the quantitative convergence criteria at a $0.5 \%$ level is shown as a function of duty cycle for several modulation frequencies. Similar to the convergence criteria results of the high-rep MIRA system for the square modulation waveform, see Section 5.3.3, the interaction of the modulation waveform and laser impulse-train creates complex dependence on $k$. Initially as the duty cycle drops the required convergence increases, most likely due to the reduction of the temporal width of the modulation envelope and the increased dependence on the Gibbs oscillations. As the duty cycle reaches a minimum however, the results in Figure 5.33 suggest that the required $k$ for convergence decreases. This trend is most likely due to the reduction in the number of pulses within the modulation envelope and similar conditions as was found for the low-rep system and the high-rep system for high modulation frequencies.

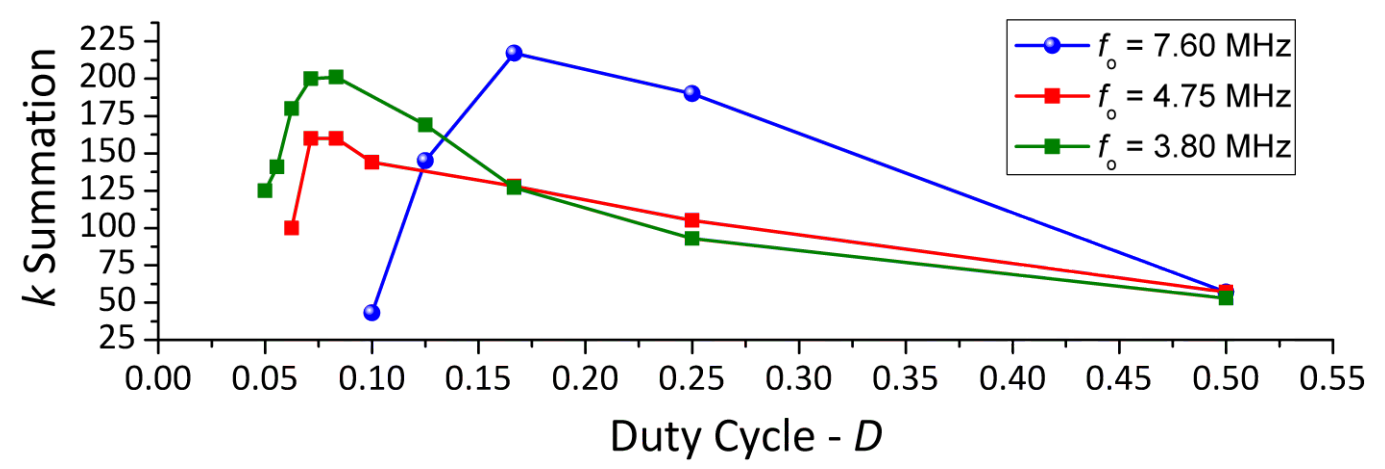

Figure 5.33: Required summation in $k$ for $0.5 \%$ convergence as a function of duty cycle from fit $h_{\mathrm{BD}}$ results from a modeled system of $100 \mathrm{~nm} \mathrm{Al} / \mathrm{Si}\left(h_{\mathrm{BD}}=215 \mathrm{MW} / \mathrm{m}^{2} \mathrm{~K}\right)$, with $M=3,500, f_{s}=76 \mathrm{MHz}$ and $f_{o}$ as shown.

For the worst case scenario the required convergence in $k$ does not appear to exceed what was established for the square modulation waveform, see Section 5.3.3, which placed fitting times on the order of 30 minutes. It should be noted that while the time to fit an individual data set is manageable, the time to complete the quantitative analysis of the convergence criteria in $k$ for the pulsed waveform, see Figure 5.33, is very computationally expensive. Therefore at this time on a small portion of modulations frequencies have been analyzed 
and these results are based on that subset of data. At a later date more simulations will have to be run to ensure that these guidelines can be applied globally.

\subsubsection{Summary of Convergence Criteria}

In this section an in-depth analysis of the convergence criteria in $M$ and $k$ required to accurately model the TTR/TDTR signal developed in Chapter 4 was presented. Quantitative guidelines were established for the summation in $M$ for both low- and high-rep laser systems, see Tables 5.1 and 5.2 respectively. The transition from the sinusoidal to square modulation waveform required additional convergence standards in $k$. The low-rep system was found to be independent of $k$ and the convergence criteria for the high-rep system as a function of modulation frequency was found, see Figure 5.28. Finally, for the pulsed modulation waveform it was established that the results of the low-rep system were still independent of $k$ and preliminary trends for the high-rep system were presented, see Figure 5.33. These results should provide quantitative guidelines to ensure accurate modeling of the TTR/TDTR signal and eliminate the "guess work" as to when the infinite summations in the thermal model are sufficiently large.

\subsection{Fitting to Deduce Thermophysical Parameters}

There are several important, practical considerations in the deduction of thermophysical parameters from TTR/TDTR data that can impact the accuracy and the efficiency of the fitted results. Here we will introduce two formulations; one focused on increasing computational efficiency and the other tailored to ensure accurate deduction of the thermophysical parameter of interest. We will begin by investing the concept of a "node spacing" formulation, to decrease the computational time required to generate the TTR/TDTR model, beyond the limits set forth by the convergence criteria in $M$ from Section 5.3. That will be followed up with an improved method of normalizing the model relative to the data for parameter deduction, to eliminate the dependence of the final result on the choice of normalization time. 


\subsubsection{Gibbs Oscillations at $t_{o}-$ M-Fourier Components}

It was established in Section 5.3, that for a finite number of $M$ in the summation term of the lock-in analysis, see Equations 4.37a-4.37b, there will be ringing in the modeled signal due to the jump discontinuity at $t_{o}$. I have developed quantitative convergence criteria where $M$ will be sufficiently large in order to shift the high amplitude oscillations before the earliest time of interest, typically 50-100 ps. While the development of the convergence criteria in $M$ made the analysis more robust, the trade off for increasing the size of $M$ is the computational time required to run the model calculations.

Figure 5.34 shows the time required to generate the TTR/TDTR model as a function of the summation ${ }^{10}$ in $M$. It is important to note that this plot is a guideline, as the actual computational time required is a function of a number of factors including how many data (time) points are in the model, and the computer system used to complete the calculations. For the data in Figure 5.34, 320 data points where contained in the model and the calculations were run in LabVIEW ${ }^{\mathrm{TM}_{2}} 2012$ on an Intel ${ }^{\circledR}$ Core $^{\mathrm{TM}_{2}}$ quad core CPU running at $2.40 \mathrm{GHz}$ with $4 \mathrm{~GB}$ of RAM.

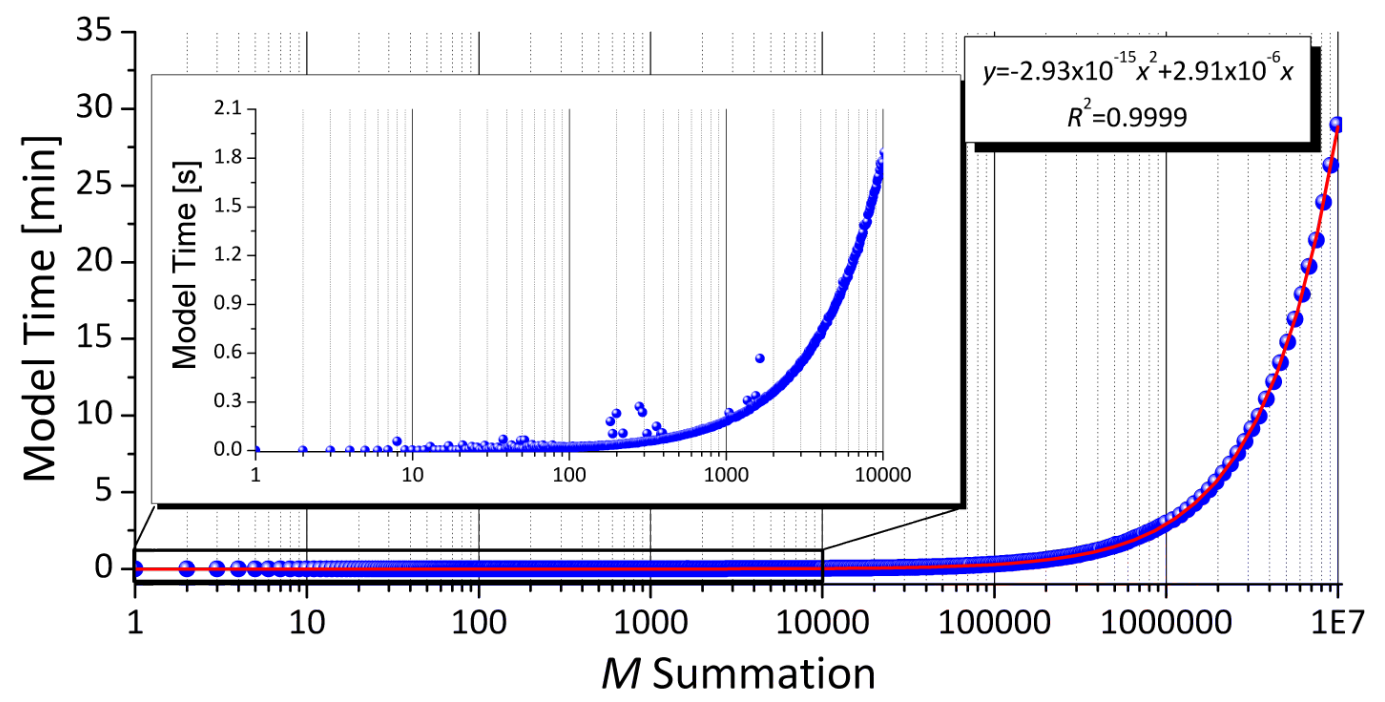

Figure 5.34: Time required to generate a TTR/TDTR model (320 data points), as a function of $M$ summation. Program run in LabVIEW ${ }^{\mathrm{TM}} 2012$ on an Intel ${ }^{\circledR}$ Core $^{\mathrm{TM}}{ }_{2}$ quad core CPU running at $2.40 \mathrm{GHz}$ with 4 GB of RAM. The red line indicates a $2^{\text {nd }}$ order polynomial fit of the data, with the fitted results shown.

\footnotetext{
${ }^{10}$ Because the summation in $M$ and $k$ are linearly couples, the time required to simulate the TTR/TDTR model with the pulsed waveform is the product of the time required for the sinusoidal waveform, i.e. $k=$ $\{-1,1\}$, of given $M$, see Figure 5.34 , and the number of summation in $k$.
} 
For the convergence criteria established in Section 5.3, depending on the choice of the high- or low-rep system and the convergence criteria, the time to generate the model ranges from on the order of $0.5 \mathrm{~s}$ to 2.5 minutes. If we assume on average 10 iterations to deduce a thermophysical parameter of interest, approximately 1-25 minutes of computational time are required to fit a collected TTR/TDTR scan. Considering that typically only small data sets are collected, and the proliferation of multi-core processors in today's market, these computational times are not unreasonable. If however, we are interested in doing large scale modeling of the thermoreflectance data ${ }^{11}$ where hundreds or thousands of fits may be required, particularly for the low-rep (RegA) system, these computational times suddenly become prohibitive.

\section{Modeling with Node Spacing}

In order to further reduce the computational time required to model the TTR/TDTR system, the concept of fitting with "node spacing" was developed. We have already established that for a finite summation in $M$, there will be oscillations or ringing in the modeled data. If $M$ is such that the convergence criteria formulated in Section 5.3 is satisfied, any inaccuracies due to this ringing will be within error tolerances. However, to speed up computational efficiency and allow for large scale modeling, it is desirable to be able to reduce the size of $M$ without introducing modeling error. Accepting the fact that reducing $M$ will increase the oscillations in the data, we have already defined $T_{\text {peak }}$ as the time between Gibbs oscillations, see Figure 5.10. However, because the data oscillates around the ideal solution, we can also define nodes in the oscillations that correspond to the correct solution, see Figure 5.35. With data points spaced properly we can take advantage of these nodes to produce the correct solution for a lower value of $M$, without impacting the error in the modeling.

To do this, rather than data with an arbitrary time configuration, the idea is to use a start time, $t_{\text {start }}$, and time step, $T_{\text {node }}$, such that the modeled data of interest will start at a node of the oscillating solution, and each subsequent data point will be at an integer multiple of the node spacing. We have already established that the frequency of the Gibbs

\footnotetext{
${ }^{11}$ Which will be done extensively in Chapter 6 .
} 


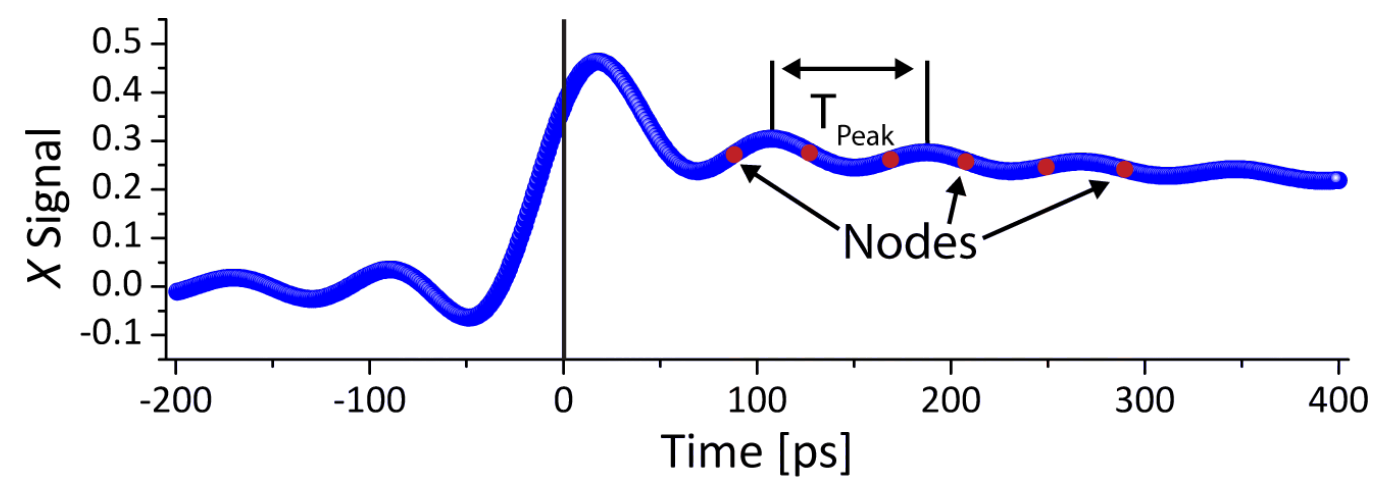

Figure 5.35: Schematic of modeled data highlighting the oscillations in the modeled data for a low summation in $M$, and defining the time period between peaks, $T_{\text {peak }}$, and the node spacing.

oscillations is dependent on the size of $M$, and therefore expect the node properties to be dependent on $M$ as well. To calculate this dependence, the temporal spacing between Gibbs peaks, $T_{\text {peak }}$, was determined as a function of the summation in $M$ for several laser repetition frequencies, $f_{s}$, see Figure 5.36. To find the peaks TTR/TDTR data was simulated with a small timestep, $0.1 \mathrm{ps}$, to capture the oscillations in the data, and then a peak finding routine was run in MATLAB to determine the temporal spacing between the peaks.

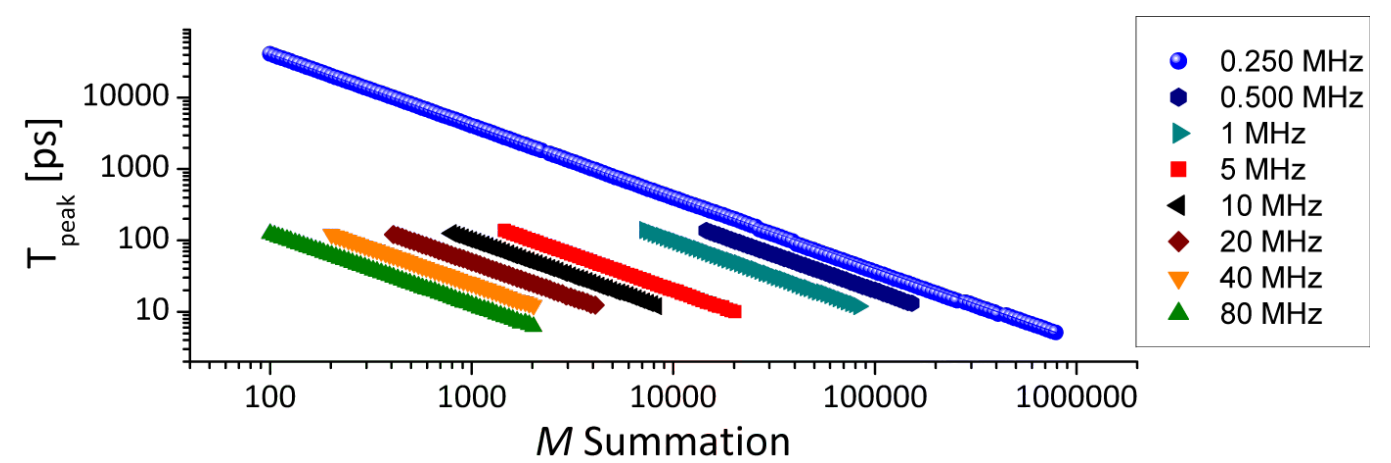

Figure 5.36: The time between the peaks in the Gibbs oscillations, $T_{\text {peak }}$, as a function of the summation bounds $M$ for several laser repetition frequencies, $f_{s}$.

Each of the data sets in Figure 5.36 were fit using a power law formulation of the form $T_{\text {peak }}=a \times M^{b}$ with the value of $b$ fixed at $b=-1$. The results of the fitting analysis are shown in Table 5.4. The data shows that the fitting coefficient corresponds almost directly to the laser repetition period $T_{s}$. There are slight errors associated with the ability to resolve $T_{\text {peak }}$, due to the finite time steps of the modeled data and the resolution of the peak picking routine that most likely accounts for the small discrepancies between $T_{s}$ and $a$. However, 
Table 5.4: Results of fitting of the data in Figure 5.36 using a power law of the form, $T_{\text {peak }}=a / M$.

\begin{tabular}{ccccc}
\hline$f_{s}[\mathrm{MHz}]$ & $T_{s}[\mathrm{~s}]$ & $a[\mathrm{~s}]$ & $b$ & $\mathrm{R}^{2}$ \\
\hline 0.250 & $4.00 \times 10^{-6}$ & $4.09 \times 10^{6} \pm 1.00 \times 10^{3}$ & -1 & 1 \\
0.500 & $2.00 \times 10^{-6}$ & $1.99 \times 10^{6} \pm 1.00 \times 10^{3}$ & -1 & 1 \\
1 & $1.00 \times 10^{-6}$ & $9.96 \times 10^{5} \pm 7.00 \times 10^{2}$ & -1 & 1 \\
5 & $2.00 \times 10^{-7}$ & $1.99 \times 10^{5} \pm 1.00 \times 10^{2}$ & -1 & 1 \\
10 & $1.00 \times 10^{-7}$ & $9.97 \times 10^{4} \pm 9.00 \times 10^{1}$ & -1 & 1 \\
20 & $5.00 \times 10^{-8}$ & $4.98 \times 10^{4} \pm 4.00 \times 10^{1}$ & -1 & 1 \\
40 & $2.50 \times 10^{-8}$ & $2.49 \times 10^{4} \pm 2.00 \times 10^{1}$ & -1 & 1 \\
80 & $1.25 \times 10^{-8}$ & $1.24 \times 10^{4} \pm 1.00 \times 10^{1}$ & -1 & 1 \\
\hline
\end{tabular}

the discrepancies are small enough that we can define a relation for the time between Gibbs oscillations, $T_{\text {peak }}$, as a function of the laser repetition period and summation in $M$, given by:

$$
T_{\text {peak }}=\frac{T_{s}}{M}
$$

With a formulation for the peak spacing established, it is possible to determine the node spacing of the oscillating solution, $T_{\text {node }}$, with is given by:

$$
T_{\text {node }}=\frac{3 T_{s}}{8 M}
$$

The time of the first node, $T_{\text {start }}$, is then given by:

$$
T_{\text {start }}=T_{\text {peak }}-T_{\text {Node }}=\frac{T_{s}}{M}-\left[\frac{\left(\frac{T_{s}}{M}-\frac{T_{s}}{4 M}\right)}{2}\right]
$$

\section{Testing the Node Spacing Formulation}

In order to test the validity of the node spacing concept, a model of TTR data was generated for $100 \mathrm{~nm}$ of $\mathrm{Al}$ on $\mathrm{Si}\left(h_{\mathrm{BD}}=215 \mathrm{MW} / \mathrm{m}^{2} \mathrm{~K}\right)$, with $M=1,000,000, f_{s}=250$ $\mathrm{kHz}, f_{s}=125 \mathrm{kHz}$, and a $50 \%$ duty cycle of the modulation waveform. Based on the work of Section 5.3, the summation in $M$ is more than sufficient to ensure that the model represents a converged solution. The data points in the model were set with a time spacing 
similar to the steps utilized in normal ${ }^{12}$ experimental data collection. The data in the region of interest was then interpolated using a cubic hermite spline interpolation routine in LabVIEW ${ }^{\mathrm{TM}}$ to set a new data spacing based on Equations 5.4 and 5.6, see Figure 5.37. The interpolation routine is setup to match the original data set as closely as possible, i.e. not changing the number of data points.

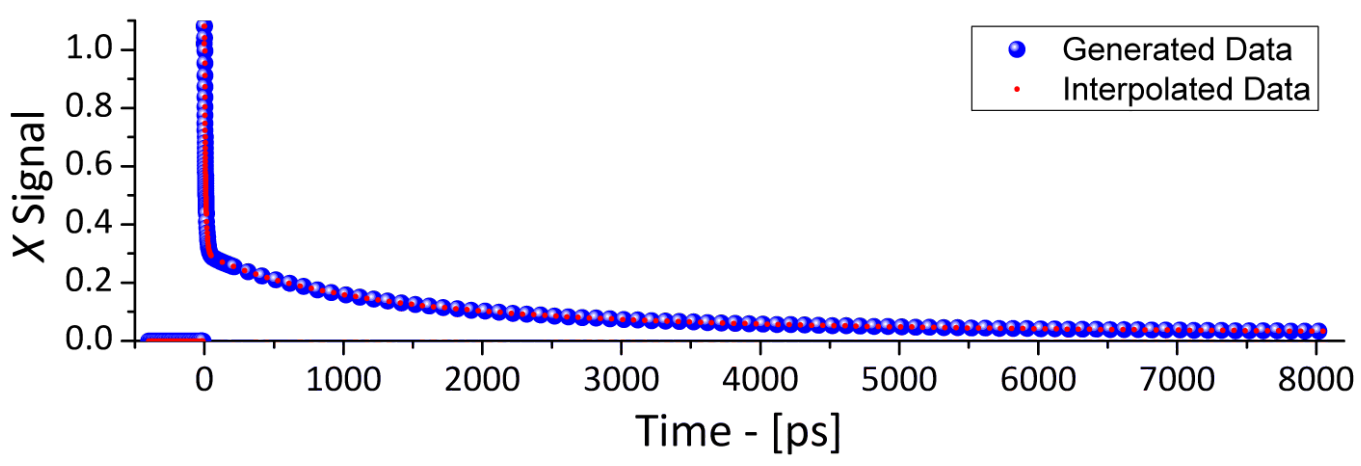

Figure 5.37: Generated TTR model of $100 \mathrm{~nm}$ of $\mathrm{Al}$ on $\mathrm{Si}\left(h_{\mathrm{BD}}=215 \mathrm{MW} / \mathrm{m}^{2} \mathrm{~K}\right)$, with $M=1,000,000$, $f_{s}=250 \mathrm{kHz}, f_{s}=125 \mathrm{kHz}$, and a $50 \%$ duty cycle of the modulation waveform (blue), and the interpolated data using Equations 5.4 and 5.6 for $M=50,000$.

The original generated data set, see Figure 5.37, was interpolated via Equations 5.4 and 5.6 for $M$ summations ranging from $M=10,000$ to $M=1,000,000$ creating a new interpolated data set for each $M$. These data sets were then fit using the standard TTR analysis and the appropriate summation in $M$ for the appropriate interpolated file, the results of which are shown in Figure 5.38. The solid red line in Figure 5.38 denotes the known $h_{\mathrm{BD}}$ value, while the horizontal dashed lines represent $1 \%$ deviation from the nominal value. For low values of $M$ the correct $h_{\mathrm{BD}}$ value is not captured. However, as $M$ increases the fitted results converge to the nominal value, reaching within $1 \%$ by $M \approx 35,000$ and by $M=50,000$ is within $0.05 \%$ of the nominal value. The continued deviation of the fitted results at low values of $M$ is most likely due to the low number of data points available. For $M=10,000 T_{\text {peak }}=400 \mathrm{ps}$, which when the original data set is interpolated, represents a reduction in the total number of data points by a factor of 4 . The reduction in the required $M$ from $M=666,200$ for normal convergence to $0.05 \%$ (see Figure 5.17) based

\footnotetext{
${ }^{12}$ The time spacing of the data collected during the experiment is a user defined quantity and is different from group to group. In our lab, typically three times regions were defined: steps on the order of 100 s of femtoseconds during the initial temperature rise, several picoseconds steps during the initial temperature decay, and 10s of picoseconds steps during the long diffusion processes.
} 


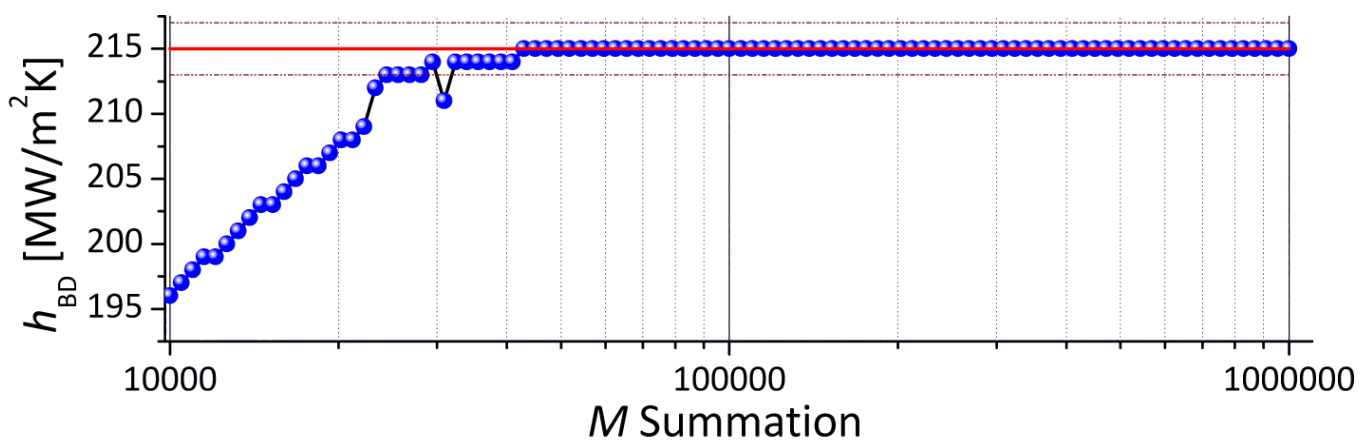

Figure 5.38: TTR fit of a modeled system of $100 \mathrm{~nm}$ of $\mathrm{Al}$ on $\mathrm{Si}\left(h_{\mathrm{BD}}=215 \mathrm{MW} / \mathrm{m}^{2} \mathrm{~K}\right)$, with $M=$ $1,000,000, f_{s}=250 \mathrm{kHz}, f_{s}=125 \mathrm{kHz}$, and a $50 \%$ duty cycle of the modulation waveform, as a function of increasing summation in $M$, with the data spacing for each $M$ adjusted based on Equations 5.4 and 5.6. The horizontal dashed lines represent plus/minus $1 \%$ deviation from the known $h_{\mathrm{BD}}$ value.

on the traditional convergence criteria, to $M=50,000$ using the node spacing formulation, represents a reduction in the required computational time from 1.94 minutes to 8.73 seconds respectively. This represents an over 13 times increase in computational efficiency, making large scale simulations of low-rep (RegA) data tractable.

While not as necessary due to the already low summation in $M$ required, the node spacing formulation can also be applied when fitting data from high-rep (MIRA) systems. Using the same methodology as was done for testing the node spacing theory for the lowrep (RegA) system, the results of testing in the high-rep (MIRA) system are shown in Figure 5.39. For almost all the data shown, the fitted $h_{\mathrm{BD}}$ is within $1 \%$ of the nominal values and drops to below $0.05 \%$ convergence for $M=1,800$. While the impact of the

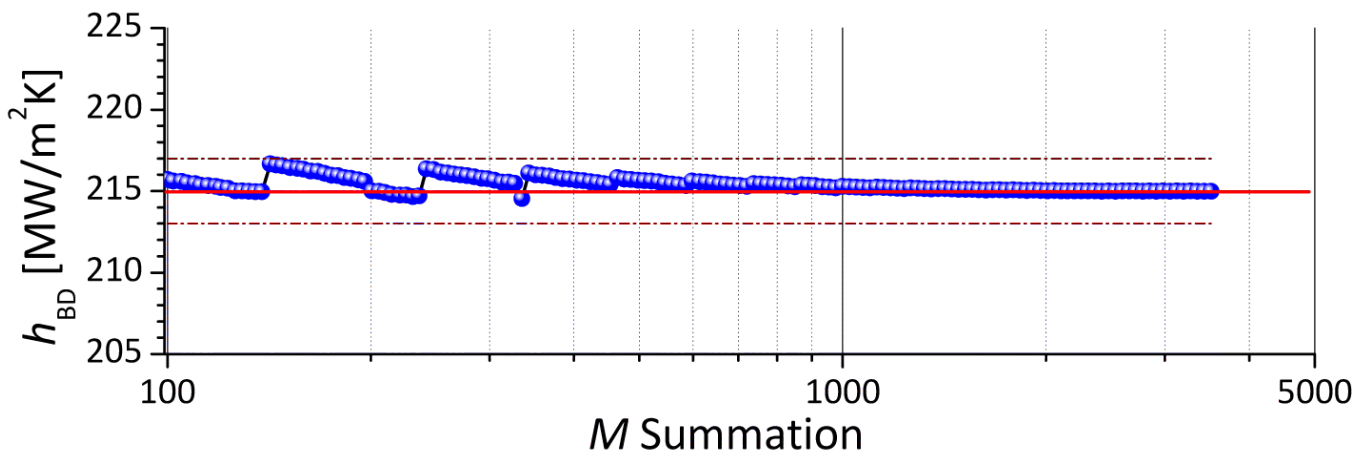

Figure 5.39: TTR fit of a modeled system of $100 \mathrm{~nm}$ of $\mathrm{Al}$ on $\mathrm{Si}\left(h_{\mathrm{BD}}=215 \mathrm{MW} / \mathrm{m}^{2} \mathrm{~K}\right)$, with $M=3,500$, $f_{s}=76 \mathrm{MHz}, f_{s}=1 \mathrm{MHz}$, and a $50 \%$ duty cycle of the modulation waveform, as a function of increasing summation in $M$, with the data spacing for each $M$ adjusted based on Equations 5.5 and 5.6. The horizontal dashed lines represent plus/minus $1 \%$ deviation from the known $h_{\mathrm{BD}}$ value. 
node spacing formulation is not as dramatic for the case of the high-rep (MIRA) system, its implementation still represents a $50 \%$ reduction in the required computational time compared to the traditional convergence criteria.

\section{Experimental Application - Sensitivity to Laser Frequencies}

While the node spacing concept was developed mostly to improve the computational efficiency of TTR/TDTR fitting to allow for large scale simulations, the natural extension is the desire to apply this methodology to the fitting of experimentally collected data. All the steps in the node spacing formulation hold for both experimental and simulated data. The challenge in applying the node spacing formulation to experimental data arises in the determination of the laser repetition frequency, $f_{s}$. Figure 5.40 shows the results of fitting simulated TTR data with a range of laser repetition frequencies, using the "mistaken" node spacing for $f_{s}=250 \mathrm{kHz}$ in each case.

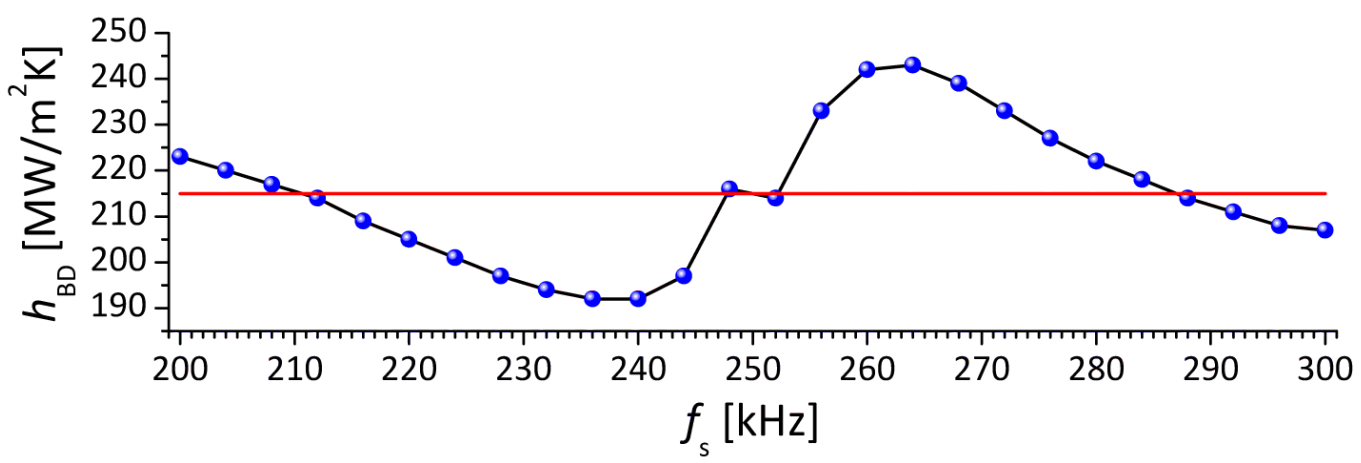

Figure 5.40: Fit $h_{\mathrm{BD}}$ as a function of repetition frequency, $f_{s}$, for modulation of $f_{o}=f_{s} / 2$, with the time spacing interpolated using Equations 5.5 and 5.6, for $f_{s}=250 \mathrm{kHz}$ in each case.

These results indicate that the implementation of the node spacing formulation is highly dependent on the knowledge of the laser repetition frequency. If the laser frequency is known to within approximately $1 \%$ the fitted results will be within $1 \%$ of the true value. However, for a miscalculation of the laser repetition frequency of $2.5 \%$ the deviation in fitted $h_{\mathrm{BD}}$ jumps to $8.5 \%$. Because the node spacing is dependent only on the laser period, $T_{s}$, the validity of the formulation for a particular system can be checked by comparing the fitted results using the node spacing to that completed using the convergence criteria established in Section 5.3 which is independent of time spacing. 


\subsubsection{Normalization of Data}

As alluded to previously, in the application of Equations $4.37 \mathrm{a}$ and $4.37 \mathrm{~b}$ it is not common practice to specify the constant $\beta$, which contains information about the thermoreflectance coefficient of the sample and the gain in the electronics, or the adsorbed laser power. Measuring the actual absorbed laser power, finding the thermoreflectance coefficient for each specific sample, and specifying the gain in the electronic components, can be challenging. Additionally if not determined accurately, these parameters will add more uncertainty than they would provide useful information. To eliminate this complexity, it is common practice when fitting the TTR/TDTR model to the experimental data, to normalize the model to the data set at some point in time, for each iteration ${ }^{13}$. The challenge in implementing this procedure is choosing an appropriate normalization time.

The method utilized when I started in the nanoscale energy transport lab at UVa, was to normalize at a specific time index, typically taken as the lower bound of the fitting time, which is on the order of 100 ps. A scaling factor would be established at that time point and multiplied by each simulated data point to normalize the model to the data during each iteration of the fitting routine. In theory this method is valid, however for realistic data, signal fluctuations due to inherent noise in the signal made the final results of the fitting procedure dependent on the choice of normalization time. Consider for example Figure $5.41 \mathrm{~b}$ which shows the results of fitting simulated data of $100 \mathrm{~nm}$ of $\mathrm{Al}$ on $\mathrm{Si}\left(h_{\mathrm{BD}}=215\right.$ $\left.\mathrm{MW} / \mathrm{m}^{2} \mathrm{~K}\right)$, with $M=1,000,000, f_{s}=250 \mathrm{kHz}, f_{o}=125 \mathrm{kHz}$, and a $50 \%$ duty cycle of the modulation waveform, with added Gaussian noise for a signal to noise parameter ${ }^{14}$ of 8.5, see Figure 5.41a, as a function of scaling times. Using a single, user defined, scaling time the fitted $h_{\mathrm{BD}}$ varied upwards of $25 \%$ from the nominal value.

To eliminate the restriction of one scaling time, the TTR/TDTR fitting program was updated to calculate an average scaling parameter. During each iteration of the fitting routine, a scaling factor is determined for every data point as the ratio of the signal and the value of the simulated model at that time index. The average scaling factor over the

\footnotetext{
${ }^{13}$ Note that when fitting the ratio, i.e. $R=(-X / Y)$, the normalization process is not needed. However, it is often advantageous to fit the $X$ signal individually, and in the case of the low-rep (RegA) system, the ratio is constant, and therefore not particularly useful.

${ }^{14}$ The formal definition of the signal to noise parameter will be provided in Section 5.6.
} 


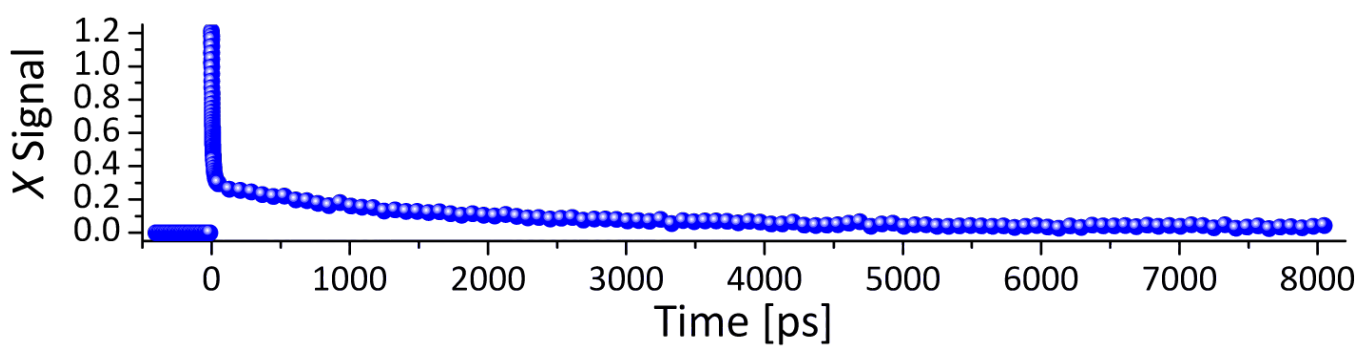

(a)

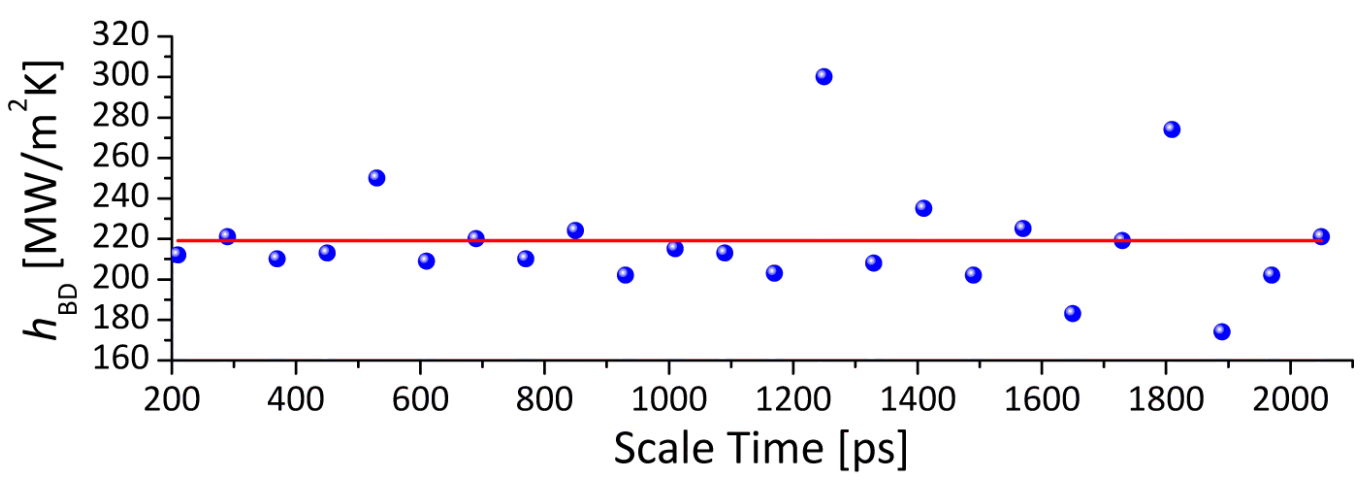

(b)

Figure 5.41: Fit of $h_{\mathrm{BD}}$ as a function of the selected scaling time between the data and the model, for simulated data of $100 \mathrm{~nm}$ of $\mathrm{Al}$ on $\mathrm{Si}\left(h_{\mathrm{BD}}=215 \mathrm{MW} / \mathrm{m}^{2} \mathrm{~K}\right)$, with $M=1,000,000, f_{s}=250 \mathrm{kHz}, f_{s}=125$ $\mathrm{kHz}$, and a $50 \%$ duty cycle of the modulation waveform, with added Gaussian noise for a signal to noise parameter of 8.5. The red line indicates the fit value using the free normalization routine.

temporal fitting window is then used to scale the model for that fitting iteration. In essence, this procedure frees the scaling time and allows it to shift during each iteration, see Figure 5.42. Using this methodology eliminates the choice of the scaling time and the dependence of the final fitting result on a quantity arbitrarily chosen by the user.

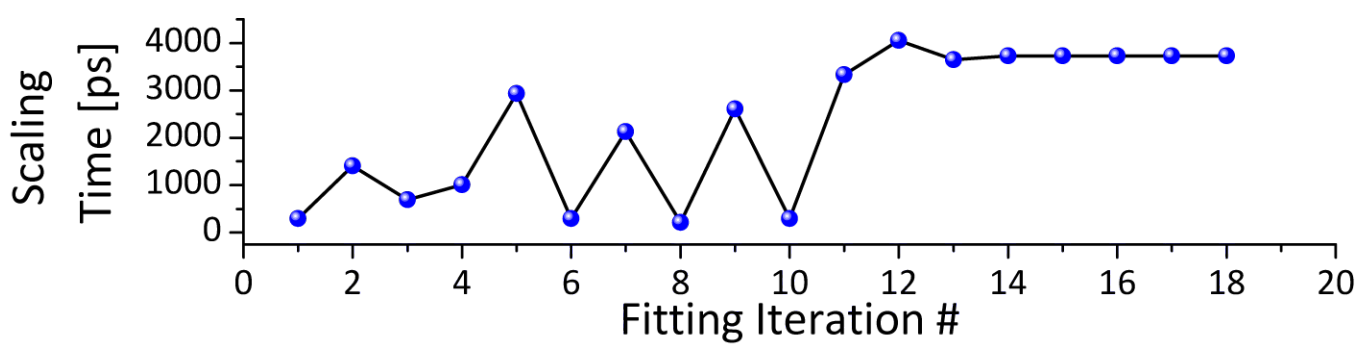

Figure 5.42: Scaling time as a function of fitting iteration from the TTR fitting program with the free normalization routine, for the fitting of $h_{\mathrm{BD}}$ for the data shown in Figure 5.41a. 


\subsection{Sensitivity Analysis}

The TTR/TDTR model developed in Chapter 4 requires a large number of input parameters to develop the output data, see Figure 5.43. In general it is desirable to have some understanding of the relationship between the input parameters and the model output. This knowledge provides a better understanding of the behavior of the model, helps to identify modeling deficiencies and unreasonable responses, and allows more focus to be placed on the input parameters that contribute most to output uncertainties. The fabrication of a sample, measurements taken via the TTR/TDTR experiment, and the characterization of the sample properties though ancillary methods all represent a significant experimental cost both in terms of real dollars and time invested. The more foreknowledge we have about the behavior of the system, the more efficiently we can direct resources and the more confidently we can propose new projects.

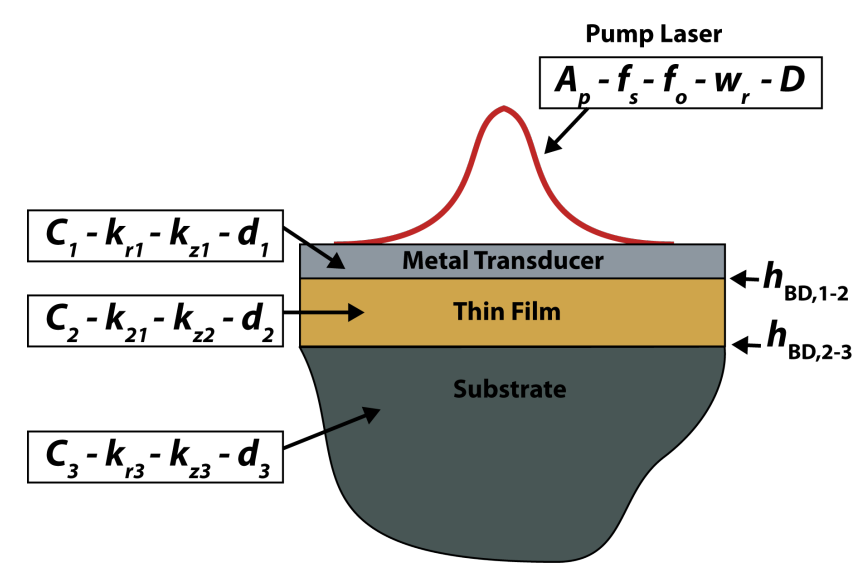

Figure 5.43: Schematic of the inputs required for the TTR/TDTR thermal model.

There are many terms that have been used in order to describe the impact that an input parameter has on the output of the model. Terms like "important", "sensitive", and "most influential" have been used in literature in order to specify the impact that an input parameter has [255]. In this work, we will generally consider the importance and sensitivity of the input parameters. To clarify, an important parameter will be one in which the variability in the input parameter has a significant impact on the variability in the output. Later in Chapter 6 we will investigate how the variability in the input parameters impacts the accuracy of deducing a parameter of interest. The sensitivity of the parameter will be 
a measure of, for a given variation in the input parameter, the variability in the model output. By these definitions, a parameter that is important is always sensitive because small changes in the input parameter can lead to large changes in the model output. On the other hand, a parameter that is sensitive is not necessarily important because the value of the parameter may be well known from some ancillary measurement.

In conducting a sensitivity analysis there are a large number of methods to choose from, all with benefits and costs. There are several good sources one can turn to to learn more about different sensitivity analysis techniques [255-258]. In general, sensitivity analysis methods can be broken down into two main categories: 1) local methods and 2) global methods. Using local methods, input variables are varied with a "one-at-a-time" (OAT) approach by some small amount from the anticipated mean value and the effect on the model output is found. In global methods all the input variables are varied simultaneously, usually selected from some form of a parameter space and the effects on the model output assessed. For this work in the discussion on parameter sensitivity we will use both local approach and quasi-global methods, and in Chapter 6 when discussing parameter importance we will also use a quasi-global methodology.

The most common of the local methods is the differential sensitivity analysis. The goal of the differential sensitivity analysis is to determine the local response of the model output to a particular input factor at a select point in the input parameter space [258]. Typically for our analyses we will work locally in the parameter space at the mean, or "best guess," of the input values. For a modeled system of $100 \mathrm{~nm}$ of $\mathrm{Al}$ on $\mathrm{Si}\left(h_{\mathrm{BD}}=1 \mathrm{MW} / \mathrm{m}^{2} \mathrm{~K}\right)$, with $k=\{-1,1\}, f_{s}=250 \mathrm{kHz}, f_{o}=125 \mathrm{kHz}$, and a $50 \%$ duty cycle of the modulation waveform, variations in thermal boundary conductance, $h_{\mathrm{BD}}$, and the specific heat capacity of the film, $L 1_{C}$, are shown in Figures 5.44 and 5.45 respectively.

In Figures 5.44a and 5.45a the baseline models and the models for the parameters reduced by $10 \%$ are shown, with both models normalized at $t=100$ ps. In Figures $5.44 \mathrm{~b}$ and $5.45 \mathrm{~b}$ the difference between the two models is shown. We can note that the thermal boundary conductance and the specific heat capacity of the film have a positive and negative response to the perturbation of the input variable respectively. In order to quantify this variation we can define a sensitivity coefficient. 


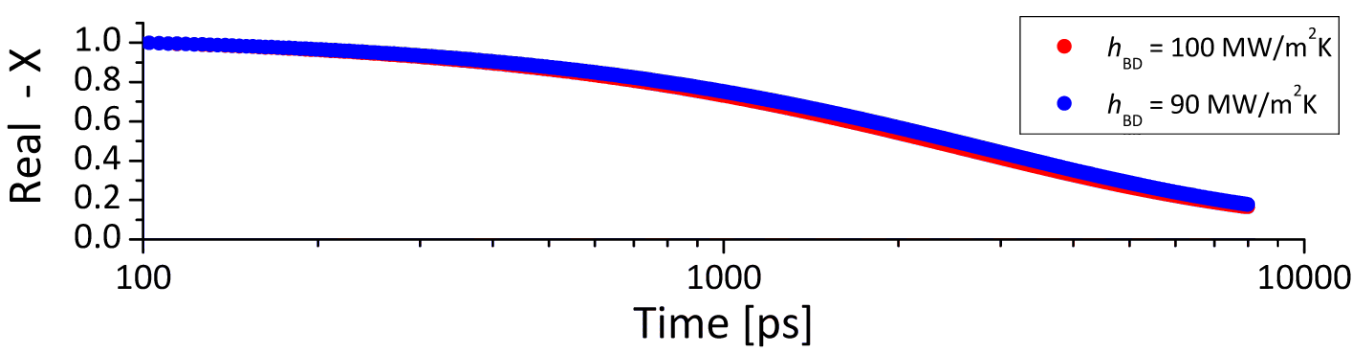

(a)

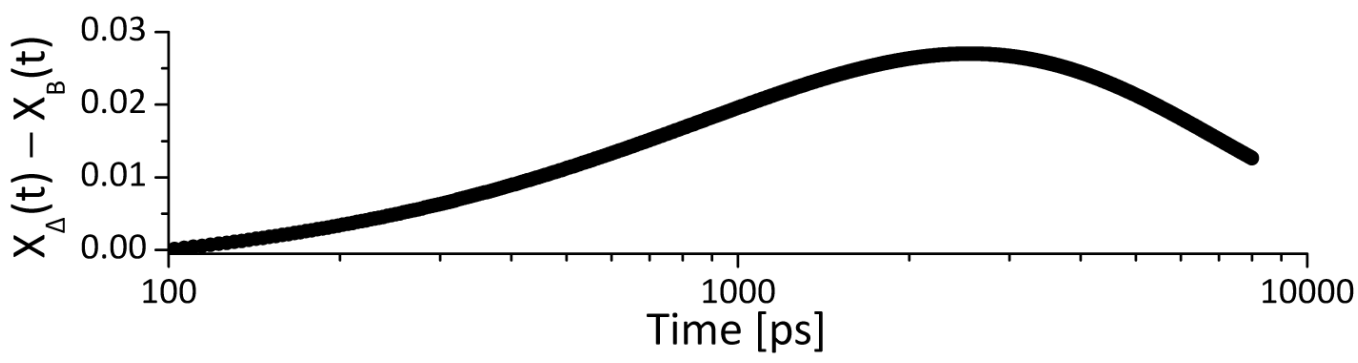

(b)

Figure 5.44: Modeled data of $100 \mathrm{~nm}$ of $\mathrm{Al}$ on $\mathrm{Si}\left(h_{\mathrm{BD}}=1 \mathrm{MW} / \mathrm{m}^{2} \mathrm{~K}\right)$, with $k=\{-1,1\}, f_{s}=250$ $\mathrm{kHz}, f_{o}=125 \mathrm{kHz}$, and a $50 \%$ duty cycle of the modulation waveform, for (a) the baseline case of $h_{\mathrm{BD}}=$ $100 \mathrm{MW} / \mathrm{m}^{2} \mathrm{~K}$ and for the perturbed case of $90 \%$ of the baseline case, and (b) the difference between the perturbed case and the baseline case as a function of time.

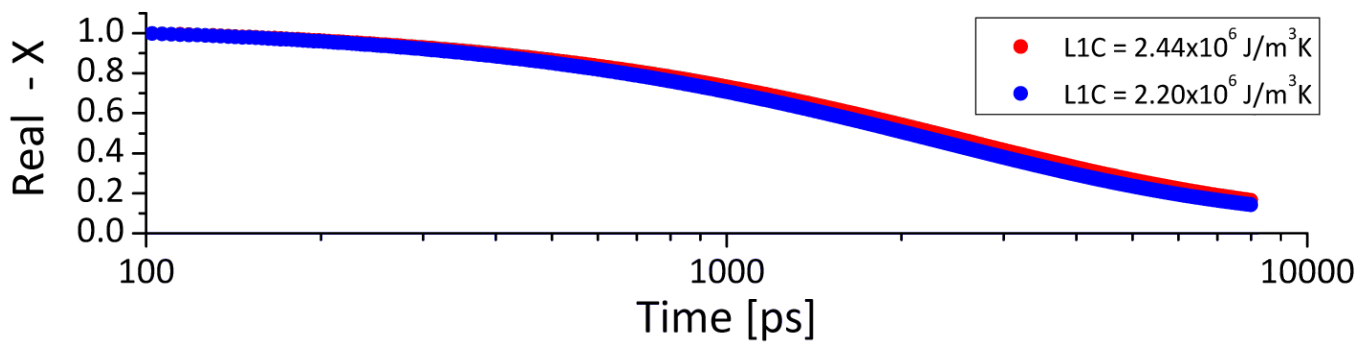

(a)

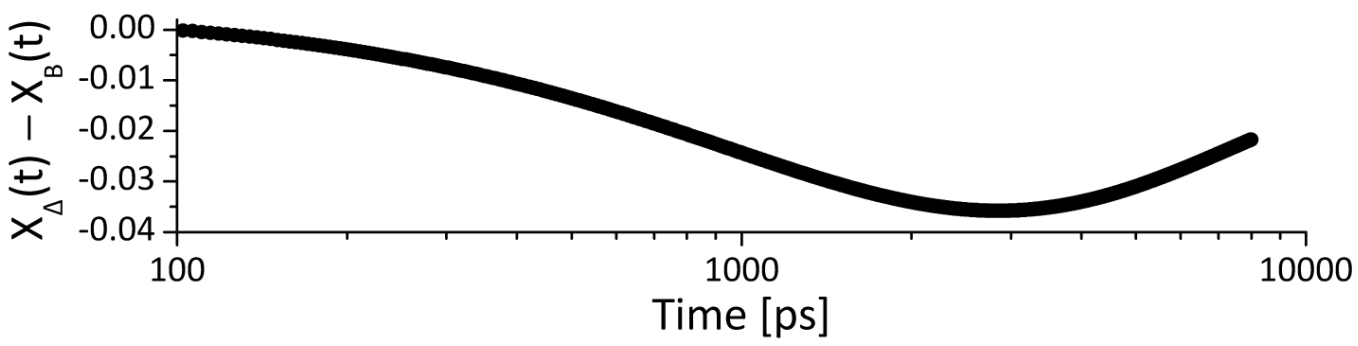

(b)

Figure 5.45: Modeled data of $100 \mathrm{~nm}$ of $\mathrm{Al}$ on $\mathrm{Si}\left(h_{\mathrm{BD}}=1 \mathrm{MW} / \mathrm{m}^{2} \mathrm{~K}\right)$, with $k=\{-1,1\}, f_{s}=250 \mathrm{kHz}$, $f_{o}=125 \mathrm{kHz}$, and a $50 \%$ duty cycle of the modulation waveform, for (a) the baseline case of $L 1_{C}=2.44 \times 10^{6}$ $\mathrm{J} / \mathrm{m}^{3} \mathrm{~K}$ and for the perturbed case of $90 \%$ of the baseline case, and (b) the difference between the perturbed case and the baseline case as a function of time. 


\subsubsection{Sensitivity Coefficient}

The sensitivity coefficient $S_{p}^{\Psi}$, is defined as the ratio of the change in the model output to the change in an input parameter, normalized by the central case and given by [258]:

$$
S_{p}^{\Psi}(t)=\frac{\partial \ln \Psi(t)}{\partial \ln p}=\frac{p_{B}}{\Psi_{B}(t)} \frac{\partial \Psi(t)}{\partial p}
$$

where $p$ is the thermophysical parameter of interest and $\Psi(t)$ is the output signal, i.e. magnitude $M$, phase $\theta$, real $X$, imaginary $Y$, or ratio $R=(-X / Y)$, and the subscript $B$ denotes the baseline case. By normalizing the sensitivity coefficient and assuming a constant perturbation of the input parameters, i.e. $p_{B} / \partial p$ is constant, we can compare the output sensitivity to different magnitudes of input perturbation and of different input magnitudes. While the differential method is straight forward and computationally efficient, one of the major draw backs is that while derivatives for simple models can be easily obtained, for more complex models differentiating the model can be quite difficult. If we assume that the perturbations to the input parameters are small, the derivatives can be approximated by a finite difference [255, 258]. Under this assumption, the sensitivity coefficient is given as:

$$
S_{p}^{\Psi}(t)=\frac{p_{B}}{\Psi(t)} \frac{\Psi_{\Delta}(t)-\Psi_{B}(t)}{p_{\Delta}-p_{B}}
$$

where the subscript $\Delta$ denotes the perturbed case. The sensitivity coefficient given in Equation 5.8 is the parameter commonly used in the nanoscale energy transport field to discuss the sensitivity of the TTR/TDTR model [75, 94, 245, 259-262].

One important consideration is that we have defined the sensitivity coefficient under the assumption of small perturbations. Therefore, we should have some quantification of when small is small enough as to not impact the results. To accomplish this, Figure 5.46 shows the sensitivity coefficient of the real portion of the lock-in amplifier signal, $X$, to $h_{\mathrm{BD}}$, as a function of the percentage that $h_{\mathrm{BD}}$ is perturbed. We will notice that above $0.1 \%$, the sensitivity begins to show nonlinearities and begins to deviate significantly for perturbations over $1 \%$. While literature typically takes the perturbation percentage to be on the order of $1-10 \%$ the sensitivity coefficient will be more valid for perturbation values under $0.1 \%$. 


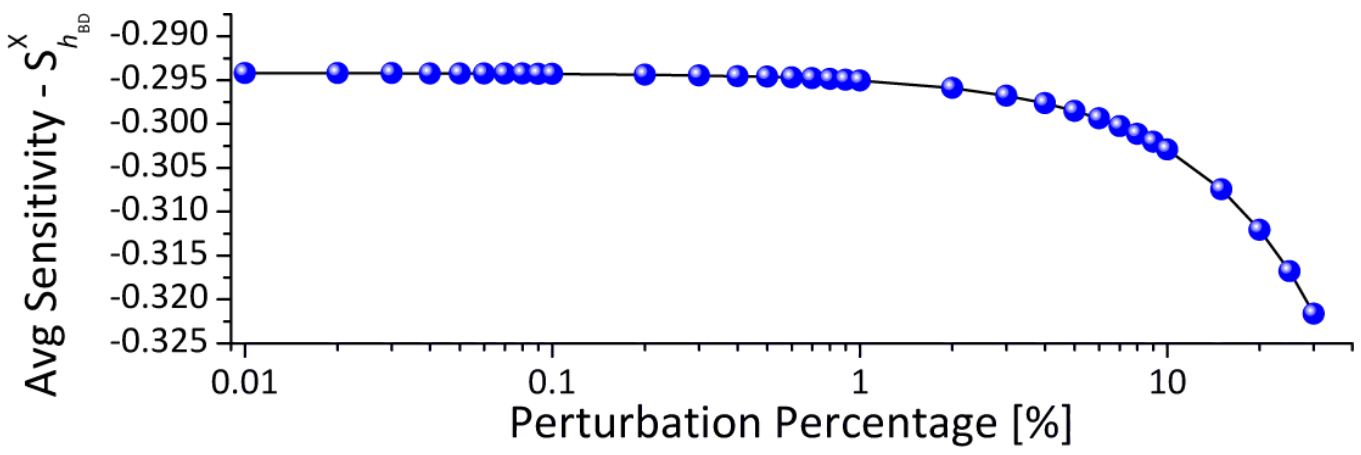

Figure 5.46: Average sensitivity parameter, $S_{h_{B D}}^{X}$, over $100-8000 \mathrm{ps}$ as a function of the percentage that $h_{\mathrm{BD}}$ is perturbed

\section{Sensitivity Coefficient as a Function of Time}

As an example of the sensitivity coefficient presented in Equation 5.8 the sensitivity as a function of time for a modeled system of $100 \mathrm{~nm}$ of $\mathrm{Al}$ on $\mathrm{Si}\left(h_{\mathrm{BD}}=1 \mathrm{MW} / \mathrm{m}^{2} \mathrm{~K}\right)$, with $k=\{-1,1\}, f_{s}=250 \mathrm{kHz}, f_{o}=125 \mathrm{kHz}$, and a $50 \%$ duty cycle of the modulation waveform is shown in Figure 5.47. In the figure, the most sensitive parameters are the pump/probe waist and the absorbed laser power. However as discussed in Section 5.4.2, it is typical to normalize the model and the data when fitting to deduce a thermophysical parameter of interest. Therefore when developing the sensitivity coefficient, and comparing the baseline model, $\Psi_{B}$, to the perturbed system, $\Psi_{\Delta}$, it is necessary to normalize these models relative to one another which is typically done at the lower time bound, see Figure $5.47 \mathrm{~b}$. With the normalization procedure, the system is no longer sensitive to the absorbed laser power, or pump/probe spot size.

While the sensitivity coefficient as formulated in Equation 5.8 and presented in Figure 5.47 provides quantitative insight into how the model changes for changes in the various input parameters as a function of time, it can be difficult to compare between different material systems. Because of the temporal dependence of $S_{p}^{\Psi}$, adding a range of input values to the sensitivity calculations yields results in three dimensions. Figure 5.48 shows the sensitivity to $h_{\mathrm{BD}}$ of a system of $100 \mathrm{~nm}$ of $\mathrm{Al}$ on $\mathrm{Si}$ as a function of pump-to-probe delay time and the $h_{\mathrm{BD}}$ of the $\mathrm{Al} / \mathrm{Si}$ interface. While there is plenty of information contained in Figure 5.48, it can be difficult to visually interpret the impact of $h_{\mathrm{BD}}$ on the sensitivity. 


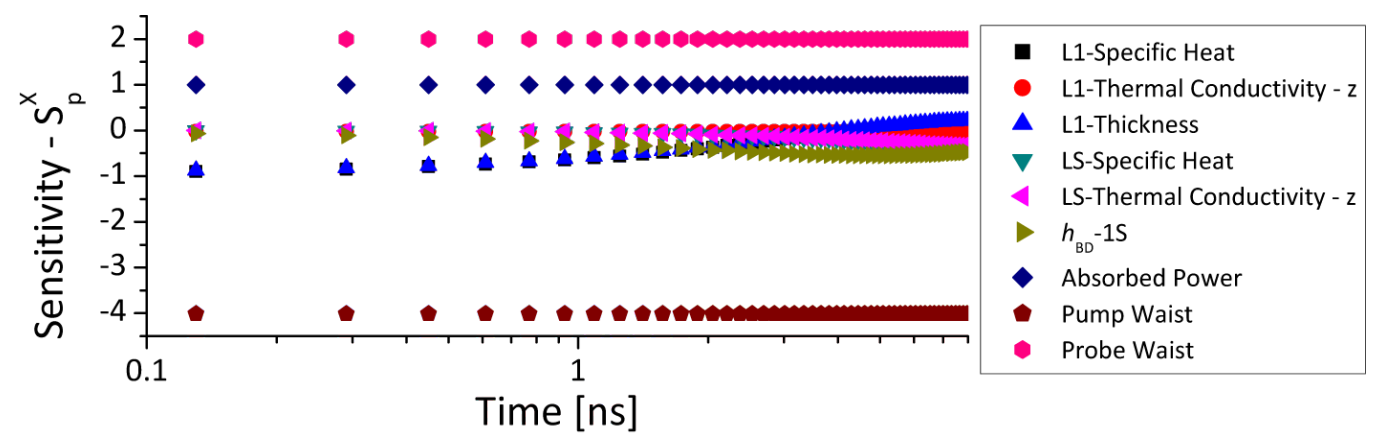

(a)

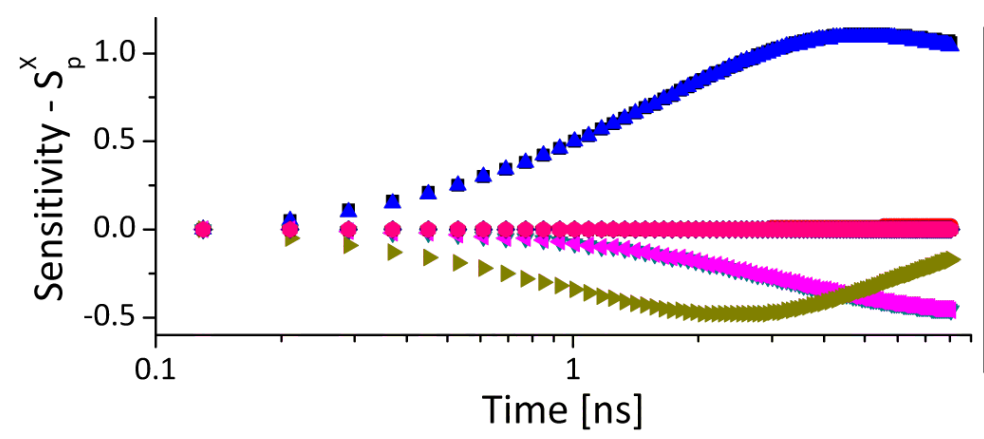

- L1-Specific Heat

- L1-Thermal Conductivity - $z$

- L1-Thickness

$\nabla$ LS-Specific Heat

4 LS-Thermal Conductivity - $z$

$h_{\mathrm{BD}}-1 \mathrm{~S}$

- Absorbed Power

- Pump Waist

- Probe Waist

(b)

Figure 5.47: Sensitivity coefficient, see Equation 5.8, as a function of time from 130-8050 ps, for (a) nonnormalized and (b) normalized data. The modeled system is $100 \mathrm{~nm}$ of $\mathrm{Al}$ on $\mathrm{Si}\left(h_{\mathrm{BD}}=1 \mathrm{MW} / \mathrm{m}^{2} \mathrm{~K}\right)$, the details of the modeled system can be found in Appendix S.1.

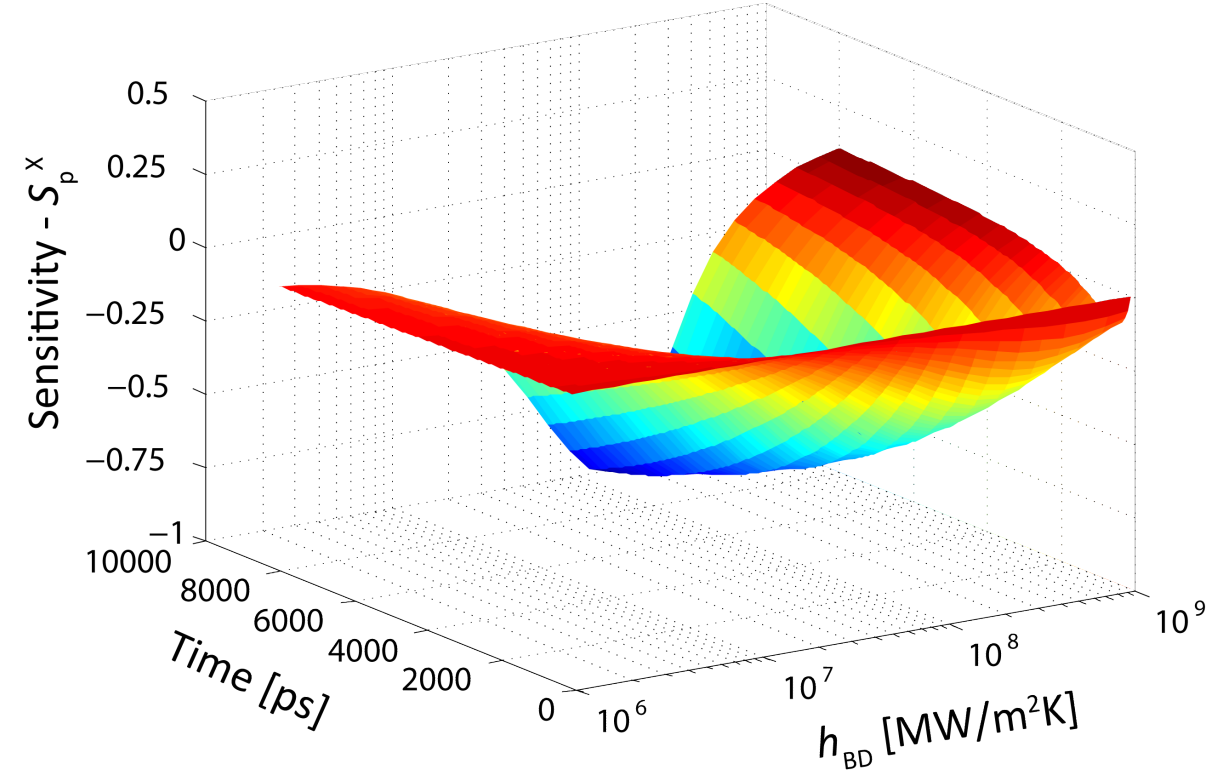

Figure 5.48: Sensitivity to $h_{\mathrm{BD}}$ of a system of $100 \mathrm{~nm}$ of $\mathrm{Al}$ on $\mathrm{Si}$ as a function of time and the assumed $h_{\mathrm{BD}}$ at the $\mathrm{Al} / \mathrm{Si}$ interface. 


\section{Sensitivity as a Function of $p$}

To simply the analysis of the data in Figure 5.48, it would be helpful to be able to remove one of the axes and reduce the information to a two-dimensional plot. This is typically done by attempting to remove the time dependence from the sensitivity. In order to remove the time dependence of the sensitivity coefficient, most literature chooses an arbitrary time, typically $100 \mathrm{ps}$, to track the sensitivity as a function of parameter [94, $245,259-262]$. While this is what is most commonly done in literature, there are two risks to using this methodology. The first is that with the normalization of the data impacting the magnitude of the sensitivity coefficient near the normalization time, see Figure 5.47, the time-independent sensitivity parameter will be dependent on the relative relationship between the normalization time and the tracking time. The second risk is that the choice of the tracking time will not sufficiently capture the trend in sensitivity. As shown in Figure 5.47, the sensitivity of a parameter can increase, decrease, and/or stay the same as a function of time. In order to make the time-independent sensitivity coefficient more universal, for the work in this dissertation rather than selecting an arbitrary track time, the sensitivity is averaged over time.

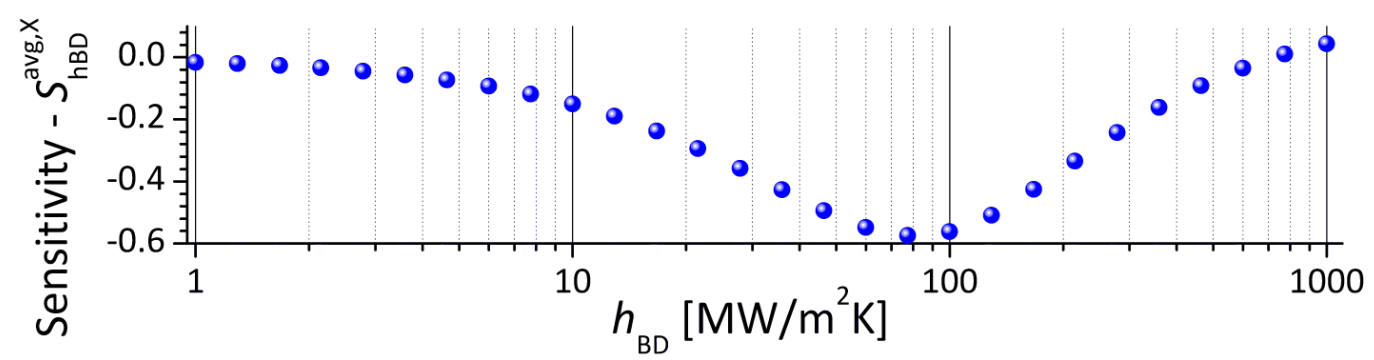

(a)

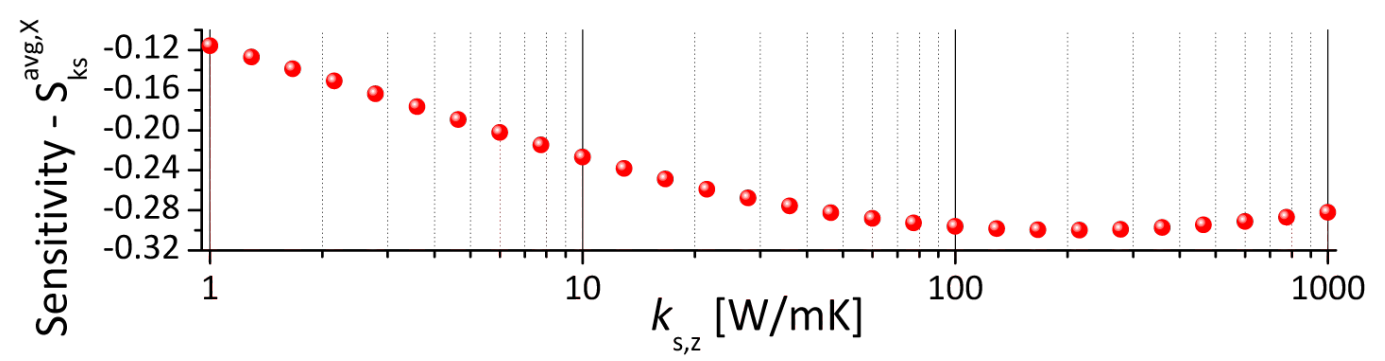

(b)

Figure 5.49: Averaged sensitivity as a function of parameter, $p$, for a modeled system of $100 \mathrm{~nm}$ of $\mathrm{Al}$ on $\mathrm{Si}$ for (a) $S_{\mathrm{hBD}}^{X}$ as a function of $h_{\mathrm{BD}}$ and (b) $S_{\mathrm{kS}}^{X}$ as a function of the substrate thermal conductivity, $k_{S z}$ 


\subsubsection{Statistical Sensitivity Analysis}

At this point the development and application of the sensitivity coefficient, either as a function of time or parameter has relied on a deterministic view of the TTR/TDTR model. That is to say we have assumed that all the input values are known exactly and only perturbed these values to generate the sensitivity coefficient. As discussed in Chapter 2, there are many factors out of the experimenters control that will impact the final thermophysical parameters of the fabricated system. Therefore, when proposing a potential system to fabricate such as when writing a new grant, it is important to take a statistical treatment of sensitivity.

In order to make the formulation of the sensitivity coefficient more stochastic in nature rather than treating each input to the thermal model as a fixed quantity, we can define each value by a probability distribution. To achieve this, the LabVIEW ${ }^{\mathrm{TM}}$ modeling program was adapted to allow the input parameters to be described by either a normal or uniform distribution centered about the anticipated value, and with the range of values described by the standard deviation given as a percentage. The program randomly selects an input parameter from the given distributions at the beginning of each iteration and then calculates the averaged sensitivity coefficient in the normal manner. With the convergence criteria and node spacing formulations developed in Sections 5.3 and 5.4.1 respectively, accurate and efficient large scale modeling of the quasi-stochastic model sensitivity is attainable.

As an example we will consider a model of $100 \mathrm{~nm}$ of $\mathrm{Al}$ on $\mathrm{Si}\left(h_{\mathrm{BD}}=215 \mathrm{MW} / \mathrm{m}^{2} \mathrm{~K}\right)$, with $k=\{-1,1\}, f_{s}=76 \mathrm{MHz}, f_{o}=1 \mathrm{MHz}$, and a $50 \%$ duty cycle of the modulation waveform. For the primary thermophysical parameters of the model the standard deviation of the normal distributions used are shown in Table 5.5. For each iteration of the sensitivity analysis the input parameters were chosen by random from each of the respective distributions, and the sensitivity to the substrate thermal conductivity and thermal boundary conductance calculated. To illustrate this, Figure 5.50 shows the distribution of the input values for the substrate thermal conductivity (Figure 5.50a) and the thermal boundary conductance (Figure 5.50b) for 5,000 trial iterations. The resulting distributions of the sensitivity coefficients for the substrate thermal conductivity and interface conductance are shown in Figure 5.51, with the red vertical lines representing the sensitivity coefficient 
Table 5.5: Uncertainty in TTR/TDTR input parameters for the example of a modeled system of $100 \mathrm{~nm}$ of $\mathrm{Al}$ on $\mathrm{Si}\left(h_{\mathrm{BD}}=215 \mathrm{MW} / \mathrm{m}^{2} \mathrm{~K}\right)$ in Section 5.5.2 demonstrating the implementation of stochastic sensitivity analysis, where $\mathbf{L} \mathbf{1}$ and $\mathbf{L S}$ denote the film and substrate layer respectively.

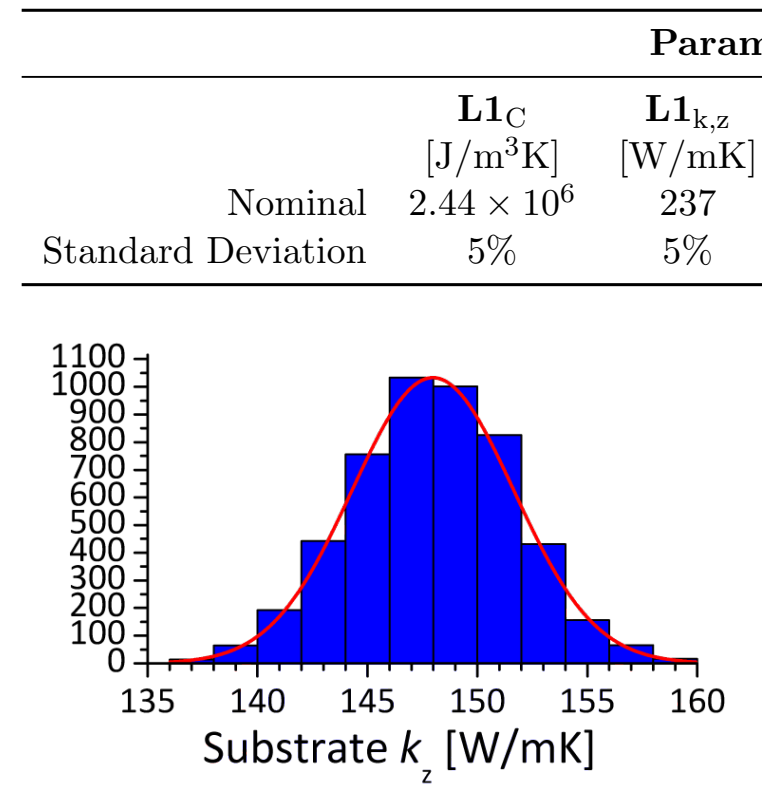

(a)

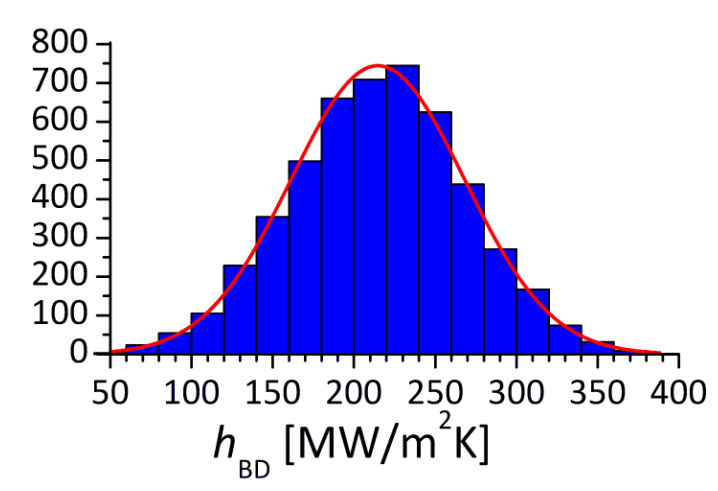

(b)

Figure 5.50: Examples of the distribution of input values for a modeled system of $100 \mathrm{~nm}$ of $\mathrm{Al}$ on $\mathrm{Si}$ with $k=\{-1,1\}, f_{s}=76 \mathrm{MHz}, f_{o}=1 \mathrm{MHz}$, and a $50 \%$ duty cycle of the modulation waveform for (a) substrate thermal conductivity with a nominal value of $k_{z}=148 \mathrm{~W} / \mathrm{mK}$ and (b) $h_{\mathrm{BD}}$ with a nominal value of $h_{\mathrm{BD}}=215 \mathrm{MW} / \mathrm{m}^{2} \mathrm{~K}$.

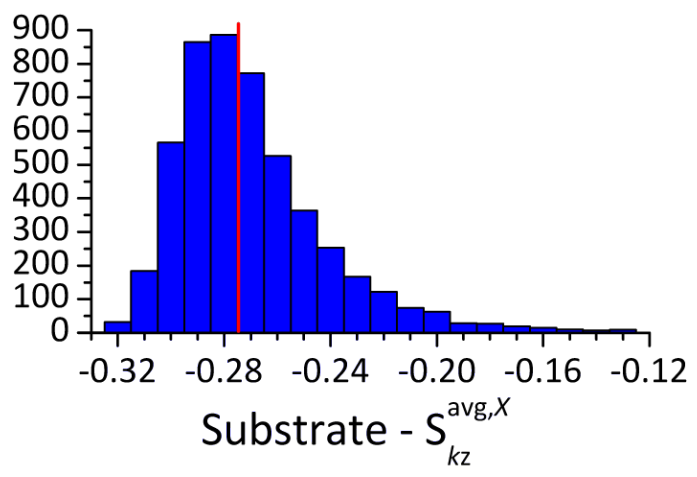

(a)

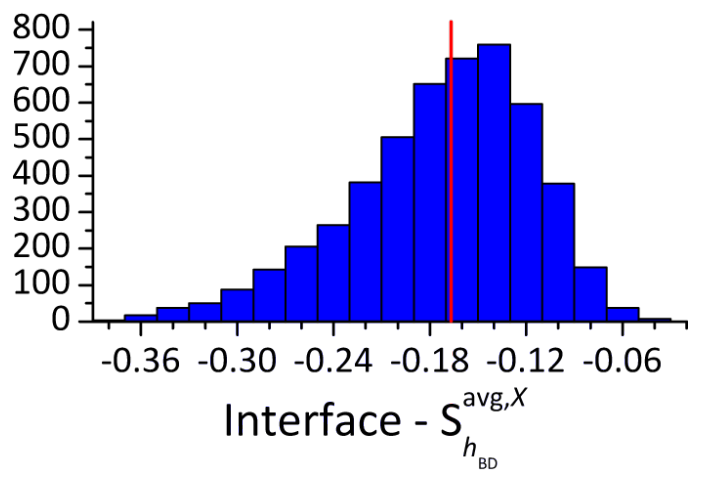

(b)

Figure 5.51: Resulting distributions of the average sensitivity coefficient for substrate thermal conductivity and thermal boundary conductance based on the stochastic sensitivity analysis example in Section 5.5.2. The distributions represent the results for $N=5,000$ iterations of the sensitivity model, and the red vertical lines represent the sensitivity results using the nominal input parameters.

calculated via the nominal thermophysical parameters. We can notice immediately that even though the uncertainty in the input parameters was assumed to be normally distributed, 
the distributions of the sensitivity coefficients for each parameter are non-normal. In the case of the substrate thermal conductivity the probability indicates that the sensitivity is likely to increase for the given input uncertainties. In contrast, the results show that for the thermal boundary conductance the variations in the input parameters will most likely decrease the sensitivity to deducing $h_{\mathrm{BD}}$. While in general, for this example the distribution of sensitivity coefficients fall within the range of values that are acceptable, we will note than when comparing the range of the distribution of sensitivity coefficients calculated for $h_{\mathrm{BD}}$, see Figure 5.51b, to those calculated as a function of $h_{\mathrm{BD}}$ over all values for the 100 $\mathrm{nm} \mathrm{Al}$ on Si system, see Figure 5.49a, that this distribution represents a significant portion of the range of possible sensitivity values. Later in Chapter 6 we will discuss how the sensitivity coefficient of the parameter of interest impacts the precision of the fitted result for a given amount of noise in the data, which we will now discuss quantitatively.

\subsection{Defining Noise in Data}

To this point we have only dealt with ideal modeled data, that is data with no sources of noise or random variation. In real systems there are several sources of intrinsic (random) noise present that affect the overall noise level in the measured signal including $1 / f$ noise ("pink noise"), Shot noise, radio frequency (RF) noise, and Johnson noise. The total random noise in the system can be defined as the square root of the sum of the squares of each of the individual noise components [263]. While a large portion of the noise is filtered out by the lock-in amplifier in TTR/TDTR signals, and several methods can be utilized to help mitigate the different sources of noise [228], it is not possible to remove all noise from the data. The remaining noise in the data will in part contribute to the variations in the fitted results and reduce the precision to which a parameter may be deduced (more details will follow in Chapter 6). Therefore, in order to estimate anticipated parameter precision and determine whether the signal is sufficient to merit data collection, it is important to have a method to quantify the amount of noise in the data. 


\subsubsection{Measuring Signal Noise via the Lock-in Amplifier}

Using the lock-in amplifier in the TTR/TDTR system (Stanford Research Systems SR844) it is possible to measure the level of noise in the signal [263]. While the lockin amplifier itself is an excellent noise filter, it will measure any signal close to the reference frequency. Therefore, any sources of noise with frequency components within the detection bandwidth of the lock-in amplifier near the reference frequency will show up in the measured signal. The simplest measure of the signal noise is the standard deviation of the measured signal. This is difficult however for the lock-in amplifier to calculate in real time. To calculate the noise in a given channel, i.e. $X$ or $Y$, the lock-in amplifier first takes a moving average of the signal at a rate of $512 \mathrm{~Hz}$ and subtracts that value from the current signal to obtain the signal deviation. The moving average of the mean of the deviations over time is then calculated and is called the Mean Average Deviation (MAD). The rate at which the MAD is collected is a function of the time constant of the lock-in amplifier. Assuming a Gaussian distribution of the noise, the MAD is scaled by a factor known as the Equivalent Noise Bandwidth (ENBW). The ENBW is a function of the slope of the lowpass filter in the lock-in amplifier. The noise measurement given by the lock-in amplifier, $X_{\text {Noise,LIA }}$, is independent of the magnitude and slope of the time constant, but fluctuations in the measured noise depend on these settings. Longer time constants and/or higher slopes provide more stable readings, however a longer time is needed for the results to settle after a variation in the input signal. The noise measurement from the lock-in amplifier can be converted to an equivalent standard deviation, given in terms of voltage, by scaling by the ENBW:

$$
V_{\text {Noise }}^{X}=X_{\text {Noise }, \text { LIA }} \times \sqrt{\mathrm{ENBW}}
$$

While the lock-in amplifier provides a good real-time measurement of the noise in the output signal, typically a measurement of noise alone is only partially useful. If the noise level is low but the signal strength is on a similar order, the ability to deduce parameters from the data and/or the confidence in said parameters will be limited. Therefore, in reporting the noise in a particular signal it is common to group the noise measurement and the signal magnitude together in a signal-to-noise quantification. 


\subsubsection{Defining a Signal-to-Noise Parameter}

Traditionally, the method of quantifying the comparison between the signal and noise in data is through the signal-to-noise ratio (SNR). Being defined for the lock-in amplifier as the ratio of the signal voltage to the noise voltage:

$$
\mathrm{SNR}=\frac{V_{\mathrm{Sig}}^{\Psi}}{V_{\text {Noise }}^{\Psi}}
$$

were $\Psi$ represents the type of signal (magnitude, phase, etc.). Typically the noise in the system is characterized by measuring the level of signal fluctuations prior to $t_{o}$, and the signal characterized by the magnitude of the signal taken at the peak around $t_{o}$.

In order to allow for the noise parameter to be calculated for data sets post-collection and independent of normalization, we define a modified version of the signal-to-noise ratio. We will define this quantity as the signal-to-noise parameter (SNP) due to its similar meaning, but modified formulation, as compared to the more traditional SNR. The SNP is an averaged parameter over all time points within the region of interest. Usually this begins at the smallest time index considered, typically $100 \mathrm{ps,}$, and includes all subsequent data points. At each time index the average signal is calculated and used to normalize all the data points at that index. The standard deviation of that data set is then taken and the average collected over all data points. The value is finally inverted to increase the scale of the final value. Mathematically the SNP is represented by:

$$
\mathrm{SNP}=\left\{\frac{1}{N} \sum_{j=1}^{N}\left[\frac{1}{n-1} \sum_{i=1}^{n}\left(\frac{x_{i}}{\bar{x}(j)}-\bar{x}(j)\right)^{2}\right]\right\}^{-1}
$$

where $n$ is the number of data sets and $N$ is the number of time points considered.

As an example, we will consider a modeled system of $100 \mathrm{~nm}$ of $\mathrm{Al}$ on $\mathrm{Si}\left(h_{\mathrm{BD}}=\right.$ $1 \mathrm{MW} / \mathrm{m}^{2} \mathrm{~K}$ ), with $k=\{-1,1\}, f_{s}=250 \mathrm{kHz}, f_{o}=125 \mathrm{kHz}$, and a $50 \%$ duty cycle of the modulation waveform. Figure 5.52 shows the modeled data with Gaussian noise added to create three different levels of noise. The noise was added by assuming that each data point was the mean value of a Gaussian distribution and with the standard deviation used to control the noise level. A point was randomly selected from each distribution to rebuild 
the modeled data with the added noise. More details on the method of simulating noise in the modeled data will be presented in Chapter 6 when discussing simulations realistic TTR/TDTR data. The added noise resulted in SNPs for plots (a)-(c) in Figure 5.52 of $4.18,8.65$ and 12.12 respectively.

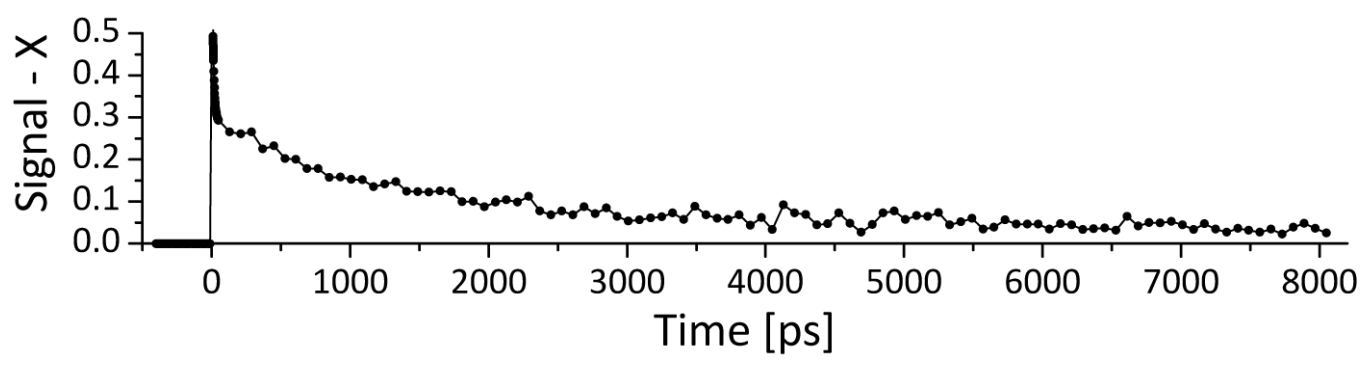

(a)

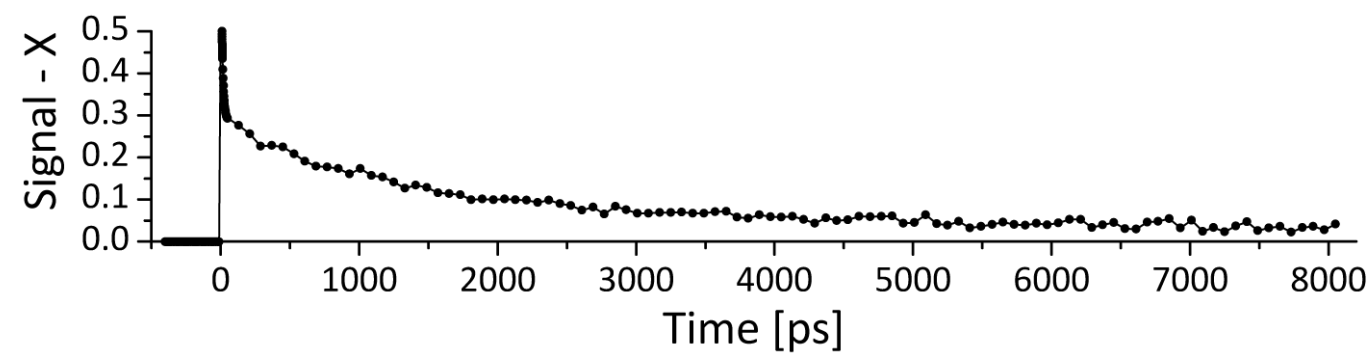

(b)

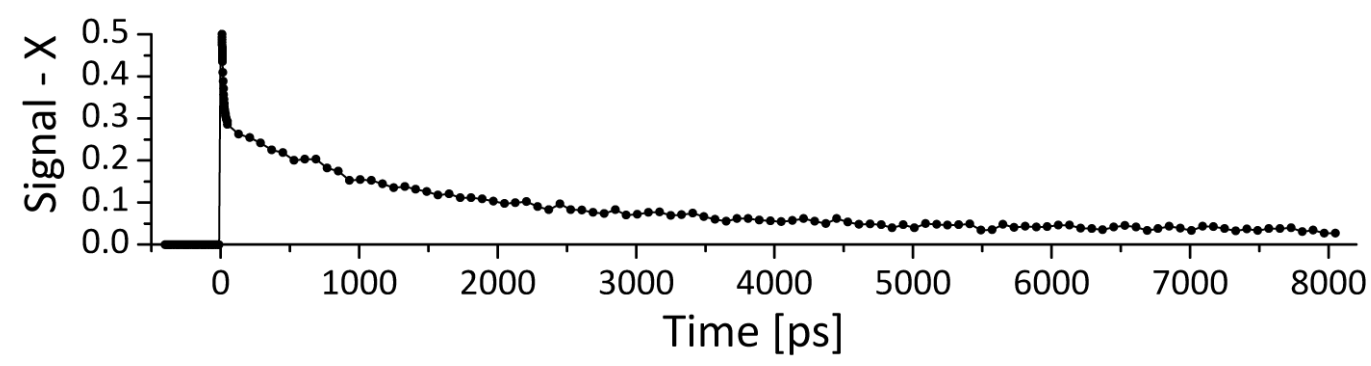

(c)

Figure 5.52: Modeled data sets of $100 \mathrm{~nm}$ of $\mathrm{Al}$ on $\mathrm{Si}$ with $h_{\mathrm{BD}}=2.15 \mathrm{MW} / \mathrm{m}^{2} \mathrm{~K}$ for 3 different signal-tonoise parameters a) 4.18 , b) 8.65 , and c) 12.12 .

To illustrate the added noise, Figure 5.53 shows the distribution of noise in 10 trials of the modeled system for the three simulated SNPs. By definition of the formulation in Equation 5.11, the data at each time index is normalized and therefore overall has an average value of one. The SNP is then calculated based on the average standard deviation across all the time indexes. This provides a statistical advantage by averaging over all time in the model, as opposed to calculating a signal-to-noise ratio based on the peak in the 
signal alone. To estimate the SNP in real time during the TTR/TDTR experiment, rather than calculating the SNP based on a collection of scans, data can be collected at a fixed time at the midpoint in the region of interest and averaged in real time using a "staring" routine until sufficient data has been collected to produce an accurate result. This will allow a real-time check before data is collected to ensure that there is sufficient signal compared to the noise to warrant data collection.

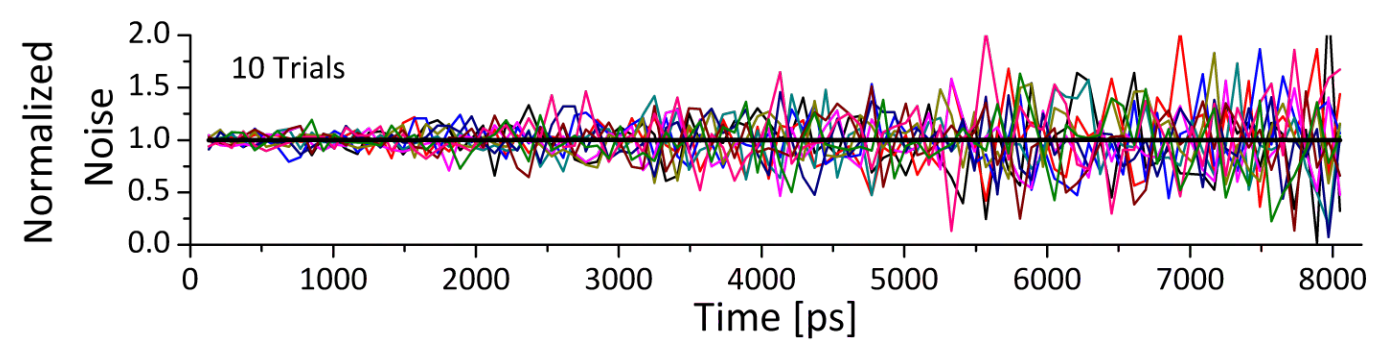

(a)

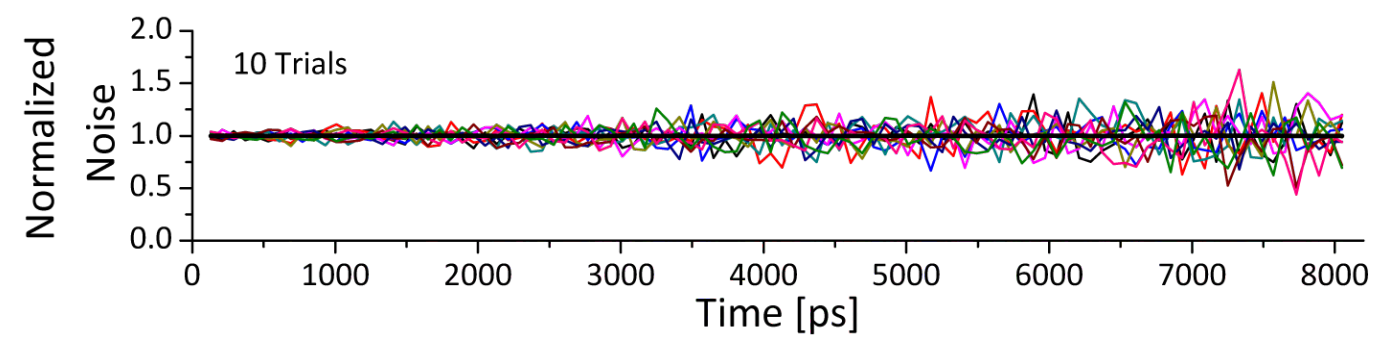

(b)

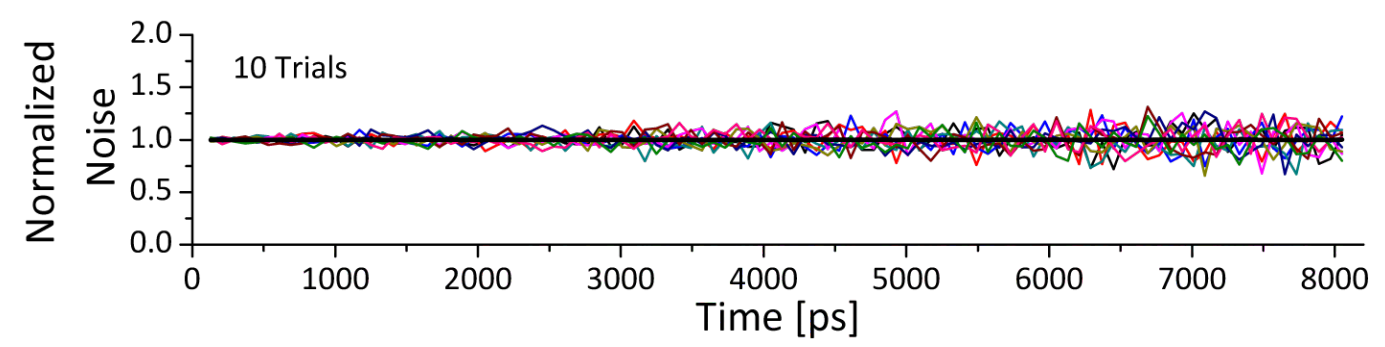

(c)

Figure 5.53: Distribution of noise in 10 trials of the modeled system of $100 \mathrm{~nm}$ of $\mathrm{Al}$ on $\mathrm{Si}\left(h_{\mathrm{BD}}=\right.$ $1 \mathrm{MW} / \mathrm{m}^{2} \mathrm{~K}$ ), with $k=\{-1,1\}, f_{s}=250 \mathrm{kHz}, f_{o}=125 \mathrm{kHz}$, and a $50 \%$ duty cycle of the modulation waveform, with Gaussian noise added to achieve signal-to-noise parameters of $4.18,8.65$, and 12.12 for panes (a)-(c) respectively. 


\subsection{Post-processing of Data to Remove Outliers}

One of the challenges in analyzing the results of the TTR/TDTR model is that in almost all instances the model will output a quantitative result. It is the job of the experimenter however to determine whether the results are valid. The obvious first check is to decide whether the result makes sense. If the result is not physical, such as a negative thermal conductivity, it is easy to attribute the data point to some error and discard it. Additionally, if a result is highly uncharacteristic in comparison to the rest of the data, it is highly likely that result can be discarded as well. The difficulty arises as a result becomes less and less uncharacteristic in comparison to the rest of the data. At that point, arbitrarily removing a result introduces the possibility of artificially influencing the final conclusions. To remove this risk we can turn to statistical methodologies in order to test the TTR/TDTR data for the presence of statistical outliers.

In 1980 D.M. Hawkins provided a definition of a statistical outlier as: “.. an observation, which so much deviates from other observations as to arouse suspicions that is was generated by a different mechanism" [264]. There are several sources that lead to fluctuations in experimental data: 1) the inherent probabilistic fluctuations in the results that cannot be removed, 2) fluctuations due to errors in the measurement devices, such as from background noise, and 3) imperfect collection and analysis of the data, such as biased sampling.

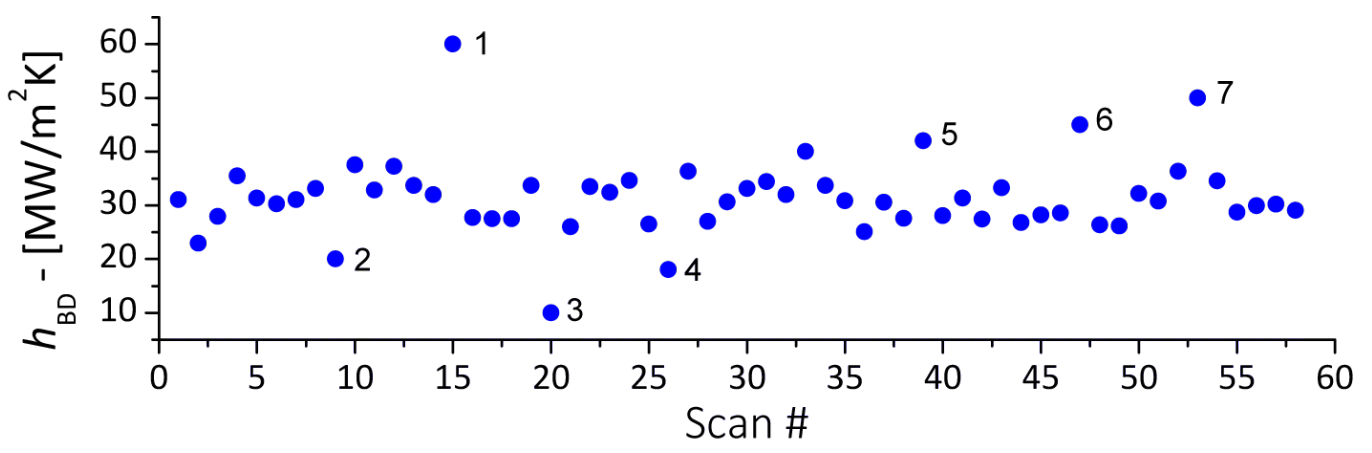

Figure 5.54: Theoretical $h_{\mathrm{BD}}$ values for a data set containing 58 data points.

Fortunately there are several statistical methods that can be used to identity whether a data point represents a statistical outlier, and these methods have be compared for various 
types of measurements $[264,265]$. Because the results of the TTR/TDTR measurement are relatively simple, we will not spend the time contrasting and discussing the various types of outlier identifying formulations. The purpose here is not to present anything novel, but rather to demonstrate how simple methods can be used to remove the arbitrary, and statistically dangerous method of throwing away data based on visual inspection and encourage a more statistically robust treatment of data.

\subsubsection{Removing Statistical Outliers}

To statistically handle the treatment of outliers the generalized extreme Studentized deviate (ESD) test developed by Rosner in 1983 is presented [266]. The generalized ESD test allows for the detection of $r$ outliers in univariant data that is assumed to follow an approximately normal distribution. If the data is not normally distributed, the generalized ESD test can falsely identify data points as outliers when they are in fact apart of the non-normal distribution. It is therefore prudent to check for normality of the data set before applying the generalized ESD routine. There are several numerical and graphical methods to test for normality, several of which are discussed in Appendix D.1. Figure 5.55 shows normality testing for the data shown in Figure 5.54 using graphical analysis of the data distribution and distribution parameters, Figure 5.55a, and using more quantitative analysis through a quantile-quantile plot and the Shaprio-Wilk normality test, Figure 5.55b. While not strictly meeting the criteria for normality, the generalized ESD test only requires that the data approximate a normal distribution and therefore the data in Figure 5.54 can be analyzed. The other major strength of the generalized ESD test is that unlike similar outlier tests such as the Grubbs test [267], the number of outliers does not need to be specified exactly but rather an upper bound for the number of potential outliers provided.

To test for statistical outliers the implementation of the generalized ESD test is based on the following hypothesis:

$$
\begin{aligned}
& \mathrm{H}_{0} \text { : There are no outliers in the data set } \\
& \mathrm{H}_{1} \text { : There are up to } r \text { outliers in the data set }
\end{aligned}
$$




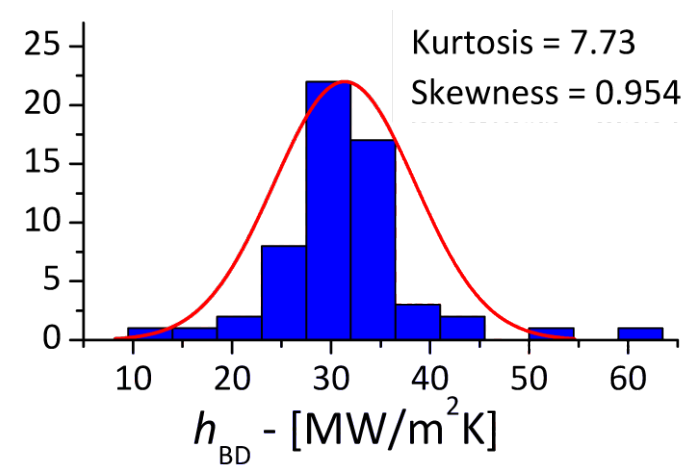

(a)

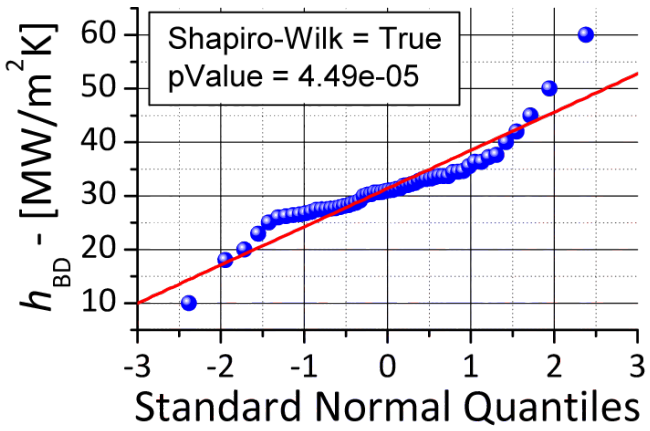

(b)

Figure 5.55: Normality testing of the data shown in Figure 5.54 by (a) a histogram plot with the distribution skew and kurtosis denoted and (b) a quantile-quantile plot with the results of the Shapiro-Wilk test for normality displayed.

where $\mathrm{H}_{0}$ and $\mathrm{H}_{1}$ are the null and alternative hypotheses respectively. To test the hypothesis, assuming a total of $r$ potential outliers, for each iteration of the routine, i.e. $i=1,2,3 \ldots r$, the test statistic, $R_{i}$, is computed where:

$$
R_{i}=\frac{\max \left|x_{i}-\bar{x}\right|}{s}
$$

and where $\bar{x}$ is the sample mean and $s$ is the sample standard deviation. The critical value, $\lambda_{i}$, is then calculated using the formulation:

$$
\lambda_{i}=\frac{(n-i) t_{p, n-i-1}}{\sqrt{\left(n-i-1+t_{p, n-i-1}^{2}\right)(n-1+1)}}
$$

where $n$ is the number of data points, $i$ is the current iteration, and $t_{p, n-i-1}$ is the $100 p$ percentage point of the Student's t-distribution with $n-i-1$ degrees of freedom with $p$ given by:

$$
p=1-\frac{\alpha}{2(n-i+1)}
$$

where $\alpha$ is the significance level. For each iteration the test statistic and critical value can be tabulated as shown in Table 5.6. After each iteration the data point that maximizes the quantity $\left|x_{i}-\bar{x}\right|$ is removed as the potential outlier and Equations 5.13-5.15 are recalculated assuming $n-1$ data points. The total number and identity of the outliers is determined by 
Table 5.6: Tabulated parameters of the generalized Studentized extreme deviate test for outlier based on the data in Figure 5.54 assuming $r=5$ potential outliers and a significance level of $\alpha=0.05$.

\begin{tabular}{ccccc}
\hline Trial, $i$ & $h_{\mathrm{BD}} \mathrm{MW} / \mathrm{m}^{2} \mathrm{~K}$ & Test Statistic, $R_{i}$ & Critical Value, $\lambda_{i}$ & $\mathrm{H}_{0}$ \\
\hline 1 & $6.00 \times 10^{7}$ & 4.015 & 3.187 & Reject \\
2 & $1.00 \times 10^{7}$ & 3.438 & 3.173 & Reject \\
3 & $5.00 \times 10^{7}$ & 3.455 & 3.159 & Reject \\
4 & $4.50 \times 10^{7}$ & 2.916 & 3.144 & Accept \\
5 & $1.80 \times 10^{7}$ & 2.827 & 3.128 & Accept \\
\hline
\end{tabular}

the maximum $i$ in which $R_{i}>\lambda_{i}$.

In the case of the data shown in Figure 5.54 the results of the generalized ESD test presented in Table 5.6 indicate that there are 3 outliers present in the data, namely the points labeled 1,3, and 7. These data points can be "safely" removed ${ }^{15}$ from this data set, and the remaining collection of data processed to produce a statistical result, which will be discussed in more depth in Chapter 6.

\subsection{Chapter Summary}

In this chapter many details were developed that are often ignored or understated in the application of the TTR/TDTR analysis. I began by presenting convergence criteria for the infinite summations present in the TTR/TDTR model that was developed and presented in Chapter 4. This included summation in $M$ for the sinusoidal, square, and pulsed modulation waveforms and the summation in $k$ for the latter two waveforms. The goal was to not only remove the "guess work" from the choice of $M$ and $k$ to ensure accurate modeling, but also to provide a lower bound to these values to make calculations as computationally efficient as possible. To further increase the computational efficiency, I demonstrated the ability to take advantage of the Gibbs oscillations in the data for values of $M$ below the established convergence criteria to allow accurate modeling with $M$ below the convergence limit. This process supports further calculations throughout this work making them more computationally feasible. To ensure accurate deduction of parameters from experimental data via fitting using the TTR/TDTR model I introduced a modified normalization scheme

\footnotetext{
${ }^{15}$ I specified that the data can be removed, rather than discarded, because while the outlier does not statistically coincide with the rest of the data, that does not mean that there is no potentially knowledge to be gained from that result.
} 
that eliminates the user defined scaling time which was shown to influence the final results. To quantify the behavior of the model output to variations in the input parameters I discussed methods of sensitivity analysis, demonstrating the small perturbation limit of the sensitivity coefficient and promoting the use of a stochastic analysis when modeling an unknown system. This stochastic approach along with the formulations in Chapter 6 should provide quantitative estimates of the precision and accuracy of a measured system. In support of this, I presented methodologies to quantify the noise in the collected data and finally introduced a statistical method to test for outliers in the collected data. Together these results will make the application of the TTR/TDTR analysis more repeatable, computationally efficient, accurate, and provide a better framework for modeling a system prior to sample fabrication to determine whether the desired measurements will be statistically feasible. 


\section{CHAPTER 6}

\section{ROBUST DATA COLLECTION AND}

MANAGEMENT FOR HIGH

PRECISION MEASUREMENTS OF

THIN FILM THERMOPHYSICAL

PROPERTIES VIA ULTRAFAST

OPTICAL PUMP-PROBE

SPECTROSCOPY

6.1 Introduction . . . . . . . . . . . . . . . . . . 177

6.2 Confidence in Results - Accuracy vs. Precision _ . . . . . . . . . . . . 180

6.3 Statistically Reporting Results . . . . . . . . . . . . . . . . . . 183

6.4 Assumptions of the Sampling Distribution . . . . . . . . . . . . . 187 
6.5 Quantification of Confidence in Prediction of the Population Mean . . . . . 190

6.6 Predicting Population Statistics of Thermoreflectance Data Through Large

Scale Simulations . . . . . . . . . . . . . . . . . . . . 194

6.7 Statistical Analysis via Bootstrapping . . . . . . . . . . . . . . . . . . 212

6.8 Accuracy of Results . . . . . . . . . . . . . . . . 225

6.9 Statistical Example $\mathrm{Pt} / \mathrm{Si} \ldots \ldots \ldots \ldots \ldots \ldots$

6.10 Chapter Summary . . . . . . . . . . . . . . . 236

\subsection{Introduction}

In Chapter 1 a brief history of computing focused on thermal management was presented and the various consequences of heat related device failure were discussed. There is little doubt that computing devices have become an integral part of our daily lives, some very apparent such as computers and smart phones, and some less obvious such as those in traffic control systems and similar infrastructures. Therefore, almost every article or grant in the thermal physics field begins by discussing the importance of proper thermal management, some even going as far as to discuss "life-threatening consequences" of thermal failure. Over the past several decades measurements of $h_{\mathrm{BD}}$ have transitioned from more broad comparisons of very different material systems, to more refined comparisons of the same material system, i.e. film and substrate combination, with minute variations in the interface structure and properties, see Chapter 2.

Unfortunately, the statistical treatment and analysis of TTR/TDTR data has remained relatively crude, remnant of the time when comparisons between measured values from different material systems could vary by an order of magnitude or more. Often times when data from experiments or simulations is reported in literature or at conferences there is no quantification of a measure of variability in the results, i.e. "error bars." If some quantification is presented via some type of error bars, there is no specification as to whether they represent a standard deviation, a standard error, or a confidence level of some significance level ${ }^{1}$. More unfortunate still, if provided the error bars are often treated as a "decoration"

\footnotetext{
${ }^{1}$ All these terms will be defined and discussed further in the coming sections.
} 
to the plot, in as much as there is no statistical interpretation of the results and conclusions are drawn regardless of the information the error bars provide.

Consider for example the data shown in Figure 6.1. The figure was reproduced from data presented in a major heat transfer journal (impact factor $\approx 2.5$ ) and depicts $h_{\mathrm{BD}}$ as a function of temperature from molecular dynamics simulations ${ }^{2}$. The results are the average of 5 independent simulations and while the type of error quantification is not specified, it is assumed that the error bars represent plus/minus one standard deviation in the results. From the data in Figure 6.1, the authors discuss an increasing trend in $h_{\mathrm{BD}}$ between 50 and $250 \mathrm{~K}$ citing various experiments and studies supporting this interpretation, and also discusses the plateau in $h_{\mathrm{BD}}$ above $250 \mathrm{~K}$ concluding that it is the result of various physical phenomena in the system.

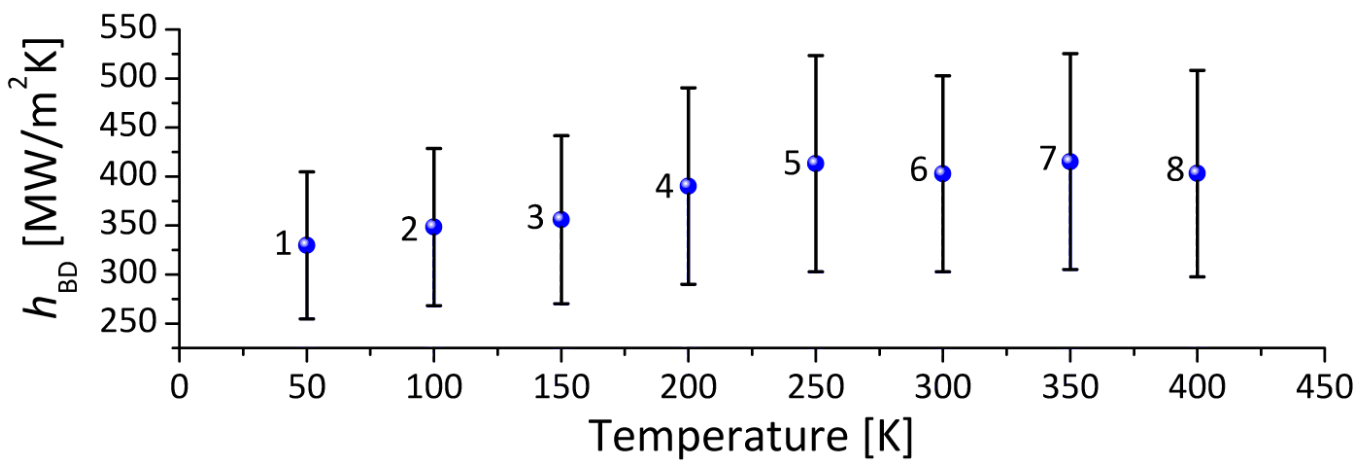

Figure 6.1: Results of molecular dynamics simulations of $h_{\mathrm{BD}}$ as a function of temperature taken from a predominate heat transfer journal (impact factor $\approx 2.5$ ). Error bars represent plus/minus one standard deviation.

While the arguments from the trends in the data presented by the authors appears valid considering the average data points, if the data in Figure 6.1 is analyzed statistically as well, a problem arises. In analyzing a trend in the data it is important to consider, for example, if the data points can be assumed to be statistically different based on the mean and variance of the data sets. As an example, for the data presented in Figure 6.1, the Welch's t-test for the equality of two means [268] was performed on the permutations of the pairings of data points 1-8 assuming a null hypothesis that the means are equivalent, see Table

\footnotetext{
${ }^{2}$ The details of the study and the citation of the work are omitted. The purpose of this demonstration is not to challenge the work of a particular group, but to advocate higher standards of statistical analysis and consideration in the thermal community.
} 
6.1. From the $p$-values shown in Table 6.1 there is no strong evidence to suggest rejection of the null hypothesis which indicates that the mean values are statistically equivalent. Therefore, there is little statistical evidence to support any trends in the data other than a constant $h_{\mathrm{BD}}$ as a function of temperature. In order to draw more robust conclusions based on experimental data and more accurately represent results, a more in-depth statistical analysis and interpretation of TTR/TDTR data is required.

Table 6.1: Calculated $p$-values based on Welch's t-test between the data points shown in Figure 6.1 where the null hypothesis is that the two means are equal.

\begin{tabular}{ccccccccc}
\hline Data \# & 1 & 2 & 3 & 4 & 5 & 6 & 7 & 8 \\
\hline 1 & 1.000 & & & & & & & \\
2 & 0.717 & 1.000 & & & & & & \\
3 & 0.624 & 0.889 & 1.000 & & & & & \\
4 & 0.316 & 0.489 & 0.579 & 1.000 & & & & \\
5 & 0.205 & 0.324 & 0.391 & 0.742 & 1.000 & & & \\
6 & 0.232 & 0.372 & 0.451 & 0.847 & 0.884 & 1.000 & & \\
7 & 0.195 & 0.309 & 0.374 & 0.719 & 0.976 & 0.859 & 1.000 & \\
8 & 0.245 & 0.385 & 0.462 & 0.848 & 0.889 & 0.997 & 0.865 & 1.000 \\
\hline
\end{tabular}

In this chapter we will focus on the statistical analysis and interpretation of the deduced thermophysical data from the TTR/TDTR experiment via the thermal modeling developed in Chapters 4 and 5. We will begin by going over some of the basic formulations and terminology of statistical inference as it applies to our thermal experiment. This is to develop a robust statistical language moving forward and then highlight some of the nuances that are often missing from the presentation of TTR/TDTR data. We will suggest the use of confidence intervals as a quantitative measure of the precision in the fitted results, and discuss how the number of samples and the standard deviation of the sample set affects the length of the confidence interval for precision. To allow for a priori estimation of the standard deviation of a sample set based on fitting sensitivity and noise in the data, see Chapter 5, we present the results of a series of large scale simulations of fitted TTR/TDTR data. Using these results, a set of empirical formulations are presented that relate the sensitivity coefficient and the signal-to-noise parameter to the estimated ideal standard deviation of a sample set. In order to test the validity of the statistical assumptions made in the analysis of the TTR/TDTR data, the non-parametric bootstrapping technique is 
introduced. Finally, as a measure of the accuracy in the modeled data, and supported by the methods to increase computational efficiency proposed in Chapter 5, quasi-stochastic methods of accuracy analysis are presented which take into account the distribution and covariance between the input parameters.

\subsection{Confidence in Results - Accuracy vs. Precision}

In terms of mathematical statistics, the goal in the collection and analysis of TTR/TDTR data is to use statistical inference to draw conclusions about parameters of a population, i.e. a material system, through the estimation of thermophysical parameters such as thermal conductivity, thermal boundary conductance, etc., taken from a random sampling of the population. We will begin by giving a brief overview of basic statistical methods, not to introduce new statistical methods, but to define and develop a robust statistical language that is often missing in TTR/TDTR data analysis.

Taking $h_{\mathrm{BD}}$ as an example ${ }^{3}$, we begin by defining the total population as all potential $h_{\mathrm{BD}}$ values from which there are collections of sub-populations which represent the various combinations and configurations of material systems ${ }^{4}$, see Figure 6.2. In our experimental methods, from these sub-populations we will wish to select a random sample set in order to attempt to create a point estimate of a statistic of the sub-population ${ }^{5}$, e.g. the average $h_{\mathrm{BD}}$.

The methods used to generate and analyze the statistical inferences made from the sampled data will depend on how the data in the sample and the overall population are distributed. If the data sample conforms to a known distribution, or is sampled from a known distribution, there are several parametric methods that can be used in the analysis. However, if the data does not conform to a defined distribution or the distribution is

\footnotetext{
${ }^{3}$ Note: We can choose to analyze any thermophysical parameter from the thermal model, see Chapter 4. However, because it is the primary focus of this dissertation, the statistical analysis will mostly discuss $h_{\mathrm{BD}}$ as the parameter of interest.

${ }^{4}$ This is further complicated due to the fact that not all film/substrate combinations are created equal. Factors such as surface preparation, material quality, deposition conditions, etc., further provide potential sub-populations, see Chapter 2.

${ }^{5}$ From this point on, we will refer to the parameter of interest of a particular TTR/TDTR sample in terms of a population, with the understanding that it represents a subset of all potential values.
} 


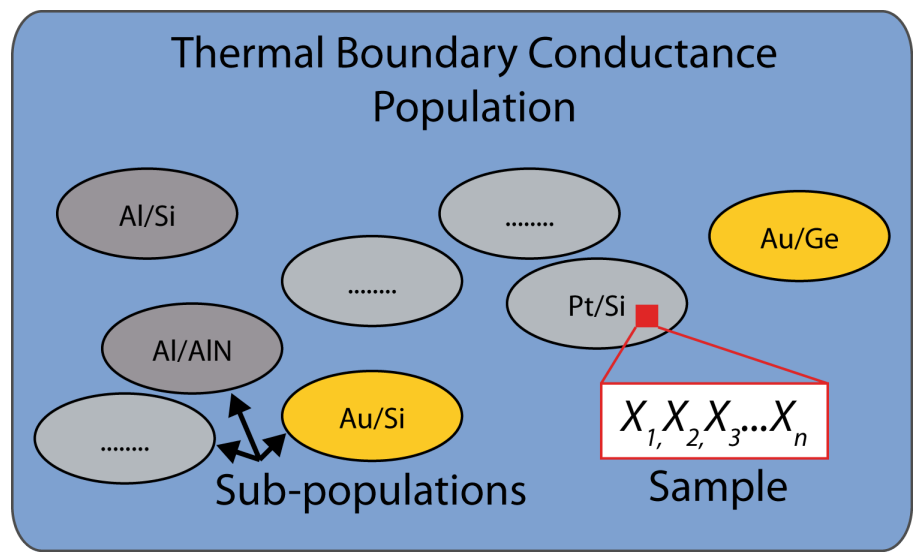

Figure 6.2: Representation of the population of all potential $h_{\mathrm{BD}}$ values and several example subpopulations of potential material systems, from which a random sample set will be selected.

unknown, more computationally expensive non-parametric methods must be used ${ }^{6}$.

For the sake of argument, we will begin by assuming that the distribution of a population of $h_{\mathrm{BD}}$ values for a given material system follows a normal distribution, which is given in its general form by [19]:

$$
f(x, \mu, \sigma)=\frac{1}{\sigma \sqrt{2 \pi}} e^{-\frac{(x-\mu)^{2}}{2 \sigma^{2}}} \quad \text { and } \quad-\infty<x<\infty
$$

where $\mu$ is the population mean, and $\sigma^{2}$ is the population variance (from which we can calculate the population standard deviation, $\sigma$ ). An example of a normal distribution of $h_{\mathrm{BD}}$ values with $\mu=100 \mathrm{MW} / \mathrm{m}^{2} \mathrm{~K}$, and with several values of standard deviation, $\sigma=1,5$, and $10 \mathrm{MW} / \mathrm{m}^{2} \mathrm{~K}$ receptively, is shown in Figure 6.3. While common, the assumption of normality is powerful and provides a large framework of tools to provide estimation and statistical significance to inferences made about the parameters of a population. Therefore if violated, and/or the wrong assumption about the distribution of the population made, inferences may not be valid or reliable. For now, we will stick with the assumption that the population is normally distributed and later will discuss the validity of this assumption and how the results would be affected if this assumption broke down.

One of the challenges in the statistical analysis of TTR/TDTR results is that we do not sample a thermophysical parameter of interest, e.g. $h_{\mathrm{BD}}$, directly. Rather we collect

\footnotetext{
${ }^{6}$ Non-parametric statistical methods will be discussed in more detail in Section 6.7
} 
information on the samples surface temperature as a function of time after short pulsed laser excitation and compare the results to a thermal model (see Chapter 4) to deduce the parameter of interest. As detailed in Chapter 4, the thermophysical model has several inputs based on the parameters of the sample and laser, and the parameter of interest is typically deduced by treating all other parameters as constants and iterating a guess of the desired parameter until the data and the model match to within some stopping criteria.

Whenever an experiment is conducted and analysis completed, there is a certain amount of variability in the results of repeated measurements. In the TTR/TDTR procedure, these variations can arise from several sources:

1. Variations within the population of $h_{\mathrm{BD}}$ values across the TTR/TDTR sample surface due to variations in the parameters affecting $h_{\mathrm{BD}}$, e.g. species mixing.

2. Uncertainty in the values of the input parameters of the thermal model leading to potential variations in the deduced parameter for a given data set.

3. Noise fluctuations in the surface temperature of the data set leading to variation in the deduced parameters for fixed inputs.

Of these three sources, the first is what we wish to capture in conducting our experiment and analysis, with the confidence we have in our results being limited by the other two. The amount of uncertainty we have in the input parameters of our model, will directly effect the accuracy of our results. While systematic errors and noise fluctuations will affect the

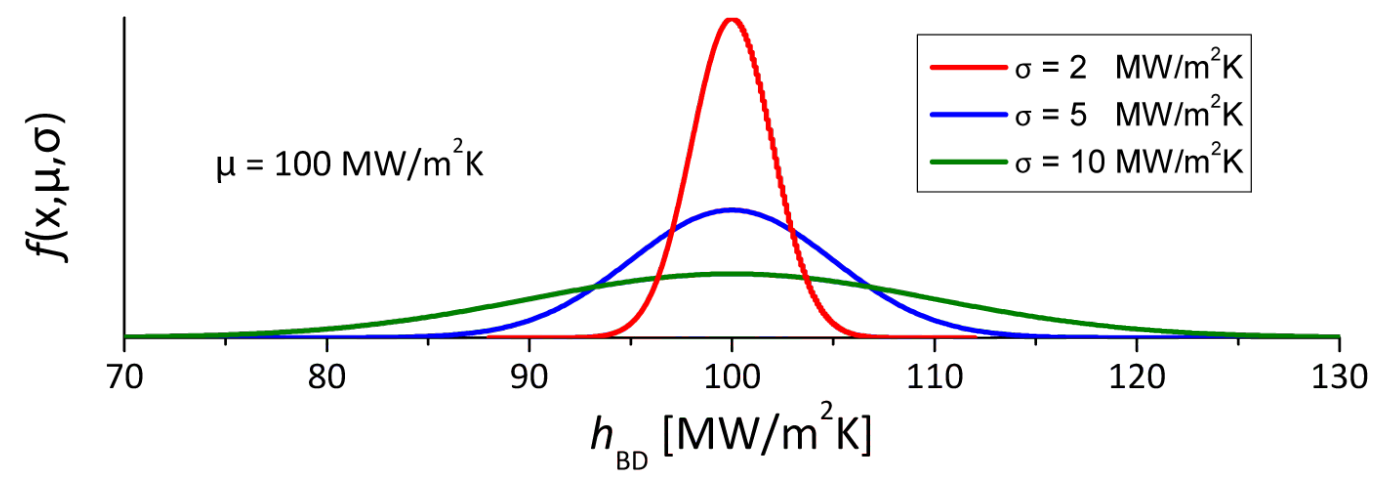

Figure 6.3: Examples of normal distributions, see Equation 6.1 , for $\mu=100 \mathrm{MW} / \mathrm{m}^{2} \mathrm{~K}$, and $\sigma=1,5$, and $10 \mathrm{MW} / \mathrm{m}^{2} \mathrm{~K}$. 
precision of the measurement. The precision of our results can be improved through repeated measurements, while the accuracy can be improved through better characterization of the input parameters to the model. Any statistical quantification of uncertainty in our final result will be subject to the larger of these two bounds, i.e. accuracy driven or precision driven. Typically, we have the most control over the precision of our results, therefore we will focus on ensuring that our bounds of precision are within the bounds dictated by the accuracy of our modeling. We will begin first by detailing the methods for high precision and then develop the methods for estimating the accuracy of our final result.

\subsection{Statistically Reporting Results}

If we were able to select an $h_{\mathrm{BD}}$ value from a TTR/TDTR sample surface, we would expect our results to fall somewhere within the bounds of the distribution with a probably dictated by $\mu$ and $\sigma$. For a single sampling, the probability of the deduced value falling within prescribed bounds, represented as integer pairs of $\sigma$, can readily be found by considering the probability distribution of the standard normal curve, see Figure 6.4. The probability of selecting a single value between $\pm 1 \sigma$ is $68.2 \%, \pm 2 \sigma$ is $95.4 \%, \pm 3 \sigma$ is $99.6 \%$.

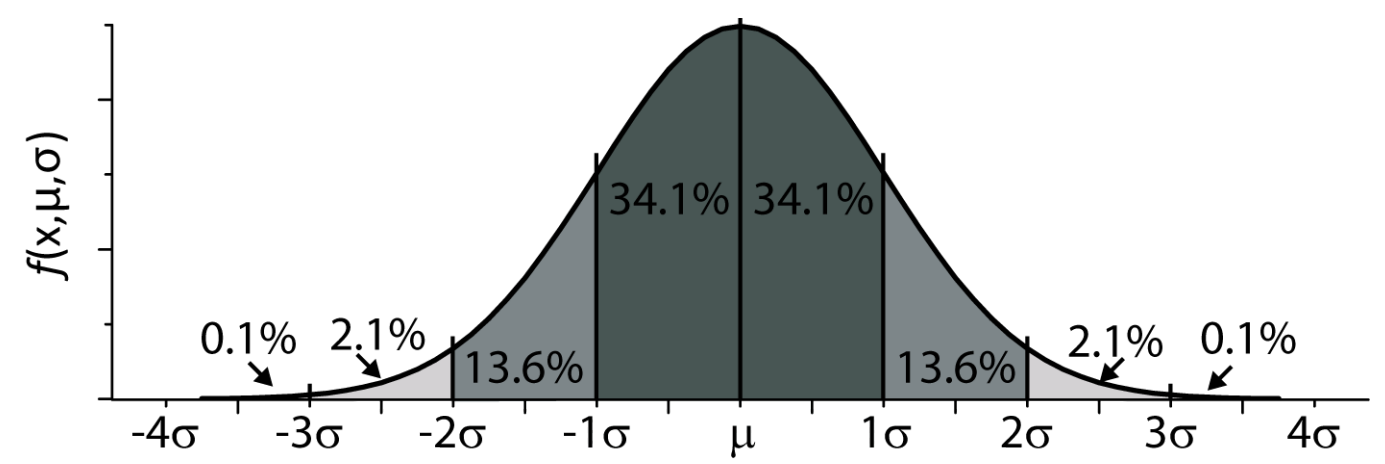

Figure 6.4: Schematic of the standard normal curve with percentages for each standard deviation denoted.

It is typically poor experimental practice to conduct only one sampling and assume that we have captured all necessary information about the population of a system ${ }^{7}$. As experimentalists it is more typical, although far from assured, to take a number of scans on each sample in hopes of building a "statistical picture" of the behavior of a population.

\footnotetext{
${ }^{7}$ And impossible to quantify the uncertainty in that estimation without knowing the variance of the underlying population.
} 
Suppose we select a random sample ${ }^{8}$ of size $n$ which we will denote $\mathbf{X}=\left\{x_{1}, x_{2}, x_{3}, \ldots x_{n}\right\}$ from a given normal population with mean $\mu$ and variance $\sigma^{2}$. From this sample set we can find the arithmetic sample mean, $\bar{x}$, which will be a point estimate of the true mean, $\mu$, given by [19]:

$$
\bar{x}=\frac{x_{1}+x_{2}+x_{3}+\cdots+x_{n}}{n}=\frac{1}{n} \sum_{i=1}^{n} x_{i}
$$

Additionally, we can also calculate the standard deviation of the sample as a measure of the dispersion of the collected data, given by [19]:

$$
s=\sqrt{\frac{1}{n-1} \sum_{i=1}^{n}\left(x_{i}-\bar{x}\right)^{2}}
$$

It is important to note that the mean and standard deviation, $\bar{x}$ and $s$, are properties of the sample of size $n$, assumed to be randomly selected from an infinite population.

\section{What is the current state of the field in reporting statistical results?}

It is important to pause a moment here and take note that in the field of nanoscale thermal measurements specifically focused on thermophysical measurements from thermoreflectance, Equations 6.2 and 6.3 represent the full extent of the statistical analysis typically employed. For those groups that provide some type of statistical uncertainty quantification $^{9}$, it is typically in the form of a mean of a fixed number of scans (arbitrarily chosen) and error bars representing plus/minus one standard deviation calculated from the data set. In terms of what has been presented so far, this provides information about the particular sample of data that was collected by the group and does not really provide any statistical estimation about the population. This only provides information on what was done and makes no attempt to predict the properties of the population, i.e the sample, or provide an estimate as to the probability of whether the collected data contains a true estimate of the parameter value.

\footnotetext{
${ }^{8}$ By random we will assume that the values selected are independent and identically distributed (i.i.d). Meaning that the selection of one variable does not affect the probability of selecting the next, and that they are drawn from the same probability distribution.

${ }^{9}$ Unfortunately, it is not uncommon to see data plotted with trends and results inferred and no statistical quantification of the significance of the results which casts doubts on the conclusions.
} 


\section{Turning to More Robust Statistical Methods of Inference}

To conduct a truly robust analysis, we would repeatedly collect random samples of size $n$ from the same population and calculate the sample mean, $\bar{x}$, for each to build a sampling distribution of the means. The sampling distribution of the means is not to be confused with the distribution of the population. Where the population distribution provides the statistical distribution of all $h_{\mathrm{BD}}$ values in the population, the sampling distribution of the means provides the statistical distribution of mean $h_{\mathrm{BD}}$ values calculated from repeated samplings of size $n$. As an example, consider a random sampling of $h_{\mathrm{BD}}$ values of size $n=10$ selected from a normal distribution with mean $\mu=100 \mathrm{MW} / \mathrm{m}^{2} \mathrm{~K}$ and standard deviation $\sigma=5 \mathrm{MW} / \mathrm{m}^{2} \mathrm{~K}$. Figure 6.5 shows the sampling distribution of the mean $h_{\mathrm{BD}}$ values for an increasing number of repeated samplings of size $n$.

If we consider $N$ random samplings of size $n$ from a population, we can define the expected mean of the sampling distribution of mean $h_{\mathrm{BD}}$ values, $\mu_{\bar{x}}$ to be:

$$
\mu_{\bar{x}}=\frac{\bar{x}_{1}+\bar{x}_{2}+\bar{x}_{3}+\cdots+\bar{x}_{N}}{N}=\frac{1}{N} \sum_{j=1}^{N} \bar{x}_{j}
$$

where $\bar{x}_{j}$ is the mean of the $j^{\text {th }}$ sampling of $n$ random $h_{\mathrm{BD}}$ values and $N$ is the total number of resamplings. In the limit that $N$ is sufficiently large ${ }^{10}$, the mean of the sampling distribution will converge to the population mean, i.e.:

$$
\lim _{N \rightarrow \infty} \frac{1}{N} \sum_{j=1}^{N} \mu_{\bar{x}}=\mu
$$

The standard deviation of the sampling distribution, $\sigma_{\bar{x}}$, also called the standard error is given by [19]:

$$
\sigma_{\bar{x}}=\frac{\sigma}{\sqrt{n}}
$$

where $\sigma$ is the standard deviation of the underlying population.

In an ideal experimental world, when using statistical inference to estimate the mean $h_{\mathrm{BD}}$ of a given population we would report the mean and standard deviation of the sampling

\footnotetext{
${ }^{10}$ The concept of "sufficiently large" will show up often in the statistical treatment and inference of data. Throughout, every attempt will be made to indicate the rough order of when large is large enough.
} 


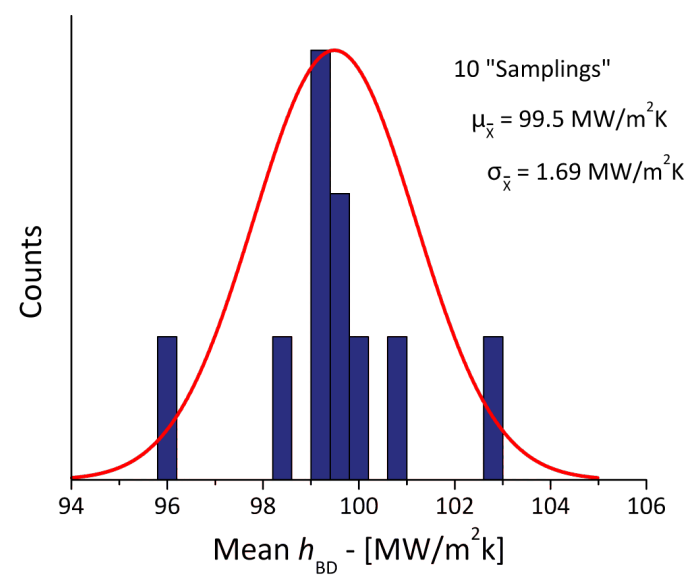

(a)

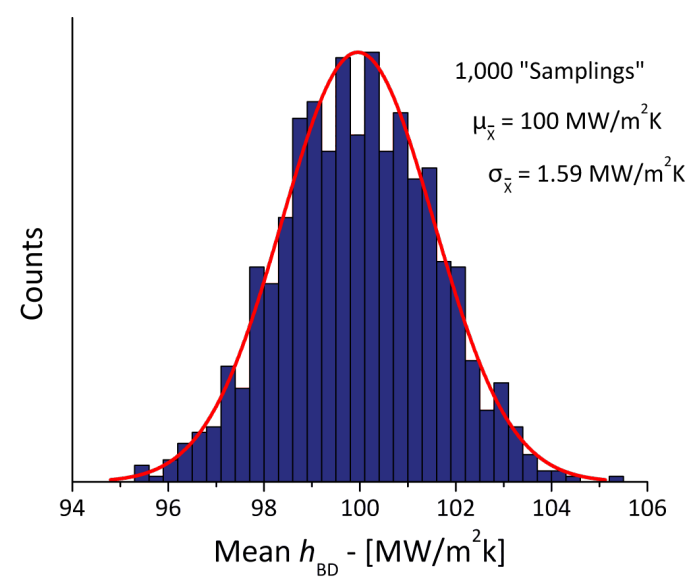

(c)

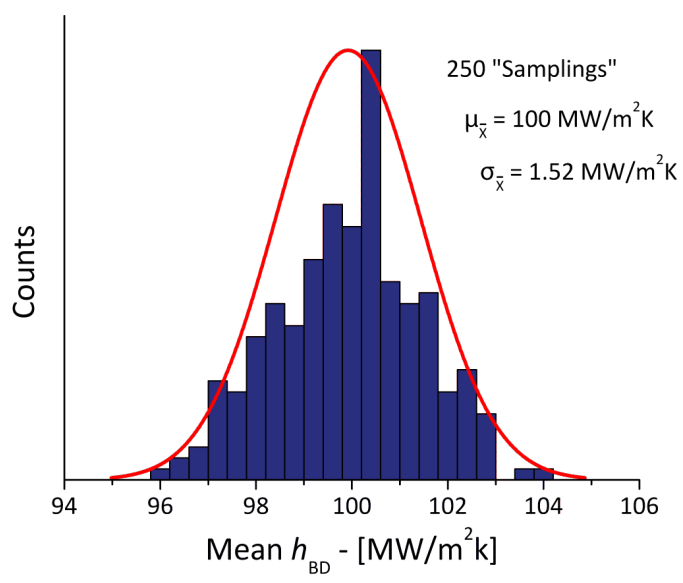

(b)

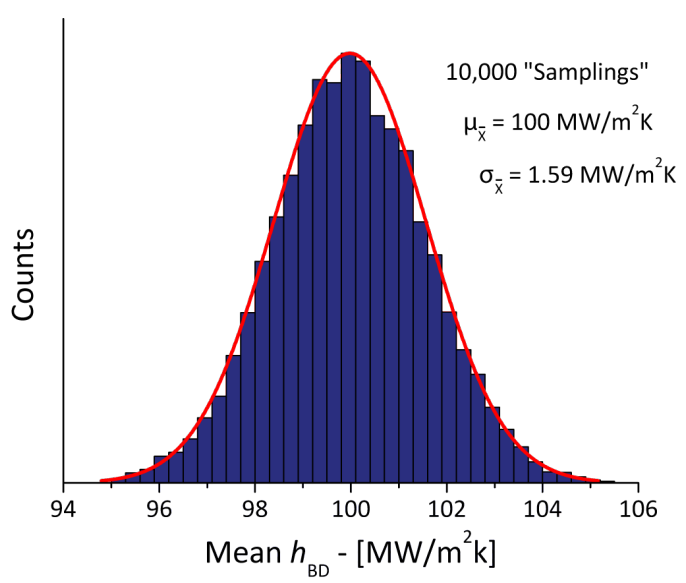

(d)

Figure 6.5: Sampling distribution of the mean $h_{\mathrm{BD}}$ values for a sampling size of $n=10$ selected from a normal population with mean $\mu=100 \mathrm{MW} / \mathrm{m}^{2} \mathrm{~K}$ and standard deviation $\sigma=5 \mathrm{MW} / \mathrm{m}^{2} \mathrm{~K}$, for a total number of repeated samplings of (a) 10, (b) 250, (c) 1,000, and (d) 10,000.

distribution of the means. Provided that $N$ was "sufficiently large" we could assume that the reported mean was an accurate representation of the population mean, see Equation 6.4, within some level of confidence dictated by the choice of sample size $n$, see Equation 6.6. This raises very important questions as to: 1) when is $N$ "sufficiently large", 2) if there are restrictions on the size of $n$, and 3) what can be assumed about the form of the distribution of the sampling means? We will begin by considering the size of $n$ and the distribution of the means as they will be closely related, and in Section 6.7 we will look further into the size of $N$. 


\subsection{Assumptions of the Sampling Distribution}

Ideally when describing the sampling distribution we would like to be able to assume that the means are distributed normally. There are a large number of relationships that can be utilized if we can use parametric methods to analyze the results, particularly if we can assume the distribution is normal. Thus far we have assumed that the distribution of the population is normal and because of that assumption we can assume directly that the sampling distribution of means is normal for any ${ }^{11} n \geq 1$. However, if we have no $a$ priori knowledge of how the population is distributed the appropriate choice of $n$ becomes somewhat more complicated.

Luckily, before giving up on the assumption of normality we can turn to the assumptions of the Central Limit Theorem which states [19]:

If a random variable $Y$ is the sum of $n$ independent random variables that satisfy certain general conditions, then for sufficiently large $n, Y$ is approximately normally distributed.

Essentially this provides that as long as $n$ is "sufficiently large", regardless of how the underlying population is distributed, the sampling distribution of the means can be assumed to be normal. This gives us hope for the assumption of normality, but still returns us to the question of when is $n$ "sufficiently large." The general "rule of thumb" that is often cited along with the central limit theorem is that $n \geq 30$ is sufficient to assume that the sampling distribution of the means will be normally distributed for an underlying population of unknown distribution. There are generally worse and better case scenarios to the rule dependent on how "well behaved ${ }^{12}$ " the underlying population is.

\section{Properties of the Distribution}

To describe how "well behaved" a distribution is beyond the properties of mean, $\mu$, and standard deviation $\sigma$, the general shape of a distribution can be defined by two dimensionless

\footnotetext{
${ }^{11}$ Assuming we have completed a sufficient number of samplings $N$.

${ }^{12}$ Well behaved referring to being close to a normal distribution.
} 
quantities, the skewness. $\beta_{\text {skew }}$, and the kurtosis, $\beta_{\text {kurt }}$, given by [19]:

$$
\begin{array}{ll}
\beta_{\text {skew }}=\frac{n}{(n-1)(n-2)} \frac{\sum_{i=1}^{n}\left(x_{i}-\bar{x}\right)^{3}}{s^{3}} & n>2 \\
\beta_{\text {kurt }}=\frac{(n)(n+1)}{(n-1)(n-2)} \frac{\sum_{i=1}^{n}\left(x_{i}-\bar{x}\right)^{4}}{s^{4}} & n>3
\end{array}
$$

where $n$ is the sample size, $x_{i}$ is the value of the $i^{\text {th }}$ sample, $\bar{x}$ is the sample mean, and $s$ is the sample standard deviation. The skewness is a measure of the distribution's symmetry and the kurtosis is a measure of the "peakedness" of the distribution and provides information on the shape of the tails of the distribution. For comparison, a perfectly normal distribution will have zero skewness, i.e. $\beta_{\text {skew }}=0$, and a kurtosis ${ }^{13}$ of 3 , i.e. $\beta_{\text {kurt }}=3$.

We can estimate the required sample size, $n$, in order to satisfy the conditions of the central limit theorem for low kurtosis, i.e. $\left|\beta_{\text {kurt }}\right|<3.75$, based on the population skewness using the relation [19]:

$$
n>25\left(\beta_{\text {skew }}\right)^{2}
$$

Investigating Equation 6.8, we see that the "rule of thumb" of $n \geq 30$ to satisfy the conditions of the central limit theorem implies a skewness of greater than 1. In Figure 6.6 two examples are shown of 1,000 random samples collected from normal distributions with similar kurtosis and a skewness of $\beta_{\text {skew }}=-0.6$ and $\beta_{\text {skew }}=-1.1$ for (a) and (b) respectively. From the plots we can see that a skewness greater than 1 , i.e. $\beta_{\text {skew }}>1$, deviates significantly from the shape of a normal distribution. Therefore, if the shape of a given distribution reasonably approximates a normal distribution, the required sample size to satisfy the central limit theorem will be significantly less than 30 . In their text, Hines et al. further expanded the general rule of thumb for the size of $n$ into 3 subgroups [19]:

- Well behaved systems: Distributions which do not deviate greatly from the bell shape of a normal distribution and are still nearly symmetric may only require a sample size of $n \geq 4$.

\footnotetext{
${ }^{13}$ Note: There are two accepted definitions of the kurtosis, one in which the kurtosis of the normal distribution is 3 , and an alternate definition of Equation $6.7 \mathrm{~b}$ with a factor of 3 subtracted such that the kurtosis of a normal distribution is zero.
} 


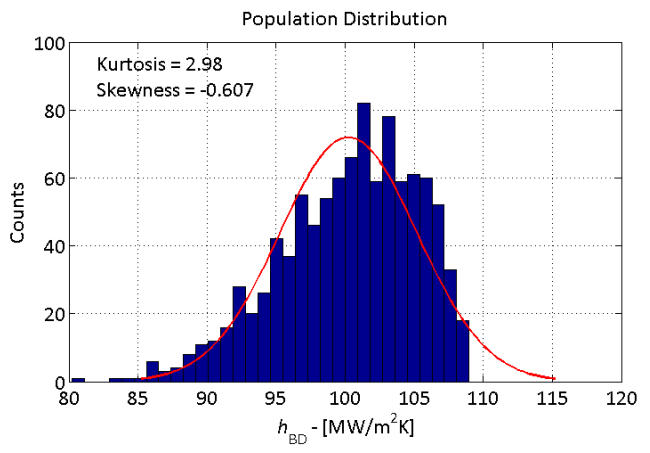

(a)

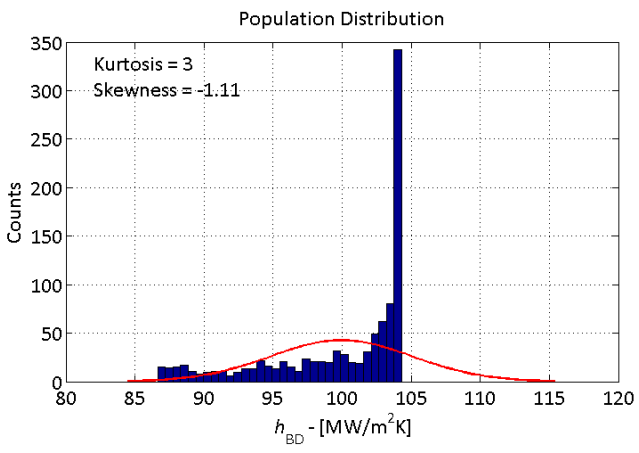

(b)

Figure 6.6: Distributions of 1,000 samples from a normal distribution with $\mu=100 \mathrm{MW} / \mathrm{m}^{2} \mathrm{~K}$ and $\sigma=5 \mathrm{MW} / \mathrm{m}^{2} \mathrm{~K}$ and (a) $\beta_{\text {skew }}=-0.6$ and (b) $\beta_{\text {skew }}=-1.1$.

- Reasonable systems: Distributions that are generally symmetric but may not have a prominent mode may require a sample size on the order of $n \geq 12$.

- Ill behaved systems: The less well behaved a distribution is the more difficult it is to estimate the required sample size. However for distributions with most of the information in the tails, such as exponential distributions $\left(\beta_{\text {skew }}=2\right)$, a sample size of $n \geq 100$ may be required.

The discussion of the central limit theorem has highlighted the importance of the shape of the underlying distribution on the appropriate choice of sample size. In fact, there will be several areas in the statistical analysis of this work and in the general statistical analysis of data, where the normality or deviation there from will be important. Therefore, to assist in the interpretation of the remainder of this work several methods to test and quantify normality of a distribution are presented in Appendix D.

Very seldom, if at all, are repeated experimental samplings of size $n$ collected in order to build the sampling distribution of the means from which we could determine confidence in the measurement via the standard deviation of the sampling distribution. Later in Section 6.7 we will discuss a mathematical method to populate the sampling distribution based on a single sample set. Without populating the sampling distribution directly, we rely on a series of analytic expressions for the standard error and the probability of the sampling distribution under the assumption that it is inherently normal or that the conditions of the central limit theorem have been satisfied. 


\subsection{Quantification of Confidence in Prediction of the Popu- lation Mean}

Assuming that we have chosen appropriate sample size $n$ to satisfy all the requirements of the central limit theorem, and have taken a "sufficiently large" number, $N$, of samplings of size $n$ we have the required information to construct the sampling distribution of the means and assume that it is distributed normally. From this distribution we will wish to report the mean of the distribution (which will be the population mean under the condition of sufficiently large $n$, and $N$ ), and provide some quantification of the confidence in that prediction. While the standard deviation provides some quantification of the dispersion of estimator values, a single value is not as effective as providing a potential range of values. What we are interested in is providing an upper and lower bound within which there is some probability that the true value of the estimator, $\theta$, is contained [19]:

$$
\begin{gathered}
L \leq \theta \leq U \\
P\{L \leq \theta \leq U\}=1-\alpha
\end{gathered}
$$

where $\theta$ is the estimator of a statistical parameter, e.g. the average $h_{\mathrm{BD}}$, the interval between $L$ and $U$ is known as the $100(1-\alpha) \%$ confidence interval, $1-\alpha$ is known as the confidence coefficient, and $\alpha$ is the significance level. For most of the cases in this dissertation we will be concerned with the $95 \%$ confidence level ${ }^{14}$.

Assuming we select a random sample of size $n$ from a normally distributed population with unknown mean $\mu$ and known variance $\sigma^{2}$, the two-sided confidence interval about the estimator of interest $\mu$, is given by [19]:

$$
\bar{x}-Z_{\alpha / 2} \frac{\sigma}{\sqrt{n}} \leq \mu \leq \bar{x}+Z_{\alpha / 2} \frac{\sigma}{\sqrt{n}}
$$

where $\bar{x}$ is the sample mean, $Z_{\alpha / 2}$ is the normal distribution at the $\alpha / 2$ point, and $\mu$ is the

\footnotetext{
${ }^{14}$ Note: There is a common misconception that a $95 \%$ confidence level means that there is a $95 \%$ chance that the value report is correct, however this is not the case. By providing a $95 \%$ confidence interval we specifying that under identical repeated experiments, i.e. the same methods used to collect the data, $95 \%$ of the time, the prescribed interval of the estimator will contain the true value.
} 
true mean. Therefore the upper and lower bound of the confidence interval is given by [19]:

$$
\begin{aligned}
L & =\bar{x}-Z_{\alpha / 2} \frac{\sigma}{\sqrt{n}} \\
U & =\bar{x}+Z_{\alpha / 2} \frac{\sigma}{\sqrt{n}}
\end{aligned}
$$

The distance between the upper and lower bound of the confidence interval is known as the confidence interval length, $L_{\mathrm{CI}}$, given by:

$$
\begin{gathered}
L_{\mathrm{CI}}=U-L=\bar{x}+Z_{\alpha / 2} \frac{\sigma}{\sqrt{n}}-\left[\bar{x}-Z_{\alpha / 2} \frac{\sigma}{\sqrt{n}}\right] \\
L_{\mathrm{CI}}=2 \times Z_{\alpha / 2} \frac{\sigma}{\sqrt{n}}
\end{gathered}
$$

The confidence interval length is a measure of the precision of the estimation, see Figure 6.7. The longer the length of the confidence interval, the more confident we can be that the interval contains the true mean. Therefore for a given variance, $\sigma^{2}$, and sample size, $n$, the $99 \%$ confidence interval will be longer than the $95 \%$ confidence interval. However, half of the interval length provides a measure of the accuracy, $U-\Theta$ (or $\Theta-L$ ). Therefore, for a long interval length the estimate is less accurate even though we are more confident the interval contains the true value. The ideal situation is one in which the confidence interval length is short, but the $100(1-\alpha) \%$ confidence interval is high.

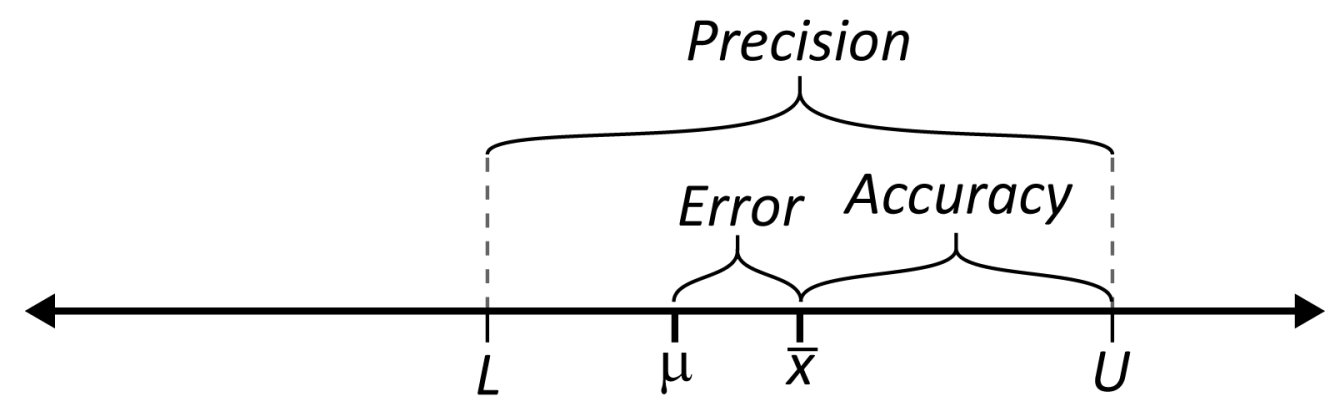

Figure 6.7: Error, precision, and accuracy of estimating the true mean of a distribution $\mu$ using the sample mean $\bar{X}[19]$. 


\subsubsection{Choice of Sample Size for Normally Distributed Data with Known Variance}

As seen in Equation 6.12, for a given confidence level the length of the interval is dependent on the variance $\sigma^{2}$ and the number of samples $n$. As discussed previously, an important experimental consideration is choosing a sample size that is sufficient to provide precise results but also be reasonable to collect. If we assume the population is normally distributed and that we know the variance, $\sigma^{2}$, it is straightforward to estimate the sample size required given bounds on the desired confidence interval and error tolerance. The number of samples required to satisfy these conditions is given by [19]:

$$
n=\left(\frac{Z_{\alpha / 2} \sigma}{E}\right)^{2}
$$

where $Z_{\alpha / 2}$ is the normal distribution at the $\alpha / 2$ point, $\sigma$ is the standard deviation of the population and $E$ is the specified error tolerance. As an example, suppose the standard deviation in $h_{\mathrm{BD}}$ is $\sigma=3 \times 10^{6} \mathrm{~W} / \mathrm{m}^{2} \mathrm{~K}$ and we want the error in our estimation to be less than $E=1 \times 10^{6} \mathrm{~W} / \mathrm{m}^{2} \mathrm{~K}$ with a confidence interval of $95 \%$, the number of samples required to reach this condition is then given by:

$$
n=\left(\frac{Z_{\alpha / 2} \sigma}{E}\right)^{2}=\left[\frac{1.96 * 3 \times 10^{6}}{1 \times 10^{6}}\right]^{2}=34.57=35
$$

If we want the same error in estimation for a $99 \%$ confidence interval the number of required samples would increase to 60 .

While this methodology appears straightforward and the required number of samples easy to calculate, the formulation in Equation 6.13 relies on a major assumption, a known variance (and therefore standard deviation). Typically in experimental TTR/TDTR measurements (and in most experimental measurements), the standard deviation is not known until the data is collected and analyzed. Without having a prior knowledge of the variance in the population, the formulation in Equation 6.13 is no longer valid, and the definition of the confidence interval must be reconsidered. The most straightforward manner to correct for the unknown variance is to replace $\sigma$ in Equation 6.10 with the standard deviation of 
the sample set, $s$, and therefore the confidence interval is given by:

$$
\bar{x}-Z_{\alpha / 2} \frac{s}{\sqrt{n}} \leq \mu \leq \bar{x}+Z_{\alpha / 2} \frac{s}{\sqrt{n}}
$$

This change is valid provided that the sample size is large enough to assume a normal distribution based on the central limit theorem, typically $n>30$, and that the sample standard deviation is a good estimate of the standard deviation of the population. However, at this point we do not want to already set a minimum of 30 samples $^{15}$, but have the ability to consider a broader range of sample sizes in a consistent manner. Assuming that the data is still normally distributed, a stronger assumption for small samples sets is to use the $t$-distribution. Replacing the distribution function in Equation 6.15 we get the two-sided confidence interval for normally distributed data of unknown variance [19]:

$$
\bar{x}-t_{\alpha / 2, n-1} \frac{s}{\sqrt{n}} \leq \mu \leq \bar{x}+t_{\alpha / 2, n-1} \frac{s}{\sqrt{n}}
$$

In Equation 6.16 the sample size $n$ shows up as $1 / \sqrt{n}$ and in the distribution $t_{\alpha / 2, n-1}$. Therefore it is not straightforward, as in Equation 6.13, to calculate the sample size required to satisfy given confidence and error tolerances.

Typically the only way to determine the required $n$ to fall within prescribed error tolerances for a distribution of unknown variance is through trial and error. We are forced to take an estimated guess as to the required sample size and hope that when we calculate $s$ and the confidence interval we reach acceptable precision tolerances. This is further complicated because TTR/TDTR measurements do not produce thermophysical quantities directly, but require deduction by comparison between the thermal model and the data adding computational expense. Ideally we would hope for some a priori estimate of the standard deviation of a sampling prior to data collection so that we could use formulations such as Equation 6.13 to determine the appropriate number of scans to collect to ensure that the precision of the results would be within prescribed accuracy bounds.

\footnotetext{
${ }^{15} \mathrm{~A}$ sample size of 30 is already well above what is typically reported in $h_{\mathrm{BD}}$ thermoreflectance measurements.
} 


\subsection{Predicting Population Statistics of Thermoreflectance Data Through Large Scale Simulations}

In an effort to gain some a priori intuition as to the standard deviation of a population a series of simulated experiments were conducted in order to take advantage of the law of large numbers as a means for predicting the standard deviation of a population from estimated thermophysical and laser parameters. The law of large numbers predicts, as is intuitively expected, that as the number of samples $n$ gets large a sample estimator will approach the estimator of the population [269]. As a visual example, consider the data shown in Figure 6.8 which represents the average $h_{\mathrm{BD}}$ of a sample set of size $n$, as the sample size is increased. The data for each sample set was randomly selected from a normal distribution with $\mu=100.00 \mathrm{MW} / \mathrm{m}^{2} \mathrm{~K}$ and $\sigma=20 \mathrm{MW} / \mathrm{m}^{2} \mathrm{~K}$. From the data in Figure 6.8 we notice that as the sample size gets large the mean and standard deviation of the sample

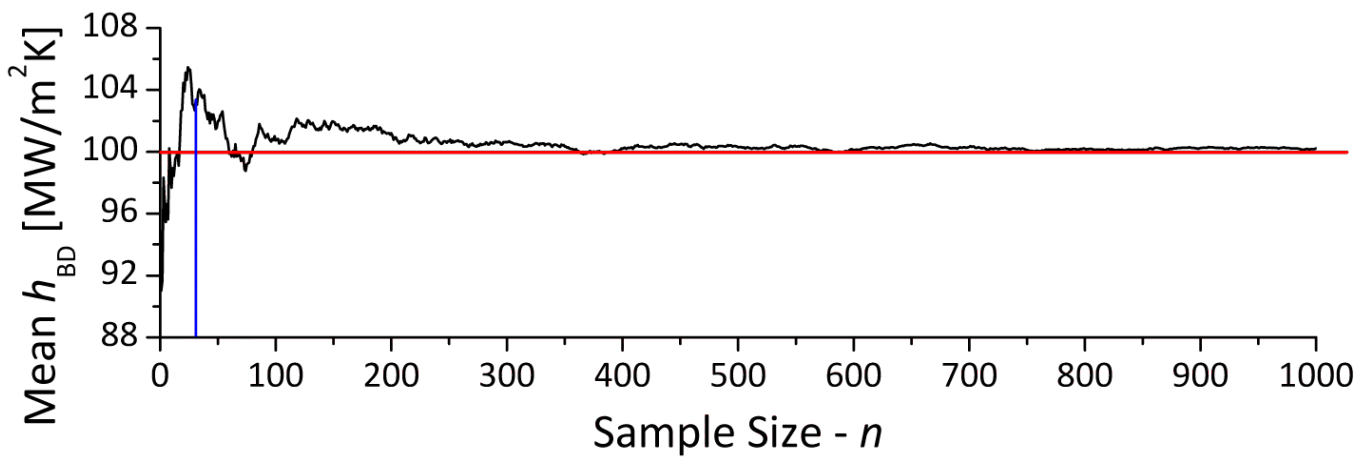

(a)

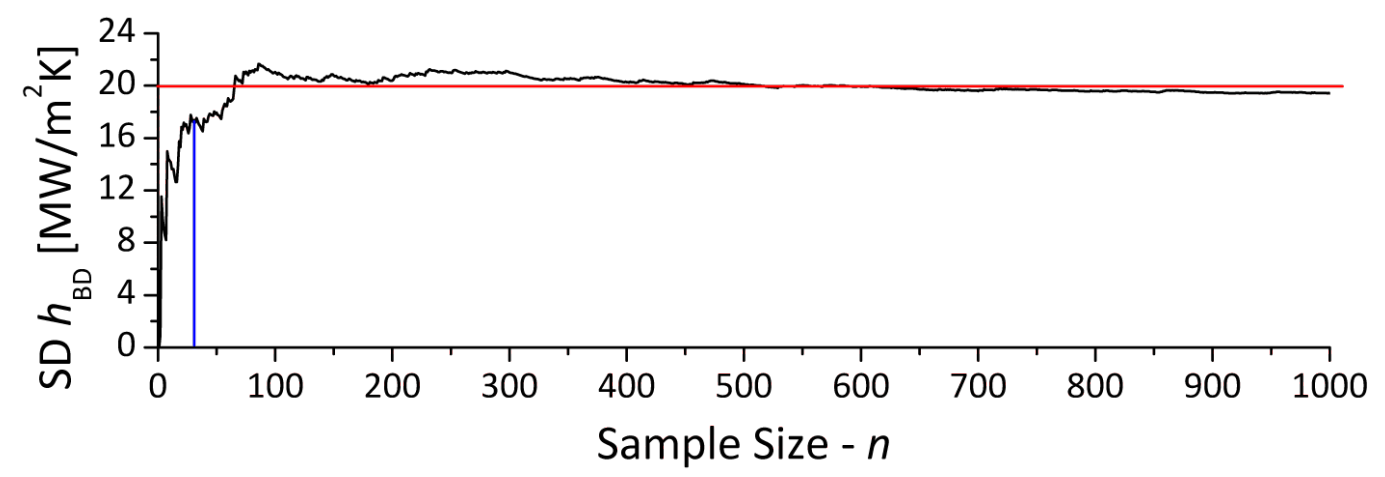

(b)

Figure 6.8: Mean (a) and standard deviation (b) of a random sampling of $h_{\mathrm{BD}}$ values from a normally distributed population of $\mu=100.00 \mathrm{MW} / \mathrm{m}^{2} \mathrm{~K}$ and $\sigma=20 \mathrm{MW} / \mathrm{m}^{2} \mathrm{~K}$ as a function of the sample size $n$. 
approximates the mean and standard deviation of the underlying population denoted by the red line as expected by the law of large numbers. Also denoted in the figure by the vertical blue line is $n=30$, the typically quoted sample size that is "sufficiently large" such that the standard deviation of a sample is a good representation of the standard deviation of the underlying population. We note that at $n=30$ the sample standard deviation is significantly lower than the nominal standard deviation of the population. It has been suggested that "the rule of 30 " should be replaced in favor of computational methods ${ }^{16}$ to test statistical assumptions [270].

By the law of large numbers, if we could collect a large enough data set and deduce a parameter of interest from each individual scan, we should be reasonably confident that our results would describe the population of these values for a particular sample independent of assumption as to how the population is distributed or analytic methods to infer the population parameters based on a smaller sample set. With TTR/TDTR data collection taking on the order of 5 minutes per scan, physically it would not be very feasible to collect on the order of 1,000 scans to take advantage of the law of large numbers and reproduce results such as in Figure 6.8. However, if we can simulate realistic data using a modeled system the primary limitation in the application of the law of large numbers analysis would be the computational time required. Using the tools developed in Chapter 5.4 to increase computational efficiency in conjunction with available computer power, this process becomes manageable.

Under the assumption that the thermophysical parameters of a TTR/TDTR sample are consistent, the dispersion in the deduced results are a function of the noise in the data and the sensitivity of the model to the parameter of interest. In order to simulate realistic TTR/TDTR data and attempt to provide a global intuition into the standard deviation in the data, a range of sensitivities and levels of noise typically found experimentally must be simulated.

\footnotetext{
${ }^{16} \mathrm{An}$ introduction to one of these methods, the bootstrap will be presented in the next section.
} 


\subsubsection{Modeling a Range of Fitting Sensitivities}

The sensitivity to deducing a thermophysical parameter of interest from the TTR/TDTR model (see Chapter 4) is dependent on the magnitude of the parameter of interest in conjunction with the magnitude of the other model parameters. The primary parameters that we will be interested in deducing will be the $h_{\mathrm{BD}}$ of the film/substrate interface and the thermal conductivity of the substrate ${ }^{17}$. To that end, and for the sake of consistency, we will consider all modeled systems to have a $100 \mathrm{~nm} \mathrm{Al} \mathrm{layer} \mathrm{as} \mathrm{the} \mathrm{film.} \mathrm{The} \mathrm{thermal}$ boundary conductance of the modeled systems were simulated from $h_{\mathrm{BD}}=1 \mathrm{MW} / \mathrm{m}^{2} \mathrm{~K}$ to $h_{\mathrm{BD}}=1,000 \mathrm{MW} / \mathrm{m}^{2} \mathrm{~K}$, representing an extreme range of possible values. These bounds on $h_{\mathrm{BD}}$ provided a range of sensitivity coefficients for both $h_{\mathrm{BD}}$ and the substrate thermal conductivity as shown for the $\mathrm{Al} / \mathrm{Si}$ system in Figure 6.9. To add further variation to the sensitivity coefficient and the modeled systems tested, a total of three substrates that are typically found in nanodevices and which represent a range of thermal conductivity values were modeled, namely Si, Ge, and AlN. A list of the thermophysical parameters used in modeling these systems along with the trends in the sensitivity coefficient as a function of time and $h_{\mathrm{BD}}$ can be found in Supplement S.

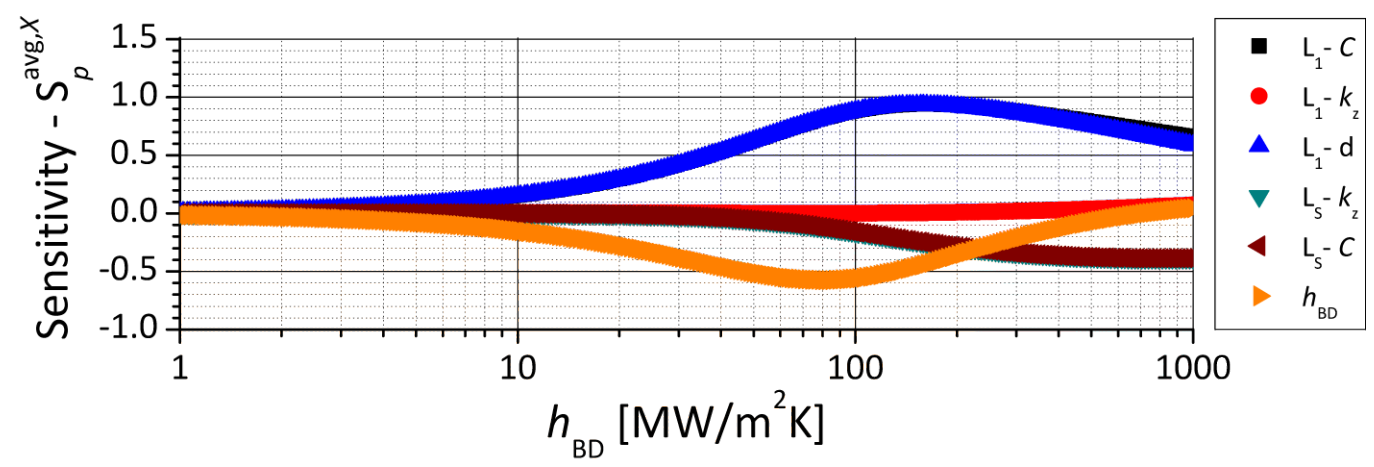

Figure 6.9: Sensitivity parameter as a function of boundary conductance for the modeled $100 \mathrm{~nm} \mathrm{Al}$ on Si system, where $\mathrm{L}_{1}$ denotes the $\mathrm{Al}$ film and $\mathrm{L}_{\mathrm{S}}$ the substrate.

Using these modeling parameters the ideal data sets were generated using $M=1,00,000$. The range of $h_{\mathrm{BD}}$ values provided a variety of temperature profiles, see Figure 6.10. Each of these data sets were then interpolated using the methodology described in Chapter 5.4

\footnotetext{
${ }^{17}$ This could also be the thermal conductivity of a layer of interest if the $\mathrm{Al}$ film is only present as a transducer layer.
} 


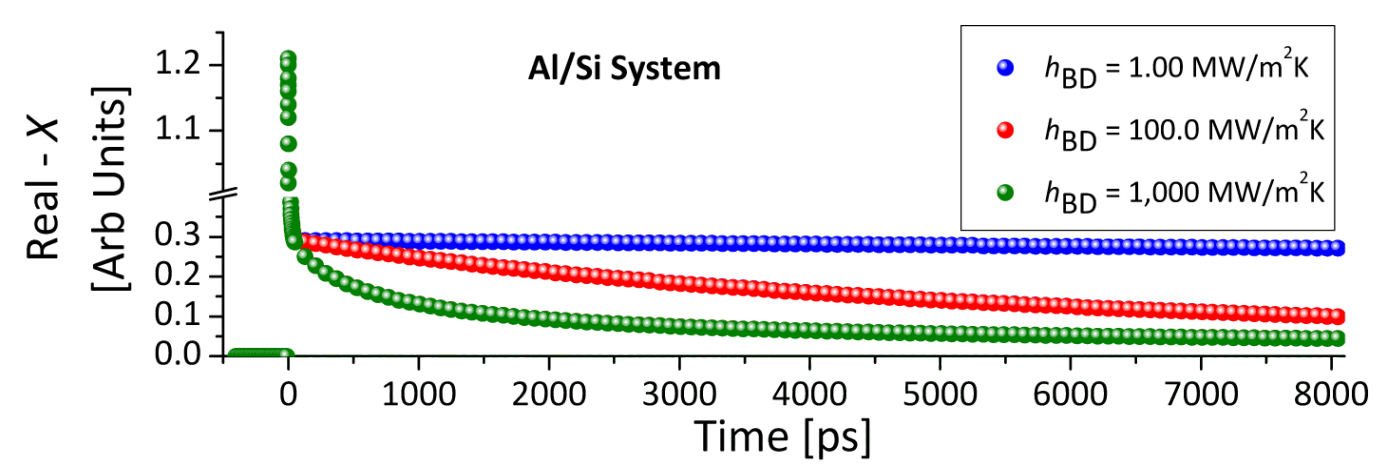

Figure 6.10: Examples of ideal data created for the $\mathrm{Al} / \mathrm{Si}$ system with various $h_{\mathrm{BD}}$ values.

to establish the proper time spacing for $M=50,000$. Each of the interpolated files then served as a basis from which statistical noise was added to create the realistic modeled data.

\subsubsection{Simulating Noise in the Data}

In order to create realistic thermoreflectance data and to ascertain the affects of noise in the raw data on the standard deviation of the deduced results, for each of the "perfect" data series of simulated scans random noise was added. To simulate experimental noise in the data we will first consider the distribution of noise found in real experimental data. Figure 6.11a provides examples of experimental data collected on a sample of $100 \mathrm{~nm}$ of $\mathrm{Pt}$ on $\mathrm{Si}$ using the low-rep TTR system configuration. In order to analyze the noise in the data, for a total of 152 scans collected at the same location on the surface of a Pt/Si sample, each time index in the collected data was normalized by the mean and standard deviation of the data at that time index using the relation:

$$
z=\frac{x-\bar{x}}{s}
$$

All the data points across the time of the scan were then collected $(n=14,288)$ to build the distribution of the noise, see Figure 6.11b. From the shape of the distribution and the analysis provided, the data appears to be distributed normally. To further confirm this assumption a Q-Q plot of the data and the results of the Shapiro-Wilk test are shown in Figure 6.11c. Based on the normality testing of the experimentally collected data, when simulating the TTR/TDTR data for testing we will assume that it is normally distributed. 


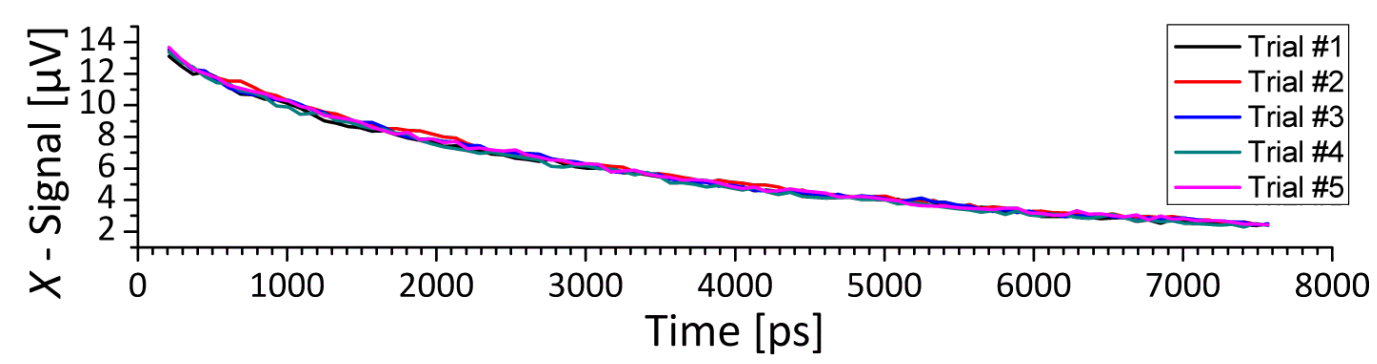

(a)

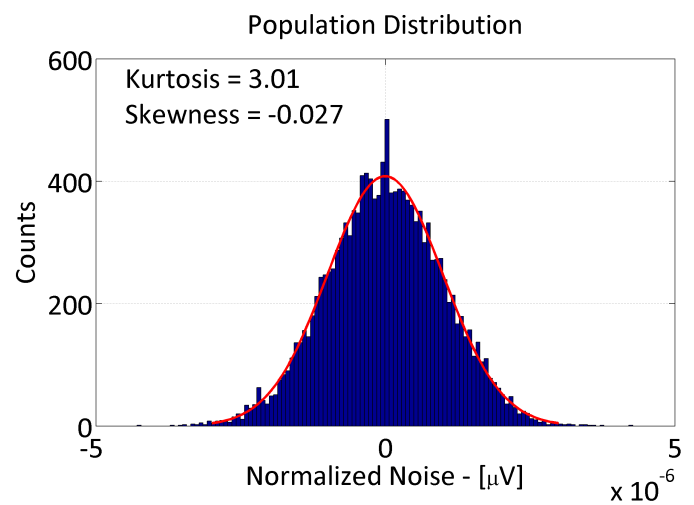

(b)

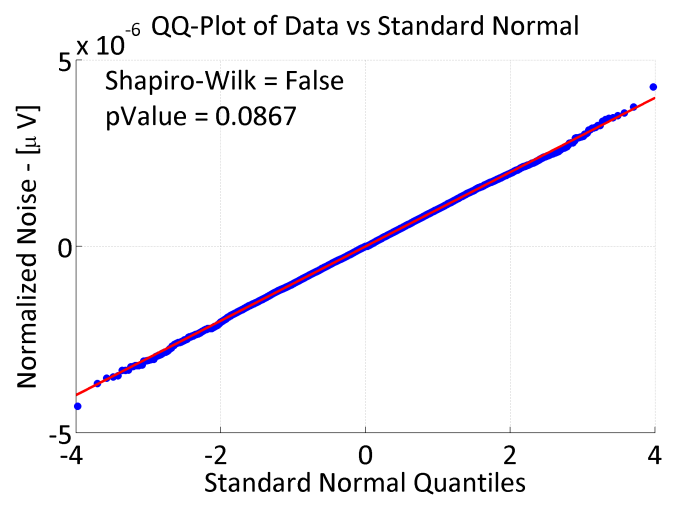

(c)

Figure 6.11: (a) Five examples of experimental data taken with the low-rep TTR system on $100 \mathrm{~nm}$ of Pt on $\mathrm{Si}$, (b) distribution of normalized noise in 152 trials of Pt/Si data and (c) Q-Q plot and Shapiro-Wilk analysis of noise in the experimental data.

Under the assumption that the noise in the data should be normally distributed we began by taking the ideal data set, i.e. the generated model using the appropriate time configuration or properly interpolated to take advantage of the node spacing routine presented in Chapter 5.4, and for each data point in the region of interest a normal distribution with a mean value equal to the signal at that time index was defined, see Figure 6.12. An initial standard deviation of the Gaussian distributions was set and a value randomly selected from the distribution for each time index to generate a new data set with added noise. A total of 1,000 simulated TTR/TDTR scans were produced and the signal-to-noise parameter for the series calculated as described in Chapter 5.4. If the stopping criteria was met, i.e. the desired SNP within a prescribed error tolerance, the individual data sets were saved. If the SNP was too high, the standard deviation of the Gaussian distributions were reduced and the process reiterated until the stopping criteria was reached. 


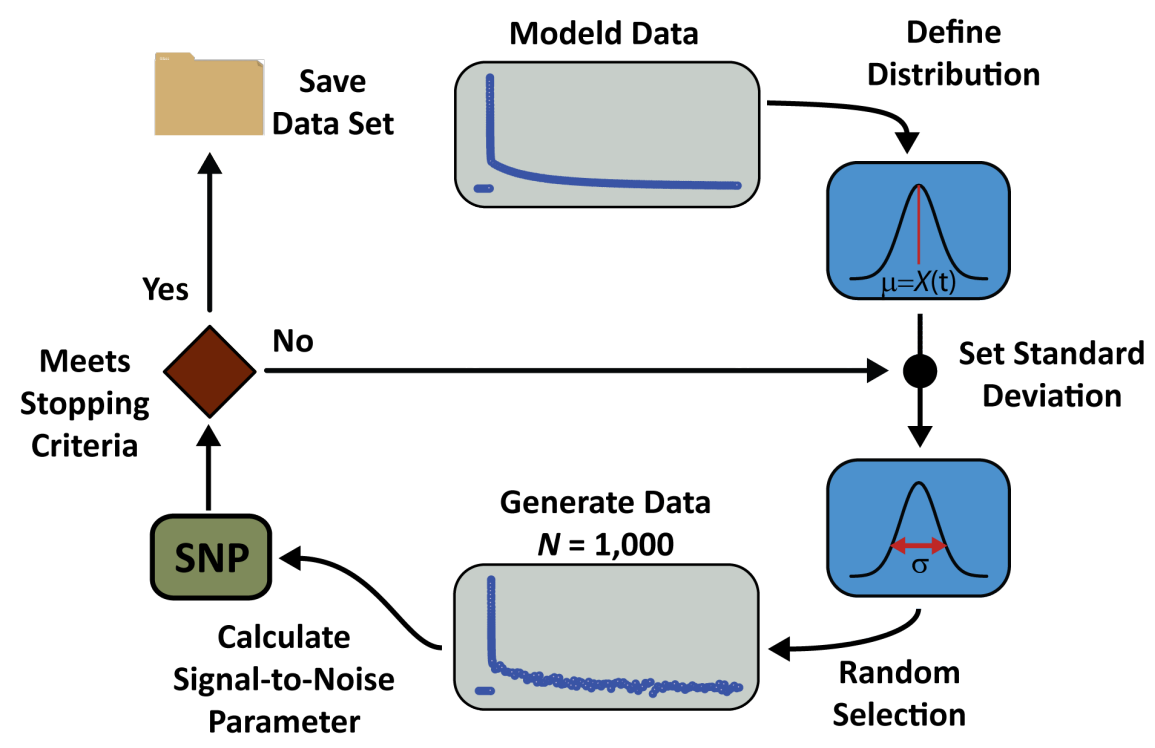

Figure 6.12: Process flow for adding normally distributed noise to modeled data.

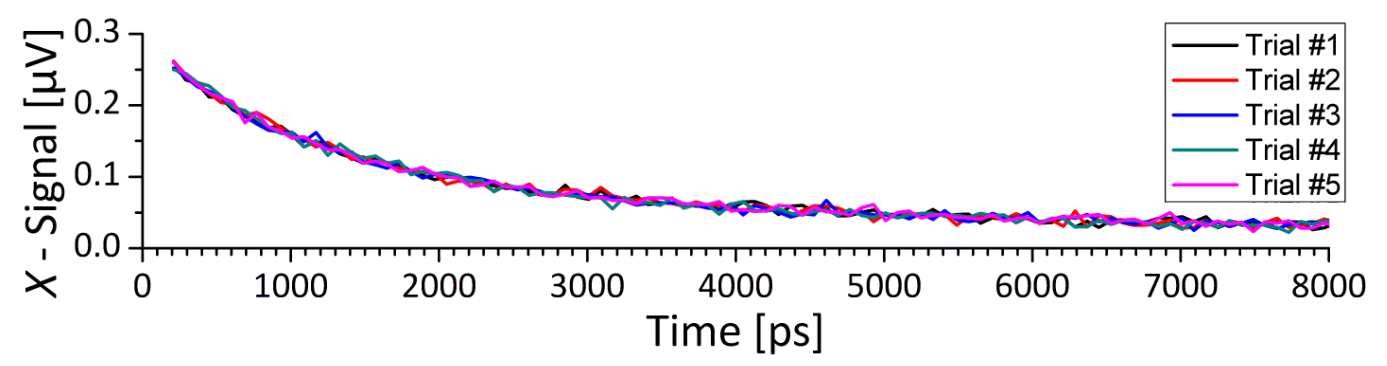

(a)

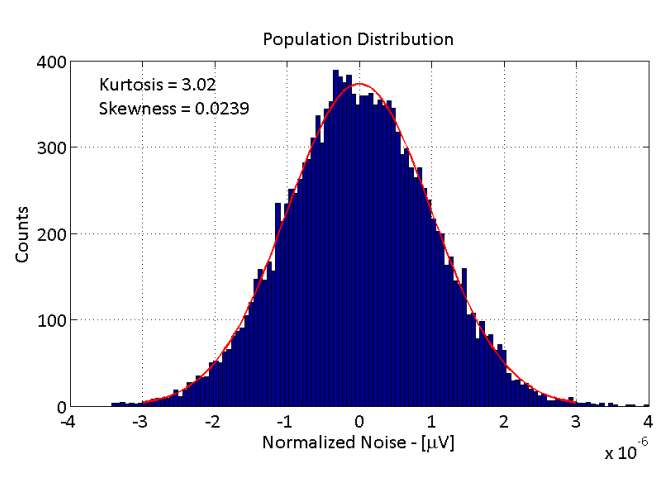

(b)

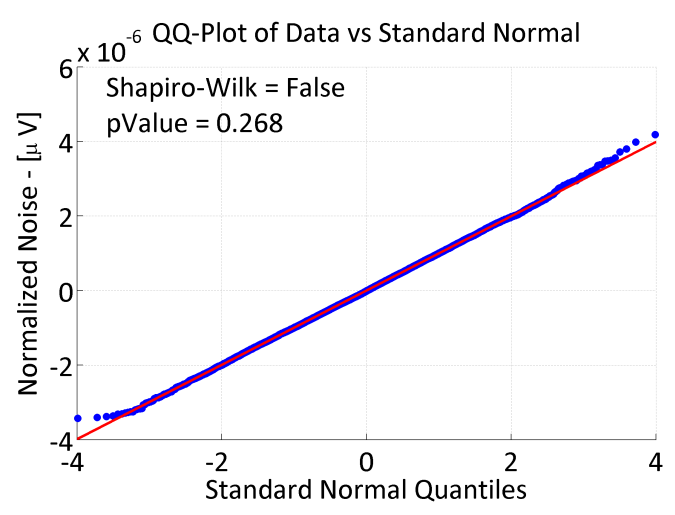

(c)

Figure 6.13: (a) Five examples of simulated data of $100 \mathrm{~nm}$ of $\mathrm{Al}$ on $\mathrm{Si}\left(h_{\mathrm{BD}}=215 \mathrm{MW} / \mathrm{m}^{2} \mathrm{~K}\right), f_{s}=250$ $\mathrm{kHz}$, and $f_{o}=125 \mathrm{kHz}$ with added noise (b) distribution of normalized noise in 152 trials of $\mathrm{Al} / \mathrm{Si}$ data and (c) Q-Q plot and Shapiro-Wilk analysis of noise in the simulated data.

To test the validity of this process as a means to simulate noise in modeled data, the distribution of noise in 152 simulated scans was tested for normality using the same pro- 
cedure that was used for the experimental data, see Figure 6.11. The results for simulated data of $100 \mathrm{~nm}$ of $\mathrm{Al}$ on $\mathrm{Si}\left(h_{\mathrm{BD}}=215 \mathrm{MW} / \mathrm{m}^{2} \mathrm{~K}\right), f_{s}=250 \mathrm{kHz}$, and $f_{o}=125 \mathrm{kHz}$ with added noise to produce a SNP of SNP $=12.70 \pm 0.04$ are shown in Figure 6.13. The results of the histogram, Figure 6.13b, and the Q-Q plot, Figure 6.13c, show that the noise in the data is indeed normally distributed as expected since it was generated using a Gaussian distribution, and follows the same trends as shown in the experimental data, see Figure 6.11. Therefore in terms of the noise we can assume that the simulated data is a valid representation of experimental TTR/TDTR data.

For the data generated in this work the SNP ranged from 1.21 to 113.53 . While there is no quantitative criteria for the selection of this range, qualitative experience has suggested that this represents a wide range of potential SNPs that have been encountered experimentally. Examples of the two extremes of the SNP are shown in Figure 6.14. For each of the modeled systems, i.e. film, substrate, and $h_{\mathrm{BD}}$ combinations, 21 different noise levels were generated with 1,000 random data sets created for each level. The generated data was run through the same LabVIEW ${ }^{\mathrm{TM}}$ program as the experimentally collected data to determine the signal-to-noise parameter for each system.

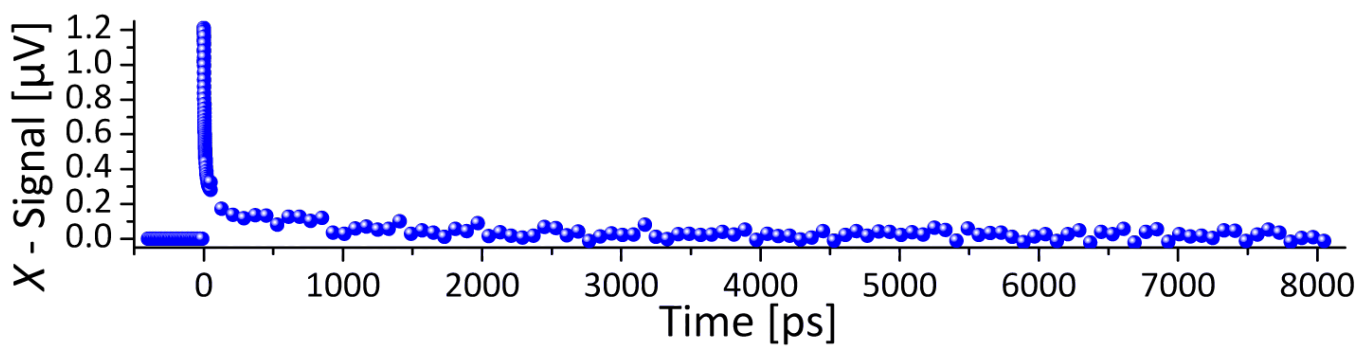

(a)

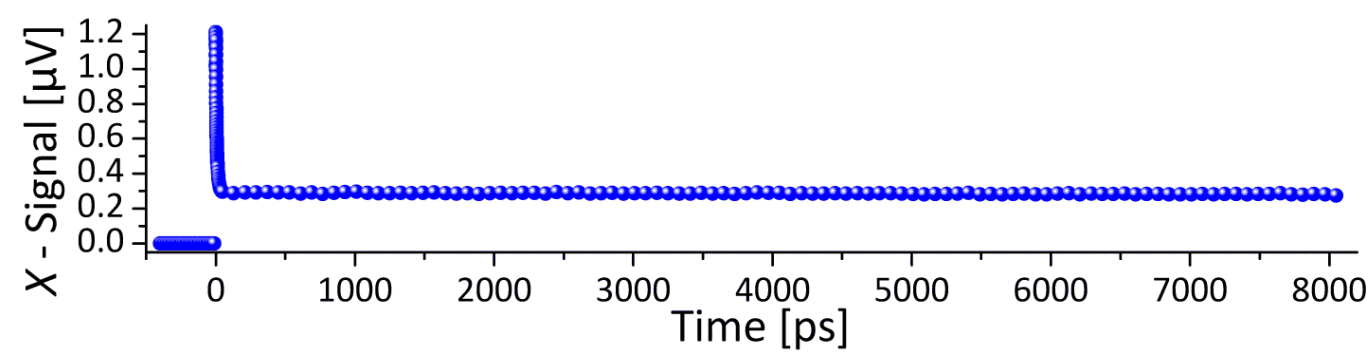

(b)

Figure 6.14: Simulated data of (a) $100 \mathrm{~nm}$ of $\mathrm{Al}$ on AlAlN $\left(h_{\mathrm{BD}}=1,000 \mathrm{MW} / \mathrm{m}^{2} \mathrm{~K}\right), f_{s}=250 \mathrm{kHz}$, and $f_{o}=125 \mathrm{kHz}$ with added noise $\mathrm{SNP}=1.21$ and (b) $100 \mathrm{~nm}$ of $\mathrm{Al}$ on $\mathrm{Si}\left(h_{\mathrm{BD}}=1 \mathrm{MW} / \mathrm{m}^{2} \mathrm{~K}\right), f_{s}=250 \mathrm{kHz}$, and $f_{o}=125 \mathrm{kHz}$ with added noise $\mathrm{SNP}=113.53$ 


\subsubsection{Fitting Simulated Data and Results}

In total 61 film, substrate, and $h_{\mathrm{BD}}$ combinations were generated with 21 SNPs and 1,000 data sets in each group for a total of over 1.28 million simulated data sets. For each of these data sets the standard TTR/TDTR analysis was completed as it would be for experiential data to deduce $h_{\mathrm{BD}}$ and other permutations of the substrate thermal conductivity and film thickness. In total, these results represent over 2.2 million simulated data fits consuming approximately 6 years of computational time ${ }^{18}$. For each system that was tested a results file was generated listing the data file name, best fit of the parameter of interest, the sum of the squared errors of the fit, and any program warnings, ${ }^{19}$ see Figure 6.15. This file was then used and edited as the data was post-processed.

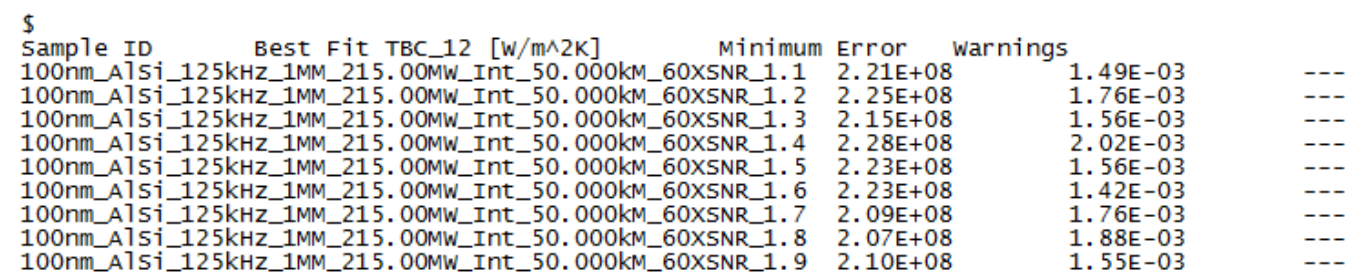

Figure 6.15: Example of the fit summary file generated by the TTR/TDTR fitting program recording the file name, best fit value, sum of squared errors, and any program warnings.

Each of the summary files from the fitted data sets were run through a post-processing program to remove any errors and/or statistical outliers. As discussed above, any fits that resulted in a program error were automatically discarded by the post-processing program. The remaining data was tested for the presence of statistical outliers using the generalized extreme studentized deviate as described in Chapter 5.7. An example of a data set before and after the removal of errors and outliers is shown in Figure 6.16.

With the errors and outliers removed we can analyze the results of the fitted $h_{\mathrm{BD}}$ data to determine how the data is distributed and if the data appears to converge following the law of large numbers. Figure 6.17 shows an example of the statistical analysis of the modeled system of $100 \mathrm{~nm}$ of $\mathrm{Al}$ on $\mathrm{Si}\left(h_{\mathrm{BD}}=215 \mathrm{MW} / \mathrm{m}^{2} \mathrm{~K}\right), f_{s}=250 \mathrm{kHz}, f_{o}=125$

\footnotetext{
${ }^{18}$ Note that without the node spacing formulation developed in Chapter 5.4 the required computational time based on brute force convergence in $M$ would be on the order of 100 years.

${ }^{19}$ The fitting routine is terminated when the supplied tolerance is met or the maximum number of iterations is reached. If the program stops due to the maximum number of iterations being reached without convergence the program produces an error flag.
} 


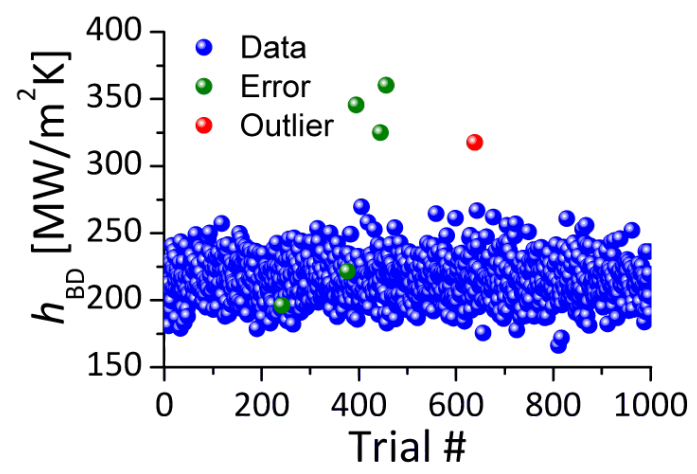

(a)

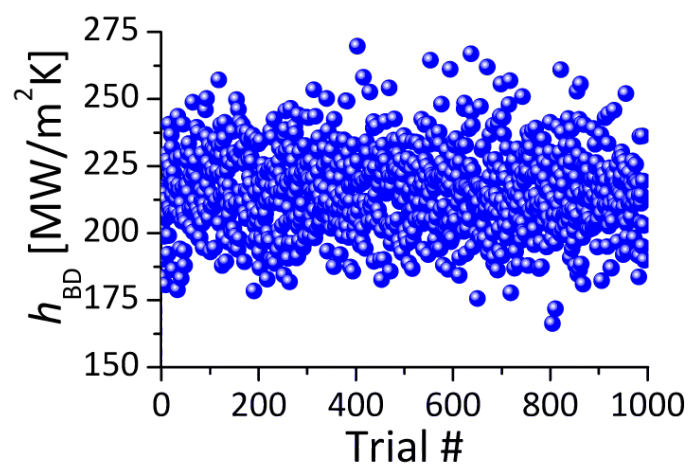

(b)

Figure 6.16: Results of fitting simulated TTR data to deduce $h_{\mathrm{BD}}$ for a system of $100 \mathrm{~nm}$ of $\mathrm{Al}$ on $\mathrm{Si}\left(h_{\mathrm{BD}}\right.$ $\left.=215 \mathrm{MW} / \mathrm{m}^{2} \mathrm{~K}\right), f_{s}=250 \mathrm{kHz}, f_{o}=125 \mathrm{kHz}$, and $\mathrm{SNP}=6.37$ (a) showing the raw data including errors and outliers and (b) with the errors and statistical outliers removed.

$\mathrm{kHz}$, for a signal-to-noise parameter of $\mathrm{SNP}=6.37$. From assessment of the normality of the data collected, see Figures $6.17 \mathrm{a}$ and $6.17 \mathrm{~b}$, it appears that in general the population of the $h_{\mathrm{BD}}$ values is approximately normally distributed. While only one example of this type of analysis is shown for brevity the analysis of the data as a whole supports this conclusion. The interpretation of the data via the law of large numbers, see Figures $6.17 \mathrm{c}$ and $6.17 \mathrm{~d}$, indicates that the mean and standard deviation of the data has converged within the number of samples tested.

To plot the results as in Figure 6.17 for each material combination and noise level would require over 1,500 figures. Therefore to summarize the results for each material system and parameter fit the data is tabulated as in Table 6.2. The table shows the resulting mean values and standard deviations of the deduced results as a function of the SNP for each series of 1,000 simulated data sets. The error quantification in the table represents a $95 \%$ confidence interval in the mean and the standard deviation respectively ${ }^{20}$. We do not expect the mean values to deviate greatly regardless of noise as all the models in this series were generated with the same $h_{\mathrm{BD}}$ value. We find that this holds in general to within the error tolerances, with only slight deviations from the ideal $h_{\mathrm{BD}}$ for very low SNPs. To visually inspect the trend in the standard deviation, the data in Table 6.2 is plotted as a function of the SNP, see Figure 6.18. Because we are interested in developing a general trend in the standard

\footnotetext{
${ }^{20}$ The confidence interval of the mean was calculated using Equation 6.16 and the confidence interval of the standard deviation is based on the Chi-square distribution, see Appendix D.
} 


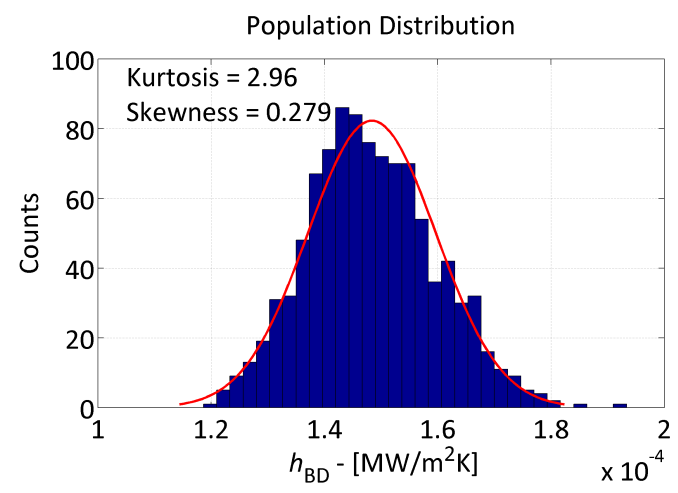

(a)

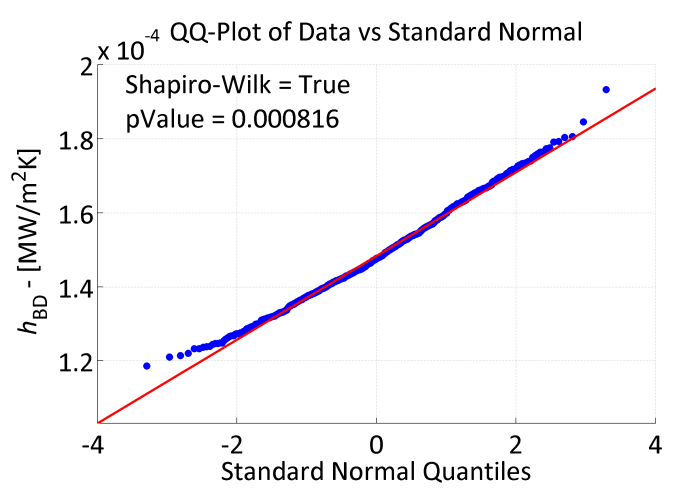

(b)

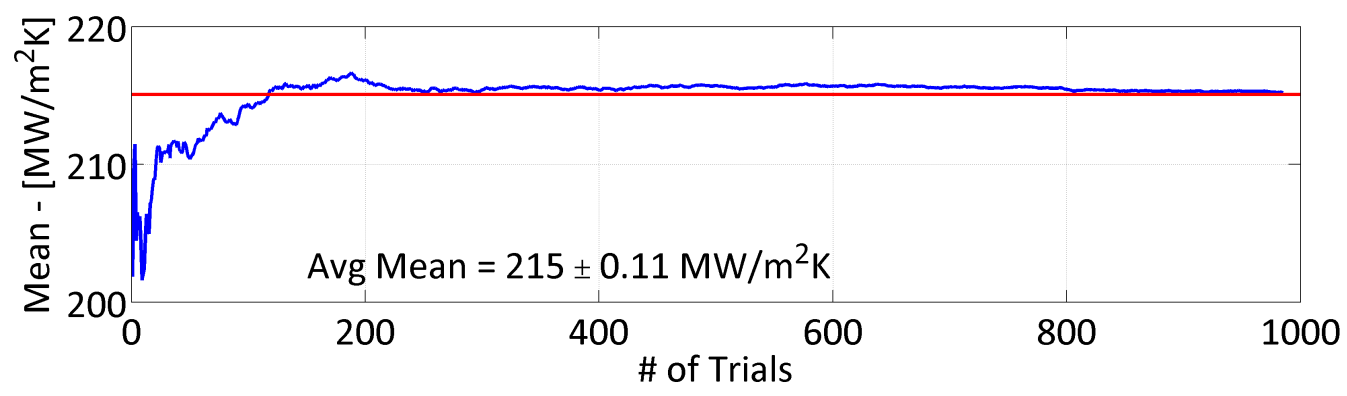

(c)

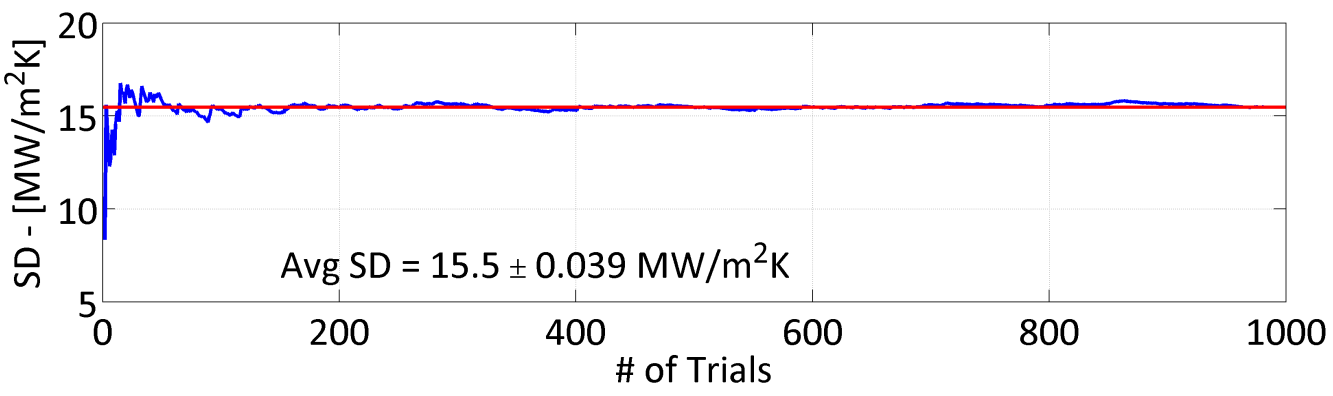

(d)

Figure 6.17: Analysis of the results of fitting simulated TTR data to deduce $h_{\mathrm{BD}}$ for a system of $100 \mathrm{~nm}$ of $\mathrm{Al}$ on $\mathrm{Si}\left(h_{\mathrm{BD}}=215 \mathrm{MW} / \mathrm{m}^{2} \mathrm{~K}\right), f_{s}=250 \mathrm{kHz}, f_{o}=125 \mathrm{kHz}$, and $\mathrm{SNP}=6.37$ for normality (a)-(b) and the mean and standard deviation of the results as a function of the number or trials considered (c)-(d). The red horizontal line in (c)-(d) represents the average mean and average standard deviation across all trials.

deviation of a parameter of interest, we will plot the relative standard deviation, i.e. the sample standard deviation normalized by the sample mean and reported as a percentage, as opposed to the absolute values shown in Table 6.2. This will allow a comparison across any deduced thermophysical parameter of interest regardless of magnitude.

The relative standard deviation of $h_{\mathrm{BD}}$ as a function of the signal-to-noise parameter 
Table 6.2: Summary of simulation data - $100 \mathrm{~nm} \mathrm{Al}$ on $\mathrm{Si} h_{\mathrm{BD}}=215 \mathrm{MW}$ Series. Error bounds represent a $95 \%$ confidence level.

\begin{tabular}{cccccc}
\hline \multicolumn{6}{c}{$\mathbf{1 0 0} \mathbf{~ n m ~ A l ~ o n ~} \mathbf{~ S i}, h_{\mathrm{BD}}=\mathbf{2 1 5} \mathbf{M W} / \mathbf{m}^{2} \mathbf{K}$} \\
\hline $\mathbf{S N P}$ & $\mu-\left[\mathbf{M W} / \mathbf{m}^{2} \mathbf{K}\right]$ & $\sigma-\left[\mathbf{M W} / \mathbf{m}^{2} \mathbf{K}\right]$ & $\mathbf{S N P}$ & $\mu-\left[\mathbf{M W} / \mathbf{m}^{2} \mathbf{K}\right]$ & $\sigma-\left[\mathbf{M W} / \mathbf{m}^{2} \mathbf{K}\right]$ \\
2.13 & $222.50 \pm 3.255$ & $50.05 \pm 0.1531$ & 13.80 & $215.04 \pm 0.456$ & $7.30 \pm 0.0206$ \\
3.21 & $218.14 \pm 2.152$ & $34.05 \pm 0.0984$ & 14.77 & $214.76 \pm 0.421$ & $6.74 \pm 0.0190$ \\
4.26 & $217.52 \pm 1.588$ & $25.29 \pm 0.0721$ & 16.01 & $215.28 \pm 0.406$ & $6.49 \pm 0.0183$ \\
5.34 & $215.72 \pm 1.188$ & $19.00 \pm 0.0537$ & 16.94 & $215.12 \pm 0.383$ & $6.12 \pm 0.0173$ \\
6.37 & $215.19 \pm 0.968$ & $15.48 \pm 0.0438$ & 18.12 & $215.03 \pm 0.337$ & $5.38 \pm 0.0152$ \\
7.45 & $215.19 \pm 0.851$ & $13.61 \pm 0.0385$ & 19.07 & $215.11 \pm 0.339$ & $5.42 \pm 0.0153$ \\
8.52 & $215.05 \pm 0.744$ & $11.91 \pm 0.0336$ & 20.25 & $215.03 \pm 0.305$ & $4.87 \pm 0.0138$ \\
9.56 & $215.06 \pm 0.645$ & $10.32 \pm 0.0292$ & 21.18 & $215.02 \pm 0.291$ & $4.66 \pm 0.0132$ \\
10.60 & $215.59 \pm 0.596$ & $9.54 \pm 0.0269$ & 22.25 & $214.91 \pm 0.281$ & $4.50 \pm 0.0127$ \\
11.71 & $215.07 \pm 0.544$ & $8.71 \pm 0.0246$ & 23.34 & $214.80 \pm 0.273$ & $4.37 \pm 0.0124$ \\
12.73 & $214.99 \pm 0.512$ & $8.20 \pm 0.0232$ & & & \\
\hline
\end{tabular}

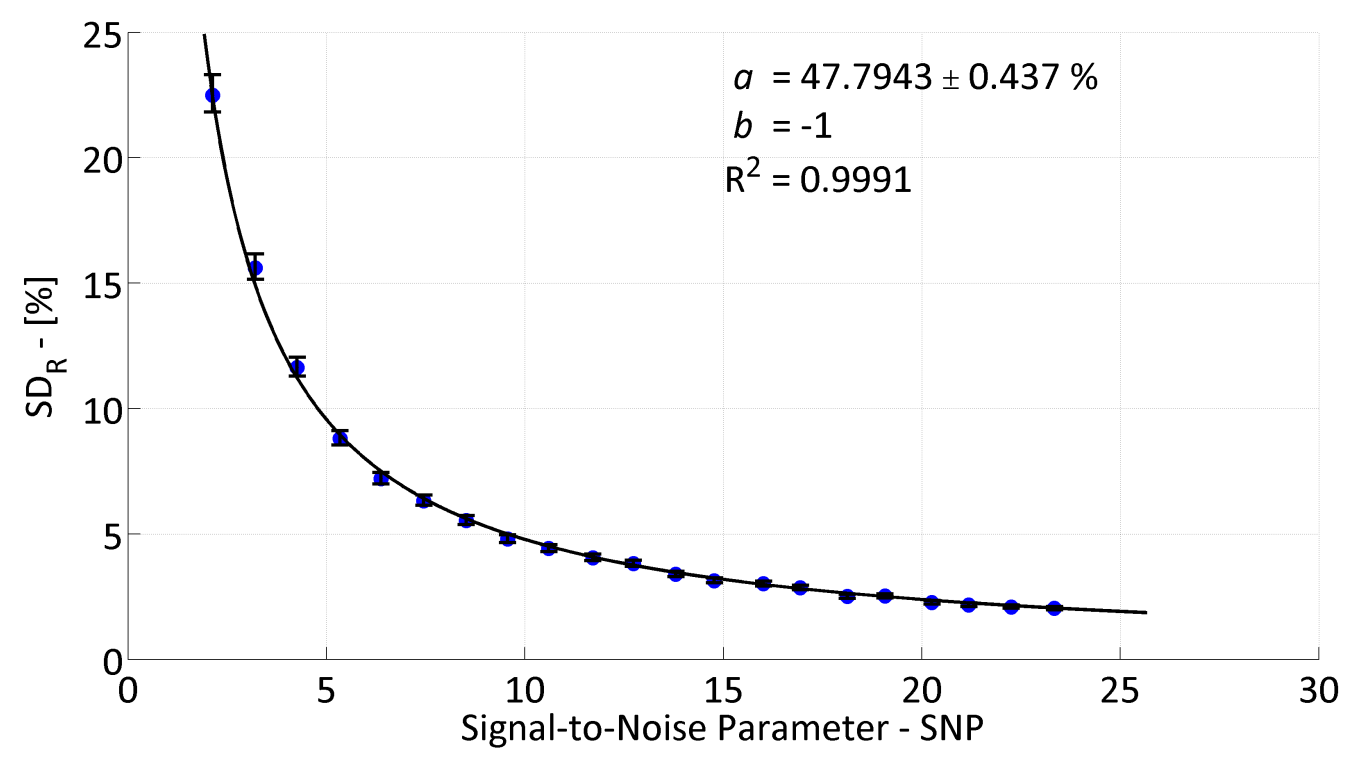

Figure 6.18: Relative population standard deviation as a function of signal-to-noise parameter for 100 $\mathrm{nm}$ of $\mathrm{Al} / \mathrm{Si}$ with $h_{\mathrm{BD}}=215.00 \mathrm{MW} / \mathrm{m}^{2} \mathrm{~K}$. Error bars represent a $95 \%$ confidence interval of the measured $\mathrm{SD}_{\mathrm{R}}$ and the solid line represents a best fit of a power law to the data, of the form $\mathrm{SD}_{\mathrm{R}}=a \times \mathrm{SNP}^{b}$, with parameter $a$ provided for a fixed $b=-1$, along with the $95 \%$ confidence level in $a$.

follows a power law trend. Therefore for each data set, i.e. film, substrate, and $h_{\mathrm{BD}}$ combination, the data collected as in Figure 6.18 was fit using a power law of the form $\mathrm{SD}_{\mathrm{R}}=a \times \mathrm{SNP}^{b}$ where $b$ was fixed at -1 and the fitting coefficient $a$ and the confidence in $a$ determined by the fitting routine. Again Figure 6.18 represents a single example of the 
94 systems analyzed. A summary of the tables of fitted values and the plots of the relative standard deviations as a function of SNP for each of the simulated systems and parameters deduced are provided in Supplement S.

\subsubsection{Standard Deviation Simulation Results}

From analysis of the statistical data of the deduced thermophysical parameters from the simulated TTR data several interesting trends have been discovered. We will begin by considering the fitting coefficient of the standard deviation analysis, $a$, as a function of $h_{\mathrm{BD}}$ as it is the primary thermophysical parameter of interest in this study, see Figure 6.19. Often times conceptually the transport of thermal energy in a film-substrate system is considered via a theoretical set of series thermal resistors for the film, interface, and substrate or sample. It is assumed that the thermal model will have the greatest sensitivity to the highest thermal resistance as it will introduce the highest temperature drop in the system. That is if $h_{\mathrm{BD}}$ is very low, i.e. thermal resistance at the interface high, compared to the substrate thermal conductivity the model will be most sensitive to $h_{\mathrm{BD}}$. In contrast, if the substrate or sample thermal conductivity is very low we would intuitively expect from the resistor analogy that it would be the most sensitive parameter. However, the results of Figure 6.19 show that for approximately $h_{\mathrm{BD}}<50 \mathrm{MW} / \mathrm{m}^{2} \mathrm{~K}$ the fitting coefficient increases with decreasing $h_{\mathrm{BD}}$, which for a given SNP translates to decreased precision in the deduced $h_{\mathrm{BD}}$.

While this conclusion seems counter intuitive compared to the resistor analysis concept,

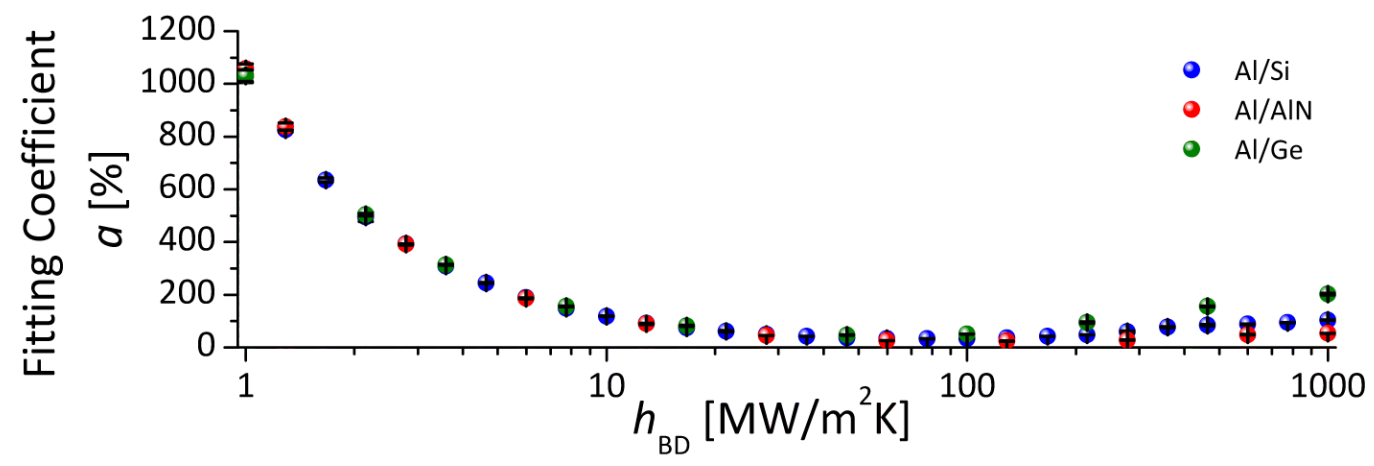

Figure 6.19: Fitting coefficient of the relative standard deviation analysis as a function of $h_{\mathrm{BD}}$ for the modeled systems of $\mathrm{Al} / \mathrm{Si}, \mathrm{Al} / \mathrm{AlN}$, and $\mathrm{Al} / \mathrm{Ge}$. 
it does follow the trends in the model sensitivity to $h_{\mathrm{BD}}$ as shown in Figure 6.9. The data in the figure indicates that for low values of $h_{\mathrm{BD}}$ the TTR/TDTR model has little sensitivity for any of the input parameters. In essence, the interface is "too resistive" and there is very little heat transported away from the surface film. Stoner and Maris provided an estimate of the interface time constant, $\tau_{\text {int }}$, for the decay of the film temperature in the pretense of an interface, given by $[8,10]$ :

$$
\tau_{\text {int }}=\frac{d C_{f}}{h_{\mathrm{BD}}}
$$

where $d$ is the film thickness and $C_{f}$ is the specific heat capacity of the film. Figure 6.20 shows the interface time constant as a function of $h_{\mathrm{BD}}$ for the interface conductances considered in this study. The maximum pump-to-probe delay time in the experimental system used is $8 \mathrm{~ns}$, which from Figure 6.20 corresponds to an interface conductance on the order of $30 \mathrm{MW} / \mathrm{m}^{2} \mathrm{~K}$. Comparing this value to Figure 6.19 we see that this roughly corresponds to the minimum in the fitting coefficient, $a$, and therefore a maximum in the precision of the ability to deduce $h_{\mathrm{BD}}$. The also explains why we find from Figure 6.19 that for $h_{\mathrm{BD}}$ values below on the order of $h_{\mathrm{BD}}=30 \mathrm{MW} / \mathrm{m}^{2} \mathrm{~K}$ that the fitting coefficient is independent of the properties of the substrate. To increase the precision in the ability to deduce $h_{\mathrm{BD}}$ the film can be made thinner, however the time constant for heat diffusion across the film is on the order of less than $1 \mathrm{~ns}$ which is small compared to the interface time constant for low $h_{\mathrm{BD}}$ values. Therefore, the only way to increase the precision in the ability to deduce $h_{\mathrm{BD}}$ for low interface conductance systems, is by increasing the maximum pump-to-probe delay time as is evident by the extended sensitivity plot shown in Figure

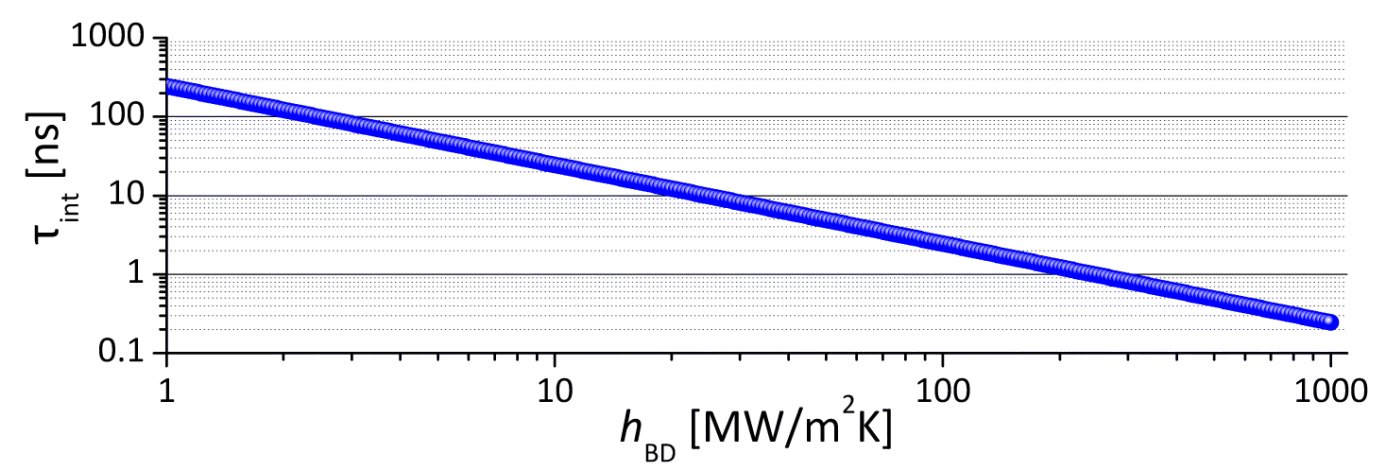

Figure 6.20: Interface time constant, $\tau_{\text {int }}$, as a function of $h_{\mathrm{BD}}$, see Equation 6.18. 
6.21. As indicated by Figure 6.20 , the sensitivity to $h_{\mathrm{BD}}$ should not increase significantly until the delay time is on the order of 100s of nanoseconds, which is shown in the sensitivity plot given in Figure 6.21.

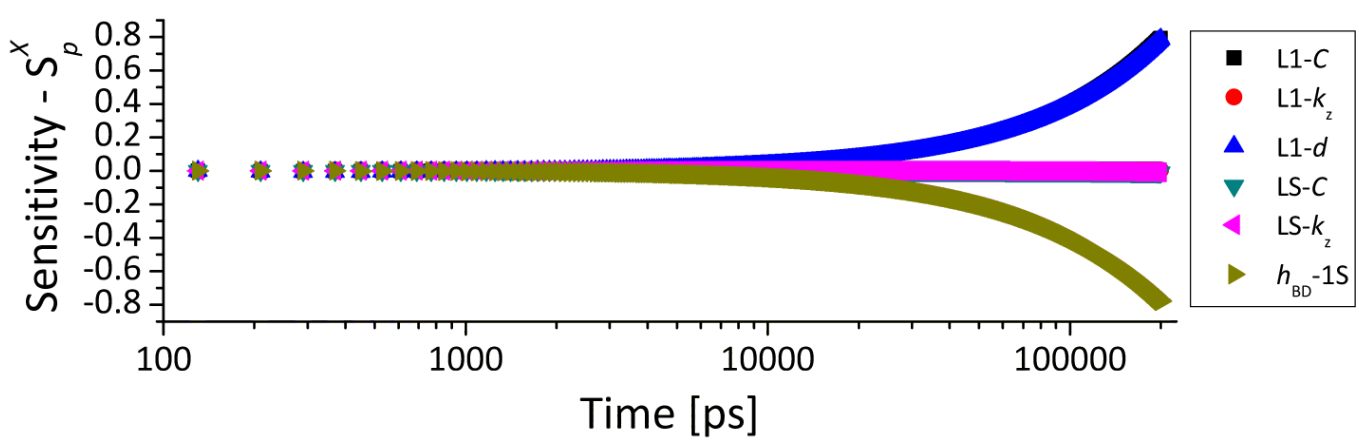

Figure 6.21: Sensitivity of the real signal, $X$, as a function of extended time for the thermal parameters in the $100 \mathrm{~nm} \mathrm{Al}$ on $\mathrm{Si}$ system for $h_{\mathrm{BD}}=1.00 \mathrm{MW} / \mathrm{m}^{2} \mathrm{~K}$.

While $h_{\mathrm{BD}}$ is the primary thermophysical parameter of interest in this work, to make the discussion more global we can consider the fitting coefficient as a function of the sensitivity coefficient, see Figure 6.22, for the $\mathrm{Al}$ on Si series. We notice that the data in Figure 6.22 appears to follow two different trends. If we recall the form of the sensitivity coefficient to $h_{\mathrm{BD}}$ as a function of $h_{\mathrm{BD}}$, see Figure 6.9 , we notice that it too appears to follow two different trends. The magnitude of the sensitivity coefficient to $h_{\mathrm{BD}}$ begins small and increases with increasing $h_{\mathrm{BD}}$ reaching a maximum around $h_{\mathrm{BD}}=75 \mathrm{MW} / \mathrm{m}^{2} \mathrm{~K}$. As $h_{\mathrm{BD}}$ increases further the sensitivity coefficient to $h_{\mathrm{BD}}$ begins to decrease again. These two different regions will produce different trends in the fitting coefficient as a function of the sensitivity coefficient.

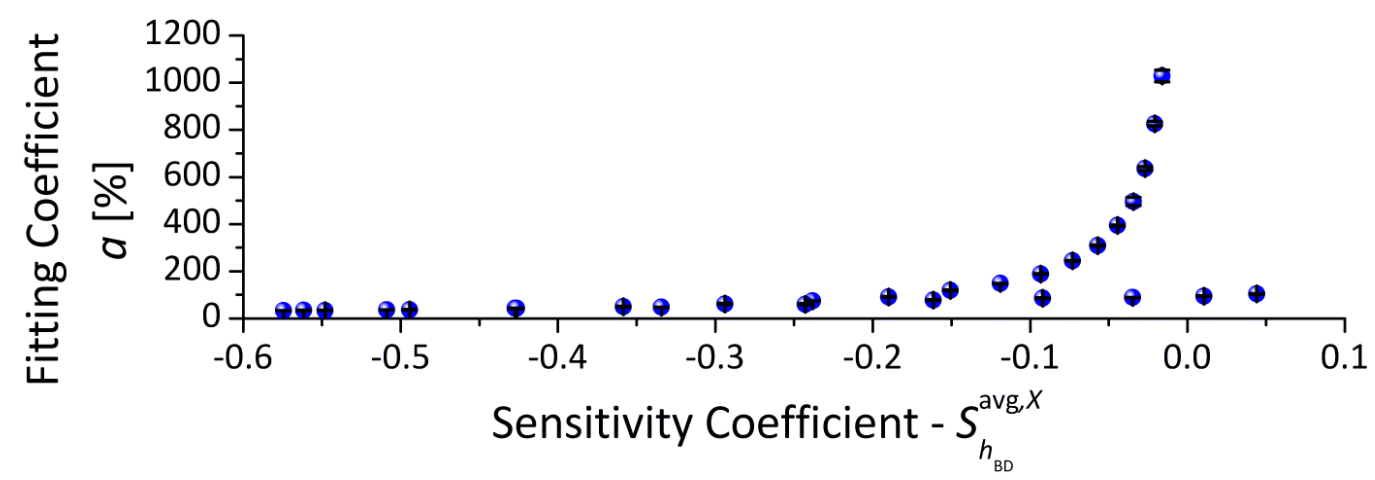

Figure 6.22: Standard deviation coefficient as a function of sensitivity coefficient for the $100 \mathrm{~nm}$ of $\mathrm{Al}$ on Si simulated data series. 
The first region we will define will be the diffusive regime. This will include sensitivity coefficients for the thermophysical properties of the film and substrate, i.e. specific heat capacity, thermal conductivity, and thickness. In addition, this regime will include the sensitivity to $h_{\mathrm{BD}}$ below the inflection point in the curve of the sensitivity coefficient for $h_{\mathrm{BD}}$ as a function of $h_{\mathrm{BD}}$, see Figure 6.9. For the systems in this study that will be typically for $h_{\mathrm{BD}}<80 \mathrm{MW} / \mathrm{m}^{2} \mathrm{~K}$, corresponding to where the fitting coefficient is independent of the substrate properties. The second region will be defined as the effusive regime where the fitting coefficient as a function of the sensitivity coefficient for $h_{\mathrm{BD}}$ will be dependent on the substrate thermal conductivity. The data indicates that the $h_{\mathrm{BD}}$ dependence on substrate thermal conductivity in the effusive regime is a special case for the sensitivity to $h_{\mathrm{BD}}$, and it will therefore be the only parameter in this regime ${ }^{21}$.

In the analysis of the data we will begin by considering the diffusive regime for the precision in the deduction of thermophysical parameters. Figure 6.23 shows the fitting

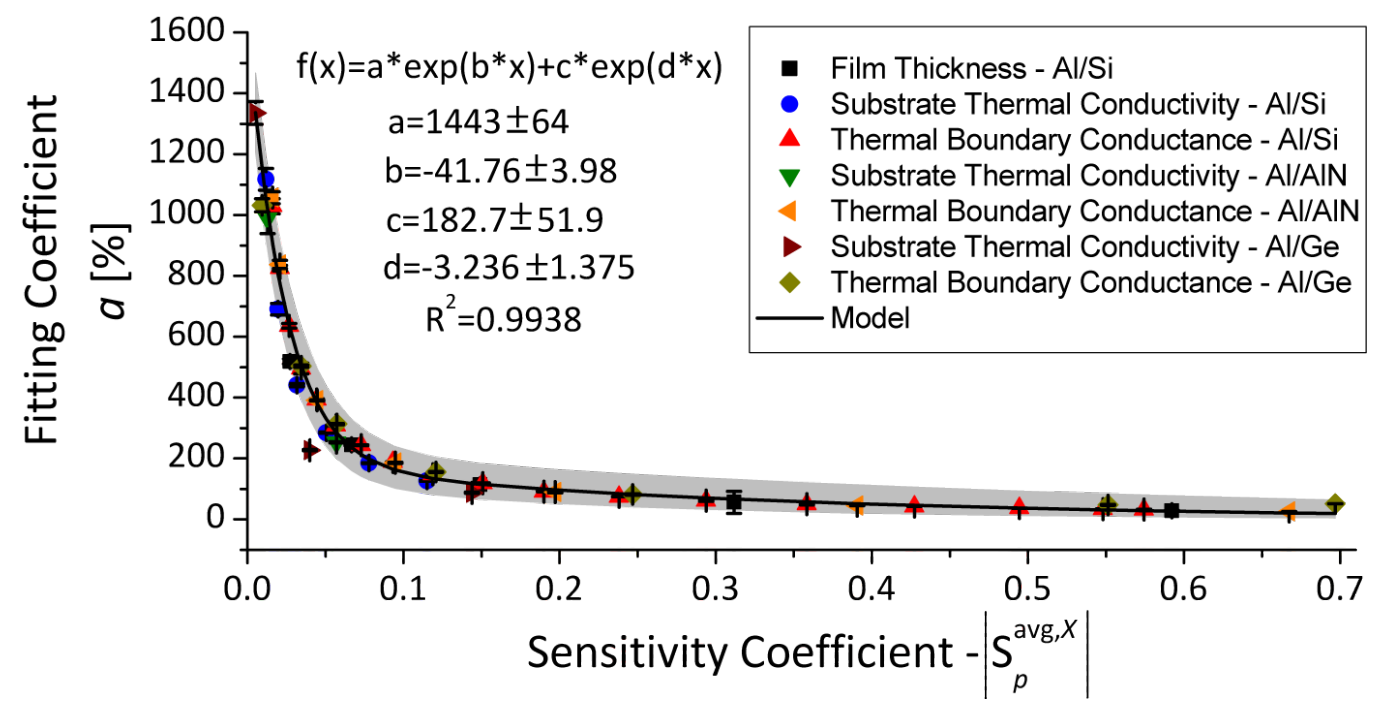

Figure 6.23: Fitting coefficient as a function of the absolute value of the sensitivity coefficient for various parameter fits in the diffusive regime. The data was fit using a double exponential of the form $f(x)=$ $a * \exp (b x)+c * \exp (d x)$ with the fitting coefficients listed. The bounds on the coefficients and the grey shaded area represent a $95 \%$ confidence interval in the fitted values and the model respectively.

\footnotetext{
${ }^{21}$ It is important to note that the bounds of these regimes were defined in part based on the inflection in the sensitivity coefficient as a function of parameter for $h_{\mathrm{BD}}$. These results indicate that this type of inflection in the trend of the sensitivity as a function of parameter may be indicative of different system behaviors. Therefore, care must be taken in analyzing sensitivity results if such points are manifested for other systems and/or parameters.
} 
coefficient as a function of the absolute value of the sensitivity coefficient ${ }^{22}$ for several different material systems and parameters of interest. As expected, as the magnitude of the sensitivity coefficient decreases the fitting coefficient increases and thereby for a given SNP the standard deviation in the deduced parameter increases. The collective data was fit using a double exponential formulation of the form $f(x)=a * \exp (b x)+c * \exp (d x)$. The trend in the model and the $95 \%$ confidence region of the prediction is shown by the black line and the gray shaded region in Figure 6.23 respectively. Using the relation for the fitting coefficient, $a$, which was developed for the relative standard deviation as a function of the $\mathrm{SNP}$, i.e. $\mathrm{SD}_{\mathrm{R}}=a \times \mathrm{SNP}^{b}$, in conjunction with the double exponential formulation, we can develop an empirical relation for the expected relative standard deviation in an deduced parameter, $\mathrm{SD}_{\mathrm{R}, \mathrm{D}}$, in the diffusive regime as a function of the sensitivity coefficient for that parameter and the SNP. This relation is given by:

$$
\mathrm{SD}_{\mathrm{R}, \mathrm{D}}=\frac{C D_{1} \times \exp \left[C D_{2} \times S_{p}^{\mathrm{avg}, X}\right]+C D_{3} \times \exp \left[C D_{4} \times S_{p}^{\mathrm{avg}, X}\right]}{\mathrm{SNP}}
$$

where the $C D_{i}$ 's are the fitting coefficients for the model in the diffusive regime given by, $C D_{1}=1443 \pm 64, C D_{2}=-41.76 \pm 3.98, C D_{3}=182.7 \pm 51.9$, and $C D_{4}=-3.236 \pm 1.375$, with the bounds on the constants representing $95 \%$ confidence intervals on the modeling parameters.

As an example we will consider the system of $100 \mathrm{~nm}$ of $\mathrm{Al}$ on $\mathrm{Si}$ with $h_{\mathrm{BD}}=30 \mathrm{MW} / \mathrm{m}^{2} \mathrm{~K}$ which has a sensitivity coefficient to $h_{\mathrm{BD}}$ of $\left|S_{h_{\mathrm{BD}}}^{\mathrm{avg}, X}\right|=0.3786$. Using Equation 6.19 the standard deviation as a function of the SNP for the Al/Si system is shown in Figure 6.24. Provided with an estimate for the SNP from experiential intuition or a real-time measurement from the experimental system, the standard deviation in the $h_{\mathrm{BD}}$ population can be estimated.

One important consideration of the sensitivity coefficient developed in Chapter 5 which has not been discussed explicitly, is the lower bound of the sensitivity coefficient for which a parameter can be deduced. From the development of the sensitivity coefficient, there is

\footnotetext{
${ }^{22}$ For analyzing the trends in the sensitivity the sign of the sensitivity coefficient is important for indicating the shift in the model relative to the shift in the input parameter. However, for calculating the uncertainty in the deduced result, the analysis depends only on the magnitude of the sensitivity coefficient.
} 


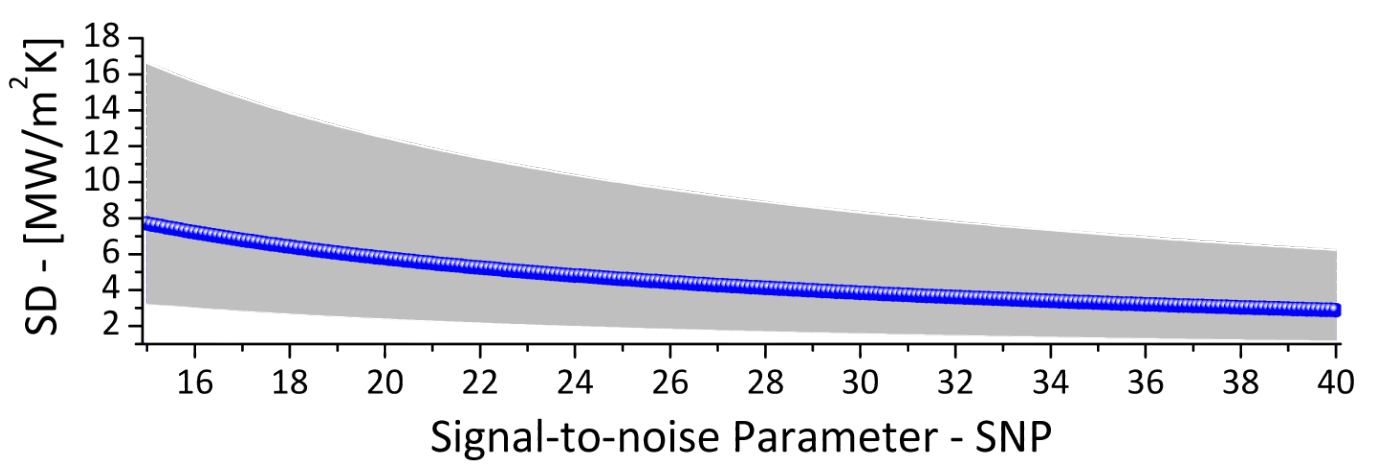

Figure 6.24: Estimated standard deviation of the population of $h_{\mathrm{BD}}$ values for a modeled system of 100 $\mathrm{nm}$ of $\mathrm{Al}$ on $\mathrm{Si}\left(h_{\mathrm{BD}}=30 \mathrm{MW} / \mathrm{m}^{2} \mathrm{~K}\right)$ as a function of the SNP in the data as calculated from Equation 6.19. The blue data points represent the analysis based on the best fit coefficients and the gray shaded region the $95 \%$ confidence region in the model.

no obvious mathematical formulation which can be employed to produce a lower bound to when a parameter can be reasonably deduced. The results of this empirical study however, see Figure 6.23, provide evidence for a usable rule of thumb. During the simulation process it was found that the results became highly dependent on the amount of noise in the data for $\left|S_{h_{\mathrm{BD}}}^{\mathrm{avg}, X}\right|<0.01$. At or below this level of sensitivity and for low SNPs, the data became erratic and the results did not converge after 1,000 iterations. The trend in Figure 6.23 shows that below $\left|S_{h_{\mathrm{BD}}}^{\text {avg } X}\right| \approx 0.05$ the fitting coefficient increases significantly, which for SNPs below 10 can result in an over 50\% uncertainty in the measured values and require a very large sample size to produce precise results. Therefore I recommend care be taken when considering a sensitivity coefficient below $\left|S_{h_{\mathrm{BD}}}^{\mathrm{avg}, X}\right| \approx 0.05$ as the experimental cost in the number of scans required and the uncertainty in the final precision may be high.

Turning to the results of the simulation data collected for the systems in the effusive regime, the fitting coefficient as a function of the absolute value of the sensitivity coefficient for these systems is shown in Figure 6.25. As mentioned previously, these results are dependent on the thermal conductivity of the substrate and therefore for each material system, i.e. film and substrate combination, a different trend is observed. The trend for each system was fit using a exponential decay model ${ }^{23}$ of the form $f(x)=a * \exp (b x)$. Similar

\footnotetext{
${ }^{23}$ It should be noted that several different mathematical formulations could be used to fit the data in Figure 6.25 including linear and polynomial models. The exponential model was chosen as we would expect that as the absolute value of the sensitivity coefficient becomes exceedingly large, the fitting coefficient, and in turn for a given SNP the standard deviation in the deduced parameter, would tend towards zero. A more in-depth investigation of $h_{\mathrm{BD}}$ in the effusive regime will be part of the future work.
} 


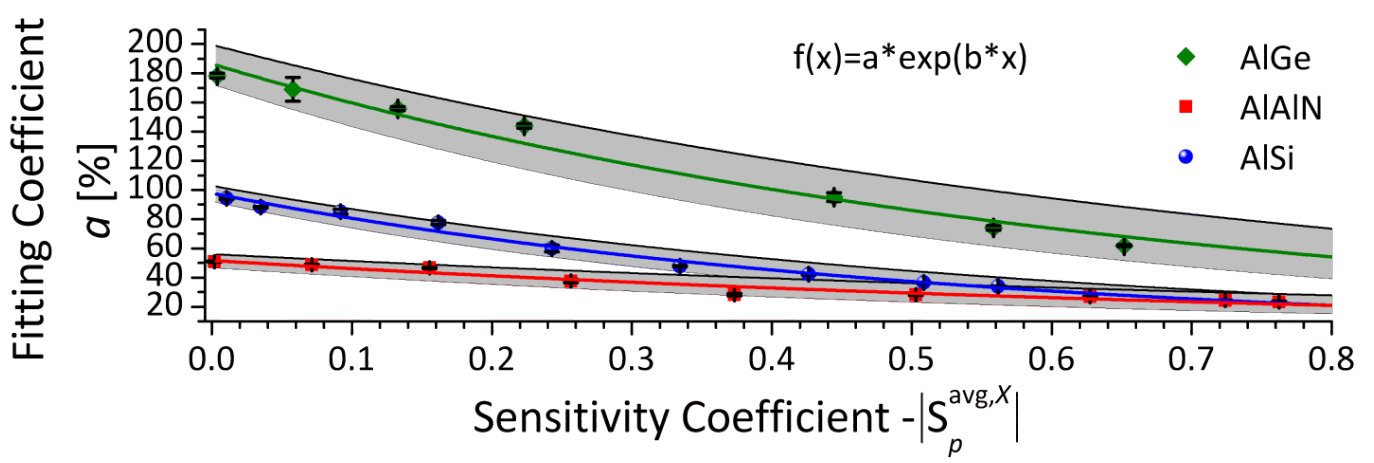

Figure 6.25: Fitting coefficient as a function of the absolute value of the sensitivity coefficient for $h_{\mathrm{BD}}$ in the AlSi, AlAlN, and AlGe sample series in the effusive regime. The data was fit using a exponential of the form $f(x)=a * \exp (b x)$ with the coefficients AlSi $(a=97.76 \pm 5.14, b=-1.93 \pm 0.25)$, AlAlN $(a=51.65 \pm 4.40, b=-1.13 \pm 0.26)$, and AlGe $(a=186.40 \pm 13.20, b=-1.55 \pm 0.30)$. The bounds on the coefficients and the grey shaded area represent a $95 \%$ confidence interval in the values and model respectively.

to the diffusive regime, using the relation for the fitting coefficient, $a$, which was developed for the relative standard deviation as a function of the $\mathrm{SNP}$, i.e. $\mathrm{SD}_{\mathrm{R}}=a \times \mathrm{SNP}^{b}$, in conjunction with the exponential fit, we can develop an empirical relation for the expected relative standard deviation in the deduced $h_{\mathrm{BD}}, \mathrm{SD}_{\mathrm{R}, \mathrm{E}}$, as a function of the sensitivity coefficient and the SNP in the effusive regime. This relation is given by:

$$
\mathrm{SD}_{\mathrm{R}, \mathrm{E}}=\frac{C E_{1, i} \times \exp \left[C E_{2, i} \times S_{p}^{\mathrm{avg}, X}\right]}{\mathrm{SNP}}
$$

where $C E_{1, i}$ and $C E_{2, i}$ are the fitting constants for the model in the effusive regime of the $i^{\text {th }}$ substrate. For the material systems investigated in this study the fitting coefficients are given in Table 6.3 .

From the developed empirical relations, see Equations 6.19 and 6.20, using the estimated sensitivity coefficient of the desired parameter and an estimation of the SNP, we can

Table 6.3: Fitting coefficients for estimating the relative standard deviation in the deduced parameters calculated for the effusive regime, see Equation 6.20, for the three system substrates used in this study along with the $R^{2}$ values of the model fits.

\begin{tabular}{rccc}
\hline & $C E_{1, i}$ & $C E_{2,1}$ & $R^{2}$ \\
\hline Silicon & $97.76 \pm 5.14$ & $-1.93 \pm 0.25$ & 0.9836 \\
Germanium & $186.40 \pm 13.20$ & $-1.55 \pm 0.30$ & 0.9801 \\
Aluminum nitride & $54.65 \pm 4.40$ & $-1.13 \pm 0.26$ & 0.9464 \\
\hline
\end{tabular}


calculate an estimate for the standard deviation in the deduced result. We will consider a more in-depth example and compare the calculated results to experiment in Section 6.9.

\subsection{Statistical Analysis via Bootstrapping}

To this point we have spent a lot of effort in determining an appropriate sample size, $n$, such that the conditions of the central limit theorem are satisfied. We can therefore assume a normal distribution of sample means and that the standard deviation of that distribution, i.e. the standard error, is such that we can define a confidence interval that falls within our accuracy tolerances. While much effort has been made to keep $n$ as small as possible to reduce experimental costs while maintaining statistical significance, there is an important consideration that has not yet been addressed. In theory, when building the sampling distribution of an estimator we assume that we choose an appropriate sample size $n$, and then conduct a sufficient number, $N$, of repeated samplings, i.e experiments, to populate the distribution. Even if we can keep the sample size $n$ small the experimental costs of repeated samplings would be prohibitive.

Therefore, in statistical practice very seldom do we actually construct the sampling distribution of an estimator. Instead we rely heavily on the assumption that the sampling distribution of the estimator is normal. Using the power of this assumption we utilize nice analytic forms for the mean of the distribution and the confidence interval of the mean based on the standard error, see Equations 6.5, 6.6, and 6.10. While this makes for simple calculations, it places all the weight of our statistical analysis and the statistical significance of our results on the assumption of normality of the sampling distribution without ever testing this assumption.

Luckily with the dramatic increase in available computational power over the last few decades methods are available in order to attempt to put these assumptions to the test. In order to provide a computational means to complete what we would ideally accomplish, repeated experiments, the concept of bootstrapping is presented ${ }^{24}$. Using the bootstrapping

\footnotetext{
${ }^{24}$ I make no claims to any new creations in terms of the bootstrapping methodology, but rather introduce the bootstrap as technique which has merits in the analysis of TTR/TDTR data and should be used in conjunction with traditional statistics to analyze results.
} 
methodology will provide a computational method to populate a statistical analog to the sampling distribution of an estimator and provide a robust manner in which to calculate accurate population estimators and their confidence levels.

\subsubsection{Notation and Principles of Bootstrapping}

The concept of bootstrapping was first proposed by Bradley Efron in 1979 [271, 272]. Efron's primary purpose in developing the bootstrap was the estimation of confidence intervals, particularly for situations of small samples sizes such that asymptotic assumptions were inaccurate [273]. We will not belabor the history of bootstrapping but will point interested readers to several literature sources with information and background on its development $[273-275]$.

The bootstrapping process begins in the same manner as did our previous statistical analysis, by assuming we have a random sample ${ }^{25}$ of size $n$ with values given by $\mathbf{X}=$ $\left\{x_{1}, x_{2}, x_{3}, \ldots x_{n}\right\}$ taken from an unknown distribution $F$. Again we will denote $\theta$ as an unknown property of $F$, e.g. mean, median, etc. of which we would like to find an estimator $\hat{\theta}$ derived from information in the sample $\mathbf{X}$. It is at this point in the previous analysis where we would assume that the distribution of repeated estimations was normal and that the properties of the distribution could be obtained, along with confidence bounds, through the information in the single sample set and a series of analytic expressions. To avoid the experimental expense of repeated trials the bootstrapping methodology suggests resampling data from a distribution, $\hat{F}$, which is similar to $F$. How we choose to define $\hat{F}$ is important and characterizes two of the main sub-categories of bootstrapping, parametric and nonparametric.

In the parametric bootstrapping technique we assume that we have some knowledge as to how $F$ is distributed. For example, we could assume that $F$ is normally distributed with an unknown mean, $\mu$, and unknown variance, $\sigma^{2}$. We would then draw our random sample from a normal distribution, $\hat{F}_{\hat{\theta}}$ whose properties are estimated from the properties of the sample $\bar{x}$ and $s^{2}$. The parametric formulation of the bootstrap resampling has been shown to work well, provided that the assumptions about the form of the underlying distribution

\footnotetext{
${ }^{25}$ Again by random we assume that the values selected are independent and identically distributed (i.i.d).
} 
are correct [273]. The appeal of the non-parametric bootstrap is that no assumptions are made about the underlying distribution. Rather, the original data set, $\mathbf{X}$, is treated as an empirical distribution, $\hat{F}_{E}$, that is a close statistical representation of $F$. In fact, no calculations are performed on $\mathbf{X}$, e.g estimation of the mean, variance, etc., but the values in the data set are themselves resampled.

Regardless of the type of bootstrapping conducted, the data is resampled with replacement ${ }^{26}$ from either the original data (non-parametric) or the assumed distribution (parametric) to create a new sample set of $\operatorname{size}^{27} n$, known as the bootstrap sample set, $\mathbf{X}^{*}=\left\{x_{1}^{*}, x_{2}^{*}, x_{3}^{*}, \ldots x_{n}^{*}\right\}$. The concept of randomly resampling with replacement has several important impacts on the bootstrap sample set, $\mathbf{X}^{*}$. It provides that the probability of an element of the bootstrap sample set, $x_{i}^{*}$, being equal to an element of the original sample set, $x_{j}$, is uniform and given by [273]:

$$
\operatorname{Prob}\left[x_{i}^{*}=x_{j} \mid \mathbf{X}\right]=n^{-1}
$$

This provides that just like the original data the bootstrap data points, the $x_{j}^{*}$ 's, are independent and identically distributed. This implies that when looking at an individual bootstrap sample, $\mathbf{X}^{*}$, some of the individual values from the original sample, $x_{j}$, may appear more than once, or not at all.

From the bootstrap data set we calculate a new estimator $\hat{\theta}^{*}$. The process of resampling and calculating a new estimator is repeated $B \operatorname{times}^{28}$ to create a distribution of estimators $\hat{\mathbf{\Theta}}^{*}=\left\{\hat{\theta}_{1}^{*}, \hat{\theta}_{2}^{*}, \hat{\theta}_{3}^{*} \ldots \hat{\theta}_{B}^{*}\right\}$. This distribution of bootstrap estimators, $\hat{\mathbf{\Theta}}^{*}$, is the bootstrap analog of the sampling distribution of estimators, $\hat{\Theta}$, which we can use for diagnostics, and for estimating standard error and confidence intervals. A schematic of the non-parametric bootstrapping procedure is shown in Figure 6.26. The only difference in the process for the parameter bootstrap procedure in comparison to the non-parametric bootstrap procedure is the resampling of data from a distribution with properties estimated from the original sample properties rather than directly from the original sample set itself.

\footnotetext{
${ }^{26}$ Sampling with replacement means that after sampling a value, it is not removed from the pool but is still available to be chosen again.

${ }^{27}$ It is important that we keep the size of the bootstrap sample the same as the original sample.

${ }^{28}$ We will discuss later how the choice of $B$ may impact the final results.
} 


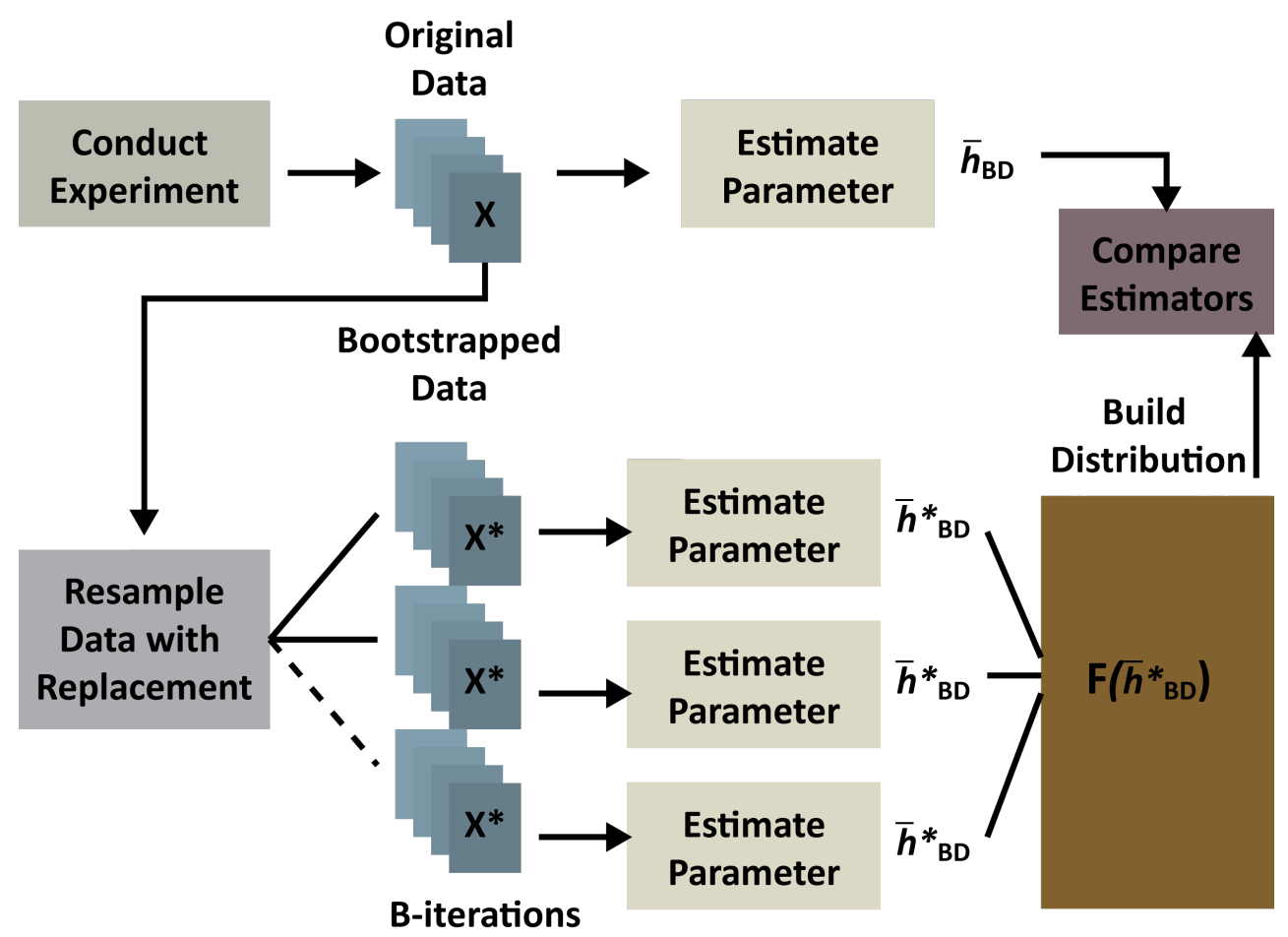

Figure 6.26: Schematic in the non-parametric bootstrapping of experimental data to deduce $h_{\mathrm{BD}}$.

\subsubsection{Bootstrapping Diagnostics of the Sampling Distribution}

One of the major strengths of the bootstrapping process is that it allows us to create with actual numbers, an analog for the sampling distribution of an estimator. As a result of this, it gives an opportunity to test the assumptions made in using the central limit theorem. That is, we assume that $n$ is large enough such that we can assume that the sampling distribution of an estimator is normal. Using the bootstrapping methods we can test the normality, see Appendix D, of the bootstrap distribution of estimators, $\hat{\mathbf{\Theta}}^{*}$, and therefore if our choice of $n$ was sufficiently large to satisfy the assumptions of the central limit theorem.

As an example of our ability to diagnosis the validity of the sample size $n$, we will consider two pseudo-normal populations with similar kurtosis values but two different levels of skewness, see Figure 6.27. From each of these populations we select a random sample of size $n=10$ and complete the bootstrapping routine using $B=10,000$ resamplings to build the sampling distribution of the bootstrapped means, see Figure 6.28. In Figure 6.28a, as expected since the underlying population was normally distributed, the sampling distribu- 


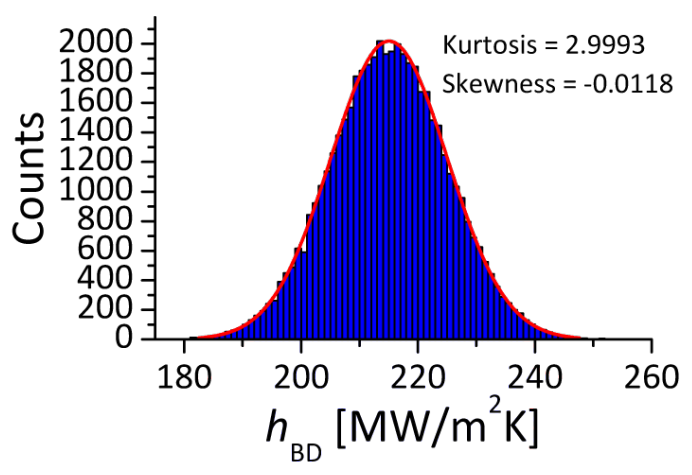

(a)

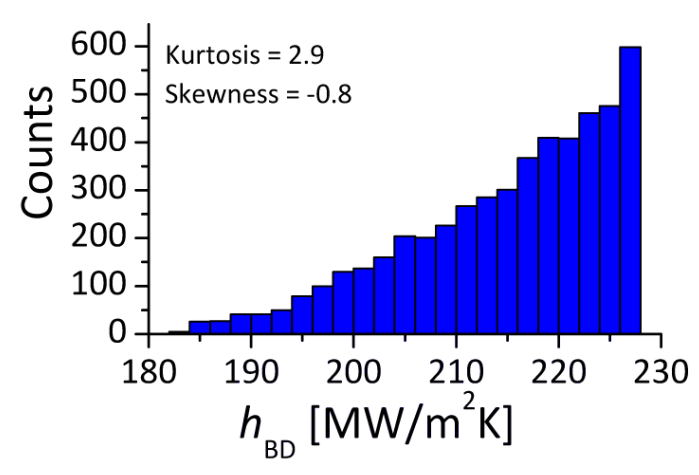

(b)

Figure 6.27: Distribution of 50,000 and 5,000 random samples respectively selected from (a) a normal distribution with $\beta_{\text {kurt }}=3$ and a skewness coefficient of $\beta_{\text {skew }}=0$ and (b) a skewed distribution with $\beta_{\text {kurt }}=2.9$ and $\beta_{\text {skew }}=-0.8$. The skew and kurtosis listed in the plots are the calculated values from the data sets.

tion of the bootstrapped means is also normal ${ }^{29}$. In Figure $6.28 \mathrm{~b}$, we notice however that the sample size $n=10$ is not sufficiently large to produce a completely normal distribution of bootstrapped means. Therefore, any analytic assumptions made about the distribution of the means, e.g. the standard error, confidence interval, etc., that were made based on the assumption of normality would be suspect. The bootstrap method therefore provides a relatively simplistic method to test whether a sample size is "sufficiently large" to produce

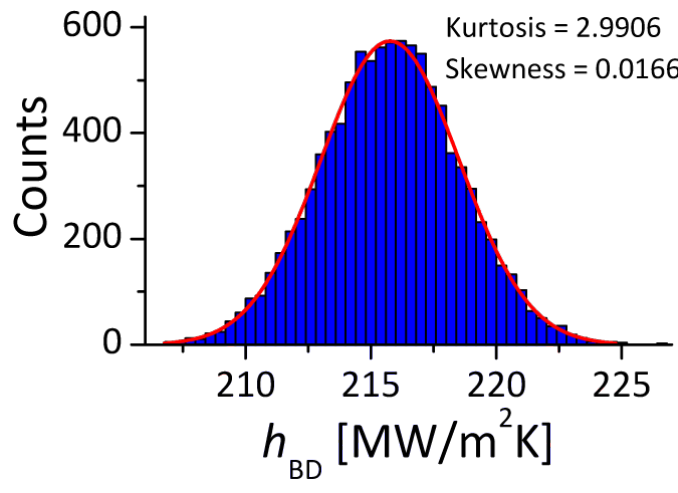

(a)

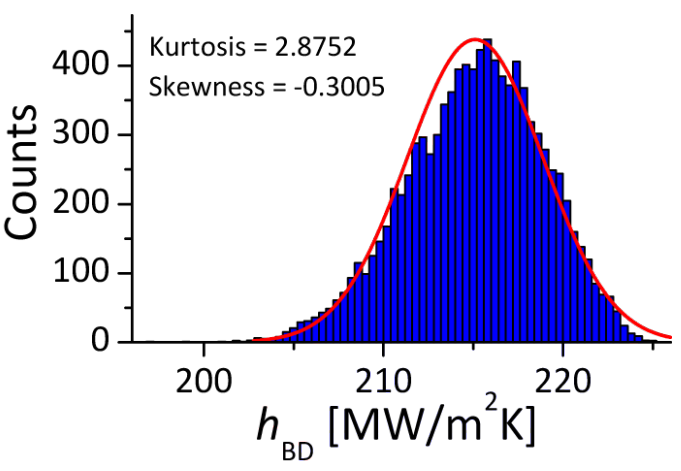

(b)

Figure 6.28: Distribution of bootstrap means from 10 random samples selected from a normal and skewed population with $\beta_{\text {kurt }}=3, \beta_{\text {skew }}=0$ and $\beta_{\text {kurt }}=2.9, \beta_{\text {skew }}=-0.8$ respectively, see Figure 6.27 , for $B=10,000$ resamplings. The skew and kurtosis listed in the plots are the calculated values from the data sets.

\footnotetext{
${ }^{29}$ Analytically we have discussed that the sampling distribution of means would be normal regardless of sample size for an underlying population that is also normal. However, as we will discuss shortly for very small $n$ there are too few combinations that can be created during the resampling.
} 
a normally distributed sampling distribution without making assumptions about the distribution of the underlying population. Once the form of the sampling distribution has been verified we can either more confidently rely on analytic descriptions of the dispersion in the data via the standard error or confidence interval and/or continue to analyze the bootstrap sampling distribution to determine these values.

\subsubsection{Bootstrapping Standard Error and Confidence Intervals}

Using the bootstrapping procedure we have been able to create a mathematical analog to the sampling distribution of an estimator, $\hat{\Theta}$, which we have denoted the bootstrap distribution of estimators, $\hat{\mathbf{\Theta}}^{*}$. Because we have an analog of the sampling distribution we can perform calculations on the distribution directly rather than relying on analytic expressions based on the assumed distribution. As an example, we will consider a population with a normal distribution, $F_{\mu, \sigma}$ with mean $\mu=215 \mathrm{MW} / \mathrm{m}^{2} \mathrm{~K}$ and standard deviation $\sigma=2 \mathrm{MW} / \mathrm{m}^{2} \mathrm{~K}$. From this distribution we will select a sample of size $n=50$, see Figure 6.29a. Analytically using Equation 6.6, the standard error of the sampling distribution of the means will be given by $\sigma_{\bar{x}}=0.283 \mathrm{MW} / \mathrm{m}^{2} \mathrm{~K}$. Using the bootstrapping procedure the original data set, see Figure 6.29a, is resampled for $B=50,000$ and the mean of each resampling used to build the bootstrap distribution of the means, see Figure 6.29b.

We know that by definition the standard error of an estimator is the standard deviation

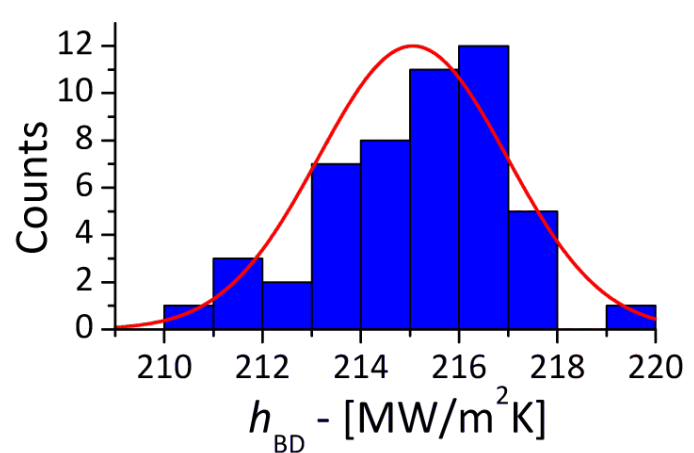

(a)

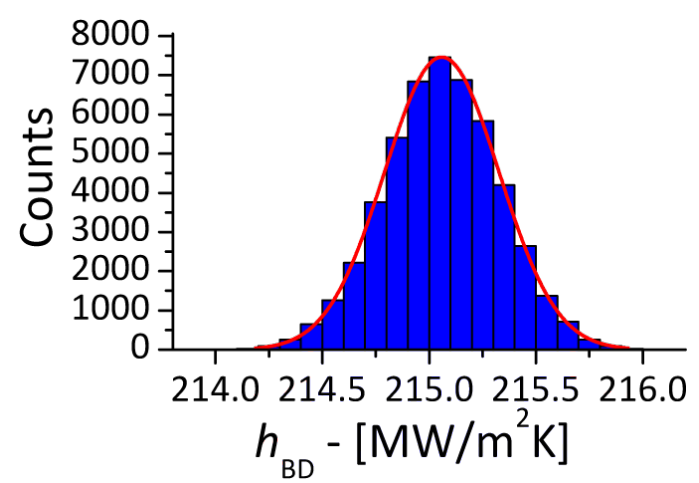

(b)

Figure 6.29: Example of the standard error of the bootstrap distribution of the means, where (a) is the distribution of the random sample of $n=50$ selected from a normal distribution, $F_{\mu, \sigma}$ with mean $\mu=215 \mathrm{MW} / \mathrm{m}^{2} \mathrm{~K}$ and standard deviation $\sigma=2 \mathrm{MW} / \mathrm{m}^{2} \mathrm{~K}$, and (b) is the bootstrap distribution of the means for $B=50,000$. 
of the sampling distribution. Therefore, for the bootstrap estimate the standard error is given by $[273,276,277]$ :

$$
\mathrm{SE}_{\text {boot }, \bar{x}}=\sqrt{\frac{1}{B-1} \sum_{i=1}^{B}\left[\bar{x}_{i}^{*}-\frac{1}{B} \sum_{j=1}^{B} \bar{x}_{j}^{*}\right]}
$$

where $B$ is the number of bootstrap resamplings and $\bar{x}_{i}^{*}$ is the average of the $i^{\text {th }}$ bootstrap sample. Using this formulation and the data in Figure 6.29b the bootstrap standard error for the distribution of the means is $\mathrm{SE}_{\mathrm{boot}, \bar{x}}=0.268 \mathrm{MW} / \mathrm{m}^{2} \mathrm{~K}$. This is a slight under predication of the true standard error. However, very seldom do we know the actual variance of an underlying population and instead use the sample standard deviation, $s$, as a point estimate of the population standard deviation. Using the standard deviation of the sample set in Figure 6.29a to analytically calculate the standard error we find $s_{\bar{x}}=0.272 \mathrm{MW} / \mathrm{m}^{2} \mathrm{~K}$ which in general agrees with the bootstrapped standard error.

With an estimate of the standard error of the bootstrap sampling distribution it is possible to develop a confidence interval for the mean. If we can assume that the bootstrap distribution of the means is approximately normal and has little bias, we can calculate the confidence interval of the mean using the bootstrap standard error in a similar manner as was done analytically, see Equation 6.16 . The bootstrap equivalent of the $t$-confidence interval is given by $[276,277]$ :

$$
\bar{\mu}^{*} \pm t_{\alpha / 2, n-1} \times \mathrm{SE}_{\mathrm{boot}, \bar{x}}
$$

where $t_{\alpha / 2, n-1}$ is the $t$-student distribution at the $\alpha / 2$ point for $n-1$ degrees of freedom. Using the bootstrap standard error calculated above, the estimated confidence in the bootstrap mean is $\bar{\mu}^{*} \pm 0.538 \mathrm{MW} / \mathrm{m}^{2} \mathrm{~K}$.

Additionally, we can again get the confidence interval directly from the data in the bootstrap sampling distribution, see Figure 6.29b. For an $100(1-\alpha) \%$ confidence level of the bootstrapped sampling distribution we are interested in the means at the upper and lower $\alpha / 2$ point of the distribution, i.e. $\bar{x}_{i, \mathrm{LO}}^{*}, \bar{x}_{i, \mathrm{HI}}^{*}$, where the lower index is given by $i, \mathrm{LO}=(B \alpha / 2)$ and the upper index given by $i, \mathrm{HI}=(B-i, \mathrm{LO}+1)$. For the above 
example, $B=50,000$ and therefore for a $95 \%$ confidence interval the upper and lower indices are 49,376 and 625 respectively. Ranking the values of the bootstrap sampling distribution of the means and finding the value at these indices gives a $95 \%$ confidence interval of $\bar{\mu}^{*} \pm 0.605 \mathrm{MW} / \mathrm{m}^{2} \mathrm{~K}$ which again shows reasonable agreement with the theoretical value.

\subsubsection{Two-sample Bootstrap Comparison}

Similar to the Welch's t-test used in the comparison of sample means in the introduction to this chapter, the method of bootstrapping can be applied to compare whether the means of two sampling distributions are statistically different ${ }^{30}$. Consider for example the two data sets shown in Table 6.4 which were randomly selected from normal distributions of $\mu=215 \mathrm{MW} / \mathrm{m}^{2} \mathrm{~K}$ and $\mu=220 \mathrm{MW} / \mathrm{m}^{2} \mathrm{~K}$ for sample 1 and sample 2 respectively with both distributions having a dispersion of $\sigma=4 \mathrm{MW} / \mathrm{m}^{2} \mathrm{~K}$. The mean and standard deviation of the sample sets is also provided. The difference between the two sample means, $\bar{x}_{1}-\bar{x}_{2}$, is $\bar{x}_{1}-\bar{x}_{2}=6.07 \mathrm{MW} / \mathrm{m}^{2} \mathrm{~K}$. Similar to the example in the introduction of this chapter, the question arises as to whether this represents a statistically significant difference.

One way to test whether there is a significant difference between the sample sets is to assume that there is not. Assuming all 20 samples were selected from the same distribution, the only factor that delineates the data in the two sets is the label of either being from sample 1 or sample 2. Assuming they are from the same distribution, we can randomly select 10

Table 6.4: Example of two sample sets of $n=10$ randomly selected from normal distributions of $\mu=$ $215 \mathrm{MW} / \mathrm{m}^{2} \mathrm{~K}$ and $\mu=220 \mathrm{MW} / \mathrm{m}^{2} \mathrm{~K}$ for sample 1 and sample 2 respectively with both distributions having a dispersion of $\sigma=4 \mathrm{MW} / \mathrm{m}^{2} \mathrm{~K}$.

\begin{tabular}{rcr|cc}
\hline \multicolumn{4}{c}{$h_{\mathrm{BD}}\left[\mathbf{M W} / \mathbf{m}^{2} \mathbf{K}\right]$} \\
\hline \multicolumn{3}{c}{ Sample 1 } & \multicolumn{3}{c}{ Sample 2 } \\
& $2.11 \times 10^{8}$ & $2.14 \times 10^{8}$ & $2.20 \times 10^{8}$ & $2.18 \times 10^{8}$ \\
$2.07 \times 10^{8}$ & $2.19 \times 10^{8}$ & $2.16 \times 10^{8}$ & $2.18 \times 10^{8}$ \\
$2.13 \times 10^{8}$ & $2.14 \times 10^{8}$ & $2.22 \times 10^{8}$ & $2.14 \times 10^{8}$ \\
& $2.12 \times 10^{8}$ & $2.12 \times 10^{8}$ & $2.17 \times 10^{8}$ & $2.18 \times 10^{8}$ \\
& $2.11 \times 10^{8}$ & $2.17 \times 10^{8}$ & $2.23 \times 10^{8}$ & $2.26 \times 10^{8}$ \\
\hline Mean & $2.13 \times 10^{8}$ & $2.19 \times 10^{8}$ \\
SD & $3.49 \times 10^{6}$ & $3.63 \times 10^{6}$ \\
\hline
\end{tabular}

\footnotetext{
${ }^{30}$ Again one of the major strengths of the bootstrapping methodology, is that we do not need to make any assumptions about how the data is distributed or the similarity of the variances between the two samples, which is a major factor in determining the form of the comparison test used.
} 


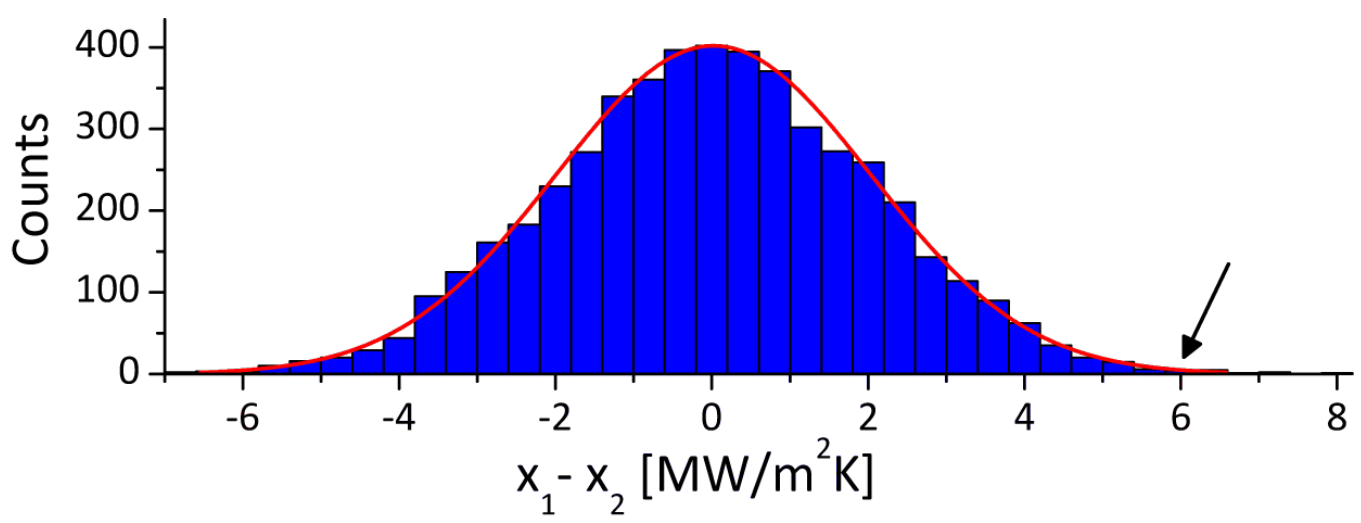

Figure 6.30: Distribution of the difference in the means between sample 1 and sample $2, \bar{x}_{1}-\bar{x}_{2}$, with the means calculated from random samplings with replacement of $n=10$ from all 20 values shown in Table 6.4. The arrow denotes the location of the difference in the means calculated for the original data samples.

values from the given 20 (with replacement) and denote them as sample 1. Similarly, we can select an additional 10 values and denote them as sample 2, and then find the difference between the sample means, $\bar{x}_{1}-\bar{x}_{2}$. If we repeat this process a large number of times we can build a bootstrap distribution of the difference of the means for the randomly selected samples, see Figure 6.30. The probability of the difference of the means for the two samples being equal to $\bar{x}_{1}-\bar{x}_{2}=6.07 \mathrm{MW} / \mathrm{m}^{2} \mathrm{~K}$ due to random chance is shown by the arrow in Figure 6.30 which corresponds to a $P$-value of $P=0.00135$. The interpretation of this result is that there is strong evidence to suggest that the two sample sets, and the respective means for each set, are not selected from the same population and are statistically different. It should be noted that we could have completed a similar analysis by individually resampling the data samples and calculating the system estimator, $\bar{x}_{1}-\bar{x}_{2}$, to build a bootstrapped sampling distribution around the calculated value, $\bar{x}_{1}-\bar{x}_{2}=6.07 \mathrm{MW} / \mathrm{m}^{2} \mathrm{~K}$. Using the bootstrapped sampling distribution a confidence interval on the estimator could be established, and if that confidence bounds did not contain zero the results would indicate a low probability of the difference in the means occurring randomly.

\subsubsection{Effect of Sample Size on Bootstrapping}

While the bootstrapping methodology is a powerful tool in helping to establish and provide confidence in the statistical analysis of data, what it does not do is provide any additional experimental information. That is to say that it does not replace the actual 
experiment or provide any new experimental evidence beyond what was collected in the original data set. For the non-parametric bootstrap it is assumed that the original data set is a good statistical representation of the underlying population. If we attempt to collect too few data samples we will run into two issues: 1) the sample set will not be a good statistical representation of the population and 2) there will not be enough possible resampling combinations to produce an accurate bootstrap.

For $n$ data points in a sample set the total number of possible combinations with repetition is given by [19]:

$$
N_{c}=\frac{(2 n-1) !}{n !(n-1) !}
$$

Figure 6.31 shows the number of potential combinations, with repetition, as a function of the original sample size $n$ for $2<n<30$. For very low values of $n$, there are insufficient combinations possible to accurately build the bootstrap sampling distribution, with the limited combinations causing the sampling distribution to mimic the discrete nature of the distribution of the original sample set.

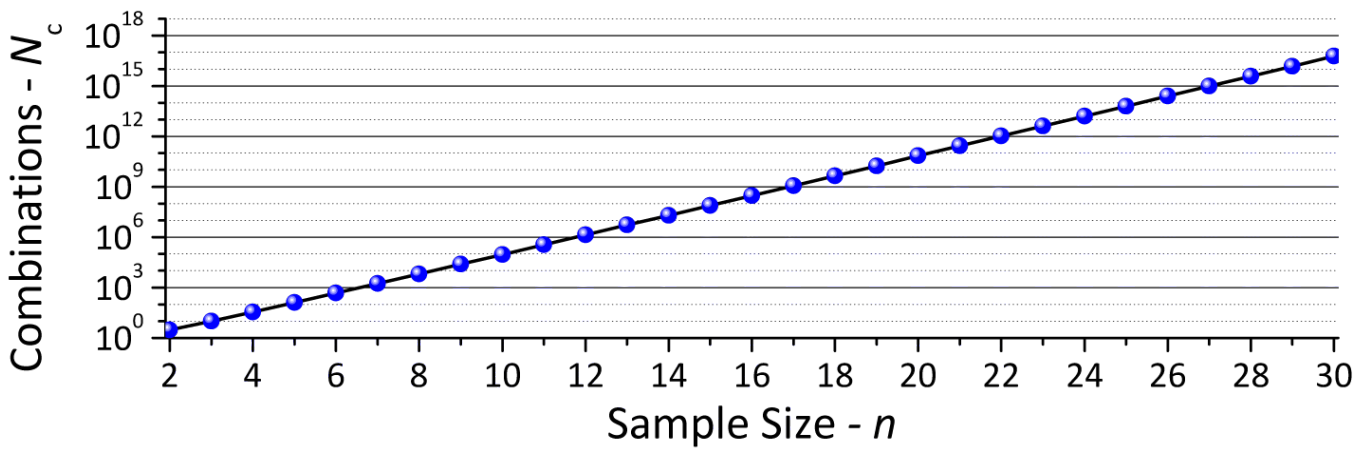

Figure 6.31: Possible number of combinations of $n$ values with repetition and independent of order, see Equation 6.24.

Small sample sizes do not have sufficient information to allow the bootstrap procedure to consistently build the bootstrap sampling distribution of an estimator. As shown in Figure 6.32, for four random samples of size $n=8$ selected from a normal distribution, $F_{\mu, \sigma}$, with mean $\mu=215 \mathrm{MW} / \mathrm{m}^{2} \mathrm{~K}$ and standard deviation $\sigma=2 \mathrm{MW} / \mathrm{m}^{2} \mathrm{~K}$, there are large variations in the skew and width of the bootstrapped sampling distribution of the means. While the mean of the sampling distribution will always follow the sample mean, the shape of the sampling distribution should be relatively consistent for each sample. We 


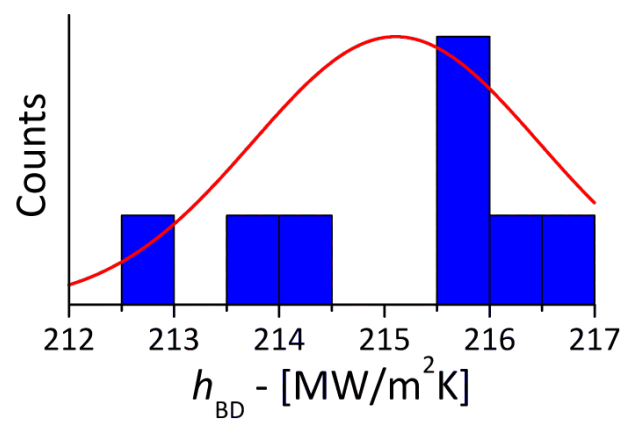

(a)

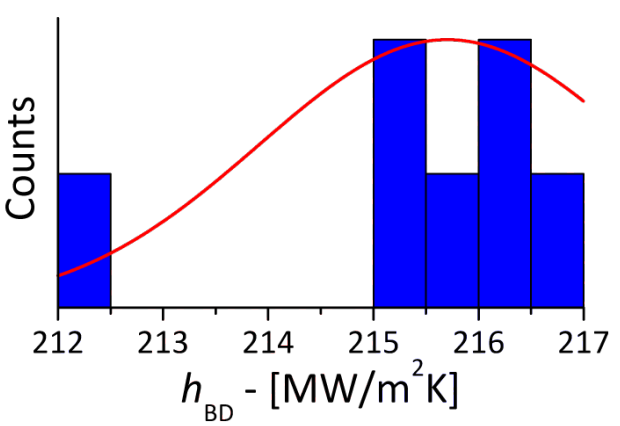

(c)

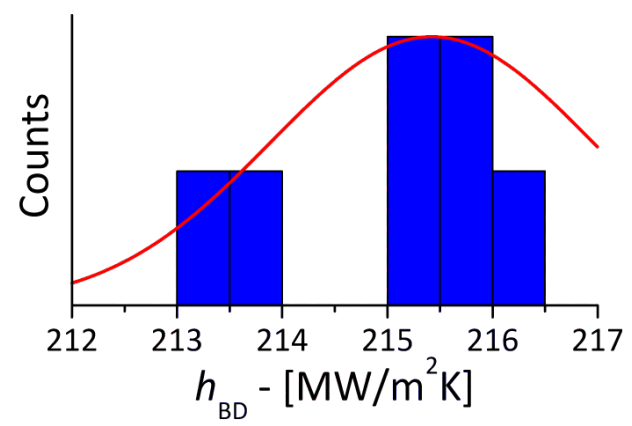

(e)

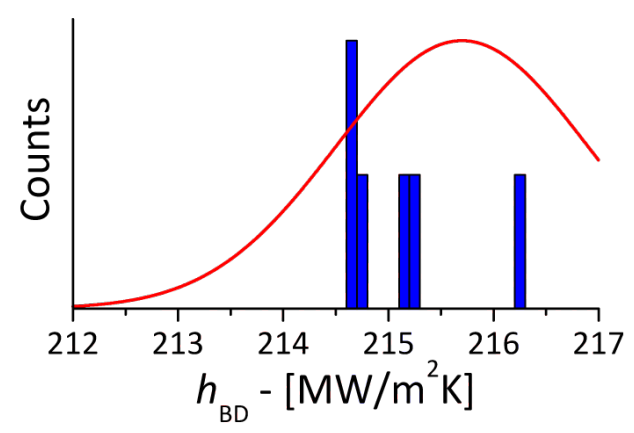

(g)

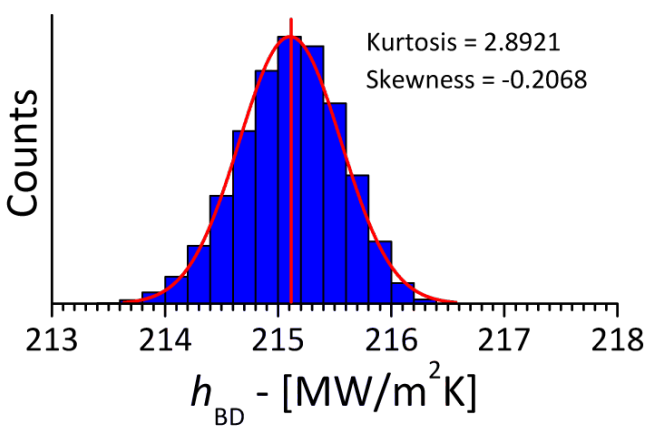

(b)

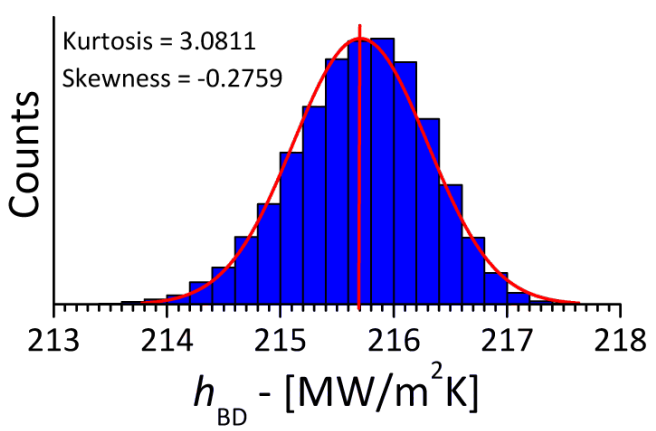

(d)

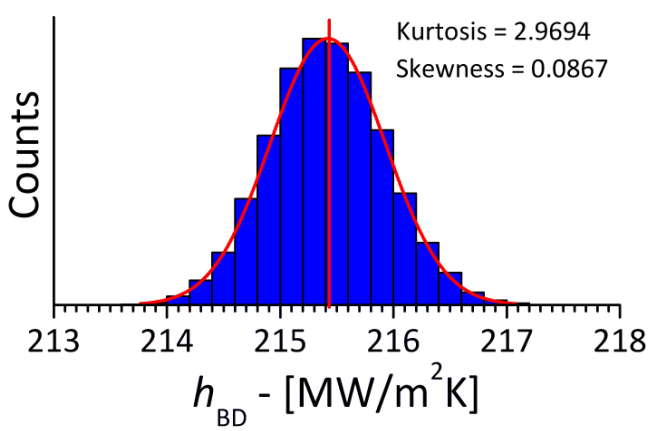

(f)

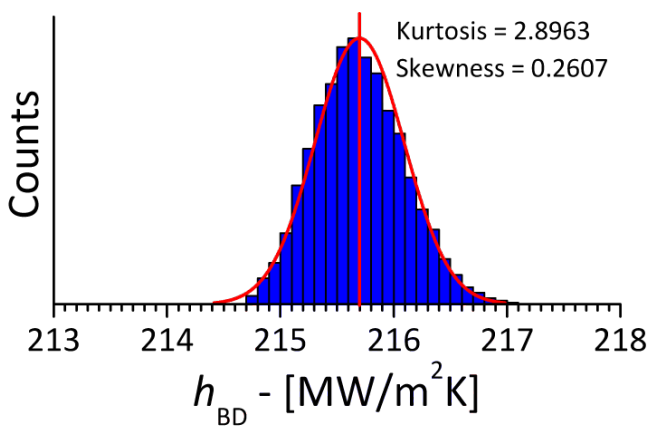

(h)

Figure 6.32: Four random samples of size $n=8$ selected from a normal distribution $F_{\mu, \sigma}$ with mean $\mu=215 \mathrm{MW} / \mathrm{m}^{2} \mathrm{~K}$ and standard deviation $\sigma=2 \mathrm{MW} / \mathrm{m}^{2} \mathrm{~K}$ are shown in the left column. In the right column, the bootstrap distribution of the sample means for each corresponding sample, $B=50,000$. 
find that this is the case when the sample size is increased to $n=30$, see Figure 6.33 .

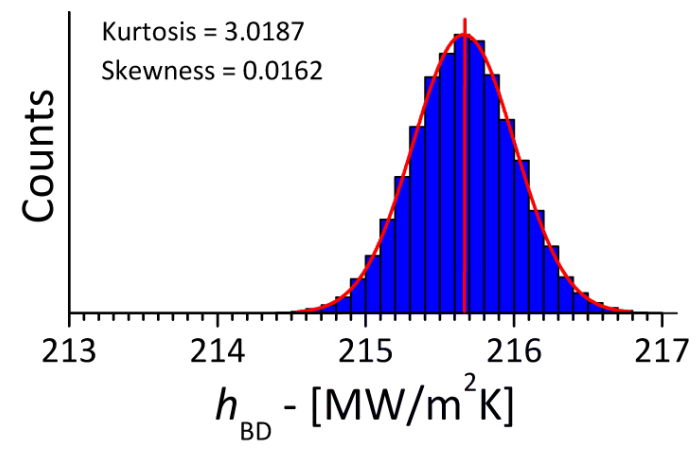

(a)

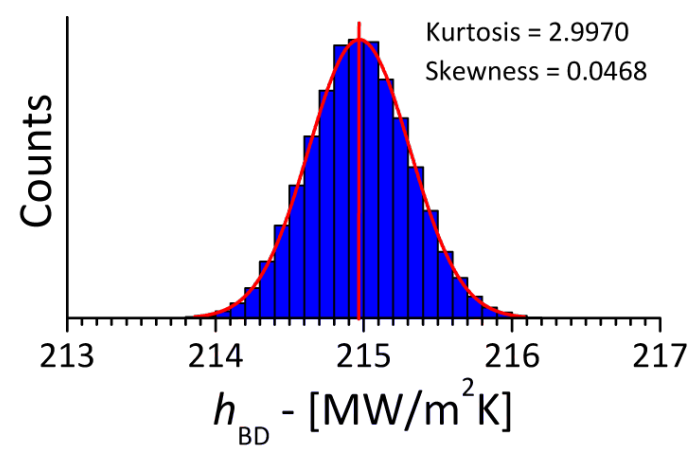

(c)

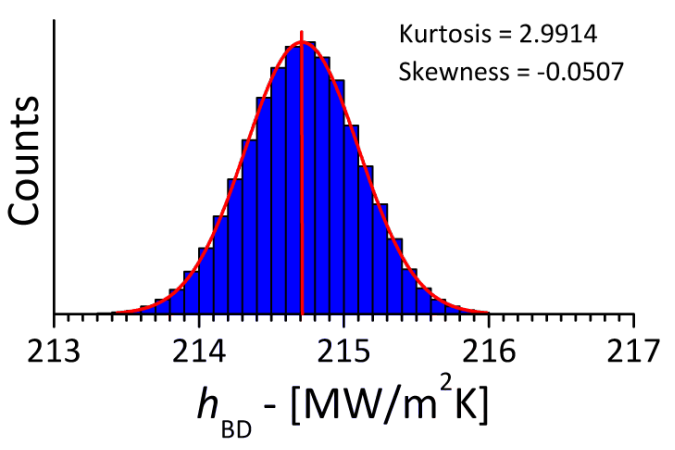

(b)

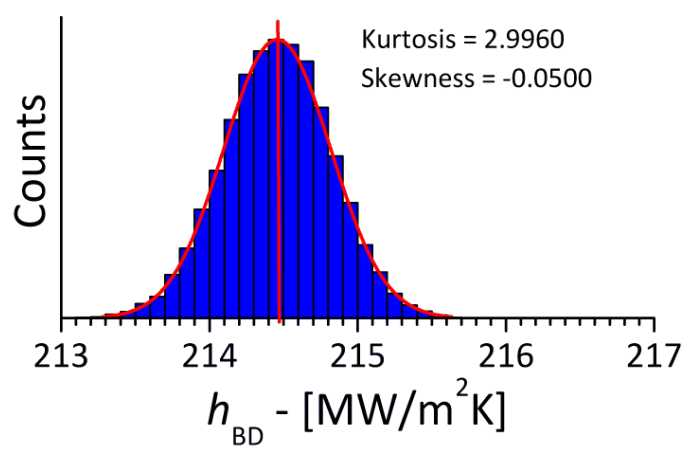

(d)

Figure 6.33: Four random samples of size $n=30$ selected from a normal distribution, $F_{\mu, \sigma}$, with mean $\mu=215 \mathrm{MW} / \mathrm{m}^{2} \mathrm{~K}$ and standard deviation $\sigma=2 \mathrm{MW} / \mathrm{m}^{2} \mathrm{~K}$ bootstrapped to establish the bootstrap distribution of sample means for each corresponding sample, $B=50,000$.

\subsubsection{How Many Bootstrap Resamplings?}

The process of resampling the data or the empirical distribution $B$ times during the non-parametric and parametric bootstrapping procedure respectively, introduces another question of how large should $B$ be to accurately apply the bootstrap? The answer to that question is dependent on the statistic one is trying to estimate with the bootstrapping procedure. For calculation of the standard error, Efron and Tibshirani [277] recommend on the order of $B=25-200$. Intuitively it makes sense that if we are interested in quantities such as the confidence interval, particularly for a low significance level where we will be interested in the information in the tails of the distribution, that the amount of bootstrap iterations must be much higher. Henderson [274] indicates that for $t$-confidence intervals $B$ may need to be as large as $B=25,000$, and Wehrens et al. [275] cite $B=100,000$ as large 
enough to ignore Monte Carlo error for the bootstrapped standard error. The fortunate answer is that modern computational power has outpaced the restrictions on $B$ and it is therefore very computationally tractable to reach these criteria for $B$.

\subsubsection{Summary: Why bootstrap?}

The presentation of the bootstrap methodology begs the question of why implement the bootstrap technique in the analysis of TTR/TDTR data? If there is no "new" information about the sample gained, what is the purpose of the added computations? The simple answers to these questions are that 1) we do not know the form of the underling distribution of the population and 2) in most cases we simply do not and/or cannot collect enough data to be "sufficiently large" to accurately meet all the criteria to use analytic statistical expressions blindly.

Assuming that the properties of a given TTR/TDTR sample are relatively uniform, i.e. uniform film thickness, interface roughness, etc., we would like to assume that the underlying population of $h_{\mathrm{BD}}$ values for that sample would be normally distributed. If we therefore randomly collected data from that sample we have a large number of analytic assumptions that can be made in defining a mean value of the measured sample and defining confidence in that estimate based on the standard error. However, in general we find in literature that only 1-15 scans are ever collected from a sample. This sample size is too small to evoke principles such as the law of large numbers to estimate the distribution of the population. It is also too small to visually inspect the distribution of the sampled data to make any determination of the underlying population. Therefore if we rely solely on "standard" analytic methods to complete a statistical analysis of our results we are basing it on an assumption that we have no way to prove or estimate.

While the bootstrap technique is not a substitute for collecting a sufficient amount of experimental data, it does provide an in depth set of tools that allows for statistical diagnosis and interpretation of the collected data outside the typically bounds of normality and "sufficiently large". These relatively simplistic and computationally tractable methods allow for the visualization and diagnosis of the sampling distribution, the estimate of standard error and the development of confidence intervals, and the comparison of different data sets 
without relying heavily on the assumptions of the underlying population.

\subsection{Accuracy of Results}

To this point we have discussed the statistical analysis of the TTR/TDTR results in terms of precision. That is the amount of dispersion in the deduced data which is dependent on the sensitivity to fitting and the noise in the collected data. The analysis of precision has assumed that the inputs to the TTR/TDTR model were known exactly. In reality, we cannot know these values exactly and they are in fact either a best guess, estimated from literature, or taken based on some ancillary measurement which will have its own uncertainty. Regardless, there is always some amount of uncertainty in the input parameters and it is these uncertainties that will carry over and affect the accuracy of the final results.

Traditionally, the uncertainty in the accuracy of the final results would be calculated through a standard propagation of error based on the uncertainly in the input parameters and the form of the thermophysical model [278]. The challenge in implementing this type of accuracy analysis is that the complexity of the thermal model makes it difficult to propagate the input errors to the final result. As a more numerical method to calculate accuracy, Malen et al. calculated the accuracy in the deduced parameters from the fiber aligned frequency domain thermoreflectance technique using a relation of the form:

$$
\Delta \Phi=\sqrt{\sum_{j}\left(\Delta \Phi_{p}\right)^{2}}
$$

where $\Delta \Phi$ is the total uncertainty in the deduced parameter of interest and $\Delta \Phi_{p}$ is the change in the deduced result for a given perturbation of the input parameter $p$. Using this type of analysis the fitting routine was implemented to determine the nominal values to produce the best fit and then using a one-at-a-time (OAT) perturbation method, each parameter, $p$, was perturbed by its uncertainty, $\Delta p$, and the change in the model, $\Delta \Phi_{p}$, determined. The final uncertainty in the parameter of interest, $\Delta \Phi$, was then found by the square root of the sum of the squares of the uncertainty based on each parameter, $\Delta \Phi_{p}$. As an example of this procedure, Table 6.5 provides uncertainties calculated based on a sample of $100 \mathrm{~nm}$ of $\mathrm{Al}$ on $\mathrm{Si}\left(h_{\mathrm{BD}}=215 \mathrm{MW} / \mathrm{m}^{2} \mathrm{~K}\right)$ fit to deduce $h_{\mathrm{BD}}$ with examples of estimated 
Table 6.5: Uncertainty in the input parameters for a modeled system of $100 \mathrm{~nm}$ of $\mathrm{Al}$ on $\mathrm{Si}\left(h_{\mathrm{BD}}=\right.$ $215 \mathrm{MW} / \mathrm{m}^{2} \mathrm{~K}$ ) and the corresponding change in the deduced $h_{\mathrm{BD}}$ using the uncertainty analysis described in Equation 6.25.

\begin{tabular}{rrrrrrr}
\hline & Baseline & L1C & L1kz & L1d & LSC & LSkz \\
\hline Perturbation & & $\mathbf{5 \%}$ & $\mathbf{5 \%}$ & $\mathbf{1 0 \%}$ & $\mathbf{5 \%}$ & $\mathbf{5 \%}$ \\
Fit $h_{\mathrm{BD}}\left[\mathrm{MW} / \mathrm{m}^{2} \mathrm{~K}\right]$ & 215.00 & 241.41 & 215.03 & 264.24 & 205.98 & 206.02 \\
$\Delta \Phi_{j}\left[\mathrm{MW} / \mathrm{m}^{2} \mathrm{~K}\right]$ & & -26.49 & -0.1160 & -49.33 & 8.94 & 8.89 \\
\hline$\Delta \Phi\left[\mathrm{MW} / \mathrm{m}^{2} \mathrm{~K}\right]$ & & \multicolumn{5}{c}{57.4} \\
\hline
\end{tabular}

uncertainty in the input parameters shown. From this example the total uncertainty in the deduced parameter is found to be $\Delta h_{\mathrm{BD}}=57.4 \mathrm{MW} / \mathrm{m}^{2} \mathrm{~K}$ or $\pm 26.7 \%$ of the nominal value.

One of the major assumptions of either the traditional propagation of error formulation or the uncertainty analysis based on the OAT perturbation of the input parameter during a fit, is that they assume there is no correlation between the input parameters. In the TTR/TDTR model the interaction of the input parameters is more complex and it is not difficult to envision from the discussion and interpretation of the sensitivity analysis provided in Chapter 5.5, that the uncertainty in multiple parameters can have a constructive or destructive affect on the overall uncertainty depending on the positive and/or negative correlation between the sensitivity coefficients. In addition, the uncertainty analysis presented above assumes that the distribution of the uncertainty is uniform. In reality it is more likely that the nominal values represent the "best guess" of the input parameters with a decreasing probability in occurrence as the input parameters are perturbed further from the nominal values. To this end, the analysis presented in Equation 6.25 represents an upper bound to the uncertainty in the deduced parameter based on the uncertainty in the input parameters.

In an effort to more accurately model the uncertainty in the deduced parameter, we will again evoke a quasi-stochastic treatment to describe the uncertainty in the input parameters. The process begins by generation of a modeled TTR/TDTR data set using the nominal input parameters ${ }^{31}$. Using a properly conditioned fitting routine, any parameter of sufficient sensitivity can be treated as an unknown parameter and the nominal value deduced by refitting the modeled data. To model the uncertainty in the input parameters,

\footnotetext{
${ }^{31} \mathrm{~A}$ modeled system is used as opposed to the collected data to eliminate the influence of noise in the data which affects the precision of the collected result and is not relevant for the discussion of the accuracy.
} 
each input is prescribed a distribution of values with the mean value being the nominal input parameter. In this case we describe the parameter input by a normal distribution assuming the nominal value represents the mean with decreasing probability as the input deviates from that value. The width of the normal distribution, given in terms of the standard deviation, represents the uncertainty in the input value given as a percentage ${ }^{32}$. For each trial iteration in the quasi-stochastic accuracy analysis, random input parameters are selected from each respective uncertainty distribution and the modeled data generated via the nominal parameters fit to deduce the parameter of interest using the standard fitting routine. Using the methods to increase computational efficiency presented in Chapter 5.4 it is possible to complete a large number of trial iterations in a reasonable amount of time. For the low-rep system, assuming 10 iterations required for each fit, it is possible to complete 1,000 trials in approximately 24 hours assuming reasonable computational power.

As an example of the quasi-stochastic accuracy analysis we will consider the same system and uncertainty described in the previous OAT analysis, see Equation 6.25 and Table 6.5. Using the quasi-stochastic uncertainty analysis for normally distributed uncertainty in the input parameters, the distribution of the deduced $h_{\mathrm{BD}}$ results are shown in Figure 6.34a. The figure shows that the data is for the most part normally distributed with a slight skew towards higher $h_{\mathrm{BD}}$. Assuming the distribution is approximately normal we can define the sample mean and standard deviation in the usual sense, $\bar{x}=2.15 \times 10^{8} \mathrm{~W} / \mathrm{m}^{2} \mathrm{~K}$ and $s=2.81 \times 10^{7} \mathrm{~W} / \mathrm{m}^{2} \mathrm{~K}$ respectively. As expected the mean value is statistically equivalent to the expected $h_{\mathrm{BD}}$. We will take note however that the standard deviation in the expected $h_{\mathrm{BD}}$ is roughly half of that given by the OAT accuracy analysis described in Equation 6.25. It is expected that this can be attributed to a more accurate representation of the input uncertainty and the inclusion of the covariance in the input parameters.

To more closely represent the type of analysis performed in Equation 6.25, the quasistochastic accuracy analysis was also completed using a uniform distribution for the uncertainty in the input parameters where the maximum and minimum bounds on the dis-

\footnotetext{
${ }^{32}$ It is important to recall that for the normal distribution $1 \sigma$ represents $68.2 \%$ of the total distribution, see Figure 6.4. Therefore for the prescribed input uncertainty, almost $32 \%$ of the trial values will be beyond those bounds. It may be more prudent to assume that the standard uncertainty in the input values represents a $2 \sigma$ uncertainty in the input distribution.
} 


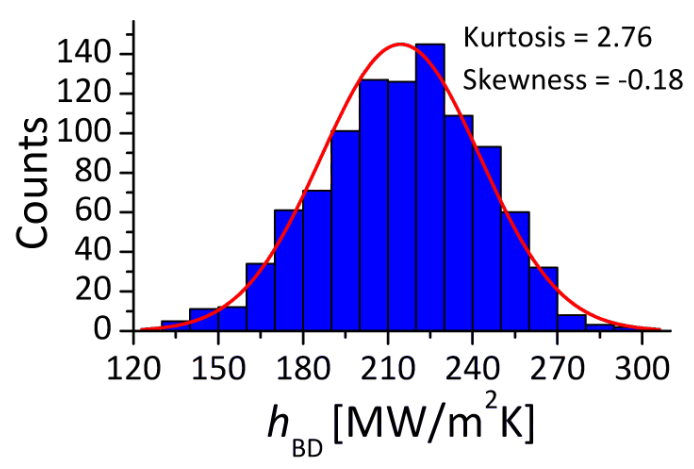

(a)

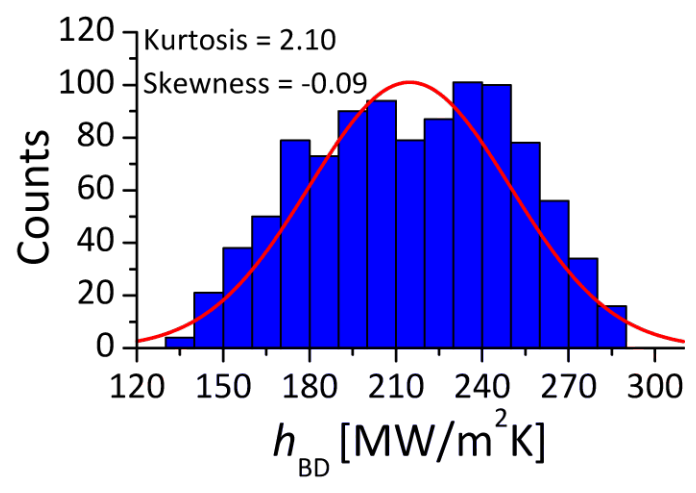

(b)

Figure 6.34: Distribution of $h_{\mathrm{BD}}$ values fit using the quasi-stochastic accuracy analysis for input parameters (a) normally distributed with a $1 \sigma$ uncertainty given by the percentages in Table 6.5 and (b) uniformly distributed between a maximum and minimum given by $\mu \pm 1 \sigma$.

tribution were given by $\mu \pm 1 \sigma$. The results for this analysis are shown in Figure 6.34b with the distribution of data showing a reduced kurtosis as compared to the analysis with normally distributed input uncertainty. However, we will notice that the overall range of the deduced $h_{\mathrm{BD}}$ values is roughly the same for both examples. The standard deviation in the $h_{\mathrm{BD}}$ results for the uniformly distributed case is $s=3.50 \times 10^{7}$ which is only slightly larger than the normally distributed case and still significantly less than the OAT analysis from Equation 6.25. This indicates that the correlation in the input parameters plays a significant role in determining the accuracy of the deduced parameter based on the input uncertainties.

\subsection{Statistical Example Pt/Si}

As an example of the concepts and methodologies developed in this chapter we will consider the system of $100 \mathrm{~nm}$ of Pt on Si through the computational analysis of sensitivity, precision estimation, and accuracy estimation and compare these calculations to experimental results. The basic thermophysical parameters used to model the initial system are shown in Table 6.6 with the properties of the film and substrate taken as bulk values and the $h_{\mathrm{BD}}$ at the $\mathrm{Pt} / \mathrm{Si}$ interface taken from previous literature [10]. 
Table 6.6: Thermophysical properties used in modeling a Pt film on Si substrate.

\begin{tabular}{rcc}
\hline Layer Properties & Layer 1 & Layer S \\
\hline Specific Heat $-C\left[\mathrm{~J} / \mathrm{m}^{3} \mathrm{~K}\right]$ & $2.88 \times 10^{6}$ & $1.66 \times 10^{6}$ \\
Thermal Conductivity $-k_{r}[\mathrm{~W} / \mathrm{mK}]$ & 71.6 & 148 \\
Thermal Conductivity $-k_{z}[\mathrm{~W} / \mathrm{mK}]$ & 71.6 & 148 \\
Thickness $-d[\mathrm{~nm}]$ & 100 & Infinite \\
$h_{\mathrm{BD}}\left[\mathrm{MW} / \mathrm{m}^{2} \mathrm{~K}\right]$ & \multicolumn{2}{c}{140} \\
\hline Laser Properties & \\
\hline Absorbed Power $[\mathbf{W}]$ & 0.03 \\
Pump Waist $[\mu \mathbf{m}]$ & \multicolumn{2}{c}{70} \\
Probe Waist $[\mu \mathbf{m}]$ & 15 \\
Rep Rate $[\mathrm{MHz}]$ & 0.250 \\
Modulation Rate $[\mathrm{MHz}]$ & 0.125 \\
Duty Cycle $[\%]$ & 50 \\
\hline
\end{tabular}

\subsubsection{Preliminary Sensitivity Analysis}

Using these parameter values as an initial best guess, the sensitivity coefficient as a function of time can be calculated as shown in Figure 6.35. With $h_{\mathrm{BD}}$ being the primary thermophysical parameter of interest we see in Figure 6.35 that the sensitivity to $h_{\mathrm{BD}}$ increases and reaches a maximum around $5 \mathrm{~ns}$ and then begins to decrease. The inflection in the sensitivity to $h_{\mathrm{BD}}$ and the increase in the sensitivity to the substrate thermal conductivity are indicators that the thermal energy has transported across the $\mathrm{Pt} / \mathrm{Si}$ boundary and into the substrate during the $8 \mathrm{~ns}$ of the TTR scan. Therefore we expect to have reasonable sensitivity to $h_{\mathrm{BD}}$. The time averaged sensitivity coefficients of the real signal, $S_{p}^{\mathrm{avg}, X}$, for the parameters in the $\mathrm{Pt} / \mathrm{Si}$ system are shown in Table 6.7. From the magnitude of the sensitivity coefficients all the parameters should have sufficient sensitivity for deduction.

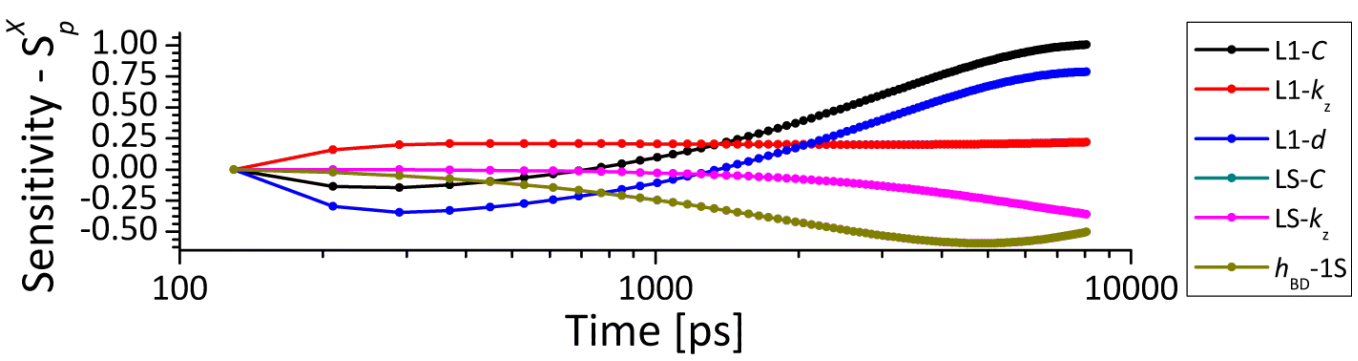

Figure 6.35: Sensitivity coefficient per parameter as a function of time for the $100 \mathrm{~nm}$ of $\mathrm{Pt}$ on Si system where $\mathrm{L}_{1}$ denotes the $\mathrm{Pt}$ film and $\mathrm{L}_{\mathrm{S}}$ the $\mathrm{Si}$ substrate. 
Table 6.7: Averaged sensitivity coefficient, $S_{p}^{\mathrm{avg}, X}$, for the parameters in the simulated Pt/Si system.

\begin{tabular}{cccccc}
\hline Property & $S_{p}^{X}$ & Property & $S_{p}^{X}$ & Property & $S_{p}^{X}$ \\
\hline L1- $C$ & 0.6457 & L1- $d$ & 0.4433 & LS- $k_{z}$ & -0.1860 \\
L1- $k_{z}$ & 0.2033 & LS- $C$ & -0.1860 & $h_{\mathrm{BD}}$ & -0.4770 \\
\hline
\end{tabular}

By estimating the uncertainty in the input parameters we find the distribution of potential sensitivity coefficients of the final system. Table 6.8 provides the estimated uncertainty in the input parameters. The specific heat capacity of the film and substrate are taken from bulk literature values and assumed to be accurate to within 5\%. The specific heat capacity should be relatively insensitive to nanoscale size effects, with the largest potential for uncertainty coming from the density of the deposited film. The thermal conductivity of the Pt film could be reduced due to thin film size effects, however for now we will assume the bulk value and that we could measure the nominal value using ancillary methods to within 10\%. For the Si thermal conductivity we will assume the bulk value to within $5 \%$. The largest uncertainty is the $h_{\mathrm{BD}}$ value itself due to the large impact that the final interface structure can have on the interface conductance, see Chapter 2. Using the uncertainty in the input parameters listed in Table 6.8, the distribution of the input $h_{\mathrm{BD}}$ parameters and the distribution of the sensitivity coefficient for $h_{\mathrm{BD}}$ is shown in Figures 6.36a and 6.36b respectively. The results of the quasi-stochastic sensitivity analysis indicate that the distribution of $h_{\mathrm{BD}}$ sensitivity coefficients skew slightly to the right, with a median value that is slightly below the sensitivity coefficient calculated from the nominal input parameters. However, the ranges of sensitivity coefficients in Figure 6.36b all indicate strong sensitivity to $h_{\mathrm{BD}}$.

Table 6.8: Uncertainty in the TTR/TDTR input parameters for the example of a system of $100 \mathrm{~nm}$ of Pt on $\mathrm{Si}\left(h_{\mathrm{BD}}=140 \mathrm{MW} / \mathrm{m}^{2} \mathrm{~K}\right)$ where $\mathbf{L} 1$ and $\mathbf{L S}$ denote the Pt film and Si substrate layer respectively.

\begin{tabular}{rcccccc}
\hline \multicolumn{7}{c}{ Parameters } \\
\hline & $\mathbf{L 1}_{\mathrm{C}}$ & $\mathbf{L 1}_{\mathrm{k}, \mathrm{z}}$ & $\mathbf{L 1}_{\mathrm{d}}$ & $\mathbf{h}_{\mathrm{BD}}$ & $\mathbf{L S}_{\mathrm{C}}$ & $\mathbf{L S}_{\mathrm{k}, \mathrm{z}}$ \\
& {$\left[\mathrm{J} / \mathrm{m}^{3} \mathrm{~K}\right]$} & {$[\mathrm{W} / \mathrm{mK}]$} & {$[\mathrm{nm}]$} & {$\left[\mathrm{W} / \mathrm{m}^{2} \mathrm{~K}\right]$} & {$\left[\mathrm{J} / \mathrm{m}^{3} \mathrm{~K}\right]$} & {$[\mathrm{W} / \mathrm{mK}]$} \\
Nominal & $2.88 \times 10^{6}$ & 71.6 & 100 & $1.40 \times 10^{8}$ & $1.66 \times 10^{6}$ & 148 \\
Standard Deviation & $5 \%$ & $10 \%$ & $10 \%$ & $50 \%$ & $5 \%$ & $5 \%$ \\
\hline
\end{tabular}




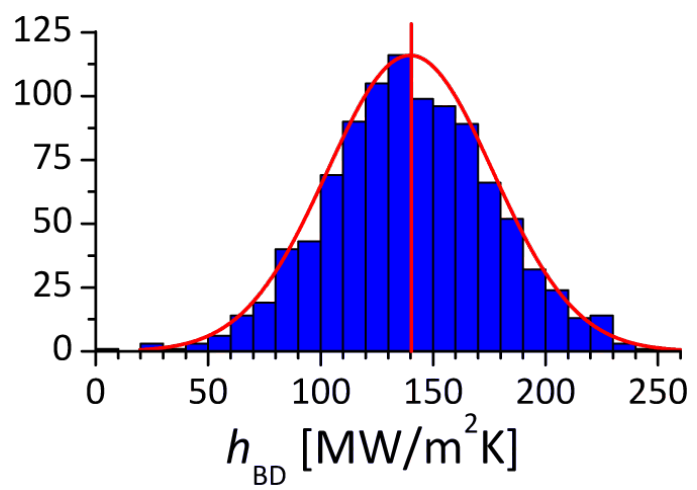

(a)

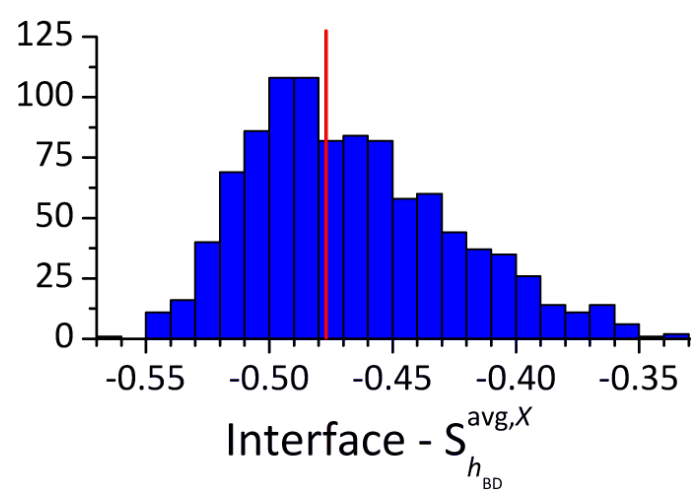

(b)

Figure 6.36: For the quasi-stochastic sensitivity analysis of the Pt/Si system (a) the distribution of the input $h_{\mathrm{BD}}$ values and (b) the resulting distribution of the average sensitivity coefficient for thermal boundary conductance based on $N=1,000$ iterations of the sensitivity model, with the red vertical line representing the sensitivity results using the nominal input parameters.

\subsubsection{Estimated Precision Analysis}

To determine the regime of the sensitivity coefficients for $h_{\mathrm{BD}}$, i.e. diffusive or effusive (see Section 6.6), the sensitivity coefficient for the input parameters of the $\mathrm{Pt} / \mathrm{Si}$ system as a function of $h_{\mathrm{BD}}$ are shown in Figure 6.37. The modeled $h_{\mathrm{BD}}$ value of $h_{\mathrm{BD}}=140 \mathrm{MW} / \mathrm{m}^{2} \mathrm{~K}$ places the sensitivity coefficient in the effusive regime. However, because we have the highest uncertainty in the $h_{\mathrm{BD}}$ value, and the nominal value is close to the boarder between the two sensitivity regimes, see Figure 6.37, for the sake of argument we will consider the calculated relative standard deviation from both regimes.

For an assumed range of likely signal-to-noise parameters, the calculated anticipated

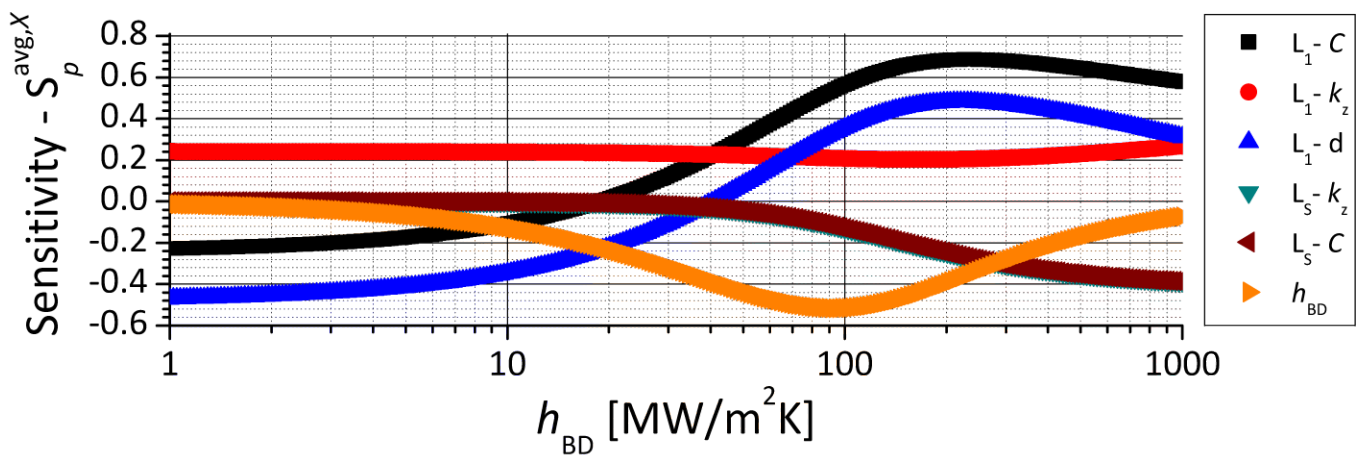

Figure 6.37: Sensitivity parameter as a function of $h_{\mathrm{BD}}$ for the modeled $100 \mathrm{~nm} \mathrm{Pt}$ on Si system, where $\mathrm{L}_{1}$ denotes the $\mathrm{Pt}$ film and $\mathrm{L}_{\mathrm{S}}$ the Si substrate. 
standard deviation in the deduced $h_{\mathrm{BD}}$ results assuming $\left|S_{h_{\mathrm{BD}}}^{\mathrm{avg}, X}\right|=0.4770$ is shown in Figure 6.38 for calculations based on both the diffusive and effusive regimes. As anticipated, both models, Equations 6.19 and 6.20, produced almost exactly the same estimate for the standard deviation because the simulated boundary conductance is very close to the regime boundary between the diffusive and effusive models, see Figure 6.37. The major difference between the two models for this example is the amount of uncertainty in the predicted results which is indicated for each model by the shaded regions in Figure 6.38.

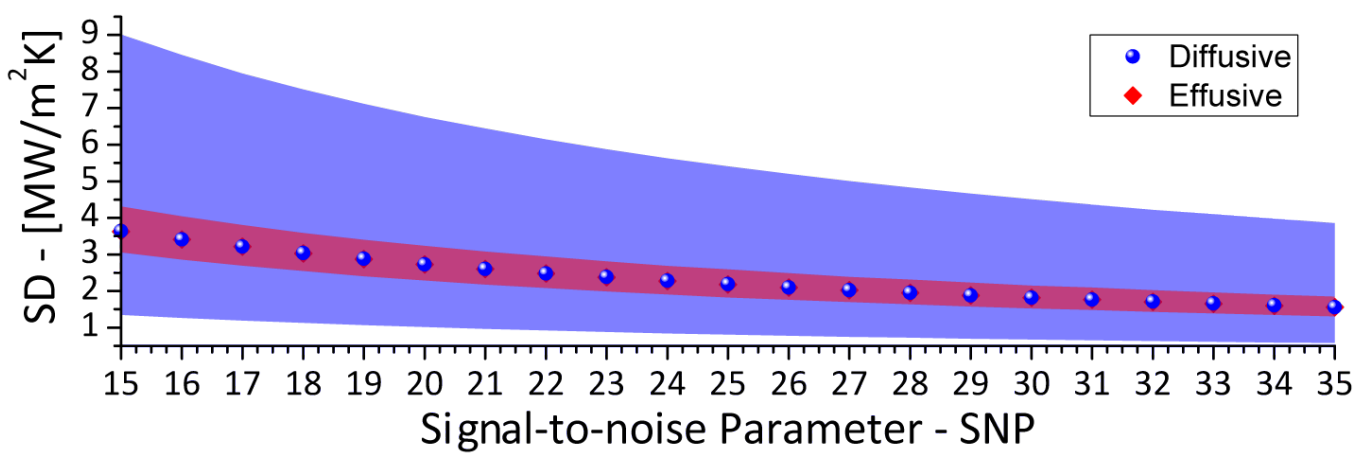

Figure 6.38: Calculated anticipated standard deviation of the population of $h_{\mathrm{BD}}$ values for a system of $100 \mathrm{~nm}$ of $\mathrm{Pt}$ on $\mathrm{Si}\left(h_{\mathrm{BD}}=140 \mathrm{MW} / \mathrm{m}^{2} \mathrm{~K}\right),\left|S_{h_{\mathrm{BD}}}^{\mathrm{avg},}\right|=0.4770$, as a function of SNP for calculations from both the diffusive and effusive regimes, given by Equations 6.19 and 6.20 respectively. The shaded regions represent a $95 \%$ confidence region in the predicted values. Note: Data points overlap.

\subsubsection{Estimated Accuracy Analysis}

To compare to the estimates of precision we can also model the anticipated accuracy of the final result using the estimates for the uncertainty in the input parameters. Using the nominal estimated parameters from Table 6.6 a model system was generated and refit using the anticipated uncertainty of the input parameters from Table 6.8 for a total of 1,000 trial fits. The resulting distribution of $h_{\mathrm{BD}}$ values is shown in Figure 6.39a. The standard deviation in the $h_{\mathrm{BD}}$ results for the accuracy analysis is $s=1.64 \times 10^{7} \mathrm{~W} / \mathrm{m}^{2} \mathrm{~K}$, which is approximately double the standard deviation found for the precision measurements depending on the assumed SNP. For comparison, the accuracy analysis was also completed assuming the uncertainty in the input parameters as listed in Table 6.8 represents a $2 \sigma$ variation, i.e. the values used in the accuracy analysis were half of those presented in the table. The results of the distributed $h_{\mathrm{BD}}$ values are shown in Figure 6.39b. The reduction in 


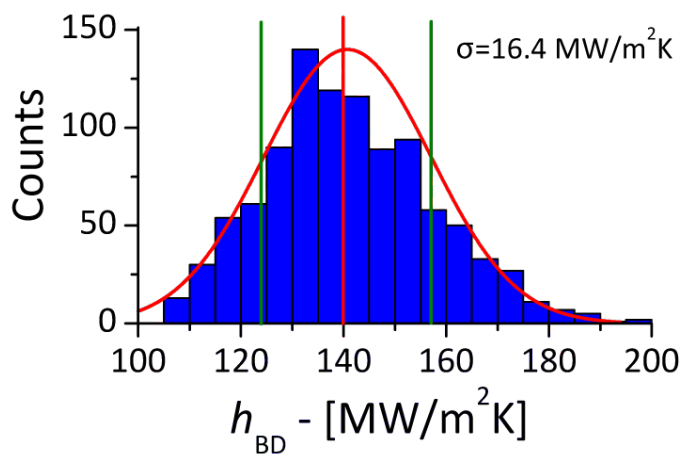

(a)

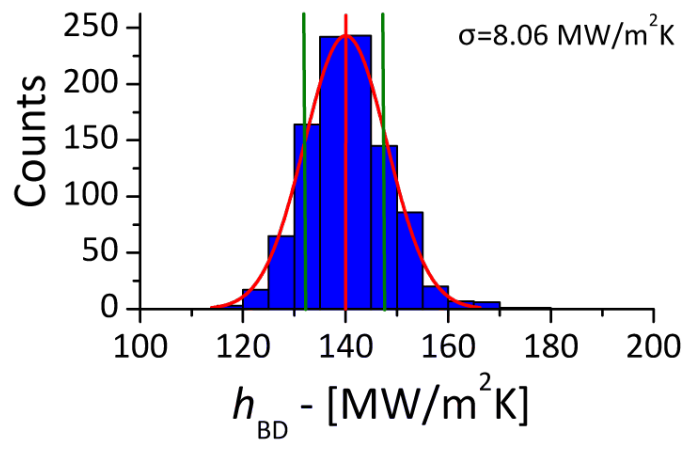

(b)

Figure 6.39: Distributed of deduced $h_{\mathrm{BD}}$ values for a modeled system of $100 \mathrm{~nm}$ of $\mathrm{Pt}$ on $\mathrm{Si}\left(h_{\mathrm{BD}}=\right.$ $140 \mathrm{MW} / \mathrm{m}^{2} \mathrm{~K}$ ) for the assumed uncertainty in the input parameters provided by Table 6.8. The red vertical line represents the nominal $h_{\mathrm{BD}}$ value, and the green lines plus/minus one standard deviation.

the uncertainty of the input parameters translated to a reduction in the standard deviation of the $h_{\mathrm{BD}}$ values to $s=8.06 \times 10^{6} \mathrm{~W} / \mathrm{m}^{2} \mathrm{~K}$.

The analysis of the initial system modeling indicates that there is sufficient sensitivity and accuracy to measure the $h_{\mathrm{BD}}$ of a modeled system of $100 \mathrm{~nm}$ of $\mathrm{Pt}$ on $\mathrm{Si}\left(h_{\mathrm{BD}}=\right.$ $140 \mathrm{MW} / \mathrm{m}^{2} \mathrm{~K}$ ) to an accuracy limited resolution on the order of $6-12 \%$.

\subsubsection{Experimental and Simulated Results}

To compare these results both experimental and simulation studies were conducted. The system of $100 \mathrm{~nm}$ of $\mathrm{Pt}$ on $\mathrm{Si}$ was simulated using the same methodology of noise generation and data analysis as was shown in Section 6.6 for 21 levels of signal-to-noise parameters and 1,000 trial data sets at each level. Experimentally $100 \mathrm{~nm}$ of Pt was deposited via electron-beam evaporation onto a Si substrate ${ }^{33}$. Thermoreflectance data was collected on the $\mathrm{Pt} / \mathrm{Si}$ sample using the low-rep configuration of the TTR/TDTR system at a single location on the sample surface for a total of 152 trials and the results analyzed to deduce $h_{\mathrm{BD}}$ using the standard TTR/TDTR data analysis program.

Considering first the simulated system, the simulation results for the deduction of $h_{\mathrm{BD}}$ for the $\mathrm{Pt} / \mathrm{Si}$ system are shown in Figure 6.40. As was observed for the $\mathrm{Al} / \mathrm{Si}$ system, the relative standard deviation in the deduced $h_{\mathrm{BD}}$ as a function of the SNP follows a power law trend. The deduced fitting coefficient, $a$, for the relative standard deviation follows the

\footnotetext{
${ }^{33}$ The details of the sample fabrication process can be found in Chapter 7.4.2.
} 
trend developed in the effusive regime, see Equation 6.20, for systems on a Si substrate, see Figure 6.41.

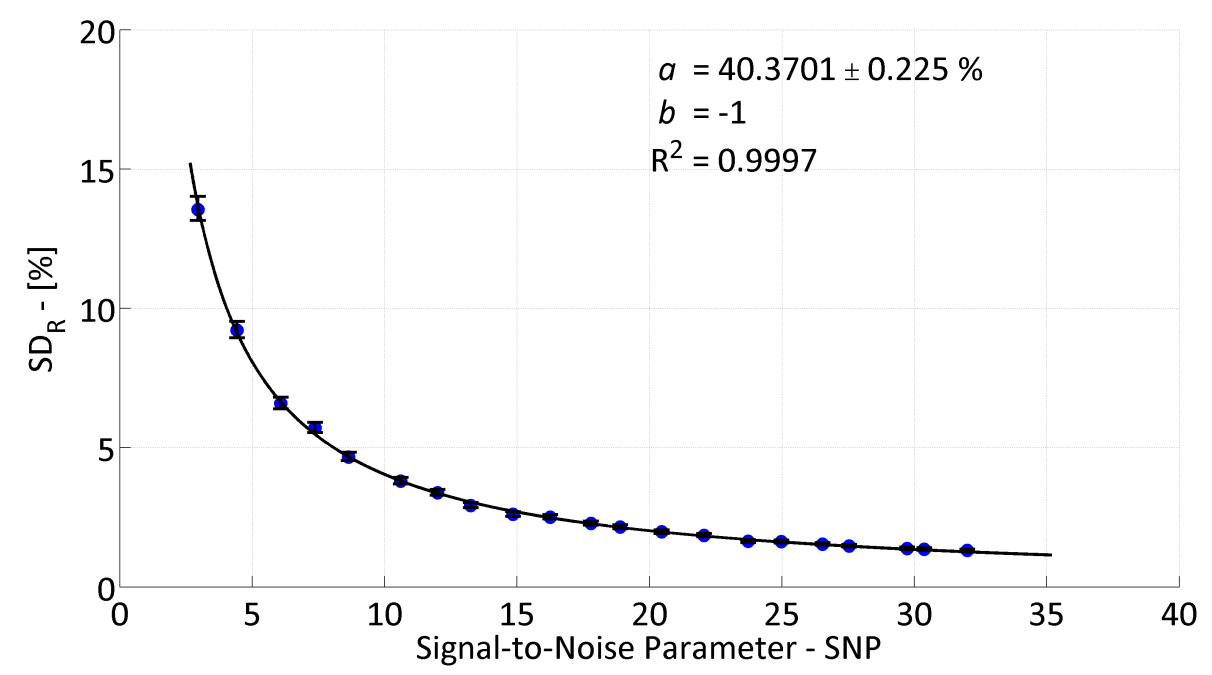

Figure 6.40: Relative population standard deviation as a function of signal-to-noise parameter for 100 $\mathrm{nm}$ of $\mathrm{Pt} / \mathrm{Si}$ with $h_{\mathrm{BD}}=140.00 \mathrm{MW} / \mathrm{m}^{2} \mathrm{~K}$. Error bars represent a $95 \%$ confidence interval of the measured $\mathrm{SD}_{\mathrm{R}}$ and the solid line represents a best fit of a power law to the data, of the form $\mathrm{SD}_{\mathrm{R}}=a \times \mathrm{SNP}^{b}$, with parameter $a$ provided for a fixed $b=-1$, along with the $95 \%$ confidence level in $a$.

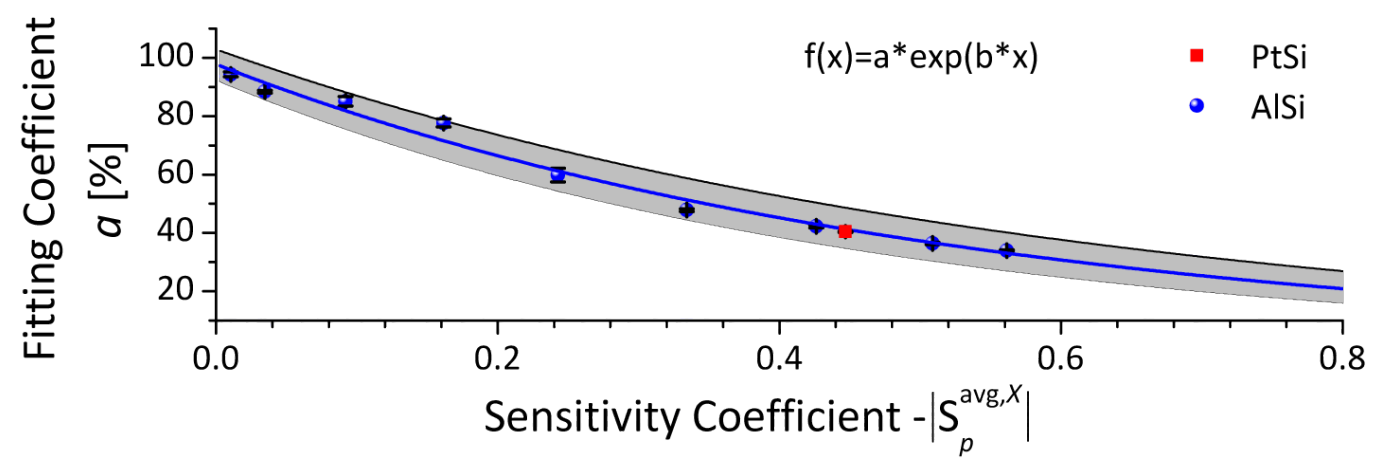

Figure 6.41: Fitting coefficient as a function of the absolute value of the sensitivity coefficient for $h_{\mathrm{BD}}$ in the $\mathrm{Al} / \mathrm{Si}$ sample series in the effusive regime. The data is fit using an exponential of the form $f(x)=a * \exp (b x)$ with the coefficients $(a=97.76 \pm 5.14, b=-1.93 \pm 0.25)$. The bounds on the coefficients and the grey shaded area represent a $95 \%$ confidence interval in the values and model respectively. The data point for the $\mathrm{Pt} / \mathrm{Si}$ system $\left(h_{\mathrm{BD}}=140 \mathrm{MW} / \mathrm{m}^{2} \mathrm{~K}\right)$ corresponds to the developed exponential trend. 
For the experimentally collected data, the resulting distribution of $h_{\mathrm{BD}}$ values deduced from the data using the parameters given in Table 6.8 is shown in Figure 6.42. The average $h_{\mathrm{BD}}$ value deduced over the 152 scans is $\bar{h}_{\mathrm{BD}}=118 \mathrm{MW} / \mathrm{m}^{2} \mathrm{~K}$ with the standard deviation of the results given by $s=7.27 \mathrm{MW} / \mathrm{m}^{2} \mathrm{~K}$. A comparison of the calculated, simulated, and experimental results in the standard deviation are summarized in Table 6.9.

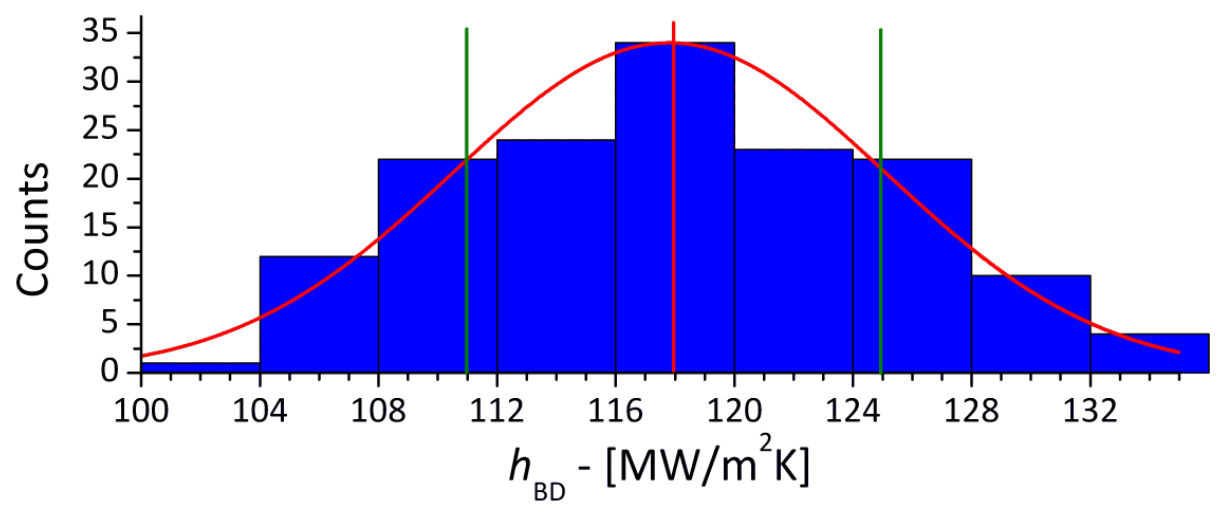

Figure 6.42: Distributed of deduced $h_{\mathrm{BD}}$ values for an experimental system of $100 \mathrm{~nm}$ of $\mathrm{Pt}$ on $\mathrm{Si}$ using the input parameters provided by Table 6.8 . The red vertical line represents the average $h_{\mathrm{BD}}$ value, and the green lines plus/minus one standard deviation.

Table 6.9: Standard deviation in $h_{\mathrm{BD}}$ calculated from Equation 6.20, simulated from 1,000 data sets of a modeled system of $100 \mathrm{~nm}$ of $\mathrm{Pt}$ on $\mathrm{Si}\left(h_{\mathrm{BD}}=140 \mathrm{MW} / \mathrm{m}^{2} \mathrm{~K}\right)$ with $\mathrm{SNP}=25$, and from 152 experimentally collected scans analyzed to deduce $h_{\mathrm{BD}}$. The upper and lower bounds represent a $95 \%$ confidence level in the respective values.

\begin{tabular}{lrccc}
\hline & & Calculated & Simulated & Data \\
\hline & High & $2.59 \times 10^{6}$ & $2.37 \times 10^{6}$ & $8.20 \times 10^{6}$ \\
Standard Deviation $\left[\mathbf{W} / \mathbf{m}^{2} \mathbf{K}\right]$ & Mean & $2.18 \times 10^{6}$ & $2.27 \times 10^{6}$ & $7.27 \times 10^{6}$ \\
& Low & $1.83 \times 10^{6}$ & $2.17 \times 10^{6}$ & $6.54 \times 10^{6}$ \\
\hline
\end{tabular}

We notice from the data in Table 6.9 that the standard deviation of the simulated results matches well with the anticipated standard deviation calculated from Equation 6.20. The standard deviation for the data however is larger than the calculated and simulated standard deviation by about a factor of 3 . There are several potential explanations for this deviation: 1) while 152 scans is a large data set in terms of typical TTR/TDTR analysis, it is much less than the number of simulated sets used in the analysis via the law of large numbers. In this case, the standard deviation of the sample may not be a completely accurate representation of the population standard deviation at this sample size, 2) the improper shape of the 
temperature decay due to inaccurate input parameters may affect the deduced standard deviation, as compared to the modeled system where the input parameters are known exactly, and 3) the noise in the data for an individual scan having induced bias compared to the ideal normal distribution of the simulated data.

Regardless of the mechanisms at work, the developed empirical model based on the simulated results provides a reasonable best case estimation of the standard deviation in the expected result. The model allows for the first time, the ability to estimate the uncertainty in the deduced result as a function of the sensitivity coefficient and the SNP parameter before any samples are fabricated and/or experiments conducted. This formulation will assist in the development of new experimental investigations and provide a metric to estimate whether the desired results can be successfully deduced given the resources at hand.

\subsection{Chapter Summary}

In this chapter we took a close look at the statistical description and analysis of data obtained via the thermoreflectance experiment. I presented the results of a large scale set of simulations directed at establishing an empirical relationship between the thermophysical parameters of a system and the properties in the measured data, i.e. the signal-to-noise parameter. The results of the simulation study brought to light two different regimes in the sensitivity to $h_{\mathrm{BD}}$, with the precision in the deduced $h_{\mathrm{BD}}$ following different trends in each regime. The developed empirical relations were shown to be consistent over a number of different material systems and match reasonably well compared to experimental data. The statistical concept of bootstrapping was introduced as a means to statistically interpret several important quantities in the analysis of TTR/TDTR data independent of the assumptions on how the data is distributed. Finally, supported by the methods developed in Chapter 5 a quasi-stochastic interpretation of the accuracy in the final results due to the input uncertainties was developed in order to more accurately model the input uncertainties and include covariance between the inputs. 


\title{
CHAPTER 7
}

\author{
VARIATIONS OF THERMAL \\ BOUNDARY CONDUCTANCE ACROSS \\ SOLID-SOLID INTERFACES VIA \\ VIBRATIONALLY MEDIATING \\ INTERMEDIATE MATERIALS
}

7.1 Introduction . . . . . . . . . . . . . . . . . . . 238

7.2 The Concept of Vibrational Bridging . . . . . . . . . . . . . . 239

7.3 Simulating Phonon Transport Across Intermediate Layers by Molecular Dynamics Simulations . . . . . . . . . . . . . . . . . . . 240

7.4 Thermal Bridging via Metallic Intermediate Layers . . . . . . . . . . . . 246

7.5 Experimental Procedure and Results . . . . . . . . . . . . . . 250

7.6 Results and Interpretation . . . . . . . . . . . . . . . . . 259

7.7 Chapter Summary . . . . . . . . . . . . . . . . . 271 


\subsection{Introduction}

As discussed in more detail in Chapter 1 and Chapter 2, interface conductance is an important quantity in the development of many modern micro- and nano-devices including optoelectroinc devices [79, 80], thermal interface materials (TIMs), [28, 86, 87], highelectron-mobility transistors (HMETs) [90], etc. To this end, a large body of work has been devoted to understanding and predicting the behavior of phonons at the interface between two solids, and therefore the resulting $h_{\mathrm{BD}}[73,94]$. However, most of the different mechanisms studied that affect $h_{\mathrm{BD}}$ as discussed in Chapter 2 , are artifacts of the deposition process or the natural interaction of the different species that comprise the film and substrate. In the majority of these semiconductor based nanodevices, it is common to find a number of metal-metal, metal-semiconductor, and semiconductor-semiconductor interfaces [279]. As the effects of these mechanisms become more well understood, focus shifts towards the concept of phononic engineering in order to enhance or reduce thermal transport to suite a particular application.

In this chapter we will focus on the addition of an intermediate layer confined between two solids with differing vibrational spectra in order to tune $h_{\mathrm{BD}}$ by altering the spectral overlap between each layer. We will begin by motivating this intermediate layer as a vibrational bridge and turn to previous molecular dynamics works which support this concept and provide a framework for the setup of experimental trials. This concept is experimentally tested utilizing a metallic intermediate layer $(\mathrm{Ni})$ of varying thickness between $\mathrm{Pt}$ films on $\mathrm{Si}$ and Ge substrates. The samples were prepared by electron beam evaporation and the elemental profile close to the interface characterized by Auger photoelectron spectroscopy (AES). The thermal boundary conductance of the $\mathrm{Pt} / \mathrm{Ni} / \mathrm{Si}(\mathrm{Ge})$ systems were characterized using the TTR technique utilizing the methodologies developed and discussed in the previous chapters. The results are presented and several issues are discussed before providing final conclusions as to the effectiveness of the $\mathrm{Ni}$ as an intermediate layer and the concept of vibrational bridging. 


\subsection{The Concept of Vibrational Bridging}

In the absence of all other affects that can alter interface conductance, i.e. bonding, inter-atomic mixing, etc., see Chapter 2, the dominate characteristic that is assumed to dictate $h_{\mathrm{BD}}$ across the solid-solid interface is the degree of overlap in the vibrational spectra of the two solids [78, 110, 120, 280].

To a first approximation, the "stiffness" of a solid material and thereby the vibrational properties, i.e. phonon density of states (PDOS), phonon group velocities, maximum frequencies, etc., are summarized in a single value, the Debye temperature, $\Theta_{\mathrm{D}}[32]$. Therefore, it is commonly held that for a solid-solid system in order to maximize the phonon transport across the interface, and thus $h_{\mathrm{BD}}$, it is desirable for the solids in the system to have similar Debye temperatures $[78,110,120,280]$. To better visualize this concept, a graphical representation of the fictitious phonon density of states in a solid-solid system is shown in Figure 7.1a.

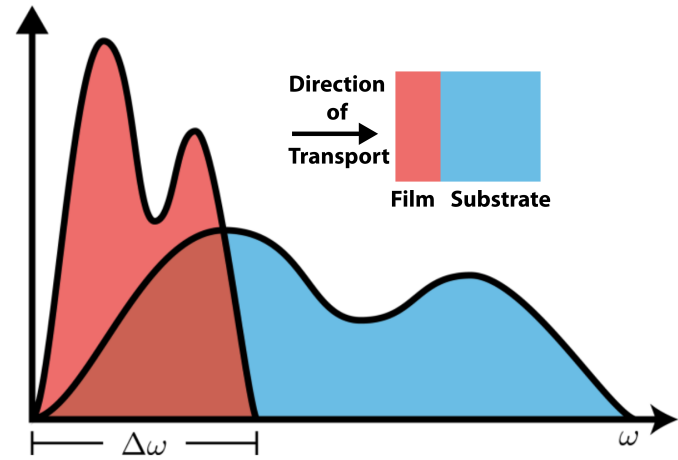

(a)

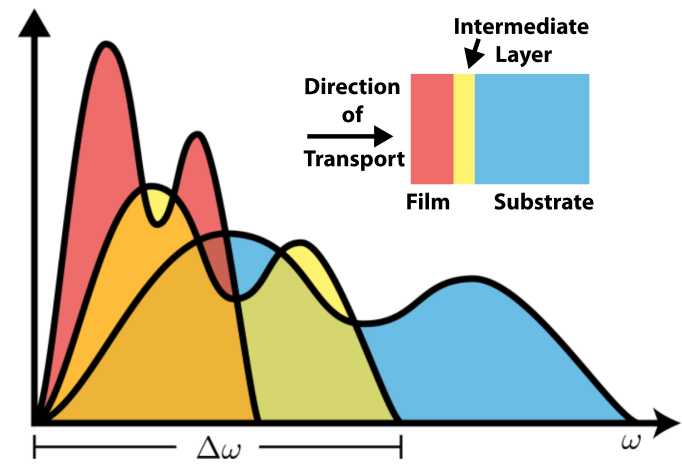

(b)

Figure 7.1: Representation of the phonon density of states as a function of frequency $\omega$ for (a) a solid-solid system in which $\Theta_{\mathrm{D}, \text { film }}($ Red $)<\Theta_{\mathrm{D} \text {,substrate }}($ Blue) and (b) a solid-solid system with an intermediate layer between the film and substrate such that $\Theta_{\mathrm{D} \text {,film }}($ Red $)<\Theta_{\mathrm{D} \text {,int }}($ Yellow $)<\Theta_{\mathrm{D} \text {, substrate }}$ (Blue).

In this representation, the shaded red area represents a solid film whose Debye temperature is lower then that of the solid substrate, represented in blue. The region of overlap between these two plots represents the spectra of phonons that can participate in elastic scattering processes. Any phonon interactions outside this region are attributed to inelastic phonon processes, which are less probable and contribute less to the overall $h_{\mathrm{BD}}$ across the interface as compared to elastic processes $[15,140]$. This thereby reinforces the concept 
that overlapping PDOS between two solids, and therefore similar Debye temperatures, is desirable for maximum interface conductance. However, non-thermal design considerations often limit the primary component materials used in the creation of micro- and nano-devices. As stated previously, the current design paradigm is to consider device performance first and then consider thermal management issues or relegate them to a post-design process altogether. In Figure 7.1b a third material is added between the two solids with mediating phononic properties. In theory, this intermediate layer will act as a "phonon bridge" helping to mediate the phonon transition between highly dissimilar phonon states by first coupling to an intermediate state. By choosing a thin metallic or semiconducting intermediate layer based on device design, the goal is to be able to tune $h_{\mathrm{BD}}$ through the device structure without significantly altering performance.

\subsection{Simulating Phonon Transport Across Intermediate Lay- ers by Molecular Dynamics Simulations}

The motivation for the intermediate layer as a vibrational bridge originally came from the basic concept of the relative ratio of the Debye temperatures between a film and substrates being a crude qualitative estimate of the anticipated thermal boundary conductance, along with measurements on a small sampling of "leftover" samples found in the back of a drawer somewhere in the UVa nanoscale energy transport lab ${ }^{1}$. The major challenge with all nanoscale experimental work is the inability to have complete control over the system parameters. However, this is an area where molecular dynamics simulations provide an invaluable tool to understanding the fundamentals of thermal transport. Therefore, motivated by the original design idea and supported by preliminary experimental work, a MD study was conducted to determine the feasibility and underlying principles of the phonon bridging effect.

\footnotetext{
${ }^{1}$ These preliminary results were first presented at IMECE in Boston in November of 2008.
} 


\section{English et al. 2012}

English et al. [14] tested and studied the concept of a vibrational bridging effect using nonequilibrium molecular dynamics simulations. Their baseline computational system consisted of two Lennard-Jones FFC crystals, denoted materials A and B, which had atomic masses of $40 \mathrm{amu}$ and $120 \mathrm{amu}$ respectively, see Figure 7.2a. The two respective masses provided mismatched vibrational properties to the two material systems which is a common technique in MD simulations to vary vibrational properties [101, 110, 112, 114, 281, 282]. The particular choice of mass ratio for this study $(R=3)$ was chosen to represent a similar ratio that is found in many devices systems such as $\mathrm{Si} / \mathrm{Ge}(R=2.6)$ and diamond/Si $(R=2.3)$.

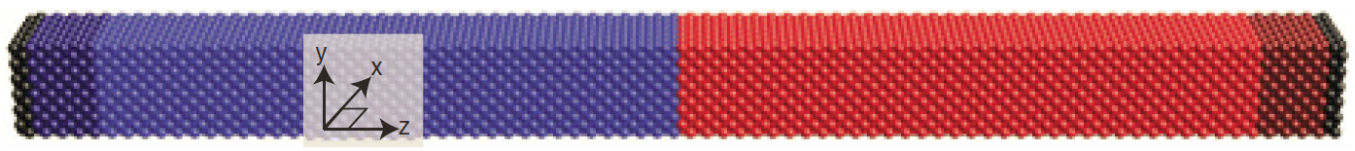

(a)

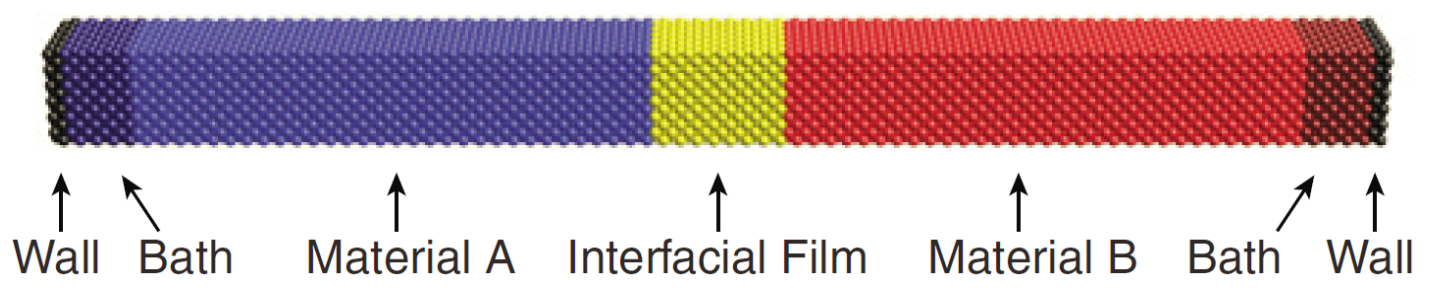

(b)

Figure 7.2: Schematic of the computational domains used by English et al. [14] for (a) the baseline case and (b) the case of an intermediate film of varying mass and thickness. Image reproduced with permission from $[14]$.

To test the concept of the vibrational bridge, a third material labeled material $\mathrm{C}$ was added between the interface of materials A and B, see Figure 7.2b. As part of the parametric study the mass of the atoms in material $\mathrm{C}$ was varied between 40 and $120 \mathrm{amu}$, the bounds of materials $\mathrm{A}$ and $\mathrm{B}$, and the width of the intermediate region was varied between 2 conventional unit cells (UCs) to 16 conventional UCs in increments of 2 UCs. The studies were completed at 0.1 and 0.5 times the crystal melting temperature, $T_{m}$, and the $h_{\mathrm{BD}}$ deduced as a total conductance from material A to material B.

The results of the parametric study at 0.1 and $0.5 T_{m}$ are shown in Figures $7.3 \mathrm{a}$ and $7.3 \mathrm{~b}$ respectively. For the high temperature simulations there is only a slight enhancement to 


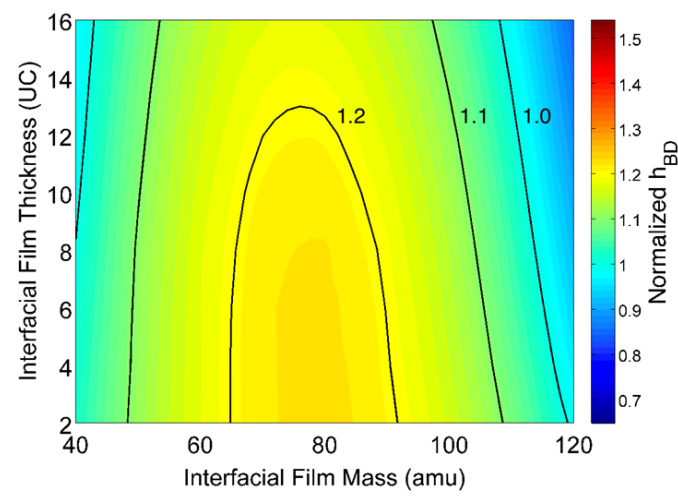

(a)

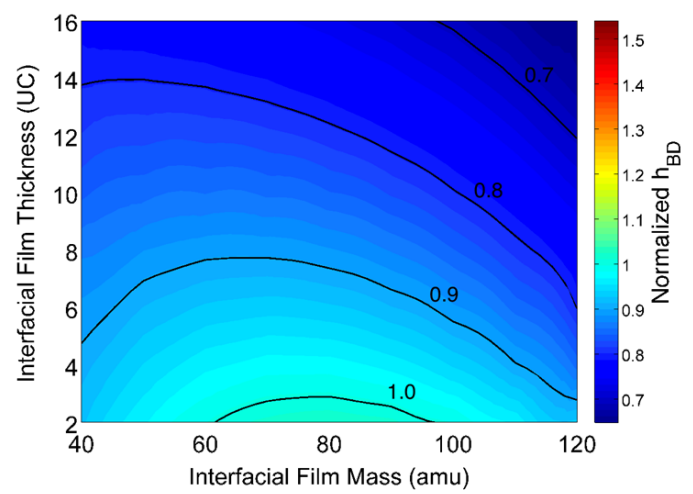

(b)

Figure 7.3: Measured ratio of $h_{\mathrm{BD}}$ with the addition of the intermediate layer to the baseline case without the intermediate layer as a function of the mass and thickness of material $\mathrm{C}$ for (a) $0.1 T_{m}$ and (b) $0.5 T_{m}$. Image reproduced with permission from [14].

$h_{\mathrm{BD}}$ for the $2 \mathrm{UC}$ layer with an atomic mass between 60 and $100 \mathrm{amu}$. As the intermediate film thickness increases the total $h_{\mathrm{BD}}$ of the system continues to drop. At high temperatures the reduction in $h_{\mathrm{BD}}$ is attributed to the reduced thermal conductivity of the intermediate layer and the increased probability of interface phonon scattering due to the reduced phonon mean free path. By contrast, at the lower simulation temperature $h_{\mathrm{BD}}$ enhancement was found over the majority of the mass and thickness range. The greatest enhancement being observed for thin intermediate layers with an atomic mass between that of the two solids.

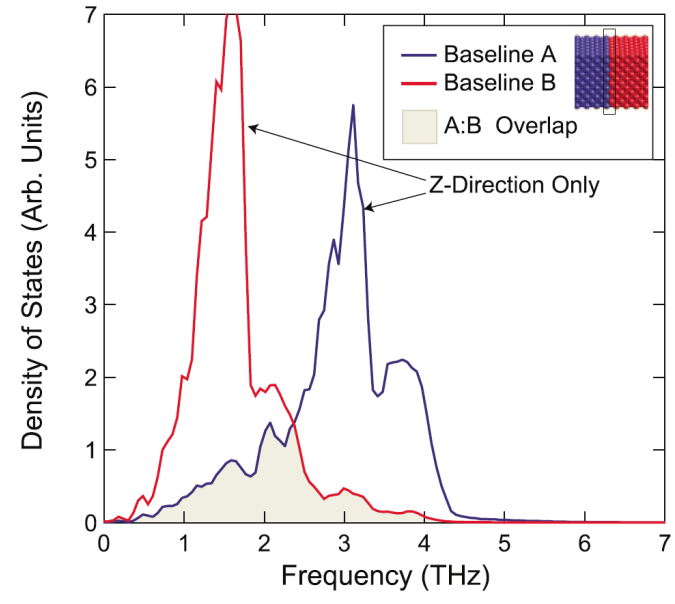

(a)

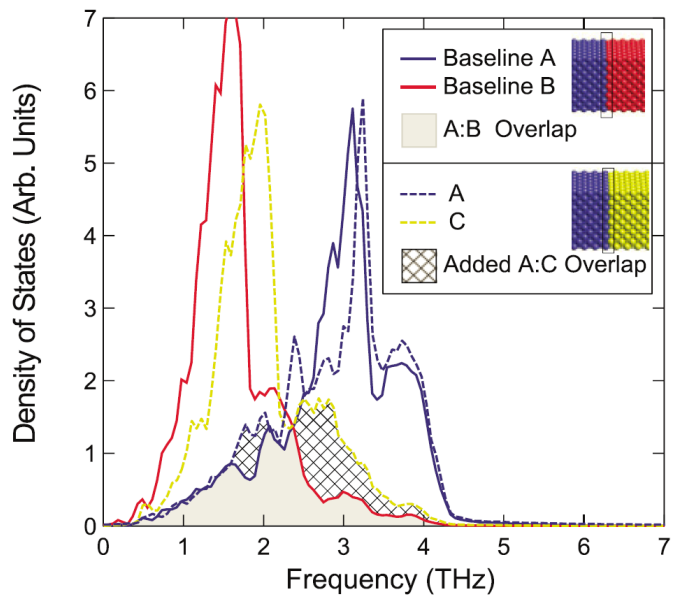

(b)

Figure 7.4: Isotropic occupies phonon density of states for (a) the baseline interface with the gray shaded region denoting the vibrational overlap and (b) with the addition of the intermediate layer at the interface between material $\mathrm{A}$ and $\mathrm{C}$ and $\mathrm{C}$ and $\mathrm{B}$ with the hatched region indication the added area of vibrational overlap. Image reproduced with permission from [14]. 
To help explain the mechanism behind the enhancement affect observed in Figure 7.3a, English et al. [14] found the occupied phonon density of states in the monolayers adjacent to the interface with and without the addition of the intermediate layer, see Figures 7.4a and $7.4 \mathrm{~b}$ respectively. It is observed that the added intermediate material alters the local phonon density of states of the constitute materials at the interface and provides additional phonon modes compared to materials $\mathrm{A}$ and $\mathrm{B}$, increasing the overall phonon spectra that can participate in elastic transport.

The initial part of the molecular dynamics work by English et al. focused on ideally sharp interfaces. To simulate more realistic interfaces they extended the simulation domains to consider diffusion at the interface, see Figure 7.5. For the high temperature simulation, see Figure 7.6b, the introduction of compositional disorder at the interface eliminated the

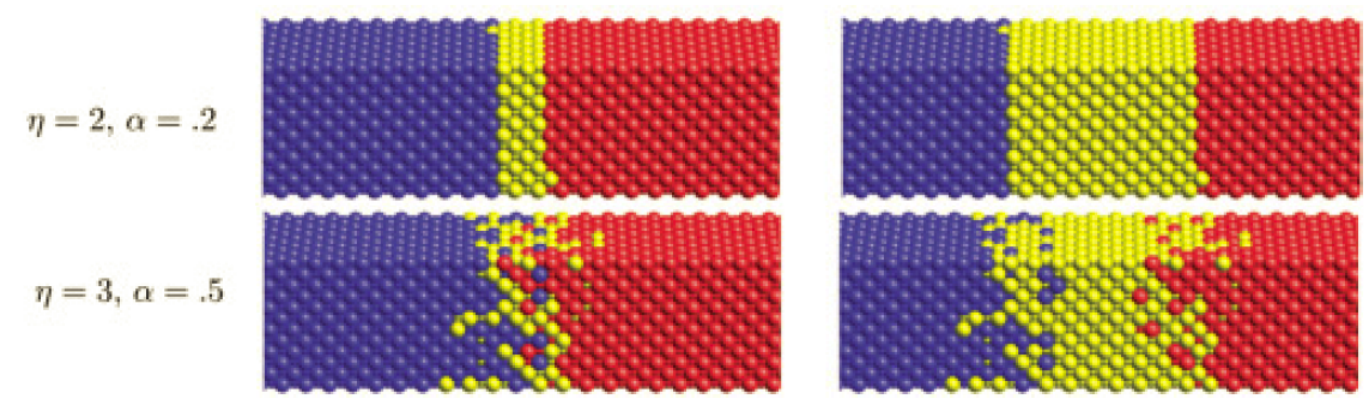

Figure 7.5: Example computation domains from the work of English et al. showing a varying degree of compositional disorder added to the interface region. Image reproduced with permission from [14].

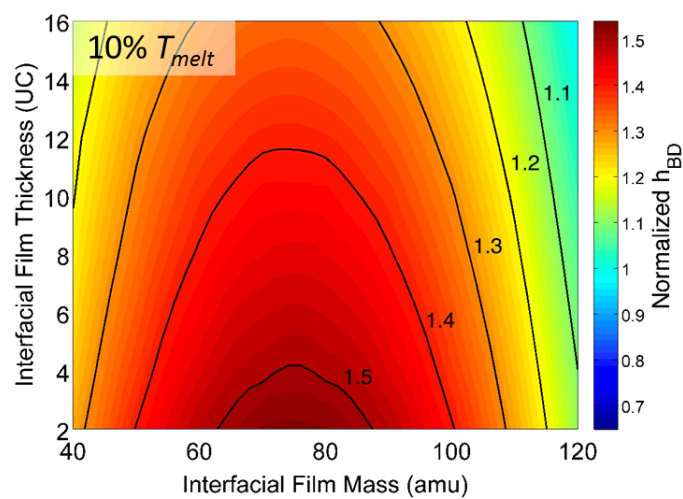

(a)

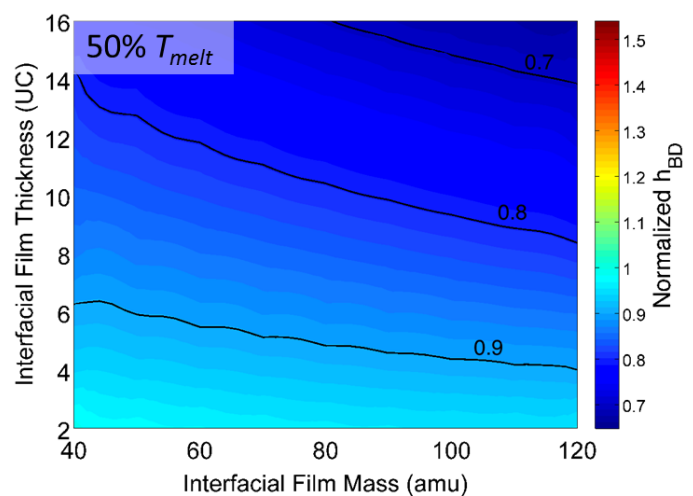

(b)

Figure 7.6: Measured ratio of $h_{\mathrm{BD}}$ with the addition of the intermediate layer to the baseline case without the intermediate layer as a function of the mass and thickness of material C for (a) $0.1 T_{m}$ and (b) $0.5 T_{m}$ for a system with simulated interface disorder. Image reproduced with permission from [14]. 
enhancement effect that was observed for the sharp interface. The addition of the intermediate layer for all masses and thicknesses reduced $h_{\mathrm{BD}}$. For the lower temperature study however, see Figure $7.6 \mathrm{a}, h_{\mathrm{BD}}$ was significantly increased compared to the sharp interface indicating that a small degree of compositional disorder actually enhanced $h_{\mathrm{BD}}$. The primary cause of this increased enhancement was again attributed to an increase in the overlap of the vibrational spectra between the materials comprising the interface by comparing the overlap in the phonon density of states with and without the added compositional disorder, see Figure 7.7.

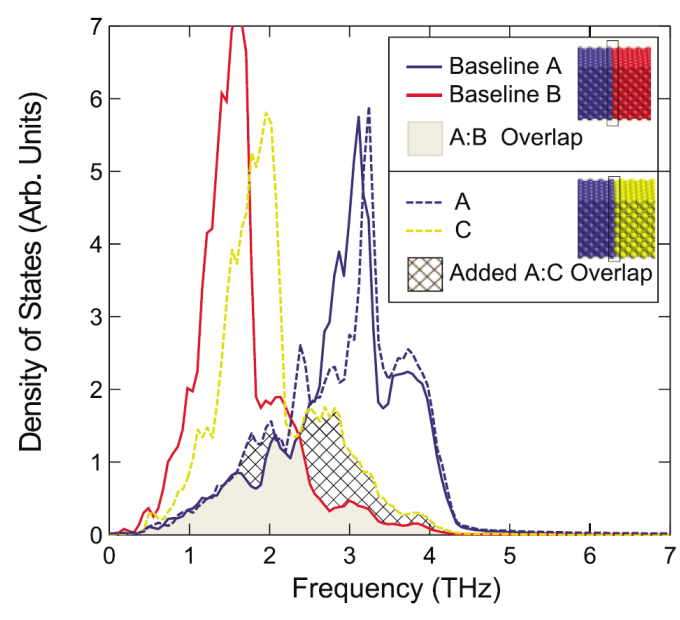

(a)

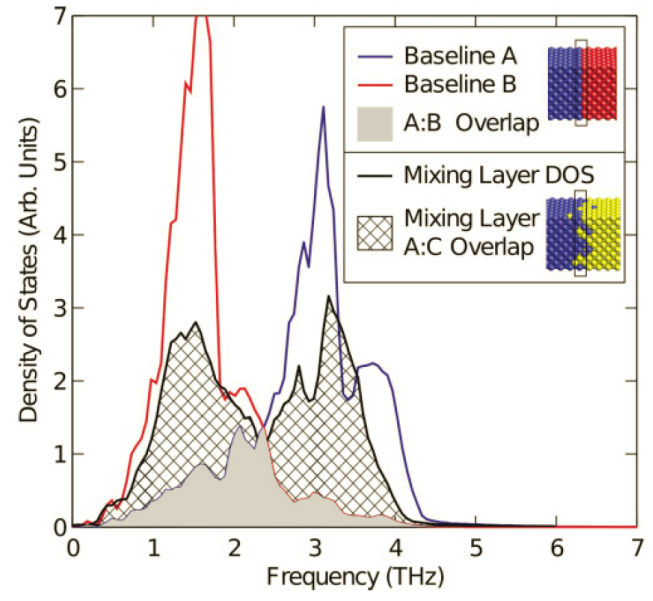

(b)

Figure 7.7: Isotropic occupied phonon density of states for (a) the added intermediate layer with an abrupt interface with the vibrational overlap denoted and (b) with the added intermediate layer with compositional disorder added with the hatched region indicating the added area of vibrational overlap. Image reproduced with permission from [14].

The simulation work of English et al. supports the concept of vibrational bridging by the addition of an intermediate layer with mediating vibrational properties as a means to increase the overlap in the phonon density of states and thereby increase $h_{\mathrm{BD}}$. Their results showed maximum enhancement for thin layers with an atomic mass ideally between the masses of the two outer layers. It was also found, contrary to previous experimental work, that a degree of compositional disorder at the interface could further increase the enhancement effect. 


\section{Liang and Tsai 2012}

A similar study was conducted by Liang and Tsai who also used molecular dynamics simulations to study the affect of an added intermediate layer between two solids on thermal conductance $^{2}[20]$. Liang and Tsai also used a Lennard-Jones system to model the crystal solids and considered an intermediate layer thickness of 1-4 UCs. The vibrational spectra of the intermediate layer was again dispersed by varying the mass of the atoms in the layer, and an effective Debye temperature, $T_{D, A}, T_{D, B}, T_{D, I L}$, was defined for material A, material $\mathrm{B}$, and the intermediate layer respectively. The Deybe temperature ratio of material $\mathrm{A}$ and material B was fixed at $T_{D, A} / T_{D, B}=3$ throughout the study.

The primary results from the simulations of Liang and Tsai are shown in Figure 7.8a as the ratio of the thermal interface resistance with and without the intermediate layer as a function of the Deybe ratio of the intermediate layer and material B. The results show an increase in $h_{\mathrm{BD}}$ across all the parameter combinations tested. The highest $h_{\mathrm{BD}}$ was found for a Debye temperature ratio between the intermediate layer and material B

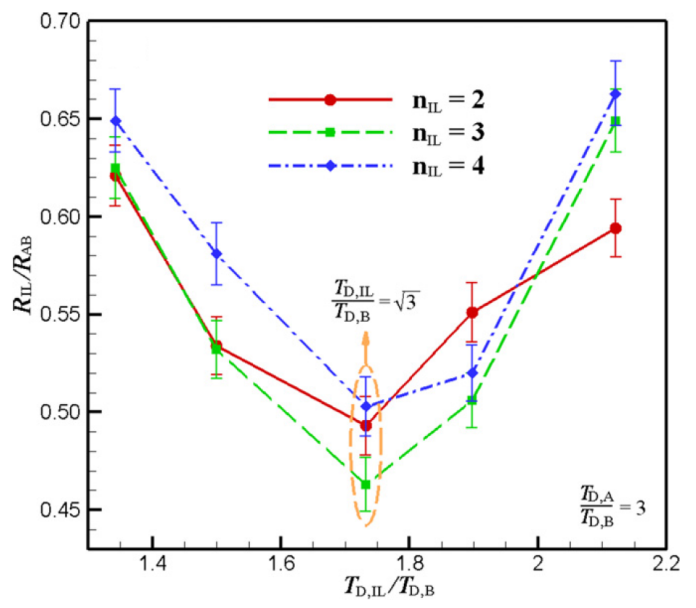

(a)

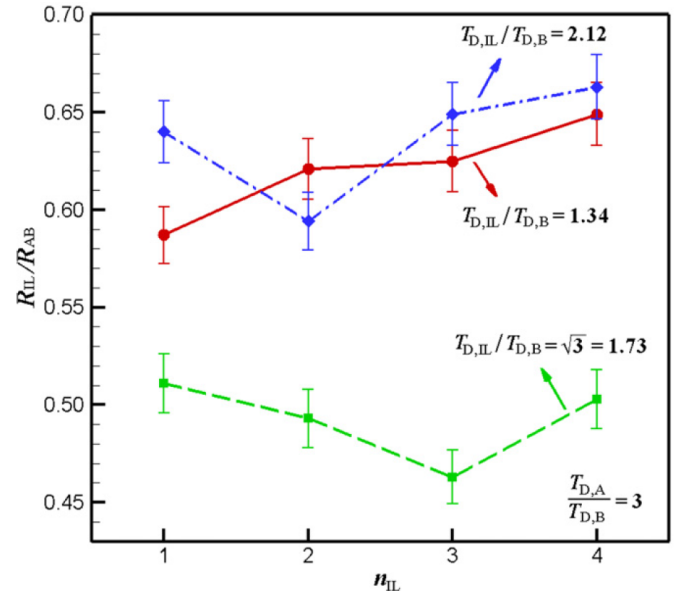

(b)

Figure 7.8: (a) The ratio of thermal boundary resistance with the intermediate layer to the baseline case as a function of the ratio of the Debye temperatures between the intermediate layer material and material B for several intermediate layer thicknesses. (b) The ratio of thermal boundary resistance with the intermediate layer to the baseline case as a function of the intermediate layer thickness for several Debye temperature ratios between the intermediate layer and material B. Image reproduced with permission from [20].

\footnotetext{
${ }^{2}$ The work of English et al. discussed their results in terms of thermal boundary conductance, while Liang and Tsai discuss their results in terms of reducing thermal resistance, which we know if the inverse of conductance.
} 
of $T_{D, I L} / T_{D, B}=\sqrt{3}$. For the range of intermediate thicknesses found there was little dependence between $h_{\mathrm{BD}}$ and layer thickness ${ }^{3}$. Using the results from the simulations Liang and Tsai quantifies the ideal Deybe temperature to maximize $h_{\mathrm{BD}}$ :

$$
T_{D, I L, \text { ideal }}=\sqrt{T_{D, A} \times T_{D, I L}}
$$

where $T_{D, I L \text {,ideal }}$ is the ideal Debye temperature of the intermediate layer and $T_{D, A}$ and $T_{D, B}$ are the Debye temperature of material A and material B respectively.

Similar to the results of English et al., Liang and Tsai were able to attribute the change in $h_{\mathrm{BD}}$ to the modification and increased overlap in the vibrational spectra of the solid materials through calculations of the occupied phonon density of states. Their results not only support the vibrational bridging concept but also provide quantification of the ideal Debye temperature material to increase $h_{\mathrm{BD}}$.

\subsection{Thermal Bridging via Metallic Intermediate Layers}

Based on the success of the preliminary experimental work in 2008 and the subsequent molecular dynamics work which promoted the possibly of a phonon bridging effect, a refined experimental study was developed and conducted to measure $h_{\mathrm{BD}}$ in real systems via the TTR/TDTR technique. Ideally, the experimental sample set would mimic the molecular dynamics simulations as closely as possible. This would dictate semiconducting materials for each layer with atomic control at the interface. Realizing this physically however is somewhat challenging.

\subsubsection{Metallic vs. Semiconductor Intermediate Layers}

The first challenge that arises is that as discussed in Chapter 3, the TTR/TDTR experiment requires a metal film on the top surface of the material system for signal detection. A thin film could be deposited on top of the prepared semiconductor samples to act as a thermal transducer, however this creates a complex sample system with 3 interface struc-

\footnotetext{
${ }^{3}$ Liang and Tsai interpret the results in Figure 7.8b to indicate a dependence of $h_{\mathrm{BD}}$ on intermediate layer thickness. However, the uncertainty in the data makes it difficult to conclude any non-constant trends from the results.
} 
tures and 4 material layers that must be modeled. A natural solution to this was to use a metallic film as the top layer.

As a highly common semiconductor substrate used in the micro- and nano-electronics industry, Si was an obvious choice for the experimental samples. With a large body of work describing the system iteration and a nearly $50 \%$ lower Debye temperature than $\mathrm{Si}, \mathrm{Ge}$ was an obvious choice for the intermediate layer. The challenge with the implementation of a Ge intermediate layer for this study was the deposition method. Using the deposition systems readily available to the UVa Nanoscale Energy Transport Lab, the Ge layer would be deposited via electron-beam evaporation of RF sputtering. Under these deposition conditions the Ge layer that would be deposited would most likely be amorphous. From previous experiments the thermal conductivity of thin film a-Ge layers has been shown to be on the order of $k \approx 0.5 \mathrm{~W} / \mathrm{mK}[283,284]$. Taking the effective conductance of a thin layer to be $h_{\text {layer }}=k / d$ where $k$ is the thermal conductivity of the layer and $d$ is the layer thickness, the total conductance of a $10 \mathrm{~nm}$ layer of $a$-Ge would be on the order of $h_{\text {layer }} \approx 5 \times 10^{7} \mathrm{~W} / \mathrm{m}^{2} \mathrm{~K}$. Typically, metal-semiconductor boundary conductance is on the order of $h_{\mathrm{BD}}=1 \times 10^{8} \mathrm{~W} / \mathrm{m}^{2}$ and therefore the $a$-Ge layer would be the dominant thermal resistance in the system and overshadow any potential enhancement effects due to phonon bridging. This in general makes semiconductor intermediate layers viable in crystalline form only. As an alternative to epitaxial grow, and based on successful preliminary studies, metallic intermediate layers were chosen due to the inherently high thermal conductivity of metals even for thin films.

To select the ideal metallic intermediate layer, using Equation 7.1, a table was generated using the readily available metals for deposition and commercially available substrates of interest to the micro- and nano-electronics industry, see Figure 7.9. The left hand column provides the available metals and their respective Debye temperatures in $\mathrm{K}$, and the row across the top the available substrates and Debye temperatures. In the field are the Debye temperatures of the ideal intermediate layers, calculated via Equation 7.1 and color coded to correspond to the available metals in the left column. For the top low Debye temperature film Pt was chosen due to its stable nature and high optical absorption at the laser wavelength which leads to a strong thermoreflectance signal [198]. Using the table in Figure 7.9 


\begin{tabular}{|c|c|c|c|c|c|c|c|c|c|c|c|c|c|c|c|}
\hline & & $\begin{array}{c}\text { InSb } \\
160\end{array}$ & $\begin{array}{c}\text { GaSb } \\
266\end{array}$ & $\begin{array}{l}\text { InAs } \\
280\end{array}$ & $\begin{array}{r}\mathrm{Ge} \\
360\end{array}$ & $\begin{array}{c}\text { GaAs } \\
360\end{array}$ & $\begin{array}{l}\operatorname{InP} \\
425\end{array}$ & $\begin{array}{l}\text { GaP } \\
445\end{array}$ & $\begin{array}{l}\mathrm{GaN} \\
600\end{array}$ & $\begin{array}{c}\mathrm{Si} \\
625\end{array}$ & $\begin{array}{l}\operatorname{InN} \\
660\end{array}$ & $\begin{array}{c}\text { HOPG CP } \\
950\end{array}$ & $\begin{array}{c}\text { AIN } \\
1150\end{array}$ & $\begin{array}{c}\mathrm{SiC} \\
1200\end{array}$ & $\begin{array}{c}\text { BN } \\
1700\end{array}$ \\
\hline $\mathrm{Bi}$ & 120 & 139 & 179 & 183 & 208 & 208 & 226 & 231 & 268 & 274 & 281 & 338 & 371 & 379 & 452 \\
\hline $\mathrm{Au}$ & 170 & 165 & 213 & 218 & 247 & 247 & 269 & 275 & 319 & 326 & 335 & 402 & 442 & 452 & 538 \\
\hline $\mathrm{Ag}$ & 215 & 185 & 239 & 245 & 278 & 278 & 302 & 309 & 359 & 367 & 377 & 452 & 497 & 508 & 605 \\
\hline Pt & 230 & 192 & 247 & 254 & 288 & 288 & 313 & 320 & 371 & 379 & 390 & 467 & 514 & 525 & 625 \\
\hline $\mathrm{Nb}$ & 275 & 210 & 270 & 277 & 315 & 315 & 342 & 350 & 406 & 415 & 426 & 511 & 562 & 574 & 684 \\
\hline Pd & 275 & 210 & 270 & 277 & 315 & 315 & 342 & 350 & 406 & 415 & 426 & 511 & 562 & 574 & 684 \\
\hline $\mathrm{Cu}$ & 315 & 224 & 289 & 297 & 337 & 337 & 366 & 374 & 435 & 444 & 456 & 547 & 602 & 615 & 732 \\
\hline $\mathrm{Ni}$ & 375 & 245 & 316 & 324 & 367 & 367 & 399 & 409 & 474 & 484 & 497 & 597 & 657 & 671 & 798 \\
\hline $\mathrm{Ti}$ & 380 & 247 & 318 & 326 & 370 & 370 & 402 & 411 & 477 & 487 & 501 & 601 & 661 & 675 & 804 \\
\hline Al & 394 & 251 & 324 & 332 & 377 & 377 & 409 & 419 & 486 & 496 & 510 & 612 & 673 & 688 & 818 \\
\hline $\mathrm{Cr}$ & 460 & 271 & 350 & 359 & 407 & 407 & 442 & 452 & 525 & 536 & 551 & 661 & 727 & 743 & 884 \\
\hline
\end{tabular}

Figure 7.9: Table of potential ideal Debye matches, see Equation 7.1, for the vibrational bridging effect. The left hand column provides the available metals and their respective Debye temperatures in $\mathrm{K}$, and the row across the top the available substrates and Debye temperatures. In the field are the Debye temperatures of the ideal intermediate layers.

the ideal intermediate layer for a Pt film on a Si substrate would have a Deybe temperature of $379 \mathrm{~K}$, which would indicate either Ni $(375 \mathrm{k})$ or Ti $(380 \mathrm{~K})$.

Ni was chosen over Ti for the intermediate layer material for several reasons: 1) Pt and Ni have the same crystal structure, FCC, as opposed to Ti which as a hexagonal structure. 2) Pt and Ni have more similar lattice constants, $a=3.92 \AA$ and $a=3.52 \AA$ respectively, to each other and $\mathrm{Si}, a=5.43 \AA$, than Ti, $a=2.95 \AA$. 3) Pt and Ni both form similar silicides at the interface with $\mathrm{Si}$, both of which have been well characterized [285-289]. To create a baseline case with the added $\mathrm{Ni}$ intermediate layer, samples were also created on Ge substrates. The Deybe temperature of $\mathrm{Ge}$ is similar to $\mathrm{Ni}$ and therefore little to no enhancement is expected due to phonon bridging. The sample sets that were prepared were $\mathrm{Pt} / \mathrm{Ni} / \mathrm{Si}$ and $\mathrm{Pt} / \mathrm{Ni} / \mathrm{Ge}$ with varying thicknesses for the Ni layer.

\subsubsection{Sample Preparation}

The samples for this study were fabricated at the US Army Research Laboratory in conjunction with the US Naval Academy. A summary of the deposition schedule is shown in Table 7.1. The primary substrates used in this study were Si and Ge with a single additional substrate of polycrystalline Ni. The Si substrates were P-type (B-doped) single crystal (100) wafers, polished on one side, $101.6 \mathrm{~mm}$ (4 inches) in diameter and $0.525 \mathrm{~mm}$ thick, with resistivities in the range of 10-20 ohm-cm, and an as received surface roughness of $<5 \AA$. The Ge substrates were P-type (Ga-doped) single crystal (100) $\pm 0.5^{\circ}$ wafers, polished on one side, $50.8 \mathrm{~mm}$ ( 2 inches) in diameter and $0.5 \mathrm{~mm}$ thick, with resistivities in 
Table 7.1: Overview of samples fabricated at the US Army Research Laboratory to test the hypothesis of the phonon bridging effect. The deposition rate was $1 \mathrm{~nm} / \mathrm{s}$ for all samples.

\begin{tabular}{|c|c|c|c|c|c|}
\hline Run \# & Dep. 1 & Thickness (nm) & Dep. 2 & Thickness (nm) & Substrates \\
\hline 1 & $\mathrm{Pt}$ & 100 & - & - & $\mathrm{Si}, \mathrm{Ge}, \mathrm{Ni}$ \\
\hline 2 & $\mathrm{Ni}$ & 100 & - & - & $\mathrm{Si}, \mathrm{Ge}$ \\
\hline 3 & $\mathrm{Ni}$ & 100 & $\mathrm{Pt}$ & 100 & $\mathrm{Si}, \mathrm{Ge}$ \\
\hline 4 & $\mathrm{Ni}$ & 50 & $\mathrm{Pt}$ & 100 & $\mathrm{Si}, \mathrm{Ge}$ \\
\hline 5 & $\mathrm{Ni}$ & 30 & $\mathrm{Pt}$ & 100 & $\mathrm{Si}, \mathrm{Ge}$ \\
\hline 6 & $\mathrm{Ni}$ & 20 & $\mathrm{Pt}$ & 100 & $\mathrm{Si}, \mathrm{Ge}$ \\
\hline 7 & $\mathrm{Ni}$ & 10 & $\mathrm{Pt}$ & 100 & $\mathrm{Si}, \mathrm{Ge}$ \\
\hline 8 & $\mathrm{Ni}$ & 5 & $\mathrm{Pt}$ & 100 & $\mathrm{Si}, \mathrm{Ge}$ \\
\hline
\end{tabular}

the range of 1-10 ohm-cm, and an as received surface roughness of $<8 \AA$. The Ni substrate was a 10x10x1 mm polycrystalline substrate with one side polished giving an as received surface roughness of $<30 \AA$, and an average grain size of 10-50 $\mu \mathrm{m}$. All substrates were purchased from the MTI corporation and all specifications listed are from MTI literature. The fabrication of the samples consisted of three sequential steps: 1) dicing of the large substrate wafers (2-4 inches in diameter) into smaller samples, 2) cleaning of the substrates to remove contaminants, and 3) deposition of the metallic films as per Table 7.1.

Thermoreflectance measurements do not in general require a large sample surface, with pump spot sizes only being on the order of a few hundred microns at most. Therefore, to prepare multiple samples the large 2-4 inch $\mathrm{Si}$ and Ge wafers were diced into smaller segments. Dicing after the thin film Pt and/or Ni was deposited would most likely damage and/or contaminate the film surface interfering with the optical measurement. To eliminate this issue, prior to film deposition the $\mathrm{Si}$ and Ge substrates were "scored" using a Disco DAD3240 dicing saw. The $500 \mu \mathrm{m}$ thick substrates were pre-cut $20 \mu \mathrm{m}$ deep in a $1 \mathrm{~cm} \mathrm{x}$ $1 \mathrm{~cm}$ grid pattern across the substrate surface using a $250 \mu \mathrm{m}$ blade and water jet coolant. The $200 \mu \mathrm{m}$ deep pre-cut into the wafer was found to provide adequate substrate durability for the ensuing cleaning and deposition steps while allowing easy cleaving post-deposition.

After dicing, the pre-cut samples were cleaned in a three step progression, which is essentially a RCA clean ${ }^{4}$ without the intermediate buffered oxide etch (BOE). The first step in the RCA process, designed to remove organic and particulate contaminants from

\footnotetext{
${ }^{4}$ The RCA clean was a basic wafer cleaning process developed by Werner Kern and David A. Puotinen in 1970 while working at the Radio Corporation of America (RCA) [290].
} 
the wafer surface, was performed by soaking the samples for 15 minutes in a solution of 5 parts deionized water, 1 part aqueous ammonium hydroxide, $\mathrm{NH}_{4} \mathrm{O}_{3}$, and 1 part aqueous hydrogen peroxide, $\mathrm{H}_{2} \mathrm{O}_{2}$. The second step, commonly referred to as piranha solution or piranha etch, is designed to remove any remaining organic or metallic (ionic) contaminants. This step was completed by soaking the samples for 15 minutes in a solution of 3 parts aqueous hydrochloric acid, $\mathrm{HCl}$, and 1 part aqueous hydrogen peroxide, $\mathrm{H}_{2} \mathrm{O}_{2}$. In the final step the samples were rinsed with running deionized water and dried with house nitrogen. The cleaning process was performed individually before each run of the deposition process listed in Table 7.1 to reduce the time between cleaning and loading into the deposition chamber and thereby reducing the risk of contamination.

Immediately after the cleaning and drying processes, the samples were loaded into an Evatec BAK 641 Electron Beam Physical Vapor Deposition (EBPVD) system. The baseline pressure of the system was maintained at $2 \times 10^{-7}$ mbar and the deposition rate was fixed at $1 \mathrm{~nm} / \mathrm{s}$ for all samples. For runs 3-8 in Table 7.1 the Ni and Pt films were deposited sequentially in situ. The thickness of the films was monitored and controlled by a quartz crystal monitor (QCM). After removal from the deposition chamber the samples were stored in an airtight Teflon ${ }^{\circledR}$ waver carrier until thermal testing.

\subsection{Experimental Procedure and Results}

The fabricated samples were tested using the low-rep TTR configuration as described in Chapter 3. For each sample a randomized pattern of 45 locations was generated, see Figure 7.10, and the pattern used to conduct a random raster of TTR scans on the sample surface. The properties of the laser system during the collection of the experimental data and the values for the input parameters used to model the systems are shown in Table 7.2. The primary value of interest that was deduced from the data is the thermal boundary conductance between the $\mathrm{Ni}$ intermediate layer and the $\mathrm{Si}$ (or Ge) substrate. The Nisubstrate $h_{\mathrm{BD}}$ was then used along with the other input parameters to calculate the total conductance from the Pt film to the semiconductor substrate. 


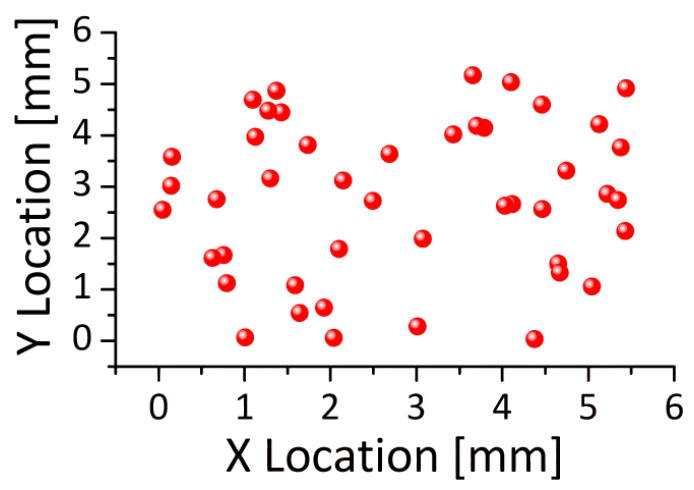

(a)

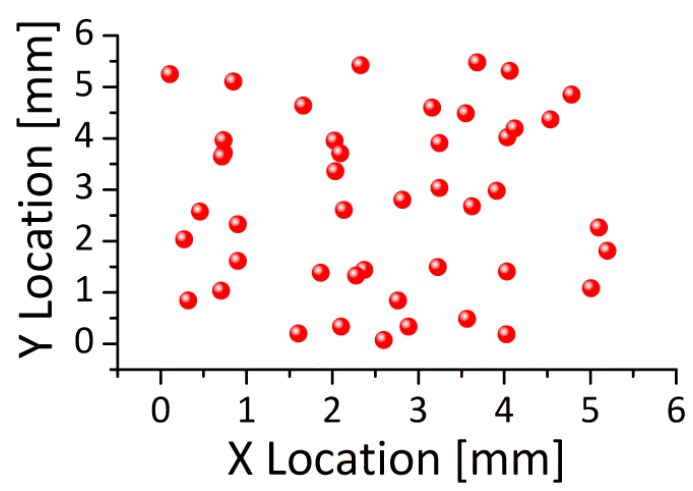

(b)

Figure 7.10: Two examples of randomized raster patterns of 45 locations used to scan the sample surfaces in the TTR/TDTR experiment.

Table 7.2: Thermophysical properties used in modeling of a Pt film on Si substrate.

\begin{tabular}{rcccc}
\hline Layer Properties & $\mathbf{P t}$ & $\mathbf{N i}$ & $\mathbf{S i}$ & $\mathbf{G e}$ \\
\hline Specific Heat $-C\left[\mathrm{~J} / \mathrm{m}^{3} \mathrm{~K}\right]$ & $2.88 \times 10^{6}$ & $3.91 \times 10^{6}$ & $1.66 \times 10^{6}$ & $1.71 \times 10^{6}$ \\
Thermal Conductivity $-k_{z}[\mathrm{~W} / \mathrm{mK}]$ & 50.12 & $42.95,43.90,45.82,47.74,51.58,61.17$ & 148 & 59.90 \\
Thickness $-d[\mathrm{~nm}]$ & 100 & $5,10,20,30,50,100$ & Infinite & Infinite \\
\hline Laser Properties & & & \\
\hline Absorbed Power $[\mathrm{mW}]$ & & $\approx 12$ & \\
Pump Waist $[\mu \mathrm{m}]$ & & $73.15 \pm 0.59$ & \\
Probe Waist $[\mu \mathrm{m}]$ & & $14.59 \pm 0.11$ & \\
Rep Rate $[\mathrm{MHz}]$ & & 0.247 & \\
Modulation Rate $[\mathrm{MHz}]$ & & 0.124 & \\
Duty Cycle [\%] & 50 & \\
Laser Wavelength $[\mathrm{nm}]$ & & 785 & \\
\hline
\end{tabular}

\subsubsection{Resistor Network Analysis}

The addition of the intermediate layer adds complexity to the deduction of parameters and analysis of the results as compared to the simple case of a single film on a bulk substrate. One of the most intuitive ways to consider the system with intermediate layers is as a network of thermal resistors. In this analysis, we consider the 3 primary resistances (conductances) to the flow of thermal energy from the Pt film to the substrate: 1) the boundary resistance at the $\mathrm{Pt} / \mathrm{Ni}$ interface, 2) the thermal resistance of the $\mathrm{Ni}$ layer, given in equivalent units of $h_{\mathrm{BD}}$ by the ratio of the film thermal conductivity and thickness, and

3) the boundary resistance between the Ni layer and the Si (or Ge) substrate, see Figure 7.11a.

While we will consider some of the conductances individually, the primary conductance of interest for comparison with the previous molecular dynamics work and the quantity 


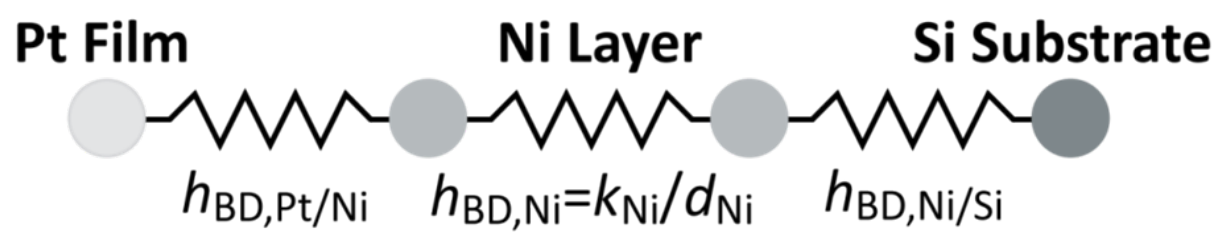

(a)

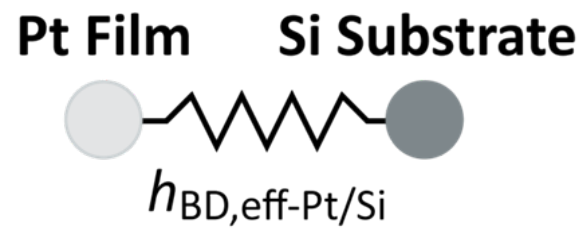

(b)

Figure 7.11: Theoretical thermal resistor network for a Pt film on a Si substrate with a Ni intermediate layer.

of most interest to industry will be the total conductance from the $\mathrm{Pt}$ film to the Si or Ge substrates. Following the thermal resistor analogy, we can treat the individual components as series resistors and define a total effective conductance, see Figure 7.11b, given mathematically by:

$$
h_{\mathrm{BD}, \mathrm{eff}}=\left[\frac{1}{h_{\mathrm{BD}, \mathrm{Pt} / \mathrm{Ni}}}+\frac{d_{\mathrm{Ni}}}{k_{\mathrm{Ni}}}+\frac{1}{h_{\mathrm{BD}, \mathrm{Ni} / \mathrm{Si}}}\right]^{-1}
$$

Assuming that the metal-metal $h_{\mathrm{BD}}$ will be high (i.e. the resistance low), the thermal transport from the Pt film to the substrate will be dominated by the resistance of the $\mathrm{Ni}$ layer and the Ni-Si interface. We know that for intermediate layer thicknesses on the order of $1 \mathrm{~s}-100 \mathrm{~s}$ of $\mathrm{nm}$ that size effects will alter the film thermal conductivity and will therefore

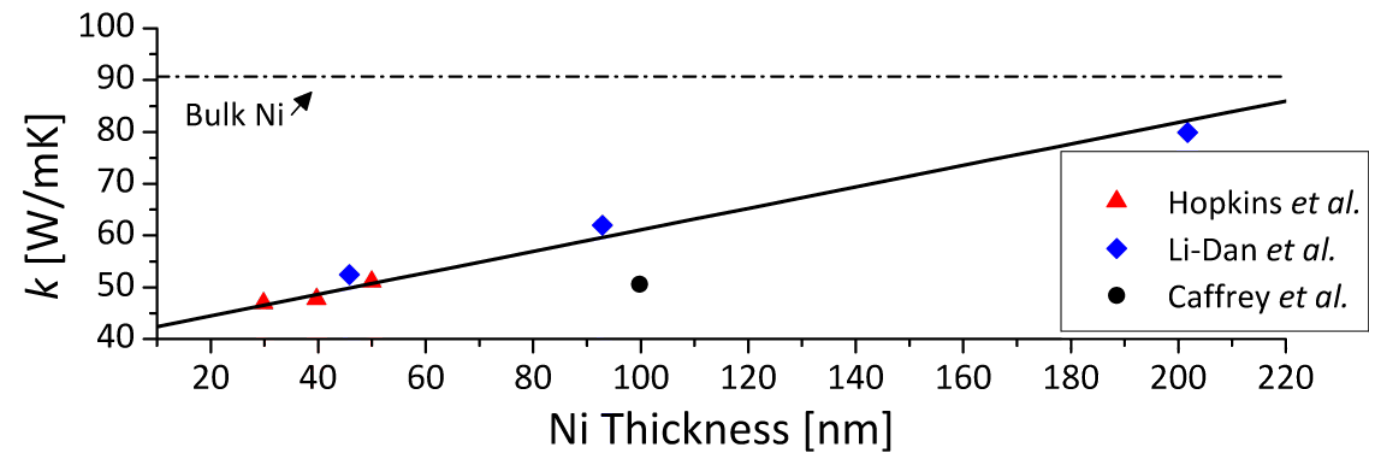

Figure 7.12: Thermal conductivity as a function of film thickness, for data from Hopkins et al. [21], Li-Dan et al. [22], and Caffrey et al. [23]. Image reproduced from Li-Dan et al. [22]. 
be a function of thickness [81, 108, 178, 251, 291-294]. The thermal conductivity of Ni as a function of film thickness has been studied in literature [21-23], see Figure 7.12. Using the data in Figure 7.12, we can estimate how the conductance of the Ni layer will vary as a function of the film thickness. The values used in the analysis of the TTR data are shown for each thickness in Table, 7.2. Assuming the thermal conductivity of Pt and Ni behave similarly at reduced thickness, based on the reduction in Ni thermal conductivity at 100 $\mathrm{nm}$ as compared to the bulk, the thermal conductivity of the $100 \mathrm{~nm} \mathrm{Pt}$ film is reduced by $30 \%$ to account for thin film effects.

\subsubsection{Sensitivity Analysis}

For each of the systems the sensitivity was calculated as a function of time and $h_{\mathrm{BD}}$ to ensure sufficient sensitivity to all parameters deduced. As an example, for the system of $100 \mathrm{~nm}$ of $\mathrm{Pt}$ on $\mathrm{Si}$ with a $5 \mathrm{~nm} \mathrm{Ni}$ intermediate layer, the sensitivity as a function of time and $h_{\mathrm{BD}}$ is shown in Figures $7.13 \mathrm{a}$ and $7.13 \mathrm{~b}$ respectively. The averaged sensitivity

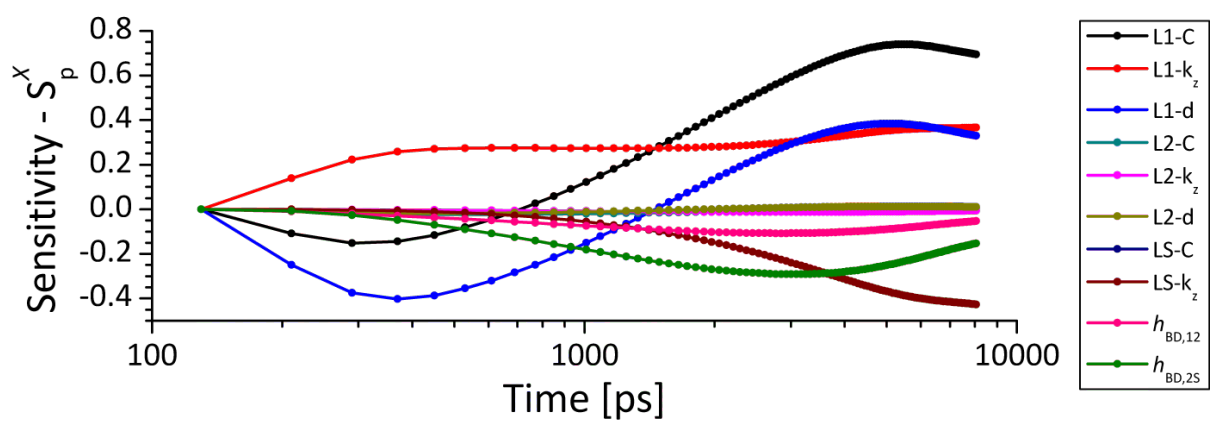

(a)

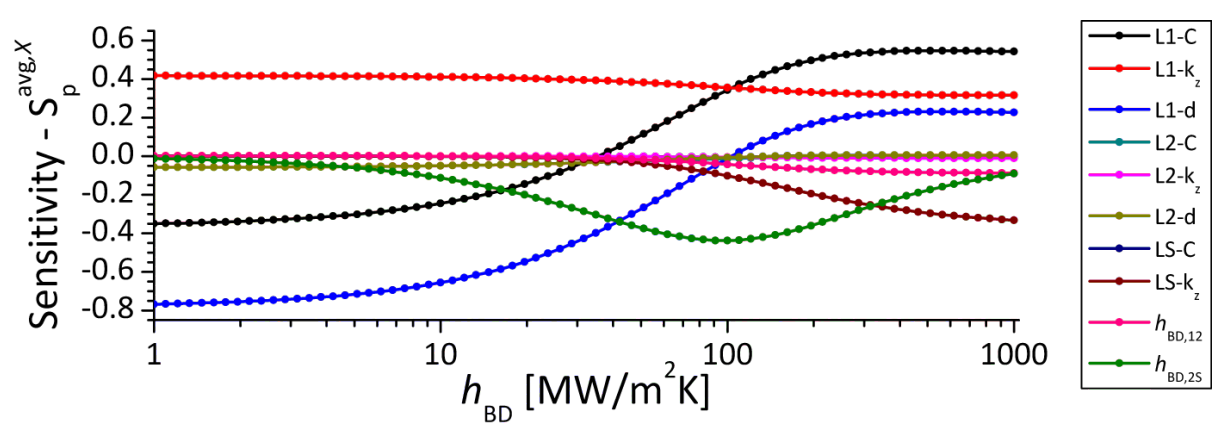

(b)

Figure 7.13: (a) The sensitivity coefficient as a function of time and parameter and (b) the sensitivity coefficient as a function of $h_{\mathrm{BD}}$ and parameter for $100 \mathrm{~nm}$ of Pt on Si with a $5 \mathrm{~nm}$ Ni intermediate layer. 
Table 7.3: Averaged sensitivity coefficient, $S_{p}^{\mathrm{avg}, X}$, for the parameters in the system of $100 \mathrm{~nm}$ of $\mathrm{Pt}$ on $\mathrm{Si}$ with a $5 \mathrm{~nm} \mathrm{Ni}$ intermediate layer.

\begin{tabular}{cccccc}
\hline Property & $S_{p}^{X}$ & Property & $S_{p}^{X}$ & Property & $S_{p}^{X}$ \\
\hline L1- $C$ & 0.5445 & L1- $d$ & 0.3191 & L1- $k_{z}$ & 0.2258 \\
L2- $C$ & -0.0040 & L2- $d$ & -0.0098 & L2- $k_{z}$ & 0.0058 \\
LS- $C$ & -0.2737 & LS- $k_{z}$ & -0.2737 & $h_{\mathrm{BD}, 12}$ & -0.0816 \\
$h_{\mathrm{BD}, 2 \mathrm{~S}}$ & -0.2209 & & & & \\
\hline
\end{tabular}

coefficients, $S_{p}^{\mathrm{avg}, X}$, for the various parameters are given in Table 7.3. A full list of the sensitivity parameters for the measured systems is given in Appendix E.

\subsubsection{Raw Data}

The results of the deduced data are shown in Figures 7.14-7.17. In Figures 7.14 and 7.16 the total effective thermal conductance from the Pt film to the substrate is shown as calculated using the resistor network analysis shown in Equation 7.2. In Figures 7.15 and 7.17 the individual $\mathrm{Ni} / \mathrm{Si}$ and $\mathrm{Ni} / \mathrm{Ge} h_{\mathrm{BD}}$ values as a function of $\mathrm{Ni}$ thickness layers are provided. The error bars shown in the plots of the data points represents a $95 \%$ confidence on the calculated mean $h_{\mathrm{BD}}$ value for the measurements distributed across the 45 random points on the sample.

\section{Total Pt-to-Si Conductance}

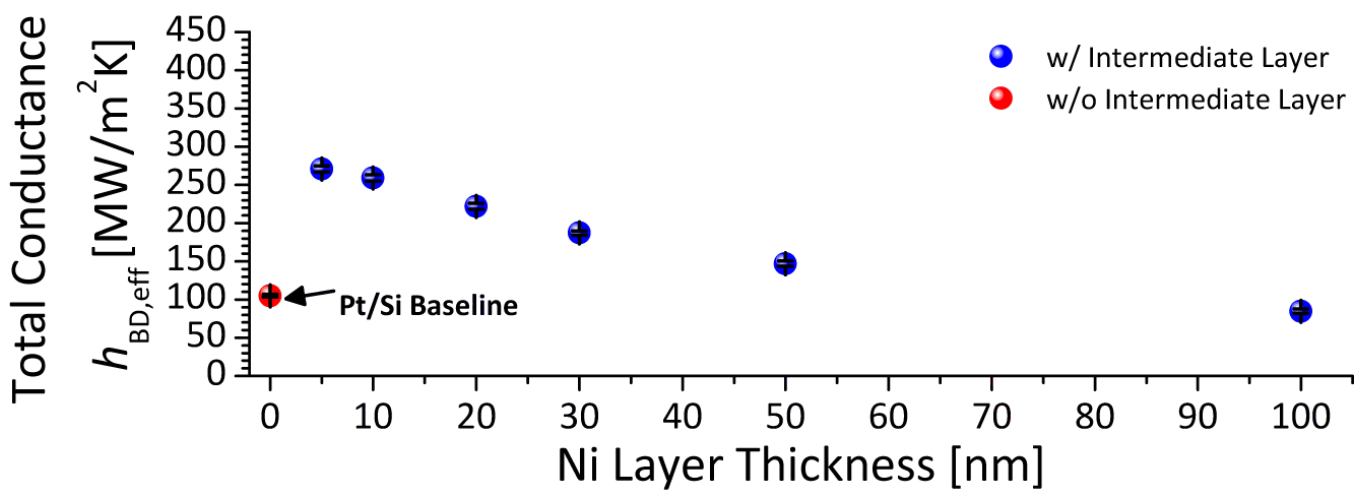

Figure 7.14: Total conductance from Pt to Si based on the thermal resistor network model, see Equation 7.2 , as a function of the $\mathrm{Ni}$ intermediate layer thickness. The data points represent the average $h_{\mathrm{BD}}$ value and the error bars represent a $95 \%$ confidence in the precision of the deduced value based on repeated samplings. 


\section{Ni-to-Si Interface Conductance}

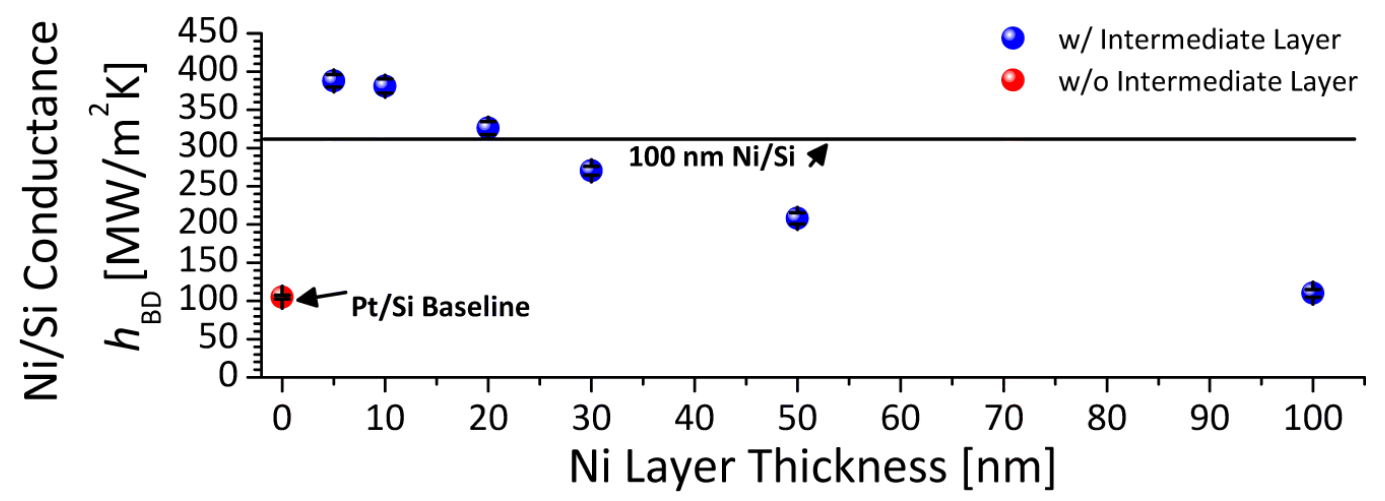

Figure 7.15: Thermal boundary conductance from the Ni intermediate layer to the Si substrate as a function of the Ni intermediate layer thickness. The horizontal line represents an independently measured system of $100 \mathrm{~nm}$ of $\mathrm{Ni}$ on $\mathrm{Si}$. The data points represent the average $h_{\mathrm{BD}}$ value and the error bars represent a $95 \%$ confidence in the precision of the deduced value based on repeated samplings.

\section{Total Pt-to-Ge Conductance}

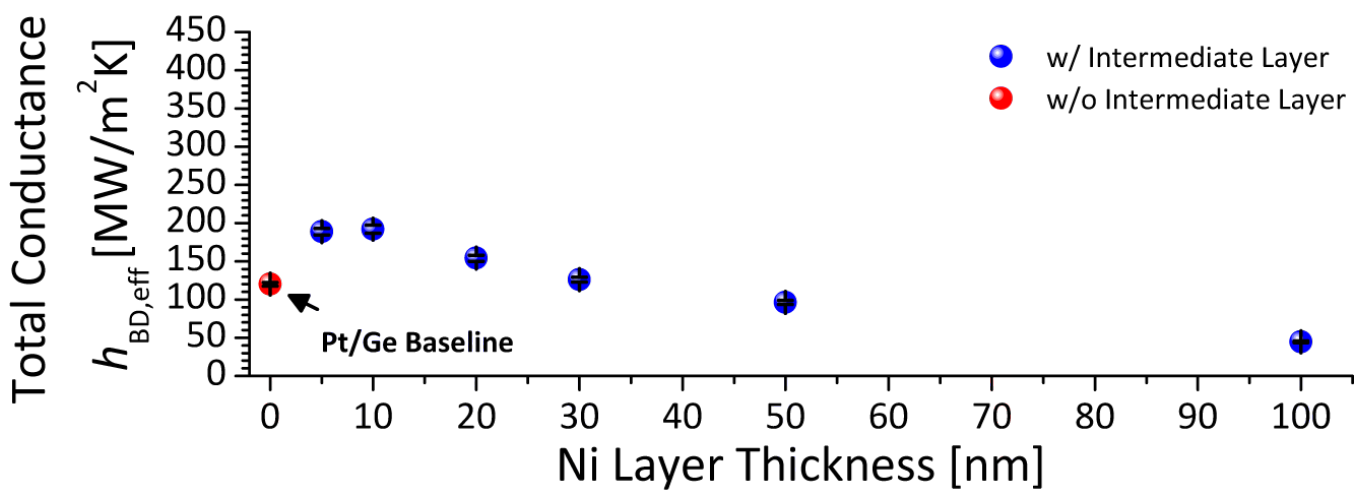

Figure 7.16: Total conductance from Pt to Ge based on the thermal resistor network model, see Equation 7.2 , as a function of the $\mathrm{Ni}$ intermediate layer thickness. The data points represent the average $h_{\mathrm{BD}}$ value and the error bars represent a $95 \%$ confidence in the precision of the deduced value based on repeated samplings. 


\section{Ni-to-Ge Interface Conductance}

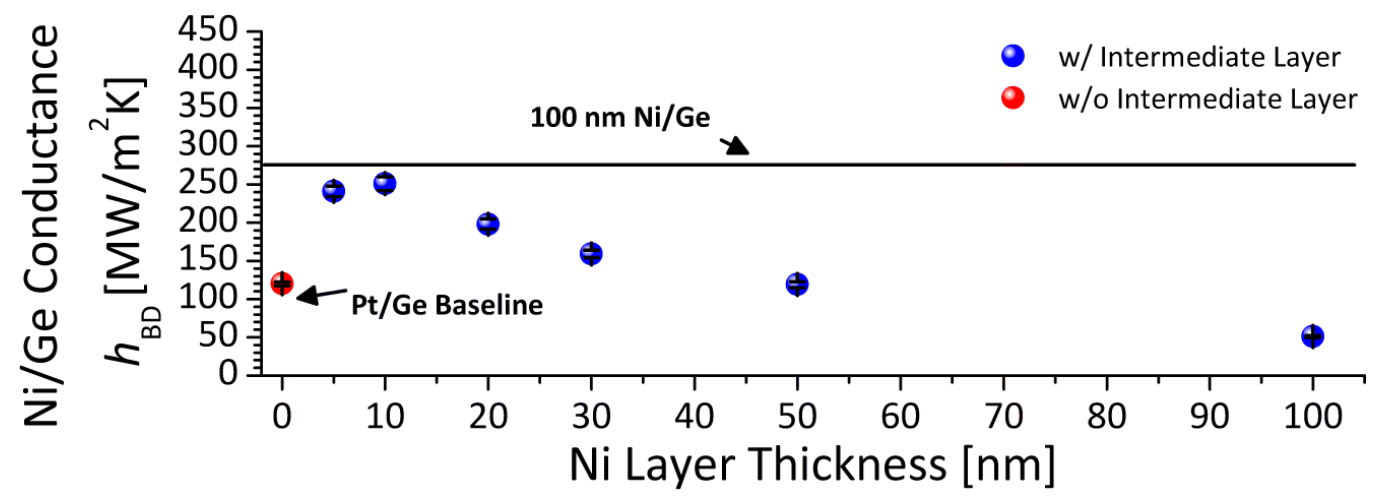

Figure 7.17: Thermal boundary conductance from the Ni intermediate layer to the Ge substrate as a function of the Ni intermediate layer thickness. The horizontal line represents an independently measured system of $100 \mathrm{~nm}$ of $\mathrm{Ni}$ on Ge. The data points represent the average $h_{\mathrm{BD}}$ value and the error bars represent a $95 \%$ confidence in the precision of the deduced value based on repeated samplings.

We will note that in Figures 7.14 and 7.15 that the baseline value for $\mathrm{Pt}$ on $\mathrm{Si}$ is below that previously reported in literature by Stevens et al. $\left(h_{\mathrm{BD}}=145 \mathrm{MW} / \mathrm{m}^{2} \mathrm{~K}\right)$ [10]. We are, however, confident in our results as they represent the average from 45 scans randomly selected across the sample surface and include confidence bounds in the overall accuracy based on a quasi-stochastic treatment of the uncertainty in the input parameters, see Chapters 5 and 6 . The difference in the results may be due to differences in the substrate preparations which would alter the presence of an oxide at the Si surface, and/or variations in the input parameters. We note that Stevens et al. used bulk properties for the Pt film which at $29 \mathrm{~nm}$ may be affected by nanoscale effects.

For each scan the thermal model was compared to the collected data in the temporal pump-to-probe delay range of $200 \mathrm{ps}$ to $7850 \mathrm{ps.}$ With the addition of the Ni layer, there is an increased possibility of electron-phonon coupling and therefore it may take a longer period of time after the initial heating for the thermal processes to become predominantly diffusive. To ensure that the inspected time period is such that the primary thermal processes are diffusive, the deduced results were compared at modeling start times out to $1000 \mathrm{ps.} \mathrm{This}$ analysis showed no major deviation in the trends observed in the results. A minor increase in the measured conductance was found for delayed start times but not enough to affect the interpretation of the results. A more in-depth analysis needs to be completed to find 
the ideal time range.

\subsubsection{Accuracy Analysis}

To determine the overall accuracy in the deduced results, the quasi-stochastic accuracy analysis that was developed in Chapter 6 was implemented for the experimental systems in this chapter. The uncertainties used for each of the input parameters are shown in Table 7.4. The values listed in the table are considered to be a $2 \sigma$ value for the uncertainty in the input parameters. That is the say that there is an approximately $95 \%$ probability that the true value of the input parameter lies within the given percentage. The distribution of the deduced $h_{\mathrm{BD}}$ values from the quasi-stochastic accuracy analysis is shown for the $100 \mathrm{~nm}$ Pt on Si system with $5 \mathrm{~nm}$ and $10 \mathrm{~nm} \mathrm{Ni}$ intermediate layers in Figures 7.18a and 7.18b, respectively.

Table 7.4: Uncertainty in the TTR/TDTR input parameters for the example of a system of $100 \mathrm{~nm}$ of Pt on $\mathrm{Si}\left(h_{\mathrm{BD}}=140 \mathrm{MW} / \mathrm{m}^{2} \mathrm{~K}\right)$ where $\mathbf{L} \mathbf{1}$ and $\mathbf{L S}$ denote the Pt film and Si substrate layer respectively.

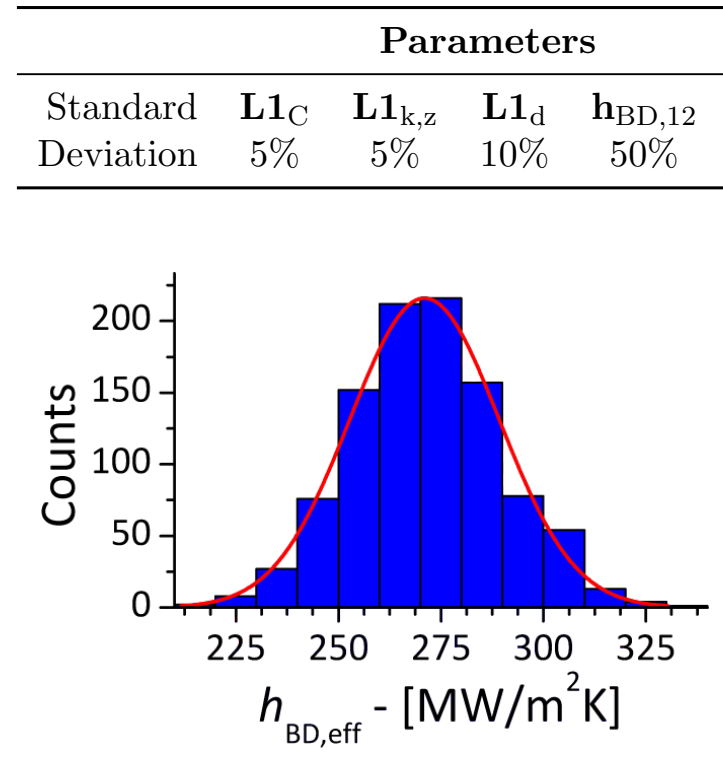

(a)

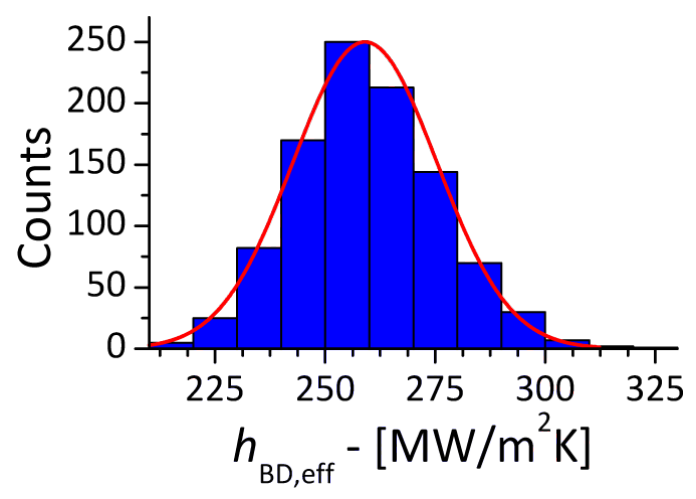

(b)

Figure 7.18: Distribution of deduced $h_{\mathrm{BD}}$ values for the system $100 \mathrm{~nm}$ of $\mathrm{Pt}$ on Si with a intermediate $\mathrm{Ni}$ of (a) $5 \mathrm{~nm}$ and (b) $10 \mathrm{~nm}$ with the assumed uncertainty in the input parameters provided by Table 6.8. 


\subsubsection{Final Results}

Using the results of the accuracy analysis the data for the total conductance between the Pt film and the semiconductor substrates, see Figures 7.14 and 7.16, are replotted to include the uncertainty in the accuracy of the results, see Figures 7.19 and 7.20. In these plots, the black error bars represent $95 \%$ confidence bounds in the precision of the average total effective conductance found for multiples measurements and analyzed using the nominal thermophysical input parameters to the TTR model.

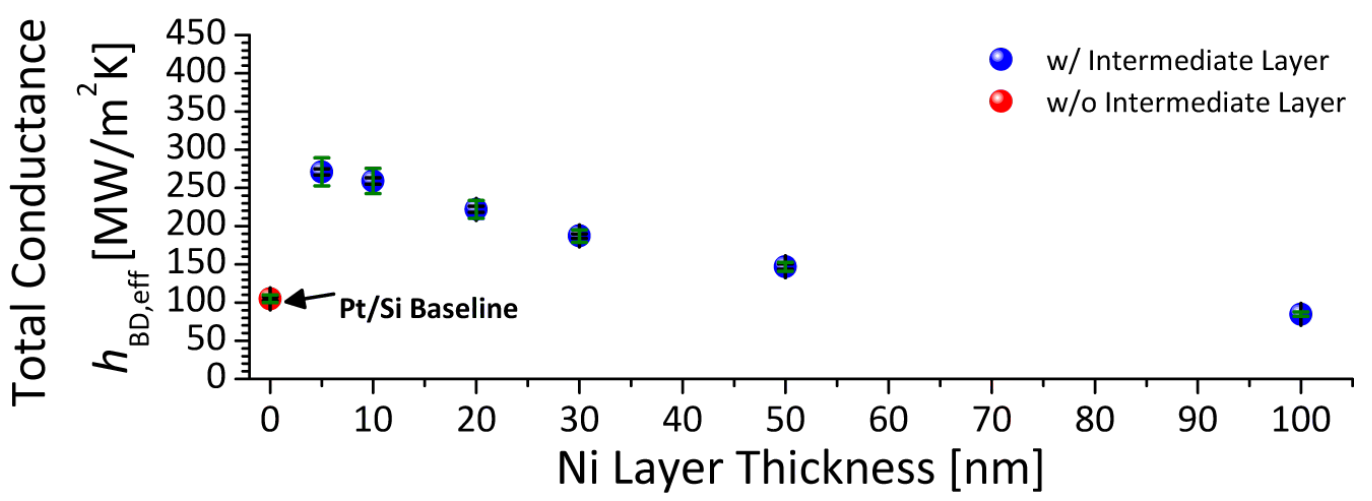

Figure 7.19: Total conductance from $\mathrm{Pt}$ to $\mathrm{Si}$ based on the thermal resistor network model, see Equation 7.2 , as a function of the $\mathrm{Ni}$ intermediate layer thickness. The data points represent the average $h_{\mathrm{BD}}$ value and the black error bars represent a $95 \%$ confidence in the precision of the deduced value based on repeated samplings. The green error bars represent the uncertainty in the accuracy of the deduced results based on uncertainties in the input parameters, see Table 7.4.

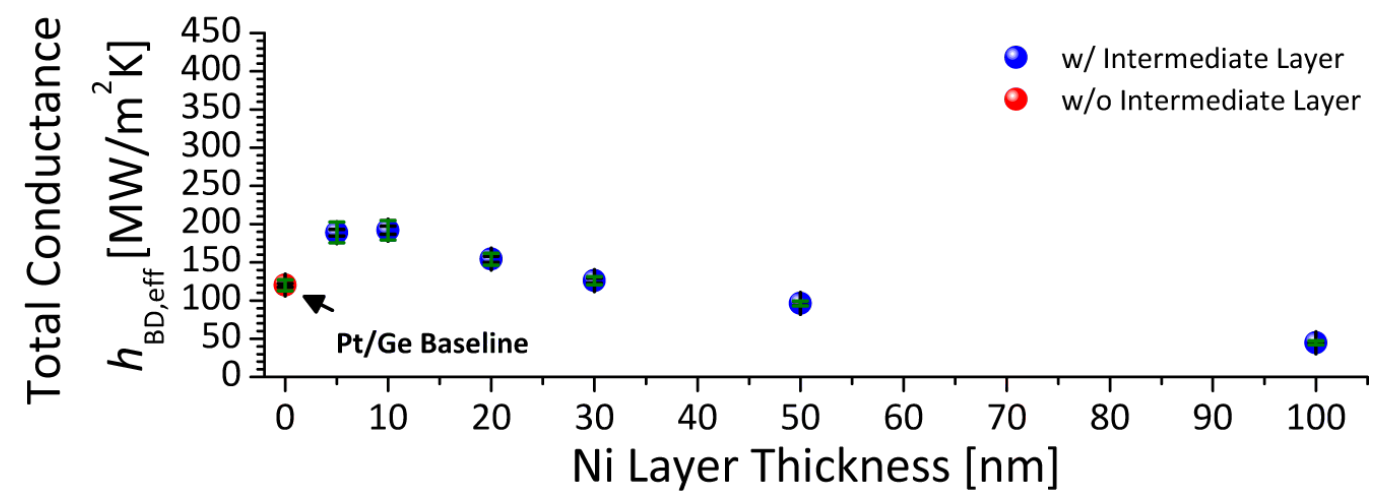

Figure 7.20: Total conductance from Pt to Ge based on the thermal resistor network model, see Equation 7.2 , as a function of the Ni intermediate layer thickness. The data point represents the average $h_{\mathrm{BD}}$ value and the black error bar represents a $95 \%$ confidence in the precision of the deduced value based on repeated samplings. The green error bar represents the uncertainty in the accuracy of the deduced results based on uncertainties in the input parameters, see Table 7.4.

The results for the $\mathrm{Pt} / \mathrm{Si}$ system shown in Figure 7.19 indicate that the addition of 
the 5-10 $\mathrm{nm}$ Ni intermediate layer ${ }^{5}$ increases the total Pt-to-Si boundary conductance by approximately a factor of 2.6 over the baseline case of $\mathrm{Pt}$ on $\mathrm{Si}$ with no Ni intermediate layer. As the thickness of the Ni intermediate layer increases the Ni-Si $h_{\mathrm{BD}}$ decreases, see Figure 7.15, as well as the conductance of the Ni layer due to the added thickness. This in turn decreases the overall conductance of the system as the thickness of the Ni layer increases. However, the total conductance remains above the baseline $\mathrm{Pt} / \mathrm{Si}$ case except for a Ni thickness of $100 \mathrm{~nm}$. For the $\mathrm{Pt} / \mathrm{Ge}$ case however, while there is an initial increase in the total Pt-to-Ge conductance with the addition of 5-10 nm of Ni of about a factor of 1.8, the total conductance drops below the baseline values for $\mathrm{Ni}$ thicknesses greater than $30 \mathrm{~nm}$.

\subsection{Results and Interpretation}

The results of the previous molecular dynamics studies $[14,151,151]$ and the trends in the experimentally measured conductance for the $\mathrm{Pt} / \mathrm{Ni} / \mathrm{Si}(\mathrm{Ge})$ system all point towards the possibility of tuning $h_{\mathrm{BD}}$ through the use of an intermediate layer between solid materials. To support the quantitative argument for the ability to tune $h_{\mathrm{BD}}$ and in order to better understand the experimental results, we will consider several mechanisms which may influence thermal transport in the measured system beyond the "bridging effect".

\subsubsection{Bonding Analysis}

While comparing the experimental work to the molecular dynamics simulations the ideal situation would be to assume that changing the elemental species comprising the solid layers would only alter the vibrational spectra. Of course in reality the pairing of elemental species can lead to any number of complex interactions. One of the most prominent changes in the system configuration besides the change in vibrational spectra, and the one that is most often brought up in an attempt to cast doubt in the vibrational bridging interpretation is the change in bonding.

\footnotetext{
${ }^{5}$ As discussed in Chapter 6, the uncertainty in the deduced results for the 5 and $10 \mathrm{~nm}$ sample prevents us from considering these averages statistically different. Therefore, we can make no distinction in the difference between these values.
} 
Both Pt and Ni have complex interactions with Si and will form several possible silicides under certain stoichiometric and thermodynamic conditions [285-289]. Due to these complex interactions it is difficult to quantitatively determine the bonding strength of the Pt-Si and Ni-Si interface, without detailed characterization. However, if for the sake of argument we assume that $\mathrm{Ni}$ creates a stronger bond to $\mathrm{Si}$, as compared to that of $\mathrm{Pt}$, we would expect an increase in the $h_{\mathrm{BD}}$ at the metal-Si interface $[16,155,157-160]$.

To consider the impact of bonding at the metal-semiconductor interface we will consider a thought experiment. Assuming that the increase in $h_{\mathrm{BD}}$ observed with the addition of the Ni layer is due to increased bonding only, we will consider the measured Ni-Si $h_{\mathrm{BD}}$ for the $5 \mathrm{~nm} \mathrm{Ni}$ to be the bonding contribution. This is a reasonable assumption as it is common practice in thin film microfabrication to use a 5-10 nm layer of an oxide forming metal to increase the adhesion of an otherwise poorly bonded film and substrate [295]. If bonding is the only factor contributing to the increased conductance, we would expect that after the addition of the $5 \mathrm{~nm}$ Ni layer, the subsequent conductance for thicker intermediate layers would only be driven by the added conductance of the Ni layer. The contribution to conductance as a function of Ni thickness is shown in Figure 7.21.

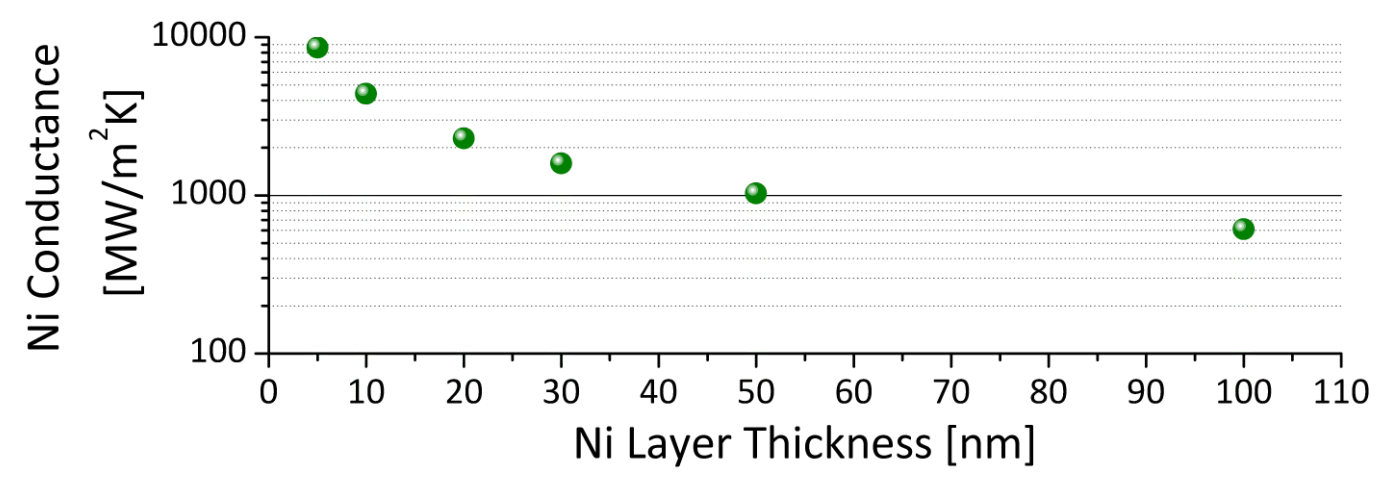

Figure 7.21: Effective conductance of a $\mathrm{Ni}$ film, $h_{\mathrm{BD}, \mathrm{eff}}=k_{\mathrm{Ni}} / d_{\mathrm{Ni}}$, as a function of film thickness, with the thermal conductivity of Ni taken from Li-Dan et al. [22], see Figure 7.12.

If we use this concept and the data shown in Figure 7.21 we can interpret the theoretical trend in the data under this assumption. Figure 7.22 shows the original total conductance measured from the experimental data along with the theoretical conductance assuming the $5 \mathrm{~nm}$ of $\mathrm{Ni}$ establishes the bonded interface and subsequently for the remaining $\mathrm{Ni}$ thicknesses the $h_{\mathrm{BD}}$ is unaffected and the total conductance is driven by the changes to $\mathrm{Ni}$ 
conductance with thickness only. Comparing the trends in the measured and theoretical data, the results show that bonding alone should not be able to account for the observed trends in the experimental results. As discussed in Chapter 2, there are many factors that can influence $h_{\mathrm{BD}}$ simultaneously and bonding is no doubt one factor in the measured samples, however the analysis of the results support the hypothesis that in part the trends in the experimental results are due to modified phonon properties.

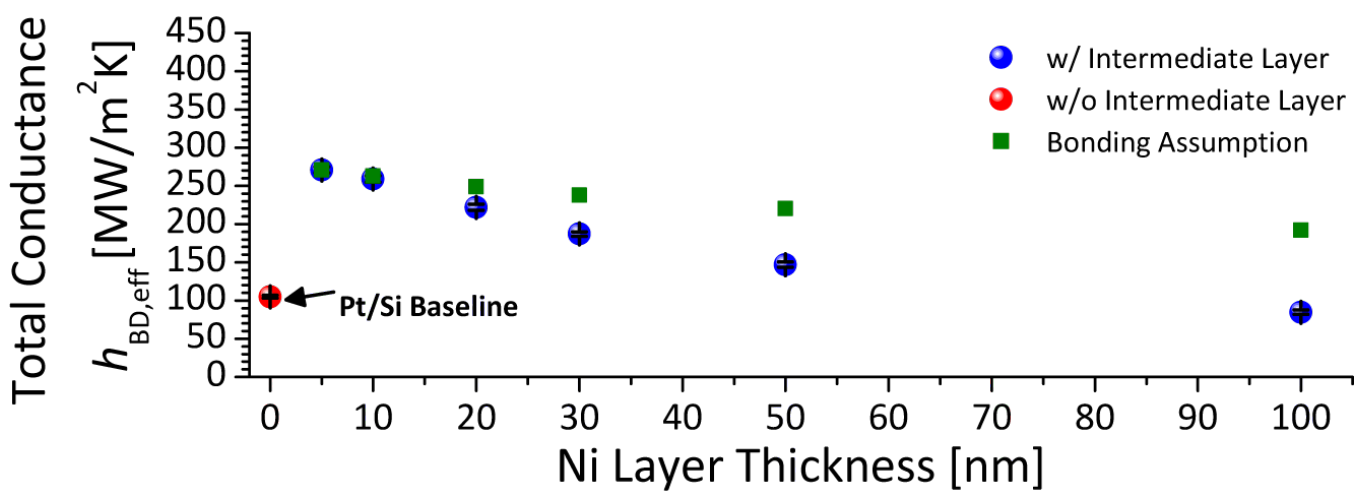

Figure 7.22: Total conductance as a function of Ni intermediate layer thickness assuming a Ni-Si boundary conductance of $h_{\mathrm{BD}}=38.8 \mathrm{MW} / \mathrm{m}^{2} \mathrm{~K}$ (the measured value for the $\mathrm{Pt} / 5 \mathrm{nmNi} / \mathrm{Si}$ sample) and the total conductance of the thicker Ni layers driven by the Ni conductance only.

\subsubsection{Electron Boundary Conductance}

One of the major differences between the MD simulations conducted and the experimental data taken is the use of metallic films and intermediate layers in the experiments as compared to the simulations. As discussed in Section 7.4.1, the choice of metallic intermediate layers was motivated by the high thermal conductivity of thin film metals as compared to thin film semiconducting layers, and as also a metallic film is necessary for TTR/TDTR measurements. This difference in material systems poses the question as to whether the results from experimental measurements can be correlated to the simulation results and if the conclusions regarding the mechanisms behind the results are the same.

Classical molecular dynamics simulations, as implemented in English et al. [14] and Liang et al. [20,151], do not include the interactions or contributions of free electrons in the material systems. In these experiments however, since transition metals were used as both the film and intermediate layer, the question is raised as to what contribution electrons 
have to the thermal transport and therefore if the effects observed are phonon or electron dominated. It is well established that electrons are the primary thermal carriers in metals and therefore boundary conductance across metal-metal interfaces is electron dominated and large compared to metal-nonmetal and nonmetal-nonmetal interfaces [75, 92, 296]. At metal-nonmetal interfaces the role of electrons in boundary conductance is not as well intuitively established. Therefore to ensure that the resulting enhancement in $h_{\mathrm{BD}}$ observed in the experimental data conforms to the original hypothesis of modified interfacial phononic properties, a more in depth assessment of electron contributions to $h_{\mathrm{BD}}$ at metal-nonmetal interfaces was undertaken.

Thermal energy carrying electrons that travel in the metal film are assumed to encounter a potential barrier at the interface formed at the metal-semiconductor junction. The height of the barrier depends on the band structure of the two materials comprising the interface and the width or thickness of the barrier depends on the metal work function, the work function of the semiconductor, along with the semiconductor doping density and permittivity. The electron-electron boundary conductance across the metal/semiconductor interface is modeled based on the work of Bartkowiak et al. and daSilva et al. [184, 297].

\section{Ideal Metal-Semiconductor Junctions}

The work function by definition is the energy difference between the Fermi level and the vacuum level in a material, and is denoted $q \phi_{m}$ and $q \phi_{s c, n}$ for a metal and semiconductor respectively [24]. Considering the case of an ideal metal-semiconductor system with no surface states, oxides, or other abnormalities, the band structure of an isolated high work-function metal, i.e. $q \phi_{m}>q \phi_{s c, n}$, and an isolated n-type semiconductor is shown in Figure 7.23a. The difference between the conduction band of the semiconductor and the vacuum level is given by $q \chi$ where $\chi$ is the electron affinity in volts. When the metal and semiconductor are brought into contact the Fermi levels of the two systems will align with the Fermi level of the semiconductor being lowered relative to the metal, in the high work-function case, where the magnitude of the lowering is equal to the difference between the work functions of the two systems, see Figure 7.23b. On contact, electrons will flow from the conduction band of the semiconductor into the metal and accumulate near the 

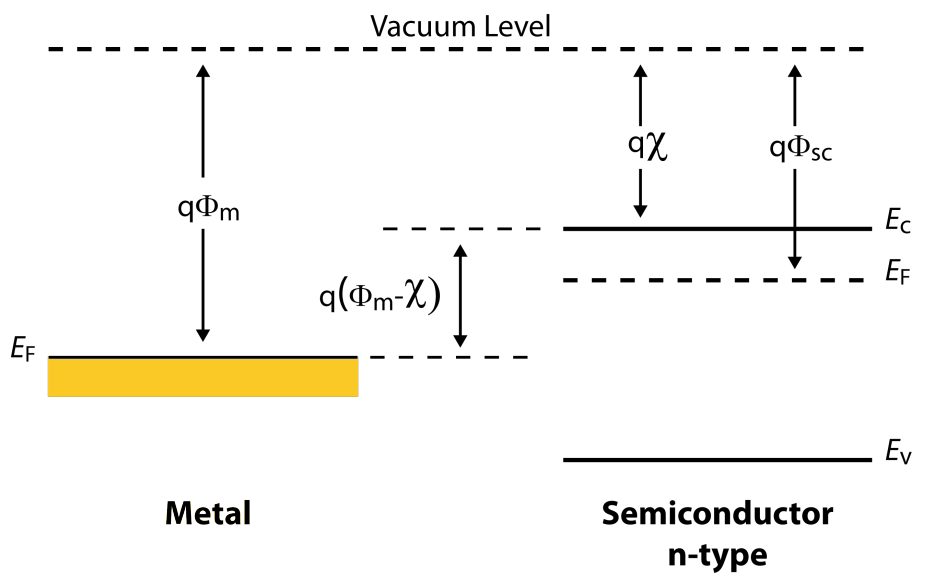

(a) Isolated systems

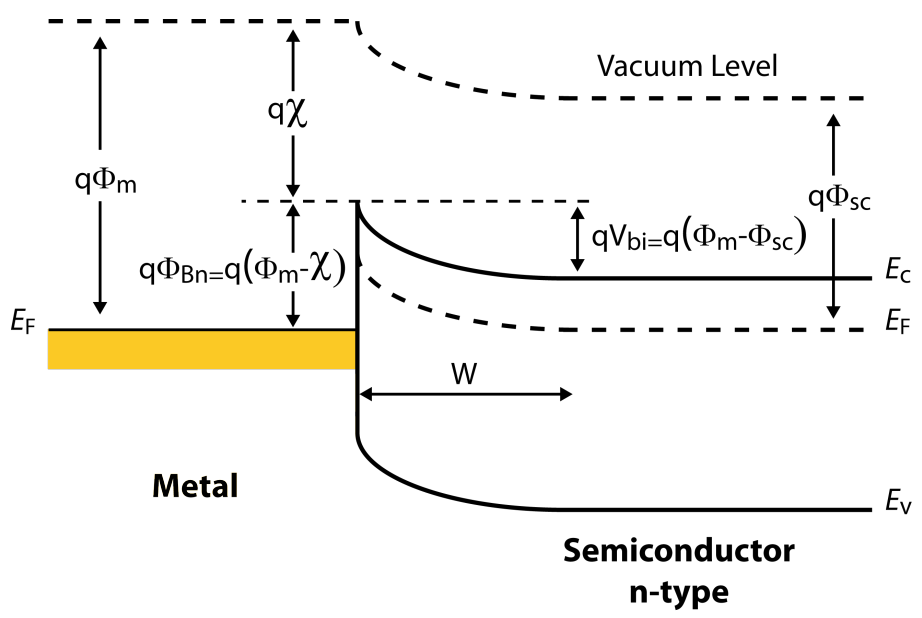

(b) Metal-semiconductor (n-type) contact

Figure 7.23: Energy band diagrams of (a) an isolated metal and isolated n-type semiconductor adjacent to each other and (b) a metal-semiconductor (n-type) contact at thermal equilibrium.

surface. This migration of electrons leaves behind an electron-depleted region of width $W$ in the semiconductor interface region. The potential barrier to electrons moving from the metal across the interface into the n-type semiconductor is given by [24]:

$$
q \phi_{B, n}=q\left(\phi_{m}-\chi\right)=E_{o}
$$

where $q$ is the electron charge, $\phi_{B, n}$ is the barrier height in $\mathrm{eV}$ for a n-type semiconductor, $\phi$, is the metal work function in $\mathrm{eV}, \chi$ is the electron affinity in volts, and $E_{o}$ is the potential barrier height in $\mathrm{eV}$. A selection of electron affinities for various semiconductors is given in Table 7.5 and the work functions for various clean metals in vacuum shown in Figure 7.24. 
Table 7.5: Electron affinity, $q \chi$, of various semiconductors.

\begin{tabular}{cccc}
\hline Semiconductor & $\begin{array}{c}\text { Electron } \\
\text { Affinity [eV] }\end{array}$ & Semiconductor & $\begin{array}{c}\text { Electron } \\
\text { Affinity [eV] }\end{array}$ \\
\hline $\mathrm{Si}$ & 4.05 & $\mathrm{InP}$ & 4.38 \\
$\mathrm{Ge}$ & 4.00 & $\mathrm{AlN}$ & 0.60 \\
$\mathrm{GaP}$ & 3.80 & $\mathrm{BN}$ & 4.50 \\
$\mathrm{GaAs}$ & 4.07 & $\mathrm{GaN}$ & 4.10 \\
$\mathrm{InAs}$ & 4.90 & $\mathrm{GaSb}$ & 4.06 \\
$\mathrm{InSb}$ & 4.59 & & \\
\hline
\end{tabular}

Being able to establish the height of the potential barrier for a metal/semiconductor system is the first major step in calculating the electron boundary conductance.

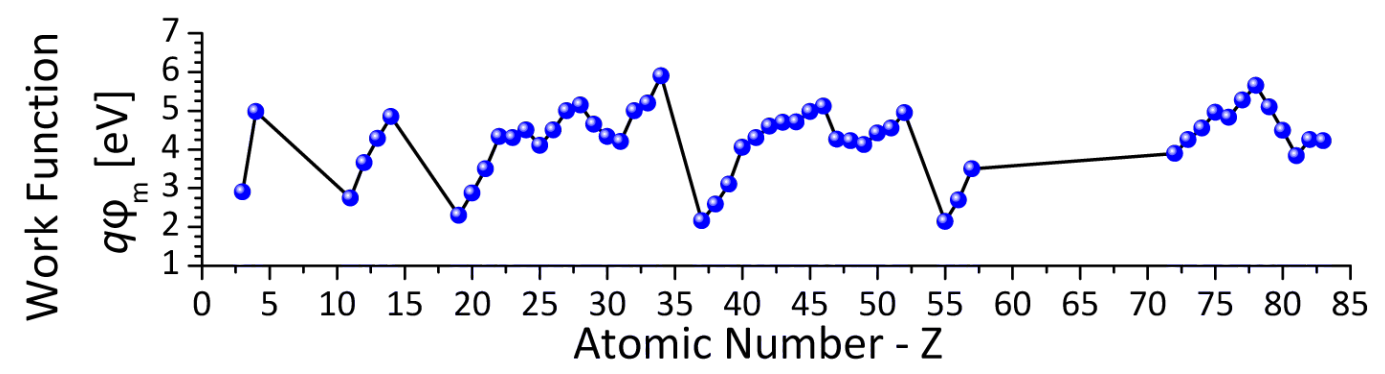

Figure 7.24: Work function for various clean metals in vacuum [24, 25].

\section{Electron Boundary Resistance}

Traditionally it is assumed that for thermal transport in metal/non-metal systems the primary thermal energy carriers are phonons, and thereby the dominant resistance to thermal boundary transport is phonon mediated boundary resistance near the metal/non-metal junction. The contribution of thermal electrons to transport and therefore the boundary affects impeding electron flow are considered to be negligible. To that end as discussed in Chapter 2, a large amount of both experimental and theoretical work has gone into understanding phonon mediated transport across solid-solid boundaries, with the primary theoretical model being the DMM [72]. It has been suggested that there is a similar resistance to the flow of thermal electrons across a boundary [184, 297-302]. To quantify this phenomena Bartkowiak et al., developed an expression for the electron boundary resistance based in analogy to the Wiedemann-Franz law [297]. 
Following the work of Silva and Kaviany, the electron boundary resistance, $R_{\mathrm{BD}, e e}$, is given by [184]:

$$
\frac{1}{R_{\mathrm{BD}, e e}}=\frac{\pi^{2}}{3} \frac{T}{R_{\mathrm{BD}, e l}}\left(\frac{k_{B}}{q}\right)^{2}
$$

where $T$ is the temperature, $R_{\mathrm{BD}, e l}$ is the electrical boundary resistance, $k_{B}$ is the Boltzmann constant, and $q$ is the electronic charge. In order to determine $R_{\mathrm{BD}, e l}$, the mechanism of electron barrier transport must be determined. In theory, there are two primary mechanisms of electron transport across the metal-semiconductor interface, either tunneling through the barrier or thermionic emission over the barrier. In practice however the two mechanisms are not easily decoupled. Depending on the shape of the barrier, i.e. if near the apex the barrier is thin, the rates of tunneling and thermionic emission may be coupled, leading to a more complex problem. In the work of Bartkowiak et al. the two primary barrier transport mechanisms are discussed: quantum mechanical tunneling, and thermionic emission. In order to determine the dominant transport mechanism, tunneling or thermionic emission, the barrier height, $E_{o}$, and width, $d_{B}$, must be estimated.

Estimation of the barrier height, $E_{o}$, was discussed for the ideal metal/semiconductor junction previously. The barrier width, $d_{B}$, is estimated from the width of the depletion region, $W$, where $d_{B}=(W / 2)$. For the case of zero applied voltage, the width of the depletion region is given by [184]:

$$
W=\left(\frac{2 \epsilon_{r} \epsilon_{o} E_{o, J}}{q^{2} N}\right)^{1 / 2}
$$

where $\epsilon_{r}$ is the relative permittivity of the semi-conductor, $\epsilon_{o}$ is the free space permittivity, $E_{o, J}$ is the barrier height in Joules, and $N$ is the donor/acceptor concentration of the semiconductor.

Assuming tunneling transport through the potential barrier, the electrical boundary resistance is given by [184]

$$
\frac{1}{R_{\mathrm{BD}, e l, t n}}=\frac{4 \pi q^{2} m_{e, s c} P}{h_{P}^{3}}\left[\frac{h_{P}^{2} E_{o, J}}{8 \pi^{2} m_{e, s c} d_{B}^{2}}\right]^{1 / 2}
$$

where $m_{e, s c}$ is the effective mass of the electrons/holes in the semiconductor, $h_{P}$ is Planck's 
constant, $E_{o, J}$ is the potential barrier height, $d_{B}$ is the potential barrier thickness and $P$ is the tunneling probability. The tunneling probability is given by [184]:

$$
P=\left\{1+\frac{E_{o, J}^{2} \sinh ^{2}\left[8 \pi^{2} m_{e, s c}\left(E_{o, J}-E\right) d_{B}^{2} / h_{P}^{2}\right]^{1 / 2}}{4 E\left(E_{o, J}-E\right)}\right\}^{-1}
$$

where $E$ is the electron energy measured from the bottom of the potential barrier, such that $E<E_{o, J}$.

Assuming the primary mechanism of electron transport is thermionic emission, the electrical boundary resistance is given by [184]:

$$
\frac{1}{R_{\mathrm{BD}, e l, t e}}=\frac{q B T \exp ^{-E_{o, J} / k_{B} T}}{k_{B}}
$$

where $B$ is equal to $B=120 \times 10^{4} \mathrm{~A} / \mathrm{m}^{2} \mathrm{~K}^{2}$, and $E_{o, J}$ is assumed to be much larger than $k_{B} T$. For the two transport mechanisms, tunneling is assumed the primary mechanism if:

$$
\left[\frac{h_{P}^{2} E_{o, J}}{\left(8 \pi^{2} m_{e, s c} d_{B}^{2}\right)}\right]^{-1 / 2} k_{B} T \ll 1
$$

otherwise thermionic emission is assumed [297].

The total thermal boundary resistance including phonons and electrons is given by:

$$
\frac{1}{R_{\mathrm{BD}}}=\frac{1}{R_{\mathrm{BD}, p p}}+\frac{1}{R_{\mathrm{BD}, e e}}+\frac{1}{R_{\mathrm{BD}, e p}}+\frac{1}{R_{\mathrm{BD}, p e}}
$$

where $R_{\mathrm{BD}, p p}$ is the phonon-dominated boundary resistance, $R_{\mathrm{BD}, e e}$ is the electron-dominated boundary resistance, and $R_{\mathrm{BD}, e p}$ and $R_{\mathrm{BD}, p e}$ is the resistance due to the interaction of phonon and electrons across the interface. For the sake of simplicity at this point it is assumed that there is no direct heat transport between electron and phonons on either side of the interface in either direction, i.e. $R_{\mathrm{BD}, e p}$ and $R_{\mathrm{BD}, p e} \rightarrow \infty$. Therefore, we will assume that the boundary resistance will be a function of the phonon-phonon and electron-electron interaction only. 


\section{Calculated Electron Thermal Conductance}

In the experimental data collected, the four primary metal-semiconductor interfaces of interest are $\mathrm{Pt} / \mathrm{Si}, \mathrm{Ni} / \mathrm{Si}, \mathrm{Pt} / \mathrm{Ge}$, and $\mathrm{Ni} / \mathrm{Ge}$. Using the formulation presented above, it was prudent to determine what changes in electron transport could be expected theoretically when the thermally restrictive interface was changed from a $\mathrm{Pt} / \mathrm{Si}(\mathrm{Pt} / \mathrm{Ge})$ to a $\mathrm{Ni} / \mathrm{Si}$ (Ni/Ge) interface. As mentioned previously, the difficulty in modeling a Schottky barrier arises in determining the value of the barrier height and the doping density of the substrate. To better understand how these variations affect the overall electronic contributions to thermal transport, various doping concentrations were modeled over a range of barrier heights. The parameters used in the calculations are shown in Table 7.6.

Table 7.6: Properties of semiconductor elements used to model electron boundary conductance.

\begin{tabular}{ccc}
\hline Property & $\mathbf{S i}$ & $\mathbf{G e}$ \\
\hline$\epsilon_{r}$ & 11.68 & 16.20 \\
$m_{e, s c}[\mathrm{~kg}]$ & $0.98 m_{o}$ & $1.60 m_{o}$ \\
$T[\mathrm{~K}]$ & 300 & 300 \\
\hline
\end{tabular}

In order to determine the electron boundary conductance, the first task is to determine the primary method that electrons utilize to cross the potential barrier, tunneling or thermionic emission. The criteria for electron tunneling is given by Equation 7.9 and is shown as a function of barrier height in Figure 7.25. To check the tunneling condition in Figure 7.25, the barrier width, $d$, is calculated via Equation 7.5, and given as a function of

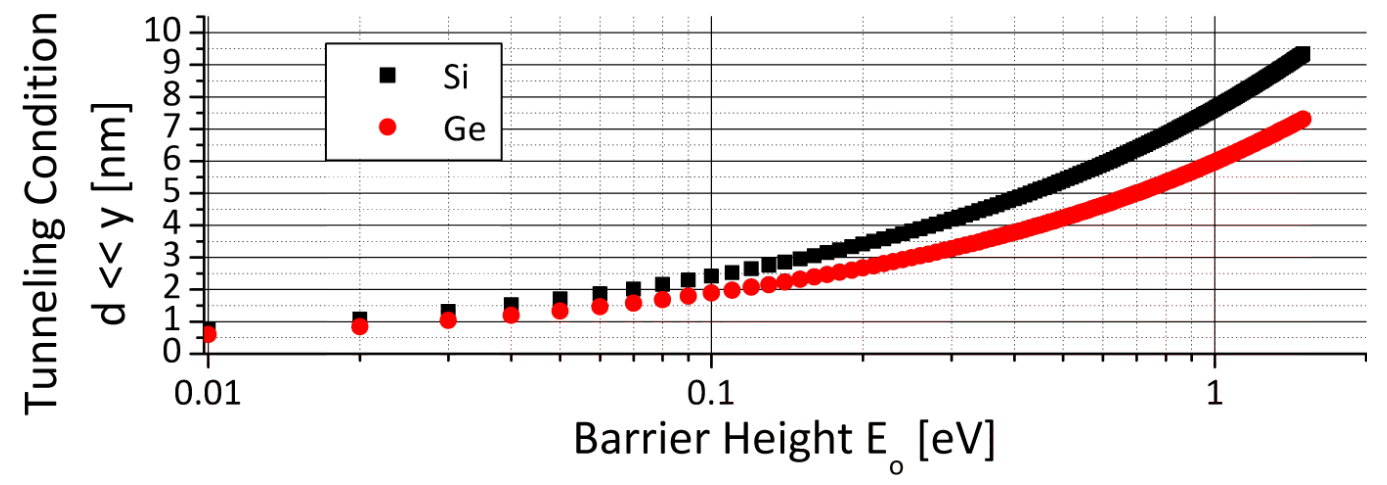

Figure 7.25: Tunneling condition as a function of barrier height. Tunneling occurs if $d$ is much less than the tunneling condition, based on Equation 7.9. 
barrier height, $E_{o}$, in Figure 7.26. Comparing the data in Figures 7.25-7.26 we find that except for the cases of the highly doped substrates and low barrier heights, the criteria for tunneling is generally not met.

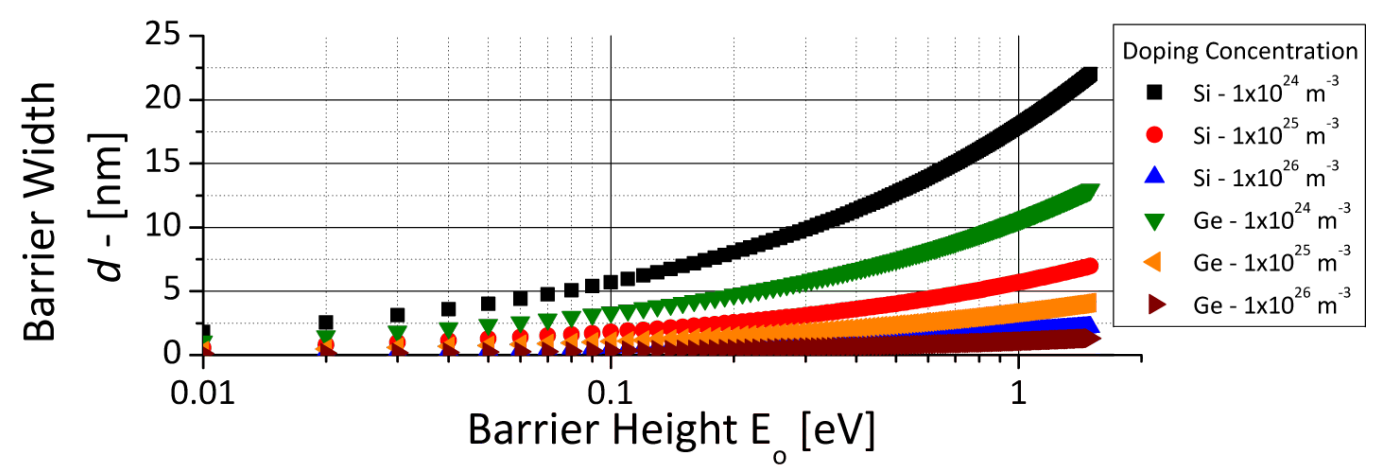

Figure 7.26: Barrier width as a function of barrier height for various substrate doping densities.

If tunneling is assumed, the tunneling probability as a function of the metal-semiconductor barrier height for various doping concentrations is shown in Figure 7.27. The tunneling probability was calculated from Equation 7.7 using the parameters shown in Table 7.6. The plot in Figure 7.27 shows that expect for a high semiconductor doping concentration the tunneling probably drops close to zero for barrier heights greater then $0.1 \mathrm{eV}$. As can be expected, as the tunneling probability is reduced the contributions of electrons to thermal boundary conductance drops significantly above $0.1 \mathrm{eV}$ for all but the cases of heavily doped semiconductors.

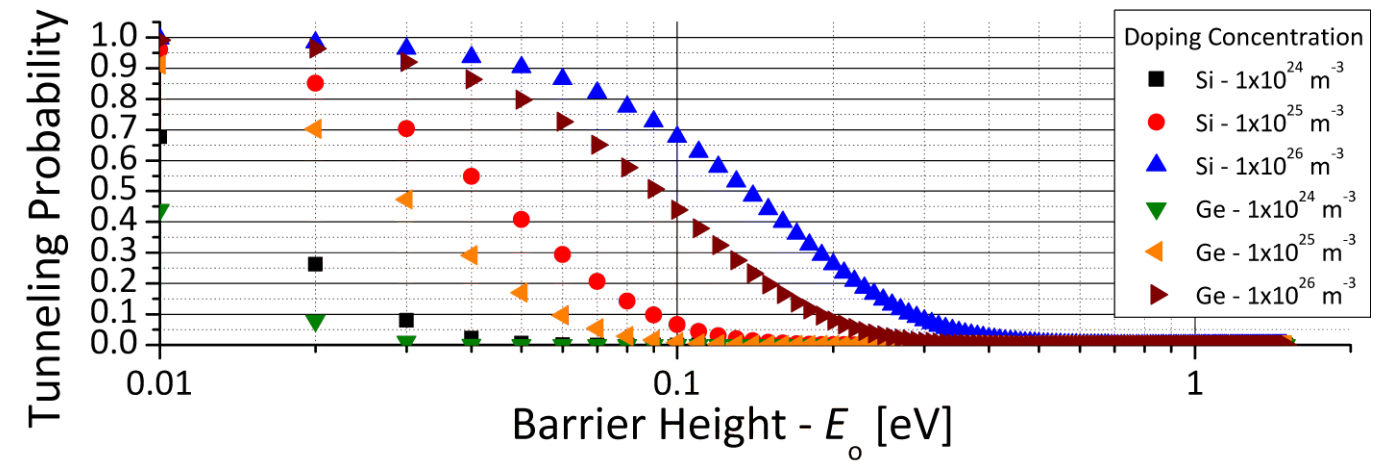

Figure 7.27: Tunneling probability as a function of metal-semiconductor interface barrier height for various semiconductor doping concentration.

As shown in Equation 7.8, when assuming that the primary mechanism for electron 


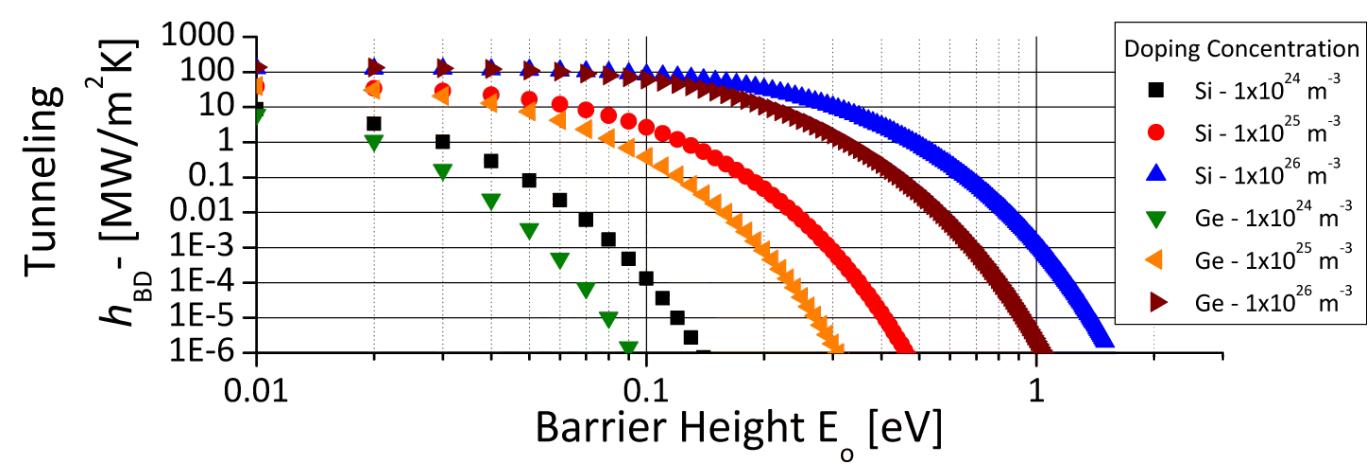

Figure 7.28: Electron thermal boundary conductance assuming electron tunneling as a function of metalsemiconductor interface barrier height, for various semiconductor doping concentrations.

transport across the interface is thermionic emission, the results are independent of the substrate doping. The primary factor of interest is the barrier height, $E_{o}$, and the contribution of electron boundary conductance due to thermionic emission as a function of barrier height is shown in Figure 7.29. Similar to the case of barrier tunneling, the continuation of electrons to boundary conductance via thermionic emission drops off significantly for barrier heights over $0.1 \mathrm{eV}$.

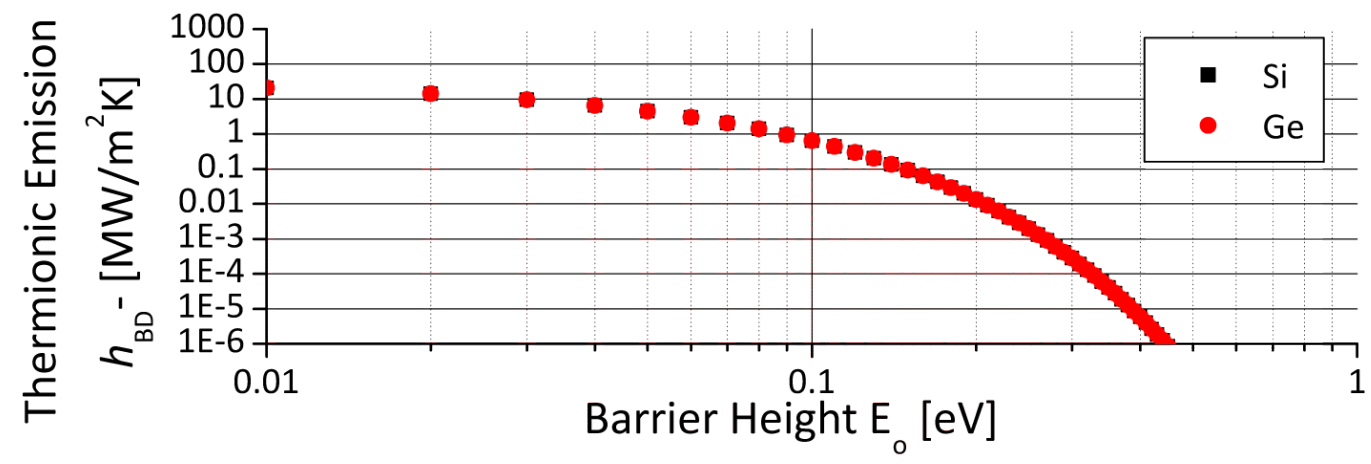

Figure 7.29: Electron thermal boundary conductance assuming electron thermionic emission as a function of metal-semiconductor interface barrier height independent of semiconductor doping concentrations.

\section{Theoretical and Experimental Barrier Heights}

In order to calculate the electron boundary resistance across a metal-semiconductor interface the major parameter that needs to be determined is the barrier height $E_{o}$. For 
the ideal case, the barrier height can be determined from the metal work function and the semiconductor electron affinity based on Equation 7.3. For the materials in this study, the work functions of the two metals are $q \phi_{m}=5.56 \mathrm{eV}$ and $q \phi_{m}=5.15 \mathrm{eV}$ for Pt and Ni, respectively. The electron affinity for the two substrates, as given in Table 7.5, are $q \chi=4.05$ $\mathrm{eV}$ and $q \chi=4.00 \mathrm{eV}$ for $\mathrm{Si}$ and $\mathrm{Ge}$, respectively. Therefore, the ideal barrier heights for metal-semiconductor interfaces of interest are: $E_{o, P t / S i}=1.51 \mathrm{eV}, E_{o, N i / S i}=1.10 \mathrm{eV}$, $E_{o, P t / G e}=1.56 \mathrm{eV}$, and $E_{o, N i / G e}=1.15 \mathrm{eV}$. With the barrier heights all being above 1 $\mathrm{eV}$, the plots in Figures 7.29 and 7.28 indicate that there is little contribution of electrons transporting across the metal-semiconductor interface.

The values given for the Schottky barrier heights of the metal-semiconductor systems, calculated from Equation 7.3, are for the ideal case only. For non-ideal interfaces, the actual barrier height has been shown to be strongly affected by conduction during fabrication, $[143,303]$ and to vary depending on the investigator [304]. The measured upper bound for Schottky barrier heights at $300 \mathrm{~K}$ on n-type semiconductor substrates was given by Sze and $\mathrm{Ni}[24]$ as $E_{o, P t / S i}=0.90 \mathrm{eV}, E_{o, N i / S i}=0.74 \mathrm{eV}, E_{o, N i / G e}=0.49 \mathrm{eV}$, and Chi et al. [305] provided measured values for $\mathrm{Pt} / \mathrm{Ge}$ systems in the range of $E_{o, P t / G e}=0.62-0.63 \mathrm{eV}$. Even for the reduced Schottky barrier height in real systems, the contributions of electrons to $h_{\mathrm{BD}}$ for either tunneling or thermionic emission are less that $1 \mathrm{MW} / \mathrm{m}^{2} \mathrm{~K}$.

The analysis of the potential contributions of electrons to thermal boundary conductance for the systems in this study indicates that the effect of electrons can be neglected at the metal-semiconductor interface. While there is little doubt that at the $\mathrm{Pt} / \mathrm{Ni}$ interface electrons will be the primary energy carriers, before transporting thermal energy into the substrate the electrons in the Ni must couple their energy to the phonon system in the Ni layer. It is therefore the modification of the phononic properties of the Ni layer as a function of thickness in comparison to the phononic properties of the Si or Ge substrate that drives the change in conductance observed in the experimental system. This supports that while transport is not $100 \%$ phonon dominated as in the molecular dynamics simulations, phonons are the primary energy carriers into the substrate. 


\subsection{Chapter Summary}

In this chapter, I presented the concept of modifying $h_{\mathrm{BD}}$ at a solid-solid interface by inserting a material with mediating vibrational properties between the two solids to act as a "vibrational bridge" and increase the probability of elastic phonon transport. The concept was motivated by previous experimental work and more detailed studies conducted using molecular dynamics simulations which demonstrated that inserting an intermediate layer of mediating vibrational properties increased $h_{\mathrm{BD}}$ by increasing the overlap in the occupied phonon density of states between the film, substrate, and intermediate layer. Using the relations developed by the MD studies, a series of $\mathrm{Pt} / \mathrm{Si}$ and $\mathrm{Pt} / \mathrm{Ge}$ samples were fabricated with $\mathrm{Ni}$ intermediate layers with thicknesses in the range of 5-100 $\mathrm{nm}$. The total conductance between the Pt film and the semiconductor substrate was measured in these system using the transient thermoreflectance technique to deduce $h_{\mathrm{BD}}$. The results indicated a strong increase in $h_{\mathrm{BD}}$ for the $\mathrm{Pt} / \mathrm{Si}$ system with the addition of the Ni intermediate layer across several Ni thicknesses. Additionally, subsequent analysis of the impact of bonding and electron thermal transport at the Ni-semiconductor indicated that electrons play a minority role in thermal transport and that while increased bonding enhanced $h_{\mathrm{BD}}$, bonding alone could not describe the observed trend in the data. In conclusion, the effective total conductance in the $\mathrm{Pt} / \mathrm{Si}$ system was shown to increase by as much as $158 \%$ with the addition of a 5 nm Ni layer, and remained above the baseline Pt-to-Si conductance for thicknesses up to $50 \mathrm{~nm}$. While the results and trends presented indicate enhanced conductance with the addition of the Ni layer, the results are still preliminary and cannot be directly attributed to a vibrational bridging effect. A more detailed analysis must be conducted to determine if the mechanism of enhanced conductance is due to vibrational bridging or another affect. 


\section{CHAPTER 8}

\section{SUMMARY, CONCLUSIONS,}

\section{OUTLOOK, AND SUGGESTIONS}

8.1 Summary and Conclusions . . . . . . . . . . . . . . . . . . 272

8.2 Outlook and Suggested Future Work . . . . . . . . . . . . . . 275

\subsection{Summary and Conclusions}

Understanding the fundamental processes dictating $h_{\mathrm{BD}}$ and developing methods to phononically engineer the thermal performance of solid structures is critical to continued advancements in the performance and proliferation of nanodevices. The pump-probe thermoreflectance experiment is a powerful tool in the measurement of nanoscale thermophysical properties and critical to the further development of phononic engineering efforts. In this dissertation several contributions were presented aimed at extending the capabilities of the thermoreflectance experiment and increasing the accuracy and precision of the deduced results.

In Chapter 1 the lineage of how thermal management limitations have at times restricted advances in device performance and have in part forced the implementation of new device 
structures was presented. The basics of thermal management across a number of length scales were presented and a summary of management techniques provided. Chapter 2 focused specially on thermal boundary conductance and the current analytical models used to predict its magnitude and trend in various systems. A review of predominate literature was provided for the major factors that have been shown to influence thermal boundary conductance. Together, Chapters 1 and 2 were developed to provide a strong introduction to the general scope and current state of the art in thermal management specifically focused on thermal boundary conductance.

Experimentally, the design and setup of the thermoreflectance system used in this work was described in detail in Chapter 3. The system was disassembled, redesigned, and rebuilt during my tenure as a graduate student with an effort to improve the overall functionality and robustness of the experiment, incorporating many design features developed since the system was first installed.

Described in Chapter 4 from a mathematical point of view, the methodology used to model the thermoreflectance signal and deduce a parameter of interest was presented. The theoretical model was also redeveloped and extended from a single film lumped capacitance model, to a multilayer model taking into account the effects of pulse accumulation and radial thermal conduction that have been previously presented by Cahill and Schmidt [230, 231]. The model was also extended to include modulation of the pump laser by a pulsed modulation waveform which takes into account higher harmonics present in the measured signal and allows for variation in the duty cycle of the modulation waveform.

Together, Chapters 3 and 4 provide the basic physical and mathematical structure of the thermoreflectance system. It is my hope that these chapters will provide a valuable reference to individuals trying to understand and/or implement the thermoreflectance technique in their own research. So few details are typically provided in literature, hopefully this work helps to fill in some of the gaps often omitted.

In Chapter 5 more details on the numerical application of the thermal model developed in Chapter 4 were presented, including convergence criteria for the infinite summations in the thermal model. These results should allow for accurate application of the model without the need for each individual to retest convergence. Methods were also presented to speed 
up convergence to allow for large scale modeling of the thermal system, and the quasistochastic treatment of sensitivity and accuracy analyses. In addition, several practical tools in the operation and analysis of the thermoreflectance experiment were developed and presented including quantification of the sensitivity of the model to the input parameters, the quantification of noise in the data, and the identification of statistical outliers in the deduced results.

The application of the thermoreflectance technique to measure and understand thermal transport at the nanoscale relies heavily on the statistical interpretation of the results to infer thermophysical properties of the measured system. Chapter 6 discussed some of the basic concepts of statistical inference as it applies to thermoreflectance data. A set of empirical relations were developed to predict the estimated standard deviation in the final results based on the sensitivity coefficient of the parameter of interest and the signal-tonoise in the data. Having a priori knowledge of the estimated standard deviation in the results will assist in the design of experimental parameters and save time and experimental resources. To test the statistical assumptions commonly used to describe collected data, the nonparametric bootstrap was introduced as a means to diagnose the statistical validity of several primary assumptions. A method to quasi-stochastically determine the accuracy of the final result based on the uncertainty of the input parameters was also established which takes into account the covariance between the input parameters, as an improvement over previous analytic models.

Finally, in Chapter 7, relying on the methodologies developed in Chapters 3-6 to ensure robust and accurate results, thermoreflectance measurements were presented on $\mathrm{Pt} / \mathrm{Si}$ and $\mathrm{Pt} / \mathrm{Ge}$ systems with the addition of a metallic intermediate layer of varying thickness added at the metal-semiconductor interface to act as a "phonon bridge" to improve the metalsemiconductor thermal conductance. The results showed that for the $\mathrm{Pt} / \mathrm{Si}$ system, the $\mathrm{Ni}$ intermediate layer dramatically increased $h_{\mathrm{BD}}$ compared to the baseline $\mathrm{Pt} / \mathrm{Si}$ conductance for thin Ni layers up to $50 \mathrm{~nm}$ in thickness. The results were compared to a theoretical analysis assuming the increased $h_{\mathrm{BD}}$ was from improved metal-semiconductor bonding and theoretical calculations of the contribution of electron transport at the metal-semiconductor interface. Overall, the results of Chapter 7 support the concept "vibrational bridging" 
via intermediate layers as a means to enhance and tune solid-solid interface conductance. However, the results are only preliminary and more in-depth work must be conducted in order to determine whether the observed increase in conductance can be attributed to a "vibrational bridging" phenomena or some other modification effect.

It is my hope that the work presented in this dissertation will provide those who study thermal transport at the nanoscale and use the thermoreflectance technique a useful guide and a basis for which new and more in depth studies can be developed in pursing a greater understanding of nanoscale thermal transport which in turn will lead in part to the next revolution in nanoelectronic devices.

\subsection{Outlook and Suggested Future Work}

While the work presented in this dissertation represents a significant effort to advance the understanding and accuracy of thermal measurements via the thermoreflectance technique and the ability to phononically engineer interface conductance, there is always more work to be done. With this in mind and based on the lessons learned in the completion of this dissertation, there are several avenues that can advance the work presented here and that merit future study:

\subsubsection{Chapter 6}

My desire is that the results of Chapter 6 will help to drive the robust statistical treatment of thermoreflectance data and encourage the inference of population parameters rather than solely the presentation of collected results. I am by no means a statistician and there is therefore always room for improvement in the statistical methodologies used to analyze the experimental results beyond what was presented in this work. In particular, the presented work on the bootstrapping technique was only an introduction to a complex and powerful statistical tool. Much more work can be done in refining the bootstrapping methodology used, particularly for the application of predicting confidence intervals. There are a number of improvements to the basic methods presented in this work, each with their own strengths and weaknesses, and more work must be undertaken to find the methods best suited for 
thermoreflectance data.

The discrepancy of the standard deviation predicted by the simulated data compared to the results of the experimentally collected data indicate that the experimental system is not reaching the theoretical limit of precision. While it may not be possible to reach this limit exactly, it is worth investigating possible reasons behind the discrepancy and potential methods to improve the experimental results. One possible avenue to investigate is the filtering of the noise in the data by the lock-in amplifier and other filters. While the noise in the data on the whole follows a normal distribution, under and/or improper filtering during the course of an individual scan may be introducing artifacts in the measured signal that impacts the results on a scan-by-scan basis and therefore the standard deviation between measurements.

The biggest challenge to determining the true value of a thermophysical parameter deduced from the thermoreflectance experiment with a high degree of accuracy is reducing the uncertainty in the input parameters. While there are ancillary methods that can be used to independently measure certain thermophysical input parameters, each have their own uncertainties and experimental costs. To reduce the dependency on outside equipment and increase the capability of the thermoreflectance experiment, it is important to know how many parameters can be "safely" deduced from the collected data. Using the established criteria for determining parameter sensitivity and the modeling methods presented, a more in depth analysis of multiparameter fitting should be completed.

\subsubsection{Chapter 7}

One challenge to the experimental work on the vibrational bridging concept will always be the use of metallic intermediate layers. While the work in this dissertation supports the concept of modified vibrational properties over electron dominated effects, experiments sometimes speak louder than words. With the proper collaboration with those who could produce epitaxially grown Ge intermediate layers on Si substrates, it would be worth reproducing this work with a $\mathrm{Pt} / \mathrm{Ge} / \mathrm{Si}$ system. In lieu of semiconducting intermediate layers, it would be useful to measure the Schottky barrier heights of the Pt/Si system with and without the addition of the Ni intermediate layer as quantitative evidence of the potential 
contribution of electrons to thermal transport. In addition, to support the tunability of the vibrational bridging effect, it would be useful to compare the relative "strength" of the bridging effect by varying the Debye temperature of the substrate to include different Debye mismatches between the film, substrate, and intermediate layer.

In as much as the top metallic film is required to complete the TTR/TDTR analysis, it creates a disconnect between the previous molecular dynamics work and the experimental results. The MD work, and the concept of "vibrational bridging," is developed on phonon properties alone, while in the experimental system phonons do not participate to an appreciable degree until considering the Ni-substrate interface. Therefore, in a system that contains metallic films the "vibrational bridging" concept may not be the best manner to picture the enhancement effect as there is no phonon "communication" between the Pt and the substrate. It may be more apt to consider the modification to thermal conductance in terms of the modified phonon properties of the Ni layer in relation to the substrate, while maintaining high overall film-to-substrate conductance due to the intrinsically high thermal conductivity of the intermediate layer. 
[This page is intentionally left blank] 


\section{APPENDIX A}

\section{FUNDAMENTALS OF SOLID-STATE \\ PHYSICS FOR MICRO/NANO-SCALE THERMAL TRANSPORT}

A.1 Introduction . . . . . . . . . . . . . . . . . . . 279

A.2 Crystallography . . . . . . . . . . . . . . . . . . . . 280

A.3 Phonons . . . . . . . . . . . . . . . . . . . . . . 289

A.4 Phonon Dispersion . . . . . . . . . . . . . . . . . . . . . 291

A.5 Phonon Density of States . . . . . . . . . . . . . . . . . 304

A.6 Phonon Scattering . . . . . . . . . . . . . . . . . . . 309

A.7 Appendix Summary _ . . . . . . . . . . . . . . . . . 317

\section{A.1 Introduction}

In this appendix, the fundamental topics of crystallography and phonons are discussed, with an emphasis on the development of the phonon dispersion relation and the phonon density of states (PDOS) from crystal properties. The general development of these relations 
from fundamental lattice dynamics is presented and the relationship between the phonon properties and thermal transport is discussed. Most, if not all, of the information provided is based on, and is readily available in, some of the major texts in solid-state and nanoscale physics, namely Kittel [306], Ashcroft and Mermin [32], Dove [307], and Chen [33]. The primary purpose of this appendix is to provide a "crash course" in phonon physics in solids, and develop the basic tenants and terminology required to better understand the discussed literature in Chapter 2 and the experimental studies and results in Chapter 7.

\section{A.2 Crystallography}

The logical starting point is to discuss the medium of interest, in this case solids, which can in whole, or in part, be crystalline in nature ${ }^{1}$. Crystallography deals extensively with the structure, properties, and organization of atoms, clusters, and molecules in crystalline solid structures. In its most basic form, a crystal can be considered a configuration of particles, whose structure can be repeated periodically in three directions. It is this periodic nature that gives crystallography its strength. Consider that a block of copper, $1 \mathrm{in}^{3}$ in volume, contains roughly $10^{24}$ atoms. If we were to assign individual coordinates to each copper atom, in three dimensions, using 6 characters for each label, at 1 byte per character, it would take over $2.5 \times 10^{25}$ bytes, or roughly 2.5 trillion terabytes of memory just to store the xyz location of each atom within the volume. Clearly if we want to investigate anything more than the smallest collections of atoms we need to have some tricks to make things more manageable. The next few subsections will introduce the basic mathematical definitions and terminologies of a crystal in real space, and introduce the concept of a reciprocal space, the crystallographic analog of the Fourier transform, which will provide the tools necessary to deal with "large" collections of atoms.

\footnotetext{
${ }^{1}$ Solids can also of course be amorphous, however this work will focus primarily on crystalline and poly-crystalline materials.
} 


\section{A.2.1 Crystals in Real Space}

The definition of a crystal's structure in space requires two components; a lattice and a basis, i.e.

$$
\text { crystal structure }=\text { lattice }+ \text { basis }
$$

The lattice is the mathematical representation of points in space that define the crystal and give it its periodicity. The basis describes how the atom, or atoms ${ }^{2}$, are distributed around each lattice point. For a basis of 1, the logical configuration is that 1 atom is located at each lattice point. For a basis greater than 1, the atoms are clustered around each lattice point in some configuration, the details of which are unimportant at this point provided that the relation between the lattice points and the basis atoms remains consistent. Due to this periodicity, every point in the lattice has identical surroundings. That is, if we were to stand on a lattice point, looking around in all directions, and taking note of the surroundings, and then were to move to a different lattice point and repeat the observation, the surroundings would look identical in both cases.

Mathematically, the translation from one lattice point to another can be represented through the set of primitive lattice vectors, $\overrightarrow{a_{1}}, \overrightarrow{a_{2}}$, and $\overrightarrow{a_{3}}$, such that [33]:

$$
\overrightarrow{\mathbf{R}}=n_{1} \overrightarrow{a_{1}}+n_{2} \overrightarrow{a_{2}}+n_{3} \overrightarrow{a_{3}}
$$

where $\overrightarrow{\mathbf{R}}$ is the translation vector and $n_{1}, n_{2}$, and $n_{3}$ are integer values. The vectors, $\vec{a}_{i}$, being defined as primitive, implies that using any integer combination of these vectors in three dimensions, one can reach every point within the lattice. As examples, Figure A.1 shows a 2D lattice structure with several regions defined, shaded in gray, with primitive lattice vectors $a_{1}$ and $a_{2}$ highlighted. We can notice, that the primitive lattice vectors are not unique, and there can be several different variations of lattice vectors, three of which are shown in Figure A.1. Additionally in Figure A.1, we have lattice vectors denoted $a_{1}^{*}$ and $a_{2}^{*}$, these are examples of non-primitive lattice vectors. Through integer combinations of $a_{1}^{*}$ and $a_{2}^{*}$, we are unable to reach all the lattice points within the $2 \mathrm{D}$ lattice, such as

\footnotetext{
${ }^{2}$ Again, the basis can also represent the organization of a collection of atomic clusters, molecules, etc., but for the sake of description we will typically refer only to atoms.
} 


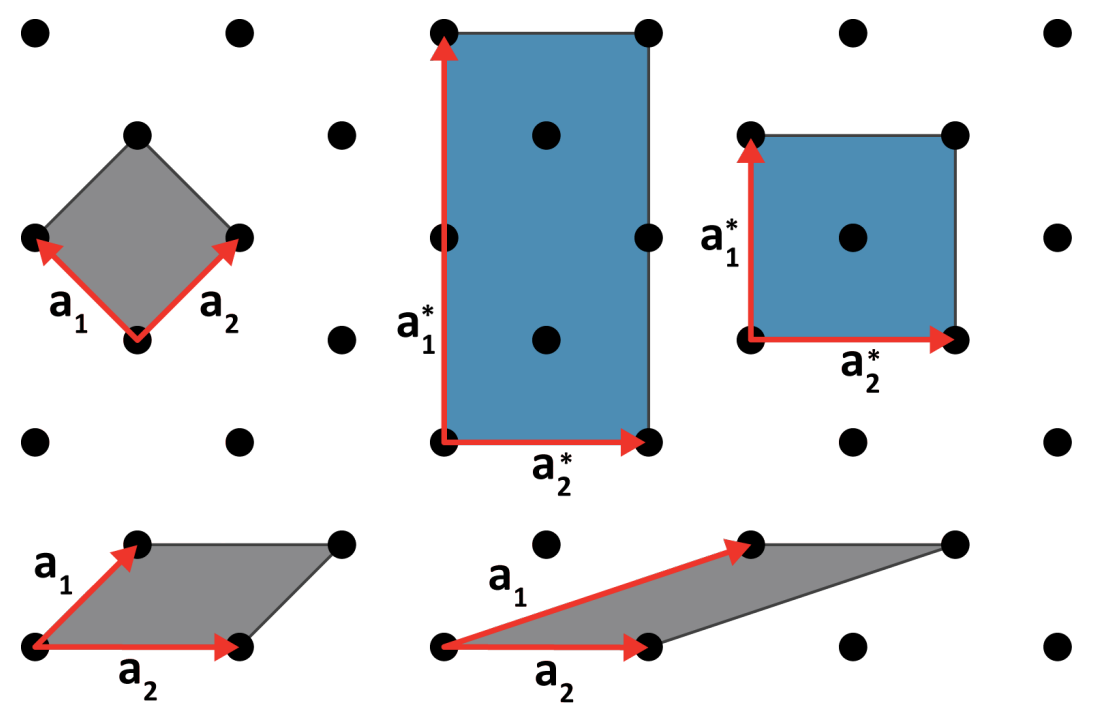

Figure A.1: A two-dimensional lattice with vectors, $a_{1}$ and $a_{2}$, highlighting three examples of primitive lattice vectors, which also define three examples of primitive unit cells, shaded in grey. Additionally vectors $a_{1}^{*}$ and $a_{2}^{*}$ provide two examples of sets of non-primitive lattice vectors, which define two examples of conventional unit cells, shaded in blue.

those lattice points located within the blue shaded regions in Figure A.1.

Sets of primitive lattice vectors define the edges of parallelepipeds that are designated as primitive unit cells, examples of which are the gray shaded regions of Figure A.1. The primitive unit cell is the smallest reproducible unit, i.e. the smallest volume, that can be used to construct the entire lattice. Each primitive unit cell contains exactly one lattice point. For crystals with a basis of 1, such as the 2D crystal shown in Figure A.1, this can include $1 / 4$ of an atom at each corner of the parallelepiped. However, as primitive lattice vectors are degenerate, so are the defined regions of the primitive unit cell. A special case of the primitive unit cell is the Wigner-Seitz unit cell, shown in Figure A.2. The Wigner-Seitz unit cell is constructed by connecting the adjacent lattice points around a point of interest, see the blue lines in Figure A.2, and bisecting each of those blue lines perpendicularly at the midpoint, see the lines denoted in red. The smallest region defined by these planes, shaded in gray, is the Wigner-Seitz unit cell for that lattice. Again, since it is a primitive unit cell, it contains only one lattice point.

The non-primitive lattice vectors in Figure A.1, $a_{1}^{*}$ and $a_{2}^{*}$, define the edges of unit cells, which are denoted as non-primitive (or conventional) unit cells. While not the smallest reproducible volume, and therefore can contain more than one lattice point, conventional 


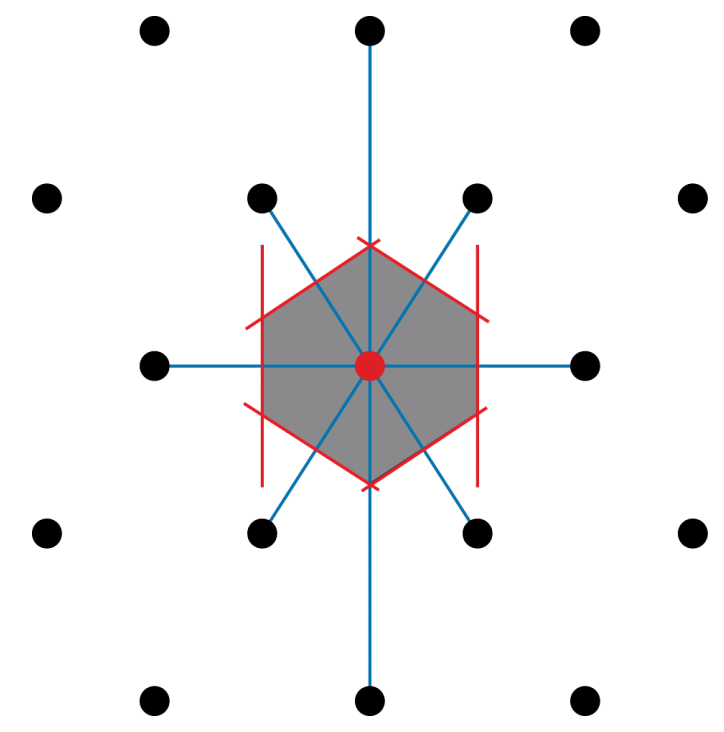

Figure A.2: Schematic of the construction of the Wigner-Seitz unit cell for a two-dimensional lattice with a monatomic basis.

unit cells are often more convenient to visualize the lattice symmetry.

The symmetry of a lattice is a property by which the system can undergo some movement or operation and arrive at a state which is indistinguishable from the original configuration [308]. Different types of crystal lattices are typically defined based on their symmetry properties. The typical properties of interest are the symmetry related to inversion, reflection, and rotation. The unit cell is said to have inversion symmetry, if there is a point in which the unit cell remains invariant under the translation $\overrightarrow{\mathbf{R}} \rightarrow-\overrightarrow{\mathbf{R}}$. The unit cell is said to have reflection symmetry, if there exits a plane such that when a mirror reflection along this plane is performed, the unit cell remains invariant. Finally, the unit cell is said to have rotational symmetry, if there exists an axis such that if the cell is rotated about that axis through some angle, the cell will remain invariant.

An infinite array of lattice points constructed via the translation vector, $\overrightarrow{\mathbf{R}}$, is an example of a Bravais lattice. In total, there are 14 different Bravais lattices in three dimensions, see Figure A.3, which can be further broken down by 7 crystal systems: triclinic, monoclinic, orthorhombic, tetragonal, cubic, hexagonal, and trigonal. Note that by definition, all the Bravais lattices have inversion symmetry, with monoclinic and cubic systems having reflection and rotation symmetry as well. The majority of elemental solids form either cubic or hexagonal lattices. 


\section{A.2.2 Crystals in Reciprocal Space}

While we have discussed the construction of a Bravais lattice through the translation vector, $\overrightarrow{\mathbf{R}}$, and provided examples of the 14 primary Bravais lattices, see Figure A.3, we have yet to discuss how we identify the types of crystal structures in real systems. We can study the crystal structure of an unknown system through the process of diffraction. The discussion of diffraction at this point will serve two purposes 1) to provide a basic introduction to the interaction of electromagnetic radiation and a crystal lattice, and perhaps more importantly 2) to develop the concept of reciprocal space. As we mentioned initially, for all but the smallest of samples, i.e. dimensions on the order of nanometers, systems will have an exceedingly large number of atoms. Attempting to track that many atoms, or unit cells, is computationally and mathematically difficult, if not impossible, depending on the system size. The definition of a reciprocal space ${ }^{3}$ allows us to invert regular space. Therefore, what was relatively large in regular space, such as the distance across a long chain of atoms, will be small in reciprocal space, making it more mathematically tractable. On the other hand, the small distances will become large, however the minimum distances have a lower bound,

Rhombohedral

Figure A.3: The 14 types of Bravais lattices, representing 7 crystal systems: triclinic, monoclinic, orthorhombic, tetragonal, cubic, hexagonal, and trigonal.

\footnotetext{
${ }^{3}$ Reciprocal space is sometimes called $k$-space or $q$-space because it related to and has the same units as a wavevector, often denoted $k$ or $q$.
} 
given by the interatomic spacing, $a$, below which there will be no new usable information. We will begin by discussing the diffraction experiment to give a physical picture of the reciprocal space, before developing the concept in a more mathematical framework.

We know from fundamental experimental physics, that electromagnetic radiation incident on a small aperture, such as a slit, with a wavelength that is on the order of the slit dimensions, will produce a diffraction pattern. In crystallography, when x-rays or electrons are incident on a periodic chain of atoms, they will undergo similar diffraction, with constructive and destructive interference creating a pattern of light and dark regions on a detector screen, see Figure A.4a. If we now consider an array of atoms, see Figure A.4b, the x-rays produce a periodic array of points on the detector screen. These points are a physical manifestation of the reciprocal lattice. Because it is a reciprocal lattice, if we were to move the atoms further apart, in one or both directions, the points on the screen, the lattice points of the reciprocal lattice, would move closer together, see Figure A.4c. Similarly, if we were to move the atomic planes closer together, the points in the reciprocal lattice would move further apart, see Figure A.4d.

Now we will consider how the periodic nature of the regular lattice factors into the mathematical description of the reciprocal lattice. To begin, we take a one-dimensional array of atoms, as in Figure A.5, assuming a uniform spacing between each atom, defined by the lattice constant, $a$. Due to the periodic nature of the array, we can also assume that many properties of the system are periodic, such as the electron density, potential energy fields, and mass density. If we consider periodicity in the mass density, we can relate the mass density at a given point $x, \rho(x)$, to the mass density at any other lattice point by:

$$
\rho(x)=\rho(x+n a)
$$

where $a$ is the lattice constant and $n$ is an integer value. Periodic systems such as this are well suited to be treated in terms of Fourier Analysis ${ }^{4}$. In terms of a Fourier series of

\footnotetext{
${ }^{4}$ Fourier analysis will be relied on heavily in Chapter 4 when discussing the interaction of the periodic laser pulses from the laser system.
} 


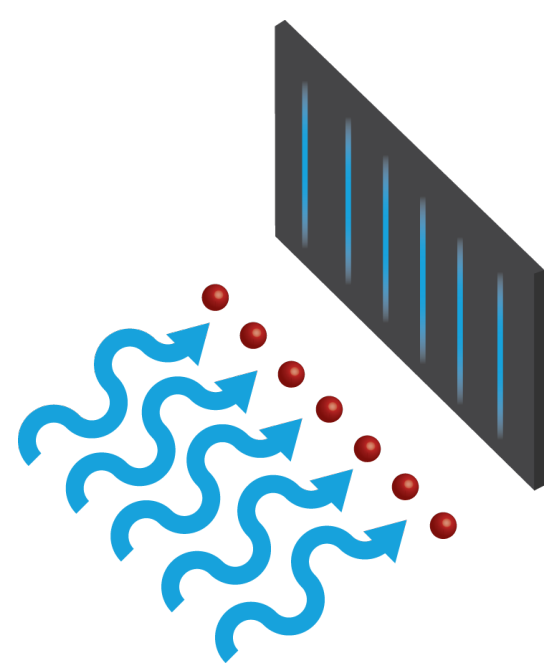

(a)

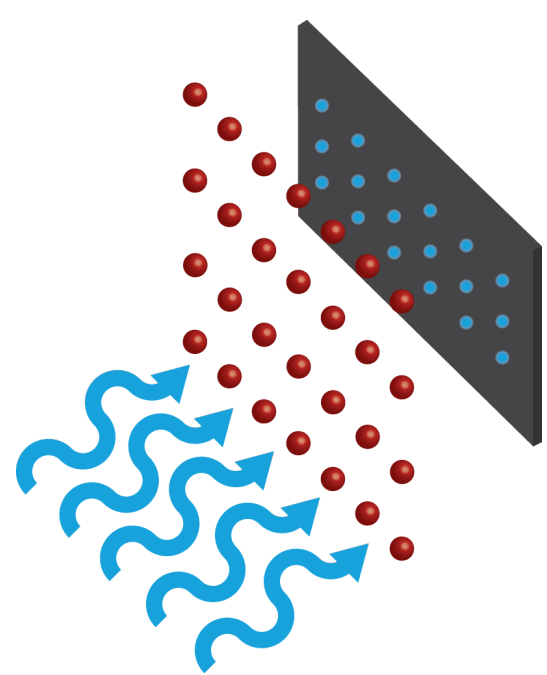

(c)

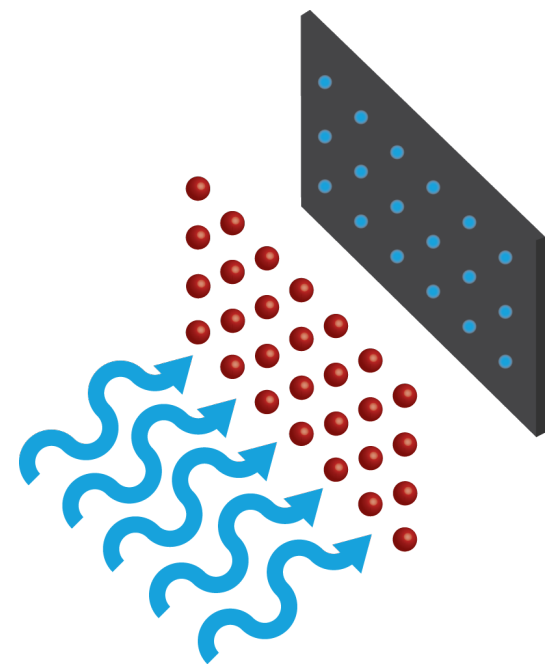

(b)

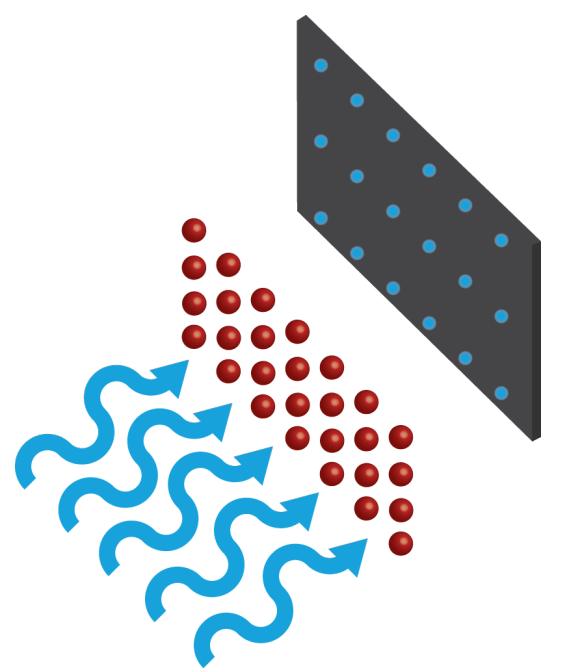

(d)

Figure A.4: A schematic representation of x-ray diffraction for (a) a single row of periodic atoms, (b) a $2 \mathrm{D}$ array of atoms in a square lattice, (c) the real space lattice stretched in the vertical direction and (d) the real space lattice compressed in the vertical direction.

complex exponentials, the relation for the mass density in Equation A.3 can be written as:

$$
\begin{gathered}
\rho(x)=\sum_{m} \rho_{m} \exp \left[i G_{m} x\right] \\
\rho(x+n a)=\sum_{m} \rho_{m} \exp \left[i G_{m}(x+n a)\right]
\end{gathered}
$$

Equation A.4b can be rewritten as: 


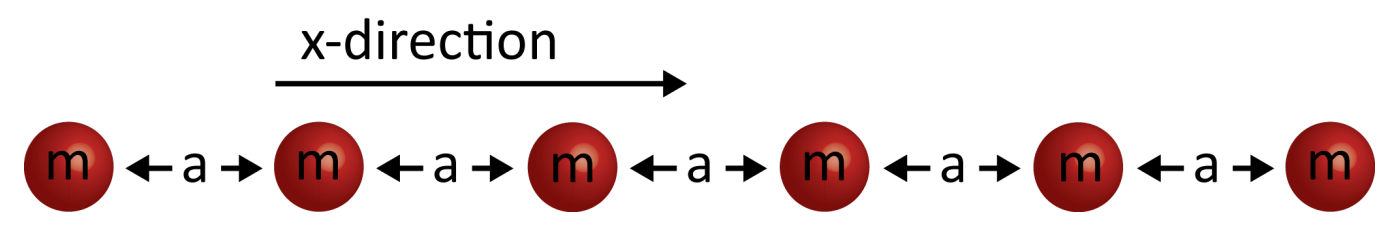

Figure A.5: Schematic of a periodic one-dimensional chain of atoms of uniform mass $m$, and interatomic spacing $a$.

$$
\rho(x+n a)=\sum_{m} \rho_{m} \exp \left[i G_{m} x\right] \exp \left[i G_{m} n a\right]
$$

Equating Equations A.4a and A.5 implies that:

$$
\exp \left[i G_{m} n a\right]=1
$$

which is the case under the condition that:

$$
G_{m} n a=2 \pi \times \text { integer }
$$

This relation can easily be extended to three dimensions by considering the vector form of $G$ and recalling that integer sums of the lattice constant $a$ was the definition of the primitive translation vector $\overrightarrow{\mathbf{R}}$. Therefore, in three-dimensional space we define:

$$
\overrightarrow{\mathbf{G}}_{m} \cdot \overrightarrow{\mathbf{R}}_{n}=2 \pi \times \text { integer }
$$

Where $\overrightarrow{\mathbf{G}}$ is defined as the reciprocal translation vector, which is given by:

$$
\overrightarrow{\mathbf{G}}=m_{1} \overrightarrow{b_{1}}+m_{2} \overrightarrow{b_{2}}+m_{3} \overrightarrow{b_{3}}
$$

where $\overrightarrow{b_{i}}$ is a primitive reciprocal lattice vector and $m_{i}$ is an integer. The relationship between the primitive lattice vectors and the primitive reciprocal lattice vectors is given by [306]:

$$
\begin{aligned}
& \overrightarrow{b_{1}}=2 \pi \frac{\overrightarrow{a_{2}} \times \overrightarrow{a_{3}}}{\overrightarrow{a_{1}} \cdot\left(\overrightarrow{a_{2}} \times \overrightarrow{a_{3}}\right)} \\
& \overrightarrow{b_{2}}=2 \pi \frac{\overrightarrow{a_{3}} \times \overrightarrow{a_{1}}}{\overrightarrow{a_{1}} \cdot\left(\overrightarrow{a_{2}} \times \overrightarrow{a_{3}}\right)}
\end{aligned}
$$




$$
\overrightarrow{b_{3}}=2 \pi \frac{\overrightarrow{a_{1}} \times \overrightarrow{a_{2}}}{\overrightarrow{a_{1}} \cdot\left(\overrightarrow{a_{2}} \times \overrightarrow{a_{3}}\right)}
$$

From Equation A.8, we can infer that if $\overrightarrow{\mathbf{R}}$ is the translation vector in real space (with units of meters), then for the equation to remain unitless, the reciprocal space translation vector $\overrightarrow{\mathbf{G}}$ must have units of meters ${ }^{-1}$. Because the reciprocal lattice vector $\overrightarrow{\mathbf{G}}$ has the same translational symmetry as the real space lattice vector $\overrightarrow{\mathbf{R}}$ used to define the Bravais lattice, see Equations A.2 and A.9, the reciprocal space representation of a real space Bravais lattice, is also a Bravais lattice ${ }^{5}$.

Just as in the case of the real space lattice, we can define a fundamental cell in reciprocal space, which is the smallest repeatable volume which contains all pertinent wave information. This reciprocal space equivalent of the Wigner-Seitz unit cell in real space, is defined as the first Brillouin zone in reciprocal space. By definition, the first Brillouin zone "is the smallest volume entirely enclosed by planes that are the perpendicular bisectors of the reciprocal lattice vectors drawn from the origin" [306]. We can construct the first Brillouin zone in reciprocal space, using this definition in a similar manner that we used to construct the Wigner-Seitz unit cell, see Figure A.2.

In Figure A.6 is a reciprocal space representation of a two-dimensional real space square lattice. To construct the first Brillouin zone from a central lattice point, we can draw an arrow (shown in red) to a first nearest-neighbor lattice point, and draw a perpendicular line bisecting that distance (see the red lines). This process is repeated for the four nearest-neighbor atoms. The smallest region bounded by these red lines represents the first Brillouin zone (shaded in red). To construct higher order Brillouin zones, we can repeat the process using the next nearest-neighbors and the third nearest-neighbors for the second and third Brillouin zones respectively. For a real space square lattice of lattice constant $a$ the dimensions of the Brillouin zone in reciprocal space is $2 \pi / a$, meaning that central to the lattice point, $k$ ranges from $-\pi / a$ to $\pi / a$. The importance and power of the first Brillouin zone will become more apparent as we discuss modes of vibration in the periodic crystal.

\footnotetext{
${ }^{5}$ Note that this does not imply that the reciprocal lattice has the same structure as the real space lattice. While the reciprocal lattice of a real space simple cubic lattice is simple cubic, the reciprocal lattice of a real space face-centered cubic lattice is body-centered cubic lattice, and conversely the reciprocal lattice of a real space body-centered lattice is a face-centered cubic lattice.
} 


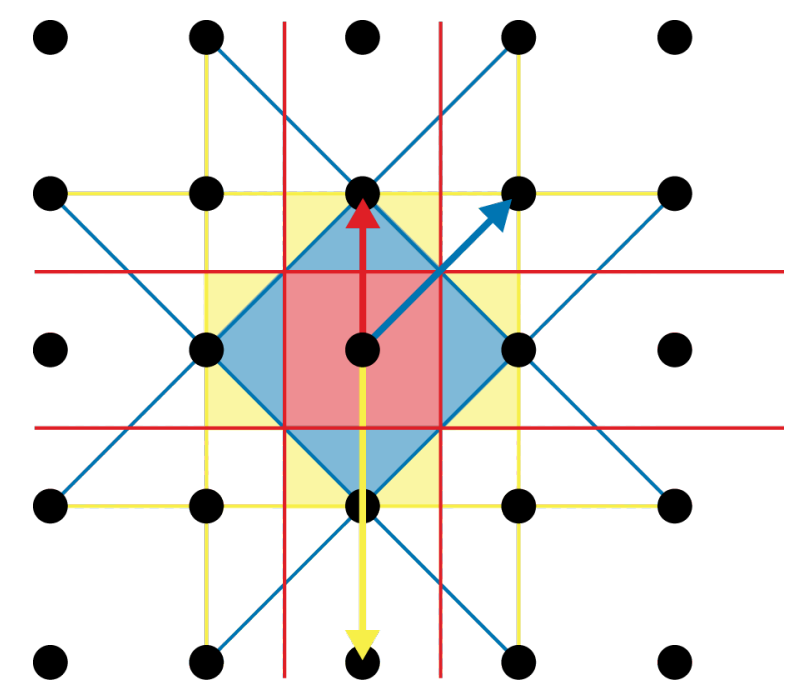

Figure A.6: Representation of a real space square lattice in reciprocal space showing the construction of the first (red), second (blue) and third (yellow) Brillouin zones.

\section{A.3 Phonons}

To this point, we have discussed crystals in terms of their structure, i.e. the lattice and basis, from a static point of view, attributing no motion to the atoms. It is this crystal structure, along with the types of chemical bonding holding the crystal together, that determines many of the physical properties of a material. Allotropes of carbon are probably one of the best examples of this idea, with the property differences between diamond and graphite arising due to the difference in bonding properties between the isometric and hexagonal lattice of each material respectively. However, if we only considered atoms in a rigid lattice, i.e. no flexibility in the bonds, we would not be able to describe such properties as the ability to store thermal energy, i.e. the specific heat, or the ability to transport thermal energy, i.e. the thermal conductivity. With our primary interest in this work being thermal transport, we seek to describe how the vibrational motion of the atoms in a crystal allow for the propagation of some finite amount of energy $E$, at a velocity $v$. As mentioned in Chapter 1, the primary energy carriers in non-metallic materials, i.e. semiconductors and insulators, are phonons.

Phonons are quantized vibrations of the crystal lattice, and are the quantum mechanical 
equivalents to normal modes of a classical system ${ }^{6}$. Therefore, a phonon of a given frequency or wavelength is expected to extend throughout the entire dimension of the system, and is linearly independent of other normal modes. A superposition of such modes will form a wavepacket with a narrow spatial extent, and if the spatial extent is on the order of, or below, the interatomic spacing of the crystal, the phonon wavepacket can be treated as a particle [33]. The properties of phonons will become more apparent as we discuss the formulation of the phonon dispersion relation.

\section{A.3.1 Phonon Polarizations}

However, before developing some of the fundamental properties of phonons in a solid, it is important to discuss two specific allowed "families," or as we will call them, polarizations of phonons. These polarizations are easiest to picture in a two-dimensional lattice, however the interpretation will hold for the three-dimensional structure as well. In the two-dimensional lattice, we will consider the displacement of entire planes of atoms. In the longitudinal polarization, the atomic planes are displaced in the same, or opposite, direction of the wavevector, $k$, see Figure A.7a. For waves that are of transverse polarization, the displacement of the atomic planes is perpendicular to the direction to $k$, see Figure A.7b. One can imagine that in the two-dimensional case there is one transverse polarization, while there are two, and none, in the three-dimensional and one-dimensional cases respectively.
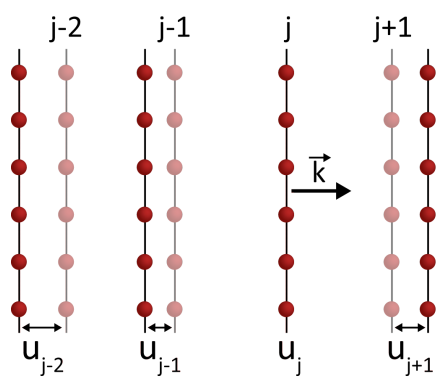

(a)

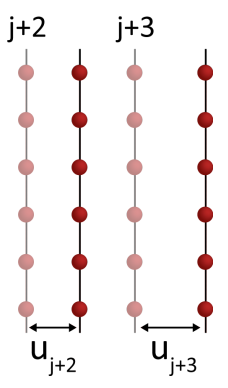

$$
\text { (1) }
$$




\section{A.4 Phonon Dispersion}

When discussing the propagation of heat, we are in essence, talking about the propagation of energy. Therefore, in the discussion of elastic waves, i.e. phonons, in a crystal, it would be convenient to describe a relationship between some property that describes the wave, and the energy the wave carries. We know from quantum mechanics that the energy of a quantum entity, such as a photon or phonon, can be directly related to the angular frequency by, $E=\hbar \omega$. Also in physics, it is common to describe a wave in terms of its wavevector $\vec{k}$, which is related to the wavelength of the wave, and is proportional to the momentum carried by the wave. We therefore seek to relate the wavevector $\vec{k}$ to the phonon frequency $\omega$, which is known as the phonon dispersion relation.

From the phonon dispersion relation, we will find that we can obtain several important quantities and principles that will serve in both the understanding and modeling of thermal transport. We will begin with the simple case of a one-dimensional crystal with a monatomic basis and later expand our analysis to a diatomic basis which will introduce additional phonon branches.

\section{A.4.1 Vibrations of a 1D Lattice with a Monatomic Basis}

To begin with a simple classical mechanics example, without the loss of generality, we will consider a one-dimensional monatomic chain of atoms connected by massless springs, see Figure A.8. Due to the restriction of the motion of the atoms to the x-direction only, this formulation will focus on only longitudinal phonons ${ }^{7}$. While this is a relatively simplistic example, it, along with the one to follow, will provide the necessary tools for a general discussion of the properties of phonons in solids. In this treatment, $m$ is the mass of the atom, $K$ is the force constant between atoms, and $a$ is the equilibrium interatomic spacing. All three of these properties are assumed to be uniform across the chain, and $j$ is provided as the atomic index. In this derivation we will make the following assumptions: 1) that we have an ensemble of $N$ atoms in total, 2) the potential energy is a minimum when the atoms are at their equilibrium positions, 3) we will only consider nearest-neighbor interactions

\footnotetext{
${ }^{7}$ We will discuss the translation of these principles to three-dimensions, including transverse phonons, in an upcoming section.
} 


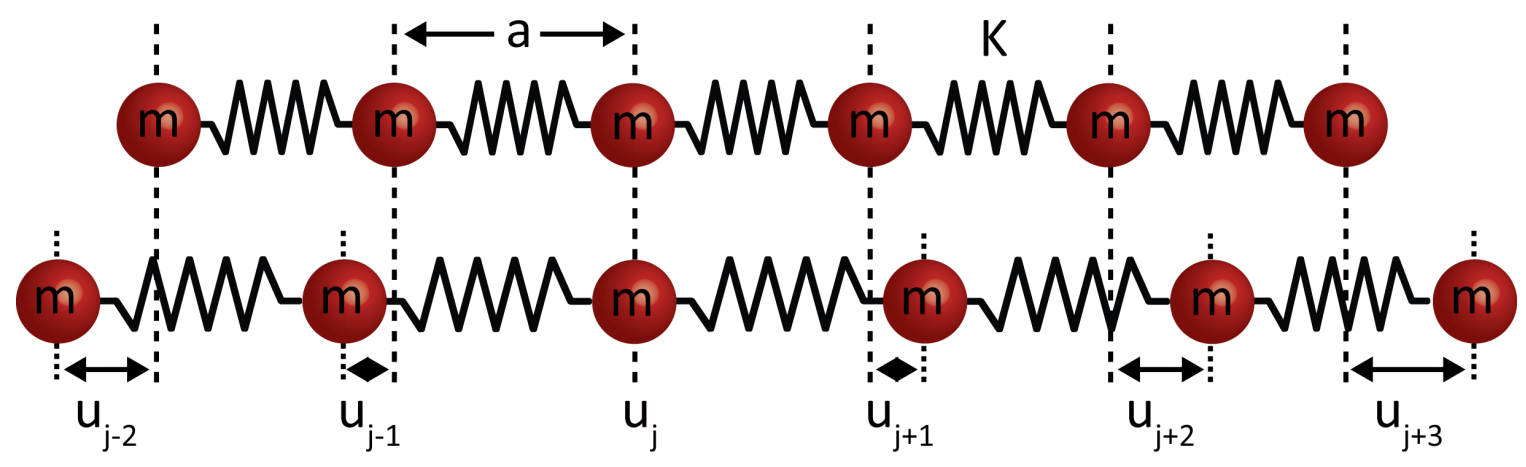

Figure A.8: Schematic of a one-dimensional monatomic chain of atoms. Here $m$ is the mass of the atoms, $K$ is the force constant between atoms, $a$ is the equilibrium interatomic spacing, and $j$ is the index of the atom. The top row of atoms is the equilibrium configuration, with the lower row depicting atoms displaced from equilibrium by a distance $u_{j+i}$.

between atoms, and 4) the average displacement of the atoms is less than their interatomic spacing, $a$.

Now considering the motion of atoms, if we move the $j^{\text {th }}$ atom from its equilibrium position, $x_{j}^{o}$, to a new position $x_{j}$, we can define the atomic displacement $u_{j}$ by:

$$
u_{j}=x_{j}-x_{j}^{o}
$$

When an atom in the linear chain is displaced, the "spring" potential between the atoms introduces a restoring force which tries to return the atoms to their equilibrium positions. Assuming only nearest-neighbor interactions, the restoring force on an atom $j$, arises from the displacement of the atoms on either side, i.e. the displacement of atom $j+1$ relative to atom $j$, and the displacement of atom $j-1$ relative to atom $j$. Using Newtonian mechanics ${ }^{8}$, the net force on atom $j$ is given by [33]:

$$
F_{m}=K\left(u_{j+1}-u_{j}\right)-K\left(u_{j}-u_{j-1}\right)
$$

Applying Newton's second law to Equation A.12 we have:

$$
F_{m}=m \frac{d^{2} u_{j}}{d t^{2}}=K\left(u_{j+1}-u_{j}\right)-K\left(u_{j}-u_{j-1}\right)
$$

\footnotetext{
${ }^{8}$ This could also be interpreted via quantum mechanics, but again the more simplistic classical treatment will suffice to elicit the phonon physics of interest.
} 


$$
m \frac{d^{2} u_{j}}{d t^{2}}=K\left[-2 u_{j}+u_{j+1}+u_{j-1}\right]
$$

Equation A.14 is a special form of the wave equation given by [33]:

$$
m \frac{\partial^{2} u}{\partial t^{2}}=K a^{2} \frac{\partial^{2} u}{\partial x^{2}}
$$

Therefore we can seek solutions of the form of normal modes of oscillation, i.e:

$$
u(j a, t) \propto u e^{i(\vec{k} j a-\omega t)}
$$

where $\vec{k}$ is the wavevector, $j a$ is the discrete location of the $j^{\text {th }}$ atom ${ }^{9}, \omega$ is the phonon frequency, and $t$ is the time in the usual sense. Substituting Equation A.16 into Equation A.14 we have:

$$
\begin{gathered}
-m \omega^{2} u e^{i(\vec{k} j a-\omega t)}=-K\left[2 u e^{i(\vec{k} j a-\omega t)}-u e^{i(\vec{k}(j+1) a-\omega t)}-u e^{i(\vec{k}(j-1) a-\omega t)}\right] \\
m \omega^{2} u e^{i(\vec{k} j a-\omega t)}=K u e^{i(\vec{k} j a-\omega t)}\left[2-e^{-i \vec{k} a}-e^{i \vec{k} a}\right] \\
m \omega^{2}=K\left[2-e^{-i \vec{k} a}-e^{i \vec{k} a}\right]
\end{gathered}
$$

Switching notation to sines and cosines we have:

$$
\begin{gathered}
m \omega^{2}=K[2-(\cos k a-i \sin k a)-(\cos k a+i \sin k a)] \\
m \omega^{2}=2 K[1-\cos k a] \\
\omega(\vec{k})=\sqrt{\frac{2 K(1-\cos k a)}{m}}
\end{gathered}
$$

Finally using the trigonometric identity $\sin ^{2} \theta=\frac{1}{2}(1-\cos 2 \theta)$, we have:

$$
\omega(\vec{k})=2 \sqrt{\frac{K}{m}}\left|\sin \frac{1}{2} k a\right|=\omega_{c}\left|\sin \frac{1}{2} k a\right|
$$

where $k$ is the wavevector, $K$ is the spring constant, $m$ is the atomic mass, $a$ is the in-

\footnotetext{
${ }^{9}$ The factor $j a$ illustrates the discrete nature of the solutions due to the discrete spacing between the atoms, as composed to the more traditional solution to the wave equation which is continuous in $x$.
} 
teratomic spacing and $w_{c}$ is defined as the cutoff frequency. Equation A.23, is the phonon dispersion relation that we sought, which is sometimes referred to as the sine-type dispersion relation.

The phonon dispersion relation from Equation A.23 is plotted in Figure A.9 in the range of $-4 \pi / a \leq k \leq 4 \pi / a$. One striking observation from Figure A.9 is that the phonon dispersion relation is repetitive over the range of $2 \pi / a$. As discussed previously in Section A.2.2, all unique wave information for the crystal is contained within the first Brillouin zone. Therefore, it is typically not necessary to plot the phonon dispersion relation beyond the first Brillouin zone, see Figure A.10. Typically of greatest importance when analyzing the phonon dispersion relation is the behavior of phonons in the long and short wavelength limits, and the slope of the dispersion relation, which provides the phonon group velocity as a function of wavevector.

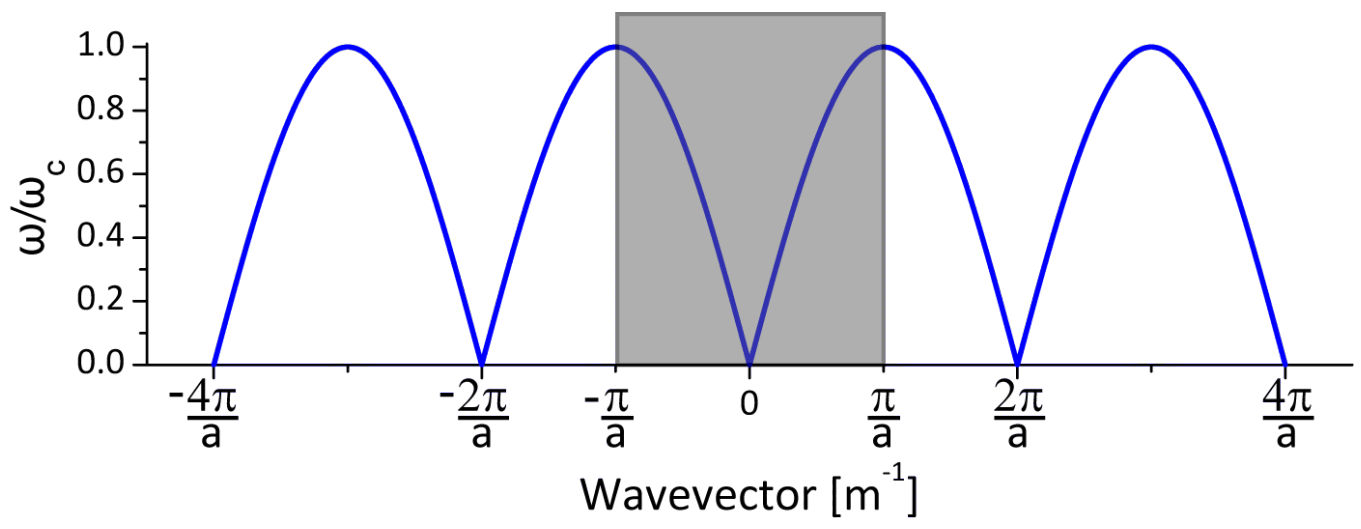

Figure A.9: Phonon dispersion relation assuming a sine-type relation, as in Equation A.23, extended from $-4 \pi / a \leq k \leq 4 \pi / a$.

\section{A.4.2 Phonon Behavior in the Brillouin Zone}

A useful exercise in understanding the behavior of phonons in a crystal, is to consider their properties, particularly the slope of the dispersion curve, at the extremes of the Brillouin zone. 


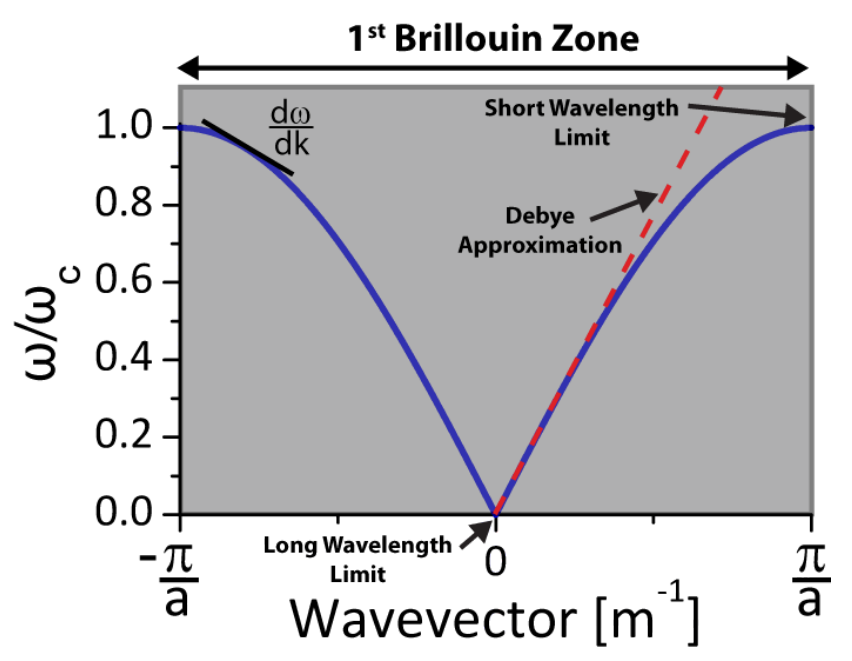

Figure A.10: Phonon dispersion relation assuming a sine-type relation, as in Equation A.23, within the extent of the first Brillouin zone.

\section{Long Wavelength Limit - Brillouin Zone Center}

We begin by considering the long phonon wavelength limit, which in reciprocal space, relates to small wavevectors, i.e. as $\vec{k}$ approaches zero, also called the zone center. If we consider the slope of the phonon dispersion at the Brillouin zone center we have:

$$
\lim _{\vec{k} \rightarrow 0} \frac{\partial \omega}{\partial \vec{k}}=\lim _{\vec{k} \rightarrow 0} \frac{\partial}{\partial k}\left[2 \sqrt{\frac{K}{m}}\left|\sin \frac{1}{2} k a\right|\right]=a \sqrt{\frac{K}{m}}
$$

Integrating we have:

$$
\omega=a \sqrt{\frac{K}{m}}|\vec{k}|=c \vec{k}
$$

where $c$ is the speed of sound in the crystal $=a \sqrt{\frac{K}{m}}$. This implies that longitudinal acoustic phonons ${ }^{10}$ at the Brillouin zone center, travel at the speed of sound in the crystal. In certain cases, to simplify analyses, rather than the sine-type dispersion (see Equation A.23), the phonon dispersion is assumed to be linear, with a slope equal to the speed of sound in the crystal, as shown by the red dashed line in Figure A.10. This is the so called Debye approximation, which holds well for long phonon wavelengths but breaks down as the phonon wavelength decreases near the Brillouin zone edge. The distribution of phonon

\footnotetext{
${ }^{10}$ The phonons discussed for the one-dimensional monatomic chain are designated as acoustic phonons due to having a similar frequency and group velocity to sound waves in a material. Later when discussing the diatomic chain we will compare these to optical phonons.
} 
wavelengths, i.e. more long or short, is a function of temperature, through the statistical mechanical application of the Bose-Einstein distribution. Typically, more long phonon wavelengths will be present at low temperatures with shorter wavelength phonons becoming more prevalent at higher temperatures. Therefore, the Debye assumption is typically more valid for transport at low temperature, breaking down as temperature increases. When describing energy storage, the Debye assumption is asymptotically correct in the high and low temperature limits, and is quite accurate at intermediate temperatures.

\section{Short Wavelength Limit - Brillouin Zone Edge}

At the opposite end of the spectrum, the Brillouin zone edge, the slope of the dispersion relation is given by:

$$
\lim _{\vec{k} \rightarrow \frac{\pi}{a}} \frac{\partial \omega}{\partial \vec{k}}=\lim _{\vec{k} \rightarrow \frac{\pi}{a}} a \sqrt{\frac{K}{m}}\left|\cos \frac{1}{2} k a\right|
$$

Which evaluates in the limit as $\vec{k}$ approaches the edge of the Brillouin zone as:

$$
\frac{\partial \omega}{\partial \vec{k}}=0=v_{g}
$$

where $v_{g}$ is the phonon group velocity. Physically, this indicates that phonons are nonpropagating at the Brillouin zone edge. That is to say, that phonons at the Brillouin zone edge form standing waves, with the atoms in the linear chain oscillating completely out of phase with each other.

\section{Interpretation in 3-dimensional Space}

Thus far, the simple example of a one-dimensional chain of atoms has served well to elicit some of the properties of phonons within the Brillouin zone, and provide a formulation of the dispersion relation for longitudinal acoustic phonons. Left unresolved however, is a discussion on how these formulations will change when considering a three-dimensional crystal structure. Potentially the largest physical change when considering phonons in a multi-dimensional space is the ability of the atoms to vibrate in more than one direction. As the analysis is extended into three dimensions, rather than single atoms vibrating independently of each other, entire planes of atoms will be displaced. This concept can be difficult 
to visualize in three dimensions, but can be readily visualized in two-dimensional space, as was alluded to in Figure A.7, when the types of phonon polarizations were discussed. Beyond the longitudinal mode that follows parallel to the wavevector, $\vec{k}$, one can envision two additional directions orthogonal to the longitudinal mode. These additional modes are defined as the transverse acoustic modes.

In these additional orthogonal directions one can imagine there will be different force constants and interatomic distances. To understand the varying interatomic spacing, recall Figure A.3, which showed the 14 types of Bravais lattices, and we notice that there will be different lattice constants, $a$, in the different crystallographic directions, dependent on the type of lattice. To understand the change in force constant, $K$, we again turn to a formulation of classical mechanics. Under the same assumptions that the dispersion relation was derived, i.e. small displacements, Hooke's law states that the strain and stress in an elastic solid are linearly related [306]. From this relation, along with some simplifying assumptions, it is possible to determine the phonon group velocities in the primary crystallographic directions. It is not necessary to belabor the derivations here, however Figure A.11 provides the proportionality of the elastic stiffness constants, $C_{i j}$, for each phonon polarization in the three primary crystallographic directions. It is this variation in elastic force constants in the primary crystallographic directions that vary the form of the dispersion relation for

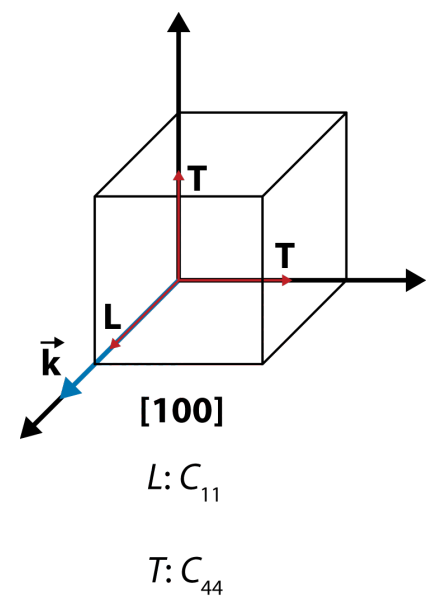

(a)

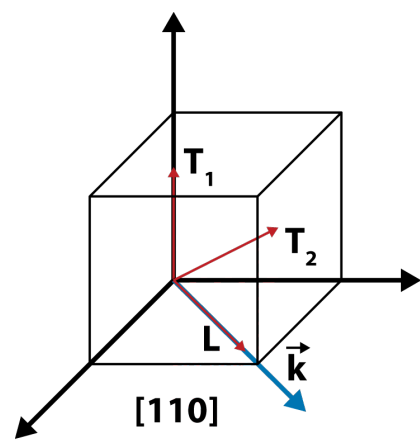

$L: 1 / 2\left(C_{11}+C_{12}+2 C_{44}\right)$

$T_{1}: C_{44}$ $T_{2}: 1 / 2\left(C_{11}-C_{12}\right)$

(b)

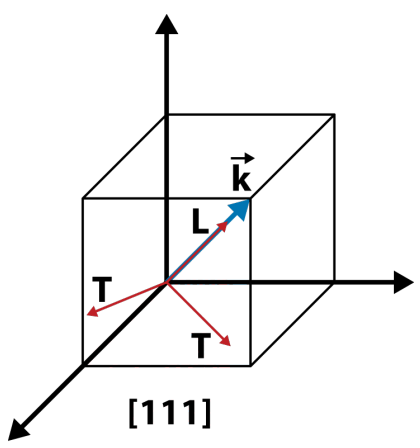

$L: 1 / 3\left(C_{11}+2 C_{12}+4 C_{44}\right)$

$T: 1 / 3\left(C_{11}-C_{12}+C_{44}\right)$

(c)

Figure A.11: Elastic stiffness constants, $C_{i j}$, for the longitudinal and transverse phonon polarizations in the 3 primary crystallographic directions. Note that in the [100] and [111] directions, the transverse polarization modes are degenerate. 
each phonon branch and/or direction. The formulation that was derived in Section A.4.1 for the one-dimensional monatomic case is still valid however, the steps simply must be repeated with the appropriate force constant $K$, and lattice constant $a$, when treating each phonon polarization and/or crystallographic direction.

To illustrate the full three-dimensional phonon dispersion relation, the phonon dispersion of solid argon at $0 \mathrm{~K}$ is shown in Figure A.12, for the crystallographic directions of high symmetry. Note that as indicated in Figure A.11, for the [100] and [111] crystallographic directions, the transverse phonon polarizations are degenerate.

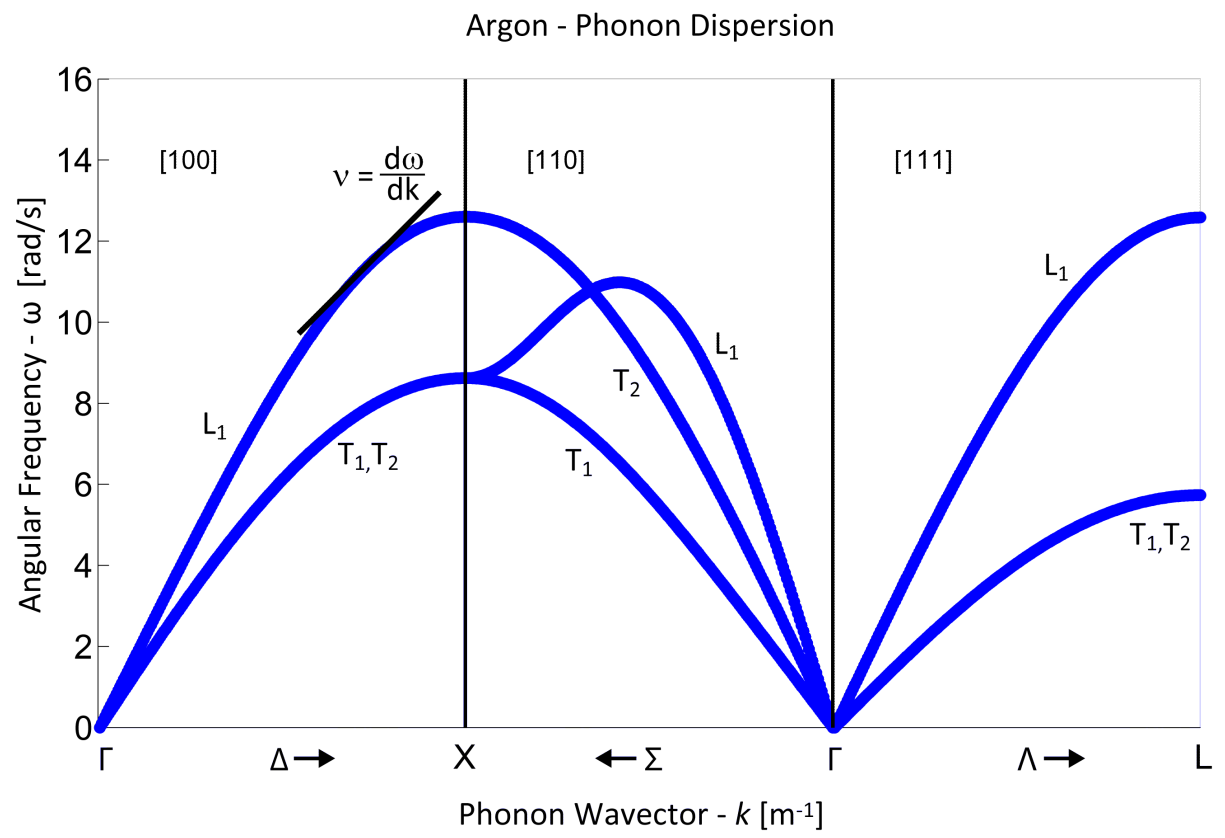

Figure A.12: The phonon dispersion relation for solid Argon in the directions of high symmetry at $0 \mathrm{~K}$, modeled with the lattice dynamics program, General Utility Lattice Program (GULP) [13].

\section{A.4.3 Vibrations of a 1D Lattice with a Diatomic Basis}

Thus far, we have only considered the simplest of examples, the one-dimensional chain of like atoms, with a monatomic basis. We will now increase the complexity by switching to a diatomic basis, on a one-dimensional chain, see Figure A.13. Here we have alternating atoms of mass $m$ and $M$, and we will assume $m<M$. In terms of the force constant $K$, we are able to assume that it is either the same between each atom, or alternates as well. For the sake of simplicity, we will assume that $K$ is uniform throughout the chain. A more 


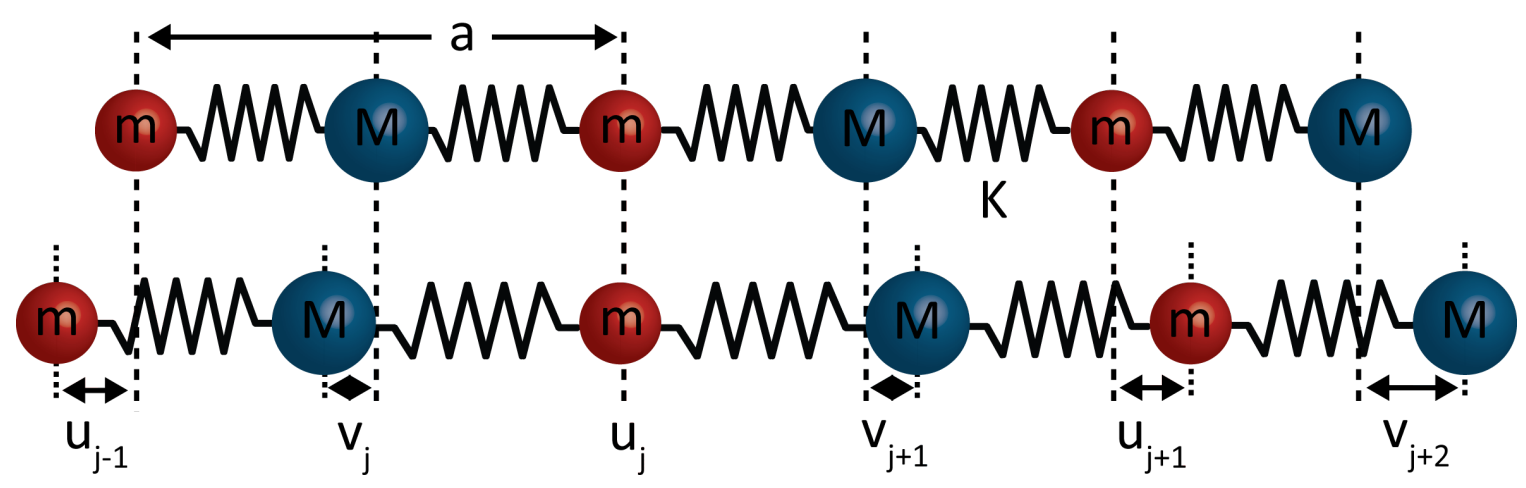

Figure A.13: Schematic of a one-dimensional diatomic chain of atoms. Here $m$ and $M$ are the masses of the two types of atoms, $K$ is the force constant between atoms, $a$ is the equilibrium interatomic spacing, and $j$ is the index of the atom. The top row of atoms is the equilibrium configuration, with the lower row depicting atoms displaced from equilibrium by a distance $u_{j+i}$ and $v_{j+i}$.

detailed view of the various permutations of mass and force constant combinations can be found in Dove [307].

Similar to the monatomic chain, expressions can be developed for the force on each type of atom:

$$
\begin{aligned}
& m \frac{d^{2} u_{j}}{d t^{2}}=K\left[-2 u_{j}+v_{j}+v_{j-1}\right] \\
& M \frac{d^{2} v_{j}}{d t^{2}}=K\left[-2 v_{j}+u_{j+1}+u_{j}\right]
\end{aligned}
$$

Again, we seek solutions of the form of normal traveling waves:

$$
\begin{aligned}
& u(j a, t) \propto u e^{i(\vec{k} j a-\omega t)} \\
& v(j a, t) \propto v e^{i(\vec{k} j a-\omega t)}
\end{aligned}
$$

Inserting Equations A.29a-A.29b into Equations A.28a-A.28b we have:

$$
\begin{gathered}
-M u \omega^{2}=K v\left(1+e^{-i k a}\right)-2 K u \\
-m v \omega^{2}=K u\left(1+e^{i k a}\right)-2 K v
\end{gathered}
$$


These two equations have solutions when their determinant is zero, therefore we have:

$$
\left|\begin{array}{cc}
2 K-m \omega^{2} & -K\left[1+e^{i k a}\right] \\
-K\left[1+e^{i k a}\right] & 2 K-M \omega^{2}
\end{array}\right|=0
$$

or

$$
m M \omega^{4}-2 K(m+M) \omega^{2}+2 K^{2}(1-\cos k a)=0
$$

Solving for $\omega$ we find the dispersion relation for the diatomic case:

$$
\omega(\vec{k})=\left[\frac{K(m+M)}{m M} \pm \frac{1}{2 m M} \sqrt{4 K^{2}(m+M)^{2}-8 m M K^{2}(1-\cos 2 k a)}\right]^{1 / 2}
$$

While still solvable, we immediately notice that the complexity of Equation A.33 is significantly increased as compared to the monatomic dispersion relation given by Equation A.23.

\section{A.4.4 Phonon Behavior in the Brillouin Zone}

As with the case of the monatomic chain, the behavior of phonons within certain regions of the Brillouin zone is potentially more telling than considering the entire dispersion itself. Therefore, we will again take a more in depth look at the behavior of phonons at the center and edge of the Brillouin zone.

\section{Long Wavelength Limit - Brillouin Zone Center}

Looking first at the Brillouin zone center, i.e. as $\vec{k} \rightarrow 0$, Equations A.30a and A.30b reduce to:

$$
\begin{aligned}
& -M u \omega^{2}=2 K v-2 K u \\
& -m v \omega^{2}=2 K u-2 K v
\end{aligned}
$$

This set of equations only has solutions when the determinant of the coefficients is zero, i.e:

$$
\left|\begin{array}{cc}
1-\frac{M \omega^{2}}{2 K} & -1 \\
1 & -\left(1-\frac{m \omega^{2}}{2 K}\right)
\end{array}\right|=0
$$


From the binomial factor in $\omega$, we know that there will be two solutions, each of which we will use to define a different phonon branch. Solving for the determinant we have:

$$
1-\frac{M \omega^{2}}{2 K}-\frac{m \omega^{2}}{2 K}+\frac{m M \omega^{2}}{4 K^{2}}=1
$$

Immediately, we see that a solution exits when $\omega=0$. This is the same result that was observed for the monatomic case, see Figure A.10, as $\vec{k}$ approaches zero, the phonon frequency does as well. This is indicative of the acoustic branch that was defined in the monatomic case. Continuing to solve Equation A.36 for $\omega$ we have:

$$
\begin{gathered}
\frac{\omega^{2}}{2 K}(M+m)=\frac{m M \omega^{2}}{2 K}\left(\frac{\omega^{2}}{2 K}\right) \\
\omega=\sqrt{\frac{2 K}{m M}(M+m)}
\end{gathered}
$$

This solution will be designated as part of the optical branch, see Figure A.15. Comparing the acoustic and optical branches, the atoms in an acoustic wave oscillate in phase with each other, see Figure A.14b, similar to sounds waves, hence the acoustic designation. The atoms in an optical branch however oscillate out of phase with each other, see Figure A.14a, at a frequency close to the infrared portion of the electromagnetic spectrum, and can therefore be excited by photons and are hence designed optical phonons [306].

\section{Short Wavelength Limit - Brillouin Zone Edge}

Now moving to the Brillouin zone edge for $k a= \pm \pi$ we have:

$$
\begin{gathered}
-M u \omega^{2}=K v\left(1+e^{-i \pi}\right)-2 K u \\
-m v \omega^{2}=K u\left(1+e^{i \pi}\right)-2 K v
\end{gathered}
$$

from which we can find two solutions:

$$
\omega=\sqrt{\frac{2 K}{M}}
$$




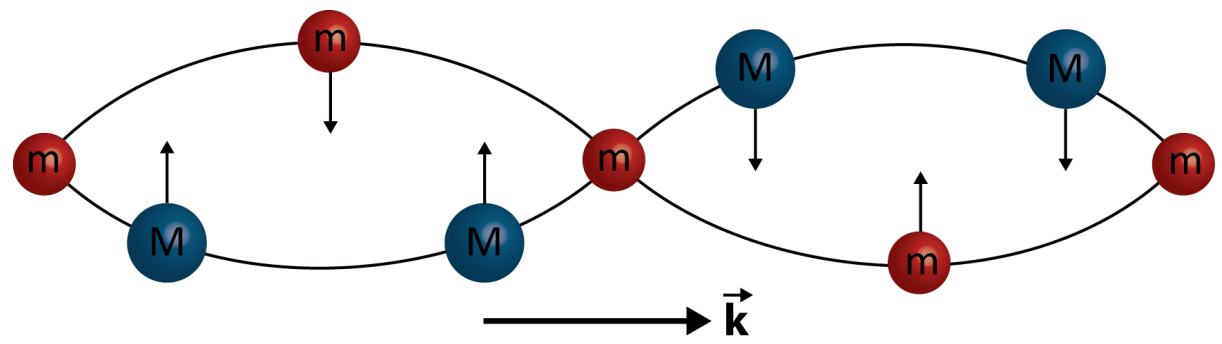

(a) Optical Mode

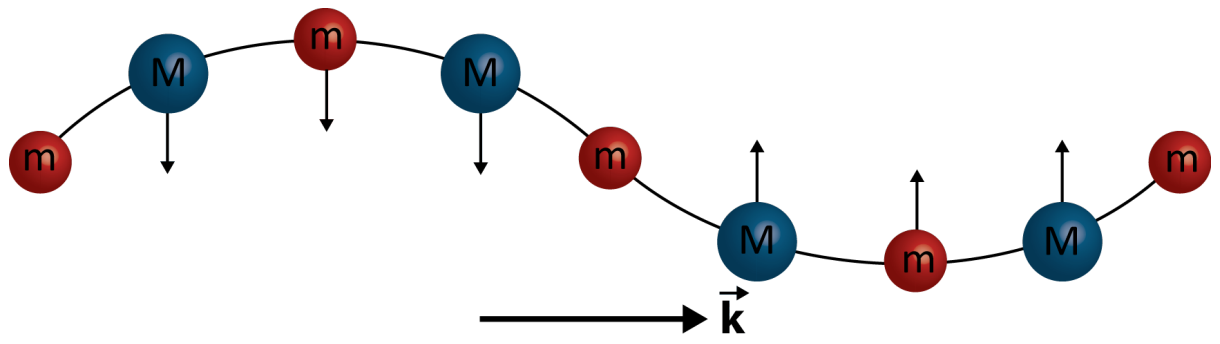

(b) Acoustic Mode

Figure A.14: Schematic of the motion in (a) a transverse optical mode, i.e the two masses moving $180^{\circ}$ out of phase with each other, and (b) a transverse acoustic mode, i.e. the two masses moving in phase with each other, assuming an equal wavelength for both modes.

$$
\omega=\sqrt{\frac{2 K}{m}}
$$

The difference between these solutions represents a phononic bandgap between the optical and acoustic branches, the magnitude of which is proportional to the mass difference between the two atoms in the diatomic chain ${ }^{11}$, see Figure A.15.

\section{A.4.5 Generalized Discussion 3D Crystal with Z Atoms in the Basis}

We have already mentioned that for three-dimensional systems, we have one longitudinal mode, and two transverse modes which may or may not be degenerate depending on the crystal system and orientation. With the addition of an atomic basis greater than one, we saw the splitting of the phonon dispersion into acoustic and optical branches. For a crystal system with basis of $Z$ atoms, there will be a total of $3 Z$ branches, 3 of which are always acoustic branches (containing 1 longitudinal and 2 transverse polarizations). There are a total of $3(Z-1)$ optical branches with $2(Z-1)$ of those branches being transverse

\footnotetext{
${ }^{11}$ In the other permutations of this derivation, i.e. for the possible combinations of mass and spring constants, it is possible for the magnitude of the phononic bandgap to be proportional to $K$ or both $m(M)$ and $K$.
} 


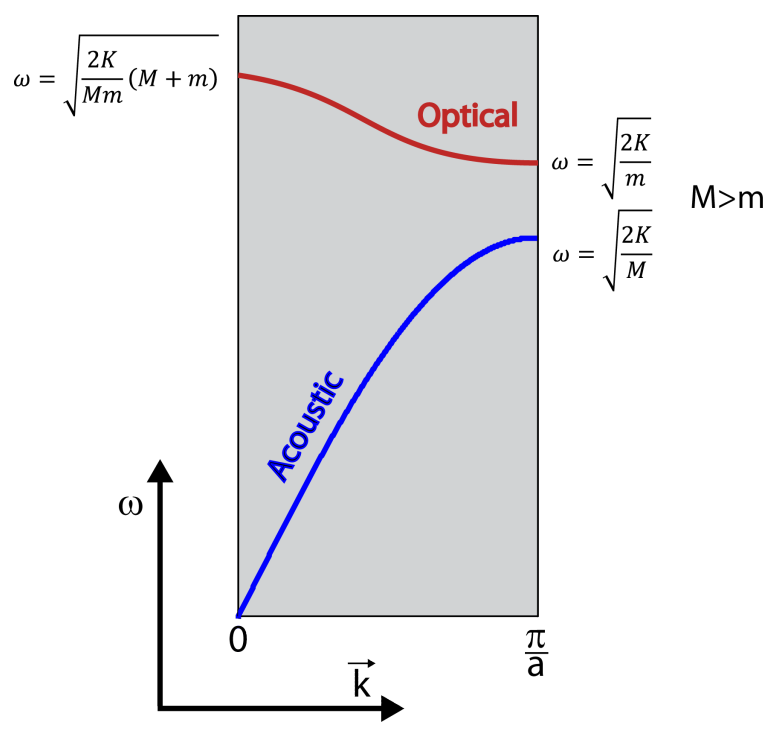

Figure A.15: Phonon dispersion in half of the Brillouin zone, for a diatomic chain of atoms of masses $m$ and $M$, connected by massless springs of force constant $K$. The acoustic branch is shown in blue and the optical branch in red, with the cutoff frequencies at the Brillouin zone boundaries denoted.

polarizations and the remaining being longitudinal polarization(s). As an example, we will consider $\mathrm{Si}$, which forms a face-centered cubic lattice with a basis of $Z=2$. We therefore expect a total of 6 branches, 3 acoustic (as with all 3D crystals) and 3 optical (1 longitudinal optical and 2 transverse optical). Figure A.16 provides the phonon dispersion relation of Si taken from literature [26]. The plotted dispersion does follow the relation for phonon branches, with 6 total branches, 1 of longitudinal polarization and 2 of transverse polarization for both the optical and acoustic branches, with the transverse polarizations being degenerate in the [100] and [111] crystallographic directions, as shown in Figure A.11.

While this methodology of determining the number, and types, of phonon branches present in the dispersion curve, based on the atomic basis, $Z$, has been straightforward, the expansion to the diatomic basis has greatly complicated the analysis of the equations of motion, to develop the dispersion curves. The dispersion relation of real three-dimensional systems is typically calculated through lattice dynamics calculations, which uses the properties of a dynamical matrix, the reciprocal space equivalent of a real space force constant matrix of an atomic system, to determine the frequency and displacement of the phonons. A general introduction to lattice dynamics calculations can be found in Dove [307]. 


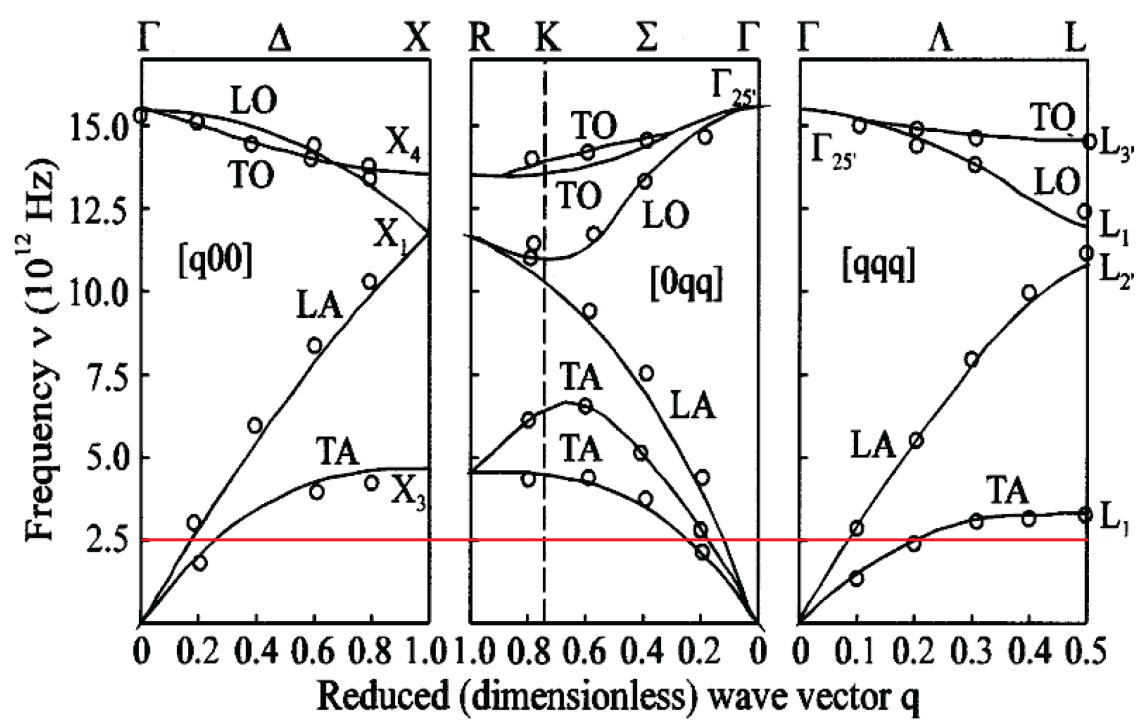

Figure A.16: Phonon dispersion of $\mathrm{Si}$ in the directions of high symmetry, image from [26]. The lines are calculated from [26], and the circles are experimental data. Note: The frequency is given in terms of $\nu$ which is related to $\omega$ by a factor of $2 \pi$, and the wavevector is denoted $q$, which is equivalent to $k$ in the notation of this dissertation.

The development of the phonon dispersion relation provides important information regarding the types, frequencies, speeds, and behaviors of phonons in a crystal system. It is an important component in understanding how the configuration of atoms in a lattice structure influences the phononic properties of a material. The dispersion relation alone however does not provide all the necessary information to discuss phonon transport. We will now discuss how energy level degeneracy is described through the phonon density of states, and later discuss the interaction of phonons with other crystal structures and other phonons.

\section{A.5 Phonon Density of States}

One of the important lessons of phonon physics that can be taken away from the dispersion relation, is that there can be multiple wavevectors, $\vec{k}$, that can correspond to the same phonon frequency, and therefore the same phonon energy. This is illustrated by the red line shown in Figure A.16. This concept is represented in Figure A.17, with a constant energy sphere represented in red, where there are numerous combinations of directional wavevectors, $k_{x, y, z}$, that can reach the energy surface. 


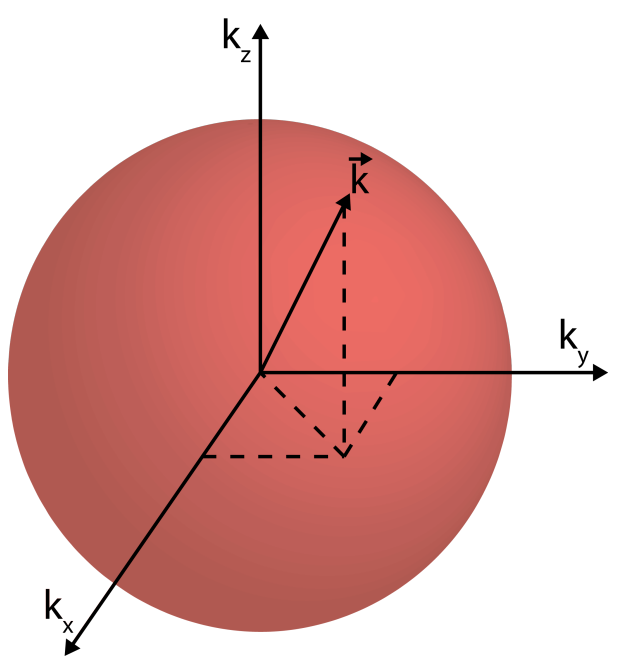

Figure A.17: Schematic representation a constant energy surface in k-space.

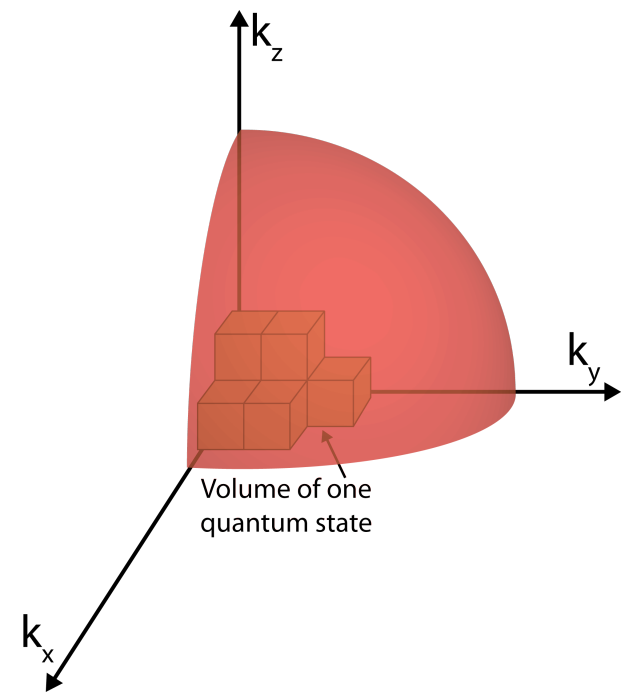

(a)

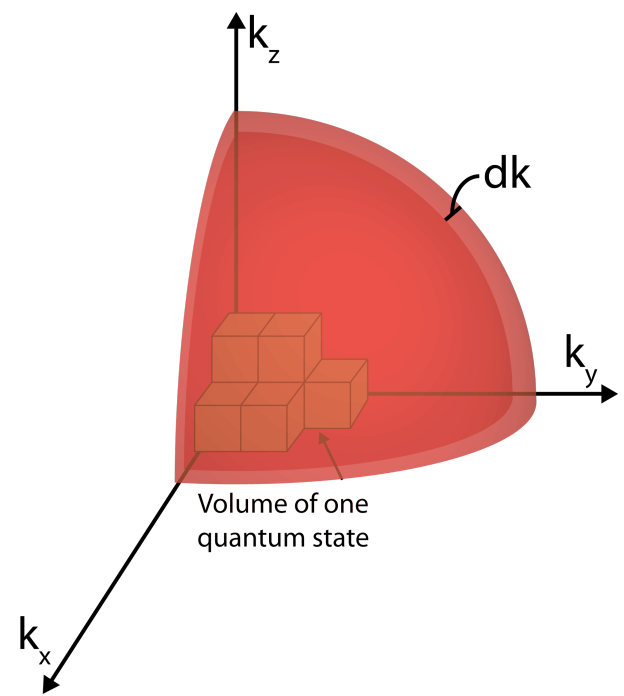

(b)

Figure A.18: Schematic of the first quadrant of a constant energy surface in k-space depicting (a) the determination of the number of quantum states that fit into that volume and (b) the differential expansion of the spherical volume in $\mathrm{k}$-space.

To understand the behavior of phonons and their relation to heat transfer, it is helpful to determine, for a given frequency, the number of quantum states that correspond to that energy level. To accomplish this, we are essentially interested in determining the number of quantum states that "fit" into a spherical volume in k-space, see Figure A.18a. Due to the fact that the energy levels in solids are quasi-continuous, we will look for the number of states between a wavevector $k$ and $k+d k$, see Figure A.18b. Therefore, for an infinitesimal 
change in the wavevector, $k$, we have:

$$
\frac{d N}{d k_{V}}=\text { Modes per unit volume in } k \text {-space }
$$

In three-dimensional space, the unit volume in $\mathrm{k}$-space is give by:

$$
d k=d k_{x} d k_{y} d k_{z}=\left(\frac{2 \pi}{L}\right)\left(\frac{2 \pi}{L}\right)\left(\frac{2 \pi}{L}\right)=\left(\frac{2 \pi}{L}\right)^{3}
$$

Therefore, for one mode per unit volume, we have:

$$
\frac{d N}{d k_{V}}=\frac{1}{\left(\frac{2 \pi}{L}\right)^{3}}
$$

Separating and integrating:

$$
\int d N=\int\left(\frac{L}{2 \pi}\right)^{3} d k_{V}
$$

where, assuming a spherical Brillouin zone, we have:

$$
\begin{gathered}
d k_{\text {volume }}=k_{r}^{2} \sin \theta d k_{r} d \theta d \phi \\
N(\vec{k})=\frac{V}{8 \pi^{3}} \int_{0}^{2 \pi} \int_{0}^{\pi} \int_{0}^{k} k_{r}^{2} \sin \theta d k_{r} d \theta d \phi
\end{gathered}
$$

Completing the integration:

$$
N(\vec{k})=\frac{V}{6 \pi^{2}} k^{3}
$$

where $N(\vec{k})$ is the \# of states between $k$ and $k+d k$ per polarization and $V$ is the volume of the quantum state. We will denote $n(\vec{k})$ as the \# of states between $k$ and $k+d k$ per unit volume per polarization, i.e.:

$$
n(\vec{k})=\frac{k^{3} d k}{6 \pi^{2}}
$$

Finally, the \# of states between $k$ and $k+d k$ per unit volume, per unit wavevector, per polarization, which is what is typically meant when referring to the phonon density of states (PDOS), in k-space, is given by:

$$
D(\vec{k})=\frac{k^{2}}{2 \pi^{2}}
$$


The advantage of deriving the PDOS in k-space, is that to this point we have made no assumptions about the form of the dispersion relation. During application, the PDOS can be converted to a function of $\omega$ through the dispersion relation.

\section{A.5.1 Debye Assumptions}

The simplest example, which will be shown for clarity, is the application of the Debye dispersion relation, which is given by:

$$
\omega=v_{D} k
$$

where $v_{D}$ is the Debye velocity. Starting from $n(\vec{k})$, and inserting the Debye dispersion solved in terms of $k$ and $d k$, we have:

$$
n(\omega)=\frac{1}{2 \pi^{2}}\left(\frac{\omega}{v_{D}}\right)^{2} \frac{1}{v_{D}} d \omega
$$

Simplifying and converting to the \# of states between $\omega$ and $\omega+d \omega$ per unit volume, per unit frequency, per polarization, the PDOS under the Debye assumption is given by:

$$
D(\omega)=\frac{\omega^{2}}{2 \pi^{2} v_{D}^{3}}
$$

Using a similar process, the pDOS assuming a sine-type phonon dispersion relation, see Equation A.33, is given by:

$$
D(\omega)=\frac{2}{a^{3} \pi^{2} \sqrt{\omega \omega_{c}}} \frac{\left(\sin ^{-1}\left[\sqrt{\frac{\omega}{\omega_{c}}}\right]\right)^{2}}{\sqrt{1-\frac{\omega}{\omega_{c}}}}
$$

Figure A.19a shows the PDOS, under the Debye assumption, for FCC Cu. As expected from Equation A.53, the PDOS has a quadratic dependence on $\omega$. Figure A.19b shows the PDOS of solid Ar at $0 \mathrm{~K}$, calculated from lattice dynamics using GULP [13]. Since both $\mathrm{Cu}$ and solid Ar are FCC structures, we would anticipate similar trends in the PDOS. At frequencies below $6 \mathrm{THz}$, the PDOS in Figure A.19b shows close to $\omega^{2}$ dependence, similar to the Debye model. However, above $6 \mathrm{THz}$ there is significant deviation from the $\omega^{2}$ trend. 


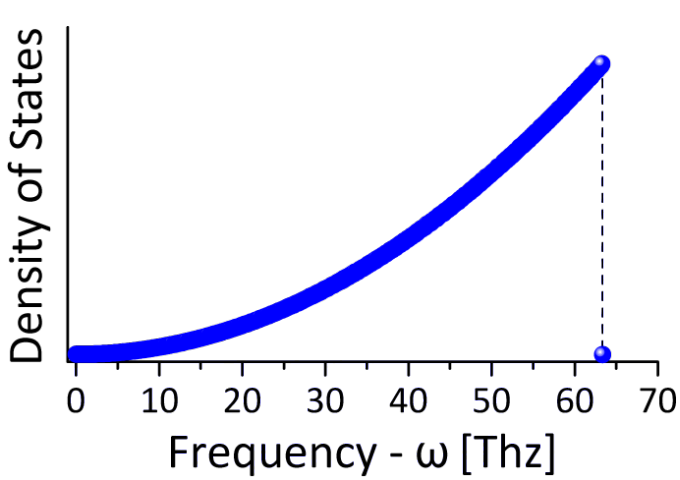

(a)

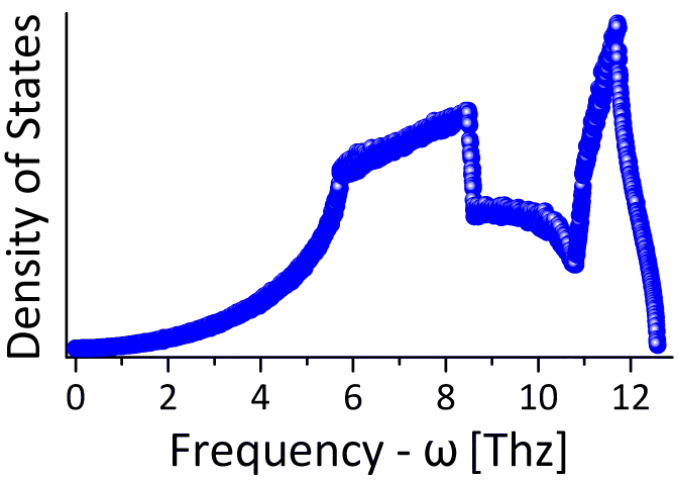

(b)

Figure A.19: (a) Debye density of states for FCC Cu from Equation A.53 and (b) DOS of solid Ar at $0 \mathrm{~K}$ calculated from lattice dynamics, GULP [13].

This is due to the deviation of the Debye (linear) model from the actual dispersion at high frequencies.

The phonon density of states provides a quantification to the number of available wavevectors, $k$, or frequencies, $\omega$, that correspond to the same phonon energy. A high value of the density of states at a given frequency implies there are many states that can be occupied with that energy. Conversely, a density of states of zero at a given frequency indicates that no phonons can occupy that energy level. However, while the PDOS provides a quantification of how many, and what frequencies are possible, it alone does not describe what phonons are present in a system. How the phonons are distributed within the PDOS at a given temperature is determined from Bose-Einstein statistics. The product of the PDOS with the statistical distribution of phonon energies, i.e. the Bose-Einstein distribution, is called the occupied phonon density of states. The occupied phonon density of states is used in the calculation of the phonon energy, heat capacity and thermal transport, which was discussed further in Chapter 2.

A useful quantity in summarizing the phononic properties of a material, to a crude but useful approximation, is the Debye temperature, $\Theta_{D}$, given by [306]:

$$
\Theta_{D}=\frac{\hbar \omega_{D}}{k_{b}}
$$


with $\omega_{D}$, the maximum allowed frequency under the Deybe assumption, given by:

$$
\omega_{D}=\left(\frac{6 \pi^{3} v^{3} N}{V}\right)^{1 / 3}
$$

where $N / V$ is the number of modes per unit volume. The Debye temperature provides an indication of the temperature at which all phonon modes are excited and a system approaches the classical limit. In the same manner, being proportional to the maximum frequency, $\omega_{D}$, the Debye temperature provides an indication to the strength of the bonds in the material. A high Debye temperature material, has a high cutoff frequency $\omega_{D}$, and therefore strong bonds are implied. In the same manner, a low Debye temperature material has a low cutoff frequency implying weak bonds. Examples of several Debye temperatures at room temperature are shown in Table A.1. In Chapters 2 and 7 we will look closer at the role of the Debye temperature in predicting thermal transport.

Table A.1: Room temperature Debye temperatures, $\Theta_{D}$, for various elements. Data taken from [32].

\begin{tabular}{cccc}
\hline Element & $\Theta_{D}[\mathbf{K}]$ & Element & $\Theta_{D}[\mathbf{K}]$ \\
\hline $\mathrm{C}$ & 1860 & $\mathrm{Nb}$ & 275 \\
$\mathrm{Ti}$ & 380 & $\mathrm{Ag}$ & 215 \\
$\mathrm{Cr}$ & 460 & $\mathrm{In}$ & 129 \\
$\mathrm{Ni}$ & 375 & $\mathrm{~W}$ & 310 \\
$\mathrm{Al}$ & 394 & $\mathrm{Pt}$ & 230 \\
$\mathrm{Si}$ & 625 & $\mathrm{Au}$ & 170 \\
$\mathrm{Ge}$ & 360 & $\mathrm{~Pb}$ & 88 \\
$\mathrm{Pd}$ & 275 & $\mathrm{Bi}$ & 120 \\
\hline
\end{tabular}

Having established the phonon dispersion relation and the density of states, it is possible to calculate energy properties of a system, such as the phonon energy, $U$, and the heat capacity, $C_{V}$. This will not be belabored here, but can be found in numerous solid-state texts $[32,306,309]$. Since the primary focus of this work is thermal transport, instead a brief focus will be placed on the interaction of phonons within a material system.

\section{A.6 Phonon Scattering}

In the development of the phonon dispersion relation and phonon density of states, we have made the major assumptions that the crystals were perfect infinite periodic structures, 
and that the forces between atoms were harmonic. When considering thermal transport within a system, these assumptions lead to an infinite thermal conductivity. We know that real systems have a finite thermal conductivity, which is due to sources of thermal resistance within the material. To understand how the interactions of phonons with a material, and themselves, affects thermal transport, we will briefly derive an expression for the thermal conductivity of a material, and discuss the various scattering mechanisms which impede phonon transport.

\section{A.6.1 Transport Processes - Boltzmann Transport Equation}

The challenge that arises when we begin to talk about thermal transport processes, is that we are no longer discussing a system at equilibrium. Due to this nonequilibrium state, we can no longer describe the system in terms of an equilibrium distribution function, e.g. the Boltzmann distribution for phonons (Bosons), but rather we must use a nonequilibrium distribution function. Therefore, in order to describe transport, we must have a way to describe the change in the nonequilibrium distribution function as a function of time. The most straightforward way to do this is to consider the position vector $\overrightarrow{\mathbf{r}}$, and the momentum vector $\overrightarrow{\mathbf{p}}$, for a system of $N$ particles. Mathematically this is accomplished through the application of the Liouville equation, and computationally this is part of the basis of molecular dynamics simulations. Both of these methods can be computationally expensive with the Liouville equation requiring $2 p \times N$ variables, where $p$ is the number of degrees of freedom, and molecular dynamics simulations taking on the order of hours to days to complete depending on the domain size.

To simplify the analysis, and significantly reduce the number of variables, Boltzmann considered one particle and its interaction with the rest of the "system", with the properties of the other $(N-1)$ particles being averaged. This simplification of the Liouville equation leads to the Boltzmann Transport Equation (BTE), given by [33]:

$$
\frac{\partial f}{\partial t}+\frac{d \vec{r}}{d t} \cdot \nabla_{r} f+\frac{d \vec{p}}{d t} \cdot \nabla_{p} f=\left(\frac{\partial f}{\partial t}\right)_{c}
$$

where $f$ is the distribution function, $\frac{\partial f}{\partial t}$ is the change in the distribution function with time, 
$\frac{d \vec{r}}{d t} \cdot \nabla_{r} f$ is the change in position, $\frac{d \vec{p}}{d t} \cdot \nabla_{p} f$ is the change in momentum, and $\left(\frac{\partial f}{\partial t}\right)_{c}$ is the "scattering term". Rather then using momentum $\vec{p}$ we can also write the BTE in terms of velocity $(\vec{p}=m \vec{v})$ :

$$
\frac{\partial f}{\partial t}+\vec{v} \cdot \nabla_{r} f+\frac{\vec{F}}{m} \cdot \nabla_{v} f=\left(\frac{\partial f}{\partial t}\right)_{c}
$$

The terms on the left side of the equation account for changes in the distribution function due to outside influences, such as external forces. The term on the right, the scattering term, lumps together the interactions of the one particle with the rest of the system. The challenge in the application of the BTE is the definition of the scattering term. The rigorous way of dealing with the scattering term is to solve the time-dependent Schrödinger equation for the two particle system, or use perturbation theory to solve for a scattering integral. However, due to this complexity, it is common to simplify the analysis of the scattering term by evoking the relaxation time approximation.

The relaxation time approximation assumes that for a quasi-equilibrium system, i.e. small perturbations from equilibrium, there is a characteristic time for the system to return to equilibrium, given by $\tau$, called the relaxation or scattering time, i.e.:

$$
\frac{\partial f}{\partial t}=\frac{f-f_{o}}{\tau}
$$

As we are interested in the distribution of phonons, we will use the Bose-Einstein distribution function, which at equilibrium is given by:

$$
f_{o}=\frac{1}{\exp \left(\frac{\hbar \omega}{k_{B} T}\right)-1}
$$

Additionally, we will define a function $g$ which represents the deviation of the system from an equilibrium state, such that:

$$
f=f_{o}+g
$$

Inserting this formulation into the BTE, see Equation A.58, we have:

$$
\frac{\partial g}{\partial t}+\frac{\partial f_{o}}{\partial t}+\vec{v} \cdot \nabla_{r} f_{o}+\vec{v} \cdot \nabla_{r} g+\frac{\vec{F}}{m} \cdot \nabla_{v} f_{o}+\frac{\vec{F}}{m} \cdot \nabla_{v} g=-\frac{g}{\tau}
$$


Using this formulation of the BTE, we can solve to find an expression for the distribution function, $f$, in terms of a small perturbation from equilibrium, $g$. Assuming perturbations from equilibrium are small, which is generally true for diffusive systems, we can assume the following: 1) the system is at steady state, i.e. the transient terms are negligible, 2) the gradient of $g$ is much smaller than the gradient of $\left.f_{o}, 3\right)$ similarly, $g$ is much smaller than $f_{o}$ and 4 ) because we are dealing with phonons, there are no external forces. Under these assumption, Equation A.62 reduces to:

$$
f=f_{o}-\tau \frac{\partial f_{o}}{\partial T} \vec{v} \cdot \nabla T
$$

where $f$ is the nonequilibrium distribution function, which can then be used to calculate transport phenomenon such as thermal conductivity, which we will now discuss. We will find that the term of major importance that will follow through in the derivation of thermal conductivity, is the scattering time, $\tau$, which we will provide examples of and discuss further in Section A.6.3.

\section{A.6.2 Heat Flux in 1-Dimension - Thermal Conductivity}

We will begin deriving the thermal conductivity by starting with a general expression for heat flux in one dimension [33]:

$$
J_{q x}(x)=\int_{0}^{\omega_{\max }} \int_{0}^{2 \pi} \int_{0}^{\pi} v_{g} \cos \theta \hbar \omega f \frac{D(\omega)}{4 \pi} \sin \theta d \omega d \theta d \phi
$$

where $\omega_{\max }$ is the highest phonon frequency, such as that given by the cutoff frequency of the Debye or sine-type phonon dispersion relation. Substituting the expression for the distribution function that was presented earlier, see Equation A.63, we have:

$$
J_{q x}(x)=\int_{0}^{\omega_{\max }} \int_{0}^{2 \pi} \int_{0}^{\pi} v_{g} \cos \theta \hbar \omega\left[f_{o}-\tau \frac{\partial f_{o}}{\partial T} \vec{v} \cdot \nabla T\right] \frac{D(\omega)}{4 \pi} \sin \theta d \omega d \theta d \phi
$$

If we now integrate over the azimuthal angle and simplify we have:

$$
J_{q x}(x)=-\frac{1}{2} \frac{d T}{d x} \int_{0}^{\omega_{\max }} \int_{0}^{\pi} \tau v_{g}^{2} \sin \theta \cos ^{2} \theta \hbar \omega D(\omega) \frac{\partial f_{o}}{\partial T} d \omega d \theta
$$


The first term in the integration containing $f_{o}$, dropped out because $f_{o}$ represents an equilibrium distribution with equal amount of energy going in opposite directions, and therefore no net flux. Equation A.66 can be related to the thermal conductivity by evoking the Fourier Law, given by [33]:

$$
J_{q x}=-k \frac{d T}{d X}
$$

Therefore we get the following equation for the thermal conductivity:

$$
k=\frac{1}{2} \int_{0}^{\omega_{\max }} \int_{0}^{\pi} \tau v_{g}^{2} C_{\omega} \sin \theta \times \cos ^{2} \theta d \theta d \omega
$$

If we assume that the material properties are isotropic, this reduces to:

$$
k=\frac{1}{3} \int_{0}^{\omega_{\max }} \tau v_{g}^{2} \hbar \omega D(\omega) \frac{d f_{o}}{d T} d \omega
$$

In terms of the energy stored in a crystal, the phonon heat capacity is given by [33]:

$$
C_{\omega}=\int_{0}^{\omega_{c}} \hbar \omega D(\omega) \frac{d f_{o}}{d T} d \omega
$$

Therefore, the thermal conductivity is given by:

$$
k=\frac{1}{3} \int_{0}^{\omega_{\max }} \tau v_{g}^{2} C_{\omega} d \omega
$$

where $\tau$ is the mean phonon scattering time and $v_{g}$ is the phonon group velocity. If we assume that both $\tau$ and $v_{g}$ are independent of frequency, the expression for thermal conductivity is reduced to the classical kinetic relation:

$$
k=\frac{1}{3} C v_{g} l
$$

where $l$ is the phonon mean free path $=\tau v_{g}$. The mean free path by definition is the average distance a phonon travels between collisions with other "objects' such as impurities, defects, other phonons, etc. Therefore, $\tau$ is the average time between successive collisions. It is the frequency of these collisions that reduces the ability to transport thermal energy and 
leads to a finite thermal conductivity. To better understand how phonons scatter, we will look at a few scattering mechanisms, and provide empirical formulations associated with each mechanism to show the functional dependency of the scattering rate, $\tau^{-1}$, on phonon frequency.

\section{A.6.3 Phonon Scattering Events}

\section{Mass-difference Scattering}

The first type of scattering that we will consider is mass-difference scattering, which is due to the presence of a different than average atomic mass within the crystal lattice. This could be due to either the presence of impurity atoms and/or the presence of atomic isotopes. The mass-difference scattering rate is given by [310]:

$$
\frac{1}{\tau_{m}}=\frac{V_{o} \omega^{4}}{4 \pi v_{g}^{3}} \Gamma_{m}
$$

where $V_{o}$ is the atomic volume, $v_{g}$ is the phonon group velocity, and $\Gamma_{M}$ is the form factor, given by [310]:

$$
\Gamma_{m}=f_{i}\left[\left(\frac{\Delta M_{i}}{\bar{M}}\right)^{2}+2\left\{\left(\frac{\Delta G_{i}}{\bar{G}}\right)-6.4 \gamma\left(\frac{\Delta \delta_{i}}{\bar{\delta}}\right)\right\}^{2}\right]
$$

where $f_{i}$ is the fractional proportion of atoms with mass $M_{i}, \bar{M}$ is the average atomic mass, $G_{i}$ is the average atomic stiffness constant between the impurity atom and the nearestneighbor atoms, $G$ is the atomic stiffness constant of the host lattice, $\gamma$ represents the average Grüneisen anharmonicity parameter in the atomic bonds, and $\delta_{i}$ is the cube root of the atomic volume for the $i^{\text {th }}$ impurity atom in its own lattice.

The scattering due to impurities can in theory be reduced by working with high quality materials, however it should be noted that $\mathrm{Si}$ for example, in nature, contains a mixture of 3 primary isotopes, $92 \%{ }^{28} \mathrm{Si}, 4.6 \%{ }^{29} \mathrm{Si}$, and $3.1 \%{ }^{30} \mathrm{Si}[310]$. Due to the strong dependence on phonon frequency, and the ever present atomic isotopes, mass-difference scattering contributes significantly to thermal resistance. 


\section{Phonon-Phonon Scattering}

Phonons can also scatter with other phonons ${ }^{12}$. This process can be categorized by two different types of events depending on the momentum, which is directly proportional to the wavevector, $k$, of the phonon pairs. These are denoted as normal and Umklapp ${ }^{13}$ processes. In a normal process, two phonon of wavevector $k_{1}$ and $k_{2}$ combine ${ }^{14}$ to form a phonon of wavevector $k_{3}$, see Figure A.20a. The combination of these two phonons preserves both energy and crystal momentum, leaving the net direction of phonon transport unchanged. Due to this conservation, normal processes do not alter the dynamic movements of phonons, and therefore normal processes alone, would lead to infinite thermal conductivities.

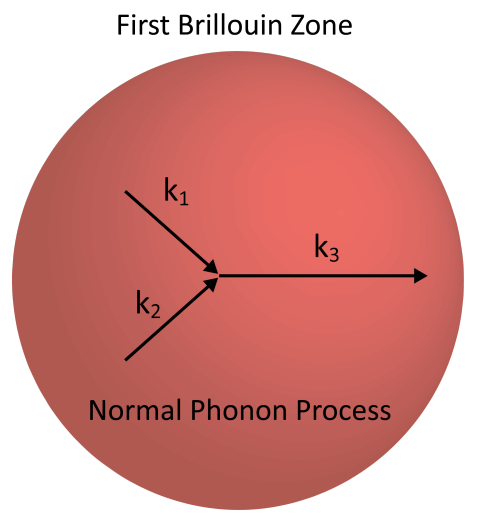

(a)

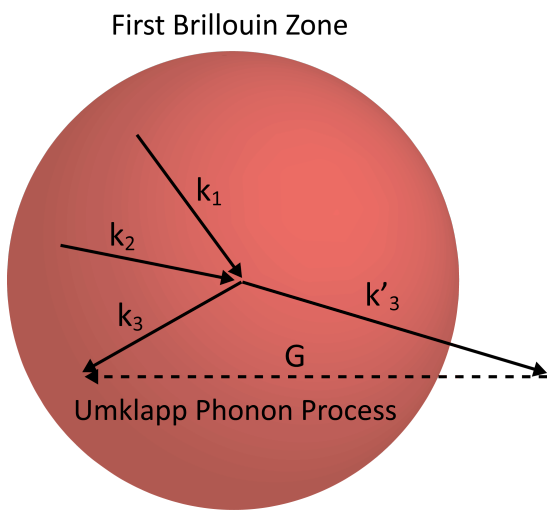

(b)

Figure A.20: Schematic of phonon-phonon scattering processes within the first Brillouin zone for (a) normal phonon processes and (b) Umklapp phonon processes.

In an Umklapp process, two phonon of wavevector $k_{1}$ and $k_{2}$ again combine to form a phonon of wavevector $k_{3}$, however in this case, the wavevector $k_{3}$ falls outside the first Brillouin zone, see Figure A.20b. We recall that phonons with wavevectors outside the first Brillouin zone carry no more information than those within the first zone, because their wavelengths are shorter than the interatomic spacing. However, we can map $k_{3}^{\prime}$ to an equivalent wavevector, $k_{3}$, within the first Brillouin zone through the addition of the reciprocal lattice vector $\overrightarrow{\mathbf{G}}$, see Equation A.9. We note however, see Figure A.20b, that

\footnotetext{
${ }^{12}$ Note that in Section A.4 we derived the dispersion of phonons based on a purely harmonic potential between the atoms, which lead to non-interacting normal modes. In real system, anharmonicity in the interatomic potential lead to interactions between phonons.

${ }^{13}$ The name Umklapp derives from the German word Umklappen, which means to turn over.

${ }^{14} \mathrm{~A}$ phonon-phonon scattering event can also be considered the splitting of a phonon into two phonons of lesser energy.
} 
the resulting wavevector $k_{3}$, has changed direction as compared to the initial phonons. It is this change in the net direction of propagation of the resulting phonon that gives rise to thermal resistance due to phonon-phonon scattering. The rate of Umklapp scattering, $\tau_{U}^{-1}$, can be difficult to calculate but an approximate relation has been given by [311]:

$$
\frac{1}{\tau_{U}} \approx \frac{\hbar \gamma^{2}}{M v_{g}^{2} \theta_{D}} \omega^{2} T \exp \left\{\frac{-\theta_{D}}{3 T}\right\}
$$

where $\gamma$ is the Grüneisen anharmonicity parameter, $M$ is the atomic mass, $v_{g}$ is the phonon group velocity, and $\Theta_{D}$ is the Debye temperature of the material. Due to an increasing population of high $k$ phonons with increasing temperature, Umklapp scattering is the dominant resistive thermal process at high temperatures, for materials with a low defect density.

\section{Matthiessen's Rule}

Mass/impurity and phonon-phonon scattering are but two examples of phonon scattering that limits thermal transport. Phonons can also scatter at boundaries, in low dimensionality structures, with electrons, etc., each with their own unique scattering rates. The combination of these processes leads to the total relaxation time, as presented in the BTE and shown in the derivation of the phononic thermal conductivity. The total relaxation time is often calculated from the combination of individual scattering times ${ }^{15}$, through Matthiessen's Rule, given by [33]:

$$
\frac{1}{\tau_{t}}=\sum_{j} \frac{1}{\tau_{j}}
$$

where $\tau_{j}$ are the individual scattering times for normal processes, Umklapp processes, mass/impurity scattering, etc:

$$
\frac{1}{\tau_{\mathrm{t}}}=\frac{1}{\tau_{n}}+\frac{1}{\tau_{u}}+\frac{1}{\tau_{m}}+\ldots
$$

The magnitude of these scattering rates, at different temperatures and frequencies, provides valuable insight into the dominate processes impacting thermal transport.

\footnotetext{
${ }^{15}$ Matthiessen's rule assumes that the individual scattering mechanisms are linearly independent from each other.
} 


\section{A.7 Appendix Summary}

In this appendix I presented a brief overview into the fundamentals of solid-state physics, as they apply particularly to thermal transport. I began by discussing the types of crystal systems, and developed the concept of a reciprocal space to aid in the mathematical description of large collections of atoms. Considering the vibrational motion of atoms within the crystal lattice, I presented the concept of the phonons, and described how they are related by energy and wavevector in a crystal through the development of the phonon dispersion relation. The types and number of modes available in a material was developed in the phonon density of states, with the occupied density of states describing the distribution of phonon frequencies at a given temperature. Finally, I discussed how phonons traveling in a material interact with features in the material and other phonons. Using the concepts developed, in Chapter 2 we will look specifically at how phonons are transported between two dissimilar materials, and how each materials phononic properties, i.e. dispersion and density of states, impact the flow of thermal energy across a boundary. 


\section{APPENDIX B}

\section{LASER SYSTEM BASICS}

B.1 Reflectivity vs Reflectance . . . . . . . . . . . . . . . . . . 318

\section{B.1 Reflectivity vs Reflectance}

There are sometimes discrepancies in using the terms reflectivity and reflectance to describe the amount of incident light that is reflected at the interface between two materials of differing optical properties. It is generally accepted, and is the convention used in this dissertation, that the reflectivity of a material is an inherent property of the material, while the reflectance is dependent on the particular configuration of the materials comprising the interface. With our particular interest in the interaction of laser energy with thin metallic films, the defining criteria in this case will be the film thickness.

The primary quantity of interest in comparing the use of reflectivity or reflectance in thin films is the optical penetration depth, OPD, given by [23]:

$$
\delta=\frac{\lambda}{4 \pi k_{f}}
$$

where $\lambda$ is the incident photon wavelength and $k_{f}$ is the extinction coefficient of the metallic film. An example of the OPD of a Pt film as a function of incident photon wavelength is 
shown in Figure B.1. For film thicknesses several times the OPD, the metallic film is considered "optically thick" and in terms of optical properties behaves like the bulk element. When the film thickness is on the order of the OPD depth, special consideration must be taken, and the properties of the metallic film alone are not sufficient to accurately represent the reflection of incident photon energy.

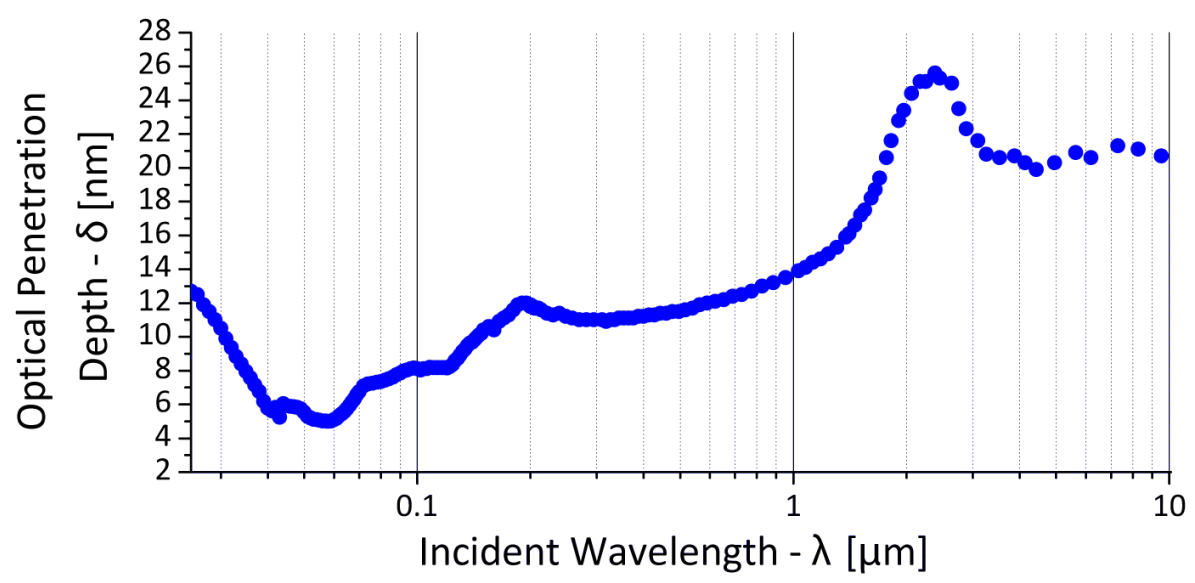

Figure B.1: Optical penetration depth in Pt as a function of incident photon wavelength, calculated using Equation B.1. Optical constant data provided from [27].

When the metallic film interacting with the laser energy is optically thick, the reflectivity as a function of the complex indicies of refraction of the system, assuming normal incidence, is given by [312]:

$$
R_{\mathrm{bulk}}=\frac{\left(n_{1}-n_{o}\right)^{2}+k_{1}^{2}}{\left(n_{1}+n_{o}\right)^{2}+k_{1}^{2}}
$$

where $n_{i}$ and $k_{i}$ are the real and imaginary components of the index of refraction respectively. Therefore, as an example, the bulk reflectivity of a Pt film is given by:

$$
R_{\text {bulk,Pt }}=\frac{(2.79-1)^{2}+4.885^{2}}{(2.79+1)^{2}+4.885^{2}}
$$

$$
R_{\text {bulk,Pt }}=0.7081
$$

However when the metallic film is optically thin, the reflectivity of the film is affected by the optical properties of the substrate, and the relectivity model given in Equation B.2 is modified to take into account these properties. The reflectivity of the optically thin system 
is given by [313]:

$$
\begin{gathered}
R_{\text {thin }}=\frac{a b e^{2 k \eta}+c d e^{-2 k \eta}+2 r \cos \left(2 n_{f} \eta\right)+2 s \sin \left(2 n_{f} \eta\right)}{b d e^{2 k \eta}+a c e^{-2 k \eta}+2 t \cos \left(2 n_{f} \eta\right)+2 u \sin \left(2 n_{f} \eta\right)} \\
a=\left(n_{f}-n_{a}\right)^{2}+k_{f}^{2} \\
b=\left(n_{f}+n_{s}\right)^{2}+k_{f}^{2} \\
c=\left(n_{f}-n_{s}\right)^{2}+k_{f}^{2} \\
d=\left(n_{f}+n_{a}\right)^{2}+k_{f}^{2} \\
r=\left(n_{a}^{2}+n_{s}^{2}\right)\left(n_{f}^{2}+k_{f}^{2}\right)-\left(n_{f}^{2}+k_{f}^{2}\right)^{2}-n_{a}^{2} n_{s}^{2}-4 n_{a} n_{s} k_{f}^{2} \\
t=\left(n_{a}^{2}+n_{s}^{2}\right)\left(n_{f}^{2}+k_{f}^{2}\right)-\left(n_{f}^{2}+k_{f}^{2}\right)^{2}-n_{a}^{2} n_{s}^{2}+4 n_{a} n_{s} k_{f}^{2} \\
s=2 k_{f}\left(n_{f}-n_{a}\right)\left(n_{f}^{2}+k_{f}^{2}+n_{a} n_{s}\right) \\
u=2 k_{f}\left(n_{s}+n_{a}\right)\left(n_{f}^{2}+k_{f}^{2}-n_{a} n_{s}\right) \\
\eta=\frac{2 \pi d}{\lambda}
\end{gathered}
$$

where the subscripts $a, f, s$ refer to the ambient (typcially air), film, and substrate respectively, $d$ is the film thickness, and $\lambda$ is the incident photon wavelength. As an example, the reflectance as a function of film thickness for $\mathrm{Al}, \mathrm{Au}, \mathrm{Pt}$, and $\mathrm{Ni}$ on a Si substrate is shown in Figure B.2 with properties used in the calculations provided in Table B.1.

\begin{tabular}{lcccccc}
\hline Property & $\mathrm{Al}$ & $\mathrm{Au}$ & $\mathrm{Pt}$ & $\mathrm{Ni}$ & $\mathrm{Si}$ & Ambient \\
\hline $\mathrm{n}$ & 2.699 & 0.177 & 2.79 & 2.45 & 3.705 & 1 \\
$\mathrm{k}$ & 9 & 4.964 & 4.885 & 4.34 & 0.088 & 0 \\
\hline$\lambda=785 \mathrm{~nm}$ & & & & & \\
\hline
\end{tabular}

Table B.1: Optical and laser properties used in the calculation of reflectance and reflectivity of $\mathrm{Al}, \mathrm{Au}, \mathrm{Pt}$, and Ni shown in Figure B.2. 


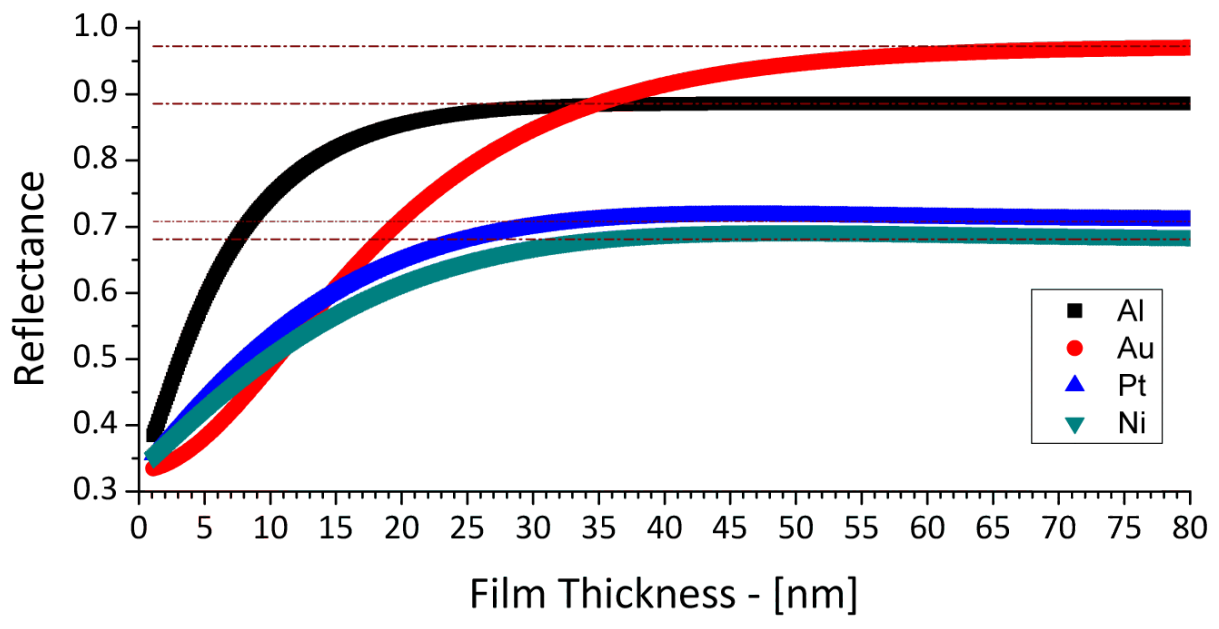

Figure B.2: Reflectance as a function of film thickness for Al, Au, Pt, and Ni on Si substrates in air, based on Equations B.4a-B.4j. The dashed lines are the reflectance of the given bulk metals using Equation B.2. Optical properties used in the calculations are shown in Table B.1. 


\section{APPENDIX C}

\section{SIGNAL ANALYSIS AND \\ INTERPRETATION}

C.1 Square Wave of Arbitrary Duty Cycle . . . . . . . . . . . . . . . . . . 322

C.2 Frequency Domain Signal Analysis - Reduction of Pulsed Modulation to Sinusoidal Modulation . . . . . . . . . . . . . . . . 326

C.3 Frequency Domain Signal Analysis - Sinusoidal Modulation . . . . . . . . . 328

C.4 Feldman Analysis - Layer Temperature . . . . . . . . . . . . . . . . . . . . 332

C.5 Feldman Analysis - Probe Through Glass . . . . . . . . . . . . . . . . 333

\section{C.1 Square Wave of Arbitrary Duty Cycle}

In order to modulate the pump beam, and thereby allow the system to lock into the probe signal, the electro-optical modulator (EOM) and the lock-in amplifier are provided with a driving function via a function generator or alternative source. The derivation of the thermal model typically assumes a sinusoidal modulation waveform for modulation of the pump beam, see Section C.3. However, as part of the work in this dissertation that formulation was extended to incorporate a pulsed waveform, i.e. a square waveform with 
an arbitrary duty cycle. The pulsed waveform supplied to the EOM for pump modulation in the TTR/TDTR experiment, $x(t)$, is of the form given by:

$$
x(t)= \begin{cases}1 & 0<t<d / 2-\tau_{d} \\ 0 & d / 2-\tau_{d}<t<T_{o} / 2-\tau_{d}\end{cases}
$$

where $T_{o}$ is the period of the function, $d$ is the time the function is "on" (High logic), $D$ is the duty cycle, and $\tau_{d}$ is the delay time, see Figure C.1. In our implementation of the pump-probe spectroscopy experiment, the pump is advanced rather than the probe delayed to obtain the pump-to-probe delay and temporal resolution of the temperature decay, see Chapter 3. Therefore, in reference to the temporal location of the modulation waveform at $t=0$, a time shift, i.e. a phase shift, will be observed as a function of the pump-probe delay time, $\tau_{d}$. When the duty cycle is $50 \%$, i.e. $D=0.5$, the pulsed waveform is more commonly referred to as a square waveform.

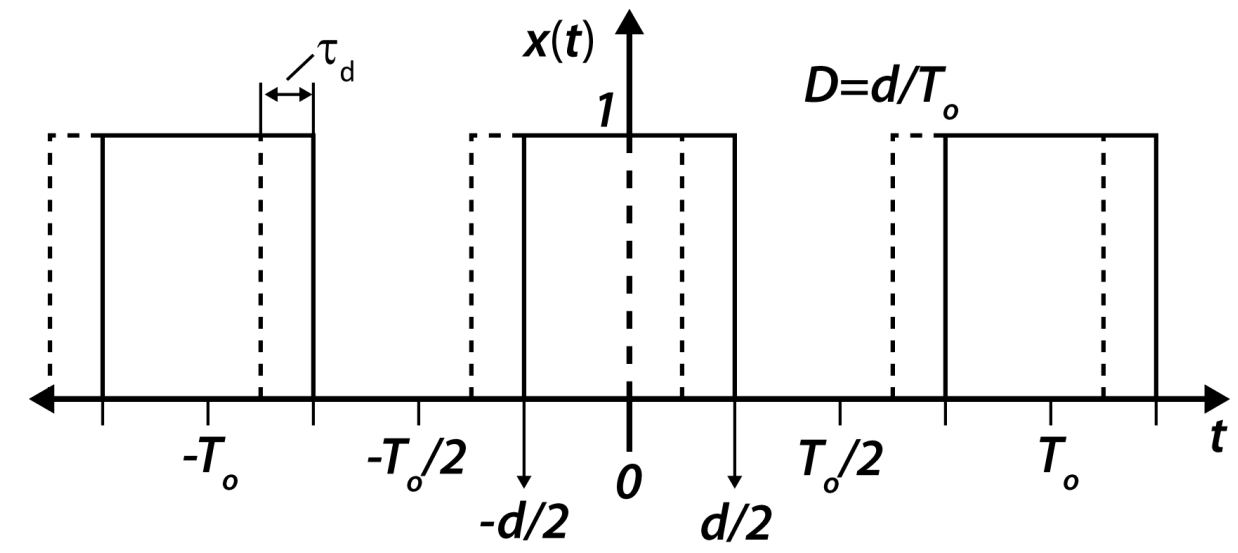

Figure C.1: Generalization of the pulsed wavefunction that we seek, given by Equation C.1.

The waveform described in Equation C.1 and represented in Figure C.1, can be formulated from Fourier Series analysis with the complex representation of the continuously periodic time signal given by [226]:

$$
x(t)=\sum_{k=-\infty}^{\infty} a_{k} e^{i k\left(2 \pi / T_{o}\right) t}
$$


where the $a_{k}$ 's are the Fourier coefficients which can be calculated by given by:

$$
a_{k}=\frac{1}{T_{o}} \int_{T_{o}} x(t) e^{-i k\left(2 \pi / T_{o}\right) t} d t
$$

The special DC component, $a_{0}$, and the general cases of the AC Fourier coefficients, $a_{k}$, can be derived for the pulsed waveform Equation C.3. Therefore we have:

\section{Fourier Coefficient - $\mathbf{a}_{0}$}

$$
\begin{gathered}
a_{0}=\frac{1}{T_{o}} \int_{T_{o}} x(t) d t \\
=\frac{1}{T_{o}} \int_{-d / 2-\tau_{d}}^{d / 2-\tau_{d}} 1 d t+\frac{1}{T_{o}} \int_{-T_{o} / 2+d / 2-\tau_{d}}^{T_{o} / 2-d / 2-\tau_{d}} 0 d t \\
a_{0}=\frac{d}{T_{o}}
\end{gathered}
$$

\section{AC Fourier Coefficients - $\mathbf{a}_{k}$}

$$
\begin{gathered}
a_{k}=\frac{1}{T_{o}} \int_{T_{o}} x(t) e^{-i k\left(2 \pi / T_{o}\right) t d t} \\
=\frac{1}{T_{o}} \int_{-d / 2-\tau_{d}}^{d / 2-\tau_{d}} e^{-i k\left(2 \pi / T_{o}\right) t} d t+\frac{1}{T_{o}} \int_{-T_{o} / 2+d / 2-\tau_{d}}^{T_{o} / 2-d / 2-\tau_{d}} 0 d t \\
=\left.\frac{1}{T_{o}} \frac{1}{-i k\left(2 \pi / T_{o}\right)} e^{-i k\left(2 \pi / T_{o}\right) t}\right|_{-d / 2-\tau_{d}} ^{d / 2-\tau_{d}} \\
a_{k}=\frac{1}{-2 \pi i k}\left[e^{-i k \pi d / T_{o}+i k \omega_{o} \tau_{d}}-e^{i k \pi d / T_{o}+i k \omega_{o} \tau_{d}}\right] \\
a_{k}=\frac{1}{-2 \pi i k}\left[e^{-i k \pi d / T_{o}}-e^{i k \pi d / T_{o}}\right] e^{i k \omega_{o} \tau_{d}}
\end{gathered}
$$

The final Fourier representation of the pulsed waveform, with an arbitrary duty cycle, $D$, 
is given by:

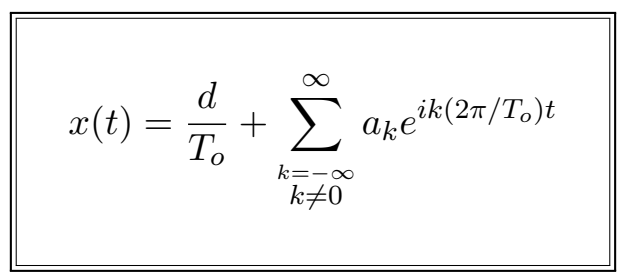

where $a_{k}$ is given by Equation C.11

In order to test the validity of the formulated waveform given in Equations C.12 and C.11, and to compare to the functional form sought in Figure C.1 the plotted wavefunction for duty cycles of 10, 25, 50, and $75 \%$ is shown in Figure C.2. Interpretation of the plots indicated that the formulation of the waveform developed, see Equation C.12, follows the criteria set forth in Equation C.1.

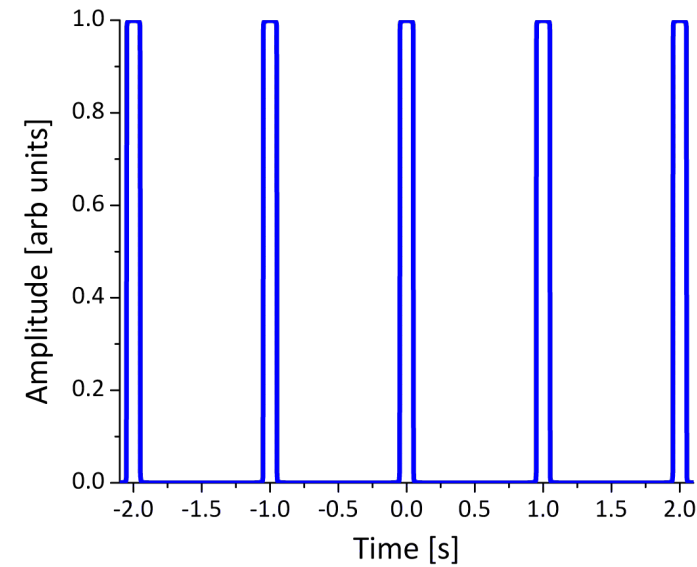

(a)

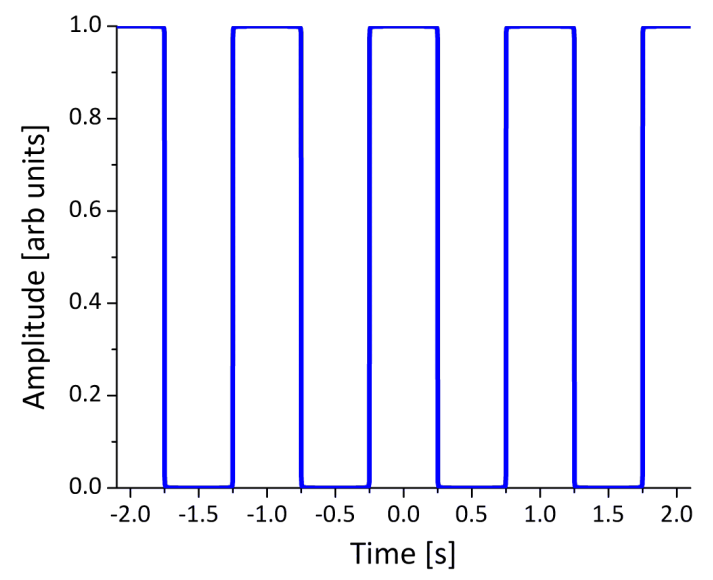

(c)

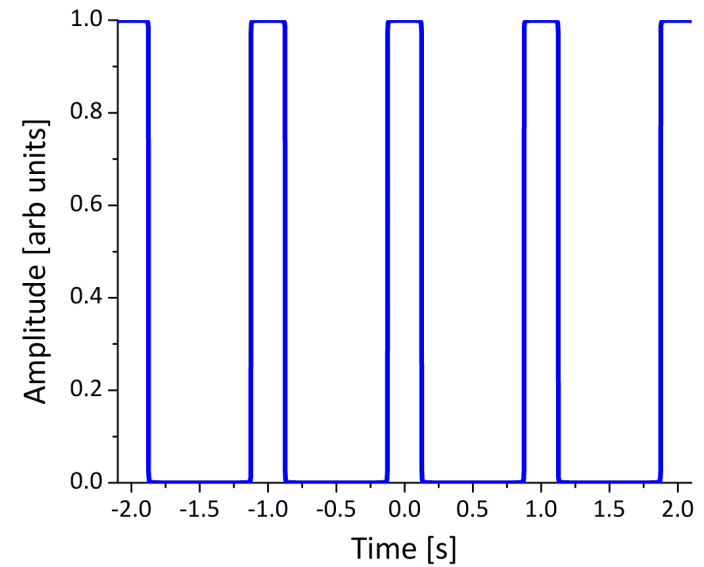

(b)

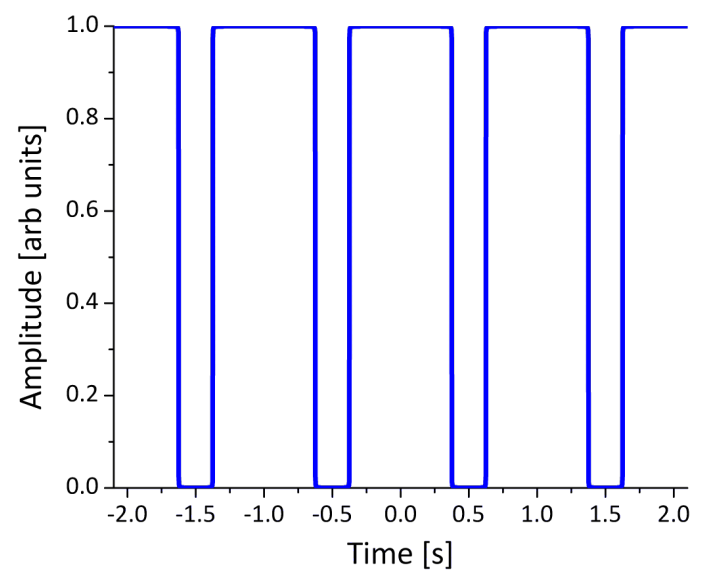

(d)

Figure C.2: Plot of the waveform given by Equations C.12 and C.11 for a frequency of $1 \mathrm{~Hz}$, i.e. $f=1$ $\mathrm{Hz}, \tau_{d}=0$, for a duty cycles (a) $D=0.10$, (b) $D=0.25$, (c) $D=0.50$, and (d) $D=0.75$. 


\section{C.2 Frequency Domain Signal Analysis - Reduction of Pulsed Modulation to Sinusoidal Modulation}

In order to test the validity of Equations C.11 and C.12, beyond the plots shown in Figure C.2, we will consider the special case where $D=0.5$ and $k=\{-1,1\}$. We know from Fourier analysis that a square wave, or pulsed wave, can be constructed from an infinite summation of complex exponentials (sines and cosines), see Figure C.3. Therefore for the case of $k=\{-1,1\}$, the solution for the pulsed waveform should reduce to the sinusoidal solution. In the case of the waveform shown in Figure C.1, since $x(t)$ is symmetric about $x=0$ for $D=0.5, x(t)$ is formed from an infinite summation of cosine waves of odd harmonics. Therefore for the case of $k=\{-1,1\}$ we expect to seek a solution of the form:

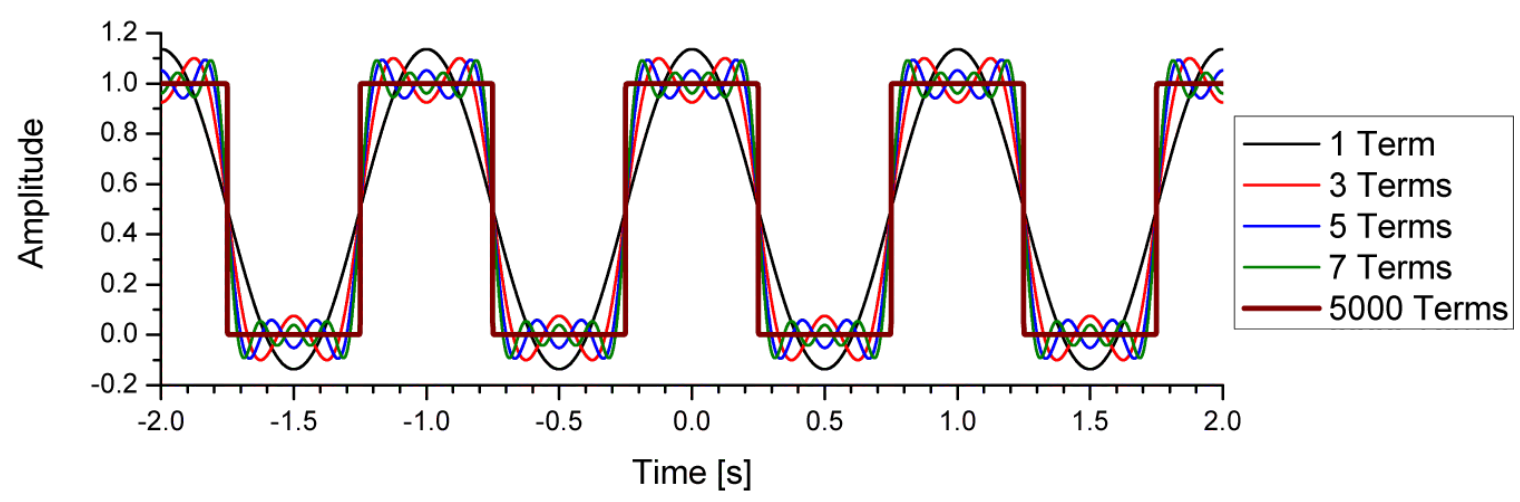

Figure C.3: Amplitude as a function of time, for the pulsed wave formulation, Equations C.11 and C.12, showing the sum of $1,3,5,7$, and 5000 harmonic terms, $k$. Assuming $f=1 \mathrm{~Hz}, \mathrm{D}=0.5$, and $\tau_{d}=0$.

$$
x(t)=\mathfrak{R e}\left\{e^{i \omega_{o} t}\right\}=\cos \left(\omega_{o} t\right)
$$

Beginning with the formulation of the Fourier coefficients from Equation C.11:

$$
a_{k}=\frac{1}{-2 \pi i k}\left[e^{-i k \pi d / T_{o}}-e^{i k \pi d / T_{o}}\right] e^{i k \omega_{o} \tau_{d}}
$$

for $D=0.5, k=\{-1,1\}$, and $\tau_{d}=0$

$$
a_{k}=\frac{1}{-2 \pi i k}\left[e^{-i k \pi / 2}-e^{i k \pi / 2}\right]
$$




$$
a_{1}=a_{-1}=\frac{1}{\pi}
$$

Therefore considering the AC component of Equation C.12 we have:

$$
\begin{gathered}
x(t)=\frac{1}{\pi} e^{i \omega_{o} t}+\frac{1}{\pi} e^{i \omega_{o} t} \\
x(t)=\frac{e^{i \omega_{o} t}+e^{i \omega_{o} t}}{\pi}
\end{gathered}
$$

This solution looks very similar to the Euler relation for a cosine wave given by:

$$
x(t)=\frac{e^{i \omega_{o} t}+e^{i \omega_{o} t}}{2}
$$

However, we note that the denominator of our derivation is a factor of $\pi$ rather than a factor of 2 as given by the Euler relation. This discrepancy can be explained by considering the formulation of the pulsed waveform in this analysis. We derived the functional form of the pulsed waveform specifically to reach a maximum value of unity during the "on" condition. Taking a look at the Fourier coefficients, shown in Figure C.4, we see that there are an equal number of harmonic components with positive and negative contributions, with amplitudes reducing as $1 / k$ to form the square wave. Therefore for a duty cycle of $50 \%$ the contributions of the first harmonic components must be greater than $1 / 2$. This is also noted in Figure C.3 where the first harmonic wave exceeds a magnitude of one, and it is only when higher harmonics are considered that the waveform converges towards unity.

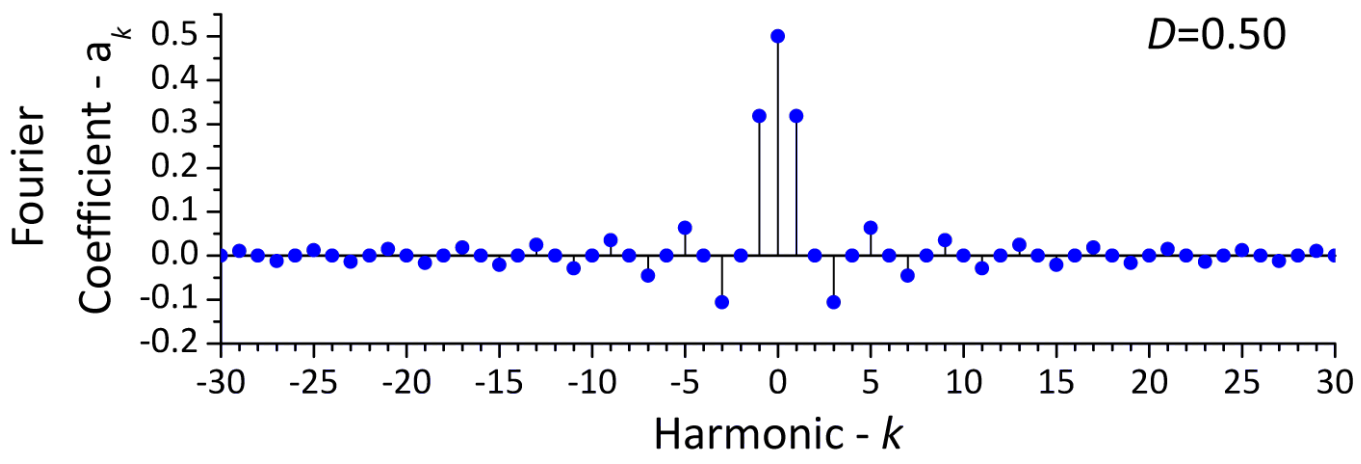

Figure C.4: Fourier coefficients, $a_{k}$, for the first 61 harmonic terms, assuming $D=0.50$ and $\tau_{d}=0$. 


\section{C.3 Frequency Domain Signal Analysis - Sinusoidal Modu- lation}

Traditionally the effects of higher harmonics and non-symmetric duty cycles are ignored and the modulation function of the pump is assumed to be sinusoidal. Here to show the parallel path and the simplified case, the derivation of the transfer function is reproduced following the work of Schmidt [228] in the notation and formulation of this work.

Again we will begin by considering a train of impulses from the laser given by:

$$
q_{\text {gen }}(t)=Q \sum_{n=-\infty}^{\infty} \delta(t-n T)
$$

and a sinusoidal modulation function given by ${ }^{1}$

$$
x(t)=e^{i \omega_{o} t} e^{i \omega_{o} \tau_{d}}
$$

Recalling from Chapter 4, the sampled function in the frequency domain assuming the pump laser to be an impulse-train of period $T_{s}$, is given by:

$$
X_{p}(\omega)=\frac{Q_{\mathrm{pm}}}{T_{s}} \sum_{k=-\infty}^{\infty} P\left(\omega-k \frac{2 \pi}{T_{s}}\right)
$$

where $P(\omega)$ is the sampling function, i.e. the modulation waveform. In this case the sampling function is the complex sinusoidal function given by Equation C.21, which in the frequency domain is given by:

$$
P(\omega)=2 \pi \delta\left(\omega-\omega_{o}\right) e^{i \omega_{o} \tau_{d}}
$$

Inserting Equation C.23 into C.22, the frequency domain solution for a periodic pump

\footnotetext{
${ }^{1}$ As was the case for the derivation of the pulsed waveform, we must also account for the delay of the pump relative to the waveform at $t=0$, which is the temporal reference of the lock-in, by including a phase factor $e^{i \omega_{0} \tau_{d}}$.
} 
impulse-train modulated by a complex sinusoid is given by:

$$
Q(\omega)=X_{p}(\omega)=\frac{2 \pi Q_{\mathrm{pm}}}{T_{s}} \sum_{M=-\infty}^{\infty} \delta\left(\omega-\omega_{o}-M \omega_{s}\right) e^{i \omega_{o} \tau_{d}}
$$

Recall that the temperature response in the time domain is the convolution of the impulse heat input and the temperature response of the system, which in the frequency domain is given by the product of the two, i.e.

$$
\Theta(t)=Q(t) * h(t) \stackrel{\mathcal{F}}{\longleftrightarrow} \Theta(\omega)=Q(\omega) H(\omega)
$$

Therefore the temperature response of a system due to thermal input from a sinusoidal modulated pump impulse-train is given by:

$$
\Theta(\omega)=\frac{2 \pi Q_{\mathrm{pm}}}{T_{s}} \sum_{M=-\infty}^{\infty} H(\omega) \delta\left(\omega-\omega_{o}-M \omega_{s}\right) e^{i \omega_{o} \tau_{d}}
$$

In order to monitor the temperature response of the system to the modulated heat input from the pump, the system must be "sampled" a second time by the probe. The functional form of the probe impulse-train is given by:

$$
p_{\mathrm{pr}}(t)=\beta Q_{\mathrm{pr}} \sum_{l=-\infty}^{\infty} \delta\left(t-n T-\tau_{d}\right)
$$

where $Q_{\mathrm{pr}}$ is the heat input of the probe, $\beta$ is a constant that contains information about the thermoreflectance coefficient of the sample and the gain in the measurement electronics, and $\tau_{d}$ is the time delay between the pump and probe impulses. In the frequency domain the probe impulse function is given by:

$$
P(\omega)=\left[\frac{2 \pi \beta Q_{\mathrm{pr}}}{T_{s}} \sum_{l=-\infty}^{\infty} \delta\left(\omega-l \frac{2 \pi}{T_{s}}\right)\right] e^{-i \omega \tau_{d}}
$$

Using the same methodology as in Chapter 4.2 .2 it can be shown that:

$$
Z(\omega)=\frac{2 \pi \beta Q_{\mathrm{pm}} Q_{\mathrm{pr}}}{T_{s}^{2}} \sum_{M=-\infty}^{\infty} \sum_{l=-\infty}^{\infty} H\left(\omega-l \omega_{s}\right) \delta\left(\omega-\omega_{o}-(l+M) \omega_{s}\right) e^{-i l \omega_{s} \tau_{d}} e^{i \omega_{o} \tau_{d}}
$$


This function will equal zero expect in cases where the contents of the delta function evaluate to zero, i.e.:

$$
\omega-\omega_{o}-(l+M) \omega_{s}=0
$$

Assuming $\omega$ is within the lock-in bandwidth, i.e. $\omega=\omega_{o}$, we find that this condition is only satisfied when $l=-M$, therefore:

$$
Z(\omega)=\frac{2 \pi \beta Q_{\mathrm{pm}} Q_{\mathrm{pr}}}{T_{s}^{2}} \sum_{M=-\infty}^{\infty} H\left(\omega+M \omega_{s}\right) \delta\left(\omega-\omega_{o}\right) e^{i M \omega_{s} \tau_{d}} e^{i \omega_{o} \tau_{d}}
$$

Taking the inverse Fourier transform we arrive at the transfer function of interest:

$$
z(t)=Z\left(\omega_{0}\right) e^{i \omega_{o} t}
$$

where

$$
Z\left(\omega_{o}\right)=\frac{\beta Q_{\mathrm{pm}} Q_{\mathrm{pr}}}{T_{s}^{2}} \sum_{M=-\infty}^{\infty} H\left(\omega_{o}+M \omega_{s}\right) e^{i M \omega_{s} \tau_{d}} e^{i \omega_{o} \tau_{d}}
$$

This is the "common" solution for the time domain system response and complex transfer function reported by various groups [230, 231, 245, 251].

\section{C.3.1 Lock-in Amplifier Signal}

As was done in Chapter 4.2.3, we are interested in the lock-in amplifiers response in channels $X$ and $Y$ to the time domain input given by Equation C.32. Both terms in Equation C.32, $Z\left(k \omega_{o}\right)$ and $e^{i \omega_{o} t}$ are complex terms and therefore $z(t)$ is of the form:

$$
z(t)=(a+i b)(c+i d)
$$

where

$$
\begin{aligned}
& a=\mathfrak{R e}\left\{Z\left(\omega_{o}\right)\right\} \\
& b=\mathfrak{I m}\left\{Z\left(\omega_{o}\right)\right\}
\end{aligned}
$$




$$
\begin{aligned}
& c=\mathfrak{R e}\left\{e^{i \omega_{o} t}\right\}=\cos \left(\omega_{o} t\right) \\
& d=\mathfrak{I m}\left\{e^{i \omega_{o} t}\right\}=\sin \left(\omega_{o} t\right)
\end{aligned}
$$

Therefore,

$$
\begin{aligned}
z(t)=\left(\mathfrak{R e}\left\{Z\left(\omega_{o}\right)\right\}\right. & \left.+i \mathfrak{I m}\left\{Z\left(\omega_{o}\right)\right\}\right)\left(\cos \left(\omega_{o} t\right)+i \sin \left(\omega_{o} t\right)\right) \\
z(t)=\mathfrak{R e}\left\{Z\left(\omega_{o}\right)\right\} \cos \left(\omega_{o} t\right)+i \mathfrak{I m}\{ & \left.Z\left(\omega_{o}\right)\right\} \cos \left(\omega_{o} t\right) \\
& +i \mathfrak{R e}\left\{Z\left(\omega_{o}\right)\right\} \sin \left(\omega_{o} t\right)-\mathfrak{I m}\left\{Z\left(\omega_{o}\right)\right\} \sin \left(\omega_{o} t\right)
\end{aligned}
$$

In terms of the lock-in analysis for the pump-probe spectroscopy experiment, we are interested in the physical signal to be measured, i.e. the real component of $z(t)$, therefore we have:

$$
z(t)=\mathfrak{R e}\left\{Z\left(\omega_{o}\right)\right\} \cos \left(\omega_{o} t\right)-\mathfrak{I m}\left\{Z\left(\omega_{o}\right)\right\} \sin \left(\omega_{o} t\right)
$$

Where the $X$ and $Y$ components of the lock-in output are given by:

$$
\begin{aligned}
& X=\mathfrak{R e}\left\{Z\left(\omega_{o}\right)\right\} \\
& Y=\mathfrak{I m}\left\{Z\left(\omega_{o}\right)\right\}
\end{aligned}
$$

In complex analysis the real, $X$, and imaginary, $Y$, components of a complex signal $Z$ can be given in terms of the complex conjugate $Z^{*}:^{2}$

$$
\begin{gathered}
\mathfrak{R e}\left\{Z\left(\omega_{o}\right)\right\}=\frac{1}{2}\left[Z+Z^{*}\right] \\
\mathfrak{I m}\left\{Z\left(\omega_{o}\right)\right\}=\frac{1}{2 i}\left[Z-Z^{*}\right]
\end{gathered}
$$

where $Z^{*}\left(\omega_{o}\right)=Z\left(-\omega_{o}\right)$. Therefore the final form of the lock-in components, $X$ and $Y$, for the system response due to the sinusoidal modulation waveform is given by:

\footnotetext{
${ }^{2}$ Note that this the same form as Equations 4.36a and 4.36b for the first harmonic terms, i.e. $k=\{-1,1\}$, expect for the magnitude of the coefficient, which is explained in Appendix C.3.
} 


$$
\begin{aligned}
X=\frac{1}{2} \frac{\beta Q_{\mathrm{pm}} Q_{\mathrm{pr}}}{T_{s}^{2}}\left[\sum_{M=-\infty}^{\infty} H\left(\omega_{o}+M \omega_{s}\right) e^{i M \omega_{s} \tau}\right. & \\
& \left.+\sum_{M=-\infty}^{\infty} H\left(-\omega_{o}+M \omega_{s}\right) e^{i M \omega_{s} \tau_{d}}\right] e^{i \omega_{o} \tau_{d}} \\
Y=\frac{-i}{2} \frac{\beta Q_{\mathrm{pm}} Q_{\mathrm{pr}}}{T_{s}^{2}}\left[\sum_{M=-\infty}^{\infty} H\left(\omega_{o}+M \omega_{s}\right) e^{i M \omega_{s} \tau}\right. & \left.-\sum_{M=-\infty}^{\infty} H\left(-\omega_{o}+M \omega_{s}\right) e^{i M \omega_{s} \tau_{d}}\right] e^{i \omega_{o} \tau_{d}}
\end{aligned}
$$

\section{C.4 Feldman Analysis - Layer Temperature}

Recalling from Chapter 4.3 we have that the temperature on either side of the heat source, located at $z=\xi$, is given by:

$$
\tilde{\mathbf{T}}_{\mathbf{n}}\left(\xi^{+}\right)-\tilde{\mathbf{T}}_{\mathbf{n}}\left(\xi^{-}\right)=-\frac{Q}{2 \gamma_{n, Q}}\left[\begin{array}{c}
1 \\
-1
\end{array}\right]
$$

Inserting Equations 4.65a and 4.65b into Equation C.43 we have:

$$
T_{N+1}\left[\begin{array}{c}
B^{+} \\
B^{-}
\end{array}\right]+T_{0}\left[\begin{array}{c}
A^{+} \\
A^{-}
\end{array}\right]=-\frac{Q}{2 \gamma_{n, Q}}\left[\begin{array}{c}
1 \\
-1
\end{array}\right]
$$

Using this we obtain two equations and two unknowns:

$$
\begin{gathered}
T_{N+1} B^{+}-T_{0} A^{+}=-\frac{Q}{2 \gamma_{n, Q}} \\
T_{N+1} B^{-}-T_{0} A^{-}=\frac{Q}{2 \gamma_{n, Q}}
\end{gathered}
$$

Equation C.45b is then solved for $T_{N+1}$ :

$$
T_{N+1}=\frac{1}{B^{-}} \frac{Q}{2 \gamma_{n, Q}}+T_{0} \frac{A^{-}}{B^{-}}
$$


Plugging Equation C.46 into Equation C.45a it follows that:

$$
\begin{gathered}
\left(\frac{1}{B^{-}} \frac{Q}{2 \gamma_{n, Q}}+T_{0} \frac{A^{-}}{B^{-}}\right) B^{+}-T_{0} A^{+}=-\frac{Q}{2 \gamma_{n, Q}} \\
T_{0}\left(\frac{B^{+} A^{-}}{B^{-}}-A^{+}\right)=-\frac{Q}{2 \gamma_{n, Q}}\left(1+\frac{B^{+}}{B^{-}}\right) \\
T_{0}=\frac{-\frac{Q}{2 \gamma_{n, Q}} \frac{B^{-}+B^{+}}{B^{-}}}{\frac{A^{-} B^{+}-B^{-} A^{+}}{B^{-}}}
\end{gathered}
$$

$$
T_{0}=\frac{Q}{2 \gamma_{n, Q}} \frac{B^{+}+B^{-}}{A^{+} B^{-}-A^{-} B^{+}}
$$

Similarly, if we solve Equation C.45a or C.45b for $T_{0}$ and insert into the other, it can be shown that:

$$
T_{N+1}=\frac{Q}{2 \gamma_{n, Q}} \frac{A^{+}+A^{-}}{A^{+} B^{-}-A^{-} B^{+}}
$$

\section{C.5 Feldman Analysis - Probe Through Glass}

In Chapter 4.3.2 we looked at two examples of sample configurations and constructed the $\tilde{\mathbf{A}}$ and $\tilde{\mathbf{B}}$ matrices to show the effects of the semi-infinite substrate assumption. Here

will we derive $\tilde{\mathbf{A}}$ and $\tilde{\mathbf{B}}$ for a more complex configuration where we are heating and probing the surface of the metal film through glass, see Figure C.5. For this configuration we assume only one layer, i.e. $\mathrm{N}=1$, and that the glass slide and the substrate are semi-infinite. This configuration has been used in order to probe the thermal properties of liquids and spin coated films [240, 314] Beginning with Equation 4.68a we can construct the $\tilde{\mathbf{A}}$ :

$$
\tilde{\mathbf{A}}=\tilde{\mathbf{U}}_{\mathbf{1}}(\mathbf{0}) \times \tilde{\boldsymbol{\Gamma}}_{\mathbf{1} \rightarrow \mathbf{0}} \times\left(\begin{array}{l}
1 \\
0
\end{array}\right)
$$




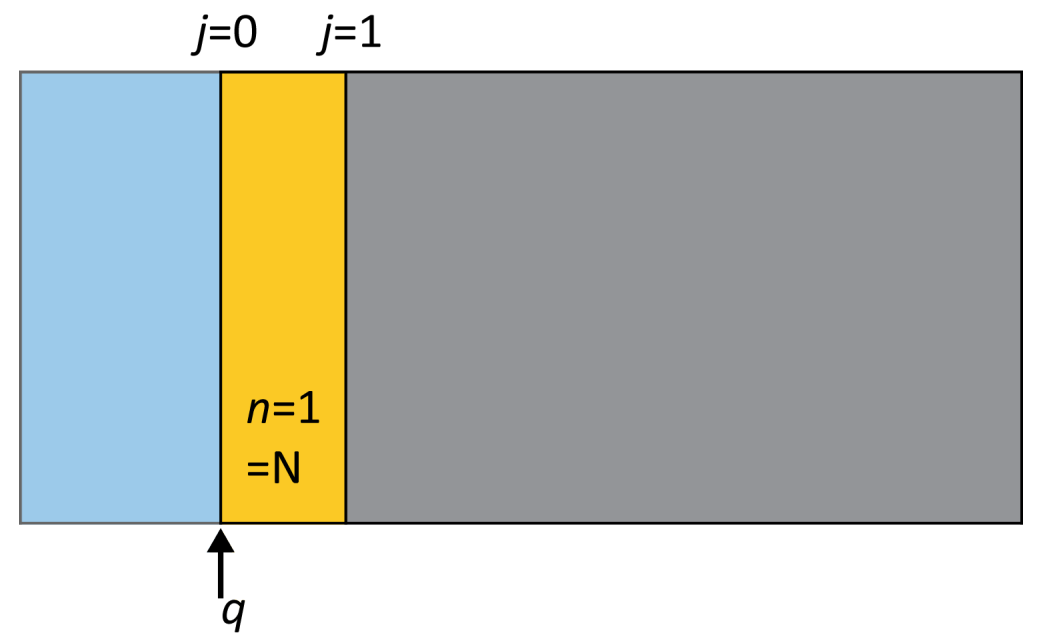

Figure C.5: Schematic of the indexing scheme used in the thermal analysis for a sample configuration where heating and probing are through a glass layer.

Expanding

$$
\tilde{\mathbf{A}}=\frac{1}{2} \times\left[\begin{array}{ll}
1 & 0 \\
0 & 1
\end{array}\right] \times\left[\begin{array}{cc}
1+\frac{\gamma_{0}}{\gamma_{1}}-\frac{\gamma_{0}}{h_{\mathrm{BD}, 1 \rightarrow 0}} & 1-\frac{\gamma_{0}}{\gamma_{1}}+\frac{\gamma_{0}}{h_{\mathrm{BD}, 1 \rightarrow 0}} \\
1-\frac{\gamma_{0}}{\gamma_{1}}-\frac{\gamma_{0}}{h_{\mathrm{BD}, 1 \rightarrow 0}} & 1+\frac{\gamma_{0}}{\gamma_{1}}+\frac{\gamma_{0}}{h_{\mathrm{BD}, 1 \rightarrow 0}}
\end{array}\right] \times\left[\begin{array}{l}
1 \\
0
\end{array}\right]
$$

Simplifying

$$
\tilde{\mathbf{A}}=\frac{1}{2} \times\left[\begin{array}{c}
1+\frac{\gamma_{0}}{\gamma_{1}}-\frac{\gamma_{0}}{h_{\mathrm{BD}, 1 \rightarrow 0}} \\
1-\frac{\gamma_{0}}{\gamma_{1}}-\frac{\gamma_{0}}{h_{\mathrm{BD}, 1 \rightarrow 0}}
\end{array}\right]
$$

In contrast to the previous examples in Chapter 4.3.2, the properties of $\gamma_{0}$ are not zero, and therefore will contain the properties of the glass. Because the substrate is assumed to be semi-infinite the formation of the $\tilde{\mathbf{B}}$ matrix is the same as in Chapter 4.3.2.

$$
\tilde{\mathbf{B}}=\tilde{\mathbf{U}}_{\mathbf{1}}\left(d_{1}\right) \times \tilde{\boldsymbol{\Gamma}}_{\mathbf{1} \rightarrow \mathbf{2}} \times\left(\begin{array}{l}
0 \\
1
\end{array}\right)
$$

Expanding

$$
\begin{aligned}
\tilde{\mathbf{B}}=\frac{1}{2} \times\left[\begin{array}{cc}
\exp \left[-q_{1} d_{1}\right] & 0 \\
0 & \exp \left[q_{1} d_{1}\right]
\end{array}\right] \\
\times\left[\begin{array}{cc}
1+\frac{\gamma_{2}}{\gamma_{1}}-\frac{\gamma_{2}}{h_{\mathrm{BD}, 1 \rightarrow 2}} & 1-\frac{\gamma_{2}}{\gamma_{1}}+\frac{\gamma_{2}}{h_{\mathrm{BD}, 1 \rightarrow 2}} \\
1-\frac{\gamma_{2}}{\gamma_{1}}-\frac{\gamma_{2}}{h_{\mathrm{BD}, 1 \rightarrow 2}} & 1+\frac{\gamma_{2}}{\gamma_{1}}+\frac{\gamma_{2}}{h_{\mathrm{BD}, 1 \rightarrow 2}}
\end{array}\right] \times\left[\begin{array}{l}
0 \\
1
\end{array}\right]
\end{aligned}
$$


Simplifying

$$
\begin{gathered}
B^{+}=\frac{1}{2} \times\left[\left(1-\frac{\gamma_{2}}{\gamma_{1}}+\frac{\gamma_{2}}{h_{\mathrm{BD}, 1 \rightarrow 2}}\right) \exp \left[-q_{1} d_{1}\right]\right] \\
B^{-}=\frac{1}{2} \times\left[\left(1+\frac{\gamma_{2}}{\gamma_{1}}+\frac{\gamma_{2}}{h_{\mathrm{BD}, 1 \rightarrow 2}}\right) \exp \left[q_{1} d_{1}\right]\right]
\end{gathered}
$$




\section{APPENDIX D}

\section{FUNDAMENTALS OF STATISTICAL \\ INFERENCE}

D.1 Testing for Normality . . . . . . . . . . . . . . 336

\section{D.1 Testing for Normality}

Through out the analysis in this chapter, there are several areas where the method in which we proceed will be dependent on being able to assume a normal distribution, or that the given distribution behaves normally to a good approximation. In theory, we could take a sufficiently large number of TTR/TDTR scans, analyze the data to deduce a thermophysical value, and create a histogram in an attempt to estimate the shape of the population of values. This of course brings us back to the problem of taking large sets of data. What we would ideally like, is a way to test for Normality from a subset of data, and make inference as to the properties of the distribution. Very seldom does a discrete sampling correspond exactly to a normal distribution so there will be a certain amount of judgment in determining normality. There is no one method that is used to test a distribution of data for Normality, and in fact there are over 40 normality tests found in literature [315, 316]. 
Generally, the different methods to test Normality can be broken down into graphical, numeric, and testing methods. We will use a combination of these 3 methods in order to test for normality. To better discuss the merits of several of the methods that will be used throughout the course of this work, we exam a simulated population with known mean and standard deviation, $\mu=100 \mathrm{MW} / \mathrm{m}^{2} \mathrm{~K}$ and $\sigma=1 \mathrm{MW} / \mathrm{m}^{2} \mathrm{~K}$ respectively.

\section{D.1.1 Graphical Methods for Testing Normality}

The simplest methods for assessing Normality, are graphical methods. Probably the most common of the graphical methods is to plot a histogram of the data. Figures D.1a and D.1c show a histogram of the model data. Figure D.1a is generated from a random sampling from a normal distribution, and Figure D.1c from a skewed normal distribution. While the histogram of the data, is a quick visual format to asses how the data is distributed, depending on the number of samples and the bin size, it is somewhat subjective to determine whether the data is distributed normally or not. Therefore, in addition to a histogram of the data, we can also consider a normal quantile-quantile plot (Q-Q pot). In the Q-Q plot, the data is plotted against the quantiles of a normal distribution with the mean and standard deviation of the data. If the distribution of the data is normal, the data points will lay along the straight line, see Figure D.1b. Deviation away from the line, is indicative of a deviation away from Normality, see Figure D.1d. While graphical comparisons such as the histograms and Q-Q plots shown in Figure D.1 provide quick analysis as to the normality of a distribution of data, as the sample size decreases, it becomes more difficult to asses the results, see Figure D.2.

\section{D.1.2 Numerical Methods for Testing Normality}

Turning to more numerical methods, we can consider the skew, $\beta_{\text {skew }}$, and kurtosis, $\beta_{\text {kurt }}$ of the distribution, given by Equations $6.7 \mathrm{a}$ and $6.7 \mathrm{~b}$ respectively. We know that for a perfectly normal distribution that the skew and kurtosis should be equal to zero and three respectively, and any deviation from these values indicates a deviation for normality. Along with the histrograms plotted in Figures D.1a and D.1c, the skew and kurtosis are also reported. We see that both distributions have similar kurtosis, however, the distribution 


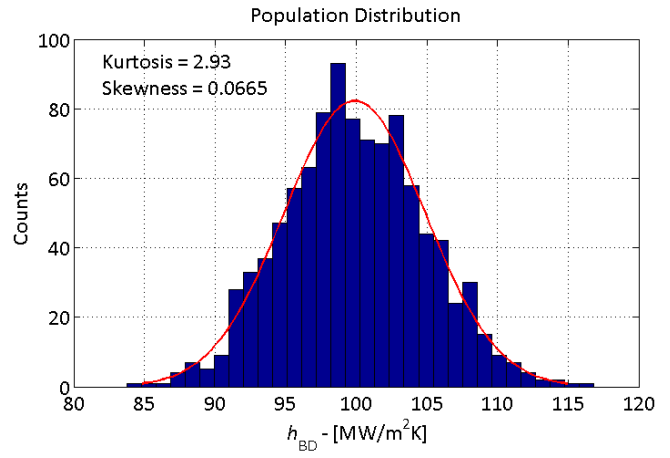

(a)

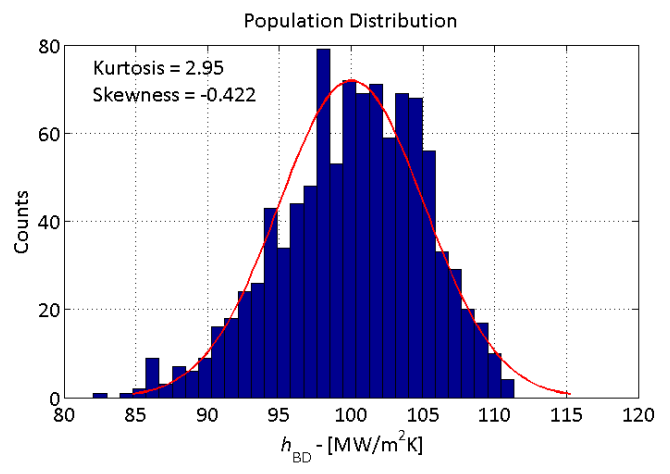

(c)

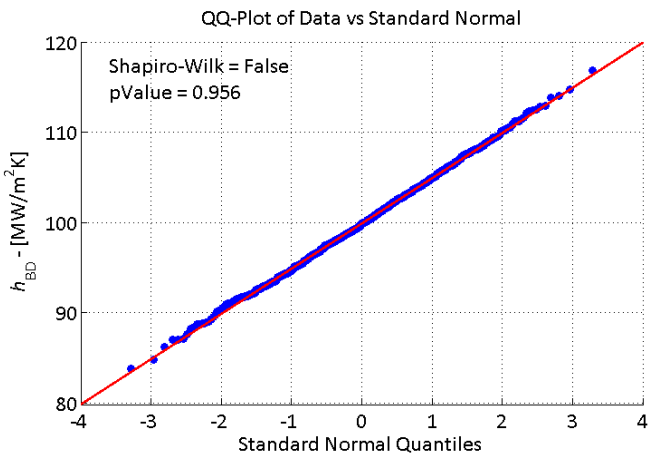

(b)

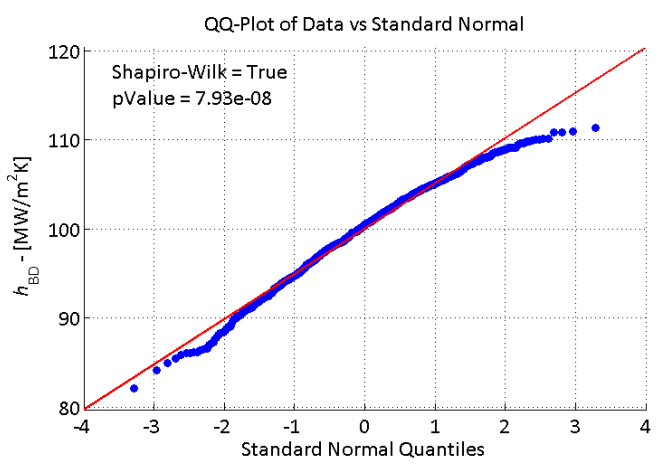

(d)

Figure D.1: Normal data, $\mu=100 \mathrm{MW} / \mathrm{m}^{2} \mathrm{~K} \sigma=1 \mathrm{MW} / \mathrm{m}^{2} \mathrm{~K}$

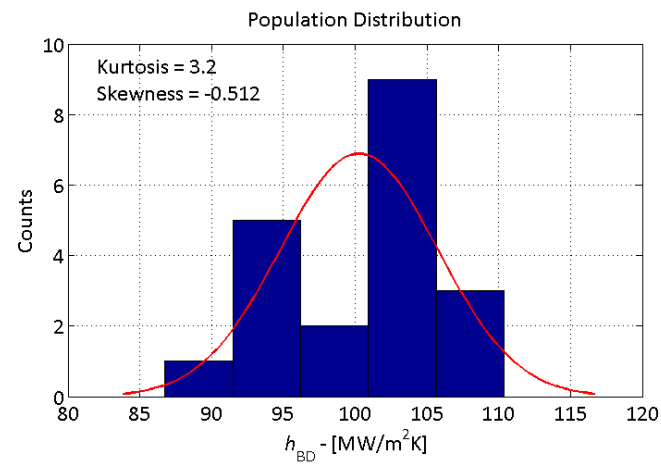

(a)

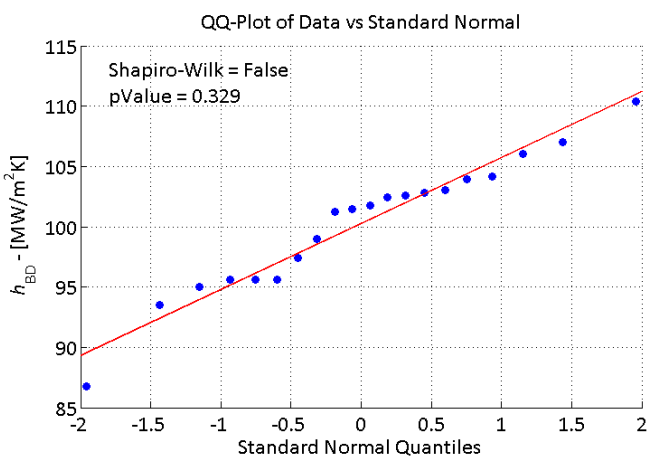

(b)

Figure D.2: Normal data, $\mu=100 \mathrm{MW} / \mathrm{m}^{2} \mathrm{~K} \sigma=1 \mathrm{MW} / \mathrm{m}^{2} \mathrm{~K}, n=20$

in Figure D.1c has a larger skew compared to Figure D.1a. While the skew and kurtosis provide a good quantitative measure of the normality of a given distribution, again care must be taken, because both the skew and kurtosis can vary for small sample sizes.

Figure D.3 illustrates how a small number of data points can effect the measurement of 
the skew and kurtosis of a distribution. Figures D.3a and D.3b give the graphical analysis of 5000 data points selected from a normal distribution, along with the skew and kurtosis of the overall population. In Figures D.3c and D.3d the skew and kurtosis as a function of the number of data points used in the calculation is shown. From the plots, it is apparent that when the number of data points is small, there is large variations in the calculated skew and kurtosis. Therefore, while providing valuable quantification of the Normality of larger data sets, care must be taken in interpreting the results as the number of data points decreases.

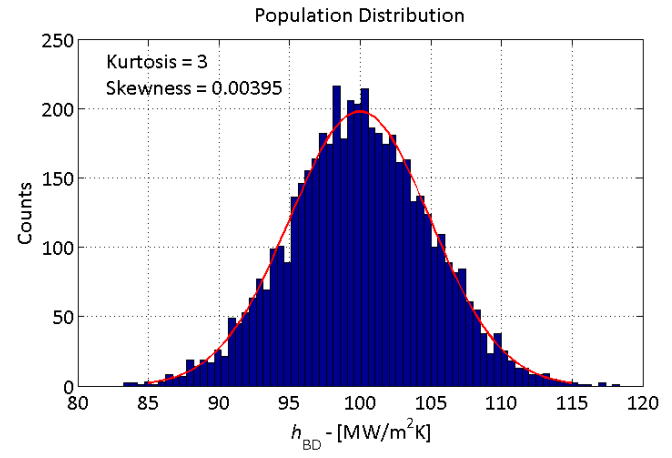

(a)

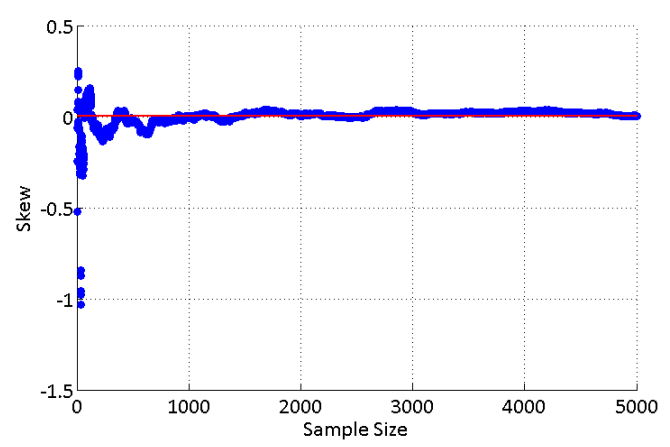

(c)

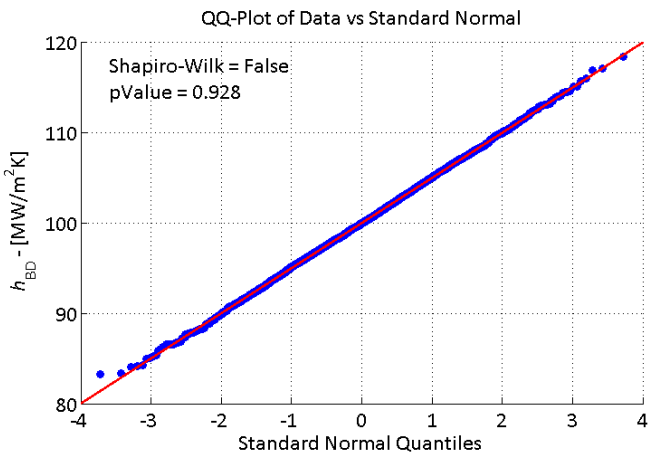

(b)

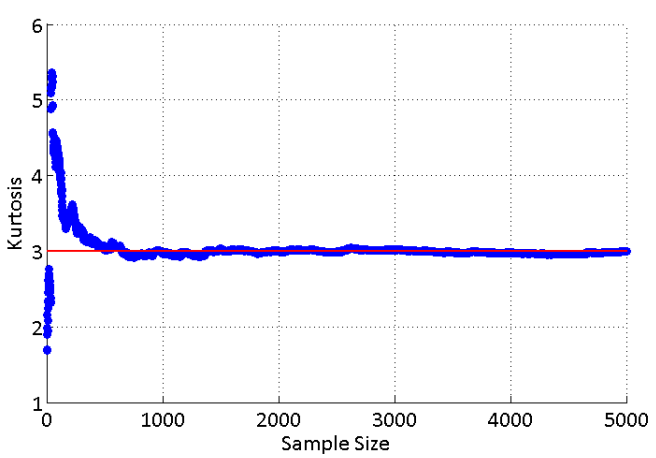

(d)

Figure D.3: Normal data, skew and kurtosis as a function of the number of data points.

\section{D.1.3 Hypothesis Testing for Normality}

The final group of methods for testing the Normality of a distribution involves the process of hypothesis testing. It is assumed that the null hypothesis is that the distribution 
is normal, and the alternate hypothesis is that the distribution is not normal, i.e.

$$
\begin{aligned}
& \mathrm{H}_{0} \text { : The distribution is normal } \\
& \mathrm{H}_{1} \text { : The distribution is not normal }
\end{aligned}
$$

The various tests that can be performed in order to test the validity of the null hypothesis can typically be separated in to three categories, 1) empirical distribution function (EDF) tests, moment tests, and correlation tests [316]. Examples of EDF tests include the Kolmogorov-Smirnov (KS), Cramer-von Mises (CV), and Andreson-Darling (AD) test, which compute a measurement of discrepancy between the hypothesized distribution of the data and the normal distribution. Moment tests compare the third and fourth moments (which we already know as the skew and kurtosis) of the normal distribution to that of the test distribution. Finally, the correlation tests compare the ratio of the weighted least squares from a normal population to the sample variance. The most common of these tests being the Shapiro-Wilk test, which is the test that will be used for this analysis.

The Shapiro-Wilk test was first developed in 1965 to test the Normality of small samples sizes in the range of $3 \leq n \leq 50$ and was later extended in 1995 to be used for sample sizes in the range of $3 \leq n \leq 5000$. The Shapiro-Wilk test calculates the test statistic, $W$ given by [317]:

$$
W=\frac{\left(\sum_{i=1}^{n} a_{i} x_{(i)}\right)^{2}}{\sum_{i=1}^{n}\left(x_{i}-\bar{x}\right)^{2}}
$$

where $x_{(i)}$ is the ordered sample values (i.e. $x_{(1)}$ is the smallest value), $\bar{x}$ is the sample mean, and $a_{i}$ is the weighting constant generated from the mean, variance, and covariance of a normal distribution and given by [317]:

$$
\mathbf{a}=\frac{\mathbf{m}^{T} \mathbf{V}^{-1}}{\left(\mathbf{m}^{T} \mathbf{V}^{-1} \mathbf{V}^{-1} \mathbf{m}\right)^{1 / 2}}
$$

where $\mathbf{m}$ are the expected values of random variables sampled from the normal distribution and $\mathbf{V}$ is the covariance matrix.

The test statistic of the Shaprio-Wilk test, $W$, ranges in value between zero and one, 
with small values of $W$ rejecting the null hypothesis (Normality). In multiple analyses, the Shapiro-Wilk test has been shown to be one of the best tests for identifying Normality and ideal for smaller sample sizes $[315,318,319]$. In fact, since the test statistic is biased based on sample size, care must be taken with large sample sets, which could produce a statistically significant p-value regardless of the underling distribution.

All the methods presented for testing for Normality, graphical, numerical, and hypothesis testing, can provide insight into how a subset of data, and/or how the underlying population is distributed. However, in each case there are also limitations to the interpretations based on several factors. Therefore, it is not recommended to rely on one method exclusively, and we will therefore compare results from each test in making a judgment of Normality. 


\section{APPENDIX E}

\section{MODELING ANALYSIS OF \\ PT/NI/SI(GE) SYSTEM}

E.1 Averaged Sensitivity Parameter . . . . . . . . . . . . . . . . . . . . . 342

E.2 Sensitivity Parameter as a Function of Time . . . . . . . . . . . . . . 343

\section{E.1 Averaged Sensitivity Parameter}

Table E.1: Averaged sensitivity parameter for the $100 \mathrm{~nm} \mathrm{Pt}$ on Si system.

\begin{tabular}{rcccccccccc}
\hline \multicolumn{10}{c}{ Pt/Si System } \\
\hline Ni Thickness & L1C & L1kz & L1d & L2C & L2kz & Lsd & LSC & LSkz & TBC_12 & TBC2S \\
0 & 0.4383 & 0.3255 & 0.1134 & - & - & - & -0.1309 & -0.1309 & -0.5018 & - \\
5 & 0.5445 & 0.3191 & 0.2258 & -0.0040 & -0.0098 & 0.0058 & -0.2737 & -0.2737 & -0.0816 & -0.2209 \\
10 & 0.5271 & 0.3267 & 0.2007 & -0.0114 & -0.0188 & 0.0073 & -0.2611 & -0.2611 & -0.0787 & -0.2227 \\
20 & 0.4957 & 0.3355 & 0.1606 & -0.0300 & -0.0339 & 0.0039 & -0.2249 & -0.2249 & -0.0729 & -0.2447 \\
30 & 0.4616 & 0.3420 & 0.1199 & -0.0541 & -0.0449 & -0.0093 & -0.1858 & -0.1858 & -0.0670 & -0.2662 \\
50 & 0.4059 & 0.3505 & 0.0557 & -0.1144 & -0.0586 & -0.0558 & -0.1273 & -0.1273 & -0.0587 & -0.2701 \\
100 & 0.3219 & 0.3643 & -0.0422 & -0.2984 & -0.0625 & -0.2359 & -0.0386 & -0.0386 & -0.0461 & -0.2019 \\
\hline
\end{tabular}


Table E.2: Averaged sensitivity parameter for the $100 \mathrm{~nm} \mathrm{Pt}$ on Ge system.

\begin{tabular}{|c|c|c|c|c|c|c|c|c|c|c|}
\hline \multirow[b]{2}{*}{ Ni Thickness } & \multicolumn{10}{|c|}{$\mathrm{Pt} /$ GeSystem } \\
\hline & L1C & L1kz & L1d & $\mathrm{L} 2 \mathrm{C}$ & $\mathrm{L} 2 \mathrm{kz}$ & Lsd & LSC & LSkz & TBC_12 & TBC2S \\
\hline 0 & 0.3439 & 0.3384 & 0.0061 & - & - & - & -0.1642 & -0.1642 & -0.3538 & - \\
\hline 5 & 0.3745 & 0.3503 & 0.0246 & -0.0114 & -0.0060 & -0.0054 & -0.2199 & -0.2199 & -0.0501 & -0.2176 \\
\hline 10 & 0.3622 & 0.3574 & 0.0051 & -0.0262 & -0.0116 & -0.0146 & -0.2131 & -0.2131 & -0.0487 & -0.2068 \\
\hline 20 & 0.3249 & 0.3662 & -0.0411 & -0.0615 & -0.0199 & -0.0416 & -0.1693 & -0.1693 & -0.0435 & -0.2275 \\
\hline 30 & 0.2925 & 0.3714 & -0.0786 & -0.1021 & -0.0254 & -0.0768 & -0.1304 & -0.1304 & -0.0394 & -0.2361 \\
\hline 50 & 0.2586 & 0.3760 & -0.1171 & -0.1863 & -0.0325 & -0.1539 & -0.0804 & -0.0804 & -0.0356 & -0.2192 \\
\hline 100 & 0.2293 & 0.3824 & -0.1529 & -0.3925 & -0.0342 & -0.3583 & -0.0133 & -0.0133 & -0.0306 & -0.1277 \\
\hline
\end{tabular}

\section{E.2 Sensitivity Parameter as a Function of Time}

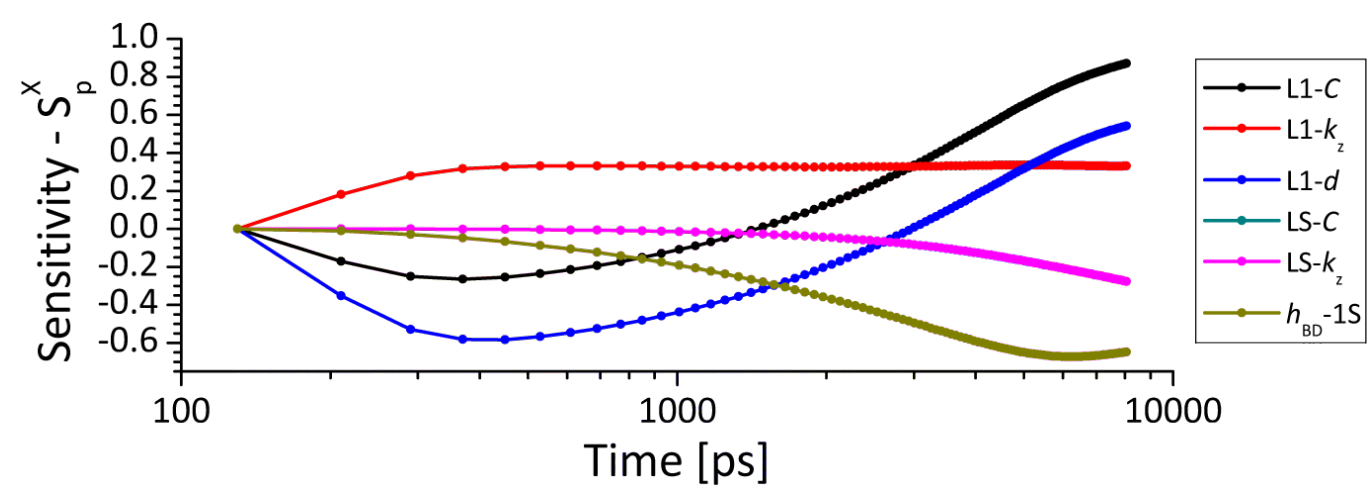

Figure E.1: The sensitivity coefficient as a function of time for $100 \mathrm{~nm}$ of $\mathrm{Pt}$ on Si.

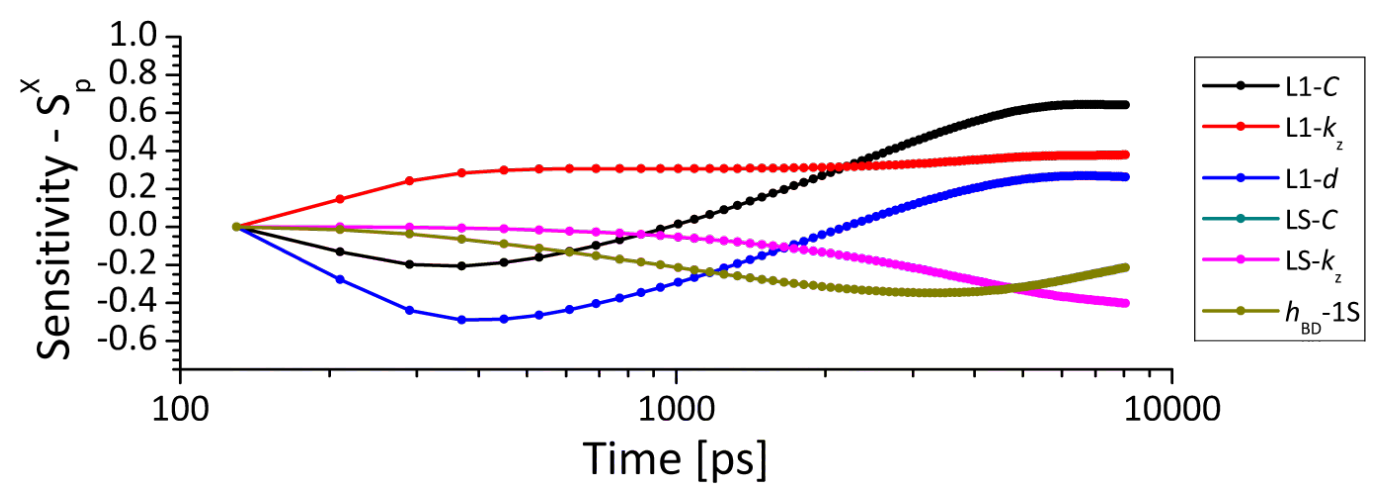

Figure E.2: The sensitivity coefficient as a function of time for $100 \mathrm{~nm}$ of $\mathrm{Ni}$ on $\mathrm{Si}$. 


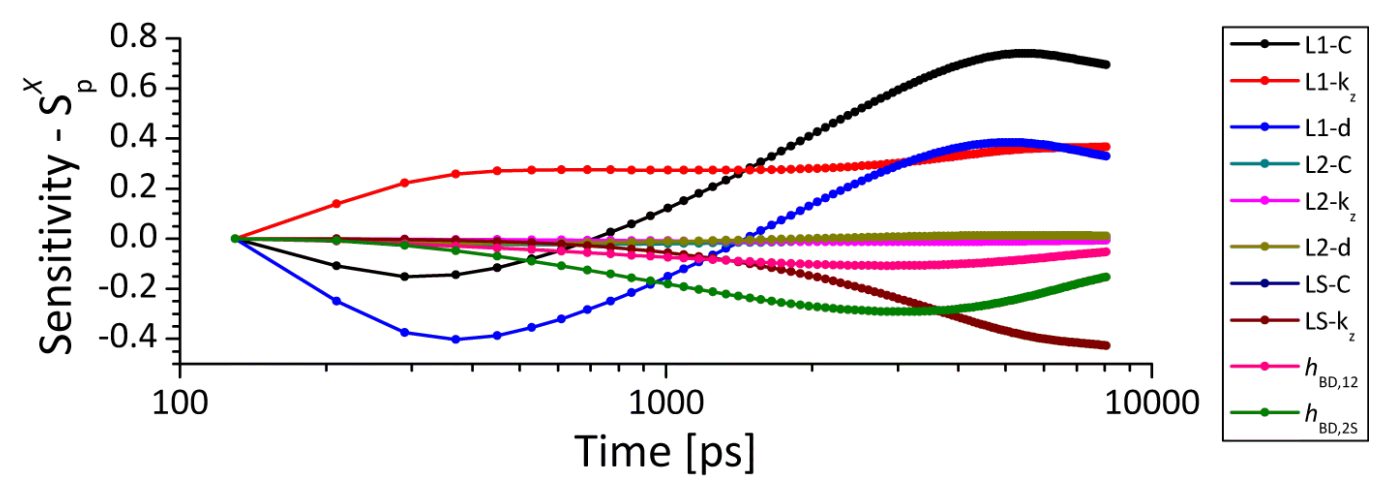

Figure E.3: The sensitivity coefficient as a function of time for $100 \mathrm{~nm}$ of $\mathrm{Pt}$ on $\mathrm{Si}$ with a $5 \mathrm{~nm} \mathrm{Ni}$ intermediate layer.

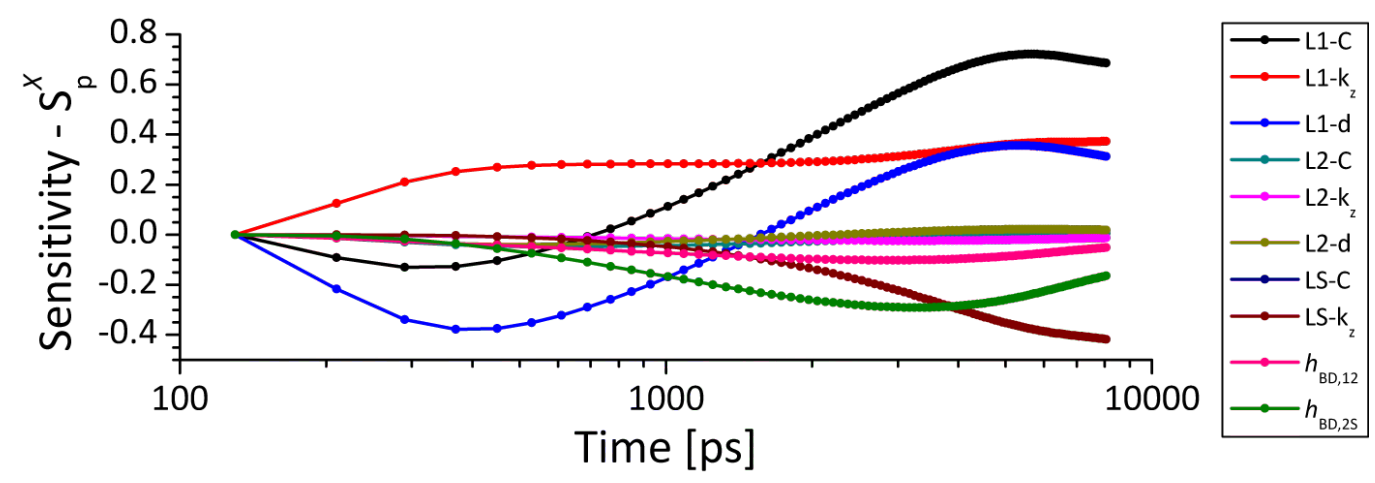

Figure E.4: The sensitivity coefficient as a function of time for $100 \mathrm{~nm}$ of Pt on Si with a $10 \mathrm{~nm} \mathrm{Ni}$ intermediate layer.

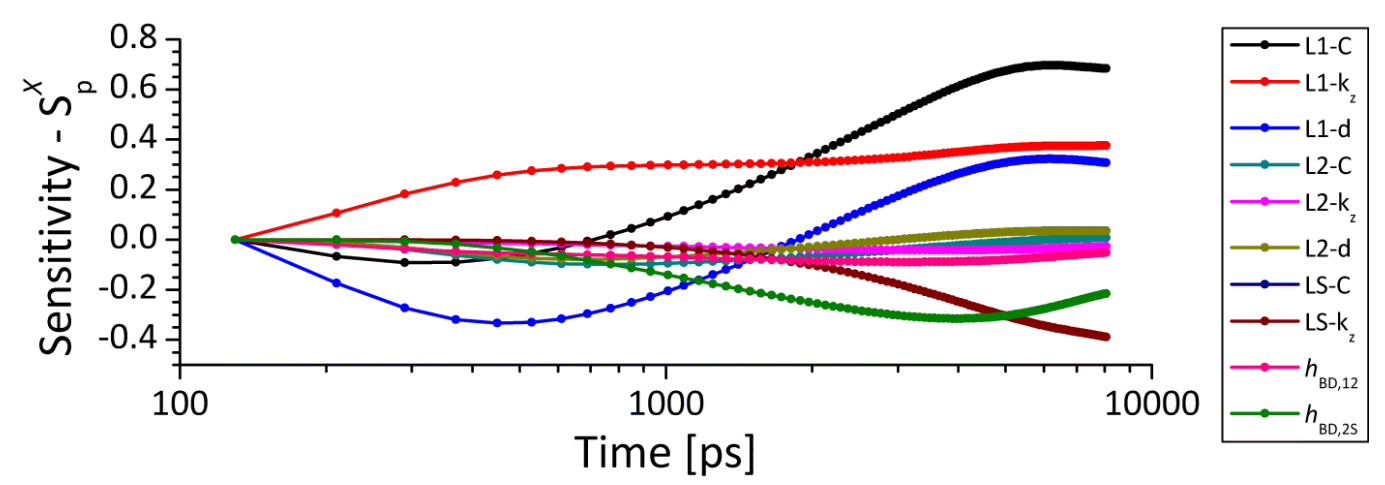

Figure E.5: The sensitivity coefficient as a function of time for $100 \mathrm{~nm}$ of $\mathrm{Pt}$ on $\mathrm{Si}$ with a $20 \mathrm{~nm} \mathrm{Ni}$ intermediate layer. 


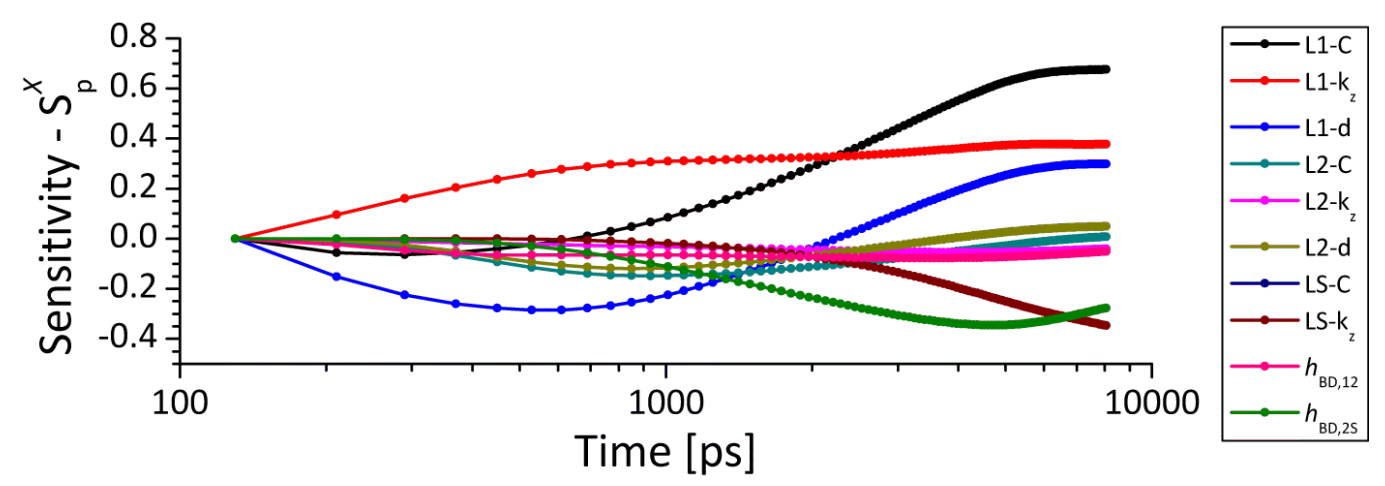

Figure E.6: The sensitivity coefficient as a function of time for $100 \mathrm{~nm}$ of Pt on Si with a $30 \mathrm{~nm} \mathrm{Ni}$ intermediate layer.

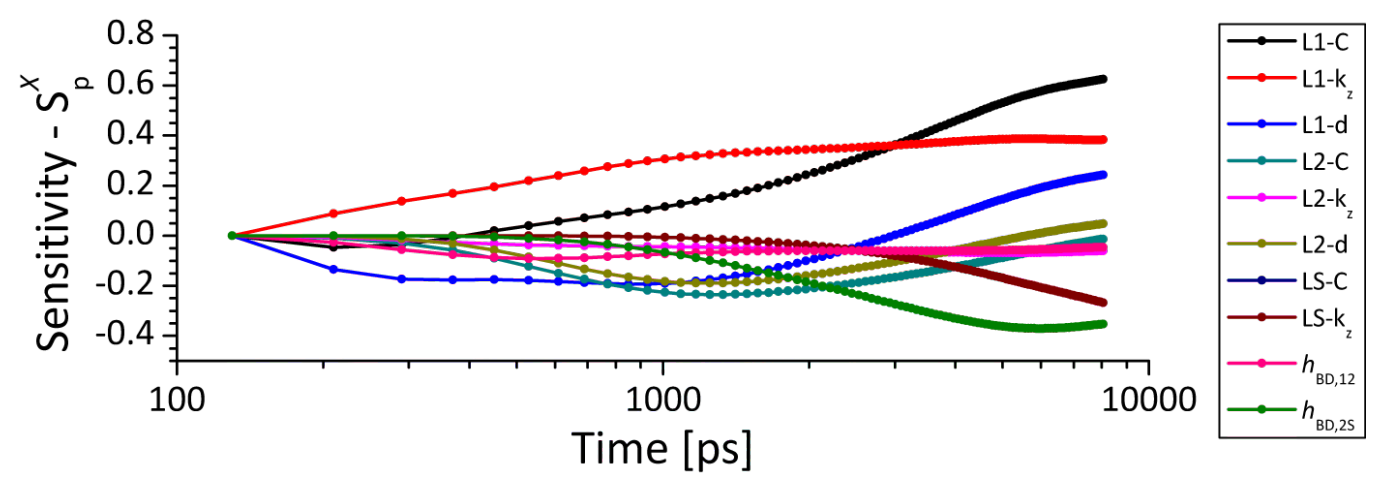

Figure E.7: The sensitivity coefficient as a function of time for $100 \mathrm{~nm}$ of Pt on Si with a $50 \mathrm{~nm} \mathrm{Ni}$ intermediate layer.

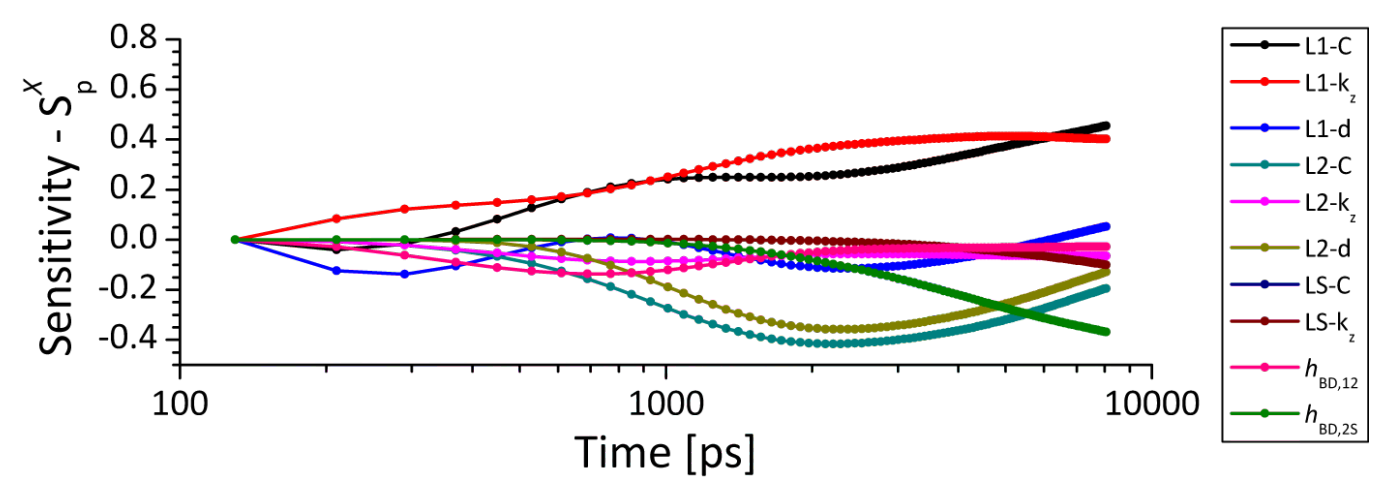

Figure E.8: The sensitivity coefficient as a function of time for $100 \mathrm{~nm}$ of Pt on Si with a $100 \mathrm{~nm} \mathrm{Ni}$ intermediate layer. 


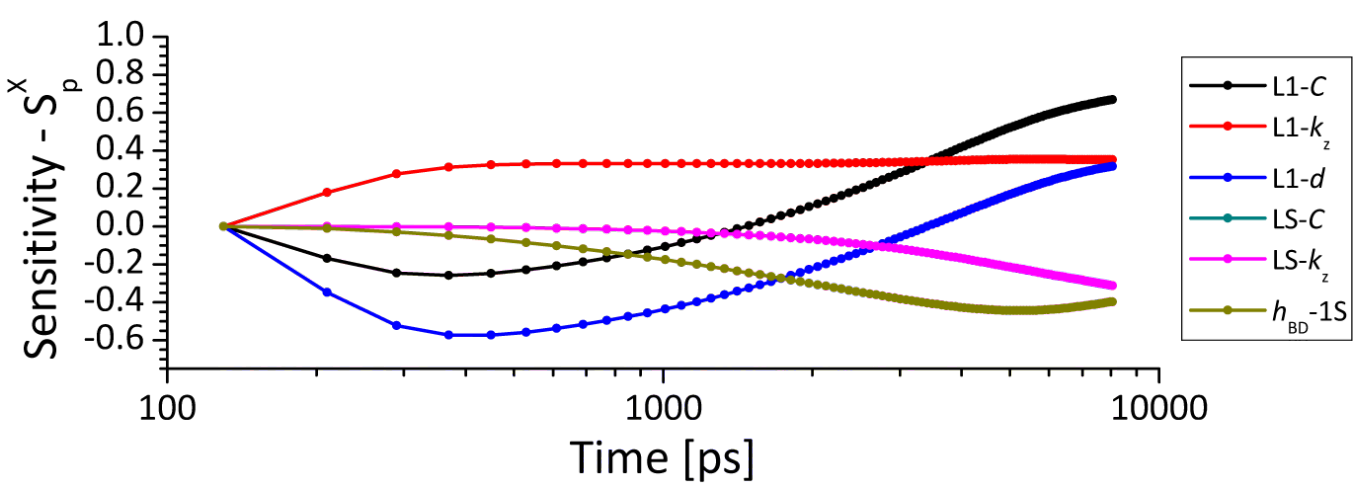

Figure E.9: The sensitivity coefficient as a function of time for $100 \mathrm{~nm}$ of $\mathrm{Pt}$ on Ge.

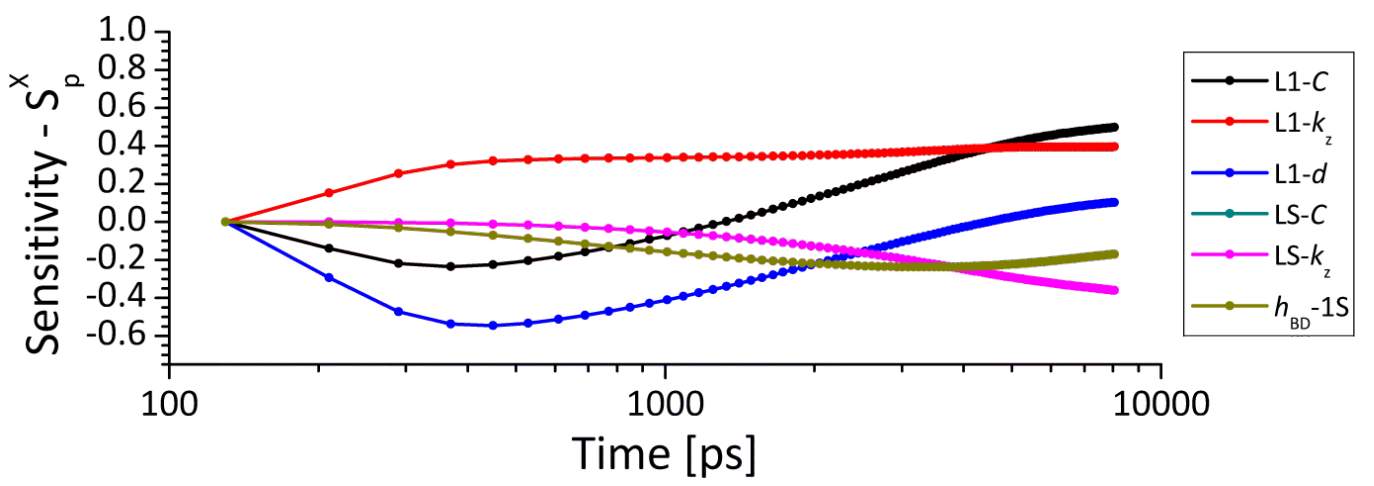

Figure E.10: The sensitivity coefficient as a function of time for $100 \mathrm{~nm}$ of $\mathrm{Ni}$ on Ge.

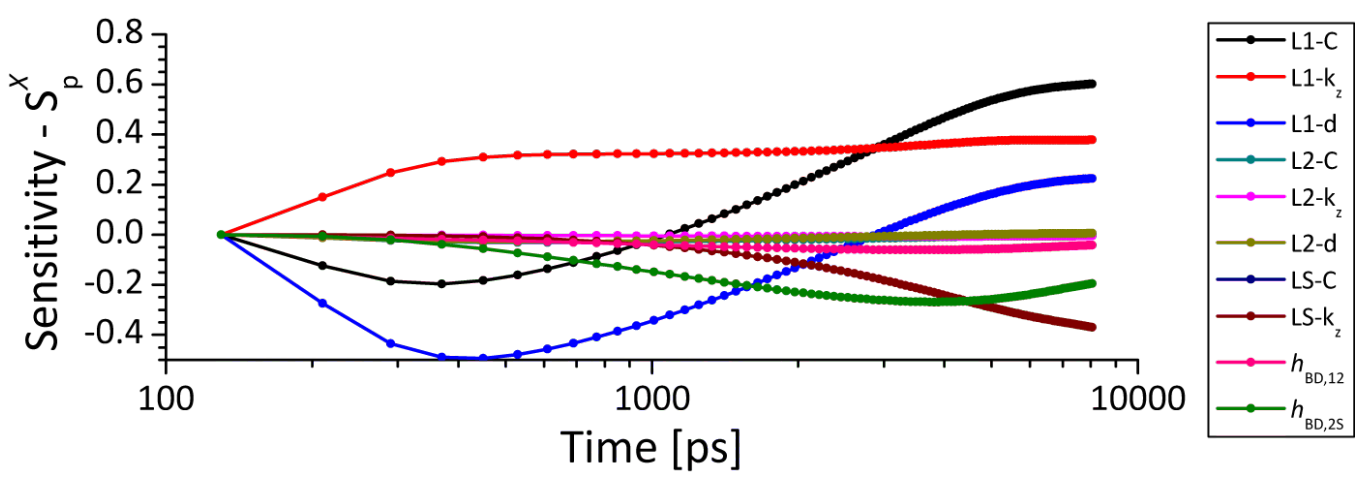

Figure E.11: The sensitivity coefficient as a function of time for $100 \mathrm{~nm}$ of $\mathrm{Pt}$ on Ge with a $5 \mathrm{~nm} \mathrm{Ni}$ intermediate layer. 


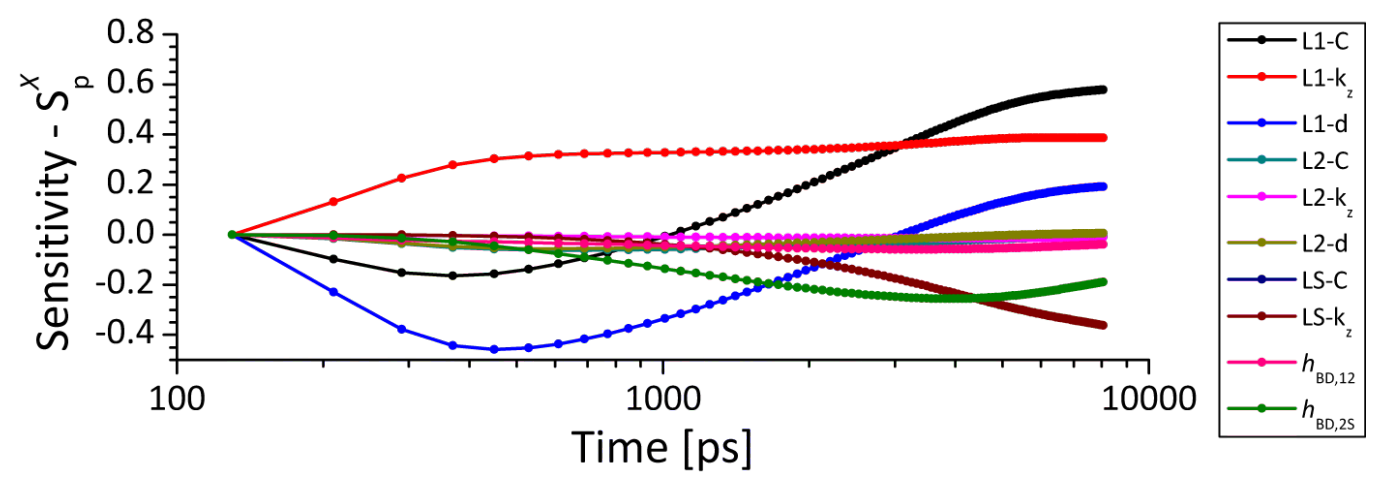

Figure E.12: The sensitivity coefficient as a function of time for $100 \mathrm{~nm}$ of $\mathrm{Pt}$ on Ge with a $10 \mathrm{~nm} \mathrm{Ni}$ intermediate layer.

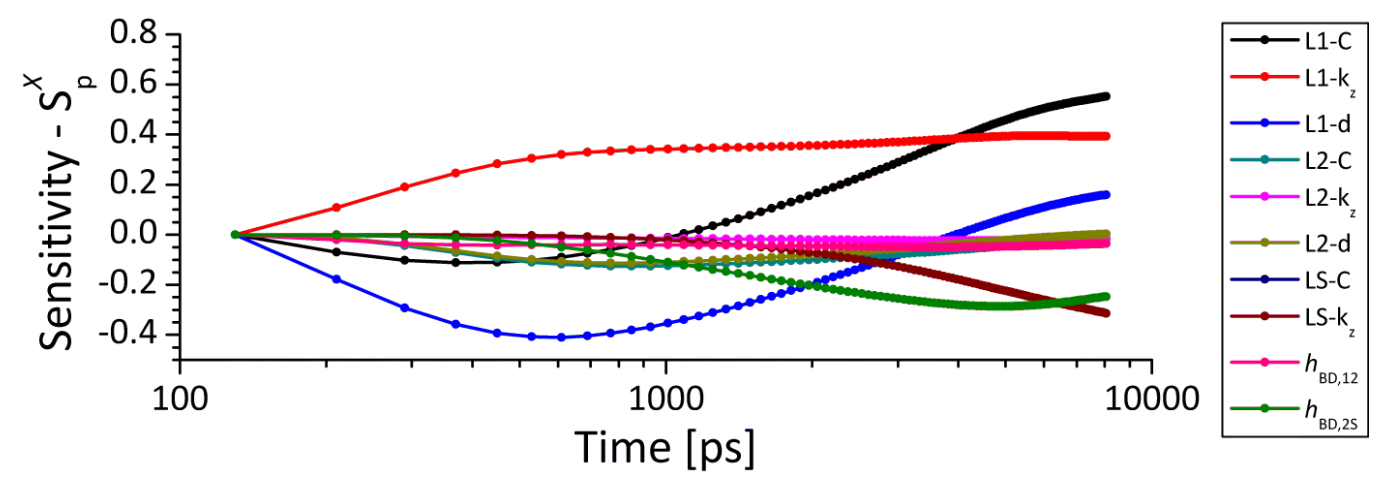

Figure E.13: The sensitivity coefficient as a function of time for $100 \mathrm{~nm}$ of $\mathrm{Pt}$ on Ge with a $20 \mathrm{~nm} \mathrm{Ni}$ intermediate layer.

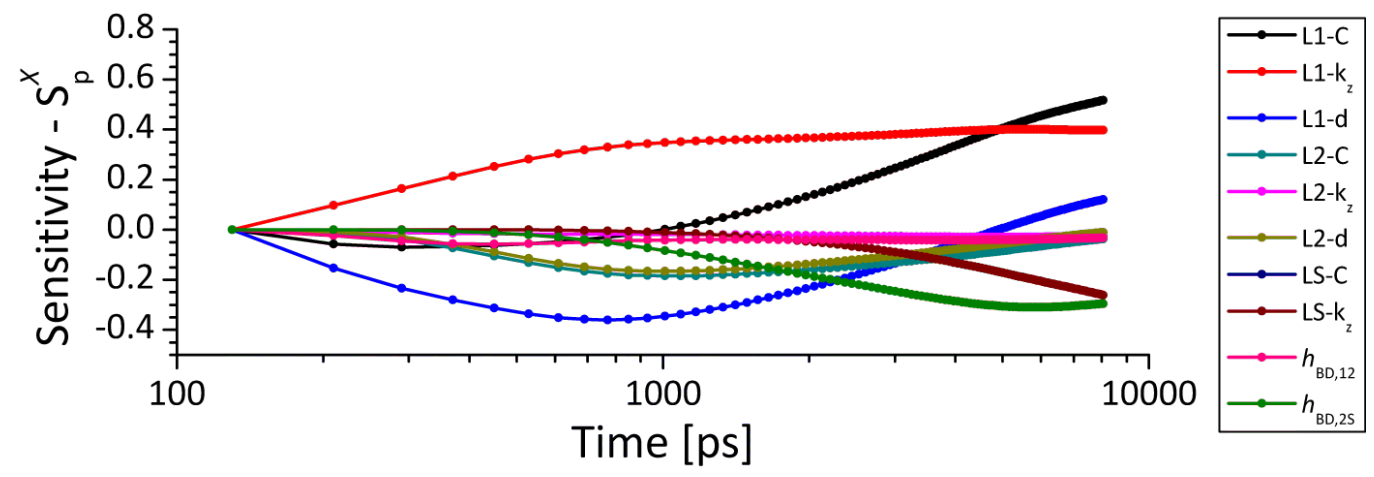

Figure E.14: The sensitivity coefficient as a function of time for $100 \mathrm{~nm}$ of $\mathrm{Pt}$ on Ge with a $30 \mathrm{~nm} \mathrm{Ni}$ intermediate layer. 


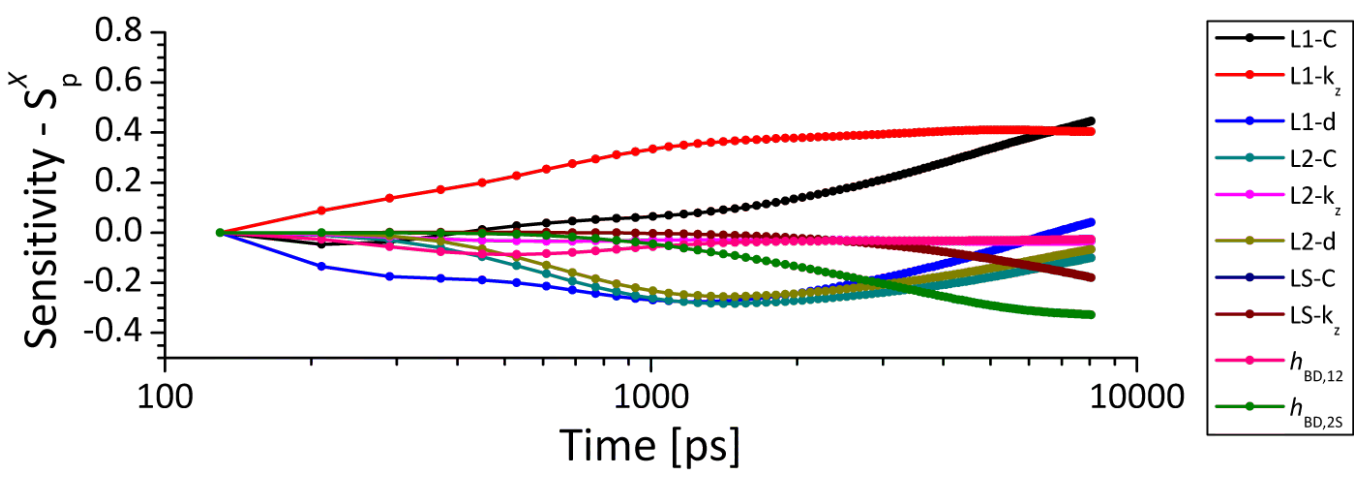

Figure E.15: The sensitivity coefficient as a function of time for $100 \mathrm{~nm}$ of $\mathrm{Pt}$ on Ge with a $50 \mathrm{~nm} \mathrm{Ni}$ intermediate layer.

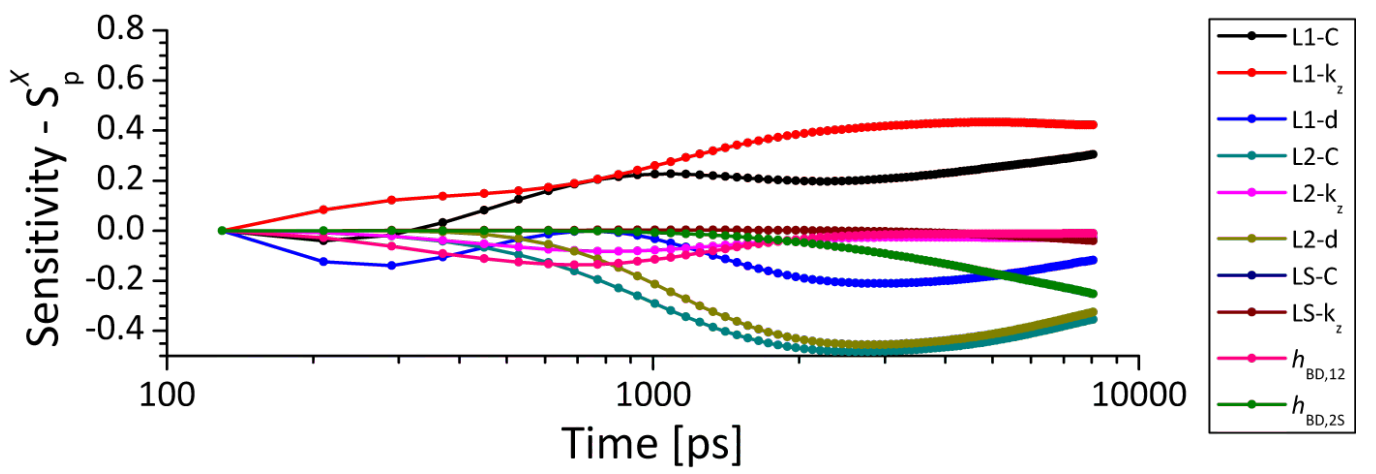

Figure E.16: The sensitivity coefficient as a function of time for $100 \mathrm{~nm}$ of $\mathrm{Pt}$ on Ge with a $100 \mathrm{~nm} \mathrm{Ni}$ intermediate layer. 


\section{BIBLIOGRAPHY}

[1] Ellsworth, JR, M. J. Thermal design and implementation of roubust liquid cooling systems for high performance computer systems. InterPACK '11, July 2007.

[2] IscI, C. Workload Adaptive Power Management with Live Phase Monitoring and Prediction. PhD thesis, Princeton Univeristy, 2007.

[3] Reda, S., Cochran, R. J., And Nowroz, A. N. Improved thermal tracking for processors using hard and soft sensor allocation techniques. IEEE Transactions on Computers 60, 6 (June 2011), 841-851.

[4] Thermal management - teraspeed consulting. http://www.teraspeed.com/, January 2015.

[5] Addison, S. Thermal analysis moves into the 21st century. http://www.electronicscooling.com/2000/01/thermal-analysis-moves-into-the-21st-century/, January 2000.

[6] Tong, X. Thermal management fundamentals and design guides in electronic packaging. In Advanced Materials for Thermal Management of Electronic Packaging, vol. 30 of Springer Series in Advanced Microelectronics. Springer New York, 2011, pp. 1-58.

[7] Pop, E., And Goodson, K. E. Thermal phenomena in nanoscale transistors. Journal of Electronic Packaging 128, 2 (2006), 102-108.

[8] Stoner, R. J., And Maris, H. J. Kapitza conductance and heat flow between solids at temperatures from 50 to 300 K. Physical Review B 48, 22 (1993), 16373-16387.

[9] Lyeo, H.-K., and Cahill, D. G. Thermal conductance of interfaces between highly dissimilar materials. Phys. Rev. B 73, 14 (April 2006), 144301.

[10] Stevens, R. J., Smith, A. N., And Norris, P. M. Measurement of thermal boundary conductance of a series of metal-dielectric interfaces by the transient thermoreflectance technique. Journal of Heat Transfer 127, 3 (2005), 315-322.

[11] Hopkins, P., Salaway, R., Stevens, R., And Norris, P. Temperature-dependent thermal boundary conductance at $\mathrm{Al} / \mathrm{Al}_{2} \mathrm{O}_{3}$ and $\mathrm{Pt} / \mathrm{Al}_{2} \mathrm{O}_{3}$ interfaces. International Journal of Thermophysics 28 (2007), 947-957. 
[12] Hopkins, P. E., Norris, P. M., And Stevens, R. J. Influence of inelastic scattering at metal-dielectric interfaces. Journal of Heat Transfer 130, 2 (2008), 022401.

[13] Gale, J. D., And Rohl, A. L. The general utility lattice program (GULP). Molecular Simulation 29, 5 (2003), 291-341.

[14] English, T. S., Duda, J. C., Smoyer, J. L., Jordan, D. A., Norris, P. M., AND ZHIGILEI, L. V. Enhancing and tuning phonon transport at vibrationally mismatched solid-solid interfaces. Phys. Rev. B 85 (January 2012), 035438.

[15] Hopkins, P. E., Duda, J. C., And Norris, P. M. Anharmonic phonon interactions at interfaces and contributions to thermal boundary conductance. Journal of Heat Transfer 133, 6 (2011), 062401.

[16] Chen, J., Zhang, G., And Li, B. Thermal contact resistance across nanoscale silicon dioxide and silicon interface. Journal of Applied Physics 112, 6 (2012), 064319.

[17] Li, X., AND YANG, R. Effect of lattice mismatch on phonon transmission and interface thermal conductance across dissimilar material interfaces. Phys. Rev. B 86 (Aug 2012), 054305.

[18] Tian, Z., Esfarjani, K., And Chen, G. Enhancing phonon transmission across a Si/Ge interface by atomic roughness: First-principles study with the Green's function method. Phys. Rev. B 86 (Dec 2012), 235304.

[19] Hines, W. W., Montgomery, D. C., Goldsman, D. M., and Borror, C. M. Probability and Statistics in Engineering, 4th ed. John Wiley \& Sons, 2003.

[20] Liang, Z., And Tsai, H.-L. Reduction of solid-solid thermal boundary resistance by inserting an interlayer. International Journal of Heat and Mass Transfer 55, 1112 (2012), $2999-3007$.

[21] Hopkins, P. E., Klopf, J. M., And Norris, P. M. Influence of interband transitions on electron-phonon coupling measurements in ni films. Appl. Opt. 46, 11 (2007), $2076-2083$.

[22] Li-Dan, Z., Fang-Yuan, S., Jie, Z., Da-Wei, T., Yu-Hua, L., and ChaOHong, G. Nano-metal film thermal conductivity measurement by using the femtosecond laser pump and probe method. Chinese Physics Letters 29, 6 (2012), 066301.

[23] Caffrey, A. P., Hopkins, P. E., Klopf, J. M., and Norris, P. M. Thin film non-noble transition metal thermophysical properties. Nanoscale and Microscale Thermophysical Engineering 9, 4 (2005), 365-377.

[24] Sze, S. M., And NG, K. K. Physics of Semiconductor Devices, 3rd ed. WileyInterscience, 2006.

[25] Michaelson, H. B. Relation between an atomic electronegativity scale and the work function. IBM Journal of Research and Devlopment 22 (1978), 72-80.

[26] Tubino, R., Piseri, L., And Zerbi, G. Lattice dynamics and spectroscopic properties by a valence force potential of diamondlike crystals: $\mathrm{C}, \mathrm{Si}, \mathrm{Ge}$, and $\mathrm{Sn}$. The Journal of Chemical Physics 56, 3 (1972), 1022-1039. 
[27] PAlik, E. D. Handbook of Optical Constants of Solids. Academic Press, 1985.

[28] Prasher, R. Thermal interface materials: Historical perspective, status, and future directions. Proceedings of the IEEE 94, 8 (August 2006), 1571-1586.

[29] Samson, E. C., Machiroutu, S. V., Chang, J.-Y., Santos, I., Hermerding, J., Dani, A., Prasher, R., And Song, D. Interface material selection and a thermal management technique in second-generation platforms built on Intel Centrino mobile technology. Intel technology journal 9, 1 (2005), 75.

[30] Narumanchi, S., Mihalic, M., Kelly, K., and Eesley, G. Thermal interface materials for power electronics applications. In Thermal and Thermomechanical Phenomena in Electronic Systems, 2008. ITHERM 2008. 11th Intersociety Conference on (May 2008), pp. 395-404.

[31] Gwinn, J., And WebB, R. Performance and testing of thermal interface materials. Microelectronics Journal 34, 3 (2003), 215 - 222.

[32] Ashcroft, N. W., And Mermin, N. D. Solid State Physics. Sanders College Publishing, 1976.

[33] Chen, G. Nanoscale energy transport and conversion: A parallel treatment of electrons, molecules, phonons, and photons. Oxford University Press, New York, 2005.

[34] Hartree, D. R. The Eniac, an electronic computing machine. Nature 158, 4015 (1946), 500-506.

[35] Bergles, A. Evolution of cooling technology for electrical, electronic, and microelectronic equipment. Components and Packaging Technologies, IEEE Transactions on 26, 1 (2003), 6-15.

[36] Chu, R., Simons, R., Ellsworth, M., Schmidt, R., and Cozzolino, V. Review of cooling technologies for computer products. Device and Materials Reliability, IEEE Transactions on 4, 4 (December 2004), 568 - 585.

[37] Eniac: Celebrating penn engineering history. http://www.seas.upenn.edu/aboutseas/eniac/operation.php, September 2014.

[38] KempF, K. Electronic computers within the ordnance corps. http://ftp.arl.mil/mike/comphist/61ordnance/, November 1961.

[39] Schmidt, R. R., And Notohardjono, B. D. High-end server low-temperature cooling. IBM Journal of Research and Devlopment 46, 6 (2002), 739.

[40] Chu, R., Simons, R., And Chrysler, G. Experimental investigation of an enhanced thermosyphon heat loop for cooling of a high performance electronics module. In Semiconductor Thermal Measurement and Management Symposium, 1999. Fifteenth Annual IEEE (1999), pp. 1-9.

[41] Moore, G. Cramming more components onto integrated circuits. Electronics 38, 8 (April 1965), 114-117.

[42] Moore, G. Progress in digital integrated electronics. In Electron Devices Meeting, 1975 International (1975), vol. 21, pp. 11-13. 
[43] Vassighi, A., And Sachdev, M. Thermal and Power Management of Integrated Circuits. Springer US, 2006.

[44] Pop, E., Sinha, S., And Goodson, K. E. Heat generation and transport in nanometer-scale transistors. Proceedings of the IEEE 94, 8 (2006), 1587-1601.

[45] Pop, E. Energy dissipation and transport in nanoscale devices. Nano Research 3 (2010), 147-169.

[46] Pedram, M., And Nazarian, S. Thermal modeling, analysis, and management in VLSI circuits: Principles and methods. Proceedings of the IEEE 94, 8 (Aug 2006), $1487-1501$.

[47] Mahajan, R., Nair, R., Wakharkar, V., Swan, J., Tang, J., and Vandentop, G. Emerging directions for packaging technologies. Intel Technology Journal 6 (2002), $62-75$.

[48] Mahajan, R., Pin Chiu, C., and Chrysler, G. Cooling a microprocessor chip. Proceedings of the IEEE 94, 8 (August 2006), 1476 -1486.

[49] Atluri, V. P., Mahajan, R. V., Patel, P. R., Mallik, D., Tang, J., Wakharkar, V. S., Chrysler, G. M., Chiu, C.-P., Choksi, G. N., And VisWANATH, R. S. Critical aspects of high-performance microprocessor packaging. MRS Bulletin 28 (0 2003), 21-34.

[50] Pop, E., On Chui, C., Dutton, R., Sinha, S., and Goodson, K. Electrothermal comparison and performance optimization of thin-body SOI and GOI MOSFETs. In Electron Devices Meeting, 2004. IEDM Technical Digest. IEEE International (2004), pp. 411-414.

[51] Fiegna, C., Yang, Y., Sangiorgi, E., and O’Neill, A. G. Analysis of selfheating effects in ultrathin-body SOI MOSFETs by device simulation. Electron Devices, IEEE Transactions on 55, 1 (Jan 2008), 233-244.

[52] Bar-Cohen, A., And Wang, P. Thermal management of on-chip hot spot. Journal of Heat Transfer 134, 5 (2012), 051017.

[53] Shakouri, A., And Zhang, Y. On-chip solid-state cooling for integrated circuits using thin-film microrefrigerators. Components and Packaging Technologies, IEEE Transactions on 28, 1 (2005), 65-69.

[54] Brooks, D., Dick, R., Joseph, R., And Shang, L. Power, thermal, and reliability modeling in nanometer-scale microprocessors. Micro, IEEE 27, 3 (May 2007), 49-62.

[55] Patterson, M. The effect of data center temperature on energy efficiency. In Thermal and Thermomechanical Phenomena in Electronic Systems, 2008. ITHERM 2008. 11th Intersociety Conference on (May 2008), pp. 1167-1174.

[56] Hamann, H., Weger, A., Lacey, J., Hu, Z., Bose, P., Cohen, E., And Wakil, J. Hotspot-limited microprocessors: Direct temperature and power distribution measurements. Solid-State Circuits, IEEE Journal of 42, 1 (2007), 56-65. 
[57] Black, J. R. Electromigration - a brief survey and some recent results. Electron Devices, IEEE Transactions on 16, 4 (Apr 1969), 338-347.

[58] Banerjee, K., Pedram, M., And Ajami, A. H. Analysis and optimization of thermal issues in high-performance VLSI. In Proceedings of the 2001 International Symposium on Physical Design (New York, NY, USA, 2001), ISPD '01, ACM, pp. 230237.

[59] Singh, R., Sahu, D., Shukla, N. K., Bhatnagar, P., Geetanjali, and Goel, A. Analysis of the effect of temperature variations on sub-threshold leakage current in P3 and P4 SRAM cells at deep sub-micron CMOS technology. International Journal of Computer Applications 35, 5 (December 2011), 8-13.

[60] Youtube viewership statistics. http://www.youtube.com/yt/press/statistics.html, May 2013.

[61] Garimella, S. V., Yeh, L.-T., and Persoons, T. Thermal management challenges in telecommunication systems and data centers. Components, Packaging and Manufacturing Technology, IEEE Transactions on 2, 8 (2012), 1307-1316.

[62] Viswanath, R., Wakharkar, V., Watwe, A., and Lebonheur, V. Thermal performance challenges from silicon to systems. Tech. rep., Intel Technology Journal, 2000 .

[63] Savija, I., Culham, J. R., Yovanovich, M. M., and Marotta, E. E. Review of thermal conductance models for joints incorporating enhancement materials. Journal of Thermophysics and Heat Transfer 17, 1 (2003), 43-52.

[64] Prasher, R. S. Surface chemistry and characteristics based model for the thermal contact resistance of fluidic interstitial thermal interface materials. Journal of Heat Transfer 123, 5 (2001), 969-975.

[65] Prasher, R. S., Shipley, J., Prstic, S., Koning, P., And lin Wang, J. Thermal resistance of particle laden polymeric thermal interface materials. Journal of Heat Transfer 125, 6 (2003), 1170-1177.

[66] Sarvar, F., Whalley, D., And Conway, P. Thermal interface materials - a review of the state of the art. In Electronics Systemintegration Technology Conference (sep. 2006), vol. 2, pp. $1292-1302$.

[67] Goyal, V., And Balandin, A. A. Thermal properties of the hybrid graphene-metal nano-micro-composites: Applications in thermal interface materials. Applied Physics Letters 100, 7 (2012), 073113.

[68] Fu, Y., Nabiollahi, N., Wang, T., Wang, S., Hu, Z., Carlberg, B., Zhang, Y., WANG, X., AND LIU, J. A complete carbon-nanotube-based on-chip cooling solution with very high heat dissipation capacity. Nanotechnology 23, 4 (2012), 045304.

[69] Garimella, S. V., Fleischer, A., Murthy, J. Y., Keshavarzi, A., Prasher, R., Patel, C., Bhavnani, S., Venkatasubramanian, R., Mahajan, R., Joshi, 
Y., Sammakia, B., Myers, B., Chorosinski, L., Baelmans, M., SathyaMURThy, P., AND RAAD, P. Thermal challenges in next-generation electronic systems. Components and Packaging Technologies, IEEE Transactions on 31, 4 (2008), 801-815.

[70] Fan, V., Harman, D., Jewett, J., Leet, B., and Speranza, D. Evaluation process for semiconductor fabrication materials that are better for the environment. Intel Technology Journal 12, 1 (February 2008), 69-76.

[71] Yovanovich, M. Four decades of research on thermal contact, gap, and joint resistance in microelectronics. Components and Packaging Technologies, IEEE Transactions on 28, 2 (June 2005), $182-206$.

[72] Swartz, E. T., And Pohl, R. O. Thermal boundary resistance. Rev. Mod. Phys. 61, 3 (July 1989), 605-668.

[73] Cahill, D. G., Ford, W. K., Goodson, K. E., Mahan, G. D., Majumdar, A., Maris, H. J., Merlin, R., And Phillpot, S. R. Nanoscale thermal transport. Journal of Applied Physics 93, 2 (2003), 793-818.

[74] Majumdar, A., And Reddy, P. Role of electron-phonon coupling in thermal conductance of metal-nonmetal interfaces. Applied Physics Letters 84, 23 (2004), 47684770 .

[75] Gundrum, B. C., Cahill, D. G., and Averback, R. S. Thermal conductance of metal-metal interfaces. Phys. Rev. B 72, 24 (December 2005), 245426.

[76] Singh, P., Seong, M., And Sinha, S. Detailed consideration of the electron-phonon thermal conductance at metal-dielectric interfaces. Applied Physics Letters 102, 18 (2013), 181906.

[77] Battabyal, M., Beffort, O., Kleiner, S., Vaucher, S., and Rohr, L. Heat transport across the metal-diamond interface. Diamond and Related Materials 17, 710 (2008), $1438-1442$.

[78] Kim, W., Wang, R., And Majumdar, A. Nanostructuring expands thermal limits. Nano Today 2, 1 (2007), 40 - 47.

[79] Capinski, W. S., Maris, H. J., Ruf, T., Cardona, M., Ploog, K., and Katzer, D. S. Thermal conductivity measurements of GaAs/AlAs superlattices using a picosecond optical pump-and-probe technique. Phys. Rev. B 59, 12 (March 1999), 8105-8113.

[80] Goodson, K. E., And Ju, Y. S. Heat conduction in novel electronic films. Annual Review of Materials Science 29, 1 (1999), 261-293.

[81] Chen, G. Size and interface effects on thermal conductivity of superlattices and periodic thin-film structures. Journal of Heat Transfer 119, 2 (May 1997), 220-229.

[82] Dames, C., and Chen, G. Theoretical phonon thermal conductivity of Si/Ge superlattice nanowires. Journal of Applied Physics 95, 2 (2004), 682-693. 
[83] Hepplestone, S. P., And Srivastava, G. P. Phononic gaps in thin semiconductor superlattices. Journal of Applied Physics 10\%, 4 (2010), 043504.

[84] Lops, A., Spagnolo, V., And Scamarcio, G. Thermal modeling of GaInAs/AlInAs quantum cascade lasers. Journal of Applied Physics 100, 4 (2006), 043109.

[85] Ezzahri, Y., Grauby, S., Dilhaire, S., Rampnoux, J. M., and Claeys, W. Cross-plan $\mathrm{Si} / \mathrm{SiGe}$ superlattice acoustic and thermal properties measurement by picosecond ultrasonics. Journal of Applied Physics 101, 1 (2007), 013705.

[86] Panzer, M. A., Zhang, G., Mann, D., Hu, X., Pop, E., Dai, H., And GoodSON, K. E. Thermal properties of metal-coated vertically aligned single-wall nanotube arrays. Journal of Heat Transfer 130, 5 (2008), 052401.

[87] Cola, B. A., Xu, X., And Fisher, T. S. Increased real contact in thermal interfaces: A carbon nanotube/foil material. Applied Physics Letters 90, 9 (2007), 093513.

[88] Reifenberg, J., Kencke, D., and Goodson, K. The impact of thermal boundary resistance in phase-change memory devices. Electron Device Letters, IEEE 29, 10 (October 2008), $1112-1114$.

[89] Reifenberg, J., Chang, K.-W., Panzer, M., Kim, S., Rowlette, J., Asheghi, M., Wong, H.-S., And Goodson, K. Thermal boundary resistance measurements for phase-change memory devices. Electron Device Letters, IEEE 31, 1 (January 2010), $56-58$.

[90] Cho, J., Bozorg-Grayeli, E., Altman, D., Asheghi, M., And Goodson, K. Low thermal resistances at GaN-SiC interfaces for HEMT technology. Electron Device Letters, IEEE 33, 3 (March 2012), 378 -380.

[91] Hopkins, P. E. Thermal transport across solid interfaces with nanoscale imperfections: Effects of roughness, disorder, dislocations, and bonding on thermal boundary conductance. ISRN Mechanical Engineering 2013 (2013), 19.

[92] Hopkins, P. E., Beechem, T. E., Duda, J. C., Smoyer, J. L., And Norris, P. M. Effects of subconduction band excitations on thermal conductance at metalmetal interfaces. Applied Physics Letters 96, 1 (2010), 011907.

[93] Wilson, R. B., And Cahill, D. G. Experimental validation of the interfacial form of the Wiedemann-Franz law. Phys. Rev. Lett. 108 (Jun 2012), 255901.

[94] Cahill, D. G., Braun, P. V., Chen, G., Clarke, D. R., Fan, S., Goodson, K. E., Keblinski, P., King, W. P., Mahan, G. D., Majumdar, A., Maris, H. J., Phillpot, S. R., Pop, E., And Shi, L. Nanoscale thermal transport. II. 2003-2012. Applied Physics Reviews 1, 1 (2014), 011305.

[95] Huberman, M. L., And Overhauser, A. W. Electronic Kapitza conductance at a diamond-Pb interface. Phys. Rev. B 50 (Aug 1994), 2865-2873.

[96] Hopkins, P. E., And Norris, P. M. Substrate influence in electron-phonon coupling measurements in thin Au films. Applied Surface Science 253, 15 (2007), 6289 - 6294. 
[97] Hopkins, P. E., Kassebaum, J. L., And Norris, P. M. Effects of electron scattering at metal-nonmetal interfaces on electron-phonon equilibration in gold films. Journal of Applied Physics 105, 2 (2009), 023710.

[98] Abramson, A. R., Tien, C.-L., and Majumdar, A. Interface and strain effects on the thermal conductivity of heterostructures: A molecular dynamics study. Journal of Heat Transfer 124, 5 (2002), 963-970.

[99] McGaughey, A., And Kaviany, M. Thermal conductivity decomposition and analysis using molecular dynamics simulations. part I. Lennard-Jones argon. International Journal of Heat and Mass Transfer 47 (2004), 1783 - 1798.

[100] Choi, S.-H., And Maruyama, S. Thermal boundary resistance at an epitaxially perfect interface of thin films. International Journal of Thermal Sciences 44, 6 (2005), $547-558$.

[101] McGaughey, A. J. H., Hussein, M. I., Landry, E. S., Kaviany, M., And Hulbert, G. M. Phonon band structure and thermal transport correlation in a layered diatomic crystal. Phys. Rev. B 74 (Sep 2006), 104304.

[102] Hegedus, P. J., And Abramson, A. R. A molecular dynamics study of interfacial thermal transport in heterogeneous systems. International Journal of Heat and Mass Transfer 49, 25-26 (2006), 4921 - 4931.

[103] Landry, E. S., Hussein, M. I., And McGaughey, A. J. H. Complex superlattice unit cell designs for reduced thermal conductivity. Phys. Rev. B 77, 18 (May 2008), 184302.

[104] Sun, L., And Murthy, J. Y. Molecular dynamics simulation of phonon scattering at silicon/germanium interfaces. Journal of Heat Transfer 132, 10 (2010), 102403.

[105] Landry, E. S., And McGaughey, A. J. H. Thermal boundary resistance predictions from molecular dynamics simulations and theoretical calculations. Phys. Rev. B 80, 16 (October 2009), 165304.

[106] Landry, E. S., And McGaughey, A. J. H. Effect of interfacial species mixing on phonon transport in semiconductor superlattices. Phys. Rev. B 79 (February 2009), 075316 .

[107] Sellan, D. P., Landry, E. S., Turney, J. E., McGaughey, A. J. H., And Amon, C. H. Size effects in molecular dynamics thermal conductivity predictions. Phys. Rev. B 81 (Jun 2010), 214305.

[108] Landry, E. S., And McGaughey, A. J. H. Effect of film thickness on the thermal resistance of confined semiconductor thin films. Journal of Applied Physics 10\%, 1 (2010), 013521.

[109] Termentzidis, K., Chantrenne, P., And Keblinski, P. Nonequilibrium molecular dynamics simulation of the in-plane thermal conductivity of superlattices with rough interfaces. Phys. Rev. B 79 (Jun 2009), 214307. 
[110] Stevens, R. J., Zhigilei, L. V., And Norris, P. M. Effects of temperature and disorder on thermal boundary conductance at solid-solid interfaces: Nonequilibrium molecular dynamics simulations. International Journal of Heat and Mass Transfer 50, 19-20 (2007), 3977-89.

[111] Twu, C.-J., And Ho, J.-R. Molecular-dynamics study of energy flow and the Kapitza conductance across an interface with imperfection formed by two dielectric thin films. Phys. Rev. B 67 (May 2003), 205422.

[112] Lyver IV, J. W., And Blaisten-Barojas, E. Effects of the interface between two Lennard-Jones crystals on the lattice vibrations: A molecular dynamics study. Journal of Physics: Condensed Matter 21, 34 (2009), 345402.

[113] Salaway, R., Hopkins, P., Norris, P., and Stevens, R. Phonon contribution to thermal boundary conductance at metal interfaces using embedded atom method simulations. International Journal of Thermophysics 29 (2008), 1987-1996.

[114] Ju, S., Liang, X., AND WANG, S. Investigation of interfacial thermal resistance of bi-layer nanofilms by nonequilibrium molecular dynamics. Journal of Physics D: Applied Physics 43, 8 (2010), 085407.

[115] Roberts, N., Walker, D., And Li, D. Molecular dynamics simulation of thermal conductivity of nanocrystalline composite films. International Journal of Heat and Mass Transfer 52, 78 (2009), 2002 - 2008.

[116] Roberts, N. A., And Walker, D. G. Phonon wave-packet simulations of Ar/Kr interfaces for thermal rectification. Journal of Applied Physics 108, 12 (2010), 123515.

[117] Zhang, W., Fisher, T., And Mingo, N. Simulation of interfacial phonon transport in Si-Ge heterostructures using an atomistic Green's function method. Journal of Heat Transfer 129 (2006), 483-491.

[118] Zhang, W., Fisher, T. S., And Mingo, N. The atomistic Green's function method: An efficient simulation approach for nanoscale phonon transport. Numerical Heat Transfer, Part B: Fundamentals 51, 4 (2007), 333-349.

[119] Kapitza, P. L. The study of heat transfer in helium II. Journal of Physics (USSR) 4 (1941), 181.

[120] Little, W. A. The transport of heat between dissimlar solids at low temperatures. Canadian Journal of Physics 37, 3 (1959), 334-349.

[121] Ziman, J. M. Electrons and phonons : The theory of transport phenomena in solids. Oxford University Press, 2001.

[122] KAZAN, M. Interpolation between the acoustic mismatch model and the diffuse mismatch model for the interface thermal conductance: Application to InN/GaN superlattice. Journal of Heat Transfer 133, 11 (2011), 112401.

[123] Swartz, E. T., And Pohl, R. O. Thermal resistance at interfaces. Applied Physics Letters 51, 26 (1987), 2200-2202. 
[124] Duda, J. C., Hopkins, P. E., Smoyer, J. L., Bauer, M. L., English, T. S., Saltonstall, C. B., And Norris, P. M. On the assumption of detailed balance in prediction of diffusive transmission probability during interfacial transport. Nanoscale and Microscale Thermophysical Engineering 14, 1 (2010), 21.

[125] Pratt, JR, W., And Bass, J. Perpendicular-current studies of electron transport across metal/metal interfaces. Applied Surface Science 256, 2 (2009), 399 - 403.

[126] Shpiro, A., And Levy, P. M. Resistance across an interface, and that measured far from it. Phys. Rev. B 63 (Dec 2000), 014419.

[127] Bauer, G. E. W., Schep, K. M., Xia, K., And Kelly, P. J. Scattering theory of interface resistance in magnetic multilayers. Journal of Physics D: Applied Physics 35, 19 (2002), 2410.

[128] Drchal, V., Kudrnovský, J., Bruno, P., Dederichs, P. H., Turek, I., And Weinberger, P. Electron transport in magnetic multilayers: Effect of disorder. Phys. Rev. B 65 (May 2002), 214414.

[129] Velev, J., And Butler, W. H. Effects of interface disorder on transmission probability in magnetic multilayer. Phys. Rev. B 69 (Jan 2004), 024404.

[130] Loh, G. C., Tay, B. K., And Teo, E. H. T. Flux-mediated diffuse mismatch model. Applied Physics Letters 97, 12 (2010), 121917.

[131] Duda, J. C., Norris, P. M., And Hopkins, P. E. On the linear temperature dependence of phonon thermal boundary conductance in the classical limit. Journal of Heat Transfer 133, 7 (2011), 074501.

[132] Beechem, T., Duda, J. C., Hopkins, P. E., and Norris, P. M. Contribution of optical phonons to thermal boundary conductance. Applied Physics Letters 97, 6 (2010), 061907.

[133] Duda, J. C., Beechem, T. E., Smoyer, J. L., Norris, P. M., And Hopkins, P. E. Role of dispersion on phononic thermal boundary conductance. Journal of Applied Physics 108, 7 (2010), 073515.

[134] Reddy, P., Castelino, K., and Majumdar, A. Diffuse mismatch model of thermal boundary conductance using exact phonon dispersion. Applied Physics Letters 87, 21 (2005), 211908.

[135] Duda, J. C., Smoyer, J. L., Norris, P. M., And Hopkins, P. E. Extension of the diffuse mismatch model for thermal boundary conductance between isotropic and anisotropic materials. Applied Physics Letters 95, 3 (2009), 031912.

[136] Hopkins, P. E., Beechem, T., Duda, J. C., Hattar, K., Ihlefeld, J. F., Rodriguez, M. A., And Piekos, E. S. Influence of anisotropy on thermal boundary conductance at solid interfaces. Phys. Rev. B 84 (September 2011), 125408.

[137] Hopkins, P. E., And Norris, P. M. Relative contributions of inelastic and elastic diffuse phonon scattering to thermal boundary conductance across solid interfaces. Journal of Heat Transfer 131, 2 (2009), 022402. 
[138] Hopkins, P. E., And Norris, P. M. Effects of joint vibrational states on thermal boundary conductance. Nanoscale and Microscale Thermophysical Engineering 11, 3-4 (2007), 247-257.

[139] Duda, J. C., Hopkins, P. E., Beechem, T. E., Smoyer, J. L., And Norris, P. M. Inelastic phonon interactions at solid-graphite interfaces. Superlattices and Microstructures 47, 4 (2010), 550 - 555.

[140] Hopkins, P. E. Multiple phonon processes contributing to inelastic scattering during thermal boundary conductance at solid interfaces. Journal of Applied Physics 106, 1 (2009), 013528.

[141] Norris, P. M., AND Hopkins, P. E. Examining interfacial diffuse phonon scattering through transient thermoreflectance measurements of thermal boundary conductance. Journal of Heat Transfer 131, 4 (2009), 043207.

[142] Costescu, R. M., Wall, M. A., and Cahill, D. G. Thermal conductance of epitaxial interfaces. Phys. Rev. B 67, 5 (February 2003), 054302.

[143] Poate, J. M., Tu, K. N., And Mayer, J. W. Thin films-interdiffusion and reactions. John Wiley \& Sons, 1978.

[144] Paddock, C. A., And Eesley, G. L. Transient thermoreflectance from thin metal films. Journal of Applied Physics 60, 1 (1986), 285-290.

[145] Norris, P. M., Le, N. Q., And Baker, C. H. Tuning phonon transport: From interfaces to nanostructures. Journal of Heat Transfer 135, HT-12-1575 (May 2013), 061604.

[146] Luo, T., And Chen, G. Nanoscale heat transfer - from computation to experiment. Phys. Chem. Chem. Phys. 15 (2013), 3389-3412.

[147] Pettersson, S., And Mahan, G. D. Theory of the thermal boundary resistance between dissimilar lattices. Phys. Rev. B 42 (Oct 1990), 7386-7390.

[148] Wang, S., And Liang, X. Thermal conductivity and interfacial thermal resistance in bilayered nanofilms by nonequilibrium molecular dynamics simulations. International Journal of Thermophysics 31, 10 (2010), 1935-1944.

[149] Hu, M., Keblinski, P., And Schelling, P. K. Kapitza conductance of siliconamorphous polyethylene interfaces by molecular dynamics simulations. Phys. Rev. B 79, 10 (Mar 2009), 104305.

[150] Shin, S., Kaviany, M., Desai, T., And Bonner, R. Roles of atomic restructuring in interfacial phonon transport. Phys. Rev. B 82 (Aug 2010), 081302.

[151] Liang, Z., and Tsai, H.-L. Effect of thin film confined between two dissimilar solids on interfacial thermal resistance. Journal of Physics: Condensed Matter 23, 49 (2011), 495303.

[152] Luo, T., AND Lloyd, J. R. Non-equilibrium molecular dynamics study of thermal energy transport in AuSAMAu junctions. International Journal of Heat and Mass Transfer 53, 13 (2010), 1 - 11. 
[153] Prasher, R. Acoustic mismatch model for thermal contact resistance of van der Waals contacts. Applied Physics Letters 94, 4 (2009), 041905.

[154] Persson, B. N. J., Volokitin, A. I., And Ueba, H. Phononic heat transfer across an interface: Thermal boundary resistance. Journal of Physics: Condensed Matter 23, 4 (2011), 045009.

[155] Shen, M., Evans, W. J., Cahill, D., and Keblinski, P. Bonding and pressuretunable interfacial thermal conductance. Phys. Rev. B 84 (Nov 2011), 195432.

[156] Liu, H., Zeng, H., Pan, T., Huang, W., and Lin, Y. Pressure dependency of thermal boundary conductance of carbon nanotube/silicon interface: A molecular dynamics study. Journal of Applied Physics 112, 5 (2012), 053501.

[157] Collins, K. C., Chen, S., And Chen, G. Effects of surface chemistry on thermal conductance at aluminum-diamond interfaces. Applied Physics Letters 97, 8 (2010), 083102.

[158] Monachon, C., And Weber, L. Influence of diamond surface termination on thermal boundary conductance between $\mathrm{Al}$ and diamond. Journal of Applied Physics 113, 18 (2013), 183504.

[159] Hopkins, P. E., Baraket, M., Barnat, E. V., Beechem, T. E., Kearney, S. P., Duda, J. C., Robinson, J. T., And Walton, S. G. Manipulating thermal conductance at metal-graphene contacts via chemical functionalization. Nano Letters 12, 2 (2012), 590-595.

[160] Losego, M. D., Grady, M. E., Sottos, N. R., Cahill, D. G., and Braun, P. V. Effects of chemical bonding on heat transport across interfaces. Nature Materials 11 (2012), 502-506.

[161] Kozorezov, A. G., Wigmore, J. K., Erd, C., Peacock, A., and Poelaert, A. Scattering-mediated transmission and reflection of high-frequency phonons at a nonideal solid-solid interface. Phys. Rev. B 57 (Apr 1998), 7411-7414.

[162] Prasher, R. S., And Phelan, P. E. A scattering-mediated acoustic mismatch model for the prediction of thermal boundary resistance. Journal of Heat Transfer 123, 1 (2001), 105-112.

[163] Meng, Q., Wu, L., And Zhu, Y. Phonon scattering of interfacial strain field between dissimilar lattices. Phys. Rev. B 87 (Feb 2013), 064102.

[164] Duda, J. C., English, T. S., Piekos, E. S., Beechem, T. E., Kenny, T. W., And Hopkins, P. E. Bidirectionally tuning Kapitza conductance through the inclusion of substitutional impurities. Journal of Applied Physics 112, 7 (2012), 073519.

[165] Ju, S.-H., AND LiAng, X.-G. Investigation on interfacial thermal resistance and phonon scattering at twist boundary of silicon. Journal of Applied Physics 113, 5 (2013), 053513 .

[166] Hopkins, P. E., Hattar, K., Beechem, T., Ihlefeld, J. F., Medlin, D. L., AND Piekos, E. S. Reduction in thermal boundary conductance due to proton implantation in silicon and sapphire. Applied Physics Letters 98, 23 (2011), 231901. 
[167] Hopkins, P. E., Duda, J. C., Clark, S. P., Hains, C. P., Rotter, T. J., Phinney, L. M., And Balakrishnan, G. Effect of dislocation density on thermal boundary conductance across GaSb/GaAs interfaces. Applied Physics Letters 98, 16 (2011), 161913.

[168] Hanisch-Blicharski, A., Krenzer, B., Wall, S., Kalus, A., Frigge, T., And Hoegen, M. H.-V. Heat transport through interfaces with and without misfit dislocation arrays. Journal of Materials Research 27, 21 (2012), 2718-2723.

[169] Poon, C. Y., And Bhushan, B. Comparison of surface roughness measurements by stylus profiler, AFM and non-contact optical profiler. Wear 190, 1 (1995), 76 - 88. Macro and Micro-Tribology and Mechanics of Magnetic Storage Systems.

[170] Logothetidis, S., Panayiotatos, Y., Gravalidis, C., Patsalas, P., and Zoy, A. X-ray diffuse scattering investigation of thin films. Materials Science and Engineering: B 102, 13 (2003), 25 - 29.

[171] Kechrakos, D. The phonon boundary scattering cross section at disordered crystalline interfaces: A simple model. Journal of Physics: Condensed Matter 2, 11 (1990), 2637.

[172] Kechrakos, D. The role of interface disorder in the thermal boundary conductivity between two crystals. Journal of Physics: Condensed Matter 3, 11 (1991), 1443.

[173] Fagas, G., Kozorezov, A. G., Lambert, C. J., Wigmore, J. K., Peacock, A., Poelaert, A., And Den Hartog, R. Lattice dynamics of a disordered solid-solid interface. Phys. Rev. B 60 (Sep 1999), 6459-6464.

[174] ZhaO, H., And Freund, J. B. Phonon scattering at a rough interface between two fcc lattices. Journal of Applied Physics 105, 1 (2009), 013515.

[175] Zhou, X. W., Jones, R. E., Kimmer, C. J., Duda, J. C., and Hopkins, P. E. Relationship of thermal boundary conductance to structure from an analytical model plus molecular dynamics simulations. Phys. Rev. B 87 (Mar 2013), 094303.

[176] Hopkins, P. E., Phinney, L. M., Serrano, J. R., and Beechem, T. E. Effects of surface roughness and oxide layer on the thermal boundary conductance at aluminum/silicon interfaces. Phys. Rev. B 82 (August 2010), 085307.

[177] Hopkins, P. E., Duda, J. C., Petz, C. W., And Floro, J. A. Controlling thermal conductance through quantum dot roughening at interfaces. Phys. Rev. B 84 (July 2011), 035438.

[178] Chen, G., And Hui, P. Thermal conductivities of evaporated gold films on silicon and glass. Applied Physics Letters 74, 20 (1999), 2942-2944.

[179] Beechem, T., Graham, S., Hopkins, P., and Norris, P. Role of interface disorder on thermal boundary conductance using a virtual crystal approach. Applied Physics Letters 90, 5 (jan. 2007), 054104.

[180] Beechem, T., And Hopkins, P. E. Predictions of thermal boundary conductance for systems of disordered solids and interfaces. Journal of Applied Physics 106, 12 (dec. 2009), 124301. 
[181] Choi, W. I., Kim, K., And Narumanchi, S. Thermal conductance at atomically clean and disordered silicon/aluminum interfaces: A molecular dynamics simulation study. Journal of Applied Physics 112, 5 (2012), 054305.

[182] Hopkins, P. E., And Norris, P. M. Thermal boundary conductance response to a change in Cr/Si interfacial properties. Applied Physics Letters 89, 13 (2006), 131909.

[183] Hopkins, P. E., Norris, P. M., Stevens, R. J., Beechem, T. E., And GraHAM, S. Influence of interfacial mixing on thermal boundary conductance across a chromium/silicon interface. Journal of Heat Transfer 130, 6 (2008), 062402.

[184] DA Silva, L. W., ANd Kaviany, M. Micro-thermoelectric cooler: Interfacial effects on thermal and electrical transport. International Journal of Heat and Mass Transfer 47, 1011 (2004), $2417-2435$.

[185] Tong, T., Zhao, Y., Delzeit, L., Kashani, A., Meyyappan, M., and MaJUMDAR, A. Dense vertically aligned multiwalled carbon nanotube arrays as thermal interface materials. Components and Packaging Technologies, IEEE Transactions on 30, 1 (mar. 2007), $92-100$.

[186] Kim, E.-K., Kwun, S.-I., Lee, S.-M., Seo, H., And Yoon, J.-G. Thermal boundary resistance at $\mathrm{Ge} 2 \mathrm{Sb} 2 \mathrm{Te} 5 / \mathrm{ZnS}: \mathrm{SiO}_{2}$ interface. Applied Physics Letters 76 , 26 (2000), 3864-3866.

[187] Sarua, A., Ji, H., Hilton, K. P., Wallis, D. J., Uren, M. J., Martin, T., And Kuball, M. Thermal boundary resistance between GaN and substrate in AlGaN/GaN electronic devices. IEEE Transactions on Electron Devices 54, 12 (December 2007), 3152-3158.

[188] Akis, R., Ayubi-Moak, J. S., Ferry, D. K., Goodnick, S. M., Faralli, N., AND SARAniti, M. Full-band cellular monte carlo simulations of terahertz high electron mobility transistors. Journal of Physics: Condensed Matter 20, 38 (2008), 384201.

[189] Liu, H. C., Aslan, B., Gupta, J. A., Wasilewski, Z. R., Aers, G. C., SpringThorpe, A. J., And Buchanan, M. Quantum dots for terahertz generation. Journal of Physics: Condensed Matter 20, 38 (2008), 384211.

[190] Horowitz, P., And Mishina, T. The Art of Electronics. Cambridge University Press, 1989.

[191] Venkatesh, M., and Raghavan, G. An overview of dielectric properties measuring techniques. Canadian Biosystems Engineering 47 (2005), 715-730.

[192] Prasankumar, R. P., And Taylor, A. J. Optical Techniques for Solid-State Materials Characterization. Taylor \& Francis, 2012.

[193] Rosenchaig, A., Opsal, J., Smith, W. L., and Willenborg, D. L. Detection of thermal waves through optical reflectance. Applied Physics Letters 46, 11 (1985), $1013-1015$. 
[194] Opsal, J., Rosencwaig, A., And Willenborg, D. L. Thermal-wave detection and thin-film thickness measurements with laser beam deflection. Appl. Opt. 22, 20 (Oct 1983), 3169-3176.

[195] Rosencwaig, A. Thermal-wave imaging. Science 218, 4569 (1982), pp. 223-228.

[196] Rosencwaig, A., And Gersho, A. Theory of the photoacoustic effect with solids. Journal of Applied Physics 47, 1 (1976), 64-69.

[197] Bell, A. G. On the production and reproduction of sound by light. American Journal of Science Series 3 Vol. 20, 118 (1880), 305-324.

[198] Wang, Y., Park, J. Y., Koh, Y. K., and Cahill, D. G. Thermoreflectance of metal transducers for time-domain thermoreflectance. Journal of Applied Physics 108, 4 (2010), 043507.

[199] Regner, K. T., Sellan, D. P., Su, Z., Amon, C. H., McGaughey, A. J., And Malen, J. A. Broadband phonon mean free path contributions to thermal conductivity measured using frequency domain thermoreflectance. Nature Communications 4 (2013), 1640.

[200] Freedman, J. P. F., Leach, J. H., Preble, E. A., Sitar, Z., Davis, R. F., And MALEN, J. A. Universal phonon mean free path spectra in crystalline semiconductors at high temperature. Scientific Reports 3 (2013), 2963.

[201] Majumdar, A. Microscale Energy Transport in Solids. Taylor \& Francis, 1998, ch. 1, pp. 3-94.

[202] Fann, W. S., Storz, R., Tom, H. W. K., And Bokor, J. Electron thermalization in gold. Phys. Rev. B 46 (Nov 1992), 13592-13595.

[203] Fann, W. S., Storz, R., Tom, H. W. K., And Bokor, J. Direct measurement of nonequilibrium electron-energy distributions in subpicosecond laser-heated gold films. Phys. Rev. Lett. 68 (May 1992), 2834-2837.

[204] Hohlfeld, J., Wellershoff, S. S., Gdde, J., Conrad, U., Jhnke, V., And Matthias, E. Electron and lattice dynamics following optical excitation of metals. Chemical Physics 251, 1-3 (2000), 237 - 258.

[205] Schoenlein, R. W., Lin, W. Z., Fujimoto, J. G., and Eesley, G. L. Femtosecond studies of nonequilibrium electronic processes in metals. Phys. Rev. Lett. 58, 16 (Apr 1987), 1680-1683.

[206] Brorson, S. D., Fujimoto, J. G., And Ippen, E. P. Femtosecond electronic heattransport dynamics in thin gold films. Phys. Rev. Lett. 59 (Oct 1987), 1962-1965.

[207] Sun, C.-K., Vallée, F., Acioli, L. H., Ippen, E. P., and Fujimoto, J. G. Femtosecond-tunable measurement of electron thermalization in gold. Phys. Rev. B 50, 20 (Nov 1994), 15337-15348.

[208] Sun, C.-K., Vallée, F., Acioli, L., Ippen, E. P., And Fujimoto, J. G. Femtosecond investigation of electron thermalization in gold. Phys. Rev. B 48 (Oct 1993), $12365-12368$. 
[209] Suarez, C., Bron, W. E., And Juhasz, T. Dynamics and transport of electronic carriers in thin gold films. Phys. Rev. Lett. 75, 24 (Dec 1995), 4536-4539.

[210] Juhasz, T., Elsayed-Ali, H. E., Smith, G. O., Suárez, C., and Bron, W. E. Direct measurements of the transport of nonequilibrium electrons in gold films with different crystal structures. Phys. Rev. B 48, 20 (Nov 1993), 15488-15491.

[211] Lisowski, M., Loukakos, P., Bovensiepen, U., Sthler, J., Gahl, C., And Wolf, M. Ultra-fast dynamics of electron thermalization, cooling and transport effects in ru(001). Applied Physics A 78, 2 (2004), 165-176.

[212] Petek, H., And Ogawa, S. Femtosecond time-resolved two-photon photoemission studies of electron dynamics in metals. Progress in Surface Science 56, 4 (1997), 239 -310 .

[213] Ogawa, S., Nagano, H., And Petek, H. Hot-electron dynamics at $\mathrm{Cu}(100)$, $\mathrm{Cu}(110)$, and $\mathrm{Cu}(111)$ surfaces: Comparison of experiment with fermi-liquid theory. Phys. Rev. B 55 (Apr 1997), 10869-10877.

[214] Smith, A. N., ANd Norris, P. M. Numerical solution for the diffusion of high intensity, ultrashort laser pulses within metal films. In Proceedings of the 11th International Heat Transfer Conference (IHTC '98) (August, 1998 1998), vol. 5, pp. 241-246.

[215] Hostetler, J. L., Smith, A. N., Czajkowsky, D. M., and Norris, P. M. Measurement of the electron-phonon coupling factor dependence on film thickness and grain size in Au, Cr, and Al. Appl. Opt. 38, 16 (Jun 1999), 3614-3620.

[216] Hopkins, P. E. Contributions of inter- and intraband excitations to electron heat capacity and electron-phonon coupling in noble metals. Journal of Heat Transfer 132, 1 (2010), 014504.

[217] Hopkins, P. E., Duda, J. C., Salaway, R. N., Smoyer, J. L., and Norris, P. M. Effects of intra-and interband transitions on electron-phonon coupling and electron heat capacity after short-pulsed laser heating. Nanoscale and Microscale Thermophysical Engineering 12, 4 (2008), 320-333.

[218] Hopkins, P. E., And Norris, P. M. Contribution of ballistic electron transport to energy transfer during electron-phonon nonequilibrium in thin metal films. Journal of Heat Transfer 131, 4 (2009), 043208.

[219] Lin, Z., Zhigilei, L. V., And Celli, V. Electron-phonon coupling and electron heat capacity of metals under conditions of strong electron-phonon nonequilibrium. Phys. Rev. B 77, 7 (Feb 2008), 075133.

[220] Anisimov, S. I., Kapeliovich, B. L., and Pereliman, T. L. Electron emission from metal surfaces exposed to ultrashort laser pulses. Sov. Phys-JETP 39, 2 (August 1974), 375-377.

[221] QIU, T. Q., AND TIEn, C. L. Heat transfer mechanisms during short-pulse laser heating of metals. Journal of Heat Transfer 115, 4 (1993), 835-841. 
[222] Brorson, S. D., Kazeroonian, A., Moodera, J. S., Face, D. W., Cheng, T. K., Ippen, E. P., Dresselhaus, M. S., and Dresselhaus, G. Femtosecond room-temperature measurement of the electron-phonon coupling constant $\gamma$ in metallic superconductors. Phys. Rev. Lett. 64, 18 (April 1990), 2172-2175.

[223] Fujimoto, J. G., Liu, J. M., Ippen, E. P., and Bloembergen, N. Femtosecond laser interaction with metallic tungsten and nonequilibrium electron and lattice temperatures. Phys. Rev. Lett. 53 (Nov 1984), 1837-1840.

[224] Elsayed-Ali, H. E., Norris, T. B., Pessot, M. A., And Mourou, G. A. Time-resolved observation of electron-phonon relaxation in copper. Phys. Rev. Lett. 58 (Mar 1987), 1212-1215.

[225] Verdeyen, J. T. Laser Electronics, third ed. Prentice-Hall, 1995.

[226] Oppenheim, A. V., and with Ian T. Young, A. S. W. Signals and Systems. Prentice-Hall, 1983.

[227] Haykin, S., And Veen, B. V. Signals and Systems. John Wiley \& Sons, 2003.

[228] Schmidt, A. J. Optical Characterization of Thermal Transport from the Nanoscale to the Macroscale. PhD thesis, Massachusetts Institute of Technology, June 2008.

[229] Capinski, W. S., And Maris, H. J. Improved apparatus for picosecond pump-andprobe optical measurements. Review of Scientific Instruments 67, 8 (1996), 2720-2726.

[230] Cahill, D. G. Analysis of heat flow in layered structures for time-domain thermoreflectance. Review of Scientific Instruments 75, 12 (2004), 5119-5122.

[231] Schmidt, A. J., Chen, X., And Chen, G. Pulse accumulation, radial heat conduction, and anisotropic thermal conductivity in pump-probe transient thermoreflectance. Review of Scientific Instruments 79, 11 (2008), 114902.

[232] Wei, C., Zheng, X., Cahill, D. G., And Zhao, J.-C. Invited article: Micron resolution spatially resolved measurement of heat capacity using dual-frequency timedomain thermoreflectance. Review of Scientific Instruments 84, 7 (2013), 071301.

[233] Schmidt, A. J. Pump-probe thermoreflectance. Annual Review of Heat Transfer 16, 16 (2013), 159-181.

[234] Wilson, R. B., Apgar, B. A., Martin, L. W., and Cahill, D. G. Thermoreflectance of metal transducers for optical pump-probe studies of thermal properties. Opt. Express 20, 27 (Dec 2012), 28829-28838.

[235] Gengler, J. J., Roy, S., Jones, J. G., And Gord, J. R. Two-color time-domain thermoreflectance of various metal transducers with an optical parametric oscillator. Measurement Science and Technology 23, 5 (2012), 055205.

[236] Incropera, F. P., And DeWitt, D. P. Fundamentals of Heat and Mass Transfer. John Wiley \& Sons, 2002.

[237] Stevens, R. J. Thermal transport at room temperature solid-solid interfaces. PhD thesis, University of Virginia, 2005. 
[238] Feldman, A. Algorithm for solutions of the thermal diffusion equation in a stratified medium with a modulated heating source. High Temperatures - High Pressures 31, 3 (1999), 293-8.

[239] Carslaw, H. S., and Jaeger, J. C. Steady Periodic Temperature in Composite Slabs, 2nd ed. Oxford University Press, New York, 1959, ch. Linear flow of heat in the solid bounded by two parallel planes, pp. 109-112.

[240] Hopkins, P. E., Kaehr, B., Phinney, L. M., Koehler, T. P., Grillet, A. M., Dunphy, D., Garcia, F., And Brinker, C. J. Measuring the thermal conductivity of porous, transparent $\mathrm{SiO}_{2}$ films with time domain thermoreflectance. Journal of Heat Transfer 133, 6 (2011), 061601.

[241] Malen, J. A., Baheti, K., Tong, T., Hudgings, J. A., Majumdar, A., And ZHAO, Y. Optical measurement of thermal conductivity using fiber aligned frequency domain thermoreflectance. Journal of Heat Transfer 133, 8 (May 2011), 081601.

[242] Kim, J. H., Feldman, A., And Novotny, D. Application of the three omega thermal conductivity measurement method to a film on a substrate of finite thickness. Journal of Applied Physics 86, 7 (1999), 3959-3963.

[243] Tong, T., And Majumdar, A. Reexamining the 3-omega technique for thin film thermal characterization. Review of Scientific Instruments 77, 10 (2006), 104902.

[244] Zheng, X., Cahill, D. G., Weaver, R., and Zhao, J.-C. Micron-scale measurements of the coefficient of thermal expansion by time-domain probe beam deflection. Journal of Applied Physics 104, 7 (2008), 073509.

[245] Hopkins, P. E., Serrano, J. R., Phinney, L. M., Kearney, S. P., Grasser, T. W., AND HARRIS, C. T. Criteria for cross-plane dominated thermal transport in multilayer thin film systems during modulated laser heating. Journal of Heat Transfer 132, 8 (2010), 081302.

[246] Brugger, K. Exact solutions for the temperature rise in a laser-heated slab. Journal of Applied Physics 43, 2 (1972), 577-583.

[247] Stevens, R. J., Smith, A. N., And Norris, P. M. Signal analysis and characterization of experimental setup for the transient thermoreflectance technique. Review of Scientific Instruments 77, 8 (2006), 084901.

[248] RiEF, B. Thermal-wave transient behaviour. Canadian Journal of Physics 64, 9 (1986), 1303-1306.

[249] Burzo, M. G., Komarov, P. L., And RaAd, P. E. Influence of the metallic absorption layer on the quality of thermal conductivity measurements by the transient thermo-reflectance method. Microelectronics Journal 33, 9 (2002), 697 - 703.

[250] Battaglia, J.-L., Kusiak, A., Rossignol, C., And Chigarev, N. Thermal diffusivity and effusivity of thin layers using time-domain thermoreflectance. Phys. Rev. B 76, 18 (November 2007), 184110. 
[251] Liu, J., Zhu, J., Tian, M., Gu, X., Schmidt, A., and Yang, R. Simultaneous measurement of thermal conductivity and heat capacity of bulk and thin film materials using frequency-dependent transient thermoreflectance method. Review of Scientific Instruments 84, 3 (2013), 034902.

[252] Wilson, R. B., Feser, J. P., Hohensee, G. T., and Cahill, D. G. Two-channel model for nonequilibrium thermal transport in pump-probe experiments. Phys. Rev. B 88 (Oct 2013), 144305.

[253] Koh, Y. K., And Cahill, D. G. Frequency dependence of the thermal conductivity of semiconductor alloys. Phys. Rev. B 76 (Aug 2007), 075207.

[254] Norris, P. M., Smoyer, J. L., Redding, M. R., and Larkin, L. S. Investigation of nanoscale heat transfer with highly versitile phase-locked thermoreflectance. In Proceedings of the 15th International Heat Transfer Conference, IHTC-15 (2014).

[255] Hamby, D. A review of techniques for parameter sensitivity analysis of environmental models. Environmental Monitoring and Assessment 32, 2 (1994), 135-154.

[256] Helton, J. C. Uncertainty and sensitivity analysis techniques for use in performance assessment for radioactive waste disposal. Reliability Engineering 85 System Safety 42, 23 (1993), $327-367$.

[257] Campolongo, F., And Saltelli, A. Sensitivity analysis of an environmental model: an application of different analysis methods. Reliability Engineering \& System Safety 57,1 (1997), $49-69$.

[258] Wei, H., Nearing, M. A., And Stone, J. J. A comprehensive sensitivity analysis framework for model evaluation and improvment using a case study of the rangrange hydrology and erosion model. Transactions of the ASABE 50, 3 (2007), 945-953.

[259] Hopkins, P., Serrano, J., And Phinney, L. Comparison of thermal conductivity and thermal boundary conductance sensitivities in continuous-wave and ultrashortpulsed thermoreflectance analyses. International Journal of Thermophysics 31 (2010), $2380-2393$.

[260] Schmidt, A. J., Cheaito, R., And Chiesa, M. Characterization of thin metal films via frequency-domain thermoreflectance. Journal of Applied Physics 107, 2 (2010), 024908 .

[261] Schmidt, A. J., Cheaito, R., And Chiesa, M. A frequency-domain thermoreflectance method for the characterization of thermal properties. Review of Scientific Instruments 80, 9 (2009), 094901.

[262] Zhu, J., Tang, D., Wang, W., Liu, J., Holub, K. W., and Yang, R. Ultrafast thermoreflectance techniques for measuring thermal conductivity and interface thermal conductance of thin films. Journal of Applied Physics 108, 9 (2010), 094315.

[263] Systems, S. R. User's Manual : Model SR844 RF Lock-in Amplifier, revision 2.8 ed. Stanford Research Systems, November 2013. 
[264] Manoj, K., and Senthamarai Kannan, K. Comparison of methods for detecting outliers. International Journal of Scientific \& Engineering Research 4, 9 (September 2013), 709-714.

[265] Barbato, G., Barini, E. M., Genta, G., And Levi, R. Features and performance of some outlier detection methods. Journal of Applied Statistics 38, 10 (2011), 21332149 .

[266] Rosner, B. Percentage points for a generalized ESD many-outlier procedure. Technometrics 25, 2 (1983), 165-172.

[267] Grubbs, F. E. Procedures for detecting outlying observations in samples. Technometrics 11, 1 (1969), 1-21.

[268] Welch, B. L. The generalization of 'students' problem when several different population variances are involved. Biometrika 34, 1-2 (1947), 28-35.

[269] Lawson, J., And ErJavec, J. Modern Statistics for Engineering and Quality Improvement. Duxbury - Thomas Learning, 2001.

[270] Hesterberg, T. It's time to retire the "n $i=30$ " rule. In Proceedings of the Joint Statistical Meetings (Alexandria VA, 2008).

[271] Efron, B. Computers and the theory of statistics: Thinking the unthinkable. SIAM Review 21, 4 (1979), 460-480.

[272] Efron, B. Bootstrap methods: Another look at the jackknife. The Annals of Statistics 7, 1 (01 1979), 1-26.

[273] Zoubir, A. M., And Iskander, D. R. Bootstrap Techniques for Signal Processing. Cambridge University Press, 2004.

[274] Henderson, A. R. The bootstrap: A technique for data-driven statistics. using computer-intensive analyses to explore experimental data. Clinica Chimica Acta 359, 12 (2005), $1-26$.

[275] Wehrens, R., Putter, H., and Buydens, L. M. The bootstrap: a tutorial. Chemometrics and Intelligent Laboratory Systems 54, 1 (2000), 35 - 52.

[276] Hesterberg, T., Moore, D. S., Monaghan, S., Clipson, A., and Epstein, R. Bootstrap Methods and Permuation Tests. W. H. Freeman and Company, 2007, ch. 18, pp. 1-85.

[277] Efron, B., And Tibshirani, R. J. An Introduction to the Bootstrap. Chapman and Hall, 1993.

[278] Squires, G. L. Practical Physics, fourth ed. Cambridge University Press, 2001.

[279] Monch, W. Electronic Properties of Semiconductor Interfaces, vol. 43. Springer Berlin Heidelberg, 2004.

[280] Young, D. A., And Maris, H. J. Lattice-dynamical calculation of the Kapitza resistance between FCC lattices. Phys. Rev. B 40,6 (Aug 1989), 3685-3693. 
[281] Schelling, P. K., Phillpot, S. R., And Keblinski, P. Phonon wave-packet dynamics at semiconductor interfaces by molecular-dynamics simulation. Applied Physics Letters 80, 14 (2002), 2484-2486.

[282] Schelling, P. K., And Phillpot, S. R. Multiscale simulation of phonon transport in superlattices. Journal of Applied Physics 93, 9 (2003), 5377-5387.

[283] Alvarez-Quintana, J., And Rodrguez-Viejo, J. Interfacial effects on the thermal conductivity of a-Ge thin films grown on Si substrates. Journal of Applied Physics 104, 7 (2008), 074903.

[284] Cahill, D. G., And Pohl, R. O. Thermal properties of a tetrahedrally bonded amorphous solid: CdGeAss. Phys. Rev. B 37 (May 1988), 8773-8780.

[285] Finstad, T. G., And Nicolet, M.-A. Silicide formation with bilayers of Pd-Pt, Pd-Ni, and Pt-Ni. Journal of Applied Physics 50, 1 (1979), 303-307.

[286] Grunthaner, P. J., Grunthaner, F. J., And Madhukar, A. Chemical bonding and charge redistribution: Valence band and core level correlations for the $\mathrm{Ni} / \mathrm{Si}$, $\mathrm{Pd} / \mathrm{Si}$, and Pt/Si systems. Journal of Vacuum Science and Technology 20, 3 (1982), 680-683.

[287] LEE, B.-J. Thermodynamic analysis of solid-state metal/Si interfacial reactions. Journal of Materials Research 14, 03 (1999), 1002-1017.

[288] Mantovani, S., Nava, F., Nobili, C., Queirolo, G., and Celotti, G. Pt-Ni bilayers on n-type silicon: Metallurgical and electrical behavior. Journal of Applied Physics 55, 4 (1984), 899-908.

[289] Matz, R., Purtell, R. J., Yokota, Y., Rubloff, G. W., And Ho, P. S. Chemical reaction and silicide formation at the Pt/Si interface. Journal of Vacuum Science \& Technology A: Vacuum, Surfaces, and Films 2, 2 (1984), 253-258.

[290] Kern, W., And Puotinen, D. A. Cleaning solutions based on hydrogen peroxide for use in silicon semiconductor technology. RCA Review 31 (1970), 187-206.

[291] Wang, Y., Huang, H., And Ruan, X. Decomposition of coherent and incoherent phonon conduction in superlattices and random multilayers. Phys. Rev. B 90 (Oct 2014), 165406.

[292] Zhu, L., And Zheng, X. Modification of the phonon thermal conductivity in spatially confined semiconductor nanofilms under stress fields. EPL (Europhysics Letters) 88,3 (2009), 36003.

[293] McGaughey, A. J. H., Landry, E. S., Sellan, D. P., And Amon, C. H. Sizedependent model for thin film and nanowire thermal conductivity. Applied Physics Letters 99, 13 (2011), 131904.

[294] Cheaito, R., Duda, J. C., Beechem, T. E., Hattar, K., Ihlefeld, J. F., Medlin, D. L., Rodriguez, M. A., Campion, M. J., Piekos, E. S., And HopKINS, P. E. Experimental investigation of size effects on the thermal conductivity of silicon-germanium alloy thin films. Phys. Rev. Lett. 109 (Nov 2012), 195901. 
[295] Franssila, S. Introduction to Microfabrication. John Wiley \& Sons, 2010.

[296] Clemens, B. M., Eesley, G. L., And Paddock, C. A. Time-resolved thermal transport in compositionally modulated metal films. Phys. Rev. B 37 (January 1988), $1085-1096$.

[297] Bartkowiak, M., and Mahan, G. Chapter 8 heat and electricity transport through interfaces. In Recent Trends in Thermoelectric Materials Research II, T. M. Tritt, Ed., vol. 70 of Semiconductors and Semimetals. Elsevier, 2001, pp. $245-271$.

[298] Zakordonets, V., And Logvinov, G. Thermoelectric figure of merit of monopolar semiconductors with finite dimensions. Semiconductors 31 (1997), 265-267.

[299] Bulat, L. P., And Yatsyuk, V. G. Thermal effects at boundaries of solids. Soviet Physics: Semiconductors 18 (1984), 383-384.

[300] Anatychuk, L. I., Bulat, L. P., Nikirsa, D. D., And G., Y. V. Influence of size effects on the properties of cooling thermoelements. Soviet Physics: Semiconductors 21 (1986), 206-207.

[301] Gurevich, Y. G., and Logvinov, G. N. Thermo-emf and thermoelectric current in unipolar semiconductors with finite dimensions. Soviet Physics: Semiconductors 26, 11 (1992), 1091-1094.

[302] Mahan, G. D. Kapitza thermal resistance between a metal and a nonmetal. Phys. Rev. B 79 (Feb 2009), 075408.

[303] MaedA, K. Nonideality of Au/Si and Au/GaAs schottky barriers due to processinduced defects. Applied Surface Science 252, 16 (2006), 5659 - 5675.

[304] Rhoderick, E., And Williams, R. Metal-semiconductor contacts. Monographs in electrical and electronic engineering. Clarendon Press, 1988.

[305] Chi, D., Yao, H., Liew, S., Tan, C., Chua, C., Chua, K., Li, R., and Lee, S. Schottky barrier height in germanide/ge contacts and its engineering through germanidation induced dopant segregation. In Junction Technology, 2007 International Workshop on (June 2007), pp. 81-86.

[306] Kittel, C. Introduction to Solid State Physics. John Wiley \& Sons, 2005.

[307] Dove, M. T. Introduction to Lattice Dynamics. Cambridge University Press, 1993.

[308] Sands, D. E. Introduction to Crystallography. Dover Publications, Inc., 1975.

[309] Srivastava, G. P. The physics of phonons. A. Hilger, 1990.

[310] Khitun, A., Balandin, A., And Wang, K. L. Modification of the lattice thermal conductivity in silicon quantum wires due to spatial confinement of acoustic phonons. Superlattices and Microstructures 26, 3 (1999), 181 - 193.

[311] Klemens, P. Thermal conductivity and lattice vibrational modes. In Solid State Physics, F. SEITZ and D. TURNBULL, Eds., vol. 7 of Solid State Physics. Academic Press, 1958, pp. $1-98$. 
[312] Hecht, E. Optics. Addison-Wesley, 1998.

[313] Abeles, F. Advanced Optical Techniques. North Holland Publishing Compnay, 1967.

[314] Schmidt, A., Chiesa, M., Chen, X., And Chen, G. An optical pump-probe technique for measuring the thermal conductivity of liquids. Review of Scientific Instruments 79, 6 (2008), 064902.

[315] Razali, N. M., And Wah, Y. B. Power comparisons of Shapiro-Wilk, KolmogorovSmirnov, Lilliefors and Anderson-Darling tests. Journal of Statistical Modeling and Analytics 2, 1 (2011), 21-33.

[316] Dufour, J.-M., Farhat, A., Gardiol, L., and Khalaf, L. Simulation-based finite sample normality tests in linear regressions. Econometrics Journal 1, 1 (1998), $154-173$.

[317] Shapiro, S. S., And Wilk, M. B. An analysis of variance test for normality (complete samples). Biometrika 52, 3-4 (1965), 591-611.

[318] Keskin, S. Comparison of several univariate normality tests regarding type I error rate and power of the test in simulation based small samples. Journal of Applied Science Research 2, 5 (May 2006), 296-300.

[319] Saculinggan, M., And Balase, E. A. Empirical power comparison of goodness of fit tests for normality in the presence of outliers. Journal of Physics: Conference Series 435, 1 (2013), 012041. 


\section{SUPPLEMENT S}

\section{MODELING AND FITTING OF DATA}

S.1 Simulation Parameters . . . . . . . . . . . . . . . . . . . 375

S.2 Simulation Parameter Sensitivity Coefficient $-h_{\mathrm{BD}} \ldots \ldots \ldots$

S.3 Simulation Parameter Sensitivity Coefficient - Time . . . . . . . . . . 378

S.4 Simulation Summary - Al/Si 1.00 MW Series . . . . . . . . . . . . . 399

S.5 Simulation Summary - Al/Si 1.29 MW Series . . . . . . . . . . . . . 400

S.6 Simulation Summary - Al/Si 1.67 MW Series . . . . . . . . . . . . . 401

S.7 Simulation Summary - Al/Si 2.15 MW Series . . . . . . . . . . . . . 402

S.8 Simulation Summary - Al/Si 2.78 MW Series . . . . . . . . . . . . . 404

S.9 Simulation Summary - Al/Si 3.59 MW Series . . . . . . . . . . . . 405

S.10 Simulation Summary - Al/Si 4.64 MW Series . . . . . . . . . . . 406

S.11 Simulation Summary - Al/Si 5.99 MW Series . . . . . . . . . . . . 408

S.12 Simulation Summary - Al/Si 7.74 MW Series . . . . . . . . . . . . . 409

S.13 Simulation Summary - Al/Si 10.00 MW Series . . . . . . . . . . . . . 410

S.14 Simulation Summary - Al/Si 12.90 MW Series . . . . . . . . . . . . . 412

S.15 Simulation Summary - Al/Si 16.70 MW Series _ . . . . . . . . . . . 413

S.16 Simulation Summary - Al/Si 21.50 MW Series . . . . . . . . . . . . 414

S.17 Simulation Summary - Al/Si 27.80 MW Series . . . . . . . . . . . 417 
S.18 Simulation Summary - Al/Si 35.90 MW Series _ . . . . . . . . . . . . . 419

S.19 Simulation Summary - Al/Si 46.40 MW Series . . . . . . . . . . . . . 421

S.20 Simulation Summary - Al/Si 59.90 MW Series . . . . . . . . . . . . . . 424

S.21 Simulation Summary - Al/Si 77.40 MW Series _ . . . . . . . . . . . . 426

S.22 Simulation Summary - Al/Si 100.00 MW Series . . . . . . . . . . . . . 428

S.23 Simulation Summary - Al/Si 129.00 MW Series . . . . . . . . . . . . . . 431

S.24 Simulation Summary - Al/Si 167.00 MW Series . . . . . . . . . . . . . 433

S.25 Simulation Summary - Al/Si 215.00 MW Series . . . . . . . . . . . . . 435

S.26 Simulation Summary - Al/Si 278.00 MW Series . . . . . . . . . . . . 438

S.27 Simulation Summary - Al/Si 359.00 MW Series . . . . . . . . . . . . . . . 440

S.28 Simulation Summary - Al/Si 464.00 MW Series . . . . . . . . . . . . . . 442

S.29 Simulation Summary - Al/Si 599.00 MW Series . . . . . . . . . . . . . . 445

S.30 Simulation Summary - Al/Si 774.00 MW Series . . . . . . . . . . . . . 447

S.31 Simulation Summary - Al/Si 1000.00 MW Series . . . . . . . . . . . . . 449

S.32 Simulation Summary - Al/AlN 1.00 MW Series . . . . . . . . . . . . . 452

S.33 Simulation Summary - Al/AlN 1.29 MW Series . . . . . . . . . . . . . 453

S.34 Simulation Summary - Al/AlN 2.78 MW Series . . . . . . . . . . . . . 454

S.35 Simulation Summary - Al/AlN 5.99 MW Series . . . . . . . . . . . . 455

S.36 Simulation Summary - Al/AlN 12.90 MW Series . . . . . . . . . . . . 456

S.37 Simulation Summary - Al/AlN 27.80 MW Series . . . . . . . . . . . . 457

S.38 Simulation Summary - Al/AlN 59.90 MW Series . . . . . . . . . . . . . 458

S.39 Simulation Summary - Al/AlN 100.00 MW Series . . . . . . . . . . . . . 460

S.40 Simulation Summary - Al/AlN 129.00 MW Series . . . . . . . . . . . . . 461

S.41 Simulation Summary - Al/AlN 167.00 MW Series . . . . . . . . . . . . 463

S.42 Simulation Summary - Al/AlN 215.00 MW Series . . . . . . . . . . . . . . 464

S.43 Simulation Summary - Al/AlN 278.00 MW Series . . . . . . . . . . . . 465

S.44 Simulation Summary - Al/AlN 359.00 MW Series . . . . . . . . . . . . . 467

S.45 Simulation Summary - Al/AlN 464.00 MW Series . . . . . . . . . . . . 468

S.46 Simulation Summary - Al/AlN 599.00 MW Series . . . . . . . . . . . . . 469

S.47 Simulation Summary - Al/AlN 774.00 MW Series . . . . . . . . . . . . . 471 
S.48 Simulation Summary - Al/AlN 1000.00 MW Series . . . . . . . . . . . . 472

S.49 Simulation Summary - Al/Ge 1.00 MW Series . . . . . . . . . . . . . . 474

S.50 Simulation Summary - Al/Ge 2.15 MW Series . . . . . . . . . . . . . 475

S.51 Simulation Summary - Al/Ge 3.59 MW Series . . . . . . . . . . . . . 476

S.52 Simulation Summary - Al/Ge 7.74 MW Series . . . . . . . . . . . . . 477

S.53 Simulation Summary - Al/Ge 16.70 MW Series . . . . . . . . . . . . 478

S.54 Simulation Summary - Al/Ge 46.40 MW Series . . . . . . . . . . . . . 480

S.55 Simulation Summary - Al/Ge 100.00 MW Series . . . . . . . . . . . . . 482

S.56 Simulation Summary - Al/Ge 129.00 MW Series . . . . . . . . . . . . . . 484

S.57 Simulation Summary - Al/Ge 167.00 MW Series . . . . . . . . . . . . 485

S.58 Simulation Summary - Al/Ge 215.00 MW Series . . . . . . . . . . . . 486

S.59 Simulation Summary - Al/Ge 359.00 MW Series _ . . . . . . . . . . . 488

S.60 Simulation Summary - Al/Ge 464.00 MW Series . . . . . . . . . . . . . 489

S.61 Simulation Summary - Al/Ge 599.00 MW Series . . . . . . . . . . . . . 491

S.62 Simulation Summary - Al/Ge 774.00 MW Series . . . . . . . . . . . . . 492

S.63 Simulation Summary - Al/Ge 1000.00 MW Series . . . . . . . . . . . . . 493 


\section{S.1 Simulation Parameters}

\section{S.1.1 100 nm Al on Si Substrate Series}

Table S.1: Thermophysical properties used in modeling of an Al film on Si substrate, with various $h_{\mathrm{BD}}$ values, used in the work of this dissertation.

\begin{tabular}{rcc}
\hline Layer Properties & Layer 1 & Layer S \\
\hline Specific Heat $-C\left[\mathrm{~J} / \mathrm{m}^{3} \mathrm{~K}\right]$ & $2.44 \times 10^{6}$ & $1.66 \times 10^{6}$ \\
Thermal Conductivity $-k_{r}[\mathrm{~W} / \mathrm{mK}]$ & 237 & 148 \\
Thermal Conductivity $-k_{z}[\mathrm{~W} / \mathrm{mK}]$ & 237 & 148 \\
Thickness $-d[\mathrm{~nm}]$ & 100 & Infinite \\
\hline Laser Properties & \\
\hline Absorbed Power $[\mathbf{W}]$ & \multicolumn{2}{c}{0.03} \\
Pump Waist $[\mu \mathbf{m}]$ & \multicolumn{2}{c}{70} \\
Probe Waist $[\mu \mathbf{m}]$ & \multicolumn{2}{c}{15} \\
Rep Rate $[\mathrm{MHz}]$ & 0.250 \\
Modulation Rate $[\mathrm{MHz}]$ & 0.125 \\
Duty Cycle $[\%]$ & \multicolumn{2}{c}{50} \\
\hline
\end{tabular}

\section{S.1.2 $100 \mathrm{~nm} \mathrm{Al} \mathrm{on} \mathrm{AlN} \mathrm{Substrate} \mathrm{Series}$}

Table S.2: Thermophysical properties used in modeling of an Al film on AlN substrate, with various $h_{\mathrm{BD}}$ values, used in the work of this dissertation.

\begin{tabular}{rcc}
\hline Layer Properties & Layer $\mathbf{1}$ & Layer S \\
\hline Specific Heat $-C\left[\mathrm{~J} / \mathrm{m}^{3} \mathrm{~K}\right]$ & $2.44 \times 10^{6}$ & $2.41 \times 10^{6}$ \\
Thermal Conductivity $-k_{r}[\mathrm{~W} / \mathrm{mK}]$ & 237 & 285 \\
Thermal Conductivity $-k_{z}[\mathrm{~W} / \mathrm{mK}]$ & 237 & 285 \\
Thickness $-d[\mathrm{~nm}]$ & 100 & Infinite \\
\hline Laser Properties & \\
\hline Absorbed Power $[\mathbf{W}]$ & \multicolumn{2}{c}{0.03} \\
Pump Waist $[\mu \mathbf{m}]$ & \multicolumn{2}{c}{70} \\
Probe Waist $[\mu \mathbf{m}]$ & \multicolumn{2}{c}{0.250} \\
Rep Rate $[\mathrm{MHz}]$ & 0.125 \\
Modulation Rate $[\mathrm{MHz}]$ & \multicolumn{2}{c}{50} \\
Duty Cycle $[\%]$ & \multicolumn{2}{c}{0}
\end{tabular}




\section{S.1.3 $100 \mathrm{~nm} \mathrm{Al} \mathrm{on} \mathrm{Ge} \mathrm{Substrate} \mathrm{Series}$}

Table S.3: Thermophysical properties used in modeling of an Al film on Ge substrate, with various $h_{\mathrm{BD}}$ values, used in the work of this dissertation.

\begin{tabular}{rcc}
\hline Layer Properties & Layer 1 & Layer S \\
\hline Specific Heat $-C\left[\mathrm{~J} / \mathrm{m}^{3} \mathrm{~K}\right]$ & $2.44 \times 10^{6}$ & $1.73 \times 10^{6}$ \\
Thermal Conductivity $-k_{r}[\mathrm{~W} / \mathrm{mK}]$ & 237 & 59.9 \\
Thermal Conductivity $-k_{z}[\mathrm{~W} / \mathrm{mK}]$ & 237 & 59.9 \\
Thickness $-d[\mathrm{~nm}]$ & 100 & Infinite \\
\hline Laser Properties & \\
\hline Absorbed Power $[\mathbf{W}]$ & 0.03 \\
Pump Waist $[\mu \mathbf{m}]$ & \multicolumn{2}{c}{70} \\
Probe Waist $[\mu \mathbf{m}]$ & \multicolumn{2}{c}{0.250} \\
Rep Rate $[\mathrm{MHz}]$ & 0.125 \\
Modulation Rate $[\mathrm{MHz}]$ & \multicolumn{2}{c}{50} \\
Duty Cycle $[\%]$ & \multicolumn{2}{c}{0}
\end{tabular}

\section{S.2 Simulation Parameter Sensitivity Coefficient $-h_{\mathrm{BD}}$}

Sensitivity parameters of the major thermophysical inputs of the modeled systems as a function of $h_{\mathrm{BD}}$.

\section{S.2.1 $100 \mathrm{~nm} \mathrm{Al}$ on Si Substrate Series}

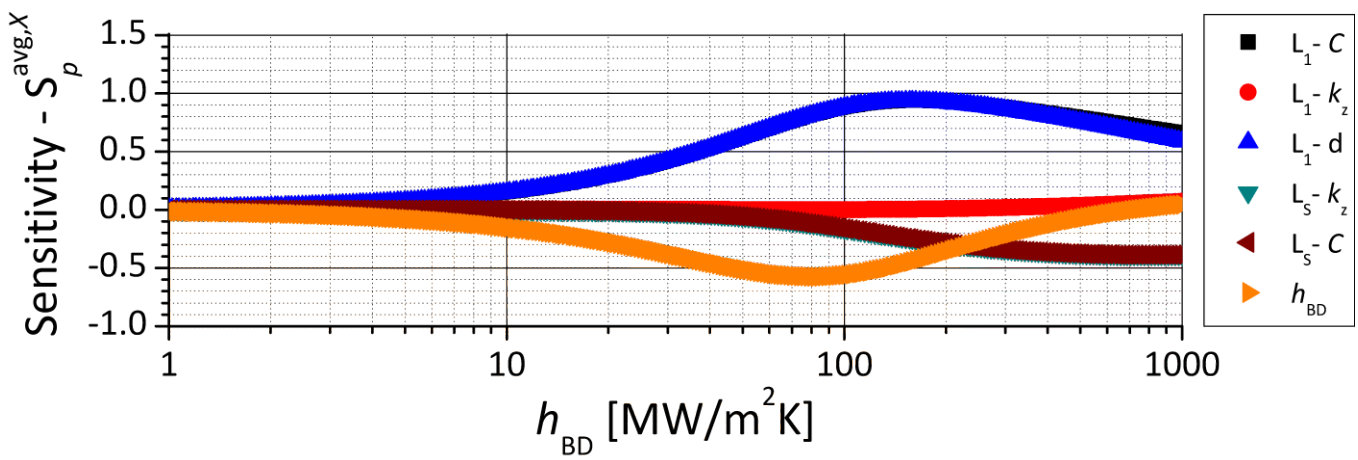

Figure S.1: Sensitivity coefficient per parameter as a function of $h_{\mathrm{BD}}$ for the $100 \mathrm{~nm}$ of $\mathrm{Al}$ on Si system where $\mathrm{L}_{1}$ denotes the $\mathrm{Al}$ film and $\mathrm{L}_{\mathrm{S}}$ the substrate. 


\section{S.2.2 $100 \mathrm{~nm} \mathrm{Al} \mathrm{on} \mathrm{AlN} \mathrm{Substrate} \mathrm{Series}$}

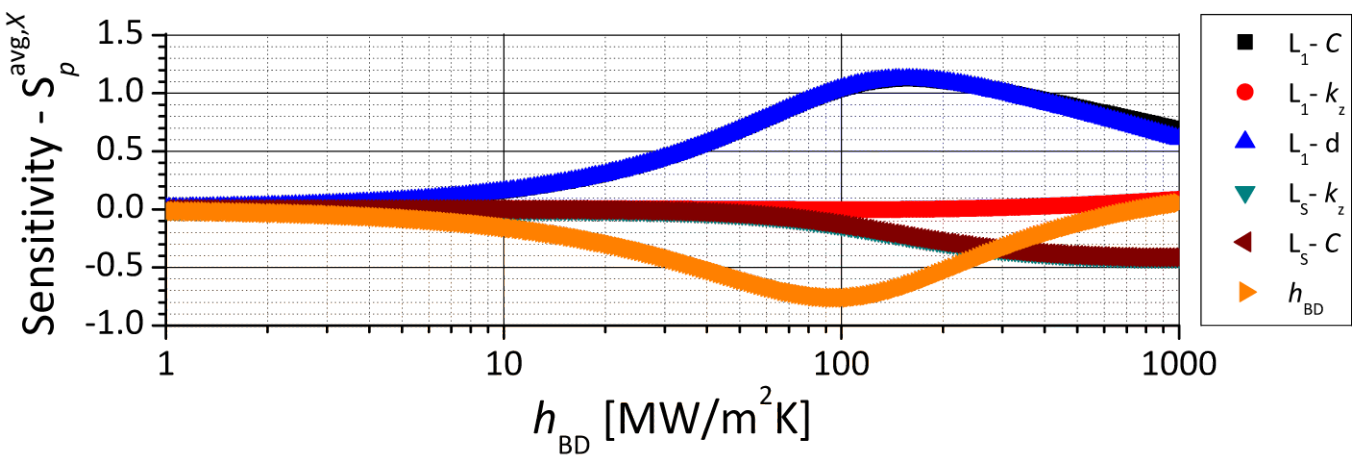

Figure S.2: Sensitivity coefficient per parameter as a function of $h_{\mathrm{BD}}$ for the $100 \mathrm{~nm}$ of $\mathrm{Al}$ on Si system where $\mathrm{L}_{1}$ denotes the $\mathrm{Al}$ film and $\mathrm{L}_{\mathrm{S}}$ the AlN substrate.

\section{S.2.3 $100 \mathrm{~nm} \mathrm{Al} \mathrm{on} \mathrm{Ge} \mathrm{Substrate} \mathrm{Series}$}

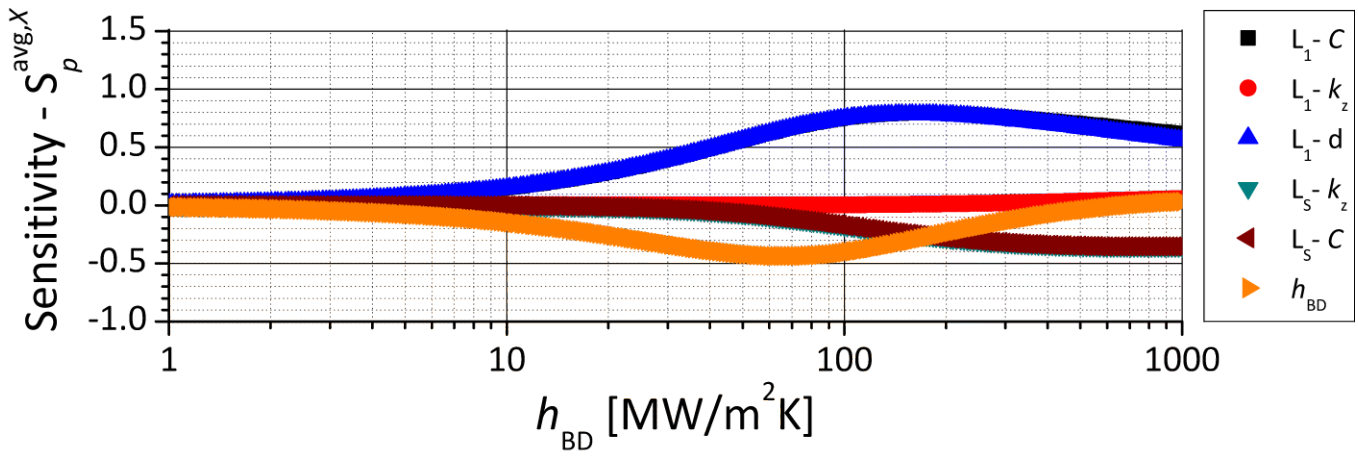

Figure S.3: Sensitivity coefficient per parameter as a function of $h_{\mathrm{BD}}$ for the $100 \mathrm{~nm}$ of $\mathrm{Al}$ on $\mathrm{Si}$ system where $\mathrm{L}_{1}$ denotes the $\mathrm{Al}$ film and $\mathrm{L}_{\mathrm{S}}$ the Ge substrate. 


\section{S.3 Simulation Parameter Sensitivity Coefficient - Time}

Sensitivity parameters of the major thermophysical inputs of the modeled systems as a function of time.

\section{S.3.1 $100 \mathrm{~nm} \mathrm{Al} \mathrm{on} \mathrm{Si} \mathrm{Substrate} \mathrm{-} \mathrm{1.00} \mathrm{MW} \mathrm{Series}$}

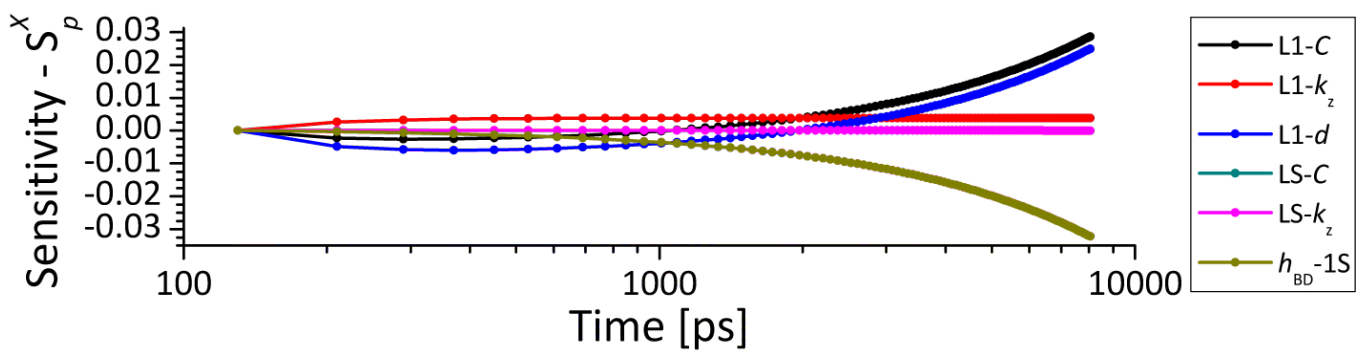

Figure S.4: Sensitivity of the real signal, $X$, as a function of time for the thermal parameters in the 100 $\mathrm{nm} \mathrm{Al} / \mathrm{Si}$ system for $h_{\mathrm{BD}}=1.00 \mathrm{MW} / \mathrm{m}^{2} \mathrm{~K}$.

\section{S.3.2 $100 \mathrm{~nm} \mathrm{Al} \mathrm{on} \mathrm{Si} \mathrm{Substrate} \mathrm{-} \mathrm{1.29} \mathrm{MW} \mathrm{Series}$}

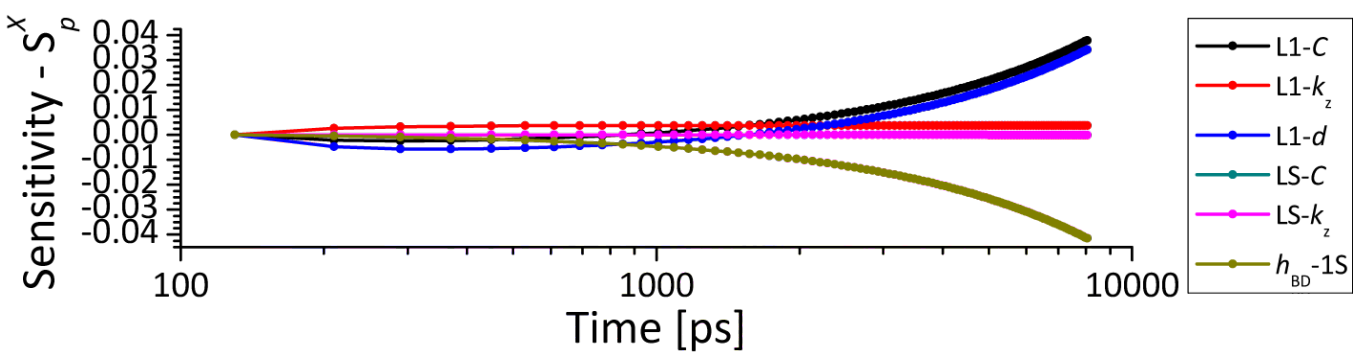

Figure S.5: Sensitivity of the real signal, $X$, as a function of time for the thermal parameters in the 100 $\mathrm{nm} \mathrm{Al} / \mathrm{Si}$ system for $h_{\mathrm{BD}}=1.29 \mathrm{MW} / \mathrm{m}^{2} \mathrm{~K}$. 


\section{S.3.3 $100 \mathrm{~nm} \mathrm{Al} \mathrm{on} \mathrm{Si} \mathrm{Substrate} \mathrm{-} \mathrm{1.67} \mathrm{MW} \mathrm{Series}$}

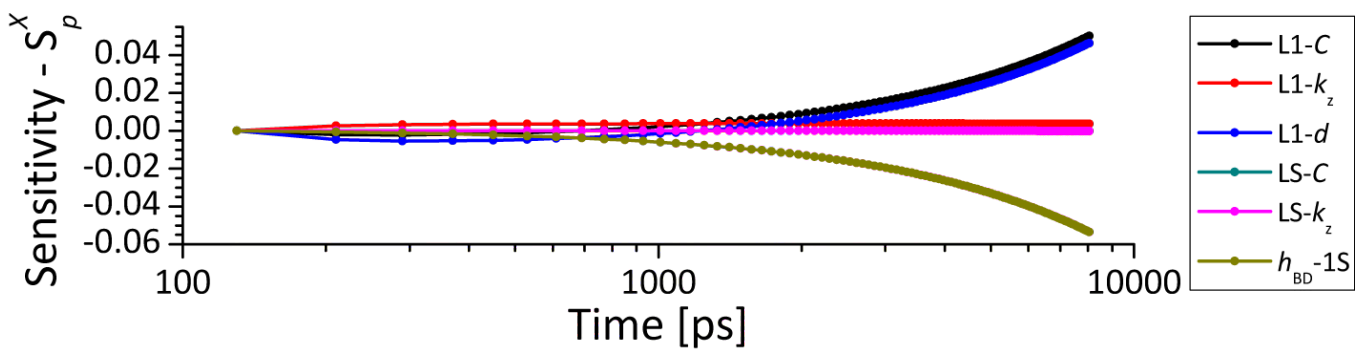

Figure S.6: Sensitivity of the real signal, $X$, as a function of time for the thermal parameters in the 100 $\mathrm{nm} \mathrm{Al} / \mathrm{Si}$ system for $h_{\mathrm{BD}}=1.67 \mathrm{MW} / \mathrm{m}^{2} \mathrm{~K}$.

\section{S.3.4 $100 \mathrm{~nm}$ Al on Si Substrate - 2.15 MW Series}

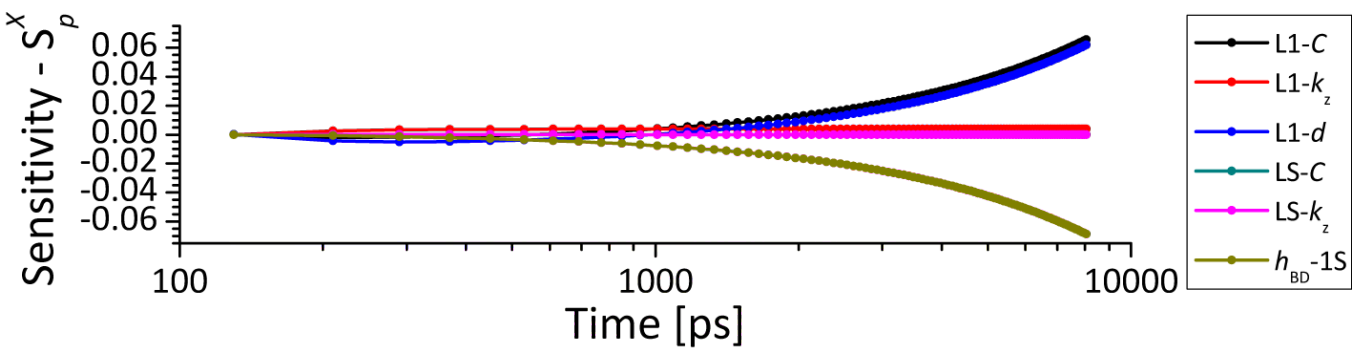

Figure S.7: Sensitivity of the real signal, $X$, as a function of time for the thermal parameters in the 100 $\mathrm{nm} \mathrm{Al} / \mathrm{Si}$ system for $h_{\mathrm{BD}}=2.15 \mathrm{MW} / \mathrm{m}^{2} \mathrm{~K}$.

\section{S.3.5 $100 \mathrm{~nm} \mathrm{Al} \mathrm{on} \mathrm{Si} \mathrm{Substrate} \mathrm{-} \mathrm{2.78} \mathrm{MW} \mathrm{Series}$}

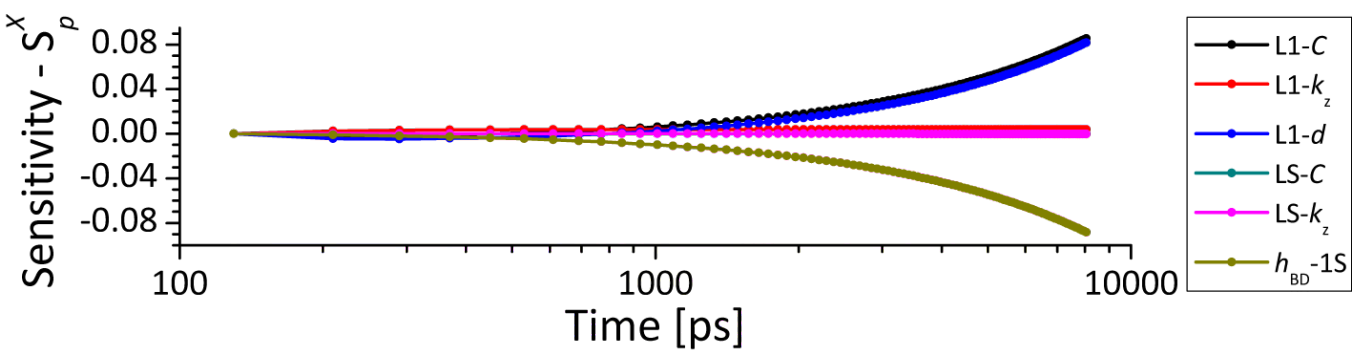

Figure S.8: Sensitivity of the real signal, $X$, as a function of time for the thermal parameters in the 100 $\mathrm{nm} \mathrm{Al} / \mathrm{Si}$ system for $h_{\mathrm{BD}}=2.78 \mathrm{MW} / \mathrm{m}^{2} \mathrm{~K}$. 


\section{S.3.6 $100 \mathrm{~nm}$ Al on Si Substrate - 3.59 MW Series}

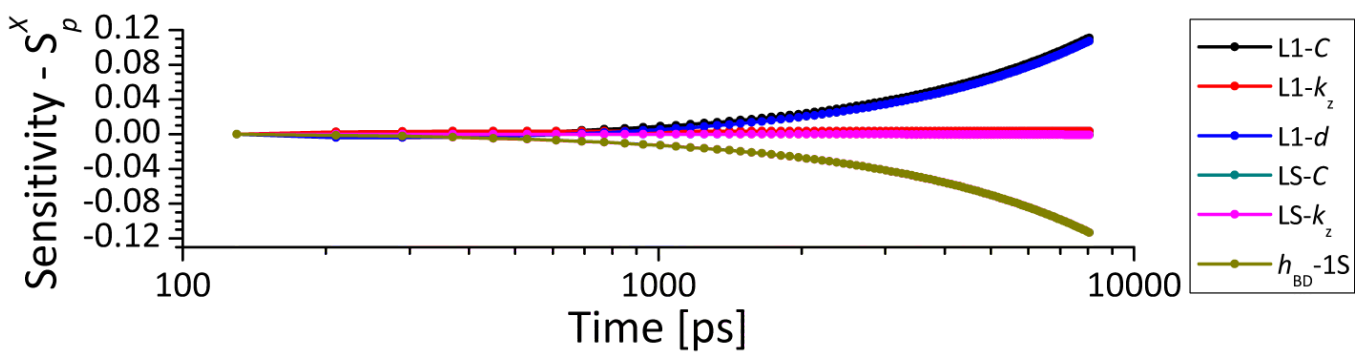

Figure S.9: Sensitivity of the real signal, $X$, as a function of time for the thermal parameters in the 100 $\mathrm{nm} \mathrm{Al} / \mathrm{Si}$ system for $h_{\mathrm{BD}}=3.59 \mathrm{MW} / \mathrm{m}^{2} \mathrm{~K}$.

\section{S.3.7 $100 \mathrm{~nm}$ Al on Si Substrate - 4.64 MW Series}

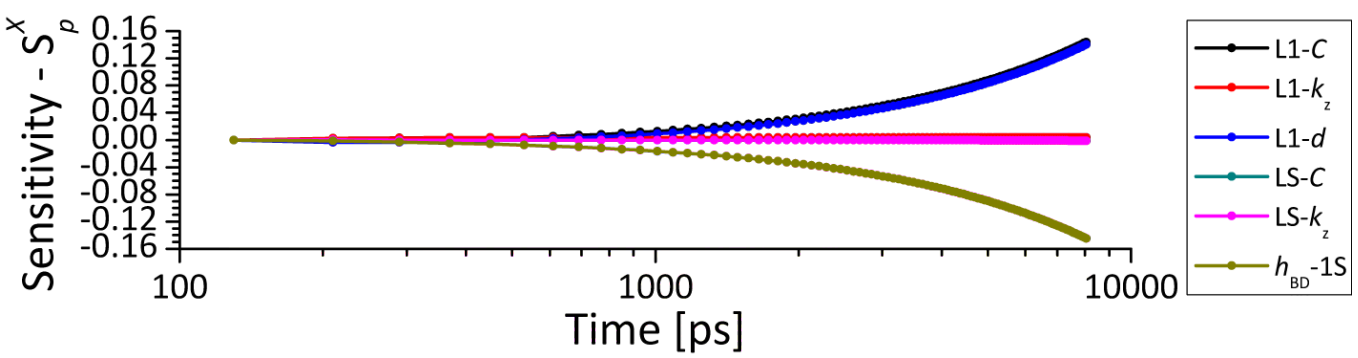

Figure S.10: Sensitivity of the real signal, $X$, as a function of time for the thermal parameters in the 100 $\mathrm{nm} \mathrm{Al} / \mathrm{Si}$ system for $h_{\mathrm{BD}}=4.64 \mathrm{MW} / \mathrm{m}^{2} \mathrm{~K}$.

\section{S.3.8 $100 \mathrm{~nm}$ Al on Si Substrate - 5.99 MW Series}

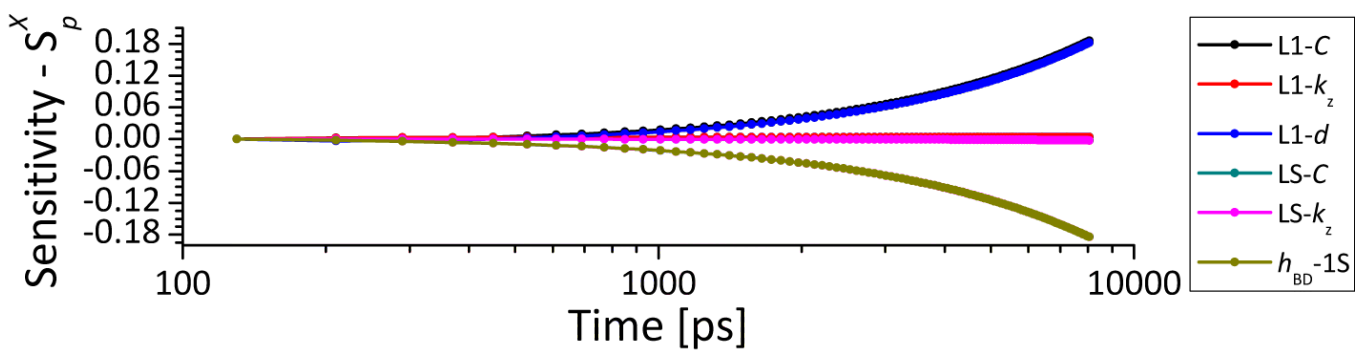

Figure S.11: Sensitivity of the real signal, $X$, as a function of time for the thermal parameters in the 100 $\mathrm{nm} \mathrm{Al} / \mathrm{Si}$ system for $h_{\mathrm{BD}}=5.99 \mathrm{MW} / \mathrm{m}^{2} \mathrm{~K}$. 


\section{S.3.9 $100 \mathrm{~nm}$ Al on Si Substrate - 7.74 MW Series}

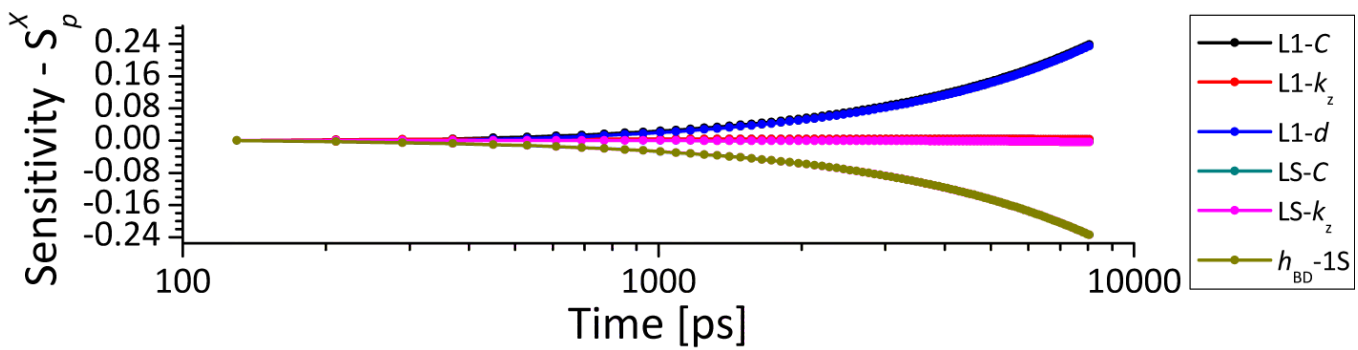

Figure S.12: Sensitivity of the real signal, $X$, as a function of time for the thermal parameters in the 100 $\mathrm{nm} \mathrm{Al} / \mathrm{Si}$ system for $h_{\mathrm{BD}}=7.74 \mathrm{MW} / \mathrm{m}^{2} \mathrm{~K}$.

\section{S.3.10 $100 \mathrm{~nm}$ Al on Si Substrate - 10.00 MW Series}

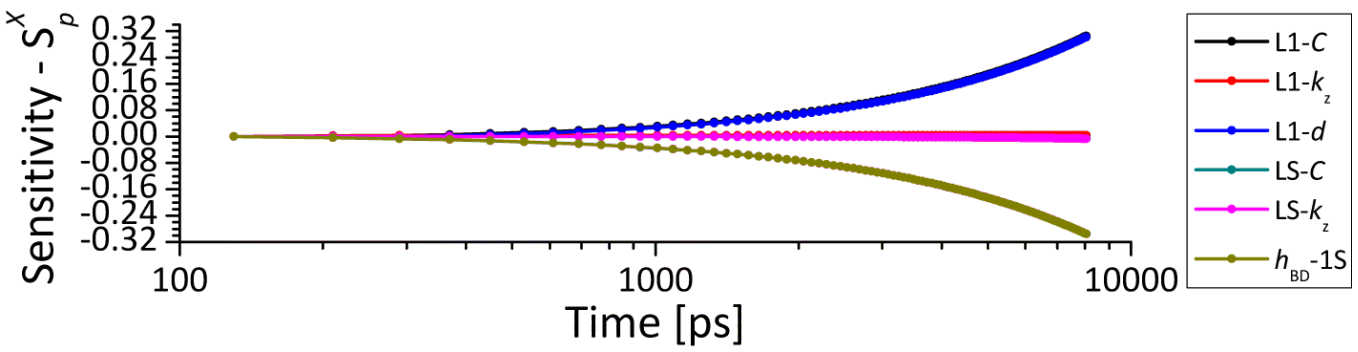

Figure S.13: Sensitivity of the real signal, $X$, as a function of time for the thermal parameters in the 100 $\mathrm{nm} \mathrm{Al} / \mathrm{Si}$ system for $h_{\mathrm{BD}}=10.00 \mathrm{MW} / \mathrm{m}^{2} \mathrm{~K}$.

\section{S.3.11 100 nm Al on Si Substrate - 12.90 MW Series}

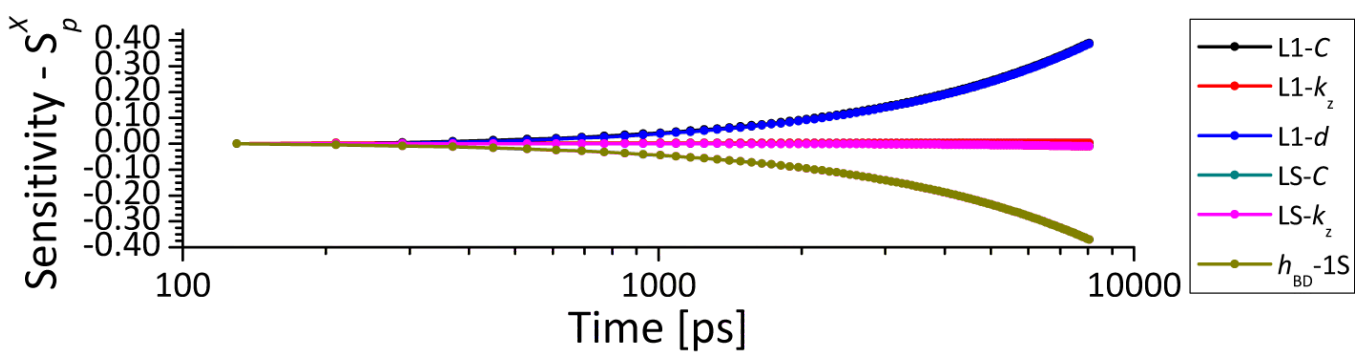

Figure S.14: Sensitivity of the real signal, $X$, as a function of time for the thermal parameters in the 100 $\mathrm{nm} \mathrm{Al} / \mathrm{Si}$ system for $h_{\mathrm{BD}}=12.90 \mathrm{MW} / \mathrm{m}^{2} \mathrm{~K}$. 


\section{S.3.12 $100 \mathrm{~nm}$ Al on Si Substrate - 16.70 MW Series}

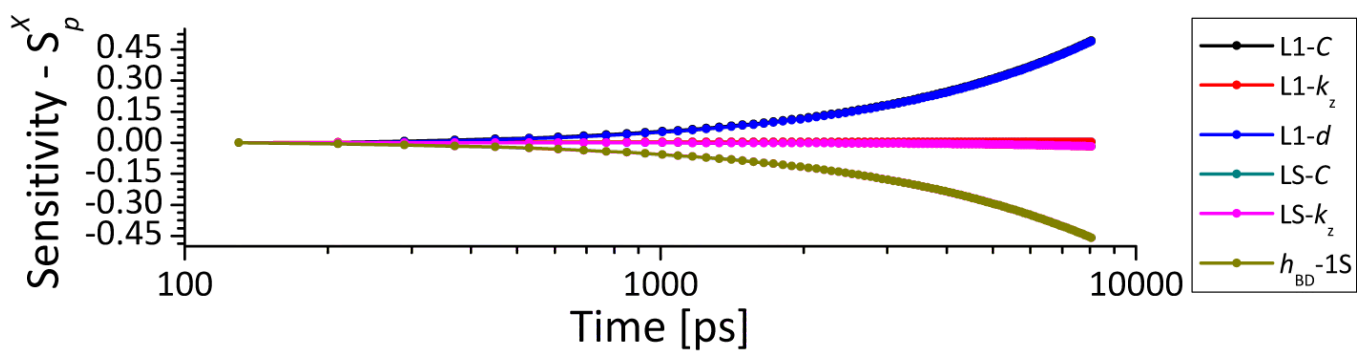

Figure S.15: Sensitivity of the real signal, $X$, as a function of time for the thermal parameters in the 100 $\mathrm{nm} \mathrm{Al} / \mathrm{Si}$ system for $h_{\mathrm{BD}}=16.70 \mathrm{MW} / \mathrm{m}^{2} \mathrm{~K}$.

\section{S.3.13 $100 \mathrm{~nm}$ Al on Si Substrate - 21.50 MW Series}

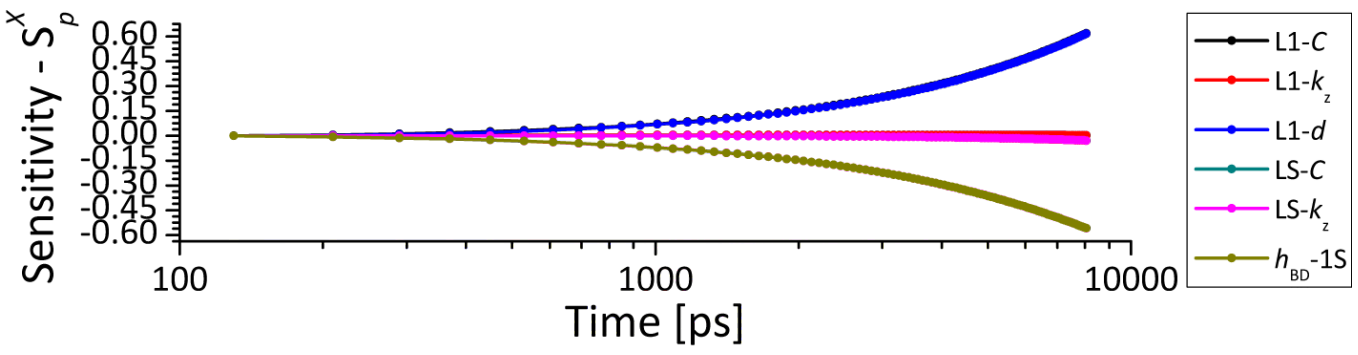

Figure S.16: Sensitivity of the real signal, $X$, as a function of time for the thermal parameters in the 100 $\mathrm{nm} \mathrm{Al} / \mathrm{Si}$ system for $h_{\mathrm{BD}}=21.50 \mathrm{MW} / \mathrm{m}^{2} \mathrm{~K}$.

\section{S.3.14 $100 \mathrm{~nm}$ Al on Si Substrate - 27.80 MW Series}

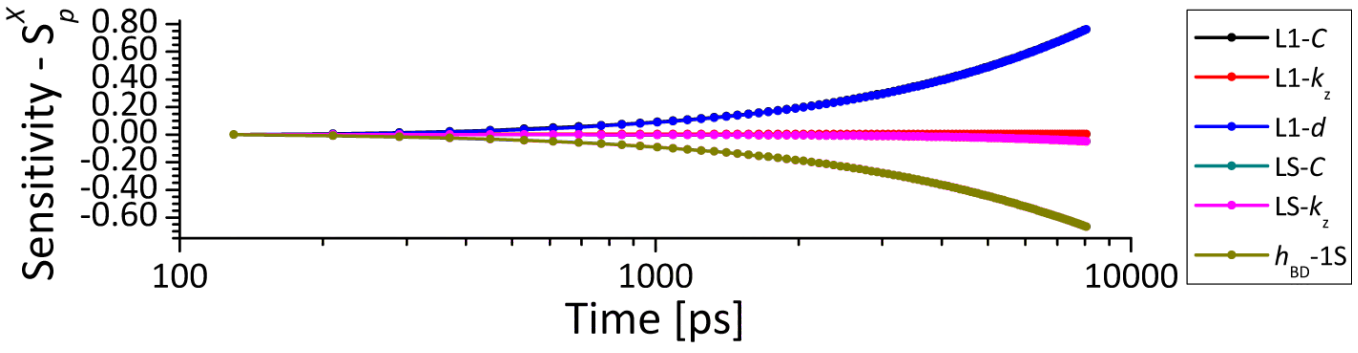

Figure S.17: Sensitivity of the real signal, $X$, as a function of time for the thermal parameters in the 100 $\mathrm{nm} \mathrm{Al} / \mathrm{Si}$ system for $h_{\mathrm{BD}}=27.80 \mathrm{MW} / \mathrm{m}^{2} \mathrm{~K}$. 


\section{S.3.15 $100 \mathrm{~nm}$ Al on Si Substrate - 35.90 MW Series}

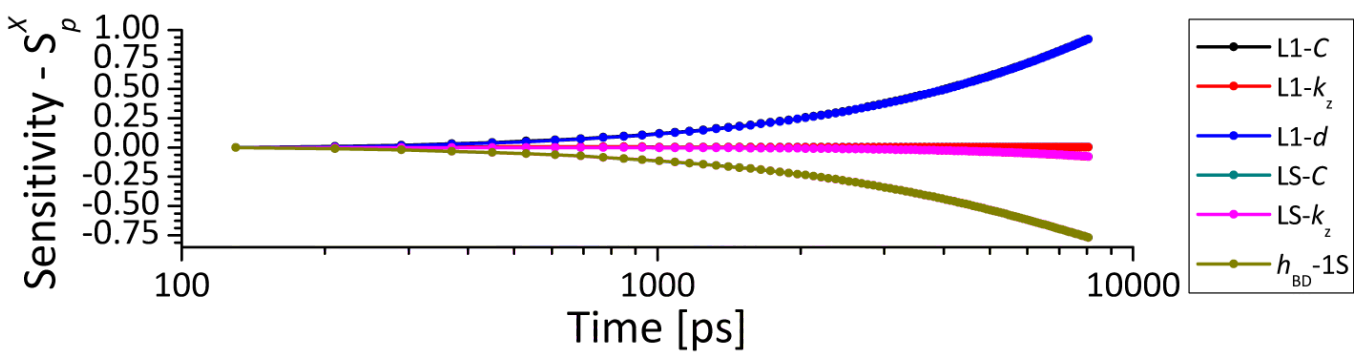

Figure S.18: Sensitivity of the real signal, $X$, as a function of time for the thermal parameters in the 100 $\mathrm{nm} \mathrm{Al} / \mathrm{Si}$ system for $h_{\mathrm{BD}}=35.90 \mathrm{MW} / \mathrm{m}^{2} \mathrm{~K}$.

\section{S.3.16 $100 \mathrm{~nm} \mathrm{Al} \mathrm{on} \mathrm{Si} \mathrm{Substrate} \mathrm{-} \mathrm{46.40} \mathrm{MW} \mathrm{Series}$}

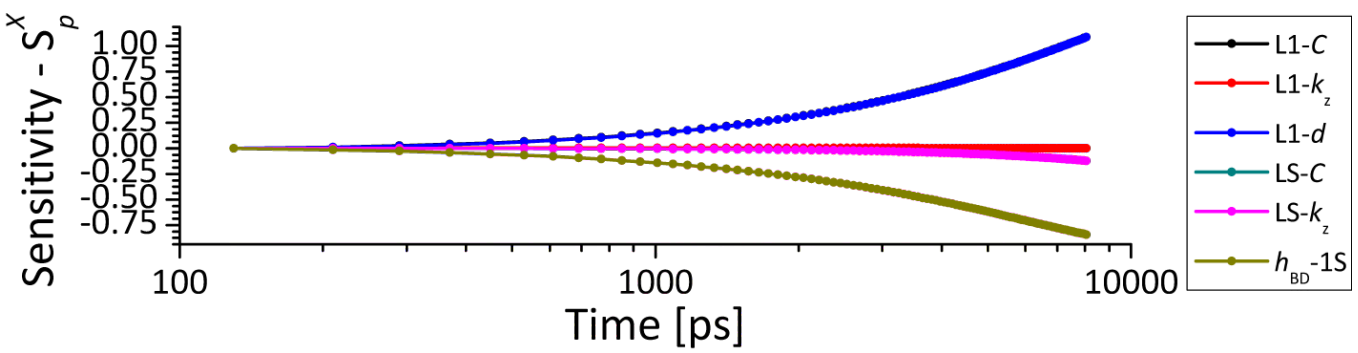

Figure S.19: Sensitivity of the real signal, $X$, as a function of time for the thermal parameters in the 100 $\mathrm{nm} \mathrm{Al} / \mathrm{Si}$ system for $h_{\mathrm{BD}}=46.40 \mathrm{MW} / \mathrm{m}^{2} \mathrm{~K}$.

\section{S.3.17 $100 \mathrm{~nm} \mathrm{Al} \mathrm{on} \mathrm{Si} \mathrm{Substrate} \mathrm{-} \mathrm{59.90} \mathrm{MW} \mathrm{Series}$}

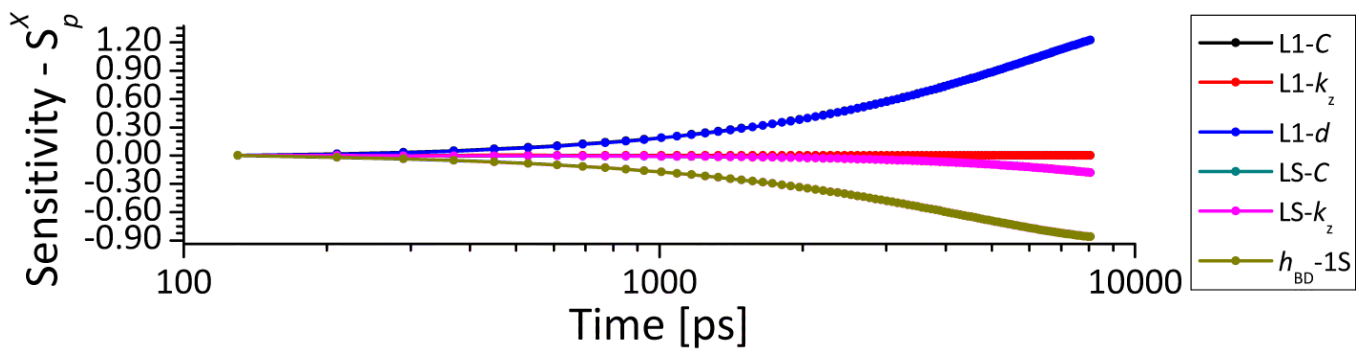

Figure S.20: Sensitivity of the real signal, $X$, as a function of time for the thermal parameters in the 100 $\mathrm{nm} \mathrm{Al} / \mathrm{Si}$ system for $h_{\mathrm{BD}}=59.90 \mathrm{MW} / \mathrm{m}^{2} \mathrm{~K}$. 


\section{S.3.18 $100 \mathrm{~nm}$ Al on Si Substrate - 77.40 MW Series}

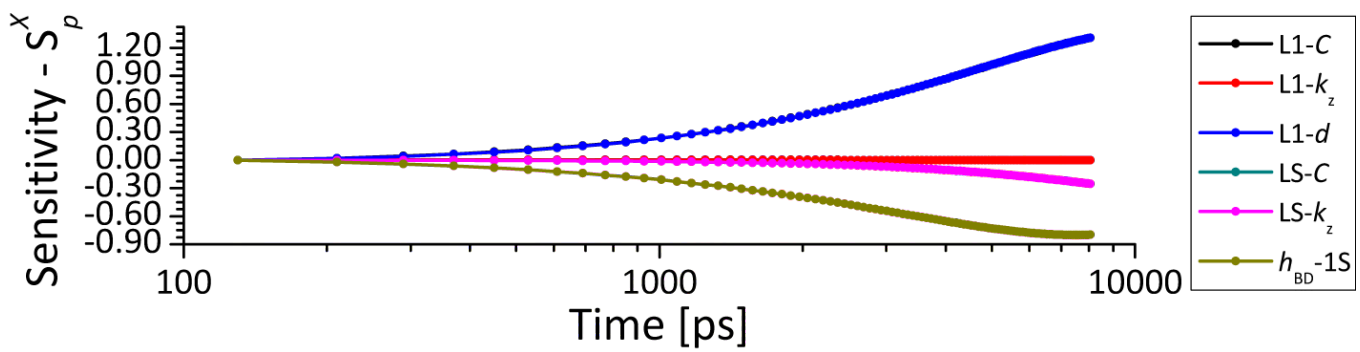

Figure S.21: Sensitivity of the real signal, $X$, as a function of time for the thermal parameters in the 100 $\mathrm{nm} \mathrm{Al} / \mathrm{Si}$ system for $h_{\mathrm{BD}}=77.40 \mathrm{MW} / \mathrm{m}^{2} \mathrm{~K}$.

\section{S.3.19 $100 \mathrm{~nm} \mathrm{Al} \mathrm{on} \mathrm{Si} \mathrm{Substrate} \mathrm{-} \mathrm{100.00} \mathrm{MW} \mathrm{Series}$}

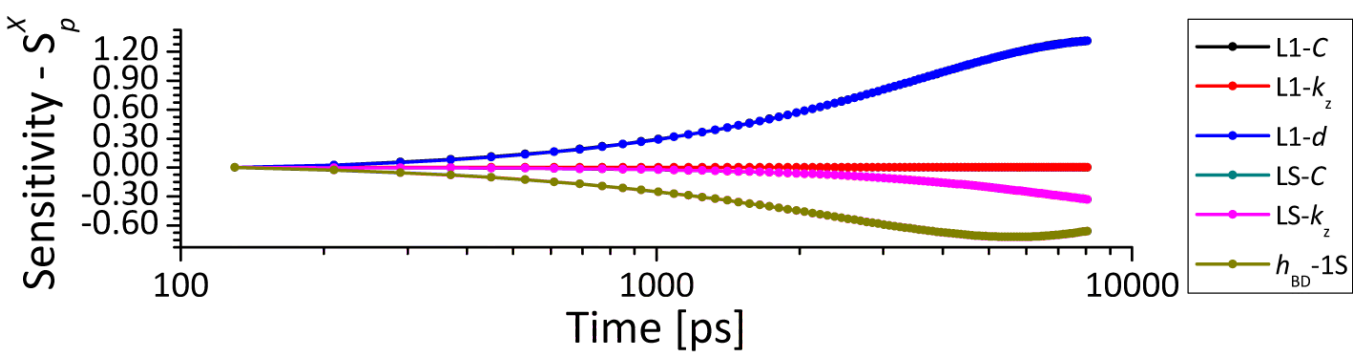

Figure S.22: Sensitivity of the real signal, $X$, as a function of time for the thermal parameters in the 100 $\mathrm{nm} \mathrm{Al} / \mathrm{Si}$ system for $h_{\mathrm{BD}}=100.00 \mathrm{MW} / \mathrm{m}^{2} \mathrm{~K}$.

\section{S.3.20 $100 \mathrm{~nm} \mathrm{Al} \mathrm{on} \mathrm{Si} \mathrm{Substrate} \mathrm{-} \mathrm{129.00} \mathrm{MW} \mathrm{Series}$}

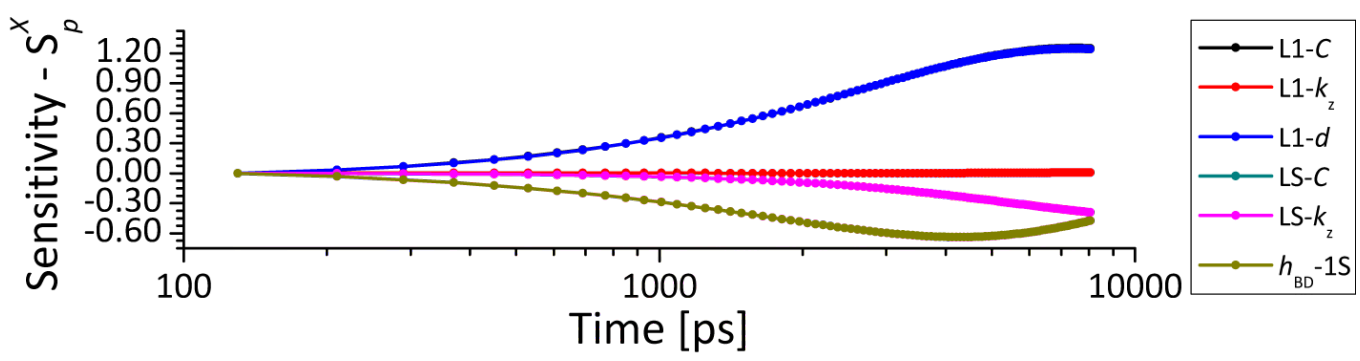

Figure S.23: Sensitivity of the real signal, $X$, as a function of time for the thermal parameters in the 100 $\mathrm{nm} \mathrm{Al} / \mathrm{Si}$ system for $h_{\mathrm{BD}}=129.00 \mathrm{MW} / \mathrm{m}^{2} \mathrm{~K}$. 


\section{S.3.21 $100 \mathrm{~nm} \mathrm{Al} \mathrm{on} \mathrm{Si} \mathrm{Substrate} \mathrm{-} \mathrm{167.00} \mathrm{MW} \mathrm{Series}$}

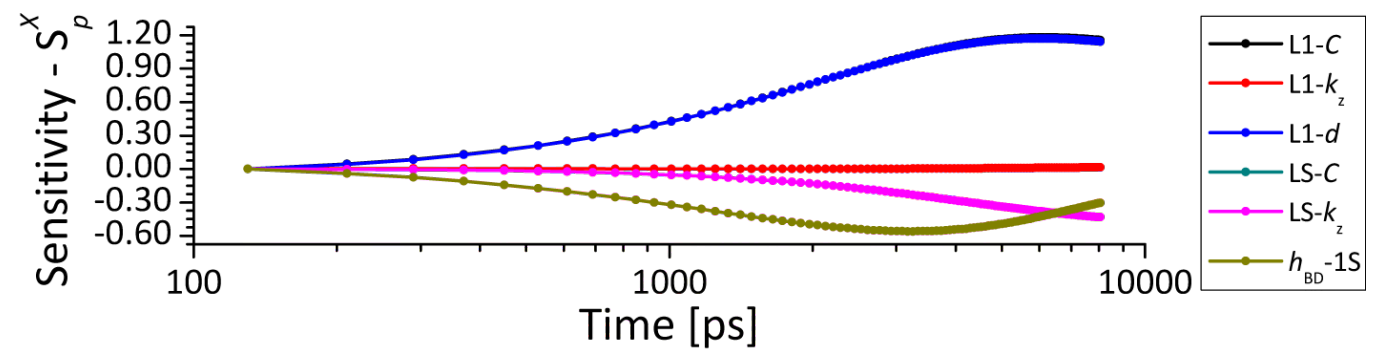

Figure S.24: Sensitivity of the real signal, $X$, as a function of time for the thermal parameters in the 100 $\mathrm{nm} \mathrm{Al} / \mathrm{Si}$ system for $h_{\mathrm{BD}}=167.00 \mathrm{MW} / \mathrm{m}^{2} \mathrm{~K}$.

\section{S.3.22 $100 \mathrm{~nm} \mathrm{Al} \mathrm{on} \mathrm{Si} \mathrm{Substrate} \mathrm{-} \mathrm{215.00} \mathrm{MW} \mathrm{Series}$}

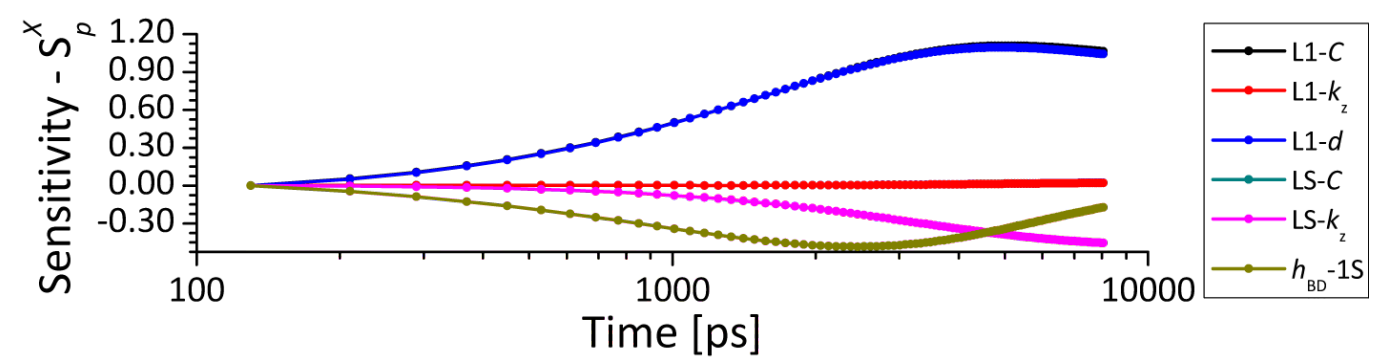

Figure S.25: Sensitivity of the real signal, $X$, as a function of time for the thermal parameters in the 100 $\mathrm{nm} \mathrm{Al} / \mathrm{Si}$ system for $h_{\mathrm{BD}}=215.00 \mathrm{MW} / \mathrm{m}^{2} \mathrm{~K}$.

\section{S.3.23 $100 \mathrm{~nm} \mathrm{Al} \mathrm{on} \mathrm{Si} \mathrm{Substrate} \mathrm{-} \mathrm{278.00} \mathrm{MW} \mathrm{Series}$}

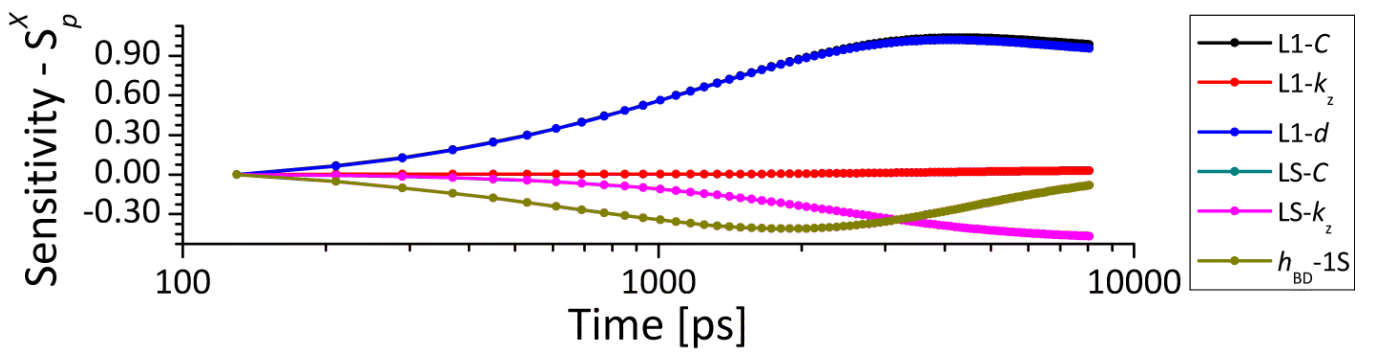

Figure S.26: Sensitivity of the real signal, $X$, as a function of time for the thermal parameters in the 100 $\mathrm{nm} \mathrm{Al} / \mathrm{Si}$ system for $h_{\mathrm{BD}}=278.00 \mathrm{MW} / \mathrm{m}^{2} \mathrm{~K}$. 


\section{S.3.24 $100 \mathrm{~nm}$ Al on Si Substrate - 359.00 MW Series}

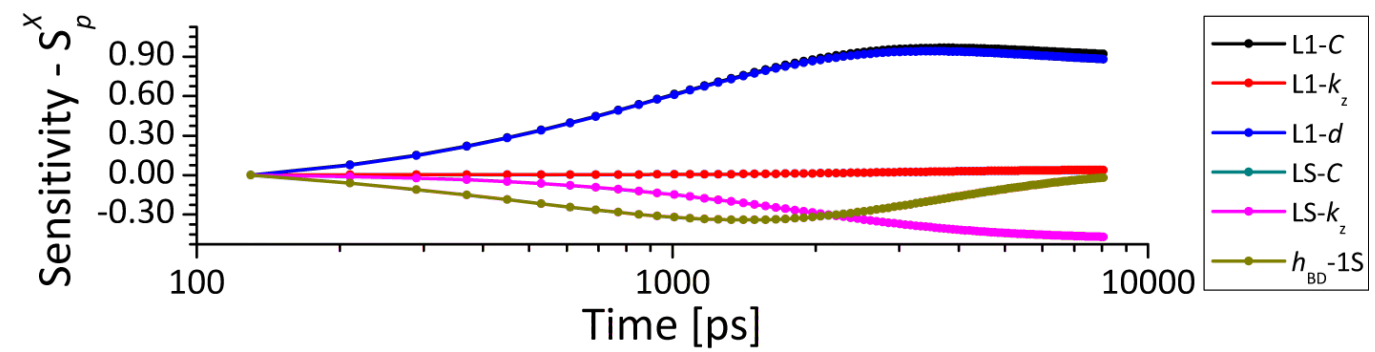

Figure S.27: Sensitivity of the real signal, $X$, as a function of time for the thermal parameters in the 100 $\mathrm{nm} \mathrm{Al} / \mathrm{Si}$ system for $h_{\mathrm{BD}}=359.00 \mathrm{MW} / \mathrm{m}^{2} \mathrm{~K}$.

\section{S.3.25 $100 \mathrm{~nm} \mathrm{Al} \mathrm{on} \mathrm{Si} \mathrm{Substrate} \mathrm{-} \mathrm{464.00} \mathrm{MW} \mathrm{Series}$}

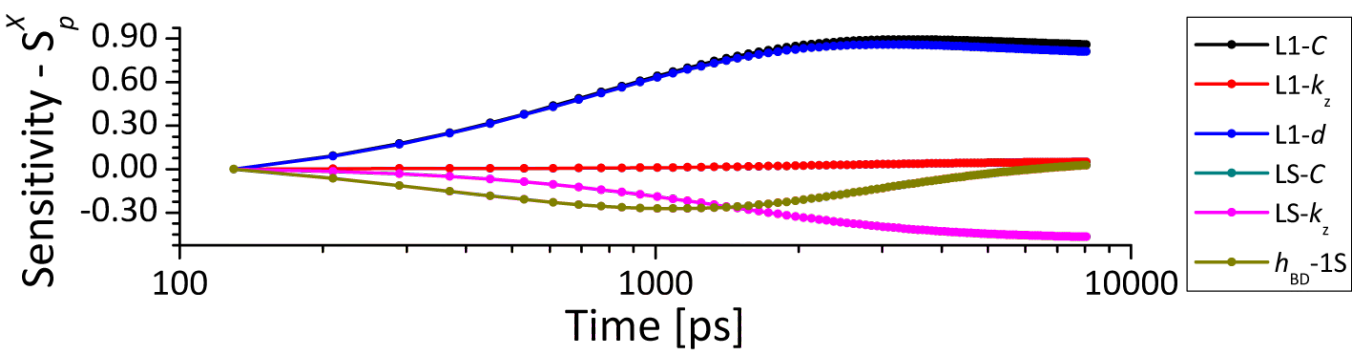

Figure S.28: Sensitivity of the real signal, $X$, as a function of time for the thermal parameters in the 100 $\mathrm{nm} \mathrm{Al} / \mathrm{Si}$ system for $h_{\mathrm{BD}}=464.00 \mathrm{MW} / \mathrm{m}^{2} \mathrm{~K}$.

\section{S.3.26 $100 \mathrm{~nm} \mathrm{Al} \mathrm{on} \mathrm{Si} \mathrm{Substrate} \mathrm{-} \mathrm{599.00} \mathrm{MW} \mathrm{Series}$}

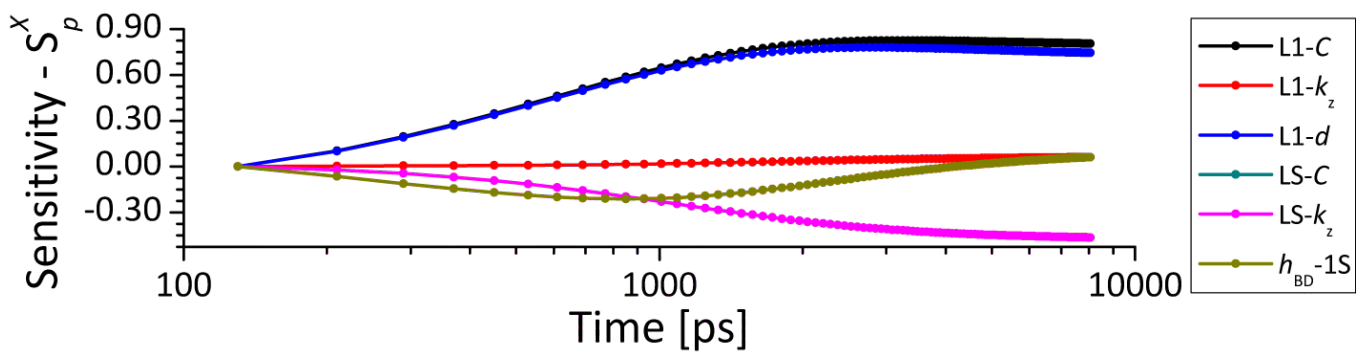

Figure S.29: Sensitivity of the real signal, $X$, as a function of time for the thermal parameters in the 100 $\mathrm{nm} \mathrm{Al} / \mathrm{Si}$ system for $h_{\mathrm{BD}}=599.00 \mathrm{MW} / \mathrm{m}^{2} \mathrm{~K}$. 


\section{S.3.27 $100 \mathrm{~nm} \mathrm{Al} \mathrm{on} \mathrm{Si} \mathrm{Substrate} \mathrm{-} \mathrm{774.00} \mathrm{MW} \mathrm{Series}$}

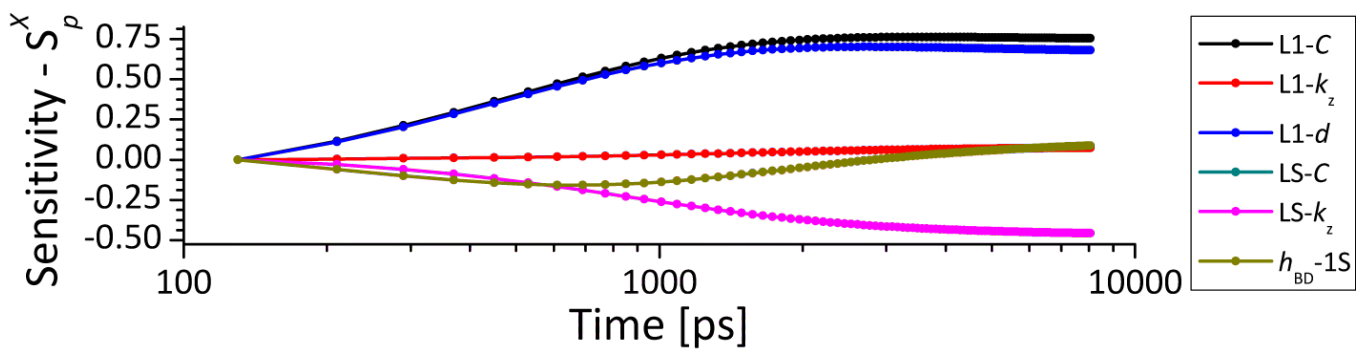

Figure S.30: Sensitivity of the real signal, $X$, as a function of time for the thermal parameters in the 100 $\mathrm{nm} \mathrm{Al} / \mathrm{Si}$ system for $h_{\mathrm{BD}}=774.00 \mathrm{MW} / \mathrm{m}^{2} \mathrm{~K}$.

\section{S.3.28 $100 \mathrm{~nm} \mathrm{Al} \mathrm{on} \mathrm{Si} \mathrm{Substrate} \mathrm{-} \mathrm{1000.00} \mathrm{MW} \mathrm{Series}$}

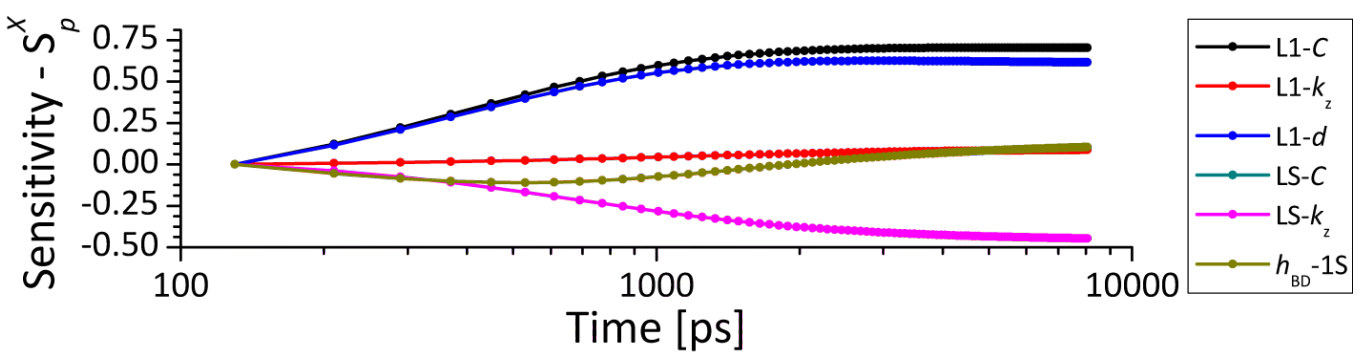

Figure S.31: Sensitivity of the real signal, $X$, as a function of time for the thermal parameters in the 100 $\mathrm{nm} \mathrm{Al} / \mathrm{Si}$ system for $h_{\mathrm{BD}}=1000.00 \mathrm{MW} / \mathrm{m}^{2} \mathrm{~K}$.

\section{S.3.29 $100 \mathrm{~nm} \mathrm{Al} \mathrm{on} \mathrm{AlN} \mathrm{Substrate} \mathrm{-} \mathrm{1.00} \mathrm{MW} \mathrm{Series}$}

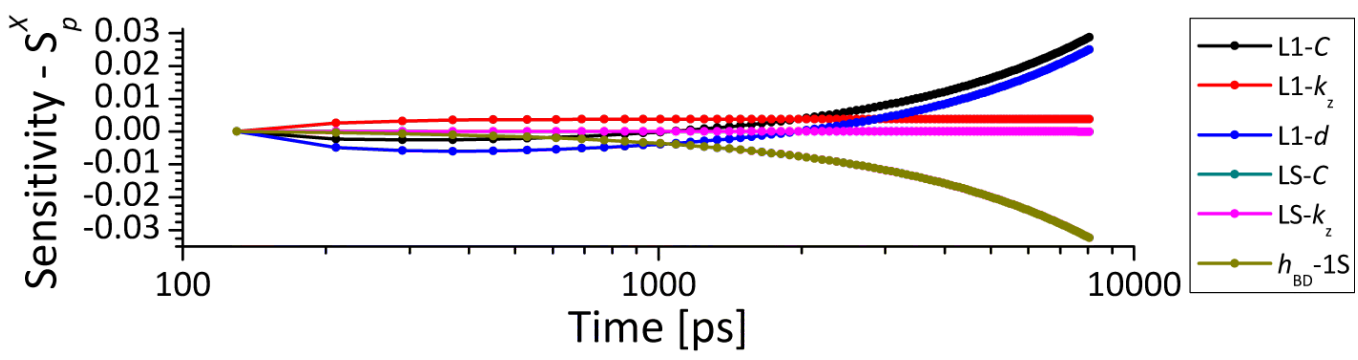

Figure S.32: Sensitivity of the real signal, $X$, as a function of time for the thermal parameters in the 100 $\mathrm{nm} \mathrm{Al} / \mathrm{AlN}$ system for $h_{\mathrm{BD}}=1.00 \mathrm{MW} / \mathrm{m}^{2} \mathrm{~K}$. 


\section{S.3.30 $100 \mathrm{~nm} \mathrm{Al} \mathrm{on} \mathrm{AlN} \mathrm{Substrate} \mathrm{-} \mathrm{1.29} \mathrm{MW} \mathrm{Series}$}

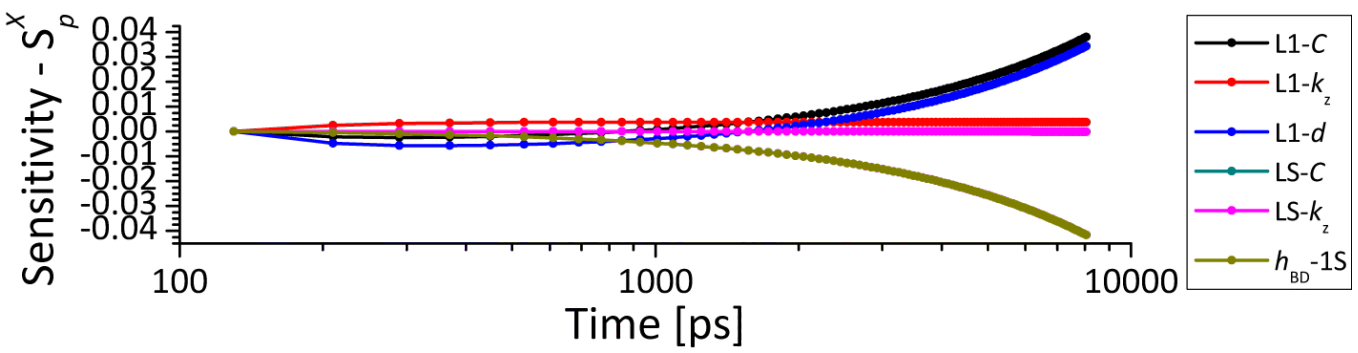

Figure S.33: Sensitivity of the real signal, $X$, as a function of time for the thermal parameters in the 100 $\mathrm{nm} \mathrm{Al} / \mathrm{AlN}$ system for $h_{\mathrm{BD}}=1.29 \mathrm{MW} / \mathrm{m}^{2} \mathrm{~K}$.

\section{S.3.31 $100 \mathrm{~nm} \mathrm{Al} \mathrm{on} \mathrm{AlN} \mathrm{Substrate} \mathrm{-} \mathrm{2.78} \mathrm{MW} \mathrm{Series}$}

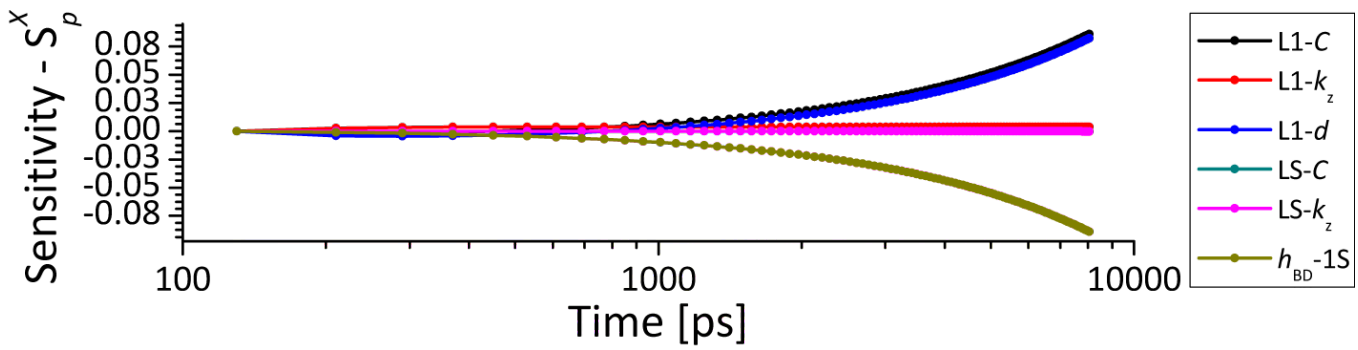

Figure S.34: Sensitivity of the real signal, $X$, as a function of time for the thermal parameters in the 100 $\mathrm{nm} \mathrm{Al} / \mathrm{AlN}$ system for $h_{\mathrm{BD}}=2.78 \mathrm{MW} / \mathrm{m}^{2} \mathrm{~K}$.

\section{S.3.32 $100 \mathrm{~nm} \mathrm{Al} \mathrm{on} \mathrm{AlN} \mathrm{Substrate} \mathrm{-} \mathrm{5.99} \mathrm{MW} \mathrm{Series}$}

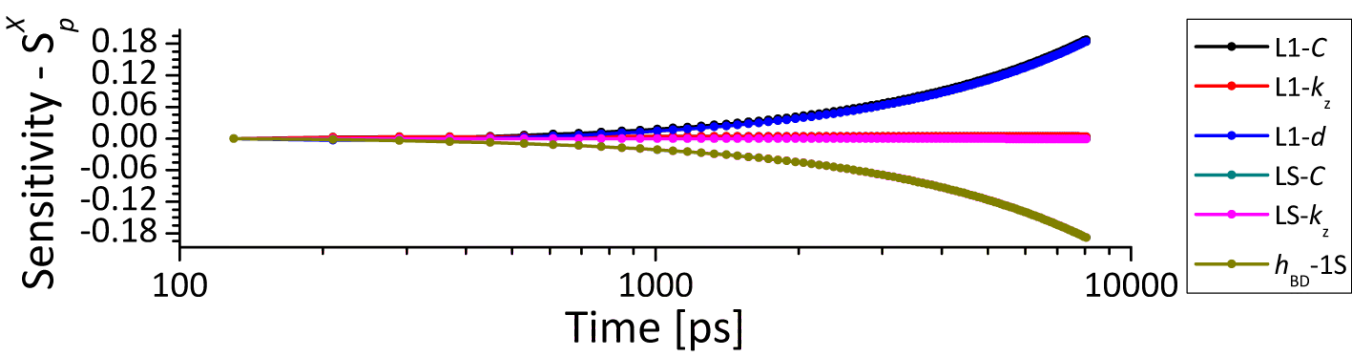

Figure S.35: Sensitivity of the real signal, $X$, as a function of time for the thermal parameters in the 100 $\mathrm{nm} \mathrm{Al} / \mathrm{AlN}$ system for $h_{\mathrm{BD}}=5.99 \mathrm{MW} / \mathrm{m}^{2} \mathrm{~K}$. 


\section{S.3.33 $100 \mathrm{~nm} \mathrm{Al} \mathrm{on} \mathrm{AlN} \mathrm{Substrate} \mathrm{-} \mathrm{12.90} \mathrm{MW} \mathrm{Series}$}

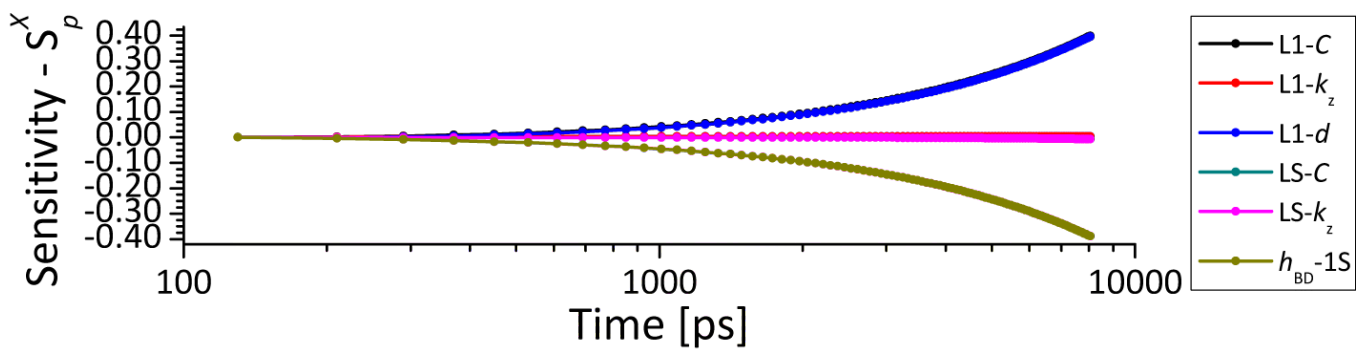

Figure S.36: Sensitivity of the real signal, $X$, as a function of time for the thermal parameters in the 100 $\mathrm{nm} \mathrm{Al} / \mathrm{AlN}$ system for $h_{\mathrm{BD}}=12.90 \mathrm{MW} / \mathrm{m}^{2} \mathrm{~K}$.

\section{S.3.34 $100 \mathrm{~nm} \mathrm{Al} \mathrm{on} \mathrm{AlN} \mathrm{Substrate} \mathrm{-} \mathrm{27.80} \mathrm{MW} \mathrm{Series}$}

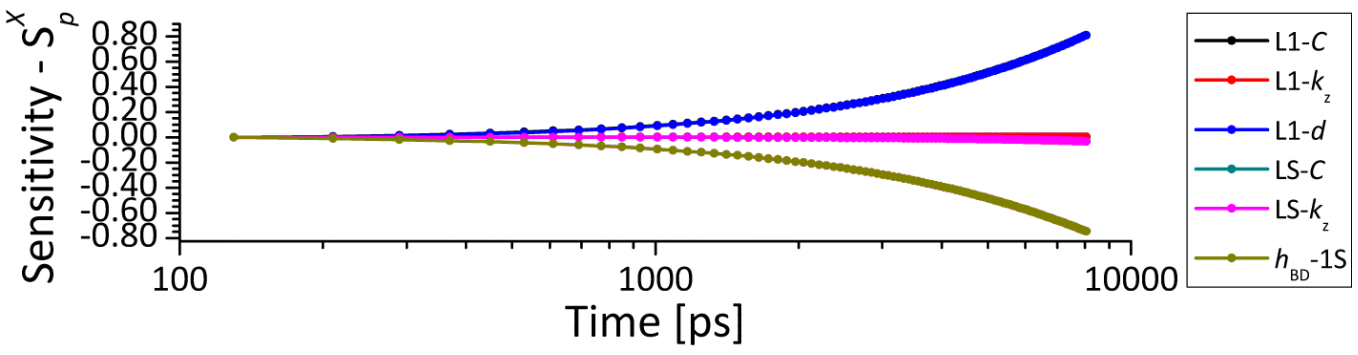

Figure S.37: Sensitivity of the real signal, $X$, as a function of time for the thermal parameters in the 100 $\mathrm{nm} \mathrm{Al} / \mathrm{AlN}$ system for $h_{\mathrm{BD}}=27.80 \mathrm{MW} / \mathrm{m}^{2} \mathrm{~K}$.

\section{S.3.35 $100 \mathrm{~nm} \mathrm{Al} \mathrm{on} \mathrm{AlN} \mathrm{Substrate} \mathrm{-} \mathrm{59.90} \mathrm{MW} \mathrm{Series}$}

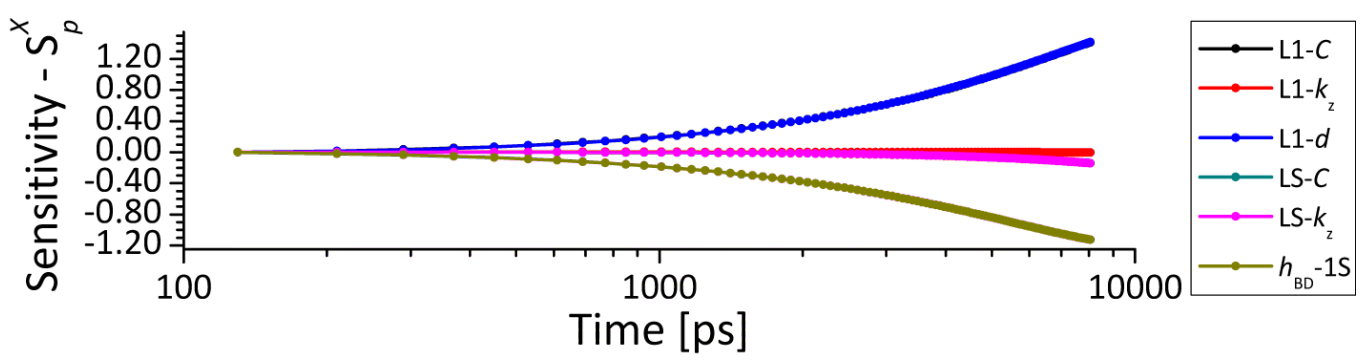

Figure S.38: Sensitivity of the real signal, $X$, as a function of time for the thermal parameters in the 100 $\mathrm{nm} \mathrm{Al} / \mathrm{AlN}$ system for $h_{\mathrm{BD}}=59.90 \mathrm{MW} / \mathrm{m}^{2} \mathrm{~K}$. 


\section{S.3.36 $100 \mathrm{~nm}$ Al on AlN Substrate - 100.00 MW Series}

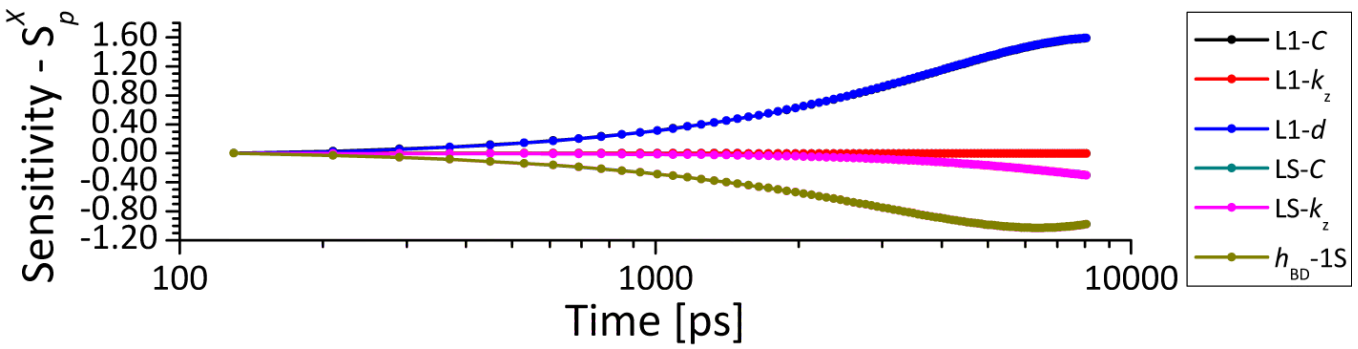

Figure S.39: Sensitivity of the real signal, $X$, as a function of time for the thermal parameters in the 100 $\mathrm{nm} \mathrm{Al} / \mathrm{AlN}$ system for $h_{\mathrm{BD}}=100.00 \mathrm{MW} / \mathrm{m}^{2} \mathrm{~K}$.

\section{S.3.37 $100 \mathrm{~nm} \mathrm{Al} \mathrm{on} \mathrm{AlN} \mathrm{Substrate} \mathrm{-} \mathrm{129.00} \mathrm{MW} \mathrm{Series}$}

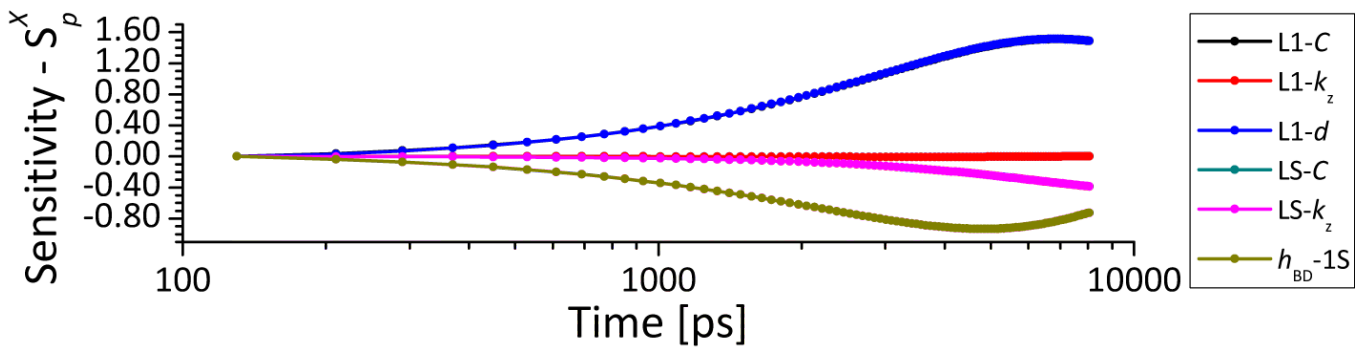

Figure S.40: Sensitivity of the real signal, $X$, as a function of time for the thermal parameters in the 100 $\mathrm{nm} \mathrm{Al} / \mathrm{AlN}$ system for $h_{\mathrm{BD}}=129.00 \mathrm{MW} / \mathrm{m}^{2} \mathrm{~K}$.

\section{S.3.38 $100 \mathrm{~nm}$ Al on AlN Substrate - 167.00 MW Series}

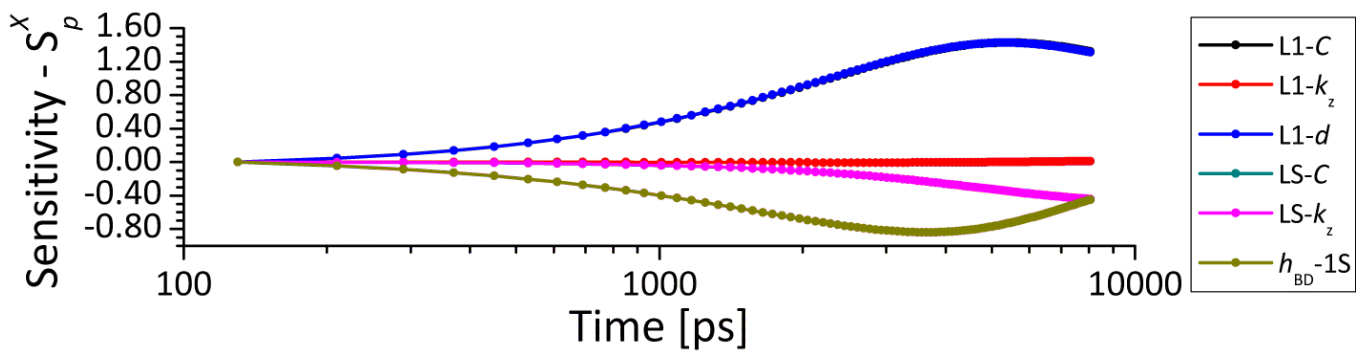

Figure S.41: Sensitivity of the real signal, $X$, as a function of time for the thermal parameters in the 100 $\mathrm{nm} \mathrm{Al} / \mathrm{AlN}$ system for $h_{\mathrm{BD}}=167.00 \mathrm{MW} / \mathrm{m}^{2} \mathrm{~K}$. 


\section{S.3.39 $100 \mathrm{~nm} \mathrm{Al} \mathrm{on} \mathrm{AlN} \mathrm{Substrate} \mathrm{-} \mathrm{215.00} \mathrm{MW} \mathrm{Series}$}

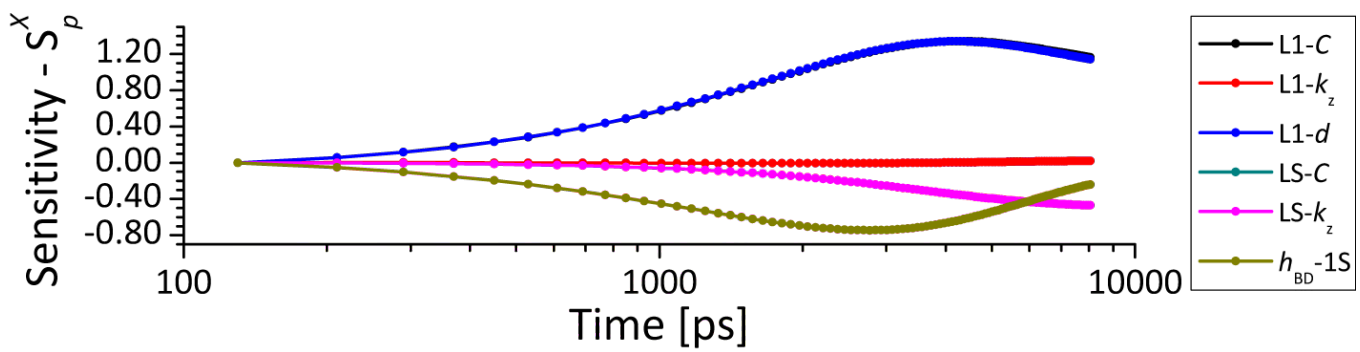

Figure S.42: Sensitivity of the real signal, $X$, as a function of time for the thermal parameters in the 100 $\mathrm{nm} \mathrm{Al} / \mathrm{AlN}$ system for $h_{\mathrm{BD}}=215.00 \mathrm{MW} / \mathrm{m}^{2} \mathrm{~K}$.

\section{S.3.40 $100 \mathrm{~nm}$ Al on AlN Substrate - 278.00 MW Series}

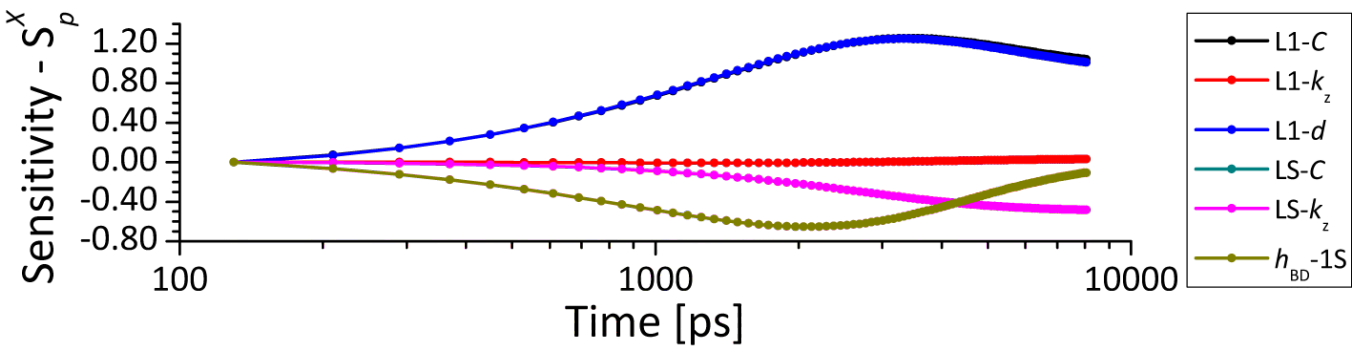

Figure S.43: Sensitivity of the real signal, $X$, as a function of time for the thermal parameters in the 100 $\mathrm{nm} \mathrm{Al} / \mathrm{AlN}$ system for $h_{\mathrm{BD}}=278.00 \mathrm{MW} / \mathrm{m}^{2} \mathrm{~K}$.

\section{S.3.41 $100 \mathrm{~nm}$ Al on AlN Substrate - 359.00 MW Series}

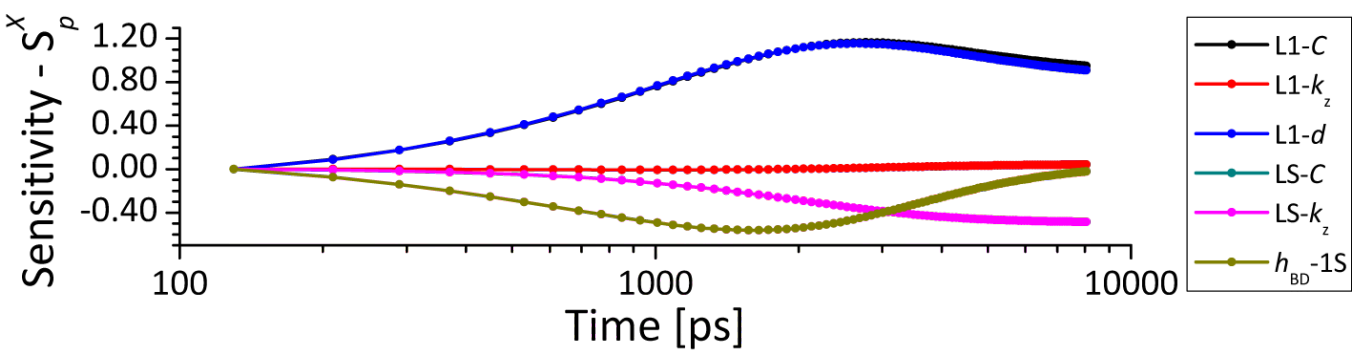

Figure S.44: Sensitivity of the real signal, $X$, as a function of time for the thermal parameters in the 100 $\mathrm{nm} \mathrm{Al} / \mathrm{AlN}$ system for $h_{\mathrm{BD}}=359.00 \mathrm{MW} / \mathrm{m}^{2} \mathrm{~K}$. 


\section{S.3.42 $100 \mathrm{~nm}$ Al on AlN Substrate - 464.00 MW Series}

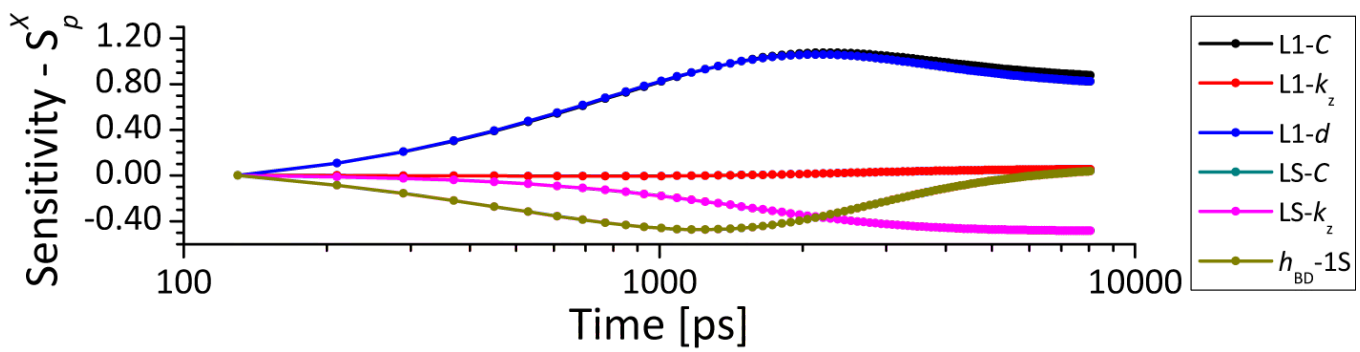

Figure S.45: Sensitivity of the real signal, $X$, as a function of time for the thermal parameters in the 100 $\mathrm{nm} \mathrm{Al} / \mathrm{AlN}$ system for $h_{\mathrm{BD}}=464.00 \mathrm{MW} / \mathrm{m}^{2} \mathrm{~K}$.

\section{S.3.43 $100 \mathrm{~nm} \mathrm{Al} \mathrm{on} \mathrm{AlN} \mathrm{Substrate} \mathrm{-} \mathrm{599.00} \mathrm{MW} \mathrm{Series}$}

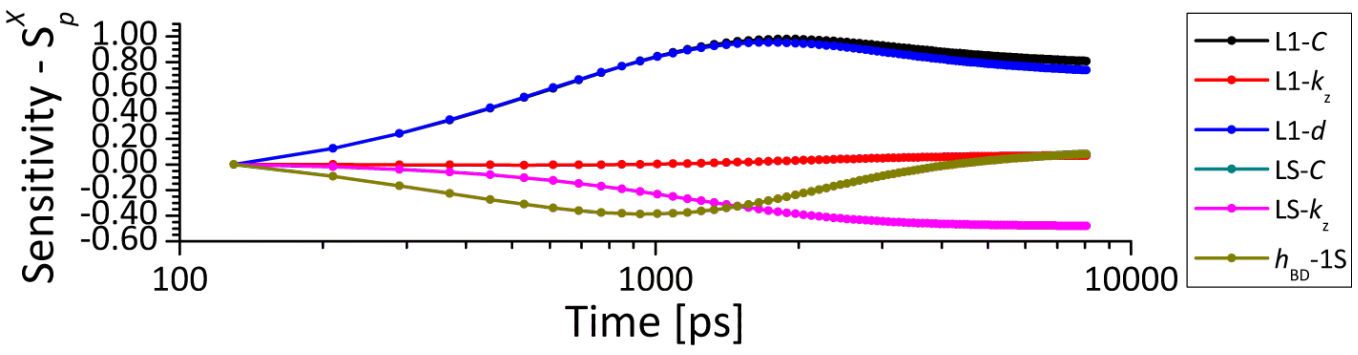

Figure S.46: Sensitivity of the real signal, $X$, as a function of time for the thermal parameters in the 100 $\mathrm{nm} \mathrm{Al} / \mathrm{AlN}$ system for $h_{\mathrm{BD}}=599.00 \mathrm{MW} / \mathrm{m}^{2} \mathrm{~K}$.

\section{S.3.44 $100 \mathrm{~nm} \mathrm{Al} \mathrm{on} \mathrm{AlN} \mathrm{Substrate} \mathrm{-} \mathrm{774.00} \mathrm{MW} \mathrm{Series}$}

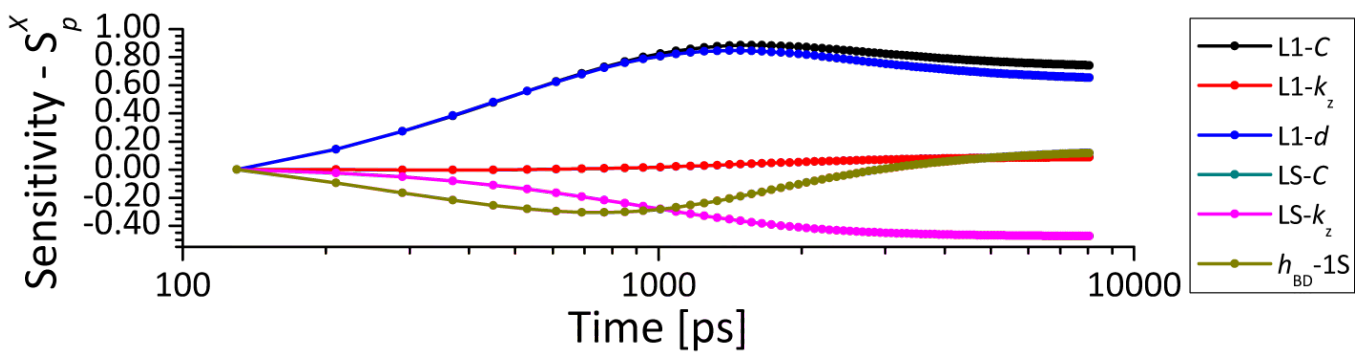

Figure S.47: Sensitivity of the real signal, $X$, as a function of time for the thermal parameters in the 100 $\mathrm{nm} \mathrm{Al} / \mathrm{AlN}$ system for $h_{\mathrm{BD}}=774.00 \mathrm{MW} / \mathrm{m}^{2} \mathrm{~K}$. 


\section{S.3.45 $100 \mathrm{~nm} \mathrm{Al} \mathrm{on} \mathrm{AlN} \mathrm{Substrate} \mathrm{-} \mathrm{1000.00} \mathrm{MW} \mathrm{Series}$}

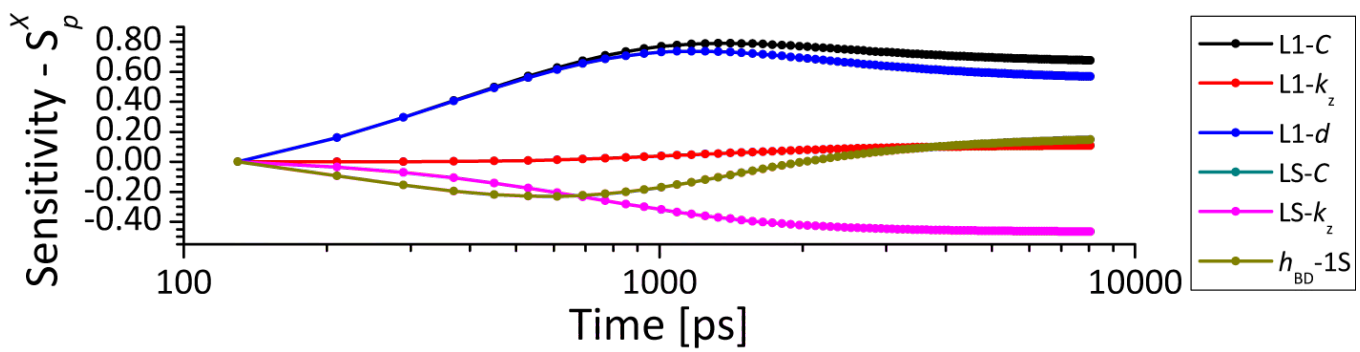

Figure S.48: Sensitivity of the real signal, $X$, as a function of time for the thermal parameters in the 100 $\mathrm{nm} \mathrm{Al} / \mathrm{AlN}$ system for $h_{\mathrm{BD}}=1000.00 \mathrm{MW} / \mathrm{m}^{2} \mathrm{~K}$.

\section{S.3.46 $100 \mathrm{~nm} \mathrm{Al} \mathrm{on} \mathrm{Ge} \mathrm{Substrate} \mathrm{-} \mathrm{1.00} \mathrm{MW} \mathrm{Series}$}

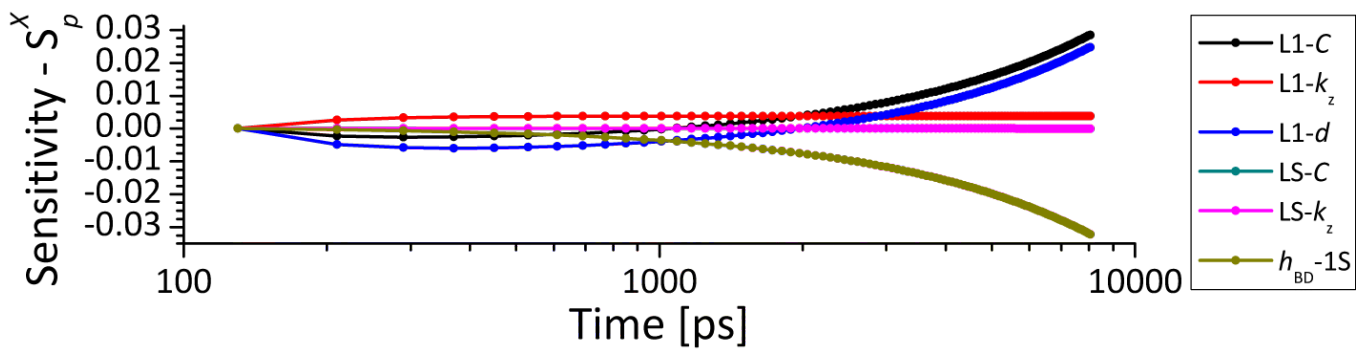

Figure S.49: Sensitivity of the real signal, $X$, as a function of time for the thermal parameters in the 100 $\mathrm{nm} \mathrm{Al} / \mathrm{Ge}$ system for $h_{\mathrm{BD}}=1.00 \mathrm{MW} / \mathrm{m}^{2} \mathrm{~K}$.

\section{S.3.47 100 nm Al on Ge Substrate - 2.15 MW Series}

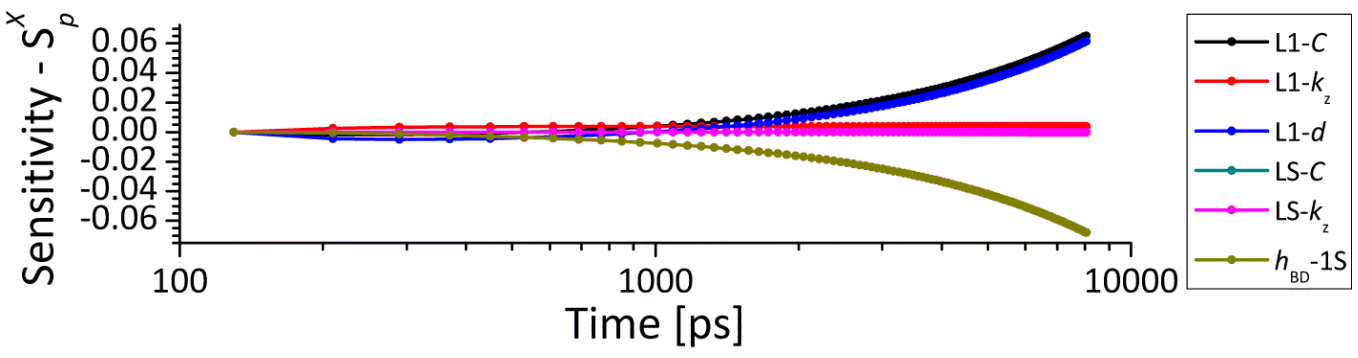

Figure S.50: Sensitivity of the real signal, $X$, as a function of time for the thermal parameters in the 100 $\mathrm{nm} \mathrm{Al} / \mathrm{Ge}$ system for $h_{\mathrm{BD}}=2.15 \mathrm{MW} / \mathrm{m}^{2} \mathrm{~K}$. 


\section{S.3.48 $100 \mathrm{~nm}$ Al on Ge Substrate - 3.59 MW Series}

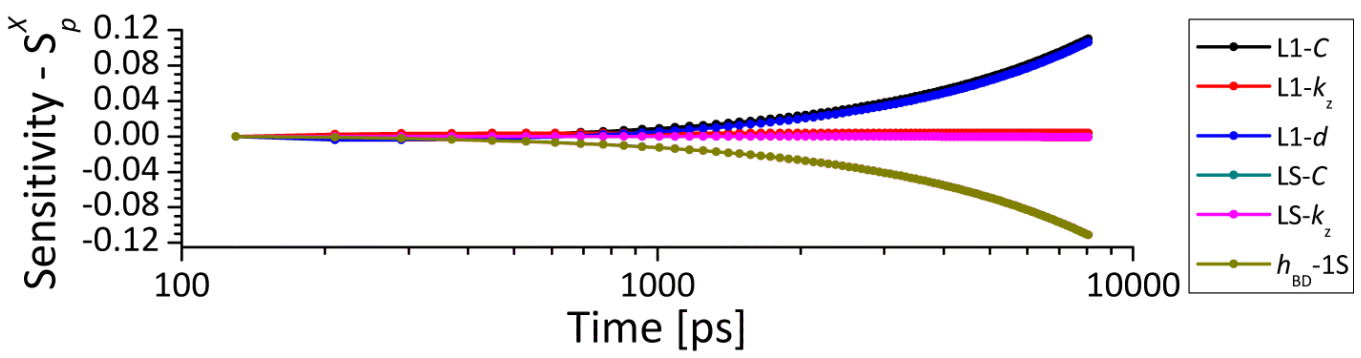

Figure S.51: Sensitivity of the real signal, $X$, as a function of time for the thermal parameters in the 100 $\mathrm{nm} \mathrm{Al} / \mathrm{Ge}$ system for $h_{\mathrm{BD}}=3.59 \mathrm{MW} / \mathrm{m}^{2} \mathrm{~K}$.

\section{S.3.49 $100 \mathrm{~nm}$ Al on Ge Substrate - 7.74 MW Series}

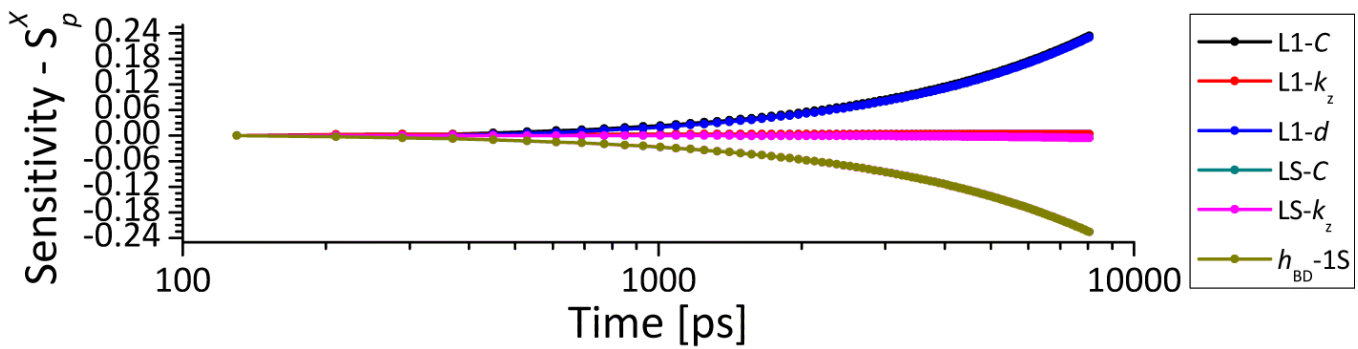

Figure S.52: Sensitivity of the real signal, $X$, as a function of time for the thermal parameters in the 100 $\mathrm{nm} \mathrm{Al} / \mathrm{Ge}$ system for $h_{\mathrm{BD}}=7.74 \mathrm{MW} / \mathrm{m}^{2} \mathrm{~K}$.

\section{S.3.50 $100 \mathrm{~nm} \mathrm{Al} \mathrm{on} \mathrm{Ge} \mathrm{Substrate} \mathrm{-} \mathrm{16.70} \mathrm{MW} \mathrm{Series}$}

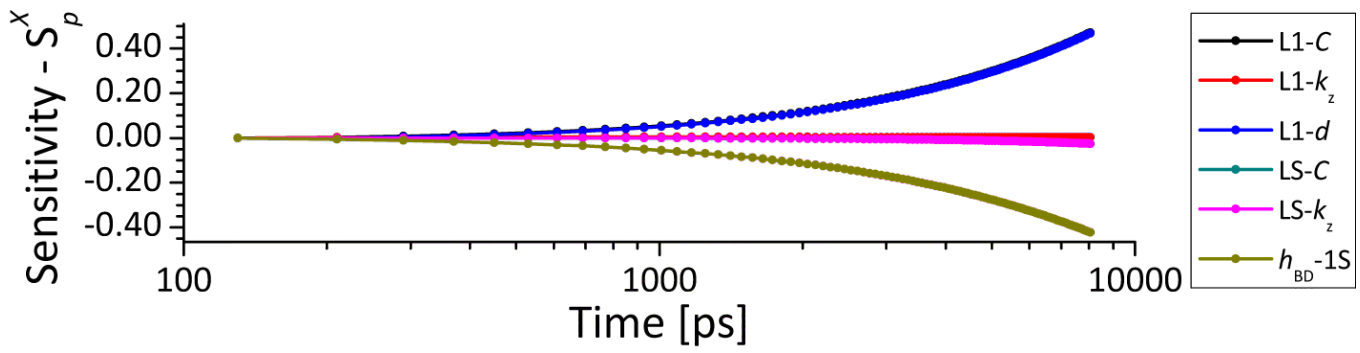

Figure S.53: Sensitivity of the real signal, $X$, as a function of time for the thermal parameters in the 100 $\mathrm{nm} \mathrm{Al} / \mathrm{Ge}$ system for $h_{\mathrm{BD}}=16.70 \mathrm{MW} / \mathrm{m}^{2} \mathrm{~K}$. 


\section{S.3.51 $100 \mathrm{~nm} \mathrm{Al} \mathrm{on} \mathrm{Ge} \mathrm{Substrate} \mathrm{-} \mathrm{46.40} \mathrm{MW} \mathrm{Series}$}

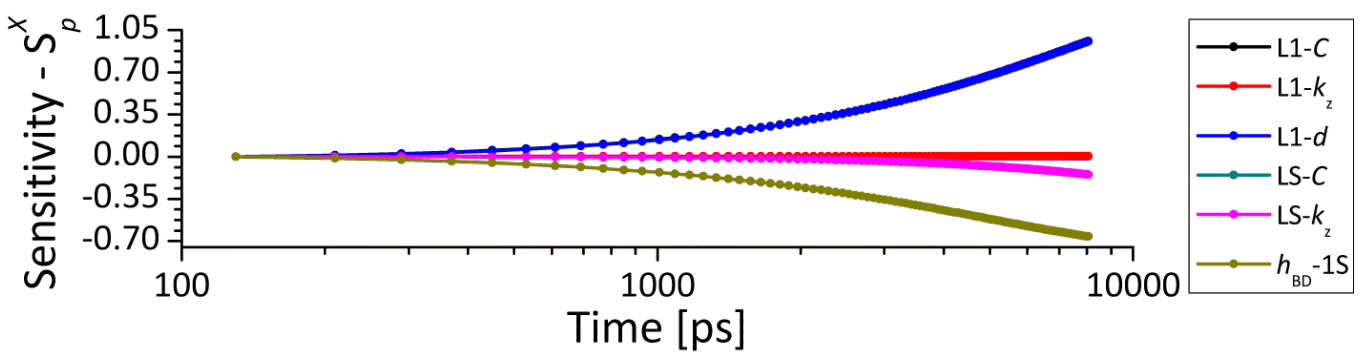

Figure S.54: Sensitivity of the real signal, $X$, as a function of time for the thermal parameters in the 100 $\mathrm{nm} \mathrm{Al} / \mathrm{Ge}$ system for $h_{\mathrm{BD}}=46.40 \mathrm{MW} / \mathrm{m}^{2} \mathrm{~K}$.

\section{S.3.52 $100 \mathrm{~nm} \mathrm{Al} \mathrm{on} \mathrm{Ge} \mathrm{Substrate} \mathrm{-} \mathrm{100.00} \mathrm{MW} \mathrm{Series}$}

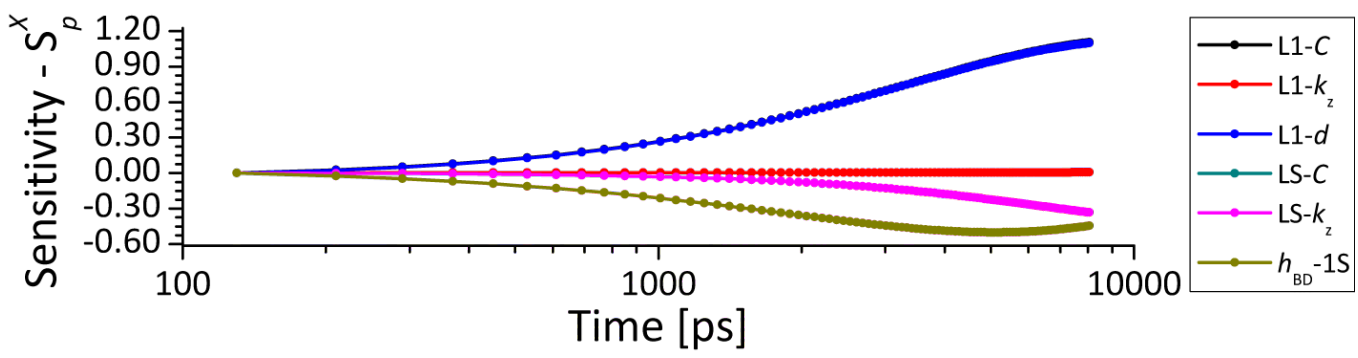

Figure S.55: Sensitivity of the real signal, $X$, as a function of time for the thermal parameters in the 100 $\mathrm{nm} \mathrm{Al} / \mathrm{Ge}$ system for $h_{\mathrm{BD}}=100.00 \mathrm{MW} / \mathrm{m}^{2} \mathrm{~K}$.

\section{S.3.53 $100 \mathrm{~nm} \mathrm{Al} \mathrm{on} \mathrm{Ge} \mathrm{Substrate} \mathrm{-} \mathrm{129.00} \mathrm{MW} \mathrm{Series}$}

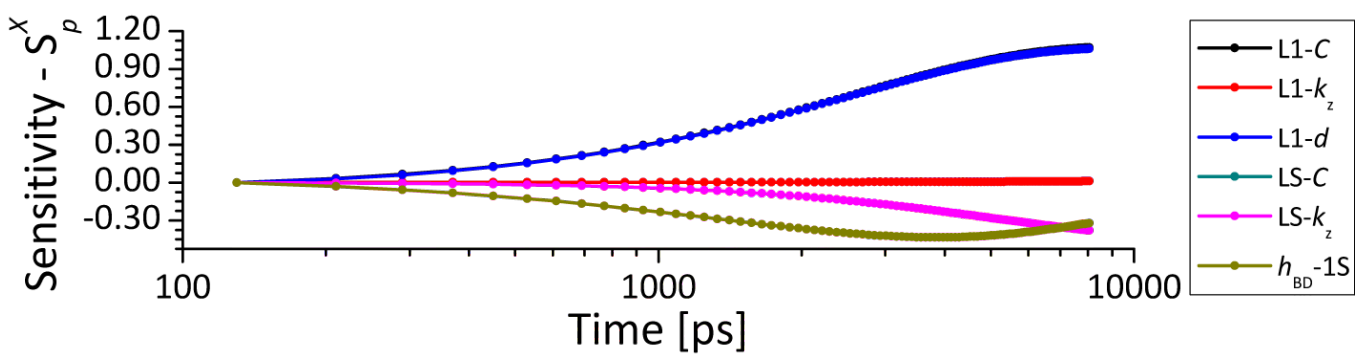

Figure S.56: Sensitivity of the real signal, $X$, as a function of time for the thermal parameters in the 100 $\mathrm{nm} \mathrm{Al} / \mathrm{Ge}$ system for $h_{\mathrm{BD}}=129.00 \mathrm{MW} / \mathrm{m}^{2} \mathrm{~K}$. 


\section{S.3.54 100 nm Al on Ge Substrate - 167.00 MW Series}

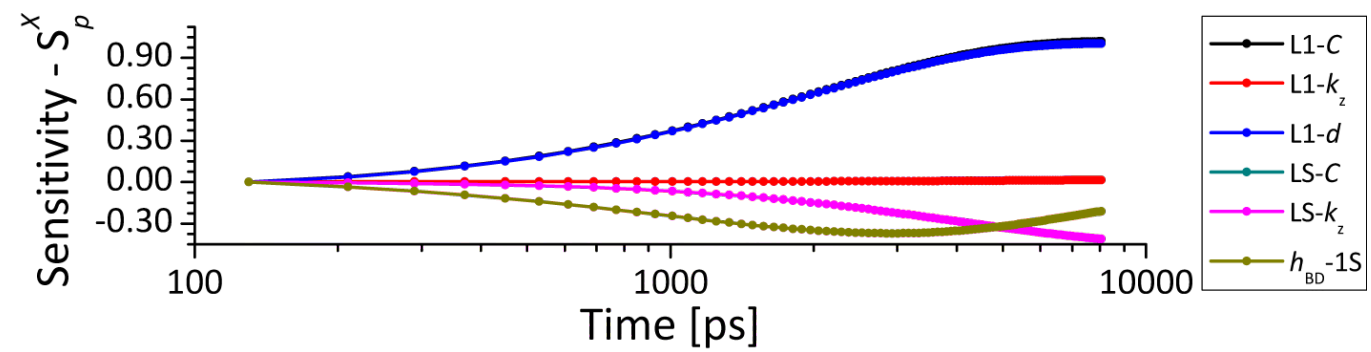

Figure S.57: Sensitivity of the real signal, $X$, as a function of time for the thermal parameters in the 100 $\mathrm{nm} \mathrm{Al} / \mathrm{Ge}$ system for $h_{\mathrm{BD}}=167.00 \mathrm{MW} / \mathrm{m}^{2} \mathrm{~K}$.

\section{S.3.55 $100 \mathrm{~nm} \mathrm{Al} \mathrm{on} \mathrm{Ge} \mathrm{Substrate} \mathrm{-} \mathrm{215.00} \mathrm{MW} \mathrm{Series}$}

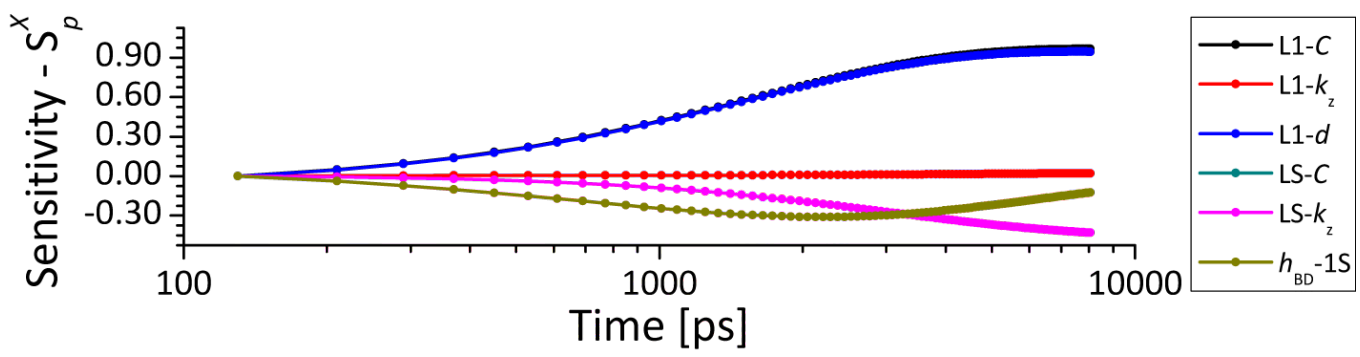

Figure S.58: Sensitivity of the real signal, $X$, as a function of time for the thermal parameters in the 100 $\mathrm{nm} \mathrm{Al} / \mathrm{Ge}$ system for $h_{\mathrm{BD}}=215.00 \mathrm{MW} / \mathrm{m}^{2} \mathrm{~K}$.

\section{S.3.56 $100 \mathrm{~nm} \mathrm{Al} \mathrm{on} \mathrm{Ge} \mathrm{Substrate} \mathrm{-} \mathrm{278.00} \mathrm{MW} \mathrm{Series}$}

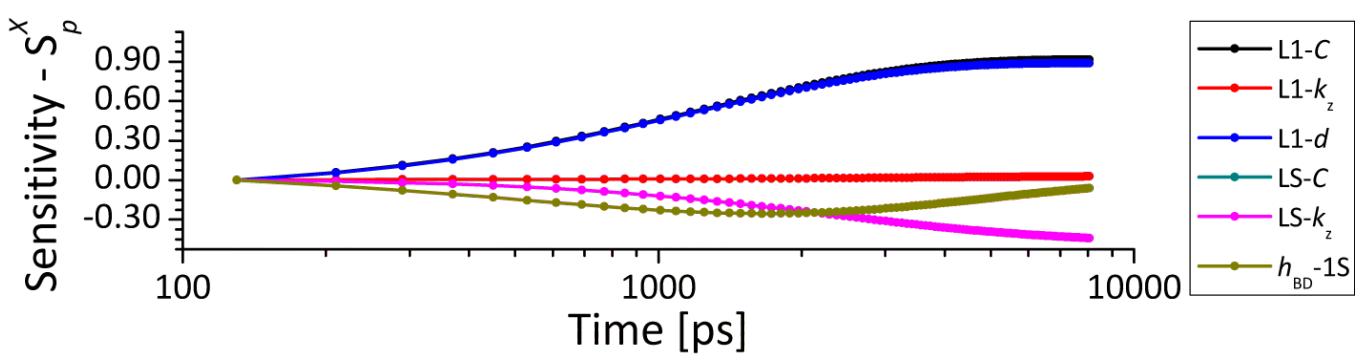

Figure S.59: Sensitivity of the real signal, $X$, as a function of time for the thermal parameters in the 100 $\mathrm{nm} \mathrm{Al} / \mathrm{Ge}$ system for $h_{\mathrm{BD}}=278.00 \mathrm{MW} / \mathrm{m}^{2} \mathrm{~K}$. 


\section{S.3.57 100 nm Al on Ge Substrate - 359.00 MW Series}

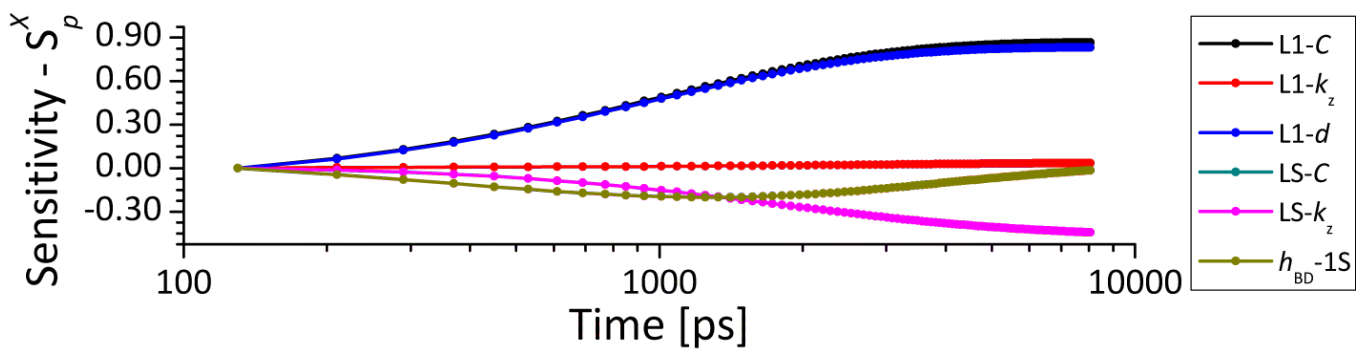

Figure S.60: Sensitivity of the real signal, $X$, as a function of time for the thermal parameters in the 100 $\mathrm{nm} \mathrm{Al} / \mathrm{Ge}$ system for $h_{\mathrm{BD}}=359.00 \mathrm{MW} / \mathrm{m}^{2} \mathrm{~K}$.

\section{S.3.58 $100 \mathrm{~nm} \mathrm{Al} \mathrm{on} \mathrm{Ge} \mathrm{Substrate} \mathrm{-} \mathrm{464.00} \mathrm{MW} \mathrm{Series}$}

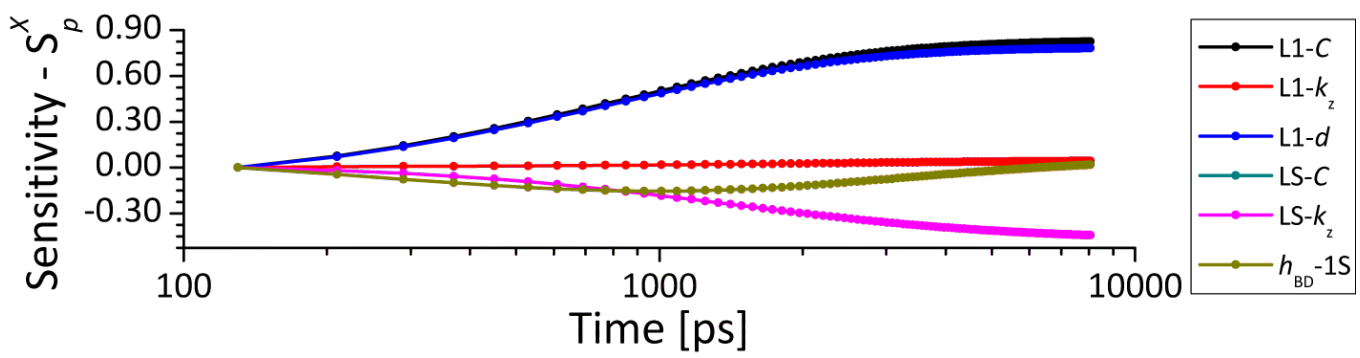

Figure S.61: Sensitivity of the real signal, $X$, as a function of time for the thermal parameters in the 100 $\mathrm{nm} \mathrm{Al} / \mathrm{Ge}$ system for $h_{\mathrm{BD}}=464.00 \mathrm{MW} / \mathrm{m}^{2} \mathrm{~K}$.

\section{S.3.59 $100 \mathrm{~nm} \mathrm{Al} \mathrm{on} \mathrm{Ge} \mathrm{Substrate} \mathrm{-} \mathrm{599.00} \mathrm{MW} \mathrm{Series}$}

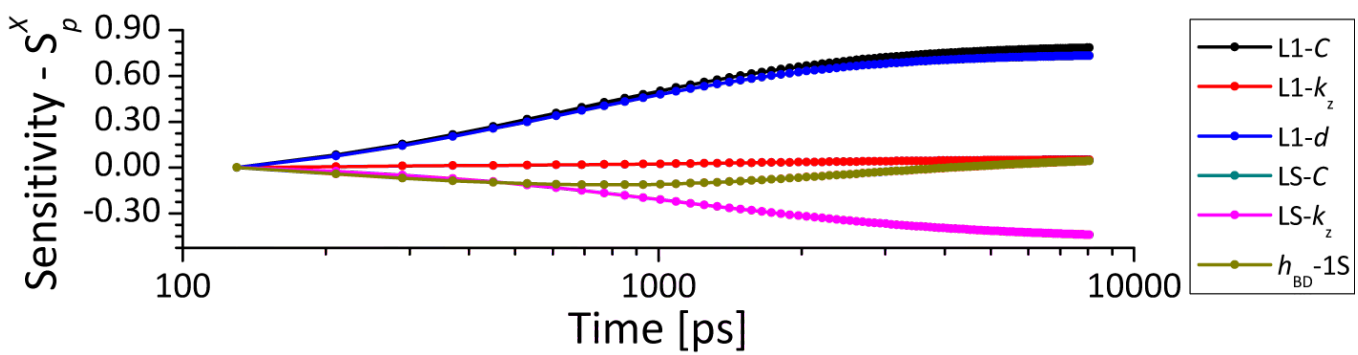

Figure S.62: Sensitivity of the real signal, $X$, as a function of time for the thermal parameters in the 100 $\mathrm{nm} \mathrm{Al} / \mathrm{Ge}$ system for $h_{\mathrm{BD}}=599.00 \mathrm{MW} / \mathrm{m}^{2} \mathrm{~K}$. 


\section{S.3.60 $100 \mathrm{~nm} \mathrm{Al} \mathrm{on} \mathrm{Ge} \mathrm{Substrate} \mathrm{-} \mathrm{774.00} \mathrm{MW} \mathrm{Series}$}

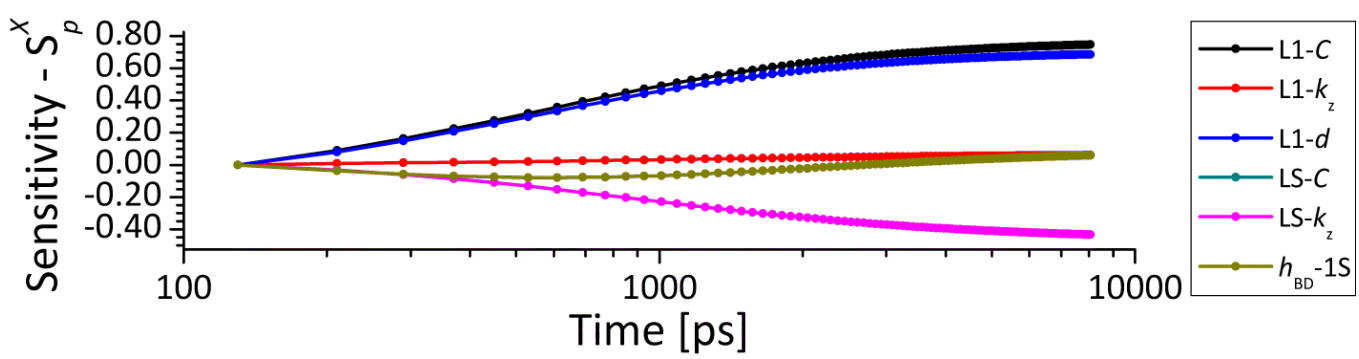

Figure S.63: Sensitivity of the real signal, $X$, as a function of time for the thermal parameters in the 100 $\mathrm{nm} \mathrm{Al} / \mathrm{Ge}$ system for $h_{\mathrm{BD}}=774.00 \mathrm{MW} / \mathrm{m}^{2} \mathrm{~K}$.

\section{S.3.61 100 nm Al on Ge Substrate - 1000.00 MW Series}

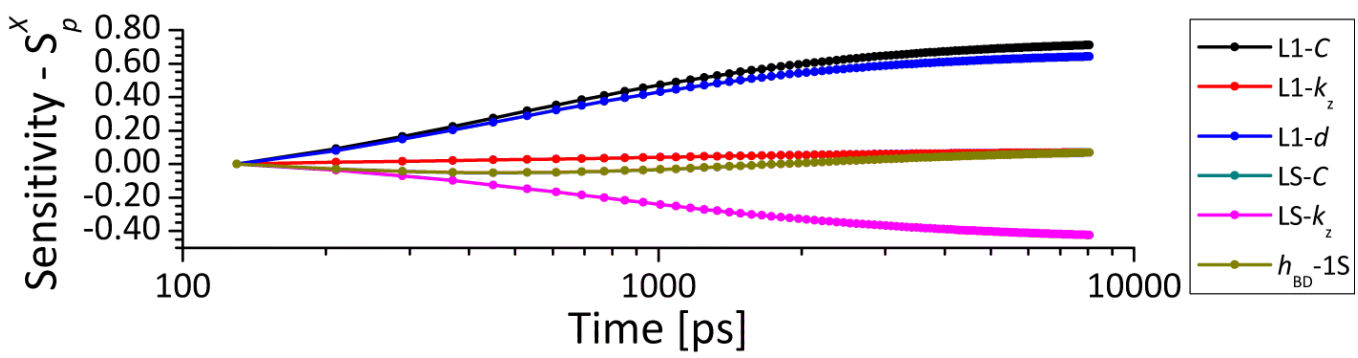

Figure S.64: Sensitivity of the real signal, $X$, as a function of time for the thermal parameters in the 100 $\mathrm{nm} \mathrm{Al} / \mathrm{Ge}$ system for $h_{\mathrm{BD}}=1000.00 \mathrm{MW} / \mathrm{m}^{2} \mathrm{~K}$. 


\section{S.4 Simulation Summary - Al/Si 1.00 MW Series}

\section{S.4.1 Fitting Film-to-Substrate Boundary Conductance $-h_{\mathrm{BD}}$}

Table S.4: Summary of simulation data - $100 \mathrm{~nm} \mathrm{Al/Si} \mathrm{1.00} \mathrm{MW} \mathrm{Series.} \mathrm{Error} \mathrm{bounds} \mathrm{represent} \mathrm{a} 95 \%$ confidence level.

\begin{tabular}{lccccc}
\hline \multicolumn{5}{c}{$\mathbf{1 0 0} \mathbf{~ n m ~ A l ~ o n ~} \mathbf{~ i}, h_{\mathrm{BD}}=\mathbf{1 . 0 0} \mathbf{~ M W} / \mathbf{m}^{2} \mathbf{K}$} \\
\hline $\mathbf{S N P}$ & $\mu-\left[\mathbf{M W} / \mathbf{m}^{2} \mathbf{K}\right]$ & $\sigma-\left[\mathbf{M W} / \mathbf{m}^{2} \mathbf{K}\right]$ & $\mathbf{S N P}$ & $\mu-\left[\mathbf{M W} / \mathbf{m}^{2} \mathbf{K}\right]$ & $\sigma-\left[\mathbf{M W} / \mathbf{m}^{2} \mathbf{K}\right]$ \\
10.62 & $1.12 \pm 0.054$ & $0.86 \pm 0.0024$ & 68.03 & $1.00 \pm 0.010$ & $0.15 \pm 0.0004$ \\
15.68 & $1.00 \pm 0.039$ & $0.64 \pm 0.0018$ & 72.35 & $1.01 \pm 0.009$ & $0.15 \pm 0.0004$ \\
21.05 & $1.04 \pm 0.030$ & $0.49 \pm 0.0014$ & 79.11 & $1.01 \pm 0.008$ & $0.14 \pm 0.0004$ \\
26.12 & $1.00 \pm 0.025$ & $0.40 \pm 0.0011$ & 82.15 & $1.01 \pm 0.008$ & $0.13 \pm 0.0004$ \\
31.80 & $1.01 \pm 0.021$ & $0.34 \pm 0.0009$ & 87.53 & $1.01 \pm 0.008$ & $0.12 \pm 0.0003$ \\
36.43 & $1.01 \pm 0.019$ & $0.31 \pm 0.0009$ & 91.73 & $1.01 \pm 0.007$ & $0.12 \pm 0.0003$ \\
41.94 & $1.01 \pm 0.016$ & $0.26 \pm 0.0007$ & 97.99 & $1.01 \pm 0.007$ & $0.11 \pm 0.0003$ \\
47.27 & $1.01 \pm 0.014$ & $0.22 \pm 0.0006$ & 102.50 & $1.01 \pm 0.007$ & $0.11 \pm 0.0003$ \\
51.68 & $1.00 \pm 0.013$ & $0.21 \pm 0.0006$ & 107.79 & $1.01 \pm 0.006$ & $0.10 \pm 0.0003$ \\
57.16 & $1.01 \pm 0.012$ & $0.19 \pm 0.0005$ & 113.53 & $1.01 \pm 0.006$ & $0.09 \pm 0.0003$ \\
62.34 & $1.01 \pm 0.011$ & $0.17 \pm 0.0005$ & & & \\
\hline
\end{tabular}

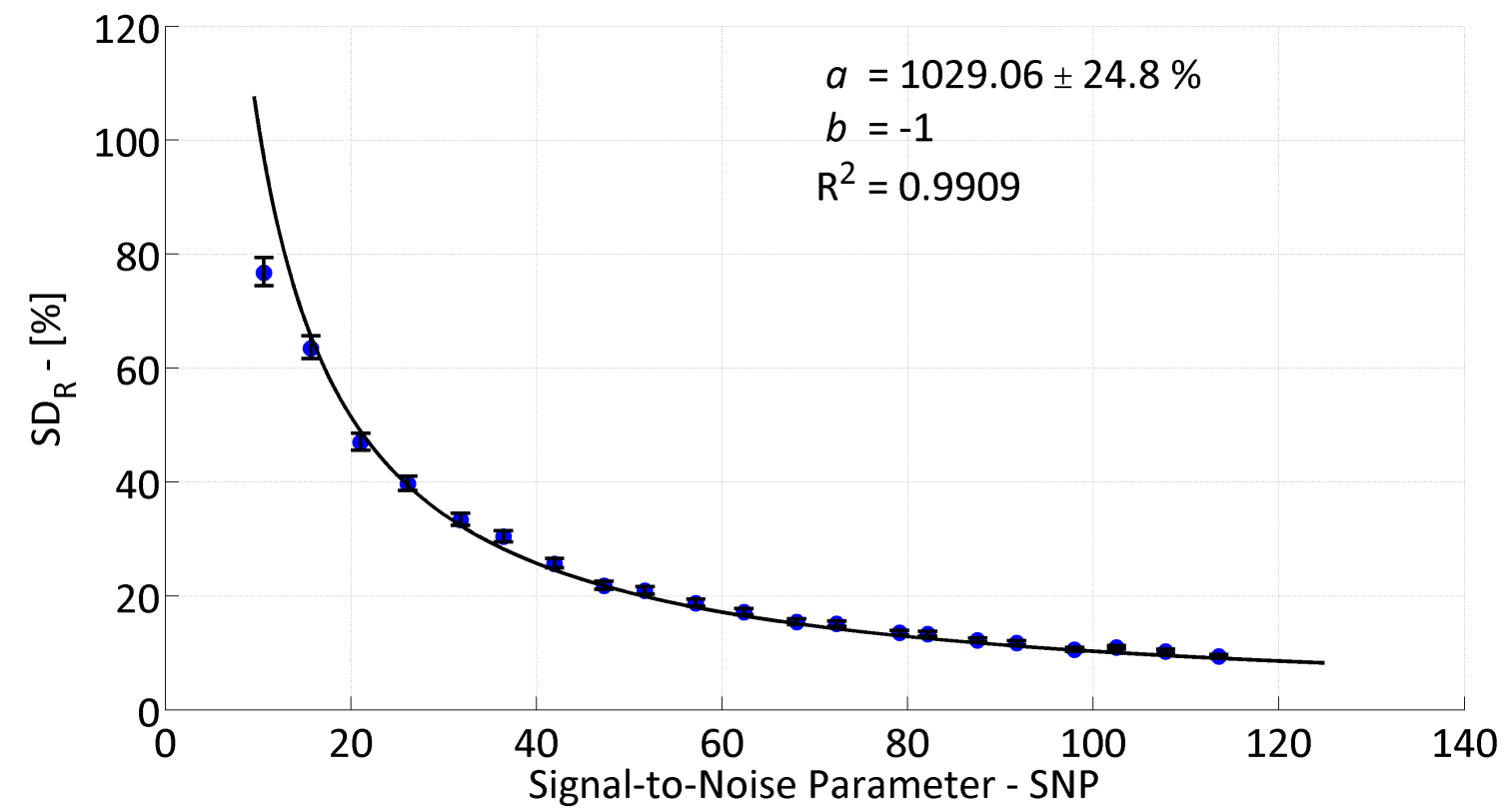

Figure S.65: Relative population standard deviation as a function of signal-to-noise parameter for 100 $\mathrm{nm}$ of $\mathrm{Al} / \mathrm{Si}$ with $h_{\mathrm{BD}}=1.00 \mathrm{MW} / \mathrm{m}^{2} \mathrm{~K}$. Error bars represent a $95 \%$ confidence interval of the measured $\mathrm{SD}_{\mathrm{R}}$ and the solid line represents a best fit of a power law to the data, of the form $\mathrm{SD}_{\mathrm{R}}=a \times \mathrm{SNP}^{b}$, with parameter $a$ provided for a fixed $b=-1$, along with the $95 \%$ confidence level in $a$. 


\section{S.5 Simulation Summary - Al/Si 1.29 MW Series}

\section{S.5.1 Fitting Film-to-Substrate Boundary Conductance $-h_{\mathrm{BD}}$}

Table S.5: Summary of simulation data - $100 \mathrm{~nm} \mathrm{Al/Si} \mathrm{1.29} \mathrm{MW} \mathrm{Series.} \mathrm{Error} \mathrm{bounds} \mathrm{represent} \mathrm{a} 95 \%$ confidence level.

\begin{tabular}{lccccc}
\hline \multicolumn{5}{c}{$\mathbf{1 0 0} \mathbf{~ n m ~ A l ~ o n ~} \mathbf{~ i}, h_{\mathrm{BD}}=\mathbf{1 . 2 9} \mathbf{~ M W} / \mathbf{m}^{2} \mathbf{K}$} \\
\hline $\mathbf{S N P}$ & $\mu-\left[\mathbf{M W} / \mathbf{m}^{2} \mathbf{K}\right]$ & $\sigma-\left[\mathbf{M W} / \mathbf{m}^{2} \mathbf{K}\right]$ & $\mathbf{S N P}$ & $\mu-\left[\mathbf{M W} / \mathbf{m}^{2} \mathbf{K}\right]$ & $\sigma-\left[\mathbf{M W} / \mathbf{m}^{2} \mathbf{K}\right]$ \\
10.53 & $1.33 \pm 0.059$ & $0.96 \pm 0.0027$ & 66.42 & $1.30 \pm 0.010$ & $0.16 \pm 0.0005$ \\
15.78 & $1.32 \pm 0.042$ & $0.68 \pm 0.0019$ & 71.41 & $1.30 \pm 0.010$ & $0.16 \pm 0.0004$ \\
20.87 & $1.28 \pm 0.032$ & $0.52 \pm 0.0014$ & 76.28 & $1.29 \pm 0.009$ & $0.15 \pm 0.0004$ \\
25.72 & $1.31 \pm 0.026$ & $0.42 \pm 0.0012$ & 82.11 & $1.30 \pm 0.008$ & $0.13 \pm 0.0004$ \\
31.59 & $1.31 \pm 0.021$ & $0.34 \pm 0.0010$ & 87.35 & $1.30 \pm 0.008$ & $0.12 \pm 0.0003$ \\
37.02 & $1.31 \pm 0.019$ & $0.30 \pm 0.0008$ & 92.39 & $1.30 \pm 0.007$ & $0.12 \pm 0.0003$ \\
40.90 & $1.30 \pm 0.016$ & $0.26 \pm 0.0007$ & 99.33 & $1.29 \pm 0.007$ & $0.11 \pm 0.0003$ \\
46.62 & $1.30 \pm 0.014$ & $0.23 \pm 0.0006$ & 101.86 & $1.30 \pm 0.007$ & $0.11 \pm 0.0003$ \\
52.30 & $1.28 \pm 0.013$ & $0.21 \pm 0.0006$ & 106.82 & $1.30 \pm 0.006$ & $0.10 \pm 0.0003$ \\
56.85 & $1.29 \pm 0.011$ & $0.18 \pm 0.0005$ & 112.35 & $1.30 \pm 0.006$ & $0.10 \pm 0.0003$ \\
62.40 & $1.30 \pm 0.011$ & $0.17 \pm 0.0005$ & & & \\
\hline
\end{tabular}

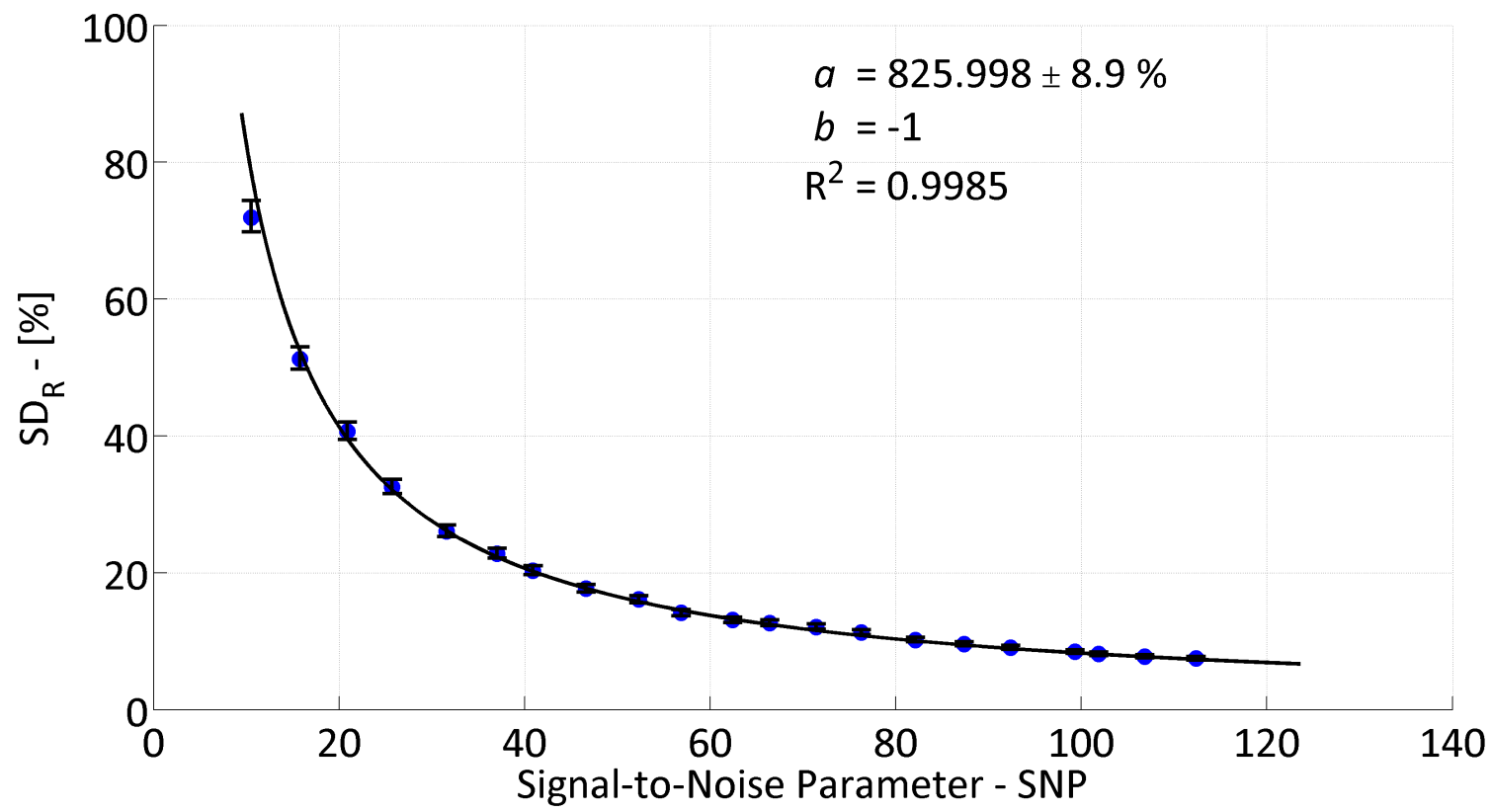

Figure S.66: Relative population standard deviation as a function of signal-to-noise parameter for 100 $\mathrm{nm}$ of $\mathrm{Al} / \mathrm{Si}$ with $h_{\mathrm{BD}}=1.29 \mathrm{MW} / \mathrm{m}^{2} \mathrm{~K}$. Error bars represent a $95 \%$ confidence interval of the measured $\mathrm{SD}_{\mathrm{R}}$ and the solid line represents a best fit of a power law to the data, of the form $\mathrm{SD}_{\mathrm{R}}=a \times \mathrm{SNP}^{b}$, with parameter $a$ provided for a fixed $b=-1$, along with the $95 \%$ confidence level in $a$. 


\section{S.6 Simulation Summary - Al/Si 1.67 MW Series}

\section{S.6.1 Fitting Film-to-Substrate Boundary Conductance $-h_{\mathrm{BD}}$}

Table S.6: Summary of simulation data - $100 \mathrm{~nm} \mathrm{Al/Si} 1.67 \mathrm{MW}$ Series. Error bounds represent a $95 \%$ confidence level.

\begin{tabular}{lccccc}
\hline \multicolumn{5}{c}{$\mathbf{1 0 0} \mathbf{~ n m ~ A l ~ o n ~} \mathbf{~ i ,} h_{\mathrm{BD}}=\mathbf{1 . 6 7} \mathbf{~} \mathbf{M W} / \mathbf{m}^{2} \mathbf{K}$} \\
\hline $\mathbf{S N P}$ & $\mu-\left[\mathbf{M W} / \mathbf{m}^{2} \mathbf{K}\right]$ & $\sigma-\left[\mathbf{M W} / \mathbf{m}^{2} \mathbf{K}\right]$ & $\mathbf{S N P}$ & $\mu-\left[\mathbf{M W} / \mathbf{m}^{2} \mathbf{K}\right]$ & $\sigma-\left[\mathbf{M W} / \mathbf{m}^{2} \mathbf{K}\right]$ \\
10.39 & $1.71 \pm 0.062$ & $1.00 \pm 0.0028$ & 66.01 & $1.68 \pm 0.011$ & $0.17 \pm 0.0005$ \\
15.52 & $1.70 \pm 0.042$ & $0.67 \pm 0.0019$ & 71.79 & $1.68 \pm 0.009$ & $0.15 \pm 0.0004$ \\
20.64 & $1.69 \pm 0.033$ & $0.53 \pm 0.0015$ & 76.72 & $1.68 \pm 0.008$ & $0.13 \pm 0.0004$ \\
25.78 & $1.65 \pm 0.025$ & $0.41 \pm 0.0011$ & 81.05 & $1.68 \pm 0.009$ & $0.14 \pm 0.0004$ \\
31.10 & $1.70 \pm 0.022$ & $0.35 \pm 0.0010$ & 85.89 & $1.68 \pm 0.007$ & $0.12 \pm 0.0003$ \\
35.75 & $1.67 \pm 0.019$ & $0.31 \pm 0.0009$ & 91.93 & $1.67 \pm 0.007$ & $0.12 \pm 0.0003$ \\
40.75 & $1.67 \pm 0.017$ & $0.27 \pm 0.0007$ & 95.38 & $1.68 \pm 0.007$ & $0.11 \pm 0.0003$ \\
46.44 & $1.69 \pm 0.015$ & $0.24 \pm 0.0007$ & 101.67 & $1.68 \pm 0.007$ & $0.11 \pm 0.0003$ \\
51.19 & $1.68 \pm 0.014$ & $0.22 \pm 0.0006$ & 105.33 & $1.68 \pm 0.006$ & $0.10 \pm 0.0003$ \\
55.72 & $1.67 \pm 0.012$ & $0.19 \pm 0.0005$ & 111.28 & $1.68 \pm 0.006$ & $0.10 \pm 0.0003$ \\
60.42 & $1.68 \pm 0.011$ & $0.19 \pm 0.0005$ & & & \\
\hline
\end{tabular}

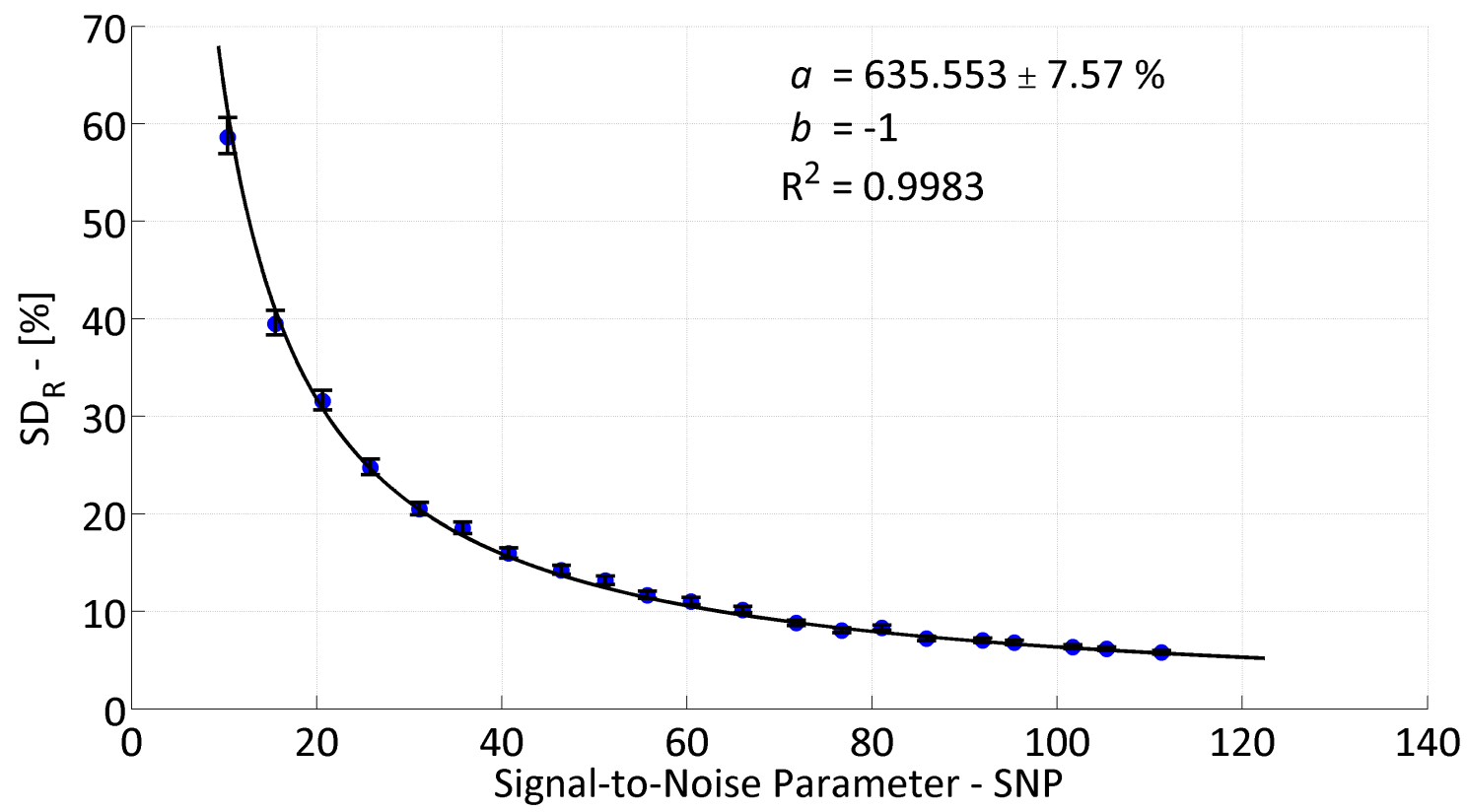

Figure S.67: Relative population standard deviation as a function of signal-to-noise parameter for 100 $\mathrm{nm}$ of $\mathrm{Al} / \mathrm{Si}$ with $h_{\mathrm{BD}}=1.67 \mathrm{MW} / \mathrm{m}^{2} \mathrm{~K}$. Error bars represent a $95 \%$ confidence interval of the measured $\mathrm{SD}_{\mathrm{R}}$ and the solid line represents a best fit of a power law to the data, of the form $\mathrm{SD}_{\mathrm{R}}=a \times \mathrm{SNP}^{b}$, with parameter $a$ provided for a fixed $b=-1$, along with the $95 \%$ confidence level in $a$. 


\section{S.7 Simulation Summary - Al/Si 2.15 MW Series}

\section{S.7.1 Fitting Film-to-Substrate Boundary Conductance $-h_{\mathrm{BD}}$}

Table S.7: Summary of simulation data - $100 \mathrm{~nm} \mathrm{Al/Si} \mathrm{2.15} \mathrm{MW} \mathrm{Series.} \mathrm{Error} \mathrm{bounds} \mathrm{represent} \mathrm{a} 95 \%$ confidence level.

\begin{tabular}{cccccc}
\hline \multicolumn{5}{c}{$\mathbf{1 0 0} \mathbf{~ n m ~ A l ~ o n ~} \mathbf{~ i}, h_{\mathrm{BD}}=\mathbf{2 . 1 5} \mathbf{~ M W} / \mathbf{m}^{2} \mathbf{K}$} \\
\hline $\mathbf{S N P}$ & $\mu-\left[\mathbf{M W} / \mathbf{m}^{2} \mathbf{K}\right]$ & $\sigma-\left[\mathbf{M W} / \mathbf{m}^{2} \mathbf{K}\right]$ & $\mathbf{S N P}$ & $\mu-\left[\mathbf{M W} / \mathbf{m}^{2} \mathbf{K}\right]$ & $\sigma-\left[\mathbf{M W} / \mathbf{m}^{2} \mathbf{K}\right]$ \\
10.39 & $2.16 \pm 0.064$ & $1.04 \pm 0.0029$ & 65.77 & $2.15 \pm 0.010$ & $0.16 \pm 0.0005$ \\
15.43 & $2.18 \pm 0.043$ & $0.69 \pm 0.0019$ & 71.37 & $2.16 \pm 0.009$ & $0.15 \pm 0.0004$ \\
20.51 & $2.16 \pm 0.033$ & $0.53 \pm 0.0015$ & 76.64 & $2.16 \pm 0.008$ & $0.13 \pm 0.0004$ \\
26.04 & $2.17 \pm 0.025$ & $0.41 \pm 0.0011$ & 80.89 & $2.16 \pm 0.009$ & $0.14 \pm 0.0004$ \\
30.75 & $2.19 \pm 0.021$ & $0.34 \pm 0.0009$ & 85.57 & $2.16 \pm 0.008$ & $0.13 \pm 0.0004$ \\
36.29 & $2.16 \pm 0.018$ & $0.29 \pm 0.0008$ & 90.73 & $2.16 \pm 0.007$ & $0.12 \pm 0.0003$ \\
40.05 & $2.16 \pm 0.017$ & $0.28 \pm 0.0008$ & 95.11 & $2.15 \pm 0.007$ & $0.12 \pm 0.0003$ \\
46.42 & $2.16 \pm 0.014$ & $0.23 \pm 0.0006$ & 101.64 & $2.16 \pm 0.007$ & $0.11 \pm 0.0003$ \\
51.49 & $2.15 \pm 0.013$ & $0.21 \pm 0.0006$ & 105.49 & $2.16 \pm 0.007$ & $0.11 \pm 0.0003$ \\
56.94 & $2.16 \pm 0.011$ & $0.19 \pm 0.0005$ & 110.34 & $2.16 \pm 0.006$ & $0.10 \pm 0.0003$ \\
60.98 & $2.16 \pm 0.011$ & $0.17 \pm 0.0005$ & & & \\
\hline
\end{tabular}

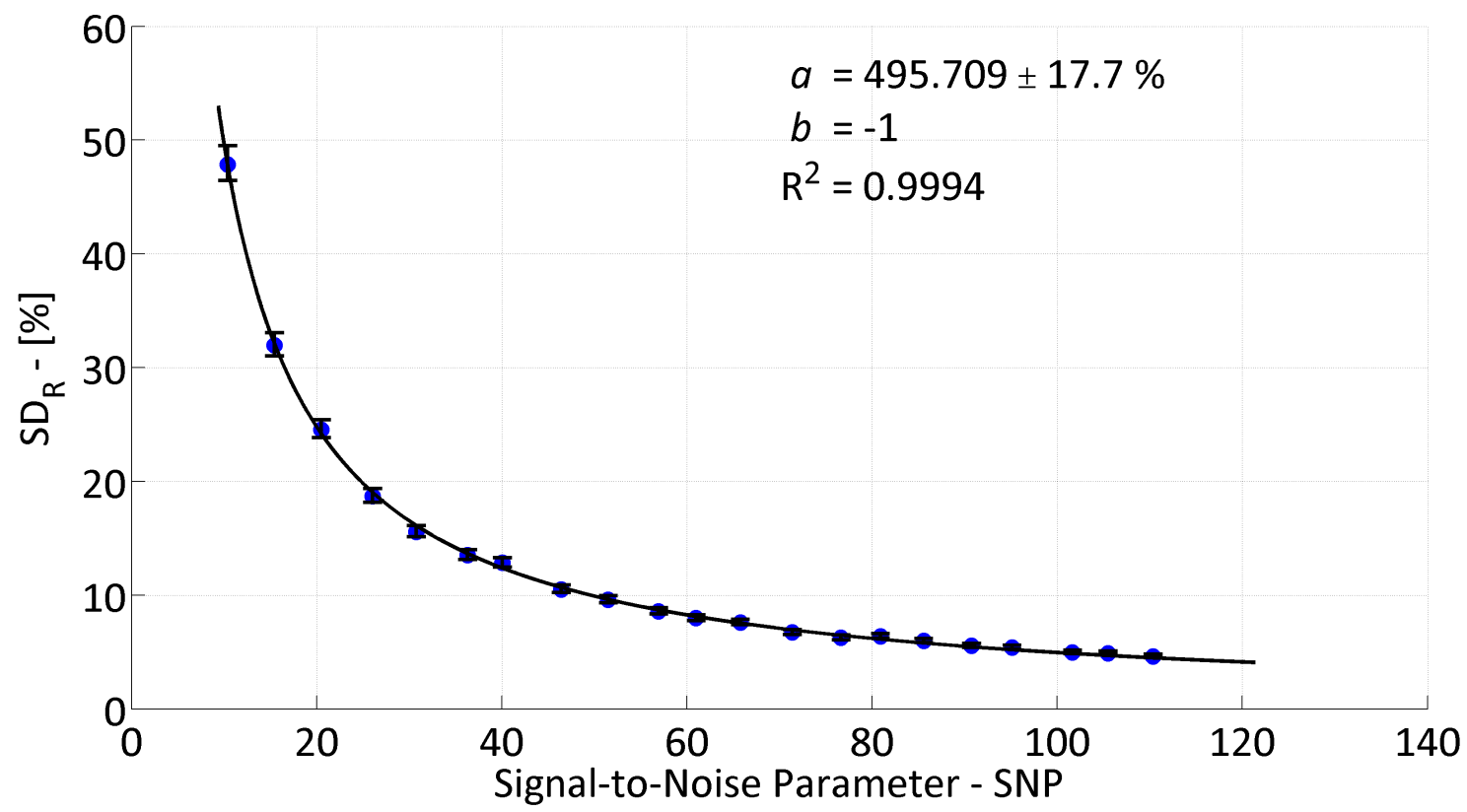

Figure S.68: Relative population standard deviation as a function of signal-to-noise parameter for 100 $\mathrm{nm}$ of $\mathrm{Al} / \mathrm{Si}$ with $h_{\mathrm{BD}}=2.15 \mathrm{MW} / \mathrm{m}^{2} \mathrm{~K}$. Error bars represent a $95 \%$ confidence interval of the measured $\mathrm{SD}_{\mathrm{R}}$ and the solid line represents a best fit of a power law to the data, of the form $\mathrm{SD}_{\mathrm{R}}=a \times \mathrm{SNP}^{b}$, with parameter $a$ provided for a fixed $b=-1$, along with the $95 \%$ confidence level in $a$. 


\section{S.7.2 Fitting Film Thickness $-d_{1}$}

Table S.8: Summary of simulation data - $100 \mathrm{~nm} \mathrm{Al/Si} \mathrm{2.15} \mathrm{MW} \mathrm{Series.} \mathrm{Error} \mathrm{bounds} \mathrm{represent} \mathrm{a} 95 \%$ confidence level.

\begin{tabular}{cccccc}
\hline \multicolumn{6}{c}{$\mathbf{1 0 0} \mathbf{n m} \mathbf{A l}$ on $\mathbf{~ S i}, h_{\mathrm{BD}}=\mathbf{2 . 1 5} \mathbf{~} \mathbf{M W} / \mathbf{m}^{2} \mathbf{K}$} \\
\hline $\mathbf{S N P}$ & $\mu-[\mathbf{n m}]$ & $\sigma-[\mathbf{n m}]$ & $\mathbf{S N P}$ & $\mu-[\mathbf{n m}]$ & $\sigma-[\mathbf{n m}]$ \\
10.39 & $120.88 \pm 3.744$ & $60.27 \pm 0.1682$ & 65.77 & $100.49 \pm 0.479$ & $7.71 \pm 0.0215$ \\
15.43 & $110.88 \pm 2.644$ & $42.57 \pm 0.1188$ & 71.37 & $100.06 \pm 0.420$ & $6.77 \pm 0.0189$ \\
20.51 & $106.17 \pm 1.901$ & $30.50 \pm 0.0857$ & 76.64 & $100.21 \pm 0.396$ & $6.38 \pm 0.0178$ \\
26.04 & $102.81 \pm 1.285$ & $20.66 \pm 0.0578$ & 80.89 & $100.10 \pm 0.398$ & $6.40 \pm 0.0179$ \\
30.75 & $100.74 \pm 1.016$ & $16.34 \pm 0.0457$ & 85.57 & $100.07 \pm 0.374$ & $6.02 \pm 0.0168$ \\
36.29 & $101.49 \pm 0.890$ & $14.33 \pm 0.0400$ & 90.73 & $100.03 \pm 0.348$ & $5.61 \pm 0.0156$ \\
40.05 & $100.99 \pm 0.806$ & $12.94 \pm 0.0363$ & 95.11 & $100.09 \pm 0.337$ & $5.42 \pm 0.0151$ \\
46.42 & $100.94 \pm 0.669$ & $10.78 \pm 0.0300$ & 101.64 & $99.99 \pm 0.310$ & $5.00 \pm 0.0139$ \\
51.49 & $100.99 \pm 0.618$ & $9.96 \pm 0.0277$ & 105.49 & $100.01 \pm 0.305$ & $4.92 \pm 0.0137$ \\
56.94 & $100.49 \pm 0.547$ & $8.81 \pm 0.0246$ & 110.34 & $99.92 \pm 0.288$ & $4.65 \pm 0.0129$ \\
60.98 & $100.07 \pm 0.503$ & $8.10 \pm 0.0226$ & & & \\
\hline
\end{tabular}

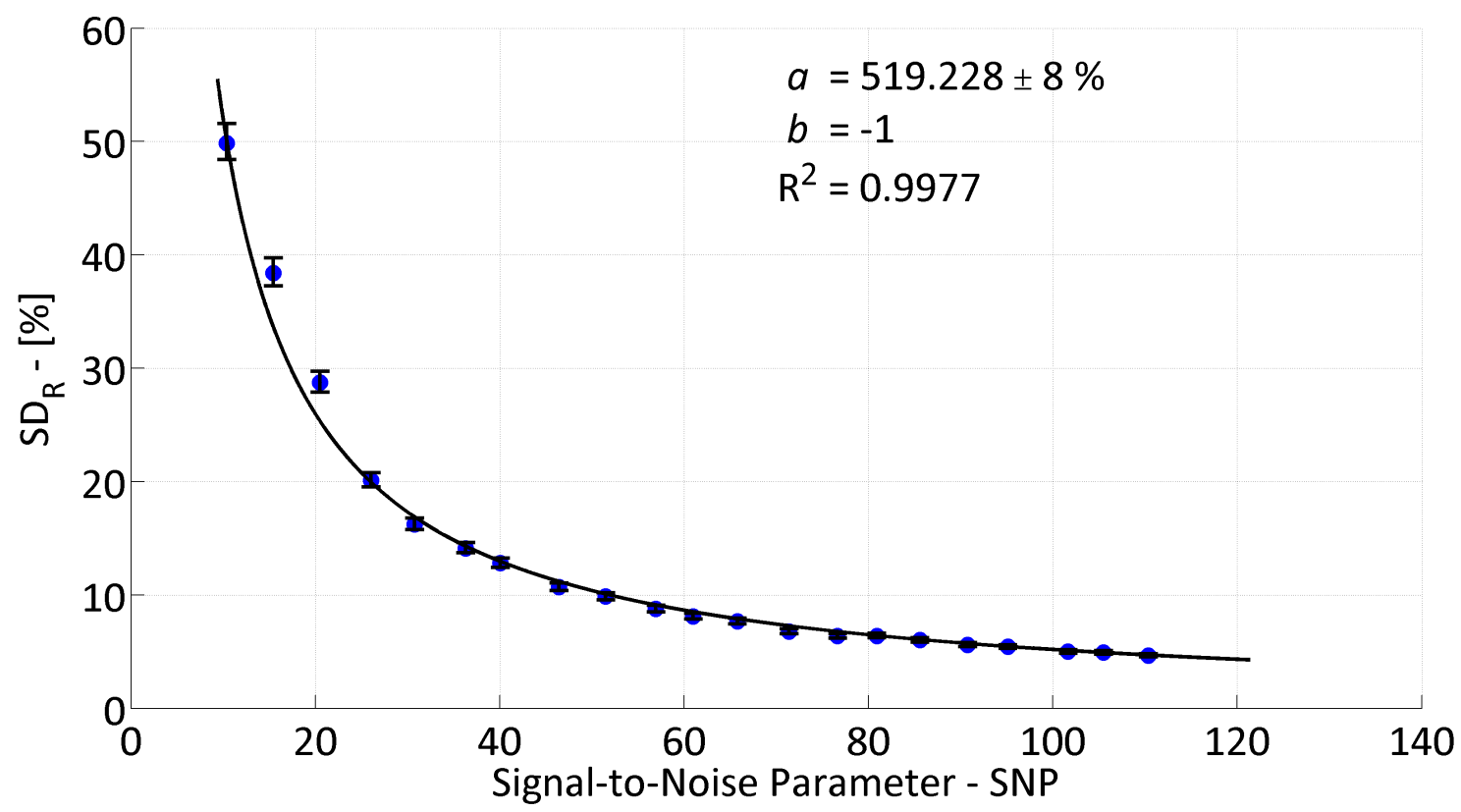

Figure S.69: Relative population standard deviation as a function of signal-to-noise parameter for 100 $\mathrm{nm}$ of $\mathrm{Al} / \mathrm{Si}$ with $h_{\mathrm{BD}}=2.15 \mathrm{MW} / \mathrm{m}^{2} \mathrm{~K}$. Error bars represent a $95 \%$ confidence interval of the measured $\mathrm{SD}_{\mathrm{R}}$ and the solid line represents a best fit of a power law to the data, of the form $\mathrm{SD}_{\mathrm{R}}=a \times \mathrm{SNP}^{b}$, with parameter $a$ provided for a fixed $b=-1$, along with the $95 \%$ confidence level in $a$. 


\section{S.8 Simulation Summary - Al/Si 2.78 MW Series}

\section{S.8.1 Fitting Film-to-Substrate Boundary Conductance $-h_{\mathrm{BD}}$}

Table S.9: Summary of simulation data - $100 \mathrm{~nm} \mathrm{Al/Si} 2.78 \mathrm{MW}$ Series. Error bounds represent a $95 \%$ confidence level.

\begin{tabular}{lccccc}
\hline \multicolumn{5}{c}{$\mathbf{1 0 0} \mathbf{~ n m ~ A l ~ o n ~} \mathbf{~ i}, h_{\mathrm{BD}}=\mathbf{2 . 7 8} \mathbf{~} \mathbf{M W} / \mathbf{m}^{2} \mathbf{K}$} \\
\hline $\mathbf{S N P}$ & $\mu-\left[\mathbf{M W} / \mathbf{m}^{2} \mathbf{K}\right]$ & $\sigma-\left[\mathbf{M W} / \mathbf{m}^{2} \mathbf{K}\right]$ & $\mathbf{S N P}$ & $\mu-\left[\mathbf{M W} / \mathbf{m}^{2} \mathbf{K}\right]$ & $\sigma-\left[\mathbf{M W} / \mathbf{m}^{2} \mathbf{K}\right]$ \\
10.27 & $2.80 \pm 0.064$ & $1.03 \pm 0.0029$ & 66.20 & $2.80 \pm 0.010$ & $0.16 \pm 0.0005$ \\
15.39 & $2.78 \pm 0.044$ & $0.71 \pm 0.0020$ & 70.29 & $2.79 \pm 0.010$ & $0.15 \pm 0.0004$ \\
20.52 & $2.80 \pm 0.035$ & $0.56 \pm 0.0016$ & 74.19 & $2.78 \pm 0.009$ & $0.15 \pm 0.0004$ \\
25.58 & $2.80 \pm 0.027$ & $0.44 \pm 0.0012$ & 80.56 & $2.78 \pm 0.009$ & $0.14 \pm 0.0004$ \\
30.86 & $2.79 \pm 0.022$ & $0.35 \pm 0.0010$ & 85.20 & $2.79 \pm 0.008$ & $0.13 \pm 0.0004$ \\
35.78 & $2.78 \pm 0.020$ & $0.32 \pm 0.0009$ & 89.31 & $2.79 \pm 0.008$ & $0.13 \pm 0.0004$ \\
40.09 & $2.80 \pm 0.017$ & $0.27 \pm 0.0008$ & 95.08 & $2.78 \pm 0.007$ & $0.11 \pm 0.0003$ \\
45.55 & $2.79 \pm 0.015$ & $0.24 \pm 0.0007$ & 100.68 & $2.79 \pm 0.007$ & $0.11 \pm 0.0003$ \\
50.51 & $2.80 \pm 0.013$ & $0.22 \pm 0.0006$ & 105.66 & $2.79 \pm 0.006$ & $0.10 \pm 0.0003$ \\
55.44 & $2.80 \pm 0.012$ & $0.20 \pm 0.0006$ & 110.15 & $2.79 \pm 0.006$ & $0.10 \pm 0.0003$ \\
60.47 & $2.79 \pm 0.011$ & $0.18 \pm 0.0005$ & & & \\
\hline
\end{tabular}

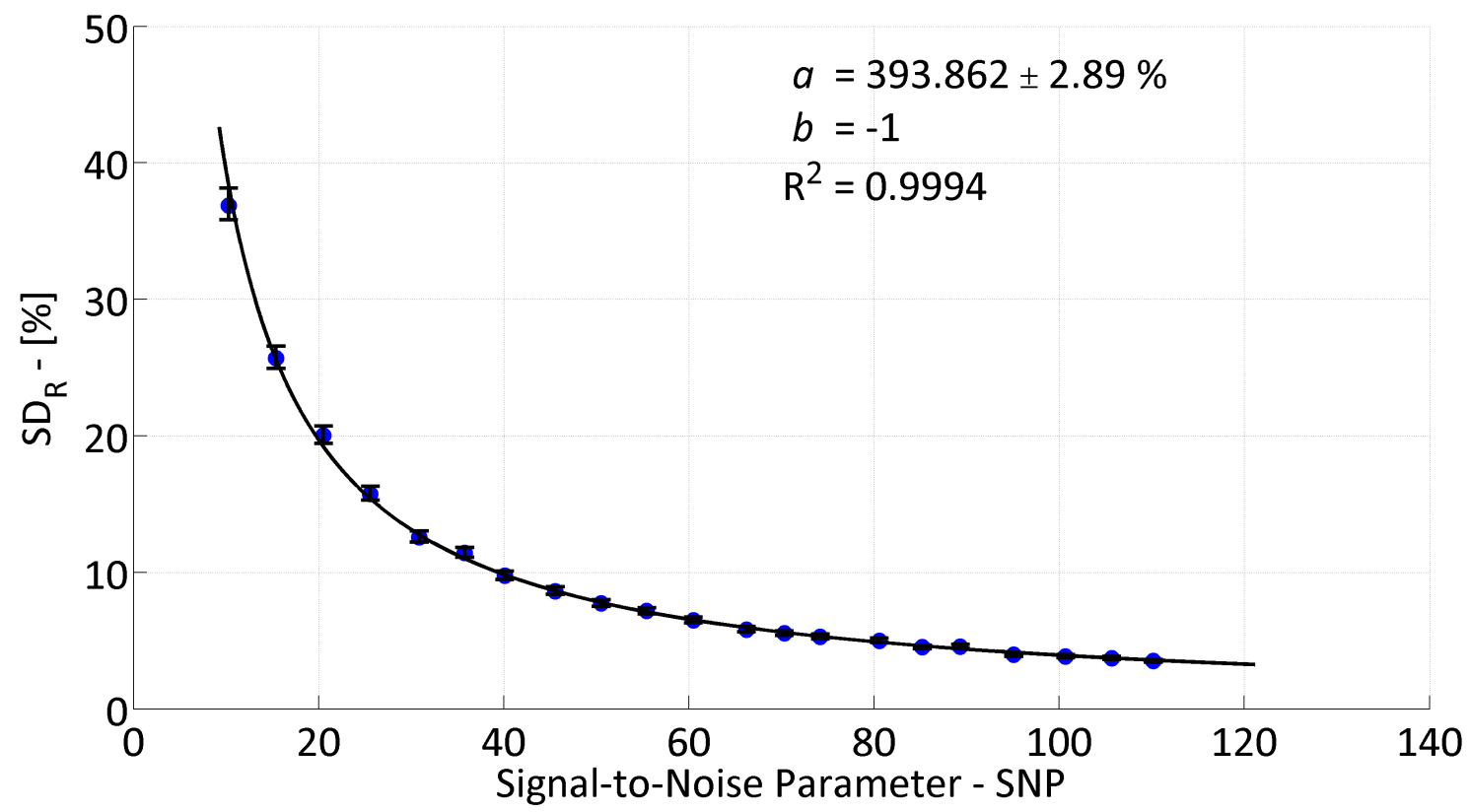

Figure S.70: Relative population standard deviation as a function of signal-to-noise parameter for 100 $\mathrm{nm}$ of $\mathrm{Al} / \mathrm{Si}$ with $h_{\mathrm{BD}}=2.78 \mathrm{MW} / \mathrm{m}^{2} \mathrm{~K}$. Error bars represent a $95 \%$ confidence interval of the measured $\mathrm{SD}_{\mathrm{R}}$ and the solid line represents a best fit of a power law to the data, of the form $\mathrm{SD}_{\mathrm{R}}=a \times \mathrm{SNP}^{b}$, with parameter $a$ provided for a fixed $b=-1$, along with the $95 \%$ confidence level in $a$. 


\section{S.9 Simulation Summary - Al/Si 3.59 MW Series}

\section{S.9.1 Fitting Film-to-Substrate Boundary Conductance $-h_{\mathrm{BD}}$}

Table S.10: Summary of simulation data - $100 \mathrm{~nm} \mathrm{Al/Si} \mathrm{3.59} \mathrm{MW} \mathrm{Series.} \mathrm{Error} \mathrm{bounds} \mathrm{represent} \mathrm{a} 95 \%$ confidence level.

\begin{tabular}{cccccc}
\hline \multicolumn{5}{c}{$\mathbf{1 0 0} \mathbf{~ n m ~} \mathbf{A l}$ on $\mathbf{~ S i}, h_{\mathrm{BD}}=\mathbf{3 . 5 9} \mathbf{~ M W} / \mathbf{m}^{2} \mathbf{K}$} \\
\hline $\mathbf{S N P}$ & $\mu-\left[\mathbf{M W} / \mathbf{m}^{2} \mathbf{K}\right]$ & $\sigma-\left[\mathbf{M W} / \mathbf{m}^{2} \mathbf{K}\right]$ & $\mathbf{S N P}$ & $\mu-\left[\mathbf{M W} / \mathbf{m}^{2} \mathbf{K}\right]$ & $\sigma-\left[\mathbf{M W} / \mathbf{m}^{2} \mathbf{K}\right]$ \\
10.04 & $3.59 \pm 0.069$ & $1.11 \pm 0.0031$ & 64.20 & $3.61 \pm 0.011$ & $0.17 \pm 0.0005$ \\
15.17 & $3.64 \pm 0.044$ & $0.70 \pm 0.0020$ & 70.39 & $3.60 \pm 0.010$ & $0.16 \pm 0.0004$ \\
20.31 & $3.59 \pm 0.035$ & $0.56 \pm 0.0016$ & 74.55 & $3.59 \pm 0.009$ & $0.15 \pm 0.0004$ \\
25.02 & $3.57 \pm 0.027$ & $0.44 \pm 0.0012$ & 78.64 & $3.60 \pm 0.009$ & $0.14 \pm 0.0004$ \\
29.64 & $3.61 \pm 0.022$ & $0.36 \pm 0.0010$ & 84.61 & $3.60 \pm 0.008$ & $0.13 \pm 0.0004$ \\
35.46 & $3.58 \pm 0.019$ & $0.31 \pm 0.0009$ & 89.04 & $3.60 \pm 0.008$ & $0.13 \pm 0.0004$ \\
40.37 & $3.59 \pm 0.017$ & $0.28 \pm 0.0008$ & 94.72 & $3.60 \pm 0.007$ & $0.12 \pm 0.0003$ \\
44.93 & $3.60 \pm 0.016$ & $0.25 \pm 0.0007$ & 99.48 & $3.60 \pm 0.007$ & $0.11 \pm 0.0003$ \\
49.54 & $3.60 \pm 0.014$ & $0.23 \pm 0.0006$ & 104.62 & $3.59 \pm 0.006$ & $0.10 \pm 0.0003$ \\
55.17 & $3.59 \pm 0.013$ & $0.21 \pm 0.0006$ & 108.83 & $3.60 \pm 0.006$ & $0.10 \pm 0.0003$ \\
60.15 & $3.60 \pm 0.012$ & $0.19 \pm 0.0005$ & & & \\
\hline
\end{tabular}

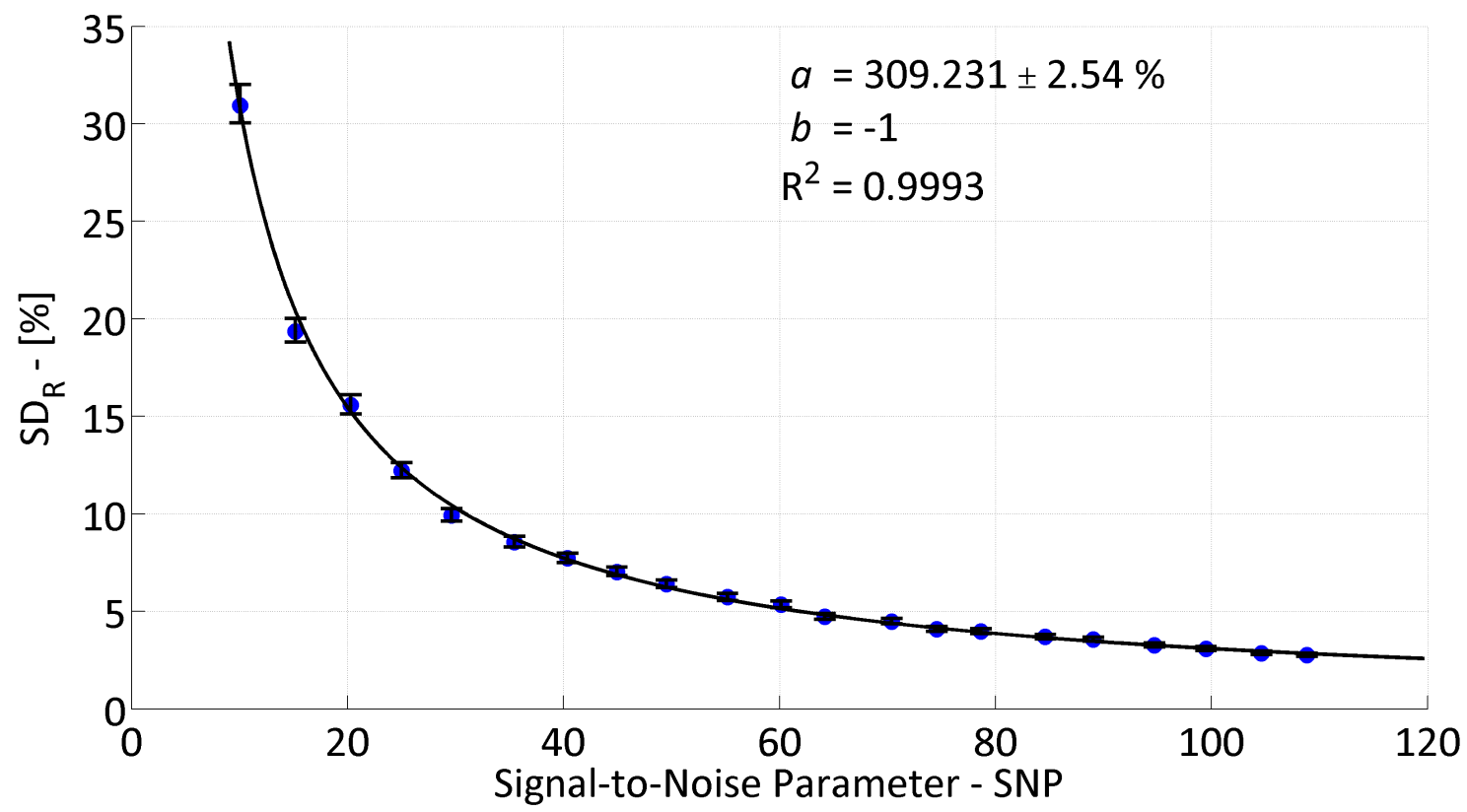

Figure S.71: Relative population standard deviation as a function of signal-to-noise parameter for 100 $\mathrm{nm}$ of $\mathrm{Al} / \mathrm{Si}$ with $h_{\mathrm{BD}}=3.59 \mathrm{MW} / \mathrm{m}^{2} \mathrm{~K}$. Error bars represent a $95 \%$ confidence interval of the measured $\mathrm{SD}_{\mathrm{R}}$ and the solid line represents a best fit of a power law to the data, of the form $\mathrm{SD}_{\mathrm{R}}=a \times \mathrm{SNP}^{b}$, with parameter $a$ provided for a fixed $b=-1$, along with the $95 \%$ confidence level in $a$. 


\section{S.10 Simulation Summary - Al/Si 4.64 MW Series}

\section{S.10.1 Fitting Film-to-Substrate Boundary Conductance - $h_{\mathrm{BD}}$}

Table S.11: Summary of simulation data - $100 \mathrm{~nm} \mathrm{Al/Si} 4.64 \mathrm{MW}$ Series. Error bounds represent a $95 \%$ confidence level.

\begin{tabular}{cccccc}
\hline \multicolumn{5}{c}{$\mathbf{1 0 0} \mathbf{~ n m ~} \mathbf{A l}$ on $\mathbf{~ S i}, h_{\mathrm{BD}}=\mathbf{4 . 6 4} \mathbf{~} \mathbf{W W} / \mathbf{m}^{2} \mathbf{K}$} \\
\hline $\mathbf{S N P}$ & $\mu-\left[\mathbf{M W} / \mathbf{m}^{2} \mathbf{K}\right]$ & $\sigma-\left[\mathbf{M W} / \mathbf{m}^{2} \mathbf{K}\right]$ & $\mathbf{S N P}$ & $\mu-\left[\mathbf{M W} / \mathbf{m}^{2} \mathbf{K}\right]$ & $\sigma-\left[\mathbf{M W} / \mathbf{m}^{2} \mathbf{K}\right]$ \\
9.81 & $4.68 \pm 0.074$ & $1.18 \pm 0.0033$ & 63.14 & $4.64 \pm 0.011$ & $0.18 \pm 0.0005$ \\
14.76 & $4.63 \pm 0.048$ & $0.77 \pm 0.0021$ & 69.21 & $4.65 \pm 0.010$ & $0.17 \pm 0.0005$ \\
19.90 & $4.64 \pm 0.034$ & $0.55 \pm 0.0015$ & 72.32 & $4.65 \pm 0.009$ & $0.15 \pm 0.0004$ \\
24.65 & $4.67 \pm 0.029$ & $0.47 \pm 0.0013$ & 78.87 & $4.65 \pm 0.009$ & $0.14 \pm 0.0004$ \\
29.42 & $4.66 \pm 0.024$ & $0.39 \pm 0.0011$ & 81.11 & $4.64 \pm 0.009$ & $0.14 \pm 0.0004$ \\
34.44 & $4.65 \pm 0.020$ & $0.32 \pm 0.0009$ & 87.43 & $4.65 \pm 0.008$ & $0.13 \pm 0.0004$ \\
38.88 & $4.65 \pm 0.018$ & $0.29 \pm 0.0008$ & 92.31 & $4.64 \pm 0.008$ & $0.13 \pm 0.0003$ \\
44.15 & $4.65 \pm 0.015$ & $0.25 \pm 0.0007$ & 97.66 & $4.65 \pm 0.007$ & $0.12 \pm 0.0003$ \\
49.42 & $4.65 \pm 0.014$ & $0.23 \pm 0.0006$ & 102.41 & $4.66 \pm 0.007$ & $0.11 \pm 0.0003$ \\
54.08 & $4.65 \pm 0.013$ & $0.21 \pm 0.0006$ & 106.17 & $4.65 \pm 0.007$ & $0.11 \pm 0.0003$ \\
58.60 & $4.65 \pm 0.012$ & $0.19 \pm 0.0005$ & & & \\
\hline
\end{tabular}

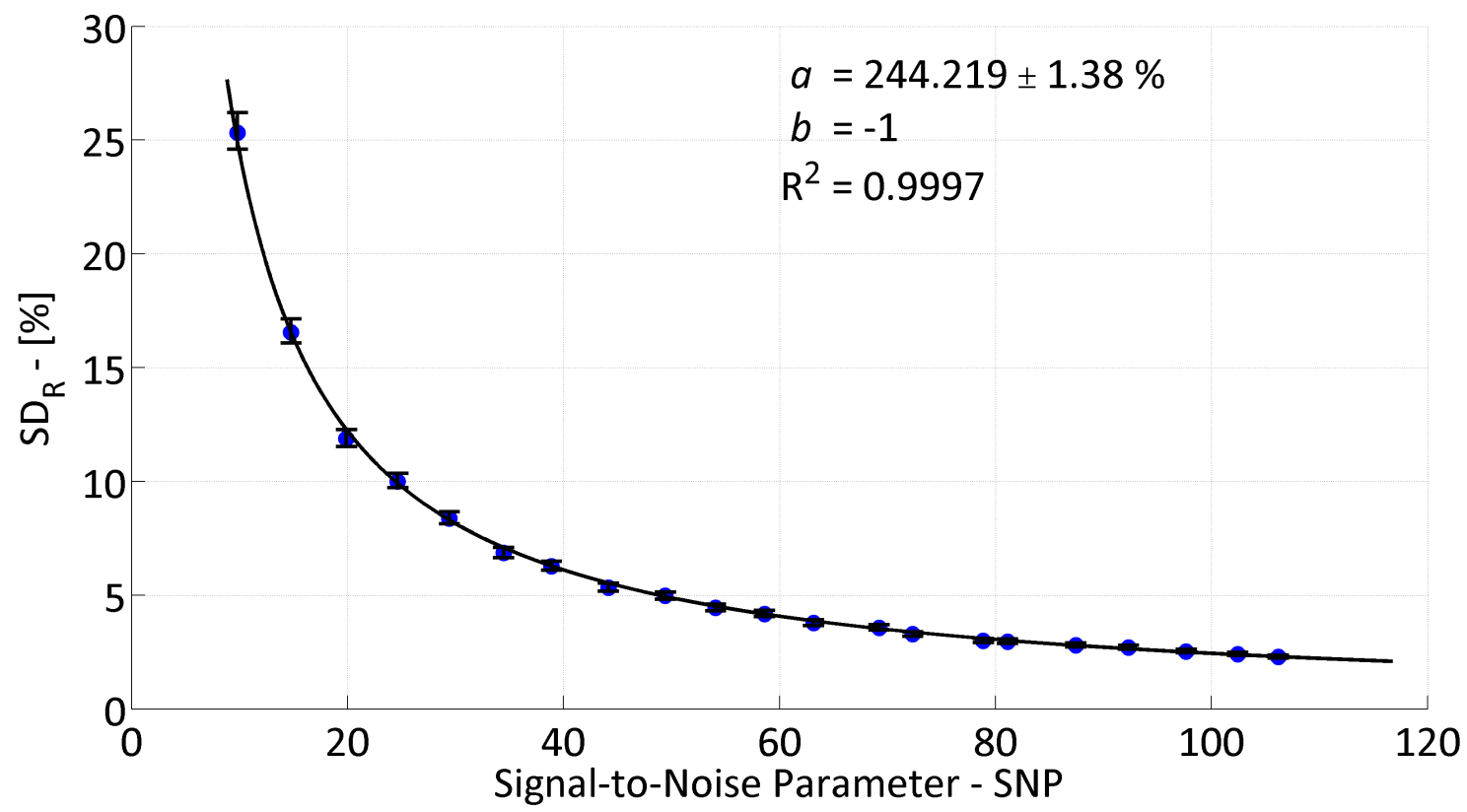

Figure S.72: Relative population standard deviation as a function of signal-to-noise parameter for 100 $\mathrm{nm}$ of $\mathrm{Al} / \mathrm{Si}$ with $h_{\mathrm{BD}}=4.64 \mathrm{MW} / \mathrm{m}^{2} \mathrm{~K}$. Error bars represent a $95 \%$ confidence interval of the measured $\mathrm{SD}_{\mathrm{R}}$ and the solid line represents a best fit of a power law to the data, of the form $\mathrm{SD}_{\mathrm{R}}=a \times \mathrm{SNP}^{b}$, with parameter $a$ provided for a fixed $b=-1$, along with the $95 \%$ confidence level in $a$. 


\section{S.10.2 Fitting Film Thickness $-d_{1}$}

Table S.12: Summary of simulation data - $100 \mathrm{~nm} \mathrm{Al} / \mathrm{Si} 4.64 \mathrm{MW}$ Series. Error bounds represent a $95 \%$ confidence level.

\begin{tabular}{cccccc}
\hline \multicolumn{6}{c}{$\mathbf{1 0 0} \mathbf{~ n m ~} \mathbf{A l}$ on $\mathbf{~ S i}, h_{\mathrm{BD}}=\mathbf{4 . 6 4} \mathbf{~} \mathbf{M W} / \mathbf{m}^{2} \mathbf{K}$} \\
\hline $\mathbf{S N P}$ & $\mu-[\mathbf{n m}]$ & $\sigma-[\mathbf{n m}]$ & $\mathbf{S N P}$ & $\mu-[\mathbf{n m}]$ & $\sigma-[\mathbf{n m}]$ \\
9.81 & $103.86 \pm 1.652$ & $26.32 \pm 0.0750$ & 63.14 & $100.09 \pm 0.231$ & $3.72 \pm 0.0103$ \\
14.76 & $102.94 \pm 1.095$ & $17.62 \pm 0.0493$ & 69.21 & $99.94 \pm 0.217$ & $3.50 \pm 0.0098$ \\
19.90 & $101.38 \pm 0.750$ & $12.07 \pm 0.0337$ & 72.32 & $99.87 \pm 0.200$ & $3.23 \pm 0.0090$ \\
24.65 & $100.45 \pm 0.629$ & $10.14 \pm 0.0283$ & 78.87 & $99.98 \pm 0.184$ & $2.96 \pm 0.0082$ \\
29.42 & $100.25 \pm 0.516$ & $8.31 \pm 0.0232$ & 81.11 & $100.06 \pm 0.182$ & $2.94 \pm 0.0082$ \\
34.44 & $100.28 \pm 0.423$ & $6.82 \pm 0.0190$ & 87.43 & $99.86 \pm 0.171$ & $2.75 \pm 0.0077$ \\
38.88 & $100.17 \pm 0.386$ & $6.22 \pm 0.0173$ & 92.31 & $99.98 \pm 0.165$ & $2.66 \pm 0.0074$ \\
44.15 & $100.02 \pm 0.329$ & $5.31 \pm 0.0148$ & 97.66 & $99.88 \pm 0.153$ & $2.47 \pm 0.0069$ \\
49.42 & $100.10 \pm 0.307$ & $4.94 \pm 0.0138$ & 102.41 & $99.74 \pm 0.146$ & $2.36 \pm 0.0066$ \\
54.08 & $100.03 \pm 0.272$ & $4.38 \pm 0.0122$ & 106.17 & $99.90 \pm 0.140$ & $2.25 \pm 0.0063$ \\
58.60 & $100.02 \pm 0.255$ & $4.12 \pm 0.0115$ & & & \\
\hline
\end{tabular}

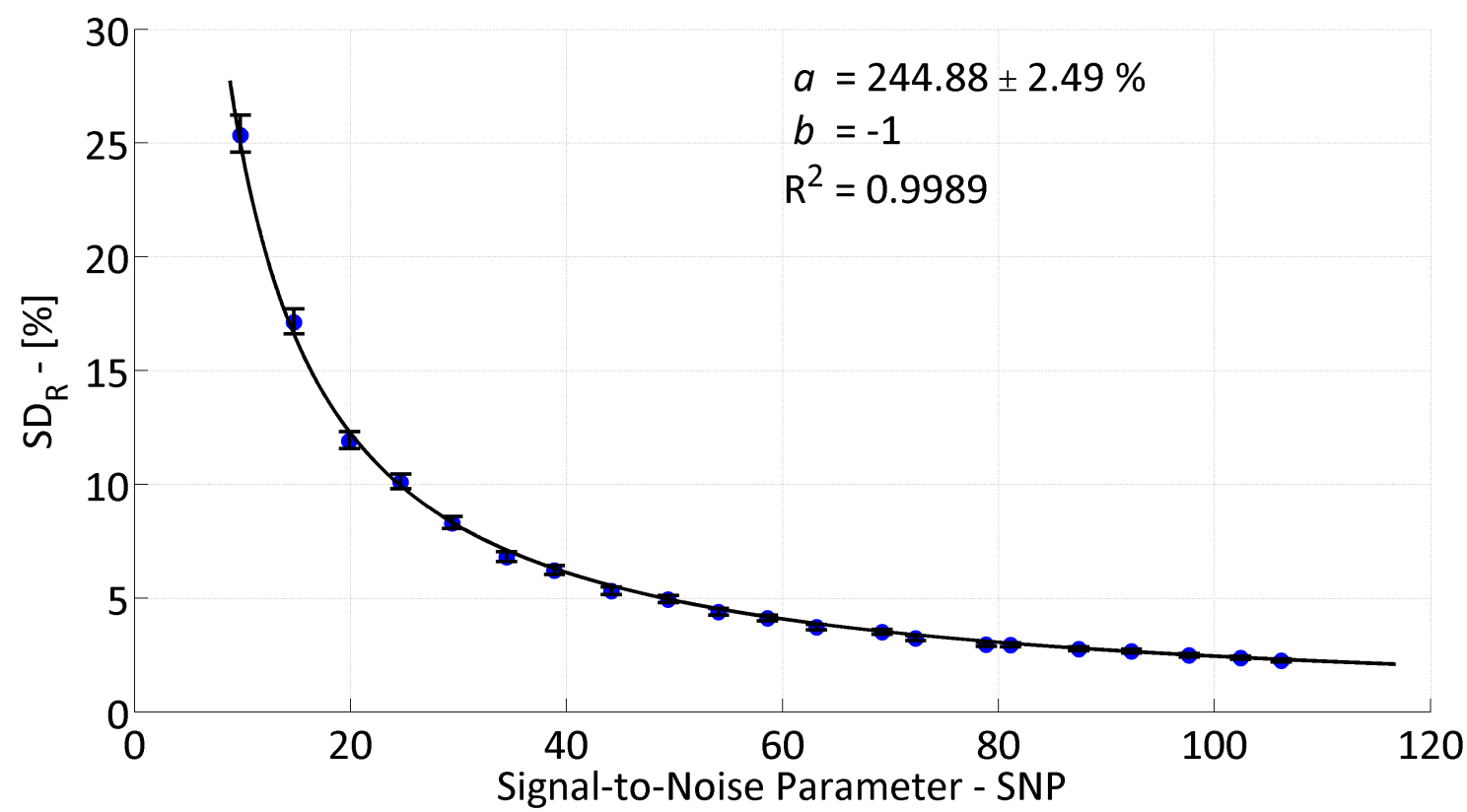

Figure S.73: Relative population standard deviation as a function of signal-to-noise parameter for 100 $\mathrm{nm}$ of $\mathrm{Al} / \mathrm{Si}$ with $h_{\mathrm{BD}}=4.64 \mathrm{MW} / \mathrm{m}^{2} \mathrm{~K}$. Error bars represent a $95 \%$ confidence interval of the measured $\mathrm{SD}_{\mathrm{R}}$ and the solid line represents a best fit of a power law to the data, of the form $\mathrm{SD}_{\mathrm{R}}=a \times \mathrm{SNP}^{b}$, with parameter $a$ provided for a fixed $b=-1$, along with the $95 \%$ confidence level in $a$. 


\section{S.11 Simulation Summary - Al/Si 5.99 MW Series}

\section{S.11.1 Fitting Film-to-Substrate Boundary Conductance - $h_{\mathrm{BD}}$}

Table S.13: Summary of simulation data - $100 \mathrm{~nm} \mathrm{Al/Si} \mathrm{5.99} \mathrm{MW} \mathrm{Series.} \mathrm{Error} \mathrm{bounds} \mathrm{represent} \mathrm{a} 95 \%$ confidence level.

\begin{tabular}{cccccc}
\hline \multicolumn{5}{c}{$\mathbf{1 0 0} \mathbf{~ n m ~} \mathbf{A l}$ on $\mathbf{~ S i}, h_{\mathrm{BD}}=\mathbf{5 . 9 9} \mathbf{~} \mathbf{M W} / \mathbf{m}^{2} \mathbf{K}$} \\
\hline $\mathbf{S N P}$ & $\mu-\left[\mathbf{M W} / \mathbf{m}^{2} \mathbf{K}\right]$ & $\sigma-\left[\mathbf{M W} / \mathbf{m}^{2} \mathbf{K}\right]$ & $\mathbf{S N P}$ & $\mu-\left[\mathbf{M W} / \mathbf{m}^{2} \mathbf{K}\right]$ & $\sigma-\left[\mathbf{M W} / \mathbf{m}^{2} \mathbf{K}\right]$ \\
9.79 & $5.99 \pm 0.071$ & $1.14 \pm 0.0032$ & 63.14 & $6.00 \pm 0.011$ & $0.18 \pm 0.0005$ \\
14.46 & $6.01 \pm 0.049$ & $0.79 \pm 0.0022$ & 66.18 & $6.00 \pm 0.010$ & $0.16 \pm 0.0005$ \\
19.43 & $5.99 \pm 0.036$ & $0.59 \pm 0.0016$ & 71.94 & $6.00 \pm 0.009$ & $0.15 \pm 0.0004$ \\
24.02 & $5.98 \pm 0.029$ & $0.47 \pm 0.0013$ & 75.72 & $6.00 \pm 0.009$ & $0.15 \pm 0.0004$ \\
28.97 & $5.98 \pm 0.024$ & $0.38 \pm 0.0011$ & 81.03 & $6.00 \pm 0.009$ & $0.14 \pm 0.0004$ \\
34.29 & $5.98 \pm 0.021$ & $0.34 \pm 0.0009$ & 85.16 & $6.00 \pm 0.008$ & $0.13 \pm 0.0004$ \\
37.97 & $6.00 \pm 0.019$ & $0.30 \pm 0.0008$ & 91.56 & $5.99 \pm 0.008$ & $0.13 \pm 0.0004$ \\
42.86 & $6.00 \pm 0.017$ & $0.27 \pm 0.0007$ & 92.91 & $6.00 \pm 0.007$ & $0.12 \pm 0.0003$ \\
48.29 & $6.00 \pm 0.015$ & $0.24 \pm 0.0007$ & 99.42 & $5.99 \pm 0.007$ & $0.11 \pm 0.0003$ \\
52.33 & $5.99 \pm 0.013$ & $0.22 \pm 0.0006$ & 105.03 & $6.00 \pm 0.007$ & $0.11 \pm 0.0003$ \\
56.90 & $6.00 \pm 0.013$ & $0.20 \pm 0.0006$ & & & \\
\hline
\end{tabular}

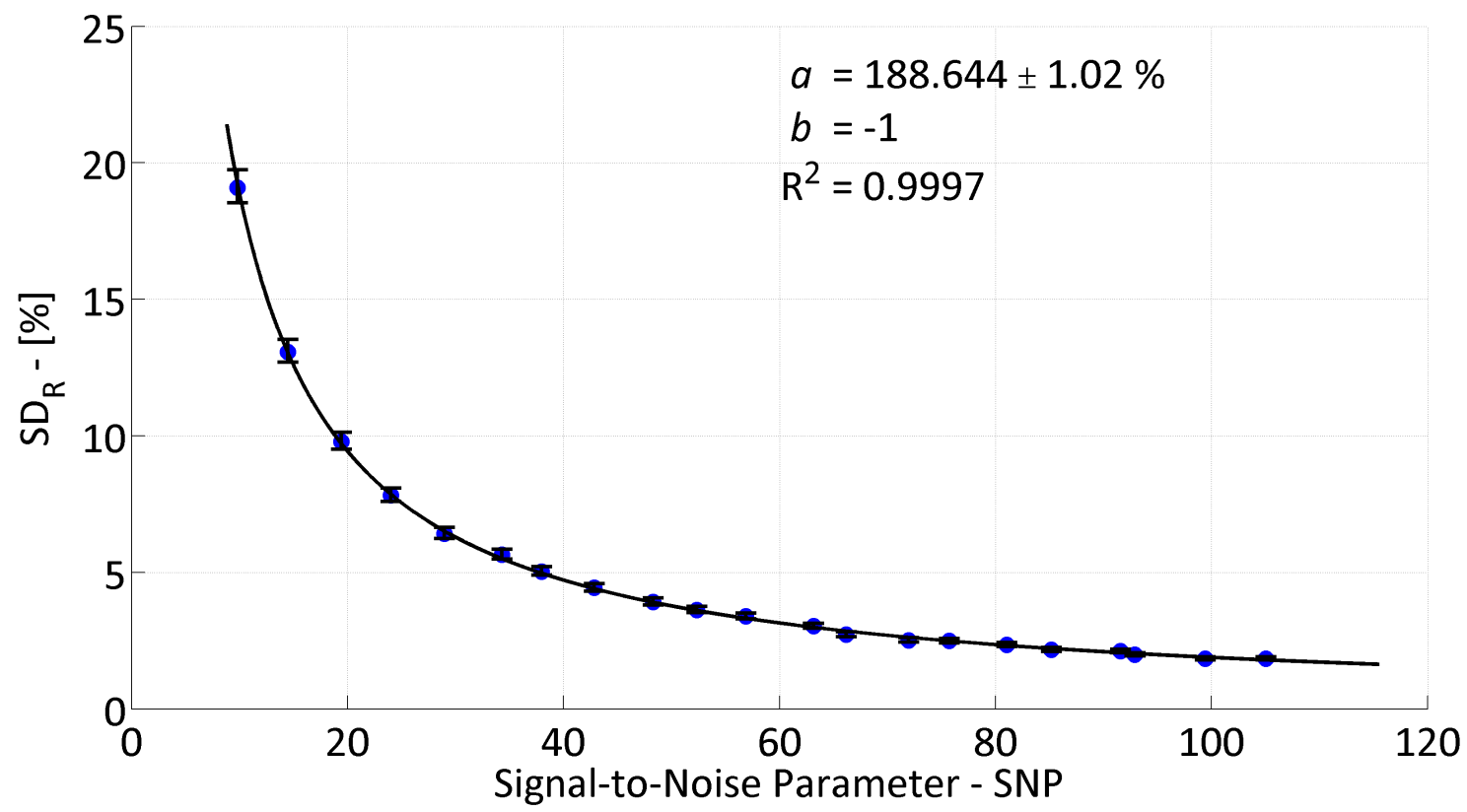

Figure S.74: Relative population standard deviation as a function of signal-to-noise parameter for 100 $\mathrm{nm}$ of $\mathrm{Al} / \mathrm{Si}$ with $h_{\mathrm{BD}}=5.99 \mathrm{MW} / \mathrm{m}^{2} \mathrm{~K}$. Error bars represent a $95 \%$ confidence interval of the measured $\mathrm{SD}_{\mathrm{R}}$ and the solid line represents a best fit of a power law to the data, of the form $\mathrm{SD}_{\mathrm{R}}=a \times \mathrm{SNP}^{b}$, with parameter $a$ provided for a fixed $b=-1$, along with the $95 \%$ confidence level in $a$. 


\section{S.12 Simulation Summary - Al/Si 7.74 MW Series}

\section{S.12.1 Fitting Film-to-Substrate Boundary Conductance - $h_{\mathrm{BD}}$}

Table S.14: Summary of simulation data - $100 \mathrm{~nm} \mathrm{Al/Si} \mathrm{7.74} \mathrm{MW} \mathrm{Series.} \mathrm{Error} \mathrm{bounds} \mathrm{represent} \mathrm{a} 95 \%$ confidence level.

\begin{tabular}{cccccc}
\hline \multicolumn{5}{c}{$\mathbf{1 0 0} \mathbf{~ n m ~ A l ~ o n ~} \mathbf{~ i ,} h_{\mathrm{BD}}=\mathbf{7 . 7 4} \mathbf{~ M W} / \mathbf{m}^{2} \mathbf{K}$} \\
\hline $\mathbf{S N P}$ & $\mu-\left[\mathbf{M W} / \mathbf{m}^{2} \mathbf{K}\right]$ & $\sigma-\left[\mathbf{M W} / \mathbf{m}^{2} \mathbf{K}\right]$ & $\mathbf{S N P}$ & $\mu-\left[\mathbf{M W} / \mathbf{m}^{2} \mathbf{K}\right]$ & $\sigma-\left[\mathbf{M W} / \mathbf{m}^{2} \mathbf{K}\right]$ \\
9.49 & $7.81 \pm 0.073$ & $1.17 \pm 0.0033$ & 60.18 & $7.74 \pm 0.012$ & $0.19 \pm 0.0005$ \\
14.29 & $7.73 \pm 0.050$ & $0.80 \pm 0.0022$ & 66.23 & $7.74 \pm 0.011$ & $0.17 \pm 0.0005$ \\
18.93 & $7.74 \pm 0.036$ & $0.58 \pm 0.0016$ & 68.30 & $7.75 \pm 0.010$ & $0.16 \pm 0.0004$ \\
23.66 & $7.74 \pm 0.030$ & $0.49 \pm 0.0014$ & 74.02 & $7.75 \pm 0.010$ & $0.16 \pm 0.0004$ \\
28.03 & $7.72 \pm 0.027$ & $0.43 \pm 0.0012$ & 79.46 & $7.74 \pm 0.009$ & $0.14 \pm 0.0004$ \\
32.66 & $7.73 \pm 0.023$ & $0.37 \pm 0.0010$ & 83.20 & $7.75 \pm 0.009$ & $0.14 \pm 0.0004$ \\
37.17 & $7.74 \pm 0.019$ & $0.31 \pm 0.0009$ & 88.50 & $7.74 \pm 0.008$ & $0.13 \pm 0.0004$ \\
41.96 & $7.74 \pm 0.017$ & $0.28 \pm 0.0008$ & 93.51 & $7.74 \pm 0.008$ & $0.12 \pm 0.0003$ \\
46.74 & $7.75 \pm 0.015$ & $0.25 \pm 0.0007$ & 96.36 & $7.75 \pm 0.007$ & $0.12 \pm 0.0003$ \\
51.15 & $7.75 \pm 0.014$ & $0.23 \pm 0.0006$ & 101.00 & $7.75 \pm 0.007$ & $0.11 \pm 0.0003$ \\
57.00 & $7.75 \pm 0.012$ & $0.20 \pm 0.0006$ & & & \\
\hline
\end{tabular}

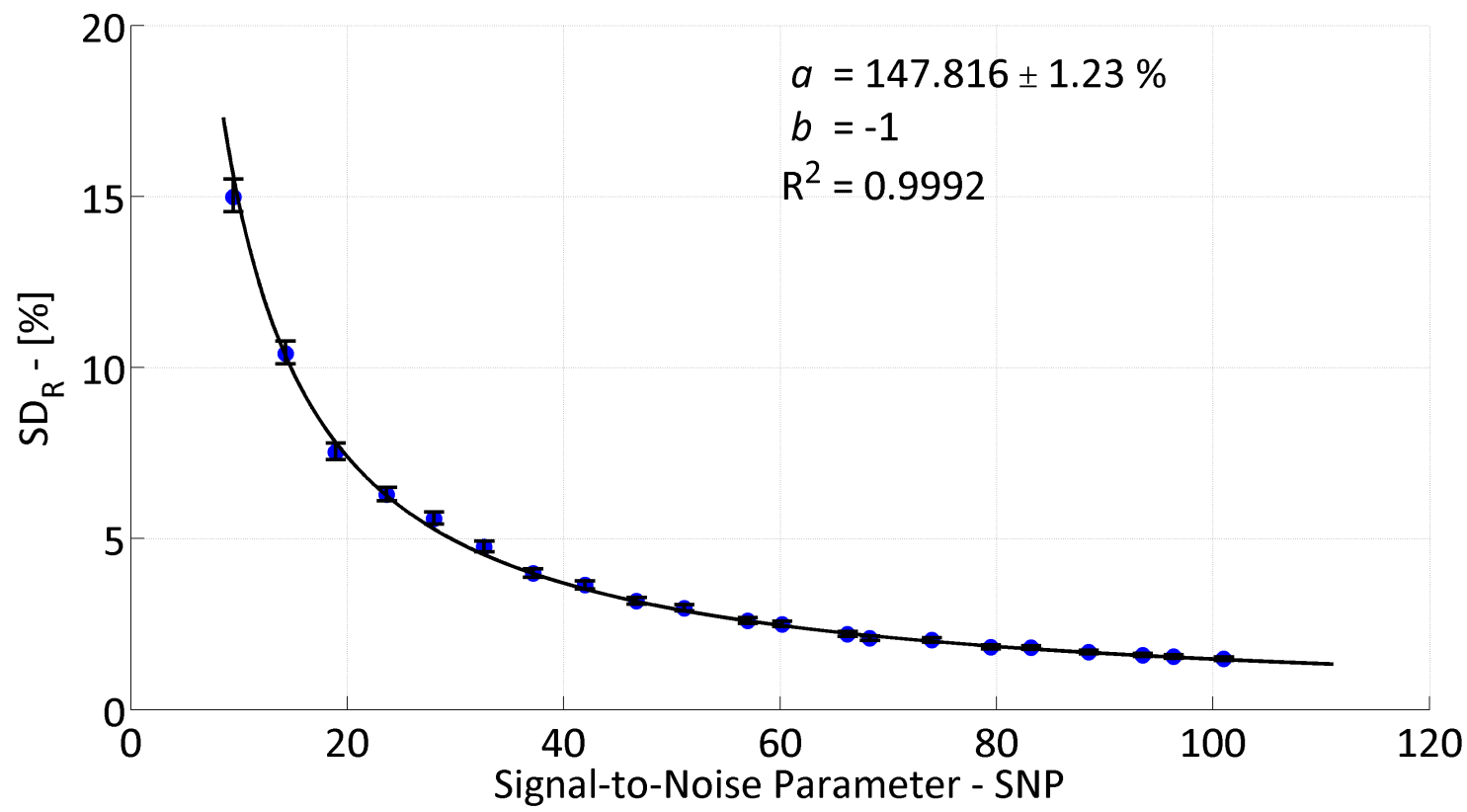

Figure S.75: Relative population standard deviation as a function of signal-to-noise parameter for 100 $\mathrm{nm}$ of $\mathrm{Al} / \mathrm{Si}$ with $h_{\mathrm{BD}}=7.74 \mathrm{MW} / \mathrm{m}^{2} \mathrm{~K}$. Error bars represent a $95 \%$ confidence interval of the measured $\mathrm{SD}_{\mathrm{R}}$ and the solid line represents a best fit of a power law to the data, of the form $\mathrm{SD}_{\mathrm{R}}=a \times \mathrm{SNP}^{b}$, with parameter $a$ provided for a fixed $b=-1$, along with the $95 \%$ confidence level in $a$. 


\section{S.13 Simulation Summary - Al/Si 10.00 MW Series}

\section{S.13.1 Fitting Film-to-Substrate Boundary Conductance - $h_{\mathrm{BD}}$}

Table S.15: Summary of simulation data - $100 \mathrm{~nm} \mathrm{Al/Si} \mathrm{10.00} \mathrm{MW} \mathrm{Series.} \mathrm{Error} \mathrm{bounds} \mathrm{represent} \mathrm{a} 95 \%$ confidence level.

\begin{tabular}{cccccc}
\hline \multicolumn{5}{c}{$\mathbf{1 0 0} \mathbf{~ n m ~ A l ~ o n ~} \mathbf{~ i}, h_{\mathrm{BD}}=\mathbf{1 0 . 0 0} \mathbf{M W} / \mathbf{m}^{2} \mathbf{K}$} \\
\hline $\mathbf{S N P}$ & $\mu-\left[\mathbf{M W} / \mathbf{m}^{2} \mathbf{K}\right]$ & $\sigma-\left[\mathbf{M W} / \mathbf{m}^{2} \mathbf{K}\right]$ & $\mathbf{S N P}$ & $\mu-\left[\mathbf{M W} / \mathbf{m}^{2} \mathbf{K}\right]$ & $\sigma-\left[\mathbf{M W} / \mathbf{m}^{2} \mathbf{K}\right]$ \\
9.15 & $9.95 \pm 0.081$ & $1.30 \pm 0.0036$ & 58.17 & $10.00 \pm 0.013$ & $0.20 \pm 0.0006$ \\
13.69 & $9.97 \pm 0.055$ & $0.88 \pm 0.0025$ & 64.09 & $10.00 \pm 0.011$ & $0.18 \pm 0.0005$ \\
18.05 & $10.02 \pm 0.040$ & $0.65 \pm 0.0018$ & 67.02 & $10.02 \pm 0.010$ & $0.17 \pm 0.0005$ \\
22.32 & $10.00 \pm 0.034$ & $0.54 \pm 0.0015$ & 70.61 & $10.01 \pm 0.010$ & $0.16 \pm 0.0005$ \\
26.93 & $10.01 \pm 0.027$ & $0.44 \pm 0.0012$ & 75.47 & $10.01 \pm 0.010$ & $0.16 \pm 0.0004$ \\
31.14 & $10.00 \pm 0.023$ & $0.37 \pm 0.0010$ & 79.78 & $10.00 \pm 0.009$ & $0.14 \pm 0.0004$ \\
35.96 & $10.01 \pm 0.019$ & $0.31 \pm 0.0009$ & 84.99 & $10.02 \pm 0.008$ & $0.14 \pm 0.0004$ \\
40.87 & $10.00 \pm 0.018$ & $0.28 \pm 0.0008$ & 88.65 & $10.01 \pm 0.008$ & $0.13 \pm 0.0004$ \\
45.12 & $10.02 \pm 0.016$ & $0.26 \pm 0.0007$ & 94.60 & $10.01 \pm 0.008$ & $0.12 \pm 0.0003$ \\
49.68 & $10.00 \pm 0.015$ & $0.24 \pm 0.0007$ & 98.34 & $10.01 \pm 0.007$ & $0.12 \pm 0.0003$ \\
53.08 & $9.99 \pm 0.013$ & $0.21 \pm 0.0006$ & & & \\
\hline
\end{tabular}

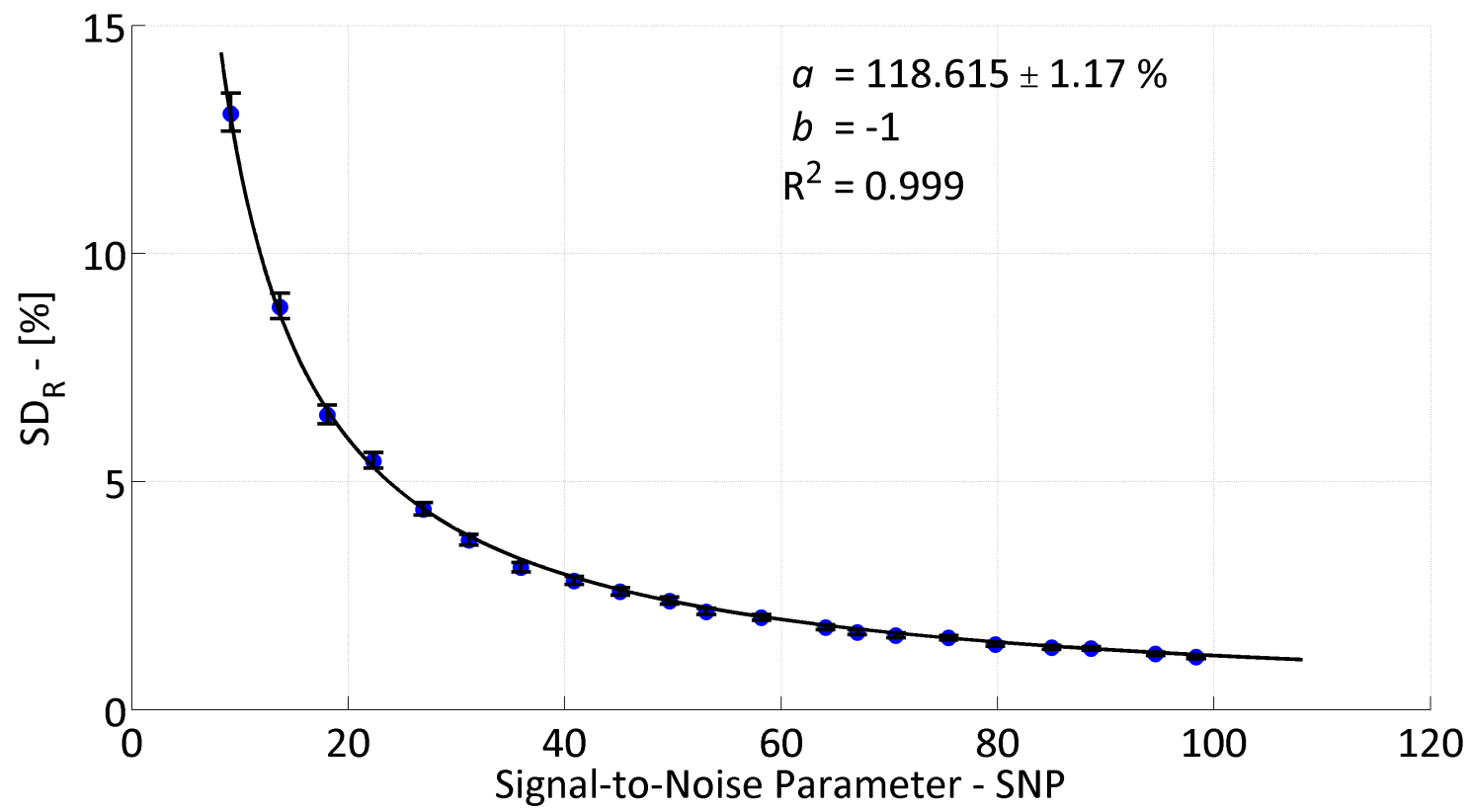

Figure S.76: Relative population standard deviation as a function of signal-to-noise parameter for 100 $\mathrm{nm}$ of $\mathrm{Al} / \mathrm{Si}$ with $h_{\mathrm{BD}}=10.00 \mathrm{MW} / \mathrm{m}^{2} \mathrm{~K}$. Error bars represent a $95 \%$ confidence interval of the measured $\mathrm{SD}_{\mathrm{R}}$ and the solid line represents a best fit of a power law to the data, of the form $\mathrm{SD}_{\mathrm{R}}=a \times \mathrm{SNP}^{b}$, with parameter $a$ provided for a fixed $b=-1$, along with the $95 \%$ confidence level in $a$. 


\section{S.13.2 Fitting Film Thickness $-d_{1}$}

Table S.16: Summary of simulation data - $100 \mathrm{~nm} \mathrm{Al/Si} \mathrm{10.00} \mathrm{MW} \mathrm{Series.} \mathrm{Error} \mathrm{bounds} \mathrm{represent} \mathrm{a} 95 \%$ confidence level.

\begin{tabular}{cccccc}
\hline \multicolumn{6}{c}{$\mathbf{1 0 0} \mathbf{~ n m ~ A l ~ o n ~} \mathbf{~ S i}, h_{\mathrm{BD}}=\mathbf{1 0 . 0 0} \mathbf{M W} / \mathbf{m}^{2} \mathbf{K}$} \\
\hline SNP & $\mu-[\mathbf{n m}]$ & $\sigma-[\mathbf{n m}]$ & $\mathbf{S N P}$ & $\mu-[\mathbf{n m}]$ & $\sigma-[\mathbf{n m}]$ \\
9.15 & $102.13 \pm 0.817$ & $13.14 \pm 0.0367$ & 58.17 & $100.00 \pm 0.120$ & $1.93 \pm 0.0054$ \\
13.69 & $100.95 \pm 0.532$ & $8.57 \pm 0.0239$ & 64.09 & $99.99 \pm 0.107$ & $1.73 \pm 0.0048$ \\
18.05 & $100.20 \pm 0.385$ & $6.20 \pm 0.0173$ & 67.02 & $99.86 \pm 0.100$ & $1.61 \pm 0.0045$ \\
22.32 & $100.27 \pm 0.324$ & $5.22 \pm 0.0146$ & 70.61 & $99.98 \pm 0.097$ & $1.56 \pm 0.0043$ \\
26.93 & $100.13 \pm 0.263$ & $4.23 \pm 0.0118$ & 75.47 & $99.93 \pm 0.093$ & $1.51 \pm 0.0042$ \\
31.14 & $100.15 \pm 0.222$ & $3.58 \pm 0.0100$ & 79.78 & $99.99 \pm 0.085$ & $1.37 \pm 0.0038$ \\
35.96 & $99.99 \pm 0.185$ & $2.99 \pm 0.0083$ & 84.99 & $99.86 \pm 0.080$ & $1.30 \pm 0.0036$ \\
40.87 & $100.09 \pm 0.168$ & $2.71 \pm 0.0076$ & 88.65 & $99.94 \pm 0.079$ & $1.28 \pm 0.0036$ \\
45.12 & $99.89 \pm 0.153$ & $2.47 \pm 0.0069$ & 94.60 & $99.96 \pm 0.073$ & $1.17 \pm 0.0033$ \\
49.68 & $100.10 \pm 0.142$ & $2.28 \pm 0.0064$ & 98.34 & $99.88 \pm 0.068$ & $1.10 \pm 0.0031$ \\
53.08 & $100.10 \pm 0.128$ & $2.06 \pm 0.0057$ & & & \\
\hline
\end{tabular}

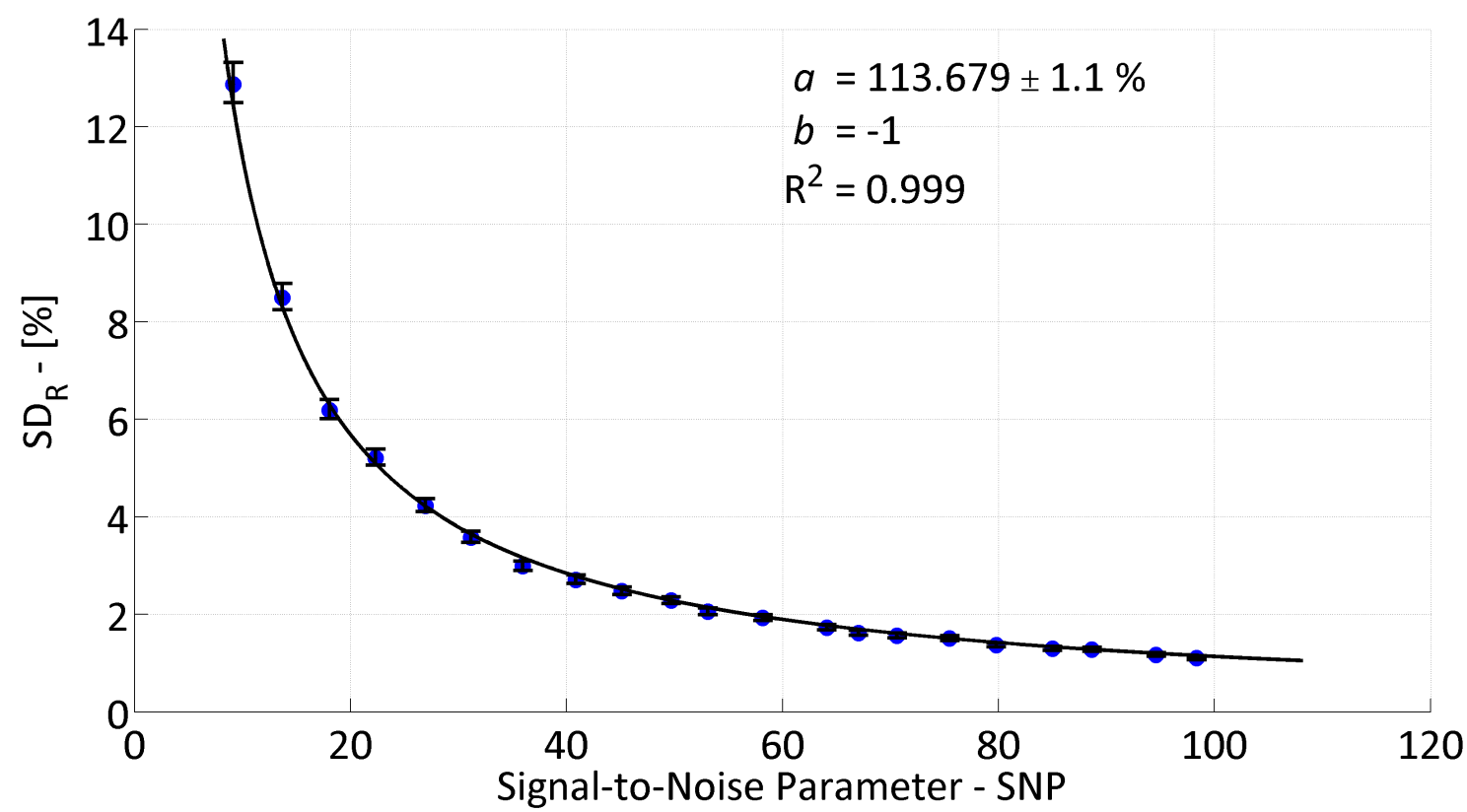

Figure S.77: Relative population standard deviation as a function of signal-to-noise parameter for 100 $\mathrm{nm}$ of $\mathrm{Al} / \mathrm{Si}$ with $h_{\mathrm{BD}}=10.00 \mathrm{MW} / \mathrm{m}^{2} \mathrm{~K}$. Error bars represent a $95 \%$ confidence interval of the measured $\mathrm{SD}_{\mathrm{R}}$ and the solid line represents a best fit of a power law to the data, of the form $\mathrm{SD}_{\mathrm{R}}=a \times \mathrm{SNP}^{b}$, with parameter $a$ provided for a fixed $b=-1$, along with the $95 \%$ confidence level in $a$. 


\section{S.14 Simulation Summary - Al/Si 12.90 MW Series}

\section{S.14.1 Fitting Film-to-Substrate Boundary Conductance $-h_{\mathrm{BD}}$}

Table S.17: Summary of simulation data - $100 \mathrm{~nm} \mathrm{Al/Si} 12.90 \mathrm{MW}$ Series. Error bounds represent a $95 \%$ confidence level.

\begin{tabular}{cccccc}
\hline \multicolumn{5}{c}{$\mathbf{1 0 0} \mathbf{~ n m ~ A l ~ o n ~} \mathbf{~ S i}, h_{\mathrm{BD}}=\mathbf{1 2 . 9 0} \mathbf{M W} / \mathbf{m}^{2} \mathbf{K}$} \\
\hline $\mathbf{S N P}$ & $\mu-\left[\mathbf{M W} / \mathbf{m}^{2} \mathbf{K}\right]$ & $\sigma-\left[\mathbf{M W} / \mathbf{m}^{2} \mathbf{K}\right]$ & $\mathbf{S N P}$ & $\mu-\left[\mathbf{M W} / \mathbf{m}^{2} \mathbf{K}\right]$ & $\sigma-\left[\mathbf{M W} / \mathbf{m}^{2} \mathbf{K}\right]$ \\
8.66 & $12.90 \pm 0.085$ & $1.36 \pm 0.0038$ & 55.79 & $12.90 \pm 0.013$ & $0.21 \pm 0.0006$ \\
13.04 & $12.93 \pm 0.056$ & $0.90 \pm 0.0025$ & 60.13 & $12.91 \pm 0.013$ & $0.20 \pm 0.0006$ \\
17.28 & $12.89 \pm 0.041$ & $0.67 \pm 0.0019$ & 64.23 & $12.92 \pm 0.012$ & $0.19 \pm 0.0005$ \\
21.51 & $12.91 \pm 0.034$ & $0.55 \pm 0.0015$ & 67.35 & $12.90 \pm 0.011$ & $0.18 \pm 0.0005$ \\
26.05 & $12.94 \pm 0.028$ & $0.46 \pm 0.0013$ & 72.69 & $12.91 \pm 0.011$ & $0.17 \pm 0.0005$ \\
30.06 & $12.92 \pm 0.026$ & $0.41 \pm 0.0011$ & 76.63 & $12.92 \pm 0.009$ & $0.15 \pm 0.0004$ \\
34.56 & $12.89 \pm 0.021$ & $0.34 \pm 0.0010$ & 81.36 & $12.91 \pm 0.009$ & $0.15 \pm 0.0004$ \\
38.42 & $12.91 \pm 0.019$ & $0.31 \pm 0.0009$ & 86.04 & $12.91 \pm 0.009$ & $0.14 \pm 0.0004$ \\
43.36 & $12.91 \pm 0.018$ & $0.28 \pm 0.0008$ & 90.43 & $12.91 \pm 0.008$ & $0.13 \pm 0.0004$ \\
46.96 & $12.92 \pm 0.015$ & $0.25 \pm 0.0007$ & 93.18 & $12.90 \pm 0.008$ & $0.14 \pm 0.0004$ \\
52.38 & $12.92 \pm 0.014$ & $0.22 \pm 0.0006$ & & & \\
\hline
\end{tabular}

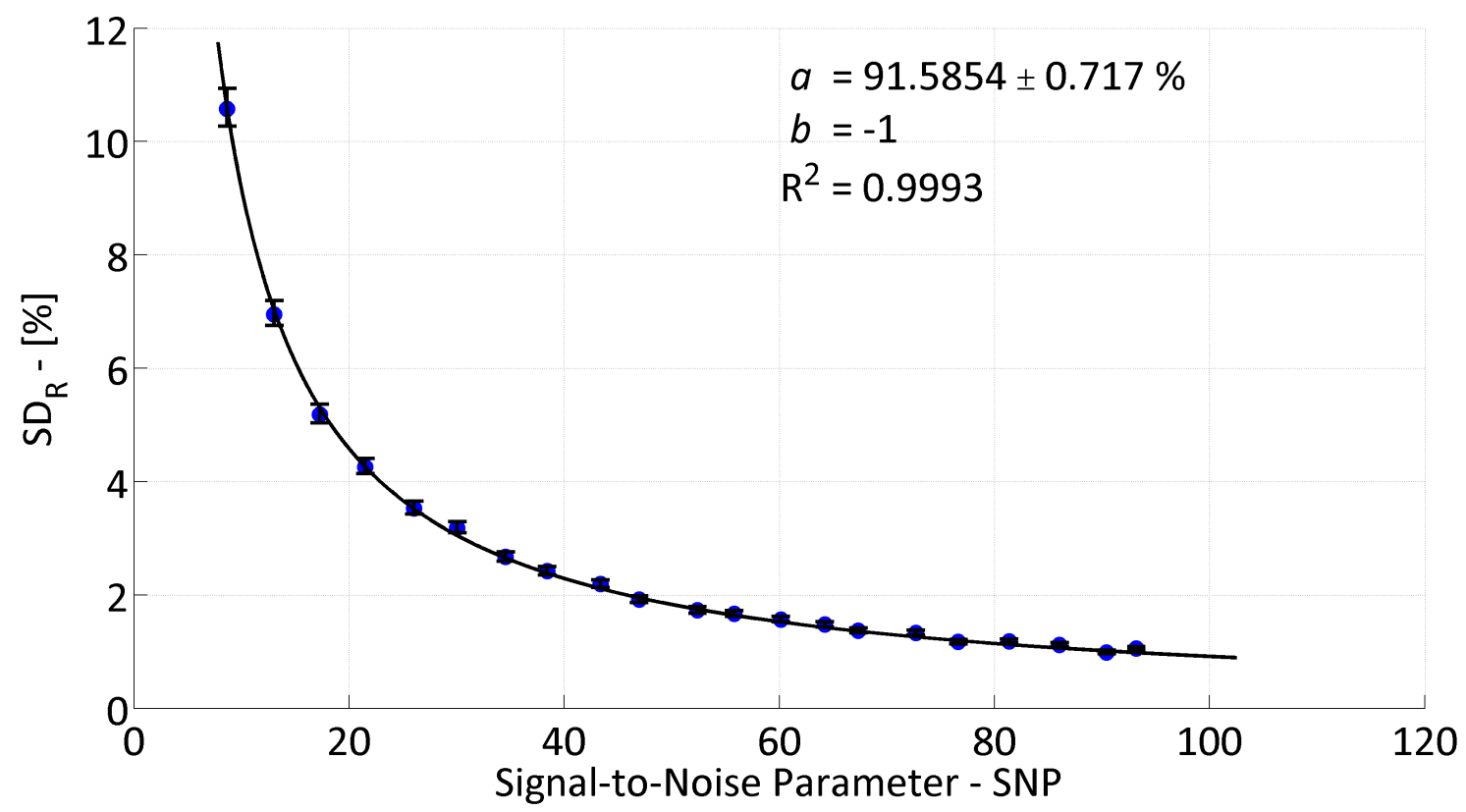

Figure S.78: Relative population standard deviation as a function of signal-to-noise parameter for 100 $\mathrm{nm}$ of $\mathrm{Al} / \mathrm{Si}$ with $h_{\mathrm{BD}}=12.90 \mathrm{MW} / \mathrm{m}^{2} \mathrm{~K}$. Error bars represent a $95 \%$ confidence interval of the measured $\mathrm{SD}_{\mathrm{R}}$ and the solid line represents a best fit of a power law to the data, of the form $\mathrm{SD}_{\mathrm{R}}=a \times \mathrm{SNP}^{b}$, with parameter $a$ provided for a fixed $b=-1$, along with the $95 \%$ confidence level in $a$. 


\section{S.15 Simulation Summary - Al/Si 16.70 MW Series}

\section{S.15.1 Fitting Film-to-Substrate Boundary Conductance $-h_{\mathrm{BD}}$}

Table S.18: Summary of simulation data - $100 \mathrm{~nm} \mathrm{Al/Si} \mathrm{16.70} \mathrm{MW} \mathrm{Series.} \mathrm{Error} \mathrm{bounds} \mathrm{represent} \mathrm{a} 95 \%$ confidence level.

\begin{tabular}{cccccc}
\hline \multicolumn{6}{c}{$\mathbf{1 0 0} \mathbf{~ n m ~ A l ~ o n ~} \mathbf{~ S i}, h_{\mathrm{BD}}=\mathbf{1 6 . 7 0} \mathbf{M W} / \mathbf{m}^{2} \mathbf{K}$} \\
\hline $\mathbf{S N P}$ & $\mu-\left[\mathbf{M W} / \mathbf{m}^{2} \mathbf{K}\right]$ & $\sigma-\left[\mathbf{M W} / \mathbf{m}^{2} \mathbf{K}\right]$ & $\mathbf{S N P}$ & $\mu-\left[\mathbf{M W} / \mathbf{m}^{2} \mathbf{K}\right]$ & $\sigma-\left[\mathbf{M W} / \mathbf{m}^{2} \mathbf{K}\right]$ \\
8.21 & $16.70 \pm 0.093$ & $1.49 \pm 0.0042$ & 52.58 & $16.72 \pm 0.015$ & $0.25 \pm 0.0007$ \\
12.24 & $16.71 \pm 0.063$ & $1.01 \pm 0.0028$ & 56.14 & $16.70 \pm 0.014$ & $0.22 \pm 0.0006$ \\
16.40 & $16.68 \pm 0.047$ & $0.76 \pm 0.0021$ & 60.28 & $16.71 \pm 0.013$ & $0.21 \pm 0.0006$ \\
20.27 & $16.72 \pm 0.038$ & $0.62 \pm 0.0017$ & 65.54 & $16.71 \pm 0.011$ & $0.18 \pm 0.0005$ \\
24.20 & $16.71 \pm 0.033$ & $0.52 \pm 0.0015$ & 68.53 & $16.71 \pm 0.011$ & $0.18 \pm 0.0005$ \\
28.54 & $16.71 \pm 0.027$ & $0.43 \pm 0.0012$ & 73.82 & $16.70 \pm 0.010$ & $0.17 \pm 0.0005$ \\
32.50 & $16.73 \pm 0.023$ & $0.38 \pm 0.0010$ & 75.51 & $16.71 \pm 0.010$ & $0.17 \pm 0.0005$ \\
36.76 & $16.70 \pm 0.022$ & $0.35 \pm 0.0010$ & 79.34 & $16.71 \pm 0.010$ & $0.16 \pm 0.0004$ \\
40.68 & $16.71 \pm 0.020$ & $0.32 \pm 0.0009$ & 83.94 & $16.71 \pm 0.009$ & $0.15 \pm 0.0004$ \\
43.86 & $16.72 \pm 0.018$ & $0.28 \pm 0.0008$ & 88.75 & $16.71 \pm 0.009$ & $0.14 \pm 0.0004$ \\
48.91 & $16.72 \pm 0.016$ & $0.26 \pm 0.0007$ & & & \\
\hline
\end{tabular}

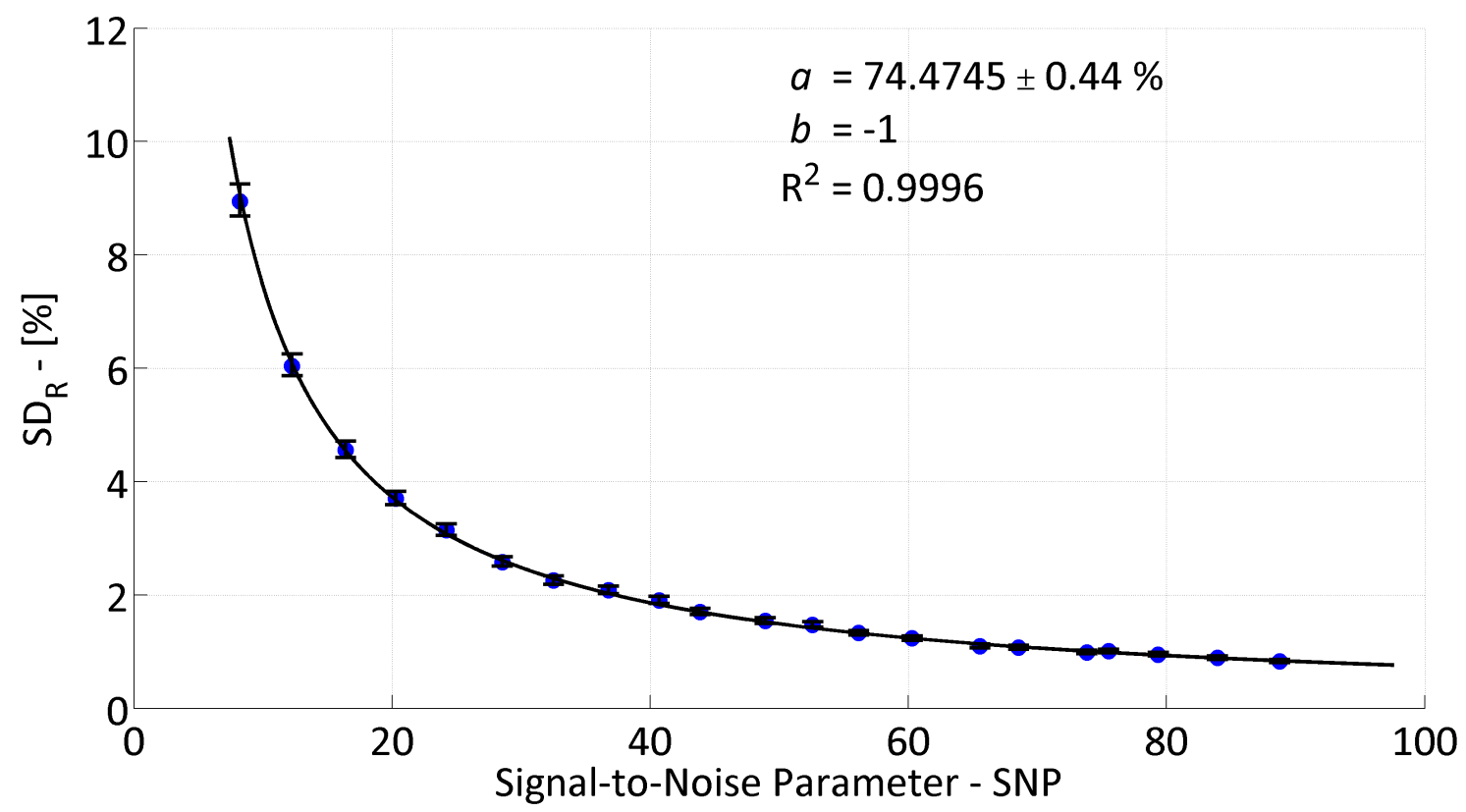

Figure S.79: Relative population standard deviation as a function of signal-to-noise parameter for 100 $\mathrm{nm}$ of $\mathrm{Al} / \mathrm{Si}$ with $h_{\mathrm{BD}}=16.70 \mathrm{MW} / \mathrm{m}^{2} \mathrm{~K}$. Error bars represent a $95 \%$ confidence interval of the measured $\mathrm{SD}_{\mathrm{R}}$ and the solid line represents a best fit of a power law to the data, of the form $\mathrm{SD}_{\mathrm{R}}=a \times \mathrm{SNP}^{b}$, with parameter $a$ provided for a fixed $b=-1$, along with the $95 \%$ confidence level in $a$. 


\section{S.16 Simulation Summary - Al/Si 21.50 MW Series}

\section{S.16.1 Fitting Film-to-Substrate Boundary Conductance - $h_{\mathrm{BD}}$}

Table S.19: Summary of simulation data - $100 \mathrm{~nm} \mathrm{Al/Si} \mathrm{21.50} \mathrm{MW} \mathrm{Series.} \mathrm{Error} \mathrm{bounds} \mathrm{represent} \mathrm{a} 95 \%$ confidence level.

\begin{tabular}{cccccc}
\hline \multicolumn{6}{c}{$\mathbf{1 0 0} \mathbf{~ n m ~ A l ~ o n ~} \mathbf{~ i}, h_{\mathrm{BD}}=\mathbf{2 1 . 5 0} \mathbf{M W} / \mathbf{m}^{2} \mathbf{K}$} \\
\hline $\mathbf{S N P}$ & $\mu-\left[\mathbf{M W} / \mathbf{m}^{2} \mathbf{K}\right]$ & $\sigma-\left[\mathbf{M W} / \mathbf{m}^{2} \mathbf{K}\right]$ & $\mathbf{S N P}$ & $\mu-\left[\mathbf{M W} / \mathbf{m}^{2} \mathbf{K}\right]$ & $\sigma-\left[\mathbf{M W} / \mathbf{m}^{2} \mathbf{K}\right]$ \\
7.63 & $21.50 \pm 0.110$ & $1.77 \pm 0.0049$ & 48.44 & $21.51 \pm 0.017$ & $0.27 \pm 0.0007$ \\
11.39 & $21.49 \pm 0.073$ & $1.18 \pm 0.0033$ & 51.69 & $21.52 \pm 0.016$ & $0.25 \pm 0.0007$ \\
15.08 & $21.51 \pm 0.054$ & $0.86 \pm 0.0024$ & 55.36 & $21.50 \pm 0.015$ & $0.24 \pm 0.0007$ \\
19.09 & $21.55 \pm 0.041$ & $0.67 \pm 0.0019$ & 60.12 & $21.51 \pm 0.014$ & $0.22 \pm 0.0006$ \\
22.81 & $21.52 \pm 0.037$ & $0.59 \pm 0.0016$ & 63.39 & $21.51 \pm 0.013$ & $0.21 \pm 0.0006$ \\
26.19 & $21.52 \pm 0.031$ & $0.49 \pm 0.0014$ & 66.31 & $21.51 \pm 0.012$ & $0.20 \pm 0.0006$ \\
29.88 & $21.52 \pm 0.028$ & $0.45 \pm 0.0012$ & 71.85 & $21.51 \pm 0.011$ & $0.19 \pm 0.0005$ \\
33.72 & $21.50 \pm 0.025$ & $0.40 \pm 0.0011$ & 75.43 & $21.51 \pm 0.010$ & $0.17 \pm 0.0005$ \\
37.64 & $21.54 \pm 0.022$ & $0.35 \pm 0.0010$ & 77.36 & $21.51 \pm 0.011$ & $0.17 \pm 0.0005$ \\
41.05 & $21.51 \pm 0.019$ & $0.31 \pm 0.0009$ & 82.38 & $21.51 \pm 0.010$ & $0.16 \pm 0.0004$ \\
44.80 & $21.48 \pm 0.018$ & $0.29 \pm 0.0008$ & & & \\
\hline
\end{tabular}

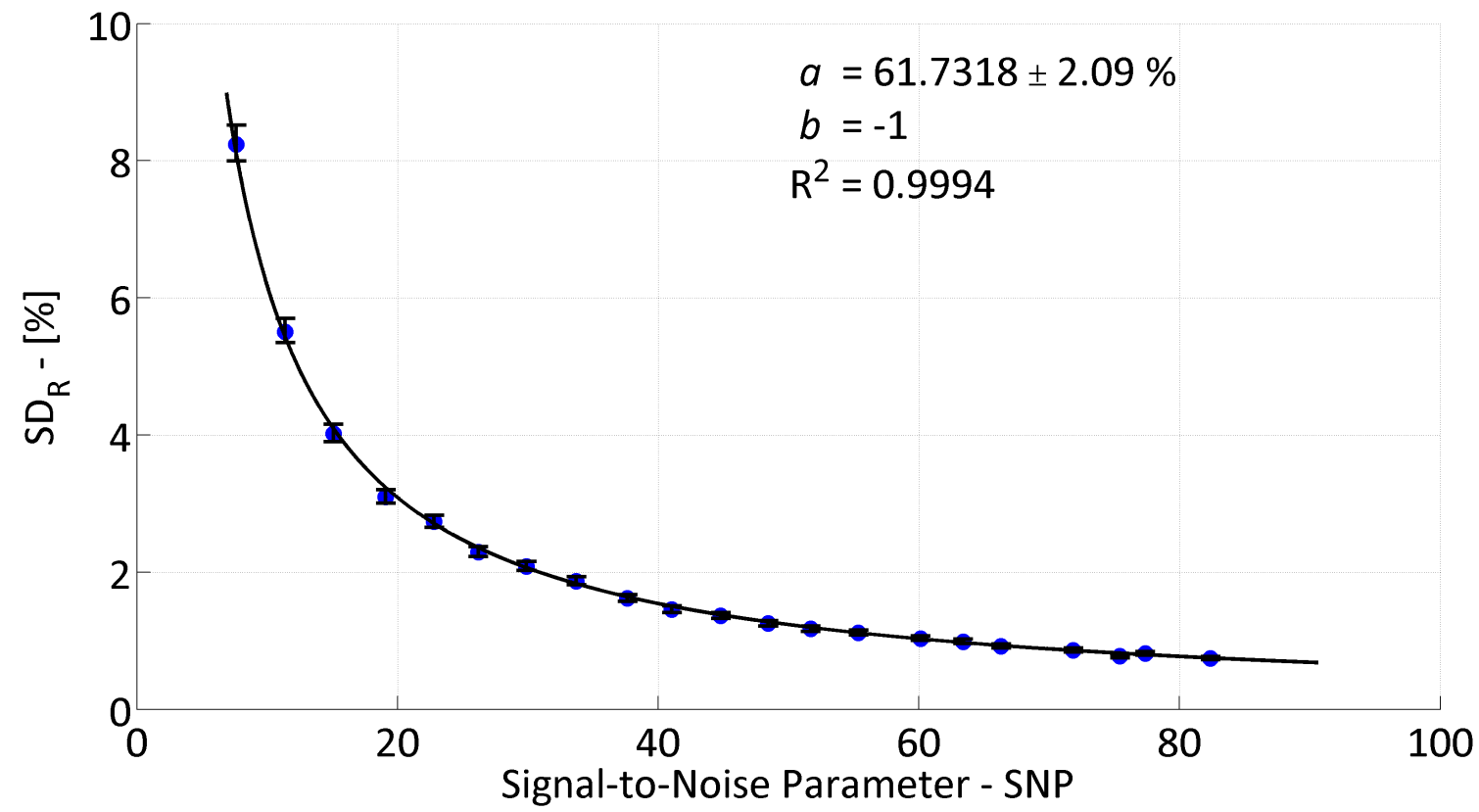

Figure S.80: Relative population standard deviation as a function of signal-to-noise parameter for 100 $\mathrm{nm}$ of $\mathrm{Al} / \mathrm{Si}$ with $h_{\mathrm{BD}}=21.50 \mathrm{MW} / \mathrm{m}^{2} \mathrm{~K}$. Error bars represent a $95 \%$ confidence interval of the measured $\mathrm{SD}_{\mathrm{R}}$ and the solid line represents a best fit of a power law to the data, of the form $\mathrm{SD}_{\mathrm{R}}=a \times \mathrm{SNP}^{b}$, with parameter $a$ provided for a fixed $b=-1$, along with the $95 \%$ confidence level in $a$. 


\section{S.16.2 Fitting Substrate Thermal Conductivity - $k_{S}$}

Table S.20: Summary of simulation data - $100 \mathrm{~nm} \mathrm{Al/Si} \mathrm{21.50} \mathrm{MW} \mathrm{Series.} \mathrm{Error} \mathrm{bounds} \mathrm{represent} \mathrm{a} 95 \%$ confidence level.

\begin{tabular}{cccccc}
\hline \multicolumn{6}{c}{$\mathbf{1 0 0} \mathbf{~ n m ~ A l ~ o n ~} \mathbf{~ i}, h_{\mathrm{BD}}=\mathbf{2 1 . 5 0} \mathbf{M W} / \mathbf{m}^{2} \mathbf{K}$} \\
\hline $\mathbf{S N P}$ & $\mu-[\mathbf{W} / \mathbf{m K}]$ & $\sigma-[\mathbf{W} / \mathbf{m K}]$ & $\mathbf{S N P}$ & $\mu-[\mathbf{W} / \mathbf{m K}]$ & $\sigma-[\mathbf{W} / \mathbf{m K}]$ \\
7.63 & $179.32 \pm 13.205$ & $192.41 \pm 0.6557$ & 48.44 & $148.80 \pm 2.205$ & $35.38 \pm 0.0994$ \\
11.39 & $185.68 \pm 11.624$ & $170.93 \pm 0.5720$ & 51.69 & $150.50 \pm 2.118$ & $34.09 \pm 0.0952$ \\
15.08 & $182.57 \pm 8.987$ & $135.45 \pm 0.4314$ & 55.36 & $147.34 \pm 1.955$ & $31.48 \pm 0.0878$ \\
19.09 & $173.38 \pm 6.283$ & $99.19 \pm 0.2878$ & 60.12 & $147.58 \pm 1.788$ & $28.82 \pm 0.0803$ \\
22.81 & $169.39 \pm 5.829$ & $92.89 \pm 0.2645$ & 63.39 & $147.69 \pm 1.719$ & $27.70 \pm 0.0771$ \\
26.19 & $158.98 \pm 4.315$ & $68.83 \pm 0.1956$ & 66.31 & $147.28 \pm 1.618$ & $26.07 \pm 0.0727$ \\
29.88 & $156.94 \pm 3.832$ & $61.26 \pm 0.1734$ & 71.85 & $146.91 \pm 1.499$ & $24.16 \pm 0.0673$ \\
33.72 & $153.29 \pm 3.387$ & $54.25 \pm 0.1529$ & 75.43 & $146.58 \pm 1.342$ & $21.62 \pm 0.0603$ \\
37.64 & $156.14 \pm 3.042$ & $48.87 \pm 0.1369$ & 77.36 & $145.78 \pm 1.387$ & $22.34 \pm 0.0623$ \\
41.05 & $151.11 \pm 2.667$ & $42.80 \pm 0.1202$ & 82.38 & $145.68 \pm 1.249$ & $20.10 \pm 0.0562$ \\
44.80 & $147.64 \pm 2.481$ & $39.93 \pm 0.1115$ & & & \\
\hline
\end{tabular}

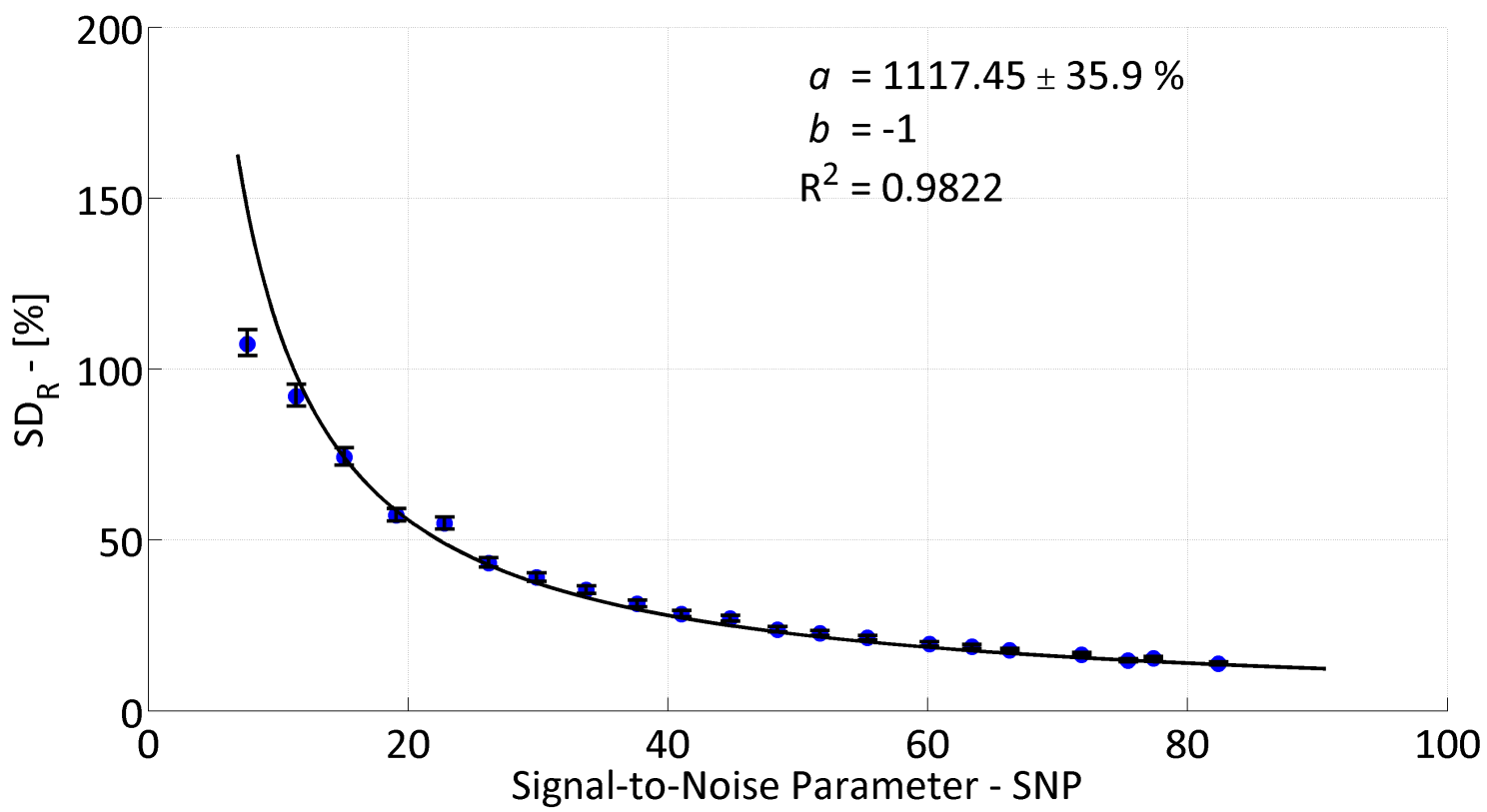

Figure S.81: Relative population standard deviation as a function of signal-to-noise parameter for 100 $\mathrm{nm}$ of $\mathrm{Al} / \mathrm{Si}$ with $h_{\mathrm{BD}}=21.50 \mathrm{MW} / \mathrm{m}^{2} \mathrm{~K}$. Error bars represent a $95 \%$ confidence interval of the measured $\mathrm{SD}_{\mathrm{R}}$ and the solid line represents a best fit of a power law to the data, of the form $\mathrm{SD}_{\mathrm{R}}=a \times \mathrm{SNP}^{b}$, with parameter $a$ provided for a fixed $b=-1$, along with the $95 \%$ confidence level in $a$. 


\section{S.16.3 Fitting Film Thickness $-d_{1}$}

Table S.21: Summary of simulation data - $100 \mathrm{~nm} \mathrm{Al/Si} \mathrm{21.50} \mathrm{MW} \mathrm{Series.} \mathrm{Error} \mathrm{bounds} \mathrm{represent} \mathrm{a} 95 \%$ confidence level.

\begin{tabular}{cccccc}
\hline \multicolumn{6}{c}{$\mathbf{1 0 0} \mathbf{~ n m ~} \mathbf{A l} \mathbf{~ o n ~} \mathbf{~ S i}, h_{\mathrm{BD}}=\mathbf{2 1 . 5 0} \mathbf{~ M W} / \mathbf{m}^{2} \mathbf{K}$} \\
\hline $\mathbf{S N P}$ & $\mu-[\mathbf{n m}]$ & $\sigma-[\mathbf{n m}]$ & $\mathbf{S N P}$ & $\mu-[\mathbf{n m}]$ & $\sigma-[\mathbf{n m}]$ \\
7.63 & $100.71 \pm 0.464$ & $7.47 \pm 0.0209$ & 48.44 & $100.14 \pm 0.070$ & $1.13 \pm 0.0031$ \\
11.39 & $100.49 \pm 0.314$ & $5.07 \pm 0.0141$ & 51.69 & $100.09 \pm 0.066$ & $1.06 \pm 0.0029$ \\
15.08 & $100.28 \pm 0.228$ & $3.67 \pm 0.0102$ & 55.36 & $100.18 \pm 0.063$ & $1.01 \pm 0.0028$ \\
19.09 & $100.05 \pm 0.174$ & $2.81 \pm 0.0078$ & 60.12 & $100.15 \pm 0.058$ & $0.93 \pm 0.0026$ \\
22.81 & $100.14 \pm 0.154$ & $2.48 \pm 0.0069$ & 63.39 & $100.13 \pm 0.055$ & $0.89 \pm 0.0025$ \\
26.19 & $100.15 \pm 0.129$ & $2.07 \pm 0.0058$ & 66.31 & $100.13 \pm 0.052$ & $0.83 \pm 0.0023$ \\
29.88 & $100.14 \pm 0.117$ & $1.88 \pm 0.0052$ & 71.85 & $100.14 \pm 0.048$ & $0.78 \pm 0.0022$ \\
33.72 & $100.20 \pm 0.105$ & $1.69 \pm 0.0047$ & 75.43 & $100.12 \pm 0.043$ & $0.70 \pm 0.0019$ \\
37.64 & $100.04 \pm 0.091$ & $1.46 \pm 0.0041$ & 77.36 & $100.15 \pm 0.046$ & $0.73 \pm 0.0020$ \\
41.05 & $100.14 \pm 0.082$ & $1.32 \pm 0.0037$ & 82.38 & $100.14 \pm 0.042$ & $0.67 \pm 0.0019$ \\
44.80 & $100.25 \pm 0.077$ & $1.24 \pm 0.0034$ & & & \\
\hline
\end{tabular}

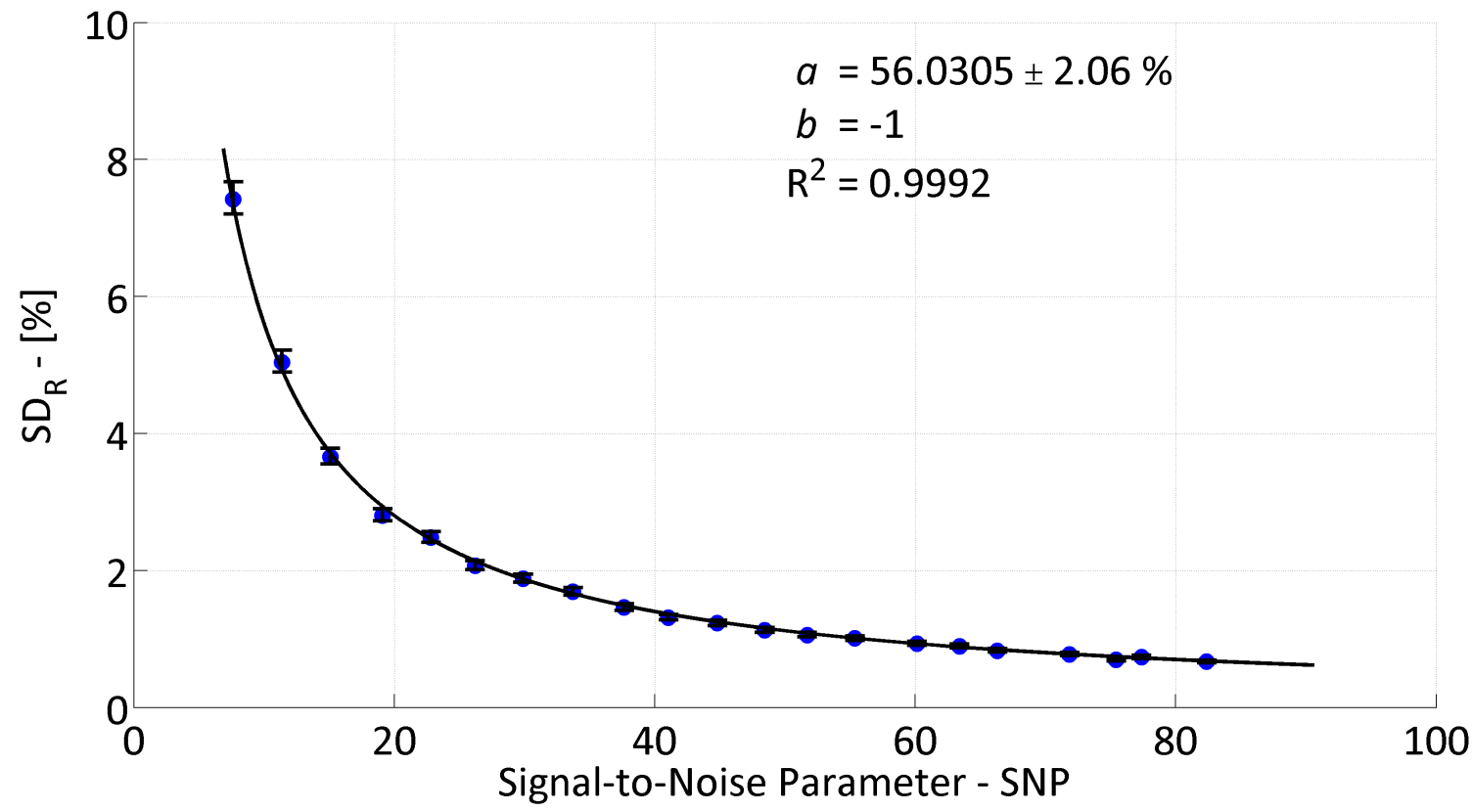

Figure S.82: Relative population standard deviation as a function of signal-to-noise parameter for 100 $\mathrm{nm}$ of $\mathrm{Al} / \mathrm{Si}$ with $h_{\mathrm{BD}}=21.50 \mathrm{MW} / \mathrm{m}^{2} \mathrm{~K}$. Error bars represent a $95 \%$ confidence interval of the measured $\mathrm{SD}_{\mathrm{R}}$ and the solid line represents a best fit of a power law to the data, of the form $\mathrm{SD}_{\mathrm{R}}=a \times \mathrm{SNP}^{b}$, with parameter $a$ provided for a fixed $b=-1$, along with the $95 \%$ confidence level in $a$. 


\section{S.17 Simulation Summary - Al/Si 27.80 MW Series}

\section{S.17.1 Fitting Film-to-Substrate Boundary Conductance $-h_{\mathrm{BD}}$}

Table S.22: Summary of simulation data - $100 \mathrm{~nm} \mathrm{Al/Si} \mathrm{27.80} \mathrm{MW} \mathrm{Series.} \mathrm{Error} \mathrm{bounds} \mathrm{represent} \mathrm{a} 95 \%$ confidence level.

\begin{tabular}{cccccc}
\hline \multicolumn{6}{c}{$\mathbf{1 0 0} \mathbf{~ n m ~ A l ~ o n ~} \mathbf{~ S i}, h_{\mathrm{BD}}=\mathbf{2 7 . 8 0} \mathbf{M W} / \mathbf{m}^{2} \mathbf{K}$} \\
\hline $\mathbf{S N P}$ & $\mu-\left[\mathbf{M W} / \mathbf{m}^{2} \mathbf{K}\right]$ & $\sigma-\left[\mathbf{M W} / \mathbf{m}^{2} \mathbf{K}\right]$ & $\mathbf{S N P}$ & $\mu-\left[\mathbf{M W} / \mathbf{m}^{2} \mathbf{K}\right]$ & $\sigma-\left[\mathbf{M W} / \mathbf{m}^{2} \mathbf{K}\right]$ \\
6.93 & $27.79 \pm 0.127$ & $2.05 \pm 0.0057$ & 43.87 & $27.81 \pm 0.020$ & $0.33 \pm 0.0009$ \\
10.38 & $27.71 \pm 0.078$ & $1.26 \pm 0.0035$ & 47.60 & $27.81 \pm 0.018$ & $0.30 \pm 0.0008$ \\
13.84 & $27.83 \pm 0.062$ & $1.00 \pm 0.0028$ & 51.10 & $27.81 \pm 0.017$ & $0.28 \pm 0.0008$ \\
17.14 & $27.81 \pm 0.050$ & $0.80 \pm 0.0022$ & 54.77 & $27.79 \pm 0.016$ & $0.26 \pm 0.0007$ \\
20.67 & $27.81 \pm 0.042$ & $0.67 \pm 0.0019$ & 58.62 & $27.80 \pm 0.016$ & $0.25 \pm 0.0007$ \\
23.73 & $27.84 \pm 0.036$ & $0.58 \pm 0.0016$ & 61.46 & $27.82 \pm 0.014$ & $0.23 \pm 0.0006$ \\
27.40 & $27.82 \pm 0.031$ & $0.50 \pm 0.0014$ & 65.07 & $27.81 \pm 0.014$ & $0.22 \pm 0.0006$ \\
30.72 & $27.84 \pm 0.028$ & $0.46 \pm 0.0013$ & 66.79 & $27.82 \pm 0.013$ & $0.21 \pm 0.0006$ \\
34.00 & $27.81 \pm 0.025$ & $0.40 \pm 0.0011$ & 70.40 & $27.81 \pm 0.013$ & $0.21 \pm 0.0006$ \\
37.74 & $27.80 \pm 0.024$ & $0.39 \pm 0.0011$ & 74.85 & $27.81 \pm 0.011$ & $0.18 \pm 0.0005$ \\
41.39 & $27.80 \pm 0.021$ & $0.34 \pm 0.0009$ & & & \\
\hline
\end{tabular}

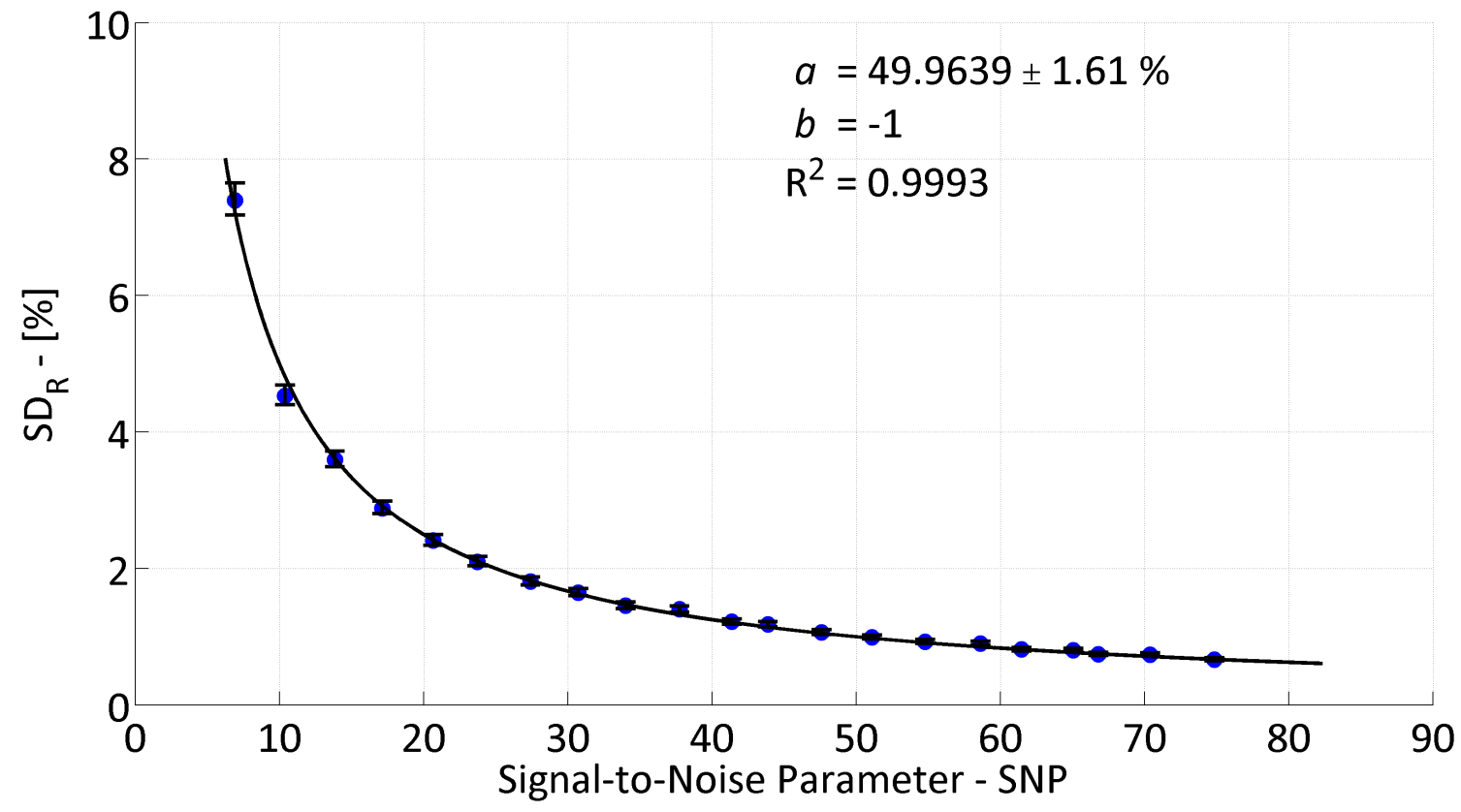

Figure S.83: Relative population standard deviation as a function of signal-to-noise parameter for 100 $\mathrm{nm}$ of $\mathrm{Al} / \mathrm{Si}$ with $h_{\mathrm{BD}}=27.80 \mathrm{MW} / \mathrm{m}^{2} \mathrm{~K}$. Error bars represent a $95 \%$ confidence interval of the measured $\mathrm{SD}_{\mathrm{R}}$ and the solid line represents a best fit of a power law to the data, of the form $\mathrm{SD}_{\mathrm{R}}=a \times \mathrm{SNP}^{b}$, with parameter $a$ provided for a fixed $b=-1$, along with the $95 \%$ confidence level in $a$. 


\section{S.17.2 Fitting Substrate Thermal Conductivity - $k_{S}$}

Table S.23: Summary of simulation data - $100 \mathrm{~nm} \mathrm{Al/Si} \mathrm{27.80} \mathrm{MW} \mathrm{Series.} \mathrm{Error} \mathrm{bounds} \mathrm{represent} \mathrm{a} 95 \%$ confidence level.

\begin{tabular}{cccccc}
\hline \multicolumn{6}{c}{$\mathbf{1 0 0} \mathbf{~ n m ~ A l ~ o n ~} \mathbf{~ S i}, h_{\mathrm{BD}}=\mathbf{2 7 . 8 0} \mathbf{M W} / \mathbf{m}^{2} \mathbf{K}$} \\
\hline $\mathbf{S N P}$ & $\mu-[\mathbf{W} / \mathbf{m K}]$ & $\sigma-[\mathbf{W} / \mathbf{m K}]$ & $\mathbf{S N P}$ & $\mu-[\mathbf{W} / \mathbf{m K}]$ & $\sigma-[\mathbf{W} / \mathbf{m K}]$ \\
6.93 & $173.00 \pm 9.694$ & $146.26 \pm 0.4648$ & 43.87 & $148.40 \pm 1.543$ & $24.81 \pm 0.0694$ \\
10.38 & $163.60 \pm 6.200$ & $97.93 \pm 0.2839$ & 47.60 & $148.74 \pm 1.415$ & $22.80 \pm 0.0635$ \\
13.84 & $170.42 \pm 5.636$ & $89.91 \pm 0.2555$ & 51.10 & $148.47 \pm 1.294$ & $20.83 \pm 0.0581$ \\
17.14 & $163.33 \pm 4.429$ & $71.05 \pm 0.1997$ & 54.77 & $146.73 \pm 1.198$ & $19.31 \pm 0.0538$ \\
20.67 & $157.18 \pm 3.489$ & $56.05 \pm 0.1570$ & 58.62 & $147.17 \pm 1.157$ & $18.65 \pm 0.0519$ \\
23.73 & $156.74 \pm 2.947$ & $47.33 \pm 0.1327$ & 61.46 & $148.27 \pm 1.063$ & $17.14 \pm 0.0477$ \\
27.40 & $153.19 \pm 2.468$ & $39.69 \pm 0.1110$ & 65.07 & $147.87 \pm 1.061$ & $17.10 \pm 0.0476$ \\
30.72 & $153.67 \pm 2.232$ & $35.90 \pm 0.1004$ & 66.79 & $148.45 \pm 0.966$ & $15.56 \pm 0.0433$ \\
34.00 & $149.89 \pm 1.907$ & $30.66 \pm 0.0858$ & 70.40 & $147.77 \pm 0.958$ & $15.43 \pm 0.0430$ \\
37.74 & $148.99 \pm 1.800$ & $28.95 \pm 0.0810$ & 74.85 & $147.30 \pm 0.866$ & $13.95 \pm 0.0389$ \\
41.39 & $148.70 \pm 1.618$ & $26.05 \pm 0.0727$ & & & \\
\hline
\end{tabular}

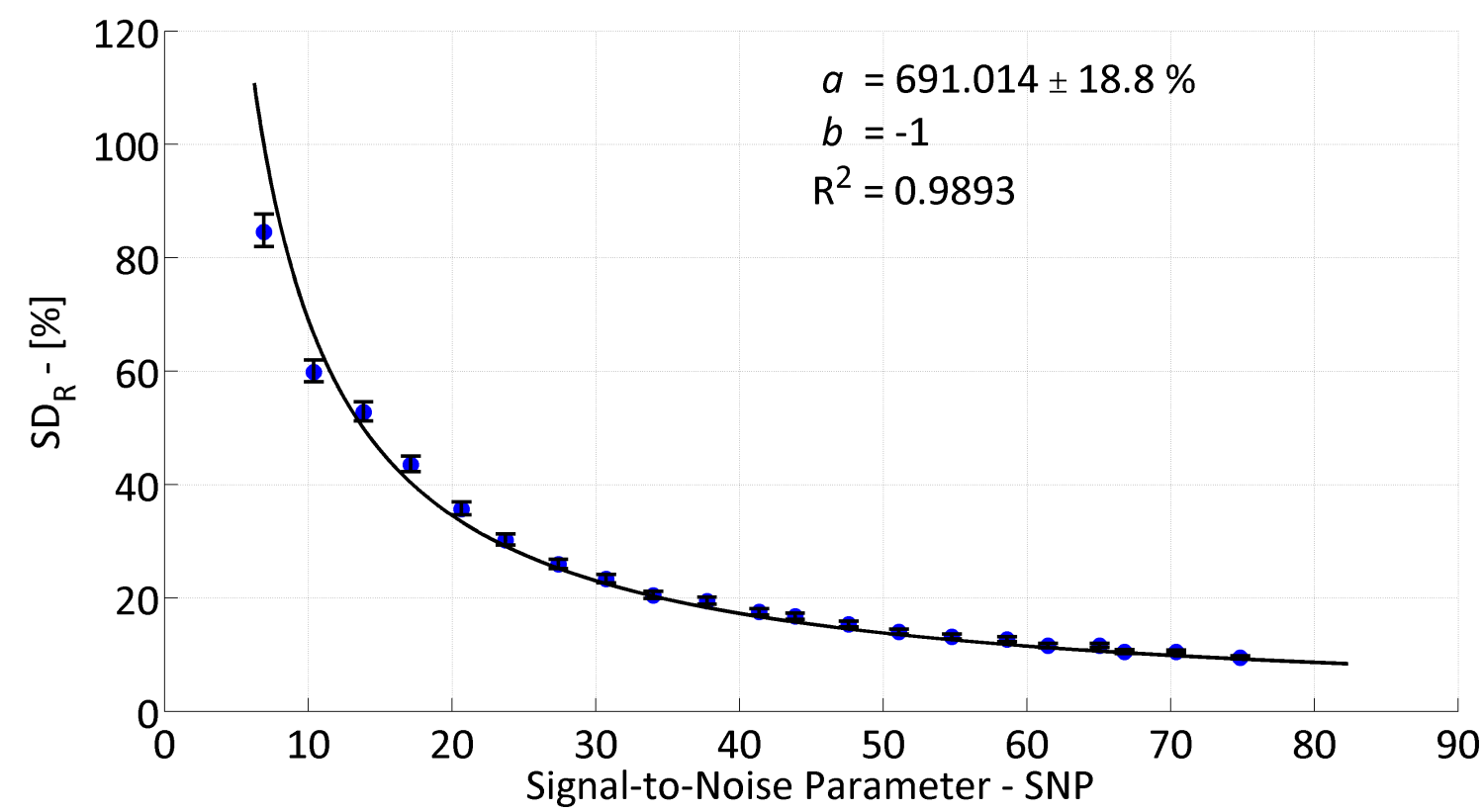

Figure S.84: Relative population standard deviation as a function of signal-to-noise parameter for 100 $\mathrm{nm}$ of $\mathrm{Al} / \mathrm{Si}$ with $h_{\mathrm{BD}}=27.80 \mathrm{MW} / \mathrm{m}^{2} \mathrm{~K}$. Error bars represent a $95 \%$ confidence interval of the measured $\mathrm{SD}_{\mathrm{R}}$ and the solid line represents a best fit of a power law to the data, of the form $\mathrm{SD}_{\mathrm{R}}=a \times \mathrm{SNP}^{b}$, with parameter $a$ provided for a fixed $b=-1$, along with the $95 \%$ confidence level in $a$. 


\section{S.18 Simulation Summary - Al/Si 35.90 MW Series}

\section{S.18.1 Fitting Film-to-Substrate Boundary Conductance - $h_{\mathrm{BD}}$}

Table S.24: Summary of simulation data - $100 \mathrm{~nm} \mathrm{Al/Si} \mathrm{35.90} \mathrm{MW} \mathrm{Series.} \mathrm{Error} \mathrm{bounds} \mathrm{represent} \mathrm{a} 95 \%$ confidence level.

\begin{tabular}{cccccc}
\hline \multicolumn{6}{c}{$\mathbf{1 0 0} \mathbf{~ n m ~ A l ~ o n ~} \mathbf{~ i}, h_{\mathrm{BD}}=\mathbf{3 5 . 9 0} \mathbf{M W} / \mathbf{m}^{2} \mathbf{K}$} \\
\hline $\mathbf{S N P}$ & $\mu-\left[\mathbf{M W} / \mathbf{m}^{2} \mathbf{K}\right]$ & $\sigma-\left[\mathbf{M W} / \mathbf{m}^{2} \mathbf{K}\right]$ & $\mathbf{S N P}$ & $\mu-\left[\mathbf{M W} / \mathbf{m}^{2} \mathbf{K}\right]$ & $\sigma-\left[\mathbf{M W} / \mathbf{m}^{2} \mathbf{K}\right]$ \\
6.16 & $35.95 \pm 0.154$ & $2.48 \pm 0.0069$ & 39.66 & $35.90 \pm 0.023$ & $0.38 \pm 0.0011$ \\
9.26 & $35.94 \pm 0.102$ & $1.65 \pm 0.0046$ & 42.02 & $35.90 \pm 0.023$ & $0.37 \pm 0.0010$ \\
12.32 & $35.96 \pm 0.076$ & $1.22 \pm 0.0034$ & 45.62 & $35.92 \pm 0.021$ & $0.34 \pm 0.0009$ \\
15.32 & $35.93 \pm 0.062$ & $1.00 \pm 0.0028$ & 48.16 & $35.91 \pm 0.019$ & $0.31 \pm 0.0009$ \\
18.27 & $35.93 \pm 0.055$ & $0.89 \pm 0.0025$ & 51.33 & $35.91 \pm 0.019$ & $0.30 \pm 0.0008$ \\
21.53 & $35.97 \pm 0.041$ & $0.67 \pm 0.0019$ & 54.62 & $35.91 \pm 0.018$ & $0.29 \pm 0.0008$ \\
24.41 & $35.90 \pm 0.039$ & $0.62 \pm 0.0017$ & 57.19 & $35.91 \pm 0.017$ & $0.27 \pm 0.0007$ \\
27.21 & $35.87 \pm 0.035$ & $0.56 \pm 0.0016$ & 59.66 & $35.93 \pm 0.016$ & $0.25 \pm 0.0007$ \\
30.89 & $35.90 \pm 0.029$ & $0.47 \pm 0.0013$ & 63.72 & $35.91 \pm 0.015$ & $0.24 \pm 0.0007$ \\
33.29 & $35.93 \pm 0.030$ & $0.48 \pm 0.0013$ & 66.85 & $35.91 \pm 0.014$ & $0.22 \pm 0.0006$ \\
35.61 & $35.92 \pm 0.026$ & $0.43 \pm 0.0012$ & & & \\
\hline
\end{tabular}

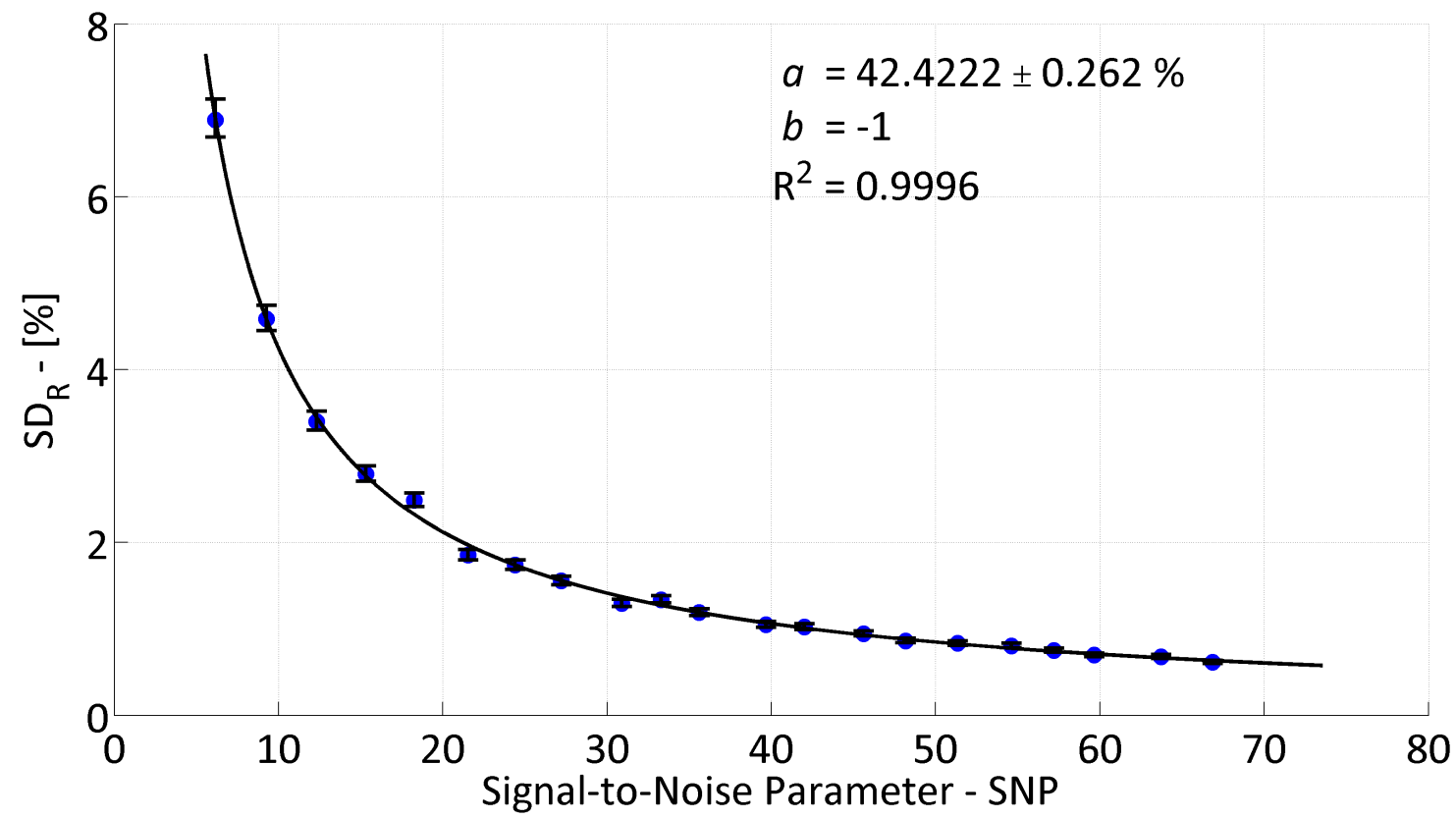

Figure S.85: Relative population standard deviation as a function of signal-to-noise parameter for 100 $\mathrm{nm}$ of $\mathrm{Al} / \mathrm{Si}$ with $h_{\mathrm{BD}}=35.90 \mathrm{MW} / \mathrm{m}^{2} \mathrm{~K}$. Error bars represent a $95 \%$ confidence interval of the measured $\mathrm{SD}_{\mathrm{R}}$ and the solid line represents a best fit of a power law to the data, of the form $\mathrm{SD}_{\mathrm{R}}=a \times \mathrm{SNP}^{b}$, with parameter $a$ provided for a fixed $b=-1$, along with the $95 \%$ confidence level in $a$. 


\section{S.18.2 Fitting Substrate Thermal Conductivity - $k_{S}$}

Table S.25: Summary of simulation data - $100 \mathrm{~nm} \mathrm{Al/Si} \mathrm{35.90} \mathrm{MW} \mathrm{Series.} \mathrm{Error} \mathrm{bounds} \mathrm{represent} \mathrm{a} 95 \%$ confidence level.

\begin{tabular}{cccccc}
\hline \multicolumn{6}{c}{$\mathbf{1 0 0} \mathbf{~ n m ~ A l ~ o n ~} \mathbf{~ S i}, h_{\mathrm{BD}}=\mathbf{3 5 . 9 0} \mathbf{M W} / \mathbf{m}^{2} \mathbf{K}$} \\
\hline $\mathbf{S N P}$ & $\mu-[\mathbf{W} / \mathbf{m K}]$ & $\sigma-[\mathbf{W} / \mathbf{m K}]$ & $\mathbf{S N P}$ & $\mu-[\mathbf{W} / \mathbf{m K}]$ & $\sigma-[\mathbf{W} / \mathbf{m K}]$ \\
6.16 & $183.40 \pm 8.155$ & $128.76 \pm 0.3736$ & 39.66 & $147.40 \pm 1.012$ & $16.31 \pm 0.0455$ \\
9.26 & $162.73 \pm 4.801$ & $76.35 \pm 0.2183$ & 42.02 & $147.34 \pm 0.971$ & $15.63 \pm 0.0436$ \\
12.32 & $159.00 \pm 3.647$ & $58.38 \pm 0.1647$ & 45.62 & $148.09 \pm 0.900$ & $14.50 \pm 0.0404$ \\
15.32 & $154.96 \pm 2.883$ & $46.31 \pm 0.1298$ & 48.16 & $147.52 \pm 0.818$ & $13.18 \pm 0.0367$ \\
18.27 & $153.86 \pm 2.524$ & $40.59 \pm 0.1135$ & 51.33 & $147.64 \pm 0.800$ & $12.89 \pm 0.0359$ \\
21.53 & $152.17 \pm 1.862$ & $29.93 \pm 0.0838$ & 54.62 & $147.44 \pm 0.772$ & $12.44 \pm 0.0346$ \\
24.41 & $149.71 \pm 1.727$ & $27.82 \pm 0.0776$ & 57.19 & $147.38 \pm 0.719$ & $11.59 \pm 0.0323$ \\
27.21 & $147.11 \pm 1.486$ & $23.88 \pm 0.0668$ & 59.66 & $148.03 \pm 0.665$ & $10.72 \pm 0.0299$ \\
30.89 & $147.82 \pm 1.246$ & $20.04 \pm 0.0560$ & 63.72 & $147.13 \pm 0.640$ & $10.31 \pm 0.0287$ \\
33.29 & $149.19 \pm 1.289$ & $20.76 \pm 0.0579$ & 66.85 & $146.96 \pm 0.582$ & $9.37 \pm 0.0261$ \\
35.61 & $148.35 \pm 1.151$ & $18.52 \pm 0.0517$ & & & \\
\hline
\end{tabular}

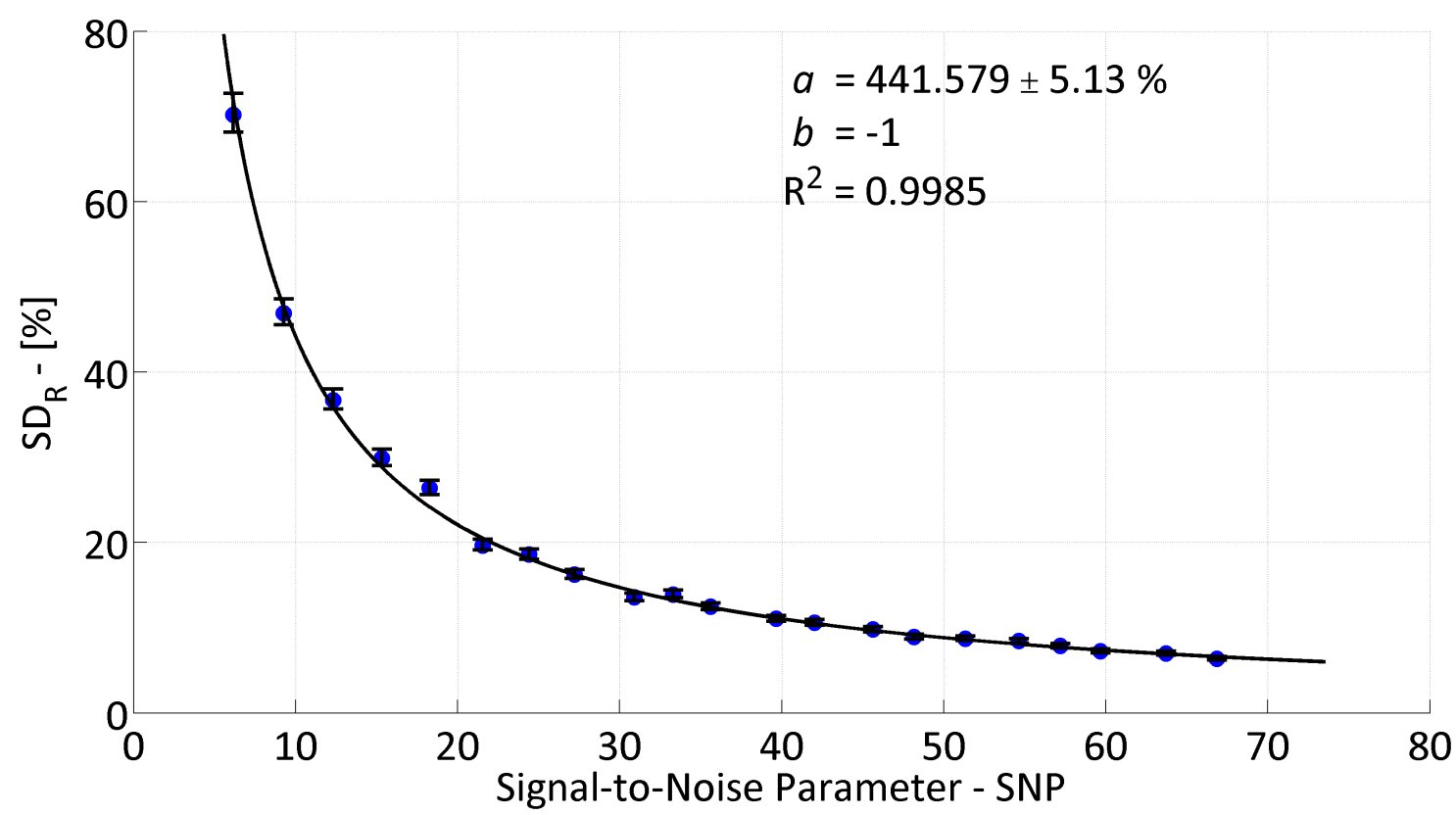

Figure S.86: Relative population standard deviation as a function of signal-to-noise parameter for 100 $\mathrm{nm}$ of $\mathrm{Al} / \mathrm{Si}$ with $h_{\mathrm{BD}}=35.90 \mathrm{MW} / \mathrm{m}^{2} \mathrm{~K}$. Error bars represent a $95 \%$ confidence interval of the measured $\mathrm{SD}_{\mathrm{R}}$ and the solid line represents a best fit of a power law to the data, of the form $\mathrm{SD}_{\mathrm{R}}=a \times \mathrm{SNP}^{b}$, with parameter $a$ provided for a fixed $b=-1$, along with the $95 \%$ confidence level in $a$. 


\section{S.19 Simulation Summary - Al/Si 46.40 MW Series}

\section{S.19.1 Fitting Film-to-Substrate Boundary Conductance - $h_{\mathrm{BD}}$}

Table S.26: Summary of simulation data - $100 \mathrm{~nm} \mathrm{Al/Si} \mathrm{46.40} \mathrm{MW} \mathrm{Series.} \mathrm{Error} \mathrm{bounds} \mathrm{represent} \mathrm{a} 95 \%$ confidence level.

\begin{tabular}{cccccc}
\hline \multicolumn{6}{c}{$\mathbf{1 0 0} \mathbf{~ n m ~ A l ~ o n ~} \mathbf{~ i}, h_{\mathrm{BD}}=\mathbf{4 6 . 4 0} \mathbf{M W} / \mathbf{m}^{2} \mathbf{K}$} \\
\hline $\mathbf{S N P}$ & $\mu-\left[\mathbf{M W} / \mathbf{m}^{2} \mathbf{K}\right]$ & $\sigma-\left[\mathbf{M W} / \mathbf{m}^{2} \mathbf{K}\right]$ & $\mathbf{S N P}$ & $\mu-\left[\mathbf{M W} / \mathbf{m}^{2} \mathbf{K}\right]$ & $\sigma-\left[\mathbf{M W} / \mathbf{m}^{2} \mathbf{K}\right]$ \\
5.38 & $46.39 \pm 0.198$ & $3.20 \pm 0.0089$ & 34.62 & $46.38 \pm 0.030$ & $0.48 \pm 0.0013$ \\
8.09 & $46.35 \pm 0.129$ & $2.08 \pm 0.0058$ & 37.08 & $46.41 \pm 0.029$ & $0.48 \pm 0.0013$ \\
10.67 & $46.46 \pm 0.095$ & $1.54 \pm 0.0043$ & 39.74 & $46.38 \pm 0.026$ & $0.42 \pm 0.0012$ \\
13.35 & $46.47 \pm 0.086$ & $1.38 \pm 0.0038$ & 42.64 & $46.43 \pm 0.024$ & $0.39 \pm 0.0011$ \\
16.04 & $46.44 \pm 0.066$ & $1.07 \pm 0.0030$ & 45.00 & $46.42 \pm 0.023$ & $0.38 \pm 0.0010$ \\
18.60 & $46.43 \pm 0.058$ & $0.93 \pm 0.0026$ & 47.57 & $46.40 \pm 0.023$ & $0.37 \pm 0.0010$ \\
21.40 & $46.42 \pm 0.049$ & $0.79 \pm 0.0022$ & 50.33 & $46.42 \pm 0.022$ & $0.35 \pm 0.0010$ \\
24.07 & $46.44 \pm 0.044$ & $0.72 \pm 0.0020$ & 52.65 & $46.41 \pm 0.021$ & $0.33 \pm 0.0009$ \\
26.48 & $46.41 \pm 0.041$ & $0.67 \pm 0.0019$ & 55.02 & $46.40 \pm 0.019$ & $0.31 \pm 0.0009$ \\
29.25 & $46.41 \pm 0.035$ & $0.57 \pm 0.0016$ & 57.85 & $46.40 \pm 0.018$ & $0.29 \pm 0.0008$ \\
31.92 & $46.40 \pm 0.033$ & $0.54 \pm 0.0015$ & & & \\
\hline
\end{tabular}

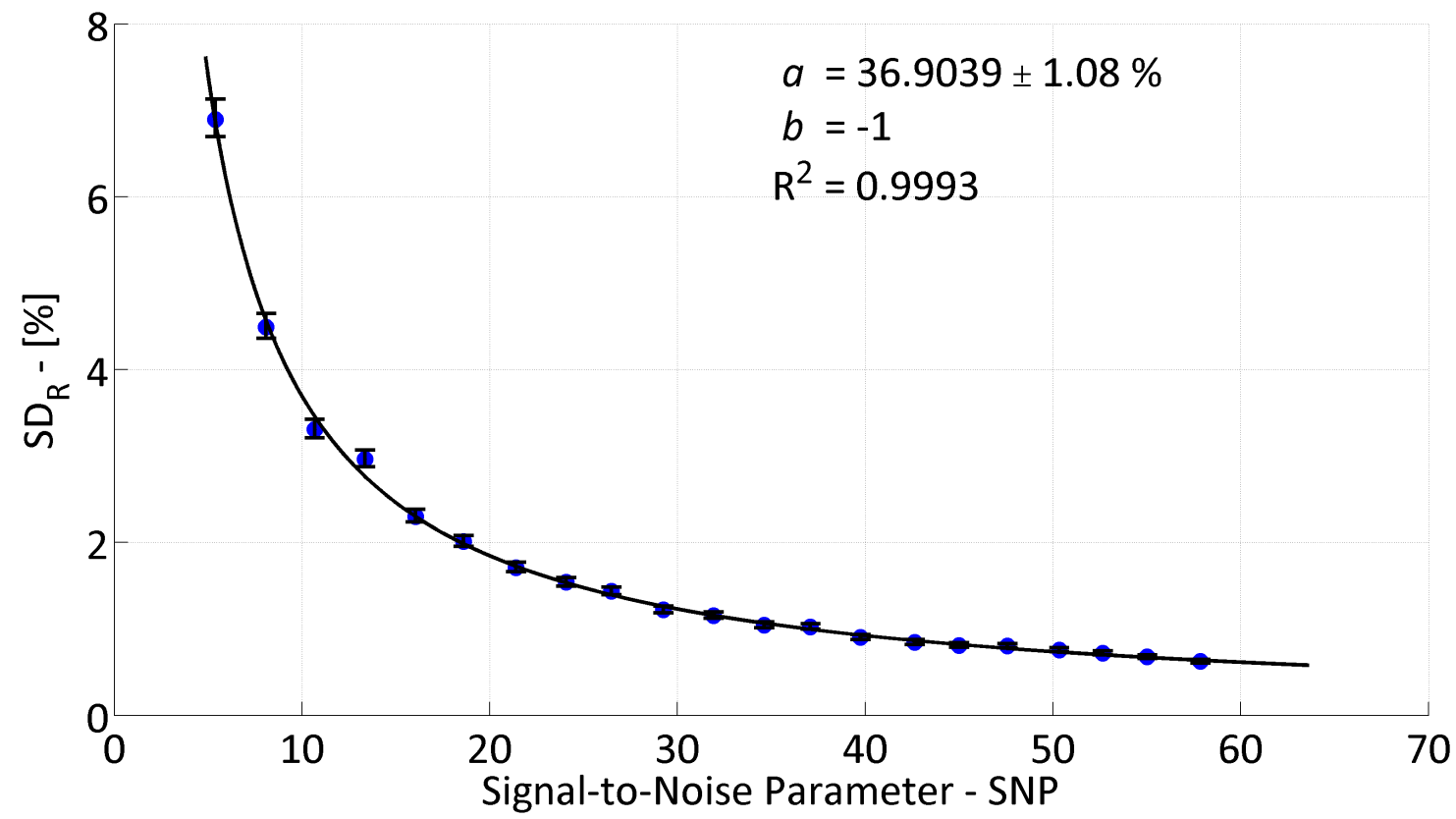

Figure S.87: Relative population standard deviation as a function of signal-to-noise parameter for 100 $\mathrm{nm}$ of $\mathrm{Al} / \mathrm{Si}$ with $h_{\mathrm{BD}}=46.40 \mathrm{MW} / \mathrm{m}^{2} \mathrm{~K}$. Error bars represent a $95 \%$ confidence interval of the measured $\mathrm{SD}_{\mathrm{R}}$ and the solid line represents a best fit of a power law to the data, of the form $\mathrm{SD}_{\mathrm{R}}=a \times \mathrm{SNP}^{b}$, with parameter $a$ provided for a fixed $b=-1$, along with the $95 \%$ confidence level in $a$. 


\section{S.19.2 Fitting Substrate Thermal Conductivity $-k_{S}$}

Table S.27: Summary of simulation data - $100 \mathrm{~nm} \mathrm{Al/Si} \mathrm{46.40} \mathrm{MW} \mathrm{Series.} \mathrm{Error} \mathrm{bounds} \mathrm{represent} \mathrm{a} 95 \%$ confidence level.

\begin{tabular}{cccccc}
\hline \multicolumn{6}{c}{$\mathbf{1 0 0} \mathbf{~ n m ~ A l ~ o n ~} \mathbf{~ S i}, h_{\mathrm{BD}}=\mathbf{4 6 . 4 0} \mathbf{M W} / \mathbf{m}^{2} \mathbf{K}$} \\
\hline $\mathbf{S N P}$ & $\mu-[\mathbf{W} / \mathbf{m K}]$ & $\sigma-[\mathbf{W} / \mathbf{m K}]$ & $\mathbf{S N P}$ & $\mu-[\mathbf{W} / \mathbf{m K}]$ & $\sigma-[\mathbf{W} / \mathbf{m K}]$ \\
5.38 & $166.43 \pm 5.566$ & $88.75 \pm 0.2525$ & 34.62 & $147.73 \pm 0.730$ & $11.76 \pm 0.0328$ \\
8.09 & $155.50 \pm 3.390$ & $54.41 \pm 0.1528$ & 37.08 & $148.36 \pm 0.727$ & $11.71 \pm 0.0326$ \\
10.67 & $154.49 \pm 2.483$ & $39.96 \pm 0.1116$ & 39.74 & $147.62 \pm 0.629$ & $10.14 \pm 0.0282$ \\
13.35 & $153.87 \pm 2.242$ & $36.09 \pm 0.1007$ & 42.64 & $148.55 \pm 0.597$ & $9.62 \pm 0.0268$ \\
16.04 & $150.86 \pm 1.659$ & $26.70 \pm 0.0745$ & 45.00 & $148.23 \pm 0.563$ & $9.07 \pm 0.0253$ \\
18.60 & $150.29 \pm 1.451$ & $23.38 \pm 0.0651$ & 47.57 & $147.79 \pm 0.550$ & $8.85 \pm 0.0247$ \\
21.40 & $149.78 \pm 1.247$ & $20.10 \pm 0.0560$ & 50.33 & $148.32 \pm 0.527$ & $8.49 \pm 0.0237$ \\
24.07 & $149.70 \pm 1.095$ & $17.64 \pm 0.0492$ & 52.65 & $148.10 \pm 0.502$ & $8.08 \pm 0.0225$ \\
26.48 & $148.98 \pm 1.023$ & $16.48 \pm 0.0459$ & 55.02 & $147.86 \pm 0.475$ & $7.66 \pm 0.0213$ \\
29.25 & $148.37 \pm 0.870$ & $14.02 \pm 0.0391$ & 57.85 & $147.69 \pm 0.433$ & $6.98 \pm 0.0194$ \\
31.92 & $148.27 \pm 0.824$ & $13.28 \pm 0.0370$ & & & \\
\hline
\end{tabular}

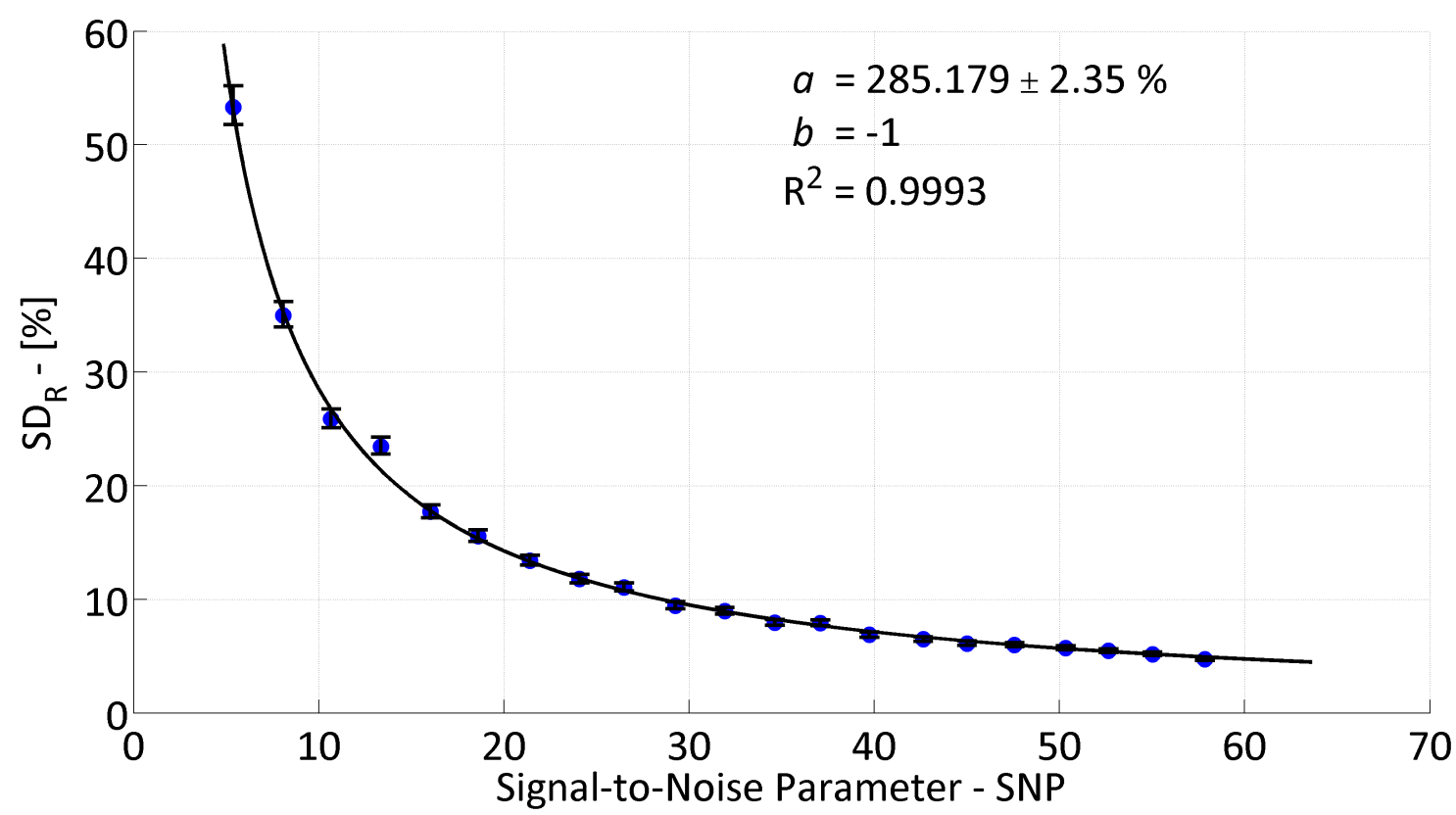

Figure S.88: Relative population standard deviation as a function of signal-to-noise parameter for 100 $\mathrm{nm}$ of $\mathrm{Al} / \mathrm{Si}$ with $h_{\mathrm{BD}}=46.40 \mathrm{MW} / \mathrm{m}^{2} \mathrm{~K}$. Error bars represent a $95 \%$ confidence interval of the measured $\mathrm{SD}_{\mathrm{R}}$ and the solid line represents a best fit of a power law to the data, of the form $\mathrm{SD}_{\mathrm{R}}=a \times \mathrm{SNP}^{b}$, with parameter $a$ provided for a fixed $b=-1$, along with the $95 \%$ confidence level in $a$. 


\section{S.19.3 Fitting Film Thickness $-d_{1}$}

Table S.28: Summary of simulation data - $100 \mathrm{~nm} \mathrm{Al/Si} 46.40 \mathrm{MW}$ Series. Error bounds represent a $95 \%$ confidence level.

\begin{tabular}{cccccc}
\hline \multicolumn{6}{c}{$\mathbf{1 0 0} \mathbf{~ n m ~} \mathbf{A l} \mathbf{~ o n ~} \mathbf{~ S i}, h_{\mathrm{BD}}=\mathbf{4 6 . 4 0} \mathbf{M W} / \mathbf{m}^{2} \mathbf{K}$} \\
\hline $\mathbf{S N P}$ & $\mu-[\mathbf{n m}]$ & $\sigma-[\mathbf{n m}]$ & $\mathbf{S N P}$ & $\mu-[\mathbf{n m}]$ & $\sigma-[\mathbf{n m}]$ \\
5.38 & $100.45 \pm 0.340$ & $5.48 \pm 0.0153$ & 34.62 & $100.07 \pm 0.052$ & $0.83 \pm 0.0023$ \\
8.09 & $100.28 \pm 0.222$ & $3.57 \pm 0.0100$ & 37.08 & $100.03 \pm 0.051$ & $0.81 \pm 0.0023$ \\
10.67 & $100.03 \pm 0.163$ & $2.62 \pm 0.0073$ & 39.74 & $100.07 \pm 0.044$ & $0.72 \pm 0.0020$ \\
13.35 & $99.99 \pm 0.145$ & $2.34 \pm 0.0065$ & 42.64 & $99.99 \pm 0.042$ & $0.67 \pm 0.0019$ \\
16.04 & $100.00 \pm 0.112$ & $1.81 \pm 0.0050$ & 45.00 & $100.01 \pm 0.040$ & $0.64 \pm 0.0018$ \\
18.60 & $100.02 \pm 0.099$ & $1.59 \pm 0.0044$ & 47.57 & $100.03 \pm 0.040$ & $0.64 \pm 0.0018$ \\
21.40 & $100.01 \pm 0.084$ & $1.35 \pm 0.0038$ & 50.33 & $100.00 \pm 0.037$ & $0.60 \pm 0.0017$ \\
24.07 & $99.98 \pm 0.076$ & $1.23 \pm 0.0034$ & 52.65 & $100.01 \pm 0.036$ & $0.57 \pm 0.0016$ \\
26.48 & $100.02 \pm 0.071$ & $1.15 \pm 0.0032$ & 55.02 & $100.03 \pm 0.033$ & $0.54 \pm 0.0015$ \\
29.25 & $100.04 \pm 0.060$ & $0.97 \pm 0.0027$ & 57.85 & $100.04 \pm 0.031$ & $0.50 \pm 0.0014$ \\
31.92 & $100.04 \pm 0.057$ & $0.92 \pm 0.0026$ & & & \\
\hline
\end{tabular}

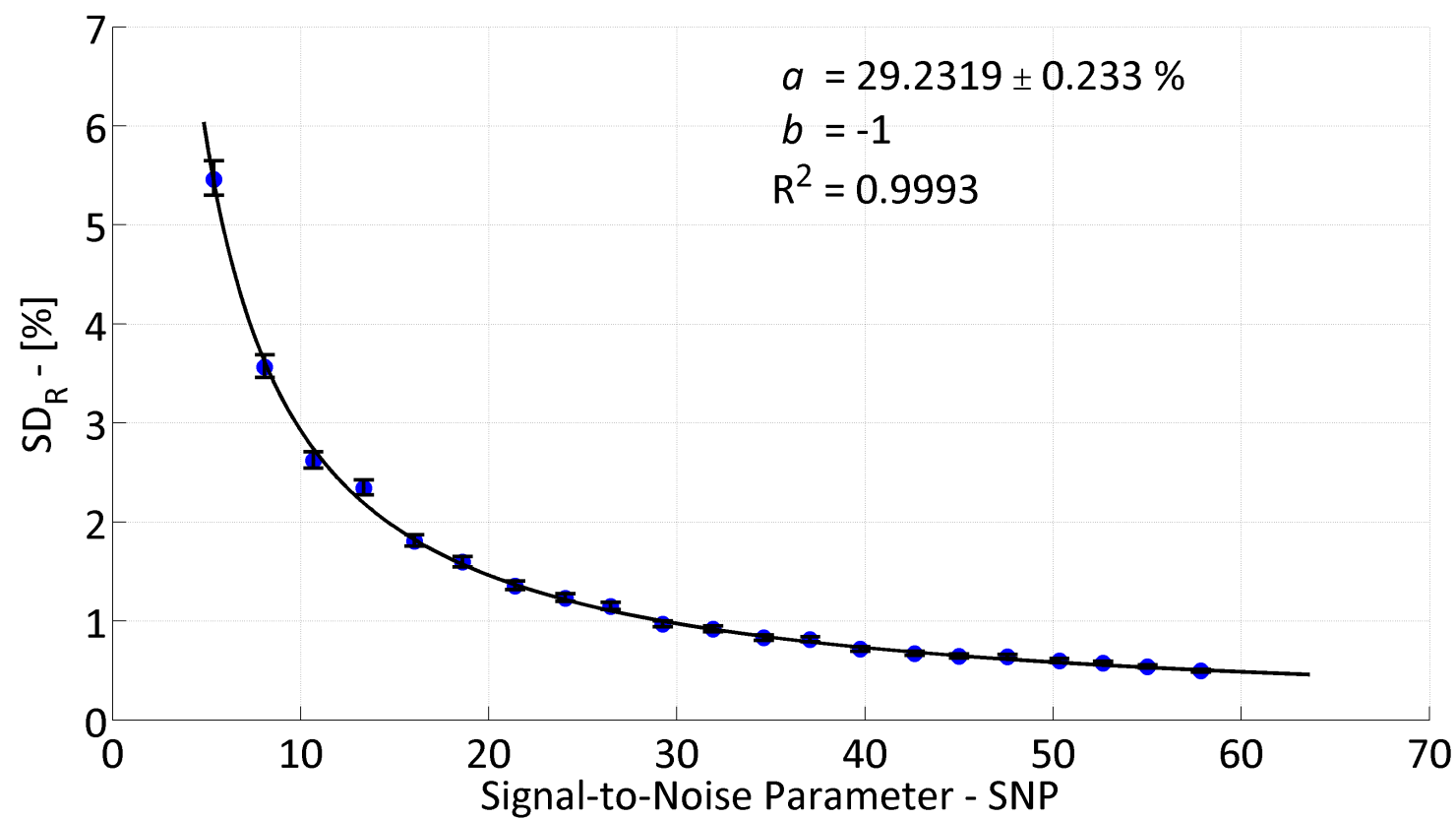

Figure S.89: Relative population standard deviation as a function of signal-to-noise parameter for 100 $\mathrm{nm}$ of $\mathrm{Al} / \mathrm{Si}$ with $h_{\mathrm{BD}}=46.40 \mathrm{MW} / \mathrm{m}^{2} \mathrm{~K}$. Error bars represent a $95 \%$ confidence interval of the measured $\mathrm{SD}_{\mathrm{R}}$ and the solid line represents a best fit of a power law to the data, of the form $\mathrm{SD}_{\mathrm{R}}=a \times \mathrm{SNP}^{b}$, with parameter $a$ provided for a fixed $b=-1$, along with the $95 \%$ confidence level in $a$. 


\section{S.20 Simulation Summary - Al/Si 59.90 MW Series}

\section{S.20.1 Fitting Film-to-Substrate Boundary Conductance - $h_{\mathrm{BD}}$}

Table S.29: Summary of simulation data - $100 \mathrm{~nm} \mathrm{Al/Si} \mathrm{59.90} \mathrm{MW} \mathrm{Series.} \mathrm{Error} \mathrm{bounds} \mathrm{represent} \mathrm{a} 95 \%$ confidence level.

\begin{tabular}{cccccc}
\hline \multicolumn{5}{c}{$\mathbf{1 0 0} \mathbf{~ n m ~ A l ~ o n ~} \mathbf{~ i}, h_{\mathrm{BD}}=\mathbf{5 9 . 9 0} \mathbf{M W} / \mathbf{m}^{2} \mathbf{K}$} \\
\hline $\mathbf{S N P}$ & $\mu-\left[\mathbf{M W} / \mathbf{m}^{2} \mathbf{K}\right]$ & $\sigma-\left[\mathbf{M W} / \mathbf{m}^{2} \mathbf{K}\right]$ & $\mathbf{S N P}$ & $\mu-\left[\mathbf{M W} / \mathbf{m}^{2} \mathbf{K}\right]$ & $\sigma-\left[\mathbf{M W} / \mathbf{m}^{2} \mathbf{K}\right]$ \\
4.94 & $60.05 \pm 0.259$ & $4.17 \pm 0.0116$ & 30.94 & $59.89 \pm 0.041$ & $0.66 \pm 0.0018$ \\
7.38 & $59.85 \pm 0.165$ & $2.67 \pm 0.0074$ & 33.70 & $59.91 \pm 0.038$ & $0.61 \pm 0.0017$ \\
9.83 & $59.97 \pm 0.129$ & $2.08 \pm 0.0058$ & 35.81 & $59.91 \pm 0.035$ & $0.56 \pm 0.0016$ \\
12.21 & $60.05 \pm 0.102$ & $1.65 \pm 0.0046$ & 38.48 & $59.91 \pm 0.033$ & $0.53 \pm 0.0015$ \\
14.78 & $59.86 \pm 0.082$ & $1.33 \pm 0.0037$ & 40.76 & $59.90 \pm 0.031$ & $0.49 \pm 0.0014$ \\
16.91 & $59.91 \pm 0.074$ & $1.20 \pm 0.0033$ & 42.51 & $59.92 \pm 0.029$ & $0.46 \pm 0.0013$ \\
19.39 & $59.93 \pm 0.066$ & $1.07 \pm 0.0030$ & 45.49 & $59.90 \pm 0.027$ & $0.44 \pm 0.0012$ \\
21.74 & $59.92 \pm 0.056$ & $0.90 \pm 0.0025$ & 47.95 & $59.92 \pm 0.026$ & $0.42 \pm 0.0012$ \\
24.03 & $59.92 \pm 0.052$ & $0.85 \pm 0.0024$ & 48.99 & $59.91 \pm 0.026$ & $0.42 \pm 0.0012$ \\
26.59 & $59.93 \pm 0.048$ & $0.78 \pm 0.0022$ & 52.46 & $59.91 \pm 0.023$ & $0.37 \pm 0.0010$ \\
28.69 & $59.91 \pm 0.042$ & $0.68 \pm 0.0019$ & & & \\
\hline
\end{tabular}

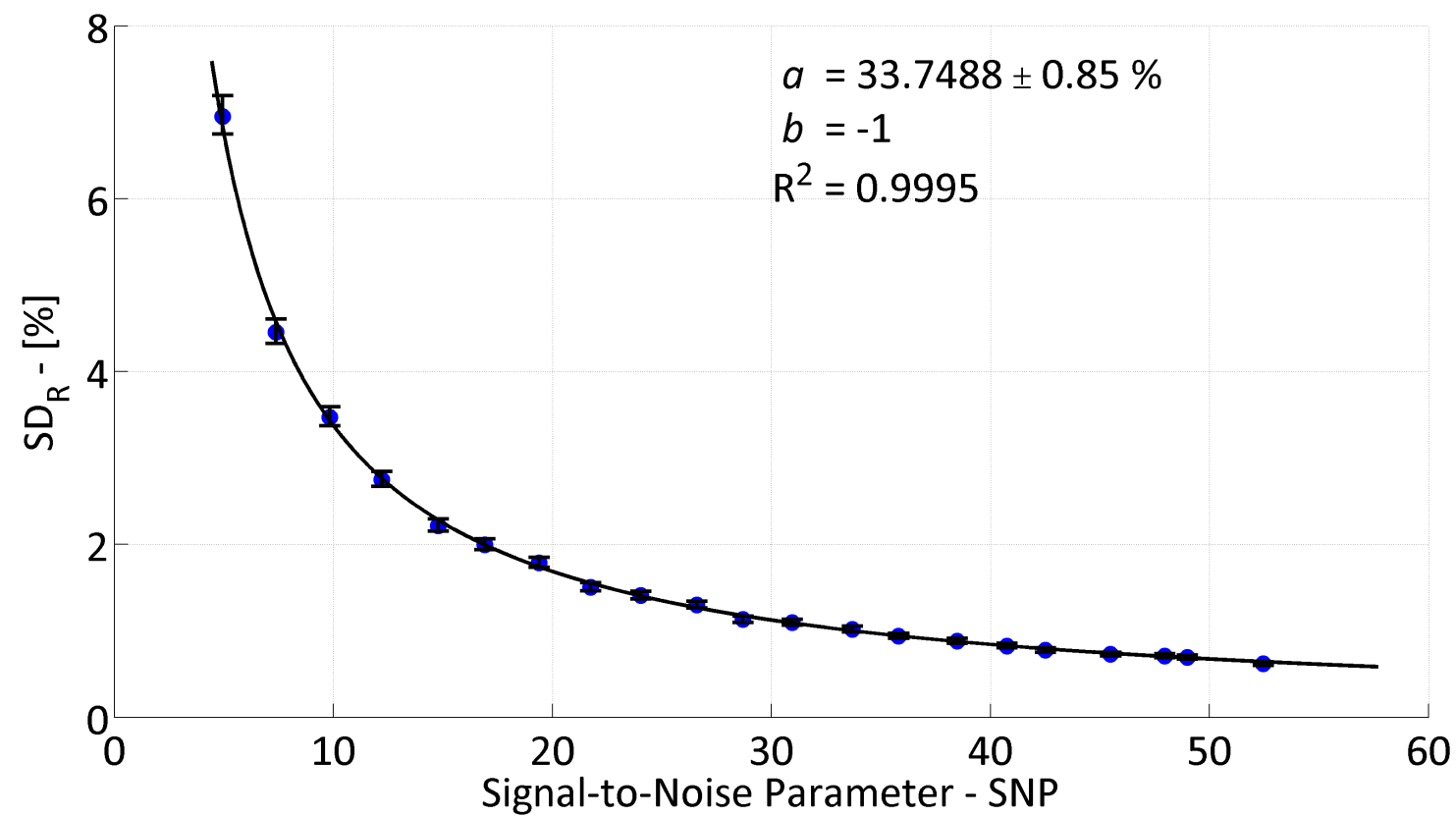

Figure S.90: Relative population standard deviation as a function of signal-to-noise parameter for 100 $\mathrm{nm}$ of $\mathrm{Al} / \mathrm{Si}$ with $h_{\mathrm{BD}}=59.90 \mathrm{MW} / \mathrm{m}^{2} \mathrm{~K}$. Error bars represent a $95 \%$ confidence interval of the measured $\mathrm{SD}_{\mathrm{R}}$ and the solid line represents a best fit of a power law to the data, of the form $\mathrm{SD}_{\mathrm{R}}=a \times \mathrm{SNP}^{b}$, with parameter $a$ provided for a fixed $b=-1$, along with the $95 \%$ confidence level in $a$. 


\section{S.20.2 Fitting Substrate Thermal Conductivity $-k_{S}$}

Table S.30: Summary of simulation data - $100 \mathrm{~nm} \mathrm{Al/Si} 59.90 \mathrm{MW}$ Series. Error bounds represent a $95 \%$ confidence level.

\begin{tabular}{cccccc}
\hline \multicolumn{6}{c}{$\mathbf{1 0 0} \mathbf{~ n m ~ A l ~ o n ~} \mathbf{~} i, h_{\mathrm{BD}}=\mathbf{5 9 . 9 0} \mathbf{M W} / \mathbf{m}^{2} \mathbf{K}$} \\
\hline SNP & $\mu-[\mathbf{W} / \mathbf{m K}]$ & $\sigma-[\mathbf{W} / \mathbf{m K}]$ & $\mathbf{S N P}$ & $\mu-[\mathbf{W} / \mathbf{m K}]$ & $\sigma-[\mathbf{W} / \mathbf{m K}]$ \\
4.94 & $158.08 \pm 3.798$ & $60.80 \pm 0.1716$ & 30.94 & $147.63 \pm 0.545$ & $8.78 \pm 0.0244$ \\
7.38 & $151.50 \pm 2.345$ & $37.69 \pm 0.1055$ & 33.70 & $147.79 \pm 0.511$ & $8.23 \pm 0.0229$ \\
9.83 & $151.29 \pm 1.808$ & $29.12 \pm 0.0812$ & 35.81 & $147.72 \pm 0.473$ & $7.62 \pm 0.0212$ \\
12.21 & $151.17 \pm 1.413$ & $22.73 \pm 0.0635$ & 38.48 & $147.69 \pm 0.441$ & $7.11 \pm 0.0198$ \\
14.78 & $147.90 \pm 1.106$ & $17.81 \pm 0.0497$ & 40.76 & $147.44 \pm 0.417$ & $6.72 \pm 0.0187$ \\
16.91 & $148.68 \pm 1.025$ & $16.51 \pm 0.0460$ & 42.51 & $147.78 \pm 0.388$ & $6.25 \pm 0.0174$ \\
19.39 & $148.62 \pm 0.917$ & $14.78 \pm 0.0412$ & 45.49 & $147.53 \pm 0.367$ & $5.92 \pm 0.0165$ \\
21.74 & $148.13 \pm 0.761$ & $12.26 \pm 0.0342$ & 47.95 & $147.72 \pm 0.356$ & $5.74 \pm 0.0160$ \\
24.03 & $147.99 \pm 0.707$ & $11.39 \pm 0.0317$ & 48.99 & $147.59 \pm 0.349$ & $5.62 \pm 0.0156$ \\
26.59 & $148.18 \pm 0.665$ & $10.72 \pm 0.0298$ & 52.46 & $147.57 \pm 0.310$ & $5.00 \pm 0.0139$ \\
28.69 & $147.93 \pm 0.566$ & $9.12 \pm 0.0254$ & & & \\
\hline
\end{tabular}

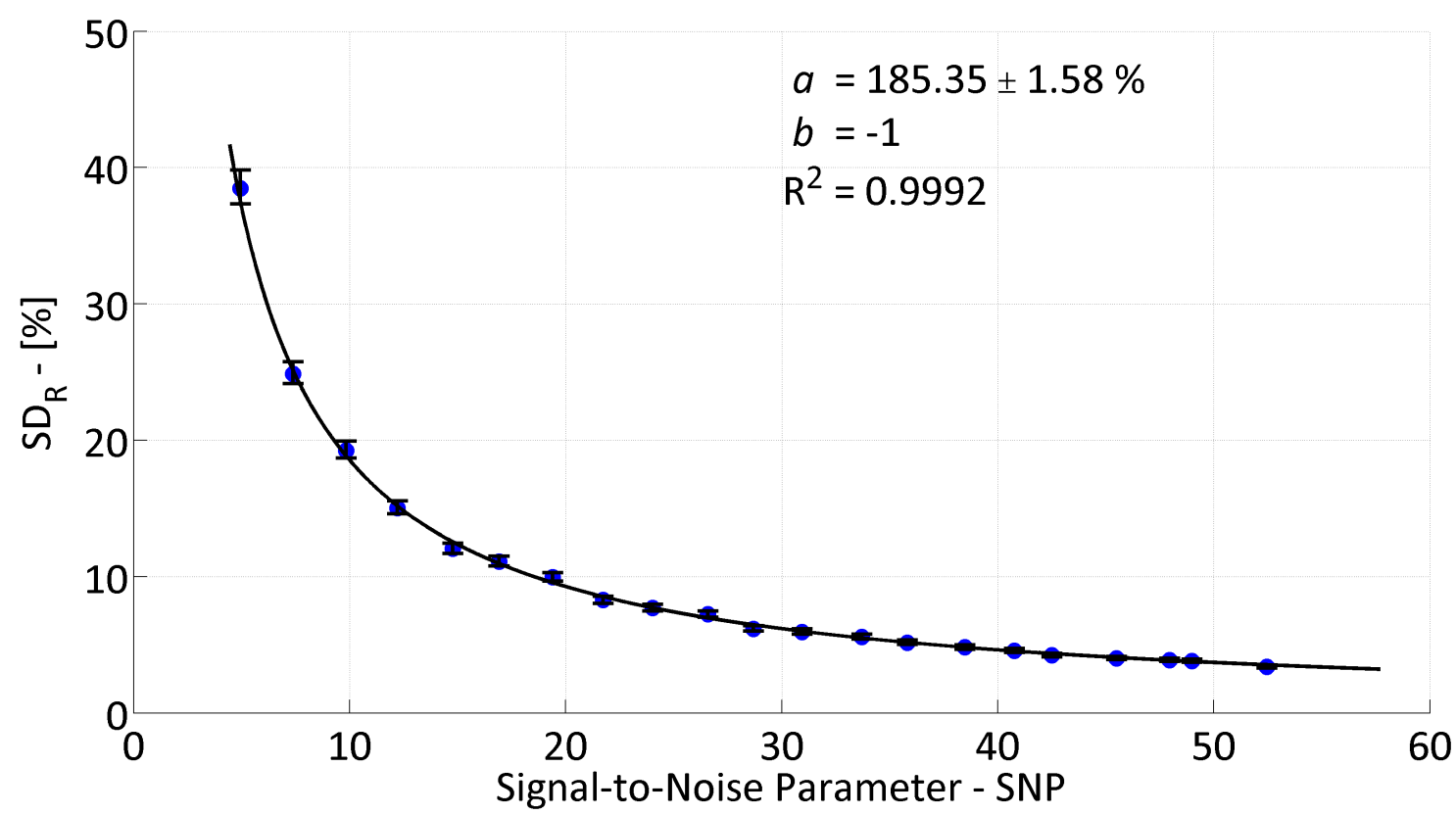

Figure S.91: Relative population standard deviation as a function of signal-to-noise parameter for 100 $\mathrm{nm}$ of $\mathrm{Al} / \mathrm{Si}$ with $h_{\mathrm{BD}}=59.90 \mathrm{MW} / \mathrm{m}^{2} \mathrm{~K}$. Error bars represent a $95 \%$ confidence interval of the measured $\mathrm{SD}_{\mathrm{R}}$ and the solid line represents a best fit of a power law to the data, of the form $\mathrm{SD}_{\mathrm{R}}=a \times \mathrm{SNP}^{b}$, with parameter $a$ provided for a fixed $b=-1$, along with the $95 \%$ confidence level in $a$. 


\section{S.21 Simulation Summary - Al/Si 77.40 MW Series}

\section{S.21.1 Fitting Film-to-Substrate Boundary Conductance $-h_{\mathrm{BD}}$}

Table S.31: Summary of simulation data - $100 \mathrm{~nm} \mathrm{Al/Si} \mathrm{77.40} \mathrm{MW} \mathrm{Series.} \mathrm{Error} \mathrm{bounds} \mathrm{represent} \mathrm{a} 95 \%$ confidence level.

\begin{tabular}{cccccc}
\hline \multicolumn{5}{c}{$\mathbf{1 0 0} \mathbf{~ n m ~ A l ~ o n ~} \mathbf{~ i}, h_{\mathrm{BD}}=\mathbf{7 7 . 4 0} \mathbf{M W} / \mathbf{m}^{2} \mathbf{K}$} \\
\hline $\mathbf{S N P}$ & $\mu-\left[\mathbf{M W} / \mathbf{m}^{2} \mathbf{K}\right]$ & $\sigma-\left[\mathbf{M W} / \mathbf{m}^{2} \mathbf{K}\right]$ & $\mathbf{S N P}$ & $\mu-\left[\mathbf{M W} / \mathbf{m}^{2} \mathbf{K}\right]$ & $\sigma-\left[\mathbf{M W} / \mathbf{m}^{2} \mathbf{K}\right]$ \\
3.84 & $77.75 \pm 0.414$ & $6.67 \pm 0.0186$ & 24.89 & $77.42 \pm 0.064$ & $1.02 \pm 0.0029$ \\
5.75 & $77.70 \pm 0.267$ & $4.30 \pm 0.0120$ & 26.90 & $77.42 \pm 0.059$ & $0.94 \pm 0.0026$ \\
7.69 & $77.50 \pm 0.204$ & $3.29 \pm 0.0092$ & 28.05 & $77.41 \pm 0.054$ & $0.88 \pm 0.0024$ \\
9.63 & $77.47 \pm 0.164$ & $2.64 \pm 0.0073$ & 30.47 & $77.41 \pm 0.052$ & $0.84 \pm 0.0023$ \\
11.38 & $77.46 \pm 0.142$ & $2.28 \pm 0.0064$ & 32.29 & $77.45 \pm 0.049$ & $0.78 \pm 0.0022$ \\
13.31 & $77.44 \pm 0.118$ & $1.91 \pm 0.0053$ & 33.69 & $77.46 \pm 0.044$ & $0.71 \pm 0.0020$ \\
15.31 & $77.41 \pm 0.101$ & $1.63 \pm 0.0045$ & 36.08 & $77.44 \pm 0.042$ & $0.68 \pm 0.0019$ \\
17.05 & $77.39 \pm 0.087$ & $1.41 \pm 0.0039$ & 37.62 & $77.42 \pm 0.040$ & $0.65 \pm 0.0018$ \\
18.94 & $77.48 \pm 0.079$ & $1.28 \pm 0.0036$ & 39.78 & $77.39 \pm 0.040$ & $0.64 \pm 0.0018$ \\
20.97 & $77.37 \pm 0.075$ & $1.20 \pm 0.0033$ & 41.72 & $77.41 \pm 0.038$ & $0.62 \pm 0.0017$ \\
22.85 & $77.35 \pm 0.068$ & $1.10 \pm 0.0031$ & & & \\
\hline
\end{tabular}

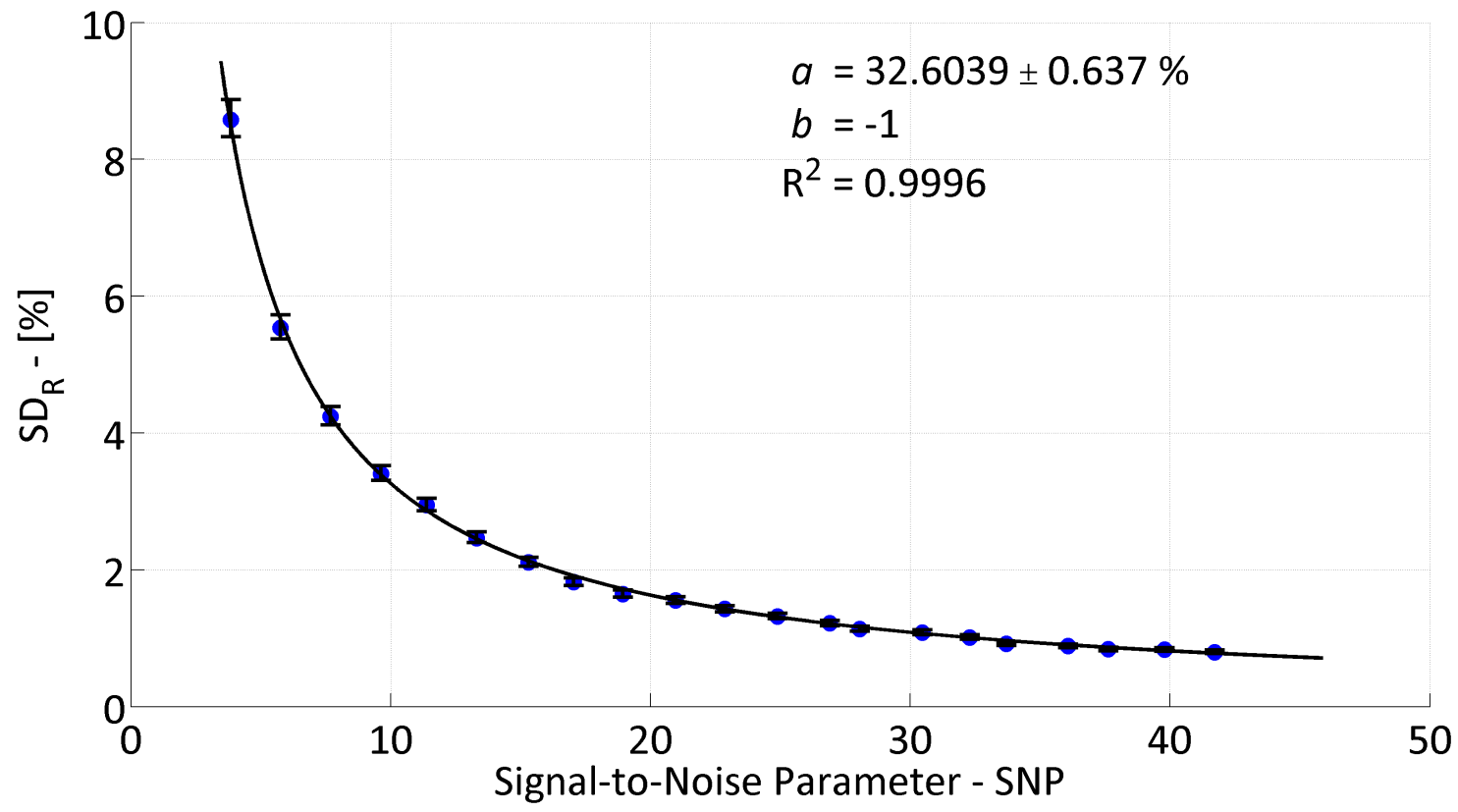

Figure S.92: Relative population standard deviation as a function of signal-to-noise parameter for 100 $\mathrm{nm}$ of $\mathrm{Al} / \mathrm{Si}$ with $h_{\mathrm{BD}}=77.40 \mathrm{MW} / \mathrm{m}^{2} \mathrm{~K}$. Error bars represent a $95 \%$ confidence interval of the measured $\mathrm{SD}_{\mathrm{R}}$ and the solid line represents a best fit of a power law to the data, of the form $\mathrm{SD}_{\mathrm{R}}=a \times \mathrm{SNP}^{b}$, with parameter $a$ provided for a fixed $b=-1$, along with the $95 \%$ confidence level in $a$. 


\section{S.21.2 Fitting Substrate Thermal Conductivity $-k_{S}$}

Table S.32: Summary of simulation data - $100 \mathrm{~nm} \mathrm{Al/Si} \mathrm{77.40} \mathrm{MW} \mathrm{Series.} \mathrm{Error} \mathrm{bounds} \mathrm{represent} \mathrm{a} 95 \%$ confidence level.

\begin{tabular}{cccccc}
\hline \multicolumn{6}{c}{$\mathbf{1 0 0} \mathbf{~ n m ~ A l ~ o n ~} \mathbf{~ i}, h_{\mathrm{BD}}=\mathbf{7 7 . 4 0} \mathbf{M W} / \mathbf{m}^{2} \mathbf{K}$} \\
\hline SNP & $\mu-[\mathbf{W} / \mathbf{m K}]$ & $\sigma-[\mathbf{W} / \mathbf{m K}]$ & $\mathbf{S N P}$ & $\mu-[\mathbf{W} / \mathbf{m K}]$ & $\sigma-[\mathbf{W} / \mathbf{m K}]$ \\
3.84 & $158.32 \pm 3.345$ & $53.79 \pm 0.1504$ & 24.89 & $148.14 \pm 0.472$ & $7.60 \pm 0.0212$ \\
5.75 & $152.88 \pm 2.068$ & $33.25 \pm 0.0930$ & 26.90 & $148.09 \pm 0.434$ & $6.99 \pm 0.0195$ \\
7.69 & $150.53 \pm 1.546$ & $24.92 \pm 0.0694$ & 28.05 & $148.04 \pm 0.407$ & $6.55 \pm 0.0182$ \\
9.63 & $149.47 \pm 1.246$ & $20.08 \pm 0.0559$ & 30.47 & $147.92 \pm 0.385$ & $6.21 \pm 0.0173$ \\
11.38 & $149.12 \pm 1.070$ & $17.24 \pm 0.0481$ & 32.29 & $148.22 \pm 0.358$ & $5.78 \pm 0.0161$ \\
13.31 & $148.63 \pm 0.879$ & $14.15 \pm 0.0394$ & 33.69 & $148.36 \pm 0.330$ & $5.32 \pm 0.0148$ \\
15.31 & $148.33 \pm 0.758$ & $12.21 \pm 0.0340$ & 36.08 & $148.17 \pm 0.313$ & $5.04 \pm 0.0140$ \\
17.05 & $148.08 \pm 0.649$ & $10.46 \pm 0.0291$ & 37.62 & $147.98 \pm 0.302$ & $4.86 \pm 0.0135$ \\
18.94 & $148.66 \pm 0.587$ & $9.45 \pm 0.0263$ & 39.78 & $147.73 \pm 0.297$ & $4.78 \pm 0.0133$ \\
20.97 & $147.77 \pm 0.556$ & $8.97 \pm 0.0250$ & 41.72 & $147.97 \pm 0.286$ & $4.61 \pm 0.0128$ \\
22.85 & $147.75 \pm 0.510$ & $8.21 \pm 0.0229$ & & & \\
\hline
\end{tabular}

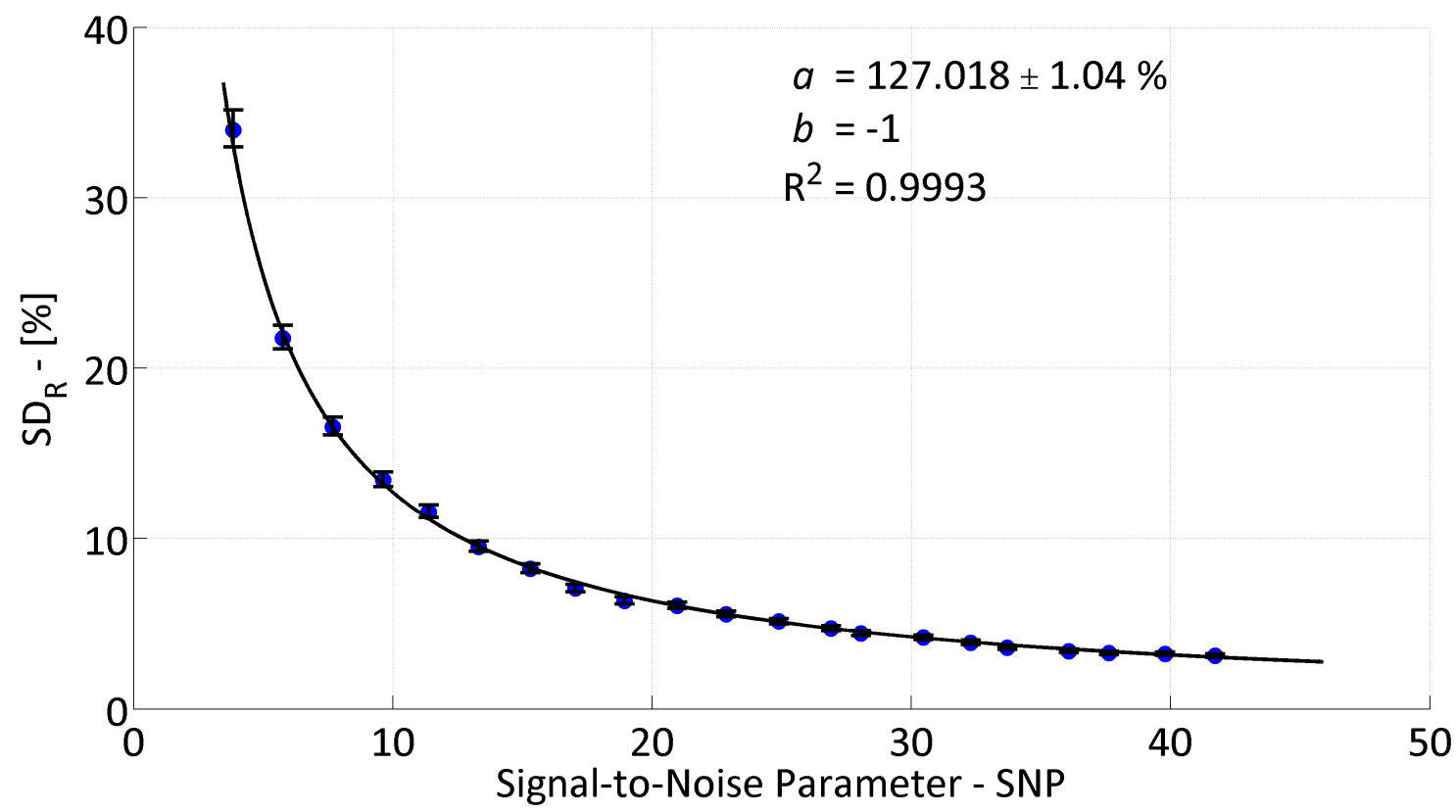

Figure S.93: Relative population standard deviation as a function of signal-to-noise parameter for 100 $\mathrm{nm}$ of $\mathrm{Al} / \mathrm{Si}$ with $h_{\mathrm{BD}}=77.40 \mathrm{MW} / \mathrm{m}^{2} \mathrm{~K}$. Error bars represent a $95 \%$ confidence interval of the measured $\mathrm{SD}_{\mathrm{R}}$ and the solid line represents a best fit of a power law to the data, of the form $\mathrm{SD}_{\mathrm{R}}=a \times \mathrm{SNP}^{b}$, with parameter $a$ provided for a fixed $b=-1$, along with the $95 \%$ confidence level in $a$. 


\section{S.22 Simulation Summary - Al/Si 100.00 MW Series}

\section{S.22.1 Fitting Film-to-Substrate Boundary Conductance - $h_{\mathrm{BD}}$}

Table S.33: Summary of simulation data - 100 nm Al/Si 100.00 MW Series. Error bounds represent a $95 \%$ confidence level.

\begin{tabular}{cccccc}
\hline \multicolumn{6}{c}{$\mathbf{1 0 0} \mathbf{~ n m ~} \mathbf{A l}$ on $\mathbf{~ S i}, h_{\mathrm{BD}}=\mathbf{1 0 0 . 0 0} \mathbf{M W} / \mathbf{m}^{2} \mathbf{K}$} \\
\hline $\mathbf{S N P}$ & $\mu-\left[\mathbf{M W} / \mathbf{m}^{2} \mathbf{K}\right]$ & $\sigma-\left[\mathbf{M W} / \mathbf{m}^{2} \mathbf{K}\right]$ & $\mathbf{S N P}$ & $\mu-\left[\mathbf{M W} / \mathbf{m}^{2} \mathbf{K}\right]$ & $\sigma-\left[\mathbf{M W} / \mathbf{m}^{2} \mathbf{K}\right]$ \\
3.22 & $100.36 \pm 0.656$ & $10.57 \pm 0.0295$ & 20.73 & $100.10 \pm 0.101$ & $1.63 \pm 0.0045$ \\
4.80 & $100.46 \pm 0.428$ & $6.90 \pm 0.0192$ & 22.12 & $100.08 \pm 0.096$ & $1.55 \pm 0.0043$ \\
6.35 & $100.37 \pm 0.332$ & $5.35 \pm 0.0149$ & 23.38 & $100.14 \pm 0.088$ & $1.42 \pm 0.0040$ \\
7.99 & $100.12 \pm 0.274$ & $4.42 \pm 0.0123$ & 25.37 & $100.05 \pm 0.080$ & $1.28 \pm 0.0036$ \\
9.58 & $100.09 \pm 0.220$ & $3.55 \pm 0.0099$ & 27.10 & $100.06 \pm 0.079$ & $1.28 \pm 0.0036$ \\
11.07 & $100.10 \pm 0.195$ & $3.13 \pm 0.0087$ & 28.54 & $100.02 \pm 0.075$ & $1.21 \pm 0.0034$ \\
12.74 & $100.09 \pm 0.167$ & $2.69 \pm 0.0075$ & 30.32 & $99.98 \pm 0.069$ & $1.12 \pm 0.0031$ \\
14.33 & $100.02 \pm 0.147$ & $2.36 \pm 0.0066$ & 31.60 & $100.03 \pm 0.066$ & $1.07 \pm 0.0030$ \\
15.98 & $100.07 \pm 0.140$ & $2.25 \pm 0.0063$ & 33.07 & $99.96 \pm 0.062$ & $1.00 \pm 0.0028$ \\
17.53 & $100.02 \pm 0.122$ & $1.97 \pm 0.0055$ & 35.01 & $100.06 \pm 0.059$ & $0.95 \pm 0.0027$ \\
18.90 & $99.97 \pm 0.110$ & $1.77 \pm 0.0049$ & & & \\
\hline
\end{tabular}

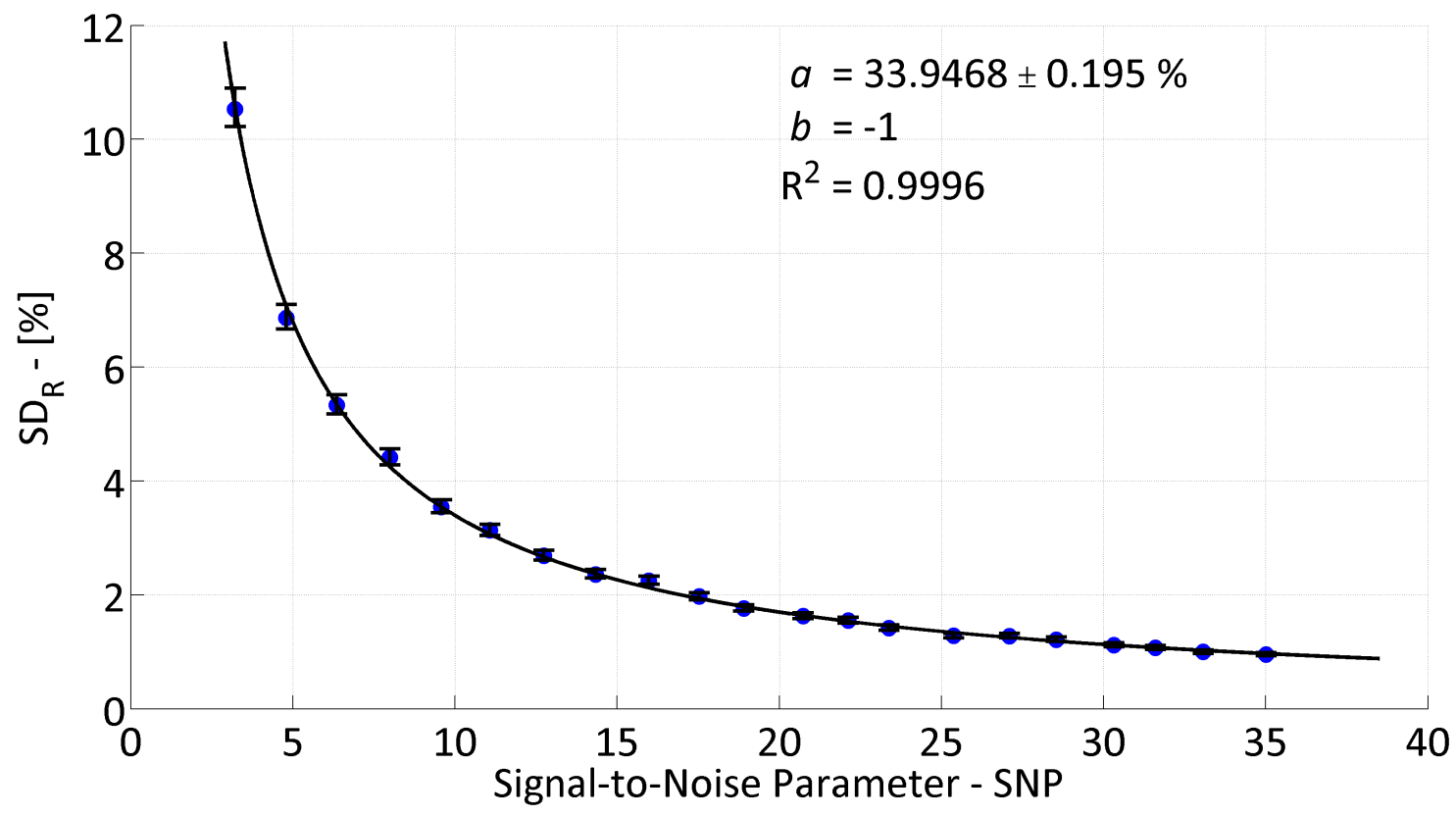

Figure S.94: Relative population standard deviation as a function of signal-to-noise parameter for $100 \mathrm{~nm}$ of $\mathrm{Al} / \mathrm{Si}$ with $h_{\mathrm{BD}}=100.00 \mathrm{MW} / \mathrm{m}^{2} \mathrm{~K}$. Error bars represent a $95 \%$ confidence interval of the measured $\mathrm{SD}_{\mathrm{R}}$ and the solid line represents a best fit of a power law to the data, of the form $\mathrm{SD}_{\mathrm{R}}=a \times \mathrm{SNP}^{b}$, with parameter $a$ provided for a fixed $b=-1$, along with the $95 \%$ confidence level in $a$. 


\section{S.22.2 Fitting Substrate Thermal Conductivity $-k_{S}$}

Table S.34: Summary of simulation data - $100 \mathrm{~nm} \mathrm{Al/Si} \mathrm{100.00} \mathrm{MW} \mathrm{Series.} \mathrm{Error} \mathrm{bounds} \mathrm{represent} \mathrm{a} 95 \%$ confidence level.

\begin{tabular}{cccccc}
\hline \multicolumn{6}{c}{$\mathbf{1 0 0} \mathbf{~ n m} \mathbf{A l}$ on $\mathbf{~ S i}, h_{\mathrm{BD}}=\mathbf{1 0 0 . 0 0} \mathbf{M W} / \mathbf{m}^{2} \mathbf{K}$} \\
\hline $\mathbf{S N P}$ & $\mu-[\mathbf{W} / \mathbf{m K}]$ & $\sigma-[\mathbf{W} / \mathbf{m K}]$ & $\mathbf{S N P}$ & $\mu-[\mathbf{W} / \mathbf{m K}]$ & $\sigma-[\mathbf{W} / \mathbf{m K}]$ \\
3.22 & $152.94 \pm 2.766$ & $44.46 \pm 0.1245$ & 20.73 & $148.51 \pm 0.402$ & $6.47 \pm 0.0180$ \\
4.80 & $151.88 \pm 1.779$ & $28.65 \pm 0.0799$ & 22.12 & $148.38 \pm 0.386$ & $6.22 \pm 0.0173$ \\
6.35 & $150.47 \pm 1.341$ & $21.58 \pm 0.0603$ & 23.38 & $148.65 \pm 0.351$ & $5.65 \pm 0.0157$ \\
7.99 & $149.28 \pm 1.097$ & $17.67 \pm 0.0493$ & 25.37 & $148.27 \pm 0.315$ & $5.07 \pm 0.0141$ \\
9.58 & $148.74 \pm 0.886$ & $14.28 \pm 0.0398$ & 27.10 & $148.26 \pm 0.313$ & $5.05 \pm 0.0141$ \\
11.07 & $148.78 \pm 0.785$ & $12.64 \pm 0.0352$ & 28.54 & $148.16 \pm 0.301$ & $4.85 \pm 0.0135$ \\
12.74 & $148.64 \pm 0.677$ & $10.90 \pm 0.0304$ & 30.32 & $147.99 \pm 0.275$ & $4.43 \pm 0.0123$ \\
14.33 & $148.21 \pm 0.582$ & $9.38 \pm 0.0261$ & 31.60 & $148.19 \pm 0.267$ & $4.30 \pm 0.0120$ \\
15.98 & $148.40 \pm 0.560$ & $9.03 \pm 0.0252$ & 33.07 & $147.90 \pm 0.247$ & $3.98 \pm 0.0111$ \\
17.53 & $148.29 \pm 0.489$ & $7.89 \pm 0.0220$ & 35.01 & $148.29 \pm 0.236$ & $3.81 \pm 0.0106$ \\
18.90 & $148.08 \pm 0.440$ & $7.09 \pm 0.0197$ & & & \\
\hline
\end{tabular}

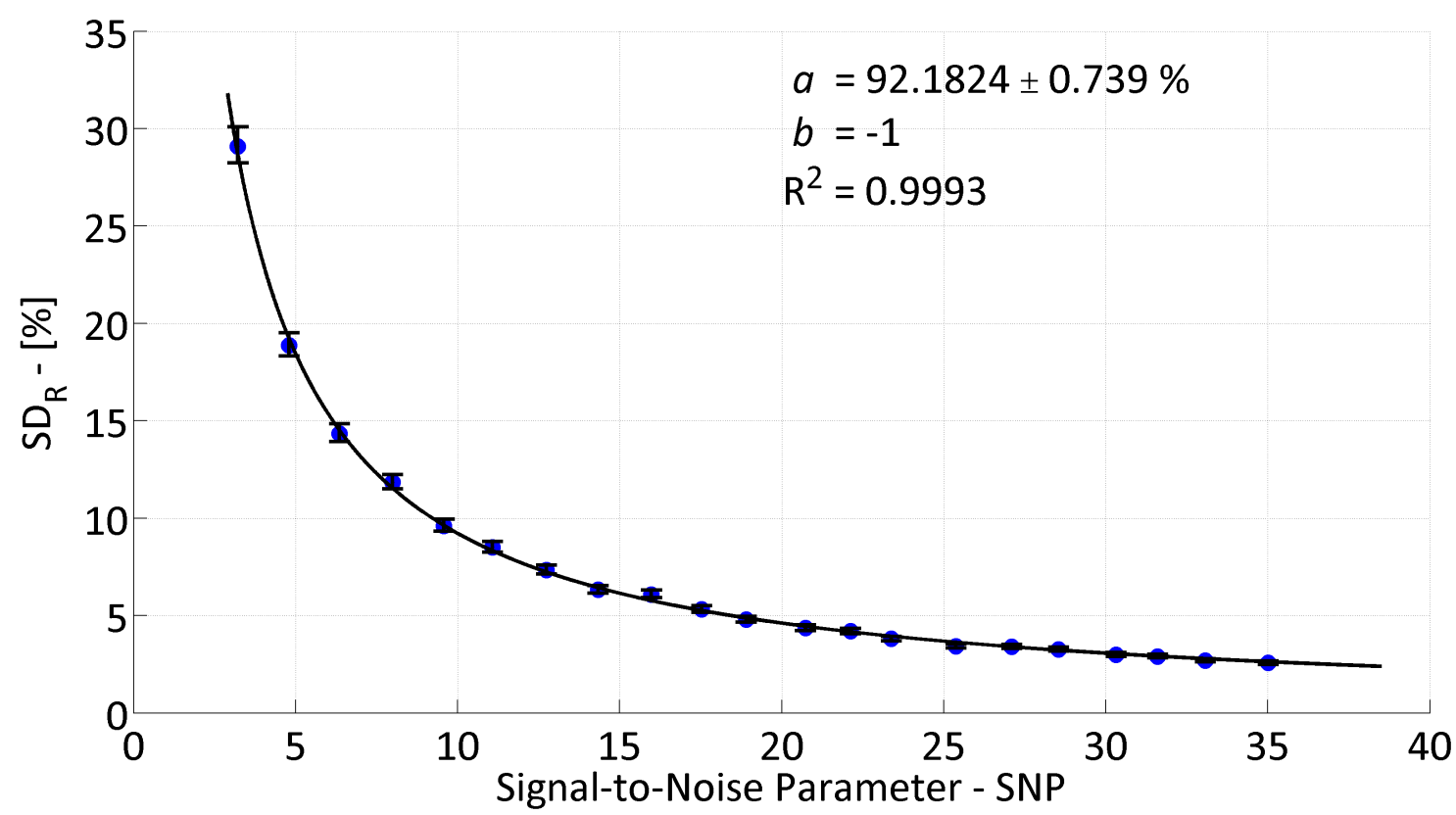

Figure S.95: Relative population standard deviation as a function of signal-to-noise parameter for $100 \mathrm{~nm}$ of $\mathrm{Al} / \mathrm{Si}$ with $h_{\mathrm{BD}}=100.00 \mathrm{MW} / \mathrm{m}^{2} \mathrm{~K}$. Error bars represent a $95 \%$ confidence interval of the measured $\mathrm{SD}_{\mathrm{R}}$ and the solid line represents a best fit of a power law to the data, of the form $\mathrm{SD}_{\mathrm{R}}=a \times \mathrm{SNP}^{b}$, with parameter $a$ provided for a fixed $b=-1$, along with the $95 \%$ confidence level in $a$. 


\section{S.22.3 Fitting Film Thickness $-d_{1}$}

Table S.35: Summary of simulation data - $100 \mathrm{~nm} \mathrm{Al/Si} \mathrm{100.00} \mathrm{MW} \mathrm{Series.} \mathrm{Error} \mathrm{bounds} \mathrm{represent} \mathrm{a} 95 \%$ confidence level.

\begin{tabular}{cccccc}
\hline \multicolumn{6}{c}{$\mathbf{1 0 0} \mathbf{~ n m} \mathbf{A l}$ on $\mathbf{~ S i}, h_{\mathrm{BD}}=\mathbf{1 0 0 . 0 0} \mathbf{M W} / \mathbf{m}^{2} \mathbf{K}$} \\
\hline $\mathbf{S N P}$ & $\mu-[\mathbf{n m}]$ & $\sigma-[\mathbf{n m}]$ & $\mathbf{S N P}$ & $\mu-[\mathbf{n m}]$ & $\sigma-[\mathbf{n m}]$ \\
3.22 & $100.48 \pm 0.392$ & $6.32 \pm 0.0176$ & 20.73 & $99.96 \pm 0.059$ & $0.95 \pm 0.0026$ \\
4.80 & $100.01 \pm 0.253$ & $4.08 \pm 0.0114$ & 22.12 & $99.97 \pm 0.056$ & $0.91 \pm 0.0025$ \\
6.35 & $99.95 \pm 0.194$ & $3.12 \pm 0.0087$ & 23.38 & $99.93 \pm 0.051$ & $0.83 \pm 0.0023$ \\
7.99 & $100.04 \pm 0.160$ & $2.58 \pm 0.0072$ & 25.37 & $99.98 \pm 0.046$ & $0.75 \pm 0.0021$ \\
9.58 & $100.03 \pm 0.129$ & $2.08 \pm 0.0058$ & 27.10 & $99.98 \pm 0.046$ & $0.75 \pm 0.0021$ \\
11.07 & $100.00 \pm 0.114$ & $1.84 \pm 0.0051$ & 28.54 & $100.00 \pm 0.044$ & $0.71 \pm 0.0020$ \\
12.74 & $99.99 \pm 0.098$ & $1.58 \pm 0.0044$ & 30.32 & $100.02 \pm 0.041$ & $0.65 \pm 0.0018$ \\
14.33 & $100.03 \pm 0.086$ & $1.38 \pm 0.0038$ & 31.60 & $99.99 \pm 0.039$ & $0.63 \pm 0.0018$ \\
15.98 & $100.00 \pm 0.082$ & $1.32 \pm 0.0037$ & 33.07 & $100.03 \pm 0.036$ & $0.59 \pm 0.0016$ \\
17.53 & $100.01 \pm 0.072$ & $1.16 \pm 0.0032$ & 35.01 & $99.97 \pm 0.035$ & $0.56 \pm 0.0016$ \\
18.90 & $100.03 \pm 0.065$ & $1.04 \pm 0.0029$ & & & \\
\hline
\end{tabular}

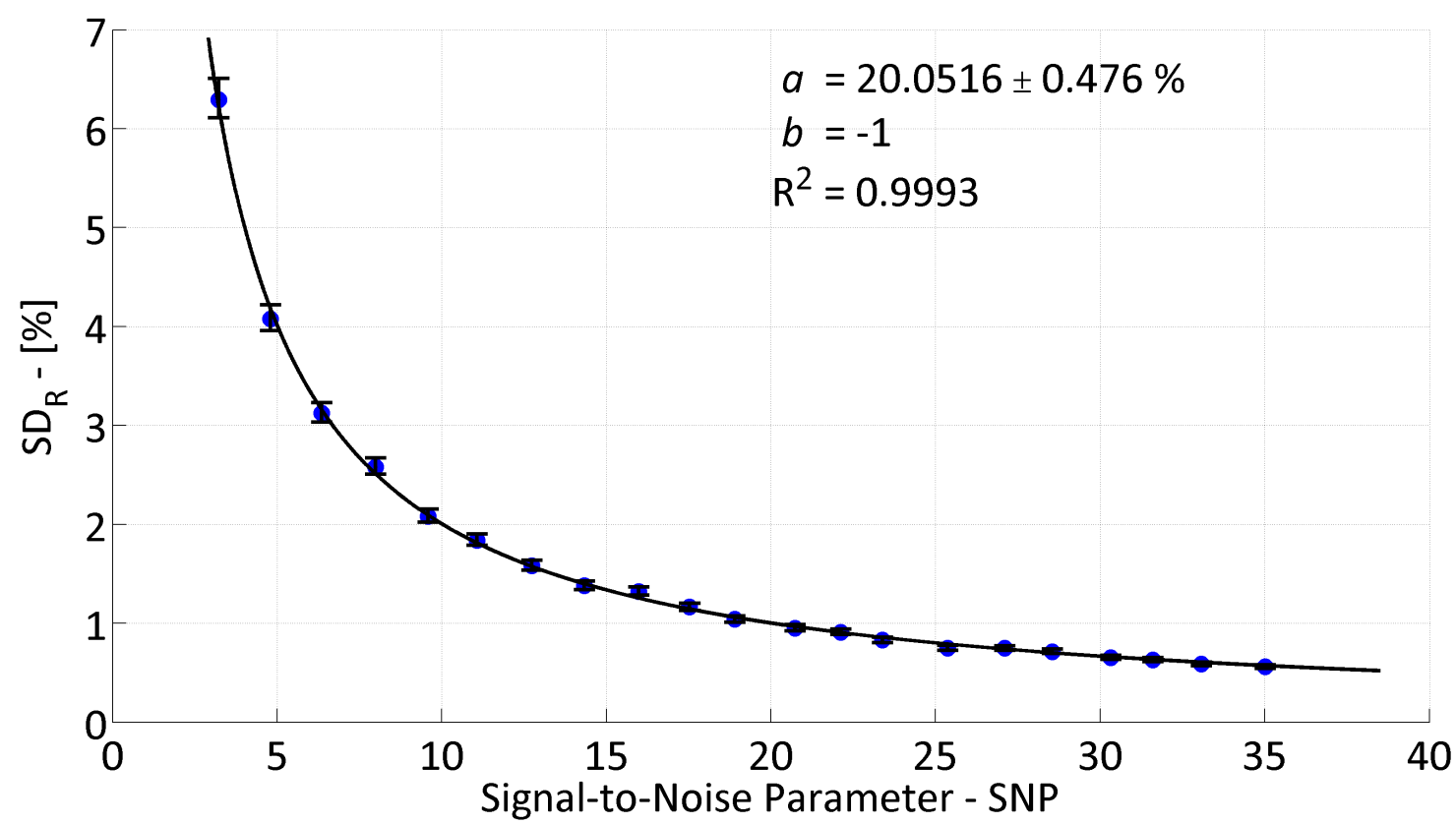

Figure S.96: Relative population standard deviation as a function of signal-to-noise parameter for $100 \mathrm{~nm}$ of $\mathrm{Al} / \mathrm{Si}$ with $h_{\mathrm{BD}}=100.00 \mathrm{MW} / \mathrm{m}^{2} \mathrm{~K}$. Error bars represent a $95 \%$ confidence interval of the measured $\mathrm{SD}_{\mathrm{R}}$ and the solid line represents a best fit of a power law to the data, of the form $\mathrm{SD}_{\mathrm{R}}=a \times \mathrm{SNP}^{b}$, with parameter $a$ provided for a fixed $b=-1$, along with the $95 \%$ confidence level in $a$. 


\section{S.23 Simulation Summary - Al/Si 129.00 MW Series}

\section{S.23.1 Fitting Film-to-Substrate Boundary Conductance - $h_{\mathrm{BD}}$}

Table S.36: Summary of simulation data - 100 nm Al/Si 129.00 MW Series. Error bounds represent a $95 \%$ confidence level.

\begin{tabular}{cccccc}
\hline \multicolumn{6}{c}{$\mathbf{1 0 0} \mathbf{~ n m ~ A l ~ o n ~} \mathbf{~ S i}, h_{\mathrm{BD}}=\mathbf{1 2 9 . 0 0} \mathbf{M W} / \mathbf{m}^{2} \mathbf{K}$} \\
\hline $\mathbf{S N P}$ & $\mu-\left[\mathbf{M W} / \mathbf{m}^{2} \mathbf{K}\right]$ & $\sigma-\left[\mathbf{M W} / \mathbf{m}^{2} \mathbf{K}\right]$ & $\mathbf{S N P}$ & $\mu-\left[\mathbf{M W} / \mathbf{m}^{2} \mathbf{K}\right]$ & $\sigma-\left[\mathbf{M W} / \mathbf{m}^{2} \mathbf{K}\right]$ \\
2.76 & $133.70 \pm 1.136$ & $17.05 \pm 0.0548$ & 17.82 & $129.12 \pm 0.164$ & $2.64 \pm 0.0074$ \\
4.12 & $130.97 \pm 0.733$ & $11.23 \pm 0.0346$ & 19.13 & $128.95 \pm 0.149$ & $2.40 \pm 0.0067$ \\
5.48 & $130.43 \pm 0.557$ & $8.79 \pm 0.0255$ & 20.45 & $128.89 \pm 0.152$ & $2.44 \pm 0.0068$ \\
6.87 & $129.82 \pm 0.439$ & $6.96 \pm 0.0200$ & 21.88 & $129.04 \pm 0.143$ & $2.29 \pm 0.0064$ \\
8.27 & $129.52 \pm 0.369$ & $5.87 \pm 0.0168$ & 23.34 & $129.08 \pm 0.130$ & $2.10 \pm 0.0059$ \\
9.57 & $129.18 \pm 0.307$ & $4.93 \pm 0.0139$ & 24.65 & $128.99 \pm 0.122$ & $1.96 \pm 0.0055$ \\
11.01 & $128.93 \pm 0.269$ & $4.31 \pm 0.0121$ & 25.90 & $129.10 \pm 0.112$ & $1.81 \pm 0.0050$ \\
12.39 & $128.98 \pm 0.243$ & $3.90 \pm 0.0109$ & 27.28 & $129.05 \pm 0.111$ & $1.79 \pm 0.0050$ \\
13.64 & $128.95 \pm 0.221$ & $3.54 \pm 0.0100$ & 28.42 & $128.95 \pm 0.101$ & $1.63 \pm 0.0046$ \\
15.06 & $129.06 \pm 0.204$ & $3.28 \pm 0.0092$ & 29.58 & $128.99 \pm 0.100$ & $1.62 \pm 0.0045$ \\
16.36 & $129.08 \pm 0.182$ & $2.91 \pm 0.0082$ & & & \\
\hline
\end{tabular}

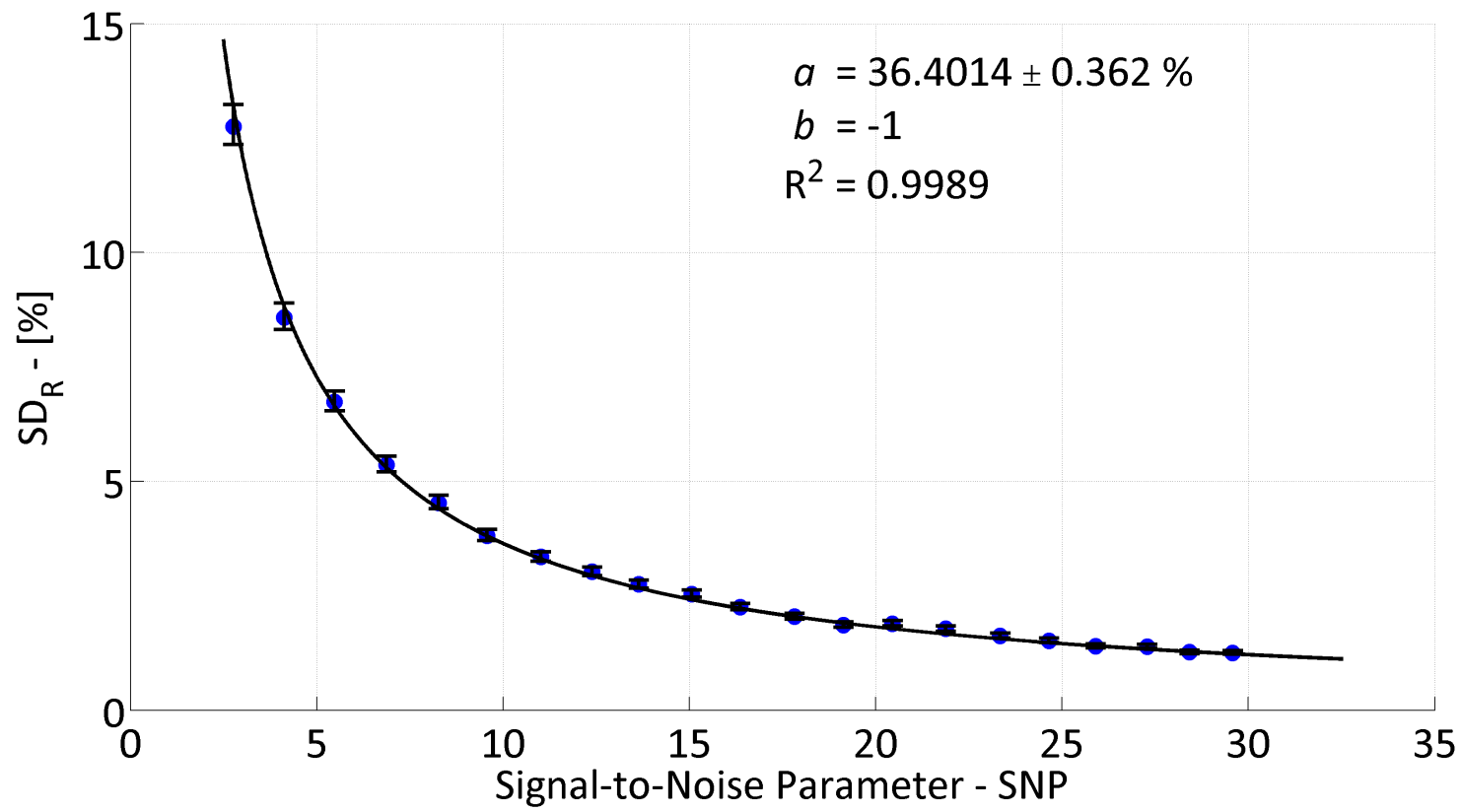

Figure S.97: Relative population standard deviation as a function of signal-to-noise parameter for $100 \mathrm{~nm}$ of $\mathrm{Al} / \mathrm{Si}$ with $h_{\mathrm{BD}}=129.00 \mathrm{MW} / \mathrm{m}^{2} \mathrm{~K}$. Error bars represent a $95 \%$ confidence interval of the measured $\mathrm{SD}_{\mathrm{R}}$ and the solid line represents a best fit of a power law to the data, of the form $\mathrm{SD}_{\mathrm{R}}=a \times \mathrm{SNP}^{b}$, with parameter $a$ provided for a fixed $b=-1$, along with the $95 \%$ confidence level in $a$. 


\section{S.23.2 Fitting Substrate Thermal Conductivity $-k_{S}$}

Table S.37: Summary of simulation data - $100 \mathrm{~nm} \mathrm{Al/Si} \mathrm{129.00} \mathrm{MW} \mathrm{Series.} \mathrm{Error} \mathrm{bounds} \mathrm{represent} \mathrm{a} 95 \%$ confidence level.

\begin{tabular}{cccccc}
\hline \multicolumn{6}{c}{$\mathbf{1 0 0} \mathbf{~ n m} \mathbf{A l}$ on $\mathbf{~ S i}, h_{\mathrm{BD}}=\mathbf{1 2 9 . 0 0} \mathbf{M W} / \mathbf{m}^{2} \mathbf{K}$} \\
\hline $\mathbf{S N P}$ & $\mu-[\mathbf{W} / \mathbf{m K}]$ & $\sigma-[\mathbf{W} / \mathbf{m K}]$ & $\mathbf{S N P}$ & $\mu-[\mathbf{W} / \mathbf{m K}]$ & $\sigma-[\mathbf{W} / \mathbf{m K}]$ \\
2.76 & $154.41 \pm 2.559$ & $41.21 \pm 0.1149$ & 17.82 & $148.05 \pm 0.352$ & $5.67 \pm 0.0158$ \\
4.12 & $149.72 \pm 1.578$ & $25.40 \pm 0.0709$ & 19.13 & $147.73 \pm 0.321$ & $5.17 \pm 0.0144$ \\
5.48 & $150.11 \pm 1.229$ & $19.80 \pm 0.0552$ & 20.45 & $147.51 \pm 0.324$ & $5.23 \pm 0.0146$ \\
6.87 & $149.23 \pm 0.958$ & $15.43 \pm 0.0430$ & 21.88 & $147.87 \pm 0.304$ & $4.89 \pm 0.0136$ \\
8.27 & $148.64 \pm 0.797$ & $12.85 \pm 0.0358$ & 23.34 & $148.08 \pm 0.279$ & $4.50 \pm 0.0125$ \\
9.57 & $148.18 \pm 0.655$ & $10.54 \pm 0.0294$ & 24.65 & $147.76 \pm 0.262$ & $4.22 \pm 0.0118$ \\
11.01 & $147.61 \pm 0.571$ & $9.20 \pm 0.0256$ & 25.90 & $148.00 \pm 0.239$ & $3.86 \pm 0.0107$ \\
12.39 & $147.65 \pm 0.516$ & $8.32 \pm 0.0232$ & 27.28 & $147.80 \pm 0.234$ & $3.78 \pm 0.0105$ \\
13.64 & $147.70 \pm 0.471$ & $7.58 \pm 0.0211$ & 28.42 & $147.66 \pm 0.213$ & $3.44 \pm 0.0096$ \\
15.06 & $147.76 \pm 0.431$ & $6.94 \pm 0.0193$ & 29.58 & $147.75 \pm 0.216$ & $3.48 \pm 0.0097$ \\
16.36 & $147.83 \pm 0.386$ & $6.22 \pm 0.0173$ & & & \\
\hline
\end{tabular}

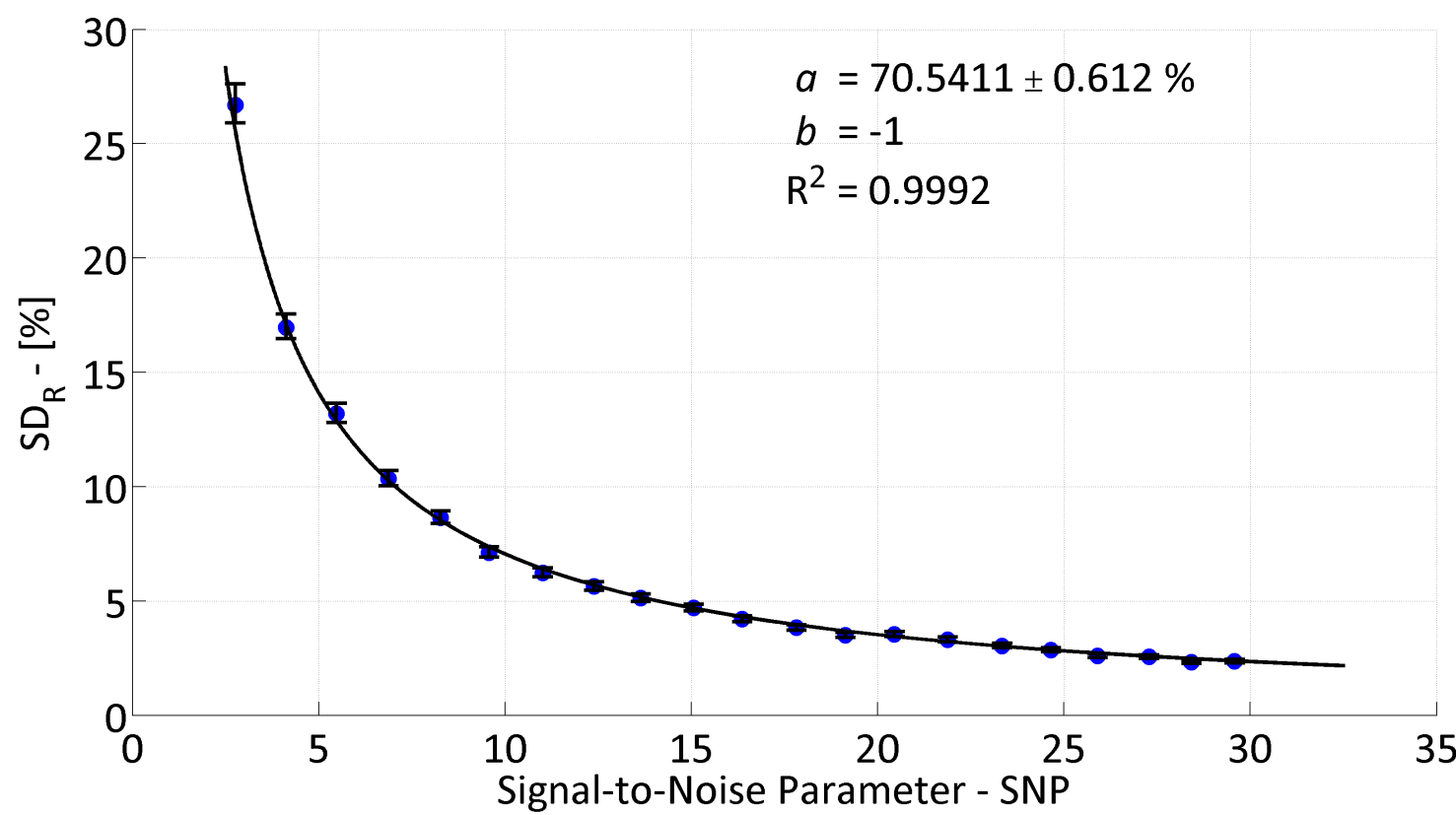

Figure S.98: Relative population standard deviation as a function of signal-to-noise parameter for $100 \mathrm{~nm}$ of $\mathrm{Al} / \mathrm{Si}$ with $h_{\mathrm{BD}}=129.00 \mathrm{MW} / \mathrm{m}^{2} \mathrm{~K}$. Error bars represent a $95 \%$ confidence interval of the measured $\mathrm{SD}_{\mathrm{R}}$ and the solid line represents a best fit of a power law to the data, of the form $\mathrm{SD}_{\mathrm{R}}=a \times \mathrm{SNP}^{b}$, with parameter $a$ provided for a fixed $b=-1$, along with the $95 \%$ confidence level in $a$. 


\section{S.24 Simulation Summary - Al/Si 167.00 MW Series}

\section{S.24.1 Fitting Film-to-Substrate Boundary Conductance - $h_{\mathrm{BD}}$}

Table S.38: Summary of simulation data - $100 \mathrm{~nm} \mathrm{Al/Si} \mathrm{167.00} \mathrm{MW} \mathrm{Series.} \mathrm{Error} \mathrm{bounds} \mathrm{represent} \mathrm{a} 95 \%$ confidence level.

\begin{tabular}{cccccc}
\hline \multicolumn{6}{c}{$\mathbf{1 0 0} \mathbf{~ n m ~ A l ~ o n ~} \mathbf{~ S i}, h_{\mathrm{BD}}=\mathbf{1 6 7 . 0 0} \mathbf{M W} / \mathbf{m}^{2} \mathbf{K}$} \\
\hline $\mathbf{S N P}$ & $\mu-\left[\mathbf{M W} / \mathbf{m}^{2} \mathbf{K}\right]$ & $\sigma-\left[\mathbf{M W} / \mathbf{m}^{2} \mathbf{K}\right]$ & $\mathbf{S N P}$ & $\mu-\left[\mathbf{M W} / \mathbf{m}^{2} \mathbf{K}\right]$ & $\sigma-\left[\mathbf{M W} / \mathbf{m}^{2} \mathbf{K}\right]$ \\
2.37 & $172.83 \pm 1.833$ & $28.51 \pm 0.0852$ & 15.38 & $166.91 \pm 0.301$ & $4.78 \pm 0.0137$ \\
3.57 & $168.25 \pm 1.232$ & $19.50 \pm 0.0563$ & 16.46 & $166.98 \pm 0.272$ & $4.39 \pm 0.0122$ \\
4.75 & $167.70 \pm 0.952$ & $15.09 \pm 0.0434$ & 17.61 & $167.01 \pm 0.248$ & $3.94 \pm 0.0113$ \\
5.93 & $167.09 \pm 0.758$ & $12.21 \pm 0.0340$ & 18.97 & $166.93 \pm 0.245$ & $3.89 \pm 0.0112$ \\
7.12 & $167.56 \pm 0.625$ & $9.91 \pm 0.0285$ & 20.15 & $166.89 \pm 0.216$ & $3.48 \pm 0.0097$ \\
8.30 & $167.27 \pm 0.537$ & $8.52 \pm 0.0245$ & 21.35 & $166.99 \pm 0.214$ & $3.39 \pm 0.0097$ \\
9.45 & $167.45 \pm 0.482$ & $7.65 \pm 0.0220$ & 22.44 & $167.02 \pm 0.200$ & $3.17 \pm 0.0091$ \\
10.61 & $167.14 \pm 0.424$ & $6.72 \pm 0.0193$ & 23.06 & $167.04 \pm 0.200$ & $3.17 \pm 0.0091$ \\
11.80 & $166.99 \pm 0.373$ & $5.92 \pm 0.0170$ & 24.54 & $166.89 \pm 0.181$ & $2.88 \pm 0.0083$ \\
13.04 & $167.31 \pm 0.334$ & $5.30 \pm 0.0152$ & 26.05 & $167.07 \pm 0.176$ & $2.80 \pm 0.0080$ \\
14.21 & $167.03 \pm 0.310$ & $4.92 \pm 0.0142$ & & & \\
\hline
\end{tabular}

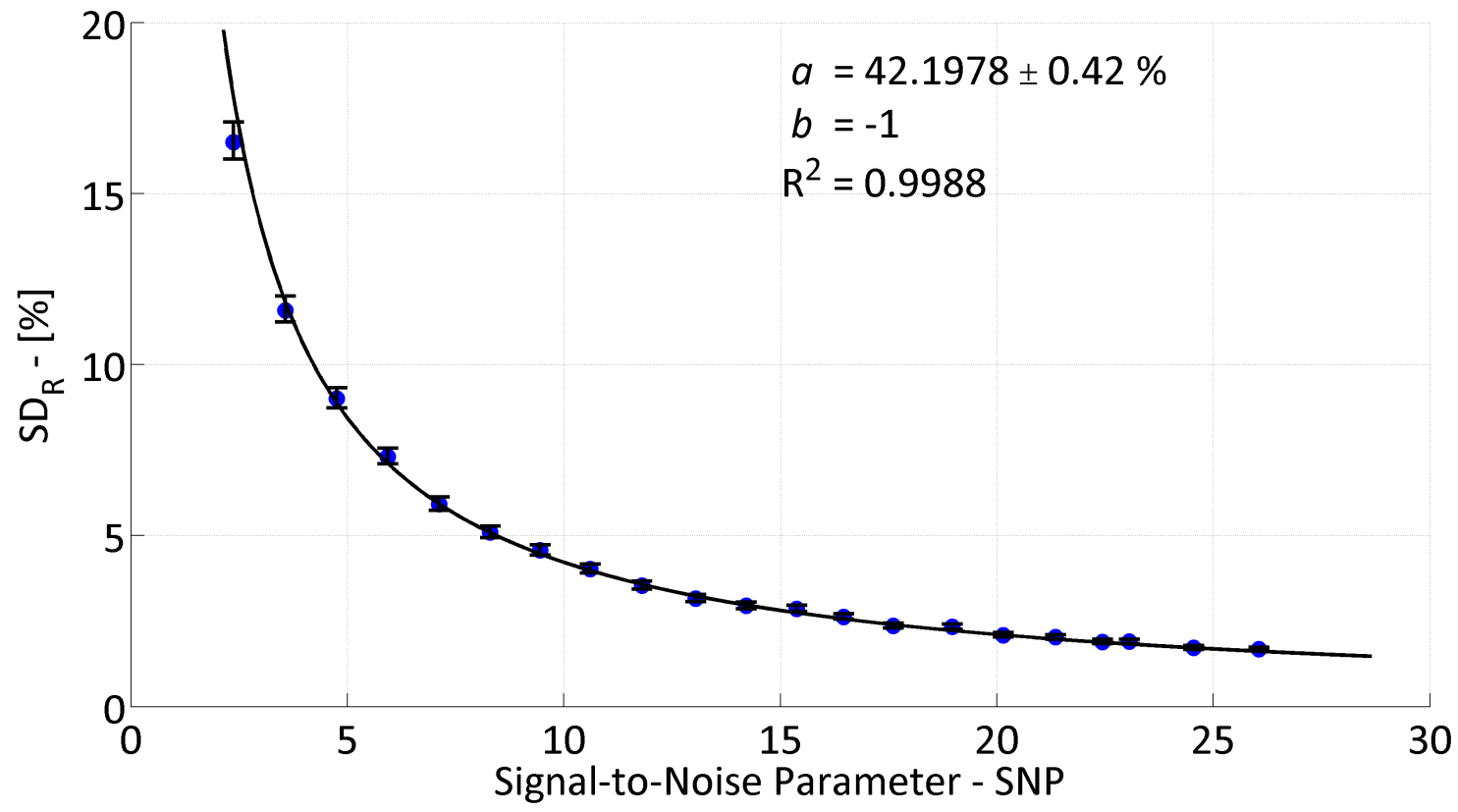

Figure S.99: Relative population standard deviation as a function of signal-to-noise parameter for $100 \mathrm{~nm}$ of $\mathrm{Al} / \mathrm{Si}$ with $h_{\mathrm{BD}}=167.00 \mathrm{MW} / \mathrm{m}^{2} \mathrm{~K}$. Error bars represent a $95 \%$ confidence interval of the measured $\mathrm{SD}_{\mathrm{R}}$ and the solid line represents a best fit of a power law to the data, of the form $\mathrm{SD}_{\mathrm{R}}=a \times \mathrm{SNP}^{b}$, with parameter $a$ provided for a fixed $b=-1$, along with the $95 \%$ confidence level in $a$. 


\section{S.24.2 Fitting Substrate Thermal Conductivity $-k_{S}$}

Table S.39: Summary of simulation data - $100 \mathrm{~nm} \mathrm{Al/Si} \mathrm{167.00} \mathrm{MW} \mathrm{Series.} \mathrm{Error} \mathrm{bounds} \mathrm{represent} \mathrm{a} 95 \%$ confidence level.

\begin{tabular}{cccccc}
\hline \multicolumn{6}{c}{$\mathbf{1 0 0} \mathbf{~ n m} \mathbf{A l}$ on $\mathbf{~ S i}, h_{\mathrm{BD}}=\mathbf{1 6 7 . 0 0} \mathbf{M W} / \mathbf{m}^{2} \mathbf{K}$} \\
\hline SNP & $\mu-[\mathbf{W} / \mathbf{m K}]$ & $\sigma-[\mathbf{W} / \mathbf{m K}]$ & $\mathbf{S N P}$ & $\mu-[\mathbf{W} / \mathbf{m K}]$ & $\sigma-[\mathbf{W} / \mathbf{m K}]$ \\
2.37 & $155.61 \pm 2.474$ & $39.79 \pm 0.1113$ & 15.38 & $148.10 \pm 0.352$ & $5.66 \pm 0.0158$ \\
3.57 & $150.11 \pm 1.511$ & $24.33 \pm 0.0678$ & 16.46 & $148.11 \pm 0.322$ & $5.18 \pm 0.0144$ \\
4.75 & $149.21 \pm 1.151$ & $18.55 \pm 0.0517$ & 17.61 & $148.11 \pm 0.286$ & $4.61 \pm 0.0128$ \\
5.93 & $148.96 \pm 0.925$ & $14.89 \pm 0.0415$ & 18.97 & $148.14 \pm 0.285$ & $4.59 \pm 0.0128$ \\
7.12 & $149.00 \pm 0.727$ & $11.72 \pm 0.0326$ & 20.15 & $148.05 \pm 0.261$ & $4.21 \pm 0.0117$ \\
8.30 & $148.40 \pm 0.622$ & $10.03 \pm 0.0279$ & 21.35 & $148.18 \pm 0.252$ & $4.05 \pm 0.0113$ \\
9.45 & $148.76 \pm 0.582$ & $9.38 \pm 0.0261$ & 22.44 & $148.11 \pm 0.235$ & $3.79 \pm 0.0106$ \\
10.61 & $148.63 \pm 0.507$ & $8.16 \pm 0.0227$ & 23.06 & $148.26 \pm 0.237$ & $3.82 \pm 0.0106$ \\
11.80 & $148.07 \pm 0.443$ & $7.13 \pm 0.0199$ & 24.54 & $148.12 \pm 0.218$ & $3.52 \pm 0.0098$ \\
13.04 & $148.91 \pm 0.398$ & $6.42 \pm 0.0179$ & 26.05 & $148.16 \pm 0.203$ & $3.27 \pm 0.0091$ \\
14.21 & $148.33 \pm 0.369$ & $5.95 \pm 0.0166$ & & & \\
\hline
\end{tabular}

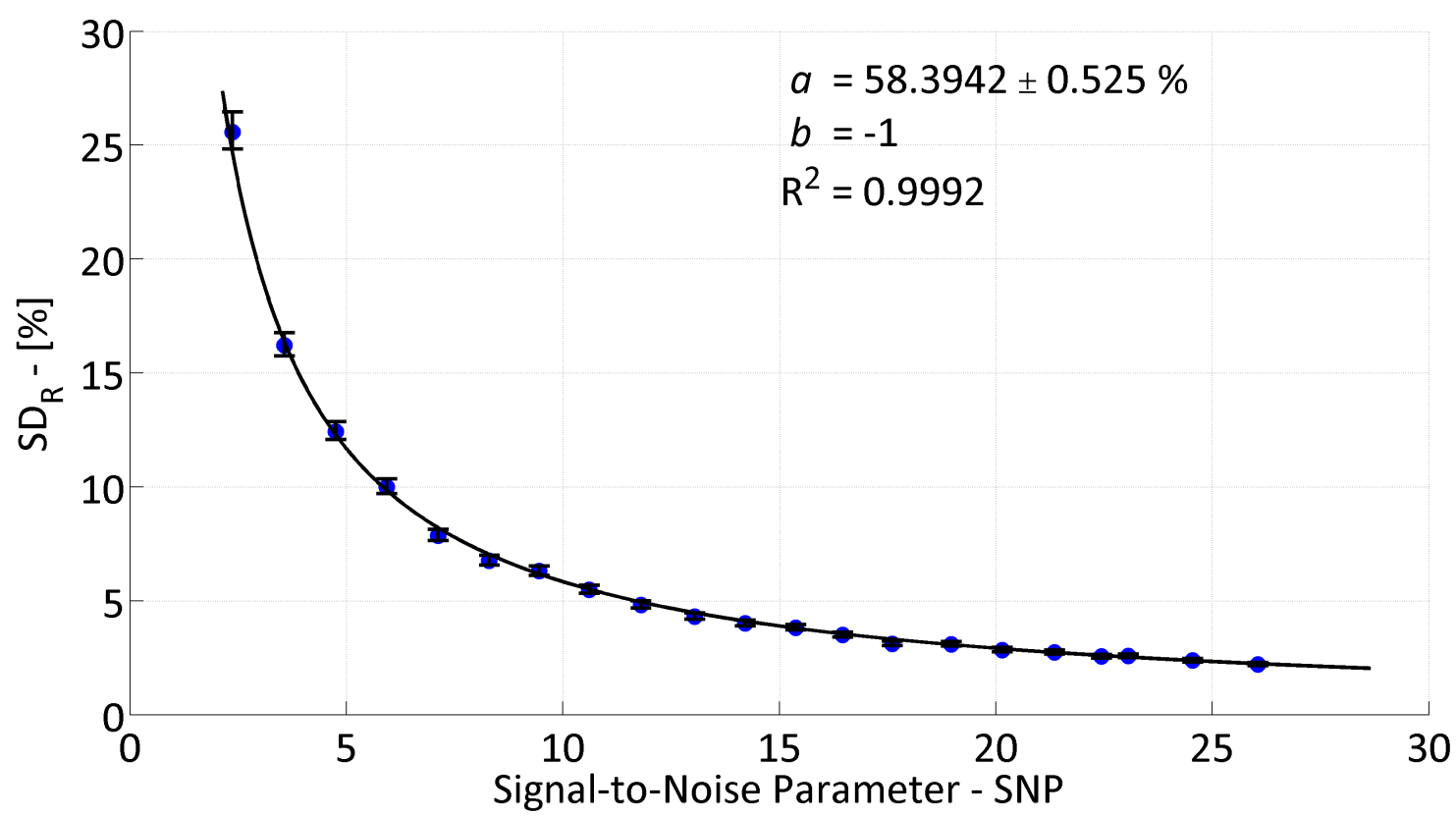

Figure S.100: Relative population standard deviation as a function of signal-to-noise parameter for 100 $\mathrm{nm}$ of $\mathrm{Al} / \mathrm{Si}$ with $h_{\mathrm{BD}}=167.00 \mathrm{MW} / \mathrm{m}^{2} \mathrm{~K}$. Error bars represent a $95 \%$ confidence interval of the measured $\mathrm{SD}_{\mathrm{R}}$ and the solid line represents a best fit of a power law to the data, of the form $\mathrm{SD}_{\mathrm{R}}=a \times \mathrm{SNP}^{b}$, with parameter $a$ provided for a fixed $b=-1$, along with the $95 \%$ confidence level in $a$. 


\section{S.25 Simulation Summary - Al/Si 215.00 MW Series}

\section{S.25.1 Fitting Film-to-Substrate Boundary Conductance - $h_{\mathrm{BD}}$}

Table S.40: Summary of simulation data - 100 nm Al/Si 215.00 MW Series. Error bounds represent a $95 \%$ confidence level.

\begin{tabular}{cccccc}
\hline \multicolumn{6}{c}{$\mathbf{1 0 0} \mathbf{~ n m ~ A l ~ o n ~} \mathbf{~ S i}, h_{\mathrm{BD}}=\mathbf{2 1 5 . 0 0} \mathbf{M W} / \mathbf{m}^{2} \mathbf{K}$} \\
\hline $\mathbf{S N P}$ & $\mu-\left[\mathbf{M W} / \mathbf{m}^{2} \mathbf{K}\right]$ & $\sigma-\left[\mathbf{M W} / \mathbf{m}^{2} \mathbf{K}\right]$ & $\mathbf{S N P}$ & $\mu-\left[\mathbf{M W} / \mathbf{m}^{2} \mathbf{K}\right]$ & $\sigma-\left[\mathbf{M W} / \mathbf{m}^{2} \mathbf{K}\right]$ \\
2.13 & $222.50 \pm 3.255$ & $50.05 \pm 0.1531$ & 13.80 & $215.04 \pm 0.456$ & $7.30 \pm 0.0206$ \\
3.21 & $218.14 \pm 2.152$ & $34.05 \pm 0.0984$ & 14.77 & $214.76 \pm 0.421$ & $6.74 \pm 0.0190$ \\
4.26 & $217.52 \pm 1.588$ & $25.29 \pm 0.0721$ & 16.01 & $215.28 \pm 0.406$ & $6.49 \pm 0.0183$ \\
5.34 & $215.72 \pm 1.188$ & $19.00 \pm 0.0537$ & 16.94 & $215.12 \pm 0.383$ & $6.12 \pm 0.0173$ \\
6.37 & $215.19 \pm 0.968$ & $15.48 \pm 0.0438$ & 18.12 & $215.03 \pm 0.337$ & $5.38 \pm 0.0152$ \\
7.45 & $215.19 \pm 0.851$ & $13.61 \pm 0.0385$ & 19.07 & $215.11 \pm 0.339$ & $5.42 \pm 0.0153$ \\
8.52 & $215.05 \pm 0.744$ & $11.91 \pm 0.0336$ & 20.25 & $215.03 \pm 0.305$ & $4.87 \pm 0.0138$ \\
9.56 & $215.06 \pm 0.645$ & $10.32 \pm 0.0292$ & 21.18 & $215.02 \pm 0.291$ & $4.66 \pm 0.0132$ \\
10.60 & $215.59 \pm 0.596$ & $9.54 \pm 0.0269$ & 22.25 & $214.91 \pm 0.281$ & $4.50 \pm 0.0127$ \\
11.71 & $215.07 \pm 0.544$ & $8.71 \pm 0.0246$ & 23.34 & $214.80 \pm 0.273$ & $4.37 \pm 0.0124$ \\
12.73 & $214.99 \pm 0.512$ & $8.20 \pm 0.0232$ & & & \\
\hline
\end{tabular}

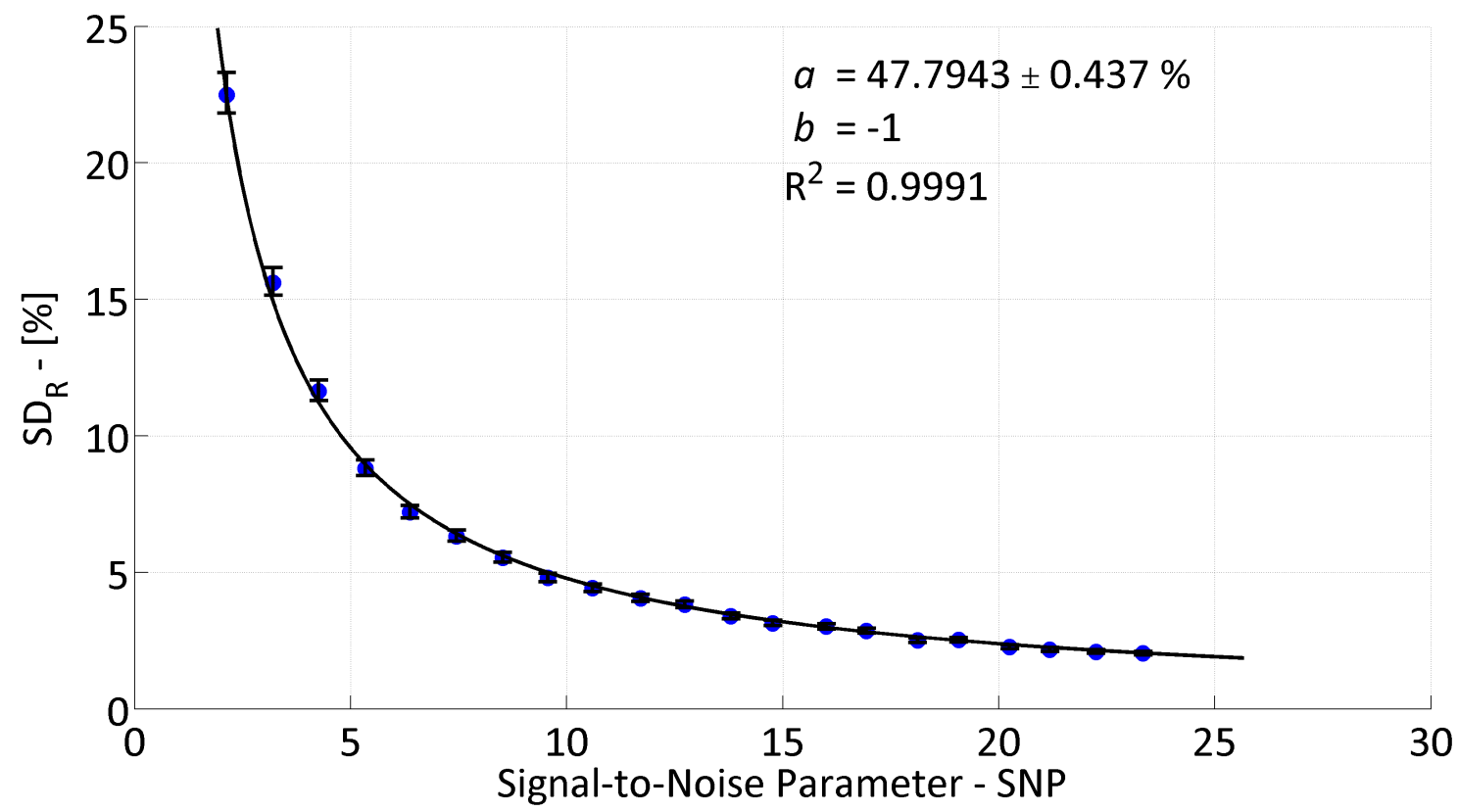

Figure S.101: Relative population standard deviation as a function of signal-to-noise parameter for 100 $\mathrm{nm}$ of $\mathrm{Al} / \mathrm{Si}$ with $h_{\mathrm{BD}}=215.00 \mathrm{MW} / \mathrm{m}^{2} \mathrm{~K}$. Error bars represent a $95 \%$ confidence interval of the measured $\mathrm{SD}_{\mathrm{R}}$ and the solid line represents a best fit of a power law to the data, of the form $\mathrm{SD}_{\mathrm{R}}=a \times \mathrm{SNP}^{b}$, with parameter $a$ provided for a fixed $b=-1$, along with the $95 \%$ confidence level in $a$. 


\section{S.25.2 Fitting Substrate Thermal Conductivity $-k_{S}$}

Table S.41: Summary of simulation data - $100 \mathrm{~nm} \mathrm{Al/Si} \mathrm{215.00} \mathrm{MW} \mathrm{Series.} \mathrm{Error} \mathrm{bounds} \mathrm{represent} \mathrm{a} 95 \%$ confidence level.

\begin{tabular}{cccccc}
\hline \multicolumn{6}{c}{$\mathbf{1 0 0} \mathbf{~ n m} \mathbf{A l}$ on $\mathbf{~ S i}, h_{\mathrm{BD}}=\mathbf{2 1 5 . 0 0} \mathbf{M W} / \mathbf{m}^{2} \mathbf{K}$} \\
\hline SNP & $\mu-[\mathbf{W} / \mathbf{m K}]$ & $\sigma-[\mathbf{W} / \mathbf{m K}]$ & $\mathbf{S N P}$ & $\mu-[\mathbf{W} / \mathbf{m K}]$ & $\sigma-[\mathbf{W} / \mathbf{m K}]$ \\
2.13 & $151.52 \pm 2.248$ & $36.18 \pm 0.1010$ & 13.80 & $147.96 \pm 0.327$ & $5.27 \pm 0.0147$ \\
3.21 & $150.14 \pm 1.475$ & $23.77 \pm 0.0662$ & 14.77 & $148.11 \pm 0.319$ & $5.14 \pm 0.0143$ \\
4.26 & $149.62 \pm 1.099$ & $17.71 \pm 0.0494$ & 16.01 & $148.05 \pm 0.289$ & $4.65 \pm 0.0130$ \\
5.34 & $148.57 \pm 0.863$ & $13.91 \pm 0.0387$ & 16.94 & $147.72 \pm 0.270$ & $4.35 \pm 0.0121$ \\
6.37 & $148.37 \pm 0.702$ & $11.32 \pm 0.0315$ & 18.12 & $148.04 \pm 0.254$ & $4.09 \pm 0.0114$ \\
7.45 & $148.06 \pm 0.630$ & $10.14 \pm 0.0283$ & 19.07 & $148.04 \pm 0.248$ & $4.00 \pm 0.0111$ \\
8.52 & $147.96 \pm 0.523$ & $8.42 \pm 0.0235$ & 20.25 & $147.99 \pm 0.228$ & $3.68 \pm 0.0102$ \\
9.56 & $148.04 \pm 0.461$ & $7.43 \pm 0.0207$ & 21.18 & $147.83 \pm 0.214$ & $3.45 \pm 0.0096$ \\
10.60 & $148.42 \pm 0.445$ & $7.17 \pm 0.0200$ & 22.25 & $147.89 \pm 0.204$ & $3.29 \pm 0.0092$ \\
11.71 & $147.87 \pm 0.386$ & $6.22 \pm 0.0173$ & 23.34 & $147.87 \pm 0.201$ & $3.23 \pm 0.0090$ \\
12.73 & $148.20 \pm 0.374$ & $6.03 \pm 0.0168$ & & & \\
\hline
\end{tabular}

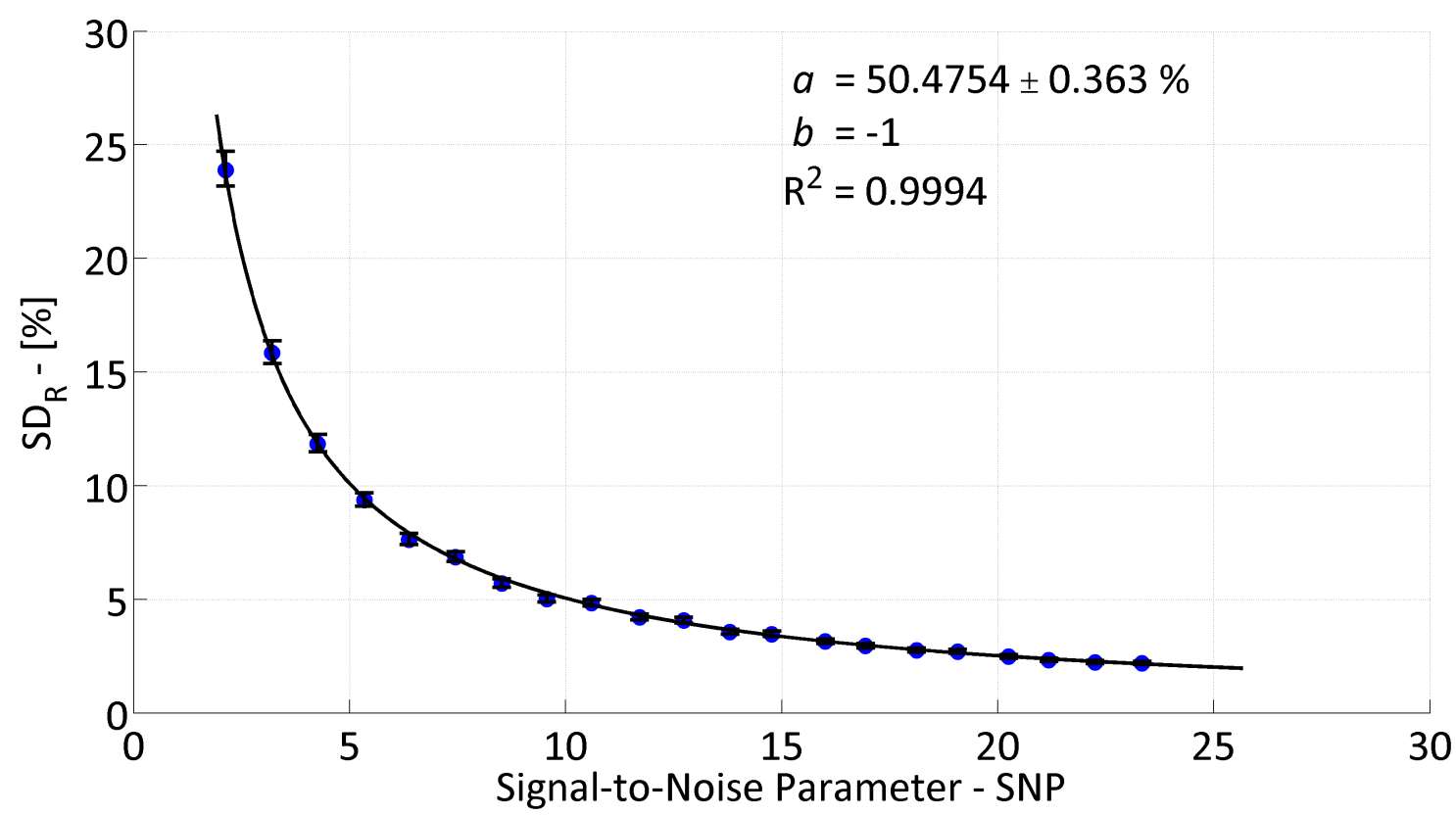

Figure S.102: Relative population standard deviation as a function of signal-to-noise parameter for 100 $\mathrm{nm}$ of $\mathrm{Al} / \mathrm{Si}$ with $h_{\mathrm{BD}}=215.00 \mathrm{MW} / \mathrm{m}^{2} \mathrm{~K}$. Error bars represent a $95 \%$ confidence interval of the measured $\mathrm{SD}_{\mathrm{R}}$ and the solid line represents a best fit of a power law to the data, of the form $\mathrm{SD}_{\mathrm{R}}=a \times \mathrm{SNP}^{b}$, with parameter $a$ provided for a fixed $b=-1$, along with the $95 \%$ confidence level in $a$. 


\section{S.25.3 Fitting Film Thickness $-d_{1}$}

Table S.42: Summary of simulation data - $100 \mathrm{~nm} \mathrm{Al/Si} 215.00 \mathrm{MW}$ Series. Error bounds represent a $95 \%$ confidence level.

\begin{tabular}{cccccc}
\hline \multicolumn{6}{c}{$\mathbf{1 0 0} \mathbf{~ n m} \mathbf{A l}$ on $\mathbf{S i}, h_{\mathrm{BD}}=\mathbf{2 1 5 . 0 0} \mathbf{M W} / \mathbf{m}^{2} \mathbf{K}$} \\
\hline SNP & $\mu-[\mathbf{n m}]$ & $\sigma-[\mathbf{n m}]$ & $\mathbf{S N P}$ & $\mu-[\mathbf{n m}]$ & $\sigma-[\mathbf{n m}]$ \\
2.13 & $100.63 \pm 0.567$ & $9.13 \pm 0.0254$ & 13.80 & $100.06 \pm 0.088$ & $1.42 \pm 0.0040$ \\
3.21 & $100.15 \pm 0.381$ & $6.14 \pm 0.0171$ & 14.77 & $100.03 \pm 0.084$ & $1.36 \pm 0.0038$ \\
4.26 & $99.96 \pm 0.293$ & $4.72 \pm 0.0131$ & 16.01 & $100.03 \pm 0.077$ & $1.25 \pm 0.0035$ \\
5.34 & $100.12 \pm 0.229$ & $3.69 \pm 0.0103$ & 16.94 & $100.10 \pm 0.072$ & $1.17 \pm 0.0032$ \\
6.37 & $100.09 \pm 0.186$ & $3.00 \pm 0.0084$ & 18.12 & $100.03 \pm 0.067$ & $1.09 \pm 0.0030$ \\
7.45 & $100.13 \pm 0.165$ & $2.66 \pm 0.0074$ & 19.07 & $100.03 \pm 0.066$ & $1.07 \pm 0.0030$ \\
8.52 & $100.12 \pm 0.140$ & $2.25 \pm 0.0063$ & 20.25 & $100.04 \pm 0.061$ & $0.98 \pm 0.0027$ \\
9.56 & $100.08 \pm 0.124$ & $1.99 \pm 0.0055$ & 21.18 & $100.07 \pm 0.057$ & $0.91 \pm 0.0025$ \\
10.60 & $99.97 \pm 0.119$ & $1.91 \pm 0.0053$ & 22.25 & $100.06 \pm 0.054$ & $0.88 \pm 0.0024$ \\
11.71 & $100.10 \pm 0.102$ & $1.65 \pm 0.0046$ & 23.34 & $100.07 \pm 0.054$ & $0.86 \pm 0.0024$ \\
12.73 & $100.02 \pm 0.099$ & $1.60 \pm 0.0045$ & & & \\
\hline
\end{tabular}

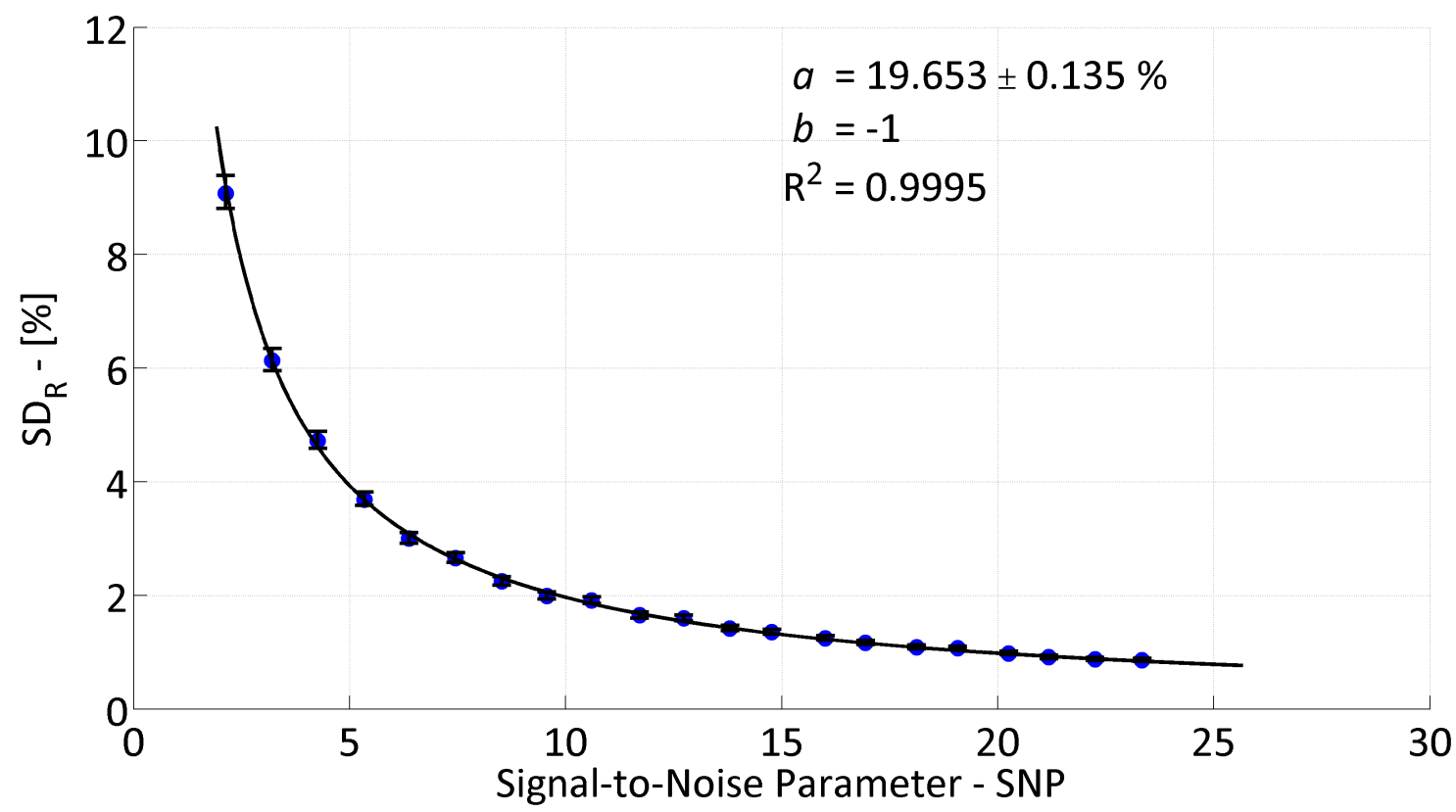

Figure S.103: Relative population standard deviation as a function of signal-to-noise parameter for 100 $\mathrm{nm}$ of $\mathrm{Al} / \mathrm{Si}$ with $h_{\mathrm{BD}}=215.00 \mathrm{MW} / \mathrm{m}^{2} \mathrm{~K}$. Error bars represent a $95 \%$ confidence interval of the measured $\mathrm{SD}_{\mathrm{R}}$ and the solid line represents a best fit of a power law to the data, of the form $\mathrm{SD}_{\mathrm{R}}=a \times \mathrm{SNP}^{b}$, with parameter $a$ provided for a fixed $b=-1$, along with the $95 \%$ confidence level in $a$. 


\section{S.26 Simulation Summary - Al/Si 278.00 MW Series}

\section{S.26.1 Fitting Film-to-Substrate Boundary Conductance - $h_{\mathrm{BD}}$}

Table S.43: Summary of simulation data - 100 nm Al/Si 278.00 MW Series. Error bounds represent a $95 \%$ confidence level.

\begin{tabular}{cccccc}
\hline \multicolumn{6}{c}{$\mathbf{1 0 0} \mathbf{~ n m ~ A l ~ o n ~} \mathbf{~ S i}, h_{\mathrm{BD}}=\mathbf{2 7 8 . 0 0} \mathbf{M W} / \mathbf{m}^{2} \mathbf{K}$} \\
\hline $\mathbf{S N P}$ & $\mu-\left[\mathbf{M W} / \mathbf{m}^{2} \mathbf{K}\right]$ & $\sigma-\left[\mathbf{M W} / \mathbf{m}^{2} \mathbf{K}\right]$ & $\mathbf{S N P}$ & $\mu-\left[\mathbf{M W} / \mathbf{m}^{2} \mathbf{K}\right]$ & $\sigma-\left[\mathbf{M W} / \mathbf{m}^{2} \mathbf{K}\right]$ \\
1.97 & $324.26 \pm 8.503$ & $134.53 \pm 0.3887$ & 12.68 & $278.79 \pm 0.752$ & $12.13 \pm 0.0338$ \\
2.95 & $290.25 \pm 3.898$ & $61.70 \pm 0.1781$ & 13.73 & $278.29 \pm 0.660$ & $10.63 \pm 0.0296$ \\
3.93 & $284.06 \pm 2.786$ & $44.45 \pm 0.1263$ & 14.76 & $278.65 \pm 0.638$ & $10.27 \pm 0.0287$ \\
4.92 & $281.18 \pm 2.189$ & $35.08 \pm 0.0988$ & 15.62 & $278.31 \pm 0.576$ & $9.28 \pm 0.0258$ \\
5.93 & $281.58 \pm 1.828$ & $29.38 \pm 0.0822$ & 16.49 & $278.28 \pm 0.569$ & $9.18 \pm 0.0256$ \\
6.86 & $280.61 \pm 1.423$ & $22.88 \pm 0.0640$ & 17.54 & $278.67 \pm 0.532$ & $8.57 \pm 0.0239$ \\
7.84 & $280.14 \pm 1.271$ & $20.48 \pm 0.0570$ & 18.67 & $277.81 \pm 0.496$ & $8.00 \pm 0.0223$ \\
8.85 & $278.22 \pm 1.077$ & $17.34 \pm 0.0483$ & 19.56 & $278.22 \pm 0.469$ & $7.55 \pm 0.0210$ \\
9.80 & $278.92 \pm 0.989$ & $15.94 \pm 0.0444$ & 20.61 & $278.16 \pm 0.456$ & $7.35 \pm 0.0205$ \\
10.84 & $279.04 \pm 0.855$ & $13.76 \pm 0.0384$ & 21.47 & $278.18 \pm 0.456$ & $7.35 \pm 0.0205$ \\
11.80 & $279.11 \pm 0.831$ & $13.38 \pm 0.0373$ & & & \\
\hline
\end{tabular}

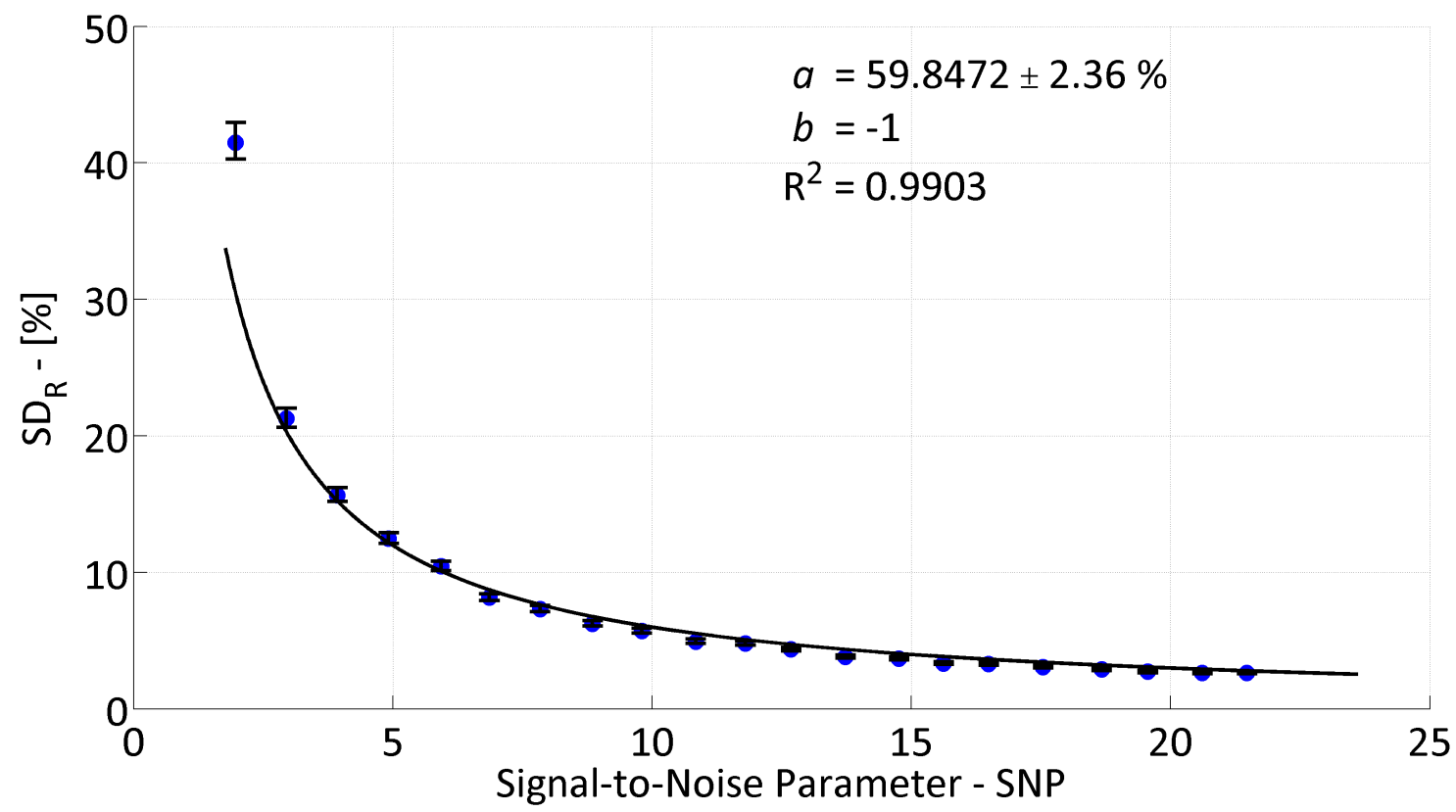

Figure S.104: Relative population standard deviation as a function of signal-to-noise parameter for 100 $\mathrm{nm}$ of $\mathrm{Al} / \mathrm{Si}$ with $h_{\mathrm{BD}}=278.00 \mathrm{MW} / \mathrm{m}^{2} \mathrm{~K}$. Error bars represent a $95 \%$ confidence interval of the measured $\mathrm{SD}_{\mathrm{R}}$ and the solid line represents a best fit of a power law to the data, of the form $\mathrm{SD}_{\mathrm{R}}=a \times \mathrm{SNP}^{b}$, with parameter $a$ provided for a fixed $b=-1$, along with the $95 \%$ confidence level in $a$. 


\section{S.26.2 Fitting Substrate Thermal Conductivity - $k_{S}$}

Table S.44: Summary of simulation data - $100 \mathrm{~nm} \mathrm{Al/Si} 278.00$ MW Series. Error bounds represent a $95 \%$ confidence level.

\begin{tabular}{cccccc}
\hline \multicolumn{6}{c}{$\mathbf{1 0 0} \mathbf{~ n m} \mathbf{A l}$ on $\mathbf{~ S i}, h_{\mathrm{BD}}=\mathbf{2 7 8 . 0 0} \mathbf{M W} / \mathbf{m}^{2} \mathbf{K}$} \\
\hline $\mathbf{S N P}$ & $\mu-[\mathbf{W} / \mathbf{m K}]$ & $\sigma-[\mathbf{W} / \mathbf{m K}]$ & $\mathbf{S N P}$ & $\mu-[\mathbf{W} / \mathbf{m K}]$ & $\sigma-[\mathbf{W} / \mathbf{m K}]$ \\
1.97 & $151.14 \pm 2.200$ & $35.39 \pm 0.0989$ & 12.68 & $148.11 \pm 0.344$ & $5.55 \pm 0.0155$ \\
2.95 & $151.42 \pm 1.542$ & $24.85 \pm 0.0692$ & 13.73 & $147.94 \pm 0.315$ & $5.07 \pm 0.0141$ \\
3.93 & $149.90 \pm 1.079$ & $17.38 \pm 0.0485$ & 14.76 & $148.05 \pm 0.286$ & $4.62 \pm 0.0129$ \\
4.92 & $148.37 \pm 0.855$ & $13.77 \pm 0.0384$ & 15.62 & $148.19 \pm 0.279$ & $4.50 \pm 0.0125$ \\
5.93 & $147.91 \pm 0.697$ & $11.23 \pm 0.0313$ & 16.49 & $148.13 \pm 0.264$ & $4.25 \pm 0.0118$ \\
6.86 & $148.19 \pm 0.610$ & $9.83 \pm 0.0274$ & 17.54 & $148.06 \pm 0.248$ & $4.00 \pm 0.0111$ \\
7.84 & $148.45 \pm 0.559$ & $9.00 \pm 0.0251$ & 18.67 & $148.00 \pm 0.236$ & $3.81 \pm 0.0106$ \\
8.85 & $148.04 \pm 0.501$ & $8.08 \pm 0.0225$ & 19.56 & $147.86 \pm 0.217$ & $3.50 \pm 0.0097$ \\
9.80 & $147.90 \pm 0.435$ & $7.01 \pm 0.0195$ & 20.61 & $148.00 \pm 0.217$ & $3.50 \pm 0.0098$ \\
10.84 & $147.67 \pm 0.391$ & $6.30 \pm 0.0176$ & 21.47 & $148.03 \pm 0.200$ & $3.22 \pm 0.0090$ \\
11.80 & $147.74 \pm 0.355$ & $5.72 \pm 0.0159$ & & & \\
\hline
\end{tabular}

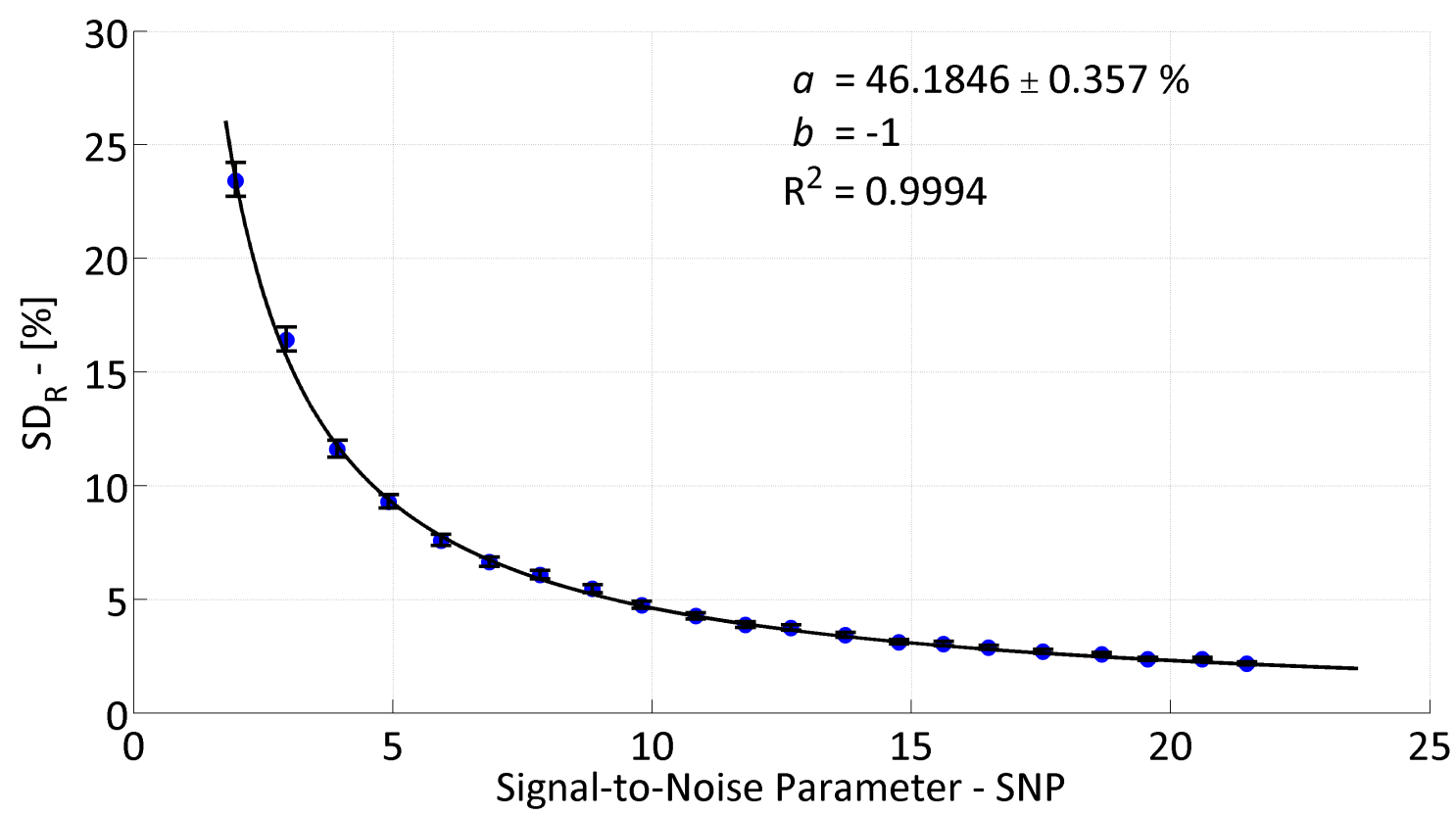

Figure S.105: Relative population standard deviation as a function of signal-to-noise parameter for 100 $\mathrm{nm}$ of $\mathrm{Al} / \mathrm{Si}$ with $h_{\mathrm{BD}}=278.00 \mathrm{MW} / \mathrm{m}^{2} \mathrm{~K}$. Error bars represent a $95 \%$ confidence interval of the measured $\mathrm{SD}_{\mathrm{R}}$ and the solid line represents a best fit of a power law to the data, of the form $\mathrm{SD}_{\mathrm{R}}=a \times \mathrm{SNP}^{b}$, with parameter $a$ provided for a fixed $b=-1$, along with the $95 \%$ confidence level in $a$. 


\section{S.27 Simulation Summary - Al/Si 359.00 MW Series}

\section{S.27.1 Fitting Film-to-Substrate Boundary Conductance - $h_{\mathrm{BD}}$}

Table S.45: Summary of simulation data - $100 \mathrm{~nm} \mathrm{Al/Si} \mathrm{359.00} \mathrm{MW} \mathrm{Series.} \mathrm{Error} \mathrm{bounds} \mathrm{represent} \mathrm{a} 95 \%$ confidence level.

\begin{tabular}{cccccc}
\hline \multicolumn{6}{c}{$\mathbf{1 0 0} \mathbf{~ n m ~ A l ~ o n ~} \mathbf{~ S i}, h_{\mathrm{BD}}=\mathbf{3 5 9 . 0 0} \mathbf{M W} / \mathbf{m}^{2} \mathbf{K}$} \\
\hline $\mathbf{S N P}$ & $\mu-\left[\mathbf{M W} / \mathbf{m}^{2} \mathbf{K}\right]$ & $\sigma-\left[\mathbf{M W} / \mathbf{m}^{2} \mathbf{K}\right]$ & $\mathbf{S N P}$ & $\mu-\left[\mathbf{M W} / \mathbf{m}^{2} \mathbf{K}\right]$ & $\sigma-\left[\mathbf{M W} / \mathbf{m}^{2} \mathbf{K}\right]$ \\
1.98 & $410.03 \pm 10.889$ & $174.24 \pm 0.4922$ & 12.79 & $359.44 \pm 1.236$ & $19.89 \pm 0.0555$ \\
2.98 & $384.26 \pm 6.335$ & $101.73 \pm 0.2853$ & 13.78 & $360.04 \pm 1.213$ & $19.54 \pm 0.0545$ \\
4.00 & $377.38 \pm 4.926$ & $79.31 \pm 0.2213$ & 14.88 & $359.72 \pm 1.109$ & $17.87 \pm 0.0498$ \\
4.96 & $366.75 \pm 3.415$ & $54.90 \pm 0.1537$ & 15.77 & $360.15 \pm 1.030$ & $16.60 \pm 0.0462$ \\
5.97 & $367.34 \pm 2.865$ & $46.09 \pm 0.1288$ & 16.82 & $359.69 \pm 0.952$ & $15.34 \pm 0.0427$ \\
6.93 & $365.50 \pm 2.503$ & $40.30 \pm 0.1124$ & 17.57 & $359.10 \pm 0.976$ & $15.74 \pm 0.0438$ \\
7.93 & $363.40 \pm 2.202$ & $35.47 \pm 0.0989$ & 18.64 & $359.35 \pm 0.884$ & $14.24 \pm 0.0397$ \\
8.93 & $361.23 \pm 1.800$ & $28.99 \pm 0.0808$ & 19.22 & $360.13 \pm 0.850$ & $13.69 \pm 0.0382$ \\
9.90 & $361.84 \pm 1.733$ & $27.93 \pm 0.0778$ & 20.71 & $359.90 \pm 0.803$ & $12.95 \pm 0.0361$ \\
10.89 & $360.13 \pm 1.519$ & $24.47 \pm 0.0682$ & 21.50 & $359.61 \pm 0.773$ & $12.45 \pm 0.0347$ \\
11.78 & $360.57 \pm 1.420$ & $22.88 \pm 0.0637$ & & & \\
\hline
\end{tabular}

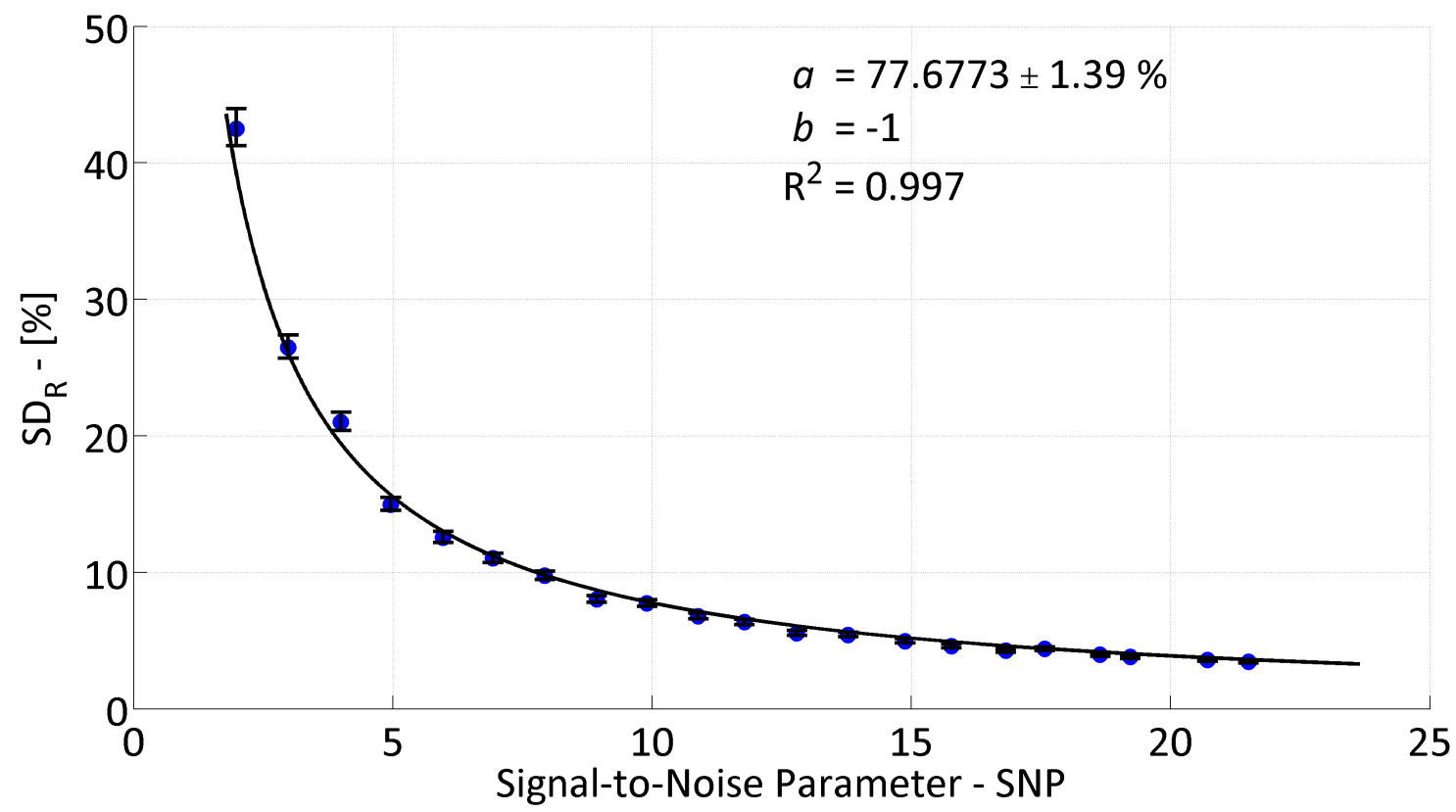

Figure S.106: Relative population standard deviation as a function of signal-to-noise parameter for 100 $\mathrm{nm}$ of $\mathrm{Al} / \mathrm{Si}$ with $h_{\mathrm{BD}}=359.00 \mathrm{MW} / \mathrm{m}^{2} \mathrm{~K}$. Error bars represent a $95 \%$ confidence interval of the measured $\mathrm{SD}_{\mathrm{R}}$ and the solid line represents a best fit of a power law to the data, of the form $\mathrm{SD}_{\mathrm{R}}=a \times \mathrm{SNP}^{b}$, with parameter $a$ provided for a fixed $b=-1$, along with the $95 \%$ confidence level in $a$. 


\section{S.27.2 Fitting Substrate Thermal Conductivity $-k_{S}$}

Table S.46: Summary of simulation data - $100 \mathrm{~nm} \mathrm{Al/Si} \mathrm{359.00} \mathrm{MW} \mathrm{Series.} \mathrm{Error} \mathrm{bounds} \mathrm{represent} \mathrm{a} 95 \%$ confidence level.

\begin{tabular}{cccccc}
\hline \multicolumn{6}{c}{$\mathbf{1 0 0} \mathbf{~ n m} \mathbf{A l}$ on $\mathbf{~ S i}, h_{\mathrm{BD}}=\mathbf{3 5 9 . 0 0} \mathbf{M W} / \mathbf{m}^{2} \mathbf{K}$} \\
\hline $\mathbf{S N P}$ & $\mu-[\mathbf{W} / \mathbf{m K}]$ & $\sigma-[\mathbf{W} / \mathbf{m K}]$ & $\mathbf{S N P}$ & $\mu-[\mathbf{W} / \mathbf{m K}]$ & $\sigma-[\mathbf{W} / \mathbf{m K}]$ \\
1.98 & $153.49 \pm 2.247$ & $36.22 \pm 0.1009$ & 12.79 & $148.05 \pm 0.316$ & $5.10 \pm 0.0142$ \\
2.98 & $149.39 \pm 1.380$ & $22.23 \pm 0.0620$ & 13.78 & $148.09 \pm 0.299$ & $4.81 \pm 0.0134$ \\
4.00 & $148.67 \pm 1.076$ & $17.34 \pm 0.0483$ & 14.88 & $148.19 \pm 0.280$ & $4.51 \pm 0.0126$ \\
4.96 & $148.51 \pm 0.801$ & $12.90 \pm 0.0359$ & 15.77 & $147.83 \pm 0.254$ & $4.10 \pm 0.0114$ \\
5.97 & $147.78 \pm 0.684$ & $11.02 \pm 0.0307$ & 16.82 & $147.99 \pm 0.239$ & $3.86 \pm 0.0107$ \\
6.93 & $148.18 \pm 0.632$ & $10.19 \pm 0.0284$ & 17.57 & $148.15 \pm 0.235$ & $3.78 \pm 0.0105$ \\
7.93 & $148.03 \pm 0.532$ & $8.58 \pm 0.0239$ & 18.64 & $148.19 \pm 0.227$ & $3.66 \pm 0.0102$ \\
8.93 & $148.39 \pm 0.447$ & $7.21 \pm 0.0201$ & 19.22 & $147.97 \pm 0.217$ & $3.50 \pm 0.0097$ \\
9.90 & $148.32 \pm 0.407$ & $6.56 \pm 0.0183$ & 20.71 & $148.01 \pm 0.202$ & $3.25 \pm 0.0091$ \\
10.89 & $148.28 \pm 0.379$ & $6.11 \pm 0.0170$ & 21.50 & $148.15 \pm 0.195$ & $3.15 \pm 0.0088$ \\
11.78 & $148.18 \pm 0.355$ & $5.72 \pm 0.0159$ & & & \\
\hline
\end{tabular}

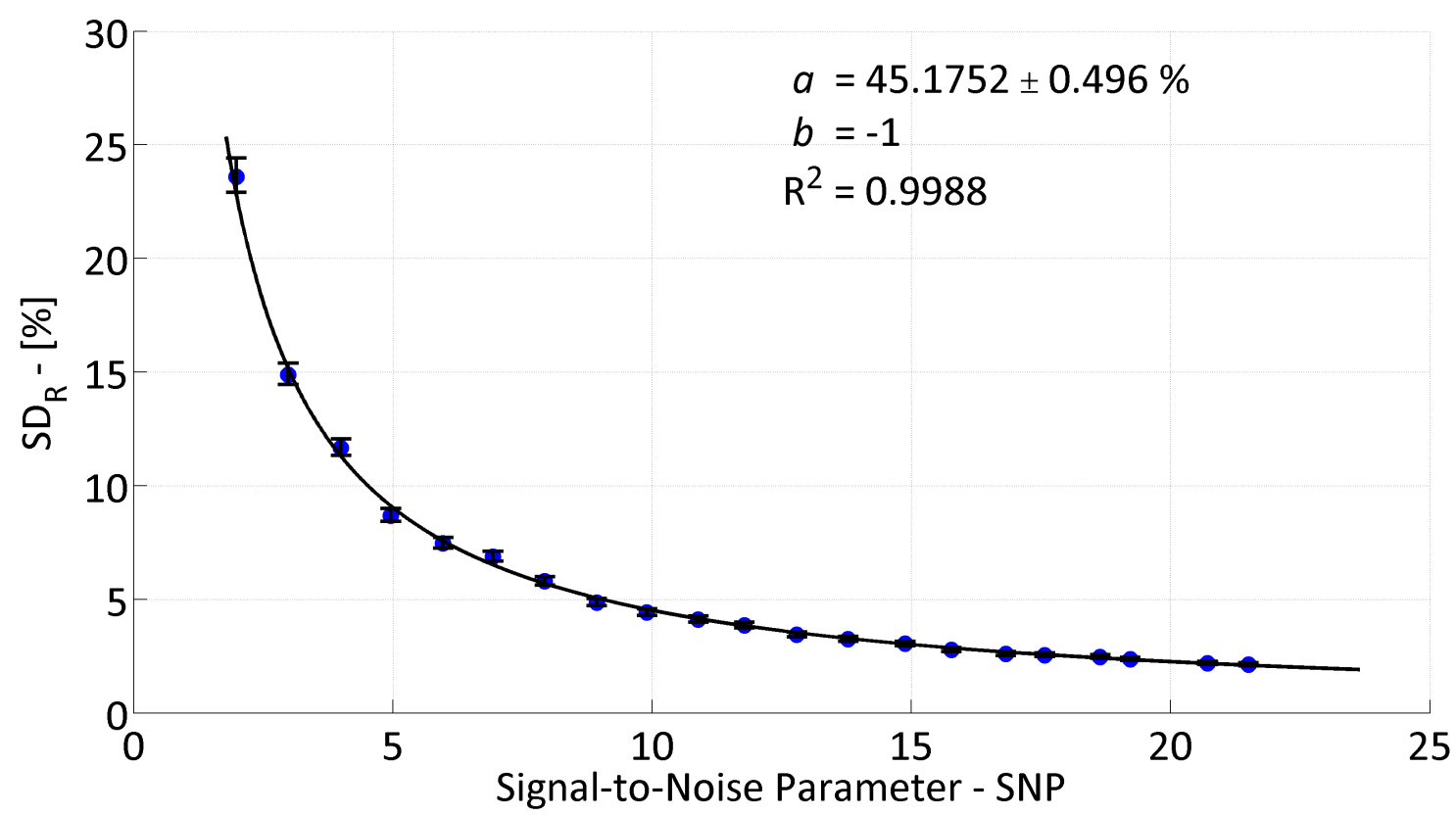

Figure S.107: Relative population standard deviation as a function of signal-to-noise parameter for 100 $\mathrm{nm}$ of $\mathrm{Al} / \mathrm{Si}$ with $h_{\mathrm{BD}}=359.00 \mathrm{MW} / \mathrm{m}^{2} \mathrm{~K}$. Error bars represent a $95 \%$ confidence interval of the measured $\mathrm{SD}_{\mathrm{R}}$ and the solid line represents a best fit of a power law to the data, of the form $\mathrm{SD}_{\mathrm{R}}=a \times \mathrm{SNP}^{b}$, with parameter $a$ provided for a fixed $b=-1$, along with the $95 \%$ confidence level in $a$. 


\section{S.28 Simulation Summary - Al/Si 464.00 MW Series}

\section{S.28.1 Fitting Film-to-Substrate Boundary Conductance - $h_{\mathrm{BD}}$}

Table S.47: Summary of simulation data - $100 \mathrm{~nm} \mathrm{Al/Si} \mathrm{464.00} \mathrm{MW} \mathrm{Series.} \mathrm{Error} \mathrm{bounds} \mathrm{represent} \mathrm{a} 95 \%$ confidence level.

\begin{tabular}{cccccc}
\hline \multicolumn{6}{c}{$\mathbf{1 0 0} \mathbf{~ n m ~ A l ~ o n ~} \mathbf{~ S i}, h_{\mathrm{BD}}=\mathbf{4 6 4 . 0 0} \mathbf{M W} / \mathbf{m}^{2} \mathbf{K}$} \\
\hline $\mathbf{S N P}$ & $\mu-\left[\mathbf{M W} / \mathbf{m}^{2} \mathbf{K}\right]$ & $\sigma-\left[\mathbf{M W} / \mathbf{m}^{2} \mathbf{K}\right]$ & $\mathbf{S N P}$ & $\mu-\left[\mathbf{M W} / \mathbf{m}^{2} \mathbf{K}\right]$ & $\sigma-\left[\mathbf{M W} / \mathbf{m}^{2} \mathbf{K}\right]$ \\
1.98 & $513.24 \pm 13.808$ & $220.61 \pm 0.6251$ & 12.69 & $466.65 \pm 1.879$ & $30.27 \pm 0.0843$ \\
3.02 & $490.11 \pm 8.238$ & $132.42 \pm 0.3707$ & 13.58 & $465.24 \pm 1.722$ & $27.75 \pm 0.0773$ \\
3.91 & $478.42 \pm 6.410$ & $103.15 \pm 0.2881$ & 14.43 & $464.81 \pm 1.696$ & $27.34 \pm 0.0761$ \\
4.85 & $476.74 \pm 5.287$ & $85.16 \pm 0.2374$ & 15.51 & $465.25 \pm 1.546$ & $24.90 \pm 0.0694$ \\
5.83 & $472.82 \pm 4.307$ & $69.37 \pm 0.1934$ & 16.24 & $463.60 \pm 1.441$ & $23.22 \pm 0.0647$ \\
6.79 & $469.03 \pm 3.707$ & $59.73 \pm 0.1663$ & 17.19 & $464.25 \pm 1.434$ & $23.11 \pm 0.0644$ \\
7.79 & $469.07 \pm 3.071$ & $49.44 \pm 0.1380$ & 18.44 & $465.29 \pm 1.369$ & $22.06 \pm 0.0614$ \\
8.66 & $469.19 \pm 2.989$ & $48.18 \pm 0.1342$ & 19.20 & $464.04 \pm 1.273$ & $20.51 \pm 0.0571$ \\
9.82 & $467.37 \pm 2.556$ & $41.19 \pm 0.1147$ & 20.30 & $464.19 \pm 1.120$ & $18.05 \pm 0.0503$ \\
10.88 & $467.53 \pm 2.364$ & $38.10 \pm 0.1061$ & 21.11 & $463.62 \pm 1.152$ & $18.56 \pm 0.0517$ \\
11.74 & $465.65 \pm 2.058$ & $33.16 \pm 0.0923$ & & & \\
\hline
\end{tabular}

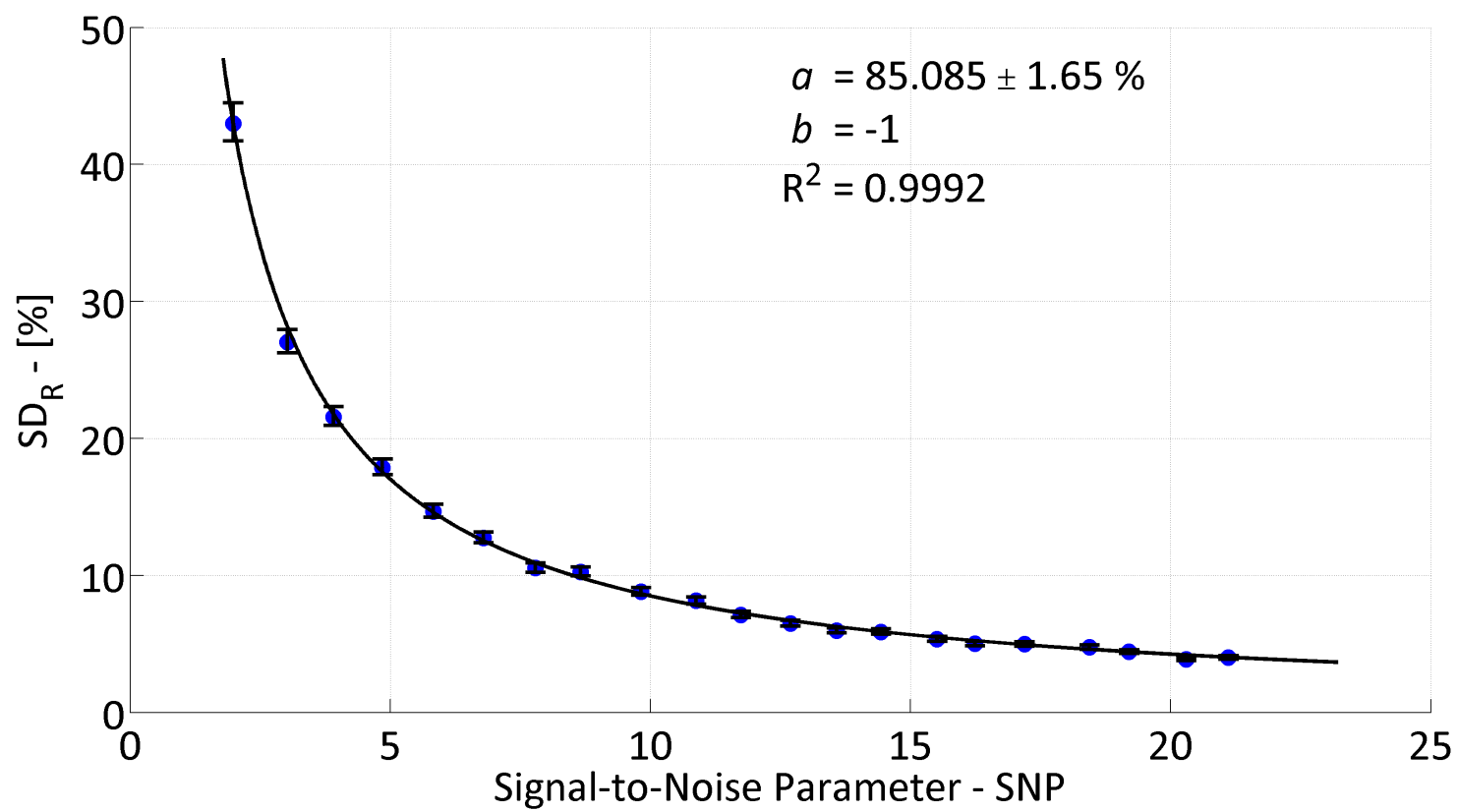

Figure S.108: Relative population standard deviation as a function of signal-to-noise parameter for 100 $\mathrm{nm}$ of $\mathrm{Al} / \mathrm{Si}$ with $h_{\mathrm{BD}}=464.00 \mathrm{MW} / \mathrm{m}^{2} \mathrm{~K}$. Error bars represent a $95 \%$ confidence interval of the measured $\mathrm{SD}_{\mathrm{R}}$ and the solid line represents a best fit of a power law to the data, of the form $\mathrm{SD}_{\mathrm{R}}=a \times \mathrm{SNP}^{b}$, with parameter $a$ provided for a fixed $b=-1$, along with the $95 \%$ confidence level in $a$. 


\section{S.28.2 Fitting Substrate Thermal Conductivity $-k_{S}$}

Table S.48: Summary of simulation data - $100 \mathrm{~nm} \mathrm{Al/Si} \mathrm{464.00} \mathrm{MW} \mathrm{Series.} \mathrm{Error} \mathrm{bounds} \mathrm{represent} \mathrm{a} 95 \%$ confidence level.

\begin{tabular}{cccccc}
\hline \multicolumn{6}{c}{$\mathbf{1 0 0} \mathbf{~ n m} \mathbf{A l}$ on $\mathbf{~ S i}, h_{\mathrm{BD}}=\mathbf{4 6 4 . 0 0} \mathbf{M W} / \mathbf{m}^{2} \mathbf{K}$} \\
\hline SNP & $\mu-[\mathbf{W} / \mathbf{m K}]$ & $\sigma-[\mathbf{W} / \mathbf{m K}]$ & $\mathbf{S N P}$ & $\mu-[\mathbf{W} / \mathbf{m K}]$ & $\sigma-[\mathbf{W} / \mathbf{m K}]$ \\
1.98 & $152.49 \pm 2.233$ & $35.93 \pm 0.1004$ & 12.69 & $147.83 \pm 0.310$ & $4.99 \pm 0.0139$ \\
3.02 & $149.32 \pm 1.353$ & $21.79 \pm 0.0607$ & 13.58 & $148.11 \pm 0.289$ & $4.65 \pm 0.0130$ \\
3.91 & $149.18 \pm 1.088$ & $17.54 \pm 0.0488$ & 14.43 & $148.15 \pm 0.286$ & $4.61 \pm 0.0128$ \\
4.85 & $148.67 \pm 0.869$ & $14.00 \pm 0.0390$ & 15.51 & $147.91 \pm 0.270$ & $4.35 \pm 0.0121$ \\
5.83 & $148.04 \pm 0.729$ & $11.74 \pm 0.0327$ & 16.24 & $148.27 \pm 0.246$ & $3.96 \pm 0.0110$ \\
6.79 & $148.26 \pm 0.625$ & $10.07 \pm 0.0280$ & 17.19 & $148.17 \pm 0.246$ & $3.97 \pm 0.0110$ \\
7.79 & $147.70 \pm 0.514$ & $8.28 \pm 0.0231$ & 18.44 & $147.87 \pm 0.225$ & $3.63 \pm 0.0101$ \\
8.66 & $147.87 \pm 0.479$ & $7.72 \pm 0.0215$ & 19.20 & $148.08 \pm 0.217$ & $3.50 \pm 0.0098$ \\
9.82 & $147.93 \pm 0.419$ & $6.76 \pm 0.0188$ & 20.30 & $148.03 \pm 0.195$ & $3.14 \pm 0.0087$ \\
10.88 & $147.93 \pm 0.385$ & $6.21 \pm 0.0173$ & 21.11 & $148.06 \pm 0.190$ & $3.06 \pm 0.0085$ \\
11.74 & $148.22 \pm 0.349$ & $5.63 \pm 0.0157$ & & & \\
\hline
\end{tabular}

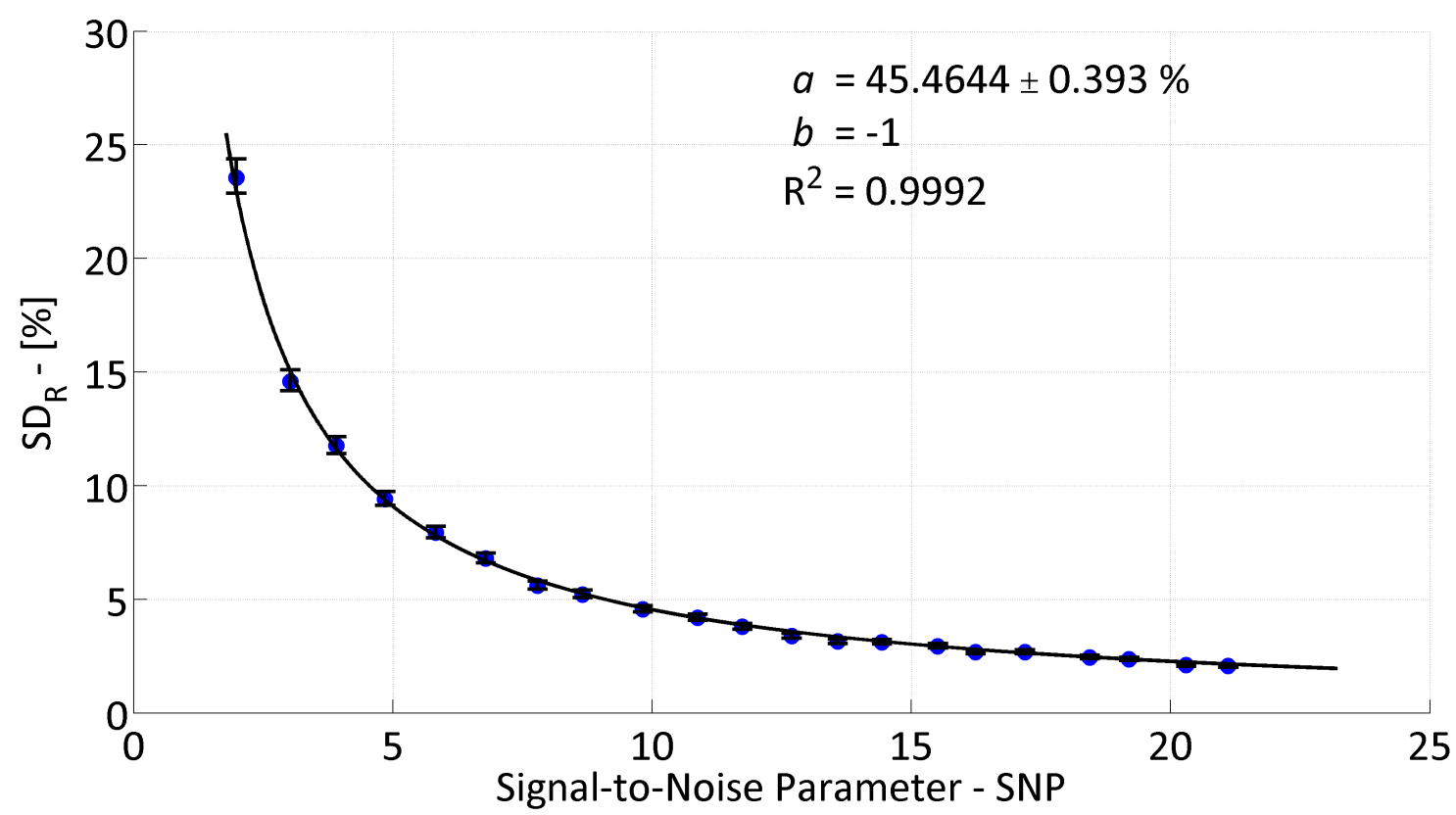

Figure S.109: Relative population standard deviation as a function of signal-to-noise parameter for 100 $\mathrm{nm}$ of $\mathrm{Al} / \mathrm{Si}$ with $h_{\mathrm{BD}}=464.00 \mathrm{MW} / \mathrm{m}^{2} \mathrm{~K}$. Error bars represent a $95 \%$ confidence interval of the measured $\mathrm{SD}_{\mathrm{R}}$ and the solid line represents a best fit of a power law to the data, of the form $\mathrm{SD}_{\mathrm{R}}=a \times \mathrm{SNP}^{b}$, with parameter $a$ provided for a fixed $b=-1$, along with the $95 \%$ confidence level in $a$. 


\section{S.28.3 Fitting Film Thickness $-d_{1}$}

Table S.49: Summary of simulation data - $100 \mathrm{~nm} \mathrm{Al/Si} \mathrm{464.00} \mathrm{MW} \mathrm{Series.} \mathrm{Error} \mathrm{bounds} \mathrm{represent} \mathrm{a} 95 \%$ confidence level.

\begin{tabular}{cccccc}
\hline \multicolumn{6}{c}{$\mathbf{1 0 0} \mathbf{~ n m} \mathbf{A l}$ on $\mathbf{~ S i}, h_{\mathrm{BD}}=\mathbf{4 6 4 . 0 0} \mathbf{~ M W} / \mathbf{m}^{2} \mathbf{K}$} \\
\hline SNP & $\mu-[\mathbf{n m}]$ & $\sigma-[\mathbf{n m}]$ & $\mathbf{S N P}$ & $\mu-[\mathbf{n m}]$ & $\sigma-[\mathbf{n m}]$ \\
1.98 & $100.65 \pm 0.859$ & $13.84 \pm 0.0385$ & 12.69 & $100.13 \pm 0.123$ & $1.99 \pm 0.0055$ \\
3.02 & $100.38 \pm 0.544$ & $8.77 \pm 0.0244$ & 13.58 & $100.00 \pm 0.116$ & $1.86 \pm 0.0052$ \\
3.91 & $100.19 \pm 0.425$ & $6.84 \pm 0.0191$ & 14.43 & $99.99 \pm 0.113$ & $1.82 \pm 0.0051$ \\
4.85 & $100.11 \pm 0.347$ & $5.60 \pm 0.0156$ & 15.51 & $100.09 \pm 0.108$ & $1.75 \pm 0.0049$ \\
5.83 & $100.29 \pm 0.290$ & $4.68 \pm 0.0130$ & 16.24 & $99.94 \pm 0.097$ & $1.57 \pm 0.0044$ \\
6.79 & $100.15 \pm 0.247$ & $3.97 \pm 0.0111$ & 17.19 & $99.97 \pm 0.097$ & $1.57 \pm 0.0044$ \\
7.79 & $100.30 \pm 0.206$ & $3.32 \pm 0.0092$ & 18.44 & $100.09 \pm 0.089$ & $1.43 \pm 0.0040$ \\
8.66 & $100.18 \pm 0.190$ & $3.06 \pm 0.0085$ & 19.20 & $100.01 \pm 0.086$ & $1.39 \pm 0.0039$ \\
9.82 & $100.14 \pm 0.168$ & $2.70 \pm 0.0075$ & 20.30 & $100.02 \pm 0.078$ & $1.25 \pm 0.0035$ \\
10.88 & $100.10 \pm 0.152$ & $2.44 \pm 0.0068$ & 21.11 & $100.02 \pm 0.075$ & $1.21 \pm 0.0034$ \\
11.74 & $99.98 \pm 0.139$ & $2.23 \pm 0.0062$ & & & \\
\hline
\end{tabular}

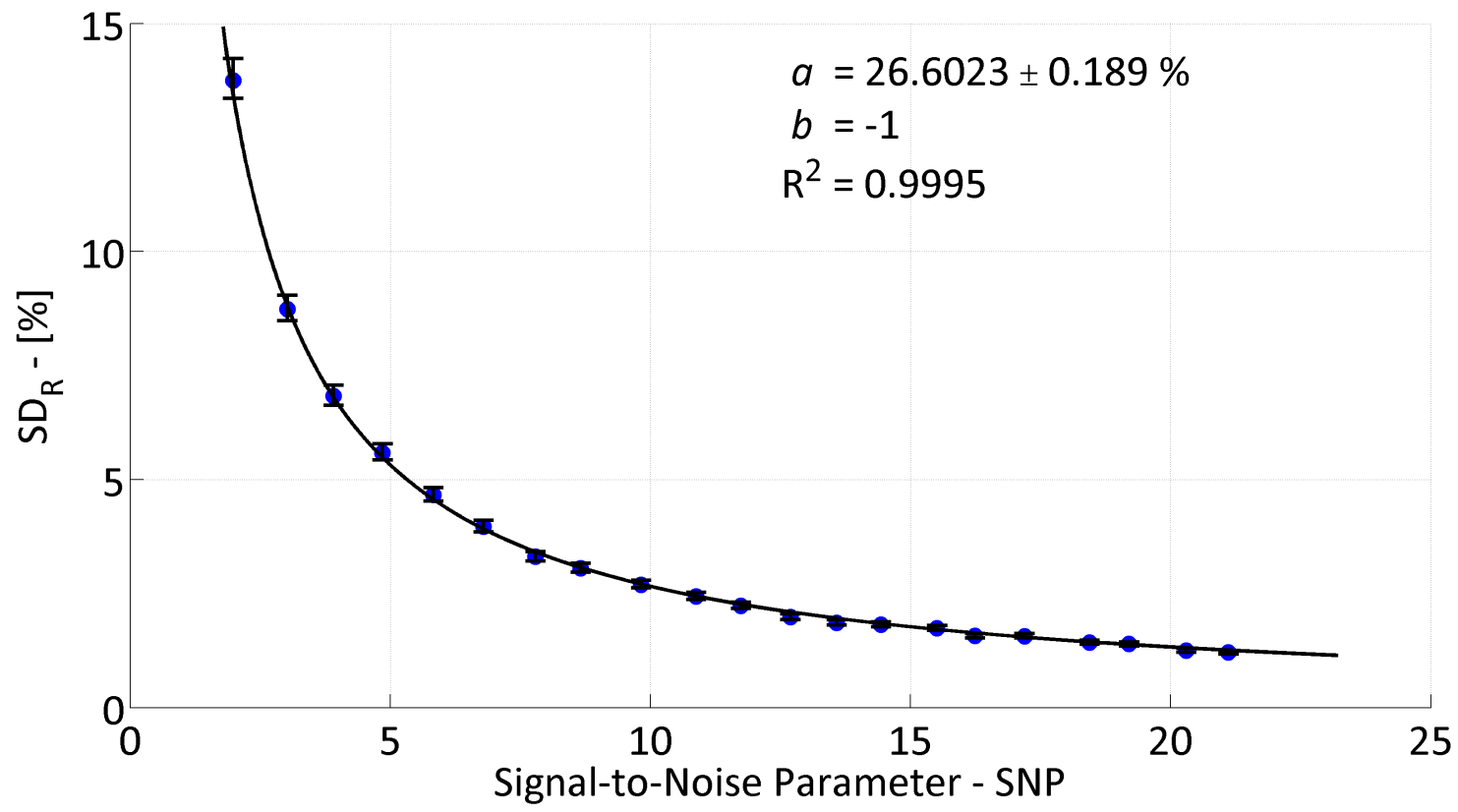

Figure S.110: Relative population standard deviation as a function of signal-to-noise parameter for 100 $\mathrm{nm}$ of $\mathrm{Al} / \mathrm{Si}$ with $h_{\mathrm{BD}}=464.00 \mathrm{MW} / \mathrm{m}^{2} \mathrm{~K}$. Error bars represent a $95 \%$ confidence interval of the measured $\mathrm{SD}_{\mathrm{R}}$ and the solid line represents a best fit of a power law to the data, of the form $\mathrm{SD}_{\mathrm{R}}=a \times \mathrm{SNP}^{b}$, with parameter $a$ provided for a fixed $b=-1$, along with the $95 \%$ confidence level in $a$. 


\section{S.29 Simulation Summary - Al/Si 599.00 MW Series}

\section{S.29.1 Fitting Film-to-Substrate Boundary Conductance - $h_{\mathrm{BD}}$}

Table S.50: Summary of simulation data - 100 nm Al/Si 599.00 MW Series. Error bounds represent a $95 \%$ confidence level.

\begin{tabular}{cccccc}
\hline \multicolumn{6}{c}{$\mathbf{1 0 0} \mathbf{~ n m ~ A l ~ o n ~} \mathbf{~ S i}, h_{\mathrm{BD}}=\mathbf{5 9 9 . 0 0} \mathbf{M W} / \mathbf{m}^{2} \mathbf{K}$} \\
\hline $\mathbf{S N P}$ & $\mu-\left[\mathbf{M W} / \mathbf{m}^{2} \mathbf{K}\right]$ & $\sigma-\left[\mathbf{M W} / \mathbf{m}^{2} \mathbf{K}\right]$ & $\mathbf{S N P}$ & $\mu-\left[\mathbf{M W} / \mathbf{m}^{2} \mathbf{K}\right]$ & $\sigma-\left[\mathbf{M W} / \mathbf{m}^{2} \mathbf{K}\right]$ \\
1.96 & $645.82 \pm 18.160$ & $290.59 \pm 0.8208$ & 12.45 & $602.71 \pm 2.631$ & $42.37 \pm 0.1181$ \\
2.91 & $629.35 \pm 11.945$ & $191.62 \pm 0.5385$ & 13.83 & $600.08 \pm 2.441$ & $39.33 \pm 0.1095$ \\
3.96 & $619.26 \pm 8.546$ & $137.51 \pm 0.3841$ & 14.47 & $600.56 \pm 2.161$ & $34.83 \pm 0.0970$ \\
4.81 & $604.98 \pm 6.864$ & $110.61 \pm 0.3080$ & 15.42 & $600.73 \pm 2.224$ & $35.85 \pm 0.0998$ \\
5.86 & $606.29 \pm 5.796$ & $93.36 \pm 0.2603$ & 16.44 & $600.23 \pm 1.962$ & $31.60 \pm 0.0881$ \\
6.80 & $603.28 \pm 4.930$ & $79.41 \pm 0.2214$ & 17.16 & $600.46 \pm 1.878$ & $30.25 \pm 0.0843$ \\
7.73 & $603.11 \pm 4.336$ & $69.87 \pm 0.1946$ & 18.12 & $600.66 \pm 1.884$ & $30.35 \pm 0.0845$ \\
8.78 & $601.12 \pm 3.658$ & $58.93 \pm 0.1643$ & 19.29 & $598.94 \pm 1.737$ & $27.99 \pm 0.0779$ \\
9.62 & $603.95 \pm 3.428$ & $55.23 \pm 0.1538$ & 19.87 & $599.97 \pm 1.672$ & $26.95 \pm 0.0751$ \\
10.48 & $601.38 \pm 3.113$ & $50.16 \pm 0.1397$ & 21.48 & $599.10 \pm 1.517$ & $24.44 \pm 0.0681$ \\
11.79 & $602.54 \pm 2.823$ & $45.49 \pm 0.1267$ & & & \\
\hline
\end{tabular}

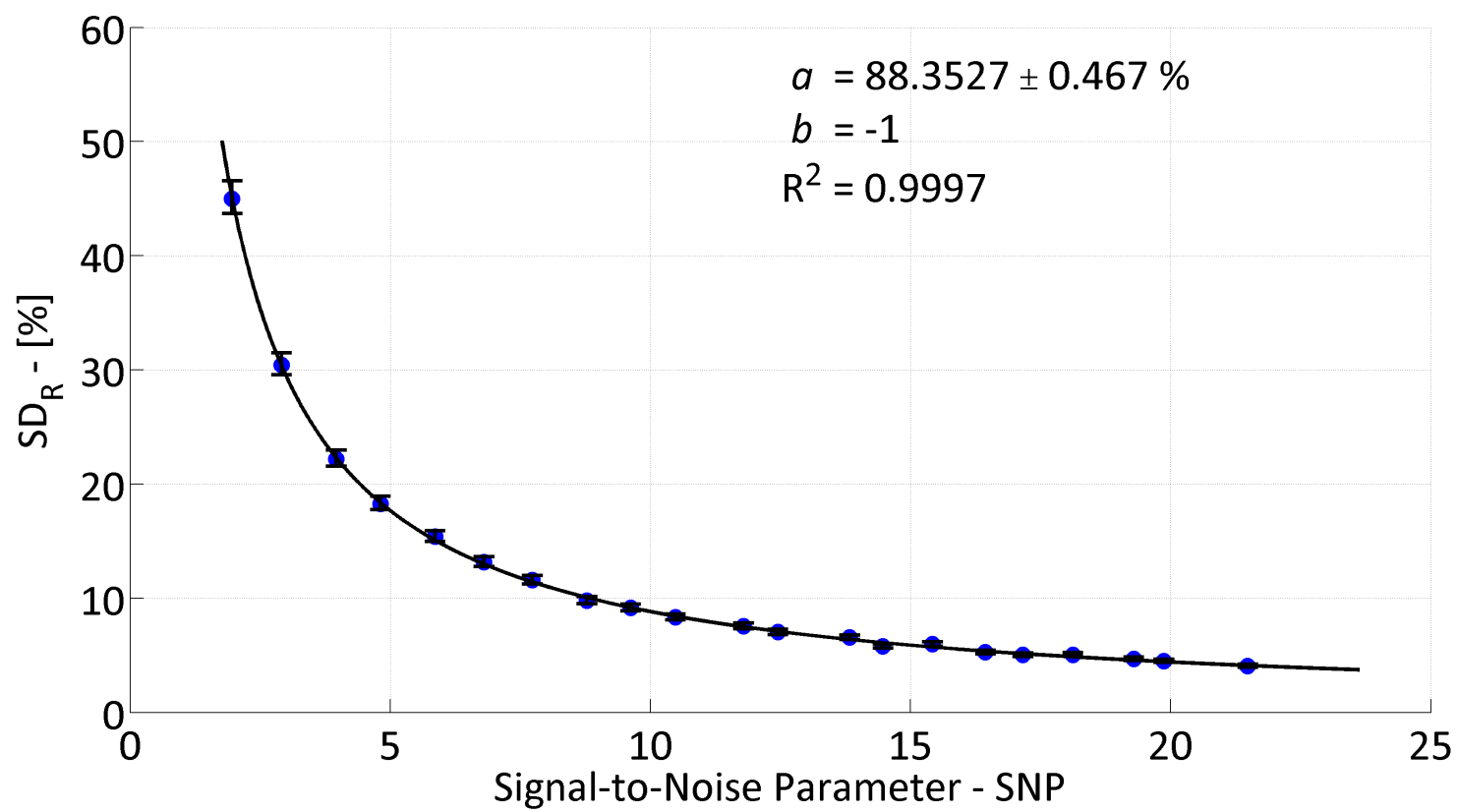

Figure S.111: Relative population standard deviation as a function of signal-to-noise parameter for 100 $\mathrm{nm}$ of $\mathrm{Al} / \mathrm{Si}$ with $h_{\mathrm{BD}}=599.00 \mathrm{MW} / \mathrm{m}^{2} \mathrm{~K}$. Error bars represent a $95 \%$ confidence interval of the measured $\mathrm{SD}_{\mathrm{R}}$ and the solid line represents a best fit of a power law to the data, of the form $\mathrm{SD}_{\mathrm{R}}=a \times \mathrm{SNP}^{b}$, with parameter $a$ provided for a fixed $b=-1$, along with the $95 \%$ confidence level in $a$. 


\section{S.29.2 Fitting Substrate Thermal Conductivity $-k_{S}$}

Table S.51: Summary of simulation data - $100 \mathrm{~nm} \mathrm{Al/Si} \mathrm{599.00} \mathrm{MW} \mathrm{Series.} \mathrm{Error} \mathrm{bounds} \mathrm{represent} \mathrm{a} 95 \%$ confidence level.

\begin{tabular}{cccccc}
\hline \multicolumn{6}{c}{$\mathbf{1 0 0} \mathbf{~ n m} \mathbf{A l}$ on $\mathbf{~ S i}, h_{\mathrm{BD}}=\mathbf{5 9 9 . 0 0} \mathbf{M W} / \mathbf{m}^{2} \mathbf{K}$} \\
\hline SNP & $\mu-[\mathbf{W} / \mathbf{m K}]$ & $\sigma-[\mathbf{W} / \mathbf{m K}]$ & $\mathbf{S N P}$ & $\mu-[\mathbf{W} / \mathbf{m K}]$ & $\sigma-[\mathbf{W} / \mathbf{m K}]$ \\
1.96 & $154.30 \pm 2.260$ & $36.42 \pm 0.1014$ & 12.45 & $147.71 \pm 0.342$ & $5.51 \pm 0.0153$ \\
2.91 & $150.08 \pm 1.568$ & $25.27 \pm 0.0704$ & 13.83 & $148.19 \pm 0.312$ & $5.03 \pm 0.0140$ \\
3.96 & $149.01 \pm 1.075$ & $17.33 \pm 0.0483$ & 14.47 & $148.00 \pm 0.279$ & $4.50 \pm 0.0125$ \\
4.81 & $149.54 \pm 0.893$ & $14.39 \pm 0.0401$ & 15.42 & $147.98 \pm 0.284$ & $4.58 \pm 0.0128$ \\
5.86 & $148.32 \pm 0.743$ & $11.98 \pm 0.0334$ & 16.44 & $147.87 \pm 0.255$ & $4.10 \pm 0.0114$ \\
6.80 & $148.42 \pm 0.632$ & $10.19 \pm 0.0284$ & 17.16 & $147.99 \pm 0.245$ & $3.94 \pm 0.0110$ \\
7.73 & $148.33 \pm 0.543$ & $8.74 \pm 0.0244$ & 18.12 & $147.92 \pm 0.239$ & $3.85 \pm 0.0107$ \\
8.78 & $148.35 \pm 0.473$ & $7.62 \pm 0.0212$ & 19.29 & $148.15 \pm 0.226$ & $3.65 \pm 0.0102$ \\
9.62 & $147.86 \pm 0.439$ & $7.08 \pm 0.0197$ & 19.87 & $147.91 \pm 0.221$ & $3.57 \pm 0.0099$ \\
10.48 & $148.16 \pm 0.404$ & $6.50 \pm 0.0181$ & 21.48 & $147.99 \pm 0.192$ & $3.10 \pm 0.0086$ \\
11.79 & $147.78 \pm 0.364$ & $5.87 \pm 0.0163$ & & & \\
\hline
\end{tabular}

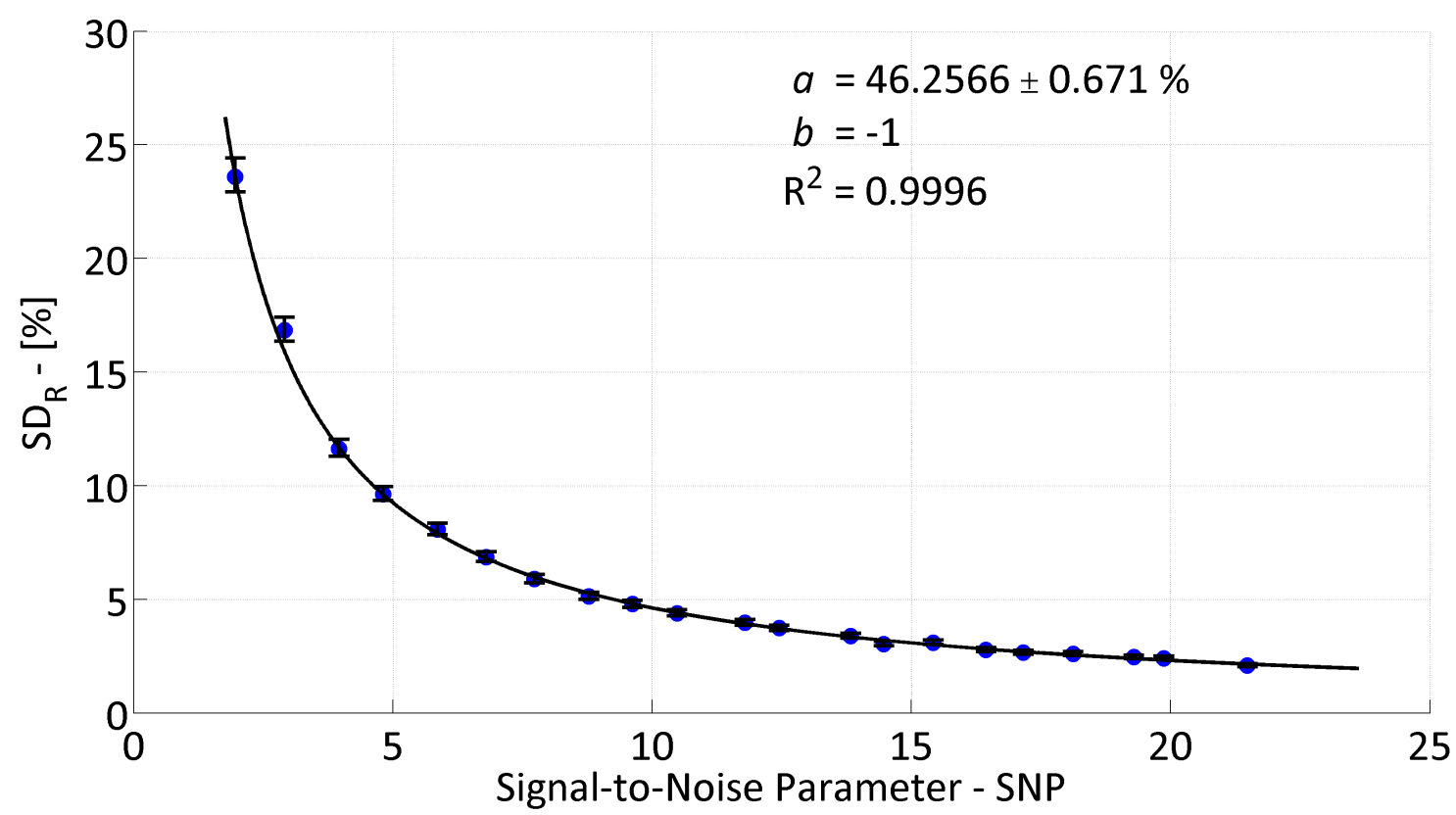

Figure S.112: Relative population standard deviation as a function of signal-to-noise parameter for 100 $\mathrm{nm}$ of $\mathrm{Al} / \mathrm{Si}$ with $h_{\mathrm{BD}}=599.00 \mathrm{MW} / \mathrm{m}^{2} \mathrm{~K}$. Error bars represent a $95 \%$ confidence interval of the measured $\mathrm{SD}_{\mathrm{R}}$ and the solid line represents a best fit of a power law to the data, of the form $\mathrm{SD}_{\mathrm{R}}=a \times \mathrm{SNP}^{b}$, with parameter $a$ provided for a fixed $b=-1$, along with the $95 \%$ confidence level in $a$. 


\section{S.30 Simulation Summary - Al/Si 774.00 MW Series}

\section{S.30.1 Fitting Film-to-Substrate Boundary Conductance - $h_{\mathrm{BD}}$}

Table S.52: Summary of simulation data - $100 \mathrm{~nm} \mathrm{Al/Si} \mathrm{774.00} \mathrm{MW} \mathrm{Series.} \mathrm{Error} \mathrm{bounds} \mathrm{represent} \mathrm{a} 95 \%$ confidence level.

\begin{tabular}{cccccc}
\hline \multicolumn{6}{c}{$\mathbf{1 0 0} \mathbf{~ n m ~ A l ~ o n ~} \mathbf{~ S i}, h_{\mathrm{BD}}=\mathbf{7 7 4 . 0 0} \mathbf{M W} / \mathbf{m}^{2} \mathbf{K}$} \\
\hline $\mathbf{S N P}$ & $\mu-\left[\mathbf{M W} / \mathbf{m}^{2} \mathbf{K}\right]$ & $\sigma-\left[\mathbf{M W} / \mathbf{m}^{2} \mathbf{K}\right]$ & $\mathbf{S N P}$ & $\mu-\left[\mathbf{M W} / \mathbf{m}^{2} \mathbf{K}\right]$ & $\sigma-\left[\mathbf{M W} / \mathbf{m}^{2} \mathbf{K}\right]$ \\
1.96 & $845.30 \pm 24.946$ & $396.93 \pm 1.1339$ & 12.58 & $772.37 \pm 3.728$ & $60.07 \pm 0.1673$ \\
3.01 & $813.87 \pm 15.815$ & $254.60 \pm 0.7105$ & 13.50 & $774.52 \pm 3.257$ & $52.46 \pm 0.1462$ \\
3.93 & $798.04 \pm 12.242$ & $197.28 \pm 0.5494$ & 14.36 & $777.96 \pm 3.110$ & $50.11 \pm 0.1396$ \\
4.92 & $791.16 \pm 9.819$ & $158.22 \pm 0.4406$ & 15.58 & $775.29 \pm 2.849$ & $45.91 \pm 0.1279$ \\
6.02 & $791.50 \pm 7.664$ & $123.50 \pm 0.3439$ & 16.48 & $775.97 \pm 2.747$ & $44.27 \pm 0.1233$ \\
6.91 & $786.92 \pm 6.754$ & $108.85 \pm 0.3031$ & 16.88 & $777.12 \pm 2.622$ & $42.25 \pm 0.1177$ \\
7.80 & $782.26 \pm 5.785$ & $93.17 \pm 0.2597$ & 17.98 & $772.54 \pm 2.511$ & $40.46 \pm 0.1127$ \\
8.88 & $779.23 \pm 5.062$ & $81.57 \pm 0.2272$ & 18.91 & $775.71 \pm 2.376$ & $38.28 \pm 0.1066$ \\
9.75 & $775.35 \pm 4.862$ & $78.35 \pm 0.2182$ & 20.95 & $774.14 \pm 2.273$ & $36.64 \pm 0.1020$ \\
10.64 & $773.21 \pm 4.158$ & $67.01 \pm 0.1866$ & 21.10 & $775.87 \pm 2.070$ & $33.34 \pm 0.0929$ \\
11.70 & $776.21 \pm 3.728$ & $60.07 \pm 0.1673$ & & & \\
\hline
\end{tabular}

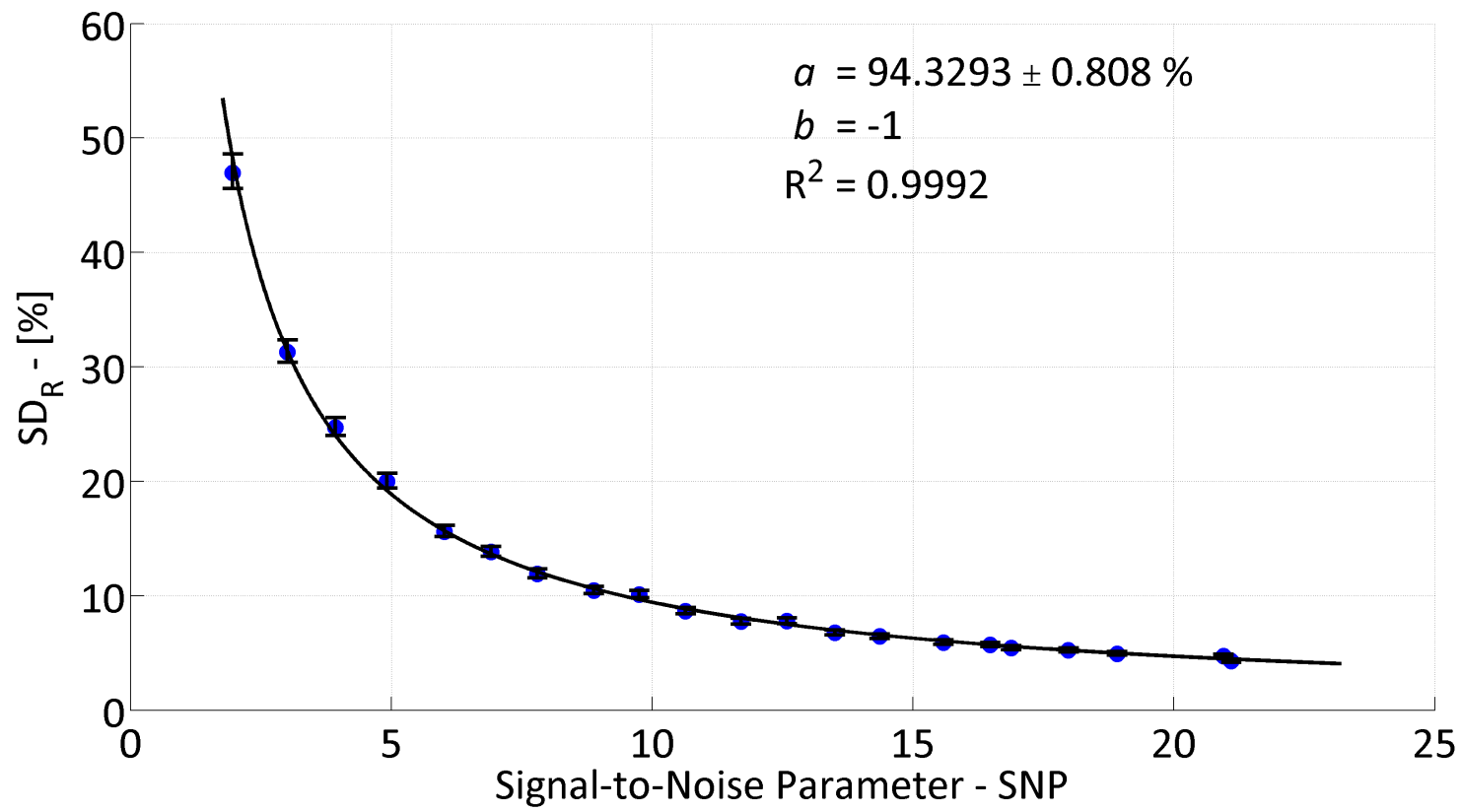

Figure S.113: Relative population standard deviation as a function of signal-to-noise parameter for 100 $\mathrm{nm}$ of $\mathrm{Al} / \mathrm{Si}$ with $h_{\mathrm{BD}}=774.00 \mathrm{MW} / \mathrm{m}^{2} \mathrm{~K}$. Error bars represent a $95 \%$ confidence interval of the measured $\mathrm{SD}_{\mathrm{R}}$ and the solid line represents a best fit of a power law to the data, of the form $\mathrm{SD}_{\mathrm{R}}=a \times \mathrm{SNP}^{b}$, with parameter $a$ provided for a fixed $b=-1$, along with the $95 \%$ confidence level in $a$. 


\section{S.30.2 Fitting Substrate Thermal Conductivity $-k_{S}$}

Table S.53: Summary of simulation data - $100 \mathrm{~nm} \mathrm{Al/Si} \mathrm{774.00} \mathrm{MW} \mathrm{Series.} \mathrm{Error} \mathrm{bounds} \mathrm{represent} \mathrm{a} 95 \%$ confidence level.

\begin{tabular}{cccccc}
\hline \multicolumn{6}{c}{$\mathbf{1 0 0} \mathbf{~ n m} \mathbf{A l}$ on $\mathbf{~ S i}, h_{\mathrm{BD}}=\mathbf{7 7 4 . 0 0} \mathbf{M W} / \mathbf{m}^{2} \mathbf{K}$} \\
\hline SNP & $\mu-[\mathbf{W} / \mathbf{m K}]$ & $\sigma-[\mathbf{W} / \mathbf{m K}]$ & $\mathbf{S N P}$ & $\mu-[\mathbf{W} / \mathbf{m K}]$ & $\sigma-[\mathbf{W} / \mathbf{m K}]$ \\
1.96 & $152.04 \pm 2.280$ & $36.73 \pm 0.1024$ & 12.58 & $148.48 \pm 0.371$ & $5.98 \pm 0.0167$ \\
3.01 & $149.88 \pm 1.481$ & $23.85 \pm 0.0665$ & 13.50 & $148.11 \pm 0.326$ & $5.26 \pm 0.0146$ \\
3.93 & $149.21 \pm 1.166$ & $18.79 \pm 0.0523$ & 14.36 & $147.68 \pm 0.301$ & $4.85 \pm 0.0135$ \\
4.92 & $148.59 \pm 0.930$ & $14.98 \pm 0.0417$ & 15.58 & $148.05 \pm 0.286$ & $4.61 \pm 0.0128$ \\
6.02 & $147.97 \pm 0.741$ & $11.94 \pm 0.0333$ & 16.48 & $147.86 \pm 0.273$ & $4.40 \pm 0.0122$ \\
6.91 & $147.99 \pm 0.654$ & $10.55 \pm 0.0294$ & 16.88 & $147.90 \pm 0.257$ & $4.15 \pm 0.0115$ \\
7.80 & $148.18 \pm 0.580$ & $9.35 \pm 0.0260$ & 17.98 & $148.21 \pm 0.254$ & $4.09 \pm 0.0114$ \\
8.88 & $148.23 \pm 0.501$ & $8.08 \pm 0.0225$ & 18.91 & $147.88 \pm 0.238$ & $3.83 \pm 0.0107$ \\
9.75 & $148.45 \pm 0.484$ & $7.79 \pm 0.0217$ & 20.95 & $148.08 \pm 0.223$ & $3.60 \pm 0.0100$ \\
10.64 & $148.47 \pm 0.415$ & $6.69 \pm 0.0186$ & 21.10 & $147.78 \pm 0.210$ & $3.38 \pm 0.0094$ \\
11.70 & $148.06 \pm 0.377$ & $6.07 \pm 0.0169$ & & & \\
\hline
\end{tabular}

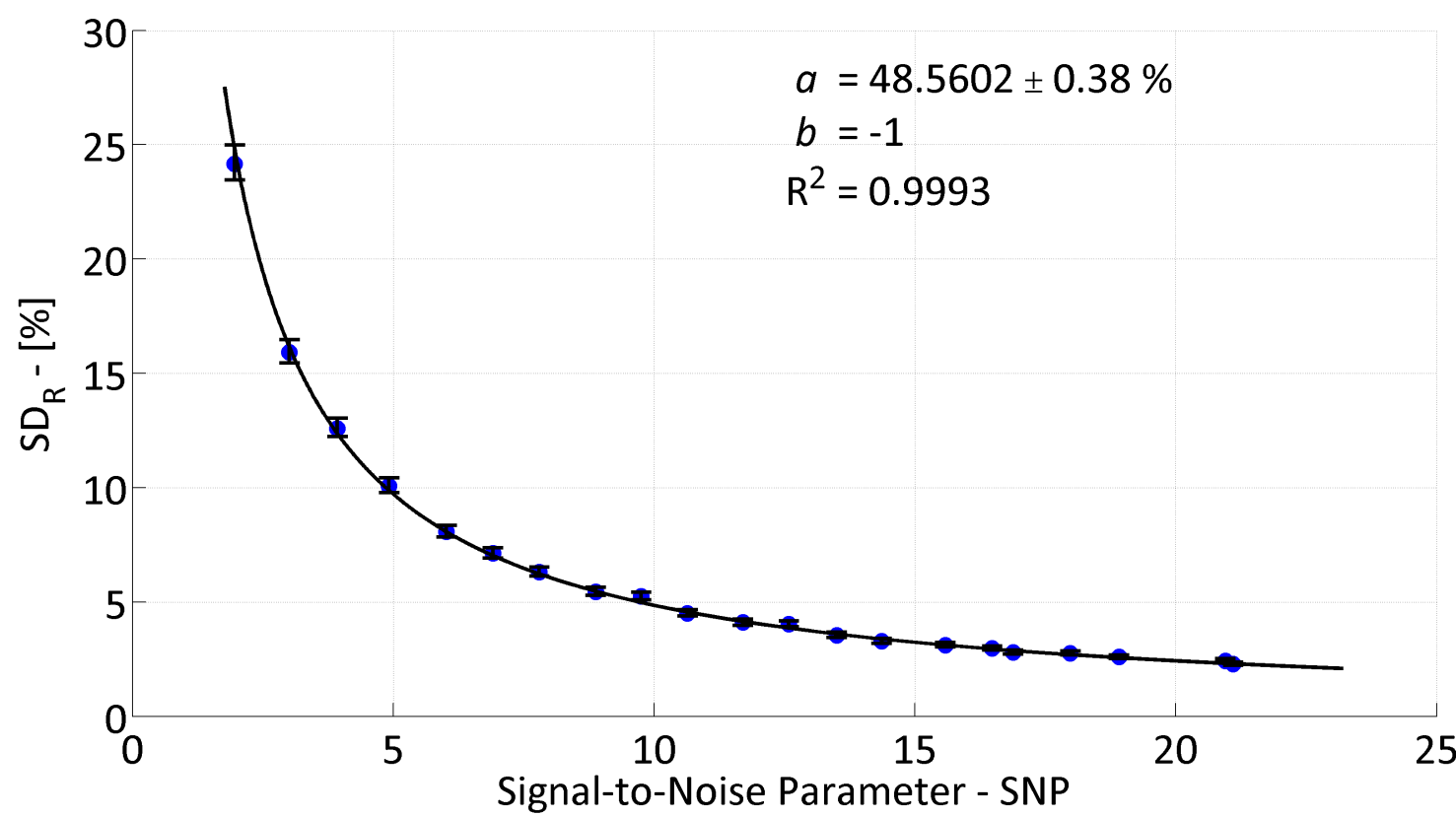

Figure S.114: Relative population standard deviation as a function of signal-to-noise parameter for 100 $\mathrm{nm}$ of $\mathrm{Al} / \mathrm{Si}$ with $h_{\mathrm{BD}}=774.00 \mathrm{MW} / \mathrm{m}^{2} \mathrm{~K}$. Error bars represent a $95 \%$ confidence interval of the measured $\mathrm{SD}_{\mathrm{R}}$ and the solid line represents a best fit of a power law to the data, of the form $\mathrm{SD}_{\mathrm{R}}=a \times \mathrm{SNP}^{b}$, with parameter $a$ provided for a fixed $b=-1$, along with the $95 \%$ confidence level in $a$. 


\section{S.31 Simulation Summary - Al/Si 1000.00 MW Series}

\section{S.31.1 Fitting Film-to-Substrate Boundary Conductance - $h_{\mathrm{BD}}$}

Table S.54: Summary of simulation data - $100 \mathrm{~nm} \mathrm{Al/Si} \mathrm{1000.00} \mathrm{MW} \mathrm{Series.} \mathrm{Error} \mathrm{bounds} \mathrm{represent} \mathrm{a}$ $95 \%$ confidence level.

\begin{tabular}{cccccc}
\hline \multicolumn{6}{c}{$\mathbf{1 0 0} \mathbf{~ n m ~ A l ~ o n ~} \mathbf{~ S i}, h_{\mathrm{BD}}=\mathbf{1 0 0 0 . 0 0} \mathbf{M W} / \mathbf{m}^{2} \mathbf{K}$} \\
\hline $\mathbf{S N P}$ & $\mu-\left[\mathbf{M W} / \mathbf{m}^{2} \mathbf{K}\right]$ & $\sigma-\left[\mathbf{M W} / \mathbf{m}^{2} \mathbf{K}\right]$ & $\mathbf{S N P}$ & $\mu-\left[\mathbf{M W} / \mathbf{m}^{2} \mathbf{K}\right]$ & $\sigma-\left[\mathbf{M W} / \mathbf{m}^{2} \mathbf{K}\right]$ \\
1.97 & $1176.73 \pm 40.124$ & $634.82 \pm 1.8343$ & 12.57 & $1002.30 \pm 5.039$ & $80.88 \pm 0.2271$ \\
2.92 & $1058.09 \pm 24.232$ & $387.94 \pm 1.0947$ & 13.61 & $1004.28 \pm 4.714$ & $75.62 \pm 0.2125$ \\
3.84 & $1031.97 \pm 16.782$ & $267.58 \pm 0.7612$ & 14.31 & $1002.88 \pm 4.242$ & $68.09 \pm 0.1912$ \\
4.85 & $1024.05 \pm 13.540$ & $216.88 \pm 0.6114$ & 15.58 & $1005.74 \pm 4.189$ & $67.23 \pm 0.1888$ \\
5.80 & $1015.56 \pm 11.577$ & $185.81 \pm 0.5217$ & 16.39 & $1005.80 \pm 3.878$ & $62.25 \pm 0.1748$ \\
6.86 & $1014.28 \pm 9.373$ & $150.82 \pm 0.4213$ & 17.41 & $1003.13 \pm 3.749$ & $60.15 \pm 0.1690$ \\
7.86 & $1008.66 \pm 8.048$ & $129.04 \pm 0.3630$ & 18.35 & $997.09 \pm 3.367$ & $53.98 \pm 0.1519$ \\
8.67 & $1009.57 \pm 7.594$ & $121.88 \pm 0.3422$ & 19.20 & $1003.29 \pm 3.121$ & $50.09 \pm 0.1406$ \\
9.77 & $1007.11 \pm 6.545$ & $105.05 \pm 0.2949$ & 20.52 & $1001.96 \pm 3.220$ & $51.68 \pm 0.1451$ \\
10.77 & $1006.63 \pm 6.037$ & $96.89 \pm 0.2720$ & 20.37 & $1004.01 \pm 3.039$ & $48.77 \pm 0.1369$ \\
11.70 & $1001.83 \pm 5.247$ & $84.22 \pm 0.2364$ & & & \\
\hline
\end{tabular}

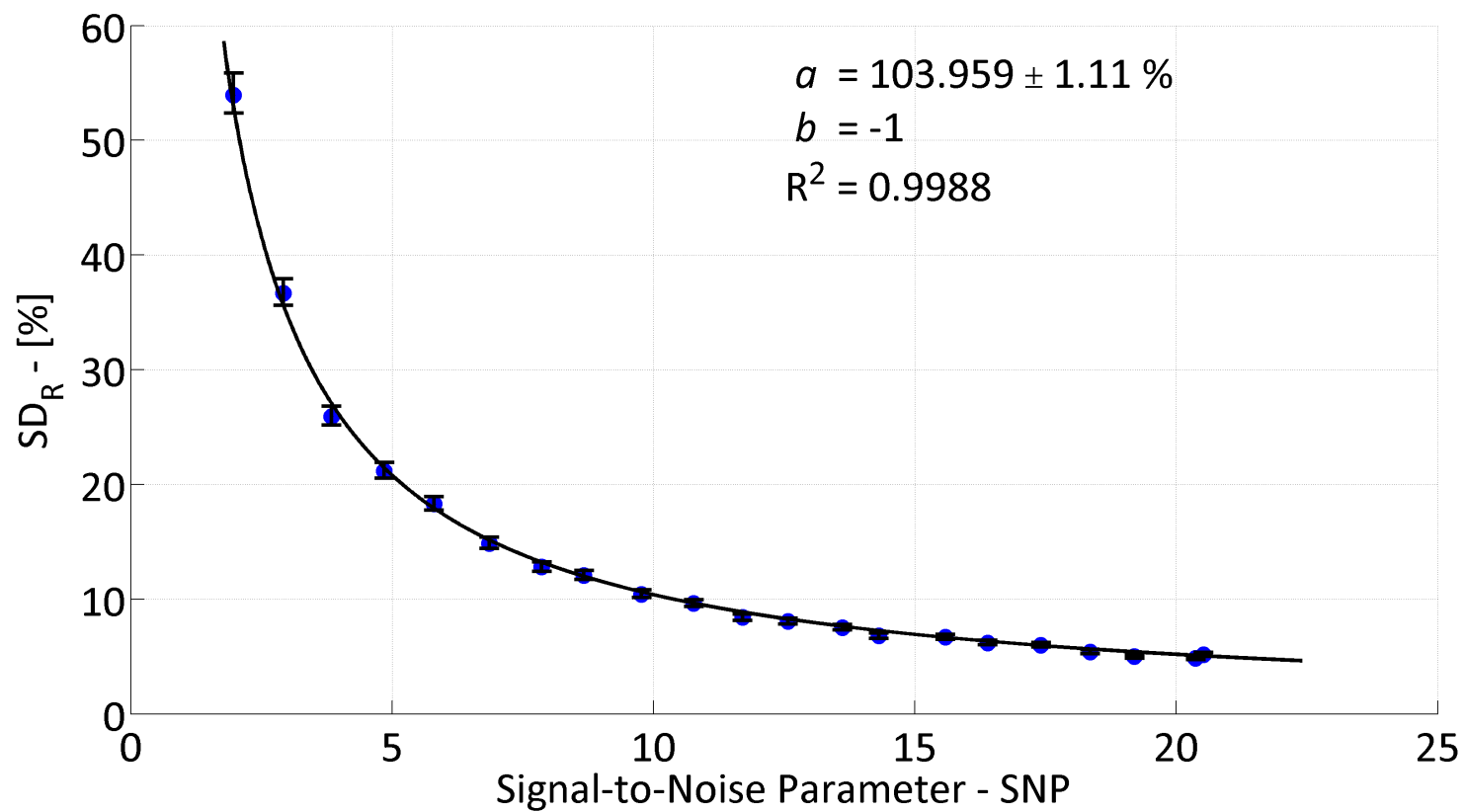

Figure S.115: Relative population standard deviation as a function of signal-to-noise parameter for 100 $\mathrm{nm}$ of $\mathrm{Al} / \mathrm{Si}$ with $h_{\mathrm{BD}}=1000.00 \mathrm{MW} / \mathrm{m}^{2} \mathrm{~K}$. Error bars represent a $95 \%$ confidence interval of the measured $\mathrm{SD}_{\mathrm{R}}$ and the solid line represents a best fit of a power law to the data, of the form $\mathrm{SD}_{\mathrm{R}}=a \times \mathrm{SNP}^{b}$, with parameter $a$ provided for a fixed $b=-1$, along with the $95 \%$ confidence level in $a$. 


\section{S.31.2 Fitting Substrate Thermal Conductivity $-k_{S}$}

Table S.55: Summary of simulation data - $100 \mathrm{~nm} \mathrm{Al/Si} \mathrm{1000.00} \mathrm{MW} \mathrm{Series.} \mathrm{Error} \mathrm{bounds} \mathrm{represent} \mathrm{a}$ $95 \%$ confidence level.

\begin{tabular}{cccccc}
\hline \multicolumn{6}{c}{$\mathbf{1 0 0} \mathbf{~ n m}$ Al on $\mathbf{~ S i}, h_{\mathrm{BD}}=\mathbf{1 0 0 0 . 0 0} \mathbf{~ M W} / \mathbf{m}^{2} \mathbf{K}$} \\
\hline $\mathbf{S N P}$ & $\mu-[\mathbf{W} / \mathbf{m K}]$ & $\sigma-[\mathbf{W} / \mathbf{m K}]$ & $\mathbf{S N P}$ & $\mu-[\mathbf{W} / \mathbf{m K}]$ & $\sigma-[\mathbf{W} / \mathbf{m K}]$ \\
1.97 & $150.08 \pm 2.540$ & $40.91 \pm 0.1141$ & 12.57 & $148.10 \pm 0.381$ & $6.15 \pm 0.0171$ \\
2.92 & $151.03 \pm 1.702$ & $27.43 \pm 0.0764$ & 13.61 & $147.90 \pm 0.353$ & $5.69 \pm 0.0159$ \\
3.84 & $149.02 \pm 1.258$ & $20.26 \pm 0.0564$ & 14.31 & $148.03 \pm 0.328$ & $5.29 \pm 0.0147$ \\
4.85 & $148.80 \pm 0.982$ & $15.82 \pm 0.0441$ & 15.58 & $147.80 \pm 0.317$ & $5.11 \pm 0.0142$ \\
5.80 & $148.72 \pm 0.847$ & $13.64 \pm 0.0380$ & 16.39 & $147.61 \pm 0.287$ & $4.63 \pm 0.0129$ \\
6.86 & $148.07 \pm 0.698$ & $11.25 \pm 0.0314$ & 17.41 & $147.88 \pm 0.282$ & $4.55 \pm 0.0127$ \\
7.86 & $148.35 \pm 0.616$ & $9.92 \pm 0.0276$ & 18.35 & $148.29 \pm 0.262$ & $4.22 \pm 0.0118$ \\
8.67 & $148.10 \pm 0.557$ & $8.98 \pm 0.0250$ & 19.20 & $147.82 \pm 0.241$ & $3.89 \pm 0.0108$ \\
9.77 & $148.05 \pm 0.489$ & $7.88 \pm 0.0219$ & 20.52 & $148.01 \pm 0.241$ & $3.88 \pm 0.0108$ \\
10.77 & $148.07 \pm 0.453$ & $7.31 \pm 0.0203$ & 20.37 & $147.76 \pm 0.233$ & $3.75 \pm 0.0105$ \\
11.70 & $148.16 \pm 0.405$ & $6.52 \pm 0.0182$ & & & \\
\hline
\end{tabular}

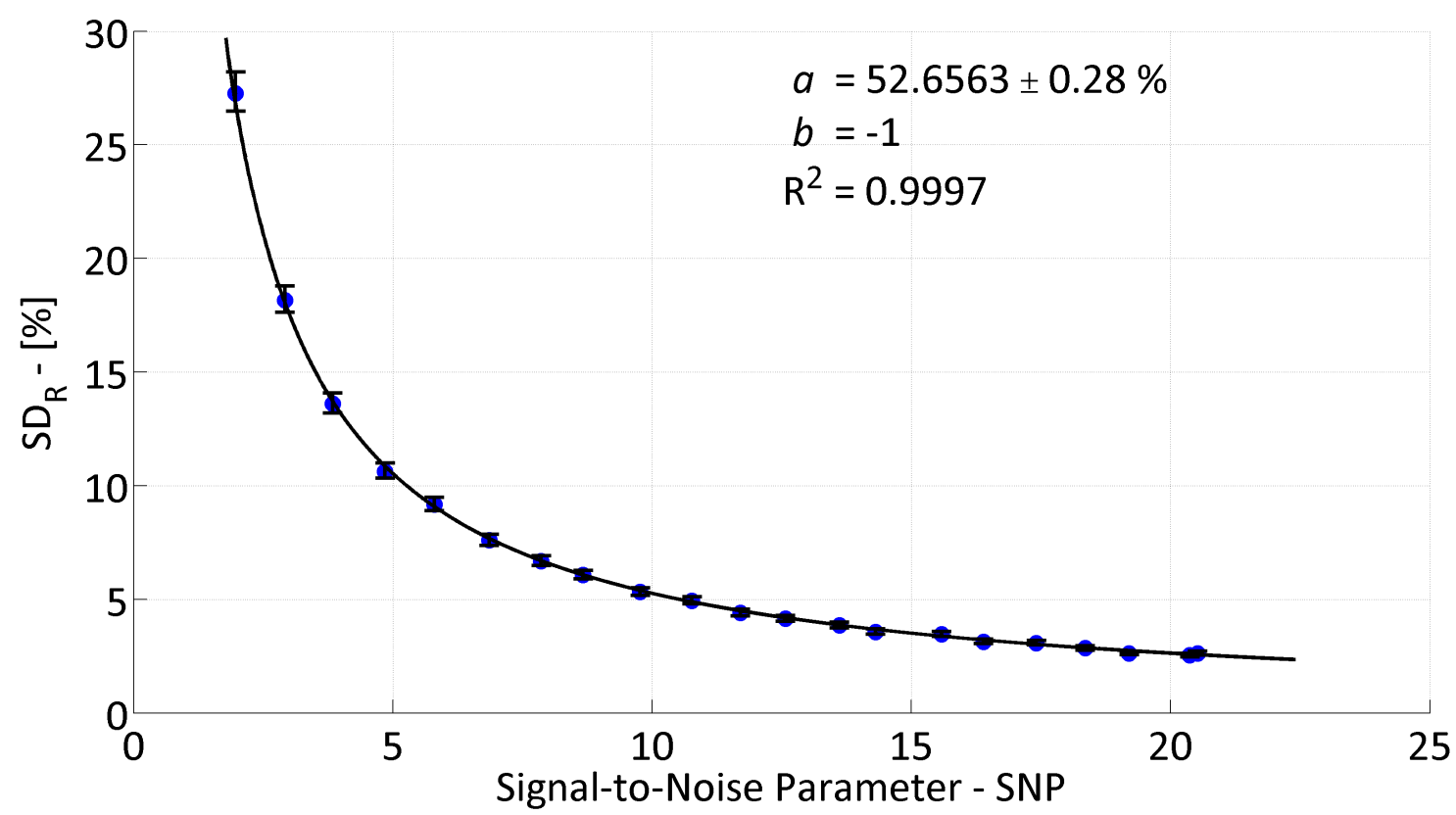

Figure S.116: Relative population standard deviation as a function of signal-to-noise parameter for 100 $\mathrm{nm}$ of $\mathrm{Al} / \mathrm{Si}$ with $h_{\mathrm{BD}}=1000.00 \mathrm{MW} / \mathrm{m}^{2} \mathrm{~K}$. Error bars represent a $95 \%$ confidence interval of the measured $\mathrm{SD}_{\mathrm{R}}$ and the solid line represents a best fit of a power law to the data, of the form $\mathrm{SD}_{\mathrm{R}}=a \times \mathrm{SNP}^{b}$, with parameter $a$ provided for a fixed $b=-1$, along with the $95 \%$ confidence level in $a$. 


\section{S.31.3 Fitting Film Thickness $-d_{1}$}

Table S.56: Summary of simulation data - $100 \mathrm{~nm} \mathrm{Al/Si} \mathrm{1000.00} \mathrm{MW} \mathrm{Series.} \mathrm{Error} \mathrm{bounds} \mathrm{represent} \mathrm{a}$ $95 \%$ confidence level.

\begin{tabular}{cccccc}
\hline \multicolumn{6}{c}{$\mathbf{1 0 0} \mathbf{~ n m} \mathbf{A l}$ on $\mathbf{~ S i}, h_{\mathrm{BD}}=\mathbf{1 0 0 0 . 0 0} \mathbf{~ M W} / \mathbf{m}^{2} \mathbf{K}$} \\
\hline SNP & $\mu-[\mathbf{n m}]$ & $\sigma-[\mathbf{n m}]$ & $\mathbf{S N P}$ & $\mu-[\mathbf{n m}]$ & $\sigma-[\mathbf{n m}]$ \\
1.97 & $103.00 \pm 1.451$ & $23.38 \pm 0.0651$ & 12.57 & $100.10 \pm 0.210$ & $3.38 \pm 0.0094$ \\
2.92 & $100.25 \pm 0.931$ & $15.00 \pm 0.0418$ & 13.61 & $100.19 \pm 0.195$ & $3.14 \pm 0.0088$ \\
3.84 & $100.56 \pm 0.688$ & $11.08 \pm 0.0309$ & 14.31 & $100.08 \pm 0.182$ & $2.93 \pm 0.0082$ \\
4.85 & $100.27 \pm 0.549$ & $8.84 \pm 0.0246$ & 15.58 & $100.19 \pm 0.174$ & $2.80 \pm 0.0078$ \\
5.80 & $100.15 \pm 0.466$ & $7.51 \pm 0.0209$ & 16.39 & $100.32 \pm 0.158$ & $2.54 \pm 0.0071$ \\
6.86 & $100.32 \pm 0.394$ & $6.34 \pm 0.0177$ & 17.41 & $100.15 \pm 0.154$ & $2.48 \pm 0.0069$ \\
7.86 & $100.11 \pm 0.339$ & $5.46 \pm 0.0152$ & 18.35 & $99.93 \pm 0.144$ & $2.32 \pm 0.0065$ \\
8.67 & $100.22 \pm 0.306$ & $4.93 \pm 0.0137$ & 19.20 & $100.16 \pm 0.134$ & $2.15 \pm 0.0060$ \\
9.77 & $100.18 \pm 0.268$ & $4.32 \pm 0.0120$ & 20.52 & $100.04 \pm 0.131$ & $2.11 \pm 0.0059$ \\
10.77 & $100.12 \pm 0.249$ & $4.01 \pm 0.0112$ & 20.37 & $100.19 \pm 0.129$ & $2.08 \pm 0.0058$ \\
11.70 & $100.08 \pm 0.224$ & $3.60 \pm 0.0100$ & & & \\
\hline
\end{tabular}

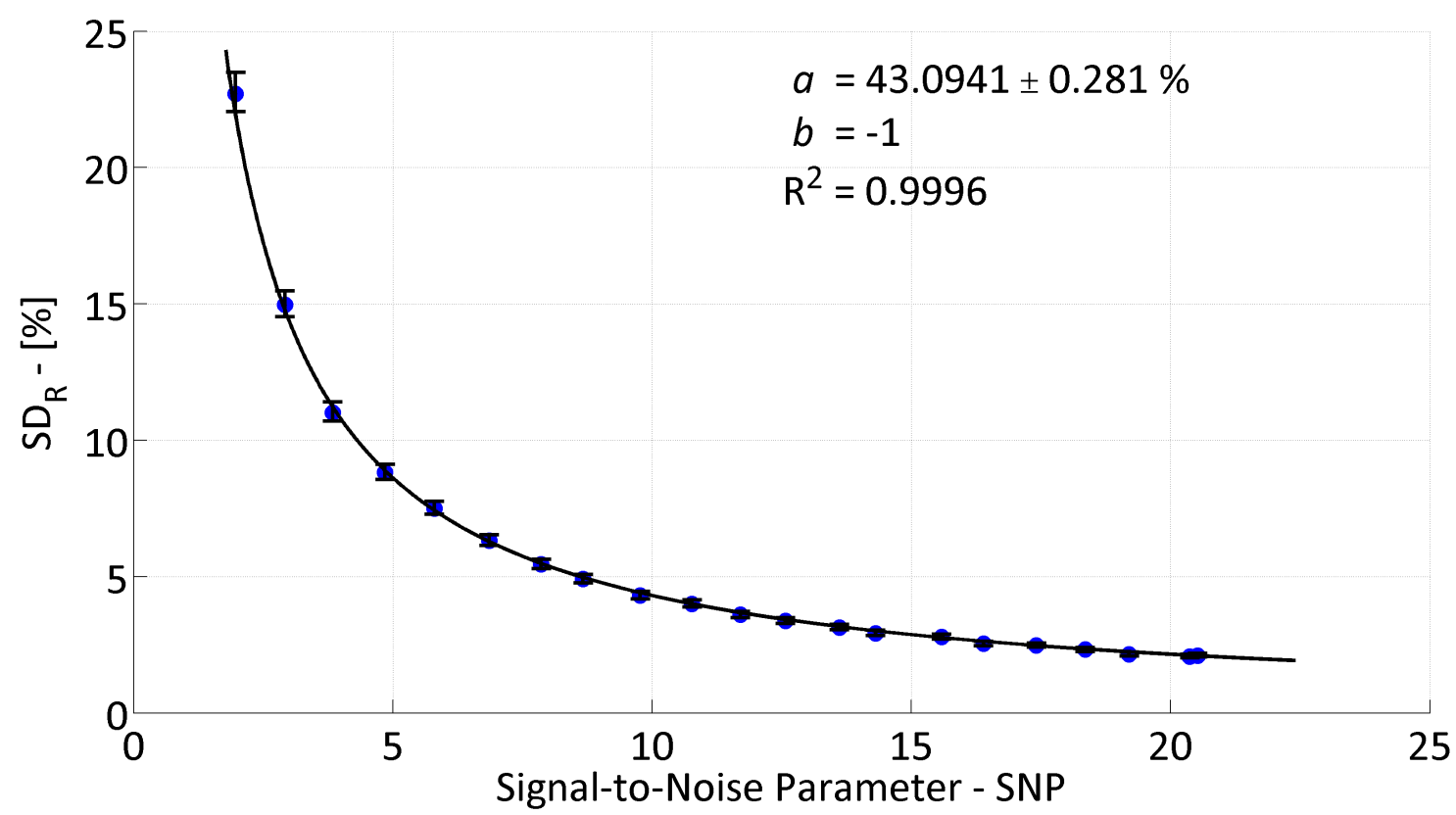

Figure S.117: Relative population standard deviation as a function of signal-to-noise parameter for 100 $\mathrm{nm}$ of $\mathrm{Al} / \mathrm{Si}$ with $h_{\mathrm{BD}}=1000.00 \mathrm{MW} / \mathrm{m}^{2} \mathrm{~K}$. Error bars represent a $95 \%$ confidence interval of the measured $\mathrm{SD}_{\mathrm{R}}$ and the solid line represents a best fit of a power law to the data, of the form $\mathrm{SD}_{\mathrm{R}}=a \times \mathrm{SNP}^{b}$, with parameter $a$ provided for a fixed $b=-1$, along with the $95 \%$ confidence level in $a$. 


\section{S.32 Simulation Summary - Al/AIN 1.00 MW Series}

\section{S.32.1 Fitting Film-to-Substrate Boundary Conductance - $h_{\mathrm{BD}}$}

Table S.57: Summary of simulation data - $100 \mathrm{~nm} \mathrm{Al/AlN} \mathrm{1.00} \mathrm{MW} \mathrm{Series.} \mathrm{Error} \mathrm{bounds} \mathrm{represent} \mathrm{a} 95 \%$ confidence level.

\begin{tabular}{cccccc}
\hline \multicolumn{5}{c}{$\mathbf{1 0 0} \mathbf{~ n m ~ A l ~ o n ~ A l N , ~} h_{\mathrm{BD}}=\mathbf{1 . 0 0} \mathbf{~ M W} / \mathbf{m}^{2} \mathbf{K}$} \\
\hline $\mathbf{S N P}$ & $\mu-\left[\mathbf{M W} / \mathbf{m}^{2} \mathbf{K}\right]$ & $\sigma-\left[\mathbf{M W} / \mathbf{m}^{2} \mathbf{K}\right]$ & $\mathbf{S N P}$ & $\mu-\left[\mathbf{M W} / \mathbf{m}^{2} \mathbf{K}\right]$ & $\sigma-\left[\mathbf{M W} / \mathbf{m}^{2} \mathbf{K}\right]$ \\
10.45 & $1.08 \pm 0.056$ & $0.90 \pm 0.0025$ & 65.42 & $1.01 \pm 0.011$ & $0.17 \pm 0.0005$ \\
15.69 & $0.99 \pm 0.041$ & $0.66 \pm 0.0018$ & 71.28 & $1.01 \pm 0.009$ & $0.15 \pm 0.0004$ \\
21.01 & $1.02 \pm 0.032$ & $0.52 \pm 0.0015$ & 76.77 & $1.01 \pm 0.008$ & $0.14 \pm 0.0004$ \\
25.86 & $1.00 \pm 0.025$ & $0.40 \pm 0.0011$ & 81.05 & $1.01 \pm 0.008$ & $0.13 \pm 0.0004$ \\
30.60 & $1.00 \pm 0.021$ & $0.34 \pm 0.0009$ & 87.96 & $1.01 \pm 0.008$ & $0.12 \pm 0.0003$ \\
36.19 & $1.01 \pm 0.018$ & $0.30 \pm 0.0008$ & 93.01 & $1.00 \pm 0.007$ & $0.12 \pm 0.0003$ \\
41.70 & $1.01 \pm 0.017$ & $0.27 \pm 0.0007$ & 99.52 & $1.01 \pm 0.006$ & $0.10 \pm 0.0003$ \\
46.71 & $1.00 \pm 0.014$ & $0.23 \pm 0.0006$ & 103.83 & $1.00 \pm 0.006$ & $0.10 \pm 0.0003$ \\
51.10 & $1.01 \pm 0.013$ & $0.21 \pm 0.0006$ & 105.64 & $1.01 \pm 0.006$ & $0.10 \pm 0.0003$ \\
56.35 & $1.01 \pm 0.012$ & $0.19 \pm 0.0005$ & 113.24 & $1.01 \pm 0.006$ & $0.09 \pm 0.0003$ \\
62.93 & $1.00 \pm 0.011$ & $0.18 \pm 0.0005$ & & & \\
\hline
\end{tabular}

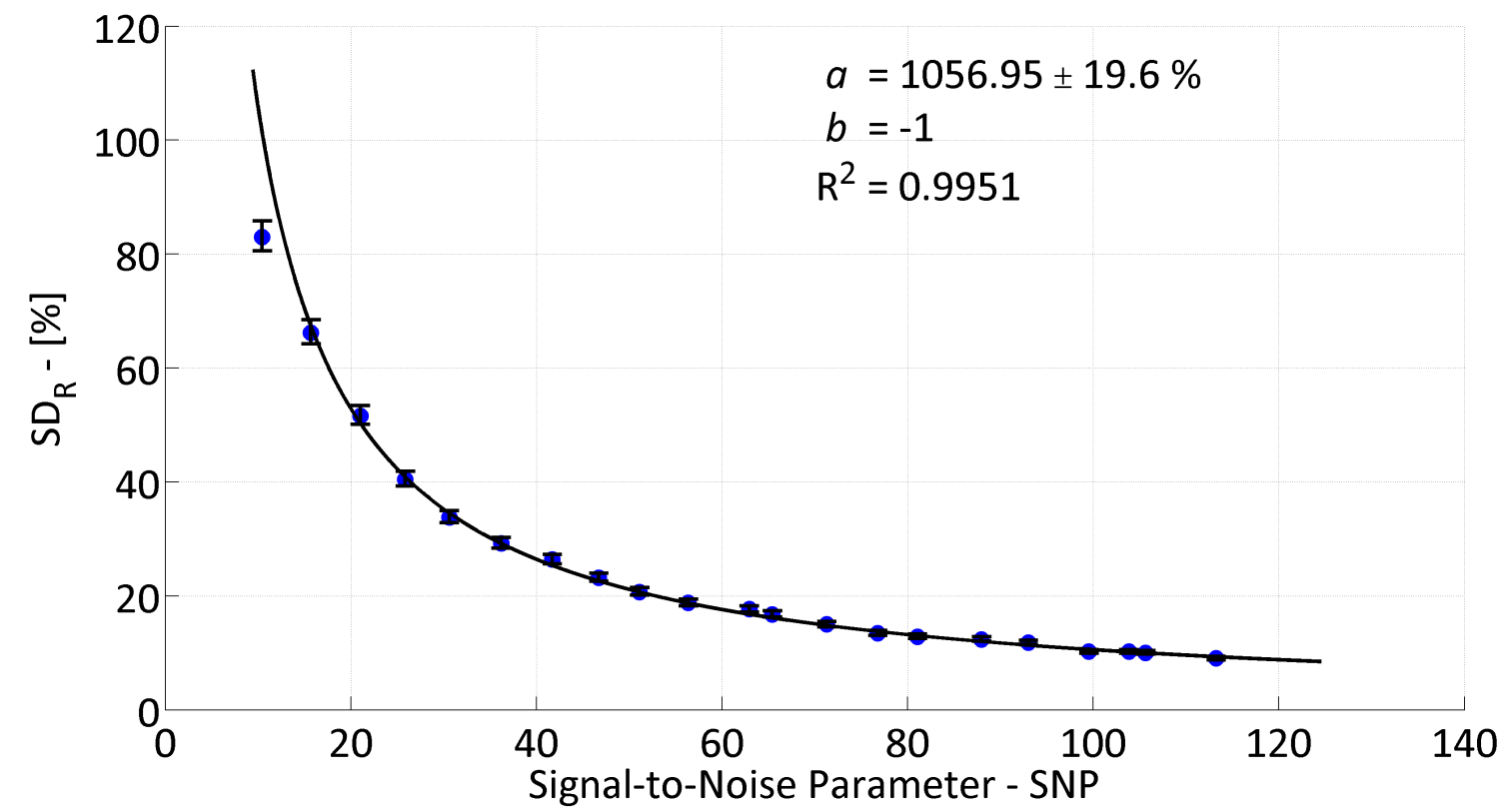

Figure S.118: Relative population standard deviation as a function of signal-to-noise parameter for 100 $\mathrm{nm}$ of $\mathrm{Al} / \mathrm{AlN}$ with $h_{\mathrm{BD}}=1.00 \mathrm{MW} / \mathrm{m}^{2} \mathrm{~K}$. Error bars represent a $95 \%$ confidence interval of the measured $\mathrm{SD}_{\mathrm{R}}$ and the solid line represents a best fit of a power law to the data, of the form $\mathrm{SD}_{\mathrm{R}}=a \times \mathrm{SNP}^{b}$, with parameter $a$ provided for a fixed $b=-1$, along with the $95 \%$ confidence level in $a$. 


\section{S.33 Simulation Summary - Al/AIN 1.29 MW Series}

\section{S.33.1 Fitting Film-to-Substrate Boundary Conductance $-h_{\mathrm{BD}}$}

Table S.58: Summary of simulation data - $100 \mathrm{~nm} \mathrm{Al/AlN} \mathrm{1.29} \mathrm{MW} \mathrm{Series.} \mathrm{Error} \mathrm{bounds} \mathrm{represent} \mathrm{a} 95 \%$ confidence level.

\begin{tabular}{cccccc}
\hline \multicolumn{5}{c}{$\mathbf{1 0 0} \mathbf{~ n m ~ A l ~ o n ~ A l N , ~} h_{\mathrm{BD}}=\mathbf{1 . 2 9} \mathbf{M W} / \mathbf{m}^{2} \mathbf{K}$} \\
\hline $\mathbf{S N P}$ & $\mu-\left[\mathbf{M W} / \mathbf{m}^{2} \mathbf{K}\right]$ & $\sigma-\left[\mathbf{M W} / \mathbf{m}^{2} \mathbf{K}\right]$ & $\mathbf{S N P}$ & $\mu-\left[\mathbf{M W} / \mathbf{m}^{2} \mathbf{K}\right]$ & $\sigma-\left[\mathbf{M W} / \mathbf{m}^{2} \mathbf{K}\right]$ \\
10.25 & $1.43 \pm 0.061$ & $0.98 \pm 0.0027$ & 66.01 & $1.30 \pm 0.010$ & $0.17 \pm 0.0005$ \\
15.34 & $1.28 \pm 0.044$ & $0.70 \pm 0.0020$ & 71.41 & $1.29 \pm 0.009$ & $0.15 \pm 0.0004$ \\
20.90 & $1.31 \pm 0.032$ & $0.51 \pm 0.0014$ & 77.96 & $1.30 \pm 0.009$ & $0.14 \pm 0.0004$ \\
26.12 & $1.28 \pm 0.026$ & $0.42 \pm 0.0012$ & 81.44 & $1.29 \pm 0.009$ & $0.14 \pm 0.0004$ \\
30.49 & $1.30 \pm 0.023$ & $0.36 \pm 0.0010$ & 87.17 & $1.30 \pm 0.008$ & $0.12 \pm 0.0003$ \\
36.18 & $1.29 \pm 0.019$ & $0.31 \pm 0.0009$ & 90.33 & $1.30 \pm 0.007$ & $0.11 \pm 0.0003$ \\
41.10 & $1.31 \pm 0.017$ & $0.27 \pm 0.0008$ & 96.24 & $1.29 \pm 0.007$ & $0.11 \pm 0.0003$ \\
46.05 & $1.29 \pm 0.014$ & $0.23 \pm 0.0006$ & 99.37 & $1.29 \pm 0.007$ & $0.11 \pm 0.0003$ \\
51.32 & $1.30 \pm 0.013$ & $0.21 \pm 0.0006$ & 104.95 & $1.29 \pm 0.006$ & $0.10 \pm 0.0003$ \\
56.02 & $1.30 \pm 0.012$ & $0.19 \pm 0.0005$ & 113.14 & $1.29 \pm 0.006$ & $0.10 \pm 0.0003$ \\
60.49 & $1.30 \pm 0.011$ & $0.17 \pm 0.0005$ & & & \\
\hline
\end{tabular}

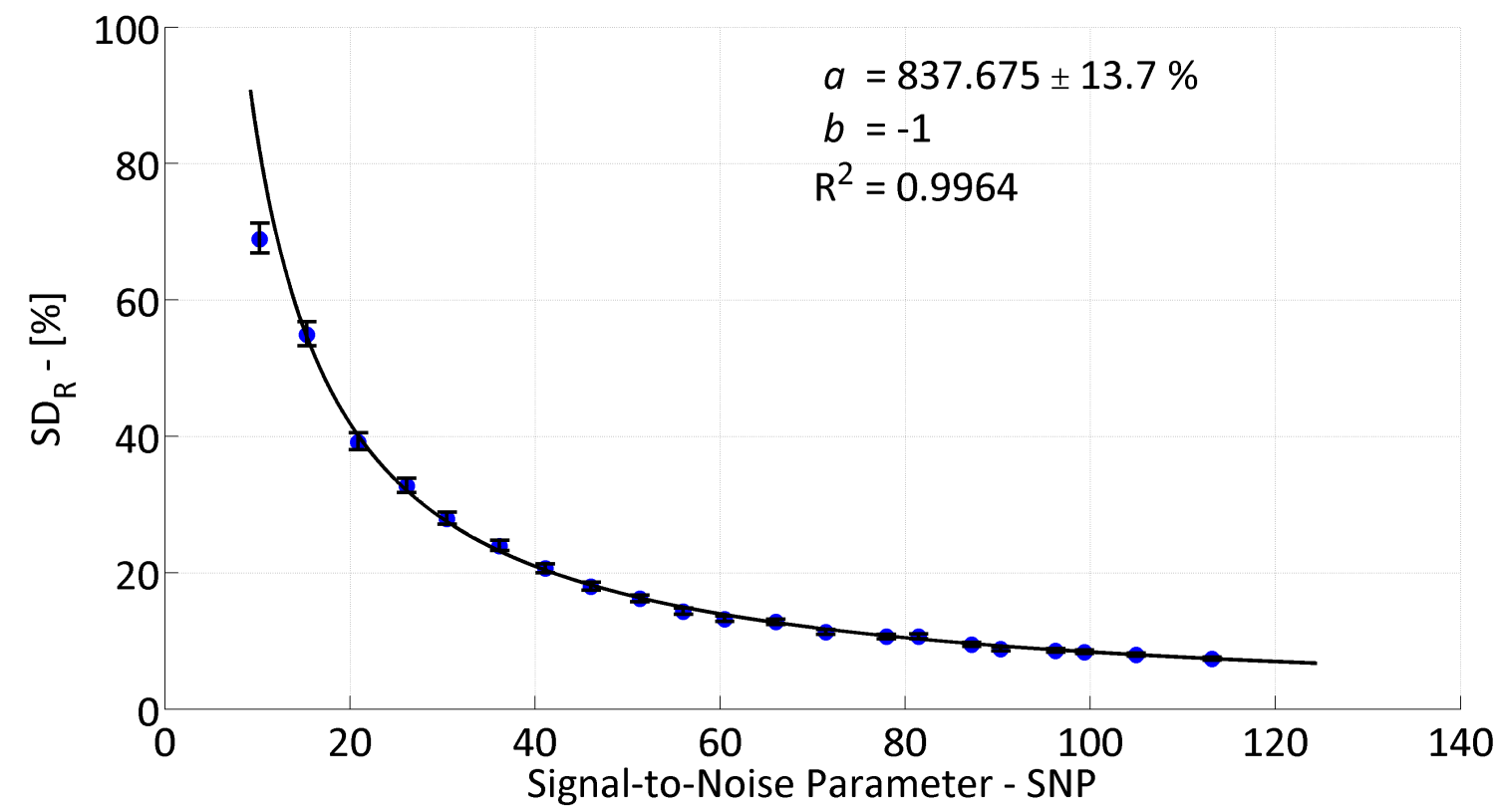

Figure S.119: Relative population standard deviation as a function of signal-to-noise parameter for 100 $\mathrm{nm}$ of $\mathrm{Al} / \mathrm{AlN}$ with $h_{\mathrm{BD}}=1.29 \mathrm{MW} / \mathrm{m}^{2} \mathrm{~K}$. Error bars represent a $95 \%$ confidence interval of the measured $\mathrm{SD}_{\mathrm{R}}$ and the solid line represents a best fit of a power law to the data, of the form $\mathrm{SD}_{\mathrm{R}}=a \times \mathrm{SNP}^{b}$, with parameter $a$ provided for a fixed $b=-1$, along with the $95 \%$ confidence level in $a$. 


\section{S.34 Simulation Summary - Al/AIN 2.78 MW Series}

\section{S.34.1 Fitting Film-to-Substrate Boundary Conductance - $h_{\mathrm{BD}}$}

Table S.59: Summary of simulation data - $100 \mathrm{~nm} \mathrm{Al/AlN} \mathrm{2.78} \mathrm{MW} \mathrm{Series.} \mathrm{Error} \mathrm{bounds} \mathrm{represent} \mathrm{a} 95 \%$ confidence level.

\begin{tabular}{cccccc}
\hline \multicolumn{5}{c}{$\mathbf{1 0 0} \mathbf{~ n m ~ A l ~ o n ~ A l N , ~} h_{\mathrm{BD}}=\mathbf{2 . 7 8} \mathbf{~ M W} / \mathbf{m}^{2} \mathbf{K}$} \\
\hline $\mathbf{S N P}$ & $\mu-\left[\mathbf{M W} / \mathbf{m}^{2} \mathbf{K}\right]$ & $\sigma-\left[\mathbf{M W} / \mathbf{m}^{2} \mathbf{K}\right]$ & $\mathbf{S N P}$ & $\mu-\left[\mathbf{M W} / \mathbf{m}^{2} \mathbf{K}\right]$ & $\sigma-\left[\mathbf{M W} / \mathbf{m}^{2} \mathbf{K}\right]$ \\
10.27 & $2.81 \pm 0.066$ & $1.06 \pm 0.0030$ & 65.87 & $2.79 \pm 0.010$ & $0.16 \pm 0.0005$ \\
15.03 & $2.77 \pm 0.046$ & $0.73 \pm 0.0020$ & 70.98 & $2.79 \pm 0.009$ & $0.15 \pm 0.0004$ \\
20.19 & $2.80 \pm 0.033$ & $0.54 \pm 0.0015$ & 74.97 & $2.78 \pm 0.009$ & $0.15 \pm 0.0004$ \\
24.86 & $2.81 \pm 0.027$ & $0.44 \pm 0.0012$ & 80.28 & $2.79 \pm 0.008$ & $0.14 \pm 0.0004$ \\
29.65 & $2.80 \pm 0.023$ & $0.37 \pm 0.0010$ & 83.89 & $2.80 \pm 0.008$ & $0.13 \pm 0.0004$ \\
34.65 & $2.78 \pm 0.020$ & $0.32 \pm 0.0009$ & 89.47 & $2.79 \pm 0.008$ & $0.12 \pm 0.0003$ \\
40.09 & $2.79 \pm 0.017$ & $0.27 \pm 0.0008$ & 94.15 & $2.79 \pm 0.007$ & $0.12 \pm 0.0003$ \\
44.72 & $2.79 \pm 0.015$ & $0.24 \pm 0.0007$ & 99.46 & $2.79 \pm 0.007$ & $0.11 \pm 0.0003$ \\
49.91 & $2.78 \pm 0.014$ & $0.22 \pm 0.0006$ & 103.32 & $2.79 \pm 0.007$ & $0.11 \pm 0.0003$ \\
54.60 & $2.79 \pm 0.013$ & $0.20 \pm 0.0006$ & 110.50 & $2.78 \pm 0.006$ & $0.10 \pm 0.0003$ \\
60.18 & $2.78 \pm 0.011$ & $0.18 \pm 0.0005$ & & & \\
\hline
\end{tabular}

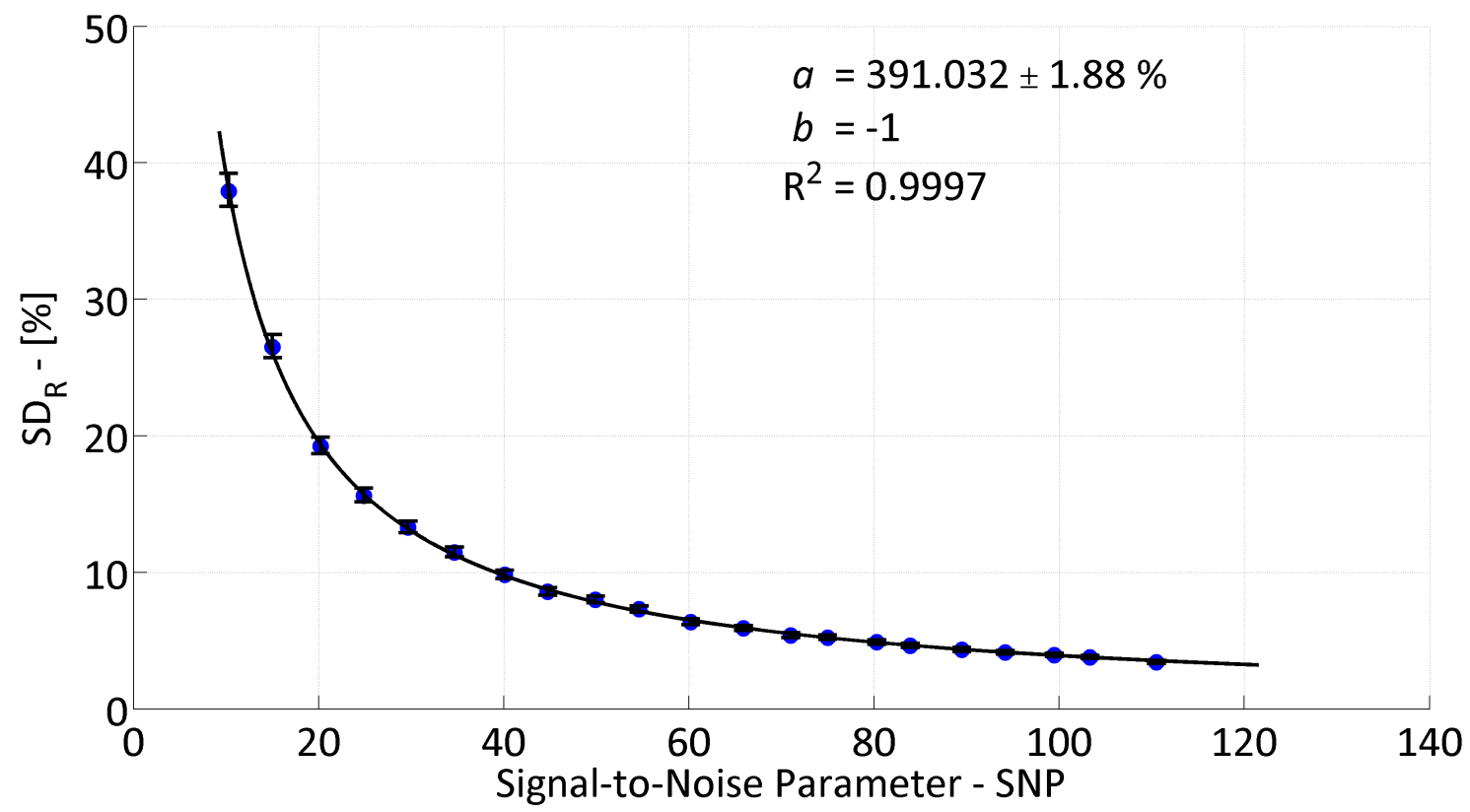

Figure S.120: Relative population standard deviation as a function of signal-to-noise parameter for 100 $\mathrm{nm}$ of $\mathrm{Al} / \mathrm{AlN}$ with $h_{\mathrm{BD}}=2.78 \mathrm{MW} / \mathrm{m}^{2} \mathrm{~K}$. Error bars represent a $95 \%$ confidence interval of the measured $\mathrm{SD}_{\mathrm{R}}$ and the solid line represents a best fit of a power law to the data, of the form $\mathrm{SD}_{\mathrm{R}}=a \times \mathrm{SNP}^{b}$, with parameter $a$ provided for a fixed $b=-1$, along with the $95 \%$ confidence level in $a$. 


\section{S.35 Simulation Summary - Al/AIN 5.99 MW Series}

\section{S.35.1 Fitting Film-to-Substrate Boundary Conductance - $h_{\mathrm{BD}}$}

Table S.60: Summary of simulation data - 100 nm Al/AlN 5.99 MW Series. Error bounds represent a $95 \%$ confidence level.

\begin{tabular}{cccccc}
\hline \multicolumn{5}{c}{$\mathbf{1 0 0} \mathbf{~ n m ~ A l ~ o n ~ A l N , ~} h_{\mathrm{BD}}=\mathbf{5 . 9 9} \mathbf{M W} / \mathbf{m}^{2} \mathbf{K}$} \\
\hline $\mathbf{S N P}$ & $\mu-\left[\mathbf{M W} / \mathbf{m}^{2} \mathbf{K}\right]$ & $\sigma-\left[\mathbf{M W} / \mathbf{m}^{2} \mathbf{K}\right]$ & $\mathbf{S N P}$ & $\mu-\left[\mathbf{M W} / \mathbf{m}^{2} \mathbf{K}\right]$ & $\sigma-\left[\mathbf{M W} / \mathbf{m}^{2} \mathbf{K}\right]$ \\
9.66 & $5.98 \pm 0.073$ & $1.18 \pm 0.0033$ & 60.81 & $6.00 \pm 0.011$ & $0.18 \pm 0.0005$ \\
14.30 & $5.98 \pm 0.049$ & $0.79 \pm 0.0022$ & 65.75 & $5.99 \pm 0.011$ & $0.17 \pm 0.0005$ \\
18.89 & $5.99 \pm 0.036$ & $0.59 \pm 0.0016$ & 70.41 & $6.00 \pm 0.010$ & $0.16 \pm 0.0004$ \\
23.61 & $6.00 \pm 0.028$ & $0.46 \pm 0.0013$ & 76.94 & $6.01 \pm 0.009$ & $0.14 \pm 0.0004$ \\
28.16 & $6.02 \pm 0.025$ & $0.40 \pm 0.0011$ & 79.68 & $5.99 \pm 0.009$ & $0.14 \pm 0.0004$ \\
33.01 & $5.99 \pm 0.020$ & $0.32 \pm 0.0009$ & 85.26 & $6.00 \pm 0.008$ & $0.13 \pm 0.0004$ \\
37.91 & $6.00 \pm 0.018$ & $0.29 \pm 0.0008$ & 89.94 & $5.99 \pm 0.008$ & $0.12 \pm 0.0003$ \\
43.54 & $5.99 \pm 0.016$ & $0.26 \pm 0.0007$ & 94.96 & $5.99 \pm 0.007$ & $0.12 \pm 0.0003$ \\
47.41 & $5.99 \pm 0.014$ & $0.23 \pm 0.0006$ & 99.49 & $6.00 \pm 0.007$ & $0.11 \pm 0.0003$ \\
51.57 & $5.99 \pm 0.013$ & $0.21 \pm 0.0006$ & 104.44 & $6.00 \pm 0.006$ & $0.10 \pm 0.0003$ \\
56.44 & $5.99 \pm 0.012$ & $0.20 \pm 0.0006$ & & & \\
\hline
\end{tabular}

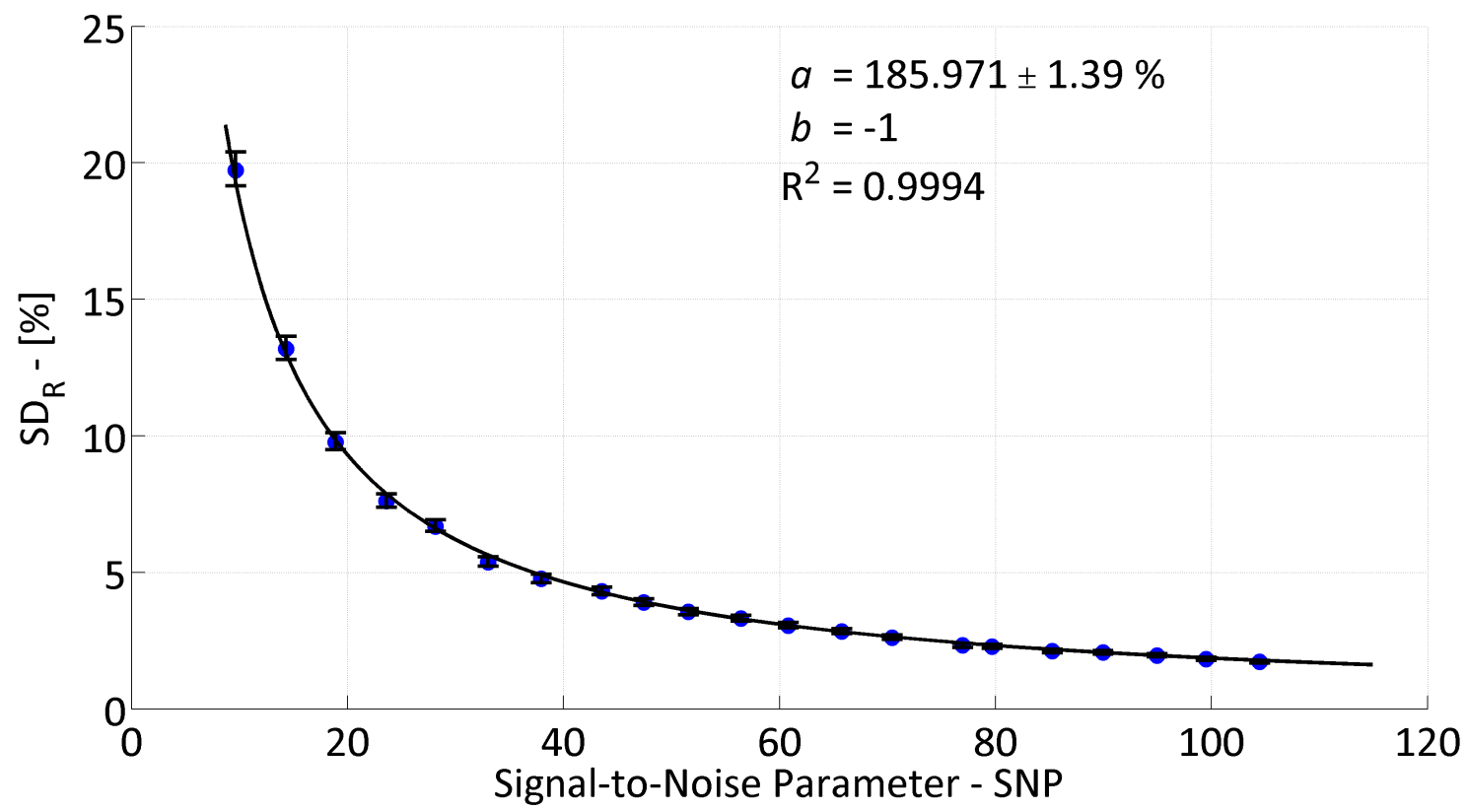

Figure S.121: Relative population standard deviation as a function of signal-to-noise parameter for 100 $\mathrm{nm}$ of $\mathrm{Al} / \mathrm{AlN}$ with $h_{\mathrm{BD}}=5.99 \mathrm{MW} / \mathrm{m}^{2} \mathrm{~K}$. Error bars represent a $95 \%$ confidence interval of the measured $\mathrm{SD}_{\mathrm{R}}$ and the solid line represents a best fit of a power law to the data, of the form $\mathrm{SD}_{\mathrm{R}}=a \times \mathrm{SNP}^{b}$, with parameter $a$ provided for a fixed $b=-1$, along with the $95 \%$ confidence level in $a$. 


\section{S.36 Simulation Summary - Al/AIN 12.90 MW Series}

\section{S.36.1 Fitting Film-to-Substrate Boundary Conductance - $h_{\mathrm{BD}}$}

Table S.61: Summary of simulation data - $100 \mathrm{~nm} \mathrm{Al/AlN} 12.90 \mathrm{MW}$ Series. Error bounds represent a $95 \%$ confidence level.

\begin{tabular}{cccccc}
\hline \multicolumn{6}{c}{$\mathbf{1 0 0} \mathbf{~ n m ~ A l ~ o n ~} \mathbf{A l N}, h_{\mathrm{BD}}=\mathbf{1 2 . 9 0} \mathbf{~ M W} / \mathbf{m}^{2} \mathbf{K}$} \\
\hline $\mathbf{S N P}$ & $\mu-\left[\mathbf{M W} / \mathbf{m}^{2} \mathbf{K}\right]$ & $\sigma-\left[\mathbf{M W} / \mathbf{m}^{2} \mathbf{K}\right]$ & $\mathbf{S N P}$ & $\mu-\left[\mathbf{M W} / \mathbf{m}^{2} \mathbf{K}\right]$ & $\sigma-\left[\mathbf{M W} / \mathbf{m}^{2} \mathbf{K}\right]$ \\
8.76 & $12.91 \pm 0.080$ & $1.29 \pm 0.0036$ & 53.31 & $12.91 \pm 0.014$ & $0.22 \pm 0.0006$ \\
12.94 & $12.89 \pm 0.055$ & $0.89 \pm 0.0025$ & 59.08 & $12.90 \pm 0.012$ & $0.19 \pm 0.0005$ \\
17.20 & $12.93 \pm 0.044$ & $0.70 \pm 0.0020$ & 62.80 & $12.90 \pm 0.011$ & $0.18 \pm 0.0005$ \\
21.45 & $12.88 \pm 0.033$ & $0.53 \pm 0.0015$ & 67.12 & $12.90 \pm 0.011$ & $0.17 \pm 0.0005$ \\
25.64 & $12.89 \pm 0.028$ & $0.45 \pm 0.0013$ & 71.21 & $12.90 \pm 0.010$ & $0.16 \pm 0.0005$ \\
30.12 & $12.91 \pm 0.024$ & $0.38 \pm 0.0011$ & 75.04 & $12.91 \pm 0.010$ & $0.16 \pm 0.0004$ \\
34.39 & $12.90 \pm 0.021$ & $0.34 \pm 0.0010$ & 82.09 & $12.91 \pm 0.008$ & $0.14 \pm 0.0004$ \\
38.03 & $12.90 \pm 0.018$ & $0.29 \pm 0.0008$ & 84.12 & $12.91 \pm 0.009$ & $0.14 \pm 0.0004$ \\
42.68 & $12.91 \pm 0.017$ & $0.28 \pm 0.0008$ & 89.42 & $12.91 \pm 0.008$ & $0.13 \pm 0.0004$ \\
48.03 & $12.90 \pm 0.015$ & $0.24 \pm 0.0007$ & 92.64 & $12.90 \pm 0.008$ & $0.12 \pm 0.0003$ \\
50.34 & $12.90 \pm 0.014$ & $0.23 \pm 0.0007$ & & & \\
\hline
\end{tabular}

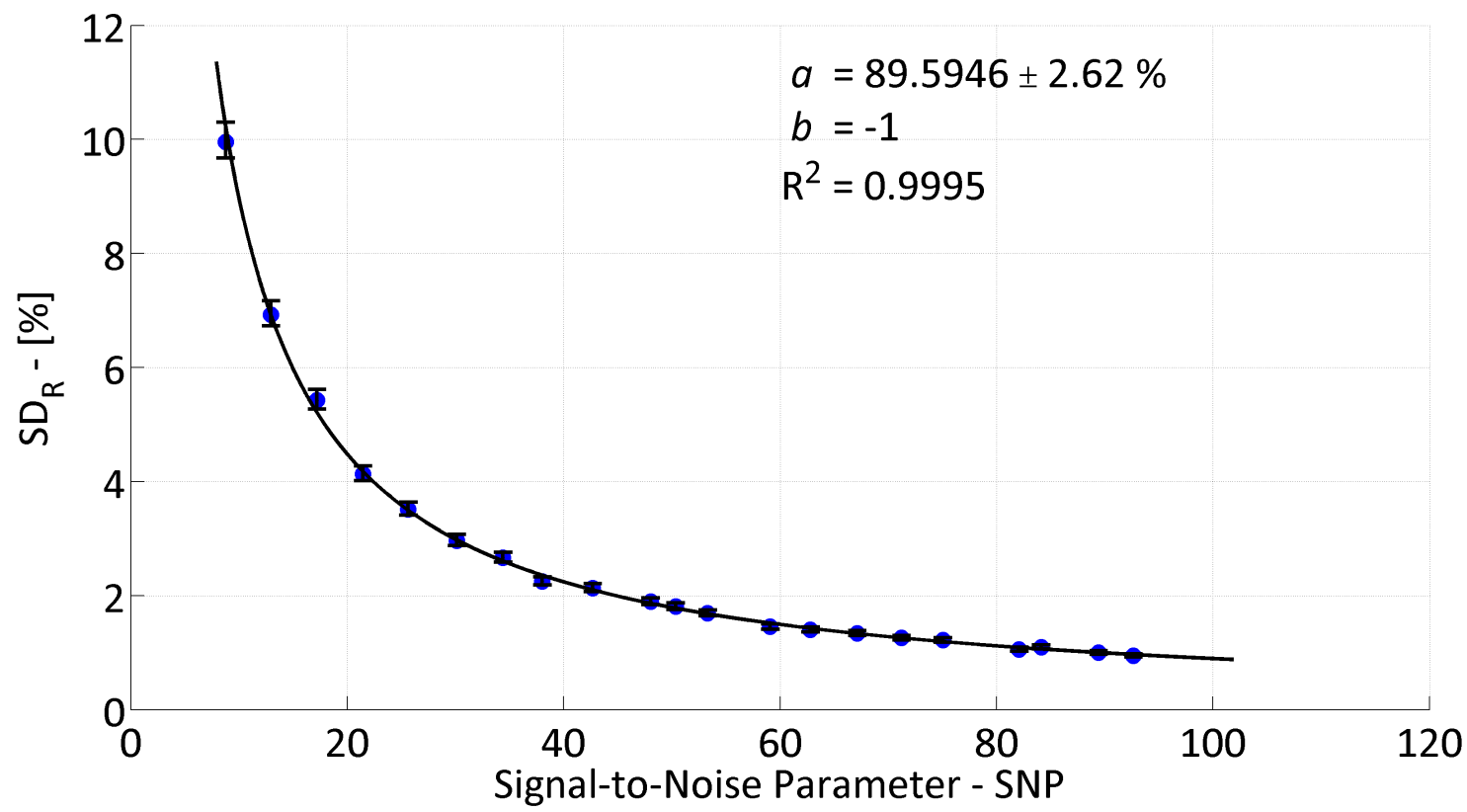

Figure S.122: Relative population standard deviation as a function of signal-to-noise parameter for 100 $\mathrm{nm}$ of $\mathrm{Al} / \mathrm{AlN}$ with $h_{\mathrm{BD}}=12.90 \mathrm{MW} / \mathrm{m}^{2} \mathrm{~K}$. Error bars represent a $95 \%$ confidence interval of the measured $\mathrm{SD}_{\mathrm{R}}$ and the solid line represents a best fit of a power law to the data, of the form $\mathrm{SD}_{\mathrm{R}}=a \times \mathrm{SNP}^{b}$, with parameter $a$ provided for a fixed $b=-1$, along with the $95 \%$ confidence level in $a$. 


\section{S.37 Simulation Summary - Al/AlN 27.80 MW Series}

\section{S.37.1 Fitting Film-to-Substrate Boundary Conductance - $h_{\mathrm{BD}}$}

Table S.62: Summary of simulation data - $100 \mathrm{~nm} \mathrm{Al/AlN} \mathrm{27.80} \mathrm{MW} \mathrm{Series.} \mathrm{Error} \mathrm{bounds} \mathrm{represent} \mathrm{a}$ $95 \%$ confidence level.

\begin{tabular}{cccccc}
\hline \multicolumn{6}{c}{$\mathbf{1 0 0} \mathbf{~ n m ~ A l ~ o n ~} \mathbf{A l N}, h_{\mathrm{BD}}=\mathbf{2 7 . 8 0} \mathbf{M W} / \mathbf{m}^{2} \mathbf{K}$} \\
\hline $\mathbf{S N P}$ & $\mu-\left[\mathbf{M W} / \mathbf{m}^{2} \mathbf{K}\right]$ & $\sigma-\left[\mathbf{M W} / \mathbf{m}^{2} \mathbf{K}\right]$ & $\mathbf{S N P}$ & $\mu-\left[\mathbf{M W} / \mathbf{m}^{2} \mathbf{K}\right]$ & $\sigma-\left[\mathbf{M W} / \mathbf{m}^{2} \mathbf{K}\right]$ \\
6.86 & $27.89 \pm 0.114$ & $1.84 \pm 0.0051$ & 43.35 & $27.80 \pm 0.018$ & $0.29 \pm 0.0008$ \\
10.06 & $27.83 \pm 0.077$ & $1.23 \pm 0.0034$ & 46.66 & $27.82 \pm 0.017$ & $0.27 \pm 0.0008$ \\
13.73 & $27.83 \pm 0.055$ & $0.89 \pm 0.0025$ & 51.47 & $27.81 \pm 0.015$ & $0.25 \pm 0.0007$ \\
16.88 & $27.83 \pm 0.049$ & $0.79 \pm 0.0022$ & 53.64 & $27.81 \pm 0.014$ & $0.23 \pm 0.0006$ \\
20.28 & $27.77 \pm 0.037$ & $0.60 \pm 0.0017$ & 57.00 & $27.81 \pm 0.013$ & $0.22 \pm 0.0006$ \\
23.23 & $27.81 \pm 0.034$ & $0.54 \pm 0.0015$ & 60.36 & $27.81 \pm 0.013$ & $0.21 \pm 0.0006$ \\
26.78 & $27.81 \pm 0.029$ & $0.47 \pm 0.0013$ & 63.89 & $27.81 \pm 0.013$ & $0.20 \pm 0.0006$ \\
30.46 & $27.81 \pm 0.026$ & $0.42 \pm 0.0012$ & 66.59 & $27.81 \pm 0.011$ & $0.18 \pm 0.0005$ \\
33.33 & $27.80 \pm 0.024$ & $0.39 \pm 0.0011$ & 69.59 & $27.80 \pm 0.011$ & $0.18 \pm 0.0005$ \\
36.69 & $27.82 \pm 0.022$ & $0.35 \pm 0.0010$ & 73.89 & $27.81 \pm 0.011$ & $0.17 \pm 0.0005$ \\
40.06 & $27.82 \pm 0.020$ & $0.32 \pm 0.0009$ & & & \\
\hline
\end{tabular}

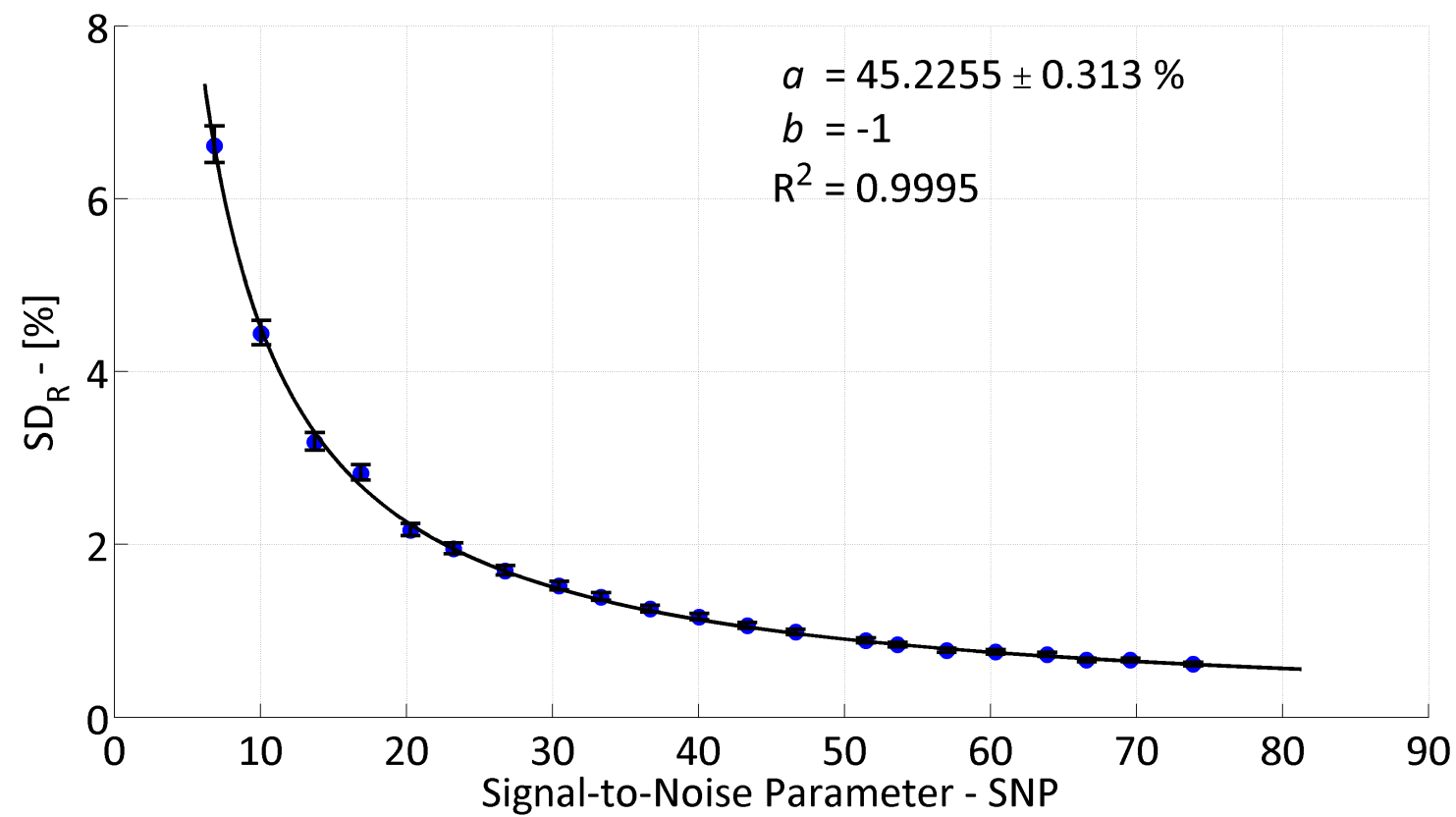

Figure S.123: Relative population standard deviation as a function of signal-to-noise parameter for 100 $\mathrm{nm}$ of $\mathrm{Al} / \mathrm{AlN}$ with $h_{\mathrm{BD}}=27.80 \mathrm{MW} / \mathrm{m}^{2} \mathrm{~K}$. Error bars represent a $95 \%$ confidence interval of the measured $\mathrm{SD}_{\mathrm{R}}$ and the solid line represents a best fit of a power law to the data, of the form $\mathrm{SD}_{\mathrm{R}}=a \times \mathrm{SNP}^{b}$, with parameter $a$ provided for a fixed $b=-1$, along with the $95 \%$ confidence level in $a$. 


\section{S.38 Simulation Summary - Al/AIN 59.90 MW Series}

\section{S.38.1 Fitting Film-to-Substrate Boundary Conductance - $h_{\mathrm{BD}}$}

Table S.63: Summary of simulation data - $100 \mathrm{~nm} \mathrm{Al/AlN} \mathrm{59.90} \mathrm{MW} \mathrm{Series.} \mathrm{Error} \mathrm{bounds} \mathrm{represent} \mathrm{a}$ $95 \%$ confidence level.

\begin{tabular}{cccccc}
\hline \multicolumn{6}{c}{$\mathbf{1 0 0} \mathbf{~ n m ~ A l ~ o n ~} \mathbf{A l N}, h_{\mathrm{BD}}=\mathbf{5 9 . 9 0} \mathbf{M W} / \mathbf{m}^{2} \mathbf{K}$} \\
\hline $\mathbf{S N P}$ & $\mu-\left[\mathbf{M W} / \mathbf{m}^{2} \mathbf{K}\right]$ & $\sigma-\left[\mathbf{M W} / \mathbf{m}^{2} \mathbf{K}\right]$ & $\mathbf{S N P}$ & $\mu-\left[\mathbf{M W} / \mathbf{m}^{2} \mathbf{K}\right]$ & $\sigma-\left[\mathbf{M W} / \mathbf{m}^{2} \mathbf{K}\right]$ \\
4.22 & $60.04 \pm 0.228$ & $3.67 \pm 0.0102$ & 26.96 & $59.94 \pm 0.037$ & $0.59 \pm 0.0016$ \\
6.36 & $59.97 \pm 0.152$ & $2.45 \pm 0.0068$ & 29.24 & $59.89 \pm 0.034$ & $0.54 \pm 0.0015$ \\
8.38 & $59.95 \pm 0.116$ & $1.87 \pm 0.0052$ & 30.99 & $59.93 \pm 0.032$ & $0.52 \pm 0.0015$ \\
10.59 & $59.95 \pm 0.094$ & $1.51 \pm 0.0042$ & 33.02 & $59.90 \pm 0.030$ & $0.49 \pm 0.0014$ \\
12.86 & $59.96 \pm 0.073$ & $1.18 \pm 0.0033$ & 35.30 & $59.91 \pm 0.028$ & $0.45 \pm 0.0012$ \\
14.60 & $59.84 \pm 0.065$ & $1.05 \pm 0.0029$ & 37.07 & $59.90 \pm 0.027$ & $0.43 \pm 0.0012$ \\
16.47 & $59.94 \pm 0.060$ & $0.97 \pm 0.0027$ & 38.55 & $59.92 \pm 0.025$ & $0.41 \pm 0.0011$ \\
18.39 & $59.92 \pm 0.054$ & $0.87 \pm 0.0024$ & 40.44 & $59.91 \pm 0.025$ & $0.40 \pm 0.0011$ \\
21.51 & $59.88 \pm 0.046$ & $0.74 \pm 0.0021$ & 43.23 & $59.91 \pm 0.023$ & $0.36 \pm 0.0010$ \\
22.76 & $59.93 \pm 0.045$ & $0.73 \pm 0.0020$ & 44.63 & $59.89 \pm 0.022$ & $0.35 \pm 0.0010$ \\
25.27 & $59.91 \pm 0.039$ & $0.63 \pm 0.0017$ & & & \\
\hline
\end{tabular}

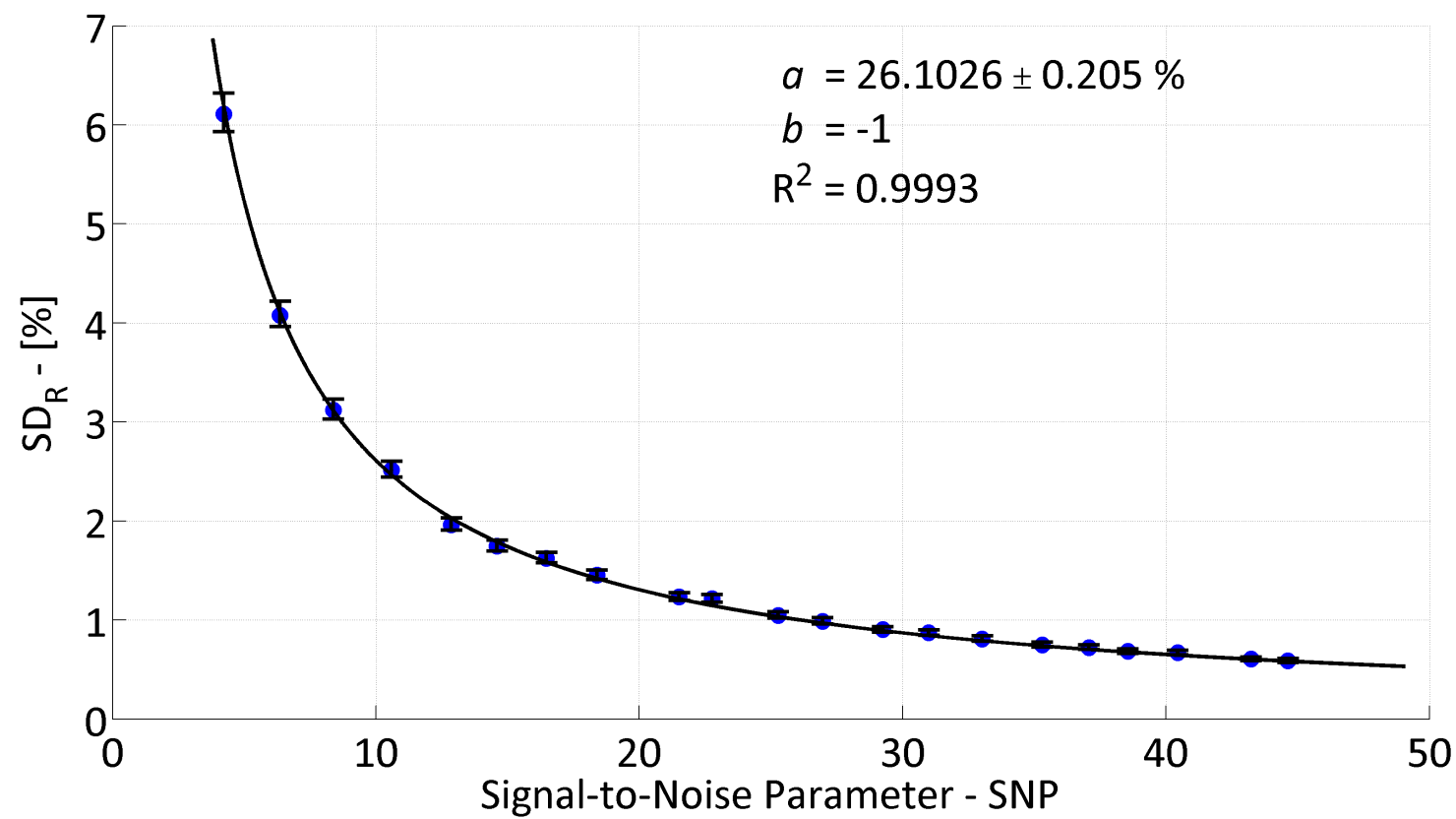

Figure S.124: Relative population standard deviation as a function of signal-to-noise parameter for 100 $\mathrm{nm}$ of $\mathrm{Al} / \mathrm{AlN}$ with $h_{\mathrm{BD}}=59.90 \mathrm{MW} / \mathrm{m}^{2} \mathrm{~K}$. Error bars represent a $95 \%$ confidence interval of the measured $\mathrm{SD}_{\mathrm{R}}$ and the solid line represents a best fit of a power law to the data, of the form $\mathrm{SD}_{\mathrm{R}}=a \times \mathrm{SNP}^{b}$, with parameter $a$ provided for a fixed $b=-1$, along with the $95 \%$ confidence level in $a$. 


\section{S.38.2 Fitting Substrate Thermal Conductivity $-k_{S}$}

Table S.64: Summary of simulation data - $100 \mathrm{~nm} \mathrm{Al/AlN} \mathrm{59.90} \mathrm{MW} \mathrm{Series.} \mathrm{Error} \mathrm{bounds} \mathrm{represent} \mathrm{a}$ $95 \%$ confidence level.

\begin{tabular}{cccccc}
\hline \multicolumn{6}{c}{$\mathbf{1 0 0} \mathbf{~ n m} \mathbf{A l}$ on $\mathbf{A l N}, h_{\mathrm{BD}}=\mathbf{5 9 . 9 0} \mathbf{M W} / \mathbf{m}^{2} \mathbf{K}$} \\
\hline $\mathbf{S N P}$ & $\mu-[\mathbf{W} / \mathbf{m K}]$ & $\sigma-[\mathbf{W} / \mathbf{m K}]$ & $\mathbf{S N P}$ & $\mu-[\mathbf{W} / \mathbf{m K}]$ & $\sigma-[\mathbf{W} / \mathbf{m K}]$ \\
4.22 & $339.80 \pm 13.189$ & $209.97 \pm 0.5992$ & 26.96 & $285.80 \pm 1.644$ & $26.46 \pm 0.0739$ \\
6.36 & $311.80 \pm 7.835$ & $125.75 \pm 0.3530$ & 29.24 & $283.30 \pm 1.493$ & $24.05 \pm 0.0670$ \\
8.38 & $298.02 \pm 5.353$ & $85.96 \pm 0.2411$ & 30.99 & $285.66 \pm 1.457$ & $23.48 \pm 0.0654$ \\
10.59 & $293.87 \pm 4.326$ & $69.60 \pm 0.1944$ & 33.02 & $283.77 \pm 1.337$ & $21.54 \pm 0.0600$ \\
12.86 & $291.71 \pm 3.460$ & $55.73 \pm 0.1554$ & 35.30 & $284.01 \pm 1.232$ & $19.85 \pm 0.0553$ \\
14.60 & $285.15 \pm 3.014$ & $48.54 \pm 0.1353$ & 37.07 & $283.53 \pm 1.184$ & $19.08 \pm 0.0531$ \\
16.47 & $288.48 \pm 2.753$ & $44.34 \pm 0.1236$ & 38.55 & $284.30 \pm 1.133$ & $18.25 \pm 0.0508$ \\
18.39 & $286.92 \pm 2.470$ & $39.80 \pm 0.1108$ & 40.44 & $284.15 \pm 1.095$ & $17.63 \pm 0.0492$ \\
21.51 & $284.33 \pm 2.064$ & $33.25 \pm 0.0927$ & 43.23 & $284.14 \pm 1.003$ & $16.17 \pm 0.0450$ \\
22.76 & $286.53 \pm 2.032$ & $32.73 \pm 0.0912$ & 44.63 & $283.00 \pm 0.983$ & $15.84 \pm 0.0441$ \\
25.27 & $285.03 \pm 1.743$ & $28.08 \pm 0.0782$ & & & \\
\hline
\end{tabular}

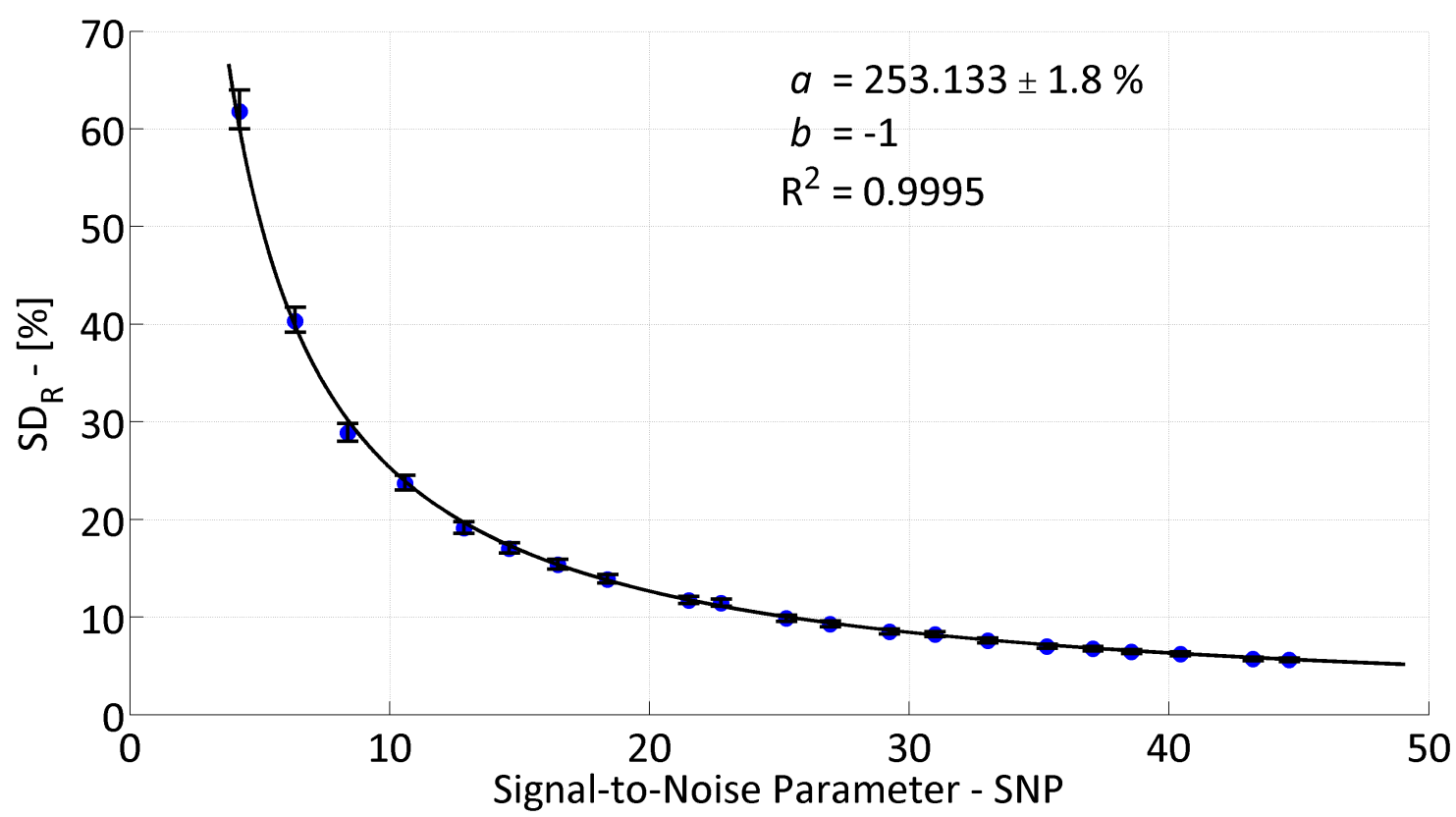

Figure S.125: Relative population standard deviation as a function of signal-to-noise parameter for 100 $\mathrm{nm}$ of $\mathrm{Al} / \mathrm{AlN}$ with $h_{\mathrm{BD}}=59.90 \mathrm{MW} / \mathrm{m}^{2} \mathrm{~K}$. Error bars represent a $95 \%$ confidence interval of the measured $\mathrm{SD}_{\mathrm{R}}$ and the solid line represents a best fit of a power law to the data, of the form $\mathrm{SD}_{\mathrm{R}}=a \times \mathrm{SNP}^{b}$, with parameter $a$ provided for a fixed $b=-1$, along with the $95 \%$ confidence level in $a$. 


\section{S.39 Simulation Summary - Al/AlN 100.00 MW Series}

\section{S.39.1 Fitting Film-to-Substrate Boundary Conductance - $h_{\mathrm{BD}}$}

Table S.65: Summary of simulation data - $100 \mathrm{~nm} \mathrm{Al/AlN} \mathrm{100.00} \mathrm{MW} \mathrm{Series.} \mathrm{Error} \mathrm{bounds} \mathrm{represent} \mathrm{a}$ $95 \%$ confidence level.

\begin{tabular}{cccccc}
\hline \multicolumn{6}{c}{$\mathbf{1 0 0} \mathbf{~ n m ~ A l ~ o n ~ A l N , ~} h_{\mathrm{BD}}=\mathbf{1 0 0 . 0 0} \mathbf{M W} / \mathbf{m}^{2} \mathbf{K}$} \\
\hline $\mathbf{S N P}$ & $\mu-\left[\mathbf{M W} / \mathbf{m}^{2} \mathbf{K}\right]$ & $\sigma-\left[\mathbf{M W} / \mathbf{m}^{2} \mathbf{K}\right]$ & $\mathbf{S N P}$ & $\mu-\left[\mathbf{M W} / \mathbf{m}^{2} \mathbf{K}\right]$ & $\sigma-\left[\mathbf{M W} / \mathbf{m}^{2} \mathbf{K}\right]$ \\
2.53 & $100.93 \pm 0.561$ & $9.03 \pm 0.0252$ & 16.55 & $99.99 \pm 0.087$ & $1.41 \pm 0.0039$ \\
3.81 & $100.35 \pm 0.391$ & $6.30 \pm 0.0176$ & 17.60 & $100.02 \pm 0.080$ & $1.29 \pm 0.0036$ \\
5.06 & $100.06 \pm 0.280$ & $4.51 \pm 0.0125$ & 18.99 & $100.04 \pm 0.075$ & $1.20 \pm 0.0033$ \\
6.34 & $100.05 \pm 0.231$ & $3.73 \pm 0.0104$ & 20.31 & $100.05 \pm 0.070$ & $1.13 \pm 0.0032$ \\
7.59 & $99.90 \pm 0.193$ & $3.10 \pm 0.0086$ & 21.60 & $100.02 \pm 0.065$ & $1.05 \pm 0.0029$ \\
8.90 & $99.96 \pm 0.168$ & $2.70 \pm 0.0075$ & 22.72 & $100.01 \pm 0.062$ & $1.00 \pm 0.0028$ \\
10.12 & $100.10 \pm 0.142$ & $2.29 \pm 0.0064$ & 24.11 & $100.05 \pm 0.059$ & $0.96 \pm 0.0027$ \\
11.41 & $99.94 \pm 0.119$ & $1.92 \pm 0.0053$ & 25.38 & $100.01 \pm 0.054$ & $0.87 \pm 0.0024$ \\
12.66 & $99.92 \pm 0.116$ & $1.87 \pm 0.0052$ & 26.38 & $100.02 \pm 0.053$ & $0.85 \pm 0.0024$ \\
13.94 & $100.04 \pm 0.103$ & $1.66 \pm 0.0046$ & 27.77 & $99.97 \pm 0.052$ & $0.83 \pm 0.0023$ \\
15.16 & $99.98 \pm 0.094$ & $1.52 \pm 0.0042$ & & & \\
\hline
\end{tabular}

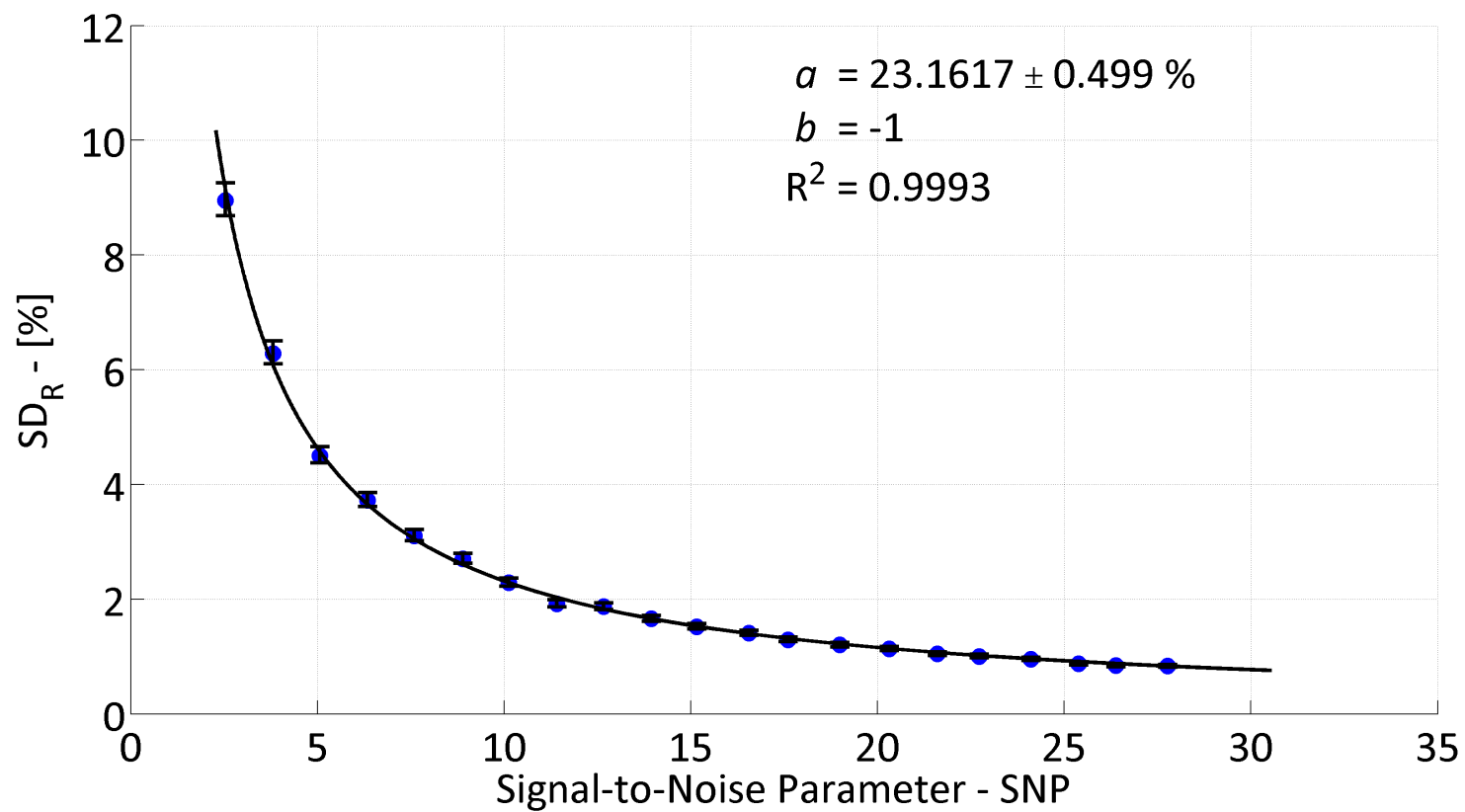

Figure S.126: Relative population standard deviation as a function of signal-to-noise parameter for 100 $\mathrm{nm}$ of $\mathrm{Al} / \mathrm{AlN}$ with $h_{\mathrm{BD}}=100.00 \mathrm{MW} / \mathrm{m}^{2} \mathrm{~K}$. Error bars represent a $95 \%$ confidence interval of the measured $\mathrm{SD}_{\mathrm{R}}$ and the solid line represents a best fit of a power law to the data, of the form $\mathrm{SD}_{\mathrm{R}}=a \times \mathrm{SNP}^{b}$, with parameter $a$ provided for a fixed $b=-1$, along with the $95 \%$ confidence level in $a$. 


\section{S.40 Simulation Summary - Al/AlN 129.00 MW Series}

\section{S.40.1 Fitting Film-to-Substrate Boundary Conductance - $h_{\mathrm{BD}}$}

Table S.66: Summary of simulation data - $100 \mathrm{~nm} \mathrm{Al/AlN} \mathrm{129.00} \mathrm{MW} \mathrm{Series.} \mathrm{Error} \mathrm{bounds} \mathrm{represent} \mathrm{a}$ $95 \%$ confidence level.

\begin{tabular}{cccccc}
\hline \multicolumn{6}{c}{$\mathbf{1 0 0} \mathbf{~ n m ~ A l ~ o n ~ A l N , ~} h_{\mathrm{BD}}=\mathbf{1 2 9 . 0 0} \mathbf{M W} / \mathbf{m}^{2} \mathbf{K}$} \\
\hline $\mathbf{S N P}$ & $\mu-\left[\mathbf{M W} / \mathbf{m}^{2} \mathbf{K}\right]$ & $\sigma-\left[\mathbf{M W} / \mathbf{m}^{2} \mathbf{K}\right]$ & $\mathbf{S N P}$ & $\mu-\left[\mathbf{M W} / \mathbf{m}^{2} \mathbf{K}\right]$ & $\sigma-\left[\mathbf{M W} / \mathbf{m}^{2} \mathbf{K}\right]$ \\
2.15 & $130.55 \pm 0.924$ & $14.80 \pm 0.0417$ & 13.56 & $129.02 \pm 0.148$ & $2.37 \pm 0.0067$ \\
3.22 & $129.66 \pm 0.600$ & $9.61 \pm 0.0271$ & 14.58 & $129.01 \pm 0.131$ & $2.09 \pm 0.0059$ \\
4.34 & $129.27 \pm 0.454$ & $7.27 \pm 0.0205$ & 15.89 & $129.09 \pm 0.126$ & $2.02 \pm 0.0057$ \\
5.31 & $129.28 \pm 0.373$ & $5.97 \pm 0.0168$ & 16.74 & $129.01 \pm 0.117$ & $1.87 \pm 0.0053$ \\
6.39 & $129.30 \pm 0.301$ & $4.82 \pm 0.0136$ & 18.18 & $128.98 \pm 0.106$ & $1.69 \pm 0.0048$ \\
7.44 & $128.98 \pm 0.252$ & $4.03 \pm 0.0114$ & 18.86 & $129.09 \pm 0.106$ & $1.70 \pm 0.0048$ \\
8.85 & $129.19 \pm 0.225$ & $3.61 \pm 0.0102$ & 19.82 & $128.95 \pm 0.100$ & $1.61 \pm 0.0045$ \\
9.56 & $129.05 \pm 0.206$ & $3.29 \pm 0.0093$ & 21.19 & $128.96 \pm 0.089$ & $1.42 \pm 0.0040$ \\
10.92 & $128.92 \pm 0.180$ & $2.88 \pm 0.0081$ & 21.98 & $128.98 \pm 0.089$ & $1.43 \pm 0.0040$ \\
11.81 & $128.95 \pm 0.164$ & $2.62 \pm 0.0074$ & 23.44 & $129.02 \pm 0.087$ & $1.40 \pm 0.0039$ \\
12.70 & $129.14 \pm 0.158$ & $2.53 \pm 0.0071$ & & & \\
\hline
\end{tabular}

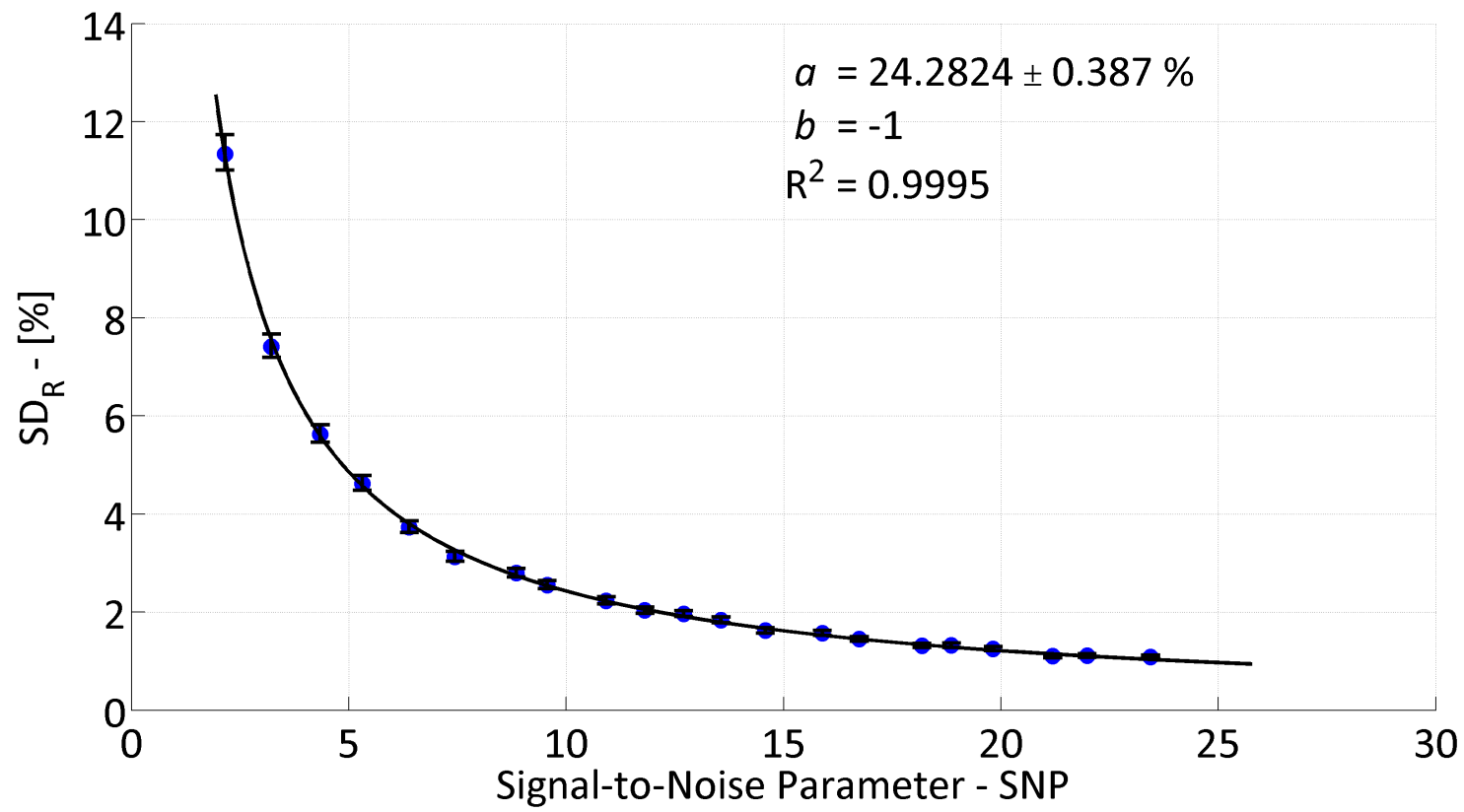

Figure S.127: Relative population standard deviation as a function of signal-to-noise parameter for 100 $\mathrm{nm}$ of $\mathrm{Al} / \mathrm{AlN}$ with $h_{\mathrm{BD}}=129.00 \mathrm{MW} / \mathrm{m}^{2} \mathrm{~K}$. Error bars represent a $95 \%$ confidence interval of the measured $\mathrm{SD}_{\mathrm{R}}$ and the solid line represents a best fit of a power law to the data, of the form $\mathrm{SD}_{\mathrm{R}}=a \times \mathrm{SNP}^{b}$, with parameter $a$ provided for a fixed $b=-1$, along with the $95 \%$ confidence level in $a$. 


\section{S.40.2 Fitting Substrate Thermal Conductivity $-k_{S}$}

Table S.67: Summary of simulation data - $100 \mathrm{~nm} \mathrm{Al/AlN} \mathrm{129.00} \mathrm{MW} \mathrm{Series.} \mathrm{Error} \mathrm{bounds} \mathrm{represent} \mathrm{a}$ 95\% confidence level.

\begin{tabular}{cccccc}
\hline \multicolumn{6}{c}{$\mathbf{1 0 0} \mathbf{~ n m} \mathbf{A l}$ on $\mathbf{A l N}, h_{\mathrm{BD}}=\mathbf{1 2 9 . 0 0} \mathbf{M W} / \mathbf{m}^{2} \mathbf{K}$} \\
\hline $\mathbf{S N P}$ & $\mu-[\mathbf{W} / \mathbf{m K}]$ & $\sigma-[\mathbf{W} / \mathbf{m K}]$ & $\mathbf{S N P}$ & $\mu-[\mathbf{W} / \mathbf{m K}]$ & $\sigma-[\mathbf{W} / \mathbf{m K}]$ \\
2.15 & $307.21 \pm 6.679$ & $107.09 \pm 0.3013$ & 13.56 & $284.57 \pm 1.009$ & $16.26 \pm 0.0453$ \\
3.22 & $293.83 \pm 4.159$ & $66.89 \pm 0.1870$ & 14.58 & $284.57 \pm 0.898$ & $14.48 \pm 0.0403$ \\
4.34 & $289.50 \pm 3.064$ & $49.30 \pm 0.1377$ & 15.89 & $284.95 \pm 0.835$ & $13.46 \pm 0.0375$ \\
5.31 & $288.22 \pm 2.578$ & $41.50 \pm 0.1158$ & 16.74 & $284.46 \pm 0.791$ & $12.74 \pm 0.0355$ \\
6.39 & $287.78 \pm 2.071$ & $33.37 \pm 0.0929$ & 18.18 & $284.16 \pm 0.708$ & $11.41 \pm 0.0318$ \\
7.44 & $285.27 \pm 1.717$ & $27.66 \pm 0.0771$ & 18.86 & $285.03 \pm 0.722$ & $11.63 \pm 0.0324$ \\
8.85 & $286.53 \pm 1.561$ & $25.16 \pm 0.0701$ & 19.82 & $284.15 \pm 0.674$ & $10.85 \pm 0.0302$ \\
9.56 & $285.37 \pm 1.415$ & $22.80 \pm 0.0635$ & 21.19 & $283.96 \pm 0.598$ & $9.63 \pm 0.0268$ \\
10.92 & $284.28 \pm 1.218$ & $19.64 \pm 0.0547$ & 21.98 & $284.14 \pm 0.600$ & $9.67 \pm 0.0269$ \\
11.81 & $284.49 \pm 1.112$ & $17.92 \pm 0.0499$ & 23.44 & $284.44 \pm 0.592$ & $9.54 \pm 0.0266$ \\
12.70 & $285.32 \pm 1.075$ & $17.31 \pm 0.0482$ & & & \\
\hline
\end{tabular}

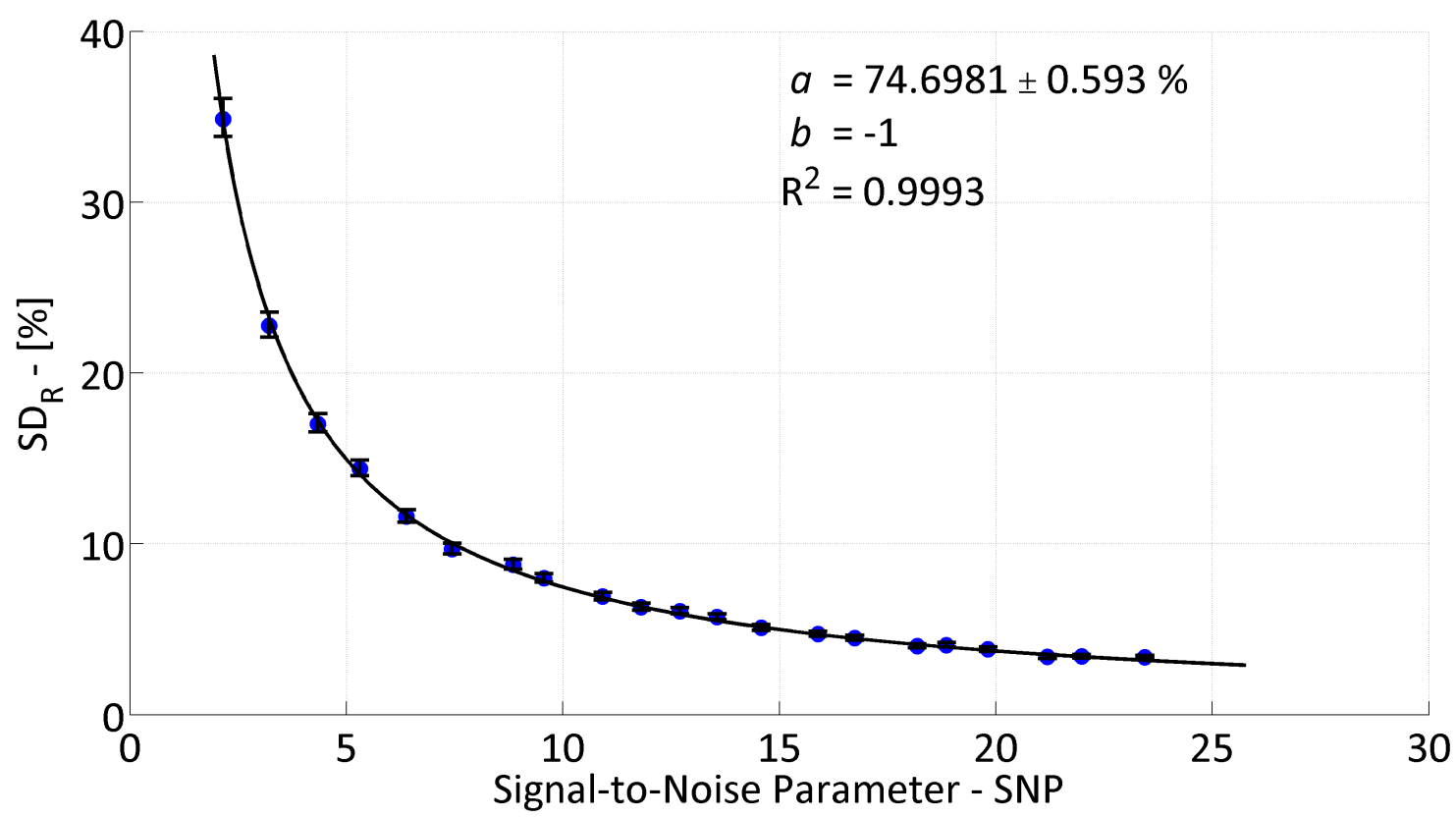

Figure S.128: Relative population standard deviation as a function of signal-to-noise parameter for 100 $\mathrm{nm}$ of $\mathrm{Al} / \mathrm{AlN}$ with $h_{\mathrm{BD}}=129.00 \mathrm{MW} / \mathrm{m}^{2} \mathrm{~K}$. Error bars represent a $95 \%$ confidence interval of the measured $\mathrm{SD}_{\mathrm{R}}$ and the solid line represents a best fit of a power law to the data, of the form $\mathrm{SD}_{\mathrm{R}}=a \times \mathrm{SNP}^{b}$, with parameter $a$ provided for a fixed $b=-1$, along with the $95 \%$ confidence level in $a$. 


\section{S.41 Simulation Summary - Al/AlN 167.00 MW Series}

\section{S.41.1 Fitting Film-to-Substrate Boundary Conductance - $h_{\mathrm{BD}}$}

Table S.68: Summary of simulation data - $100 \mathrm{~nm} \mathrm{Al/AlN} \mathrm{167.00} \mathrm{MW} \mathrm{Series.} \mathrm{Error} \mathrm{bounds} \mathrm{represent} \mathrm{a}$ $95 \%$ confidence level.

\begin{tabular}{cccccc}
\hline \multicolumn{6}{c}{$\mathbf{1 0 0} \mathbf{~ n m ~ A l ~ o n ~ A l N , ~} h_{\mathrm{BD}}=\mathbf{1 6 7 . 0 0} \mathbf{M W} / \mathbf{m}^{2} \mathbf{K}$} \\
\hline $\mathbf{S N P}$ & $\mu-\left[\mathbf{M W} / \mathbf{m}^{2} \mathbf{K}\right]$ & $\sigma-\left[\mathbf{M W} / \mathbf{m}^{2} \mathbf{K}\right]$ & $\mathbf{S N P}$ & $\mu-\left[\mathbf{M W} / \mathbf{m}^{2} \mathbf{K}\right]$ & $\sigma-\left[\mathbf{M W} / \mathbf{m}^{2} \mathbf{K}\right]$ \\
1.77 & $169.71 \pm 1.563$ & $25.16 \pm 0.0702$ & 11.21 & $167.05 \pm 0.246$ & $3.94 \pm 0.0111$ \\
2.63 & $167.68 \pm 1.064$ & $17.11 \pm 0.0478$ & 12.18 & $166.97 \pm 0.230$ & $3.68 \pm 0.0104$ \\
3.54 & $167.66 \pm 0.786$ & $12.62 \pm 0.0354$ & 13.07 & $166.93 \pm 0.223$ & $3.56 \pm 0.0101$ \\
4.37 & $167.04 \pm 0.642$ & $10.30 \pm 0.0289$ & 14.13 & $167.24 \pm 0.208$ & $3.33 \pm 0.0094$ \\
5.26 & $166.99 \pm 0.539$ & $8.65 \pm 0.0243$ & 14.59 & $167.12 \pm 0.207$ & $3.32 \pm 0.0094$ \\
6.15 & $166.77 \pm 0.459$ & $7.37 \pm 0.0207$ & 15.72 & $166.94 \pm 0.184$ & $2.93 \pm 0.0083$ \\
6.92 & $167.22 \pm 0.414$ & $6.63 \pm 0.0187$ & 16.63 & $167.03 \pm 0.174$ & $2.78 \pm 0.0078$ \\
7.85 & $166.75 \pm 0.354$ & $5.67 \pm 0.0160$ & 17.58 & $166.90 \pm 0.156$ & $2.49 \pm 0.0071$ \\
8.82 & $166.96 \pm 0.339$ & $5.43 \pm 0.0153$ & 18.39 & $166.88 \pm 0.153$ & $2.45 \pm 0.0069$ \\
9.66 & $167.17 \pm 0.286$ & $4.58 \pm 0.0129$ & 18.90 & $166.87 \pm 0.153$ & $2.45 \pm 0.0069$ \\
10.42 & $166.83 \pm 0.274$ & $4.39 \pm 0.0124$ & & & \\
\hline
\end{tabular}

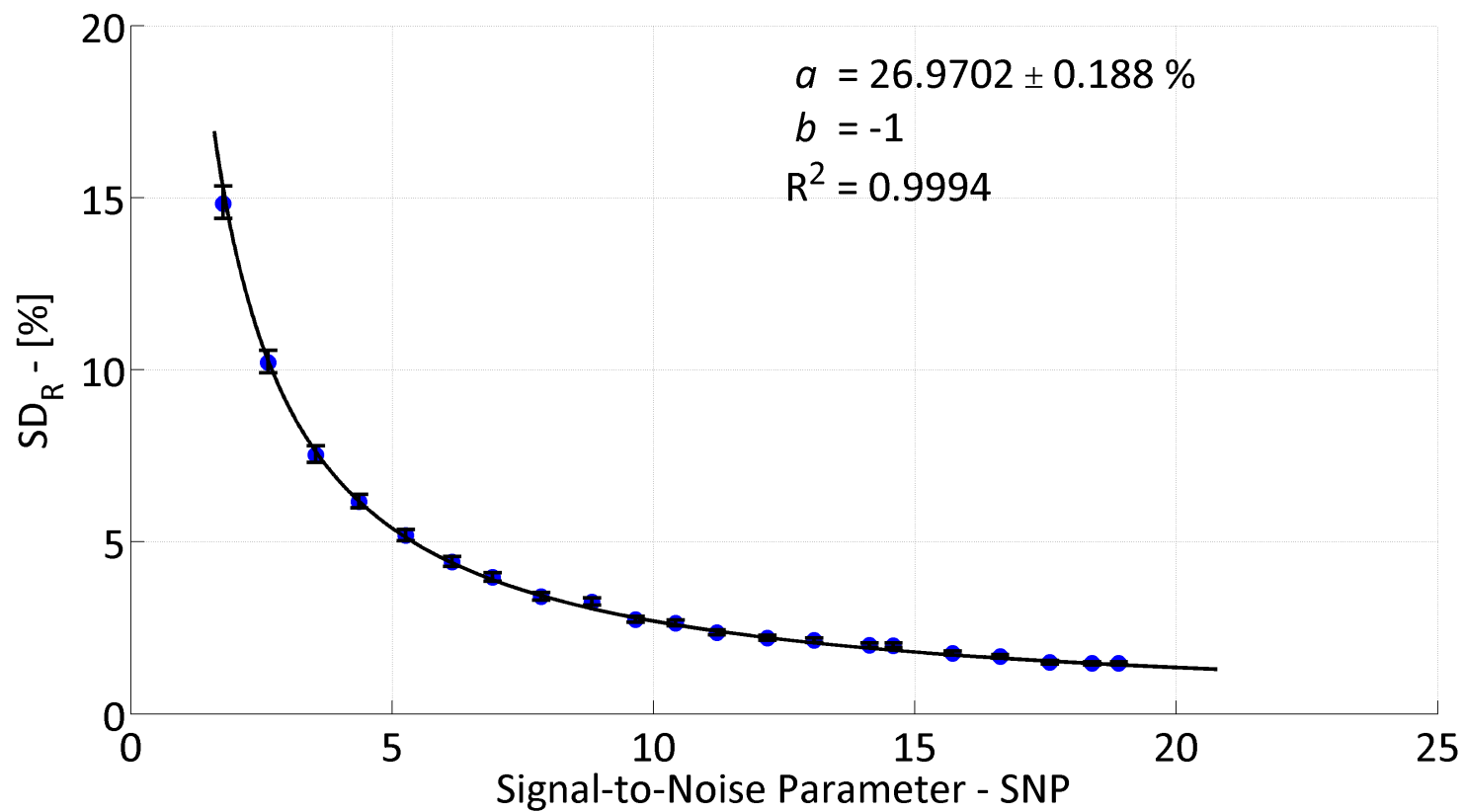

Figure S.129: Relative population standard deviation as a function of signal-to-noise parameter for 100 $\mathrm{nm}$ of $\mathrm{Al} / \mathrm{AlN}$ with $h_{\mathrm{BD}}=167.00 \mathrm{MW} / \mathrm{m}^{2} \mathrm{~K}$. Error bars represent a $95 \%$ confidence interval of the measured $\mathrm{SD}_{\mathrm{R}}$ and the solid line represents a best fit of a power law to the data, of the form $\mathrm{SD}_{\mathrm{R}}=a \times \mathrm{SNP}^{b}$, with parameter $a$ provided for a fixed $b=-1$, along with the $95 \%$ confidence level in $a$. 


\section{S.42 Simulation Summary - Al/AlN 215.00 MW Series}

\section{S.42.1 Fitting Film-to-Substrate Boundary Conductance - $h_{\mathrm{BD}}$}

Table S.69: Summary of simulation data - $100 \mathrm{~nm} \mathrm{Al/AlN} \mathrm{215.00} \mathrm{MW} \mathrm{Series.} \mathrm{Error} \mathrm{bounds} \mathrm{represent} \mathrm{a}$ $95 \%$ confidence level.

\begin{tabular}{cccccc}
\hline \multicolumn{6}{c}{$\mathbf{1 0 0} \mathbf{~ n m ~ A l ~ o n ~ A l N , ~} h_{\mathrm{BD}}=\mathbf{2 1 5 . 0 0} \mathbf{M W} / \mathbf{m}^{2} \mathbf{K}$} \\
\hline $\mathbf{S N P}$ & $\mu-\left[\mathbf{M W} / \mathbf{m}^{2} \mathbf{K}\right]$ & $\sigma-\left[\mathbf{M W} / \mathbf{m}^{2} \mathbf{K}\right]$ & $\mathbf{S N P}$ & $\mu-\left[\mathbf{M W} / \mathbf{m}^{2} \mathbf{K}\right]$ & $\sigma-\left[\mathbf{M W} / \mathbf{m}^{2} \mathbf{K}\right]$ \\
1.51 & $219.42 \pm 2.357$ & $37.12 \pm 0.1083$ & 9.79 & $214.71 \pm 0.414$ & $6.61 \pm 0.0187$ \\
2.27 & $216.03 \pm 1.614$ & $25.76 \pm 0.0731$ & 10.36 & $215.18 \pm 0.381$ & $6.09 \pm 0.0173$ \\
3.01 & $215.30 \pm 1.286$ & $20.55 \pm 0.0582$ & 11.23 & $214.70 \pm 0.339$ & $5.42 \pm 0.0154$ \\
3.79 & $214.61 \pm 1.000$ & $15.98 \pm 0.0453$ & 11.97 & $214.96 \pm 0.320$ & $5.11 \pm 0.0145$ \\
4.50 & $214.62 \pm 0.856$ & $13.66 \pm 0.0387$ & 12.63 & $214.60 \pm 0.310$ & $4.95 \pm 0.0140$ \\
5.29 & $214.63 \pm 0.721$ & $11.52 \pm 0.0326$ & 13.49 & $214.87 \pm 0.286$ & $4.56 \pm 0.0129$ \\
6.09 & $214.83 \pm 0.636$ & $10.15 \pm 0.0288$ & 14.26 & $215.05 \pm 0.265$ & $4.23 \pm 0.0120$ \\
6.78 & $214.49 \pm 0.564$ & $9.02 \pm 0.0255$ & 15.03 & $214.71 \pm 0.254$ & $4.05 \pm 0.0115$ \\
7.55 & $214.83 \pm 0.520$ & $8.31 \pm 0.0235$ & 15.86 & $214.91 \pm 0.257$ & $4.10 \pm 0.0116$ \\
8.28 & $215.33 \pm 0.461$ & $7.37 \pm 0.0209$ & 16.56 & $214.70 \pm 0.237$ & $3.78 \pm 0.0107$ \\
8.91 & $215.16 \pm 0.436$ & $6.96 \pm 0.0197$ & & & \\
\hline
\end{tabular}

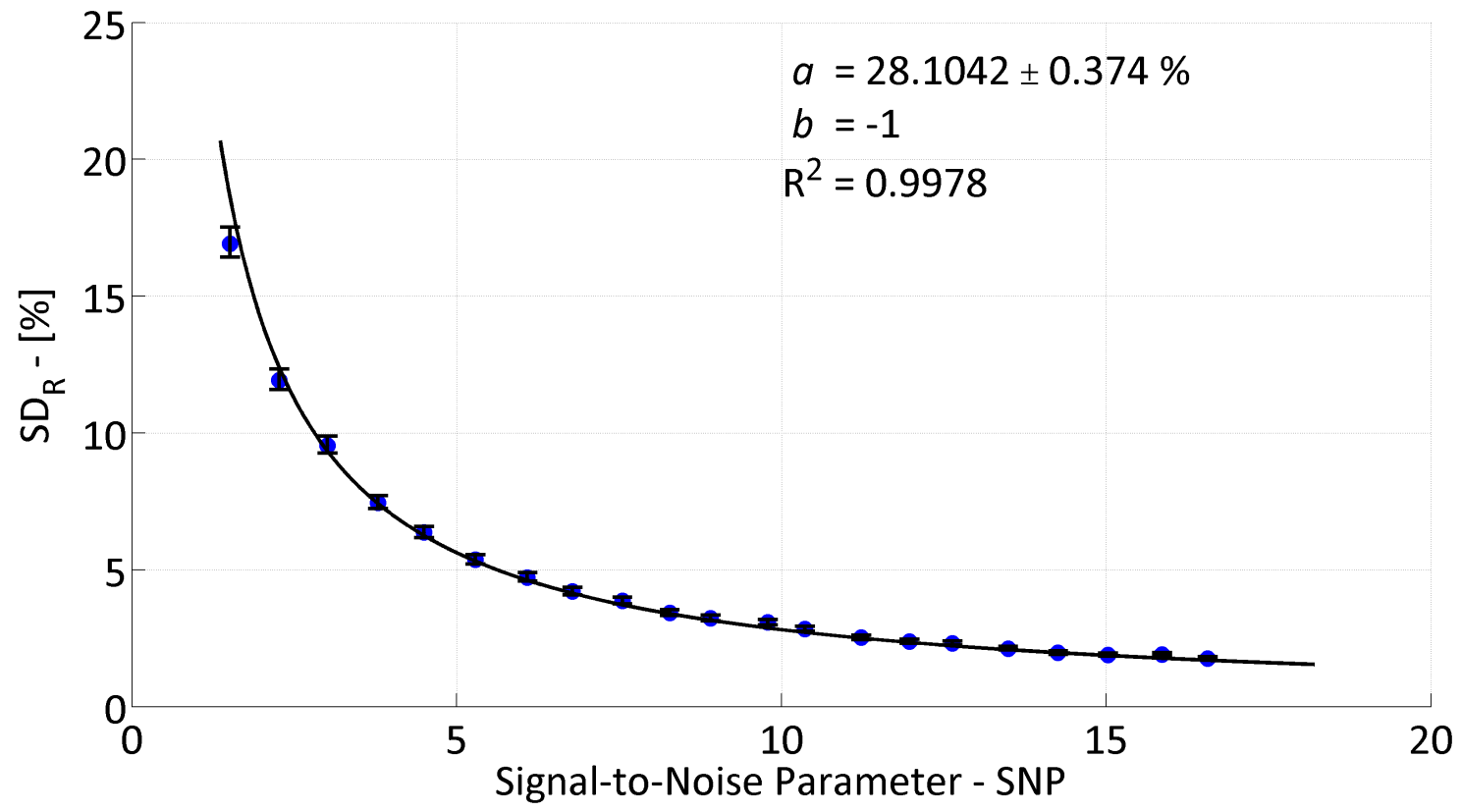

Figure S.130: Relative population standard deviation as a function of signal-to-noise parameter for 100 $\mathrm{nm}$ of $\mathrm{Al} / \mathrm{AlN}$ with $h_{\mathrm{BD}}=215.00 \mathrm{MW} / \mathrm{m}^{2} \mathrm{~K}$. Error bars represent a $95 \%$ confidence interval of the measured $\mathrm{SD}_{\mathrm{R}}$ and the solid line represents a best fit of a power law to the data, of the form $\mathrm{SD}_{\mathrm{R}}=a \times \mathrm{SNP}^{b}$, with parameter $a$ provided for a fixed $b=-1$, along with the $95 \%$ confidence level in $a$. 


\section{S.43 Simulation Summary - Al/AlN 278.00 MW Series}

\section{S.43.1 Fitting Film-to-Substrate Boundary Conductance - $h_{\mathrm{BD}}$}

Table S.70: Summary of simulation data - $100 \mathrm{~nm} \mathrm{Al/AlN} \mathrm{278.00} \mathrm{MW} \mathrm{Series.} \mathrm{Error} \mathrm{bounds} \mathrm{represent} \mathrm{a}$ $95 \%$ confidence level.

\begin{tabular}{cccccc}
\hline \multicolumn{6}{c}{$\mathbf{1 0 0} \mathbf{~ n m ~ A l ~ o n ~} \mathbf{A l N}, h_{\mathrm{BD}}=\mathbf{2 7 8 . 0 0} \mathbf{M W} / \mathbf{m}^{2} \mathbf{K}$} \\
\hline $\mathbf{S N P}$ & $\mu-\left[\mathbf{M W} / \mathbf{m}^{2} \mathbf{K}\right]$ & $\sigma-\left[\mathbf{M W} / \mathbf{m}^{2} \mathbf{K}\right]$ & $\mathbf{S N P}$ & $\mu-\left[\mathbf{M W} / \mathbf{m}^{2} \mathbf{K}\right]$ & $\sigma-\left[\mathbf{M W} / \mathbf{m}^{2} \mathbf{K}\right]$ \\
1.38 & $285.96 \pm 4.387$ & $68.58 \pm 0.2030$ & 8.83 & $277.81 \pm 0.498$ & $8.03 \pm 0.0224$ \\
2.06 & $281.32 \pm 2.588$ & $40.97 \pm 0.1183$ & 9.43 & $277.87 \pm 0.440$ & $7.09 \pm 0.0197$ \\
2.75 & $281.10 \pm 1.922$ & $30.65 \pm 0.0872$ & 10.18 & $277.93 \pm 0.430$ & $6.92 \pm 0.0193$ \\
3.42 & $277.85 \pm 1.406$ & $22.48 \pm 0.0636$ & 10.81 & $277.82 \pm 0.410$ & $6.61 \pm 0.0184$ \\
4.11 & $278.73 \pm 1.145$ & $18.43 \pm 0.0515$ & 11.62 & $277.64 \pm 0.367$ & $5.92 \pm 0.0165$ \\
4.67 & $277.74 \pm 0.982$ & $15.82 \pm 0.0441$ & 12.12 & $277.65 \pm 0.346$ & $5.58 \pm 0.0155$ \\
5.45 & $279.02 \pm 0.830$ & $13.36 \pm 0.0373$ & 12.91 & $277.76 \pm 0.339$ & $5.46 \pm 0.0152$ \\
6.09 & $278.43 \pm 0.709$ & $11.42 \pm 0.0319$ & 13.31 & $277.95 \pm 0.313$ & $5.04 \pm 0.0140$ \\
6.84 & $277.95 \pm 0.635$ & $10.23 \pm 0.0285$ & 14.45 & $277.82 \pm 0.294$ & $4.73 \pm 0.0132$ \\
7.47 & $278.12 \pm 0.602$ & $9.69 \pm 0.0270$ & 14.94 & $277.87 \pm 0.296$ & $4.77 \pm 0.0133$ \\
8.15 & $277.33 \pm 0.552$ & $8.90 \pm 0.0248$ & & & \\
\hline
\end{tabular}

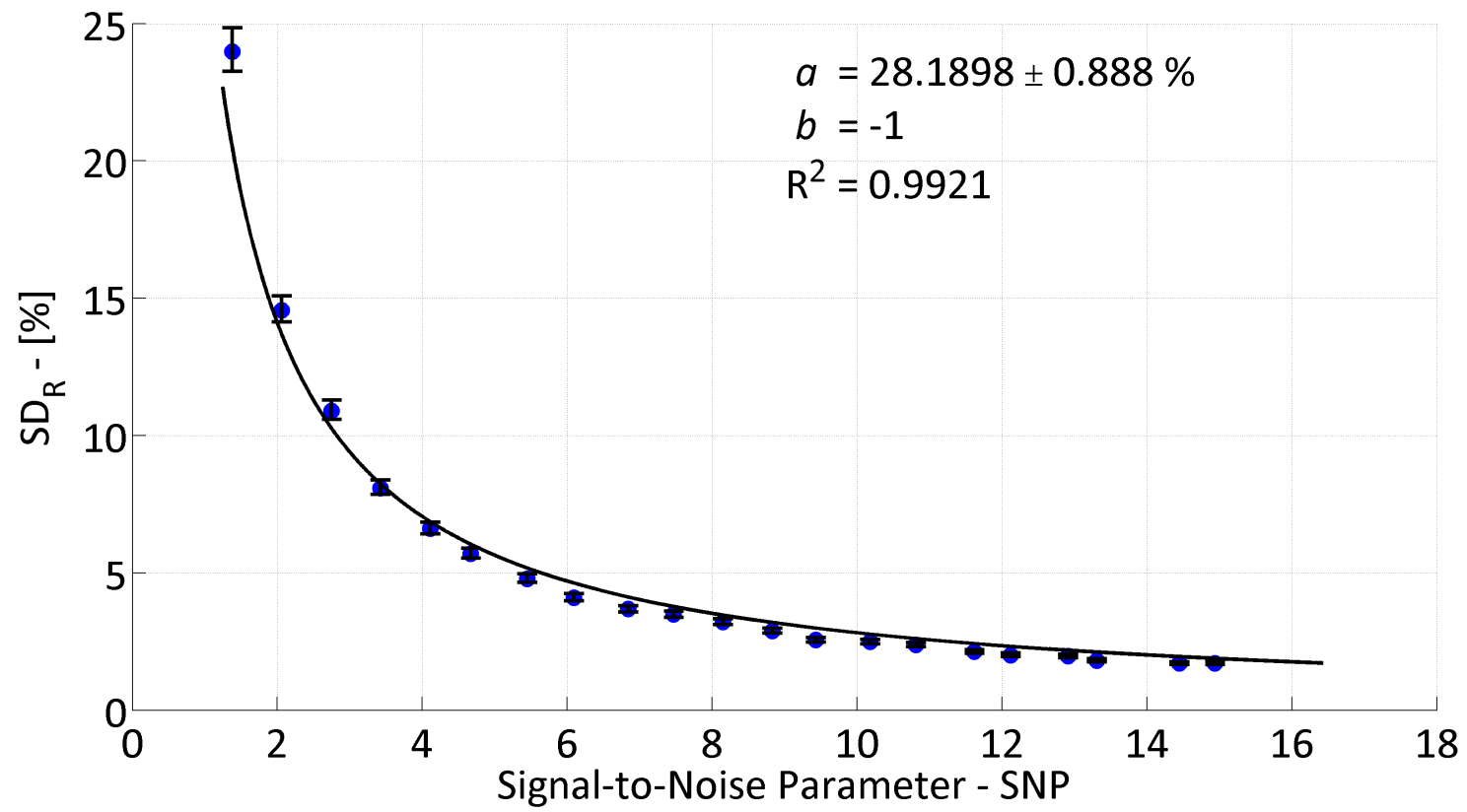

Figure S.131: Relative population standard deviation as a function of signal-to-noise parameter for 100 $\mathrm{nm}$ of $\mathrm{Al} / \mathrm{AlN}$ with $h_{\mathrm{BD}}=278.00 \mathrm{MW} / \mathrm{m}^{2} \mathrm{~K}$. Error bars represent a $95 \%$ confidence interval of the measured $\mathrm{SD}_{\mathrm{R}}$ and the solid line represents a best fit of a power law to the data, of the form $\mathrm{SD}_{\mathrm{R}}=a \times \mathrm{SNP}^{b}$, with parameter $a$ provided for a fixed $b=-1$, along with the $95 \%$ confidence level in $a$. 


\section{S.43.2 Fitting Substrate Thermal Conductivity $-k_{S}$}

Table S.71: Summary of simulation data - $100 \mathrm{~nm} \mathrm{Al/AlN} \mathrm{278.00} \mathrm{MW} \mathrm{Series.} \mathrm{Error} \mathrm{bounds} \mathrm{represent} \mathrm{a}$ $95 \%$ confidence level.

\begin{tabular}{cccccc}
\hline \multicolumn{6}{c}{$\mathbf{1 0 0} \mathbf{~ n m ~} \mathbf{A l}$ on AlN, $h_{\mathrm{BD}}=\mathbf{2 7 8 . 0 0 ~} \mathbf{M W} / \mathbf{m}^{2} \mathbf{K}$} \\
\hline $\mathbf{S N P}$ & $\mu-[\mathbf{W} / \mathbf{m K}]$ & $\sigma-[\mathbf{W} / \mathbf{m K}]$ & $\mathbf{S N P}$ & $\mu-[\mathbf{W} / \mathbf{m K}]$ & $\sigma-[\mathbf{W} / \mathbf{m K}]$ \\
1.38 & $291.46 \pm 5.360$ & $85.80 \pm 0.2421$ & 8.83 & $284.98 \pm 0.858$ & $13.83 \pm 0.0385$ \\
2.06 & $290.01 \pm 3.746$ & $60.24 \pm 0.1684$ & 9.43 & $285.32 \pm 0.776$ & $12.50 \pm 0.0348$ \\
2.75 & $289.47 \pm 2.723$ & $43.87 \pm 0.1222$ & 10.18 & $284.37 \pm 0.758$ & $12.20 \pm 0.0341$ \\
3.42 & $287.39 \pm 2.175$ & $35.03 \pm 0.0977$ & 10.81 & $285.18 \pm 0.689$ & $11.10 \pm 0.0309$ \\
4.11 & $288.36 \pm 1.800$ & $28.99 \pm 0.0808$ & 11.62 & $284.62 \pm 0.633$ & $10.20 \pm 0.0284$ \\
4.67 & $287.57 \pm 1.658$ & $26.73 \pm 0.0744$ & 12.12 & $284.68 \pm 0.623$ & $10.04 \pm 0.0280$ \\
5.45 & $287.02 \pm 1.428$ & $23.01 \pm 0.0641$ & 12.91 & $284.47 \pm 0.566$ & $9.11 \pm 0.0254$ \\
6.09 & $286.26 \pm 1.228$ & $19.80 \pm 0.0551$ & 13.31 & $284.89 \pm 0.544$ & $8.77 \pm 0.0244$ \\
6.84 & $286.00 \pm 1.043$ & $16.81 \pm 0.0468$ & 14.45 & $284.49 \pm 0.500$ & $8.06 \pm 0.0224$ \\
7.47 & $285.63 \pm 1.004$ & $16.18 \pm 0.0451$ & 14.94 & $284.79 \pm 0.512$ & $8.25 \pm 0.0230$ \\
8.15 & $284.26 \pm 0.900$ & $14.48 \pm 0.0404$ & & & \\
\hline
\end{tabular}

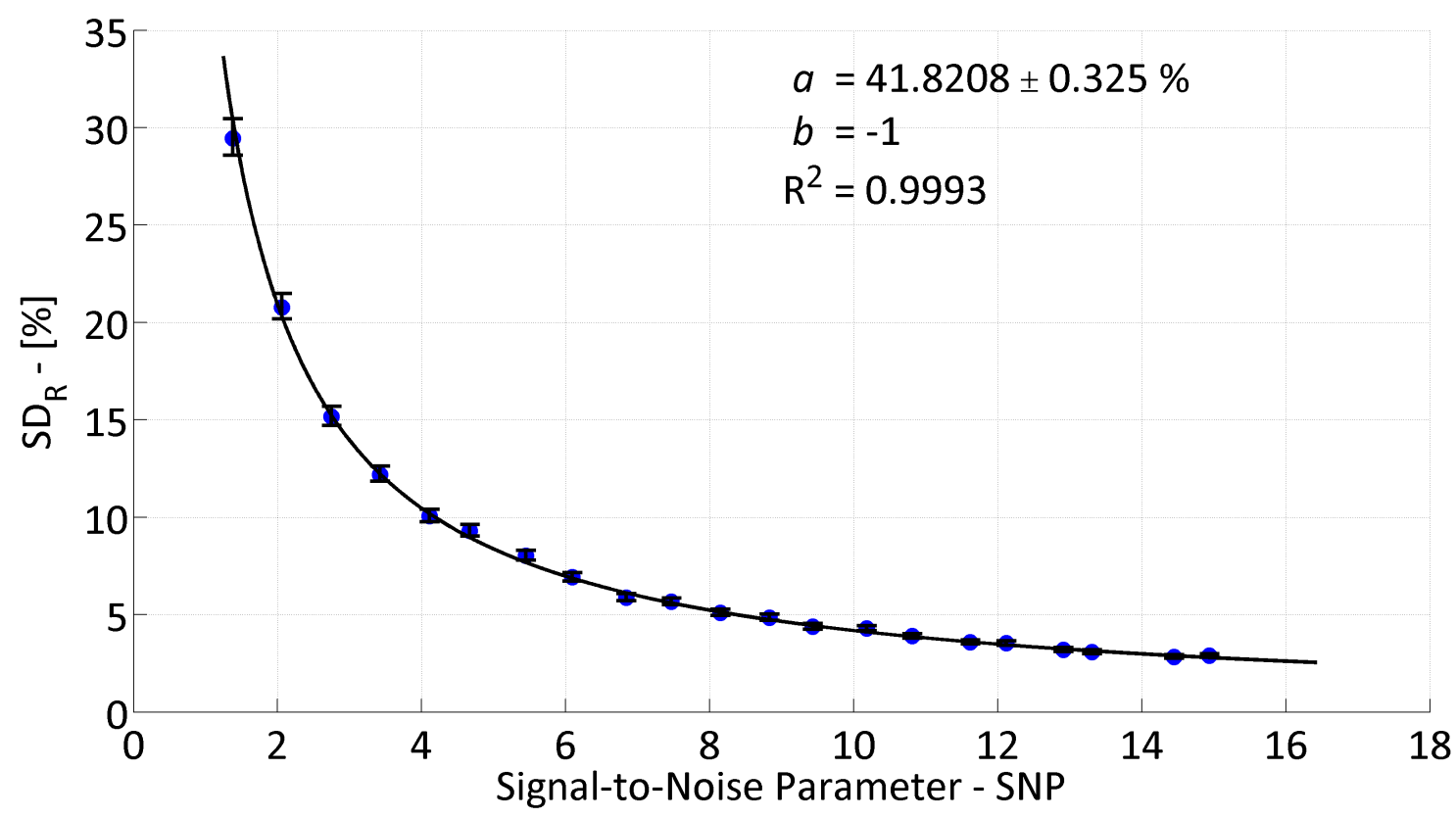

Figure S.132: Relative population standard deviation as a function of signal-to-noise parameter for 100 $\mathrm{nm}$ of $\mathrm{Al} / \mathrm{AlN}$ with $h_{\mathrm{BD}}=278.00 \mathrm{MW} / \mathrm{m}^{2} \mathrm{~K}$. Error bars represent a $95 \%$ confidence interval of the measured $\mathrm{SD}_{\mathrm{R}}$ and the solid line represents a best fit of a power law to the data, of the form $\mathrm{SD}_{\mathrm{R}}=a \times \mathrm{SNP}^{b}$, with parameter $a$ provided for a fixed $b=-1$, along with the $95 \%$ confidence level in $a$. 


\section{S.44 Simulation Summary - Al/AlN 359.00 MW Series}

\section{S.44.1 Fitting Film-to-Substrate Boundary Conductance $-h_{\mathrm{BD}}$}

Table S.72: Summary of simulation data - $100 \mathrm{~nm} \mathrm{Al/AlN} \mathrm{359.00} \mathrm{MW} \mathrm{Series.} \mathrm{Error} \mathrm{bounds} \mathrm{represent} \mathrm{a}$ $95 \%$ confidence level.

\begin{tabular}{cccccc}
\hline \multicolumn{6}{c}{$\mathbf{1 0 0} \mathbf{~ n m ~ A l ~ o n ~} \mathbf{A l N}, h_{\mathrm{BD}}=\mathbf{3 5 9 . 0 0} \mathbf{M W} / \mathbf{m}^{2} \mathbf{K}$} \\
\hline $\mathbf{S N P}$ & $\mu-\left[\mathbf{M W} / \mathbf{m}^{2} \mathbf{K}\right]$ & $\sigma-\left[\mathbf{M W} / \mathbf{m}^{2} \mathbf{K}\right]$ & $\mathbf{S N P}$ & $\mu-\left[\mathbf{M W} / \mathbf{m}^{2} \mathbf{K}\right]$ & $\sigma-\left[\mathbf{M W} / \mathbf{m}^{2} \mathbf{K}\right]$ \\
1.29 & $402.69 \pm 9.024$ & $143.95 \pm 0.4091$ & 8.28 & $359.84 \pm 0.924$ & $14.87 \pm 0.0415$ \\
1.91 & $376.01 \pm 4.736$ & $75.51 \pm 0.2148$ & 8.88 & $359.79 \pm 0.837$ & $13.48 \pm 0.0376$ \\
2.56 & $371.10 \pm 3.610$ & $57.94 \pm 0.1627$ & 9.59 & $360.41 \pm 0.792$ & $12.75 \pm 0.0356$ \\
3.21 & $371.18 \pm 2.919$ & $46.99 \pm 0.1311$ & 10.17 & $359.96 \pm 0.780$ & $12.58 \pm 0.0350$ \\
3.80 & $365.21 \pm 2.218$ & $35.72 \pm 0.0996$ & 10.84 & $359.13 \pm 0.692$ & $11.16 \pm 0.0311$ \\
4.47 & $361.50 \pm 1.712$ & $27.51 \pm 0.0771$ & 11.48 & $360.10 \pm 0.674$ & $10.87 \pm 0.0303$ \\
5.11 & $362.12 \pm 1.513$ & $24.34 \pm 0.0680$ & 12.12 & $360.19 \pm 0.635$ & $10.24 \pm 0.0285$ \\
5.67 & $361.16 \pm 1.390$ & $22.35 \pm 0.0625$ & 12.66 & $359.78 \pm 0.608$ & $9.78 \pm 0.0273$ \\
6.35 & $360.37 \pm 1.212$ & $19.50 \pm 0.0545$ & 13.40 & $359.76 \pm 0.581$ & $9.36 \pm 0.0261$ \\
6.97 & $360.30 \pm 1.113$ & $17.93 \pm 0.0500$ & 14.07 & $359.44 \pm 0.552$ & $8.90 \pm 0.0248$ \\
7.60 & $360.23 \pm 0.999$ & $16.09 \pm 0.0449$ & & & \\
\hline
\end{tabular}

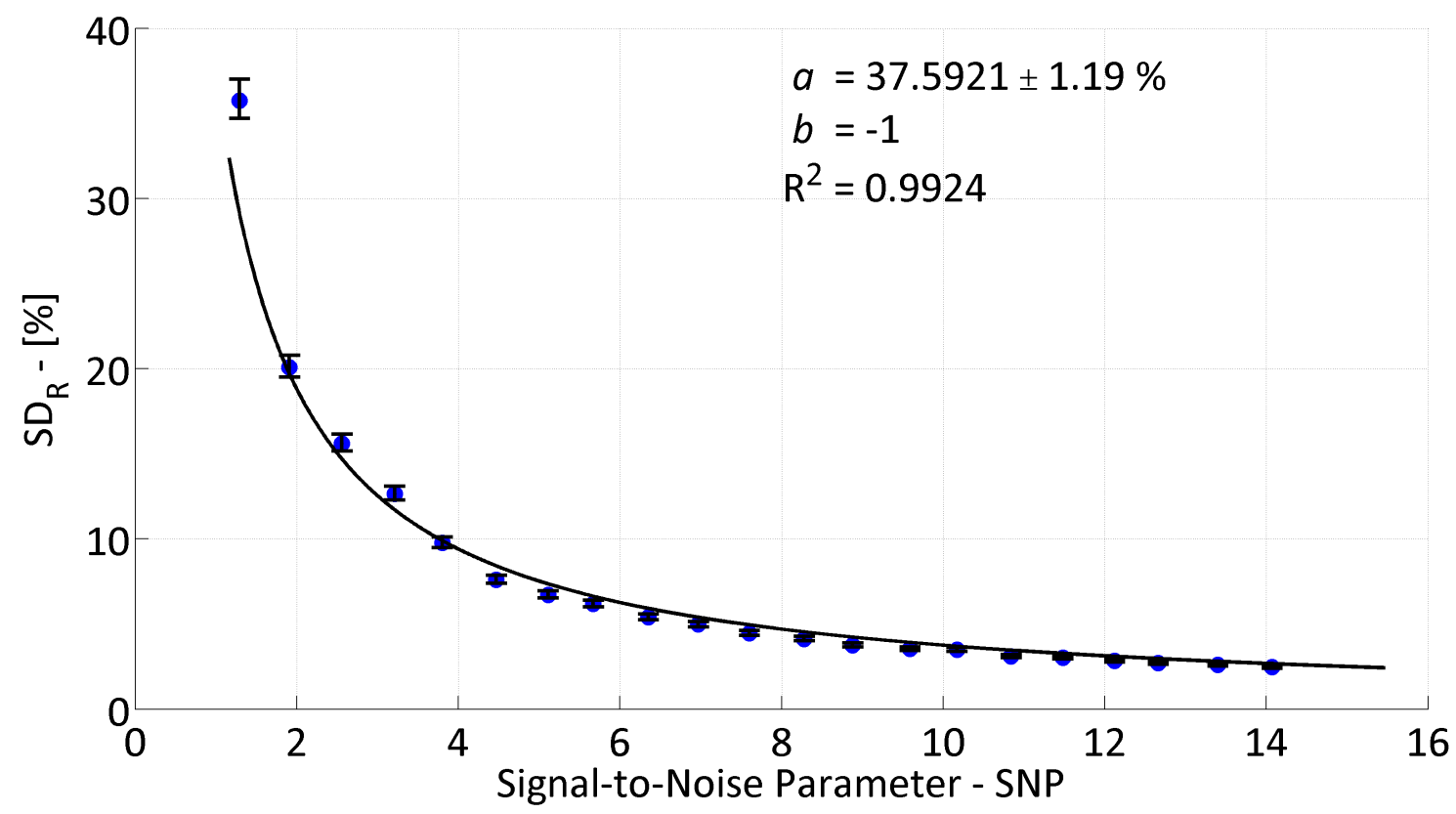

Figure S.133: Relative population standard deviation as a function of signal-to-noise parameter for 100 $\mathrm{nm}$ of $\mathrm{Al} / \mathrm{AlN}$ with $h_{\mathrm{BD}}=359.00 \mathrm{MW} / \mathrm{m}^{2} \mathrm{~K}$. Error bars represent a $95 \%$ confidence interval of the measured $\mathrm{SD}_{\mathrm{R}}$ and the solid line represents a best fit of a power law to the data, of the form $\mathrm{SD}_{\mathrm{R}}=a \times \mathrm{SNP}^{b}$, with parameter $a$ provided for a fixed $b=-1$, along with the $95 \%$ confidence level in $a$. 


\section{S.45 Simulation Summary - Al/AlN 464.00 MW Series}

\section{S.45.1 Fitting Film-to-Substrate Boundary Conductance - $h_{\mathrm{BD}}$}

Table S.73: Summary of simulation data - $100 \mathrm{~nm} \mathrm{Al/AlN} \mathrm{464.00} \mathrm{MW} \mathrm{Series.} \mathrm{Error} \mathrm{bounds} \mathrm{represent} \mathrm{a}$ $95 \%$ confidence level.

\begin{tabular}{cccccc}
\hline \multicolumn{6}{c}{$\mathbf{1 0 0} \mathbf{~ n m ~ A l ~ o n ~ A l N , ~} h_{\mathrm{BD}}=\mathbf{4 6 4 . 0 0} \mathbf{M W} / \mathbf{m}^{2} \mathbf{K}$} \\
\hline $\mathbf{S N P}$ & $\mu-\left[\mathbf{M W} / \mathbf{m}^{2} \mathbf{K}\right]$ & $\sigma-\left[\mathbf{M W} / \mathbf{m}^{2} \mathbf{K}\right]$ & $\mathbf{S N P}$ & $\mu-\left[\mathbf{M W} / \mathbf{m}^{2} \mathbf{K}\right]$ & $\sigma-\left[\mathbf{M W} / \mathbf{m}^{2} \mathbf{K}\right]$ \\
1.25 & $519.91 \pm 12.208$ & $196.03 \pm 0.5498$ & 8.03 & $466.06 \pm 1.630$ & $26.26 \pm 0.0731$ \\
1.88 & $494.01 \pm 7.836$ & $126.15 \pm 0.3520$ & 8.54 & $466.57 \pm 1.556$ & $25.07 \pm 0.0699$ \\
2.50 & $479.95 \pm 5.492$ & $88.41 \pm 0.2467$ & 9.33 & $464.28 \pm 1.374$ & $22.14 \pm 0.0617$ \\
3.13 & $473.42 \pm 4.185$ & $67.37 \pm 0.1880$ & 9.90 & $465.06 \pm 1.397$ & $22.51 \pm 0.0627$ \\
3.72 & $473.38 \pm 3.648$ & $58.76 \pm 0.1638$ & 10.57 & $464.94 \pm 1.316$ & $21.20 \pm 0.0590$ \\
4.33 & $470.53 \pm 3.116$ & $50.14 \pm 0.1401$ & 11.02 & $465.21 \pm 1.220$ & $19.65 \pm 0.0547$ \\
4.94 & $467.11 \pm 2.759$ & $44.46 \pm 0.1238$ & 11.65 & $464.33 \pm 1.145$ & $18.45 \pm 0.0514$ \\
5.56 & $468.24 \pm 2.416$ & $38.91 \pm 0.1085$ & 12.28 & $465.38 \pm 1.089$ & $17.53 \pm 0.0489$ \\
6.17 & $466.72 \pm 2.158$ & $34.77 \pm 0.0968$ & 12.93 & $464.72 \pm 1.077$ & $17.36 \pm 0.0483$ \\
6.85 & $467.43 \pm 1.915$ & $30.86 \pm 0.0859$ & 13.53 & $463.88 \pm 0.958$ & $15.44 \pm 0.0430$ \\
7.38 & $465.35 \pm 1.785$ & $28.75 \pm 0.0801$ & & & \\
\hline
\end{tabular}

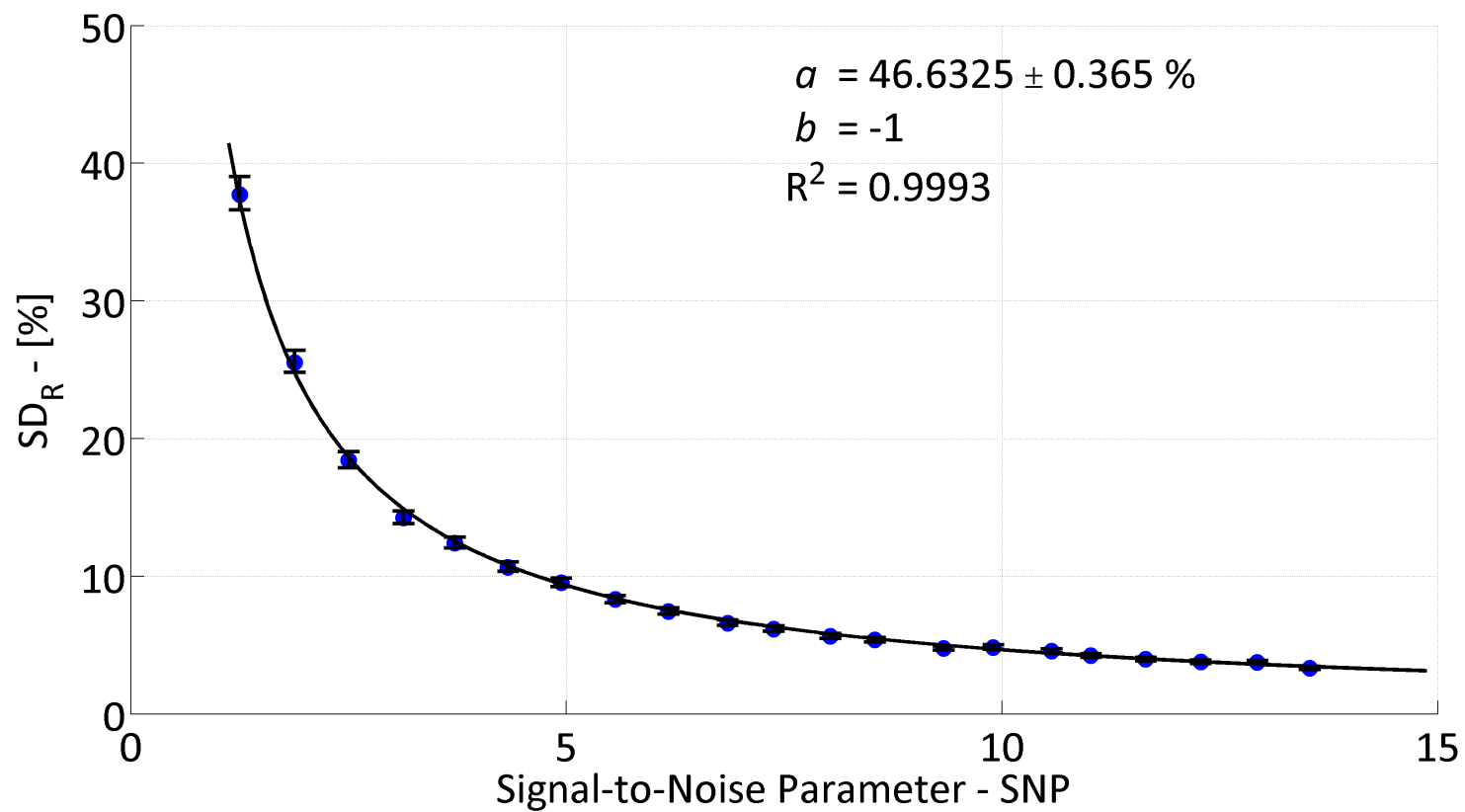

Figure S.134: Relative population standard deviation as a function of signal-to-noise parameter for 100 $\mathrm{nm}$ of $\mathrm{Al} / \mathrm{AlN}$ with $h_{\mathrm{BD}}=464.00 \mathrm{MW} / \mathrm{m}^{2} \mathrm{~K}$. Error bars represent a $95 \%$ confidence interval of the measured $\mathrm{SD}_{\mathrm{R}}$ and the solid line represents a best fit of a power law to the data, of the form $\mathrm{SD}_{\mathrm{R}}=a \times \mathrm{SNP}^{b}$, with parameter $a$ provided for a fixed $b=-1$, along with the $95 \%$ confidence level in $a$. 


\section{S.46 Simulation Summary - Al/AIN 599.00 MW Series}

\section{S.46.1 Fitting Film-to-Substrate Boundary Conductance - $h_{\mathrm{BD}}$}

Table S.74: Summary of simulation data - $100 \mathrm{~nm} \mathrm{Al/AlN} \mathrm{599.00} \mathrm{MW} \mathrm{Series.} \mathrm{Error} \mathrm{bounds} \mathrm{represent} \mathrm{a}$ $95 \%$ confidence level.

\begin{tabular}{cccccc}
\hline \multicolumn{6}{c}{$\mathbf{1 0 0} \mathbf{~ n m ~ A l ~ o n ~ A l N , ~} h_{\mathrm{BD}}=\mathbf{5 9 9 . 0 0} \mathbf{M W} / \mathbf{m}^{2} \mathbf{K}$} \\
\hline $\mathbf{S N P}$ & $\mu-\left[\mathbf{M W} / \mathbf{m}^{2} \mathbf{K}\right]$ & $\sigma-\left[\mathbf{M W} / \mathbf{m}^{2} \mathbf{K}\right]$ & $\mathbf{S N P}$ & $\mu-\left[\mathbf{M W} / \mathbf{m}^{2} \mathbf{K}\right]$ & $\sigma-\left[\mathbf{M W} / \mathbf{m}^{2} \mathbf{K}\right]$ \\
1.18 & $661.90 \pm 16.837$ & $269.69 \pm 0.7602$ & 7.71 & $600.25 \pm 2.500$ & $40.29 \pm 0.1122$ \\
1.80 & $628.45 \pm 10.491$ & $168.72 \pm 0.4718$ & 8.33 & $600.07 \pm 2.135$ & $34.41 \pm 0.0958$ \\
2.39 & $615.55 \pm 7.717$ & $124.17 \pm 0.3469$ & 8.91 & $599.91 \pm 2.097$ & $33.80 \pm 0.0941$ \\
2.98 & $610.87 \pm 6.098$ & $98.22 \pm 0.2738$ & 9.49 & $602.15 \pm 2.010$ & $32.39 \pm 0.0902$ \\
3.57 & $603.87 \pm 5.428$ & $87.47 \pm 0.2436$ & 10.01 & $598.53 \pm 1.819$ & $29.30 \pm 0.0817$ \\
4.17 & $599.56 \pm 4.439$ & $71.53 \pm 0.1992$ & 10.70 & $600.13 \pm 1.722$ & $27.74 \pm 0.0773$ \\
4.75 & $600.83 \pm 3.850$ & $62.01 \pm 0.1729$ & 11.28 & $598.67 \pm 1.623$ & $26.16 \pm 0.0729$ \\
5.34 & $600.84 \pm 3.562$ & $57.41 \pm 0.1599$ & 11.89 & $600.86 \pm 1.546$ & $24.90 \pm 0.0694$ \\
5.92 & $601.09 \pm 3.081$ & $49.65 \pm 0.1383$ & 12.50 & $601.16 \pm 1.468$ & $23.66 \pm 0.0659$ \\
6.55 & $600.68 \pm 2.790$ & $44.96 \pm 0.1252$ & 13.07 & $599.04 \pm 1.384$ & $22.30 \pm 0.0621$ \\
7.15 & $599.53 \pm 2.681$ & $43.20 \pm 0.1203$ & & & \\
\hline
\end{tabular}

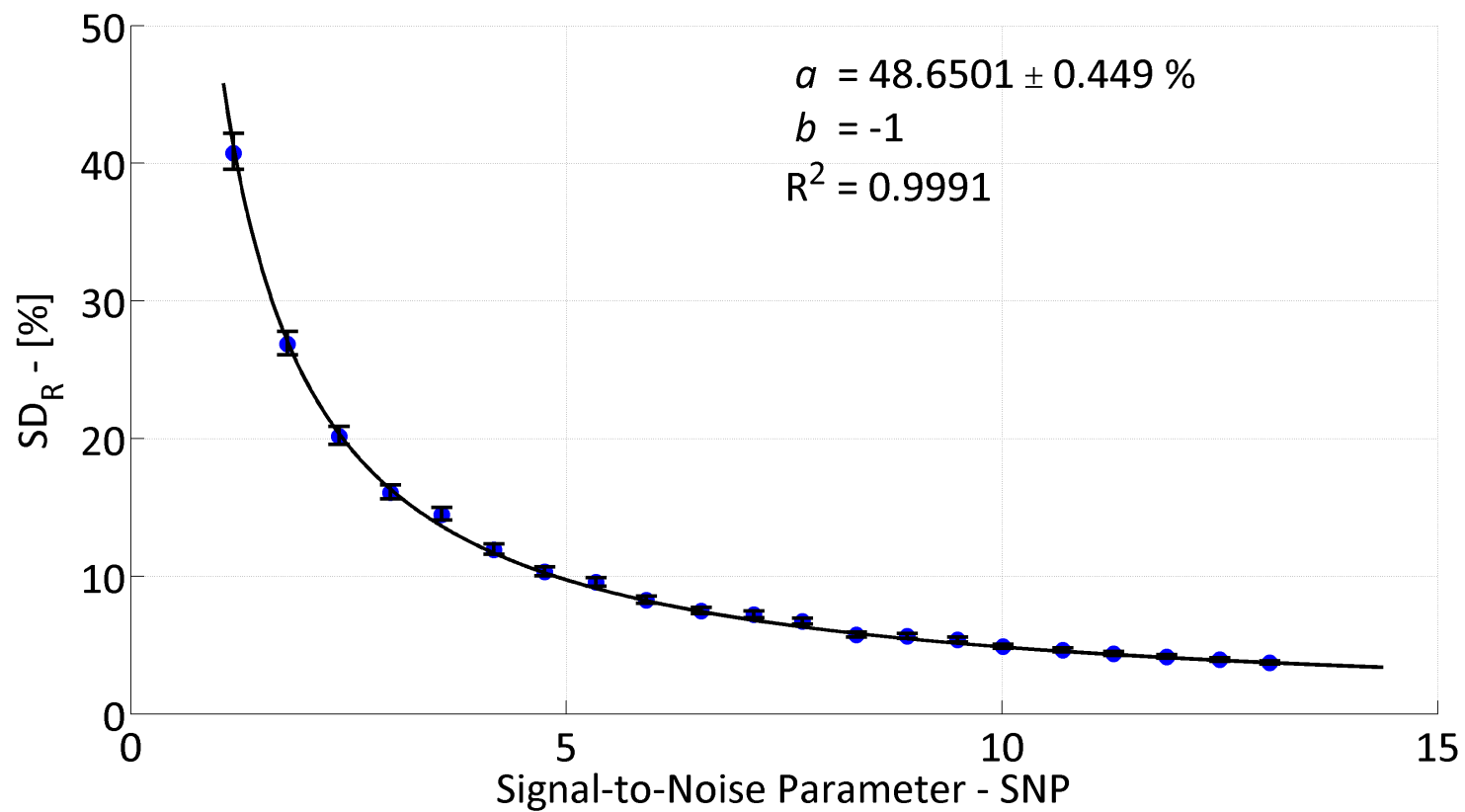

Figure S.135: Relative population standard deviation as a function of signal-to-noise parameter for 100 $\mathrm{nm}$ of $\mathrm{Al} / \mathrm{AlN}$ with $h_{\mathrm{BD}}=599.00 \mathrm{MW} / \mathrm{m}^{2} \mathrm{~K}$. Error bars represent a $95 \%$ confidence interval of the measured $\mathrm{SD}_{\mathrm{R}}$ and the solid line represents a best fit of a power law to the data, of the form $\mathrm{SD}_{\mathrm{R}}=a \times \mathrm{SNP}^{b}$, with parameter $a$ provided for a fixed $b=-1$, along with the $95 \%$ confidence level in $a$. 


\section{S.46.2 Fitting Substrate Thermal Conductivity - $k_{S}$}

Table S.75: Summary of simulation data - $100 \mathrm{~nm} \mathrm{Al/AlN} \mathrm{599.00} \mathrm{MW} \mathrm{Series.} \mathrm{Error} \mathrm{bounds} \mathrm{represent} \mathrm{a}$ $95 \%$ confidence level.

\begin{tabular}{cccccc}
\hline \multicolumn{6}{c}{$\mathbf{1 0 0} \mathbf{~ n m} \mathbf{A l}$ on $\mathbf{A l N}, h_{\mathrm{BD}}=\mathbf{5 9 9 . 0 0} \mathbf{M W} / \mathbf{m}^{2} \mathbf{K}$} \\
\hline $\mathbf{S N P}$ & $\mu-[\mathbf{W} / \mathbf{m K}]$ & $\sigma-[\mathbf{W} / \mathbf{m K}]$ & $\mathbf{S N P}$ & $\mu-[\mathbf{W} / \mathbf{m K}]$ & $\sigma-[\mathbf{W} / \mathbf{m K}]$ \\
1.18 & $302.46 \pm 6.428$ & $103.43 \pm 0.2889$ & 7.71 & $285.42 \pm 0.909$ & $14.64 \pm 0.0408$ \\
1.80 & $288.72 \pm 3.726$ & $59.98 \pm 0.1674$ & 8.33 & $285.35 \pm 0.781$ & $12.59 \pm 0.0351$ \\
2.39 & $288.45 \pm 2.780$ & $44.77 \pm 0.1248$ & 8.91 & $285.07 \pm 0.733$ & $11.82 \pm 0.0329$ \\
2.98 & $286.82 \pm 2.185$ & $35.19 \pm 0.0981$ & 9.49 & $284.50 \pm 0.699$ & $11.26 \pm 0.0314$ \\
3.57 & $287.90 \pm 1.962$ & $31.62 \pm 0.0881$ & 10.01 & $285.73 \pm 0.664$ & $10.69 \pm 0.0298$ \\
4.17 & $287.88 \pm 1.597$ & $25.73 \pm 0.0717$ & 10.70 & $284.82 \pm 0.618$ & $9.95 \pm 0.0277$ \\
4.75 & $286.00 \pm 1.363$ & $21.96 \pm 0.0612$ & 11.28 & $285.37 \pm 0.583$ & $9.40 \pm 0.0262$ \\
5.34 & $285.87 \pm 1.277$ & $20.58 \pm 0.0573$ & 11.89 & $284.51 \pm 0.562$ & $9.06 \pm 0.0252$ \\
5.92 & $285.70 \pm 1.124$ & $18.11 \pm 0.0504$ & 12.50 & $284.52 \pm 0.529$ & $8.52 \pm 0.0237$ \\
6.55 & $285.71 \pm 1.030$ & $16.60 \pm 0.0462$ & 13.07 & $285.18 \pm 0.502$ & $8.09 \pm 0.0225$ \\
7.15 & $286.09 \pm 0.965$ & $15.55 \pm 0.0433$ & & & \\
\hline
\end{tabular}

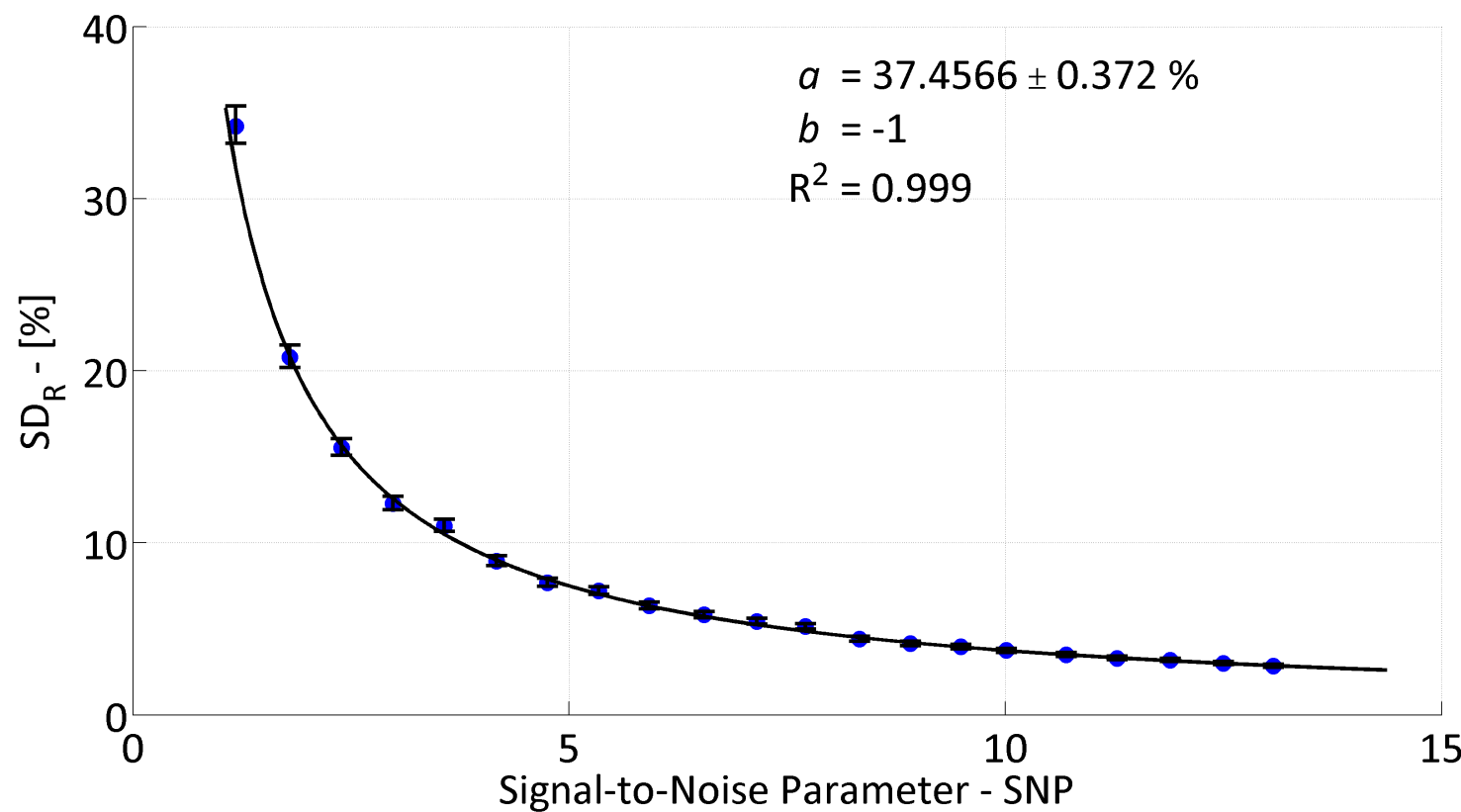

Figure S.136: Relative population standard deviation as a function of signal-to-noise parameter for 100 $\mathrm{nm}$ of $\mathrm{Al} / \mathrm{AlN}$ with $h_{\mathrm{BD}}=599.00 \mathrm{MW} / \mathrm{m}^{2} \mathrm{~K}$. Error bars represent a $95 \%$ confidence interval of the measured $\mathrm{SD}_{\mathrm{R}}$ and the solid line represents a best fit of a power law to the data, of the form $\mathrm{SD}_{\mathrm{R}}=a \times \mathrm{SNP}^{b}$, with parameter $a$ provided for a fixed $b=-1$, along with the $95 \%$ confidence level in $a$. 


\section{S.47 Simulation Summary - Al/AlN 774.00 MW Series}

\section{S.47.1 Fitting Film-to-Substrate Boundary Conductance $-h_{\mathrm{BD}}$}

Table S.76: Summary of simulation data - $100 \mathrm{~nm} \mathrm{Al/AlN} \mathrm{774.00} \mathrm{MW} \mathrm{Series.} \mathrm{Error} \mathrm{bounds} \mathrm{represent} \mathrm{a}$ $95 \%$ confidence level.

\begin{tabular}{cccccc}
\hline \multicolumn{6}{c}{$\mathbf{1 0 0} \mathbf{~ n m ~ A l ~ o n ~ A l N , ~} h_{\mathrm{BD}}=\mathbf{7 7 4 . 0 0} \mathbf{M W} / \mathbf{m}^{2} \mathbf{K}$} \\
\hline $\mathbf{S N P}$ & $\mu-\left[\mathbf{M W} / \mathbf{m}^{2} \mathbf{K}\right]$ & $\sigma-\left[\mathbf{M W} / \mathbf{m}^{2} \mathbf{K}\right]$ & $\mathbf{S N P}$ & $\mu-\left[\mathbf{M W} / \mathbf{m}^{2} \mathbf{K}\right]$ & $\sigma-\left[\mathbf{M W} / \mathbf{m}^{2} \mathbf{K}\right]$ \\
1.24 & $859.10 \pm 22.412$ & $359.17 \pm 1.0114$ & 8.11 & $774.70 \pm 2.956$ & $47.62 \pm 0.1327$ \\
1.86 & $800.06 \pm 13.355$ & $214.78 \pm 0.6006$ & 8.44 & $777.08 \pm 2.896$ & $46.68 \pm 0.1300$ \\
2.46 & $777.46 \pm 9.948$ & $160.15 \pm 0.4469$ & 9.26 & $775.76 \pm 2.678$ & $43.16 \pm 0.1202$ \\
3.09 & $773.65 \pm 7.838$ & $126.24 \pm 0.3519$ & 9.91 & $774.89 \pm 2.520$ & $40.61 \pm 0.1131$ \\
3.71 & $775.52 \pm 6.912$ & $111.27 \pm 0.3105$ & 10.46 & $774.13 \pm 2.354$ & $37.93 \pm 0.1056$ \\
4.26 & $785.03 \pm 5.917$ & $95.36 \pm 0.2656$ & 10.95 & $773.86 \pm 2.227$ & $35.89 \pm 0.0999$ \\
4.90 & $778.97 \pm 5.131$ & $82.69 \pm 0.2303$ & 11.57 & $774.87 \pm 2.086$ & $33.61 \pm 0.0936$ \\
5.55 & $776.80 \pm 4.509$ & $72.66 \pm 0.2023$ & 12.43 & $776.86 \pm 2.038$ & $32.84 \pm 0.0914$ \\
6.13 & $781.95 \pm 3.984$ & $64.20 \pm 0.1788$ & 12.70 & $774.07 \pm 1.906$ & $30.70 \pm 0.0856$ \\
6.79 & $778.18 \pm 3.583$ & $57.73 \pm 0.1608$ & 13.58 & $775.26 \pm 1.793$ & $28.88 \pm 0.0805$ \\
7.32 & $775.05 \pm 3.443$ & $55.49 \pm 0.1545$ & & & \\
\hline
\end{tabular}

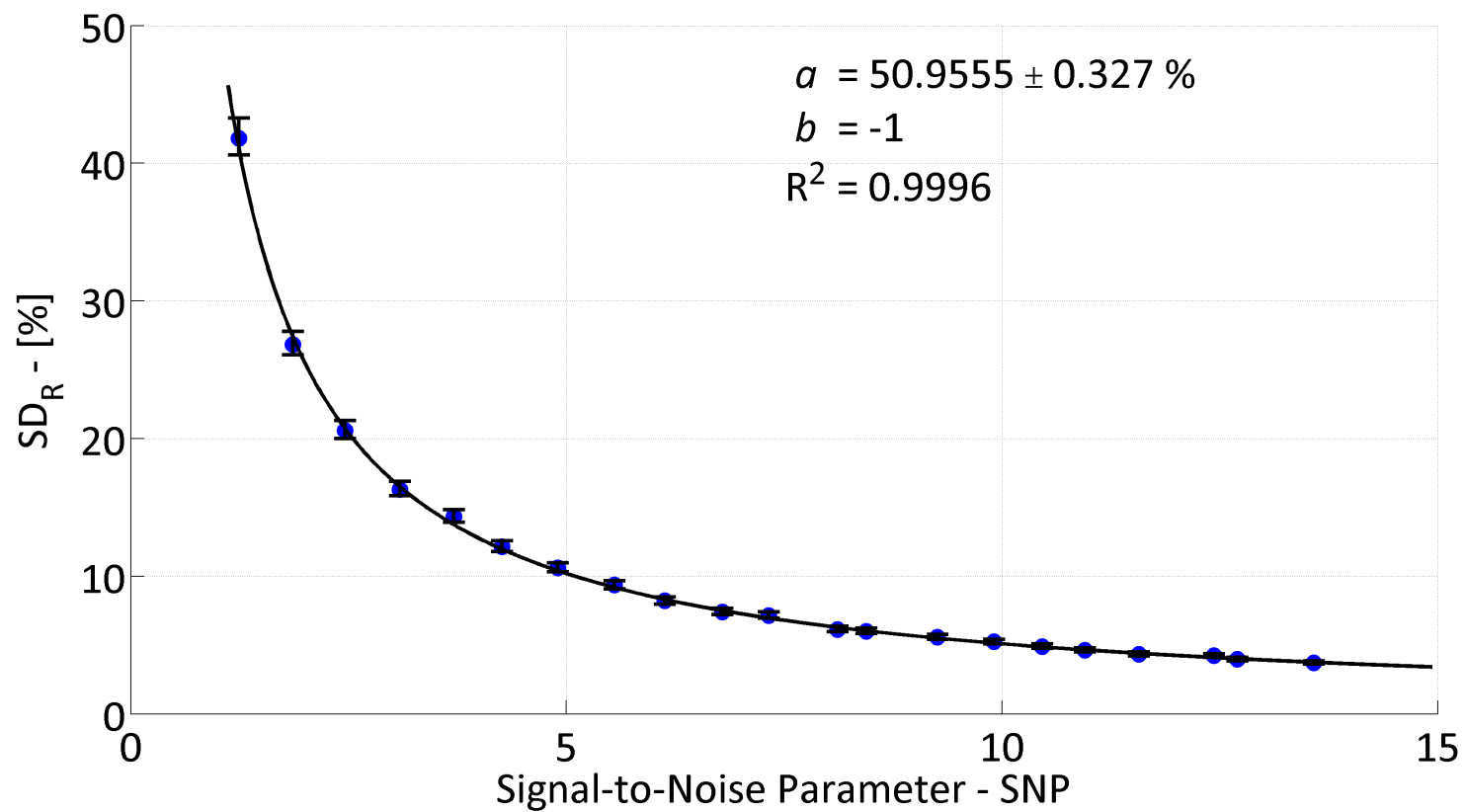

Figure S.137: Relative population standard deviation as a function of signal-to-noise parameter for 100 $\mathrm{nm}$ of $\mathrm{Al} / \mathrm{AlN}$ with $h_{\mathrm{BD}}=774.00 \mathrm{MW} / \mathrm{m}^{2} \mathrm{~K}$. Error bars represent a $95 \%$ confidence interval of the measured $\mathrm{SD}_{\mathrm{R}}$ and the solid line represents a best fit of a power law to the data, of the form $\mathrm{SD}_{\mathrm{R}}=a \times \mathrm{SNP}^{b}$, with parameter $a$ provided for a fixed $b=-1$, along with the $95 \%$ confidence level in $a$. 


\section{S.48 Simulation Summary - Al/AIN 1000.00 MW Series}

\section{S.48.1 Fitting Film-to-Substrate Boundary Conductance - $h_{\mathrm{BD}}$}

Table S.77: Summary of simulation data - $100 \mathrm{~nm} \mathrm{Al/AlN} \mathrm{1000.00} \mathrm{MW} \mathrm{Series.} \mathrm{Error} \mathrm{bounds} \mathrm{represent} \mathrm{a}$ $95 \%$ confidence level.

\begin{tabular}{cccccc}
\hline \multicolumn{6}{c}{$\mathbf{1 0 0} \mathbf{~ n m ~ A l ~ o n ~ A l N , ~} h_{\mathrm{BD}}=\mathbf{1 0 0 0 . 0 0} \mathbf{M W} / \mathbf{m}^{2} \mathbf{K}$} \\
\hline $\mathbf{S N P}$ & $\mu-\left[\mathbf{M W} / \mathbf{m}^{2} \mathbf{K}\right]$ & $\sigma-\left[\mathbf{M W} / \mathbf{m}^{2} \mathbf{K}\right]$ & $\mathbf{S N P}$ & $\mu-\left[\mathbf{M W} / \mathbf{m}^{2} \mathbf{K}\right]$ & $\sigma-\left[\mathbf{M W} / \mathbf{m}^{2} \mathbf{K}\right]$ \\
1.21 & $1098.71 \pm 29.548$ & $473.05 \pm 1.3348$ & 7.89 & $1002.52 \pm 4.130$ & $66.49 \pm 0.1855$ \\
1.82 & $1029.32 \pm 19.196$ & $308.10 \pm 0.8650$ & 8.51 & $1003.91 \pm 4.063$ & $65.40 \pm 0.1825$ \\
2.44 & $1015.25 \pm 13.598$ & $218.58 \pm 0.6118$ & 9.14 & $1002.40 \pm 3.686$ & $59.27 \pm 0.1657$ \\
3.04 & $1009.09 \pm 11.551$ & $185.95 \pm 0.5189$ & 9.72 & $1001.84 \pm 3.394$ & $54.64 \pm 0.1525$ \\
3.63 & $1007.24 \pm 9.078$ & $146.15 \pm 0.4078$ & 10.36 & $1003.32 \pm 3.194$ & $51.41 \pm 0.1435$ \\
4.23 & $1005.61 \pm 8.131$ & $130.90 \pm 0.3653$ & 10.97 & $1002.49 \pm 2.897$ & $46.57 \pm 0.1304$ \\
4.88 & $1004.25 \pm 6.993$ & $112.58 \pm 0.3142$ & 11.53 & $1003.11 \pm 3.016$ & $48.53 \pm 0.1356$ \\
5.45 & $1003.69 \pm 6.162$ & $99.20 \pm 0.2768$ & 12.16 & $999.97 \pm 2.791$ & $44.97 \pm 0.1252$ \\
6.09 & $999.60 \pm 5.702$ & $91.80 \pm 0.2562$ & 12.71 & $1003.61 \pm 2.580$ & $41.51 \pm 0.1159$ \\
6.68 & $999.87 \pm 4.979$ & $80.16 \pm 0.2237$ & 13.35 & $1002.23 \pm 2.541$ & $40.87 \pm 0.1143$ \\
7.30 & $1006.87 \pm 4.702$ & $75.78 \pm 0.2110$ & & & \\
\hline
\end{tabular}

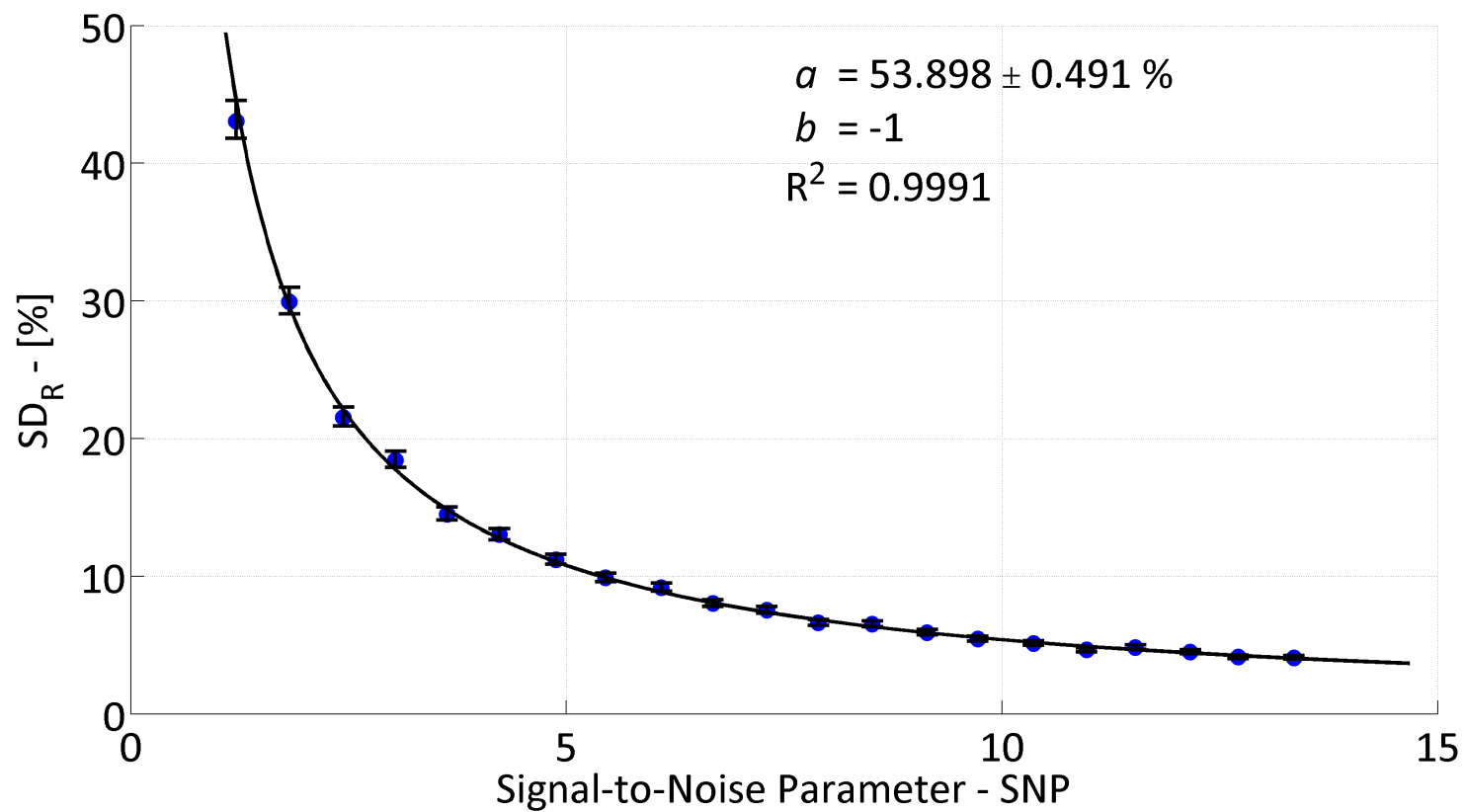

Figure S.138: Relative population standard deviation as a function of signal-to-noise parameter for $100 \mathrm{~nm}$ of $\mathrm{Al} / \mathrm{AlN}$ with $h_{\mathrm{BD}}=1000.00 \mathrm{MW} / \mathrm{m}^{2} \mathrm{~K}$. Error bars represent a $95 \%$ confidence interval of the measured $\mathrm{SD}_{\mathrm{R}}$ and the solid line represents a best fit of a power law to the data, of the form $\mathrm{SD}_{\mathrm{R}}=a \times \mathrm{SNP}^{b}$, with parameter $a$ provided for a fixed $b=-1$, along with the $95 \%$ confidence level in $a$. 


\section{S.48.2 Fitting Substrate Thermal Conductivity $-k_{S}$}

Table S.78: Summary of simulation data - $100 \mathrm{~nm} \mathrm{Al/AlN} \mathrm{1000.00} \mathrm{MW} \mathrm{Series.} \mathrm{Error} \mathrm{bounds} \mathrm{represent} \mathrm{a}$ 95\% confidence level.

\begin{tabular}{cccccc}
\hline \multicolumn{6}{c}{$\mathbf{1 0 0} \mathbf{~ n m} \mathbf{A l}$ on $\mathbf{A l N}, h_{\mathrm{BD}}=\mathbf{1 0 0 0 . 0 0} \mathbf{~ M W} / \mathbf{m}^{2} \mathbf{K}$} \\
\hline $\mathbf{S N P}$ & $\mu-[\mathbf{W} / \mathbf{m K}]$ & $\sigma-[\mathbf{W} / \mathbf{m K}]$ & $\mathbf{S N P}$ & $\mu-[\mathbf{W} / \mathbf{m K}]$ & $\sigma-[\mathbf{W} / \mathbf{m K}]$ \\
1.21 & $295.81 \pm 6.001$ & $96.42 \pm 0.2701$ & 7.89 & $285.23 \pm 0.895$ & $14.41 \pm 0.0401$ \\
1.82 & $293.80 \pm 4.096$ & $66.01 \pm 0.1838$ & 8.51 & $284.86 \pm 0.899$ & $14.48 \pm 0.0403$ \\
2.44 & $289.55 \pm 2.967$ & $47.79 \pm 0.1332$ & 9.14 & $284.59 \pm 0.805$ & $12.97 \pm 0.0361$ \\
3.04 & $289.45 \pm 2.504$ & $40.35 \pm 0.1124$ & 9.72 & $284.96 \pm 0.747$ & $12.04 \pm 0.0335$ \\
3.63 & $287.50 \pm 2.011$ & $32.41 \pm 0.0903$ & 10.36 & $284.59 \pm 0.701$ & $11.29 \pm 0.0314$ \\
4.23 & $286.85 \pm 1.798$ & $28.97 \pm 0.0807$ & 10.97 & $284.67 \pm 0.632$ & $10.18 \pm 0.0283$ \\
4.88 & $286.20 \pm 1.498$ & $24.13 \pm 0.0673$ & 11.53 & $284.79 \pm 0.663$ & $10.68 \pm 0.0297$ \\
5.45 & $285.82 \pm 1.377$ & $22.19 \pm 0.0618$ & 12.16 & $285.08 \pm 0.614$ & $9.89 \pm 0.0275$ \\
6.09 & $286.38 \pm 1.248$ & $20.10 \pm 0.0560$ & 12.71 & $284.36 \pm 0.568$ & $9.16 \pm 0.0255$ \\
6.68 & $286.03 \pm 1.110$ & $17.89 \pm 0.0498$ & 13.35 & $284.56 \pm 0.565$ & $9.11 \pm 0.0254$ \\
7.30 & $284.43 \pm 1.023$ & $16.49 \pm 0.0459$ & & & \\
\hline
\end{tabular}

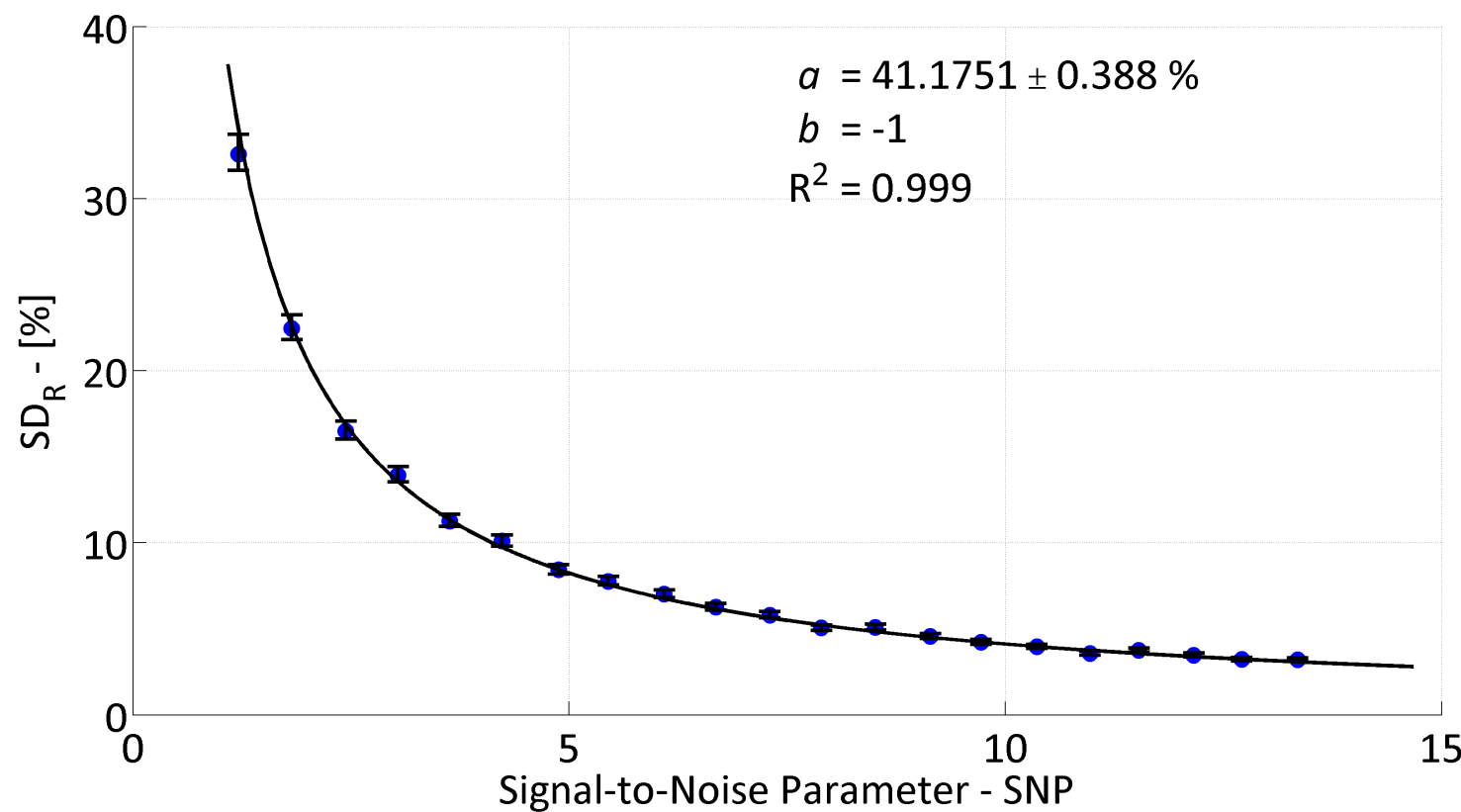

Figure S.139: Relative population standard deviation as a function of signal-to-noise parameter for $100 \mathrm{~nm}$ of $\mathrm{Al} / \mathrm{AlN}$ with $h_{\mathrm{BD}}=1000.00 \mathrm{MW} / \mathrm{m}^{2} \mathrm{~K}$. Error bars represent a $95 \%$ confidence interval of the measured $\mathrm{SD}_{\mathrm{R}}$ and the solid line represents a best fit of a power law to the data, of the form $\mathrm{SD}_{\mathrm{R}}=a \times \mathrm{SNP}^{b}$, with parameter $a$ provided for a fixed $b=-1$, along with the $95 \%$ confidence level in $a$. 


\section{S.49 Simulation Summary - Al/Ge 1.00 MW Series}

\section{S.49.1 Fitting Film-to-Substrate Boundary Conductance - $h_{\mathrm{BD}}$}

Table S.79: Summary of simulation data - $100 \mathrm{~nm} \mathrm{Al/Ge} 1.00 \mathrm{MW}$ Series. Error bounds represent a $95 \%$ confidence level.

\begin{tabular}{cccccc}
\hline \multicolumn{5}{c}{$\mathbf{1 0 0} \mathbf{~ n m ~ A l ~ o n ~ G e , ~} h_{\mathrm{BD}}=\mathbf{1 . 0 0} \mathbf{M W} / \mathbf{m}^{2} \mathbf{K}$} \\
\hline $\mathbf{S N P}$ & $\mu-\left[\mathbf{M W} / \mathbf{m}^{2} \mathbf{K}\right]$ & $\sigma-\left[\mathbf{M W} / \mathbf{m}^{2} \mathbf{K}\right]$ & $\mathbf{S N P}$ & $\mu-\left[\mathbf{M W} / \mathbf{m}^{2} \mathbf{K}\right]$ & $\sigma-\left[\mathbf{M W} / \mathbf{m}^{2} \mathbf{K}\right]$ \\
10.61 & $1.09 \pm 0.056$ & $0.91 \pm 0.0025$ & 68.15 & $1.01 \pm 0.010$ & $0.16 \pm 0.0004$ \\
15.68 & $1.02 \pm 0.040$ & $0.64 \pm 0.0018$ & 72.38 & $1.00 \pm 0.009$ & $0.15 \pm 0.0004$ \\
20.81 & $1.01 \pm 0.031$ & $0.49 \pm 0.0014$ & 75.62 & $1.02 \pm 0.009$ & $0.14 \pm 0.0004$ \\
26.22 & $1.02 \pm 0.025$ & $0.41 \pm 0.0011$ & 81.25 & $1.01 \pm 0.008$ & $0.14 \pm 0.0004$ \\
31.05 & $1.01 \pm 0.022$ & $0.36 \pm 0.0010$ & 87.53 & $1.01 \pm 0.008$ & $0.12 \pm 0.0003$ \\
35.79 & $1.01 \pm 0.019$ & $0.31 \pm 0.0009$ & 93.37 & $1.01 \pm 0.007$ & $0.11 \pm 0.0003$ \\
42.27 & $1.00 \pm 0.016$ & $0.26 \pm 0.0007$ & 95.28 & $1.00 \pm 0.007$ & $0.11 \pm 0.0003$ \\
46.74 & $1.00 \pm 0.014$ & $0.22 \pm 0.0006$ & 104.03 & $1.01 \pm 0.006$ & $0.10 \pm 0.0003$ \\
51.54 & $1.00 \pm 0.013$ & $0.21 \pm 0.0006$ & 105.68 & $1.01 \pm 0.006$ & $0.10 \pm 0.0003$ \\
57.60 & $1.01 \pm 0.012$ & $0.19 \pm 0.0005$ & 114.56 & $1.01 \pm 0.006$ & $0.10 \pm 0.0003$ \\
62.27 & $1.01 \pm 0.011$ & $0.17 \pm 0.0005$ & & & \\
\hline
\end{tabular}

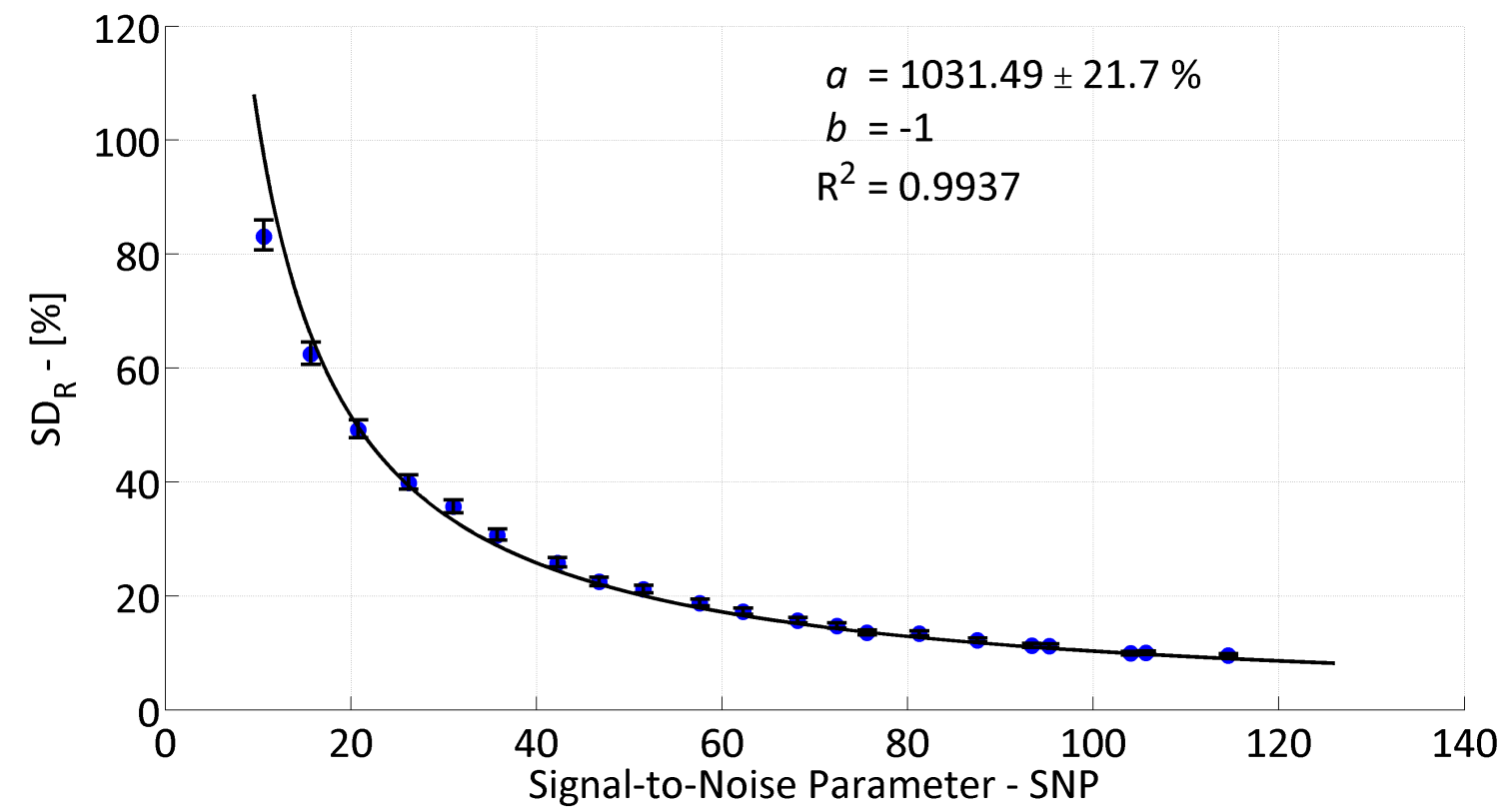

Figure S.140: Relative population standard deviation as a function of signal-to-noise parameter for 100 $\mathrm{nm}$ of $\mathrm{Al} / \mathrm{Ge}$ with $h_{\mathrm{BD}}=1.00 \mathrm{MW} / \mathrm{m}^{2} \mathrm{~K}$. Error bars represent a $95 \%$ confidence interval of the measured $\mathrm{SD}_{\mathrm{R}}$ and the solid line represents a best fit of a power law to the data, of the form $\mathrm{SD}_{\mathrm{R}}=a \times \mathrm{SNP}^{b}$, with parameter $a$ provided for a fixed $b=-1$, along with the $95 \%$ confidence level in $a$. 


\section{S.50 Simulation Summary - Al/Ge 2.15 MW Series}

\section{S.50.1 Fitting Film-to-Substrate Boundary Conductance - $h_{\mathrm{BD}}$}

Table S.80: Summary of simulation data - $100 \mathrm{~nm} \mathrm{Al/Ge} \mathrm{2.15} \mathrm{MW} \mathrm{Series.} \mathrm{Error} \mathrm{bounds} \mathrm{represent} \mathrm{a} 95 \%$ confidence level.

\begin{tabular}{cccccc}
\hline \multicolumn{5}{c}{$\mathbf{1 0 0} \mathbf{~ n m ~ A l ~ o n ~ G e , ~} h_{\mathrm{BD}}=\mathbf{2 . 1 5} \mathbf{M W} / \mathbf{m}^{2} \mathbf{K}$} \\
\hline $\mathbf{S N P}$ & $\mu-\left[\mathbf{M W} / \mathbf{m}^{2} \mathbf{K}\right]$ & $\sigma-\left[\mathbf{M W} / \mathbf{m}^{2} \mathbf{K}\right]$ & $\mathbf{S N P}$ & $\mu-\left[\mathbf{M W} / \mathbf{m}^{2} \mathbf{K}\right]$ & $\sigma-\left[\mathbf{M W} / \mathbf{m}^{2} \mathbf{K}\right]$ \\
10.26 & $2.19 \pm 0.064$ & $1.03 \pm 0.0029$ & 65.76 & $2.16 \pm 0.011$ & $0.17 \pm 0.0005$ \\
15.39 & $2.15 \pm 0.043$ & $0.70 \pm 0.0019$ & 72.64 & $2.16 \pm 0.009$ & $0.15 \pm 0.0004$ \\
20.55 & $2.17 \pm 0.034$ & $0.55 \pm 0.0015$ & 76.64 & $2.16 \pm 0.009$ & $0.14 \pm 0.0004$ \\
25.48 & $2.13 \pm 0.026$ & $0.42 \pm 0.0012$ & 81.15 & $2.16 \pm 0.009$ & $0.14 \pm 0.0004$ \\
31.31 & $2.17 \pm 0.022$ & $0.36 \pm 0.0010$ & 86.47 & $2.16 \pm 0.008$ & $0.13 \pm 0.0004$ \\
35.63 & $2.14 \pm 0.020$ & $0.32 \pm 0.0009$ & 89.39 & $2.16 \pm 0.008$ & $0.12 \pm 0.0003$ \\
40.44 & $2.15 \pm 0.017$ & $0.27 \pm 0.0007$ & 95.85 & $2.16 \pm 0.007$ & $0.11 \pm 0.0003$ \\
45.98 & $2.16 \pm 0.015$ & $0.24 \pm 0.0007$ & 105.19 & $2.15 \pm 0.006$ & $0.10 \pm 0.0003$ \\
50.89 & $2.18 \pm 0.013$ & $0.21 \pm 0.0006$ & 107.10 & $2.16 \pm 0.007$ & $0.11 \pm 0.0003$ \\
55.63 & $2.16 \pm 0.012$ & $0.20 \pm 0.0005$ & 109.88 & $2.15 \pm 0.006$ & $0.10 \pm 0.0003$ \\
61.24 & $2.16 \pm 0.011$ & $0.18 \pm 0.0005$ & & & \\
\hline
\end{tabular}

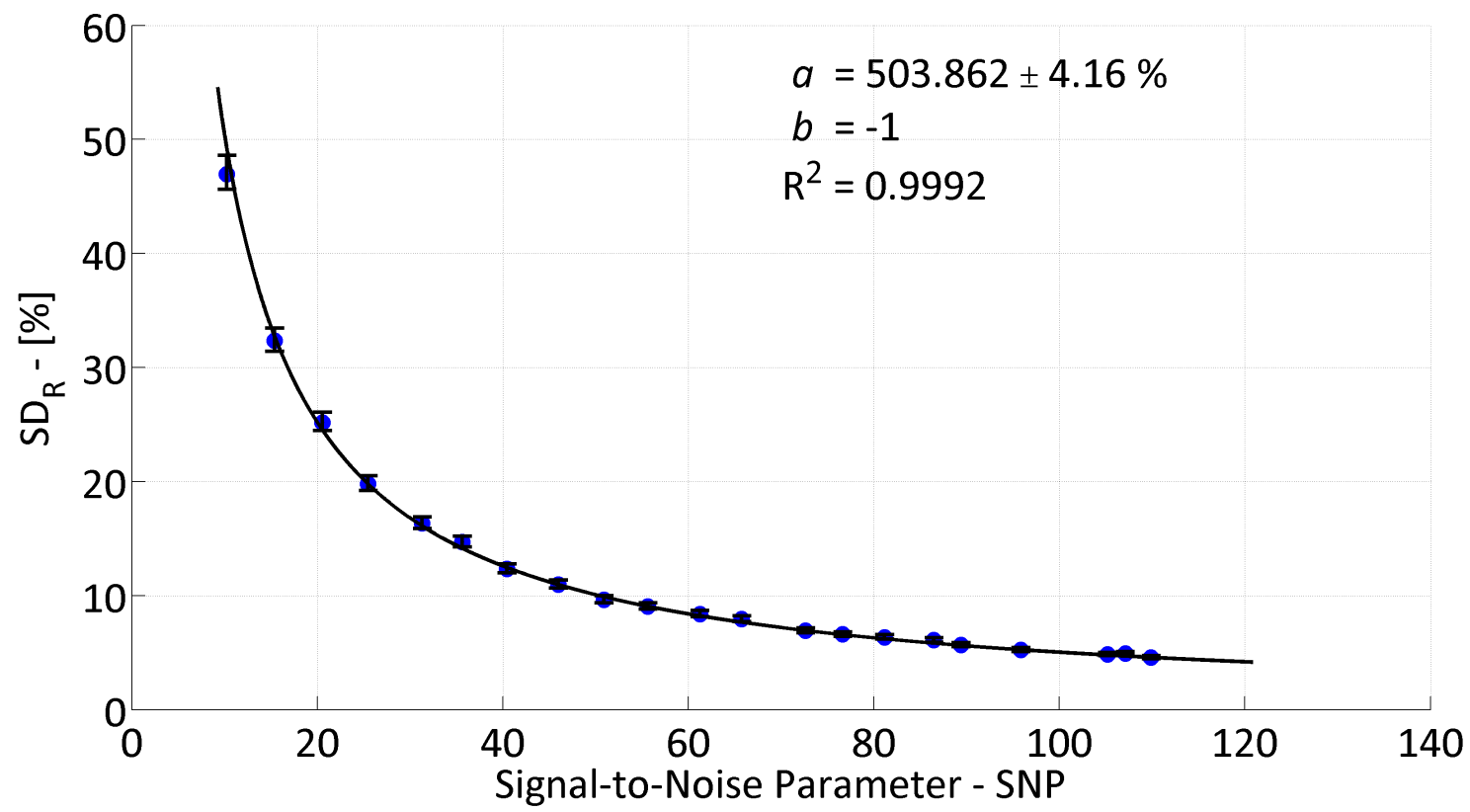

Figure S.141: Relative population standard deviation as a function of signal-to-noise parameter for 100 $\mathrm{nm}$ of $\mathrm{Al} / \mathrm{Ge}$ with $h_{\mathrm{BD}}=2.15 \mathrm{MW} / \mathrm{m}^{2} \mathrm{~K}$. Error bars represent a $95 \%$ confidence interval of the measured $\mathrm{SD}_{\mathrm{R}}$ and the solid line represents a best fit of a power law to the data, of the form $\mathrm{SD}_{\mathrm{R}}=a \times \mathrm{SNP}^{b}$, with parameter $a$ provided for a fixed $b=-1$, along with the $95 \%$ confidence level in $a$. 


\section{S.51 Simulation Summary - Al/Ge 3.59 MW Series}

\section{S.51.1 Fitting Film-to-Substrate Boundary Conductance - $h_{\mathrm{BD}}$}

Table S.81: Summary of simulation data - $100 \mathrm{~nm} \mathrm{Al/Ge} \mathrm{3.59} \mathrm{MW} \mathrm{Series.} \mathrm{Error} \mathrm{bounds} \mathrm{represent} \mathrm{a} 95 \%$ confidence level.

\begin{tabular}{cccccc}
\hline \multicolumn{5}{c}{$\mathbf{1 0 0} \mathbf{~ n m ~ A l ~ o n ~} \mathbf{G e}, h_{\mathrm{BD}}=\mathbf{3 . 5 9} \mathbf{M W} / \mathbf{m}^{2} \mathbf{K}$} \\
\hline $\mathbf{S N P}$ & $\mu-\left[\mathbf{M W} / \mathbf{m}^{2} \mathbf{K}\right]$ & $\sigma-\left[\mathbf{M W} / \mathbf{m}^{2} \mathbf{K}\right]$ & $\mathbf{S N P}$ & $\mu-\left[\mathbf{M W} / \mathbf{m}^{2} \mathbf{K}\right]$ & $\sigma-\left[\mathbf{M W} / \mathbf{m}^{2} \mathbf{K}\right]$ \\
10.09 & $3.60 \pm 0.070$ & $1.12 \pm 0.0031$ & 64.07 & $3.59 \pm 0.011$ & $0.17 \pm 0.0005$ \\
14.95 & $3.63 \pm 0.047$ & $0.76 \pm 0.0021$ & 70.00 & $3.60 \pm 0.010$ & $0.16 \pm 0.0004$ \\
20.26 & $3.58 \pm 0.034$ & $0.55 \pm 0.0015$ & 73.79 & $3.60 \pm 0.009$ & $0.15 \pm 0.0004$ \\
24.78 & $3.59 \pm 0.029$ & $0.47 \pm 0.0013$ & 80.57 & $3.61 \pm 0.008$ & $0.14 \pm 0.0004$ \\
29.06 & $3.60 \pm 0.024$ & $0.38 \pm 0.0011$ & 84.24 & $3.59 \pm 0.008$ & $0.14 \pm 0.0004$ \\
35.56 & $3.60 \pm 0.020$ & $0.31 \pm 0.0009$ & 89.22 & $3.60 \pm 0.008$ & $0.13 \pm 0.0004$ \\
39.63 & $3.61 \pm 0.018$ & $0.28 \pm 0.0008$ & 93.45 & $3.60 \pm 0.007$ & $0.12 \pm 0.0003$ \\
44.49 & $3.60 \pm 0.016$ & $0.26 \pm 0.0007$ & 97.48 & $3.59 \pm 0.007$ & $0.11 \pm 0.0003$ \\
50.36 & $3.59 \pm 0.014$ & $0.23 \pm 0.0006$ & 102.21 & $3.59 \pm 0.007$ & $0.11 \pm 0.0003$ \\
53.99 & $3.60 \pm 0.013$ & $0.21 \pm 0.0006$ & 108.06 & $3.60 \pm 0.006$ & $0.10 \pm 0.0003$ \\
58.87 & $3.61 \pm 0.012$ & $0.20 \pm 0.0005$ & & & \\
\hline
\end{tabular}

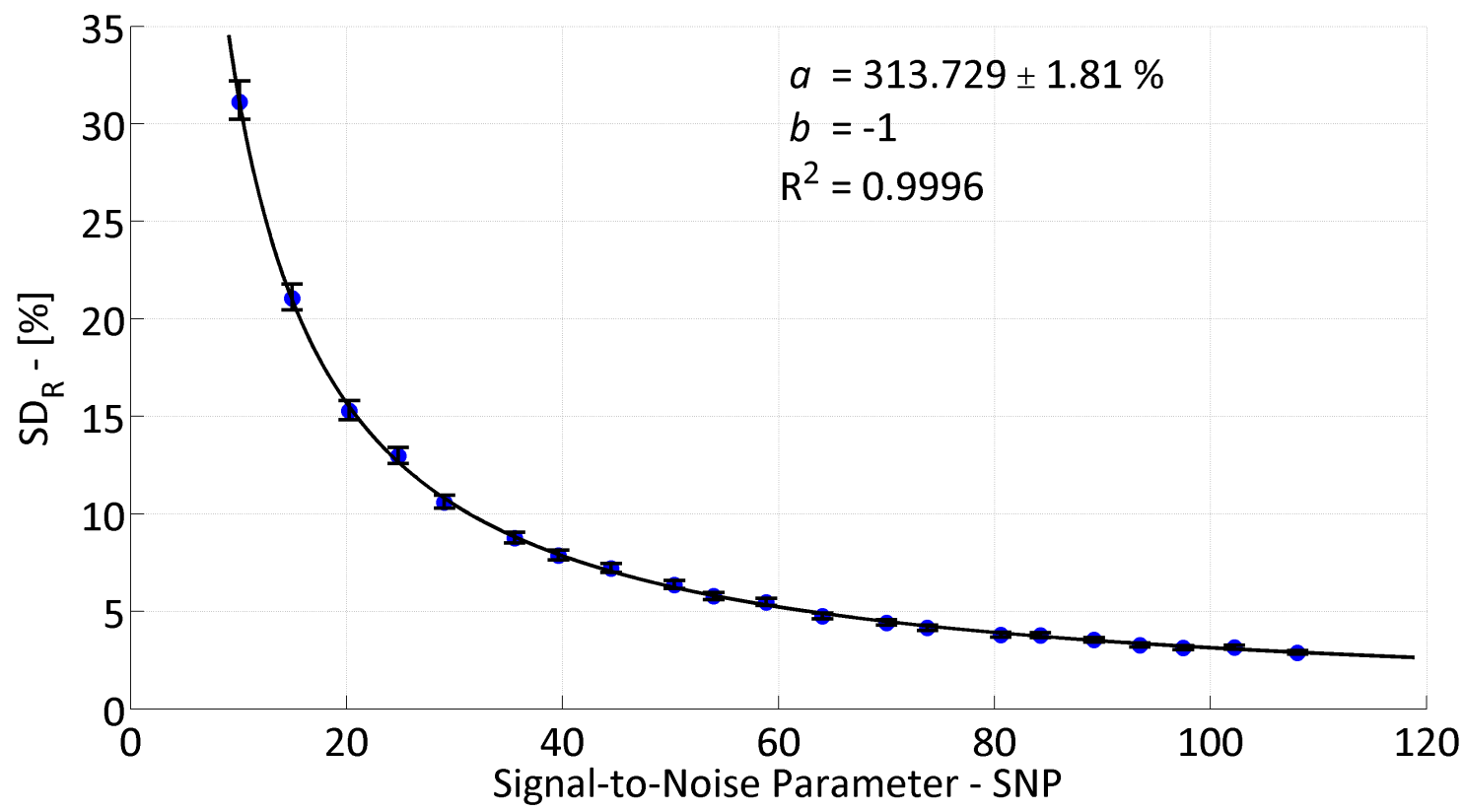

Figure S.142: Relative population standard deviation as a function of signal-to-noise parameter for 100 $\mathrm{nm}$ of $\mathrm{Al} / \mathrm{Ge}$ with $h_{\mathrm{BD}}=3.59 \mathrm{MW} / \mathrm{m}^{2} \mathrm{~K}$. Error bars represent a $95 \%$ confidence interval of the measured $\mathrm{SD}_{\mathrm{R}}$ and the solid line represents a best fit of a power law to the data, of the form $\mathrm{SD}_{\mathrm{R}}=a \times \mathrm{SNP}^{b}$, with parameter $a$ provided for a fixed $b=-1$, along with the $95 \%$ confidence level in $a$. 


\section{S.52 Simulation Summary - Al/Ge 7.74 MW Series}

\section{S.52.1 Fitting Film-to-Substrate Boundary Conductance - $h_{\mathrm{BD}}$}

Table S.82: Summary of simulation data - $100 \mathrm{~nm} \mathrm{Al/Ge} \mathrm{7.74} \mathrm{MW} \mathrm{Series.} \mathrm{Error} \mathrm{bounds} \mathrm{represent} \mathrm{a} 95 \%$ confidence level.

\begin{tabular}{cccccc}
\hline \multicolumn{6}{c}{$\mathbf{1 0 0} \mathbf{~ n m ~ A l ~ o n ~ G e , ~} h_{\mathrm{BD}}=\mathbf{7 . 7 4} \mathbf{M W} / \mathbf{m}^{2} \mathbf{K}$} \\
\hline $\mathbf{S N P}$ & $\mu-\left[\mathbf{M W} / \mathbf{m}^{2} \mathbf{K}\right]$ & $\sigma-\left[\mathbf{M W} / \mathbf{m}^{2} \mathbf{K}\right]$ & $\mathbf{S N P}$ & $\mu-\left[\mathbf{M W} / \mathbf{m}^{2} \mathbf{K}\right]$ & $\sigma-\left[\mathbf{M W} / \mathbf{m}^{2} \mathbf{K}\right]$ \\
9.38 & $7.73 \pm 0.081$ & $1.30 \pm 0.0036$ & 59.88 & $7.74 \pm 0.013$ & $0.21 \pm 0.0006$ \\
14.01 & $7.71 \pm 0.053$ & $0.86 \pm 0.0024$ & 64.07 & $7.76 \pm 0.012$ & $0.19 \pm 0.0005$ \\
18.57 & $7.73 \pm 0.040$ & $0.65 \pm 0.0018$ & 69.49 & $7.74 \pm 0.011$ & $0.17 \pm 0.0005$ \\
23.28 & $7.76 \pm 0.033$ & $0.53 \pm 0.0015$ & 75.15 & $7.75 \pm 0.010$ & $0.16 \pm 0.0004$ \\
27.69 & $7.75 \pm 0.027$ & $0.44 \pm 0.0012$ & 78.14 & $7.75 \pm 0.010$ & $0.15 \pm 0.0004$ \\
33.38 & $7.75 \pm 0.022$ & $0.35 \pm 0.0010$ & 82.40 & $7.75 \pm 0.009$ & $0.15 \pm 0.0004$ \\
37.02 & $7.77 \pm 0.020$ & $0.33 \pm 0.0009$ & 86.77 & $7.75 \pm 0.008$ & $0.13 \pm 0.0004$ \\
43.65 & $7.75 \pm 0.016$ & $0.26 \pm 0.0007$ & 96.91 & $7.75 \pm 0.007$ & $0.12 \pm 0.0003$ \\
46.50 & $7.76 \pm 0.016$ & $0.25 \pm 0.0007$ & 97.74 & $7.75 \pm 0.008$ & $0.13 \pm 0.0003$ \\
50.52 & $7.76 \pm 0.015$ & $0.24 \pm 0.0007$ & 99.00 & $7.76 \pm 0.008$ & $0.12 \pm 0.0003$ \\
55.15 & $7.76 \pm 0.013$ & $0.22 \pm 0.0006$ & & & \\
\hline
\end{tabular}

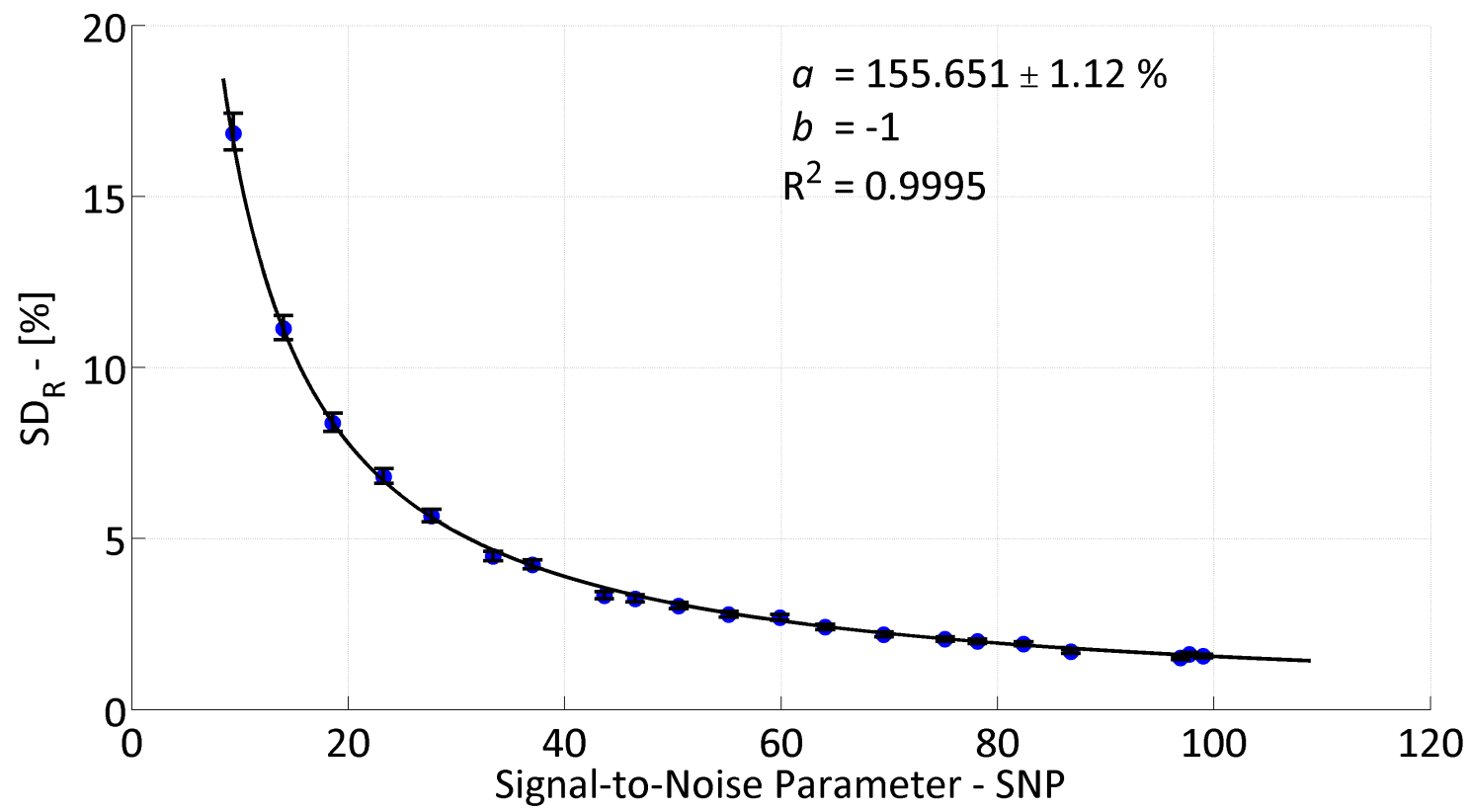

Figure S.143: Relative population standard deviation as a function of signal-to-noise parameter for 100 $\mathrm{nm}$ of $\mathrm{Al} / \mathrm{Ge}$ with $h_{\mathrm{BD}}=7.74 \mathrm{MW} / \mathrm{m}^{2} \mathrm{~K}$. Error bars represent a $95 \%$ confidence interval of the measured $\mathrm{SD}_{\mathrm{R}}$ and the solid line represents a best fit of a power law to the data, of the form $\mathrm{SD}_{\mathrm{R}}=a \times \mathrm{SNP}^{b}$, with parameter $a$ provided for a fixed $b=-1$, along with the $95 \%$ confidence level in $a$. 


\section{S.53 Simulation Summary - Al/Ge 16.70 MW Series}

\section{S.53.1 Fitting Film-to-Substrate Boundary Conductance $-h_{\mathrm{BD}}$}

Table S.83: Summary of simulation data - 100 nm Al/Ge 16.70 MW Series. Error bounds represent a $95 \%$ confidence level.

\begin{tabular}{cccccc}
\hline \multicolumn{6}{c}{$\mathbf{1 0 0} \mathbf{~ n m ~ A l ~ o n ~ G e , ~} h_{\mathrm{BD}}=\mathbf{1 6 . 7 0} \mathbf{~ M W} / \mathbf{m}^{2} \mathbf{K}$} \\
\hline $\mathbf{S N P}$ & $\mu-\left[\mathbf{M W} / \mathbf{m}^{2} \mathbf{K}\right]$ & $\sigma-\left[\mathbf{M W} / \mathbf{m}^{2} \mathbf{K}\right]$ & $\mathbf{S N P}$ & $\mu-\left[\mathbf{M W} / \mathbf{m}^{2} \mathbf{K}\right]$ & $\sigma-\left[\mathbf{M W} / \mathbf{m}^{2} \mathbf{K}\right]$ \\
8.06 & $16.79 \pm 0.106$ & $1.71 \pm 0.0048$ & 51.85 & $16.71 \pm 0.016$ & $0.26 \pm 0.0007$ \\
12.29 & $16.72 \pm 0.069$ & $1.11 \pm 0.0031$ & 56.04 & $16.71 \pm 0.015$ & $0.23 \pm 0.0007$ \\
16.44 & $16.79 \pm 0.052$ & $0.85 \pm 0.0024$ & 61.53 & $16.71 \pm 0.014$ & $0.22 \pm 0.0006$ \\
20.32 & $16.69 \pm 0.042$ & $0.68 \pm 0.0019$ & 63.80 & $16.71 \pm 0.013$ & $0.21 \pm 0.0006$ \\
24.59 & $16.69 \pm 0.037$ & $0.60 \pm 0.0017$ & 68.81 & $16.70 \pm 0.012$ & $0.20 \pm 0.0005$ \\
28.04 & $16.73 \pm 0.031$ & $0.50 \pm 0.0014$ & 71.28 & $16.71 \pm 0.012$ & $0.20 \pm 0.0005$ \\
32.19 & $16.72 \pm 0.026$ & $0.42 \pm 0.0012$ & 75.45 & $16.70 \pm 0.011$ & $0.18 \pm 0.0005$ \\
36.36 & $16.70 \pm 0.024$ & $0.38 \pm 0.0011$ & 81.93 & $16.70 \pm 0.010$ & $0.17 \pm 0.0005$ \\
40.15 & $16.71 \pm 0.022$ & $0.35 \pm 0.0010$ & 85.90 & $16.71 \pm 0.010$ & $0.16 \pm 0.0004$ \\
43.84 & $16.69 \pm 0.019$ & $0.31 \pm 0.0009$ & 87.02 & $16.72 \pm 0.010$ & $0.16 \pm 0.0004$ \\
48.94 & $16.71 \pm 0.017$ & $0.28 \pm 0.0008$ & & & \\
\hline
\end{tabular}

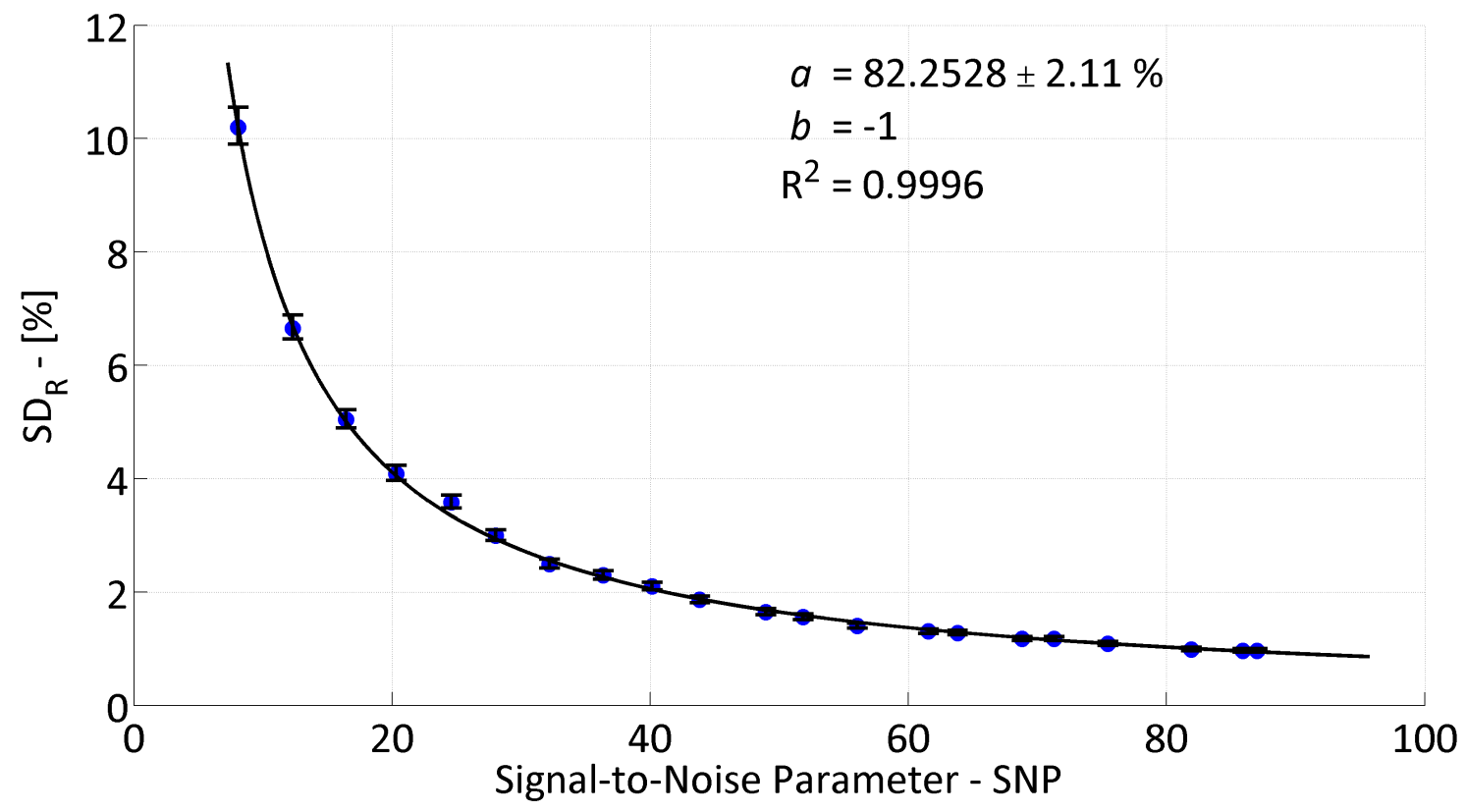

Figure S.144: Relative population standard deviation as a function of signal-to-noise parameter for 100 $\mathrm{nm}$ of $\mathrm{Al} / \mathrm{Ge}$ with $h_{\mathrm{BD}}=16.70 \mathrm{MW} / \mathrm{m}^{2} \mathrm{~K}$. Error bars represent a $95 \%$ confidence interval of the measured $\mathrm{SD}_{\mathrm{R}}$ and the solid line represents a best fit of a power law to the data, of the form $\mathrm{SD}_{\mathrm{R}}=a \times \mathrm{SNP}^{b}$, with parameter $a$ provided for a fixed $b=-1$, along with the $95 \%$ confidence level in $a$. 


\section{S.53.2 Fitting Substrate Thermal Conductivity $-k_{S}$}

Table S.84: Summary of simulation data - $100 \mathrm{~nm} \mathrm{Al/Ge} \mathrm{16.70} \mathrm{MW} \mathrm{Series.} \mathrm{Error} \mathrm{bounds} \mathrm{represent} \mathrm{a} 95 \%$ confidence level.

\begin{tabular}{cccccc}
\hline \multicolumn{6}{c}{$\mathbf{1 0 0} \mathbf{~ n m ~ A l ~ o n ~ G e , ~} h_{\mathrm{BD}}=\mathbf{1 6 . 7 0} \mathbf{~ M W} / \mathbf{m}^{2} \mathbf{K}$} \\
\hline SNP & $\mu-[\mathbf{W} / \mathbf{m K}]$ & $\sigma-[\mathbf{W} / \mathbf{m K}]$ & $\mathbf{S N P}$ & $\mu-[\mathbf{W} / \mathbf{m K}]$ & $\sigma-[\mathbf{W} / \mathbf{m K}]$ \\
8.06 & $89.69 \pm 7.605$ & $109.58 \pm 0.3819$ & 51.85 & $63.83 \pm 1.032$ & $16.57 \pm 0.0465$ \\
12.29 & $86.94 \pm 6.188$ & $90.33 \pm 0.3067$ & 56.04 & $64.05 \pm 0.953$ & $15.33 \pm 0.0429$ \\
16.44 & $84.23 \pm 4.622$ & $67.88 \pm 0.2277$ & 61.53 & $63.81 \pm 0.880$ & $14.17 \pm 0.0395$ \\
20.32 & $72.92 \pm 2.989$ & $47.07 \pm 0.1373$ & 63.80 & $63.22 \pm 0.836$ & $13.46 \pm 0.0375$ \\
24.59 & $70.73 \pm 2.608$ & $41.22 \pm 0.1194$ & 68.81 & $62.19 \pm 0.751$ & $12.08 \pm 0.0338$ \\
28.04 & $71.02 \pm 2.228$ & $35.49 \pm 0.1012$ & 71.28 & $63.26 \pm 0.775$ & $12.50 \pm 0.0348$ \\
32.19 & $69.01 \pm 1.828$ & $29.26 \pm 0.0826$ & 75.45 & $62.35 \pm 0.699$ & $11.25 \pm 0.0314$ \\
36.36 & $66.44 \pm 1.620$ & $25.94 \pm 0.0732$ & 81.93 & $62.29 \pm 0.634$ & $10.20 \pm 0.0285$ \\
40.15 & $66.47 \pm 1.474$ & $23.65 \pm 0.0664$ & 85.90 & $62.59 \pm 0.619$ & $9.97 \pm 0.0278$ \\
43.84 & $64.04 \pm 1.263$ & $20.28 \pm 0.0569$ & 87.02 & $62.91 \pm 0.627$ & $10.08 \pm 0.0282$ \\
48.94 & $64.45 \pm 1.117$ & $17.94 \pm 0.0503$ & & & \\
\hline
\end{tabular}

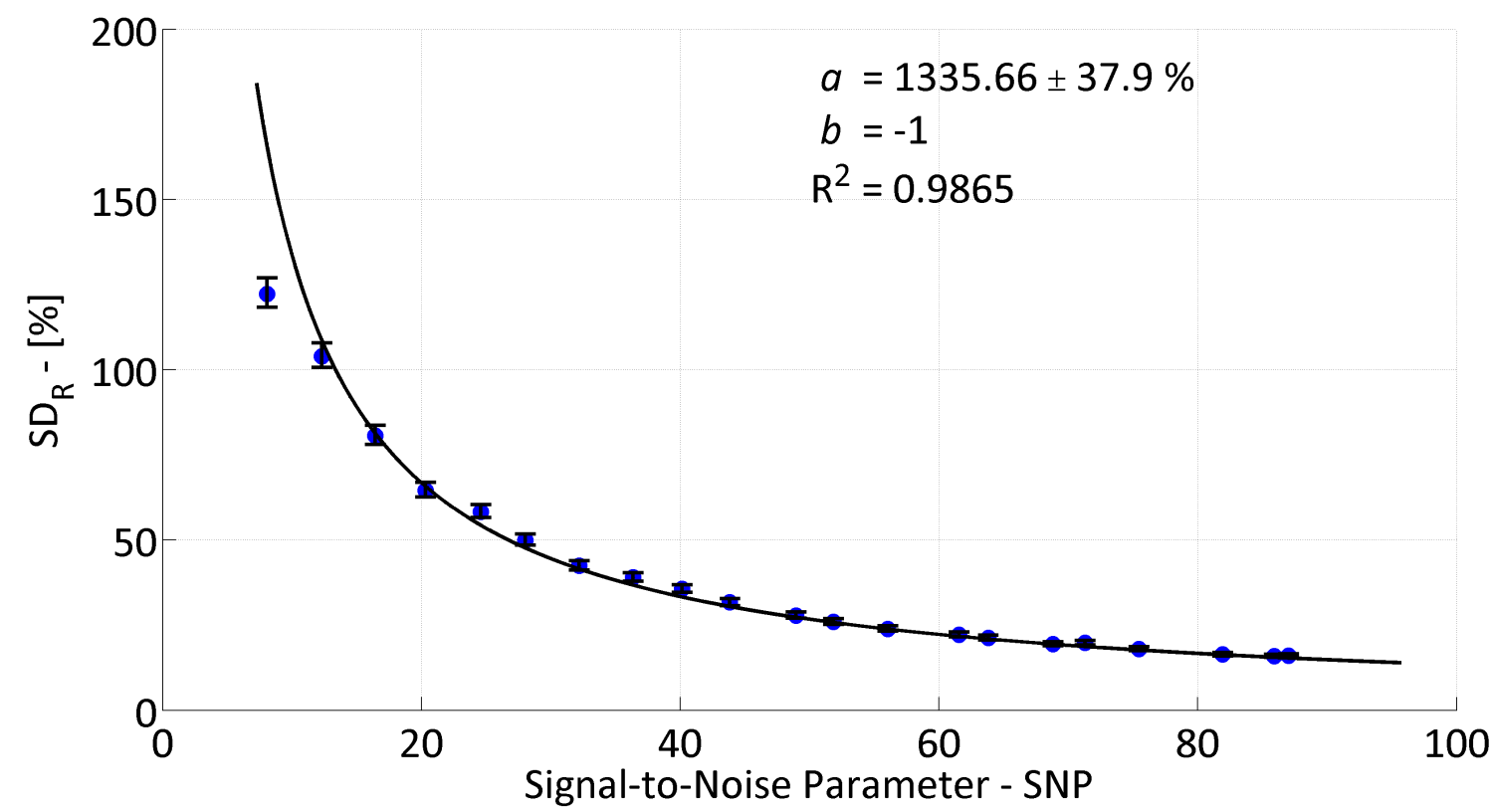

Figure S.145: Relative population standard deviation as a function of signal-to-noise parameter for 100 $\mathrm{nm}$ of $\mathrm{Al} / \mathrm{Ge}$ with $h_{\mathrm{BD}}=16.70 \mathrm{MW} / \mathrm{m}^{2} \mathrm{~K}$. Error bars represent a $95 \%$ confidence interval of the measured $\mathrm{SD}_{\mathrm{R}}$ and the solid line represents a best fit of a power law to the data, of the form $\mathrm{SD}_{\mathrm{R}}=a \times \mathrm{SNP}^{b}$, with parameter $a$ provided for a fixed $b=-1$, along with the $95 \%$ confidence level in $a$. 


\section{S.54 Simulation Summary - Al/Ge 46.40 MW Series}

\section{S.54.1 Fitting Film-to-Substrate Boundary Conductance $-h_{\mathrm{BD}}$}

Table S.85: Summary of simulation data - $100 \mathrm{~nm} \mathrm{Al/Ge} \mathrm{46.40} \mathrm{MW} \mathrm{Series.} \mathrm{Error} \mathrm{bounds} \mathrm{represent} \mathrm{a} 95 \%$ confidence level.

\begin{tabular}{cccccc}
\hline \multicolumn{6}{c}{$\mathbf{1 0 0} \mathbf{~ n m ~ A l ~ o n ~ G e , ~} h_{\mathrm{BD}}=\mathbf{4 6 . 4 0} \mathbf{~ M W} / \mathbf{m}^{2} \mathbf{K}$} \\
\hline $\mathbf{S N P}$ & $\mu-\left[\mathbf{M W} / \mathbf{m}^{2} \mathbf{K}\right]$ & $\sigma-\left[\mathbf{M W} / \mathbf{m}^{2} \mathbf{K}\right]$ & $\mathbf{S N P}$ & $\mu-\left[\mathbf{M W} / \mathbf{m}^{2} \mathbf{K}\right]$ & $\sigma-\left[\mathbf{M W} / \mathbf{m}^{2} \mathbf{K}\right]$ \\
5.74 & $46.56 \pm 0.243$ & $3.91 \pm 0.0109$ & 36.72 & $46.42 \pm 0.038$ & $0.61 \pm 0.0017$ \\
8.60 & $46.44 \pm 0.158$ & $2.55 \pm 0.0071$ & 38.99 & $46.42 \pm 0.036$ & $0.59 \pm 0.0016$ \\
11.39 & $46.43 \pm 0.119$ & $1.92 \pm 0.0054$ & 41.15 & $46.39 \pm 0.033$ & $0.53 \pm 0.0015$ \\
14.20 & $46.44 \pm 0.093$ & $1.50 \pm 0.0042$ & 44.91 & $46.41 \pm 0.030$ & $0.48 \pm 0.0013$ \\
17.09 & $46.44 \pm 0.080$ & $1.29 \pm 0.0036$ & 47.60 & $46.40 \pm 0.029$ & $0.47 \pm 0.0013$ \\
20.24 & $46.40 \pm 0.066$ & $1.06 \pm 0.0030$ & 50.61 & $46.41 \pm 0.027$ & $0.44 \pm 0.0012$ \\
22.82 & $46.44 \pm 0.061$ & $0.98 \pm 0.0027$ & 53.44 & $46.41 \pm 0.025$ & $0.41 \pm 0.0011$ \\
25.61 & $46.43 \pm 0.055$ & $0.89 \pm 0.0025$ & 56.53 & $46.40 \pm 0.024$ & $0.38 \pm 0.0011$ \\
28.29 & $46.39 \pm 0.048$ & $0.77 \pm 0.0022$ & 58.49 & $46.40 \pm 0.024$ & $0.38 \pm 0.0011$ \\
31.51 & $46.40 \pm 0.044$ & $0.72 \pm 0.0020$ & 60.90 & $46.41 \pm 0.022$ & $0.35 \pm 0.0010$ \\
33.91 & $46.40 \pm 0.040$ & $0.64 \pm 0.0018$ & & & \\
\hline
\end{tabular}

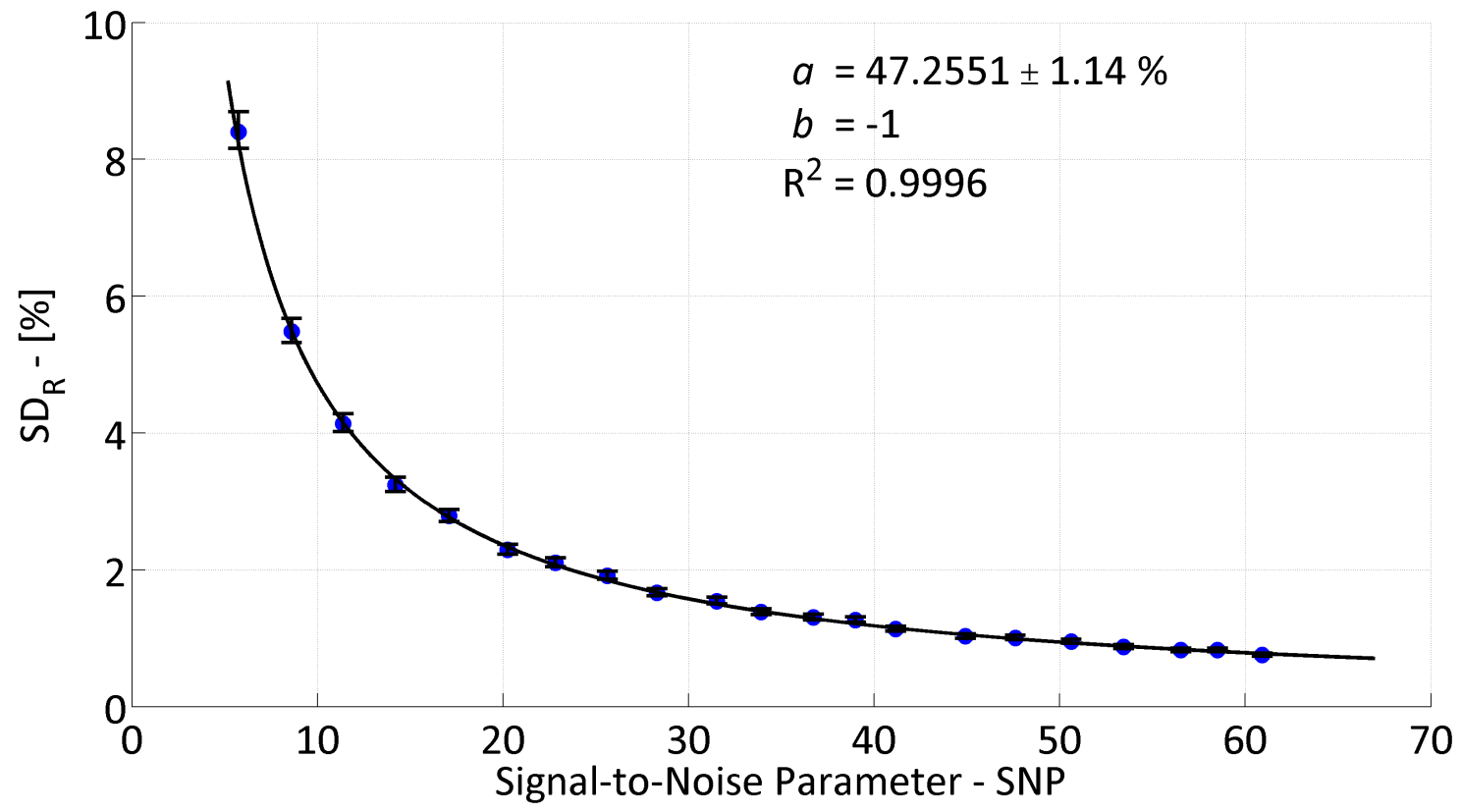

Figure S.146: Relative population standard deviation as a function of signal-to-noise parameter for 100 $\mathrm{nm}$ of $\mathrm{Al} / \mathrm{Ge}$ with $h_{\mathrm{BD}}=46.40 \mathrm{MW} / \mathrm{m}^{2} \mathrm{~K}$. Error bars represent a $95 \%$ confidence interval of the measured $\mathrm{SD}_{\mathrm{R}}$ and the solid line represents a best fit of a power law to the data, of the form $\mathrm{SD}_{\mathrm{R}}=a \times \mathrm{SNP}^{b}$, with parameter $a$ provided for a fixed $b=-1$, along with the $95 \%$ confidence level in $a$. 


\section{S.54.2 Fitting Substrate Thermal Conductivity - $k_{S}$}

Table S.86: Summary of simulation data - $100 \mathrm{~nm} \mathrm{Al/Ge} 46.40 \mathrm{MW}$ Series. Error bounds represent a $95 \%$ confidence level.

\begin{tabular}{cccccc}
\hline \multicolumn{6}{c}{$\mathbf{1 0 0} \mathbf{~ n m} \mathbf{A l}$ on $\mathbf{G e}, h_{\mathrm{BD}}=\mathbf{4 6 . 4 0} \mathbf{~} \mathbf{W W} / \mathbf{m}^{2} \mathbf{K}$} \\
\hline $\mathbf{S N P}$ & $\mu-[\mathbf{W} / \mathbf{m K}]$ & $\sigma-[\mathbf{W} / \mathbf{m K}]$ & $\mathbf{S N P}$ & $\mu-[\mathbf{W} / \mathbf{m K}]$ & $\sigma-[\mathbf{W} / \mathbf{m K}]$ \\
5.74 & $66.01 \pm 1.780$ & $28.60 \pm 0.0801$ & 36.72 & $59.98 \pm 0.232$ & $3.74 \pm 0.0104$ \\
8.60 & $61.89 \pm 1.010$ & $16.21 \pm 0.0455$ & 38.99 & $59.99 \pm 0.227$ & $3.65 \pm 0.0102$ \\
11.39 & $61.14 \pm 0.769$ & $12.38 \pm 0.0346$ & 41.15 & $59.85 \pm 0.205$ & $3.31 \pm 0.0092$ \\
14.20 & $60.75 \pm 0.597$ & $9.62 \pm 0.0268$ & 44.91 & $59.87 \pm 0.181$ & $2.92 \pm 0.0081$ \\
17.09 & $60.57 \pm 0.506$ & $8.15 \pm 0.0227$ & 47.60 & $59.81 \pm 0.180$ & $2.90 \pm 0.0081$ \\
20.24 & $60.14 \pm 0.414$ & $6.67 \pm 0.0186$ & 50.61 & $59.91 \pm 0.171$ & $2.75 \pm 0.0077$ \\
22.82 & $60.35 \pm 0.376$ & $6.05 \pm 0.0169$ & 53.44 & $59.88 \pm 0.156$ & $2.52 \pm 0.0070$ \\
25.61 & $60.29 \pm 0.345$ & $5.56 \pm 0.0155$ & 56.53 & $59.86 \pm 0.147$ & $2.38 \pm 0.0066$ \\
28.29 & $59.95 \pm 0.298$ & $4.80 \pm 0.0134$ & 58.49 & $59.83 \pm 0.147$ & $2.37 \pm 0.0066$ \\
31.51 & $59.90 \pm 0.276$ & $4.45 \pm 0.0124$ & 60.90 & $59.90 \pm 0.136$ & $2.19 \pm 0.0061$ \\
33.91 & $59.92 \pm 0.245$ & $3.95 \pm 0.0110$ & & & \\
\hline
\end{tabular}

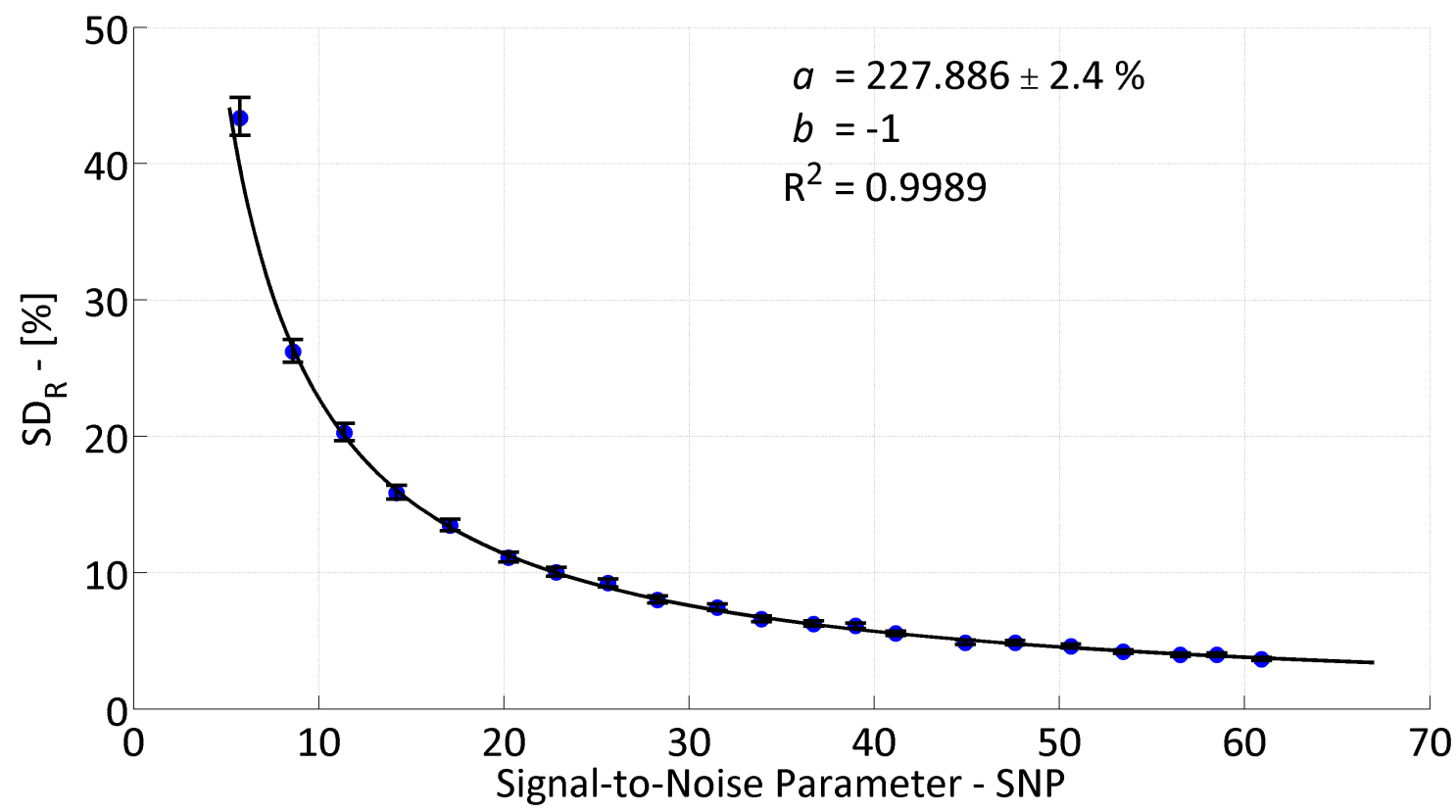

Figure S.147: Relative population standard deviation as a function of signal-to-noise parameter for 100 $\mathrm{nm}$ of $\mathrm{Al} / \mathrm{Ge}$ with $h_{\mathrm{BD}}=46.40 \mathrm{MW} / \mathrm{m}^{2} \mathrm{~K}$. Error bars represent a $95 \%$ confidence interval of the measured $\mathrm{SD}_{\mathrm{R}}$ and the solid line represents a best fit of a power law to the data, of the form $\mathrm{SD}_{\mathrm{R}}=a \times \mathrm{SNP}^{b}$, with parameter $a$ provided for a fixed $b=-1$, along with the $95 \%$ confidence level in $a$. 


\section{S.55 Simulation Summary - Al/Ge 100.00 MW Series}

\section{S.55.1 Fitting Film-to-Substrate Boundary Conductance $-h_{\mathrm{BD}}$}

Table S.87: Summary of simulation data - $100 \mathrm{~nm} \mathrm{Al/Ge} \mathrm{100.00} \mathrm{MW} \mathrm{Series.} \mathrm{Error} \mathrm{bounds} \mathrm{represent} \mathrm{a}$ $95 \%$ confidence level.

\begin{tabular}{cccccc}
\hline \multicolumn{6}{c}{$\mathbf{1 0 0} \mathbf{~ n m ~ A l ~ o n ~ G e , ~} h_{\mathrm{BD}}=\mathbf{1 0 0 . 0 0} \mathbf{M W} / \mathbf{m}^{2} \mathbf{K}$} \\
\hline $\mathbf{S N P}$ & $\mu-\left[\mathbf{M W} / \mathbf{m}^{2} \mathbf{K}\right]$ & $\sigma-\left[\mathbf{M W} / \mathbf{m}^{2} \mathbf{K}\right]$ & $\mathbf{S N P}$ & $\mu-\left[\mathbf{M W} / \mathbf{m}^{2} \mathbf{K}\right]$ & $\sigma-\left[\mathbf{M W} / \mathbf{m}^{2} \mathbf{K}\right]$ \\
3.97 & $101.54 \pm 0.809$ & $13.01 \pm 0.0364$ & 24.78 & $100.04 \pm 0.128$ & $2.06 \pm 0.0057$ \\
6.03 & $100.78 \pm 0.526$ & $8.47 \pm 0.0236$ & 26.84 & $100.07 \pm 0.121$ & $1.95 \pm 0.0054$ \\
7.87 & $100.33 \pm 0.408$ & $6.58 \pm 0.0183$ & 29.48 & $100.09 \pm 0.107$ & $1.73 \pm 0.0048$ \\
10.08 & $100.12 \pm 0.323$ & $5.20 \pm 0.0145$ & 31.20 & $100.01 \pm 0.101$ & $1.63 \pm 0.0045$ \\
11.82 & $100.11 \pm 0.266$ & $4.29 \pm 0.0120$ & 32.84 & $100.01 \pm 0.095$ & $1.53 \pm 0.0043$ \\
13.61 & $100.29 \pm 0.238$ & $3.83 \pm 0.0107$ & 34.73 & $100.05 \pm 0.093$ & $1.51 \pm 0.0042$ \\
15.72 & $100.23 \pm 0.210$ & $3.38 \pm 0.0094$ & 36.27 & $100.09 \pm 0.091$ & $1.46 \pm 0.0041$ \\
17.72 & $100.16 \pm 0.183$ & $2.94 \pm 0.0082$ & 38.78 & $100.05 \pm 0.085$ & $1.36 \pm 0.0038$ \\
19.79 & $100.12 \pm 0.162$ & $2.61 \pm 0.0073$ & 40.32 & $100.06 \pm 0.080$ & $1.29 \pm 0.0036$ \\
21.63 & $99.93 \pm 0.150$ & $2.42 \pm 0.0067$ & 42.69 & $100.02 \pm 0.073$ & $1.17 \pm 0.0033$ \\
23.51 & $100.03 \pm 0.139$ & $2.24 \pm 0.0062$ & & & \\
\hline
\end{tabular}

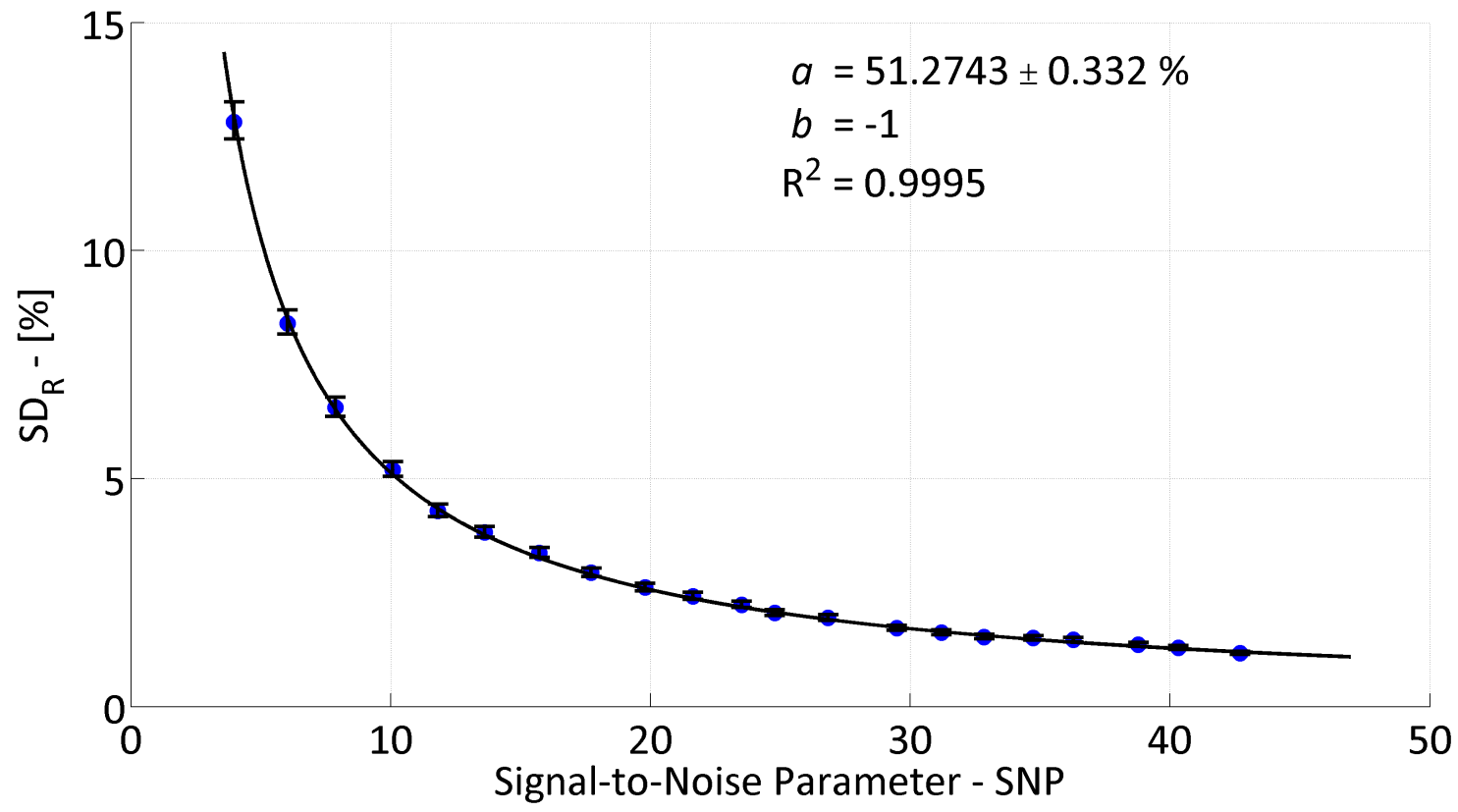

Figure S.148: Relative population standard deviation as a function of signal-to-noise parameter for 100 $\mathrm{nm}$ of $\mathrm{Al} / \mathrm{Ge}$ with $h_{\mathrm{BD}}=100.00 \mathrm{MW} / \mathrm{m}^{2} \mathrm{~K}$. Error bars represent a $95 \%$ confidence interval of the measured $\mathrm{SD}_{\mathrm{R}}$ and the solid line represents a best fit of a power law to the data, of the form $\mathrm{SD}_{\mathrm{R}}=a \times \mathrm{SNP}^{b}$, with parameter $a$ provided for a fixed $b=-1$, along with the $95 \%$ confidence level in $a$. 


\section{S.55.2 Fitting Substrate Thermal Conductivity - $k_{S}$}

Table S.88: Summary of simulation data - $100 \mathrm{~nm} \mathrm{Al/Ge} \mathrm{100.00} \mathrm{MW} \mathrm{Series.} \mathrm{Error} \mathrm{bounds} \mathrm{represent} \mathrm{a}$ $95 \%$ confidence level.

\begin{tabular}{cccccc}
\hline \multicolumn{6}{c}{$\mathbf{1 0 0} \mathbf{~ n m} \mathbf{A l}$ on Ge, $h_{\mathrm{BD}}=\mathbf{1 0 0 . 0 0} \mathbf{M W} / \mathbf{m}^{2} \mathbf{K}$} \\
\hline $\mathbf{S N P}$ & $\mu-[\mathbf{W} / \mathbf{m K}]$ & $\sigma-[\mathbf{W} / \mathbf{m K}]$ & $\mathbf{S N P}$ & $\mu-[\mathbf{W} / \mathbf{m K}]$ & $\sigma-[\mathbf{W} / \mathbf{m K}]$ \\
3.97 & $61.98 \pm 0.850$ & $13.68 \pm 0.0382$ & 24.78 & $59.90 \pm 0.130$ & $2.10 \pm 0.0058$ \\
6.03 & $60.81 \pm 0.531$ & $8.55 \pm 0.0239$ & 26.84 & $59.96 \pm 0.122$ & $1.96 \pm 0.0055$ \\
7.87 & $60.26 \pm 0.408$ & $6.58 \pm 0.0183$ & 29.48 & $60.00 \pm 0.108$ & $1.74 \pm 0.0048$ \\
10.08 & $60.08 \pm 0.333$ & $5.37 \pm 0.0149$ & 31.20 & $59.91 \pm 0.102$ & $1.64 \pm 0.0046$ \\
11.82 & $60.09 \pm 0.273$ & $4.39 \pm 0.0122$ & 32.84 & $59.94 \pm 0.095$ & $1.53 \pm 0.0043$ \\
13.61 & $60.23 \pm 0.244$ & $3.94 \pm 0.0110$ & 34.73 & $59.93 \pm 0.093$ & $1.50 \pm 0.0042$ \\
15.72 & $60.12 \pm 0.209$ & $3.38 \pm 0.0094$ & 36.27 & $60.00 \pm 0.093$ & $1.49 \pm 0.0042$ \\
17.72 & $60.14 \pm 0.187$ & $3.01 \pm 0.0084$ & 38.78 & $59.95 \pm 0.085$ & $1.37 \pm 0.0038$ \\
19.79 & $60.02 \pm 0.163$ & $2.62 \pm 0.0073$ & 40.32 & $59.97 \pm 0.082$ & $1.32 \pm 0.0037$ \\
21.63 & $59.84 \pm 0.154$ & $2.48 \pm 0.0069$ & 42.69 & $59.92 \pm 0.074$ & $1.19 \pm 0.0033$ \\
23.51 & $59.96 \pm 0.136$ & $2.19 \pm 0.0061$ & & & \\
\hline
\end{tabular}

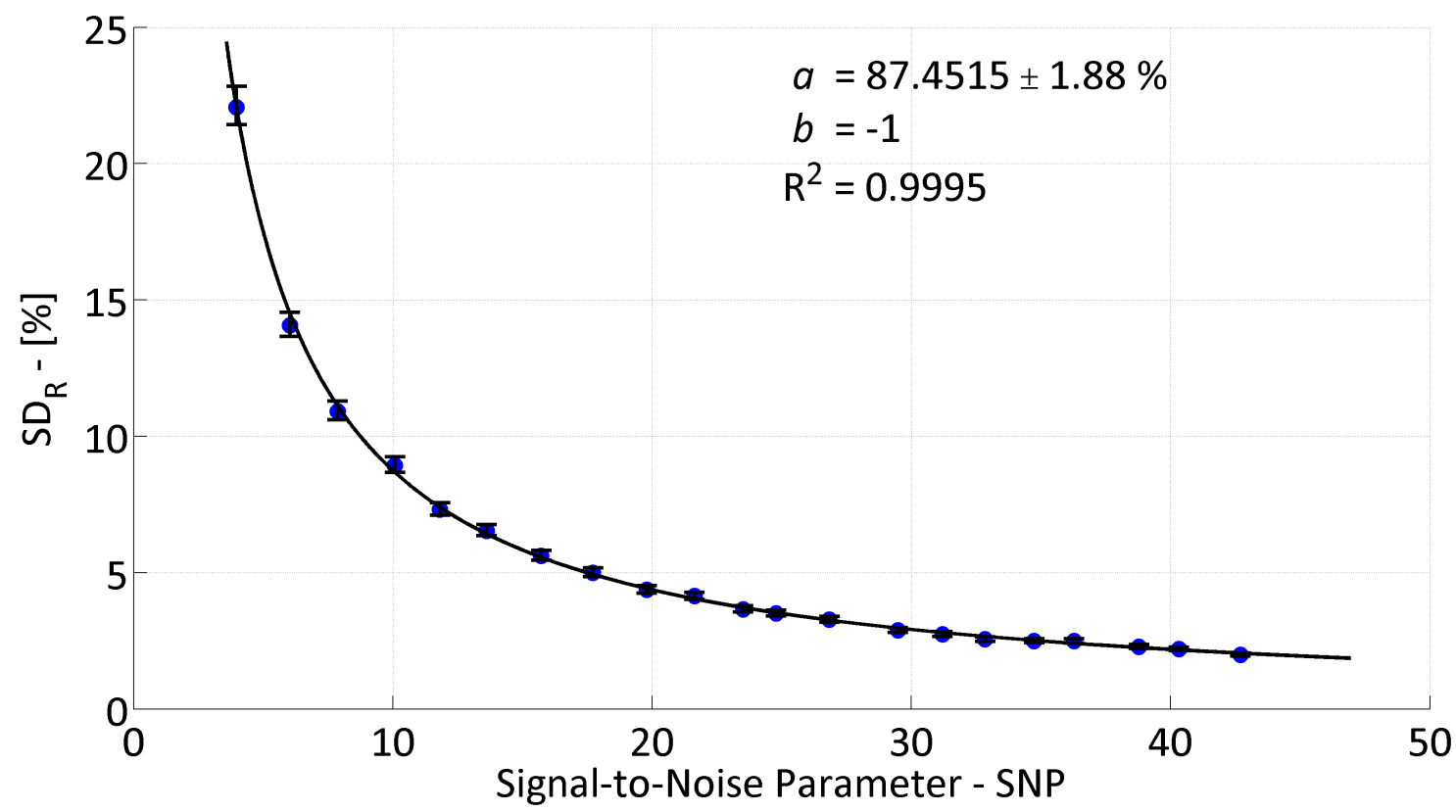

Figure S.149: Relative population standard deviation as a function of signal-to-noise parameter for 100 $\mathrm{nm}$ of $\mathrm{Al} / \mathrm{Ge}$ with $h_{\mathrm{BD}}=100.00 \mathrm{MW} / \mathrm{m}^{2} \mathrm{~K}$. Error bars represent a $95 \%$ confidence interval of the measured $\mathrm{SD}_{\mathrm{R}}$ and the solid line represents a best fit of a power law to the data, of the form $\mathrm{SD}_{\mathrm{R}}=a \times \mathrm{SNP}^{b}$, with parameter $a$ provided for a fixed $b=-1$, along with the $95 \%$ confidence level in $a$. 


\section{S.56 Simulation Summary - Al/Ge 129.00 MW Series}

\section{S.56.1 Fitting Film-to-Substrate Boundary Conductance $-h_{\mathrm{BD}}$}

Table S.89: Summary of simulation data - $100 \mathrm{~nm} \mathrm{Al/Ge} \mathrm{129.00} \mathrm{MW} \mathrm{Series.} \mathrm{Error} \mathrm{bounds} \mathrm{represent} \mathrm{a}$ $95 \%$ confidence level.

\begin{tabular}{cccccc}
\hline \multicolumn{6}{c}{$\mathbf{1 0 0} \mathbf{~ n m ~} \mathbf{A l}$ on $\mathbf{G e}, h_{\mathrm{BD}}=\mathbf{1 2 9 . 0 0} \mathbf{M W} / \mathbf{m}^{2} \mathbf{K}$} \\
\hline $\mathbf{S N P}$ & $\mu-\left[\mathbf{M W} / \mathbf{m}^{2} \mathbf{K}\right]$ & $\sigma-\left[\mathbf{M W} / \mathbf{m}^{2} \mathbf{K}\right]$ & $\mathbf{S N P}$ & $\mu-\left[\mathbf{M W} / \mathbf{m}^{2} \mathbf{K}\right]$ & $\sigma-\left[\mathbf{M W} / \mathbf{m}^{2} \mathbf{K}\right]$ \\
3.47 & $131.51 \pm 1.534$ & $24.18 \pm 0.0704$ & 22.53 & $129.03 \pm 0.213$ & $3.39 \pm 0.0097$ \\
5.17 & $130.33 \pm 0.963$ & $15.31 \pm 0.0438$ & 24.10 & $129.28 \pm 0.207$ & $3.30 \pm 0.0094$ \\
6.90 & $130.33 \pm 0.750$ & $11.93 \pm 0.0341$ & 25.64 & $129.01 \pm 0.194$ & $3.08 \pm 0.0088$ \\
8.62 & $129.20 \pm 0.597$ & $9.50 \pm 0.0272$ & 27.39 & $129.06 \pm 0.179$ & $2.85 \pm 0.0081$ \\
10.34 & $129.55 \pm 0.470$ & $7.47 \pm 0.0214$ & 29.07 & $129.03 \pm 0.173$ & $2.75 \pm 0.0079$ \\
12.04 & $129.06 \pm 0.416$ & $6.62 \pm 0.0189$ & 31.24 & $129.06 \pm 0.163$ & $2.59 \pm 0.0074$ \\
13.81 & $129.56 \pm 0.361$ & $5.75 \pm 0.0164$ & 32.53 & $129.13 \pm 0.148$ & $2.36 \pm 0.0067$ \\
15.52 & $128.73 \pm 0.311$ & $4.95 \pm 0.0141$ & 34.49 & $129.20 \pm 0.146$ & $2.32 \pm 0.0066$ \\
17.22 & $129.13 \pm 0.295$ & $4.69 \pm 0.0134$ & 35.31 & $129.12 \pm 0.144$ & $2.29 \pm 0.0066$ \\
18.97 & $129.18 \pm 0.266$ & $4.22 \pm 0.0121$ & 37.28 & $129.04 \pm 0.133$ & $2.12 \pm 0.0061$ \\
20.66 & $129.14 \pm 0.237$ & $3.77 \pm 0.0108$ & & & \\
\hline
\end{tabular}

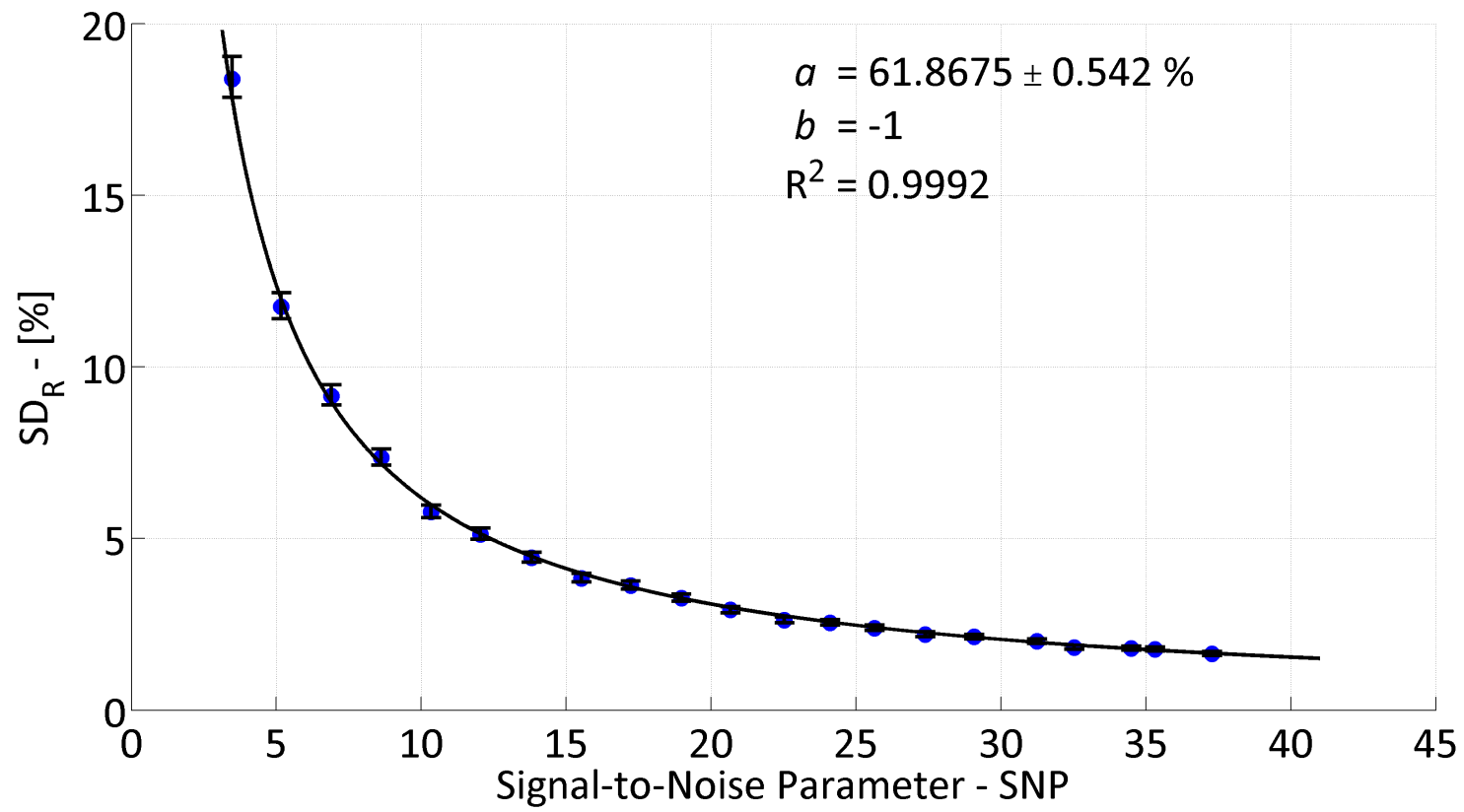

Figure S.150: Relative population standard deviation as a function of signal-to-noise parameter for 100 $\mathrm{nm}$ of $\mathrm{Al} / \mathrm{Ge}$ with $h_{\mathrm{BD}}=129.00 \mathrm{MW} / \mathrm{m}^{2} \mathrm{~K}$. Error bars represent a $95 \%$ confidence interval of the measured $\mathrm{SD}_{\mathrm{R}}$ and the solid line represents a best fit of a power law to the data, of the form $\mathrm{SD}_{\mathrm{R}}=a \times \mathrm{SNP}^{b}$, with parameter $a$ provided for a fixed $b=-1$, along with the $95 \%$ confidence level in $a$. 


\section{S.57 Simulation Summary - Al/Ge 167.00 MW Series}

\section{S.57.1 Fitting Film-to-Substrate Boundary Conductance $-h_{\mathrm{BD}}$}

Table S.90: Summary of simulation data - $100 \mathrm{~nm} \mathrm{Al/Ge} \mathrm{167.00} \mathrm{MW} \mathrm{Series.} \mathrm{Error} \mathrm{bounds} \mathrm{represent} \mathrm{a}$ 95\% confidence level.

\begin{tabular}{cccccc}
\hline \multicolumn{6}{c}{$\mathbf{1 0 0} \mathbf{~ n m ~} \mathbf{A l}$ on $\mathbf{G e}, h_{\mathrm{BD}}=\mathbf{1 6 7 . 0 0} \mathbf{M W} / \mathbf{m}^{2} \mathbf{K}$} \\
\hline $\mathbf{S N P}$ & $\mu-\left[\mathbf{M W} / \mathbf{m}^{2} \mathbf{K}\right]$ & $\sigma-\left[\mathbf{M W} / \mathbf{m}^{2} \mathbf{K}\right]$ & $\mathbf{S N P}$ & $\mu-\left[\mathbf{M W} / \mathbf{m}^{2} \mathbf{K}\right]$ & $\sigma-\left[\mathbf{M W} / \mathbf{m}^{2} \mathbf{K}\right]$ \\
3.25 & $171.47 \pm 2.535$ & $39.09 \pm 0.1189$ & 20.65 & $167.36 \pm 0.388$ & $6.17 \pm 0.0176$ \\
4.84 & $169.51 \pm 1.611$ & $25.52 \pm 0.0736$ & 22.18 & $167.08 \pm 0.341$ & $5.43 \pm 0.0155$ \\
6.47 & $169.67 \pm 1.246$ & $19.78 \pm 0.0568$ & 24.03 & $166.99 \pm 0.331$ & $5.26 \pm 0.0150$ \\
8.12 & $167.89 \pm 0.961$ & $15.28 \pm 0.0437$ & 25.98 & $167.13 \pm 0.303$ & $4.82 \pm 0.0138$ \\
9.56 & $167.96 \pm 0.844$ & $13.42 \pm 0.0384$ & 27.74 & $167.01 \pm 0.283$ & $4.50 \pm 0.0129$ \\
11.25 & $167.58 \pm 0.702$ & $11.16 \pm 0.0319$ & 28.16 & $166.71 \pm 0.274$ & $4.35 \pm 0.0124$ \\
12.85 & $167.26 \pm 0.582$ & $9.27 \pm 0.0265$ & 30.11 & $166.92 \pm 0.248$ & $3.95 \pm 0.0113$ \\
14.49 & $167.22 \pm 0.523$ & $8.31 \pm 0.0238$ & 31.44 & $167.11 \pm 0.241$ & $3.84 \pm 0.0109$ \\
16.01 & $167.87 \pm 0.487$ & $7.75 \pm 0.0221$ & 33.41 & $166.94 \pm 0.228$ & $3.63 \pm 0.0104$ \\
17.77 & $167.31 \pm 0.443$ & $7.05 \pm 0.0201$ & 34.81 & $167.01 \pm 0.223$ & $3.55 \pm 0.0101$ \\
19.29 & $167.06 \pm 0.397$ & $6.31 \pm 0.0181$ & & & \\
\hline
\end{tabular}

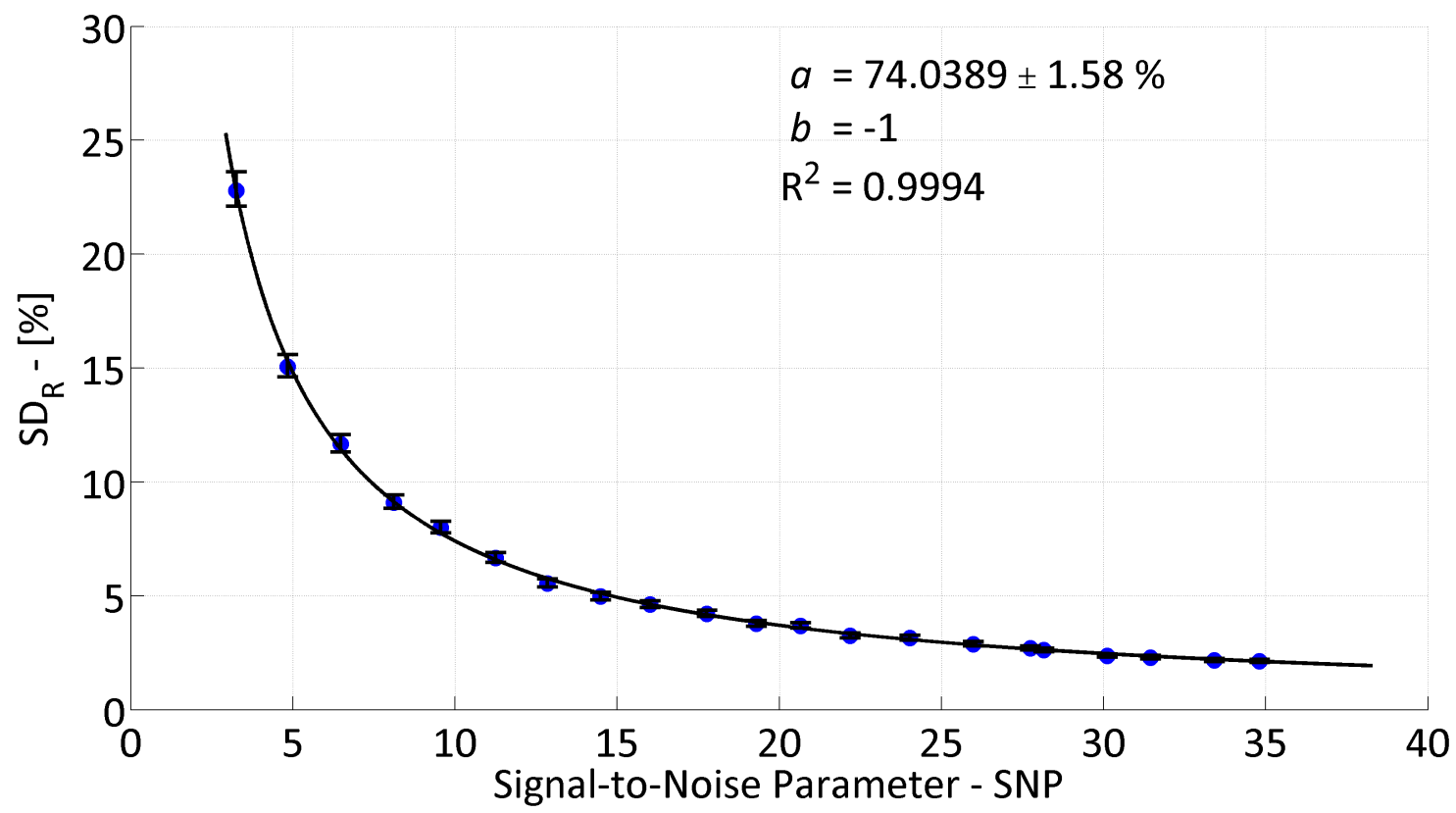

Figure S.151: Relative population standard deviation as a function of signal-to-noise parameter for 100 $\mathrm{nm}$ of $\mathrm{Al} / \mathrm{Ge}$ with $h_{\mathrm{BD}}=167.00 \mathrm{MW} / \mathrm{m}^{2} \mathrm{~K}$. Error bars represent a $95 \%$ confidence interval of the measured $\mathrm{SD}_{\mathrm{R}}$ and the solid line represents a best fit of a power law to the data, of the form $\mathrm{SD}_{\mathrm{R}}=a \times \mathrm{SNP}^{b}$, with parameter $a$ provided for a fixed $b=-1$, along with the $95 \%$ confidence level in $a$. 


\section{S.58 Simulation Summary - Al/Ge 215.00 MW Series}

\section{S.58.1 Fitting Film-to-Substrate Boundary Conductance $-h_{\mathrm{BD}}$}

Table S.91: Summary of simulation data - $100 \mathrm{~nm} \mathrm{Al/Ge} \mathrm{215.00} \mathrm{MW} \mathrm{Series.} \mathrm{Error} \mathrm{bounds} \mathrm{represent} \mathrm{a}$ $95 \%$ confidence level.

\begin{tabular}{cccccc}
\hline \multicolumn{6}{c}{$\mathbf{1 0 0} \mathbf{~ n m ~ A l ~ o n ~ G e , ~} h_{\mathrm{BD}}=\mathbf{2 1 5 . 0 0} \mathbf{M W} / \mathbf{m}^{2} \mathbf{K}$} \\
\hline $\mathbf{S N P}$ & $\mu-\left[\mathbf{M W} / \mathbf{m}^{2} \mathbf{K}\right]$ & $\sigma-\left[\mathbf{M W} / \mathbf{m}^{2} \mathbf{K}\right]$ & $\mathbf{S N P}$ & $\mu-\left[\mathbf{M W} / \mathbf{m}^{2} \mathbf{K}\right]$ & $\sigma-\left[\mathbf{M W} / \mathbf{m}^{2} \mathbf{K}\right]$ \\
3.07 & $244.93 \pm 6.285$ & $97.41 \pm 0.2934$ & 19.74 & $215.45 \pm 0.603$ & $9.68 \pm 0.0272$ \\
4.57 & $222.42 \pm 3.095$ & $48.76 \pm 0.1421$ & 21.12 & $215.38 \pm 0.582$ & $9.34 \pm 0.0262$ \\
6.16 & $218.37 \pm 2.134$ & $34.04 \pm 0.0967$ & 22.59 & $215.46 \pm 0.536$ & $8.60 \pm 0.0241$ \\
7.50 & $218.45 \pm 1.754$ & $28.06 \pm 0.0793$ & 23.68 & $214.87 \pm 0.500$ & $8.03 \pm 0.0225$ \\
9.17 & $216.03 \pm 1.326$ & $21.23 \pm 0.0599$ & 25.70 & $214.88 \pm 0.464$ & $7.48 \pm 0.0208$ \\
10.55 & $217.15 \pm 1.170$ & $18.83 \pm 0.0526$ & 27.00 & $215.04 \pm 0.457$ & $7.34 \pm 0.0206$ \\
11.92 & $214.90 \pm 1.020$ & $16.37 \pm 0.0460$ & 28.34 & $215.08 \pm 0.418$ & $6.73 \pm 0.0188$ \\
13.33 & $215.67 \pm 0.920$ & $14.75 \pm 0.0415$ & 30.31 & $215.44 \pm 0.394$ & $6.33 \pm 0.0178$ \\
15.16 & $215.36 \pm 0.823$ & $13.26 \pm 0.0369$ & 31.89 & $215.26 \pm 0.378$ & $6.06 \pm 0.0170$ \\
16.63 & $216.07 \pm 0.717$ & $11.51 \pm 0.0323$ & 33.41 & $215.20 \pm 0.368$ & $5.90 \pm 0.0166$ \\
17.85 & $215.60 \pm 0.678$ & $10.93 \pm 0.0304$ & & & \\
\hline
\end{tabular}

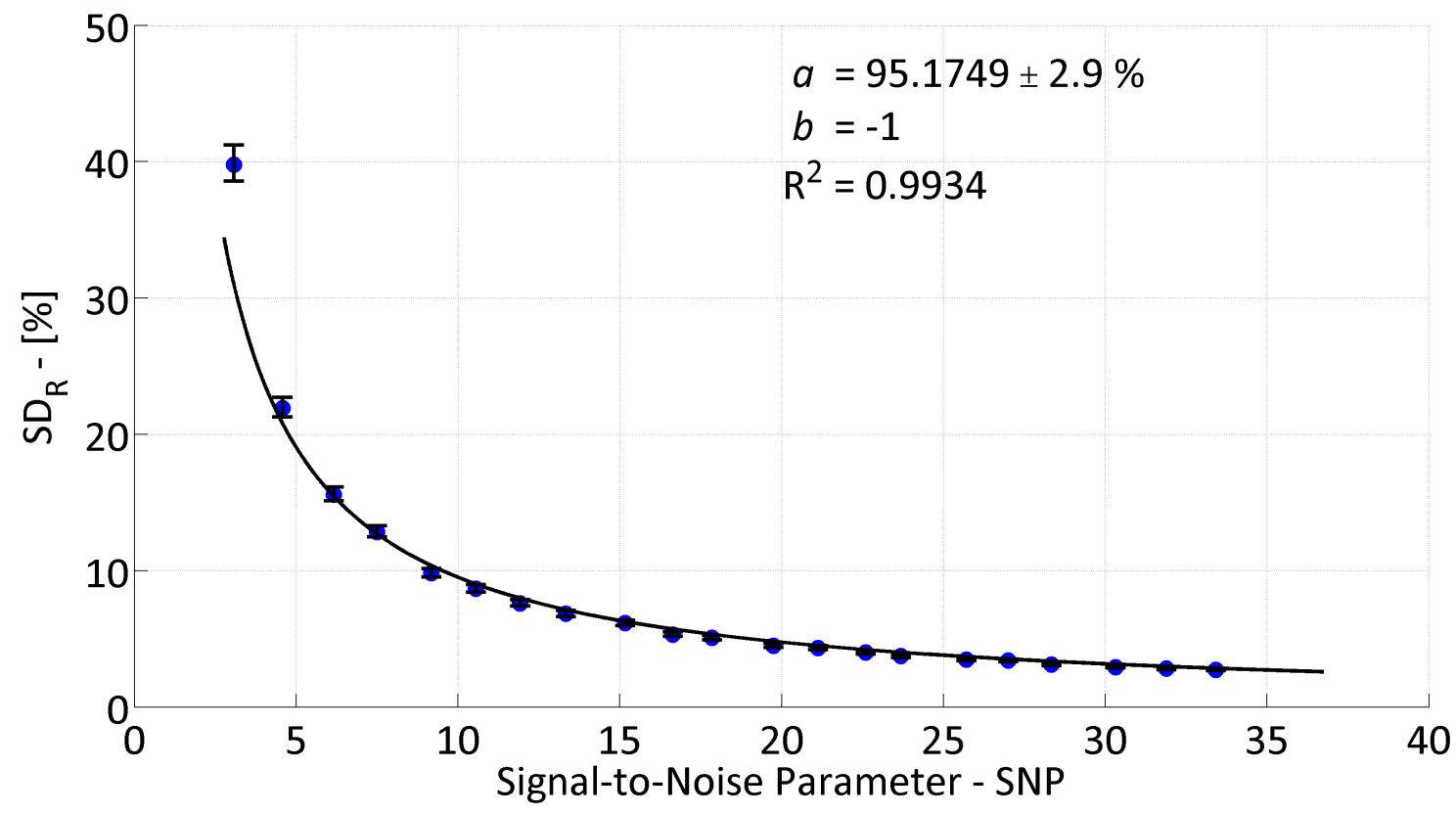

Figure S.152: Relative population standard deviation as a function of signal-to-noise parameter for 100 $\mathrm{nm}$ of $\mathrm{Al} / \mathrm{Ge}$ with $h_{\mathrm{BD}}=215.00 \mathrm{MW} / \mathrm{m}^{2} \mathrm{~K}$. Error bars represent a $95 \%$ confidence interval of the measured $\mathrm{SD}_{\mathrm{R}}$ and the solid line represents a best fit of a power law to the data, of the form $\mathrm{SD}_{\mathrm{R}}=a \times \mathrm{SNP}^{b}$, with parameter $a$ provided for a fixed $b=-1$, along with the $95 \%$ confidence level in $a$. 


\section{S.58.2 Fitting Substrate Thermal Conductivity - $k_{S}$}

Table S.92: Summary of simulation data - $100 \mathrm{~nm} \mathrm{Al/Ge} \mathrm{215.00} \mathrm{MW} \mathrm{Series.} \mathrm{Error} \mathrm{bounds} \mathrm{represent} \mathrm{a}$ $95 \%$ confidence level.

\begin{tabular}{cccccc}
\hline \multicolumn{6}{c}{$\mathbf{1 0 0} \mathbf{~ n m}$ Al on Ge, $h_{\mathrm{BD}}=\mathbf{2 1 5 . 0 0 ~} \mathbf{M W} / \mathbf{m}^{2} \mathbf{K}$} \\
\hline $\mathbf{S N P}$ & $\mu-[\mathbf{W} / \mathbf{m K}]$ & $\sigma-[\mathbf{W} / \mathbf{m K}]$ & $\mathbf{S N P}$ & $\mu-[\mathbf{W} / \mathbf{m K}]$ & $\sigma-[\mathbf{W} / \mathbf{m K}]$ \\
3.07 & $60.98 \pm 0.695$ & $11.19 \pm 0.0312$ & 19.74 & $59.86 \pm 0.106$ & $1.71 \pm 0.0048$ \\
4.57 & $60.33 \pm 0.488$ & $7.87 \pm 0.0219$ & 21.12 & $59.85 \pm 0.097$ & $1.56 \pm 0.0043$ \\
6.16 & $60.14 \pm 0.341$ & $5.49 \pm 0.0153$ & 22.59 & $59.95 \pm 0.094$ & $1.51 \pm 0.0042$ \\
7.50 & $60.15 \pm 0.282$ & $4.54 \pm 0.0127$ & 23.68 & $59.87 \pm 0.089$ & $1.44 \pm 0.0040$ \\
9.17 & $60.08 \pm 0.239$ & $3.85 \pm 0.0107$ & 25.70 & $59.85 \pm 0.080$ & $1.29 \pm 0.0036$ \\
10.55 & $60.10 \pm 0.205$ & $3.30 \pm 0.0092$ & 27.00 & $59.85 \pm 0.075$ & $1.21 \pm 0.0034$ \\
11.92 & $59.99 \pm 0.186$ & $3.00 \pm 0.0084$ & 28.34 & $59.93 \pm 0.076$ & $1.23 \pm 0.0034$ \\
13.33 & $59.95 \pm 0.162$ & $2.61 \pm 0.0073$ & 30.31 & $59.90 \pm 0.070$ & $1.13 \pm 0.0031$ \\
15.16 & $59.90 \pm 0.146$ & $2.35 \pm 0.0065$ & 31.89 & $59.87 \pm 0.067$ & $1.07 \pm 0.0030$ \\
16.63 & $59.99 \pm 0.130$ & $2.09 \pm 0.0058$ & 33.41 & $59.95 \pm 0.064$ & $1.03 \pm 0.0029$ \\
17.85 & $59.93 \pm 0.119$ & $1.92 \pm 0.0053$ & & & \\
\hline
\end{tabular}

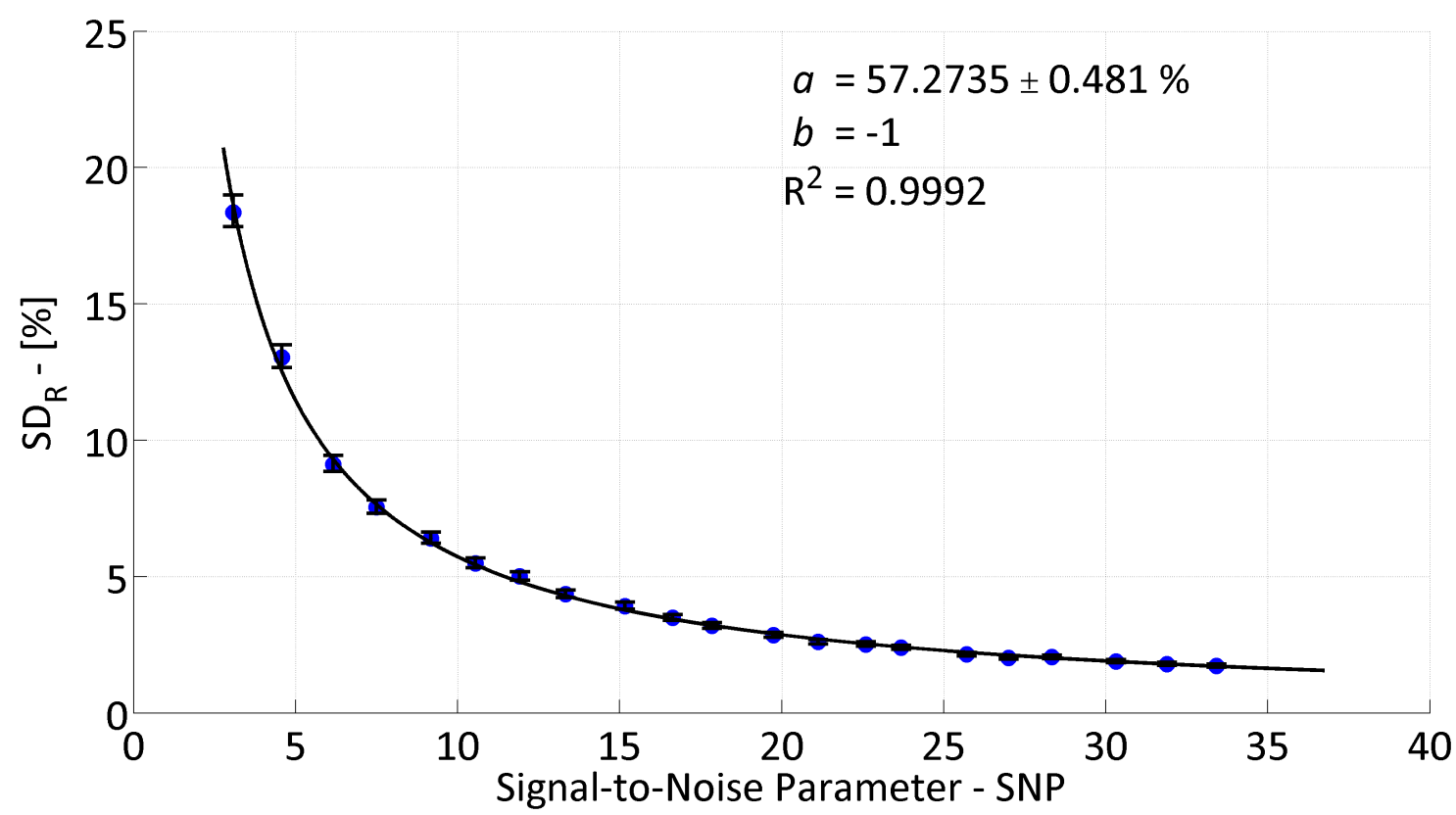

Figure S.153: Relative population standard deviation as a function of signal-to-noise parameter for 100 $\mathrm{nm}$ of $\mathrm{Al} / \mathrm{Ge}$ with $h_{\mathrm{BD}}=215.00 \mathrm{MW} / \mathrm{m}^{2} \mathrm{~K}$. Error bars represent a $95 \%$ confidence interval of the measured $\mathrm{SD}_{\mathrm{R}}$ and the solid line represents a best fit of a power law to the data, of the form $\mathrm{SD}_{\mathrm{R}}=a \times \mathrm{SNP}^{b}$, with parameter $a$ provided for a fixed $b=-1$, along with the $95 \%$ confidence level in $a$. 


\section{S.59 Simulation Summary - Al/Ge 359.00 MW Series}

\section{S.59.1 Fitting Film-to-Substrate Boundary Conductance - $h_{\mathrm{BD}}$}

Table S.93: Summary of simulation data - $100 \mathrm{~nm} \mathrm{Al/Ge} \mathrm{359.00} \mathrm{MW} \mathrm{Series.} \mathrm{Error} \mathrm{bounds} \mathrm{represent} \mathrm{a}$ 95\% confidence level.

\begin{tabular}{cccccc}
\hline \multicolumn{6}{c}{$\mathbf{1 0 0} \mathbf{~ n m ~} \mathbf{A l}$ on $\mathbf{G e}, h_{\mathrm{BD}}=\mathbf{3 5 9 . 0 0} \mathbf{M W} / \mathbf{m}^{2} \mathbf{K}$} \\
\hline $\mathbf{S N P}$ & $\mu-\left[\mathbf{M W} / \mathbf{m}^{2} \mathbf{K}\right]$ & $\sigma-\left[\mathbf{M W} / \mathbf{m}^{2} \mathbf{K}\right]$ & $\mathbf{S N P}$ & $\mu-\left[\mathbf{M W} / \mathbf{m}^{2} \mathbf{K}\right]$ & $\sigma-\left[\mathbf{M W} / \mathbf{m}^{2} \mathbf{K}\right]$ \\
2.82 & $418.09 \pm 13.350$ & $211.44 \pm 0.6097$ & 18.14 & $362.18 \pm 1.708$ & $27.52 \pm 0.0767$ \\
4.22 & $393.74 \pm 8.780$ & $140.84 \pm 0.3958$ & 19.58 & $360.51 \pm 1.576$ & $25.39 \pm 0.0707$ \\
5.61 & $381.01 \pm 6.338$ & $101.89 \pm 0.2852$ & 20.55 & $359.26 \pm 1.476$ & $23.78 \pm 0.0662$ \\
6.94 & $372.29 \pm 4.851$ & $78.02 \pm 0.2181$ & 21.98 & $360.42 \pm 1.383$ & $22.28 \pm 0.0620$ \\
8.35 & $366.61 \pm 3.876$ & $62.28 \pm 0.1745$ & 23.69 & $360.37 \pm 1.331$ & $21.45 \pm 0.0597$ \\
9.81 & $365.62 \pm 3.291$ & $52.93 \pm 0.1480$ & 25.11 & $359.23 \pm 1.294$ & $20.86 \pm 0.0581$ \\
11.24 & $363.64 \pm 2.843$ & $45.77 \pm 0.1277$ & 26.42 & $360.29 \pm 1.224$ & $19.73 \pm 0.0549$ \\
12.55 & $361.24 \pm 2.461$ & $39.64 \pm 0.1105$ & 27.25 & $358.41 \pm 1.166$ & $18.78 \pm 0.0524$ \\
13.99 & $362.27 \pm 2.307$ & $37.15 \pm 0.1036$ & 29.40 & $359.33 \pm 1.028$ & $16.57 \pm 0.0461$ \\
15.36 & $361.23 \pm 2.116$ & $34.08 \pm 0.0950$ & 30.66 & $359.64 \pm 1.047$ & $16.88 \pm 0.0470$ \\
16.83 & $361.86 \pm 1.893$ & $30.50 \pm 0.0849$ & & & \\
\hline
\end{tabular}

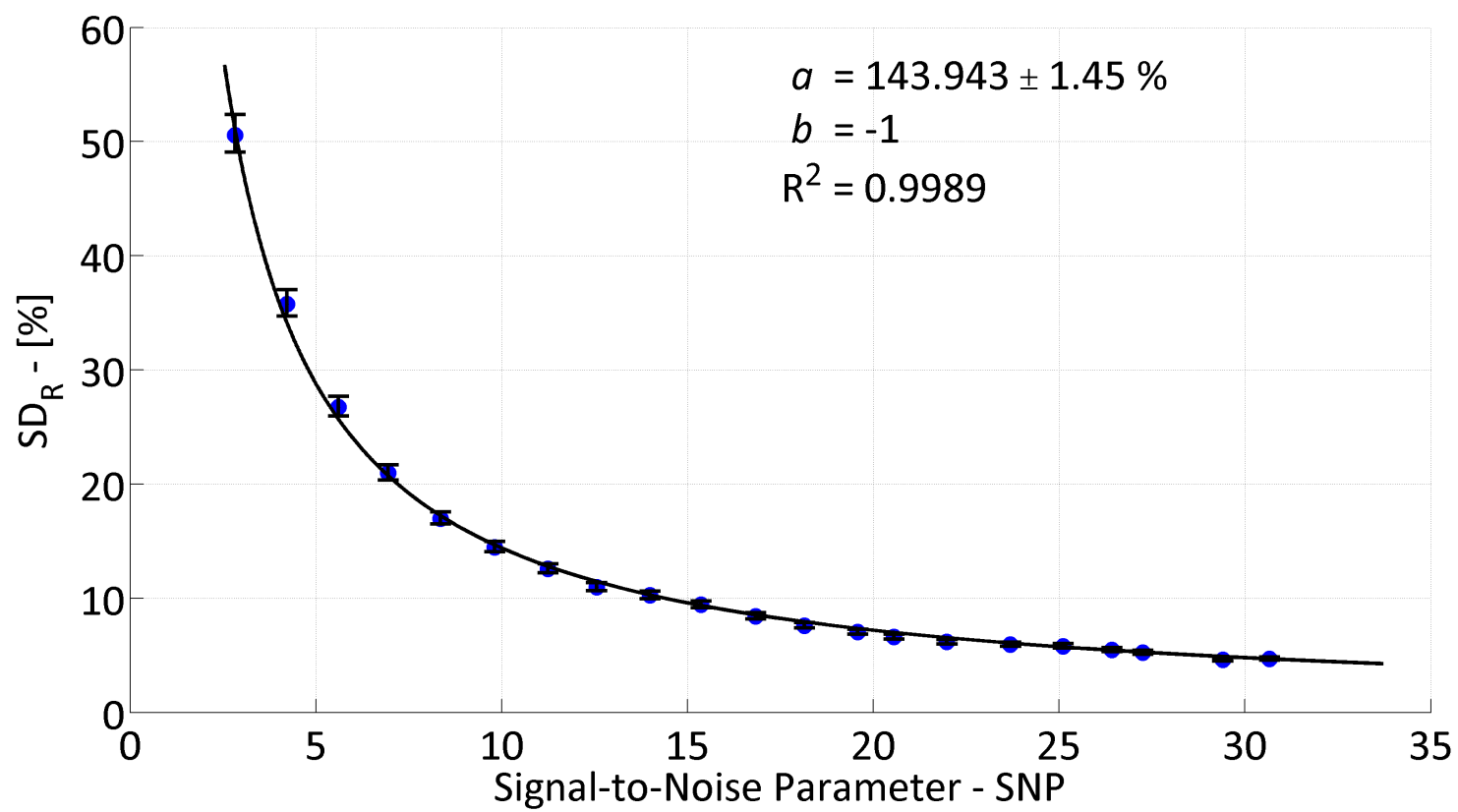

Figure S.154: Relative population standard deviation as a function of signal-to-noise parameter for 100 $\mathrm{nm}$ of $\mathrm{Al} / \mathrm{Ge}$ with $h_{\mathrm{BD}}=359.00 \mathrm{MW} / \mathrm{m}^{2} \mathrm{~K}$. Error bars represent a $95 \%$ confidence interval of the measured $\mathrm{SD}_{\mathrm{R}}$ and the solid line represents a best fit of a power law to the data, of the form $\mathrm{SD}_{\mathrm{R}}=a \times \mathrm{SNP}^{b}$, with parameter $a$ provided for a fixed $b=-1$, along with the $95 \%$ confidence level in $a$. 


\section{S.60 Simulation Summary - Al/Ge 464.00 MW Series}

\section{S.60.1 Fitting Film-to-Substrate Boundary Conductance $-h_{\mathrm{BD}}$}

Table S.94: Summary of simulation data - $100 \mathrm{~nm} \mathrm{Al/Ge} \mathrm{464.00} \mathrm{MW} \mathrm{Series.} \mathrm{Error} \mathrm{bounds} \mathrm{represent} \mathrm{a}$ $95 \%$ confidence level.

\begin{tabular}{cccccc}
\hline \multicolumn{6}{c}{$\mathbf{1 0 0} \mathbf{~ n m ~} \mathbf{A l}$ on $\mathbf{G e}, h_{\mathrm{BD}}=\mathbf{4 6 4 . 0 0} \mathbf{M W} / \mathbf{m}^{2} \mathbf{K}$} \\
\hline $\mathbf{S N P}$ & $\mu-\left[\mathbf{M W} / \mathbf{m}^{2} \mathbf{K}\right]$ & $\sigma-\left[\mathbf{M W} / \mathbf{m}^{2} \mathbf{K}\right]$ & $\mathbf{S N P}$ & $\mu-\left[\mathbf{M W} / \mathbf{m}^{2} \mathbf{K}\right]$ & $\sigma-\left[\mathbf{M W} / \mathbf{m}^{2} \mathbf{K}\right]$ \\
2.78 & $540.87 \pm 20.595$ & $326.18 \pm 0.9405$ & 17.93 & $466.51 \pm 2.426$ & $39.10 \pm 0.1089$ \\
4.18 & $499.44 \pm 11.695$ & $186.37 \pm 0.5308$ & 19.51 & $466.45 \pm 2.279$ & $36.71 \pm 0.1023$ \\
5.57 & $479.78 \pm 8.145$ & $130.34 \pm 0.3682$ & 20.55 & $465.18 \pm 2.225$ & $35.86 \pm 0.0999$ \\
6.87 & $484.64 \pm 6.966$ & $112.09 \pm 0.3131$ & 21.75 & $466.37 \pm 2.057$ & $33.13 \pm 0.0924$ \\
8.30 & $474.15 \pm 5.559$ & $89.54 \pm 0.2496$ & 23.33 & $464.94 \pm 1.853$ & $29.86 \pm 0.0831$ \\
9.78 & $470.87 \pm 4.646$ & $74.86 \pm 0.2085$ & 24.70 & $465.28 \pm 1.735$ & $27.94 \pm 0.0779$ \\
11.06 & $470.44 \pm 4.199$ & $67.63 \pm 0.1885$ & 25.60 & $463.78 \pm 1.804$ & $29.07 \pm 0.0810$ \\
12.37 & $471.66 \pm 3.609$ & $58.15 \pm 0.1619$ & 27.28 & $464.31 \pm 1.668$ & $26.88 \pm 0.0749$ \\
13.97 & $466.80 \pm 3.193$ & $51.40 \pm 0.1434$ & 28.59 & $463.90 \pm 1.565$ & $25.20 \pm 0.0703$ \\
15.09 & $467.10 \pm 3.002$ & $48.36 \pm 0.1348$ & 29.69 & $463.60 \pm 1.477$ & $23.80 \pm 0.0663$ \\
16.99 & $467.55 \pm 2.645$ & $42.62 \pm 0.1187$ & & & \\
\hline
\end{tabular}

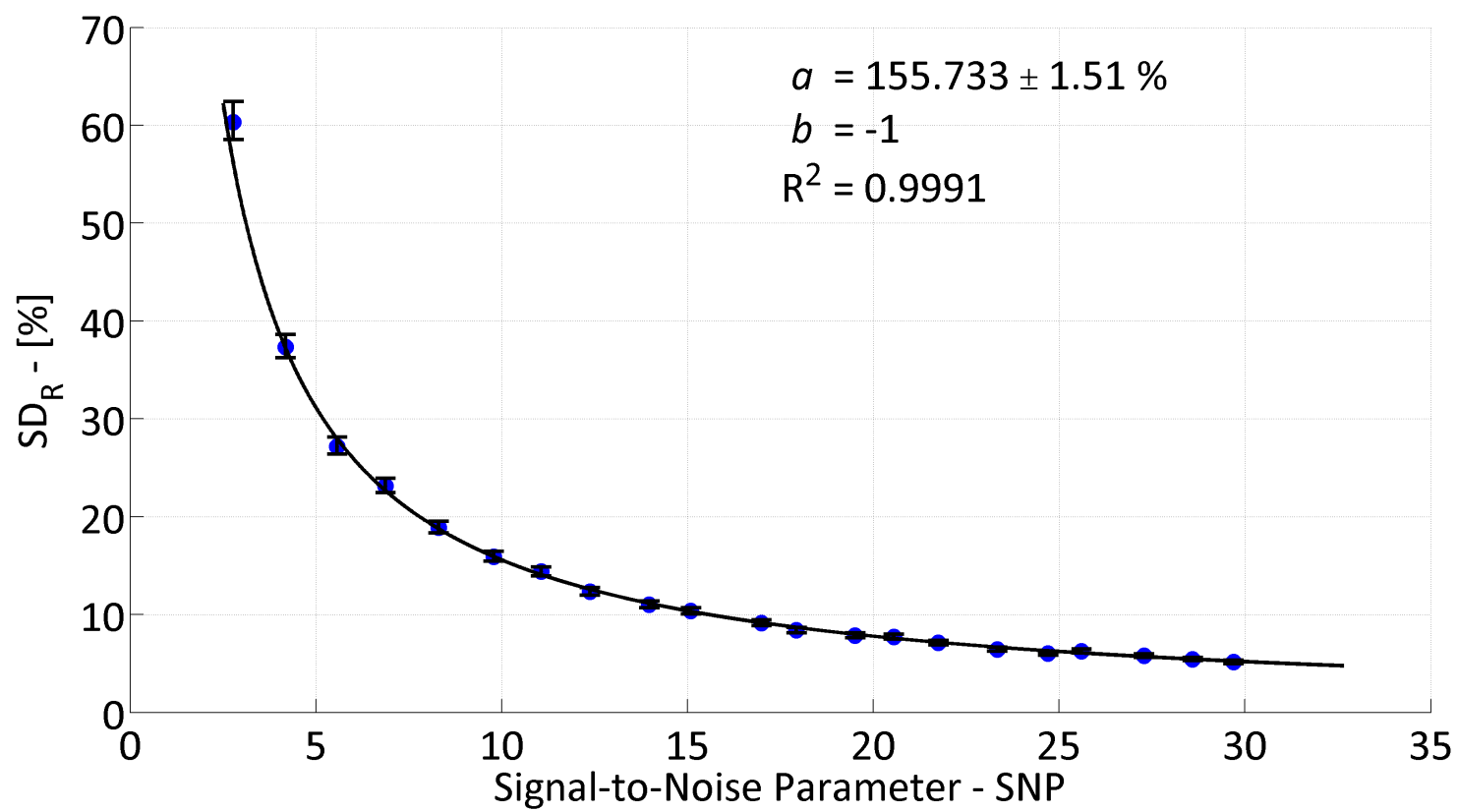

Figure S.155: Relative population standard deviation as a function of signal-to-noise parameter for 100 $\mathrm{nm}$ of $\mathrm{Al} / \mathrm{Ge}$ with $h_{\mathrm{BD}}=464.00 \mathrm{MW} / \mathrm{m}^{2} \mathrm{~K}$. Error bars represent a $95 \%$ confidence interval of the measured $\mathrm{SD}_{\mathrm{R}}$ and the solid line represents a best fit of a power law to the data, of the form $\mathrm{SD}_{\mathrm{R}}=a \times \mathrm{SNP}^{b}$, with parameter $a$ provided for a fixed $b=-1$, along with the $95 \%$ confidence level in $a$. 


\section{S.60.2 Fitting Substrate Thermal Conductivity $-k_{S}$}

Table S.95: Summary of simulation data - $100 \mathrm{~nm} \mathrm{Al/Ge} \mathrm{464.00} \mathrm{MW} \mathrm{Series.} \mathrm{Error} \mathrm{bounds} \mathrm{represent} \mathrm{a}$ $95 \%$ confidence level.

\begin{tabular}{cccccc}
\hline \multicolumn{6}{c}{$\mathbf{1 0 0} \mathbf{~ n m ~} \mathbf{A l}$ on Ge, $h_{\mathrm{BD}}=\mathbf{4 6 4 . 0 0} \mathbf{M W} / \mathbf{m}^{2} \mathbf{K}$} \\
\hline $\mathbf{S N P}$ & $\mu-[\mathbf{W} / \mathbf{m K}]$ & $\sigma-[\mathbf{W} / \mathbf{m K}]$ & $\mathbf{S N P}$ & $\mu-[\mathbf{W} / \mathbf{m K}]$ & $\sigma-[\mathbf{W} / \mathbf{m K}]$ \\
2.78 & $61.12 \pm 0.762$ & $12.27 \pm 0.0342$ & 17.93 & $59.92 \pm 0.115$ & $1.85 \pm 0.0052$ \\
4.18 & $60.11 \pm 0.513$ & $8.26 \pm 0.0230$ & 19.51 & $59.88 \pm 0.108$ & $1.74 \pm 0.0048$ \\
5.57 & $60.04 \pm 0.353$ & $5.68 \pm 0.0158$ & 20.55 & $59.91 \pm 0.101$ & $1.62 \pm 0.0045$ \\
6.87 & $59.95 \pm 0.306$ & $4.94 \pm 0.0138$ & 21.75 & $59.89 \pm 0.097$ & $1.56 \pm 0.0044$ \\
8.30 & $59.88 \pm 0.245$ & $3.94 \pm 0.0110$ & 23.33 & $59.92 \pm 0.087$ & $1.41 \pm 0.0039$ \\
9.78 & $60.06 \pm 0.207$ & $3.34 \pm 0.0093$ & 24.70 & $59.88 \pm 0.083$ & $1.35 \pm 0.0037$ \\
11.06 & $59.87 \pm 0.192$ & $3.10 \pm 0.0086$ & 25.60 & $59.93 \pm 0.084$ & $1.35 \pm 0.0038$ \\
12.37 & $59.81 \pm 0.164$ & $2.65 \pm 0.0074$ & 27.28 & $59.91 \pm 0.076$ & $1.23 \pm 0.0034$ \\
13.97 & $60.02 \pm 0.152$ & $2.46 \pm 0.0068$ & 28.59 & $59.94 \pm 0.073$ & $1.18 \pm 0.0033$ \\
15.09 & $59.95 \pm 0.136$ & $2.19 \pm 0.0061$ & 29.69 & $59.92 \pm 0.068$ & $1.09 \pm 0.0031$ \\
16.99 & $59.91 \pm 0.120$ & $1.93 \pm 0.0054$ & & & \\
\hline
\end{tabular}

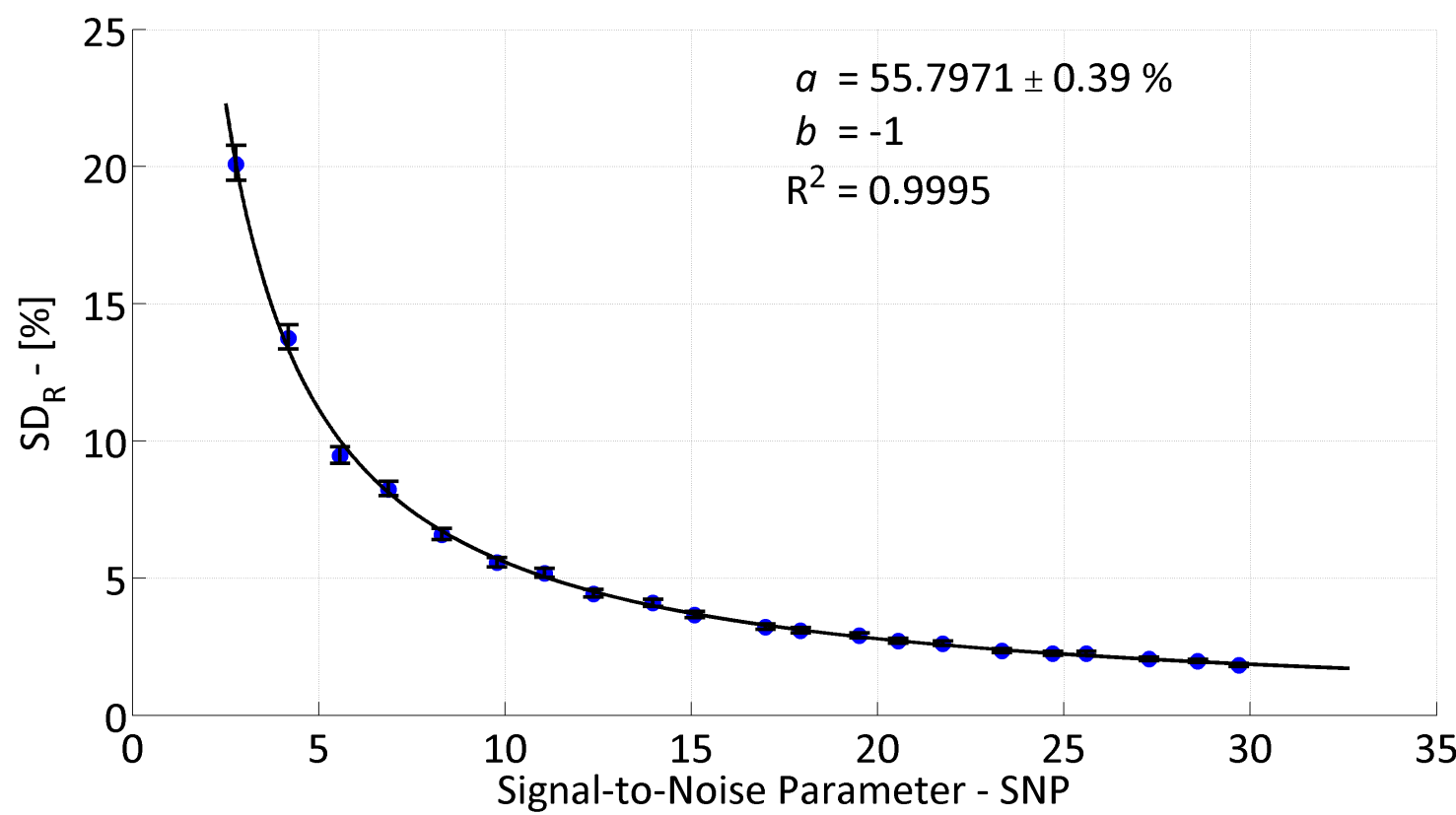

Figure S.156: Relative population standard deviation as a function of signal-to-noise parameter for 100 $\mathrm{nm}$ of $\mathrm{Al} / \mathrm{Ge}$ with $h_{\mathrm{BD}}=464.00 \mathrm{MW} / \mathrm{m}^{2} \mathrm{~K}$. Error bars represent a $95 \%$ confidence interval of the measured $\mathrm{SD}_{\mathrm{R}}$ and the solid line represents a best fit of a power law to the data, of the form $\mathrm{SD}_{\mathrm{R}}=a \times \mathrm{SNP}^{b}$, with parameter $a$ provided for a fixed $b=-1$, along with the $95 \%$ confidence level in $a$. 


\section{S.61 Simulation Summary - Al/Ge 599.00 MW Series}

\section{S.61.1 Fitting Film-to-Substrate Boundary Conductance $-h_{\mathrm{BD}}$}

Table S.96: Summary of simulation data - $100 \mathrm{~nm} \mathrm{Al/Ge} \mathrm{599.00} \mathrm{MW} \mathrm{Series.} \mathrm{Error} \mathrm{bounds} \mathrm{represent} \mathrm{a}$ $95 \%$ confidence level.

\begin{tabular}{cccccc}
\hline \multicolumn{6}{c}{$\mathbf{1 0 0} \mathbf{~ n m ~} \mathbf{A l}$ on $\mathbf{G e}, h_{\mathrm{BD}}=\mathbf{5 9 9 . 0 0} \mathbf{M W} / \mathbf{m}^{2} \mathbf{K}$} \\
\hline $\mathbf{S N P}$ & $\mu-\left[\mathbf{M W} / \mathbf{m}^{2} \mathbf{K}\right]$ & $\sigma-\left[\mathbf{M W} / \mathbf{m}^{2} \mathbf{K}\right]$ & $\mathbf{S N P}$ & $\mu-\left[\mathbf{M W} / \mathbf{m}^{2} \mathbf{K}\right]$ & $\sigma-\left[\mathbf{M W} / \mathbf{m}^{2} \mathbf{K}\right]$ \\
2.73 & $816.81 \pm 46.759$ & $750.11 \pm 2.1081$ & 17.49 & $600.22 \pm 3.373$ & $54.36 \pm 0.1514$ \\
4.05 & $652.28 \pm 18.325$ & $294.56 \pm 0.8245$ & 18.91 & $599.03 \pm 3.063$ & $49.34 \pm 0.1375$ \\
5.46 & $621.83 \pm 11.579$ & $186.13 \pm 0.5210$ & 20.25 & $602.97 \pm 3.134$ & $50.51 \pm 0.1407$ \\
6.80 & $617.73 \pm 9.281$ & $149.26 \pm 0.4173$ & 21.66 & $600.05 \pm 2.696$ & $43.42 \pm 0.1210$ \\
8.16 & $610.58 \pm 7.608$ & $122.30 \pm 0.3423$ & 22.94 & $598.77 \pm 2.629$ & $42.37 \pm 0.1180$ \\
9.55 & $606.24 \pm 6.342$ & $102.05 \pm 0.2851$ & 24.58 & $598.23 \pm 2.437$ & $39.27 \pm 0.1094$ \\
10.95 & $610.83 \pm 5.514$ & $88.72 \pm 0.2478$ & 25.63 & $600.23 \pm 2.386$ & $38.45 \pm 0.1071$ \\
12.25 & $604.28 \pm 4.876$ & $78.54 \pm 0.2190$ & 27.02 & $601.24 \pm 2.288$ & $36.87 \pm 0.1027$ \\
13.46 & $602.01 \pm 4.496$ & $72.45 \pm 0.2018$ & 28.76 & $598.37 \pm 2.141$ & $34.50 \pm 0.0961$ \\
14.91 & $600.80 \pm 4.143$ & $66.77 \pm 0.1859$ & 29.21 & $600.46 \pm 2.032$ & $32.74 \pm 0.0912$ \\
16.45 & $603.75 \pm 3.752$ & $60.46 \pm 0.1684$ & & & \\
\hline
\end{tabular}

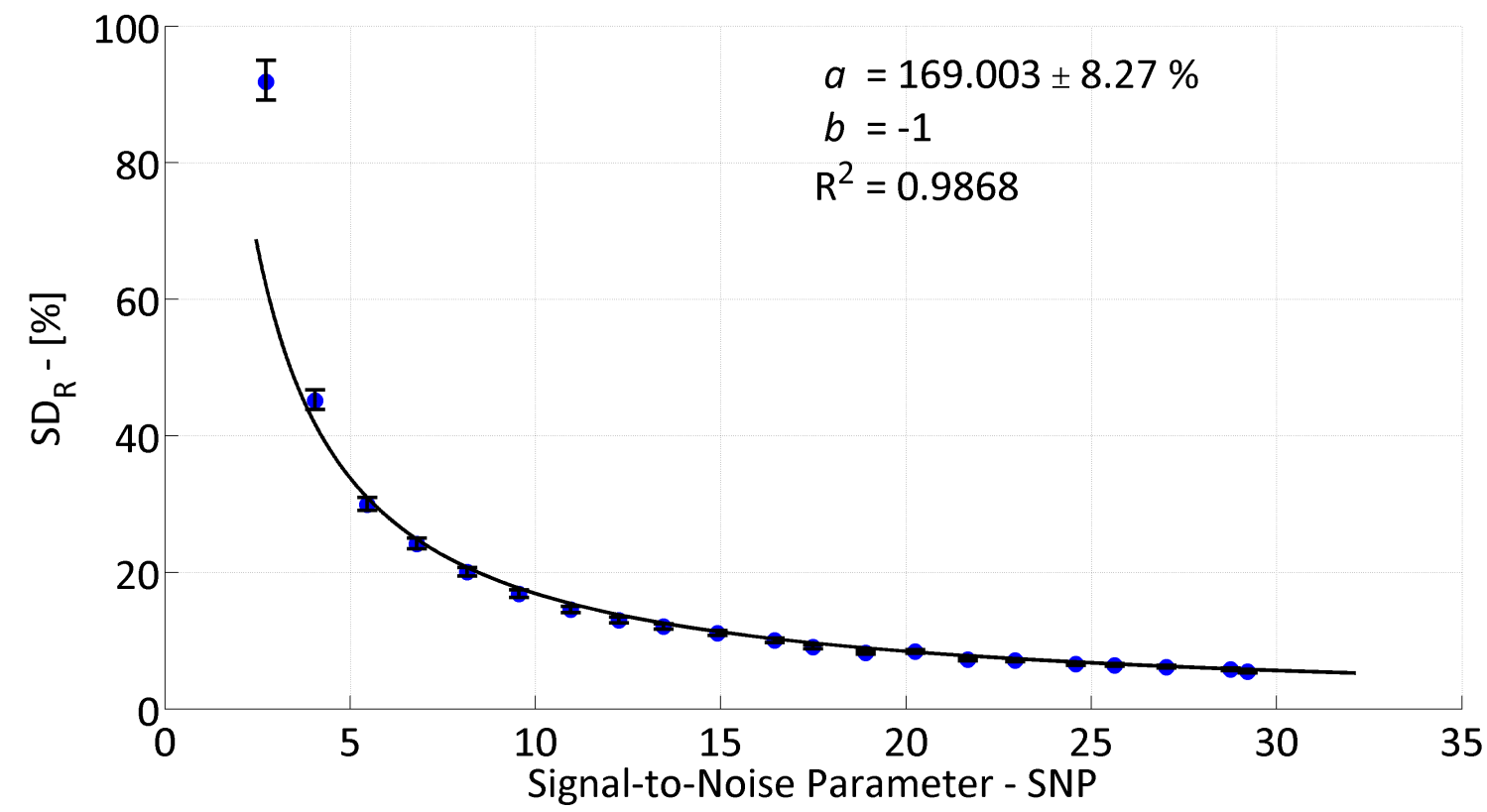

Figure S.157: Relative population standard deviation as a function of signal-to-noise parameter for 100 $\mathrm{nm}$ of $\mathrm{Al} / \mathrm{Ge}$ with $h_{\mathrm{BD}}=599.00 \mathrm{MW} / \mathrm{m}^{2} \mathrm{~K}$. Error bars represent a $95 \%$ confidence interval of the measured $\mathrm{SD}_{\mathrm{R}}$ and the solid line represents a best fit of a power law to the data, of the form $\mathrm{SD}_{\mathrm{R}}=a \times \mathrm{SNP}^{b}$, with parameter $a$ provided for a fixed $b=-1$, along with the $95 \%$ confidence level in $a$. 


\section{S.62 Simulation Summary - Al/Ge 774.00 MW Series}

\section{S.62.1 Fitting Film-to-Substrate Boundary Conductance $-h_{\mathrm{BD}}$}

Table S.97: Summary of simulation data - $100 \mathrm{~nm} \mathrm{Al/Ge} \mathrm{774.00} \mathrm{MW} \mathrm{Series.} \mathrm{Error} \mathrm{bounds} \mathrm{represent} \mathrm{a}$ $95 \%$ confidence level.

\begin{tabular}{cccccc}
\hline \multicolumn{6}{c}{$\mathbf{1 0 0} \mathbf{~ n m ~} \mathbf{A l}$ on $\mathbf{G e}, h_{\mathrm{BD}}=\mathbf{7 7 4 . 0 0} \mathbf{M W} / \mathbf{m}^{2} \mathbf{K}$} \\
\hline $\mathbf{S N P}$ & $\mu-\left[\mathbf{M W} / \mathbf{m}^{2} \mathbf{K}\right]$ & $\sigma-\left[\mathbf{M W} / \mathbf{m}^{2} \mathbf{K}\right]$ & $\mathbf{S N P}$ & $\mu-\left[\mathbf{M W} / \mathbf{m}^{2} \mathbf{K}\right]$ & $\sigma-\left[\mathbf{M W} / \mathbf{m}^{2} \mathbf{K}\right]$ \\
2.68 & $874.29 \pm 36.889$ & $575.69 \pm 1.7098$ & 17.34 & $777.28 \pm 5.001$ & $79.61 \pm 0.2272$ \\
4.02 & $865.98 \pm 26.465$ & $413.89 \pm 1.2240$ & 18.71 & $778.08 \pm 4.739$ & $76.36 \pm 0.2127$ \\
5.39 & $823.35 \pm 18.821$ & $297.47 \pm 0.8613$ & 20.15 & $772.01 \pm 4.312$ & $68.65 \pm 0.1959$ \\
6.68 & $808.62 \pm 13.369$ & $215.01 \pm 0.6012$ & 21.35 & $778.46 \pm 4.128$ & $65.71 \pm 0.1875$ \\
8.10 & $801.85 \pm 11.343$ & $180.11 \pm 0.5166$ & 22.60 & $777.41 \pm 3.803$ & $60.55 \pm 0.1728$ \\
9.39 & $780.73 \pm 8.885$ & $141.01 \pm 0.4049$ & 23.95 & $777.64 \pm 3.624$ & $57.69 \pm 0.1646$ \\
10.67 & $786.46 \pm 8.330$ & $132.40 \pm 0.3790$ & 25.45 & $778.15 \pm 3.229$ & $52.01 \pm 0.1450$ \\
11.96 & $783.16 \pm 7.290$ & $117.48 \pm 0.3272$ & 26.57 & $775.43 \pm 3.187$ & $50.73 \pm 0.1448$ \\
13.37 & $787.02 \pm 6.407$ & $103.14 \pm 0.2878$ & 28.11 & $777.15 \pm 3.057$ & $49.26 \pm 0.1372$ \\
14.67 & $780.03 \pm 5.946$ & $94.51 \pm 0.2705$ & 29.45 & $774.31 \pm 2.928$ & $46.61 \pm 0.1330$ \\
15.98 & $781.86 \pm 5.352$ & $85.16 \pm 0.2433$ & & & \\
\hline
\end{tabular}

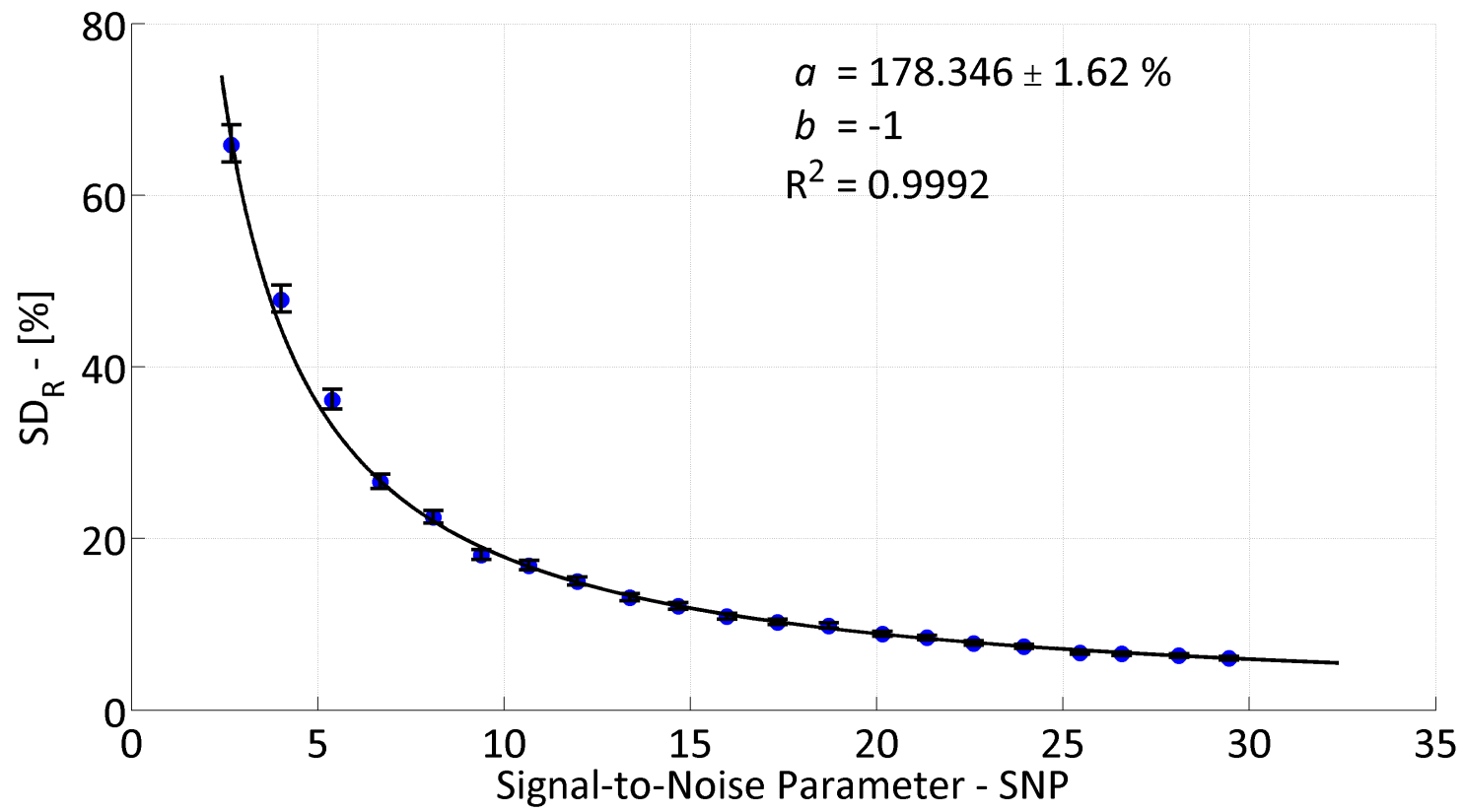

Figure S.158: Relative population standard deviation as a function of signal-to-noise parameter for 100 $\mathrm{nm}$ of $\mathrm{Al} / \mathrm{Ge}$ with $h_{\mathrm{BD}}=774.00 \mathrm{MW} / \mathrm{m}^{2} \mathrm{~K}$. Error bars represent a $95 \%$ confidence interval of the measured $\mathrm{SD}_{\mathrm{R}}$ and the solid line represents a best fit of a power law to the data, of the form $\mathrm{SD}_{\mathrm{R}}=a \times \mathrm{SNP}^{b}$, with parameter $a$ provided for a fixed $b=-1$, along with the $95 \%$ confidence level in $a$. 


\section{S.63 Simulation Summary - Al/Ge 1000.00 MW Series}

\section{S.63.1 Fitting Film-to-Substrate Boundary Conductance - $h_{\mathrm{BD}}$}

Table S.98: Summary of simulation data - $100 \mathrm{~nm} \mathrm{Al/Ge} 1000.00 \mathrm{MW}$ Series. Error bounds represent a $95 \%$ confidence level.

\begin{tabular}{cccccc}
\hline \multicolumn{6}{c}{$\mathbf{1 0 0} \mathbf{~ n m ~ A l ~ o n ~ G e , ~} h_{\mathrm{BD}}=\mathbf{1 0 0 0 . 0 0} \mathbf{M W} / \mathbf{m}^{2} \mathbf{K}$} \\
\hline $\mathbf{S N P}$ & $\mu-\left[\mathbf{M W} / \mathbf{m}^{2} \mathbf{K}\right]$ & $\sigma-\left[\mathbf{M W} / \mathbf{m}^{2} \mathbf{K}\right]$ & $\mathbf{S N P}$ & $\mu-\left[\mathbf{M W} / \mathbf{m}^{2} \mathbf{K}\right]$ & $\sigma-\left[\mathbf{M W} / \mathbf{m}^{2} \mathbf{K}\right]$ \\
2.82 & $1153.97 \pm 50.812$ & $782.73 \pm 2.3860$ & 18.09 & $1007.78 \pm 7.021$ & $110.91 \pm 0.3215$ \\
4.19 & $1165.48 \pm 40.046$ & $624.63 \pm 1.8571$ & 19.45 & $1007.22 \pm 6.602$ & $104.23 \pm 0.3024$ \\
5.60 & $1092.56 \pm 25.575$ & $401.04 \pm 1.1797$ & 20.62 & $1014.44 \pm 6.223$ & $98.41 \pm 0.2846$ \\
7.08 & $1066.94 \pm 20.163$ & $316.84 \pm 0.9281$ & 22.32 & $1008.18 \pm 5.461$ & $86.35 \pm 0.2498$ \\
8.17 & $1053.05 \pm 16.809$ & $270.05 \pm 0.7566$ & 23.50 & $1010.34 \pm 5.449$ & $86.11 \pm 0.2493$ \\
9.75 & $1021.06 \pm 12.821$ & $205.99 \pm 0.5772$ & 23.91 & $1006.75 \pm 5.092$ & $80.48 \pm 0.2330$ \\
10.95 & $1017.51 \pm 11.565$ & $182.31 \pm 0.5307$ & 26.01 & $1006.87 \pm 4.962$ & $78.46 \pm 0.2270$ \\
12.38 & $1014.42 \pm 10.415$ & $164.61 \pm 0.4766$ & 27.28 & $1004.98 \pm 4.766$ & $75.36 \pm 0.2180$ \\
13.96 & $1013.47 \pm 9.362$ & $147.88 \pm 0.4286$ & 28.97 & $1001.68 \pm 4.311$ & $68.21 \pm 0.1971$ \\
15.41 & $1007.73 \pm 8.527$ & $134.62 \pm 0.3906$ & 29.89 & $1000.62 \pm 4.194$ & $66.25 \pm 0.1920$ \\
16.65 & $1007.76 \pm 7.583$ & $119.79 \pm 0.3472$ & & & \\
\hline
\end{tabular}

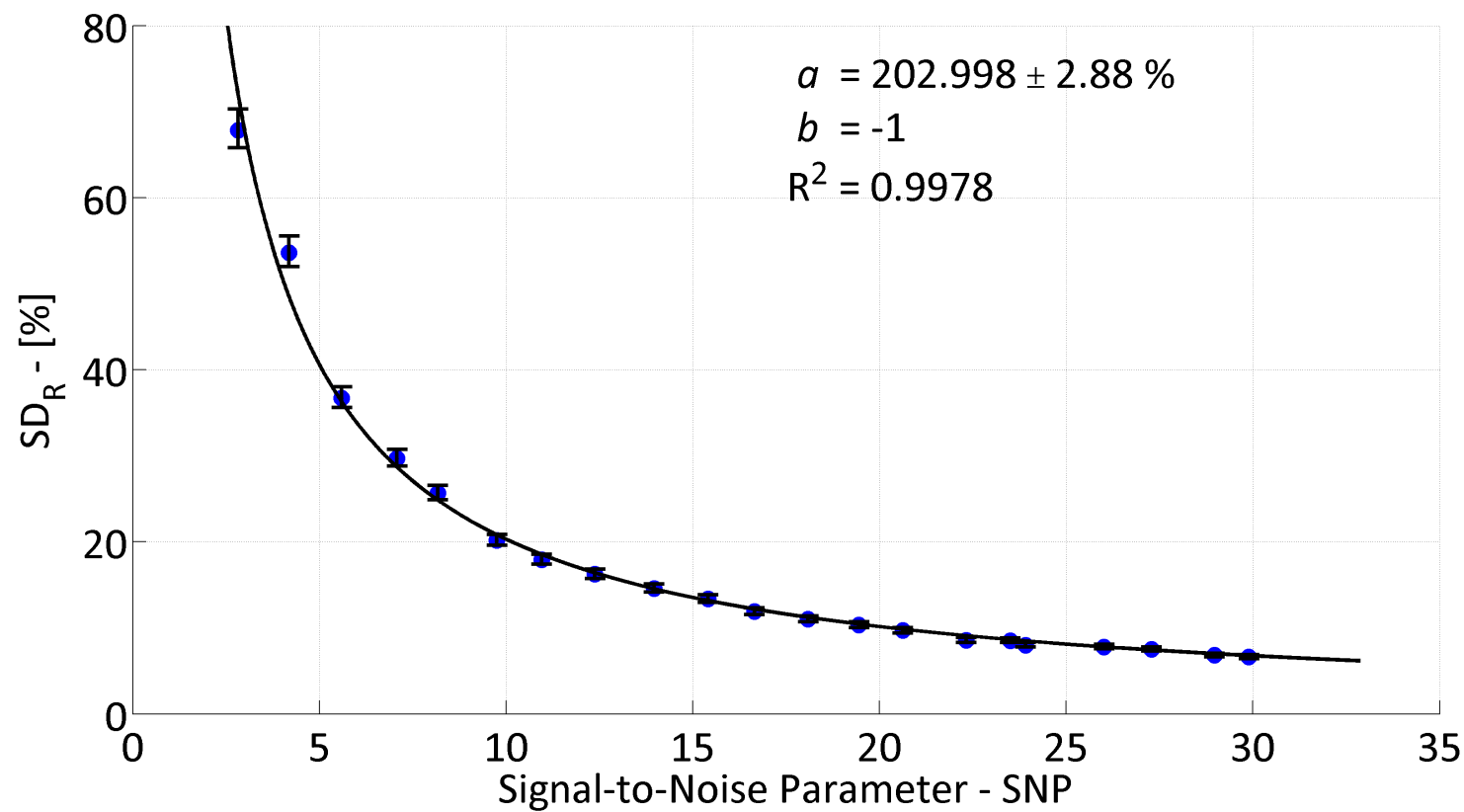

Figure S.159: Relative population standard deviation as a function of signal-to-noise parameter for 100 $\mathrm{nm}$ of $\mathrm{Al} / \mathrm{Ge}$ with $h_{\mathrm{BD}}=1000.00 \mathrm{MW} / \mathrm{m}^{2} \mathrm{~K}$. Error bars represent a $95 \%$ confidence interval of the measured $\mathrm{SD}_{\mathrm{R}}$ and the solid line represents a best fit of a power law to the data, of the form $\mathrm{SD}_{\mathrm{R}}=a \times \mathrm{SNP}^{b}$, with parameter $a$ provided for a fixed $b=-1$, along with the $95 \%$ confidence level in $a$. 